\title{
A Three-Component, Interrupted Radical Heck/Allylic Substitution Cascade Involving Unactivated Alkyl Bromides
}

Huan-Ming Huang, a Peter Bellotti,, Philipp M. Pflüger, a J. Luca Schwarz, ${ }^{\text {a Bastian }}$ Heidrich, ${ }^{\mathrm{b}}$ and Frank Glorius ${ }^{\mathrm{a} *}$

${ }^{a}$ Organisch-Chemisches Institut, Westfälische Wilhelms-Universität Münster, Corrensstraße 40, 48149 Münster, Germany; ${ }^{b}$ MEET Battery Research Center, Institute of Physical Chemistry, Westfälische Wilhelms-Universität Münster, Corrensstraße 46, 48149, Münster, Germany.

*Correspondence author. Email: glorius@uni-muenster.de

\section{SUPPORTING INFORMATION}




\section{Contents}

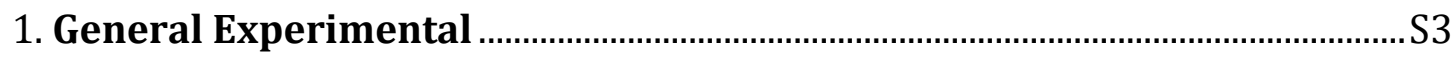

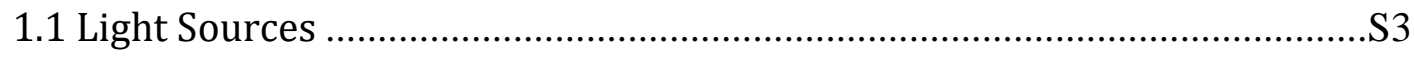

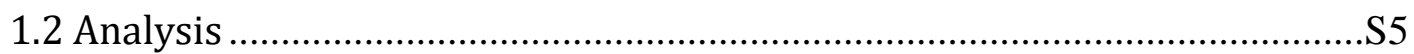

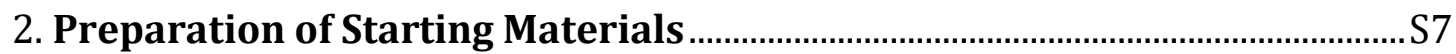

2.1 General Procedure A: Preparation of Tertiary Alcohols with Ether Linkage ..S7

2.2 General Procedure B: Preparation of Teriary Alkyl Bromides....................S7

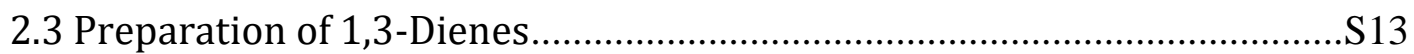

2.4 General Procedure C: Synthesis of Aldehydes via Heck Reaction ..............S14

2.5 General Procedure D: Synthesis of 1,3-Dienes via Wittig Reaction ............S15

2.6 General Procedure E: Synthesis of Phenols ..............................................S26

2.7 General Procedure F: Preparation of Sodium Sulfinate Salts ....................S30

3. Radical Interrupted Heck/Allylic Substitution Cascade ............................... S36

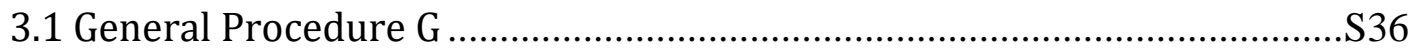

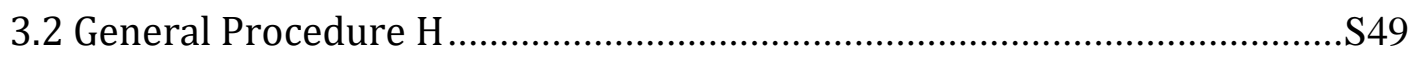

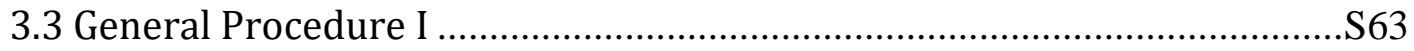

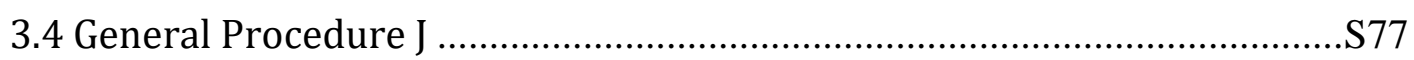

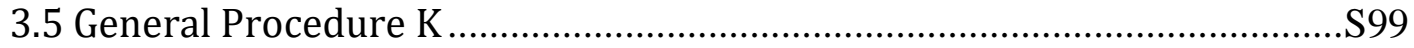

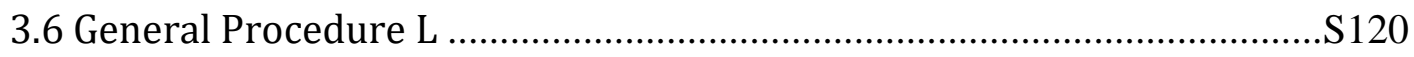

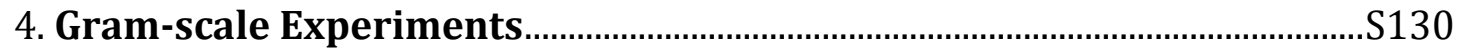

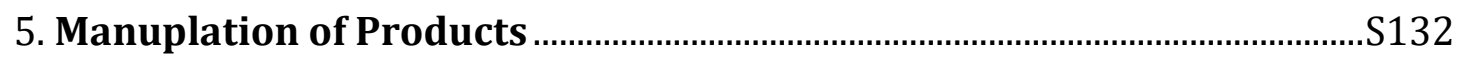

6. Mechanistic Studies ..................................................................................... 135

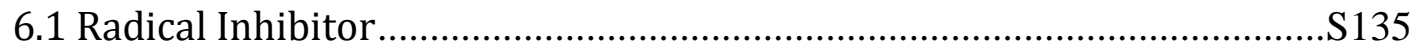

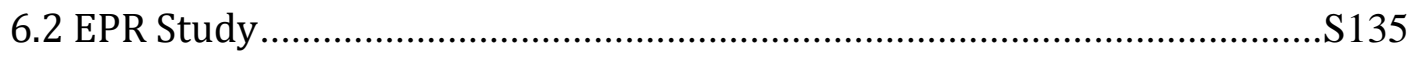

6.3 X-Ray Photoelectron Spectroscopy (XPS) …........................................S136

6.4 UV-visible Absorption Analysis...........................................................S140

6.5 Stern-Volmer Luminescence Quenching Analysis.................................S140

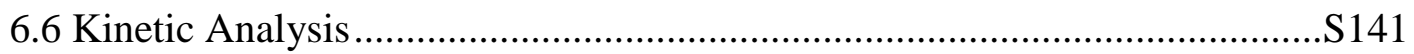

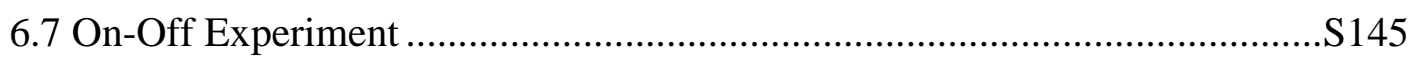




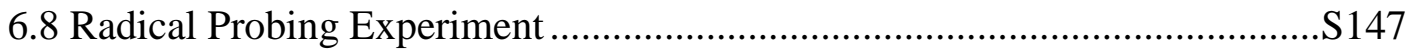

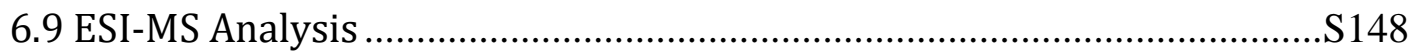

6.9 Reaction of Pd(II)-allyl complexes with nucleophiles ............................S149

6.10 Alternative Proposed Mechanism .......................................................S151

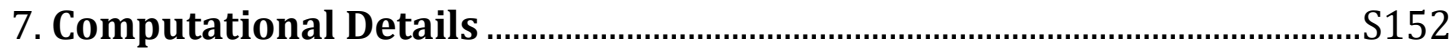

8. Condition-based Sensitivity Screen ……..........................................................S178

9. ${ }^{1} \mathrm{H},{ }^{13} \mathrm{C}$ and ${ }^{19} \mathrm{~F}$ NMR Spectra of Compounds ................................................. 185

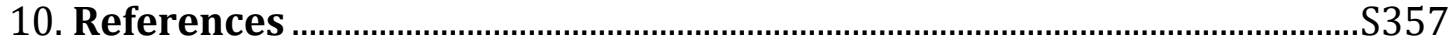




\section{General Experimental}

All experiments were performed under argon unless otherwise stated. The reported reaction temperature refers to the one of the heating media (for instance air or oil bath), unless otherwise stated. All reagents were purchased from Alfa Aesar, Sigma-Aldrich, Merck, TCI, Fluorochem, Combi-Blocks, VWR and used without further purification, unless otherwise stated. Dry solvents were purchased from ACROS organics (Extra Dry, $\mathrm{H}_{2} \mathrm{O}<50 \mathrm{ppm}$ ) in AcroSeal ${ }^{\circledR}$ bottles, stored under molecular sieves and used as received or obtained from commercial sources and freshly distilled over standard drying agents (THF and $\mathrm{Et}_{2} \mathrm{O}$ over $\mathrm{Na}$ /benzophenone ketyl; $\mathrm{CH}_{2} \mathrm{Cl}_{2}$, toluene and hexane over $\mathrm{CaH}_{2}$ ) and stored under argon. Flash chromatography was carried out using Merck silica gel (40-63 mesh) under a light positive pressure (0.3-0.5 bar), eluting with the specified solvent system. Ethyl acetate, $n$-pentane and $\mathrm{CH}_{2} \mathrm{Cl}_{2}$ used for column chromatography were purchased of technical grade and purified by atmospheric pressure distillation, $\mathrm{Et}_{3} \mathrm{~N}$ (reagent grade) for column chromatography was purchased from ACROS Organics and used as received. Thin layer chromatography was carried out on Merck silica gel $60 \mathrm{~F}_{254}$ pre-coated aluminum sheets and were visualized using UV light (254 nm) and stained with basic aqueous potassium permanganate $\left(2 \mathrm{~g} \mathrm{KMnO}_{4}, 10 \mathrm{~g} \mathrm{~K}_{2} \mathrm{CO}_{3}, 0.3 \mathrm{~g}\right.$ of $\mathrm{NaOH}$ in 200 $\mathrm{ml}$ of deionized water) or alcoholic solution of phosphomolybdic acid (10 g of PMA in $100 \mathrm{ml}$ of absolute EtOH).

\subsection{Light Sources}

Blue LEDs ( $5 \mathrm{~W}, \lambda_{\max }=455 \mathrm{~nm} ; 3 \mathrm{~W}, \lambda_{\max }=420 \mathrm{~nm}$ or $3 \mathrm{~W}, \lambda_{\max }=400 \mathrm{~nm}$, purchased from AVONEC, Germany, https://avonec.de/5w-high-power-led/5whigh-power-led-auf-starplatine-10000k-20000k-kaltweiss-65-66.html) or $32 \mathrm{~W}$ Kessil H150 lights (purchased from https://www.kessil.com/horticulture/H150.php) were used for the light irradiation (see Emission spectra of the used LEDs.). 

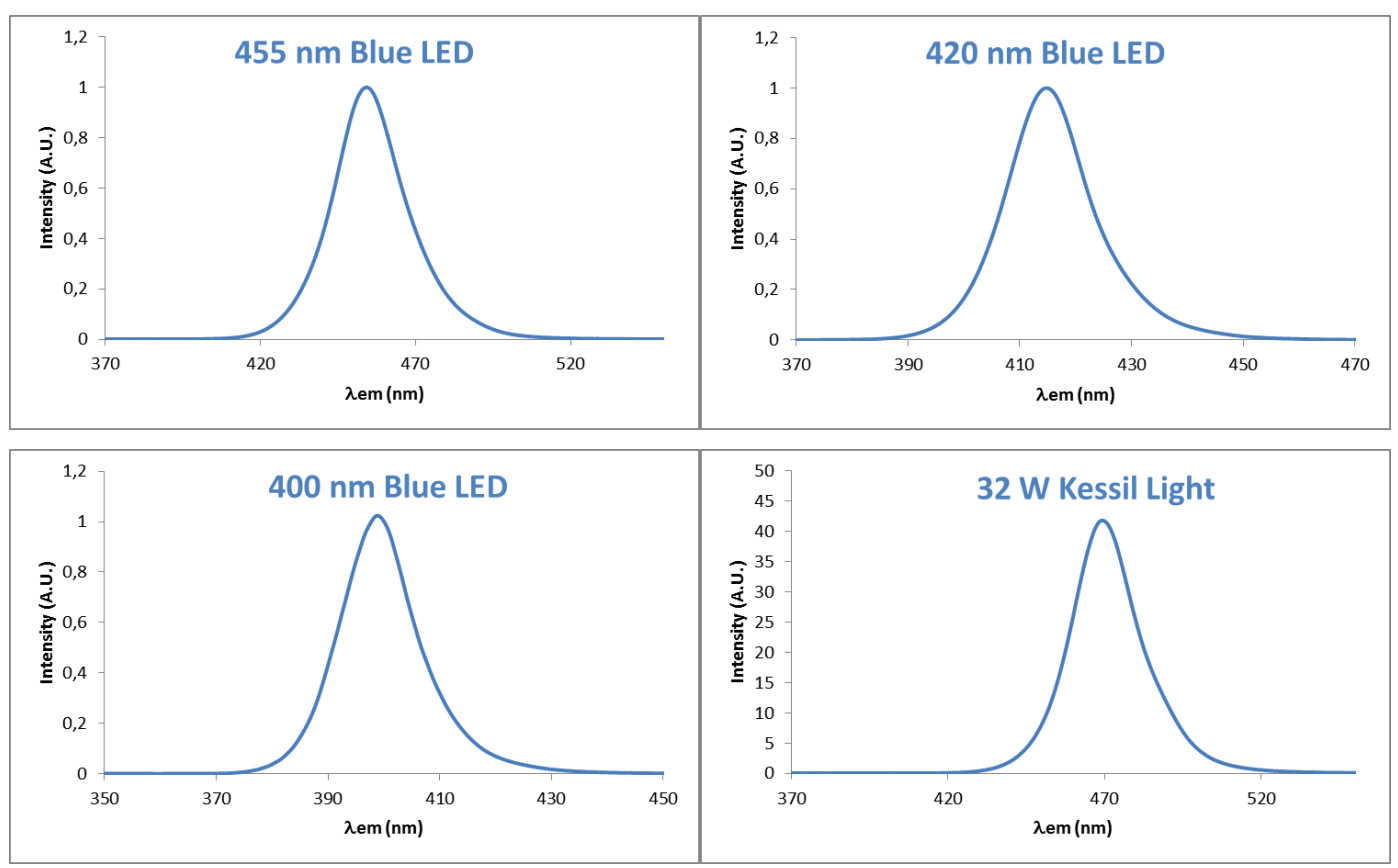

Fig. S1 Emission spectra of the used LEDs.

The light source was placed $\sim 5 \mathrm{~cm}$ from the reaction vessel. A custom made "light box" was used with 6 LEDs arranged around the reaction vessels (see Fig. S2). A fan attached to the apparatus was used to maintain the temperature inside the "box" at no more than $9^{\circ} \mathrm{C}$ above room temperature. The temperature in the box is $\sim 30{ }^{\circ} \mathrm{C}$. The temperature inside the reaction mixture is $35 \sim 40{ }^{\circ} \mathrm{C}$. For irradiation with Kessil lights, the reaction vessel was placed between two light sources ( $\sim 3 \mathrm{~cm}$ distance between the light source and the vessel).
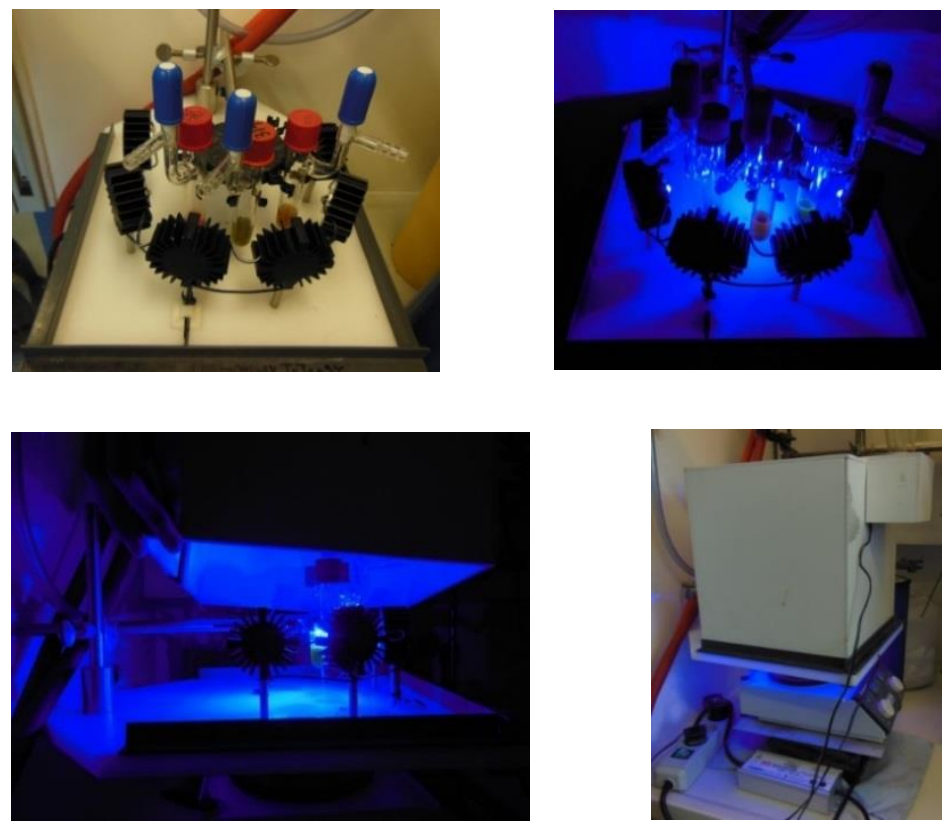
Fig. S2 Photographs of the custom-made light box used for irradiation of the reaction solutions.

\subsection{Analysis}

NMR-spectra were recorded on a Bruker ARX-300, AV-300, AV-400 MHz or on a Varian Associated, Varian 500 or 600 unity plus spectrometer. All spectral data were acquired at $295 \mathrm{~K}$. Chemicals shifts $(\delta)$ are quoted in parts per million (ppm) against tetramethylsilane (TMS, $\delta=0.00 \mathrm{ppm}$ ). The following residual solvent signals were used as references for ${ }^{1} \mathrm{H}$ and ${ }^{13} \mathrm{C}$ NMR spectra: $\mathrm{CDCl}_{3}, \delta_{\mathrm{H}} 7.26 \mathrm{ppm}$, $\delta_{\mathrm{C}} 77.16 \mathrm{ppm} ; \mathrm{CD}_{2} \mathrm{Cl}_{2}, \delta_{\mathrm{H}} 5.32 \mathrm{ppm}, \delta_{\mathrm{C}} 54.00 \mathrm{ppm} ; \mathrm{DMSO}-d_{6}, \delta_{\mathrm{H}} 2.50 \mathrm{ppm}, \delta_{\mathrm{C}}$ $39.52 \mathrm{ppm} ; \mathrm{C}_{6} \mathrm{D}_{6}, \delta_{\mathrm{H}} 7.16 \mathrm{ppm}, \delta_{\mathrm{C}} 128.06 \mathrm{ppm} ; \mathrm{D}_{2} \mathrm{O}, \delta_{\mathrm{H}} 4.79 \mathrm{ppm}, \delta_{\mathrm{C}}$ calibrated using absolute referencing to the ${ }^{1} \mathrm{H}$ spectrum). ${ }^{19} \mathrm{~F}$ NMR spectra were calibrated using absolute referencing to the ${ }^{1} \mathrm{H}$ NMR spectrum, as suggested by IUPAC. Coupling constants $(J)$ are reported in Hertz $(\mathrm{Hz})$ to the nearest $0.1 \mathrm{~Hz}$. The multiplicity abbreviations used (or combinations thereof) are $\mathrm{s}=$ singlet, $\mathrm{d}=$ doublet, $\mathrm{t}=$ triplet, $\mathrm{q}=$ quartet, hept $=$ heptet, $\mathrm{m}=$ multiplet.

Note: When site-selectivity was observed to be $>20: 1$ and $E / Z>95: 5$, only the major isomer was assigned. In the other cases, diagnostic/non-overlapping signals have been reported (extensive overlap, in particular in the ${ }^{1} \mathrm{H}$ spectra, prevents complete assignment of the minor isomer).

High-resolution mass spectra (HRMS) were obtained by the MS service of the Organisch-Chemisches Institut, Westfälische Wilhelms Universität Münster, using electrospray ionisation (ESI) on a Bruker Daltonics, MicroToF spectrometer.

GC-FID analysis was performed on an Agilent Technologies 6890A equipment featured with a HP-5 quartz column (0.32 mm x $30 \mathrm{~m}$, film: $0.25 \mu \mathrm{m})$ using flame ionization detection.

GC-MS spectra were recorded on an Agilent Technologies 7890A GC-system with an Agilent 5975C VL MSD or an Agilent 5975 inert Mass Selective Detector (EI) 
and a HP-5MS column ( $0.25 \mathrm{~mm} \times 30 \mathrm{~m}$, film: $0.25 \mu \mathrm{m})$. The major signals are quoted in $m / z$ with the relative intensity in parentheses. The method indicated as '50_40_4.8 long' starts with the injection temperature $\mathrm{T}_{0}\left(50^{\circ} \mathrm{C}\right)$; after holding this temperature for $3 \mathrm{~min}$, the column is heated by $40{ }^{\circ} \mathrm{C} / \mathrm{min}$ to temperature $\mathrm{T}_{1}$ $\left(290{ }^{\circ} \mathrm{C}\right.$ or $\left.320^{\circ} \mathrm{C}\right)$ and this temperature is held for an additional time.

UV/vis absorption spectra were recorded on a Jasco V-650 spectrophotometer, equipped with a temperature control unit at $25{ }^{\circ} \mathrm{C}$. The samples were measured in Hellma fluorescence QS quartz cuvettes (chamber volume $=1.4 \mathrm{~mL}, \mathrm{H} \times \mathrm{W} \times \mathrm{D}$ $=46 \mathrm{~mm} \times 12.5 \mathrm{~mm}, 12.5 \mathrm{~mm}$ ) fitted with a PTFE stopper.

Stern-Volmer luminescence quenching analyses were conducted using a Jasco FP-8300 spectrofluorometer. The following parameters were employed: excitation bandwidth $=5 \mathrm{~nm}$, data interval $=0.2 \mathrm{~nm}$, scan speed $=500 \mathrm{~nm} / \mathrm{min}$, response time $=0.2 \mathrm{sec}$. The samples were measured in Hellma fluorescence QS quartz cuvettes (chamber volume $=1.4 \mathrm{~mL}, \mathrm{H} \times \mathrm{W} \times \mathrm{D}=46 \mathrm{~mm} \times 12.5 \mathrm{~mm}, 12.5$ $\mathrm{mm}$ ) fitted with a PTFE stopper. 


\section{Preparation of Starting Materials}

\subsection{General Procedure A: Preparation of Tertiary Alcohols with Ether Linkage}

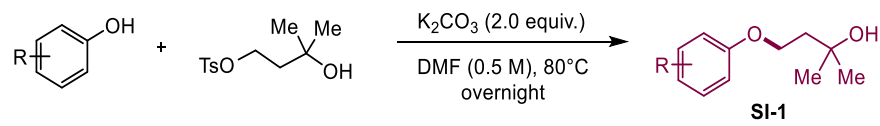

The tertiary alcohols with aryl ether linkage were prepared according to the following procedure. In an oven-dried Schlenk tube equipped with a PTFEcoated stirring bar, 3-hydroxy-3-methylbutyl 4-methylbenzenesulfonate (1.2 equiv.), the corresponding phenol (1.0 equiv.), $\mathrm{K}_{2} \mathrm{CO}_{3}$ (2.0 equiv.) and dry DMF $(0.5 \mathrm{M})$ were charged under argon, then the reaction was stirred at $80^{\circ} \mathrm{C}$ overnight. The reaction was cooled to room temperature, then most of the solvent was removed in vacuo $\left(<1\right.$ mbar @ $\left.60^{\circ} \mathrm{C}\right)$, then the residue was taken-up with water and extracted twice with $\mathrm{CH}_{2} \mathrm{Cl}_{2}$ or EtOAc (depending upon the polarity of the product), then the combined organic extracts were washed twice with water, dried over $\mathrm{MgSO}_{4}$ and the solvent was removed in vacuo. The product SI-1 was either purified by flash column chromatography on silica or used for the next step without further purification.

\subsection{General Procedure B: Preparation of Tertiary Alkyl Bromides}

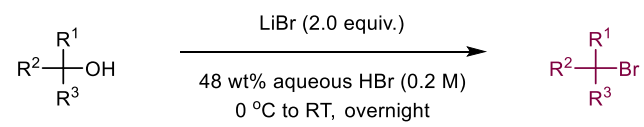

The tertiary alkyl bromides were prepared according to a literature procedure from the corresponding tertiary alcohol precursors which reported by the groups of $\mathrm{Fu}$ and Gong. ${ }^{1-4}$ The corresponding tertiary alcohol precursor (10 mmol, 1.0 equiv., neat or dissolved in a minimal amount of $\mathrm{CH}_{2} \mathrm{Cl}_{2}$ ) was added $\operatorname{LiBr}\left(1.80 \mathrm{~g}, 20 \mathrm{mmol}, 2.0\right.$ equiv.) in $48 \mathrm{wt} \%$ aqueous $\mathrm{HBr}(0.2 \mathrm{M}, 20 \mathrm{~mL})$ at $0{ }^{\circ} \mathrm{C}$. The reaction mixture was allowed to warm to room temperature and stirred for overnight. The reaction mixture was diluted with ethyl acetate, washed with water and saturated $\mathrm{NaHCO}_{3}$. The organic layer was collected, washed with brine, 
dried over $\mathrm{MgSO}_{4}$, and concentrated. The residue was purified by column chromatography to afford the desired tertiary bromide $\mathbf{1}$.

Note: To prevent decomposition of the tertiary bromides, neutralization of the column with $E t_{3} N$ and elution with $0.5-5 \%$ of $E t_{3} N$ proved to be a suitable method.

Compound 1a, 1ag, 1ai-1am are commercially available, and used as received.

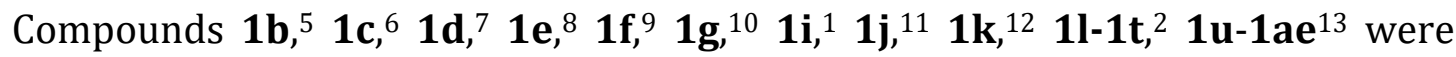
prepared according to the above procedure or the ones specified below.<smiles>CC(C)(C)Br</smiles>

1a

$1 \mathrm{~b}$<smiles>CCC(C)(Br)CCc1ccccc1</smiles><smiles>CC(C)(C)CCc1ccc2c(c1)OCO2</smiles><smiles>COc1ccc(CC(C)(C)Br)cc1OC</smiles>

$1 \mathrm{e}$
$\underbrace{\mathrm{Me}} X_{\mathrm{Br}}^{\mathrm{Me}}$ 1f<smiles>Cc1ccc(S(=O)(=O)OCCC(C)(C)Br)cc1</smiles>

$1 \mathrm{~g}$<smiles>CC(C)(Br)CCOC(=O)c1ccccc1N</smiles><smiles>CC(C)(C)CCOC(=O)c1ccc(C(F)(F)F)cc1</smiles>

$1 \mathrm{r}$<smiles>BrC1(CCc2ccccc2)CCC1</smiles>

$1 \mathrm{~h}$

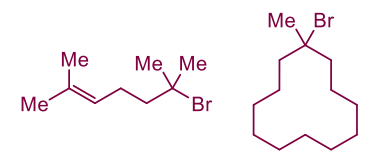

1j

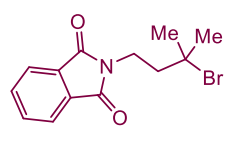

$1 \mathrm{k}$

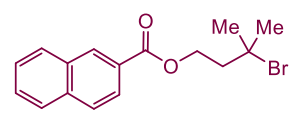

11<smiles>Cc1cc([N+](=O)[O-])c(C(=O)OCCC(C)(C)Br)c([N+](=O)[O-])c1</smiles><smiles>CC(C)(C)CCOC(=O)c1cccs1</smiles>

10<smiles>CCC(C)(C)CCOC(=O)c1ccccc1</smiles>

$1 \mathrm{p}$<smiles>CC(C)(Br)CCOC(=O)c1ccc(Cl)cc1</smiles>

$1 q$<smiles>COC(=O)c1ccc(OCCC(C)(C)Br)c(OCCC(C)(C)Br)c1</smiles><smiles>CC(C)(Br)CCOc1ccc2ccc(=O)oc2c1</smiles><smiles>CC(C)(Br)CCOc1cc([N+](=O)[O-])c(Cl)c([N+](=O)[O-])c1</smiles><smiles>CC(C)(C)CCOc1ccccc1-c1ccccc1</smiles><smiles>CC(=O)c1cc(-c2ccc(F)cc2F)ccc1OCCC(C)(C)Br</smiles>

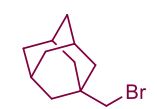

1 af

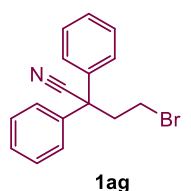

1 ag

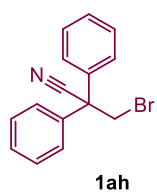

1ah

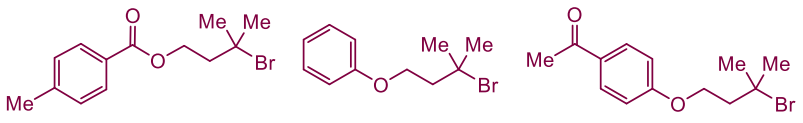

$1 \mathrm{u}$

1aa

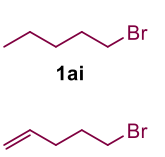

1aj

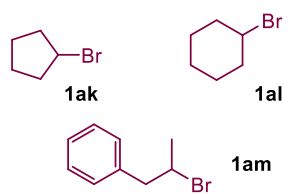

\section{西}

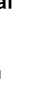


(2-(1-Bromocyclobutyl)ethyl)benzene (1h). The compound was synthesized according to the following two step procedure.

1h Step 1. In an oven-dried Schlenk tube equipped with a PTFE-coated stirring bar, magnesium turnings (365 mg, $15.0 \mathrm{mmol}, 1.5$ equiv.) were charged, then the vessel was flame-dried under vacuum. Dry THF (12 mL) was added, followed by a small crystal of iodine, and the suspension stirred until disappearance of the red-brown color. Phenylethylbromide $(1.77 \mathrm{ml}, 13.0 \mathrm{mmol}$, 1.3 equiv.) was added dropwise (the exothermic reaction, which is a sign of Grignard formation, could be controlled using a water bath), then upon completion of the addition the reaction was stirred at room temperature for 45 minutes (grey solution). The solution of phenylethylmagnesium bromide was transferred to a clean flame-dried Schlenk tube (to remove the unreacted magnesium), cooled to $0^{\circ} \mathrm{C}$ and cyclobutanone (734 $\mu \mathrm{L}, 10.0 \mathrm{mmol}, 1.0$ equiv.) in dry THF (12 mL) was added dropwise, then the reaction was warmed at room temperature and stirred for 2 hours, then quenched by the addition of a saturated $\mathrm{NH}_{4} \mathrm{Cl}$ solution $(15 \mathrm{~mL})$. The reaction was diluted with $\mathrm{Et}_{2} \mathrm{O}(50 \mathrm{~mL})$, then the layers separated. The organic layer was extracted twice with $\mathrm{Et}_{2} \mathrm{O}$ (50 $\mathrm{mL}$ each time), then the combined organic layers were dried over $\mathrm{MgSO}_{4}$ and the solvent was removed under reduced pressure. The crude was purified by flash column chromatography on silica (pentane/EtOAc $=6 / 1 \rightarrow 4 / 1$ ) to afford S1h (1.37 g, $7.79 \mathrm{mmol}, 78 \%$ ) as a colorless liquid. ${ }^{\mathbf{1}} \mathbf{H}$ NMR (300 $\mathbf{M H z}, \mathbf{C D C l}_{3}$ ): $\delta \mathrm{ppm}$ $7.41-7.09$ (m, 5H), $2.80-2.64(\mathrm{~m}, 2 \mathrm{H}), 2.19-1.99(\mathrm{~m}, 4 \mathrm{H}), 2.00-1.88(\mathrm{~m}, 3 \mathrm{H})$, $1.87-1.70(\mathrm{~m}, 1 \mathrm{H}), 1.60-1.47(\mathrm{~m}, 1 \mathrm{H})$. Note: Due to the relatively high volatility of the product, attention must be paid to avoid drying the compound under high vacuum. The experimental data are in agreement with the previous reports. ${ }^{14}$

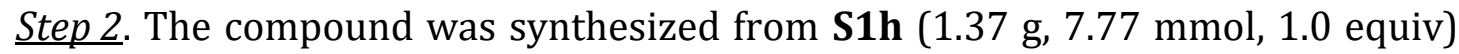
according to the general procedure B, purified by flash column chromatography on silica neutralized with $\mathrm{Et}_{3} \mathrm{~N}$ (pentane $\left.=100 \%+0.5 \% \mathrm{Et}_{3} \mathrm{~N}\right)$, affording $\mathbf{1 h}(1.58$ g, $6.62 \mathrm{mmol}, 85 \%$ ) as a colorless oil. $\mathbf{R}_{\boldsymbol{f}}$ (pentane $=100 \%+0.5 \% \mathrm{Et}_{3} \mathrm{~N}$ ) $0.45 .{ }^{1} \mathbf{H}$ NMR (400 MHz, $\left.\mathbf{C D C l}_{3}\right): \delta$ ppm $7.36-7.28(\mathrm{~m}, 2 \mathrm{H}), 7.27-7.16(\mathrm{~m}, 3 \mathrm{H}), 2.89-$ 
$2.80(\mathrm{~m}, 2 \mathrm{H}), 2.78-2.65(\mathrm{~m}, 2 \mathrm{H}), 2.48-2.36(\mathrm{~m}, 2 \mathrm{H}), 2.31-2.16(\mathrm{~m}, 3 \mathrm{H}), 1.90$ (dtd, $J=11.1,8.6,4.3 \mathrm{~Hz}, 1 \mathrm{H}) .{ }^{13} \mathbf{C}$ NMR (101 MHz, $\mathbf{C D C l}_{3}$ ): $\delta$ ppm 141.7, 128.64 , 128.60, 126.1, 68.2, 46.1, 40.2, 32.8, 17.3. GC-MS (EI): 39 (10), 51 (7), 65 (20), 91 (100), 117 (23), 129 (10), 130 (9), 131 (14), 154 (13). Note: The compound did not ionize under the available ESI conditions, therefore no HRMS could be recorded.

7-(3-Bromo-3-methylbutoxy)-2H-chromen-2-one (1ab). The compound was

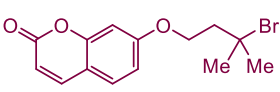

$1 \mathrm{ab}$ synthesized according to the following two step procedure.

Step 1. The compound was synthesized from umbelliferone

(973 mg, $6.00 \mathrm{mmol}, 1.0$ equiv.) according to general procedure A, purified by flash column chromatography on silica (pentane/EtOAc $=1 / 1 \rightarrow 2 / 3$ ) to afford S1ab (1.23 g, $4.94 \mathrm{mmol}, 82 \%$ ) as a white waxy solid. $\mathbf{R}_{\boldsymbol{f}}$ (pentane/EtOAc $=2: 3$ ) 0.31. ${ }^{1} \mathrm{H}$ NMR (400 MHz, $\left.\mathrm{CDCl}_{3}\right): \delta \mathrm{ppm} 7.62(\mathrm{~d}, J=9.5 \mathrm{~Hz}, 1 \mathrm{H}), 7.35(\mathrm{~d}, J=8.1 \mathrm{~Hz}$, $1 \mathrm{H}), 6.86-6.78(\mathrm{~m}, 2 \mathrm{H}), 6.23(\mathrm{~d}, J=9.5 \mathrm{~Hz}, 1 \mathrm{H}), 4.22(\mathrm{t}, J=6.5 \mathrm{~Hz}, 2 \mathrm{H}), 2.02(\mathrm{t}, J=$ $6.5 \mathrm{~Hz}, 2 \mathrm{H}), 1.31$ (s, 6H). ${ }^{13} \mathrm{C}$ NMR (101 MHz, $\mathbf{C D C l}_{3}$ ): $\delta$ ppm 162.0, 161.3, 156.0, 143.5, 128.9, 113.2, 113.0, 112.7, 101.6, 70.2, 65.7, 41.7, 29.9. HRMS (ESI+) calc. for $\mathrm{C}_{14} \mathrm{H}_{16} \mathrm{O}_{4} \mathrm{Na}\left(\mathrm{M}+\mathrm{Na}^{+}\right): 271.0946$. Found: 271.0949 .

Step 2. The compound was synthesized from $\mathbf{S 1 a b}(1.19 \mathrm{~g}, 4.77 \mathrm{mmol}, 1.0$ equiv.) according to general procedure B, purified by flash column chromatography on silica neutralized with $\mathrm{Et}_{3} \mathrm{~N}$ (pentane/EtOAc $=7 / 3+1 \% \mathrm{Et}_{3} \mathrm{~N}$ ) to afford 1ab (864 $\mathrm{mg}, 2.78 \mathrm{mmol}, 58 \%$ ) as a white waxy solid. $\mathbf{R}_{f}$ (pentane $/ \mathrm{EtOAc}=7 / 3+1 \% \mathrm{Et}_{3} \mathrm{~N}$ ) 0.40. ${ }^{1} \mathrm{H}$ NMR (400 MHz, $\left.\mathbf{C D C l}_{3}\right): \delta$ ppm $7.63(\mathrm{~d}, J=9.5 \mathrm{~Hz}, 1 \mathrm{H}), 7.42-7.32(\mathrm{~m}$, 1H), $6.89-6.76(\mathrm{~m}, 2 \mathrm{H}), 6.24(\mathrm{~d}, J=9.5 \mathrm{~Hz}, 1 \mathrm{H}), 4.30(\mathrm{t}, J=6.6 \mathrm{~Hz}, 2 \mathrm{H}), 2.32(\mathrm{t}, J=$ $6.6 \mathrm{~Hz}, 2 \mathrm{H}), 1.85$ (s, 6H). ${ }^{13} \mathrm{C}$ NMR (101 MHz, CDCl $_{3}$ ): $\delta$ ppm 161.9, 161.2, 156.0, 143.5, 129.0, 113.4, 112.8, 101.8, 66.9, 64.7, 45.6, 35.0. HRMS (ESI+) calc. for $\mathrm{C}_{14} \mathrm{H}_{15} \mathrm{O}_{3}{ }^{79} \mathrm{BrNa}\left(\mathrm{M}+\mathrm{Na}^{+}\right)$: 333.0097. Found, 333.0096; calc. for $\mathrm{C}_{14} \mathrm{H}_{15} \mathrm{O}_{3}{ }^{81} \mathrm{BrNa}$ $\left(\mathrm{M}+\mathrm{Na}^{+}\right): 335.0077$. Found: 335.0075 .

5-(3-Bromo-3-methylbutoxy)-2-chloro-1,3-dimethylbenzene (1ac). The

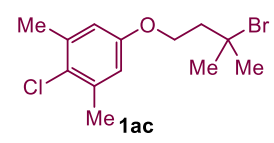


compound was synthesized from 4-chloro-3,5-dimethylphenol (940 mg, 6.00 mmol, 1.0 equiv.) according to general procedure A, followed by bromination according to general procedure B. The crude product purified by flash column chromatography on silica neutralized with $\mathrm{Et}_{3} \mathrm{~N}$ (pentane $=100 \%+0.5 \% \mathrm{Et}_{3} \mathrm{~N} \rightarrow$ pentane/EtOAc 100/1 + 1\% $\mathrm{Et}_{3} \mathrm{~N}$ ), affording 1 ac (934 mg, $3.06 \mathrm{mmol}$, 51\% over two steps) as a colorless oil. $\mathbf{R}_{\boldsymbol{f}}$ (pentane $=100 \%+0.5 \% \mathrm{Et}_{3} \mathrm{~N}$ ) 0.27. ${ }^{1} \mathbf{H} \mathbf{~ N M R}$ (300 MHz, $\mathrm{CDCl}_{3}$ ): $\delta$ ppm $6.65(\mathrm{br} \mathrm{s}, 2 \mathrm{H}), 4.19(\mathrm{t}, J=6.5 \mathrm{~Hz}, 2 \mathrm{H}), 2.37-2.32(\mathrm{~m}$, 6H), $2.29(\mathrm{t}, J=6.6 \mathrm{~Hz}, 2 \mathrm{H}), 1.85(\mathrm{~s}, 6 \mathrm{H}) .{ }^{13} \mathrm{C} \mathrm{NMR}$ (75 MHz, CDCl 3 ): $\delta$ ppm 156.5, $137.3,126.5,114.6,66.3,65.5,46.0,35.0,21.1$. Note: The compound did not ionize under the available ESI conditions, therefore no HRMS could be recorded.

2-(3-Bromo-3-methylbutoxy)-1,1'-biphenyl (1ad). The compound was

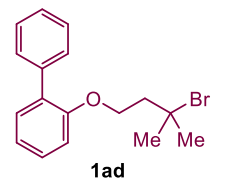
synthesized from 2-phenylphenol (901 mg, $6.00 \mathrm{mmol}, 1.0$ equiv.) according to general procedure A, followed by bromination according to general procedure B. The crude product was purified by flash column chromatography on silica neutralized with $\mathrm{Et}_{3} \mathrm{~N}$ (pentane/EtOAc $=40 / 1+1 \% \mathrm{Et}_{3} \mathrm{~N}$ ), affording 1ad (658 mg, $2.06 \mathrm{mmol}, 34 \%$ over two steps) as a light pink oil. $\mathbf{R}_{\boldsymbol{f}}$ (pentane $/$ EtOAc $=15 / 1+1 \% \mathrm{Et}_{3} \mathrm{~N}$ ) 0.60. ${ }^{\mathbf{1}} \mathbf{H} \mathbf{N M R}(\mathbf{3 0 0} \mathbf{~ M H z}$, $\mathbf{C D C l}_{3}$ ): $\delta$ ppm $7.54-7.47(\mathrm{~m}, 2 \mathrm{H}), 7.44-7.35(\mathrm{~m}, 2 \mathrm{H}), 7.35-7.28(\mathrm{~m}, 3 \mathrm{H}), 7.08$ - $7.00(\mathrm{~m}, 2 \mathrm{H}), 4.23(\mathrm{t}, J=6.5 \mathrm{~Hz}, 2 \mathrm{H}), 2.24(\mathrm{t}, J=6.5 \mathrm{~Hz}, 2 \mathrm{H}), 1.74(\mathrm{~s}, 6 \mathrm{H}) .{ }^{13} \mathrm{C}$ NMR (101 MHz, $\mathbf{C D C l}_{3}$ ): $\delta$ ppm 155.7, 138.7, 131.2, 131.1, 129.7, 128.8, 128.0, $126.9,121.2,112.5,66.7,65.7,46.0,34.9$. Note: The compound did not ionize under the available ESI conditions, therefore no HRMS could be recorded.

\section{Methyl 4-(3-bromo-3-methylbutoxy)-2',4'-difluoro-[1,1'-biphenyl]-3-}

carboxylate (1ae). The compound was synthesized according to the following

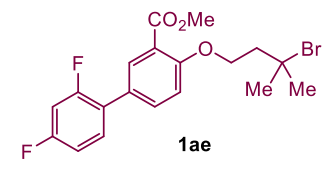
three step procedure.

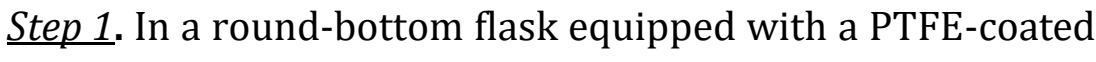
stirring bar, Diflunisal (1.52 g, 6.05 mmol, 1.0 equiv.) was dissolved in $\mathrm{MeOH}$ (20 $\mathrm{mL}$ ), then $\mathrm{H}_{2} \mathrm{SO}_{4} 96 \%$ (1.0 mL) was slowly added, then the reaction was heated at 
reflux for 20 hours. The solution was cooled to room temperature, causing the precipitation of a solid, which was collected by filtration, washed with ice-cold methanol and dried under high vacuum, affording the corresponding ester (1.42 g, 5.38 mmol, 89\%) as a white solid. ${ }^{1} \mathbf{H}$ NMR (300 $\mathbf{~ M H z}, \mathbf{C D C l}_{3}$ ): $\delta$ ppm 10.83 (s, $1 \mathrm{H}$ ), 7.98 (dd, $J=2.4,1.3 \mathrm{~Hz}, 1 \mathrm{H}$ ), 7.60 (ddd, $J=8.6,2.4,1.7 \mathrm{~Hz}, 1 \mathrm{H}$ ), 7.37 (ddd, $J=$ 9.5, 7.9, 6.4 Hz, 1H), 7.06 (d, J = 8.6 Hz, 1H), $7.02-6.82(\mathrm{~m}, 2 \mathrm{H}), 3.97$ (s, 3H). The experimental data are in agreement with the literature reports. ${ }^{15}$

Step 2-3. The tertiary alcohol was synthesized from the intermediate ester (1.41 g, 5.34 mmol, 1.0 equiv.) according to general procedure A. The crude was used for the next step according to general procedure B, affording after flash column chromatography on silica neutralized with $\mathrm{Et}_{3} \mathrm{~N}$ (pentane/EtOAc $=15 / 1+1 \%$ $\mathrm{Et}_{3} \mathrm{~N}$ ) the compound 1ae (559 mg, $1.35 \mathrm{mmol}, 25 \%$ over two steps) as a colorless viscous oil. $\mathbf{R}_{\boldsymbol{f}}$ (pentane/EtOAc $\left.=15 / 1+1 \% \mathrm{Et}_{3} \mathrm{~N}\right)$ 0.26. ${ }^{1} \mathbf{H}$ NMR $(400 \mathrm{MHz}$, $\mathbf{C D C l}_{3}$ ): $\delta$ ppm $7.92(\mathrm{dd}, J=2.5,1.2 \mathrm{~Hz}, 1 \mathrm{H}), 7.61(\mathrm{ddd}, J=8.7,2.5,1.8 \mathrm{~Hz}, 1 \mathrm{H})$, $7.38(\mathrm{td}, J=8.7,6.4 \mathrm{~Hz}, 1 \mathrm{H}), 7.09(\mathrm{~d}, J=8.7 \mathrm{~Hz}, 1 \mathrm{H}), 7.01-6.85(\mathrm{~m}, 2 \mathrm{H}), 4.36(\mathrm{t}, J$ $=6.5 \mathrm{~Hz}, 2 \mathrm{H}), 3.88(\mathrm{~s}, 3 \mathrm{H}), 2.39(\mathrm{t}, J=6.5 \mathrm{~Hz}, 2 \mathrm{H}), 1.88(\mathrm{~s}, 6 \mathrm{H}) .{ }^{13} \mathrm{C}$ NMR (101 MHz, $\mathbf{C D C l}_{3}$ ): $\delta$ ppm 166.5, $163.6(\mathrm{~d}, J=11.8 \mathrm{~Hz}), 161.1$ (dd, $J=11.8,4.9 \mathrm{~Hz}$ ), $158.6(\mathrm{~d}, J=11.8 \mathrm{~Hz}), 157.8,133.9(\mathrm{~d}, J=3.5 \mathrm{~Hz}), 132.2(\mathrm{~d}, J=2.5 \mathrm{~Hz}), 131.3(\mathrm{dd}, J$ = 9.5, 4.8 Hz), 127.3 (d, $J=1.3 \mathrm{~Hz}), 124.1$ (dd, $J=13.7,3.8 \mathrm{~Hz}), 120.7,113.3,111.8$ (dd, $J=21.1,3.8 \mathrm{~Hz}$ ), 104.5 (dd, $J=26.7,25.2 \mathrm{~Hz}$ ), 67.4, 65.5, 52.2, 45.9, 35.0. ${ }^{19} \mathbf{F}$ NMR (376 MHz, CDCl $_{3}$ ): $\delta$ ppm -111.37 (d, $J=7.3 \mathrm{~Hz}$ ), -113.69 (d, $J=7.2 \mathrm{~Hz}$ ). HRMS (ESI') calc. for $\mathrm{C}_{19} \mathrm{H}_{19} \mathrm{O}_{3}{ }^{79} \mathrm{BrF}_{2} \mathrm{Na}\left(\mathrm{M}+\mathrm{Na}^{+}\right)$: 435.0378. Found: 435.0377; calc. for $\mathrm{C}_{19} \mathrm{H}_{19} \mathrm{O}_{3}{ }^{81} \mathrm{BrF}_{2} \mathrm{Na}\left(\mathrm{M}+\mathrm{Na}^{+}\right)$: 437.0360 . Found: 437.0356 .

1-(bromomethyl)adamantane (1af). The title compound was synthesized according to a modified procedure from Wilkinson et al. ${ }^{16}$

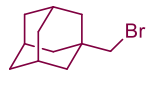

In a Schlenk tube equipped with a PTFE-coated stirring bar, 1adamantylmethanol (665 mg, $4.00 \mathrm{mmol}, 1.00$ equiv.), $\mathrm{ZnBr}_{2}$ (1.35 g, 6.00 mmol, 1.50 equiv.) and $\mathrm{HBr} 48 \%$ ( $2 \mathrm{ml})$ were charged, then the reaction was heated at $110^{\circ} \mathrm{C}$ for 90 minutes. The reaction was cooled to room temperature, then diluted with water $(10 \mathrm{ml})$ and $\mathrm{CH}_{2} \mathrm{Cl}_{2}(10 \mathrm{ml})$ and the layers were separated. The 
aqueous layer was extracted twice with $\mathrm{CH}_{2} \mathrm{Cl}_{2}$ (10 ml each time), then the combined organic layers were dried over $\mathrm{MgSO}_{4}$ and the solvent was removed in vacuo. The crude was purified by flash column chromatography on silica (pentane 100\%), affording the title compound as a colorless crystalline solid $(789 \mathrm{mg}, 3.44 \mathrm{mmol}$, 86\%). ${ }^{1} \mathbf{H}$ NMR (300 MHz, $\left.\mathbf{C D C l}_{3}\right): \delta$ ppm 3.15 (s, 2H), $2.03-1.94$ (m, 3H), $1.74-$ $1.58(\mathrm{~m}, 6 \mathrm{H}), 1.58-1.51(\mathrm{~m}, 6 \mathrm{H})$. The experimental data are in agreement with the literature report. ${ }^{16}$

3-bromo-2,2-diphenylpropanenitrile (1ah). The title compound was synthesized

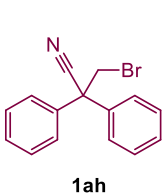
according to a modified procedure from Li et al. ${ }^{17}$

In a Schlenk tube equipped with a PTFE-coated stirring bar, 2,2diphenylacetonitrile (966 mg, $5.00 \mathrm{mmol}, 1.00$ equiv.), TBAB (161 mg, $0.5 \mathrm{mmol}, 10 \mathrm{~mol} \%)$ and $\mathrm{CH}_{2} \mathrm{Br}_{2}(1.8 \mathrm{ml})$ were charged, then $\mathrm{NaOH} 50 \% \mathrm{w} / \mathrm{w}$ in water $(4.0 \mathrm{ml})$ was added and the reaction was heated at $40^{\circ} \mathrm{C}$ overnight, then at $70^{\circ} \mathrm{C}$ for additional 3 hours. The reaction was cooled to room temperature, then the organic layer was separated and washed twice with water ( $4 \mathrm{ml}$ each time). The organic layer was dried over $\mathrm{MgSO}_{4}$ and the solvent was removed in vacuo. The crude was purified by flash column chromatography on silica (pentane/EtOAc $=40 / 1 \rightarrow 20 / 1$ ), affording the title compound as a white crystalline solid (1.34 g, $4.67 \mathrm{mmol}$, 93\%). $\mathbf{R}_{\boldsymbol{f}}$ $($ pentane $/$ EtOAc $=50 / 1)$ 0.30. ${ }^{\mathbf{1}} \mathbf{H}$ NMR (300 $\left.\mathbf{~ M H z}, \mathbf{C D C l}_{\mathbf{3}}\right): \delta \mathrm{ppm} 7.48-7.30(\mathrm{~m}$, 10H), 4.10 (s, 2H). ${ }^{\mathbf{1 3}} \mathbf{C}$ NMR (75 MHz, $\left.\mathbf{C D C l}_{3}\right): \delta$ ppm 137.6, 129.2, 128.8, 127.2, 120.9, 54.2, 36.3. $\mathbf{R}_{f}$ (pentane/EtOAc $=$ 40/1) 0.20. HRMS $\left(\mathbf{E S I}^{+}\right)$: calcd. for $\mathrm{C}_{15} \mathrm{H}_{12}{ }^{79} \mathrm{BrNNa}\left(\mathrm{M}+\mathrm{Na}^{+}\right)$: 308.0045. Found: 308.0046 .

\subsection{Preparation of 1,3-Dienes}

1,3-Butadiene (2a, $2 \mathrm{M}$ in THF), isoprene (2m), $\mathbf{2 p}$ are commercially available and used as received. All other 1,3-dienes used were synthesized according to known literature procedures through Wittig reaction. 1,3-dienes (2b and 2o) 
were synthesized according to known reports by Njardarson ${ }^{18}$ and $\mathrm{Yu}^{19}$ respectively.

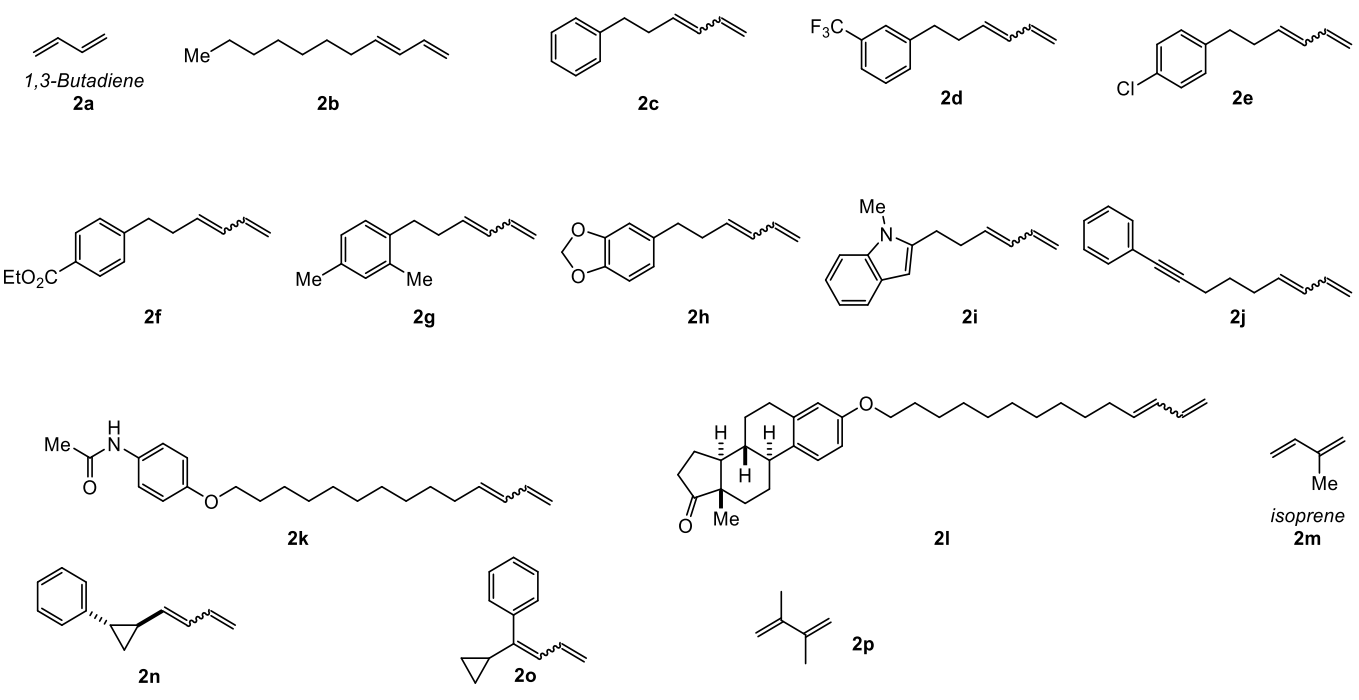

\subsection{General Procedure C: Synthesis of Aldehydes via Heck Reaction}

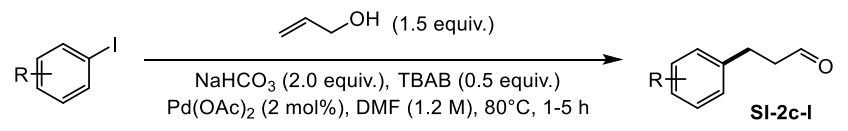

In an oven-dried Schlenk tube equipped with a PTFE-coated stirring bar, TBAB (0.50 equiv.), $\mathrm{NaHCO}_{3}$ (2.00 equiv.), $\mathrm{Pd}(\mathrm{OAc})_{2}(2.0 \mathrm{~mol} \%)$ were charged under argon, then dry DMF (1.2 $M$ ) was added, followed by the appropriate iodide $(1.0$ equiv.) and allyl alcohol (1.50 equiv.). The reaction was stirred at $80^{\circ} \mathrm{C}$ until disappearance of the starting material (usually within 3 hours), then most of the solvent was removed under high vacuum. The crude was taken-up with EtOAc, washed twice with water and the organic layer was dried over $\mathrm{MgSO}_{4}$. After removal of the solvent under reduced pressure, the crude was purified by flash column chromatography on silica (pentane/EtOAc) to afford S2c-1 Note: aldehydes, due to their proclivity towards oxidation, were stored in the fridge at $4^{\circ} \mathrm{C}$ and used as soon as possible. 


\subsection{General Procedure D: Synthesis of 1,3-Dienes via Wittig Reaction}

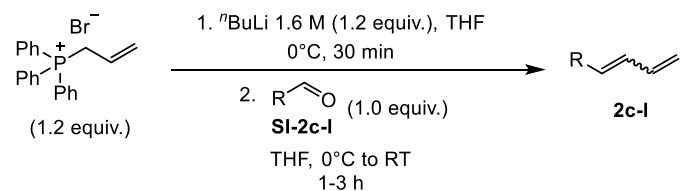

The corresponding 1,3-dienes (2c-l) were synthesized by the above method.

In an oven- and flame-dried Schlenk tube equipped with a PTFE-coated stirring bar, allyltriphenylphosphonium bromide (1.20 equiv.) was suspended in dry THF $(0.17 \mathrm{M})$ under argon, then cooled to $0^{\circ} \mathrm{C}$. A solution of ${ }^{n} \mathrm{BuLi} 1.6 \mathrm{M}$ in hexanes (1.20 equiv.) was added dropwise, then the reaction was stirred at the same temperature for additional 30 minutes (intense orange-red clear solution). A solution of S2c-1 (1.00 equiv.) in dry THF $(0.5 M)$, then the reaction was warmed at room temperature and stirred overnight. After quenching with an aqueous saturated solution of $\mathrm{NH}_{4} \mathrm{Cl}$ (approx. $4 \mathrm{~mL} / \mathrm{mmol}$ ), the water layer was extracted twice with $\mathrm{Et}_{2} \mathrm{O}$ (approx. $9 \mathrm{~mL} / \mathrm{mmol}$ each time), then the combined organic phases were dried over $\mathrm{MgSO}_{4}$ and the solvent was removed under reduced pressure. Most of the triphenylphosphine oxide was removed by precipitation with pentane, followed by filtration. The mother liquors were subsequently evaporated in vacuo, and the crude was purified by flash column chromatography on silica (pentane/EtOAc or pentane $/ \mathrm{CH}_{2} \mathrm{Cl}_{2}$ ). Note: 1,3-dienes were stored in the freezer at $-26^{\circ} \mathrm{C}$ without showing appreciable decomposition over time.

3-(3-(Trifluoromethyl)phenyl)propanal (S2d). The compound was ${ }_{0}{ }_{3} \mathrm{C}$ synthesized according to general procedure $\mathrm{C}$, starting from 3s2d trifluoromethyliodobenzene (1.44 mL, $10.0 \mathrm{mmol}, 1.0$ equiv.), which reacted for 3 hours. The product was purified by flash column chromatography on silica (pentane/EtOAc $=11 / 1 \rightarrow 10 / 1)$, affording S2d (1.59 g, $7.84 \mathrm{mmol}, 78 \%$ ) as a light-yellow oil. ${ }^{1} \mathrm{H}$ NMR (300 $\mathbf{M H z}, \mathbf{C D C l}_{3}$ ): $\delta$ ppm $9.83(\mathrm{t}$, $J=1.2 \mathrm{~Hz}, 1 \mathrm{H}), 7.55-7.33(\mathrm{~m}, 4 \mathrm{H}), 3.02(\mathrm{t}, J=7.6 \mathrm{~Hz}, 2 \mathrm{H}), 2.90-2.75(\mathrm{~m}, 2 \mathrm{H})$. The experimental data are in agreement with the literature reports. ${ }^{20}$ 

compound was synthesized according to general procedure D starting from S2d (1.54 g, $7.61 \mathrm{mmol}, 1.0$ equiv.). The product was purified by flash column chromatography on silica (pentane $=100 \%$ ), affording 2d (526 mg, $2.32 \mathrm{mmol}, 30 \%, E / Z$ approx. 1/1) as a colorless liquid. $\mathbf{R}_{\boldsymbol{f}}$ $($ pentane $=100 \%)$ 0.60. ${ }^{1} \mathbf{H}$ NMR (400 $\left.\mathbf{M H z}, \mathbf{C D C l}_{3}\right): \delta$ ppm $7.52-7.31(\mathrm{~m}, 8 \mathrm{H})$, $6.56(\mathrm{dtd}, J=16.8,11.2,10.1,1.1 \mathrm{~Hz}, 1 \mathrm{H}), 6.31(\mathrm{dt}, J=17.0,10.3 \mathrm{~Hz}, 1 \mathrm{H}), 6.15-$ $5.97(\mathrm{~m}, 2 \mathrm{H}), 5.71(\mathrm{dt}, J=14.5,6.9 \mathrm{~Hz}, 1 \mathrm{H}), 5.52-5.39(\mathrm{~m}, 1 \mathrm{H}), 5.19(\mathrm{dd}, J=16.9$, $2.0 \mathrm{~Hz}, 1 \mathrm{H}), 5.16-5.06(\mathrm{~m}, 2 \mathrm{H}), 5.00(\mathrm{dd}, J=10.1,1.7 \mathrm{~Hz}, 1 \mathrm{H}), 2.81-2.73(\mathrm{~m}$, 4H), 2.53 (qd, $J=7.6,1.5 \mathrm{~Hz}, 2 \mathrm{H}), 2.47-2.36(\mathrm{~m}, 2 \mathrm{H}) .{ }^{13} \mathrm{C}$ NMR (101 MHz, $\mathbf{C D C l}_{3}$ ): $\delta$ ppm 142.7, 142.6, 137.1, 133.5, 132.1, 132.0, 131.9, 130.7, 130.4, 128.9, 125.4 - 125.2 (m), 123.1 - 122.9 (m), 117.8, 115.7, 35.7, 35.6, 34.2, 29.4. Note: $\mathrm{CF}_{3}$ carbon signal could not be observed due to reduction in the intensity caused by $C$-F coupling. ${ }^{19}$ F NMR (376 $\mathbf{M H z}, \mathbf{C D C l}_{3}$ ): $\delta$ ppm -62.60. HRMS (GC-EI) calc. for $\mathrm{C}_{13} \mathrm{H}_{13} \mathrm{~F}_{3}\left(\mathrm{M}^{+}\right):$226.0964. Found: 226.0964 .

3-(4-Chlorophenyl)propanal (S2e). The compound was synthesized according c1 0 to general procedure $C$ starting from 4-chloroiodobenzene (2.39 g, s2e $\quad 10.0 \mathrm{mmol}, 1.0$ equiv.), which reacted for 2 hours. The product was purified by flash column chromatography on silica $($ pentane $/$ EtOAc $=10 / 1 \rightarrow$ 9/1), affording S2e (1.32 g, 7.80 mmol, 78\%) as a light-yellow oil. ${ }^{\mathbf{1}} \mathbf{H}$ NMR (300 MHz, $\left.\mathbf{C D C l}_{3}\right): \delta$ ppm $9.81(\mathrm{t}, J=1.3 \mathrm{~Hz}, 1 \mathrm{H}), 7.30-7.21(\mathrm{~m}, 2 \mathrm{H}), 7.17-7.08(\mathrm{~m}$, 2H), $2.98-2.87(\mathrm{~m}, 2 \mathrm{H}), 2.77(\mathrm{ddt}, J=8.3,7.1,1.2 \mathrm{~Hz}, 2 \mathrm{H})$. The experimental data are in agreement with the literature reports. ${ }^{21}$

(Z/E)-1-Chloro-4-(hexa-3,5-dien-1-yl)benzene (2e): The compound was synthesized according to general procedure D starting from SI2e (1.26 g, $7.47 \mathrm{mmol}, 1.0$ equiv.). The product was purified by flash column chromatography on silica (pentane $=100 \%$ ), affording 2 e $(450 \mathrm{mg}$, 
$2.34 \mathrm{mmol}, 31 \%, E / Z$ approx. $1 / 1$ ) as a colorless liquid. $\mathbf{R}_{\boldsymbol{f}}($ pentane $=100 \%) 0.65$. ${ }^{1}$ H NMR (300 MHz, CDCl$_{3}$ ): $\delta$ ppm 7.28 - $7.22(\mathrm{~m}, 4 \mathrm{H}), 7.16-7.08(\mathrm{~m}, 4 \mathrm{H}), 6.58$ (dddd, $J=16.8,11.2,10.1,1.1 \mathrm{~Hz}, 1 \mathrm{H}), 6.30$ (dt, $J=16.9,10.2 \mathrm{~Hz}, 1 \mathrm{H}$ ), $6.15-5.97$ (m, 2H), $5.70(\mathrm{dt}, J=14.5,6.9 \mathrm{~Hz}, 1 \mathrm{H}), 5.51-5.39(\mathrm{~m}, 1 \mathrm{H}), 5.25-5-05(\mathrm{~m}, 3 \mathrm{H})$, $4.99(\mathrm{dd}, J=10.1,1.7 \mathrm{~Hz}, 1 \mathrm{H}), 2.74-2.63(\mathrm{~m}, 4 \mathrm{H}), 2.51-2.44(\mathrm{~m}, 2 \mathrm{H}), 2.39$ (q, $J=$ $7.4 \mathrm{~Hz}, 2 \mathrm{H}) .{ }^{13} \mathrm{C}$ NMR (75 MHz, $\mathbf{C D C l}_{3}$ ): $\delta$ ppm 140.24, 140.18, 137.1, 133.8, 132.0, 131.9, 131.74, 131.70, 131.1, 130.1, 129.9, 128.6, 117.7, 115.6, 35.3, 35.1, 34.4, 29.5. Note: The compound does not ionize to provide a molecular ion under the available conditions.

Ethyl 4-(3-oxopropyl)benzoate (S2f): The compound was synthesized s2f according to general procedure $C$ starting from ethyl 4for 3 hours. The product was purified by flash column chromatography on silica (pentane/EtOAc = 6/1 $\rightarrow$ 5/1), affording S2f (1.63 g, $7.90 \mathrm{mmol}, 79 \%$ ) as a lightyellow oil. ${ }^{1} \mathbf{H}$ NMR (300 MHz, $\mathbf{C D C l}_{3}$ ): $\delta$ ppm $9.82(\mathrm{t}, J=1.2 \mathrm{~Hz}, 1 \mathrm{H}), 8.04-7.92$ $(\mathrm{m}, 2 \mathrm{H}), 7.31-7.18(\mathrm{~m}, 2 \mathrm{H}), 4.36(\mathrm{q}, J=7.1 \mathrm{~Hz}, 2 \mathrm{H}), 3.01(\mathrm{t}, J=7.4 \mathrm{~Hz}, 2 \mathrm{H}), 2.89-$ $2.70(\mathrm{~m}, 2 \mathrm{H}), 1.38(\mathrm{t}, J=7.1 \mathrm{~Hz}, 3 \mathrm{H})$. The experimental data are in agreement with the literature reports. ${ }^{22}$

Ethyl (Z/E)-4-(hexa-3,5-dien-1-yl)benzoate (2f). The compound was S2f $(1.61 \mathrm{~g}, 7.81 \mathrm{mmol}, 1.0$ equiv.). The product was purified
Sf by flash column chromatography on silica (pentane/EtOAc $=120 / 1 \rightarrow 50 / 1$ ), affording 2 f (687 $\mathrm{mg}, 2.98 \mathrm{mmol}, 38 \%, Z / E$ approx. 1/1) as a colorless oil. $\mathbf{R}_{\boldsymbol{f}}$ $($ pentane $/$ EtOAc $=120 / 1)$ 0.28. ${ }^{1} \mathbf{H}$ NMR (300 $\left.\mathbf{M H z}, \mathbf{C D C l}_{3}\right): \delta$ ppm $7.95-7.82$ (m, 4H), $7.23-7.12(\mathrm{~m}, 4 \mathrm{H}), 6.50$ (dddd, $J=16.9,11.2,10.2,1.1 \mathrm{~Hz}, 1 \mathrm{H}), 6.22$ (dt, $J=16.9,10.2 \mathrm{~Hz}, 1 \mathrm{H}), 6.08-5.86(\mathrm{~m}, 2 \mathrm{H}), 5.63(\mathrm{dt}, J=14.6,6.9 \mathrm{~Hz}, 1 \mathrm{H}), 5.48-$ $5.28(\mathrm{~m}, 1 \mathrm{H}), 5.18-4.86(\mathrm{~m}, 4 \mathrm{H}), 4.29$ (q, $J=7.1 \mathrm{~Hz}, 4 \mathrm{H}), 2.69(\mathrm{td}, J=8.2,7.8,3.2$ $\mathrm{Hz}, 4 \mathrm{H}), 2.55-2.26(\mathrm{~m}, 4 \mathrm{H}), 1.32(\mathrm{t}, J=7.1 \mathrm{~Hz}, 6 \mathrm{H}) .{ }^{13} \mathbf{C}$ NMR (75 MHz, $\left.\mathbf{C D C l}_{3}\right): \delta$ 
ppm 166.8, 147.2, 137.1, 133.7, 132.0, 131.9, 131.0, 130.2, 129.8, 128.6, 117.7, 115.6, 60.9, 36.0, 35.8, 34.1, 29.3, 14.5. HRMS (ESI+) calc. for $\mathrm{C}_{15} \mathrm{H}_{18} \mathrm{O}_{2} \mathrm{Na}$ $\left(\mathrm{M}+\mathrm{Na}^{+}\right):$253.1199. Found, 253.1212.

3-(2,4-Dimethylphenyl)propanal (S2g). The compound was synthesized

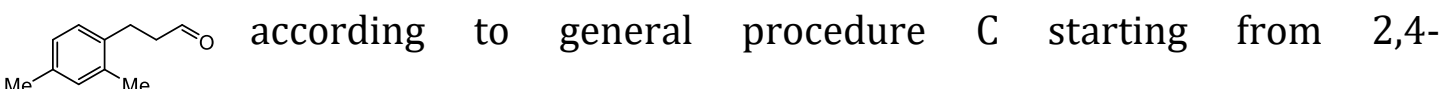
Me dimethyliodobenzene $(1.43 \mathrm{~mL}, 10.0 \mathrm{mmol}$, 1.0 equiv.), which reacted for 5 hours. The product was purified by flash column chromatography on silica (pentane/EtOAc $=18 / 1 \rightarrow 16 / 1 \rightarrow 15 / 1)$, affording S2g (1.42 g, 8.73 mmol, 87\%) as a yellow oil. ${ }^{1} \mathbf{H}$ NMR (300 $\left.\mathbf{~ M H z}, \mathbf{C D C l}_{3}\right): \delta$ ppm $9.84(\mathrm{t}, J=1.5 \mathrm{~Hz}$, 1H), $7.12-6.86(\mathrm{~m}, 3 \mathrm{H}), 3.00-2.83(\mathrm{~m}, 2 \mathrm{H}), 2.80-2.66(\mathrm{~m}, 2 \mathrm{H}), 2.29(\mathrm{~s}, 3 \mathrm{H})$, $2.28(\mathrm{~s}, 3 \mathrm{H})$. The experimental data are in agreement with the literature reports..$^{23}$

(Z/E)-1-(Hexa-3,5-dien-1-yl)-2,4-dimethylbenzene (2g). The compound was synthesized according to general procedure D starting from SI$2 \mathrm{~g}$ (1.40 g, $8.63 \mathrm{mmol}, 1.0$ equiv.). The product was purified by flash column chromatography on silica (pentane $=100 \%$ ), affording $2 \mathbf{g}(559 \mathrm{mg}$, $2.99 \mathrm{mmol}, 35 \%, E / Z$ approx. $1 / 1$ ) as a colorless liquid. $\mathbf{R}_{f}$ (pentane $\left.=100 \%\right) 0.65$. ${ }^{1} \mathrm{H}$ NMR (400 MHz, CDCl $_{3}$ ): $\delta$ ppm $7.10-7.01(\mathrm{~m}, 2 \mathrm{H}), 7.02-6.92(\mathrm{~m}, 4 \mathrm{H}), 6.65$ (dddd, $J=16.8,11.2,10.1,1.1 \mathrm{~Hz}, 1 \mathrm{H}), 6.34(\mathrm{dt}, J=16.9,10.3 \mathrm{~Hz}, 1 \mathrm{H}), 6.20-5.98$ $(\mathrm{m}, 2 \mathrm{H}), 5.80(\mathrm{dt}, J=14.5,6.9 \mathrm{~Hz}, 1 \mathrm{H}), 5.63-5.46(\mathrm{~m}, 1 \mathrm{H}), 5.22(\mathrm{dd}, J=16.9,2.0$ $\mathrm{Hz}, 1 \mathrm{H}), 5.17-5.07(\mathrm{~m}, 2 \mathrm{H}), 5.00$ (d, $J=10.1 \mathrm{~Hz}, 1 \mathrm{H}), 2.73-2.61(\mathrm{~m}, 4 \mathrm{H}), 2.53-$ $2.42(\mathrm{~m}, 2 \mathrm{H}), 2.40-2.32(\mathrm{~m}, 2 \mathrm{H}), 2.32-2.27(\mathrm{~m}, 12 \mathrm{H}) .{ }^{13} \mathbf{C}$ NMR (101 MHz, $\mathbf{C D C l}_{3}$ ): $\delta$ ppm 137.3, 137.0, 136.9, 135.8, 135.61, 135.56, 134.7, 132.2, 132.0, 131.4, 131.14, 131.13, 129.7, 128.9, 128.8, 126.73, 126.72, 117.4, 115.3, 33.5, $32.9,32.7,28.7,21.0,19.40,19.37$. Note: The compound does not ionize to provide a molecular ion under the available conditions. 
3-(Benzo[d][1,3]dioxol-5-yl)propanal (S2h). The compound was synthesized so according to general procedure $\mathrm{C}$ starting from 5iodobenzo[d][1,3]dioxole (1.27 mL, $10.0 \mathrm{mmol}, 1.0$ equiv.), which reacted for 5 hours. The product was purified by flash column chromatography on silica (pentane/EtOAc = 9/1), affording S2h (1.38 g, $7.73 \mathrm{mmol}, 77 \%)$ as a colorless oil. ${ }^{1} \mathbf{H}$ NMR (400 $\mathbf{M H z}, \mathbf{C D C l}_{3}$ ): $\delta$ ppm $9.80(\mathrm{t}, J=1.4 \mathrm{~Hz}, 1 \mathrm{H}), 6.73$ (d, $J$ $=7.9 \mathrm{~Hz}, 1 \mathrm{H}), 6.70-6.61(\mathrm{~m}, 2 \mathrm{H}), 5.92(\mathrm{~s}, 2 \mathrm{H}), 2.98-2.82(\mathrm{~m}, 2 \mathrm{H}), 2.81-2.64(\mathrm{~m}$, $2 \mathrm{H})$. The experimental data are in agreement with the literature reports. ${ }^{24}$

(Z/E)-5-(Hexa-3,5-dien-1-yl)benzo[d][1,3]dioxole (2h). The compound was synthesized according to general procedure D starting $\mathbf{S} 2 \mathbf{h}$ (1.40 g, $8.63 \mathrm{mmol}, 1.0$ equiv.). The product was purified by flash column chromatography on silica $($ pentane $/$ EtOAc $=130 / 1 \rightarrow 100 / 1 \rightarrow$ 80/1) affording $\mathbf{2 h}$ (535 mg, $2.15 \mathrm{mmol}, 28 \%, E / Z$ approx. 1/1) as a colorless liquid. Note: Due to the presence of an unknown apolar impurity very close in polarity to the product, a substantial amount of product was lost during purification by column chromatography. $\mathbf{R}_{\boldsymbol{f}}$ (pentane $/$ EtOAc $=100 / 1$ ) $0.40 .{ }^{1} \mathbf{H}$ NMR (400 MHz, $\mathbf{C D C l}_{3}$ ): $\delta$ ppm $6.74(\mathrm{~d}, J=7.9 \mathrm{~Hz}, 2 \mathrm{H}), 6.69$ (dd, $J=5.0,1.7 \mathrm{~Hz}$, 2H), $6.67-6.53(\mathrm{~m}, 3 \mathrm{H}), 6.31(\mathrm{dt}, J=17.0,10.2 \mathrm{~Hz}, 1 \mathrm{H}), 6.17-5.97(\mathrm{~m}, 2 \mathrm{H}), 5.92$ (s, 4H), $5.72(\mathrm{dt}, J=14.5,6.9 \mathrm{~Hz}, 1 \mathrm{H}), 5.47$ (dddd, $J=10.3,7.8,6.5,1.3 \mathrm{~Hz}, 1 \mathrm{H}$ ), $5.19(\mathrm{dd}, J=16.9,2.0 \mathrm{~Hz}, 1 \mathrm{H}), 5.16-5.05(\mathrm{~m}, 2 \mathrm{H}), 4.98$ (dd, $J=10.2,1.7 \mathrm{~Hz}, 1 \mathrm{H})$, $2.70-2.58(\mathrm{~m}, 4 \mathrm{H}), 2.52-2.41(\mathrm{~m}, 2 \mathrm{H}), 2.41-2.32(\mathrm{~m}, 2 \mathrm{H}) .{ }^{13} \mathrm{C}$ NMR (101 MHz, $\mathbf{C D C l}_{3}$ ): $\delta$ ppm 147.7, 145.79, 145.75, 137.3, 135.73, 135.67, 134.2, 132.2, 131.6, $131.5,129.9,121.28,121.25,117.4,115.4$, 109.02, 108.99, 108.3, 100.9, 35.7, 35.5, 34.8, 30.0. Note: The compound does not ionize to provide a molecular ion under the available conditions.

3-(1-Methyl-1H-indol-3-yl)propanal (S2i). The compound was synthesized according to the procedure reported by Stoltz et al., ${ }^{25}$ using $N$ - 
methylindole ( $1.25 \mathrm{~mL}, 10.0 \mathrm{mmol}, 1.0$ equiv.) as starting material. The crude was purified by flash column chromatography on silica (pentane/EtOAc $=8 / 1 \rightarrow$ 6/1), providing S2i (923 mg, $4.93 \mathrm{mmol}, 49 \%)$ as a pale-yellow oil. ${ }^{1} \mathbf{H}$ NMR (300 MHz, $\mathbf{C D C l}_{3}$ ): $\delta$ ppm $9.84(\mathrm{t}, J=1.7 \mathrm{~Hz}, 1 \mathrm{H}), 7.58(\mathrm{dt}, J=7.9,1.0 \mathrm{~Hz}, 1 \mathrm{H}), 7.33-$ $7.20(\mathrm{~m}, 2 \mathrm{H}), 7.12$ (ddd, $J=8.0,6.8,1.3 \mathrm{~Hz}, 1 \mathrm{H}), 6.87-6.83(\mathrm{br}, 1 \mathrm{H}), 3.74(\mathrm{~s}, 3 \mathrm{H}$ ), $3.18-3.05(\mathrm{~m}, 2 \mathrm{H}), 2.84(\mathrm{tdd}, J=7.3,1.7,0.6 \mathrm{~Hz}, 2 \mathrm{H})$. The experimental data are in agreement with the literature reports. ${ }^{26}$

(Z/E)-3-(Hexa-3,5-dien-1-yl)-1-methyl-1H-indole (2i). The compound was $\underbrace{i}_{n}$ synthesized according to general procedure D starting from S2i (908 mg, $4.85 \mathrm{mmol}, 1.0$ equiv.). The product was purified by flash column chromatography on silica (pentane/EtOAc $=100 / 1$ ) affording $2 \mathbf{i}$ (559 mg, $2.99 \mathrm{mmol}, 35 \%, Z / E$ approx. 1/1) as a light-yellow liquid. $\mathbf{R}_{\boldsymbol{f}}$ (pentane/EtOAc $=40 / 1)$ 0.50. ${ }^{1} \mathbf{H}$ NMR $\left(400 \mathbf{~ M H z}, \mathbf{C D C l}_{3}\right): \delta \mathrm{ppm} 7.61(\mathrm{ddt}, J=$ 7.9, 4.4, 1.0 Hz, 2H), $7.30(\mathrm{~d}, J=8.2 \mathrm{~Hz}, 2 \mathrm{H}), 7.23(\mathrm{t}, J=7.4 \mathrm{~Hz}, 2 \mathrm{H}), 7.18-7.05(\mathrm{~m}$, 2H), $6.85(\mathrm{~d}, J=3.7 \mathrm{~Hz}, 1 \mathrm{H}), 6.67$ (dddd, $J=16.9,11.2,10.2,1.0 \mathrm{~Hz}, 1 \mathrm{H}), 6.34(\mathrm{dt}, J$ $=17.0,10.2 \mathrm{~Hz}, 1 \mathrm{H}), 6.15(\mathrm{dd}, J=15.1,10.4 \mathrm{~Hz}, 1 \mathrm{H}), 6.05(\mathrm{t}, J=11.0 \mathrm{~Hz}, 1 \mathrm{H}), 5.84$ $(\mathrm{dt}, J=14.6,6.9 \mathrm{~Hz}, 1 \mathrm{H}), 5.69-5.53(\mathrm{~m}, 1 \mathrm{H}), 5.21(\mathrm{dd}, J=16.9,2.0 \mathrm{~Hz}, 1 \mathrm{H}), 5.16-$ $5.07(\mathrm{~m}, 2 \mathrm{H}), 4.99(\mathrm{~d}, J=10.0 \mathrm{~Hz}, 1 \mathrm{H}), 3.75(\mathrm{~s}, 6 \mathrm{H}), 2.91-2.81(\mathrm{~m}, 4 \mathrm{H}), 2.61(\mathrm{qd}, J$ $=7.5,1.6 \mathrm{~Hz}, 2 \mathrm{H}), 2.51(\mathrm{q}, J=7.2 \mathrm{~Hz}, 2 \mathrm{H}) \cdot{ }^{13} \mathbf{C} \mathbf{N M R}\left(101 \mathbf{M H z}, \mathbf{C D C l}_{3}\right): \delta \mathrm{ppm}$ 137.5, 137.2, 135.3, 132.7, 132.5, 131.3, 129.6, 128.02, 128.00, 126.3, 126.2, $121.62,121.61,119.13,119.10,118.73,118.70,117.2,115.1,114.74,114.72$, 109.27, 109.26, 33.5, 32.7, 28.7, 25.3, 25.1. HRMS (ESI+) calc. for $\mathrm{C}_{15} \mathrm{H}_{17} \mathrm{NNa}$ $\left(\mathrm{M}+\mathrm{Na}^{+}\right): 234.1253$. Found, 234.1266.

6-Phenylhex-5-ynal (S2j). The title compound was obtained via the following

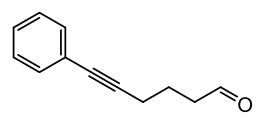

s2j two step procedure. The intermediate 6-phenylhex-5-ynol (1.72 g, $9.85 \mathrm{mmol}$, 99\%) was obtained as a yellow oil according to the literature procedure reported by Paquin et al. ${ }^{27}{ }^{1} \mathbf{H}$ NMR $\left(300 \mathrm{MHz}, \mathrm{CDCl}_{3}\right) \delta 7.43-7.35(\mathrm{~m}, 2 \mathrm{H}), 7.34-7.22(\mathrm{~m}, 3 \mathrm{H}), 3.72(\mathrm{q}, J=5.5 \mathrm{~Hz}$, 
2H), $2.46(\mathrm{t}, J=6.7 \mathrm{~Hz}, 2 \mathrm{H}), 1.86-1.63(\mathrm{~m}, 4 \mathrm{H}), 1.39-1.28(\mathrm{~m}, 1 \mathrm{H})$. The experimental data are in agreement with the previous reports. ${ }^{28}$

In an oven-dried Schlenk tube equipped with a PTFE-coated stirring bar, the intermediate alcohol (1.70 g, $9.75 \mathrm{mmol}, 1.00$ equiv.) was dissolved in dry $\mathrm{CH}_{2} \mathrm{Cl}_{2}$ (20 mL) under argon, then PCC (2.31 g, $10.7 \mathrm{mmol}, 1.10$ equiv.) was added portionwise and the reaction was stirred at room temperature for 60 minutes, then at $40^{\circ} \mathrm{C}$ for additional 60 minutes. After cooling to room temperature, then solvent was removed, and the residue directly loaded on silica for flash column chromatography (pentane/EtOAc = 12/1), affording S2j $(1.19 \mathrm{~g}, 6.93 \mathrm{mmol}, 71 \%)$ as a light-yellow oil. ${ }^{\mathbf{1}} \mathbf{H}$ NMR (300 $\mathbf{~ M H z , ~} \mathbf{C D C l}_{3}$ ): $\delta$ ppm $9.85(\mathrm{t}, J=1.4 \mathrm{~Hz}, 1 \mathrm{H})$, $7.43-7.35(\mathrm{~m}, 2 \mathrm{H}), 7.34-7.24(\mathrm{~m}, 3 \mathrm{H}), 2.66(\mathrm{td}, J=7.2,1.4 \mathrm{~Hz}, 2 \mathrm{H}), 2.50(\mathrm{t}, J=$ $6.8 \mathrm{~Hz}, 2 \mathrm{H}), 1.95(\mathrm{p}, J=7.0 \mathrm{~Hz}, 2 \mathrm{H})$. The experimental data are in agreement with the previous reports. ${ }^{29}$

(Z/E)-Nona-6,8-dien-1-yn-1-ylbenzene (2j). The compound was synthesized

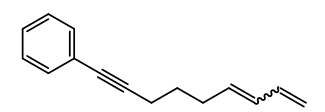

2j according to general procedure D starting from S2j (1.17 g, $6.79 \mathrm{mmol}, 1.0$ equiv.). The product was purified by flash column chromatography on silica (pentane $=100 \%$ ) affording $2 \mathbf{j}$ (321 mg, 1.64 mmol, 24\%, Z/E approx. 1/1) as a colorless liquid. $\mathbf{R}_{\boldsymbol{f}}$ (pentane $=100 \%$ ) $0.40 .{ }^{1} \mathbf{H}$ NMR (400 MHz, CDCl $_{3}$ ): $\delta$ ppm 7.49 - $7.36(\mathrm{~m}, 4 \mathrm{H}), 7.35-7.27(\mathrm{~m}, 6 \mathrm{H}), 6.71$ (dddd, $J=16.9,11.2,10.1,1.2 \mathrm{~Hz}, 1 \mathrm{H}), 6.34(\mathrm{dt}, J=16.9,10.2 \mathrm{~Hz}, 1 \mathrm{H}), 6.22-6.00$ $(\mathrm{m}, 2 \mathrm{H}), 5.73(\mathrm{dt}, J=14.7,7.0 \mathrm{~Hz}, 1 \mathrm{H}), 5.57-5.40(\mathrm{~m}, 1 \mathrm{H}), 5.22(\mathrm{dd}, J=16.9,2.0$ $\mathrm{Hz}, 1 \mathrm{H}), 5.17-5.08(\mathrm{~m}, 2 \mathrm{H}), 4.99$ (d, $J=10.0 \mathrm{~Hz}, 1 \mathrm{H}), 2.47-2.41(\mathrm{~m}, 4 \mathrm{H}), 2.41-$ $2.35(\mathrm{~m}, 2 \mathrm{H}), 2.27(\mathrm{qd}, J=7.2,1.4 \mathrm{~Hz}, 2 \mathrm{H}), 1.72(\mathrm{pd}, J=7.2,2.5 \mathrm{~Hz}, 4 \mathrm{H}) .{ }^{13} \mathrm{C} \mathrm{NMR}$ (101 MHz, $\mathbf{C D C l}_{3}$ ): $\delta$ ppm 137.3, 134.2, 132.4, 131.9, 131.69, 131.68, 131.6, 128.3, 127.7, 124.1, 117.4, 115.3, 89.993, 89.987, 81.1, 31.8, 28.7, 28.3, 26.9, 19.01, 18.99. HRMS (GC-EI) calc. for $\mathrm{C}_{15} \mathrm{H}_{16}\left(\mathrm{M}^{+}\right)$: 196.1246. Found, 196.1246.

(Z/E)-14-Bromotetradeca-1,3-diene (S2k). The compound was synthesized

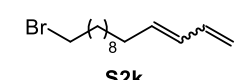


according to a slightly modified version of the procedure reported by Martin et $a{ }^{30}$

In an oven-dried Schlenk tube equipped with a PTFE-coated stirring bar, 1bromoundecanol (5.02 g, $20.0 \mathrm{mmol}, 1.0$ equiv.) was dissolved in dry $\mathrm{CH}_{2} \mathrm{Cl}_{2}$ under argon, then pyridinium chlorocromate (4.74 g, $22.0 \mathrm{mmol}, 1.1$ equiv.) was added portionwise. The reaction was stirred for 3 hours, then the reaction was filtered through a short pad of Celite ${ }^{\circledR}$, then purified by flash column chromatography on silica (pentane/EtOAc $=30: 1$ ), affording the product as a colorless oil, which solidified upon cooling (3.59 g, $14.4 \mathrm{mmol}, 72 \%$ ). According to general procedure $\mathrm{D}$, aldehyde was converted to the 1,3-diene and the crude was purified by flash column chromatography on silica (pentane $=100 \%$ ), affording S2k (1.68 g, $6.13 \mathrm{mmol}, 35 \%, E / Z$ approx. 1:1) as a colorless liquid. $\mathbf{R}_{\boldsymbol{f}}$ $($ pentane $=100 \%)$ 0.50. ${ }^{1} \mathbf{H}$ NMR (400 MHz, $\left.\mathbf{C D C l}_{3}\right): \delta$ ppm $6.64(\mathrm{dddd}, J=16.8$, 11.1, 10.0, $1.1 \mathrm{~Hz}, 1 \mathrm{H}), 6.31(\mathrm{dt}, J=17.0,10.2 \mathrm{~Hz}, 1 \mathrm{H}), 6.12-5.92(\mathrm{~m}, 2 \mathrm{H}), 5.70$ $(\mathrm{dt}, J=14.6,7.0 \mathrm{~Hz}, 1 \mathrm{H}), 5.53-5.41(\mathrm{~m}, 1 \mathrm{H}), 5.18(\mathrm{dd}, J=17.0,2.0 \mathrm{~Hz}, 1 \mathrm{H}), 5.14-$ $5.02(\mathrm{~m}, 2 \mathrm{H}), 4.95(\mathrm{dd}, J=10.1,1.8 \mathrm{~Hz}, 1 \mathrm{H}), 3.41(\mathrm{t}, J=6.9 \mathrm{~Hz}, 4 \mathrm{H}), 2.18(\mathrm{qd}, J=$ 7.4, 1.6 Hz, 2H), 2.07 (qd, J = 7.1, 1.4 Hz, 2H), $1.85(\mathrm{dt}, J=14.5,7.0 \mathrm{~Hz}, 4 \mathrm{H}), 1.48$ $1.19(\mathrm{~m}, 32 \mathrm{H}) .{ }^{13} \mathbf{C}$ NMR (101 $\left.\mathbf{M H z}, \mathbf{C D C l}_{3}\right): \delta$ ppm 137.5, 135.7, 133.2, 132.5, 131.0, 129.3, 116.8, 114.7, 34.2, 33.0, 32.7, 29.7, 29.60, 29.57, 29.56, 29.34, 29.32, 28.9, 28.3, 27.9. HRMS (ESI+) calc. for $\mathrm{C}_{14} \mathrm{H}_{25}{ }^{79} \mathrm{BrAg}\left(\mathrm{M}+\mathrm{Ag}^{+}\right)$: 379.0185. Found, 379.0187; calc. for $\mathrm{C}_{14} \mathrm{H}_{25}{ }^{81} \mathrm{BrAg}\left(\mathrm{M}+\mathrm{Ag}^{+}\right)$: 381.0173. Found, 381.0173. Note: the compound is volatile, therefore drying under high vacuum must be avoided in order to prevent product loss.

$(Z / E)-N$-(4-(Tetradeca-11,13-dien-1-yloxy)phenyl)acetamide $\quad(2 \mathrm{k}) . \quad$ The compound was synthesized according to a slightly modified version of the procedure reported by Martin et al. ${ }^{30}$ In an oven-dried Schlenk tube equipped with a PTFE-coated stirring bar, bromide S2k (683 mg, 2.5 mmol, 1.0 equiv.), 4-acetamidophenol (416 mg, $2.75 \mathrm{mmol}, 1.1$ equiv.) and $\mathrm{K}_{2} \mathrm{CO}_{3}$ (518 mg, $3.75 \mathrm{mmol}, 1.5$ equiv.) were suspended in dry DMF 
(4.2 mL) under argon, then stirred at room temperature for 2 days. Most of the solvent was removed under high vacuum, then the residue was taken-up with EtOAc $(30 \mathrm{~mL})$ and washed with water $(20 \mathrm{~mL})$. The water layer was extracted once with EtOAc $(20 \mathrm{~mL})$, then the combined organic layers were washed once with water (20 mL each time), dried over $\mathrm{MgSO}_{4}$ and the solvent was removed under reduced pressure. The crude was purified by flash column chromatography on silica (pentane/EtOAc $=1 / 1)$, affording 2k $(767 \mathrm{mg}, 2.23$ mmol, 89\%, $E / Z$ approx. $1 / 1$ ) as a white solid. $\mathbf{R}_{\boldsymbol{f}}$ (pentane/EtOAc $\left.=1 / 1\right) 0.28 .{ }^{1} \mathbf{H}$ NMR (400 MHz, CDCl $_{3}$ ): $\delta$ ppm $7.62(\mathrm{~s}, 2 \mathrm{H}), 7.45-7.30(\mathrm{~m}, 4 \mathrm{H}), 6.87-6.76(\mathrm{~m}$, 4H), 6.64 (dddd, $J=16.9,11.1,10.1,1.1 \mathrm{~Hz}, 1 \mathrm{H}), 6.30(\mathrm{dt}, J=17.0,10.2 \mathrm{~Hz}, 1 \mathrm{H}$ ), $6.16-5.90(\mathrm{~m}, 2 \mathrm{H}), 5.70(\mathrm{dt}, J=14.6,6.9 \mathrm{~Hz}, 1 \mathrm{H}), 5.56-5.29(\mathrm{~m}, 1 \mathrm{H}), 5.17(\mathrm{dd}, J$ $=16.9,2.1 \mathrm{~Hz}, 1 \mathrm{H}), 5.12-5.03(\mathrm{~m}, 2 \mathrm{H}), 4.94(\mathrm{dd}, J=10.0,1.8 \mathrm{~Hz}, 1 \mathrm{H}), 3.90(\mathrm{t}, J=$ $6.6 \mathrm{~Hz}, 4 \mathrm{H}), 2.17(\mathrm{qd}, J=7.4,1.6 \mathrm{~Hz}, 2 \mathrm{H}), 2.11(\mathrm{~s}, 6 \mathrm{H}), 2.07(\mathrm{qd}, J=7.5,1.8 \mathrm{~Hz}, 2 \mathrm{H})$, $1.80-1.69$ (m, 4H), $1.52-1.17$ (m, 28H). ${ }^{13} \mathbf{C}$ NMR (101 MHz, CDCl $\left._{3}\right): \delta$ ppm 168.6, 156.1, 137.5, 135.7, 133.2, 132.5, 131.0, 130.9, 129.2, 122.1, 116.8, 114.8, 114.7, 68.4, 32.7, 29.7, 29.64, 29.61, 29.56, 29.5, 29.4, 29.31, 29.29, 29.28, 27.8, 26.1, 24.3. HRMS (ESI+) calc. for $\mathrm{C}_{22} \mathrm{H}_{33} \mathrm{NO}_{2} \mathrm{Na}\left(\mathrm{M}^{+} \mathrm{Na}^{+}\right)$: 366.2404. Found, 366.2403 .

\section{(Z/E)-(8R,9S,13S,14S)-13-Methyl-3-(tetradeca-11,13-dien-1-yloxy)-}

\section{6,7,8,9,11,12,13,14,15,16-decahydro-17H-cyclopenta[a]phenanthren-17-}

one (21). The compound was synthesized according to a slightly modified

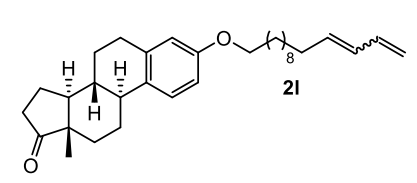

version of the procedure reported by Martin et al. ${ }^{30} \mathrm{In}$ an oven-dried Schlenk tube equipped with a PTFE-coated stirring bar, bromide $\mathbf{S} \mathbf{2 k}$ (683 mg, $2.5 \mathrm{mmol}, 1.0$ equiv.),

estrone (676 mg, $2.5 \mathrm{mmol}, 1.0$ equiv.) and $\mathrm{K}_{2} \mathrm{CO}_{3}(518 \mathrm{mg}, 3.75 \mathrm{mmol}, 1.5$ equiv.) were suspended in dry DMF (4.2 mL) under argon, then stirred at room temperature for 3 days. Most of the solvent was removed under high vacuum, then the residue was taken-up with EtOAc ( $30 \mathrm{~mL}$ ) and washed with water (20 $\mathrm{mL})$. The water layer was extracted once with EtOAc $(20 \mathrm{~mL})$, then the combined 
organic layers were washed once with water (20 mL each time), dried over $\mathrm{MgSO}_{4}$ and the solvent was removed under reduced pressure. The crude was purified by flash column chromatography on silica $($ pentane $/$ EtOAc $=9 / 1 \rightarrow$ 17/3), affording 21 (959 $\mathrm{mg}, 2.07 \mathrm{mmol}, 83 \%, Z / E$ approx. 1:1) as a colorless glass. $\mathbf{R}_{\boldsymbol{f}}$ (pentane $/$ EtOAc $\left.=9 / 1\right)$ 0.32. ${ }^{\mathbf{1}} \mathbf{H}$ NMR (400 $\left.\mathbf{M H z}, \mathbf{C D C l}_{3}\right): \delta$ ppm $7.23-$ $7.14(\mathrm{~m}, 4 \mathrm{H}), 6.71(\mathrm{dd}, J=8.6,2.8 \mathrm{~Hz}, 4 \mathrm{H}), 6.70-6.59(\mathrm{~m}, 6 \mathrm{H}), 6.31(\mathrm{dt}, J=17.0$, $10.2 \mathrm{~Hz}, 1 \mathrm{H}), 6.12-5.93(\mathrm{~m}, 2 \mathrm{H}), 5.71(\mathrm{dt}, J=14.6,7.0 \mathrm{~Hz}, 1 \mathrm{H}), 5.52-5.34(\mathrm{~m}$, 1H), $5.18(\mathrm{dd}, J=16.8,2.1 \mathrm{~Hz}, 1 \mathrm{H}), 5.14-5.03(\mathrm{~m}, 2 \mathrm{H}), 4.95(\mathrm{dd}, J=10.1,1.8 \mathrm{~Hz}$, $1 \mathrm{H}), 3.92(\mathrm{t}, J=6.6 \mathrm{~Hz}, 4 \mathrm{H}), 2.98-2.81(\mathrm{~m}, 4 \mathrm{H}), 2.51(\mathrm{dd}, J=18.8,8.6 \mathrm{~Hz}, 2 \mathrm{H})$, 2.40 (ddd, $J=11.7,6.3,3.5 \mathrm{~Hz}, 2 \mathrm{H}), 2.26(\mathrm{td}, J=10.5,4.0 \mathrm{~Hz}, 2 \mathrm{H}), 2.21-1.91(\mathrm{~m}$, 12H), $1.81-1.70(\mathrm{~m}, 4 \mathrm{H}), 1.70-1.22(\mathrm{~m}, 40 \mathrm{H}), 0.91$ (s, 6H). ${ }^{13}$ C NMR (101 MHz, $\mathbf{C D C l}_{3}$ ): $\delta$ ppm 221.1, 157.3, 137.8, 137.5, 135.7, 133.2, 132.5, 131.9, 131.0, 129.2, 126.4, 116.8, 114.69, 114.67, 112.2, 68.0, 50.5, 48.1, 44.1, 38.5, 36.0, 32.7, 31.7, $29.8,29.73,29.68,29.64,29.59,29.50,29.46,29.34,29.32,29.31,27.9,26.7,26.2$, 26.1, 21.7, 14.0. HRMS (ESI+) calc. for $\mathrm{C}_{32} \mathrm{H}_{46} \mathrm{O}_{2} \mathrm{Na}\left(\mathrm{M}+\mathrm{Na}^{+}\right)$: 485.3390. Found, 485.3377 .

(trans)-(2-Phenylcyclopropyl)methanol (S2n). In a flame-dried round-bottom $A_{\mathrm{s} 2 \mathrm{n}} \mathrm{OH}_{\mathrm{OH}}$ flask equipped with an argon inlet and a PTFE-coated stirring bar, $\mathrm{Et}_{2} \mathrm{Zn} 1.0 \mathrm{M}$ in hexane (18.8 mL, $18.8 \mathrm{mmol}, 1.26$ equiv.) was diluted with dry $\mathrm{CH}_{2} \mathrm{Cl}_{2}(60 \mathrm{~mL})$ under argon, then cooled to $-10 /-15^{\circ} \mathrm{C}$ using an ice- $\mathrm{NaCl}$ bath. $\mathrm{CH}_{2} \mathrm{I}_{2}$ (2.89 mL, $35.3 \mathrm{mmol}, 2.37$ equiv.) was added dropwise, then the reaction was gently warmed at room temperature (formation of a thick white precipitate) and stirred for 20 minutes, then (E)-cinnamyl alcohol (1.92 mL, 14.9 mmol, 1.00 equiv.) was added dropwise, followed by Ti(OEt) 4 (188 $\mu \mathrm{L}, 0.90$ mmol, $6 \mathrm{~mol} \%$ ) (disappearance of the white precipitate). The reaction was stirred at room temperature for 3 hours, then carefully quenched with saturated $\mathrm{NH}_{4} \mathrm{Cl}$ solution $(50 \mathrm{~mL})$. The biphasic mixture was filtered through a pad of Celite ${ }^{\circledR}$, rinsing with $\mathrm{CH}_{2} \mathrm{Cl}_{2}$, then the layers were separated, then the water layer was extracted twice with $\mathrm{CH}_{2} \mathrm{Cl}_{2}$ (50 mL each time), the combined organic extracts 
were dried over $\mathrm{MgSO}_{4}$ and the solvent was removed under reduced pressure. The crude was purified by flash column chromatography on silica (pentane/EtOAc $=4 / 1 \rightarrow 3 / 1$ ), affording $\mathbf{S 2 n}$ as a colorless viscous oil (1.75 g, 11.8 mmol, 79\%). ${ }^{1} \mathbf{H}$ NMR (300 MHz, $\mathbf{C D C l}_{3}$ ): $\delta$ ppm $7.24-7.15(\mathrm{~m}, 2 \mathrm{H}), 7.13-$ $7.05(\mathrm{~m}, 1 \mathrm{H}), 7.04-6.96(\mathrm{~m}, 2 \mathrm{H}), 3.56(\mathrm{dt}, J=7.3,3.8 \mathrm{~Hz}, 2 \mathrm{H}), 1.76(\mathrm{dt}, J=8.7,4.9$ $\mathrm{Hz}, 1 \mathrm{H}), 1.47-1.32(\mathrm{~m}, 2 \mathrm{H}), 0.96-0.80(\mathrm{~m}, 2 \mathrm{H})$. The experimental data are in agreement with the literature reports. ${ }^{31}$

(trans)-2-Phenylcyclopropane-1-carbaldehyde (S2n'). In an oven-dried

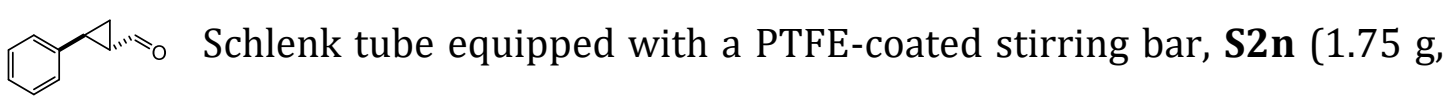
s2n' $\quad 11.8$ mmol, 1.00 equiv.) was dissolved in dry $\mathrm{CH}_{2} \mathrm{Cl}_{2}(60 \mathrm{~mL})$, then pyridinium chlorochromate $(5.09 \mathrm{~g}, 23.6 \mathrm{mmol}, 2.00$ equiv.) was added portionwise under vigorous stirring. The reaction was stirred at room temperature for 3 hours, then filtered over a pad of Celite ${ }^{\circledR}$, rinsing with $\mathrm{CH}_{2} \mathrm{Cl}_{2}$. The organic layer was evaporated in vacuo, then the crude was purified by flash column chromatography on silica (pentane/EtOAc =15/1), affording S2n' as a colorless low-melting crystalline solid (1.36 g, 9.29 mmol, 79\%). ${ }^{\mathbf{1}} \mathbf{H}$ NMR (300 MHz, $\mathbf{C D C l}_{3}$ ): $\delta$ ppm $9.33(\mathrm{~d}, J=4.6 \mathrm{~Hz}, 1 \mathrm{H}), 7.35-7.20(\mathrm{~m}, 3 \mathrm{H}), 7.17-7.06(\mathrm{~m}$, 2H), $2.63(\mathrm{ddd}, J=9.2,6.7,4.0 \mathrm{~Hz}, 1 \mathrm{H}), 2.24-2.11(\mathrm{~m}, 1 \mathrm{H}), 1.74(\mathrm{dt}, J=9.2,5.1 \mathrm{~Hz}$, $1 \mathrm{H}), 1.54$ (ddd, $J=8.3,6.7,4.9 \mathrm{~Hz}, 1 \mathrm{H}$ ). The experimental data are in agreement with the literature reports. ${ }^{32}$

(trans)-(Z/E)-2-(Buta-1,3-dien-1-yl)cyclopropyl)benzene (2n). The Dtan compound was synthesized according to general procedure D was purified by flash column chromatography on silica (pentane $=100 \%$ ) affording 2n (1.06 g, $6.20 \mathrm{mmol}, 68 \%, Z / E$ approx. 1/1) as a colorless liquid. ${ }^{\mathbf{1}} \mathbf{H}$ NMR (300 MHz, CDCl $\left._{3}\right): \delta$ ppm $7.31-7.03(\mathrm{~m}, 10 \mathrm{H}), 6.75(\mathrm{dt}, J=16.5,10.6 \mathrm{~Hz}$, $1 \mathrm{H}), 6.31(\mathrm{dt}, J=16.7,10.3 \mathrm{~Hz}, 1 \mathrm{H}), 6.16(\mathrm{dd}, J=14.9,10.5 \mathrm{~Hz}, 1 \mathrm{H}), 6.01(\mathrm{t}, J=$ $10.9 \mathrm{~Hz}, 1 \mathrm{H}$ ), 5.42 (dd, $J=14.9,8.8 \mathrm{~Hz}, 1 \mathrm{H}$ ), 5.19 (d, $J=17.0 \mathrm{~Hz}, 1 \mathrm{H}$ ), $5.13-4.87$ 
(m, 3H), $2.06-1.90(\mathrm{~m}, 2 \mathrm{H}), 1.69(\mathrm{tt}, J=9.0,4.9 \mathrm{~Hz}, 1 \mathrm{H}), 1.29(\mathrm{ddt}, J=14.1,8.5$, $5.5 \mathrm{~Hz}, 2 \mathrm{H}), 1.11(\mathrm{ddt}, J=13.5,8.7,5.3 \mathrm{~Hz}, 2 \mathrm{H})$. The analytical data are in accordance with the literature reports. ${ }^{33}$

\subsection{General Procedure E: Synthesis of Phenols}

Compounds S3a-g were prepared according to the following synthetic procedures.

1-(3-Hydroxyphenyl)pyrrolidine-2,5-dione (S3a). In a pressure-proof Schlenk tube equipped with a PFTE-coated stirring bar, 3$\pi^{\mathrm{N}} \mathrm{Y}_{\mathrm{s} 3 \mathrm{a}}^{\mathrm{OH}}$ aminophenol (1.83 g, $16.8 \mathrm{mmol}, 1.0$ equiv.) and succinic anhydride (1.68 g, $16.8 \mathrm{mmol}, 1.0$ equiv.) were dissolved in glacial acetic acid (22 $\mathrm{mL}$ ), then the reaction was heated to $120^{\circ} \mathrm{C}$ for 24 hours. The solution was cooled down to room temperature, then poured into ice-cold water ( $80 \mathrm{~mL}$ ). The precipitation was initiated by scratching the vessel with a spatula, then the solid was collected by filtration, washed with water and $n$-pentane, then dried under high vacuum to afford S3a (1.48 g, $6.72 \mathrm{mmol}, 46 \%$ ), as a white solid. $\boldsymbol{R}_{\boldsymbol{f}}$ (pentane/EtOAc = 1/1) 0.16. ${ }^{1}$ H NMR (300 MHz, DMSO-d $): \delta$ ppm $9.71(\mathrm{~s}, 1 \mathrm{H})$, $7.25(\mathrm{t}, J=8.2 \mathrm{~Hz}, 1 \mathrm{H}), 6.89-6.73(\mathrm{~m}, 1 \mathrm{H}), 6.71-6.48(\mathrm{~m}, 2 \mathrm{H}), 2.75(\mathrm{~s}, 4 \mathrm{H})$. The experimental data are in accordance with the literature report. ${ }^{34}$

4-(Adamantan-1-yl)phenol (S3b). In a round-bottom flask equipped with a

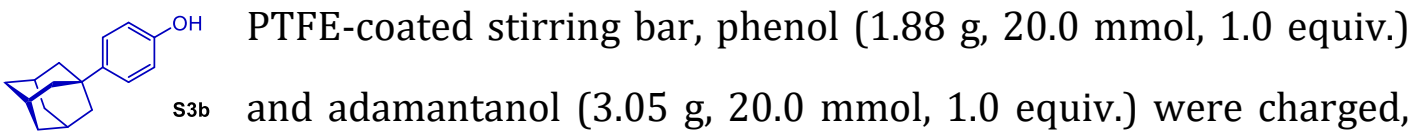
then $\mathrm{CH}_{2} \mathrm{Cl}_{2}(10 \mathrm{~mL})$ was added and the suspension stirred until full dissolution of the reagents. $\mathrm{H}_{2} \mathrm{SO}_{4} 96 \%$ (1.07 mL, $20.0 \mathrm{mmol}, 1.0$ equiv.) was slowly added dropwise (Warning: the process is exothermic, a slight reflux might be observed) and the reaction was stirred overnight at room temperature. The reaction was diluted with $\mathrm{CH}_{2} \mathrm{Cl}_{2}(100 \mathrm{~mL})$, washed with a saturated $\mathrm{NaHCO}_{3}$ solution (50 mL), then the water layer was back-extracted twice with $\mathrm{CH}_{2} \mathrm{Cl}_{2}(50 \mathrm{~mL}$ each time). The combined organic layers were dried over $\mathrm{MgSO}_{4}$, then the solvent was 
removed under reduced pressure. The crude was purified by flash column chromatography on silica (pentane $/ \mathrm{CH}_{2} \mathrm{Cl}_{2}=4 / 1$, then pentane/EtOAc $=10 / 1$ ), to afford a light pink solid which contained approx. $20 \%$ of residual phenol. The residual starting material was removed by crystallization from hot EtOH/ $\mathrm{H}_{2} \mathrm{O}$ $1 / 1$, the crystalline solid collected and washed with ice-cold EtOH/ $\mathrm{H}_{2} \mathrm{O} 1 / 1$, then dried under reduced pressure to afford S3b (1.04 g, $4.53 \mathrm{mmol}, 23 \%)$. Note: the mother liquors still contain considerable amounts of product and can be concentrated and further re-crystallized to maximize the yield. ${ }^{1} \mathbf{H} \mathbf{N M R}(400 \mathbf{M H z}$, $\left.\mathbf{C D C l}_{3}\right): \delta$ ppm $7.25-7.20(\mathrm{~m}, 2 \mathrm{H}), 6.83-6.74(\mathrm{~m}, 2 \mathrm{H}), 4.58(\mathrm{~s}, 1 \mathrm{H}), 2.12-2.04$ $(\mathrm{m}, 3 \mathrm{H}), 1.88(\mathrm{~d}, J=2.9 \mathrm{~Hz}, 6 \mathrm{H}), 1.83-1.68(\mathrm{~m}, 6 \mathrm{H})$. The experimental data are in accordance with the literature report. 35

4-(1-methyl-1H-indol-2-yl)phenol (S3c). The compound was synthesized

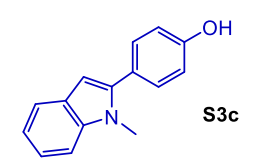
using a slightly modified procedure from Larrosa et al. ${ }^{36}$ In a flame-dried Schlenk tube equipped with a PTFE-coated stirring bar, 4-iodophenol (1.32 g, $6.0 \mathrm{mmol}, 2.0$ equiv.), $N$-Me-indole (375 $\mu \mathrm{L}, 3.0 \mathrm{mmol}, 1.0$ equiv.), $\mathrm{Pd}(\mathrm{OAc})_{2}$ (34 mg, $0.15 \mathrm{mmol}, 5 \mathrm{~mol} \%$ ), $\mathrm{Ag}_{2} \mathrm{O}$ (508 mg, $2.19 \mathrm{mmol}, 0.73$ equiv.) and dry DMF ( $6 \mathrm{~mL}$ ) were charged under argon, then the reaction was stirred at room temperature for 15 hours. The reaction was filtered through a short pad of silica, rinsing with EtOAc. The solvent was removed under reduced pressure, then the residual DMF under high vacuum. The residue was purified by flash column chromatography on silica (pentane/EtOAc $=6 / 1 \rightarrow 5 / 1$ $\rightarrow$ 4/1), affording S3c (343 mg, $1.58 \mathrm{mmol}, 53 \%$ ) as a light-yellow solid. ${ }^{\mathbf{1}} \mathbf{H}$ NMR (400 MHz, $\mathbf{C D C l}_{3}$ ): $\delta$ ppm $7.63(\mathrm{dt}, J=7.8,1.0 \mathrm{~Hz}, 1 \mathrm{H}), 7.42-7.37(\mathrm{~m}, 2 \mathrm{H}), 7.36$ (d, $J=8.3 \mathrm{~Hz}, 1 \mathrm{H}), 7.26-7.21(\mathrm{~m}, 1 \mathrm{H}), 7.14$ (ddd $J=7.9,7.0,1.1 \mathrm{~Hz}, 1 \mathrm{H}), 6.96-$ $6.89(\mathrm{~m}, 2 \mathrm{H}), 6.50(\mathrm{br} \mathrm{s}, 1 \mathrm{H}), 4.91(\mathrm{~s}, 1 \mathrm{H}), 3.73(\mathrm{~s}, 3 \mathrm{H})$. The experimental data are in agreement with the literature reports. ${ }^{37}$

4-(1H-Imidazol-1-yl)phenol (S3d). The compound was synthesized using a S3d 
slightly modified procedure from You et al. ${ }^{38}$ In a flame-dried Schlenk tube equipped with a PTFE-coated stirring bar, 4-bromophenol (519 mg, $3.0 \mathrm{mmol}$, 1.00 equiv.), $1 H$-imidazole (286 mg, $4.2 \mathrm{mmol}, 1.40$ equiv.), CuI (114 mg, 0.6 mmol, $20 \mathrm{~mol} \%$ ), $\mathrm{Cs}_{2} \mathrm{CO}_{3}$ (1.95 g, $6.0 \mathrm{mmol}, 2.00$ equiv.) and dry DMF (6 mL) were charged under argon, then the reaction was stirred at room temperature for 30 minutes and heated at $120^{\circ} \mathrm{C}$ for 40 hours. The reaction mixture was diluted with $\mathrm{HCl} 2 M(60 \mathrm{~mL})$, then washed once with $\mathrm{Et}_{2} \mathrm{O}(60 \mathrm{~mL})$. The water layer was basified up to $\mathrm{pH} 8$ with solid $\mathrm{Na}_{2} \mathrm{CO}_{3}$, then extracted five times with $\mathrm{CH}_{2} \mathrm{Cl}_{2}$ (60 mL each time). The combined organic layers were dried over $\mathrm{MgSO}_{4}$ and the solvent was removed under reduced pressure, the residual DMF under high vacuum. The crude was purified by flash column chromatography on silica $\left(\mathrm{CH}_{2} \mathrm{Cl}_{2} / \mathrm{MeOH}=20 / 1\right)$, affording S3d (223 mg, $\left.1.39 \mathrm{mmol}, 46 \%\right)$ as an off-white solid. ${ }^{1}$ H NMR (400 MHz, DMSO-d6): $\delta$ ppm 9.72 (br s, 1H), 8.07 (br s, 1H), 7.59 (br s, 1H), $7.45-7.34(\mathrm{~m}, 2 \mathrm{H}), 7.07$ (br s, 1H), 6.91 - 6.82 (m, 2H). The experimental data are in agreement with the literature reports. ${ }^{39}$

4-(1H-Pyrrol-1-yl)phenol (S3e). In a Schlenk tube equipped with a PTFEcoated stirring bar, 4-aminophenol (1.09 g, $10.0 \mathrm{mmol}, 1.00$ equiv.), s3e 2,4-dimethoxytetrahydrofuran (1.42 mL, $11.0 \mathrm{mmol}, 1.10$ equiv.) and $\mathrm{AcOH}(15 \mathrm{~mL})$ were charged, then the reaction was stirred at $115^{\circ} \mathrm{C}$ for 90 minutes, then cooled to room temperature. The reaction was quenched by pouring in $100 \mathrm{~mL}$ of ice:water 1:1, then the suspension was neutralized with solid $\mathrm{Na}_{2} \mathrm{CO}_{3}$. The slurry was filtered through a short pad of silica, rinsing with $\mathrm{CH}_{2} \mathrm{Cl}_{2} / \mathrm{MeOH} 9 / 1$. The biphasic mixture was separated, and the organic layer dried over $\mathrm{MgSO}_{4}$, then the solvent was removed under reduced pressure. The brown residue was taken-up in $\mathrm{CH}_{2} \mathrm{Cl}_{2}$ and loaded on silica, then purified by flash column chromatography on silica (pentane/EtOAc $=9 / 1 \rightarrow 7 / 1$ ), affording S3e (780 mg, 4.90 mmol, 49\%) as a blush pink solid. ${ }^{1} \mathbf{H}$ NMR (400 $\mathbf{~ M H z , ~} \mathbf{C D C l}_{3}$ ): $\delta$ ppm $7.29-7.23(\mathrm{~m}, 2 \mathrm{H}), 6.99(\mathrm{t}, J=2.2 \mathrm{~Hz}, 2 \mathrm{H}), 6.91-6.84(\mathrm{~m}, 2 \mathrm{H}), 6.32(\mathrm{t}, J=$ 
$2.2 \mathrm{~Hz}, 2 \mathrm{H}), 4.79(\mathrm{~s}, 1 \mathrm{H})$. The experimental data are in agreement with the literature reports. ${ }^{40}$

4-(Pyridin-2-yl)phenol (S3f). The compound was synthesized using a slightly OH modified procedure from Fairlamb et al.41 In a Schlenk tube
s3f equipped with a PTFE-coated stirring bar, 4-hydroxyphenylboronic acid (1.28 g, 9.28 mmol, 1.50 equiv.), $\mathrm{K}_{3} \mathrm{PO}_{4}$ (2.64 g, $12.4 \mathrm{mmol}, 2.00$ equiv.), 2bromopyridine (590 $\mu \mathrm{L}, 6.19 \mathrm{mmol}, 1.00$ equiv.) and 1,2-propandiol (35 mL) were charged under argon, then the reaction was stirred at $80^{\circ} \mathrm{C}$ for 30 minutes, then cooled to room temperature. The slurry was poured in $1 / 1$ brine $/ \mathrm{H}_{2} \mathrm{O}(120$ $\mathrm{mL})$, then the water layer was extracted four times with $\mathrm{CH}_{2} \mathrm{Cl}_{2}(75 \mathrm{~mL}$ each time). The combined organic layers were dried over $\mathrm{MgSO}_{4}$ and the solvent was removed under reduced pressure. The crude was purified by flash column chromatography on silica (pentane/EtOAc $=7 / 3 \rightarrow 3 / 2 \rightarrow 1 / 1$ ), affording S3f (763 mg, $4.46 \mathrm{mmol}, 73 \%$ ) as a white solid. ${ }^{1} \mathbf{H}$ NMR (400 $\mathbf{M H z}, \mathbf{C D C l}_{3}$ ): $\delta$ ppm $8.63(\mathrm{ddd}, J=4.9,1.8,0.9 \mathrm{~Hz}, 1 \mathrm{H}), 7.85-7.78(\mathrm{~m}, 2 \mathrm{H}), 7.74(\mathrm{ddd}, J=8.0,7.4,1.8$ $\mathrm{Hz}, 1 \mathrm{H}), 7.65(\mathrm{dt}, J=8.0,1.1 \mathrm{~Hz}, 1 \mathrm{H}), 7.20$ (ddd, $J=7.4,4.9,1.2 \mathrm{~Hz}, 1 \mathrm{H}), 6.93-$ $6.76(\mathrm{~m}, 2 \mathrm{H}), 5.30(\mathrm{~s}, 1 \mathrm{H})$. The experimental data are in agreement with the literature reports. ${ }^{42}$

4-(benzo[d]thiazol-2-yl)phenol (S3g). The compound was synthesized according to a procedure reported by Lam et al. ${ }^{43}$ In a round-

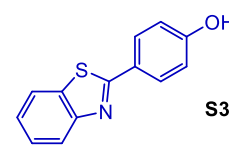
bottom flask equipped with a PTFE-coated stirring bar, 4trifluoromethylphenol (277 mg, $1.71 \mathrm{mmol}, 1.00$ equiv.), 2aminothiophenol (232 $\mu \mathrm{L}, 2.22 \mathrm{mmol}, 1.30$ equiv.) and $\mathrm{NaOH}$ (205 mg, 5.13 mmol, 3.00 equiv.) were charged, then water $(6 \mathrm{~mL})$ was added and the reaction was stirred at $80^{\circ} \mathrm{C}$ overnight. The reaction was cooled to room temperature, then poured into $\mathrm{HCl} 1 M(10 \mathrm{~mL})$ and the precipitated solid was filtered, washed three times with $\mathrm{HCl} 1 M(5 \mathrm{~mL}$ each time), twice with water (5 mL each time) and dried in vacuo, affording S3g as an off-white powder (389 mg, $1.71 \mathrm{mmol}$, > 
99\%). ${ }^{1}$ H NMR (300 MHz, DMSO-d $) \delta$ ppm 10.24 (s, 1H), 8.11 - 7.96 (m, 1H), $7.93(\mathrm{~d}, J=8.5 \mathrm{~Hz}, 2 \mathrm{H}), 7.61-7.29(\mathrm{~m}, 2 \mathrm{H}), 6.93(\mathrm{~d}, J=8.3 \mathrm{~Hz}, 2 \mathrm{H})$. The experimental data are in agreement with the literature reports. ${ }^{44}$

\subsection{General Procedure F: Preparation of Sodium Sulfinate Salts}

Compounds S3h-q were prepared according to the following procedure.

In a round-bottom flask equipped with a PTFE-coated stirring bar, the corresponding sulfonyl chloride (1.00 equiv.), $\mathrm{Na}_{2} \mathrm{SO}_{3}$ (2.00 equiv.), $\mathrm{NaHCO}_{3}$ (2.00 equiv.) and deionized water $(1.0 M)$ were charged, then the reaction was heated at $80^{\circ} \mathrm{C}$ for 4 hours (Warning: $\mathrm{CO}_{2}$ is released during the first minutes of the reaction, do not use sealed vessels.). After cooling at room temperature, the solvent was removed in vacuo, then the residue was taken-up with ethanol (approx. $2.5 \mathrm{~mL} / \mathrm{mmol}$ ), the insoluble inorganic salts were recovered by filtration and the filtrate was evaporated under reduced pressure, affording the corresponding aryl-sulfinate $\mathbf{S 3 h - q .}$

Sodium 4-methoxybenzenesulfinate (S3h). The compound was synthesized is ona according to general procedure F, starting from the corresponding ssh sulfonyl chloride (620 mg, $3.0 \mathrm{mmol}, 1.00$ equiv.), which afforded S3h (398 mg, $2.05 \mathrm{mmol}, 68 \%$ ) as a white solid. ${ }^{1} \mathrm{H}$ NMR (400 MHz, DMSO-d6): $\delta$ ppm $7.39-7.32(\mathrm{~m}, 2 \mathrm{H}), 6.88-6.81(\mathrm{~m}, 2 \mathrm{H}), 3.73(\mathrm{~s}, 3 \mathrm{H})$. The experimental data are in agreement with the literature reports. ${ }^{45}$

Sodium 4-chlorobenzenesulfinate (S3i). The compound was synthesized according to general procedure F, starting from the corresponding
sulfonyl chloride (633 mg, $3.0 \mathrm{mmol}, 1.00$ equiv.), which afforded S3i (410 mg, $2.06 \mathrm{mmol}, 69 \%$ ) as a white solid. ${ }^{\mathbf{1}} \mathbf{H}$ NMR (400 MHz, D 20 ): $\delta \mathrm{ppm}$ $7.62-7.56(\mathrm{~m}, 2 \mathrm{H}), 7.55-7.50(\mathrm{~m}, 2 \mathrm{H})$. The experimental data are in agreement with the literature reports. ${ }^{46}$ 
Sodium 4-(trifluoromethoxy)benzenesulfinate (S3j). The compound was Fis synthesized according to general procedure $F$, starting from the corresponding sulfonyl chloride (782 mg, $3.0 \mathrm{mmol}, 1.00$ equiv.), which afforded S3j (472 mg, $1.90 \mathrm{mmol}, 63 \%$ ) as a white solid. ${ }^{\mathbf{1}} \mathbf{H}$ NMR (500 MHz, DMSO-d $): \delta$ ppm $7.60-7.55(\mathrm{~m}, 2 \mathrm{H}), 7.31-7.26(\mathrm{~m}, 2 \mathrm{H})$. ${ }^{13} \mathbf{C}\left\{{ }^{1} \mathbf{H},{ }^{19}\right.$ F $\}$ NMR (126 MHz, DMSO-d 6 ): $\delta$ 159.5, 147.8, 126.2, 120.2, 120.1. ${ }^{13} \mathbf{C}$ NMR (126 MHz, DMSO-d $)$ ): $\delta$ 159.5, 147.8, 126.2, 120.2, 120.1 (q, ${ }^{1} J_{C-F}=255.8$ $\mathrm{Hz}$ ). The experimental data are in agreement with the literature reports. ${ }^{47}$

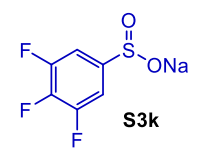

sodium 3,4,5-trifluorobenzenesulfinate (S3k). The compound was synthesized according to general procedure F, starting from the corresponding sulfonyl chloride (692 mg, $3.0 \mathrm{mmol}, 1.00$ equiv.), which afforded S3k (557 mg, $2.55 \mathrm{mmol}, 85 \%$ ) as a white solid. ${ }^{\mathbf{1}} \mathbf{H}$ NMR (500

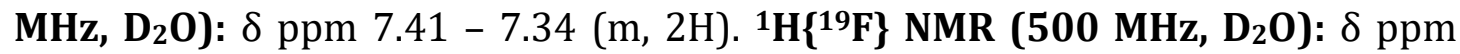
7.38 (s, 2H). ${ }^{19}$ F NMR (470 MHz, D 20$): \delta$ ppm -132.72 (d, ${ }^{3} J_{F-F}=20.2 \mathrm{~Hz}$ ), $158.21\left(\mathrm{t},{ }^{3} J_{F-F}=20.1 \mathrm{~Hz}\right) \cdot{ }^{13} \mathbf{C}\left\{{ }^{\mathbf{1}} \mathbf{H},{ }^{19} \mathbf{F}\right\}$ NMR (126 MHz, $\left.\mathbf{D}_{2} \mathbf{0}\right): \delta$ ppm 151.0, 150.3, 140.6, 108.2. ${ }^{13}$ C NMR (126 MHz, D 20 ): $\delta$ ppm 151.0 (ddd, $J=253.1,10.7,2.6$ Hz), $150.4-150.20$ (m), 140.6 (dt, $J=251.2,15.7 \mathrm{~Hz}$ ), 108.2 (dd, $J=16.9,5.1 \mathrm{~Hz}$ ). HRMS (ESI-): calcd. for $\mathrm{C}_{6} \mathrm{H}_{2} \mathrm{~F}_{3} \mathrm{O}_{2} \mathrm{~S}$ (M-): 194.9733. Found: 194.9756.

3,4-dimethoxybenzenesulfonyl chloride (S3I'). The compound was

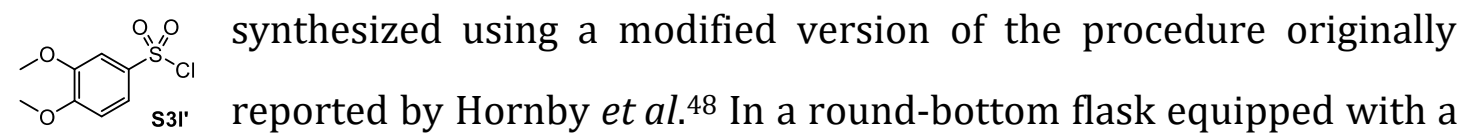
PTFE-coated stirring bar, 1,2-dimethoxybenzene (1.38 g, $10.0 \mathrm{mmol}, 1.00$ equiv.) was treated under vigorous stirring with chlorosulfonic acid (1.43 mL, 21.5 mmol, 2.15 equiv.) (WARNING: exothermic reaction with liberation of $\mathrm{HCl}$ ). Upon completion of the addition, the reaction was cooled to $0^{\circ} \mathrm{C}$ and ice $(20 \mathrm{~g}$ ) was added, then the suspension was warmed at room temperature, extracted twice with $\mathrm{CH}_{2} \mathrm{Cl}_{2}$ (40 mL each time) and the combined organic layers were dried over $\mathrm{MgSO}_{4}$. The solvent was removed under reduced pressure, then the crude was 
purified by flash column chromatography on silica (pentane/EtOAc 5/1), to afford S3l' (1.64 g, 6.93 mmol, 69\%) as a light-yellow crystalline solid. ${ }^{1} \mathbf{H}$ NMR (300 MHz, $\mathbf{C D C l}_{3}$ ): $\delta$ ppm 7.69 (dd, $\left.J=8.6,2.3 \mathrm{~Hz}, 1 \mathrm{H}\right), 7.44(\mathrm{~d}, J=2.3 \mathrm{~Hz}, 1 \mathrm{H}$ ), $7.00(\mathrm{~d}, J=8.7 \mathrm{~Hz}, 1 \mathrm{H}), 3.99(\mathrm{~s}, 3 \mathrm{H}), 3.98(\mathrm{~s}, 3 \mathrm{H})$. The experimental data are in agreement with the literature reports. ${ }^{49}$

Sodium 3,4-dimethoxybenzenesulfinate (S31). The compound was synthesized according to general procedure $\mathrm{F}$, starting from the corresponding sulfonyl chloride (1.63 g, $6.88 \mathrm{mmol}, 1.00$ equiv.), which afforded S31 (279 mg, $1.25 \mathrm{mmol}, 18 \%)$ as an off-white solid. Note: The somehow reduced yield is caused by the increased polarity of the product, which caused difficult solid phase extraction with the organic solvent. ${ }^{1} \mathbf{H}$ NMR $(400 \mathbf{M H z}$, $\mathbf{D}_{2} \mathbf{0}$ ): $\delta$ ppm $7.23-7.20$ (m, 1H), $7.18(\mathrm{dd}, J=8.2,1.9 \mathrm{~Hz}, 1 \mathrm{H}$ ), 7.04 (dd, $J=8.4$, $2.0 \mathrm{~Hz}, 1 \mathrm{H}), 3.87$ (s, 3H), 3.83 (s, 3H). ${ }^{13} \mathbf{C}$ NMR (101 MHz, D 20$): \delta$ ppm 149.7, 148.3, 146.4, 117.1, 111.5, 106.2, 55.8, 55.7. HRMS (ESI-): calcd. for $\mathrm{C}_{8} \mathrm{H}_{9} \mathrm{O}_{4} \mathrm{~S}\left(\mathrm{M}^{-}\right)$: 201.0216. Found: 201.0245 .

2,4-Dimethylbenzenesulfonyl chloride (S3m'). The compound was synthesized using a modified version of the procedure originally with a PTFE-coated stirring bar, $m$-xylene $(1.06 \mathrm{~g}, 10.0 \mathrm{mmol}$, 1.00 equiv.) was cooled to $0^{\circ} \mathrm{C}$, then treated under vigorous stirring with chlorosulfonic acid (2.04 mL, $30.6 \mathrm{mmol}, 3.06$ equiv.) (WARNING: exothermic reaction with liberation of $\mathrm{HCl}$. Upon completion of the addition, the reaction was stirred at the same temperature for 5 minutes and ice $(20 \mathrm{~g})$ was added, then the suspension was warmed at room temperature, extracted twice with $\mathrm{CH}_{2} \mathrm{Cl}_{2}$ (40 mL each time) and the combined organic layers were dried over $\mathrm{MgSO}_{4}$. The solvent was removed under reduced pressure, then the crude was purified by flash column chromatography on silica (pentane/Et0Ac 50/1), to afford SI-S3m (1.53 g, 7.49 mmol, 75\%) as a colorless liquid. ${ }^{1} \mathbf{H}$ NMR (300 MHz, 
$\mathbf{C D C l}_{3}$ ): $\delta$ ppm $7.94(\mathrm{~d}, J=8.1 \mathrm{~Hz}, 1 \mathrm{H}), 7.24-7.16(\mathrm{~m}, 2 \mathrm{H}), 2.74(\mathrm{~s}, 3 \mathrm{H}), 2.43(\mathrm{~s}$, $3 \mathrm{H})$. The experimental data are in agreement with the literature reports. ${ }^{51}$

Sodium 2,4-dimethylbenzenesulfinate (S3m). The compound was synthesized

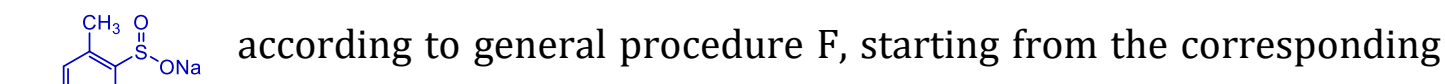
s3m sulfonyl chloride (1.52 g, $7.43 \mathrm{mmol}, 1.0$ equiv.), which afforded S3m (890 mg, $4.63 \mathrm{mmol}, 62 \%$ ) as a white solid. ${ }^{1} \mathrm{H}$ NMR (400 MHz, D 2 ): $\delta$ ppm 7.55 (d, $J=7.9 \mathrm{~Hz}, 1 \mathrm{H}), 7.16(\mathrm{~d}, J=7.9 \mathrm{~Hz}, 1 \mathrm{H}), 7.04(\mathrm{~s}, 1 \mathrm{H}), 2.43(\mathrm{~s}, 3 \mathrm{H}), 2.28$ (s, 3H). ${ }^{13}$ C NMR (101 MHz, D 20$): \delta$ ppm 147.8, 140.9, 135.6, 131.4, 127.1, 121.4, 20.3, 17.2. HRMS (ESI-): calcd. for $\mathrm{C}_{8} \mathrm{H}_{9} \mathrm{O}_{2} \mathrm{~S}\left(\mathrm{M}^{-}\right)$: 169.0318 . Found: 169.0350 .

4-Cyclohexylbenzenesulfonyl chloride (S3n'). The compound was synthesized

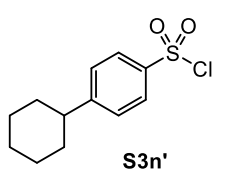
using a modified version of the procedure originally reported by Jha et al. ${ }^{52}$ In a round-bottom flask equipped with a PTFE-coated stirring bar, cyclohexylbenzene (1.60 g, $10.0 \mathrm{mmol}, 1.00$ equiv.) was dissolved in dry $\mathrm{CH}_{2} \mathrm{Cl}_{2}(3 \mathrm{~mL})$ and cooled to $0^{\circ} \mathrm{C}$, then treated under vigorous stirring with chlorosulfonic acid (2.00 mL, $30.0 \mathrm{mmol}, 3.00$ equiv.) (WARNING: exothermic reaction with liberation of $\mathrm{HCl}$ ). Upon completion of the addition, the reaction was stirred at the same temperature for 5 minutes and ice (20 g) was added, then the suspension was warmed at room temperature, extracted twice with $\mathrm{CH}_{2} \mathrm{Cl}_{2}$ (40 mL each time) (Note: Formation of an emulsion due can be reduced using large amounts of water to dissolve the generated salts) and the combined organic layers were dried over $\mathrm{MgSO}_{4}$. The solvent was removed under reduced pressure, then the crude was purified by flash column chromatography on silica (pentane/EtOAc 50/1), to afford S3n' (520 mg, 2.01 mmol, 20\%) as a colorless viscous oil, which solidified upon standing. ${ }^{\mathbf{1}} \mathbf{H}$ NMR (300 MHz, $\mathbf{C D C l}_{3}$ ): $\delta$ ppm $7.99-7.90(\mathrm{~m}, 2 \mathrm{H}), 7.47-7.39(\mathrm{~m}, 2 \mathrm{H}), 2.71-2.56(\mathrm{~m}$, $0 \mathrm{H}), 1.96-1.72(\mathrm{~m}, 6 \mathrm{H}), 1.54-1.20(\mathrm{~m}, 4 \mathrm{H})$. The experimental data are in agreement with the literature reports. ${ }^{53}$ 
Sodium 4-cyclohexylbenzenesulfinate (S3n). The compound was synthesized $\underbrace{\mathrm{O} 3 \mathrm{ONa}}_{\mathrm{S3n}}$ according to general procedure $F$, starting from the corresponding sulfonyl chloride (515 mg, $1.99 \mathrm{mmol}, 1.00$ equiv.), which afforded S3n (334 mg, $1.36 \mathrm{mmol}, 68 \%$ ) as a white solid.

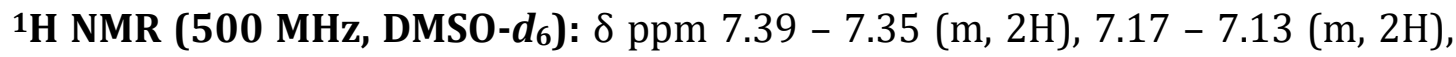
$2.49-2.43(\mathrm{~m}, 1 \mathrm{H}), 1.81-1.73(\mathrm{~m}, 4 \mathrm{H}), 1.70(\mathrm{ddq}, J=12.6,3.1,1.6 \mathrm{~Hz}, 1 \mathrm{H}), 1.45$ - 1.30 (m, 4H), 1.29 - 1.19 (m, 1H). ${ }^{13}$ C NMR (126 MHz, DMSO-d $): \delta$ ppm 157.4, 147.1, 125.9, 124.2, 43.7, 34.1, 26.4, 25.6. HRMS (ESI+): calcd. for $\mathrm{C}_{12} \mathrm{H}_{15} \mathrm{O}_{2} \mathrm{SNa}_{2}$ $\left(\mathrm{M}+2 \mathrm{Na}^{+}\right): 269.0583$. Found: 269.0584 .

2-Methoxy-5-methylbenzenesulfonyl chloride (S3o'). The compound was O O synthesized using a modified version of the procedure originally sзo' reported by Carreira et al. ${ }^{54}$ In oven-dried Schlenk tube with a PTFEcoated stirring bar, chlorosulfonic acid (6.65 mL, $100.0 \mathrm{mmol}, 5.00$ equiv.) was charged under argon, then cooled to $0^{\circ} \mathrm{C}$, then 4-methylanisole $(2.55 \mathrm{~mL}, 20.0$ mmol, 1.00 equiv.) was added dropwise over 20 minutes (Warning: strong evolution of $\mathrm{HCl}$ ). The reaction was stirred at $0^{\circ} \mathrm{C}$ for 30 minutes, then at room temperature overnight. The solution was cooled to $0^{\circ} \mathrm{C}$ and carefully quenched by the addition of ice ( $20 \mathrm{~g}$ ), extracted twice with $\mathrm{CH}_{2} \mathrm{Cl}_{2}$ (40 mL each time) and the combined organic layers were dried over $\mathrm{MgSO}_{4}$. The solvent was removed under reduced pressure, then the crude was purified by flash column chromatography on silica (pentane/EtOAc 6/1 $\rightarrow$ 5/1), to afford SI-S3o (2.83 g, $12.8 \mathrm{mmol}, 64 \%$ ) as a white crystalline solid. ${ }^{1} \mathbf{H}$ NMR (300 $\mathbf{M H z}, \mathbf{C D C l}_{3}$ ): $\delta$ ppm $7.76(\mathrm{~d}, J=1.4 \mathrm{~Hz}, 1 \mathrm{H}), 7.47(\mathrm{ddd}, J=8.5,2.2,0.6 \mathrm{~Hz}, 1 \mathrm{H}), 7.02(\mathrm{~d}, J=8.5 \mathrm{~Hz}, 1 \mathrm{H})$, $4.03(\mathrm{~s}, 3 \mathrm{H}), 2.48-2.17(\mathrm{~m}, 7 \mathrm{H})$. The experimental data are in agreement with the literature reports. ${ }^{54}$

Sodium 2-methoxy-5-methylbenzenesulfinate (S30). The compound was $\underbrace{11}_{\text {S3o }}$ synthesized according to general procedure F, starting from the 
corresponding sulfonyl chloride (2.81 g, $12.7 \mathrm{mmol}, 1.00$ equiv.), which afforded S3o (890 mg, $4.28 \mathrm{mmol}, 34 \%$ ) as a white solid. ${ }^{\mathbf{1}} \mathbf{H}$ NMR (500 MHz, $\mathbf{D}_{2}$ O): $\delta$ ppm $7.47(\mathrm{~d}, J=8.3 \mathrm{~Hz}, 1 \mathrm{H}), 7.31(\mathrm{ddd}, J=8.4,2.3,0.6 \mathrm{~Hz}, 1 \mathrm{H}), 7.03(\mathrm{~d}, J=8.4 \mathrm{~Hz}, 1 \mathrm{H})$, 3.88 (s, 3H), 2.35 (br s, 3H). ${ }^{13}$ C NMR (126 MHz, D 20$): \delta$ ppm 154.6, 140.4, 132.3, 131.3, 122.9, 112.4, 56.0, 19.7. HRMS (ESI+): calcd. for $\mathrm{C}_{8} \mathrm{H}_{9} \mathrm{O}_{3} \mathrm{SNa}_{2}\left(\mathrm{M}+2 \mathrm{Na}^{+}\right)$: 231.0062. Found: 231.0066.

Sodium propane-2-sulfinate (S3p). The compound was synthesized according $Y_{\text {ONa }}^{\text {I. }}$ to general procedure $F$, starting from the corresponding sulfonyl s3p $\quad$ chloride (1.43 mg, $10.0 \mathrm{mmol}, 1.00$ equiv.), which afforded S3p (1.26 g, 9.64 mmol, 96\%) as a white solid. ${ }^{1} \mathbf{H}$ NMR (300 MHz, $\left.\mathbf{D}_{2} \mathbf{0}\right): \delta$ ppm 2.16 (hept, $J$ $=6.9 \mathrm{~Hz}, 1 \mathrm{H}), 1.03(\mathrm{~d}, J=7.0 \mathrm{~Hz}, 6 \mathrm{H})$. The experimental data are in agreement with the literature reports. ${ }^{55}$

Sodium

$((1 R, 4 R)-7,7-d i m e t h y l-2-o x o b i c y c l o[2.2 .1]$ heptan-1yl)methanesulfinate (S3q). The compound was synthesized according to ona general procedure $\mathrm{F}$, starting from the corresponding sulfonyl chloride (1.25 g, 5.00 mmol, 1.00 equiv.), which afforded S3q (831 mg, $3.86 \mathrm{mmol}$, 77\%) as a white solid. ${ }^{\mathbf{1}} \mathbf{H}$ NMR (400 MHz, $\left.\mathbf{D}_{2} \mathbf{0}\right): \delta$ ppm $2.67(\mathrm{~d}, \mathrm{~J}=13.4$ Hz, 1H), $2.52-2.45$ (m, 1H), 2.22 (d, J =13.4 Hz, 1H), $2.20-2.15$ (m, 1H), $2.15-$ $2.11(\mathrm{~m}, 1 \mathrm{H}), 2.10-2.01(\mathrm{~m}, 1 \mathrm{H}), 1.98(\mathrm{~d}, J=9.4 \mathrm{~Hz}, 1 \mathrm{H}), 1.62-1.52(\mathrm{~m}, 1 \mathrm{H})$, $1.50-1.42(\mathrm{~m}, 1 \mathrm{H}), 1.03(\mathrm{~s}, 3 \mathrm{H}), 0.92(\mathrm{~s}, 2 \mathrm{H})$. The experimental data are in agreement with the literature reports. ${ }^{55}$ 


\section{Radical Interrupted Heck/Allylic Substitution Cascade}

\subsection{General Procedure G}

An oven-dried Schlenk tube containing a stir bar was charged under argon with the corresponding alkyl bromide 1 (0.3 mmol, 1.5 equiv.), $\mathrm{Pd}\left(\mathrm{PPh}_{3}\right)_{4}(10 \mathrm{~mol} \%)$, BINAP (12 mol\%), nitrogen based nucleophile (0.2 mmol, 1.0 equiv.), KOAc or $\mathrm{NaOH}(150 \mathrm{~mol} \%)$ and DMA $(0.85 \mathrm{~mL})$. Subsequently, butadiene 2a (0.3 mmol, $0.15 \mathrm{~mL}, 2 \mathrm{M}$ in THF, 1.5 equiv.) was added. The reaction mixture was stirred at room temperate under blue LEDs irradiation (5W, $455 \mathrm{~nm})$ for $48 \mathrm{~h}$. Then, the reaction was filtered through a pad of silica gel and washed with EtOAc $(3 \times 5$ $\mathrm{mL}$ ). The obtained solution was concentrated in vacuo and the diastereomeric ratio determined by ${ }^{1} \mathrm{H}$ NMR spectroscopy of the crude reaction mixture. The reaction mixture was purified by column chromatography on silica gel (pentane/EtOAc) to afford the corresponding compounds.

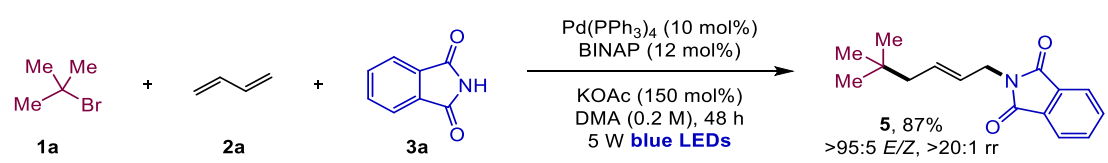

(E)-2-(5,5-Dimethylhex-2-en-1-yl)isoindoline-1,3-dione (5). According to the general procedure $\mathrm{G}$, using the corresponding alkyl bromide 1 a $(0.3 \mathrm{mmol}, 1.5$ equiv.), butadiene 2a ( $0.3 \mathrm{mmol}, 0.15 \mathrm{~mL}, 2 \mathrm{M}$ in THF, 1.5 equiv.), phthalimide (0.2 mmol, 1.0 equiv.), $\mathrm{Pd}\left(\mathrm{PPh}_{3}\right)_{4}(10 \mathrm{~mol} \%)$, BINAP (12 mol\%), KOAc (150 mol\%), and stirring at room temperature for $48 \mathrm{~h}$ under $5 \mathrm{~W}$ blue LEDs irradiation. The reaction mixture was filtered through a pad of silica gel and concentrated in vacuo, then purified by chromatography (pentane/EtOAc $=20 / 1)$ to give $\mathbf{5}(44.8$ mg, $0.174 \mathrm{mmol}, 87 \%,>95: 5 \mathrm{E} / Z$, > 20:1 rr) as a colorless oil. $\mathbf{R}_{\boldsymbol{f}}$ (pentane/EtOAc = 9/1) 0.36. ${ }^{1} \mathrm{H}$ NMR (400 MHz, $\mathbf{C D C l}_{3}$ ): $\delta$ ppm $7.83(\mathrm{dd}, J=5.4,3.0 \mathrm{~Hz}, 2 \mathrm{H}), 7.70$ $(\mathrm{dd}, J=5.4,3.0 \mathrm{~Hz}, 2 \mathrm{H}), 5.86-5.67(\mathrm{~m}, 1 \mathrm{H}), 5.55-5.38(\mathrm{~m}, 1 \mathrm{H}), 4.24(\mathrm{dt}, J=6.4$, $1.1 \mathrm{~Hz}, 2 \mathrm{H}), 1.87$ (dd, $J=7.5,1.2 \mathrm{~Hz}, 2 \mathrm{H}), 0.84(\mathrm{~s}, 9 \mathrm{H}) .{ }^{13} \mathbf{C} \mathbf{~ N M R}\left(101 \mathrm{MHz}, \mathbf{C D C l}_{3}\right)$ : $\delta$ ppm $168.1(2 \times \mathrm{C}), 134.0(2 \times \mathrm{C}), 132.6,132.3(2 \times \mathrm{C}), 125.4,123.3(2 \times \mathrm{C}), 46.7$, 
39.8, 31.0, $29.4(3 \times \mathrm{C})$. HRMS (ESI $\left.{ }^{+}\right)$: calcd. for $\mathrm{C}_{16} \mathrm{H}_{19} \mathrm{NO}_{2} \mathrm{Na}\left(\mathrm{M}+\mathrm{Na}^{+}\right)$: 280.1308. Found: 280.1306.

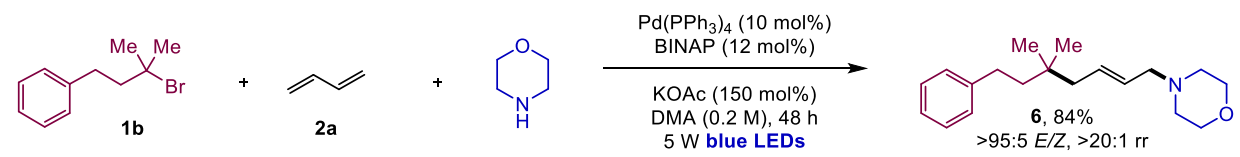

(E)-4-(5,5-Dimethyl-7-phenylhept-2-en-1-yl)morpholine (6). According to the general procedure G, using the corresponding alkyl bromide $\mathbf{1 b}(0.3 \mathrm{mmol}$, 1.5 equiv.), butadiene 2 a ( $0.3 \mathrm{mmol}, 0.15 \mathrm{~mL}, 2 \mathrm{M}$ in THF, 1.5 equiv.), morpholine (0.2 mmol, 1.0 equiv.), $\mathrm{Pd}\left(\mathrm{PPh}_{3}\right)_{4}(10 \mathrm{~mol} \%)$, BINAP (12 mol\%), KOAc (150 mol\%), and stirring at room temperature for $48 \mathrm{~h}$ under $5 \mathrm{~W}$ blue LEDs irradiation. The reaction mixture was filtered through a pad of silica gel and concentrated in vacuo, then purified by chromatography (pentane/EtOAc/ $\mathrm{Et}_{3} \mathrm{~N}=9 / 1 / 0.5$ ) to give 6 (48.3 mg, $0.168 \mathrm{mmol}, 84 \%,>95: 5 \mathrm{E} / \mathrm{Z},>20: 1 \mathrm{rr}$ ) as a colorless oil. $\mathbf{R}_{\boldsymbol{f}}$ (pentane/EtOAc/Et $\left.{ }_{3} \mathrm{~N}=9 / 1 / 0.5\right)$ 0.36. ${ }^{1} \mathbf{H}$ NMR (599 MHz, $\left.\mathbf{C D C l}_{3}\right) \delta 7.36(\mathrm{dd}, J=$ 8.6, 6.7 Hz, 2H), $7.28-7.24(\mathrm{~m}, 3 \mathrm{H}), 5.78-5.72(\mathrm{~m}, 1 \mathrm{H}), 5.63-5.57(\mathrm{~m}, 1 \mathrm{H}), 3.80$ $(\mathrm{t}, J=4.7 \mathrm{~Hz}, 4 \mathrm{H}), 3.11-3.04(\mathrm{~m}, 2 \mathrm{H}), 2.72-2.61(\mathrm{~m}, 2 \mathrm{H}), 2.54(\mathrm{~s}, 4 \mathrm{H}), 2.13-$ $2.10(\mathrm{~m}, 2 \mathrm{H}), 1.60-1.56(\mathrm{~m}, 2 \mathrm{H}), 1.03(\mathrm{~s}, 6 \mathrm{H}) .{ }^{13} \mathbf{C}$ NMR (151 MHz, $\left.\mathbf{C D C l}_{3}\right) \delta$ 143.4, 131.8, 128.4, 128.3, 125.7, 67.0, 61.5, 53.6, 44.9, 44.2, 33.6, 30.7, 27.2. HRMS (ESI+): calcd. for $\mathrm{C}_{19} \mathrm{H}_{30} \mathrm{NO}\left(\mathrm{M}+\mathrm{H}^{+}\right)$: 288.2333. Found: 288.2337.

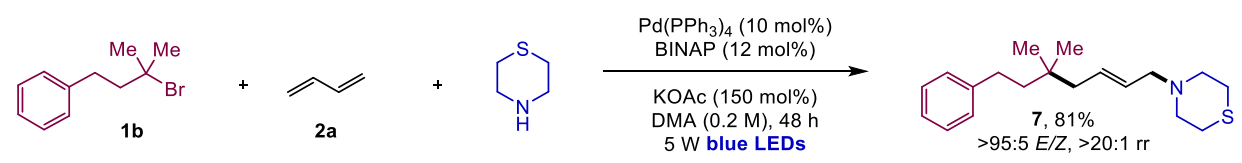

(E)-4-(5,5-Dimethyl-7-phenylhept-2-en-1-yl)thiomorpholine (7). According to the general procedure $G$, using the corresponding alkyl bromide $\mathbf{1 b}(0.3 \mathrm{mmol}$, 1.5 equiv.), butadiene $2 \mathrm{a}$ ( $0.3 \mathrm{mmol}, 0.15 \mathrm{~mL}, 2 M$ in THF, 1.5 equiv.), thiomorpholine (0.2 mmol, 1.0 equiv.), $\mathrm{Pd}\left(\mathrm{PPh}_{3}\right)_{4}(10 \mathrm{~mol} \%)$, BINAP (12 mol\%), KOAc (150 mol\%), and stirring at room temperature for $48 \mathrm{~h}$ under $5 \mathrm{~W}$ blue LEDs irradiation. The reaction mixture was filtered through a pad of silica gel and concentrated in vacuo, then purified by chromatography (pentane/EtOAc/Et $3 \mathrm{~N}=$ 9/1/0.5) to give 7 (48.9 $\mathrm{mg}, 0.161 \mathrm{mmol}, 81 \%,>95: 5 E / Z,>20: 1 \mathrm{rr}$ ) as a 
colorless oil. $\mathbf{R}_{\boldsymbol{f}}$ (pentane/EtOAc/Et $\left.{ }_{3} \mathrm{~N}=9 / 1 / 0.5\right)$ 0.53. ${ }^{\mathbf{1}} \mathbf{H} \mathbf{~ N M R}\left(599 \mathbf{~ M H z}, \mathbf{C D C l}_{3}\right.$ ) $\delta 7.38-7.32(\mathrm{~m}, 2 \mathrm{H}), 7.29-7.24(\mathrm{~m}, 3 \mathrm{H}), 5.71(\mathrm{ddt}, J=14.8,7.4,1.2 \mathrm{~Hz}, 1 \mathrm{H}), 5.61$ - $5.55(\mathrm{~m}, 1 \mathrm{H}), 3.08(\mathrm{dq}, J=6.6,0.9 \mathrm{~Hz}, 2 \mathrm{H}), 2.83-2.72(\mathrm{~m}, 8 \mathrm{H}), 2.68-2.61(\mathrm{~m}$, 2H), $2.11(\mathrm{dt}, J=7.5,1.1 \mathrm{~Hz}, 2 \mathrm{H}), 1.62-1.55(\mathrm{~m}, 2 \mathrm{H}), 1.03(\mathrm{~s}, 6 \mathrm{H}) .{ }^{13}$ C NMR (151 MHz, $\left.\mathbf{C D C l}_{3}\right) \delta 143.4,131.5,128.8,128.4,128.4,125.7,61.9,54.9,44.9,44.2,33.6$, 30.7, 28.1, 27.2. HRMS (ESI'): calcd. for $\mathrm{C}_{19} \mathrm{H}_{30} \mathrm{NS}\left(\mathrm{M}+\mathrm{H}^{+}\right)$: 304.2093. Found: 304.2112 .

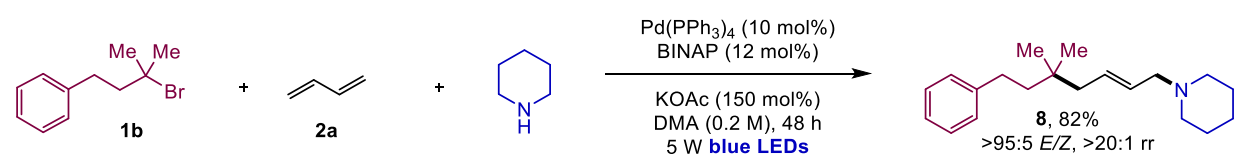

(E)-1-(5,5-Dimethyl-7-phenylhept-2-en-1-yl)piperidine (8). According to the general procedure $\mathrm{G}$, using the corresponding alkyl bromide $\mathbf{1 b}(0.3 \mathrm{mmol}, 1.5$ equiv.), butadiene 2 a ( $0.3 \mathrm{mmol}, 0.15 \mathrm{~mL}, 2 \mathrm{M}$ in THF, 1.5 equiv.), piperidine $(0.2$ mmol, 1.0 equiv.), $\mathrm{Pd}\left(\mathrm{PPh}_{3}\right)_{4}(10 \mathrm{~mol} \%)$, BINAP (12 mol\%), KOAc (150 mol\%), and stirring at room temperature for $48 \mathrm{~h}$ under $5 \mathrm{~W}$ blue LEDs irradiation. The reaction mixture was filtered through a pad of silica gel and concentrated in vacuo, then purified by chromatography (pentane/EtOAc/ $\mathrm{Et}_{3} \mathrm{~N}=9 / 1 / 0.5$ ) to give 8 (46.9 mg, $0.165 \mathrm{mmol}, 82 \%,>95: 5 \mathrm{E} / \mathrm{Z},>20: 1 \mathrm{rr}$ ) as a colorless oil. $\mathbf{R}_{\boldsymbol{f}}$ (pentane/EtOAc/Et $3 \mathrm{~N}=$ 9/1/0.5) 0.67. ${ }^{1} \mathbf{H}$ NMR (599 $\left.\mathbf{~ M H z , ~} \mathbf{C D C l}_{3}\right) \delta 7.38-7.32$ $(\mathrm{m}, 2 \mathrm{H}), 7.32-7.23(\mathrm{~m}, 3 \mathrm{H}), 5.76-5.61(\mathrm{~m}, 2 \mathrm{H}), 3.08-2.99(\mathrm{~m}, 2 \mathrm{H}), 2.69-2.59$ (m, 2H), $2.55-2.40(\mathrm{~m}, 4 \mathrm{H}), 2.15-2.08$ (m, 2H), 1.67 (p, J = 5.6 Hz, 4H), $1.64-$ $1.57(\mathrm{~m}, 2 \mathrm{H}), 1.56-1.48(\mathrm{~m}, 2 \mathrm{H}), 1.03(\mathrm{~s}, 6 \mathrm{H}) .{ }^{13} \mathbf{C}$ NMR (151 $\left.\mathbf{M H z}, \mathbf{C D C l}_{3}\right) \delta$ 143.5, 130.7, 129.6, 128.4, 128.4, 125.6, 62.0, 54.5, 44.9, 44.2, 33.6, 30.8, 27.2, 26.1, 24.5. HRMS (ESI+): calcd. for $\mathrm{C}_{20} \mathrm{H}_{32} \mathrm{~N}\left(\mathrm{M}+\mathrm{H}^{+}\right)$: 286.2529. Found: 286.2548.

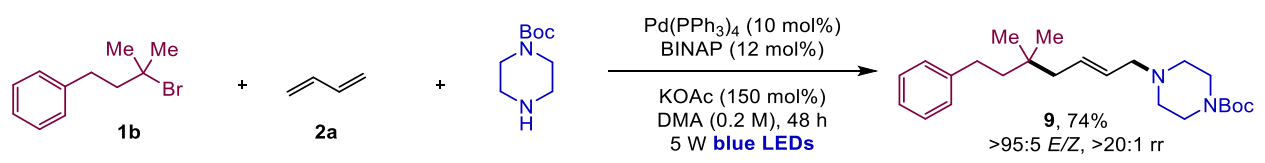

tert-Butyl

(E)-4-(5,5-dimethyl-7-phenylhept-2-en-1-yl)piperazine-1-

carboxylate (9). According to the general procedure G, using the corresponding alkyl bromide $\mathbf{1 b}$ ( $0.3 \mathrm{mmol}, 1.5$ equiv.), butadiene $\mathbf{2 a}(0.3 \mathrm{mmol}, 0.15 \mathrm{~mL}, 2 \mathrm{M}$ in S 38 
THF, 1.5 equiv.), Boc-piperazine ( $0.2 \mathrm{mmol}, 1.0$ equiv.), $\mathrm{Pd}\left(\mathrm{PPh}_{3}\right)_{4}(10 \mathrm{~mol} \%)$, BINAP (12 mol\%), KOAc (150 mol\%), and stirring at room temperature for $48 \mathrm{~h}$ under $5 \mathrm{~W}$ blue LEDs irradiation. The reaction mixture was filtered through a pad of silica gel and concentrated in vacuo, then purified by chromatography (pentane/EtOAc/Et $\left.{ }_{3} \mathrm{~N}=9 / 1 / 0.5\right)$ to give 9 (57,4 mg, 0.149 mmol, 74\%, > 95:5 $E / Z,>20: 1 \mathrm{rr}$ ) as a colorless oil. $\mathbf{R}_{\boldsymbol{f}}$ (pentane/EtOAc/Et $\left.3 \mathrm{~N}=9 / 1 / 0.5\right) 0.50 .{ }^{1} \mathbf{H}$ NMR (599 MHz, $\left.\mathbf{C D C l}_{3}\right) \delta 7.42-7.35(\mathrm{~m}, 2 \mathrm{H}), 7.30-7.21(\mathrm{~m}, 3 \mathrm{H}), 5.81-5.70(\mathrm{~m}$, 1H), $5.64-5.55(\mathrm{~m}, 1 \mathrm{H}), 3.52(\mathrm{t}, J=5.3 \mathrm{~Hz}, 4 \mathrm{H}), 3.07(\mathrm{dd}, J=6.6,1.2 \mathrm{~Hz}, 2 \mathrm{H}), 2.73$ - $2.62(\mathrm{~m}, 2 \mathrm{H}), 2.47$ (s, 4H), 2.11 (dd, J = 7.4, $1.2 \mathrm{~Hz}, 2 \mathrm{H}), 1.61-1.56(\mathrm{~m}, 2 \mathrm{H}), 1.55$ (s, 9H), 1.03 (s, 6H). ${ }^{13}$ C NMR (151 $\left.\mathbf{~ M H z , ~} \mathbf{C D C l}_{3}\right) \delta$ 154.9, 143.5, 131.6, 128.8, 128.5, 128.4, 125.7, 79.7, 61.2, 53.0, 44.9, 44.2, 33.6, 30.8, 28.6, 27.2. HRMS (ESI ${ }^{+}$): calcd. for $\mathrm{C}_{24} \mathrm{H}_{39} \mathrm{~N}_{2} \mathrm{O}_{2}\left(\mathrm{M}+\mathrm{H}^{+}\right)$: 387.3006. Found: 387.3017.
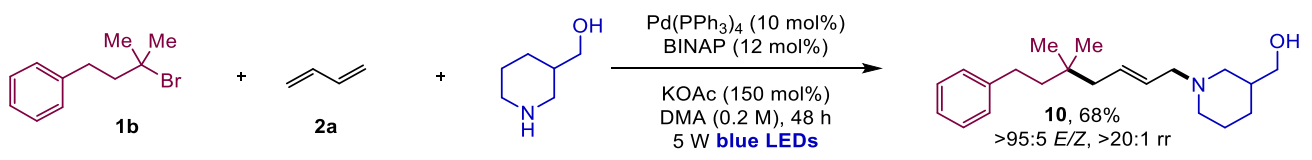

(E)-(1-(5,5-Dimethyl-7-phenylhept-2-en-1-yl)piperidin-3-yl)methanol (10).

According to the general procedure G, using the corresponding alkyl bromide $\mathbf{1 b}$ ( $0.3 \mathrm{mmol}, 1.5$ equiv.), butadiene $2 \mathrm{a}(0.3 \mathrm{mmol}, 0.15 \mathrm{~mL}, 2 \mathrm{M}$ in THF, 1.5 equiv.), the nitrogen based nucleophile 3 (0.2 mmol, 1.0 equiv.), $\mathrm{Pd}\left(\mathrm{PPh}_{3}\right)_{4}(10 \mathrm{~mol} \%)$, BINAP (12 mol\%), KOAc (150 mol\%), and stirring at room temperature for $48 \mathrm{~h}$ under $5 \mathrm{~W}$ blue LEDs irradiation. The reaction mixture was filtered through a pad of silica gel and concentrated in vacuo, then purified by chromatography (pentane/EtOAc/Et ${ }_{3} \mathrm{~N}=9 / 1 / 0.5$ ) to give 10 (43.0 mg, $0.137 \mathrm{mmol}, 68 \%$, > 95:5 $E / Z,>20: 1 \mathrm{rr}$ ) as a colorless oil. $\mathbf{R}_{\boldsymbol{f}}$ (pentane/EtOAc/Et $\left.{ }_{3} \mathrm{~N}=9 / 1 / 0.5\right) 0.10 .{ }^{1} \mathbf{H}$ NMR (400 MHz, $\left.\mathbf{C D C l}_{3}\right) \delta 7.30-7.24(\mathrm{~m}, 2 \mathrm{H}), 7.19-7.14(\mathrm{~m}, 3 \mathrm{H}), 5.67-5.47(\mathrm{~m}$, 2H), $3.60(\mathrm{dd}, J=10.6,5.2 \mathrm{~Hz}, 1 \mathrm{H}), 3.48$ (dd, $J=10.6,6.0 \mathrm{~Hz}, 1 \mathrm{H}), 2.96$ (d, J = 6.7 $\mathrm{Hz}, 2 \mathrm{H}), 2.84(\mathrm{~d}, J=10.7 \mathrm{~Hz}, 1 \mathrm{H}), 2.73-2-63(\mathrm{br}, 1 \mathrm{H}), 2.60-2.51$ (m, 2H), $2.13-$ 2.05 (br, 1H), $2.04-1.99$ (m, 2H), $1.99-1.88$ (br, 1H), $1.83-1.65$ (m, 3H), 1.59 (td, $J=9.7,4.7 \mathrm{~Hz}, 1 \mathrm{H}), 1.53-1.45(\mathrm{~m}, 2 \mathrm{H}), 1.17-1.04(\mathrm{br}, 1 \mathrm{H}), 0.94(\mathrm{~s}, 6 \mathrm{H}) .{ }^{13} \mathrm{C}$ NMR (101 MHz, $\mathbf{C D C l}_{3}$ ): $\delta$ ppm 143.6, 131.2, 129.1, 128.5, 128.4, 125.7, 67.4, 
61.7, 57.4, 54.3, 45.0, 44.2, 38.2, 33.6, 30.8, 27.8, 27.2, 24.8. HRMS (ESI+): calcd. for $\mathrm{C}_{21} \mathrm{H}_{34} \mathrm{NO}\left(\mathrm{M}+\mathrm{H}^{+}\right)$: 316.2635. Found: 316.2645 .

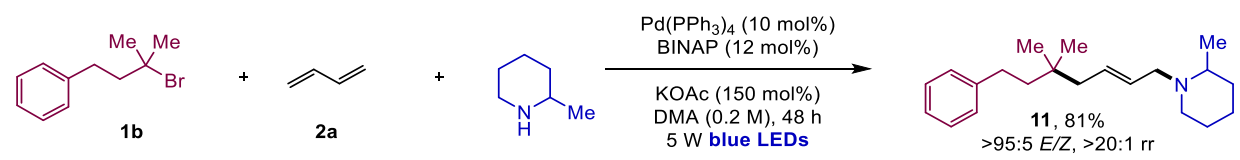

(E)-1-(5,5-Dimethyl-7-phenylhept-2-en-1-yl)-2-methylpiperidine

(11).

According to the general procedure G, using the corresponding alkyl bromide $\mathbf{1 b}$ ( $0.3 \mathrm{mmol}, 1.5$ equiv.), butadiene $2 \mathrm{a}(0.3 \mathrm{mmol}, 0.15 \mathrm{~mL}, 2 \mathrm{M}$ in THF, 1.5 equiv.), 2-methylpirimidine (0.2 mmol, 1.0 equiv.), $\mathrm{Pd}\left(\mathrm{PPh}_{3}\right)_{4}$ (10 mol\%), BINAP (12 mol\%), KOAc (150 mol\%), and stirring at room temperature for $48 \mathrm{~h}$ under 5W blue LEDs irradiation. The reaction mixture was filtered through a pad of silica gel and concentrated in vacuo, then purified by chromatography (pentane/EtOAc/Et $3 \mathrm{~N}=$ 9/1/0.5) to give 11 (48.2 mg, 0.165 mmol, 81\%, > 95:5 $E / Z,>20: 1 \mathrm{rr}$ ) as a colorless oil. $\mathbf{R}_{\boldsymbol{f}}$ (pentane/EtOAc/Et $3 \mathrm{~N}=9 / 1 / 0.5$ ) $0.81 .{ }^{1} \mathbf{H}$ NMR (599 MHz, $\mathbf{C D C l}_{3}$ ) $\delta 7.35(\mathrm{dd}, J=8.3,7.0 \mathrm{~Hz}, 2 \mathrm{H}), 7.25(\mathrm{~d}, J=7.7 \mathrm{~Hz}, 3 \mathrm{H})$, $5.72-5.63(\mathrm{~m}, 2 \mathrm{H}), 3.50-3.41(\mathrm{~m}, 1 \mathrm{H}), 3.07$ (dd, $J=13.7,7.3 \mathrm{~Hz}, 1 \mathrm{H}), 2.94(\mathrm{dt}, J$ $=12.9,4.2 \mathrm{~Hz}, 1 \mathrm{H}), 2.67-2.62(\mathrm{~m}, 2 \mathrm{H}), 2.38-2.31(\mathrm{~m}, 1 \mathrm{H}), 2.20(\mathrm{td}, J=11.4,3.1$ $\mathrm{Hz}, 1 \mathrm{H}$ ), 2.10 (d, $J=6.7 \mathrm{~Hz}, 2 \mathrm{H}), 1.74$ (ddd, $J=11.9,4.0,2.2 \mathrm{~Hz}, 1 \mathrm{H}), 1.72-1.65$ (m, 2H), $1.64-1.55$ (m, 3H), 1.36 (dddd, $J=20.7,11.9,8.7,3.5 \mathrm{~Hz}, 2 \mathrm{H}$ ), 1.17 (d, $J$ $=6.2 \mathrm{~Hz}, 3 \mathrm{H}), 1.03(\mathrm{~s}, 6 \mathrm{H}) \cdot{ }^{13} \mathbf{C}$ NMR (151 MHz, $\left.\mathbf{C D C l}_{3}\right) \delta 143.5,130.7,128.7$, 128.39, 128.38, 125.6, 56.6, 55.8, 52.4, 45.0, 44.2, 34.9, 33.6, 30.8, 27.22, 27.21, 26.3, 24.3. HRMS (ESI+): calcd. for $\mathrm{C}_{21} \mathrm{H}_{34} \mathrm{~N}\left(\mathrm{M}+\mathrm{H}^{+}\right)$: 300.2686. Found: 300.2702.
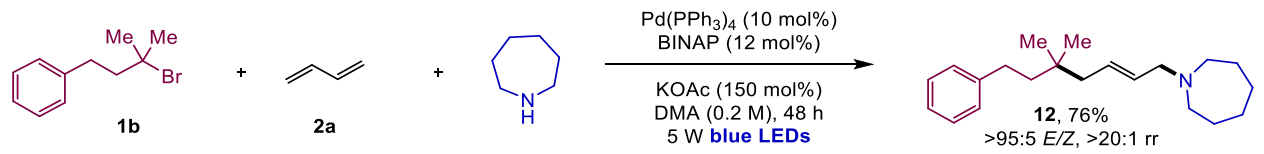

(E)-1-(5,5-Dimethyl-7-phenylhept-2-en-1-yl)azepane (12). According to the general procedure G, using the corresponding alkyl bromide $\mathbf{1 b}(0.3 \mathrm{mmol}, 1.5$ equiv.), butadiene 2 a ( $0.3 \mathrm{mmol}, 0.15 \mathrm{~mL}, 2 M$ in THF, 1.5 equiv.), azepane (0.2 
mmol, 1.0 equiv.), $\mathrm{Pd}\left(\mathrm{PPh}_{3}\right)_{4}(10 \mathrm{~mol} \%)$, BINAP (12 mol\%), KOAc (150 mol\%), and stirring at room temperature for $48 \mathrm{~h}$ under $5 \mathrm{~W}$ blue LEDs irradiation. The reaction mixture was filtered through a pad of silica gel and concentrated in vacuo, then purified by chromatography (pentane/EtOAc/ $\mathrm{Et}_{3} \mathrm{~N}=9 / 1 / 0.5$ ) to give 12 (45.4 mg, $0.152 \mathrm{mmol}, 76 \%,>95: 5 \mathrm{E} / \mathrm{Z},>20: 1 \mathrm{rr}$ ) as a colorless oil. $\mathbf{R}_{\boldsymbol{f}}$ (pentane/EtOAc/Et $3 \mathrm{~N}=$ 9/1/0.5) 0.63. ${ }^{1} \mathbf{H}$ NMR (599 $\mathbf{~ M H z , ~} \mathbf{C D C l}_{3}$ ) $\delta 7.41-7.34$ (m, 2H), $7.25(\mathrm{~d}, J=7.5 \mathrm{~Hz}, 3 \mathrm{H}), 5.66(\mathrm{dt}, J=14.6,6.5 \mathrm{~Hz}, 2 \mathrm{H}), 3.17(\mathrm{~d}, J=6.0 \mathrm{~Hz}$, 2H), $2.71-2.68(\mathrm{~m}, 4 \mathrm{H}), 2.67-2.62(\mathrm{~m}, 2 \mathrm{H}), 2.10(\mathrm{~d}, J=6.8 \mathrm{~Hz}, 2 \mathrm{H}), 1.78-1.67$ (m, 8H), $1.60-1.56(\mathrm{~m}, 2 \mathrm{H}), 1.02(\mathrm{~s}, 6 \mathrm{H}) .{ }^{13} \mathbf{C}$ NMR (151 MHz, $\left.\mathbf{C D C l}_{3}\right) \delta$ 143.6, 130.4 , 130.1, 128.4, 125.6, 61.1, 55.6, 45.0, 44.2, 33.6, 30.8, 28.1, 27.2, 27.0. HRMS (ESI'): calcd. for $\mathrm{C}_{21} \mathrm{H}_{34} \mathrm{~N}\left(\mathrm{M}+\mathrm{H}^{+}\right)$: 300.2686. Found: 300.2704.

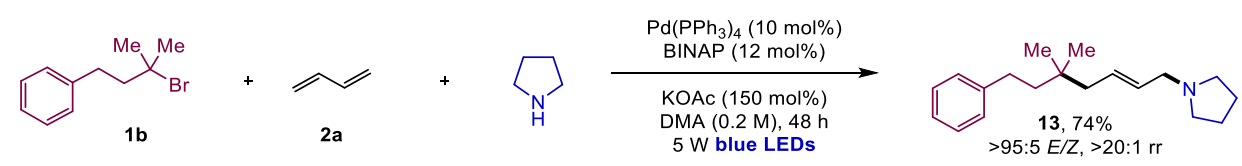

(E)-1-(5,5-Dimethyl-7-phenylhept-2-en-1-yl)pyrrolidine (13). According to the general procedure $G$, using the corresponding alkyl bromide $\mathbf{1 b}(0.3 \mathrm{mmol}$, 1.5 equiv.), butadiene 2 a ( $0.3 \mathrm{mmol}, 0.15 \mathrm{~mL}, 2 \mathrm{M}$ in THF, 1.5 equiv.), pyrrolidine (0.2 mmol, 1.0 equiv.), $\mathrm{Pd}\left(\mathrm{PPh}_{3}\right)_{4}$ (10 mol\%), BINAP (12 mol\%), KOAc (150 mol\%), and stirring at room temperature for $48 \mathrm{~h}$ under $5 \mathrm{~W}$ blue LEDs irradiation. The reaction mixture was filtered through a pad of silica gel and concentrated in vacuo, then purified by chromatography (pentane/EtOAc/Et $3 \mathrm{~N}=9 / 1 / 0.5$ ) to give 13 (40.1 mg, $0.148 \mathrm{mmol}, 74 \%,>95: 5 \mathrm{E} / \mathrm{Z},>20: 1 \mathrm{rr}$ ) as a colorless oil. $\mathbf{R}_{\boldsymbol{f}}$ (pentane/EtOAc/Et $3 \mathrm{~N}=9 / 1 / 0.5)$ 0.60. ${ }^{1} \mathbf{H}$ NMR (599 $\mathbf{~ M H z , ~} \mathbf{C D C l}_{3}$ ) $7.41-7.34$ $(\mathrm{m}, 2 \mathrm{H}), 7.25(\mathrm{~d}, J=7.7 \mathrm{~Hz}, 3 \mathrm{H}), 5.77-5.65(\mathrm{~m}, 2 \mathrm{H}), 3.16(\mathrm{~d}, J=6.1 \mathrm{~Hz}, 2 \mathrm{H}), 2.68$ - 2.62 (m, 2H), 2.57 (td, $J=5.3,4.0,2.5 \mathrm{~Hz}, 4 \mathrm{H}$ ), 2.09 (d, $J=6.8 \mathrm{~Hz}, 2 \mathrm{H}), 1.90-$ $1.83(\mathrm{~m}, 4 \mathrm{H}), 1.62-1.55(\mathrm{~m}, 2 \mathrm{H}), 1.02(\mathrm{~s}, 6 \mathrm{H})$.

${ }^{13}$ C NMR (151 MHz, CDCl $\left._{3}\right) \delta 143.6,130.4,129.8,128.4,128.4,125.6,58.5,54.1$, 44.9, 44.3, 33.6, 30.8, 27.2, 23.6. HRMS (ESI+): calcd. for $\mathrm{C}_{19} \mathrm{H}_{30} \mathrm{~N}\left(\mathrm{M}+\mathrm{H}^{+}\right)$: 272.2373. Found: 272.2389 . 


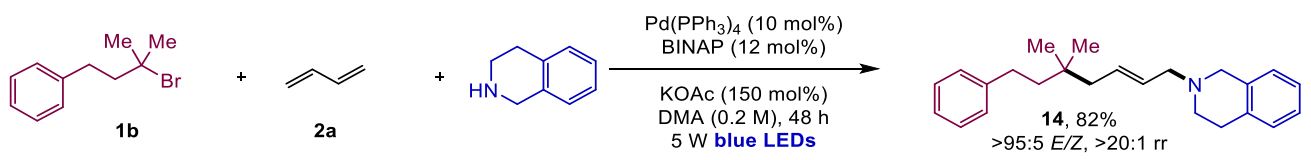

(E)-2-(5,5-Dimethyl-7-phenylhept-2-en-1-yl)-1,2,3,4-

tetrahydroisoquinoline (14). According to the general procedure G, using the corresponding alkyl bromide $\mathbf{1 b}(0.3 \mathrm{mmol}, 1.5$ equiv.), butadiene $2 \mathbf{a}(0.3 \mathrm{mmol}$, $0.15 \mathrm{~mL}, 2 \mathrm{M}$ in THF, 1.5 equiv.), the nitrogen based nucleophile 3 (0.2 mmol, 1.0 equiv.), $\mathrm{Pd}\left(\mathrm{PPh}_{3}\right)_{4}(10 \mathrm{~mol} \%)$, BINAP (12 mol\%), KOAc (150 mol\%), and stirring at room temperature for $48 \mathrm{~h}$ under $5 \mathrm{~W}$ blue LEDs irradiation. The reaction mixture was filtered through a pad of silica gel and concentrated in vacuo, then purified by chromatography (pentane/EtOAc/Et $3 \mathrm{~N}=9 / 1 / 0.5)$ to give $14(54,4$ mg, $0.163 \mathrm{mmol}, 82 \%,>95: 5 \mathrm{E} / \mathrm{Z},>20: 1 \mathrm{rr}$ ) as a colorless oil. $\mathbf{R}_{\boldsymbol{f}}$ (pentane/EtOAc/Et $3 \mathrm{~N}=$ 9/1/0.5) 0.32. ${ }^{1} \mathbf{H}$ NMR (599 $\mathbf{~ M H z , ~} \mathbf{C D C l}_{3}$ ) $\delta 7.37-7.32$ (m, 2H), $7.29-7.23(\mathrm{~m}, 3 \mathrm{H}), 7.22-7.16(\mathrm{~m}, 3 \mathrm{H}), 7.07-7.01(\mathrm{~m}, 1 \mathrm{H}), 5.78(\mathrm{dt}, J=$ 14.9, 7.4 Hz, 1H), 5.69 (dt, $J=15.2,6.6 \mathrm{~Hz}, 1 \mathrm{H}), 3.69$ (s, 2H), 3.23 (d, $J=6.6 \mathrm{~Hz}$, $2 \mathrm{H}), 2.97(\mathrm{t}, J=6.0 \mathrm{~Hz}, 2 \mathrm{H}), 2.81(\mathrm{t}, J=6.0 \mathrm{~Hz}, 2 \mathrm{H}), 2.69-2.64(\mathrm{~m}, 2 \mathrm{H}), 2.13(\mathrm{~d}, J=$ $7.3 \mathrm{~Hz}, 2 \mathrm{H}), 1.66-1.56(\mathrm{~m}, 2 \mathrm{H}), 1.05(\mathrm{~s}, 6 \mathrm{H}) .{ }^{13} \mathbf{C}$ NMR (151 MHz, CDCl $) \delta 143.5$, 134.9, 134.4, 131.1, 129.3, 128.7, 128.4, 128.4, 126.8, 126.2, 125.7, 125.6, 60.8, 56.1, 50.7, 45.0, 44.3, 33.6, 30.8, 29.2, 27.2. HRMS (ESI+): calcd. for $\mathrm{C}_{24} \mathrm{H}_{32} \mathrm{~N}(\mathrm{M}+$ $\mathrm{H}^{+}$): 334.2529. Found: 334.2551 .

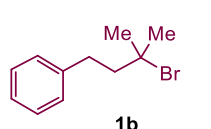

$1 \mathrm{~b}$

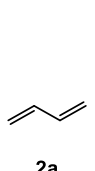

2a

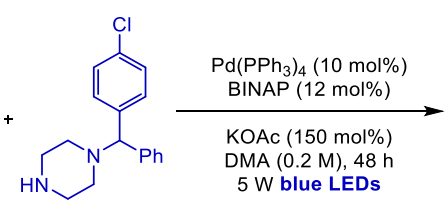

$5 \mathrm{~W}$ blue LEDs
DMA $(0.2 \mathrm{M}), 48 \mathrm{~h}$

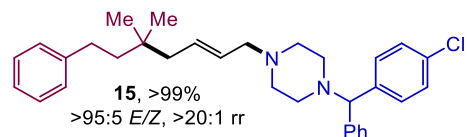

(E)-1-((4-Chlorophenyl)(phenyl)methyl)-4-(5,5-dimethyl-7-phenylhept-2-

en-1-yl)piperazine (15). According to the general procedure G, using the corresponding alkyl bromide $\mathbf{1 b}(0.3 \mathrm{mmol}, 1.5$ equiv.), butadiene $\mathbf{2 a}(0.3 \mathrm{mmol}$, $0.15 \mathrm{~mL}, 2 \mathrm{M}$ in THF, 1.5 equiv.), the nitrogen based nucleophile 3 (0.2 mmol, 1.0 equiv.), $\mathrm{Pd}\left(\mathrm{PPh}_{3}\right)_{4}(10 \mathrm{~mol} \%)$, BINAP (12 mol\%), KOAc (150 mol\%), and stirring at room temperature for $48 \mathrm{~h}$ under $5 \mathrm{~W}$ blue LEDs irradiation. The reaction mixture was filtered through a pad of silica gel and concentrated in vacuo, then 
purified by chromatography (pentane/EtOAc/Et $\left.{ }_{3} \mathrm{~N}=9 / 1 / 0.5\right)$ to give $15(96.8$ $\mathrm{mg}, 0.199 \mathrm{mmol}$, $>99 \%$, $>95: 5 E / Z,>20: 1 \mathrm{rr}$ ) as a colorless oil. $\mathbf{R}_{f}$ (pentane/EtOAc/Et $3 \mathrm{~N}=9 / 1 / 0.5)$ 0.62. ${ }^{1} \mathbf{H}$ NMR (599 $\left.\mathbf{~ M H z , ~} \mathbf{C D C l}_{3}\right) \delta 7.40-7.35$ (m, 4H), $7.29-7.19(\mathrm{~m}, 7 \mathrm{H}), 7.17-7.10(\mathrm{~m}, 3 \mathrm{H}), 5.62(\mathrm{dt}, J=14.8,7.3 \mathrm{~Hz}, 1 \mathrm{H})$, $5.56-5.49(\mathrm{~m}, 1 \mathrm{H}), 4.21(\mathrm{~s}, 1 \mathrm{H}), 2.98(\mathrm{~d}, J=6.7 \mathrm{~Hz}, 2 \mathrm{H}), 2.59-2.33(\mathrm{~m}, 10 \mathrm{H})$, $2.00(\mathrm{~d}, J=7.3 \mathrm{~Hz}, 2 \mathrm{H}), 1.50-1.43(\mathrm{~m}, 2 \mathrm{H}), 0.92(\mathrm{~s}, 6 \mathrm{H}) .{ }^{13} \mathbf{C}$ NMR (151 MHz, $\left.\mathbf{C D C l}_{3}\right) \delta 142.3,141.5,131.2,129.3,129.1,128.7,128.7,128.7,128.6,128.4$, 128.3, 128.0, 127.2, 125.6, 75.6, 61.1, 53.4, 51.9, 44.9, 44.1, 33.6, 30.7, 27.2. HRMS (ESI'): calcd. for $\mathrm{C}_{32} \mathrm{H}_{40} \mathrm{~N}_{2} \mathrm{Cl}\left(\mathrm{M}+\mathrm{H}^{+}\right)$: 487.2875. Found: 487.2882.
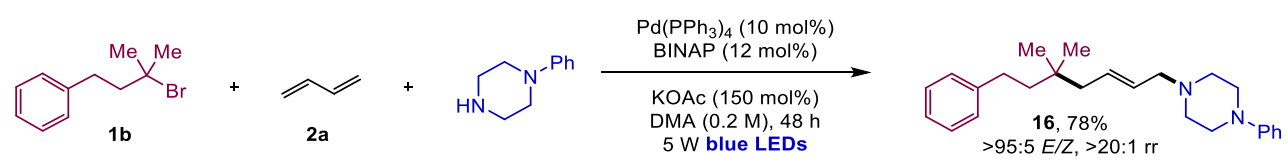

(E)-1-(5,5-Dimethyl-7-phenylhept-2-en-1-yl)-4-phenylpiperazine

(16).

According to the general procedure $G$, using the corresponding alkyl bromide $\mathbf{1 b}$ ( $0.3 \mathrm{mmol}, 1.5$ equiv.), butadiene $2 \mathrm{a}(0.3 \mathrm{mmol}, 0.15 \mathrm{~mL}, 2 \mathrm{M}$ in THF, 1.5 equiv.), the nitrogen based nucleophile 3 (0.2 mmol, 1.0 equiv.), $\mathrm{Pd}\left(\mathrm{PPh}_{3}\right)_{4}$ (10 mol\%), BINAP (12 mol\%), KOAc (150 mol\%), and stirring at room temperature for $48 \mathrm{~h}$ under $5 \mathrm{~W}$ blue LEDs irradiation. The reaction mixture was filtered through a pad of silica gel and concentrated in vacuo, then purified by chromatography (pentane/EtOAc/Et $3 \mathrm{~N}=9 / 1 / 0.5)$ to give 16 (56.4 mg, $0.156 \mathrm{mmol}$, 78\%, > 95:5 $E / Z,>20: 1 \mathrm{rr}$ ) as a colorless oil. $\mathbf{R}_{f}$ (pentane/EtOAc/Et $\left.{ }_{3} \mathrm{~N}=9 / 1 / 0.5\right)$ 0.57. ${ }^{1} \mathbf{H}$ NMR (599 MHz, $\mathbf{C D C l}_{3}$ ) $\delta 7.38-7.31(\mathrm{~m}, 4 \mathrm{H}), 7.27-7.23(\mathrm{~m}, 3 \mathrm{H}), 7.02-6.99(\mathrm{~m}$, $2 \mathrm{H}), 6.95-6.91(\mathrm{~m}, 1 \mathrm{H}), 5.84-5.73(\mathrm{~m}, 1 \mathrm{H}), 5.70-5.59(\mathrm{~m}, 1 \mathrm{H}), 3.32-3.21(\mathrm{~m}$, $4 \mathrm{H}), 3.12(\mathrm{dd}, J=6.7,1.2 \mathrm{~Hz}, 2 \mathrm{H}), 2.75-2.62(\mathrm{~m}, 6 \mathrm{H}), 2.12(\mathrm{dd}, J=7.3,1.2 \mathrm{~Hz}, 2 \mathrm{H})$, 1.67 - $1.56(\mathrm{~m}, 2 \mathrm{H}), 1.04(\mathrm{~s}, 6 \mathrm{H}) .{ }^{13} \mathrm{C}$ NMR (151 MHz, $\left.\mathbf{C D C l}_{3}\right) \delta$ 151.4, 143.5, 131.5, 129.2, 128.9, 128.4, 128.4, 125.7, 119.7, 116.1, 61.2, 53.2, 49.2, 44.9, 44.2, 33.6, 30.7, 27.2. HRMS (ESI+): calcd. for $\mathrm{C}_{25} \mathrm{H}_{35} \mathrm{~N}_{2}\left(\mathrm{M}+\mathrm{H}^{+}\right)$: 363.2820. Found: 363.2817. 


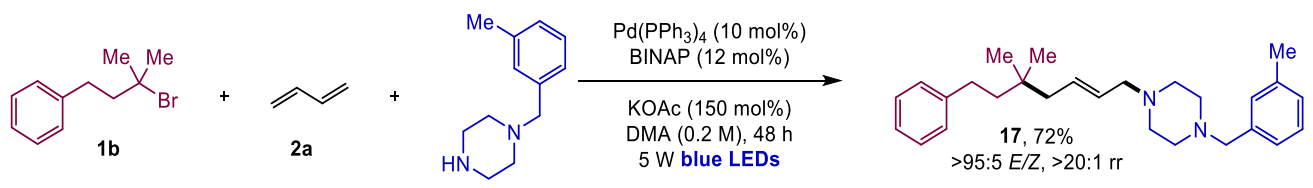

(E)-1-(5,5-Dimethyl-7-phenylhept-2-en-1-yl)-4-(3-

methylbenzyl)piperazine (17). According to the general procedure $G$, using the corresponding alkyl bromide $\mathbf{1 b}(0.3 \mathrm{mmol}, 1.5$ equiv.), butadiene $2 \mathbf{a}(0.3 \mathrm{mmol}$, $0.15 \mathrm{~mL}, 2 \mathrm{M}$ in THF, 1.5 equiv.), the nitrogen based nucleophile 3 (0.2 mmol, 1.0 equiv.), $\mathrm{Pd}\left(\mathrm{PPh}_{3}\right)_{4}(10 \mathrm{~mol} \%)$, BINAP (12 mol\%), KOAc (150 mol\%), and stirring at room temperature for $48 \mathrm{~h}$ under $5 \mathrm{~W}$ blue LEDs irradiation. The reaction mixture was filtered through a pad of silica gel and concentrated in vacuo, then purified by chromatography (pentane/EtOAc/Et $3 \mathrm{~N}=9 / 1 / 0.5)$ to give 17 (56.2 mg, $0.144 \mathrm{mmol}, 72 \%,>95: 5 \mathrm{E} / Z$, > 20:1 rr) as a colorless oil. $\mathbf{R}_{\boldsymbol{f}}$ (pentane/EtOAc/Et $3 \mathrm{~N}=$ 9/1/0.5) 0.49. ${ }^{1} \mathbf{H}$ NMR (599 $\mathbf{~ M H z , ~} \mathbf{C D C l}_{3}$ ) $\delta 7.37-7.33$ (m, 2H), 7.29 (t, J = 7.5 Hz, 1H), $7.27-7.23(\mathrm{~m}, 4 \mathrm{H}), 7.22-7.20(\mathrm{~m}, 1 \mathrm{H}), 7.16$ (ddt, $J=7.5,1.9,1.0 \mathrm{~Hz}, 1 \mathrm{H}), 5.76-5.70(\mathrm{~m}, 1 \mathrm{H}), 5.66-5.60(\mathrm{~m}, 1 \mathrm{H}), 3.58(\mathrm{~s}, 2 \mathrm{H}), 3.08$ (dd, $J=6.7,1.1 \mathrm{~Hz}, 2 \mathrm{H}), 2.82-2.30(\mathrm{~m}, 13 \mathrm{H}), 2.10$ (dd, $J=7.4,1.1 \mathrm{~Hz}, 2 \mathrm{H}), 1.67-$ $1.53(\mathrm{~m}, 2 \mathrm{H}), 1.03$ (s, 6H). ${ }^{13} \mathrm{C}$ NMR (151 MHz, $\left.\mathbf{C D C l}_{3}\right) \delta$ 143.5, 138.1, 137.9, 131.1, 130.1, 129.2, 128.4, 128.4, 128.2, 127.8, 126.5, 125.6, 63.2, 61.2, 53.2, 53.2, 44.9, 44.2, 33.6, 30.7, 27.2, 21.5. HRMS (ESI+): calcd. for $\mathrm{C}_{27} \mathrm{H}_{39} \mathrm{~N}_{2}\left(\mathrm{M}+\mathrm{H}^{+}\right)$: 391.3108. Found: 391.3119.

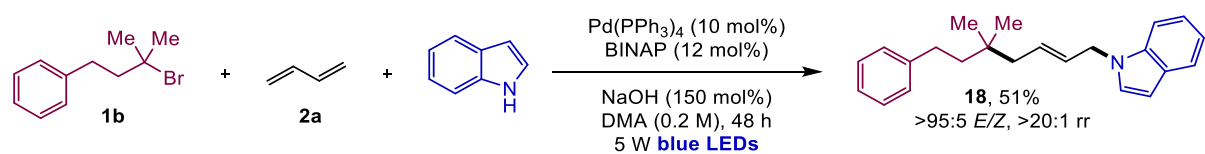

(E)-1-(5,5-Dimethyl-7-phenylhept-2-en-1-yl)-1H-indole (18). According to the general procedure $G$, using the corresponding alkyl bromide $\mathbf{1 b}(0.3 \mathrm{mmol}$, 1.5 equiv.), butadiene 2 a ( $0.3 \mathrm{mmol}, 0.15 \mathrm{~mL}, 2 M$ in THF, 1.5 equiv.), $1 \mathrm{H}$-indole (0.2 mmol, 1.0 equiv.), Pd(PPh $)_{4}$ (10 mol\%), BINAP (12 mol\%), NaOH (150 mol\%), and stirring at room temperature for $48 \mathrm{~h}$ under $5 \mathrm{~W}$ blue LEDs irradiation. The reaction mixture was filtered through a pad of silica gel and concentrated in vacuo, then purified by chromatography (pentane $/$ EtOAc $=96 / 4)$ to give 18 
(32.3 mg, $0.101 \mathrm{mmol}, 51 \%,>95: 5 \mathrm{E} / \mathrm{Z},>20: 1 \mathrm{rr}$ ) as a colorless oil. $\mathbf{R}_{\boldsymbol{f}}$

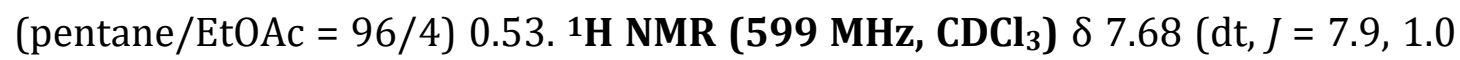
$\mathrm{Hz}, 1 \mathrm{H}), 7.40-7.36(\mathrm{~m}, 2 \mathrm{H}), 7.32-7.27(\mathrm{~m}, 2 \mathrm{H}), 7.24-7.19(\mathrm{~m}, 2 \mathrm{H}), 7.16-7.12$ (m, 4H), $6.54(\mathrm{dd}, J=3.2,0.9 \mathrm{~Hz}, 1 \mathrm{H}), 5.76-5.64(\mathrm{~m}, 2 \mathrm{H}), 4.79-4.70(\mathrm{~m}, 2 \mathrm{H})$, $2.62-2.53(\mathrm{~m}, 2 \mathrm{H}), 2.04(\mathrm{dt}, J=6.0,1.1 \mathrm{~Hz}, 2 \mathrm{H}), 1.56-1.47(\mathrm{~m}, 2 \mathrm{H}), 0.96(\mathrm{~s}, 6 \mathrm{H})$. ${ }^{13}$ C NMR (151 MHz, $\left.\mathbf{C D C l}_{3}\right) \delta 143.3,136.1,133.9,133.8,131.0,128.9,128.5$, 128.4, 127.7, 127.7, 125.7, 121.5, 121.0, 119.4, 109.7, 101.3, 48.5, 44.7, 44.2, 33.7, 30.8, 27.2. HRMS (ESI+): calcd. for $\mathrm{C}_{23} \mathrm{H}_{27} \mathrm{NNa}\left(\mathrm{M}+\mathrm{Na}^{+}\right)$: 340.2036. Found: 340.2034 .

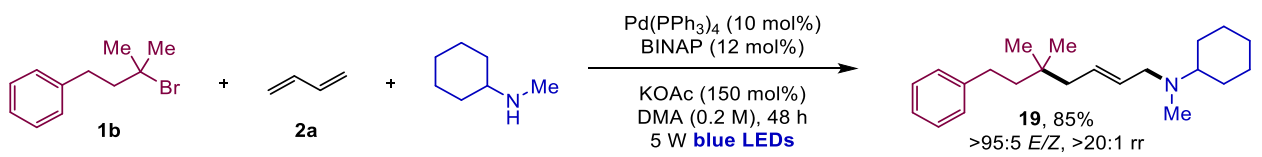

(E)-N-(5,5-dimethyl-7-phenylhept-2-en-1-yl)-N-methylcyclohexanamine

(19). According to the general procedure G, using the corresponding alkyl bromide $\mathbf{1 b}$ ( $0.3 \mathrm{mmol}, 1.5$ equiv.), butadiene $2 \mathrm{a}(0.3 \mathrm{mmol}, 0.15 \mathrm{~mL}, 2 \mathrm{M}$ in THF, 1.5 equiv.), the nitrogen based nucleophile 3 (0.2 mmol, 1.0 equiv.), $\mathrm{Pd}\left(\mathrm{PPh}_{3}\right)_{4}$ (10 mol\%), BINAP (12 mol\%), KOAc (150 mol\%), and stirring at room temperature for $48 \mathrm{~h}$ under $5 \mathrm{~W}$ blue LEDs irradiation. The reaction mixture was filtered through a pad of silica gel and concentrated in vacuo, then purified by chromatography (pentane/EtOAc/Et $\left.{ }_{3} \mathrm{~N}=9 / 1 / 0.5\right)$ to give 19 (53.1 mg, 0.170 mmol, 85\%, > 95:5 E/Z, > 20:1 rr) as a colorless oil. $\mathbf{R}_{\boldsymbol{f}}$ (pentane/EtOAc/Et ${ }_{3} \mathrm{~N}=$ 9/1/0.5) 0.35. ${ }^{1} \mathbf{H}$ NMR (599 $\left.\mathbf{M H z}, \mathbf{C D C l}_{3}\right) \delta 7.26(\mathrm{dd}, J=8.2,7.1 \mathrm{~Hz}, 2 \mathrm{H}), 7.21-$ 7.07 (m, 3H), $5.65-5.57$ (m, 1H), 5.51 (dddd, $J=15.2,6.6,5.5,1.4 \mathrm{~Hz}, 1 \mathrm{H}$ ), 3.08 $(\mathrm{d}, J=6.6 \mathrm{~Hz}, 2 \mathrm{H}), 2.61-2.52(\mathrm{~m}, 2 \mathrm{H}), 2.40(\mathrm{ddd}, J=11.1,7.4,3.3 \mathrm{~Hz}, 1 \mathrm{H}), 2.22(\mathrm{~s}$, 3H), $2.01(\mathrm{~d}, J=7.3 \mathrm{~Hz}, 2 \mathrm{H}), 1.84-1.73(\mathrm{~m}, 4 \mathrm{H}), 1.64-1.57(\mathrm{~m}, 1 \mathrm{H}), 1.54-1.47$ (m, 2H), $1.26-1.14(\mathrm{~m}, 4 \mathrm{H}), 1.12-1.04(\mathrm{~m}, 1 \mathrm{H}), 0.94(\mathrm{~s}, 6 \mathrm{H}) .{ }^{13}$ C NMR (151 MHz, $\left.\mathbf{C D C l}_{3}\right) \delta 143.5,130.8,129.9,128.41,128.40,125.6,61.7,56.4,45.0,44.3$, 37.4, 33.7, 30.8, 28.8, 27.2, 26.5, 26.1. HRMS (ESI+): calcd. for $\mathrm{C}_{22} \mathrm{H}_{36} \mathrm{~N}\left(\mathrm{M}+\mathrm{H}^{+}\right)$: 314.2842. Found: 314.2861. 


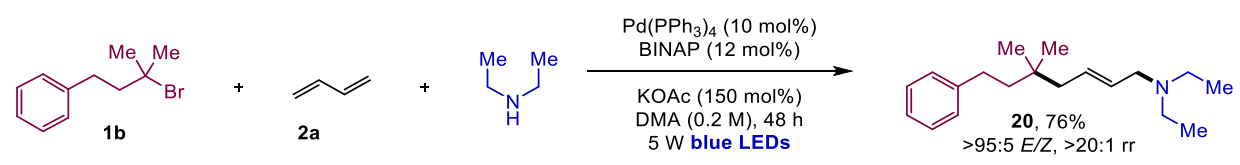

(E)-N,N-Diethyl-5,5-dimethyl-7-phenylhept-2-en-1-amine (20). According to the general procedure G, using the corresponding alkyl bromide $\mathbf{1 b}(0.3 \mathrm{mmol}$, 1.5 equiv.), butadiene $2 \mathrm{a}(0.3 \mathrm{mmol}, 0.15 \mathrm{~mL}, 2 M$ in THF, 1.5 equiv.), diethylamine (0.2 mmol, 1.0 equiv.), $\mathrm{Pd}\left(\mathrm{PPh}_{3}\right)_{4}(10 \mathrm{~mol} \%)$, BINAP (12 mol\%), KOAc (150 mol\%), and stirring at room temperature for $48 \mathrm{~h}$ under $5 \mathrm{~W}$ blue LEDs irradiation. The reaction mixture was filtered through a pad of silica gel and concentrated in vacuo, then purified by chromatography (pentane/EtOAc/Et $3 \mathrm{~N}=$ 9/1/0.5) to give 20 (41.5 mg, $0.152 \mathrm{mmol}, 85 \%,>95: 5 E / Z,>20: 1 \mathrm{rr}$ ) as a colorless oil. $\mathbf{R}_{f}$ (pentane/EtOAc/Et $3 \mathrm{~N}=9 / 1 / 0.5$ ) 0.54. ${ }^{1} \mathbf{H} \mathbf{~ N M R}\left(599 \mathbf{~ M H z}, \mathbf{C D C l}_{3}\right.$ ) $\delta 7.43-7.32(\mathrm{~m}, 2 \mathrm{H}), 7.27$ (d, $J=7.5 \mathrm{~Hz}, 3 \mathrm{H}), 5.72$ (dd, $J=15.1,7.4 \mathrm{~Hz}, 1 \mathrm{H}), 5.62$ $(\mathrm{dt}, J=14.7,6.6 \mathrm{~Hz}, 1 \mathrm{H}), 3.18(\mathrm{~d}, J=6.6 \mathrm{~Hz}, 2 \mathrm{H}), 2.77-2.59(\mathrm{~m}, 6 \mathrm{H}), 2.11(\mathrm{~d}, J=$ $7.3 \mathrm{~Hz}, 2 \mathrm{H}$ ), 1.60 (dd, $J=8.6,4.4 \mathrm{~Hz}, 2 \mathrm{H}), 1.12$ (t, $J=7.2 \mathrm{~Hz}, 6 \mathrm{H}), 1.04(\mathrm{~s}, 6 \mathrm{H}) .{ }^{13} \mathrm{C}$ NMR (151 MHz, $\left.\mathbf{C D C l}_{3}\right) \delta$ 143.5, 130.3, 129.7, 128.4, 128.4, 125.6, 55.4, 46.6, 45.0, 44.3, 33.6, 30.8, 27.2, 11.8. HRMS (ESI'): calcd. for $\mathrm{C}_{19} \mathrm{H}_{32} \mathrm{~N}\left(\mathrm{M}+\mathrm{H}^{+}\right)$: 274.2529. Found: 274.2547.

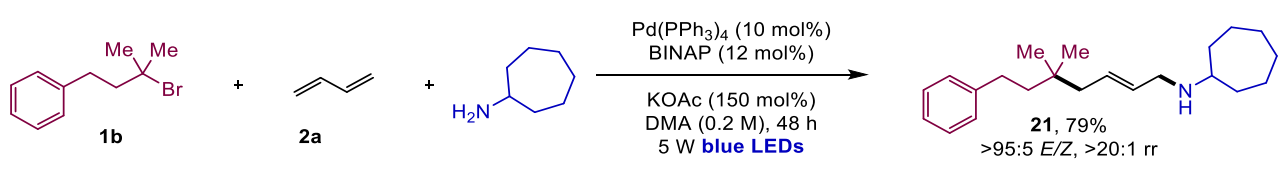

(E)-N-(5,5-dimethyl-7-phenylhept-2-en-1-yl)cycloheptanamine

According to the general procedure $\mathrm{G}$, using the corresponding alkyl bromide $\mathbf{1 b}$ ( $0.3 \mathrm{mmol}$, 1.5 equiv.), butadiene $2 \mathrm{a}$ ( $0.3 \mathrm{mmol}, 0.15 \mathrm{~mL}, 2 \mathrm{M}$ in THF, 1.5 equiv.), the nitrogen based nucleophile 3 (0.2 mmol, 1.0 equiv.), $\mathrm{Pd}\left(\mathrm{PPh}_{3}\right)_{4}(10 \mathrm{~mol} \%)$, BINAP (12 mol\%), KOAc (150 mol\%), and stirring at room temperature for $48 \mathrm{~h}$ under $5 \mathrm{~W}$ blue LEDs irradiation. The reaction mixture was filtered through a pad of silica gel and concentrated in vacuo, then purified by chromatography (pentane/EtOAc/Et ${ }_{3} \mathrm{~N}=9 / 1 / 0.5$ ) to give 21 (49.5 mg, $0.158 \mathrm{mmol}, 79 \%$, > 95:5 $E / Z,>20: 1 \mathrm{rr}$ ) as a colorless oil. $\mathbf{R}_{\boldsymbol{f}}$ (pentane/EtOAc/Et $\left.3 \mathrm{~N}=9 / 1 / 0.5\right) 0.51 .{ }^{1} \mathbf{H}$ NMR (599 MHz, $\left.\mathbf{C D C l}_{3}\right) \delta 7.38-7.33(\mathrm{~m}, 2 \mathrm{H}), 7.28-7.20(\mathrm{~m}, 3 \mathrm{H}), 5.73-5.59(\mathrm{~m}$, 
2H), $3.29(\mathrm{dt}, J=6.0,1.0 \mathrm{~Hz}, 2 \mathrm{H}), 2.75(\mathrm{dt}, J=8.5,4.3 \mathrm{~Hz}, 1 \mathrm{H}), 2.68-2.60(\mathrm{~m}, 2 \mathrm{H})$, $2.08(\mathrm{dd}, J=7.0,1.0 \mathrm{~Hz}, 2 \mathrm{H}), 1.94-1.86(\mathrm{~m}, 2 \mathrm{H}), 1.77-1.69(\mathrm{~m}, 2 \mathrm{H}), 1.66-1.54$ (m, 6H), $1.53-1.42(\mathrm{~m}, 4 \mathrm{H}), 1.02(\mathrm{~d}, J=2.0 \mathrm{~Hz}, 6 \mathrm{H}) .{ }^{13} \mathbf{C}$ NMR (151 MHz, $\left.\mathbf{C D C l}_{3}\right)$ $\delta 143.5,131.4,128.9,128.4,128.4,125.6$, 58.2, 49.5, 45.0, 44.2, 35.0, 33.5, 30.8, 28.4, 27.1, 24.6. HRMS (ESI+): calcd. for $\mathrm{C}_{22} \mathrm{H}_{36} \mathrm{~N}\left(\mathrm{M}+\mathrm{H}^{+}\right)$: 314.2847. Found: 314.2855 .

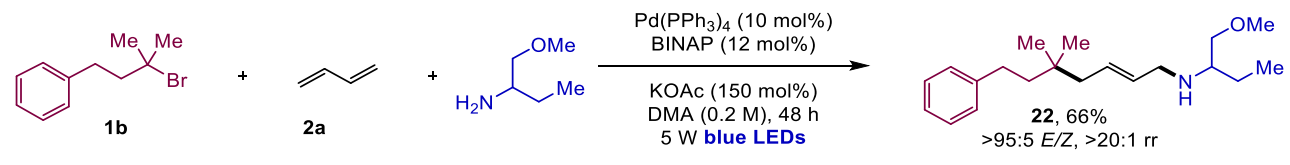

(E)-N-(1-methoxybutan-2-yl)-5,5-dimethyl-7-phenylhept-2-en-1-amine

(22). According to the general procedure G, using the corresponding alkyl bromide $1 \mathbf{b}$ ( $0.3 \mathrm{mmol}, 1.5$ equiv.), butadiene $2 \mathrm{a}(0.3 \mathrm{mmol}, 0.15 \mathrm{~mL}, 2 \mathrm{M}$ in THF, 1.5 equiv.), the nitrogen based nucleophile 3 (0.2 mmol, 1.0 equiv.), $\mathrm{Pd}\left(\mathrm{PPh}_{3}\right)_{4}$ (10 mol\%), BINAP (12 mol\%), KOAc (150 mol\%), and stirring at room temperature for $48 \mathrm{~h}$ under $5 \mathrm{~W}$ blue LEDs irradiation. The reaction mixture was filtered through a pad of silica gel and concentrated in vacuo, then purified by chromatography (pentane/EtOAc/Et $3 \mathrm{~N}=9 / 1 / 0.5$ ) to give 22 (39.7 mg, 0.131 mmol, 66\%, > 95:5 E/Z, > 20:1 rr) as a colorless oil. $\mathbf{R}_{\boldsymbol{f}}$ (pentane/EtOAc/Et $3 \mathrm{~N}=$ 9/1/0.5) 0.60. ${ }^{1} \mathbf{H}$ NMR (599 MHz, $\mathbf{C D C l}_{3}$ ) $\delta 7.42-7.31(\mathrm{~m}, 2 \mathrm{H}), 7.25$ (dtd, $J=7.6$, 1.9, $1.1 \mathrm{~Hz}, 3 \mathrm{H}), 5.77-5.61(\mathrm{~m}, 2 \mathrm{H}), 3.47$ (dd, $J=9.4,4.1 \mathrm{~Hz}, 1 \mathrm{H}), 3.42(\mathrm{~s}, 3 \mathrm{H})$, $3.39-3.32(\mathrm{~m}, 2 \mathrm{H}), 3.32-3.26(\mathrm{~m}, 1 \mathrm{H}), 2.79-2.74(\mathrm{~m}, 1 \mathrm{H}), 2.67-2.62(\mathrm{~m}, 2 \mathrm{H})$, $2.10-2.06(\mathrm{~m}, 2 \mathrm{H}), 1.61-1.55(\mathrm{~m}, 3 \mathrm{H}), 1.49(\mathrm{dt}, J=13.9,7.4 \mathrm{~Hz}, 1 \mathrm{H}), 1.02$ (s, 6H), $0.98(\mathrm{t}, J=7.5 \mathrm{~Hz}, 3 \mathrm{H}) .{ }^{13} \mathbf{C}$ NMR (151 MHz, $\left.\mathbf{C D C l}_{3}\right) \delta 143.6,131.4,129.0$, 128.4, 128.4, 125.6, 74.9, 59.0, 57.9, 49.4, 45.0, 44.3, 33.6, 30.8, 27.1, 24.2, 10.3. HRMS (ESI ${ }^{+}$): calcd. for $\mathrm{C}_{20} \mathrm{H}_{34} \mathrm{NO}\left(\mathrm{M}+\mathrm{H}^{+}\right)$: 304.2635. Found: 304.2647 .
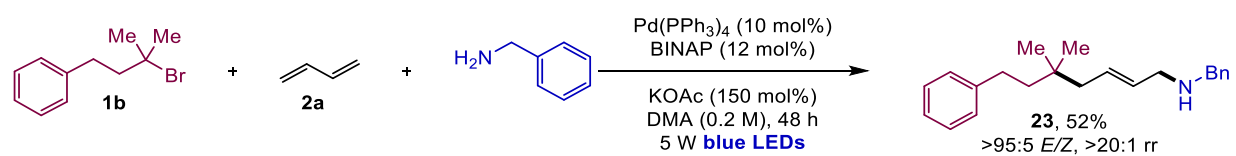

(E)-N-benzyl-5,5-dimethyl-7-phenylhept-2-en-1-amine (23). According to the general procedure $G$, using the corresponding alkyl bromide $\mathbf{1 b}(0.3 \mathrm{mmol}$, 
1.5 equiv.), butadiene $2 \mathrm{a}(0.3 \mathrm{mmol}, 0.15 \mathrm{~mL}, 2 M$ in THF, 1.5 equiv.), benzylamine (0.2 mmol, 1.0 equiv.), $\mathrm{Pd}\left(\mathrm{PPh}_{3}\right)_{4}(10 \mathrm{~mol} \%)$, BINAP (12 mol\%), KOAc (150 mol\%), and stirring at room temperature for $48 \mathrm{~h}$ under $5 \mathrm{~W}$ blue LEDs irradiation. The reaction mixture was filtered through a pad of silica gel and concentrated in vacuo, then purified by chromatography (pentane/EtOAc/Et ${ }_{3} \mathrm{~N}=$ 9/1/0.5) to give 23 (48.3 $\mathrm{mg}, 0.105 \mathrm{mmol}, 52 \%,>95: 5 E / Z,>20: 1 \mathrm{rr}$ ) as a colorless oil. $\mathbf{R}_{\boldsymbol{f}}$ (pentane/EtOAc/Et $3 \mathrm{~N}=9 / 1 / 0.5$ ) 0.24. ${ }^{\mathbf{1}} \mathbf{H} \mathbf{~ N M R}\left(599 \mathbf{~ M H z}, \mathbf{C D C l}_{3}\right.$ ) $\delta 7.32(\mathrm{~d}, J=5.2 \mathrm{~Hz}, 4 \mathrm{H}), 7.28-7.24(\mathrm{~m}, 3 \mathrm{H}), 7.17$ (ddd, $J=7.9,1.4,0.7 \mathrm{~Hz}, 3 \mathrm{H}$ ), $5.67-5.55$ (m, 2H), $3.79(\mathrm{~s}, 2 \mathrm{H}), 3.28$ - $3.24(\mathrm{~m}, 2 \mathrm{H}), 2.60-2.54(\mathrm{~m}, 2 \mathrm{H}), 2.04-$ $2.00(\mathrm{~m}, 2 \mathrm{H}), 1.52-1.49(\mathrm{~m}, 2 \mathrm{H}), 0.95(\mathrm{~s}, 6 \mathrm{H}) .{ }^{13} \mathrm{C}$ NMR (151 MHz, $\left.\mathbf{C D C l}_{3}\right) \delta$ $143.5,140.5,130.9,129.5,128.5,128.4,128.4,128.3,127.0,125.7,53.3,51.3$, 45.0, 44.3, 33.6, 30.8, 27.2. HRMS (ESI $\left.{ }^{+}\right)$: calcd. for $\mathrm{C}_{22} \mathrm{H}_{30} \mathrm{~N}\left(\mathrm{M}+\mathrm{H}^{+}\right)$: 308.2384. Found: 308.2385.
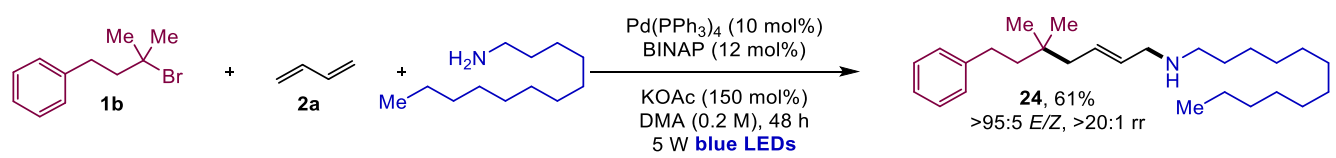

(E)-N-(5,5-dimethyl-7-phenylhept-2-en-1-yl)dodecan-1-amine

According to the general procedure G, using the corresponding alkyl bromide $\mathbf{1 b}$ ( $0.3 \mathrm{mmol}, 1.5$ equiv.), butadiene $2 \mathrm{a}$ ( $0.3 \mathrm{mmol}, 0.15 \mathrm{~mL}, 2 M$ in THF, 1.5 equiv.), the nitrogen based nucleophile 3 (0.2 mmol, 1.0 equiv.), $\mathrm{Pd}\left(\mathrm{PPh}_{3}\right)_{4}(10 \mathrm{~mol} \%)$, BINAP (12 mol\%), KOAc (150 mol\%), and stirring at room temperature for $48 \mathrm{~h}$ under $5 \mathrm{~W}$ blue LEDs irradiation. The reaction mixture was filtered through a pad of silica gel and concentrated in vacuo, then purified by chromatography (pentane/EtOAc/Et ${ }_{3} \mathrm{~N}=9 / 1 / 0.5$ ) to give 24 (47.3 mg, $0.123 \mathrm{mmol}, 61 \%$, > 95:5 $E / Z,>20: 1 \mathrm{rr}$ ) as a colorless oil. $\mathbf{R}_{\boldsymbol{f}}$ (pentane/EtOAc/Et $3 \mathrm{~N}=9 / 1 / 0.5$ ) 0.40. ${ }^{1} \mathbf{H}$ NMR (599 MHz, CDCl$\left._{3}\right) \delta 7.37-7.33(\mathrm{~m}, 2 \mathrm{H}), 7.25(\mathrm{ddd}, J=8.8,5.5,3.2 \mathrm{~Hz}, 3 \mathrm{H})$, $5.76-5.60(\mathrm{~m}, 2 \mathrm{H}), 3.30(\mathrm{dd}, J=6.0,1.1 \mathrm{~Hz}, 2 \mathrm{H}), 2.71-2.51(\mathrm{~m}, 4 \mathrm{H}), 2.09$ (dd, $J=$ 7.0, $1.0 \mathrm{~Hz}, 2 \mathrm{H}), 1.64-1.51(\mathrm{~m}, 4 \mathrm{H}), 1.43-1.26(\mathrm{~m}, 18 \mathrm{H}), 1.02$ (d, J = 2.2 Hz, 6H), $0.97(\mathrm{t}, J=7.0 \mathrm{~Hz}, 3 \mathrm{H}) .{ }^{13} \mathbf{C} \mathbf{N M R}\left(151 \mathbf{M H z} \mathbf{C D C l}_{3}\right) \delta 143.6,131.2,129.1,128.4$, 128.4, 125.6, 52.1, 49.5, 45.0, 44.2, 32.0, 30.8, 30.3, 29.8, 29.8, 29.8, 29.7, 29.7, 
29.5, 27.6, 27.1, 22.8, 14.2. HRMS (ESI'): calcd. for $\mathrm{C}_{27} \mathrm{H}_{48} \mathrm{~N}\left(\mathrm{M}+\mathrm{H}^{+}\right): 386.3792$. Found: 386.3805.

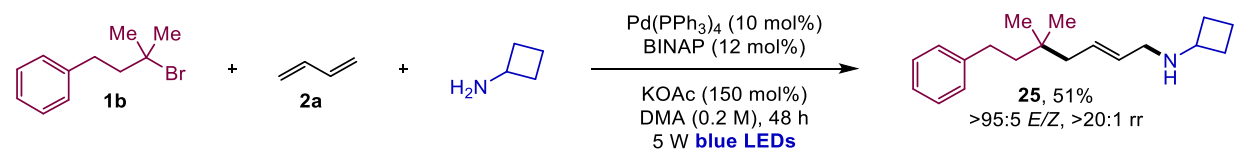

(E)-N-(5,5-dimethyl-7-phenylhept-2-en-1-yl)cyclobutanamine

(25).

According to the general procedure G, using the corresponding alkyl bromide $\mathbf{1 b}$ ( 0.3 mmol, 1.5 equiv.), butadiene 2 a ( $0.3 \mathrm{mmol}, 0.15 \mathrm{~mL}, 2 M$ in THF, 1.5 equiv.), the nitrogen based nucleophile 3 (0.2 mmol, 1.0 equiv.), $\mathrm{Pd}\left(\mathrm{PPh}_{3}\right)_{4}(10 \mathrm{~mol} \%)$, BINAP (12 mol\%), KOAc (150 mol\%), and stirring at room temperature for $48 \mathrm{~h}$ under $5 \mathrm{~W}$ blue LEDs irradiation. The reaction mixture was filtered through a pad of silica gel and concentrated in vacuo, then purified by chromatography (pentane/EtOAc/Et $3 \mathrm{~N}=$ 9/1/0.5) to give 25 (27.4 mg, 0.101 mmol, 51\%, > 95:5 $E / Z,>20: 1 \mathrm{rr}$ ) as a colorless oil. $\mathbf{R}_{\boldsymbol{f}}$ (pentane/EtOAc/Et ${ }_{3} \mathrm{~N}=9 / 1 / 0.5$ ) $0.38 .{ }^{1} \mathbf{H}$ NMR (599 MHz, $\left.\mathbf{C D C l}_{3}\right) \delta 7.40-7.33(\mathrm{~m}, 2 \mathrm{H}), 7.28-7.16(\mathrm{~m}, 3 \mathrm{H}), 5.72-5.60(\mathrm{~m}$, $2 \mathrm{H}), 3.39-3.29(\mathrm{~m}, 1 \mathrm{H}), 3.22(\mathrm{dt}, J=5.9,1.0 \mathrm{~Hz}, 2 \mathrm{H}), 2.71-2.59(\mathrm{~m}, 2 \mathrm{H}), 2.34-$ $2.25(\mathrm{~m}, 2 \mathrm{H}), 2.07(\mathrm{dd}, J=7.0,1.0 \mathrm{~Hz}, 2 \mathrm{H}), 1.82-1.66(\mathrm{~m}, 4 \mathrm{H}), 1.62-1.55(\mathrm{~m}$, 2H), 1.01 (s, 6H). ${ }^{13}$ C NMR (151 MHz, CDCl $\left._{3}\right) \delta$ 143.6, 131.3, 128.9, 128.4, 125.7, 53.7, 49.1, 45.0, 44.2, 33.6, 31.4, 30.8, 27.1, 14.9. HRMS (ESI+): calcd. for $\mathrm{C}_{19} \mathrm{H}_{30} \mathrm{~N}$ $\left(\mathrm{M}+\mathrm{H}^{+}\right):$272.2373. Found: 272.2392 .

\subsection{General Procedure H}

An oven-dried Schlenk tube containing a stir bar was charged under argon with the corresponding alkyl bromide 1 (0.2 mmol, 1.0 equiv.), $\mathrm{Pd}\left(\mathrm{PPh}_{3}\right)_{4}(10 \mathrm{~mol} \%)$, BINAP (12 mol\%), carbon based nucleophile (0.6 mmol, 3.0 equiv.), KOAc (150 mol\%) and DMA (0.85 mL). Subsequently, butadiene 2a (0.3 mmol, $0.15 \mathrm{~mL}, 2 \mathrm{M}$ in THF, 1.5 equiv.) was added. The reaction mixture was stirred at room temperate under blue LEDs irradiation ( $5 \mathrm{~W}, 455 \mathrm{~nm}$ ) for $48 \mathrm{~h}$. Then, the reaction was filtered through a pad of silica gel and washed with EtOAc $(3 \times 5 \mathrm{~mL})$. The obtained solution was concentrated in vacuo and the diastereomeric ratio 
determined by ${ }^{1} \mathrm{H}$ NMR spectroscopy of the crude reaction mixture. The reaction mixture was purified by column chromatography on silica gel (pentane/EtOAc) to afford the corresponding compounds.

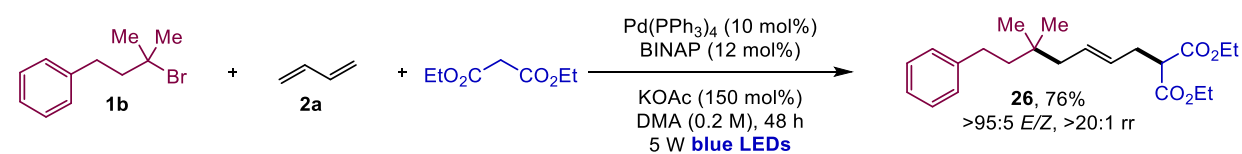

Diethyl (E)-2-(5,5-dimethyl-7-phenylhept-2-en-1-yl)malonate

(26).

According to the general procedure $\mathrm{H}$, using the corresponding alkyl bromide $\mathbf{1 b}$ ( $0.2 \mathrm{mmol}, 1.0$ equiv.), butadiene $2 \mathrm{a}(0.3 \mathrm{mmol}, 0.15 \mathrm{~mL}, 2 \mathrm{M}$ in THF, 1.5 equiv.), diethyl malonate (0.6 mmol, 3.0 equiv.), $\mathrm{Pd}\left(\mathrm{PPh}_{3}\right)_{4}(10 \mathrm{~mol} \%)$, BINAP (12 mol\%), KOAc (150 mol\%), and stirring at room temperature for $48 \mathrm{~h}$ under $5 \mathrm{~W}$ blue LEDs irradiation. The reaction mixture was filtered through a pad of silica gel and concentrated in vacuo, then purified by chromatography (pentane/EtOAc $=20 / 1$ ) to give 26 (27.4 mg, $0.101 \mathrm{mmol}, 51 \%,>95: 5 E / Z,>20: 1 \mathrm{rr}$ ) as a colorless oil. $\mathbf{R}_{\boldsymbol{f}}$ (pentane/EtOAc = 9/1) 0.43. ${ }^{1} \mathbf{H}$ NMR (599 $\left.\mathbf{~ M H z , ~} \mathbf{C D C l}_{3}\right) \delta 7.38-7.31(\mathrm{~m}, 2 \mathrm{H})$, $7.28-7.22(\mathrm{~m}, 3 \mathrm{H}), 5.71-5.61(\mathrm{~m}, 1 \mathrm{H}), 5.52-5.45(\mathrm{~m}, 1 \mathrm{H}), 4.30-4.22(\mathrm{~m}, 4 \mathrm{H})$, $3.48(\mathrm{t}, J=7.6 \mathrm{~Hz}, 1 \mathrm{H}), 2.74-2.69(\mathrm{~m}, 2 \mathrm{H}), 2.66-2.58(\mathrm{~m}, 2 \mathrm{H}), 2.03$ (dd, $J=7.5$, $1.2 \mathrm{~Hz}, 2 \mathrm{H}), 1.58-1.53(\mathrm{~m}, 2 \mathrm{H}), 1.33$ (t, $J=7.1 \mathrm{~Hz}, 6 \mathrm{H}), 0.99(\mathrm{~s}, 6 \mathrm{H}) .{ }^{13} \mathbf{C}$ NMR (151 MHz, $\mathrm{CDCl}_{3}$ ) $\delta$ 169.2, 143.5, 130.5, 128.4, 128.0, 125.7, 61.4, 52.4, 45.0, 44.3, 33.5, 32.1, 30.8, 27.0, 14.2. HRMS (ESI $\left.{ }^{+}\right)$: calcd. for $\mathrm{C}_{22} \mathrm{H}_{32} \mathrm{O}_{4} \mathrm{Na}\left(\mathrm{M}+\mathrm{Na}^{+}\right)$: 383.2193. Found: 383.2200.

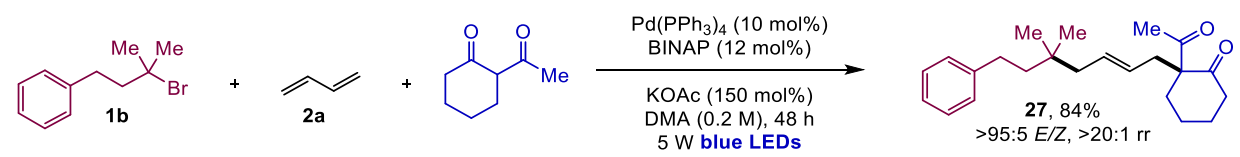

(E)-2-Acetyl-2-(5,5-dimethyl-7-phenylhept-2-en-1-yl)cyclohexan-1-one

(27). According to the general procedure $\mathrm{H}$, using the corresponding alkyl bromide 1b (0.2 mmol, 1.0 equiv.), butadiene 2a $(0.3 \mathrm{mmol}, 0.15 \mathrm{~mL}, 2 \mathrm{M}$ in THF, 1.5 equiv.), the carbon based nucleophile 3 ( $0.6 \mathrm{mmol}, 3.0$ equiv.), $\mathrm{Pd}\left(\mathrm{PPh}_{3}\right)_{4}(10$ mol\%), BINAP (12 mol\%), KOAc (150 mol\%), and stirring at room temperature for $48 \mathrm{~h}$ under $5 \mathrm{~W}$ blue LEDs irradiation. The reaction mixture was filtered 
through a pad of silica gel and concentrated in vacuo, then purified by chromatography (pentane $/$ EtOAc $=20 / 1)$ to give $27(56.9 \mathrm{mg}, 0.167 \mathrm{mmol}$, 84\%, > 95:5E/Z, > 20:1 rr) as a colorless oil. $\mathbf{R}_{\boldsymbol{f}}$ (pentane/EtOAc $=9 / 1$ ) 0.36. ${ }^{1} \mathbf{H}$ NMR (400 MHz, CDCl $) 7.38-7.31(\mathrm{~m}, 2 \mathrm{H}), 7.25(\mathrm{~d}, J=7.5 \mathrm{~Hz}, 3 \mathrm{H}), 5.56(\mathrm{qd}, J=$ 7.5, $1.2 \mathrm{~Hz}, 1 \mathrm{H}), 5.39-5.23(\mathrm{~m}, 1 \mathrm{H}), 2.71-2.44(\mathrm{~m}, 6 \mathrm{H}), 2.33$ (ddd, $J=14.1,11.7$, $6.0 \mathrm{~Hz}, 1 \mathrm{H}), 2.16$ (d, J = 1.0 Hz, 3H), $2.10-1.97$ (m, 3H), $1.83-1.68(\mathrm{~m}, 3 \mathrm{H}), 1.53$ (dq, $J=12.6,3.6,3.1 \mathrm{~Hz}, 3 \mathrm{H}), 0.98$ (s, 6H). ${ }^{13} \mathbf{C}$ NMR (101 $\left.\mathbf{M H z}, \mathbf{C D C l}_{3}\right) \delta 209.9$, 206.2, 143.5, 131.4, 128.6, 128.5, 128.4, 126.5, 126.1, 125.6, 67.9, 45.0, 44.3, 41.9, 37.9, 34.4, 34.1, 33.5, 30.8, 27.2, 27.1, 26.5, 22.2. HRMS (ESI+): calcd. for $\mathrm{C}_{23} \mathrm{H}_{32} \mathrm{O}_{2} \mathrm{Na}\left(\mathrm{M}+\mathrm{Na}^{+}\right): 363.2300$. Found: 363.2301 .

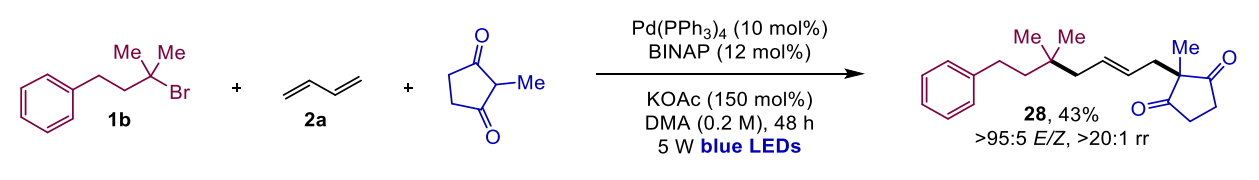

(E)-2-(5,5-Dimethyl-7-phenylhept-2-en-1-yl)-2-methylcyclopentane-1,3-

dione (28). According to the general procedure $\mathrm{H}$, using the corresponding alkyl bromide $\mathbf{1 b}$ ( $0.2 \mathrm{mmol}, 1.0$ equiv.), butadiene $\mathbf{2 a}(0.3 \mathrm{mmol}, 0.15 \mathrm{~mL}, 2 \mathrm{M}$ in THF, 1.5 equiv.), the carbon based nucleophile 3 ( 0.6 mmol, 3.0 equiv.), $\mathrm{Pd}\left(\mathrm{PPh}_{3}\right)_{4}(10$ mol\%), BINAP (12 mol\%), KOAc (150 mol\%), and stirring at room temperature for $48 \mathrm{~h}$ under $5 \mathrm{~W}$ blue LEDs irradiation. The reaction mixture was filtered through a pad of silica gel and concentrated in vacuo, then purified by chromatography (pentane $/$ EtOAc $=9 / 1)$ to give $28(26.9 \mathrm{mg}, 0.086 \mathrm{mmol}, 43 \%$, > 95:5E/Z, > 20:1 rr) as a colorless oil. $\mathbf{R}_{\boldsymbol{f}}$ (pentane/EtOAc $=8 / 1$ ) 0.57. ${ }^{1} \mathbf{H} \mathbf{~ N M R}$ (599 $\mathbf{M H z}, \mathbf{C D C l}_{3}$ ) $\delta 7.39-7.34(\mathrm{~m}, 2 \mathrm{H}), 7.27-7.24(\mathrm{~m}, 3 \mathrm{H}), 5.64-5.58(\mathrm{~m}, 1 \mathrm{H})$, $5.34-5.27(\mathrm{~m}, 1 \mathrm{H}), 2.83-2.76(\mathrm{~m}, 2 \mathrm{H}), 2.72-2.65(\mathrm{~m}, 2 \mathrm{H}), 2.64-2.57(\mathrm{~m}, 2 \mathrm{H})$, $2.42(\mathrm{dd}, J=7.4,1.1 \mathrm{~Hz}, 2 \mathrm{H}), 2.01$ (dd, $J=7.5,1.3 \mathrm{~Hz}, 2 \mathrm{H}), 1.55-1.49(\mathrm{~m}, 2 \mathrm{H})$, 1.18 (s, 3H), 0.98 (s, 6H). ${ }^{13}$ C NMR (151 MHz, CDCl 3 ) $\delta 216.6,143.4,132.8,128.5$, $128.4,125.7,125.3,57.2,45.2,44.2,39.4,35.5,33.5,30.8,27.0,19.0$. HRMS (ESI ${ }^{+}$): calcd. for $\mathrm{C}_{21} \mathrm{H}_{28} \mathrm{O}_{2} \mathrm{Na}\left(\mathrm{M}+\mathrm{Na}^{+}\right)$: 355.1982. Found: 355.1980 . 


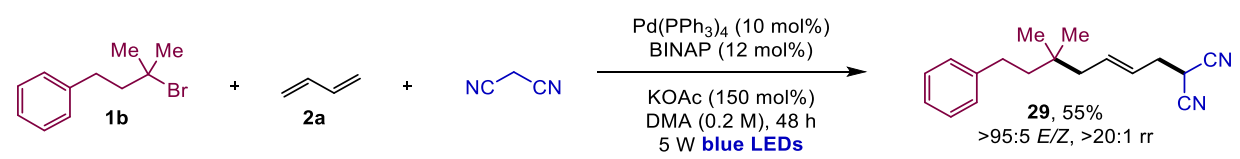

(E)-2-(5,5-Dimethyl-7-phenylhept-2-en-1-yl)malononitrile (29). According to the general procedure $\mathrm{H}$, using the corresponding alkyl bromide $\mathbf{1 b}(0.2 \mathrm{mmol}$, 1.0 equiv.), butadiene $2 \mathrm{a}$ ( $0.3 \mathrm{mmol}, 0.15 \mathrm{~mL}, 2 \mathrm{M}$ in THF, 1.5 equiv.), the carbon based nucleophile 3 (0.6 mmol, 3.0 equiv.), $\mathrm{Pd}\left(\mathrm{PPh}_{3}\right)_{4}(10 \mathrm{~mol} \%)$, BINAP (12 mol\%), KOAc (150 mol\%), and stirring at room temperature for $48 \mathrm{~h}$ under $5 \mathrm{~W}$ blue LEDs irradiation. The reaction mixture was filtered through a pad of silica gel and concentrated in vacuo, then purified by chromatography $($ pentane $/$ EtOAc $=$ 20/1) to give 29 (29.4 mg, $0.111 \mathrm{mmol}, 55 \%,>95: 5 E / Z,>20: 1 \mathrm{rr}$ ) as a colorless oil. $\mathbf{R}_{\boldsymbol{f}}$ (pentane/EtOAc $\left.=9 / 1\right)$ 0.39. ${ }^{\mathbf{1}} \mathbf{H}$ NMR (400 $\left.\mathbf{M H z}, \mathbf{C D C l}_{3}\right) \delta 7.40-7.31(\mathrm{~m}$, 2H), $7.25(\mathrm{dt}, J=6.5,1.5 \mathrm{~Hz}, 3 \mathrm{H}), 5.97-5.88(\mathrm{~m}, 1 \mathrm{H}), 5.63-5.52(\mathrm{~m}, 1 \mathrm{H}), 3.78(\mathrm{t}$, $J=6.5 \mathrm{~Hz}, 1 \mathrm{H}), 2.79(\mathrm{t}, J=6.9 \mathrm{~Hz}, 2 \mathrm{H}), 2.69-2.59(\mathrm{~m}, 2 \mathrm{H}), 2.19-2.10(\mathrm{~m}, 2 \mathrm{H})$, $1.65-1.57(\mathrm{~m}, 2 \mathrm{H}), 1.04(\mathrm{~s}, 6 \mathrm{H}) .{ }^{13} \mathrm{C}$ NMR (101 MHz, $\left.\mathbf{C D C l}_{3}\right) \delta$ 143.3, 136.2, 128.5, 128.4, 125.8, 123.1, 112.5, 45.1, 44.3, 34.2, 33.7, 30.8, 27.0, 23.6. HRMS (ESI+): calcd. for $\mathrm{C}_{18} \mathrm{H}_{22} \mathrm{~N}_{2} \mathrm{Na}\left(\mathrm{M}+\mathrm{Na}^{+}\right)$: 289.1675. Found: 289.1683 .

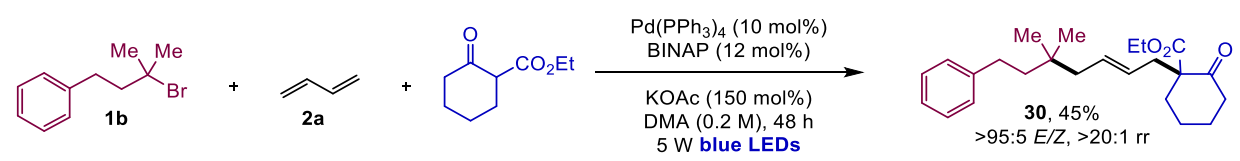

Ethyl (E)-1-(5,5-dimethyl-7-phenylhept-2-en-1-yl)-2-oxocyclohexane-1carboxylate (30). According to the general procedure $H$, using the corresponding alkyl bromide $\mathbf{1 b}(0.2 \mathrm{mmol}, 1.0$ equiv.), butadiene $2 \mathbf{a}(0.3 \mathrm{mmol}$, $0.15 \mathrm{~mL}, 2 M$ in THF, 1.5 equiv.), the carbon based nucleophile 3 (0.6 mmol, 3.0 equiv.), $\mathrm{Pd}\left(\mathrm{PPh}_{3}\right)_{4}(10 \mathrm{~mol} \%)$, BINAP (12 mol\%), KOAc (150 mol\%), and stirring at room temperature for $48 \mathrm{~h}$ under $5 \mathrm{~W}$ blue LEDs irradiation. The reaction mixture was filtered through a pad of silica gel and concentrated in vacuo, then purified by chromatography (pentane/EtOAc $=20 / 1)$ to give 30 (33.6 mg, 0.091 mmol, 45\%, > 95:5 E/Z, > 20:1 rr) as a colorless oil. $\mathbf{R}_{\boldsymbol{f}}$ (pentane/EtOAc $=19 / 1$ ) 0.41. ${ }^{1} \mathrm{H}$ NMR (599 MHz, $\left.\mathbf{C D C l}_{3}\right) \delta 7.37-7.32(\mathrm{~m}, 2 \mathrm{H}), 7.25(\mathrm{dt}, J=8.2,2.1 \mathrm{~Hz}$, $3 \mathrm{H}), 5.65-5.52(\mathrm{~m}, 1 \mathrm{H}), 5.52-5.41(\mathrm{~m}, 1 \mathrm{H}), 4.24(\mathrm{qd}, J=7.1,4.5 \mathrm{~Hz}, 2 \mathrm{H}), 2.69-$ 
$2.59(\mathrm{~m}, 3 \mathrm{H}), 2.58-2.49(\mathrm{~m}, 3 \mathrm{H}), 2.43$ (ddd, $J=13.9,7.6,1.0 \mathrm{~Hz}, 1 \mathrm{H}), 2.08$ (dddd, $J=13.0,7.8,3.6,2.0 \mathrm{~Hz}, 1 \mathrm{H}), 2.03(\mathrm{dd}, J=7.4,1.3 \mathrm{~Hz}, 2 \mathrm{H}), 1.86-1.75(\mathrm{~m}, 2 \mathrm{H})$, $1.74-1.61(\mathrm{~m}, 2 \mathrm{H}), 1.57-1.54(\mathrm{~m}, 2 \mathrm{H}), 1.31(\mathrm{t}, J=7.1 \mathrm{~Hz}, 3 \mathrm{H}), 0.99(\mathrm{~s}, 6 \mathrm{H}) .{ }^{13} \mathrm{C}$ NMR (151 MHz, $\left.\mathbf{C D C l}_{3}\right) \delta$ 207.8, 171.7, 143.6, 131.2, 128.4, 128.4, 127.1, 125.6, $61.3,61.2,45.1,44.3,41.3,38.3,35.8,33.6,30.8,27.6,27.1,27.1,22.6,14.3$. HRMS (ESI ${ }^{+}$): calcd. for $\mathrm{C}_{24} \mathrm{H}_{34} \mathrm{O}_{3} \mathrm{Na}\left(\mathrm{M}+\mathrm{Na}^{+}\right)$: 393.2406. Found: 393.2401 .

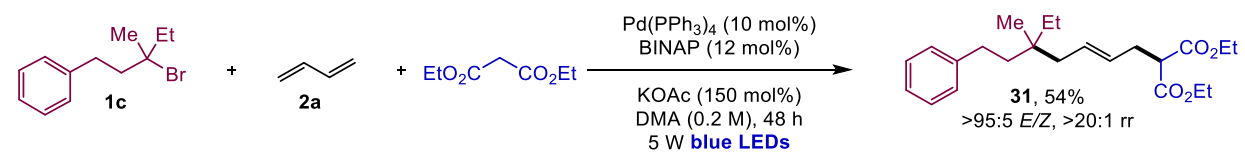

Diethyl (E)-2-(5-ethyl-5-methyl-7-phenylhept-2-en-1-yl)malonate (31). According to the general procedure $\mathrm{H}$, using the corresponding alkyl bromide 1c ( $0.2 \mathrm{mmol}, 1.0$ equiv.), butadiene $2 \mathrm{a}$ ( $0.3 \mathrm{mmol}, 0.15 \mathrm{~mL}, 2 \mathrm{M}$ in THF, 1.5 equiv.), diethyl malonate (0.6 mmol, 3.0 equiv.), $\mathrm{Pd}\left(\mathrm{PPh}_{3}\right)_{4}$ (10 mol\%), BINAP (12 mol\%), KOAc (150 mol\%), and stirring at room temperature for $48 \mathrm{~h}$ under $5 \mathrm{~W}$ blue LEDs irradiation. The reaction mixture was filtered through a pad of silica gel and concentrated in vacuo, then purified by chromatography (pentane/EtOAc $=20 / 1$ ) to give 31 (40.4 mg, $0.108 \mathrm{mmol}, 54 \%$, > 95:5 E/Z, > 20:1 rr) as a colorless oil. $\mathbf{R}_{\boldsymbol{f}}$ (pentane/EtOAc = 19/1) 0.71. ${ }^{1} \mathbf{H}$ NMR (400 MHz, CDCl $\left._{3}\right) \delta 7.42-7.33(\mathrm{~m}, 2 \mathrm{H})$, 7.32 - $7.19(\mathrm{~m}, 3 \mathrm{H}), 5.71-5.58(\mathrm{~m}, 1 \mathrm{H}), 5.57-5.46(\mathrm{~m}, 1 \mathrm{H}), 4.35-4.16(\mathrm{~m}, 4 \mathrm{H})$, $3.47(\mathrm{t}, J=7.6 \mathrm{~Hz}, 1 \mathrm{H}), 2.70(\mathrm{ddd}, J=7.9,6.8,1.1 \mathrm{~Hz}, 2 \mathrm{H}), 2.66-2.56(\mathrm{~m}, 2 \mathrm{H}), 2.10$ - 2.01 (m, 2H), $1.57-1.47$ (m, 2H), $1.44-1.24$ (m, 8H), $0.98-0.83$ (m, 6H). ${ }^{13} \mathrm{C}$ NMR (101 MHz, CDCl $\left._{3}\right) \delta 169.2,143.6,130.3,128.4,128.4,127.9,125.7,61.5$, 52.4, 42.1, 41.3, 35.9, 32.1, 31.4, 30.3, 24.4, 14.2, 8.1. HRMS (ESI'): calcd. for $\mathrm{C}_{23} \mathrm{H}_{34} \mathrm{O}_{4} \mathrm{Na}\left(\mathrm{M}+\mathrm{Na}^{+}\right): 397.2349$. Found: 397.2362 .
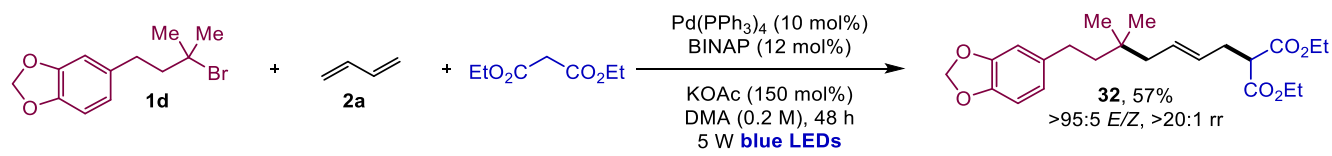

Diethyl

(E)-2-(7-(benzo[d][1,3]dioxol-5-yl)-5,5-dimethylhept-2-en-1yl)malonate (32). According to the general procedure $H$, using the corresponding alkyl bromide $1 \mathbf{d}(0.2 \mathrm{mmol}$, 1.0 equiv.), butadiene $2 \mathbf{a}(0.3 \mathrm{mmol}$, 
$0.15 \mathrm{~mL}, 2 M$ in THF, 1.5 equiv.), diethyl malonate ( $0.6 \mathrm{mmol}, 3.0$ equiv.), $\mathrm{Pd}\left(\mathrm{PPh}_{3}\right)_{4}(10 \mathrm{~mol} \%)$, BINAP (12 mol\%), KOAc (150 mol\%), and stirring at room temperature for $48 \mathrm{~h}$ under $5 \mathrm{~W}$ blue LEDs irradiation. The reaction mixture was filtered through a pad of silica gel and concentrated in vacuo, then purified by chromatography (pentane $/$ EtOAc $=20 / 1)$ to give $32(46.0 \mathrm{mg}, 0.114 \mathrm{mmol}$, $57 \%,>95: 5 E / Z,>20: 1 \mathrm{rr}$ ) as a colorless oil. $\mathbf{R}_{\boldsymbol{f}}$ (pentane/EtOAc $=9 / 1$ ) $0.45 .{ }^{1} \mathbf{H}$ NMR (599 MHz, CDCl M $_{3} \delta 6.71(\mathrm{~d}, J=7.9 \mathrm{~Hz}, 1 \mathrm{H}), 6.65(\mathrm{~d}, J=1.7 \mathrm{~Hz}, 1 \mathrm{H}), 6.60(\mathrm{dd}$, $J=7.9,1.7 \mathrm{~Hz}, 1 \mathrm{H}), 5.90(\mathrm{~s}, 2 \mathrm{H}), 5.61-5.52(\mathrm{~m}, 1 \mathrm{H}), 5.42-5.34(\mathrm{~m}, 1 \mathrm{H}), 4.23-$ $4.12(\mathrm{~m}, 4 \mathrm{H}), 3.38(\mathrm{t}, J=7.6 \mathrm{~Hz}, 1 \mathrm{H}), 2.67-2.58(\mathrm{~m}, 2 \mathrm{H}), 2.48-2.42(\mathrm{~m}, 2 \mathrm{H}), 1.92$ (dd, $J=7.4,1.2 \mathrm{~Hz}, 2 \mathrm{H}), 1.45-1.37(\mathrm{~m}, 2 \mathrm{H}), 1.25(\mathrm{t}, J=7.1 \mathrm{~Hz}, 6 \mathrm{H}), 0.88(\mathrm{~s}, 6 \mathrm{H})$. ${ }^{13} \mathrm{C}$ NMR (151 MHz, $\left.\mathbf{C D C l}_{3}\right) \delta$ 169.1, 147.6, 145.5, 137.4, 130.4, 128.0, 121.0, 108.9, 108.2, 100.8, 61.4, 52.4, 45.0, 44.5, 33.5, 32.1, 30.5, 27.0, 14.2. HRMS (ESI+): calcd. for $\mathrm{C}_{23} \mathrm{H}_{32} \mathrm{O}_{6} \mathrm{Na}\left(\mathrm{M}+\mathrm{Na}^{+}\right): 427.2091$. Found: 427.2095.

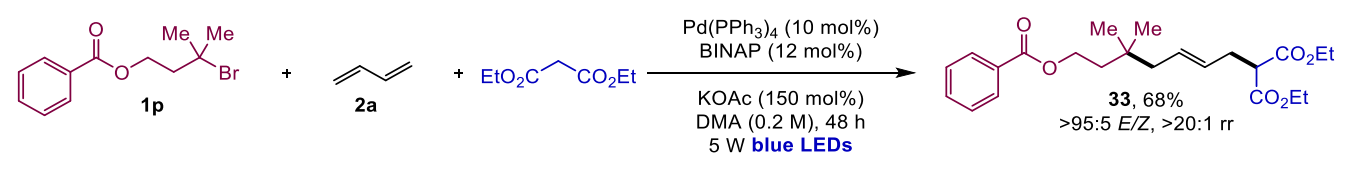

Diethyl (E)-2-(7-(benzoyloxy)-5,5-dimethylhept-2-en-1-yl)malonate (33). According to the general procedure $\mathrm{H}$, using the corresponding alkyl bromide $\mathbf{1 p}$ ( $0.2 \mathrm{mmol}, 1.0$ equiv.), butadiene $2 \mathrm{a}$ ( $0.3 \mathrm{mmol}, 0.15 \mathrm{~mL}, 2 \mathrm{M}$ in THF, 1.5 equiv.), diethyl malonate (0.6 mmol, 3.0 equiv.), $\mathrm{Pd}\left(\mathrm{PPh}_{3}\right)_{4}$ (10 mol\%), BINAP (12 mol\%), KOAc (150 mol\%), and stirring at room temperature for $48 \mathrm{~h}$ under $5 \mathrm{~W}$ blue LEDs irradiation. The reaction mixture was filtered through a pad of silica gel and concentrated in vacuo, then purified by chromatography (pentane $/$ EtOAc $=20 / 1$ ) to give 33 (54.7 mg, 0.135 mmol, 68\%, > 95:5 E/Z, > 20:1 rr) as a colorless oil. $\mathbf{R}_{\boldsymbol{f}}$ $($ pentane $/$ EtOAc $=9 / 1)$ 0.45. ${ }^{1} \mathbf{H}$ NMR (599 $\left.\mathbf{~ M H z , ~} \mathbf{C D C l}_{3}\right) \delta 8.09-8.00(\mathrm{~m}, 2 \mathrm{H})$, $7.62-7.51(\mathrm{~m}, 1 \mathrm{H}), 7.42(\mathrm{t}, J=7.8 \mathrm{~Hz}, 2 \mathrm{H}), 5.57(\mathrm{dtt}, J=15.0,7.4,1.3 \mathrm{~Hz}, 1 \mathrm{H})$, 5.40 (ddd, $J=15.1,7.5,6.2 \mathrm{~Hz}, 1 \mathrm{H}), 4.35$ (t, $J=7.3 \mathrm{~Hz}, 2 \mathrm{H}), 4.23-4.14(\mathrm{~m}, 4 \mathrm{H}$ ), $3.38(\mathrm{t}, J=7.6 \mathrm{~Hz}, 1 \mathrm{H}), 2.63-2.58(\mathrm{~m}, 2 \mathrm{H}), 1.95(\mathrm{~d}, J=7.2 \mathrm{~Hz}, 2 \mathrm{H}), 1.66(\mathrm{t}, J=7.3$ $\mathrm{Hz}, 2 \mathrm{H}), 1.24(\mathrm{t}, J=7.1 \mathrm{~Hz}, 6 \mathrm{H}), 0.93(\mathrm{~s}, 6 \mathrm{H}) .{ }^{13} \mathbf{C}$ NMR (151 MHz, CDCl 3 ) $\delta$ 169.1, 166.8, 132.9, 130.6, 129.9, 129.6, 128.5, 128.4, 62.4, 61.4, 52.3, 45.5, 39.8, 32.8, 
32.0, 27.2, 14.2. HRMS (ESI+): calcd. for $\mathrm{C}_{23} \mathrm{H}_{32} \mathrm{O}_{6} \mathrm{Na}\left(\mathrm{M}+\mathrm{Na}^{+}\right)$: 427.2091. Found: 427.2100.

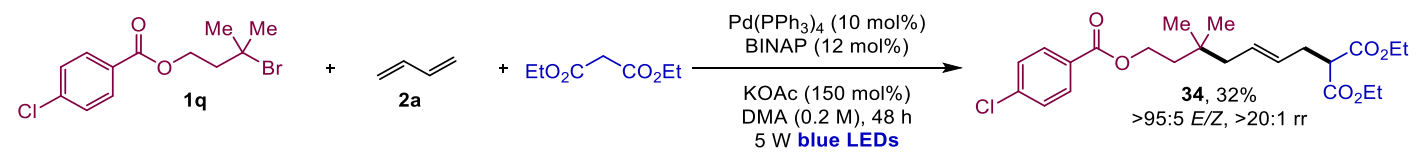

Diethyl

(E)-2-(7-((4-chlorobenzoyl)oxy)-5,5-dimethylhept-2-en-1-

yl)malonate (34). According to the general procedure $H$, using the corresponding alkyl bromide 1q (0.2 mmol, 1.0 equiv.), butadiene 2a (0.3 mmol, $0.15 \mathrm{~mL}, 2 M$ in THF, 1.5 equiv.), diethyl malonate (0.6 mmol, 3.0 equiv.), $\mathrm{Pd}\left(\mathrm{PPh}_{3}\right)_{4}(10 \mathrm{~mol} \%)$, BINAP (12 mol\%), KOAc (150 mol\%), and stirring at room temperature for $48 \mathrm{~h}$ under $5 \mathrm{~W}$ blue LEDs irradiation. The reaction mixture was filtered through a pad of silica gel and concentrated in vacuo, then purified by chromatography (pentane $/$ EtOAc $=9 / 1)$ to give $34(28.3 \mathrm{mg}, 0.065 \mathrm{mmol}, 32 \%$, > 95:5 E/Z, $>20: 1 \mathrm{rr})$ as a colorless oil. $\mathbf{R}_{\boldsymbol{f}}$ (pentane/EtOAc $\left.=9 / 1\right)$ 0.45. ${ }^{\mathbf{1}} \mathbf{H} \mathbf{~ N M R}$ $\left(599 \mathbf{M H z} \mathbf{C D C l}_{3}\right) \delta 7.95(\mathrm{~d}, J=8.6 \mathrm{~Hz}, 2 \mathrm{H}), 7.40(\mathrm{~d}, J=8.6 \mathrm{~Hz}, 2 \mathrm{H}), 5.63-5.52$ (m, 1H), $5.44-5.37(\mathrm{~m}, 1 \mathrm{H}), 4.34(\mathrm{t}, J=7.4 \mathrm{~Hz}, 2 \mathrm{H}), 4.17$ (p, J= $7.1 \mathrm{~Hz}, 4 \mathrm{H}), 3.38$ (t, $J=7.6 \mathrm{~Hz}, 1 \mathrm{H}), 2.70-2.57(\mathrm{~m}, 2 \mathrm{H}), 1.94(\mathrm{dd}, J=7.4,1.2 \mathrm{~Hz}, 2 \mathrm{H}), 1.66$ (d, $J=7.4$ $\mathrm{Hz}, 2 \mathrm{H}), 1.24(\mathrm{t}, J=7.1 \mathrm{~Hz}, 6 \mathrm{H}), 0.92$ (d, $J=4.8 \mathrm{~Hz}, 6 \mathrm{H}) .{ }^{13} \mathrm{C}$ NMR (151 MHz, $\left.\mathrm{CDCl}_{3}\right) \delta 169.1,165.9,139.4,131.0,129.9,129.0,128.8,128.6,62.6,61.5,52.3$, 45.5, 39.7, 32.8, 32.0, 27.2, 14.2. HRMS (ESI ${ }^{+}$): calcd. for $\mathrm{C}_{23} \mathrm{H}_{31} \mathrm{O}_{6} \mathrm{ClNa}\left(\mathrm{M}+\mathrm{Na}^{+}\right)$: 461.1701. Found: 461.1721.

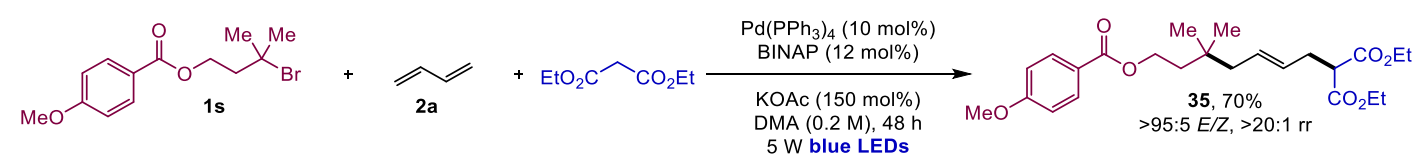

Diethyl

(E)-2-(7-((4-methoxybenzoyl)oxy)-5,5-dimethylhept-2-en-1yl)malonate (35). According to the general procedure $H$, using the corresponding alkyl bromide $1 \mathrm{~s}(0.2 \mathrm{mmol}, 1.0$ equiv.), butadiene 2 a $(0.3 \mathrm{mmol}$, $0.15 \mathrm{~mL}, 2 M$ in THF, 1.5 equiv.), diethyl malonate (0.6 mmol, 3.0 equiv.), $\mathrm{Pd}\left(\mathrm{PPh}_{3}\right)_{4}(10 \mathrm{~mol} \%)$, BINAP (12 mol\%), KOAc (150 mol\%), and stirring at room temperature for $48 \mathrm{~h}$ under $5 \mathrm{~W}$ blue LEDs irradiation. The reaction mixture was 
filtered through a pad of silica gel and concentrated in vacuo, then purified by chromatography $($ pentane $/$ EtOAc $=9 / 1)$ to give $34(60.4 \mathrm{mg}, 0.139 \mathrm{mmol}, 70 \%,>$ 95:5E/Z, > 20:1 rr) as a colorless oil. $\mathbf{R}_{\boldsymbol{f}}$ (pentane/EtOAc $=9 / 1$ ) 0.25. ${ }^{1} \mathbf{H} \mathbf{~ N M R}$ (300 MHz, $\left.\mathbf{C D C l}_{3}\right) \delta 8.02-7.89(\mathrm{~m}, 2 \mathrm{H}), 6.97-6.82(\mathrm{~m}, 2 \mathrm{H}), 5.66-5.47(\mathrm{~m}, 1 \mathrm{H})$, $5.47-5.31(\mathrm{~m}, 1 \mathrm{H}), 4.31(\mathrm{t}, J=7.3 \mathrm{~Hz}, 2 \mathrm{H}), 4.21-3.98(\mathrm{~m}, 4 \mathrm{H}), 3.83(\mathrm{~d}, J=1.0 \mathrm{~Hz}$, $3 \mathrm{H}), 3.37(\mathrm{td}, J=7.6,0.9 \mathrm{~Hz}, 1 \mathrm{H}), 2.60(\mathrm{t}, J=7.2 \mathrm{~Hz}, 2 \mathrm{H}), 1.94(\mathrm{~d}, J=7.3 \mathrm{~Hz}, 2 \mathrm{H})$, $1.63(\mathrm{t}, J=7.3 \mathrm{~Hz}, 2 \mathrm{H}), 1.23(\mathrm{td}, J=7.1,1.0 \mathrm{~Hz}, 6 \mathrm{H}), 1.03-0.80(\mathrm{~m}, 6 \mathrm{H}) .{ }^{13} \mathrm{C} \mathrm{NMR}$ (75 $\left.\mathrm{MHz}^{\mathrm{CDCl}}{ }_{3}\right) \delta 169.1,166.5,163.3,131.6,130.0,128.5,123.0,113.6,62.0$, 61.4, 55.5, 52.3, 45.5, 39.8, 32.8, 32.0, 27.1, 14.2. HRMS (ESI+): calcd. for $\mathrm{C}_{24} \mathrm{H}_{34} \mathrm{O}_{7} \mathrm{Na}\left(\mathrm{M}+\mathrm{Na}^{+}\right): 457.2197$. Found: 457.2204.
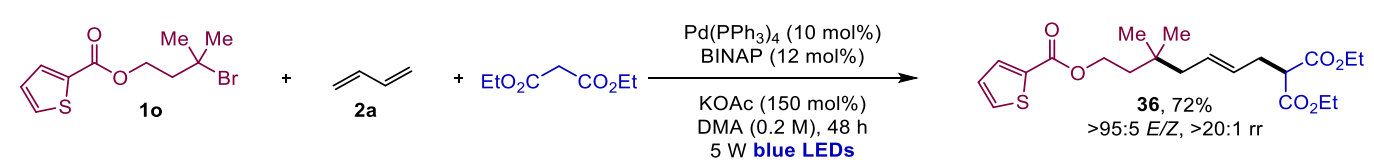

Diethyl (E)-2-(5,5-dimethyl-7-((thiophene-2-carbonyl)oxy)hept-2-en-1yl)malonate (36). According to the general procedure $H$, using the corresponding alkyl bromide 10 ( $0.2 \mathrm{mmol}, 1.0$ equiv.), butadiene 2 a $(0.3 \mathrm{mmol}$, $0.15 \mathrm{~mL}, 2 M$ in THF, 1.5 equiv.), diethyl malonate (0.6 mmol, 3.0 equiv.), $\mathrm{Pd}\left(\mathrm{PPh}_{3}\right)_{4}(10 \mathrm{~mol} \%)$, BINAP (12 mol\%), KOAc (150 mol\%), and stirring at room temperature for $48 \mathrm{~h}$ under $5 \mathrm{~W}$ blue LEDs irradiation. The reaction mixture was filtered through a pad of silica gel and concentrated in vacuo, then purified by chromatography $($ pentane $/$ EtOAc $=9 / 1)$ to give $35(59.2 \mathrm{mg}, 0.144 \mathrm{mmol}, 72 \%$, > 95:5E/Z, > 20:1 rr) as a colorless oil. $\mathbf{R}_{\boldsymbol{f}}$ (pentane/EtOAc $=9 / 1$ ) 0.32. ${ }^{1} \mathbf{H} \mathbf{~ N M R}$ (300 MHz, $\left.\mathbf{C D C l}_{3}\right) \delta 7.77(\mathrm{dd}, J=3.7,1.3 \mathrm{~Hz}, 1 \mathrm{H}), 7.53(\mathrm{dd}, J=5.0,1.3 \mathrm{~Hz}, 1 \mathrm{H})$, $7.08(\mathrm{dd}, J=5.0,3.8 \mathrm{~Hz}, 1 \mathrm{H}), 5.63-5.49(\mathrm{~m}, 1 \mathrm{H}), 5.44-5.30(\mathrm{~m}, 1 \mathrm{H}), 4.32(\mathrm{t}, J=$ $7.3 \mathrm{~Hz}, 2 \mathrm{H}$ ), 4.16 (qd, $J=7.1,2.0 \mathrm{~Hz}, 4 \mathrm{H}), 3.37$ (t, $J=7.6 \mathrm{~Hz}, 1 \mathrm{H}$ ), 2.60 (ddd, $J=7.8$, 6.7, $1.1 \mathrm{~Hz}, 2 \mathrm{H}), 1.93(\mathrm{dd}, J=7.3,1.2 \mathrm{~Hz}, 2 \mathrm{H}), 1.63(\mathrm{t}, J=7.3 \mathrm{~Hz}, 2 \mathrm{H}), 1.25(\mathrm{~d}, J=$ $7.1 \mathrm{~Hz}, 6 \mathrm{H}), 0.91$ (s, 6H). ${ }^{13} \mathbf{C}$ NMR (75 MHz, $\mathbf{C D C l}_{3}$ ) $\delta 169.1,162.4,134.2,133.4$, 132.3, 129.9, 128.5, 127.8, 62.5, 61.4, 52.3, 45.5, 39.7, 32.8, 32.0, 27.1, 14.2. HRMS (ESI+): calcd. for $\mathrm{C}_{21} \mathrm{H}_{30} \mathrm{O}_{6} \mathrm{SNa}\left(\mathrm{M}+\mathrm{Na}^{+}\right)$: 433.1655. Found: 433.1655 . 


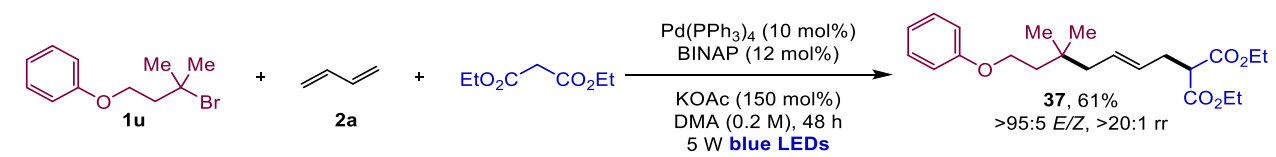

Diethyl (E)-2-(5,5-dimethyl-7-phenoxyhept-2-en-1-yl)malonate

(37).

According to the general procedure $\mathrm{H}$, using the corresponding alkyl bromide $\mathbf{1} \mathbf{u}$ ( $0.2 \mathrm{mmol}, 1.0$ equiv.), butadiene $2 \mathrm{a}$ ( $0.3 \mathrm{mmol}, 0.15 \mathrm{~mL}, 2 M$ in THF, 1.5 equiv.), diethyl malonate (0.6 mmol, 3.0 equiv.), $\mathrm{Pd}\left(\mathrm{PPh}_{3}\right)_{4}(10 \mathrm{~mol} \%)$, BINAP (12 mol\%), KOAc (150 mol\%), and stirring at room temperature for $48 \mathrm{~h}$ under $5 \mathrm{~W}$ blue LEDs irradiation. The reaction mixture was filtered through a pad of silica gel and concentrated in vacuo, then purified by chromatography (pentane $/$ EtOAc $=9 / 1)$ to give 37 (45.7 mg, $0.122 \mathrm{mmol}, 61 \%$, > 95:5 E/Z, > 20:1 rr) as a colorless oil. $\mathbf{R}_{\boldsymbol{f}}$ $($ pentane $/$ EtOAc $=9 / 1)$ 0.36. ${ }^{1} \mathbf{H}$ NMR (599 $\left.\mathbf{~ M H z , ~} \mathbf{C D C l}_{3}\right) \delta 7.66-7.59(\mathrm{~m}, 2 \mathrm{H})$, $7.27(\mathrm{tt}, J=7.3,1.0 \mathrm{~Hz}, 1 \mathrm{H}), 7.25-7.19(\mathrm{~m}, 2 \mathrm{H}), 5.98-5.89(\mathrm{~m}, 1 \mathrm{H}), 5.80-5.70$ (m, 1H), $4.58-4.48(\mathrm{~m}, 4 \mathrm{H}), 4.34(\mathrm{t}, J=7.2 \mathrm{~Hz}, 2 \mathrm{H}), 3.74(\mathrm{t}, J=7.6 \mathrm{~Hz}, 1 \mathrm{H}), 3.02-$ $2.94(\mathrm{~m}, 2 \mathrm{H}), 2.34-2.29(\mathrm{~m}, 2 \mathrm{H}), 2.04(\mathrm{t}, J=7.2 \mathrm{~Hz}, 3 \mathrm{H}), 1.60(\mathrm{t}, J=7.1 \mathrm{~Hz}, 6 \mathrm{H})$, 1.28 (s, 6H). ${ }^{13}$ C NMR (151 MHz, CDCl$\left._{3}\right) \delta$ 169.1, 159.1, 130.1, 129.5, 128.4, 120.6, 114.6, 64.8, 61.4, 52.4, 45.6, 40.4, 32.8, 32.0, 27.3, 14.2. HRMS (ESI+): calcd. for $\mathrm{C}_{22} \mathrm{H}_{32} \mathrm{O}_{5} \mathrm{Na}\left(\mathrm{M}+\mathrm{Na}^{+}\right): 399.2142$. Found: 399.2148.

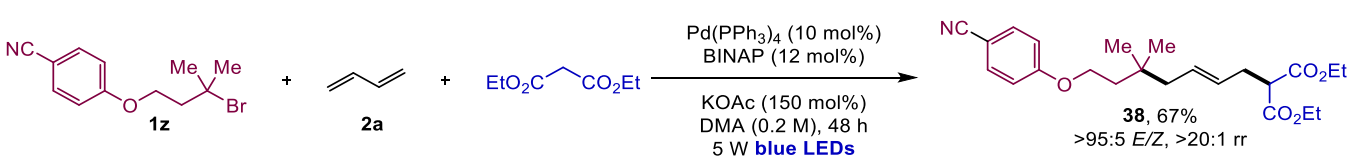

Diethyl (E)-2-(7-(4-cyanophenoxy)-5,5-dimethylhept-2-en-1-yl)malonate (38). According to the general procedure $H$, using the corresponding alkyl bromide $1 z$ ( $0.2 \mathrm{mmol}, 1.0$ equiv.), butadiene $2 \mathrm{a}(0.3 \mathrm{mmol}, 0.15 \mathrm{~mL}, 2 \mathrm{M}$ in THF, 1.5 equiv.), diethyl malonate ( $0.6 \mathrm{mmol}, 3.0$ equiv.), $\mathrm{Pd}\left(\mathrm{PPh}_{3}\right)_{4}(10 \mathrm{~mol} \%)$, BINAP (12 mol\%), KOAc (150 mol\%), and stirring at room temperature for $48 \mathrm{~h}$ under 5W blue LEDs irradiation. The reaction mixture was filtered through a pad of silica gel and concentrated in vacuo, then purified by chromatography (pentane/EtOAc $=9 / 1)$ to give $38(53.4 \mathrm{mg}, 0.133 \mathrm{mmol}, 67 \%,>95: 5 \mathrm{E} / \mathrm{Z},>20: 1$ rr) as a colorless oil. $\mathbf{R}_{\boldsymbol{f}}$ (pentane/EtOAc $\left.=4 / 1\right)$ 0.25. ${ }^{\mathbf{1}} \mathbf{H} \mathbf{~ N M R}\left(599 \mathbf{~ M H z}, \mathbf{C D C l}_{3}\right.$ ) $\delta 7.61-7.53(\mathrm{~m}, 2 \mathrm{H}), 6.95-6.87(\mathrm{~m}, 2 \mathrm{H}), 5.61-5.50(\mathrm{~m}, 1 \mathrm{H}), 5.42-5.34(\mathrm{~m}$, 
1H), $4.25-4.13(\mathrm{~m}, 4 \mathrm{H}), 4.02(\mathrm{t}, J=7.2 \mathrm{~Hz}, 2 \mathrm{H}), 3.37(\mathrm{t}, J=7.5 \mathrm{~Hz}, 1 \mathrm{H}), 2.60$ (ddd, $J=8.0,6.9,1.2 \mathrm{~Hz}, 2 \mathrm{H}), 1.94(\mathrm{dd}, J=7.5,1.3 \mathrm{~Hz}, 2 \mathrm{H}), 1.69(\mathrm{t}, J=7.2 \mathrm{~Hz}, 2 \mathrm{H}), 1.24(\mathrm{t}$, $J=7.1 \mathrm{~Hz}, 6 \mathrm{H}), 0.91(\mathrm{~s}, 6 \mathrm{H}) \cdot{ }^{13} \mathbf{C}$ NMR (151 MHz, $\left.\mathbf{C D C l}_{3}\right) \delta$ 169.0, 162.4, 134.0, 129.8, 128.6, 119.4, 115.3, 103.8, 65.5, 61.4, 52.3, 45.5, 40.0, 32.8, 32.0, 27.2, 14.2. HRMS (ESI ${ }^{+}$): calcd. for $\mathrm{C}_{23} \mathrm{H}_{31} \mathrm{NO}_{5} \mathrm{Na}\left(\mathrm{M}+\mathrm{Na}^{+}\right)$: 424.2094. Found: 424.2096.

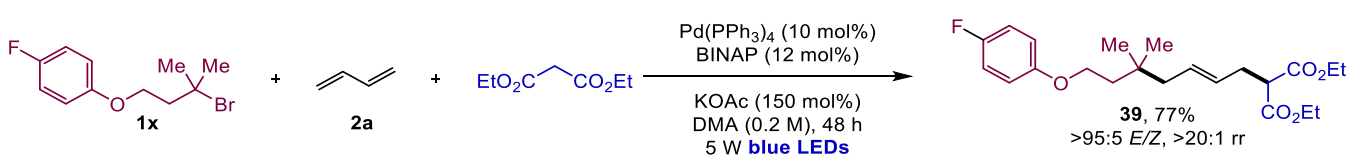

Diethyl (E)-2-(7-(4-fluorophenoxy)-5,5-dimethylhept-2-en-1-yl)malonate (39). According to the general procedure $H$, using the corresponding alkyl bromide 1x (0.2 mmol, 1.0 equiv.), butadiene $2 \mathrm{a}(0.3 \mathrm{mmol}, 0.15 \mathrm{~mL}, 2 \mathrm{M}$ in THF, 1.5 equiv.), diethyl malonate ( $0.6 \mathrm{mmol}, 3.0$ equiv.), $\mathrm{Pd}\left(\mathrm{PPh}_{3}\right)_{4}(10 \mathrm{~mol} \%)$, BINAP (12 mol\%), KOAc (150 mol\%), and stirring at room temperature for $48 \mathrm{~h}$ under 5W blue LEDs irradiation. The reaction mixture was filtered through a pad of silica gel and concentrated in vacuo, then purified by chromatography (pentane/EtOAc = 9/1) to give 39 (60.4 mg, 0.153 mmol, 77\%, > 95:5 E/Z, > 20:1 rr) as a colorless oil. $\mathbf{R}_{\boldsymbol{f}}$ (pentane/EtOAc $=4 / 1$ ) 0.43. ${ }^{\mathbf{1}} \mathbf{H} \mathbf{~ N M R}\left(599 \mathbf{~ M H z}, \mathbf{C D C l}_{3}\right.$ ) $\delta 6.99$ - $6.93(\mathrm{~m}, 2 \mathrm{H}), 6.85$ - $6.78(\mathrm{~m}, 2 \mathrm{H}), 5.63-5.54(\mathrm{~m}, 1 \mathrm{H}), 5.40(\mathrm{dtd}, J=15.1$, 6.9, $1.2 \mathrm{~Hz}, 1 \mathrm{H}), 4.24-4.13(\mathrm{~m}, 4 \mathrm{H}), 3.98-3.91(\mathrm{~m}, 2 \mathrm{H}), 3.38(\mathrm{tt}, J=7.6,0.7 \mathrm{~Hz}$, 1H), $2.64-2.58(\mathrm{~m}, 2 \mathrm{H}), 1.94(\mathrm{~d}, J=7.4 \mathrm{~Hz}, 2 \mathrm{H}), 1.67(\mathrm{t}, J=7.2 \mathrm{~Hz}, 2 \mathrm{H}), 1.25(\mathrm{tt}, J$ = 7.1, 0.7 Hz, 6H), $0.95-0.89$ (m, 6H). ${ }^{13} \mathbf{C}$ NMR (151 MHz, CDCl 3 ) $\delta 169.1,157.2$ $U=237.1 \mathrm{~Hz}), 155.2,130.1,128.5,115.8(J=22.7 \mathrm{~Hz}), 115.5(J=7.6 \mathrm{~Hz}), 65.6$, 61.5, 52.4, 45.6, 40.3, 32.8, 32.0, 27.3, 14.2. ${ }^{\mathbf{1}} \mathbf{F}\left\{{ }^{\mathbf{1}} \mathbf{H}\right\}$ NMR (564 $\left.\mathbf{M H z}, \mathbf{C D C l}_{3}\right) \delta-$ 124.5. HRMS (ESI+): calcd. for $\mathrm{C}_{22} \mathrm{H}_{31} \mathrm{O}_{5} \mathrm{FNa}\left(\mathrm{M}+\mathrm{Na}^{+}\right)$: 417.2048. Found: 417.2062 .

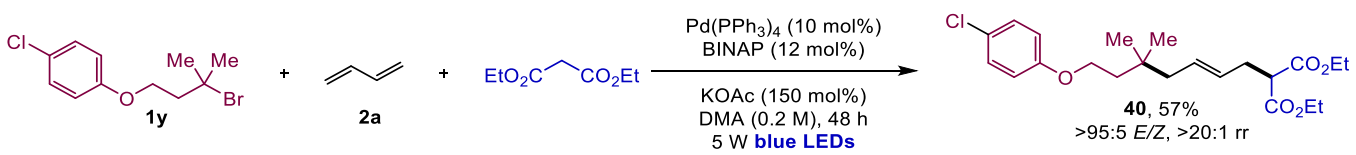

Diethyl (E)-2-(7-(4-chlorophenoxy)-5,5-dimethylhept-2-en-1-yl)malonate (40). According to the general procedure $\mathrm{H}$, using the corresponding alkyl 
bromide $1 y$ ( $0.2 \mathrm{mmol}, 1.0$ equiv.), butadiene $2 \mathrm{a}(0.3 \mathrm{mmol}, 0.15 \mathrm{~mL}, 2 \mathrm{M}$ in THF, 1.5 equiv.), diethyl malonate (0.6 mmol, 3.0 equiv.), $\mathrm{Pd}\left(\mathrm{PPh}_{3}\right)_{4}(10 \mathrm{~mol} \%)$, BINAP (12 mol\%), KOAc (150 mol\%), and stirring at room temperature for $48 \mathrm{~h}$ under 5W blue LEDs irradiation. The reaction mixture was filtered through a pad of silica gel and concentrated in vacuo, then purified by chromatography (pentane/EtOAc = 9/1) to give $40(46.9 \mathrm{mg}, 0.114 \mathrm{mmol}, 57 \%,>95: 5 E / Z,>20: 1$ rr) as a colorless oil. $\mathbf{R}_{\boldsymbol{f}}$ (pentane/EtOAc $\left.=4 / 1\right)$ 0.68. ${ }^{\mathbf{1}} \mathbf{H} \mathbf{~ N M R}\left(599 \mathbf{~ M H z}, \mathbf{C D C l}_{3}\right.$ ) $\delta 7.21(\mathrm{~d}, J=9.0 \mathrm{~Hz}, 2 \mathrm{H}), 6.79(\mathrm{~d}, J=9.0 \mathrm{~Hz}, 2 \mathrm{H}), 5.61-5.52(\mathrm{~m}, 1 \mathrm{H}), 5.44-5.36$ (m, 1H), 4.19 (dd, $J=13.3,7.2 \mathrm{~Hz}, 4 \mathrm{H}), 3.95$ (t, $J=7.2 \mathrm{~Hz}, 2 \mathrm{H}), 3.43-3.38(\mathrm{~m}, 1 \mathrm{H})$, $2.66-2.51(\mathrm{~m}, 2 \mathrm{H}), 1.94(\mathrm{dt}, J=7.5,1.1 \mathrm{~Hz}, 2 \mathrm{H}), 1.66(\mathrm{t}, J=7.2 \mathrm{~Hz}, 2 \mathrm{H}), 1.30-$ $1.24(\mathrm{~m}, 6 \mathrm{H}), 0.91$ (s, 6H). ${ }^{13} \mathbf{C}$ NMR (151 MHz, $\left.\mathbf{C D C l}_{3}\right) \delta$ 169.1, 157.7, 130.0, 129.4, 128.5, 125.5, 115.8, 65.3, 61.5, 52.3, 45.6, 40.2, 32.8, 32.0, 27.3, 14.2. HRMS (ESI ${ }^{+}$): calcd. for $\mathrm{C}_{22} \mathrm{H}_{31} \mathrm{O}_{5} \mathrm{ClNa}\left(\mathrm{M}+\mathrm{Na}^{+}\right)$: 433.1752. Found: 433.1751.

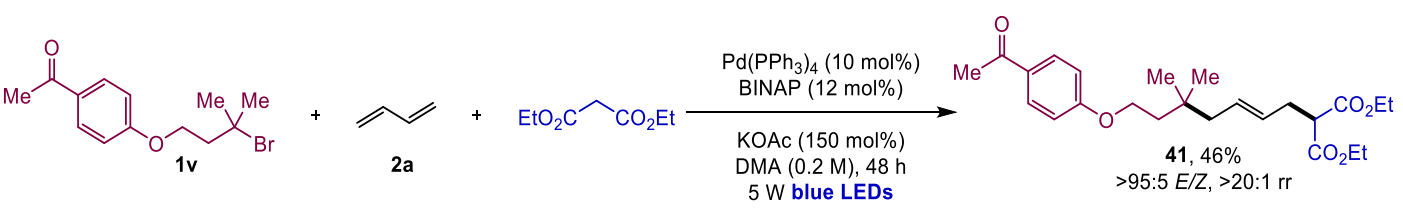

Diethyl (E)-2-(7-(4-acetylphenoxy)-5,5-dimethylhept-2-en-1-yl)malonate

(41). According to the general procedure $H$, using the corresponding alkyl bromide $1 v$ ( $0.2 \mathrm{mmol}, 1.0$ equiv.), butadiene $2 \mathrm{a}(0.3 \mathrm{mmol}, 0.15 \mathrm{~mL}, 2 \mathrm{M}$ in THF, 1.5 equiv.), diethyl malonate (0.6 mmol, 3.0 equiv.), $\mathrm{Pd}\left(\mathrm{PPh}_{3}\right)_{4}(10 \mathrm{~mol} \%)$, BINAP (12 mol\%), KOAc (150 mol\%), and stirring at room temperature for $48 \mathrm{~h}$ under 5W blue LEDs irradiation. The reaction mixture was filtered through a pad of silica gel and concentrated in vacuo, then purified by chromatography (pentane/EtOAc = 9/1) to give $41(38.4 \mathrm{mg}, 0.092 \mathrm{mmol}, 46 \%,>95: 5 \mathrm{E} / \mathrm{Z},>20: 1$ rr) as a colorless oil. $\mathbf{R}_{\boldsymbol{f}}$ (pentane/EtOAc $\left.=4 / 1\right)$ 0.27. ${ }^{\mathbf{1}} \mathbf{H} \mathbf{~ N M R}\left(\mathbf{4 0 0} \mathbf{~ M H z}, \mathbf{C D C l}_{3}\right)$ $\delta 7.97-7.86(\mathrm{~m}, 2 \mathrm{H}), 6.93-6.84(\mathrm{~m}, 2 \mathrm{H}), 5.66-5.53(\mathrm{~m}, 1 \mathrm{H}), 5.47-5.30(\mathrm{~m}$, 1H), 4.17 (qt, $J=7.3,3.7 \mathrm{~Hz}, 4 \mathrm{H}), 4.05(\mathrm{t}, J=7.2 \mathrm{~Hz}, 2 \mathrm{H}), 3.38(\mathrm{t}, J=7.6 \mathrm{~Hz}, 1 \mathrm{H})$, $2.67-2.56(\mathrm{~m}, 2 \mathrm{H}), 2.54(\mathrm{~s}, 3 \mathrm{H}), 2.02-1.87(\mathrm{~m}, 2 \mathrm{H}), 1.70(\mathrm{t}, J=7.2 \mathrm{~Hz}, 2 \mathrm{H}), 1.24$ $(\mathrm{t}, J=7.1 \mathrm{~Hz}, 6 \mathrm{H}), 0.92(\mathrm{~s}, 6 \mathrm{H}) .{ }^{13} \mathbf{C}$ NMR (101 MHz, $\left.\mathbf{C D C l}_{3}\right) \delta$ 196.9, 169.1, 163.1, 
$130.7,130.3,130.0,128.6,114.2,65.3,61.5,52.3,45.6,40.1,32.8,32.0,27.3,26.4$, 14.2. HRMS (ESI ${ }^{+}$): calcd. for $\mathrm{C}_{24} \mathrm{H}_{34} \mathrm{O}_{6} \mathrm{Na}\left(\mathrm{M}+\mathrm{Na}^{+}\right)$: 441.2248. Found: 441.2249.

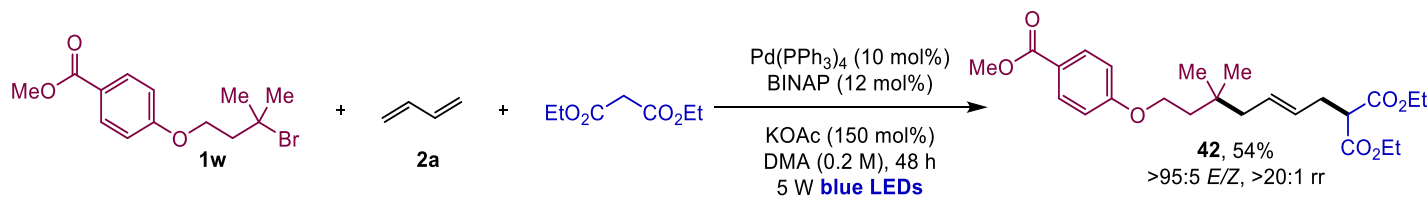

Diethyl (E)-2-(7-(4-(methoxycarbonyl)phenoxy)-5,5-dimethylhept-2-en-1yl)malonate (42). According to the general procedure $H$, using the corresponding alkyl bromide $\mathbf{1} \mathbf{w}(0.2 \mathrm{mmol}, 1.0$ equiv.), butadiene $\mathbf{2 a}(0.3 \mathrm{mmol}$, $0.15 \mathrm{~mL}, 2 M$ in THF, 1.5 equiv.), diethyl malonate ( $0.6 \mathrm{mmol}, 3.0$ equiv.), $\mathrm{Pd}\left(\mathrm{PPh}_{3}\right)_{4}(10 \mathrm{~mol} \%)$, BINAP (12 mol\%), KOAc (150 mol\%), and stirring at room temperature for $48 \mathrm{~h}$ under $5 \mathrm{~W}$ blue LEDs irradiation. The reaction mixture was filtered through a pad of silica gel and concentrated in vacuo, then purified by chromatography (pentane $/$ EtOAc $=9 / 1)$ to give $42(46.7 \mathrm{mg}, 0.108 \mathrm{mmol}, 54 \%,>$ 95:5E/Z, > 20:1 rr) as a colorless oil. $\mathbf{R}_{\boldsymbol{f}}$ (pentane/EtOAc $=4 / 1$ ) 0.42. ${ }^{1} \mathbf{H} \mathbf{~ N M R}$ $\left(400 \mathrm{MHz} \mathrm{CDCl}_{3}\right) \delta 7.97(\mathrm{~d}, J=8.9 \mathrm{~Hz}, 2 \mathrm{H}), 6.88(\mathrm{~d}, J=9.0 \mathrm{~Hz}, 2 \mathrm{H}), 5.64-5.52$ $(\mathrm{m}, 1 \mathrm{H}), 5.44-5.32(\mathrm{~m}, 1 \mathrm{H}), 4.17(\mathrm{qd}, J=7.1,3.6 \mathrm{~Hz}, 4 \mathrm{H}), 4.04(\mathrm{t}, J=7.2 \mathrm{~Hz}, 2 \mathrm{H})$, $3.87(\mathrm{~s}, 3 \mathrm{H}), 3.38(\mathrm{t}, J=7.6 \mathrm{~Hz}, 1 \mathrm{H}), 2.66-2.54(\mathrm{~m}, 2 \mathrm{H}), 1.99-1.88(\mathrm{~m}, 2 \mathrm{H}), 1.69$ (t, $J=7.2 \mathrm{~Hz}, 2 \mathrm{H}), 1.25(\mathrm{t}, J=7.1 \mathrm{~Hz}, 6 \mathrm{H}), 0.92(\mathrm{~s}, 6 \mathrm{H}) .{ }^{13} \mathbf{C} \mathbf{~ N M R}\left(101 \mathbf{~ M H z}, \mathbf{C D C l}_{3}\right)$ $\delta 169.1,167.0,162.9,131.7,130.0,128.6,122.5,114.2,65.3,61.5,52.3,51.9,45.6$, 40.2, 32.8, 32.0, 27.3, 14.2. HRMS (ESI $\left.{ }^{+}\right)$: calcd. for $\mathrm{C}_{24} \mathrm{H}_{34} \mathrm{O}_{7} \mathrm{Na}\left(\mathrm{M}+\mathrm{Na}^{+}\right)$: 457.2197. Found: 457.2206.

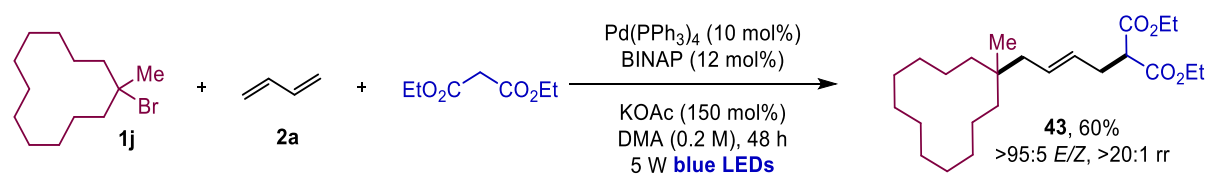

Diethyl (E)-2-(4-(1-methylcyclododecyl)but-2-en-1-yl)malonate (43). According to the general procedure $\mathrm{H}$, using the corresponding alkyl bromide $\mathbf{1 j}$ ( $0.2 \mathrm{mmol}, 1.0$ equiv.), butadiene $2 \mathrm{a}(0.3 \mathrm{mmol}, 0.15 \mathrm{~mL}, 2 \mathrm{M}$ in THF, 1.5 equiv.), diethyl malonate (0.6 mmol, 3.0 equiv.), $\mathrm{Pd}\left(\mathrm{PPh}_{3}\right)_{4}(10 \mathrm{~mol} \%)$, BINAP (12 mol\%), 
KOAc (150 mol\%), and stirring at room temperature for $48 \mathrm{~h}$ under $5 \mathrm{~W}$ blue LEDs irradiation. The reaction mixture was filtered through a pad of silica gel and concentrated in vacuo, then purified by chromatography (pentane $/$ EtOAc $=9 / 1)$ to give 43 (47.5 mg, $0.121 \mathrm{mmol}, 60 \%,>95: 5 \mathrm{E} / Z$, > 20:1 rr) as a colorless oil. $\mathbf{R}_{\boldsymbol{f}}$ (pentane/EtOAc $=9 / 1)$ 0.64. ${ }^{1} \mathbf{H}$ NMR (400 $\left.\mathbf{M H z} \mathbf{C D C l}_{3}\right) \delta 5.60-5.53(\mathrm{~m}, 1 \mathrm{H})$, $5.43-5.28(\mathrm{~m}, 1 \mathrm{H}), 4.18$ (qq, $J=7.1,3.7 \mathrm{~Hz}, 4 \mathrm{H}), 3.38$ (t, $J=7.6 \mathrm{~Hz}, 1 \mathrm{H}), 2.69-$ $2.50(\mathrm{~m}, 2 \mathrm{H}), 1.80(\mathrm{~d}, J=7.1 \mathrm{~Hz}, 2 \mathrm{H}), 1.45-0.99(\mathrm{~m}, 28 \mathrm{H}), 0.73(\mathrm{~s}, 3 \mathrm{H}) .{ }^{13} \mathbf{C}$ NMR (101 MHz, $\left.\mathbf{C D C l}_{3}\right) \delta 169.2,130.7,127.6,61.4,52.5,44.0,35.9,34.3,32.1,27.0$, 26.3, 25.2, 22.8, 22.3, 19.2, 14.2. HRMS (ESI $\left.{ }^{+}\right)$: calcd. for $\mathrm{C}_{24} \mathrm{H}_{42} \mathrm{O}_{4} \mathrm{Na}\left(\mathrm{M}+\mathrm{Na}^{+}\right)$: 417.2975. Found: 417.2979.

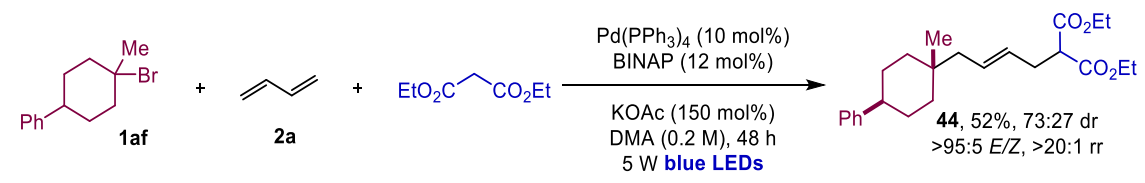

Diethyl 2-((E)-4-(-1-methyl-4-phenylcyclohexyl)but-2-en-1-yl)malonate (44). According to the general procedure $\mathrm{H}$, using the corresponding alkyl bromide 1af ( $0.2 \mathrm{mmol}, 1.0$ equiv.), butadiene $2 \mathrm{a}(0.3 \mathrm{mmol}, 0.15 \mathrm{~mL}, 2 \mathrm{M}$ in THF, 1.5 equiv.), diethyl malonate ( $0.6 \mathrm{mmol}, 3.0$ equiv.), $\mathrm{Pd}\left(\mathrm{PPh}_{3}\right)_{4}(10 \mathrm{~mol} \%)$, BINAP (12 mol\%), KOAc (150 mol\%), and stirring at room temperature for $48 \mathrm{~h}$ under 5W blue LEDs irradiation. The reaction mixture was filtered through a pad of silica gel and concentrated in vacuo, then purified by chromatography (pentane/EtOAc = 20/1) to give $44(40.4 \mathrm{mg}, 0.105 \mathrm{mmol}, 52 \%, 73: 27 \mathrm{dr},>95: 5$ $E / Z,>20: 1 \mathrm{rr}$ ) as a colorless oil. $\mathbf{R}_{\boldsymbol{f}}$ (pentane/EtOAc $\left.=9 / 1\right)$ 0.57. ${ }^{1} \mathbf{H}$ NMR (400 MHz, $\mathbf{C D C l}_{3}$ ) (major isomer only) $\delta 7.37-7.20(\mathrm{~m}, 5 \mathrm{H}), 5.68-5.55(\mathrm{~m}, 1 \mathrm{H}), 5.51$ - $5.38(\mathrm{~m}, 1 \mathrm{H}), 4.30-4.15(\mathrm{~m}, 4 \mathrm{H}), 3.43(\mathrm{td}, J=7.6,3.8 \mathrm{~Hz}, 1 \mathrm{H}), 2.71-2.60(\mathrm{~m}$, 2H), $2.46(\mathrm{td}, J=10.2,8.8,4.6 \mathrm{~Hz}, 1 \mathrm{H}), 2.12(\mathrm{~d}, J=7.3 \mathrm{~Hz}, 1 \mathrm{H}), 1.94(\mathrm{dd}, J=6.5,1.7$ $\mathrm{Hz}, 1 \mathrm{H}), 1.75-1.56(\mathrm{~m}, 6 \mathrm{H}), 1.30(\mathrm{td}, J=7.1,3.3 \mathrm{~Hz}, 8 \mathrm{H}), 0.89$ (s, 3H). ${ }^{13} \mathbf{C}$ NMR (101 $\mathbf{~ M H z , ~} \mathbf{C D C l}_{3}$ ) (major isomer only) 169.2, 147.7, 130.3, 128.4, 127.9, 126.9, 126.0, 61.4, 52.4, 44.3, 38.7, 37.8, 37.8, 32.6, 32.1, 29.9, 29.6, 29.5, 14.2. HRMS (ESI ${ }^{+}$): calcd. for $\mathrm{C}_{24} \mathrm{H}_{34} \mathrm{O}_{4} \mathrm{Na}\left(\mathrm{M}+\mathrm{Na}^{+}\right): 409.2349$. Found: 409.2351. 


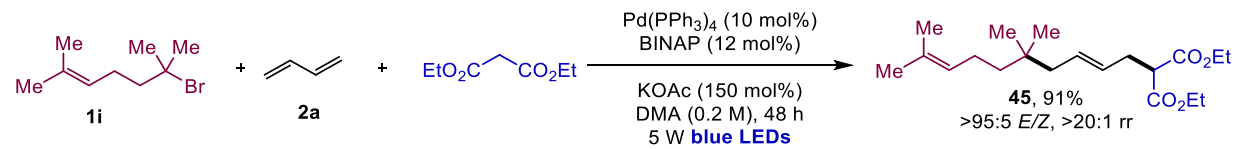

Diethyl (E)-2-(5,5,9-trimethyldeca-2,8-dien-1-yl)malonate (45). According to the general procedure $\mathrm{H}$, using the corresponding alkyl bromide $1 \mathrm{i}(0.2 \mathrm{mmol}$, 1.0 equiv.), butadiene $2 \mathrm{a}$ ( $0.3 \mathrm{mmol}, 0.15 \mathrm{~mL}, 2 M$ in THF, 1.5 equiv.), diethyl malonate (0.6 mmol, 3.0 equiv.), $\mathrm{Pd}\left(\mathrm{PPh}_{3}\right)_{4}(10 \mathrm{~mol} \%)$, BINAP (12 mol\%), KOAc (150 mol\%), and stirring at room temperature for $48 \mathrm{~h}$ under $5 \mathrm{~W}$ blue LEDs irradiation. The reaction mixture was filtered through a pad of silica gel and concentrated in vacuo, then purified by chromatography (pentane $/$ EtOAc $=9 / 1$ ) to give 45 (61.3 mg, 0.181 mmol, 91\%, > 95:5 E/Z, > 20:1 rr) as a colorless oil. $\mathbf{R}_{\boldsymbol{f}}$ (pentane $/$ EtOAc $=9 / 1)$ 0.42. ${ }^{1} \mathbf{H}$ NMR (599 $\left.\mathbf{~ M H z , ~} \mathbf{C D C l}_{3}\right) \delta 5.60-5.46(\mathrm{~m}, 1 \mathrm{H})$, $5.40-5.28(\mathrm{~m}, 1 \mathrm{H}), 5.07(\mathrm{ddt}, J=8.8,5.7,1.5 \mathrm{~Hz}, 1 \mathrm{H}), 4.27-4.11(\mathrm{~m}, 4 \mathrm{H}), 3.37$ (t, $J=7.6 \mathrm{~Hz}, 1 \mathrm{H}), 2.66-2.54(\mathrm{~m}, 2 \mathrm{H}), 1.92-1.81(\mathrm{~m}, 4 \mathrm{H}), 1.67(\mathrm{~s}, 3 \mathrm{H}), 1.59(\mathrm{~s}, 3 \mathrm{H})$, $1.25(\mathrm{td}, J=7.1,0.6 \mathrm{~Hz}, 6 \mathrm{H}), 1.19-1.13(\mathrm{~m}, 2 \mathrm{H}), 0.81(\mathrm{~s}, 6 \mathrm{H}) .{ }^{13} \mathrm{C}$ NMR (151 MHz, $\left.\mathrm{CDCl}_{3}\right) \delta 169.2,131.0,130.7,127.7,125.3,61.4,52.5,45.1,42.0,33.4,32.1,26.9$, 25.8, 22.9, 17.7, 14.2. HRMS (ESI $\left.{ }^{+}\right)$: calcd. for $\mathrm{C}_{20} \mathrm{H}_{34} \mathrm{O}_{4} \mathrm{Na}\left(\mathrm{M}+\mathrm{Na}^{+}\right): 361.2349$. Found: 361.2363.

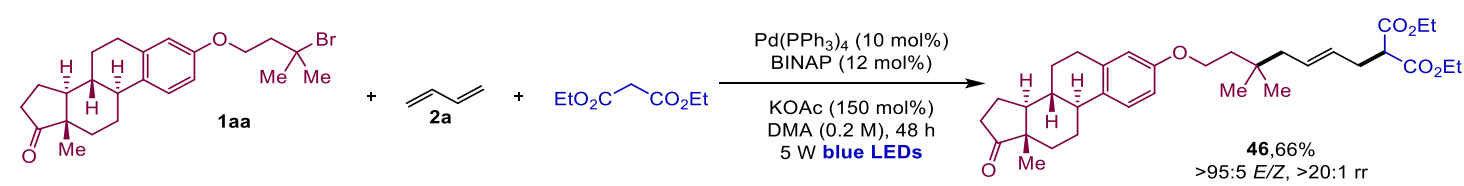

Diethyl 2-((E)-5,5-dimethyl-7-c((8R,9S,13S,14S)-13-methyl-17-oxo7,8,9,11,12,13,14,15,16,17-decahydro-6H-cyclopenta[a]phenanthren-3yl)oxy)hept-2-en-1-yl)malonate (46). According to the general procedure $H$, using the corresponding alkyl bromide $\mathbf{1 a a}(0.2 \mathrm{mmol}, 1.0$ equiv.), butadiene $\mathbf{2 a}$ (0.3 mmol, $0.15 \mathrm{~mL}, 2 \mathrm{M}$ in THF, 1.5 equiv.), the diethyl malonate (0.6 mmol, 3.0 equiv.), $\mathrm{Pd}\left(\mathrm{PPh}_{3}\right)_{4}(10 \mathrm{~mol} \%)$, BINAP (12 mol\%), KOAc (150 mol\%), and stirring at room temperature for $48 \mathrm{~h}$ under $5 \mathrm{~W}$ blue LEDs irradiation. The reaction 
mixture was filtered through a pad of silica gel and concentrated in vacuo, then purified by chromatography (pentane/EtOAc $=4 / 1)$ to give $46(72.7 \mathrm{mg}, 0.132$ mmol, 66\%, > 95:5 E/Z, > 20:1 rr) as a colorless oil. $\mathbf{R}_{\boldsymbol{f}}$ (pentane/EtOAc $=4 / 1$ ) 0.26. ${ }^{1} \mathrm{H}$ NMR (599 MHz, $\mathrm{CDCl}_{3}$ ) $\delta 7.18(\mathrm{~d}, J=8.6 \mathrm{~Hz}, 1 \mathrm{H}), 6.69$ (dd, $J=8.6,2.8 \mathrm{~Hz}$, $1 \mathrm{H}), 6.61(\mathrm{~d}, J=2.7 \mathrm{~Hz}, 1 \mathrm{H}), 5.61-5.54(\mathrm{~m}, 1 \mathrm{H}), 5.46-5.36(\mathrm{~m}, 1 \mathrm{H}), 4.23-4.09$ $(\mathrm{m}, 4 \mathrm{H}), 3.96(\mathrm{t}, J=7.2 \mathrm{~Hz}, 2 \mathrm{H}), 3.42-3.35(\mathrm{~m}, 1 \mathrm{H}), 2.89(\mathrm{td}, J=7.4,4.0 \mathrm{~Hz}, 2 \mathrm{H})$, $2.61(\mathrm{t}, J=7.4 \mathrm{~Hz}, 2 \mathrm{H}), 2.49(\mathrm{dd}, J=19.0,8.8 \mathrm{~Hz}, 1 \mathrm{H}), 2.42-2.35(\mathrm{~m}, 1 \mathrm{H}), 2.29-$ $2.21(\mathrm{~m}, 1 \mathrm{H}), 2.13(\mathrm{dt}, J=18.6,8.9 \mathrm{~Hz}, 1 \mathrm{H}), 2.07-2.00(\mathrm{~m}, 2 \mathrm{H}), 1.97-1.92(\mathrm{~m}$, 2H), $1.66(\mathrm{t}, J=7.2 \mathrm{~Hz}, 2 \mathrm{H}), 1.61-1.39(\mathrm{~m}, 5 \mathrm{H}), 1.25(\mathrm{td}, J=7.1,0.7 \mathrm{~Hz}, 6 \mathrm{H}), 0.92$ - 0.90 (m, 6H). ${ }^{13}$ C NMR (151 MHz, $\left.\mathbf{C D C l}_{3}\right) \delta$ 221.0, 169.1, 157.1, 137.7, 131.9, 130.1, 128.3, 126.3, 114.5, 112.2, 64.8, 61.4, 52.3, 50.5, 48.1, 45.6, 44.1, 40.4, 38.5, 35.9, 32.8, 32.0, 31.7, 29.7, 27.2, 26.6, 26.0, 21.7, 14.2, 13.9. HRMS (ESI+): calcd. for $\mathrm{C}_{34} \mathrm{H}_{48} \mathrm{O}_{6} \mathrm{Na}\left(\mathrm{M}+\mathrm{Na}^{+}\right): 575.3343$. Found: 575.3346 .

\subsection{General Procedure I}

An oven-dried Schlenk tube containing a stir bar was charged under argon with the corresponding alkyl bromide 1 (0.3 mmol, 1.5 equiv.), $\mathrm{Pd}\left(\mathrm{PPh}_{3}\right)_{4}(10 \mathrm{~mol} \%)$, BINAP (12 mol\%), oxygen based nucleophile ( $0.2 \mathrm{mmol}, 1.0$ equiv.), $\mathrm{K}_{2} \mathrm{CO}_{3}$ (150 mol\%) and DMA (0.85 mL). Subsequently, butadiene 2a (0.3 mmol, $0.15 \mathrm{~mL}, 2 \mathrm{M}$ in THF, 1.5 equiv.) was added. The reaction mixture was stirred at room temperate under blue LEDs irradiation (5W, $455 \mathrm{~nm}$ ) for $48 \mathrm{~h}$. Then, the reaction was filtered through a pad of silica gel and washed with EtOAc $(3 \times 5 \mathrm{~mL})$. The obtained solution was concentrated in vacuo and the diastereomeric ratio determined by ${ }^{1} \mathrm{H}$ NMR spectroscopy of the crude reaction mixture. The reaction mixture was purified by column chromatography on silica gel (pentane/EtOAc) to afford the corresponding compounds.

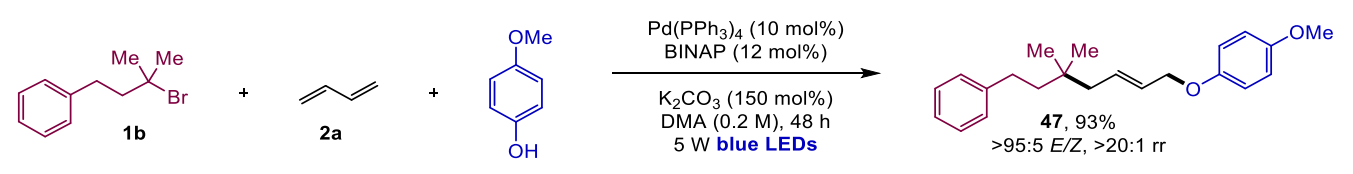




\section{(E)-1-((5,5-Dimethyl-7-phenylhept-2-en-1-yl)oxy)-4-methoxybenzene (47).}

According to the general procedure I, using the corresponding alkyl bromide $\mathbf{1 b}$ ( $0.3 \mathrm{mmol}, 1.5$ equiv.), butadiene $2 \mathrm{a}(0.3 \mathrm{mmol}, 0.15 \mathrm{~mL}, 2 \mathrm{M}$ in THF, 1.5 equiv.), 4-methoxyphenol (0.2 mmol, 1.0 equiv.), $\mathrm{Pd}\left(\mathrm{PPh}_{3}\right)_{4}$ (10 mol\%), BINAP (12 mol\%), $\mathrm{K}_{2} \mathrm{CO}_{3}(150 \mathrm{~mol} \%)$, and stirring at room temperature for $48 \mathrm{~h}$ under $5 \mathrm{~W}$ blue LEDs irradiation. The reaction mixture was filtered through a pad of silica gel and concentrated in vacuo, then purified by chromatography $($ pentane $/$ EtOAc $=$ 20/1) to give 47 (60.4 mg, $0.186 \mathrm{mmol}, 93 \%,>95: 5 E / Z,>20: 1 \mathrm{rr}$ ) as a colorless oil. $\mathbf{R}_{\boldsymbol{f}}$ (pentane/EtOAc $\left.=9 / 1\right)$ 0.76. ${ }^{\mathbf{1}} \mathbf{H}$ NMR (599 $\mathbf{M H z}, \mathbf{C D C l}_{3}$ ): $\delta$ ppm $7.31-$ $7.27(\mathrm{~m}, 2 \mathrm{H}), 7.21-7.18(\mathrm{~m}, 3 \mathrm{H}), 6.91-6.81(\mathrm{~m}, 4 \mathrm{H}), 5.90-5.85(\mathrm{~m}, 1 \mathrm{H}), 5.76-$ $5.70(\mathrm{~m}, 1 \mathrm{H}), 4.49(\mathrm{dd}, J=5.9,1.1 \mathrm{~Hz}, 2 \mathrm{H}), 3.78(\mathrm{~s}, 3 \mathrm{H}), 2.60-2.56(\mathrm{~m}, 2 \mathrm{H}), 2.09$ (dd, $J=7.4,1.1 \mathrm{~Hz}, 2 \mathrm{H}), 1.55-1.49$ (m, 2H), 0.97 (s, 6H). ${ }^{13} \mathrm{C}$ NMR (151 MHz, $\mathbf{C D C l}_{3}$ ): $\delta$ ppm 153.9, 153.0, 143.5, 131.9, 128.4, 127.8, 125.7, 115.9, 114.7, 69.5, 55.8, 44.9, 44.2, 33.7, 30.8, 27.2. HRMS (ESI $\left.{ }^{+}\right)$: calcd. for $\mathrm{C}_{22} \mathrm{H}_{28} \mathrm{O}_{2} \mathrm{Na}\left(\mathrm{M}+\mathrm{Na}^{+}\right)$: 347.1982. Found: 347.1977.
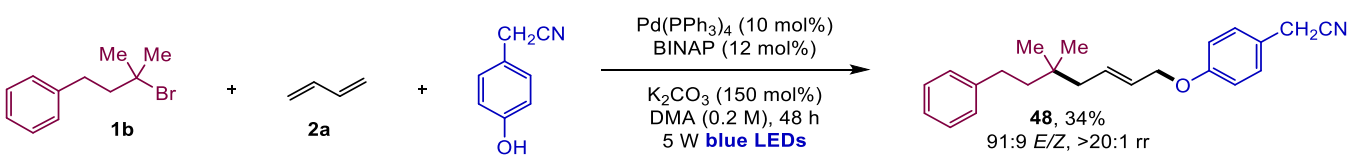

(E)-2-(4-((5,5-Dimethyl-7-phenylhept-2-en-1-yl)oxy)phenyl)acetonitrile

(48). According to the general procedure I, using the bromide $\mathbf{1 b}(0.3 \mathrm{mmol}, 1.5$ equiv.), butadiene 2a (0.3 mmol, $0.15 \mathrm{~mL}, 2 M$ in THF, 1.5 equiv.), 2-(4hydroxyphenyl)acetonitrile (0.2 mmol, 1.0 equiv.), $\mathrm{Pd}\left(\mathrm{PPh}_{3}\right)_{4}(0.020 \mathrm{mmol}, 10$ mol\%), BINAP (0.024 mmol, $12 \mathrm{~mol} \%), \mathrm{K}_{2} \mathrm{CO}_{3}$ (150 mol\%), and stirring at room temperature for $48 \mathrm{~h}$ under $5 \mathrm{~W}$ blue LEDs irradiation. The reaction mixture was filtered through a pad of silica gel and concentrated in vacuo, then purified by chromatography (pentane/EtOAc $=8 / 1 \rightarrow 7 / 1)$ to give $48(22.4 \mathrm{mg}, 0.067 \mathrm{mmol}$, $34 \%, 91: 9 E / Z$, > 20:1 rr) as a colorless gum. $\mathbf{R}_{\boldsymbol{f}}$ (pentane/EtOAc=8/1) 0.25. ${ }^{1} \mathbf{H}$ NMR (400 MHz, $\mathbf{C D C l}_{3}$, major isomer): $\delta$ ppm $7.31-7.27(\mathrm{~m}, 2 \mathrm{H}), 7.27-7.16$ (m, 5H), $6.97-6.91(\mathrm{~m}, 2 \mathrm{H}), 5.90(\mathrm{dtt}, J=15.3,7.4,1.2 \mathrm{~Hz}, 1 \mathrm{H}), 5.74(\mathrm{dtt}, J=15.3$, 5.7, $1.2 \mathrm{~Hz}, 1 \mathrm{H}), 4.55(\mathrm{~d}, J=5.9 \mathrm{~Hz}, 2 \mathrm{H}), 3.70(\mathrm{~s}, 2 \mathrm{H}), 2.64-2.54(\mathrm{~m}, 2 \mathrm{H}), 2.11(\mathrm{~d}, J$ 
$=7.3 \mathrm{~Hz}, 2 \mathrm{H}), 1.57-1.48(\mathrm{~m}, 2 \mathrm{H}), 0.99(\mathrm{~s}, 6 \mathrm{H}) .{ }^{1} \mathbf{H}$ NMR (400 $\mathbf{M H z}, \mathbf{C D C l}_{3}, \mathbf{m i n o r}^{-}$ isomer, diagnostic signals): $\delta$ ppm $5.81-5.79(\mathrm{~m}, 2 \mathrm{H}), 4.62(\mathrm{~d}, J=4.4 \mathrm{~Hz}, 3 \mathrm{H})$, $2.15(\mathrm{~d}, J=5.9 \mathrm{~Hz}, 3 \mathrm{H}), 1.03$ (s, 6H). ${ }^{13} \mathrm{C}$ NMR (101 $\mathbf{~ M H z} \mathbf{C D C l}_{3}$, major isomer): $\delta$ ppm 158.6, 143.4, 132.4, 129.15, 128.46, 128.44, 127.2, 125.7, 121.9, 115.6, $68.9,44.9,44.2,33.7,30.8,27.2,23.0$. Note: One aromatic signal is missing. ${ }^{13} \mathrm{C}$ NMR (101 MHz, $\mathbf{C D C l}_{3}$, minor isomer, non-overlapping signals): $\delta$ ppm 143.3, 129.19, 128.50, 126.4, 125.8, 115.5, 64.2, 44.3, 40.0, 34.0, 30.9, 27.0. HRMS (ESI ${ }^{+}$): calcd. for $\mathrm{C}_{23} \mathrm{H}_{27} \mathrm{NONa}\left(\mathrm{M}+\mathrm{Na}^{+}\right): 356.1985$. Found: 356.1972 .

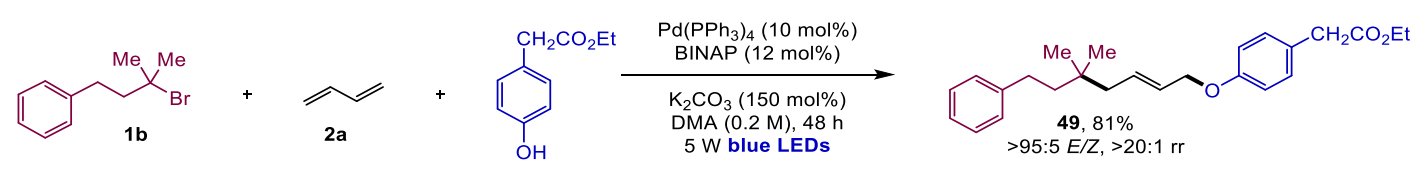

Ethyl (E)-2-(4-((5,5-dimethyl-7-phenylhept-2-en-1-yl)oxy)phenyl)acetate (49). According to the general procedure I, using the bromide $\mathbf{1 b}(0.3 \mathrm{mmol}, 1.5$ equiv.), butadiene (0.3 mmol, $2 M$ in THF, 1.5 equiv.), ethyl 2-(4hydroxyphenyl)acetate (0.2 mmol, 1.0 equiv.), $\mathrm{Pd}\left(\mathrm{PPh}_{3}\right)_{4}(0.020 \mathrm{mmol}, 10 \mathrm{~mol} \%)$, BINAP (0.024 mmol, $12 \mathrm{~mol} \%), \mathrm{K}_{2} \mathrm{CO}_{3}$ (150 mol\%), and stirring at room temperature for $48 \mathrm{~h}$ under $5 \mathrm{~W}$ blue LEDs irradiation. The reaction mixture was filtered through a pad of silica gel and concentrated in vacuo, then purified by chromatography (pentane $/$ EtOAc $=30 / 1 \rightarrow 20 / 1)$ to give $49(61.6 \mathrm{mg}, 0.162$ mmol, 81\%, > 95:5E/Z, > 20:1 rr) as a colorless viscous oil. $\mathbf{R}_{\boldsymbol{f}}$ (pentane/EtOAc $=$ 30/1) 0.30. ${ }^{1} \mathbf{H}$ NMR (300 MHz, $\mathbf{C D C l}_{3}$ ): $\delta$ ppm 7.35 - $7.26(\mathrm{~m}, 2 \mathrm{H}), 7.25$ - 7.14 (m, 5H), $6.94-6.85(\mathrm{~m}, 2 \mathrm{H}), 5.89(\mathrm{dt}, J=14.2,7.0,1 \mathrm{H}), 5.75(\mathrm{dt}, J=15.3,5.7 \mathrm{~Hz}$, $1 \mathrm{H}), 4.52(\mathrm{~d}, J=5.7 \mathrm{~Hz}, 2 \mathrm{H}), 4.16(\mathrm{q}, J=7.2 \mathrm{~Hz}, 2 \mathrm{H}), 3.56(\mathrm{~s}, 2 \mathrm{H}), 2.64-2.53(\mathrm{~m}$, 2H), 2.09 (d, J = 7.2 Hz, 2H), $1.57-1.46(\mathrm{~m}, 2 \mathrm{H}), 1.27$ (t, $J=7.1 \mathrm{~Hz}, 3 \mathrm{H}), 0.97$ (s, 6H). ${ }^{13}$ C NMR (75 MHz, CDCl$_{3}$ ): $\delta$ ppm 172.0, 157.9, 143.4, 132.1, 130.3, 128.4, $127.5,126.3,125.7,114.9,68.8,60.9,44.9,44.2,40.6,33.6,30.8,27.1,14.3$. Note: One aromatic proton missing, most likely due to overlap. HRMS (ESI+): calcd. for $\mathrm{C}_{25} \mathrm{H}_{32} \mathrm{O}_{3} \mathrm{Na}\left(\mathrm{M}+\mathrm{Na}^{+}\right): 403.2244$. Found: 403.2247. 


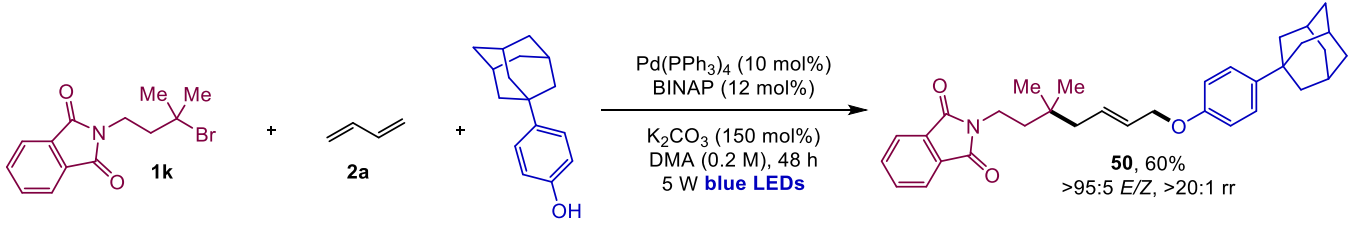

\section{2-((E)-7-(4-(Adamantan-1-yl)phenoxy)-3,3-dimethylhept-5-en-1-}

yl)isoindoline-1,3-dione (50). According to the general procedure I, using the bromide 1k (0.3 mmol, 1.50 equiv.), butadiene (0.3 mmol, $2 M$ in THF, 1.50 equiv.), phenol S3b (0.2 mmol, 1.00 equiv.), $\mathrm{Pd}\left(\mathrm{PPh}_{3}\right)_{4}(0.020 \mathrm{mmol}, 10 \mathrm{~mol} \%)$, BINAP (0.024 mmol, $12 \mathrm{~mol} \%), \mathrm{K}_{2} \mathrm{CO}_{3}(150 \mathrm{~mol} \%)$, and stirring at room temperature for $48 \mathrm{~h}$ under $5 \mathrm{~W}$ blue LEDs irradiation. The reaction mixture was filtered through a pad of silica gel and concentrated in vacuo, then purified by chromatography (pentane/EtOAc $=12 / 1 \rightarrow 10 / 1$ ) to give an inseparable mixture of product and remaining phenol ( $88.5 \mathrm{mg}$ in total). The yield was determined by ${ }^{1} \mathrm{H}$ NMR analysis $(0.120 \mathrm{mmol}, 60 \%,>95: 5 \mathrm{E} / Z,>20: 1 \mathrm{rr}) . \mathbf{R}_{\boldsymbol{f}}$ (pentane $/$ EtOAc $=$ 12/1) 0.27. ${ }^{1} \mathbf{H}$ NMR (400 MHz, $\left.\mathbf{C D C l}_{3}\right): \delta$ ppm $7.87-7.80(\mathrm{~m}, 2 \mathrm{H}), 7.73-7.67$ (m, 2H), $7.28-7.18\left(\mathrm{~m}, 2 \mathrm{H}^{*}\right), 6.90-6.84(\mathrm{~m}, 2 \mathrm{H}), 5.87(\mathrm{dt}, J=15.4,7.3 \mathrm{~Hz}, 1 \mathrm{H})$, $5.75(\mathrm{dt}, J=15.3,5.7 \mathrm{~Hz}, 1 \mathrm{H}), 4.51(\mathrm{~d}, J=5.6 \mathrm{~Hz}, 2 \mathrm{H}), 3.73-3.65(\mathrm{~m}, 2 \mathrm{H}), 2.12-$ $2.02\left(\mathrm{~m}, 3 \mathrm{H}^{*}\right), 1.90-1.82\left(\mathrm{~m}, 6 \mathrm{H}^{*}\right), 1.81-1.67\left(\mathrm{~m}, 6 \mathrm{H}^{*}\right), 1.58-1.50(\mathrm{~m}, 2 \mathrm{H})$, 0.97 (s, 6H). Note: Protons belonging to the adamantyl moiety and partially to the aromatic ring overlap with the starting phenol, therefore the observed integrations are bigger than expected (*). ${ }^{13} \mathbf{C}$ NMR (101 $\left.\mathbf{M H z}, \mathbf{C D C l}_{3}\right): \delta$ ppm 168.4, 156.5, 143.8, 134.0, 132.3, 131.1, 126.0, 125.8, 123.2, 114.5, 68.7, 44.8, 43.5, $43.4\left(^{*}\right)$, 39.6, $36.9\left(^{*}\right), 35.6,34.3,33.0,29.1\left(^{*}\right), 26.9$. Note: Aliphatic signals belonging to the starting phenol overlap with the one of the product $\left(^{*}\right)$, while the aromatic resonances can be distinguished. HRMS (ESI+): calcd. for $\mathrm{C}_{33} \mathrm{H}_{39} \mathrm{NO}_{3} \mathrm{Na}\left(\mathrm{M}+\mathrm{Na}^{+}\right)$: 520.2822. Found: 520.2812.
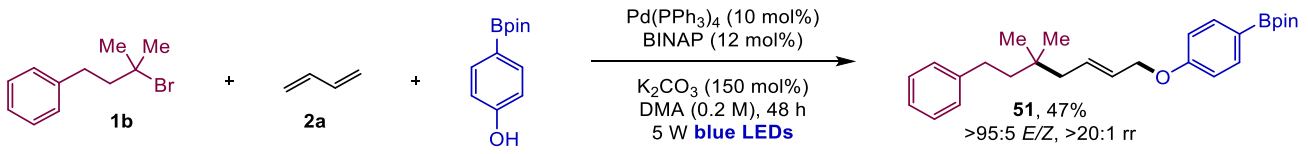


\section{(E)-2-(4-((5,5-Dimethyl-7-phenylhept-2-en-1-yl)oxy)phenyl)-4,4,5,5-}

tetramethyl-1,3,2-dioxaborolane (51). According to the general procedure I, using the bromide $\mathbf{1 b}$ ( $0.3 \mathrm{mmol}, 1.5$ equiv.), butadiene $2 \mathbf{a}(0.3 \mathrm{mmol}, 0.15 \mathrm{~mL}, 2$ $M$ in THF, 1.5 equiv.), 4-(4,4,5,5-tetramethyl-1,3,2-dioxaborolan-2-yl)phenol (0.2 mmol, 1.0 equiv.), $\mathrm{Pd}\left(\mathrm{PPh}_{3}\right)_{4}(0.020 \mathrm{mmol}, 10 \mathrm{~mol} \%)$, BINAP (0.024 mmol, 12 mol\%), $\mathrm{K}_{2} \mathrm{CO}_{3}(150 \mathrm{~mol} \%$ ), and stirring at room temperature for $48 \mathrm{~h}$ under $5 \mathrm{~W}$ blue LEDs irradiation. The reaction mixture was filtered through a pad of silica gel and concentrated in vacuo, then purified by chromatography (pentane/EtOAc $=50 / 1 \rightarrow 45 / 1 \rightarrow 40 / 1)$ to give $51(39.4 \mathrm{mg}, 0.094 \mathrm{mmol}, 47 \%,>95: 5 \mathrm{E} / \mathrm{Z},>20: 1$ rr) as a colorless gum. $\mathbf{R}_{\boldsymbol{f}}$ (pentane/EtOAc $\left.=40 / 1\right)$ 0.35. ${ }^{1} \mathbf{H}$ NMR (400 $\mathbf{~ M H z}$, $\mathrm{CDCl}_{3}$, major isomer): $\delta$ ppm $7.76(\mathrm{~d}, J=8.7 \mathrm{~Hz}, 2 \mathrm{H}), 7.31-7.25(\mathrm{~m}, 2 \mathrm{H}), 7.21-$ $7.14(\mathrm{~m}, 3 \mathrm{H}), 6.93(\mathrm{~d}, J=8.6 \mathrm{~Hz}, 1 \mathrm{H}), 5.88(\mathrm{dt}, J=15.5,7.2 \mathrm{~Hz}, 1 \mathrm{H}), 5.74(\mathrm{dt}, J=$ 15.5, $5.7 \mathrm{~Hz}, 1 \mathrm{H}), 4.55(\mathrm{~d}, J=5.7 \mathrm{~Hz}, 2 \mathrm{H}), 2.61-2.53(\mathrm{~m}, 2 \mathrm{H}), 2.08$ (d, $J=7.2 \mathrm{~Hz}$, 2H), $1.54-1.47(\mathrm{~m}, 2 \mathrm{H}), 1.35(\mathrm{~s}, 12 \mathrm{H}), 0.96(\mathrm{~s}, 6 \mathrm{H}) .{ }^{13} \mathbf{C}$ NMR (101 MHz, $\left.\mathbf{C D C l}_{3}\right)$ : $\delta$ ppm 161.4, 143.4, 136.6, 132.2, 128.46, 128.45, 127.3, 125.7, 114.2, 83.7, 68.6, $44.9,44.3,33.7,30.8,27.2,25.0$. The quaternary carbon attached to boron cannot be observed due to quadrupolar broadening. HRMS (ESI ${ }^{+}$): calcd. for $\mathrm{C}_{27} \mathrm{H}_{37} \mathrm{O}_{3} \mathrm{BNa}$ $\left(\mathrm{M}+\mathrm{Na}^{+}\right):$443.2733. Found: 443.2739 .

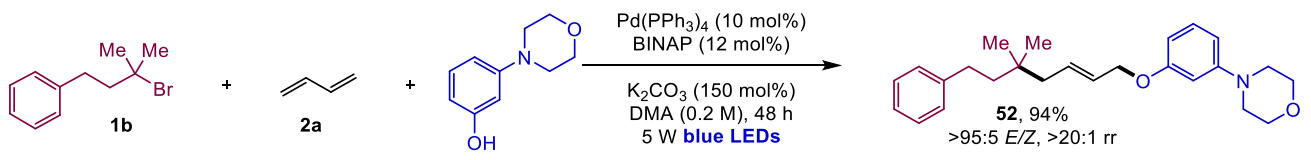

(E)-4-(3-((5,5-Dimethyl-7-phenylhept-2-en-1-yl)oxy)phenyl)morpholine

(52). According to the general procedure I, using the bromide $\mathbf{1 b}(0.3 \mathrm{mmol}, 1.5$ equiv.), butadiene 2a (0.3 mmol, $2 M$ in THF, 1.5 equiv.), 3-morpholinophenol (0.2 mmol, 1.0 equiv.), $\mathrm{Pd}\left(\mathrm{PPh}_{3}\right)_{4}(0.020 \mathrm{mmol}, 10 \mathrm{~mol} \%)$, BINAP (0.024 mmol, $12 \mathrm{~mol} \%), \mathrm{K}_{2} \mathrm{CO}_{3}$ (150 mol\%), and stirring at room temperature for $48 \mathrm{~h}$ under 5W blue LEDs irradiation. The reaction mixture was filtered through a pad of silica gel and concentrated in vacuo, then purified by chromatography (pentane $/$ EtOAc $=8 / 1 \rightarrow 7 / 1$ ) to give $52(71.3 \mathrm{mg}, 0.188 \mathrm{mmol}, 94 \%,>95: 5 \mathrm{E} / \mathrm{Z}$, > 20:1 rr) as a colorless gum. $\mathbf{R}_{\boldsymbol{f}}$ (pentane/EtOAc $=8 / 1$ ) 0.25. ${ }^{\mathbf{1}} \mathbf{H} \mathbf{~ N M R}(\mathbf{3 0 0} \mathbf{~ M H z}$, 
$\mathbf{C D C l}_{3}$ ): $\delta$ ppm $7.34-7.26(\mathrm{~m}, 2 \mathrm{H}), 7.20(\mathrm{ddd}, J=8.2,4.8,3.4 \mathrm{~Hz}, 4 \mathrm{H}), 6.59-6.44$ $(\mathrm{m}, 3 \mathrm{H}), 5.90(\mathrm{dt}, J=15.4,7.3 \mathrm{~Hz}, 1 \mathrm{H}), 5.76(\mathrm{dt}, J=15.4,5.6 \mathrm{~Hz}, 1 \mathrm{H}), 4.53(\mathrm{~d}, J=$ $5.7 \mathrm{~Hz}, 2 \mathrm{H}), 3.91-3.82(\mathrm{~m}, 4 \mathrm{H}), 3.21-3.12$ (m, 4H), $2.65-2.53(\mathrm{~m}, 2 \mathrm{H}), 2.10$ (d, $J=7.1 \mathrm{~Hz}, 2 \mathrm{H}), 1.59-1.48(\mathrm{~m}, 2 \mathrm{H}), 0.98(\mathrm{~s}, 6 \mathrm{H}) .{ }^{13} \mathbf{C}$ NMR (75 MHz, $\left.\mathbf{C D C l}_{3}\right): \delta$ ppm 159.8, 152.8, 143.4, 132.0, 129.9, 128.4, 127.6, 125.7, 108.6, 105.7, 103.1, 68.7, 67.0, 49.4, 44.8, 44.2, 33.6, 30.8, 27.1. HRMS (ESI+): calcd. for $\mathrm{C}_{25} \mathrm{H}_{33} \mathrm{NO}_{2} \mathrm{Na}$ $\left(\mathrm{M}+\mathrm{Na}^{+}\right):$402.2404. Found: 402.2391 .

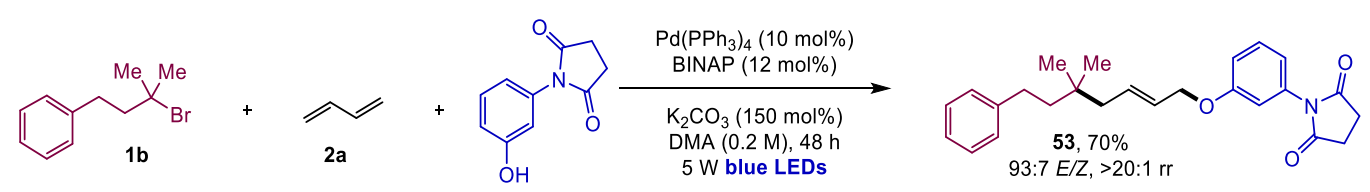

(E)-1-(3-((5,5-Dimethyl-7-phenylhept-2-en-1-yl)oxy)phenyl)pyrrolidine-

2,5-dione (53). According to the general procedure I, using the bromide $\mathbf{1 b} 0.3$ mmol, 1.5 equiv.), butadiene 2a ( $0.3 \mathrm{mmol}, 2 \mathrm{M}$ in THF, 1.5 equiv.), phenol S3a (0.2 mmol, 1.0 equiv.), $\mathrm{Pd}\left(\mathrm{PPh}_{3}\right)_{4}$ (0.020 mmol, $\left.10 \mathrm{~mol} \%\right)$, BINAP (0.024 mmol, $12 \mathrm{~mol} \%), \mathrm{K}_{2} \mathrm{CO}_{3}$ (150 mol\%), and stirring at room temperature for $48 \mathrm{~h}$ under 5W blue LEDs irradiation. The reaction mixture was filtered through a pad of silica gel and concentrated in vacuo, then purified by chromatography (pentane/EtOAc $=4 / 1 \rightarrow 2 / 1)$ to give $53(55.4 \mathrm{mg}, 0.142 \mathrm{mmol}, 71 \%, 93: 7 \mathrm{E} / \mathrm{Z}$, > 20:1 rr) as a pale yellow gum. $\mathbf{R}_{\boldsymbol{f}}$ (pentane/EtOAc $=2 / 1$ ) 0.25. ${ }^{\mathbf{1}} \mathbf{H} \mathbf{~ N M R}(\mathbf{4 0 0} \mathbf{~ M H z}$, $\mathbf{C D C l}_{3}$, major isomer): $\delta$ ppm $7.37(\mathrm{t}, J=7.9 \mathrm{~Hz}, 1 \mathrm{H}), 7.32-7.25(\mathrm{~m}, 2 \mathrm{H}), 7.23-$ $7.15(\mathrm{~m}, 3 \mathrm{H}), 6.96(\mathrm{ddd}, J=8.4,2.4,1.0 \mathrm{~Hz}, 1 \mathrm{H}), 6.91-6.83(\mathrm{~m}, 2 \mathrm{H}), 5.88(\mathrm{dtt}, J=$ 15.4, 7.3, $1.1 \mathrm{~Hz}, 1 \mathrm{H}), 5.73(\mathrm{dtt}, J=15.4,5.9,1.0 \mathrm{~Hz}, 1 \mathrm{H}), 4.52$ (d, $J=5.9 \mathrm{~Hz}, 2 \mathrm{H}$ ), $2.85(\mathrm{~s}, 4 \mathrm{H}), 2.62-2.53(\mathrm{~m}, 2 \mathrm{H}), 2.08(\mathrm{~d}, J=7.5 \mathrm{~Hz}, 2 \mathrm{H}), 1.54-1.46(\mathrm{~m}, 2 \mathrm{H}), 0.96$

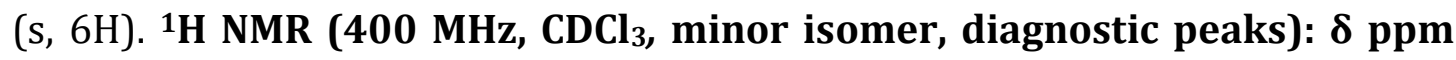
$5.82-5.78(\mathrm{~m}, 1 \mathrm{H}), 4.60(\mathrm{~d}, J=4.7 \mathrm{~Hz}, 2 \mathrm{H}), 0.99$ (s, 6H). ${ }^{13} \mathrm{C}$ NMR (101 MHz, $\mathbf{C D C l}_{3}$, major isomer): $\delta$ ppm 176.2, 159.3, 143.4, 132.9, 132.4, 123.0, 128.4, 127.0, 125.7, 118.8, 115.4, 113.3, 69.0, 44.8, 44.3, 33.6, 30.8, 28.5, 27.1. Note: One aromatic signal missing, most likely due to overlap. ${ }^{13} \mathrm{C}$ NMR (101 $\mathbf{~ M H z , ~} \mathbf{C D C l}_{3}$, non-overlapping peaks): $\delta$ ppm 129.5, 126.2, 120.2, 118.9, 64.3, 39.8, 33.9, 30.9, 
27.0. HRMS (ESI+): calcd. for $\mathrm{C}_{25} \mathrm{H}_{29} \mathrm{NO}_{3} \mathrm{Na}\left(\mathrm{M}+\mathrm{Na}^{+}\right)$: 414.2040. Found: 414.2032.

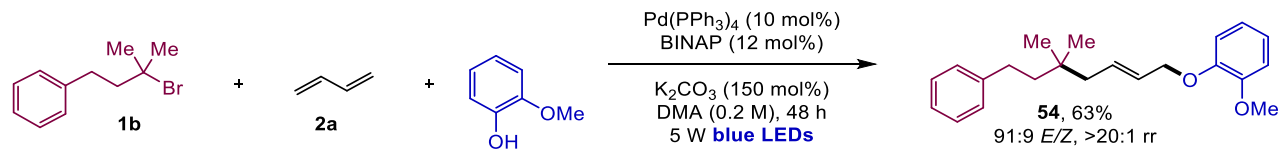

(E)-1-((5,5-Dimethyl-7-phenylhept-2-en-1-yl)oxy)-2-methoxybenzene (54). According to the general procedure I, using the bromide $\mathbf{1 b}(0.3 \mathrm{mmol}, 1.5$ equiv.), butadiene 2 a $(0.3 \mathrm{mmol}, 0.15 \mathrm{~mL}, 2 M$ in THF, 1.5 equiv.), 2methoxyphenol (0.2 mmol, 1.0 equiv.), $\mathrm{Pd}\left(\mathrm{PPh}_{3}\right)_{4}(0.020 \mathrm{mmol}, 10 \mathrm{~mol} \%)$, BINAP (0.024 mmol, $12 \mathrm{~mol} \%), \mathrm{K}_{2} \mathrm{CO}_{3}(150 \mathrm{~mol} \%)$, and stirring at room temperature for $48 \mathrm{~h}$ under $5 \mathrm{~W}$ blue LEDs irradiation. The reaction mixture was filtered through a pad of silica gel and concentrated in vacuo, then purified by chromatography (pentane $/$ EtOAc $=50 / 1 \rightarrow 40 / 1)$ to give $54(40.5 \mathrm{mg}, 0.125$ mmol, 63\%, 91:9 E/Z, > 20:1 rr) as a colorless gum. $\mathbf{R}_{\boldsymbol{f}}$ (pentane/EtOAc $=50 / 1$ ) 0.30. ${ }^{1} \mathrm{H}$ NMR (400 $\mathbf{M H z} \mathbf{C D C l}_{3}$, major isomer): $\delta$ ppm $7.32-7.25(\mathrm{~m}, 2 \mathrm{H}), 7.22$ - $7.14(\mathrm{~m}, 3 \mathrm{H}), 6.96-6.86(\mathrm{~m}, 4 \mathrm{H}), 5.88(\mathrm{dt}, J=15.4,7.2 \mathrm{~Hz}, 1 \mathrm{H}), 5.78(\mathrm{dt}, J=15.4$, $5.6 \mathrm{~Hz}, 1 \mathrm{H}), 4.62(\mathrm{~d}, J=5.7 \mathrm{~Hz}, 2 \mathrm{H}), 3.88(\mathrm{~s}, 3 \mathrm{H}), 2.61-2.52(\mathrm{~m}, 2 \mathrm{H}), 2.07$ (d, $J=$ $7.1 \mathrm{~Hz}, 2 \mathrm{H}), 1.53-1.45(\mathrm{~m}, 2 \mathrm{H}), 0.95(\mathrm{~s}, 6 \mathrm{H}) .{ }^{1} \mathbf{H}$ NMR (400 $\mathbf{~ M H z}, \mathbf{C D C l}_{3}$, minor isomer, diagnostic peaks): $\delta$ ppm $4.70(\mathrm{dd}, J=6.2,1.6 \mathrm{~Hz}, 2 \mathrm{H}), 2.13(\mathrm{dd}, J=7.7$, $1.4 \mathrm{~Hz}, 2 \mathrm{H}), 1.59-1.54(\mathrm{~m}, 2 \mathrm{H}), 1.00(\mathrm{~s}, 6 \mathrm{H}) .{ }^{13} \mathbf{C}$ NMR (101 MHz, $\mathbf{C D C l}_{3}$, major isomer): $\delta$ ppm 149.6, 148.1, 143.4, 132.2, 128.43, 128.42, 127.6, 125.7, 121.2, 120.8, 113.8, 111.8, 69.7, 56.0, 44.9, 44.2, 33.7, 30.8, 27.1. ${ }^{13}$ C NMR (101 MHz, $\mathrm{CDCl}_{3}$, minor isomer, non -overlapping peaks): $\delta$ ppm 130.4, 127.2, 125.7, 121.3, 113.6, 65.2, 44.3, 39.9, 34.0, 30.9. HRMS (ESI $\left.{ }^{+}\right)$: calcd. for $\mathrm{C}_{22} \mathrm{H}_{28} \mathrm{O}_{2} \mathrm{Na}(\mathrm{M}+$ $\mathrm{Na}^{+}$): 347.1982. Found: 347.1986.
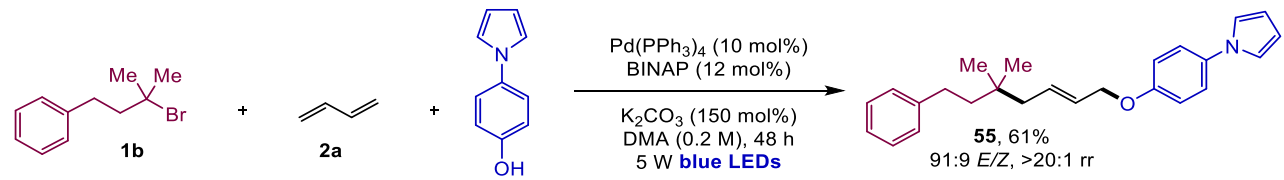

(E)-1-(4-((5,5-Dimethyl-7-phenylhept-2-en-1-yl)oxy)phenyl)-1H-imidazole

(55). According to the general procedure I, using the bromide $\mathbf{1 b}(0.3 \mathrm{mmol}, 1.5$ 
equiv.), butadiene 2a ( $0.3 \mathrm{mmol}, 0.15 \mathrm{~mL}, 2 M$ in THF, 1.5 equiv.), phenol S3e (0.2 mmol, 1.0 equiv.), $\mathrm{Pd}\left(\mathrm{PPh}_{3}\right)_{4}$ (0.020 mmol, $\left.10 \mathrm{~mol} \%\right)$, BINAP (0.024 mmol, $12 \mathrm{~mol} \%$ ), $\mathrm{K}_{2} \mathrm{CO}_{3}$ (150 mol\%), and stirring at room temperature for $48 \mathrm{~h}$ under 5W blue LEDs irradiation. The reaction mixture was filtered through a pad of silica gel and concentrated in vacuo, then purified by chromatography (pentane $/ \mathrm{CH}_{2} \mathrm{Cl}_{2}=10 / 1 \rightarrow 6 / 1 \rightarrow 3 / 1 \rightarrow 1 / 1$ ) to give 55 (43.4 mg, $0.121 \mathrm{mmol}$, $61 \%, 91: 9 E / Z,>20: 1 \mathrm{rr})$ as a low-melting colorless crystalline solid. $\mathbf{R}_{\boldsymbol{f}}$ (pentane $/ \mathrm{CH}_{2} \mathrm{Cl}_{2}=4 / 1$ ) 0.30. ${ }^{1} \mathbf{H}$ NMR (599 $\mathbf{M H z}, \mathbf{C D C l}_{3}$, major isomer): $\delta \mathrm{ppm}$ $7.32-7.28(\mathrm{~m}, 3 \mathrm{H}), 7.22-7.18(\mathrm{~m}, 3 \mathrm{H}), 7.03-7.02(\mathrm{~m}, 2 \mathrm{H}), 7.00-6.97(\mathrm{~m}, 2 \mathrm{H})$, $6.35(\mathrm{t}, J=2.2 \mathrm{~Hz}, 2 \mathrm{H}), 5.91(\mathrm{dtt}, J=15.3,7.6,1.3 \mathrm{~Hz}, 1 \mathrm{H}), 5.76(\mathrm{dtt}, J=15.4,5.9$, $1.3 \mathrm{~Hz}, 1 \mathrm{H}), 4.56(\mathrm{~d}, J=5.9 \mathrm{~Hz}, 2 \mathrm{H}), 2.62-2.57(\mathrm{~m}, 2 \mathrm{H}), 2.11(\mathrm{~d}, J=7.5 \mathrm{~Hz}, 2 \mathrm{H})$,

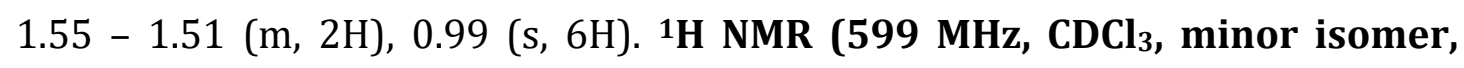
diagnostic peaks): $\delta$ ppm $5.87-5.80(\mathrm{~m}, 2 \mathrm{H}), 4.64(\mathrm{~d}, J=5.6 \mathrm{~Hz}, 2 \mathrm{H}), 2.16(\mathrm{~d}, J=$ $6.5 \mathrm{~Hz}, 2 \mathrm{H}), 1.62-1.57(\mathrm{~m}, 2 \mathrm{H}), 1.03(\mathrm{~s}, 6 \mathrm{H}) .{ }^{13} \mathrm{C}$ NMR (151 MHz, $\mathbf{C D C l}_{3}$, major isomer): $\delta$ ppm 156.85, 143.4, 134.6, 132.4, 128.46, 128.43, 127.3, 125.7, 122.2, 119.79, 115.7, 110.0, 69.2, 44.9, 44.2, 33.7, 30.8, 27.2. ${ }^{13}$ C NMR (151 MHz, CDCl$_{3}$, minor isomer, non-overlapping peaks): $\delta$ ppm 156.90, 143.3, 134.7, 131.1, 128.50, 126.5, 125.8, 122.3, 119.80, 115.6, 64.5, 44.3, 40.0, 34.0, 30.9, 27.1. HRMS (ESI+): calcd. for $\mathrm{C}_{25} \mathrm{H}_{29} \mathrm{NONa}\left(\mathrm{M}+\mathrm{Na}^{+}\right): 382.2141$. Found: 382.2146 .

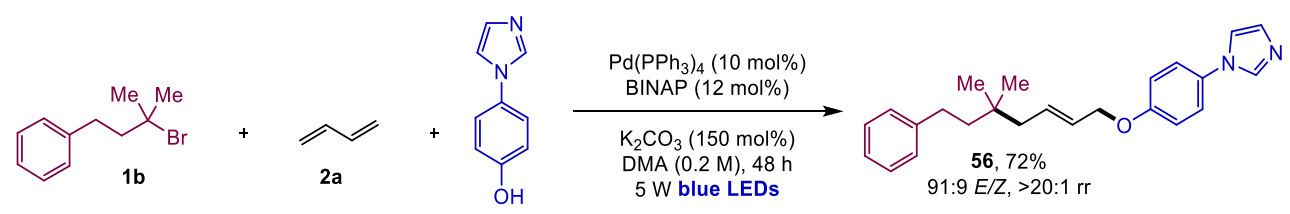

(E)-1-(4-((5,5-Dimethyl-7-phenylhept-2-en-1-yl)oxy)phenyl)-1H-imidazole

(56). According to the general procedure I, using the bromide $\mathbf{1 b}(0.3 \mathrm{mmol}, 1.5$ equiv.), butadiene 2a ( $0.3 \mathrm{mmol}, 0.15 \mathrm{~mL}, 2 \mathrm{M}$ in THF, 1.5 equiv.), phenol S3d (0.2 mmol, 1.0 equiv.), $\mathrm{Pd}\left(\mathrm{PPh}_{3}\right)_{4}(0.020 \mathrm{mmol}, 10 \mathrm{~mol} \%)$, BINAP (0.024 mmol, $12 \mathrm{~mol} \%), \mathrm{K}_{2} \mathrm{CO}_{3}$ (150 mol\%), and stirring at room temperature for $48 \mathrm{~h}$ under 5W blue LEDs irradiation. The reaction mixture was filtered through a pad of silica gel and concentrated in vacuo, then purified by chromatography 
(pentane $/ \mathrm{EtOAc}=3 / 7+0.1 \% \mathrm{Et}_{3} \mathrm{~N}$ ) to give $56(51.5 \mathrm{mg}, 0.142 \mathrm{mmol}, 72 \%, 91: 9$ $E / Z,>20: 1 \mathrm{rr}$ ) as a colorless gum. $\mathbf{R}_{f}($ pentane/EtOAc $=3 / 7) 0.30 .{ }^{1} \mathbf{H}$ NMR (599 MHz, $\mathbf{C D C l}_{3}$, major isomer): $\delta \mathrm{ppm} 7.74(\mathrm{t}, J=1.1 \mathrm{~Hz}, 1 \mathrm{H}), 7.27-7.23(\mathrm{~m}, 4 \mathrm{H})$, $7.19-7.13(\mathrm{~m}, 5 \mathrm{H}), 6.99-6.96(\mathrm{~m}, 2 \mathrm{H}), 5.88(\mathrm{dtt}, J=15.1,7.4,1.3 \mathrm{~Hz}, 1 \mathrm{H}), 5.75-$ $5.69(\mathrm{~m}, 1 \mathrm{H}), 4.54(\mathrm{~d}, J=5.9 \mathrm{~Hz}, 2 \mathrm{H}), 2.58-2.53(\mathrm{~m}, 2 \mathrm{H}), 2.08(\mathrm{~d}, J=7.5 \mathrm{~Hz}, 2 \mathrm{H})$, $1.51-1.46(\mathrm{~m}, 2 \mathrm{H}), 0.95(\mathrm{~s}, 6 \mathrm{H}) .{ }^{1} \mathrm{H}$ NMR $\left(599 \mathrm{MHz}, \mathrm{CDCl}_{3}\right.$, minor isomer, diagnostic peaks): $\delta$ ppm $5.81-5.78(\mathrm{~m}, 2 \mathrm{H}), 4.62(\mathrm{~d}, J=4.4 \mathrm{~Hz}, 2 \mathrm{H}), 2.12(\mathrm{~d}, J=$ $5.8 \mathrm{~Hz}, 2 \mathrm{H}), 1.58-1.52(\mathrm{~m}, 2 \mathrm{H}), 0.99(\mathrm{~s}, 6 \mathrm{H}) .{ }^{13} \mathrm{C}$ NMR (151 MHz, CDCl , major isomer): $\delta$ ppm 158.1, 143.3, 135.9, 132.6, 130.8, 130.1, 128.42, 128.37, 127.0, 125.7, 123.2, 118.8, 116.0, 69.1, 44.9, 44.2, 33.7, 30.8, 27.1. Note: One aromatic signal missing. ${ }^{13} \mathrm{C}$ NMR (151 $\mathrm{MHz}, \mathrm{CDCl}_{3}$, minor isomer, non-overlapping peaks): $\delta$ ppm 131.4, 128.5, 126.2, 125.8, 123.3, 115.8, 64.5, 44.2, 40.00, 34.0, 30.9, 27.0. HRMS (ESI+): calcd. for $\mathrm{C}_{24} \mathrm{H}_{29} \mathrm{~N}_{2} \mathrm{O}\left(\mathrm{M}+\mathrm{H}^{+}\right)$: 361.2080. Found: 361.2085.

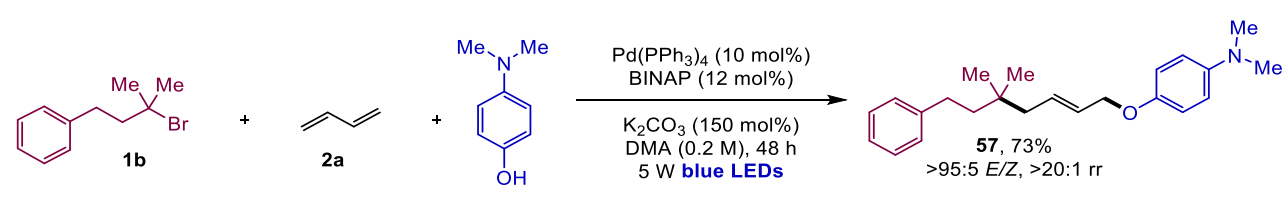

(E)-4-((5,5-Dimethyl-7-phenylhept-2-en-1-yl)oxy)- $N, N$-dimethylaniline

(57). According to the general procedure I, using the bromide $\mathbf{1 b}(0.3 \mathrm{mmol}, 1.5$ equiv.), butadiene ( $0.3 \mathrm{mmol}, 2 \mathrm{M}$ in THF, 1.5 equiv.), 4-(dimethylamino)phenol (0.2 mmol, 1.0 equiv.), $\mathrm{Pd}\left(\mathrm{PPh}_{3}\right)_{4}(0.020 \mathrm{mmol}, 10 \mathrm{~mol} \%)$, BINAP $(0.024 \mathrm{mmol}$, $12 \mathrm{~mol} \%), \mathrm{K}_{2} \mathrm{CO}_{3}$ (150 mol\%), and stirring at room temperature for $48 \mathrm{~h}$ under 5W blue LEDs irradiation. The reaction mixture was filtered through a pad of silica gel and concentrated in vacuo, then purified by chromatography (pentane/EtOAc = 20/1 $\rightarrow$ 16/1) to give $57(49.1 \mathrm{mg}, 0.145 \mathrm{mmol}, 73 \%$, > 95:5 $E / Z,>20: 1 \mathrm{rr}$ ) as a colorless gum. $\mathbf{R}_{f}($ pentane $/ E t O A c=20 / 1) 0.28 .{ }^{1} \mathbf{H}$ NMR (300 MHz, $\left.\mathbf{C D C l}_{3}\right): \delta$ ppm $7.36-7.26(\mathrm{~m}, 2 \mathrm{H}), 7.25-7.16(\mathrm{~m}, 3 \mathrm{H}), 6.93-6.85(\mathrm{~m}, 2 \mathrm{H})$, $6.81-6.72(\mathrm{~m}, 2 \mathrm{H}), 5.88(\mathrm{dt}, J=15.3,7.2 \mathrm{~Hz}, 1 \mathrm{H}), 5.76(\mathrm{dt}, J=15.3,5.6 \mathrm{~Hz}, 1 \mathrm{H})$, $4.49(\mathrm{~d}, J=5.6 \mathrm{~Hz}, 2 \mathrm{H}), 2.89(\mathrm{~s}, 6 \mathrm{H}), 2.65-2.54(\mathrm{~m}, 2 \mathrm{H}), 2.09(\mathrm{~d}, J=7.1 \mathrm{~Hz}, 2 \mathrm{H})$, 
1.58 - 1.48 (m, 2H), 0.98 (s, 6H). ${ }^{13} \mathbf{C}$ NMR (75 MHz, $\left.\mathbf{C D C l}_{3}\right): \delta$ ppm 151.2, 145.9, 143.5, 131.7, 128.5, 128.4, 128.1, 125.7, 115.9, 114.9, 69.5, 44.9, 44.3, 41.9, 33.7, 30.8, 27.2. HRMS (ESI $\left.{ }^{+}\right)$: calcd. for $\mathrm{C}_{24} \mathrm{H}_{31} \mathrm{NO}\left(\mathrm{M}+\mathrm{H}^{+}\right)$: 338.2478. Found: 338.2511.

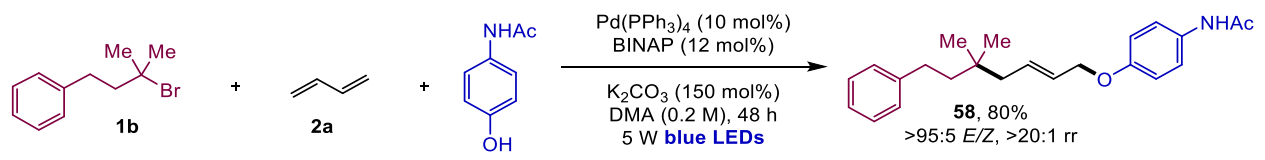

(E)-N-(4-((5,5-Dimethyl-7-phenylhept-2-en-1-yl)oxy)phenyl)acetamide

(58). According to the general procedure I, using the bromide $\mathbf{1 b}(0.3 \mathrm{mmol}, 1.5$ equiv.), butadiene 2 a ( $0.3 \mathrm{mmol}, 2 \mathrm{M}$ in THF, 1.5 equiv.), paracetamol ( $0.2 \mathrm{mmol}$, 1.0 equiv.), $\mathrm{Pd}\left(\mathrm{PPh}_{3}\right)_{4}(0.020 \mathrm{mmol}, 10 \mathrm{~mol} \%)$, BINAP (0.024 mmol, $12 \mathrm{~mol} \%$ ), $\mathrm{K}_{2} \mathrm{CO}_{3}(150 \mathrm{~mol} \%)$, and stirring at room temperature for $48 \mathrm{~h}$ under $5 \mathrm{~W}$ blue LEDs irradiation. The reaction mixture was filtered through a pad of silica gel and concentrated in vacuo, then purified by chromatography $($ pentane $/$ EtOAc $=$ $1 / 1)$ to give $58(56.3 \mathrm{mg}, 0.160 \mathrm{mmol}, 80 \%,>95: 5 E / Z,>20: 1 \mathrm{rr}$ ) as a yellow gum. $\mathbf{R}_{\boldsymbol{f}}$ (pentane/EtOAc = 1/1) 0.30. ${ }^{\mathbf{1}} \mathbf{H}$ NMR (300 $\left.\mathbf{M H z}, \mathbf{C D C l}_{3}\right): \delta$ ppm $7.51(\mathrm{br}$ s, $1 \mathrm{H}), 7.33-7.24(\mathrm{~m}, 2 \mathrm{H}), 7.23-7.14(\mathrm{~m}, 2 \mathrm{H}), 7.13-7.03(\mathrm{~m}, 3 \mathrm{H}), 6.80-6.72$ $(\mathrm{m}, 2 \mathrm{H}), 5.77(\mathrm{dt}, J=15.4,7.3 \mathrm{~Hz}, 1 \mathrm{H}), 5.61(\mathrm{dt}, J=15.3,5.7 \mathrm{~Hz}, 1 \mathrm{H}), 4.39$ (d, $J=$ $5.8 \mathrm{~Hz}, 2 \mathrm{H}), 2.52-2.41(\mathrm{~m}, 2 \mathrm{H}), 2.03(\mathrm{~s}, 3 \mathrm{H}), 1.97(\mathrm{~d}, J=7.0 \mathrm{~Hz}, 2 \mathrm{H}), 1.45-1.34$ (m, 2H), 0.86 (s, 6H). ${ }^{13}$ C NMR (75 MHz, $\mathbf{C D C l}_{3}$ ): $\delta$ ppm 168.5, 155.5, 143.4, 132.1, 131.2, 128.4, 127.4, 125.7, 121.9, 115.1, 69.0, 44.9, 44.2, 33.6, 30.8, 27.1, 24.4. HRMS (ESI+): calcd. for $\mathrm{C}_{23} \mathrm{H}_{29} \mathrm{NO}_{2} \mathrm{Na}\left(\mathrm{M}+\mathrm{Na}^{+}\right): 374.2091$. Found: 374.2095.

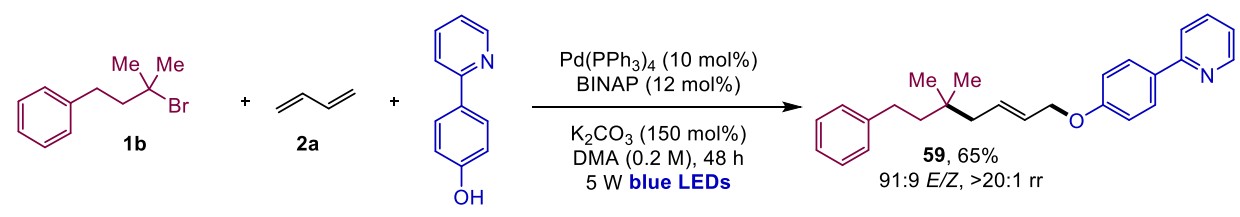

(E)-2-(4-((5,5-Dimethyl-7-phenylhept-2-en-1-yl)oxy)phenyl)pyridine (59). According to the general procedure I, using the bromide $\mathbf{1 b}(0.3 \mathrm{mmol}, 1.5$ equiv.), butadiene 2 a ( $0.3 \mathrm{mmol}, 0.15 \mathrm{~mL}, 2 \mathrm{M}$ in THF, 1.5 equiv.), phenol S3f $(0.2$ 
mmol, 1.0 equiv.), $\mathrm{Pd}\left(\mathrm{PPh}_{3}\right)_{4}(0.020 \mathrm{mmol}, 10 \mathrm{~mol} \%)$, BINAP (0.024 mmol, 12 mol\%), $\mathrm{K}_{2} \mathrm{CO}_{3}$ (150 mol\%), and stirring at room temperature for $48 \mathrm{~h}$ under $5 \mathrm{~W}$ blue LEDs irradiation. The reaction mixture was filtered through a pad of silica gel and concentrated in vacuo, then purified by chromatography (pentane/EtOAc $=12 / 1 \rightarrow 10 / 1$ ) to give 59 (48.1 $\mathrm{mg}, 0.129 \mathrm{mmol}, 65 \%, 91: 9 E / Z,>20: 1 \mathrm{rr}$ ) as a colorless gum. $\mathbf{R}_{\boldsymbol{f}}$ (pentane/EtOAc= 12/1) 0.25. ${ }^{\mathbf{1}} \mathbf{H}$ NMR (400 $\mathbf{~ M H z ,} \mathbf{C D C l}_{3}$, major isomer): $\delta$ ppm $8.66(\mathrm{ddd}, J=4.8,1.9,1.0 \mathrm{~Hz}, 1 \mathrm{H}), 7.97-7.91(\mathrm{~m}, 2 \mathrm{H})$, $7.70(\mathrm{dd}, J=8.0,7.2,1.8 \mathrm{~Hz}, 1 \mathrm{H}), 7.66(\mathrm{dt}, J=8.0,1,2 \mathrm{~Hz}, 1 \mathrm{H}), 7.30-7.21(\mathrm{~m}, 2 \mathrm{H})$, $7.21-7.14(\mathrm{~m}, 4 \mathrm{H}), 7.02(\mathrm{dq}, J=8.9,2.5,1.9 \mathrm{~Hz}, 2 \mathrm{H}), 5.90(\mathrm{dtt}, J=15.2,7.5,1.3 \mathrm{~Hz}$, 1H), $5.76(\mathrm{dtt}, J=15.3,5.9,1.2 \mathrm{~Hz}, 1 \mathrm{H}), 4.57(\mathrm{~d}, J=5.8 \mathrm{~Hz}, 2 \mathrm{H}), 2.60-2.53(\mathrm{~m}$, 2H), $2.08(\mathrm{~d}, J=7.3 \mathrm{~Hz}, 2 \mathrm{H}), 1.54-1.47(\mathrm{~m}, 2 \mathrm{H}), 0.96(\mathrm{~s}, 6 \mathrm{H}) .{ }^{1} \mathbf{H}$ NMR (400 MHz, $\mathrm{CDCl}_{3}$, minor isomer, diagnostic peaks): $\delta$ ppm $5.85-5.80(\mathrm{~m}, 2 \mathrm{H}), 4.65(\mathrm{~d}, J=$ $5.4 \mathrm{~Hz}, 2 \mathrm{H}), 2.14(\mathrm{~d}, J=6.8 \mathrm{~Hz}, 2 \mathrm{H}), 1.60-1.54(\mathrm{~m}, 2 \mathrm{H}), 1.00(\mathrm{~s}, 6 \mathrm{H}) .{ }^{13} \mathrm{C}$ NMR (101 MHz, $\mathbf{C D C l}_{3}$, major isomer): $\delta$ ppm 159.7, 157.3, 149.6, 143.4, 136.8, 132.3, 132.1, 128.4, 128.2, 127.3, 125.68, 121.5, 119.9, 115.1, 68.8, 44.9, 44.3, 33.7, 30.8, 27.2. Note: One aromatic signal is missing. ${ }^{13} \mathbf{C} \mathbf{~ N M R ~}\left(101 \mathbf{~} \mathbf{H z}, \mathbf{C D C l}_{3}, \mathbf{m i n o r}\right.$ isomer, diagnostic peaks): $\delta$ ppm 131.0, 126.5, 125.74, 64.2, 39.9, 34.0, 30.9, 27.0. HRMS (ESI ${ }^{+}$): calcd. for $\mathrm{C}_{26} \mathrm{H}_{39} \mathrm{NO}\left(\mathrm{M}+\mathrm{H}^{+}\right)$: 372.2322. Found: 372.2327 .

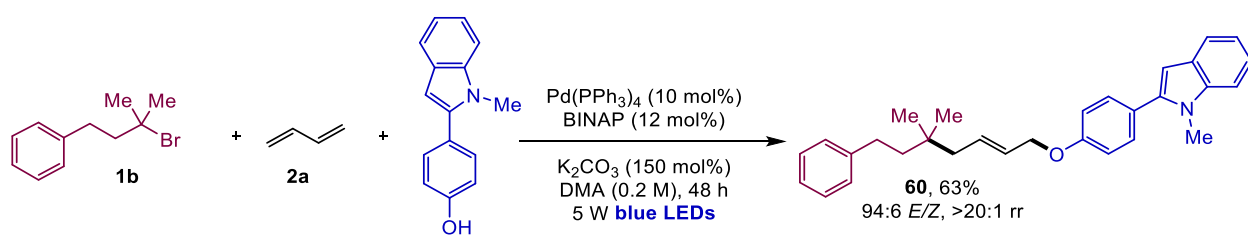

(E)-2-(4-((5,5-Dimethyl-7-phenylhept-2-en-1-yl)oxy)phenyl)-1-methyl-1H-

indole (60). According to the general procedure I, using the bromide $\mathbf{1 b}[0.3$ mmol, 1.5 equiv.), butadiene 2 a ( 0.3 mmol, $2 M$ in THF, 1.5 equiv.), phenol S3c (0.2 mmol, 1.0 equiv.), $\mathrm{Pd}\left(\mathrm{PPh}_{3}\right)_{4}(0.020 \mathrm{mmol}, 10 \mathrm{~mol} \%)$, BINAP (0.024 mmol, $12 \mathrm{~mol} \%), \mathrm{K}_{2} \mathrm{CO}_{3}$ (150 mol\%), and stirring at room temperature for $48 \mathrm{~h}$ under $5 \mathrm{~W}$ blue LEDs irradiation. The reaction mixture was filtered through a pad of silica gel and concentrated in vacuo, then purified by chromatography 
(pentane/EtOAc $=10 / 1 \rightarrow 1 / 1)$ to give $60(52.8 \mathrm{mg}, 0.115 \mathrm{mmol}, 63 \%, 94: 6 \mathrm{E} / \mathrm{Z}, \mathrm{>}$ 20:1 rr) as a white solid. $\mathbf{R}_{\boldsymbol{f}}$ (pentane/EtOAc $=4 / 1$ ) 0.30. ${ }^{\mathbf{1}} \mathbf{H}$ NMR (300 MHz, $\mathrm{CDCl}_{3}$, major isomer): $\delta$ ppm $7.68(\mathrm{dt}, J=7.7,1.0 \mathrm{~Hz}, 1 \mathrm{H}), 7.50-7.43(\mathrm{~m}, 2 \mathrm{H})$, $7.40(\mathrm{~d}, J=8.2 \mathrm{~Hz}, 1 \mathrm{H}), 7.36-7.28(\mathrm{~m}, 3 \mathrm{H}), 7.28-7.16(\mathrm{~m}, 4 \mathrm{H}), 7.10-7.02(\mathrm{~m}$, 2H), $6.56(\mathrm{~s}, 1 \mathrm{H}), 5.96(\mathrm{dt}, J=15.3,7.4 \mathrm{~Hz}, 1 \mathrm{H}), 5.81(\mathrm{dt}, J=15.3,5.6 \mathrm{~Hz}, 1 \mathrm{H}), 4.62$ (d, $J=5.7 \mathrm{~Hz}, 2 \mathrm{H}), 3.75(\mathrm{~s}, 3 \mathrm{H}), 2.69-2.56(\mathrm{~m}, 2 \mathrm{H}), 2.15$ (d, J = 7.3 Hz, 2H), $1.61-$ $1.50(\mathrm{~m}, 2 \mathrm{H}), 1.02(\mathrm{~s}, 6 \mathrm{H}) .{ }^{1} \mathrm{H}$ NMR (300 $\mathbf{~ M H z}, \mathrm{CDCl}_{3}$, minor isomer, diagnostic peaks): $\delta$ ppm $4.70(\mathrm{~d}, J=5.1 \mathrm{~Hz}, 2 \mathrm{H}), 3.76(\mathrm{~s}, 3 \mathrm{H}), 1.06(\mathrm{~s}, 6 \mathrm{H}) .{ }^{13} \mathrm{C}$ NMR (75 MHz, $\mathbf{C D C l}_{3}$, major isomer): $\delta$ ppm 158.6, 143.4, 141.6, 138.2, 132.4, 130.7, 128.5, 128.4, 128.1, 127.3, 125.7, 125.3, 121.5, 120.4, 119.9, 115.0, 109.6, 101.1, 68.9, 44.9, 44.3, 33.7, 31.2, 30.8, 27.2. ${ }^{13} \mathbf{C}$ NMR (75 $\mathbf{~ M H z , ~} \mathbf{C D C l}_{3}$, minor isomer, non-overlapping peaks) $\delta$ 138.4, 131.1, 126.5, 64.2, 39.9, 34.0, 30.9, 27.1. HRMS (ESI+): calcd. for $\mathrm{C}_{25} \mathrm{H}_{33} \mathrm{NO}_{2} \mathrm{Na}\left(\mathrm{M}+\mathrm{Na}^{+}\right)$: 402.2404. Found: 402.2391 .

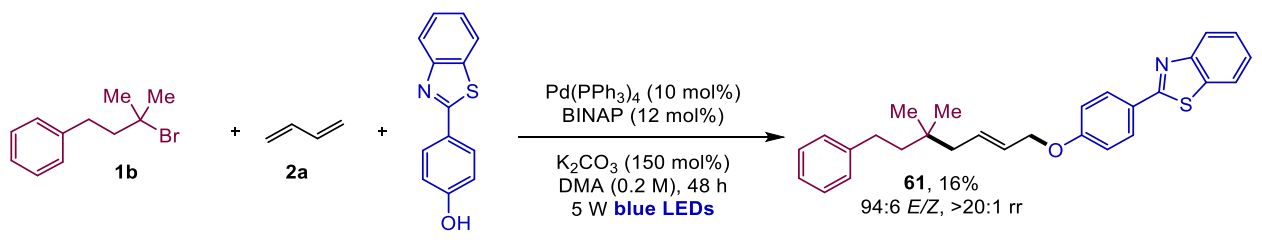

(E)-2-(4-((5,5-Dimethyl-7-phenylhept-2-en-1-

yl)oxy)phenyl)benzo[d]thiazole (61). According to the general procedure I, using the bromide $\mathbf{1 b}(0.3 \mathrm{mmol}, 1.5$ equiv.), butadiene $2 \mathbf{a}(0.3 \mathrm{mmol}, 0.15 \mathrm{~mL}, 2$ $M$ in THF, 1.5 equiv.), phenol S3g (0.2 mmol, 1.0 equiv.), $\mathrm{Pd}\left(\mathrm{PPh}_{3}\right)_{4}(0.020 \mathrm{mmol}$, $10 \mathrm{~mol} \%)$, BINAP (0.024 mmol, $12 \mathrm{~mol} \%), \mathrm{K}_{2} \mathrm{CO}_{3}$ (150 mol\%), and stirring at room temperature for $48 \mathrm{~h}$ under $5 \mathrm{~W}$ blue LEDs irradiation. The reaction mixture was filtered through a pad of silica gel and concentrated in vacuo, then purified by chromatography (pentane/EtOAc $=40 / 1 \rightarrow 35 / 1 \rightarrow 30 / 1$ ) to give 61 (13.5 mg, $0.032 \mathrm{mmol}, 16 \%, 94: 6 \mathrm{E} / Z$, > 20:1 rr) as a white solid. $\mathbf{R}_{\boldsymbol{f}}$ (pentane/EtOAc $=40 / 1)$ 0.30. ${ }^{1} \mathbf{H}$ NMR (300 $\mathbf{~ M H z}, \mathbf{C D C l}_{3}$, major isomer): $\delta$ ppm $8.03-7.93$ (m, 3H), 7.84 (ddd, $J=7.9,1.3,0.6 \mathrm{~Hz}, 1 \mathrm{H}$ ), 7.44 (ddd, $J=8.3,7.2,1.3$ $\mathrm{Hz}, 1 \mathrm{H}), 7.37-7.29(\mathrm{~m}, 1 \mathrm{H}), 7.25-7.19$ (m, 2H), 7.18 - 7.08 (m, 3H), 7.01 - 6.94 
(m, 2H), $5.87(\mathrm{dtt}, J=15.4,7.4,1.1 \mathrm{~Hz}, 1 \mathrm{H}), 5.71(\mathrm{dtt}, J=15.4,5.9,1.1 \mathrm{~Hz}, 1 \mathrm{H})$, $4.55(\mathrm{~d}, J=5.8 \mathrm{~Hz}, 2 \mathrm{H}), 2.58-2.47(\mathrm{~m}, 2 \mathrm{H}), 2.05$ (d, J = $7.3 \mathrm{~Hz}, 2 \mathrm{H}), 1.52-1.42(\mathrm{~m}$, 2H), 0.92 (s, 6H). ${ }^{1} \mathrm{H}$ NMR (300 $\mathbf{~ M H z} \mathbf{C D C l}_{3}$, minor isomer, diagnostic peaks): $\delta$ ppm $4.63(\mathrm{~d}, J=4.5 \mathrm{~Hz}, 2 \mathrm{H}), 0.97(\mathrm{~s}, 6 \mathrm{H}) .{ }^{13} \mathrm{C}$ NMR (75 MHz, CDCl$_{3}$, major isomer): $\delta$ ppm 168.1, 161.2, 154.3, 143.4, 135.0, 132.7, 129.2, 128.5, 128.4, $126.9,126.49,126.3,125.7,124.9,122.9,121.7,115.3,69.0,44.9,44.3,33.7,30.8$, 27.2. HRMS (ESI $\left.{ }^{+}\right)$: calcd. for $\mathrm{C}_{28} \mathrm{H}_{30} \mathrm{NOS}\left(\mathrm{M}+\mathrm{H}^{+}\right)$: 428.2043. Found: 428.2044 . Note: Due to the small amount of compound available, no carbon signals belonging to the minor isomer could be observed.

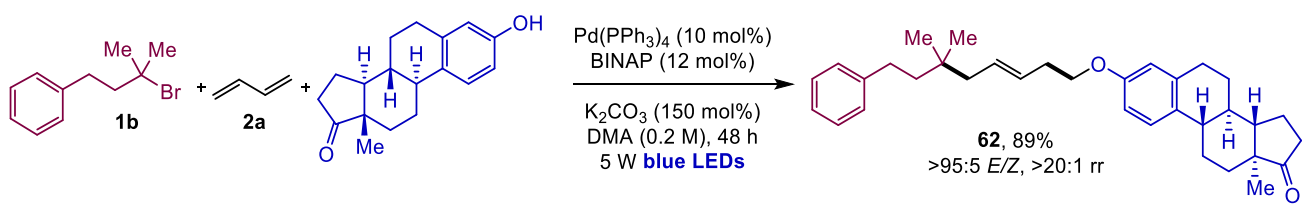

$(8 R, 9 S, 13 S, 14 S)-3-(((E)-5,5-D i m e t h y l-7-p h e n y l h e p t-2-e n-1-y l) o x y)-13-$ methyl-6,7,8,9,11,12,13,14,15,16-decahydro-17Hcyclopenta[a]phenanthren-17-one (62). According to the general procedure I, using the bromide $\mathbf{1 b}(0.3 \mathrm{mmol}, 1.5$ equiv.), butadiene $\mathbf{2 a}(0.3 \mathrm{mmol}, 0.15 \mathrm{~mL}, 2$ $M$ in THF, 1.5 equiv.), estrone (0.2 mmol, 1.0 equiv.), $\mathrm{Pd}\left(\mathrm{PPh}_{3}\right)_{4}(0.020 \mathrm{mmol}, 10$ mol\%), BINAP (0.024 mmol, $12 \mathrm{~mol} \%), \mathrm{K}_{2} \mathrm{CO}_{3}$ (150 mol\%), and stirring at room temperature for $48 \mathrm{~h}$ under $5 \mathrm{~W}$ blue LEDs irradiation. The reaction mixture was filtered through a pad of silica gel and concentrated in vacuo, then purified by chromatography (pentane $/$ EtOAc $=7 / 1 \rightarrow 6 / 1)$ to give $62(83.9 \mathrm{mg}, 0.178 \mathrm{mmol}$, $89 \%,>95: 5 E / Z,>20: 1 \mathrm{rr}$ ) as a colorless low-melting crystalline solid. $\mathbf{R}_{\boldsymbol{f}}$ (pentane/EtOAc= 19/1) 0.27. ${ }^{1} \mathbf{H}$ NMR (300 $\left.\mathbf{~ M H z , ~} \mathbf{C D C l}_{3}\right): \delta$ ppm $7.34-7.26(\mathrm{~m}$, 2H), $7.25-7.14(\mathrm{~m}, 4 \mathrm{H}), 6.76(\mathrm{dd}, J=8.6,2.8 \mathrm{~Hz}, 1 \mathrm{H}), 6.70(\mathrm{~d}, J=2.7 \mathrm{~Hz}, 1 \mathrm{H}), 5.89$ (dt, $J=15.4,7.1 \mathrm{~Hz}, 1 \mathrm{H}), 5.74(\mathrm{dt}, J=15.4,5.7 \mathrm{~Hz}, 1 \mathrm{H}), 4.52(\mathrm{dd}, J=5.7,1.1 \mathrm{~Hz}$, 2H), $2.96-2.83(\mathrm{~m}, 2 \mathrm{H}), 2.64-2.56(\mathrm{~m}, 2 \mathrm{H}), 2.57-2.35(\mathrm{~m}, 2 \mathrm{H}), 2.33-1.92(\mathrm{~m}$, 7H), $1.74-1.36(\mathrm{~m}, 8 \mathrm{H}), 0.98(\mathrm{~s}, 6 \mathrm{H}), 0.93(\mathrm{~s}, 3 \mathrm{H}) .{ }^{13} \mathrm{C}$ NMR (75 MHz, $\left.\mathbf{C D C l}_{3}\right): \delta$ ppm 221.0, 156.8, 143.4, 137.7, 132.1, 131.8, 128.4, 127.7, 126.4, 125.6, 114.9, $112.5,68.7,50.5,48.1,44.8,44.2$, 44.0, 38.4, 35.9, 33.6, 31.7, 30.8, 29.7, 27.1, 26.6, 
26.0, 21.7, 13.9. Note: One aromatic signal is missing, most likely due to overlap. HRMS (ESI ${ }^{+}$): calcd. for $\mathrm{C}_{33} \mathrm{H}_{42} \mathrm{O}_{2} \mathrm{Na}\left(\mathrm{M}+\mathrm{Na}^{+}\right)$: 493.3077. Found: 493.3079 .

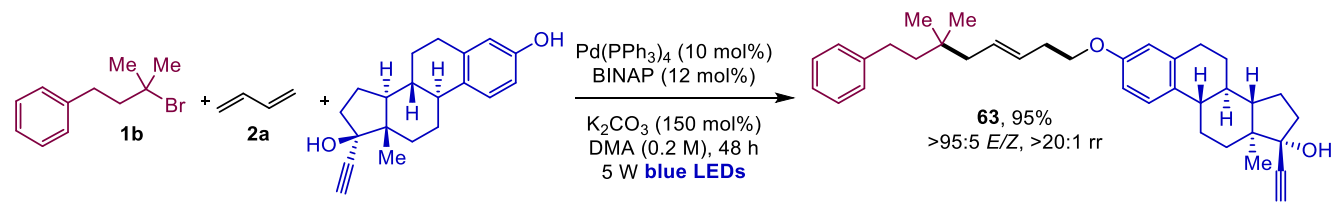

$(8 R, 9 S, 13 S, 14 S, 17 R)-3-(((E)-5,5-D i m e t h y l-7-p h e n y l h e p t-2-e n-1-y l) o x y)-17-$ ethynyl-13-methyl-7,8,9,11,12,13,14,15,16,17-decahydro-6H-

cyclopenta[a]phenanthren-17-ol (63). According to the general procedure I, using the bromide $\mathbf{1 b}(0.3 \mathrm{mmol}, 1.5$ equiv.), butadiene $\mathbf{2 a}(0.3 \mathrm{mmol}, 0.15 \mathrm{~mL}, 2$ $M$ in THF, 1.5 equiv.), ethynylestradiol ( $0.2 \mathrm{mmol}, 1.0$ equiv. $), \mathrm{Pd}\left(\mathrm{PPh}_{3}\right)_{4}(0.020$ mmol, 10 mol\%), BINAP (0.024 mmol, $12 \mathrm{~mol} \%$ ), $\mathrm{K}_{2} \mathrm{CO}_{3}$ (150 mol\%), and stirring at room temperature for $48 \mathrm{~h}$ under $5 \mathrm{~W}$ blue LEDs irradiation. The reaction mixture was filtered through a pad of silica gel and concentrated in vacuo, then purified by chromatography (pentane/EtOAc $=7 / 1 \rightarrow 6 / 1)$ to give $63(94.4 \mathrm{mg}$, $0.190 \mathrm{mmol}, 95 \%,>95: 5 \mathrm{E} / \mathrm{Z},>20: 1 \mathrm{rr}$ ) as a colorless gum. $\mathbf{R}_{\boldsymbol{f}}$ (pentane/EtOAc= 6/1) 0.35. ${ }^{1} \mathrm{H}$ NMR (400 MHz, $\left.\mathbf{C D C l}_{3}\right): \delta$ ppm $7.34-7.27(\mathrm{~m}, 2 \mathrm{H}), 7.25$ - $7.15(\mathrm{~m}$, 4H), $6.76(\mathrm{dd}, J=8.6,2.8 \mathrm{~Hz}, 1 \mathrm{H}), 6.69$ (d, $J=2.7 \mathrm{~Hz}, 1 \mathrm{H}), 5.89(\mathrm{dt}, J=15.4,7.6 \mathrm{~Hz}$, $1 \mathrm{H}), 5.76(\mathrm{dt}, J=15.4,6.0 \mathrm{~Hz}, 1 \mathrm{H}), 4.52(\mathrm{~d}, J=5.8 \mathrm{~Hz}, 2 \mathrm{H}), 2.91-2.80(\mathrm{~m}, 2 \mathrm{H})$, $2.64(\mathrm{~s}, 1 \mathrm{H}), 2.62-2.56(\mathrm{~m}, 2 \mathrm{H}), 2.44-2.32$ (m, 2H), $2.30-2.19$ (m, 1H), 2.10 (d, $J=7.4 \mathrm{~Hz}, 2 \mathrm{H}), 2.07-2.01(\mathrm{~m}, 2 \mathrm{H}), 2.00-1.65(\mathrm{~m}, 5 \mathrm{H}), 1.59-1.34(\mathrm{~m}, 5 \mathrm{H}), 0.99$ (s, 6H), 0.92 (s, 3H). ${ }^{13}$ C NMR (101 $\left.\mathbf{~ M H z , ~} \mathbf{C D C l}_{3}\right): \delta$ ppm 156.7, 143.4, 137.9, 132.6, 131.8, 128.4, 127.8, 126.4, 125.7, 114.9, 112.5, 87.7, 80.0, 74.2, 68.8, 49.6, 47.2, 44.9, 44.2, 43.6, 39.5, 39.1, 33.7, 32.9, 30.8, 29.9, 27.4, 27.2, 26.5, 22.9, 12.8. HRMS (ESI ${ }^{+}$): calcd. for $\mathrm{C}_{35} \mathrm{H}_{44} \mathrm{O}_{2} \mathrm{Na}\left(\mathrm{M}+\mathrm{Na}^{+}\right)$: 519.3234 . Found: 519.3243 .

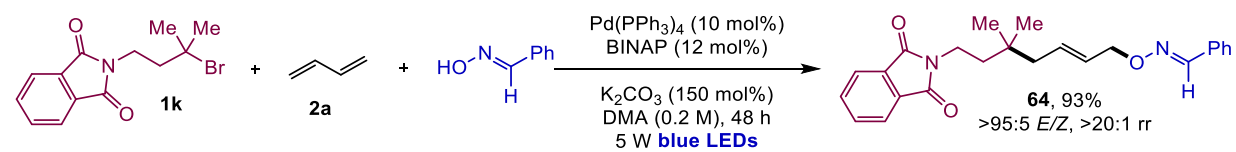

(E)-Benzaldehyde 0-((E)-7-(1,3-dioxoisoindolin-2-yl)-5,5-dimethylhept-2en-1-yl) oxime (64). According to the general procedure I, using the 
corresponding alkyl bromide $\mathbf{1 k}(0.3 \mathrm{mmol}, 1.5$ equiv.), butadiene $\mathbf{2 a}(0.3 \mathrm{mmol}$, $0.15 \mathrm{~mL}, 2 M$ in THF, 1.5 equiv.), benzaldehyde oxime ( $0.2 \mathrm{mmol}, 1.0$ equiv.), $\mathrm{Pd}\left(\mathrm{PPh}_{3}\right)_{4}(10 \mathrm{~mol} \%), \mathrm{BINAP}(12 \mathrm{~mol} \%), \mathrm{K}_{2} \mathrm{CO}_{3}(150 \mathrm{~mol} \%)$, and stirring at room temperature for $48 \mathrm{~h}$ under $5 \mathrm{~W}$ blue LEDs irradiation. The reaction mixture was filtered through a pad of silica gel and concentrated in vacuo, then purified by chromatography (pentane $/$ EtOAc $=9 / 1)$ to give $64(72.2 \mathrm{mg}, 0.185 \mathrm{mmol}$, 93\%, $>$ 95:5 E/Z, $>20: 1 \mathrm{rr})$ as a colorless oil. $\mathbf{R}_{\boldsymbol{f}}($ pentane/EtOAc $=4 / 1) 0.42 .{ }^{1} \mathbf{H}$ NMR (599 MHz, $\left.\mathbf{C D C l}_{3}\right): \delta$ ppm $8.08(\mathrm{~s}, 1 \mathrm{H}), 7.81(\mathrm{dd}, J=5.4,3.0 \mathrm{~Hz}, 2 \mathrm{H}), 7.68$ $(\mathrm{dd}, J=5.4,3.0 \mathrm{~Hz}, 2 \mathrm{H}), 7.59-7.54(\mathrm{~m}, 2 \mathrm{H}), 7.35-7.30(\mathrm{~m}, 3 \mathrm{H}), 5.88-5.80(\mathrm{~m}$, 1H), $5.78-5.70(\mathrm{~m}, 1 \mathrm{H}), 4.64(\mathrm{dd}, J=6.2,1.1 \mathrm{~Hz}, 2 \mathrm{H}), 3.73-3.67(\mathrm{~m}, 2 \mathrm{H}), 2.07$ (dd, J = 7.4, $1.2 \mathrm{~Hz}, 2 \mathrm{H}), 1.84(\mathrm{~s}, 1 \mathrm{H}), 1.60-1.53(\mathrm{~m}, 2 \mathrm{H}), 0.99$ (s, 6H). ${ }^{13} \mathbf{C}$ NMR (151 MHz, $\mathbf{C D C l}_{3}$ ): $\delta$ ppm 168.3, 148.7, 133.9, 132.5, 132.4, 131.7, 129.8, 128.7, 128.7, 127.1, 123.3, 123.2, 75.0, 45.0, 39.7, 34.4, 34.4, 33.1, 26.9. HRMS (ESI+): calcd. for $\mathrm{C}_{24} \mathrm{H}_{26} \mathrm{~N}_{2} \mathrm{O}_{3} \mathrm{Na}\left(\mathrm{M}+\mathrm{Na}^{+}\right)$: 413.1836. Found: 413.1821.

\subsection{General Procedure J}

An oven-dried Schlenk tube containing a stir bar was charged under argon with the corresponding alkyl bromide 1 (0.3 mmol, 1.5 equiv.), $\mathrm{Pd}\left(\mathrm{PPh}_{3}\right)_{4}$ (10 mol\%), BINAP (12 mol\%), sulfur based nucleophile ( $0.2 \mathrm{mmol}, 1.0$ equiv.), $\mathrm{K}_{2} \mathrm{CO}_{3}$ (150 mol\%) and DMA (0.85 mL). Subsequently, butadiene 2a $(0.3 \mathrm{mmol}, 0.15 \mathrm{~mL}, 2 \mathrm{M}$ in THF, 1.5 equiv.) was added. The reaction mixture was stirred at room temperate under blue LEDs irradiation ( $5 \mathrm{~W}, 455 \mathrm{~nm}$ ) for $48 \mathrm{~h}$. Then, the reaction was filtered through a pad of silica gel and washed with EtOAc $(3 \times 5 \mathrm{~mL})$. The obtained solution was concentrated in vacuo and the diastereomeric ratio determined by ${ }^{1} \mathrm{H}$ NMR spectroscopy of the crude reaction mixture. The reaction mixture was purified by column chromatography on silica gel (pentane/EtOAc) to afford the corresponding compounds. 


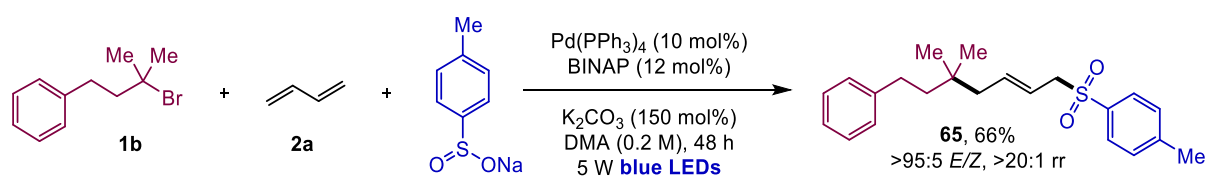

\section{(E)-1-((5,5-Dimethyl-7-phenylhept-2-en-1-yl)sulfonyl)-4-methylbenzene}

(65). According to the general procedure J, using the corresponding alkyl bromide 1 b ( $0.3 \mathrm{mmol}, 1.5$ equiv.), butadiene $2 \mathrm{a}(0.3 \mathrm{mmol}, 0.15 \mathrm{~mL}, 2 \mathrm{M}$ in THF, 1.5 equiv.), the sodium 4-methylbenzenesulfinate (0.2 mmol, 1.0 equiv.), $\mathrm{Pd}\left(\mathrm{PPh}_{3}\right)_{4}(10 \mathrm{~mol} \%), \operatorname{BINAP}(12 \mathrm{~mol} \%), \mathrm{K}_{2} \mathrm{CO}_{3}(150 \mathrm{~mol} \%)$, and stirring at room temperature for $48 \mathrm{~h}$ under $5 \mathrm{~W}$ blue LEDs irradiation. The reaction mixture was filtered through a pad of silica gel and concentrated in vacuo, then purified by chromatography (pentane $/$ EtOAc $=9 / 1)$ to give $65(46.8 \mathrm{mg}, 0.131 \mathrm{mmol}$, $66 \%,>95: 5 E / Z,>20: 1 \mathrm{rr}$ ) as a colorless oil. $\mathbf{R}_{\boldsymbol{f}}$ (pentane/EtOAc $\left.=9 / 1\right) 0.35 .{ }^{1} \mathbf{H}$ NMR (599 MHz, $\mathbf{C D C l}_{3}$ ): $\delta$ ppm $7.74-7.70$ (m, 2H), 7.30 - 7.26 (m, 4H), 7.20 $7.14(\mathrm{~m}, 3 \mathrm{H}), 5.60(\mathrm{dtt}, J=15.2,7.5,1.2 \mathrm{~Hz}, 1 \mathrm{H}), 5.40(\mathrm{dtt}, J=15.2,7.4,1.2 \mathrm{~Hz}, 1 \mathrm{H})$, $3.77(\mathrm{dd}, J=7.3,1.1 \mathrm{~Hz}, 2 \mathrm{H}), 2.53-2.48(\mathrm{~m}, 2 \mathrm{H}), 2.40$ (s, 3H), 1.98 (dd, $J=7.6,1.4$ $\mathrm{Hz}, 2 \mathrm{H}), 1.42-1.37(\mathrm{~m}, 2 \mathrm{H}), 0.85(\mathrm{~s}, 6 \mathrm{H}) .{ }^{13} \mathbf{C}$ NMR (151 MHz, $\left.\mathbf{C D C l}_{3}\right): \delta$ ppm 144.6, 143.2, 138.4, 135.7, 129.8, 128.5, 128.5, 128.4, 125.8, 118.5, 60.3, 45.2, 44.1, 33.7, 30.7, 26.9, 21.7. HRMS (ESI ${ }^{+}$): calcd. for $\mathrm{C}_{22} \mathrm{H}_{28} \mathrm{O}_{2} \mathrm{SNa}\left(\mathrm{M}+\mathrm{Na}^{+}\right)$: 379.1702. Found: 379.1704 .
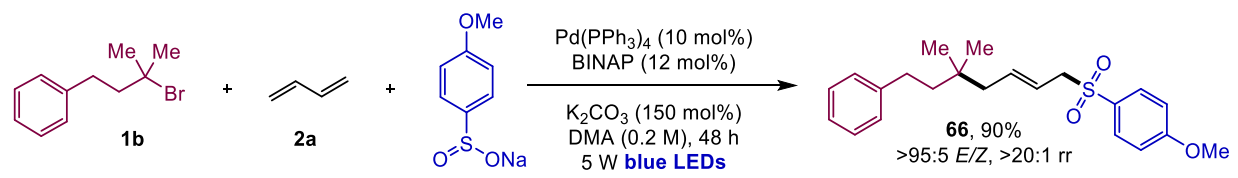

(E)-1-((5,5-dimethyl-7-phenylhept-2-en-1-yl)sulfonyl)-4-methoxybenzene

(66). According to the general procedure J, using the bromide $\mathbf{2 b}(0.3 \mathrm{mmol}, 1.5$ equiv.), butadiene 2a (0.3 mmol, $0.15 \mathrm{~mL}, 2 M$ in THF, 1.5 equiv.), sulfinate $\mathbf{S 3 h}$ (0.2 mmol, 1.0 equiv.), $\mathrm{Pd}\left(\mathrm{PPh}_{3}\right)_{4}(0.020 \mathrm{mmol}, 10 \mathrm{~mol} \%)$, BINAP (0.024 mmol, $12 \mathrm{~mol} \%), \mathrm{K}_{2} \mathrm{CO}_{3}$ (150 mol\%), and stirring at room temperature for $48 \mathrm{~h}$ under 5W blue LEDs irradiation. The reaction mixture was filtered through a pad of silica gel and concentrated in vacuo, then purified by chromatography 
(pentane/EtOAc $=7 / 1 \rightarrow 6 / 1 \rightarrow 5 / 1)$ to give $66(67.0 \mathrm{mg}, 0.180 \mathrm{mmol}, 90 \%$, > 95:5 E/Z, $>20: 1 \mathrm{rr}$ ) as a light-yellow gum. $\mathbf{R}_{\boldsymbol{f}}$ (pentane/EtOAc= 7/1) 0.25. ${ }^{\mathbf{1}} \mathbf{H}$ NMR (500 MHz, $\mathbf{C D C l}_{3}$ ): $\delta$ ppm $7.79-7.75(\mathrm{~m}, 2 \mathrm{H}), 7.32$ - $7.26(\mathrm{~m}, 2 \mathrm{H}), 7.21-$ $7.18(\mathrm{~m}, 1 \mathrm{H}), 7.18-7.15(\mathrm{~m}, 2 \mathrm{H}), 6.97-6.92(\mathrm{~m}, 2 \mathrm{H}), 5.62(\mathrm{dtt}, J=15.4,7.5,1.2$ $\mathrm{Hz}, 1 \mathrm{H}), 5.41(\mathrm{dtt}, J=15.3,7.3,1.2 \mathrm{~Hz}, 1 \mathrm{H}), 3.84(\mathrm{~s}, 3 \mathrm{H}), 3.78(\mathrm{~d}, J=7.3 \mathrm{~Hz}, 2 \mathrm{H})$, $2.56-2.49(\mathrm{~m}, 2 \mathrm{H}), 2.00(\mathrm{~d}, J=7.5 \mathrm{~Hz}, 2 \mathrm{H}), 1.46-1.39(\mathrm{~m}, 2 \mathrm{H}), 0.88(\mathrm{~s}, 6 \mathrm{H}) .{ }^{13} \mathrm{C}$ NMR (126 MHz, $\mathrm{CDCl}_{3}$ ): $\delta$ ppm 163.7, 143.2, 138.3, 130.6, 130.2, 128.4, 128.4, 125.7, 118.7, 114.3, 60.5, 55.7, 45.2, 44.1, 33.7, 30.7, 26.9. HRMS (ESI+): calcd. for $\mathrm{C}_{21} \mathrm{H}_{28} \mathrm{O}_{3} \mathrm{SNa}\left(\mathrm{M}+\mathrm{Na}^{+}\right)$: 395.1651. Found: 395.1657 .

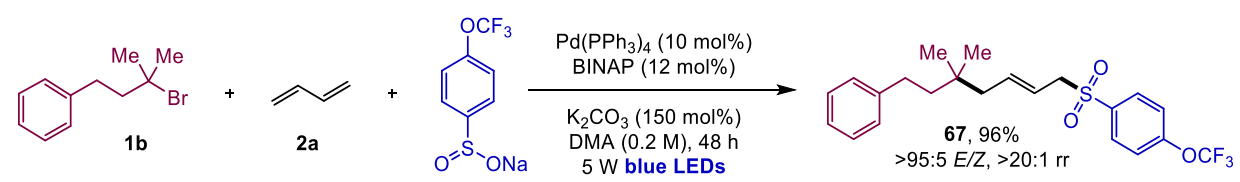

(E)-1-((5,5-Dimethyl-7-phenylhept-2-en-1-yl)sulfonyl)-4-

(trifluoromethoxy)benzene (67). According to the general procedure J, using the bromide $\mathbf{1 b}(0.3 \mathrm{mmol}, 1.5$ equiv.), butadiene $2 \mathbf{a}(0.3 \mathrm{mmol}, 0.15 \mathrm{~mL}, 2 \mathrm{M}$ in THF, 1.5 equiv.), sulfinate S3j (0.2 mmol, 1.0 equiv.), $\mathrm{Pd}\left(\mathrm{PPh}_{3}\right)_{4}(0.020 \mathrm{mmol}, 10$ mol\%), BINAP (0.024 mmol, $12 \mathrm{~mol} \%), \mathrm{K}_{2} \mathrm{CO}_{3}(150 \mathrm{~mol} \%)$, and stirring at room temperature for $48 \mathrm{~h}$ under $5 \mathrm{~W}$ blue LEDs irradiation. The reaction mixture was filtered through a pad of silica gel and concentrated in vacuo, then purified by chromatography (pentane $/$ EtOAc $=10 / 1)$ to give $67(81.9 \mathrm{mg}, 0.192 \mathrm{mmol}$, $96 \%,>95: 5 E / Z,>20: 1 \mathrm{rr}$ ) as a colorless gum. $\mathbf{R}_{\boldsymbol{f}}$ (pentane/EtOAc=10/1) 0.30. 1H NMR (599 MHz, CDCl $_{3}$ ): $\delta$ ppm $7.90-7.87(\mathrm{~m}, 2 \mathrm{H}), 7.31-7.27(\mathrm{~m}, 4 \mathrm{H}), 7.21$ - $7.18(\mathrm{~m}, 1 \mathrm{H}), 7.18$ - $7.15(\mathrm{~m}, 2 \mathrm{H}), 5.67-5.56(\mathrm{~m}, 1 \mathrm{H}), 5.47$ - $5.36(\mathrm{~m}, 1 \mathrm{H}), 3.82$ (d, $J=7.3 \mathrm{~Hz}, 2 \mathrm{H}), 2.55-2.50(\mathrm{~m}, 2 \mathrm{H}), 1.99(\mathrm{~d}, J=7.6 \mathrm{~Hz}, 2 \mathrm{H}), 1.43-1.38(\mathrm{~m}, 2 \mathrm{H})$, 0.85 (s, 6H). ${ }^{13} \mathbf{C}\left\{{ }^{1} \mathbf{H},{ }^{19}\right.$ F $\}$ NMR (151 MHz, $\left.\mathbf{C D C l}_{3}\right): \delta$ ppm 153.0, 143.1, 139.1, 136.8, 130.8, 128.5, 128.4, 125.8, 121.0, 120.3, 118.0, 60.3, 45.2, 44.1, 33.7, 30.7, 26.8. ${ }^{13} \mathbf{C}\left\{{ }^{1} \mathbf{H}\right\}$ NMR (151 MHz, $\left.\mathbf{C D C l}_{3}\right): \delta$ ppm $153.0(\mathrm{q}, J=1.9 \mathrm{~Hz}), 143.1,139.1$, 136.8, 130.8, 128.5, 128.4, 125.8, 120.96 (q, $J=1.3 \mathrm{~Hz}), 120.3$ (q, $\left.{ }^{1} J_{C-F}=249.1 \mathrm{~Hz}\right)$, 118.0, 60.3, 45.2, 44.1, 33.7, 30.7, 26.8. ${ }^{19} \mathbf{F}\left\{{ }^{1} \mathbf{H}\right\}$ NMR (564 MHz, $\left.\mathbf{C D C l}_{3}\right): \delta$ ppm - 
57.69. HRMS (ESI'): calcd. for $\mathrm{C}_{22} \mathrm{H}_{25} \mathrm{O}_{3} \mathrm{SF}_{3} \mathrm{Na}\left(\mathrm{M}+\mathrm{Na}^{+}\right)$: 449.1369. Found: 449.1373.
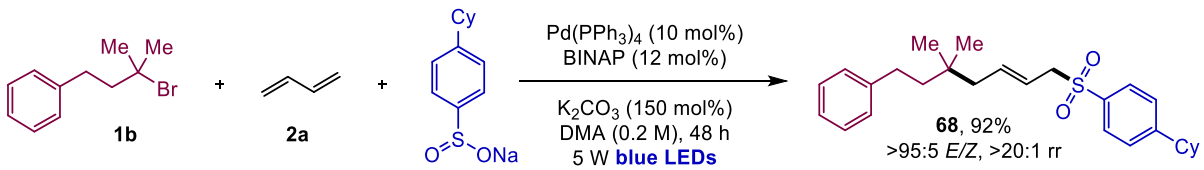

(E)-1-Cyclohexyl-4-((5,5-dimethyl-7-phenylhept-2-en-1-

yl)sulfonyl)benzene (68). According to the general procedure $J$, using the bromide $2 \mathbf{b}$ ( $0.3 \mathrm{mmol}, 1.5$ equiv.), butadiene $2 \mathbf{a}(0.3 \mathrm{mmol}, 0.15 \mathrm{~mL}, 2 \mathrm{M}$ in THF, 1.5 equiv.), sulfinate S3n (0.2 mmol, 1.0 equiv.), $\mathrm{Pd}\left(\mathrm{PPh}_{3}\right)_{4}(0.020 \mathrm{mmol}, 10$ mol\%), BINAP (0.024 mmol, $12 \mathrm{~mol} \%), \mathrm{K}_{2} \mathrm{CO}_{3}(150 \mathrm{~mol} \%)$, and stirring at room temperature for $48 \mathrm{~h}$ under $5 \mathrm{~W}$ blue LEDs irradiation. The reaction mixture was filtered through a pad of silica gel and concentrated in vacuo, then purified by chromatography (pentane $/$ EtOAc $=11 / 1 \rightarrow 10 / 1)$ to give $68(77.5 \mathrm{mg}, 0.183$ mmol, 92\%, > 95:5E/Z, > 20:1 rr) as a colorless gum. $\mathbf{R}_{\boldsymbol{f}}$ (pentane/EtOAc=10/1) 0.40. ${ }^{1} \mathrm{H}$ NMR (300 $\left.\mathbf{M H z} \mathbf{C D C l}_{3}\right): \delta \mathrm{ppm} 7.78-7.70(\mathrm{~m}, 2 \mathrm{H}), 7.34-7.24(\mathrm{~m}, 4 \mathrm{H})$, $7.23-7.12(\mathrm{~m}, 3 \mathrm{H}), 5.59(\mathrm{dt}, J=15.2,7.5 \mathrm{~Hz}, 1 \mathrm{H}), 5.42(\mathrm{dt}, J=15.2,7.2 \mathrm{~Hz}, 1 \mathrm{H})$, $3.79(\mathrm{~d}, J=7.1 \mathrm{~Hz}, 2 \mathrm{H}), 2.62-2.45(\mathrm{~m}, 3 \mathrm{H}), 1.98(\mathrm{~d}, J=7.4 \mathrm{~Hz}, 2 \mathrm{H}), 1.92-1.58(\mathrm{~m}$, 5H), $1.51-1.16(\mathrm{~m}, 7 \mathrm{H}), 0.82(\mathrm{~s}, 6 \mathrm{H}) .{ }^{13} \mathrm{C}$ NMR (75 MHz, $\mathbf{C D C l}_{3}$ ): $\delta$ ppm 154.4, 143.2, 138.4, 135.8, 128.6, 128.5, 128.4, 127.7, 125.8, 118.5, 60.3, 45.1, 44.7, 44.1, 34.2, 33.7, 30.7, 27.0, 26.8, 26.0. HRMS (ESI $\left.{ }^{+}\right)$: calcd. for $\mathrm{C}_{27} \mathrm{H}_{36} \mathrm{O}_{2} \mathrm{SNa}\left(\mathrm{M}+\mathrm{Na}^{+}\right)$: 447.2328. Found: 447.2331.
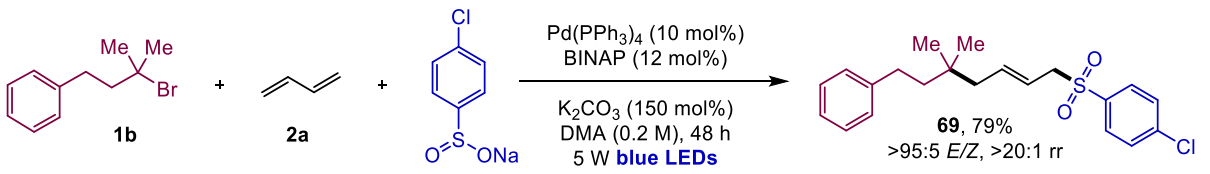

(E)-1-Chloro-4-((5,5-dimethyl-7-phenylhept-2-en-1-yl)sulfonyl)benzene

(69). According to the general procedure J, using the bromide $\mathbf{1 b}(0.3 \mathrm{mmol}, 1.5$ equiv.), butadiene $2 \mathrm{a}(0.3 \mathrm{mmol}, 0.15 \mathrm{~mL}, 2 \mathrm{M}$ in THF, 1.5 equiv.), sulfinate S3i (0.2 mmol, 1.0 equiv.), $\mathrm{Pd}\left(\mathrm{PPh}_{3}\right)_{4}(0.020 \mathrm{mmol}, 10 \mathrm{~mol} \%)$, BINAP (0.024 mmol, 
$12 \mathrm{~mol} \%), \mathrm{K}_{2} \mathrm{CO}_{3}$ (150 mol\%), and stirring at room temperature for $48 \mathrm{~h}$ under 5W blue LEDs irradiation. The reaction mixture was filtered through a pad of silica gel and concentrated in vacuo, then the yield was determined by ${ }^{1} \mathrm{H}$ NMR $(79 \%,>95: 5 E / Z,>20: 1 \mathrm{rr})$. An analytically pure sample was obtained by flash column chromatography on silica (pentane/EtOAc $=8 / 1 \rightarrow 7 / 1$ ), affording 69 as a colorless gum. $\mathbf{R}_{\boldsymbol{f}}$ (pentane/EtOAc $\left.=6 / 1\right)$ 0.45. ${ }^{1} \mathbf{H}$ NMR (500 $\left.\mathbf{~ M H z}, \mathbf{C D C l}_{3}\right): \delta$ ppm 7.78 - $7.74(\mathrm{~m}, 2 \mathrm{H}), 7.46-7.43(\mathrm{~m}, 2 \mathrm{H}), 7.31$ - $7.26(\mathrm{~m}, 2 \mathrm{H}), 7.21$ - 7.18 (m, 1H), $7.18-7.14(\mathrm{~m}, 2 \mathrm{H}), 5.63(\mathrm{dtt}, J=15.2,7.6,1.1 \mathrm{~Hz}, 1 \mathrm{H}), 5.39(\mathrm{dtt}, J=15.2,7.4$, $1.2 \mathrm{~Hz}, 1 \mathrm{H}), 3.79$ (d, $J=7.5 \mathrm{~Hz}, 2 \mathrm{H}), 2.55-2.48(\mathrm{~m}, 2 \mathrm{H}), 1.99$ (d, $J=7.5 \mathrm{~Hz}, 2 \mathrm{H})$, $1.44-1.38(\mathrm{~m}, 2 \mathrm{H}), 0.86(\mathrm{~s}, 6 \mathrm{H}) .{ }^{13} \mathbf{C}$ NMR (126 MHz, CDCl 3 ): $\delta$ ppm 143.1, 140.5, 139.0, 137.1, 130.0, 129.5, 128.5, 128.4, 125.8, 118.1, 60.4, 45.3, 44.1, 33.8, 30.7, 26.9. HRMS (ESI ${ }^{+}$): calcd. for $\mathrm{C}_{21} \mathrm{H}_{25} \mathrm{O}_{2} \mathrm{~S}^{35} \mathrm{ClNa}\left(\mathrm{M}+\mathrm{Na}^{+}\right)$: 399.1156. Found: 399.1153; calcd. for $\mathrm{C}_{21} \mathrm{H}_{25} \mathrm{O}_{2} \mathrm{~S}^{37} \mathrm{ClNa}\left(\mathrm{M}+\mathrm{Na}^{+}\right)$: 401.1128. Found: 401.1123 .

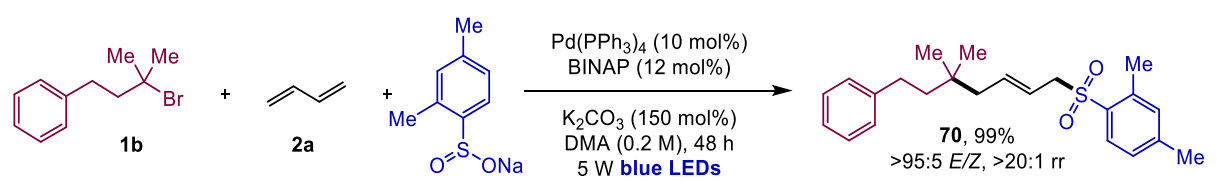

(E)-1-((5,5-Dimethyl-7-phenylhept-2-en-1-yl)sulfonyl)-2,4-

dimethylbenzene (70). According to the general procedure J, using the bromide 1 b (0.3 mmol, 1.5 equiv.), butadiene 2a (0.3 mmol, $0.15 \mathrm{~mL}, 2 \mathrm{M}$ in THF, 1.5 equiv.), sulfinate S3m (0.2 mmol, 1.0 equiv.), $\mathrm{Pd}\left(\mathrm{PPh}_{3}\right)_{4}(0.020 \mathrm{mmol}, 10 \mathrm{~mol} \%)$, BINAP (0.024 mmol, $12 \mathrm{~mol} \%), \mathrm{K}_{2} \mathrm{CO}_{3}(150 \mathrm{~mol} \%)$, and stirring at room temperature for $48 \mathrm{~h}$ under $5 \mathrm{~W}$ blue LEDs irradiation. The reaction mixture was filtered through a pad of silica gel and concentrated in vacuo, then purified by chromatography (pentane $/$ EtOAc $=12 / 1 \rightarrow 10 / 1)$ to give $70(73.0 \mathrm{mg}, 0.197$ mmol, 99\%, > 95:5 E/Z, > 20:1 rr) as a colorless gum. $\mathbf{R}_{\boldsymbol{f}}($ pentane $/$ EtOAc $=7 / 1)$ 0.50. 1H NMR (500 MHz, $\left.\mathbf{C D C l}_{3}\right): \delta$ ppm $7.78(\mathrm{~d}, J=8.1 \mathrm{~Hz}, 1 \mathrm{H}), 7.31-7.26(\mathrm{~m}$, 2H), $7.21-7.16(\mathrm{~m}, 1 \mathrm{H}), 7.16-7.13(\mathrm{~m}, 2 \mathrm{H}), 7.09$ (br s, 1H), 7.05 (d, J = 8.0 Hz, $1 \mathrm{H}), 5.61(\mathrm{dtt}, J=15.1,7.5,1.2 \mathrm{~Hz}, 1 \mathrm{H}), 5.38(\mathrm{dtt}, J=15.0,7.4,1.3 \mathrm{~Hz}, 1 \mathrm{H}), 3.82(\mathrm{~d}$, $J=7.4 \mathrm{~Hz}, 2 \mathrm{H}), 2.65(\mathrm{~s}, 3 \mathrm{H}), 2.53-2.46(\mathrm{~m}, 2 \mathrm{H}), 2.34(\mathrm{~s}, 3 \mathrm{H}), 1.96(\mathrm{~d}, J=7.5 \mathrm{~Hz}$, 
2H), 1.40 - 1.35 (m, 2H), 0.82 (s, 6H). ${ }^{13} \mathbf{C}$ NMR (126 MHz, $\mathbf{C D C l}_{3}$ ): $\delta$ ppm 144.4, 143.2, 138.1, 137.8, 133.8, 133.3, 130.9, 128.4, 128.4, 127.2, 125.7, 118.5, 59.5, 45.0, 44.0, 33.7, 30.7, 26.8, 21.4, 20.5. HRMS (ESI $\left.{ }^{+}\right)$: calcd. for $\mathrm{C}_{23} \mathrm{H}_{30} \mathrm{O}_{2} \mathrm{SNa}(\mathrm{M}+$ $\mathrm{Na}^{+}$): 393.1859. Found: 393.1864 .

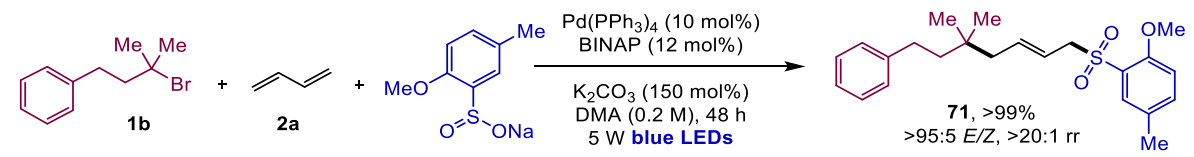

(E)-2-((5,5-Dimethyl-7-phenylhept-2-en-1-yl)sulfonyl)-1-methoxy-4-

methylbenzene (71). According to the general procedure I, using the bromide 1b (0.3 mmol, 1.5 equiv.), butadiene 2a ( $0.3 \mathrm{mmol}, 0.15 \mathrm{~mL}, 2 M$ in THF, 1.5 equiv.), sulfinate S3o (0.2 mmol, 1.0 equiv.), $\mathrm{Pd}\left(\mathrm{PPh}_{3}\right)_{4}(0.020 \mathrm{mmol}, 10 \mathrm{~mol} \%)$, BINAP (0.024 mmol, $12 \mathrm{~mol} \%), \mathrm{K}_{2} \mathrm{CO}_{3}$ (150 mol\%), and stirring at room temperature for $48 \mathrm{~h}$ under $5 \mathrm{~W}$ blue LEDs irradiation. The reaction mixture was filtered through a pad of silica gel and concentrated in vacuo, then purified by chromatography (pentane/EtOAc $=5 / 1 \rightarrow 4 / 1)$ to give $71(77.2 \mathrm{mg}, 0.200$ mmol, $>99 \%,>95: 5 E / Z,>20: 1 \mathrm{rr}$ ) as a pale yellow gum. $\mathbf{R}_{\boldsymbol{f}}$ (pentane/EtOAc= 6/1) 0.20. ${ }^{1} \mathrm{H}$ NMR (400 MHz, $\mathbf{C D C l}_{3}$ ): $\delta$ ppm 7.68 (d, J = 2.0 Hz, 1H), $7.33-7.26$ (m, 3H), $7.22-7.18(\mathrm{~m}, 1 \mathrm{H}), 7.18-7.13(\mathrm{~m}, 2 \mathrm{H}), 6.87$ (d, J = 8.4 Hz, 1H), 5.62 (dtt, $J=15.2,7.6,1.1 \mathrm{~Hz}, 1 \mathrm{H}), 5.42(\mathrm{dtt}, J=15.3,7.4,1.1 \mathrm{~Hz}, 1 \mathrm{H}), 4.08(\mathrm{~d}, J=7.4 \mathrm{~Hz}, 2 \mathrm{H})$, $3.94(\mathrm{~s}, 3 \mathrm{H}), 2.51-2.43(\mathrm{~m}, 2 \mathrm{H}), 2.24(\mathrm{~s}, 3 \mathrm{H}), 1.93$ (d, J = $7.5 \mathrm{~Hz}, 2 \mathrm{H}), 1.33-1.25$ (m, 2H), 0.73 (s, 6H). ${ }^{13} \mathbf{C}$ NMR (101 $\left.\mathbf{M H z}, \mathbf{C D C l}_{3}\right): \delta$ ppm 155.0, 143.2, 137.8, 135.9, 131.1, 130.6, 128.39, 128.37, 125.8, 125.7, 119.0, 112.2, 56.4, 44.7, 43.9, 33.6, 30.6, 26.7, 20.2. HRMS (ESI+): calcd. for $\mathrm{C}_{23} \mathrm{H}_{30} \mathrm{O}_{3} \mathrm{SNa}\left(\mathrm{M}+\mathrm{Na}^{+}\right): 409.1808$. Found: 409.1818.

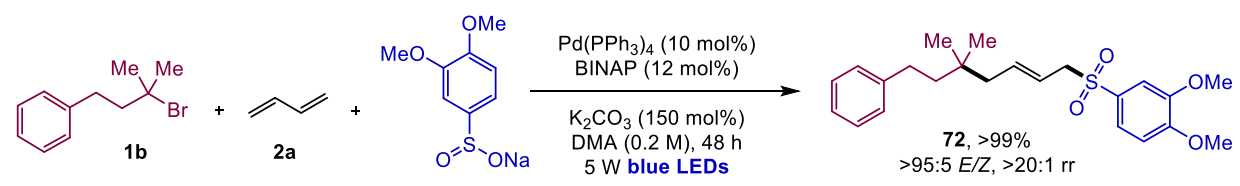

(E)-4-((5,5-Dimethyl-7-phenylhept-2-en-1-yl)sulfonyl)-1,2-

dimethoxybenzene (72). According to the general procedure J, using the 
bromide $\mathbf{1 b}$ ( $0.3 \mathrm{mmol}, 1.5$ equiv.), butadiene $2 \mathbf{a}(0.3 \mathrm{mmol}, 0.15 \mathrm{~mL}, 2 \mathrm{M}$ in THF, 1.5 equiv.), sulfinate S3l (0.2 mmol, 1.0 equiv.), $\mathrm{Pd}\left(\mathrm{PPh}_{3}\right)_{4}(0.020 \mathrm{mmol}, 10$ mol\%), BINAP (0.024 mmol, $12 \mathrm{~mol} \%), \mathrm{K}_{2} \mathrm{CO}_{3}(150 \mathrm{~mol} \%)$, and stirring at room temperature for $48 \mathrm{~h}$ under $5 \mathrm{~W}$ blue LEDs irradiation. The reaction mixture was filtered through a pad of silica gel and concentrated in vacuo, then purified by chromatography (pentane/EtOAc $=4 / 1 \rightarrow 3 / 1$ ) to give $72(80.4 \mathrm{mg}, 0.200$ mmol, $>99 \%,>95: 5 E / Z,>20: 1 \mathrm{rr}$ ) as a colorless gum. $\mathbf{R}_{\boldsymbol{f}}$ (pentane/EtOAc $=4 / 1$ ) 0.27. ${ }^{1} \mathrm{H}$ NMR (500 MHz, $\left.\mathbf{C D C l}_{3}\right): \delta$ ppm $7.46(\mathrm{dd}, J=8.4,2.1 \mathrm{~Hz}, 1 \mathrm{H}), 7.31(\mathrm{~d}, J=$ $2.1 \mathrm{~Hz}, 1 \mathrm{H}$ ), $7.31-7.26(\mathrm{~m}, 2 \mathrm{H}), 7.20-7.15(\mathrm{~m}, 3 \mathrm{H}), 6.90$ (d, J = $8.5 \mathrm{~Hz}, 1 \mathrm{H}), 5.65$ $(\mathrm{dtt}, J=15.2,7.5,1.1 \mathrm{~Hz}, 1 \mathrm{H}), 5.43(\mathrm{dtt}, J=15.4,7.3,1.2 \mathrm{~Hz}, 1 \mathrm{H}), 3.92(\mathrm{~s}, 3 \mathrm{H}), 3.90$ (s, 3H), $3.80(\mathrm{~d}, J=7.3 \mathrm{~Hz}, 2 \mathrm{H}), 2.56-2.50(\mathrm{~m}, 2 \mathrm{H}), 2.01(\mathrm{~d}, J=7.5 \mathrm{~Hz}, 2 \mathrm{H}), 1.47-$ $1.41(\mathrm{~m}, 2 \mathrm{H}), 0.88(\mathrm{~s}, 6 \mathrm{H}) .{ }^{13} \mathbf{C}$ NMR (126 MHz, $\left.\mathbf{C D C l}_{3}\right): \delta$ ppm 153.3, 149.1, 143.1, 138.3, 130.3, 128.4, 128.3, 125.7, 122.6, 118.6, 110.7, 110.6, 60.5, 56.3, 45.1, 44.1, 33.7, 30.7, 26.9. HRMS (ESI $\left.{ }^{+}\right)$: calcd. for $\mathrm{C}_{23} \mathrm{H}_{30} \mathrm{O}_{4} \mathrm{SNa}\left(\mathrm{M}+\mathrm{Na}^{+}\right): 425.1757$. Found: 425.1768.
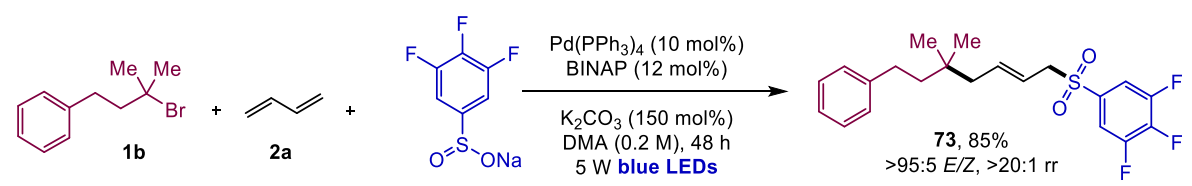

\section{(E)-5-((5,5-Dimethyl-7-phenylhept-2-en-1-yl)sulfonyl)-1,2,3-}

trifluorobenzene (73). According to the general procedure J, using the bromide 1 b (0.3 mmol, 1.5 equiv.), butadiene 2a ( $0.3 \mathrm{mmol}, 0.15 \mathrm{~mL}, 2 M$ in THF, 1.5 equiv.), sulfinate S3k (0.2 mmol, 1.0 equiv.), $\mathrm{Pd}\left(\mathrm{PPh}_{3}\right)_{4}$ (0.020 mmol, $\left.10 \mathrm{~mol} \%\right)$, BINAP (0.024 mmol, $12 \mathrm{~mol} \%), \mathrm{K}_{2} \mathrm{CO}_{3}$ (150 mol\%), and stirring at room temperature for $48 \mathrm{~h}$ under $5 \mathrm{~W}$ blue LEDs irradiation. The reaction mixture was filtered through a pad of silica gel and concentrated in vacuo, then purified by chromatography (pentane $/$ EtOAc $=10 / 1)$ to give $73(67.2 \mathrm{mg}, 0.169 \mathrm{mmol}$, $85 \%,>95: 5 E / Z,>20: 1 \mathrm{rr}$ ) as a colorless gum. $\mathbf{R}_{\boldsymbol{f}}$ (pentane/EtOAc=10/1) 0.35. ${ }^{1}$ H NMR (599 MHz, $\left.\mathbf{C D C l}_{3}\right): \delta$ ppm $7.54(\mathrm{t}, J=6.1 \mathrm{~Hz}, 2 \mathrm{H}), 7.30-7.26(\mathrm{~m}, 2 \mathrm{H})$, $7.20-7.17(\mathrm{~m}, 1 \mathrm{H}), 7.17-7.14(\mathrm{~m}, 2 \mathrm{H}), 5.69(\mathrm{dt}, J=15.2,7.6 \mathrm{~Hz}, 1 \mathrm{H}), 5.42(\mathrm{dt}, J=$ 
15.2, 7.5 Hz, 1H), 3.81 (d, $J=7.3 \mathrm{~Hz}, 2 \mathrm{H}), 2.57-2.51(\mathrm{~m}, 2 \mathrm{H}), 2.03$ (d, $J=7.6 \mathrm{~Hz}$, 2H), $1.48-1.43(\mathrm{~m}, 2 \mathrm{H}), 0.89(\mathrm{~s}, 6 \mathrm{H}) \cdot{ }^{13} \mathbf{C}\left\{{ }^{1} \mathrm{H},{ }^{19} \mathrm{~F}\right\}$ NMR (151 MHz, $\left.\mathbf{C D C l}_{3}\right): \delta$ ppm 151.2, 143.5, 143.0, 139.7, 134.7, 128.5, 128.3, 125.8, 117.5, 113.9, 60.3, 45.2, 44.3, 33.8, 30.7, 26.8. ${ }^{13} \mathbf{C}\left\{{ }^{\mathbf{H}} \mathbf{H}\right\}$ NMR (151 $\mathbf{M H z}, \mathbf{C D C l}_{3}$ ): $\delta$ ppm 151.2 (ddd, $J$ = 257.8, 10.6, $3.1 \mathrm{~Hz}), 143.4(\mathrm{dt}, J=262.1,15.7 \mathrm{~Hz}), 143.0,139.7,134.7(\mathrm{dd}, J=$ 10.7, $6.0 \mathrm{~Hz}$ ), 128.5, 128.3, 125.8, 117.5, 113.9 (dd, $J=18.0,5.6 \mathrm{~Hz}$ ), 60.3, 45.2, 44.3, 33.8, 30.7, 26.8. ${ }^{19} \mathbf{F}\left\{{ }^{1} \mathbf{H}\right\}$ NMR (564 $\mathbf{M H z}, \mathbf{C D C l}_{3}$ ): $\delta$ ppm -129.19 (dd, $J=$ 19.8, $5.9 \mathrm{~Hz}$ ), $-150.06\left(\mathrm{tt}, J=19.9,6.1 \mathrm{~Hz}\right.$ ). HRMS (ESI+): calcd. for $\mathrm{C}_{21} \mathrm{H}_{23} \mathrm{O}_{2} \mathrm{SF}_{3} \mathrm{Na}$ $\left(\mathrm{M}+\mathrm{Na}^{+}\right): 419.1263$. Found: 419.1266.

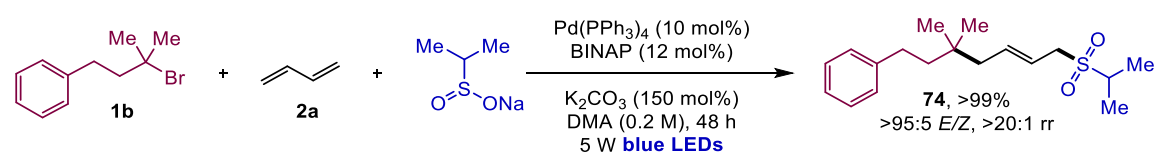

(E)-(7-(Isopropylsulfonyl)-3,3-dimethylhept-5-en-1-yl)benzene

(74).

According to the general procedure J, using the bromide $\mathbf{1 b}(0.3 \mathrm{mmol}, 1.5$ equiv.), butadiene 2a ( $0.3 \mathrm{mmol}, 0.15 \mathrm{~mL}, 2 \mathrm{M}$ in THF, 1.5 equiv.), sulfinate S3p (0.2 mmol, 1.0 equiv.), $\mathrm{Pd}\left(\mathrm{PPh}_{3}\right)_{4}$ (0.020 mmol, $\left.10 \mathrm{~mol} \%\right)$, BINAP (0.024 mmol, $12 \mathrm{~mol} \%), \mathrm{K}_{2} \mathrm{CO}_{3}$ (150 mol\%), and stirring at room temperature for $48 \mathrm{~h}$ under $5 \mathrm{~W}$ blue LEDs irradiation. The reaction mixture was filtered through a pad of silica gel and concentrated in vacuo, then purified by chromatography (pentane/EtOAc $=6 / 1 \rightarrow 5 / 1)$ to give $74(61.9 \mathrm{mg}, 0.200 \mathrm{mmol},>99 \%,>95: 5$ $E / Z,>20: 1 \mathrm{rr}$ ) as a light yellow gum. $\mathbf{R}_{\boldsymbol{f}}$ (pentane/EtOAc= 7/1) 0.25. ${ }^{\mathbf{1}} \mathbf{H} \mathbf{~ N M R}$ $\left(400 \mathbf{~ M H z}, \mathbf{C D C l}_{3}\right): \delta$ ppm $7.32-7.26(\mathrm{~m}, 2 \mathrm{H}), 7.22-7.15(\mathrm{~m}, 3 \mathrm{H}), 5.90(\mathrm{dtt}, J=$ 15.2, 7.5, $1.3 \mathrm{~Hz}, 1 \mathrm{H}), 5.58(\mathrm{dtt}, J=15.3,7.4,1.2 \mathrm{~Hz}, 1 \mathrm{H}), 3.69$ (d, $J=7.2 \mathrm{~Hz}, 2 \mathrm{H}$ ), 3.15 (hept, $J=6.9 \mathrm{~Hz}, 1 \mathrm{H}), 2.62-2.54(\mathrm{~m}, 2 \mathrm{H}), 2.11$ (d, $J=7.5 \mathrm{~Hz}, 2 \mathrm{H}), 1.55-1.47$ $(\mathrm{m}, 2 \mathrm{H}), 1.35$ (d, $J=6.9 \mathrm{~Hz}, 6 \mathrm{H}), 0.98(\mathrm{~s}, 6 \mathrm{H}) \cdot{ }^{13} \mathbf{C}$ NMR (101 MHz, $\left.\mathbf{C D C l}_{3}\right): \delta \mathrm{ppm}$ 143.1, 138.0, 128.5, 128.3, 125.7, 118.5, 54.0, 51.2, 45.3, 44.3, 33.8, 30.7, 27.0, 15.1. HRMS (ESI+): calcd. for $\mathrm{C}_{18} \mathrm{H}_{28} \mathrm{O}_{2} \mathrm{SNa}\left(\mathrm{M}+\mathrm{Na}^{+}\right)$: 331.1708. Found: 331.1706 . 


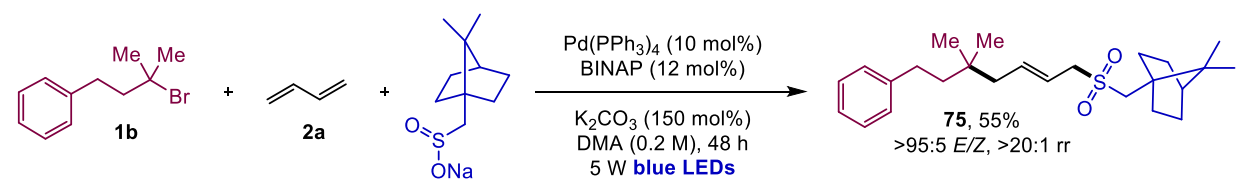

(1S,4R)-1-(((CE)-5,5-Dimethyl-7-phenylhept-2-en-1-yl)sulfonyl)methyl)-

7,7-dimethylbicyclo[2.2.1]heptan-2-one (75). According to the general procedure J, using the bromide $\mathbf{1 b}(0.3 \mathrm{mmol}, 1.5$ equiv.), butadiene 2a $(0.3$ mmol, $0.15 \mathrm{~mL}, 2 M$ in THF, 1.5 equiv.), sulfinate S3q (0.2 mmol, 1.0 equiv.), $\mathrm{Pd}\left(\mathrm{PPh}_{3}\right)_{4}(0.020 \mathrm{mmol}, 10 \mathrm{~mol} \%)$, BINAP (0.024 mmol, $\left.12 \mathrm{~mol} \%\right), \mathrm{K}_{2} \mathrm{CO}_{3}(150$ mol\%), and stirring at room temperature for $48 \mathrm{~h}$ under $5 \mathrm{~W}$ blue LEDs irradiation. The reaction mixture was filtered through a pad of silica gel and concentrated in vacuo, then purified by chromatography (pentane/EtOAc $=11 / 2$ $\rightarrow 5 / 1$ ) to give 75 (45.8 $\mathrm{mg}, 0.110 \mathrm{mmol}, 55 \%,>95: 5 E / Z,>20: 1 \mathrm{rr}$ ) as a colorless gum. $\mathbf{R}_{\boldsymbol{f}}$ (pentane $/$ EtOAc $\left.=11 / 2\right)$ 0.35. ${ }^{\mathbf{1}} \mathbf{H}$ NMR (400 $\left.\mathbf{~ M H z}, \mathbf{C D C l}_{3}\right): \delta$ ppm $7.31-7.24(\mathrm{~m}, 2 \mathrm{H}), 7.21-7.13(\mathrm{~m}, 3 \mathrm{H}), 5.98(\mathrm{dtt}, J=15.2,7.6,1.1 \mathrm{~Hz}, 1 \mathrm{H})$, $5.65(\mathrm{dtt}, J=15.0,8.1,1.4 \mathrm{~Hz}, 1 \mathrm{H}), 4.10(\mathrm{dd}, J=14.0,7.9 \mathrm{~Hz}, 1 \mathrm{H}), 3.75(\mathrm{dd}, J=14.0$, $6.7 \mathrm{~Hz}, 1 \mathrm{H}), 3.45(\mathrm{~d}, J=14.9 \mathrm{~Hz}, 1 \mathrm{H}), 2.70(\mathrm{~d}, J=14.9 \mathrm{~Hz}, 1 \mathrm{H}), 2.62-2.53(\mathrm{~m}, 2 \mathrm{H})$, $2.46-2.32$ (m, 2H), $2.16-2.10(\mathrm{~m}, 3 \mathrm{H}), 2.09-1.99(\mathrm{~m}, 1 \mathrm{H}), 1.93$ (d, J = $18.5 \mathrm{~Hz}$, 1H), 1.84 (ddd, $J=14.2,9.3,4.7 \mathrm{~Hz}, 1 \mathrm{H}), 1.55-1.49$ (m, 2H), 1.45 (ddd, $J=13.0$, 9.3, $3.9 \mathrm{~Hz}, 1 \mathrm{H}), 1.04$ (s, 3H), 0.97 (s, 6H), 0.83 (s, 3H). ${ }^{13} \mathrm{C}$ NMR (101 MHz, $\mathbf{C D C l}_{3}$ ): $\delta$ ppm 215.5, 143.2, 138.6, 128.44, 128.43, 125.7, 119.0, 59.9, 59.1, 49.0, 48.7, 45.2, 44.4, 42.8, 42.7, 33.9, 30.8, 27.2, 27.10, 27.05, 25.5, 19.84, 19.78. HRMS (ESI+): calcd. for $\mathrm{C}_{25} \mathrm{H}_{36} \mathrm{O}_{3} \mathrm{SNa}\left(\mathrm{M}+\mathrm{Na}^{+}\right)$: 439.2277. Found: 439.2272 .

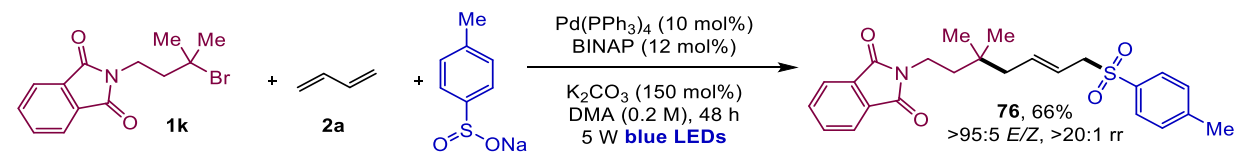

(E)-2-(3,3-Dimethyl-7-tosylhept-5-en-1-yl)isoindoline-1,3-dione

(76).

According to the general procedure J, using the corresponding alkyl bromide $\mathbf{1 k}$ ( 0.3 mmol, 1.5 equiv.), butadiene 2 a ( $0.3 \mathrm{mmol}, 0.15 \mathrm{~mL}, 2 M$ in THF, 1.5 equiv.), sodium 4-methylbenzenesulfinate (0.2 mmol, 1.0 equiv.), $\mathrm{Pd}\left(\mathrm{PPh}_{3}\right)_{4}(10 \mathrm{~mol} \%)$, BINAP (12 mol\%), $\mathrm{K}_{2} \mathrm{CO}_{3}$ (150 mol\%), and stirring at room temperature for $48 \mathrm{~h}$ 
under $5 \mathrm{~W}$ blue LEDs irradiation. The reaction mixture was filtered through a pad of silica gel and concentrated in vacuo, then purified by chromatography (pentane/EtOAc $=4 / 1)$ to give $76(55.7 \mathrm{mg}, 0.131 \mathrm{mmol}, 66 \%,>95: 5 \mathrm{E} / \mathrm{Z},>20: 1$ rr) as a colorless oil. $\mathbf{R}_{f}$ (pentane/EtOAc $=7 / 3$ ) 0.25. ${ }^{1} \mathbf{H}$ NMR (599 $\left.\mathbf{M H z}, \mathbf{C D C l}_{3}\right)$ : $\delta$ ppm $7.82(\mathrm{dd}, J=5.4,3.0 \mathrm{~Hz}, 2 \mathrm{H}), 7.76-7.66(\mathrm{~m}, 4 \mathrm{H}), 7.33-7.29(\mathrm{~m}, 2 \mathrm{H}), 5.69$ - $5.57(\mathrm{~m}, 1 \mathrm{H}), 5.48$ - $5.35(\mathrm{~m}, 1 \mathrm{H}), 3.78(\mathrm{dd}, J=7.3,1.1 \mathrm{~Hz}, 2 \mathrm{H}), 3.68$ - $3.55(\mathrm{~m}$, 2H), 2.39 (s, 3H), 1.98 (dd, $J=7.6,1.3 \mathrm{~Hz}, 2 \mathrm{H}), 1.47-1.37(\mathrm{~m}, 2 \mathrm{H}), 0.86(\mathrm{~s}, 6 \mathrm{H})$. 13C NMR (151 MHz, CDCl$_{3}$ ): $\delta$ ppm 168.3, 144.6, 137.7, 135.7, 134.0, 132.3, 129.8, 128.5, 128.5, 123.2, 119.2, 60.3, 45.0, 39.7, 34.2, 33.1, 26.7. HRMS (ESI+): calcd. for $\mathrm{C}_{24} \mathrm{H}_{27} \mathrm{NO}_{4} \mathrm{Na}\left(\mathrm{M}+\mathrm{Na}^{+}\right): 448.1553$. Found: 448.1553 .

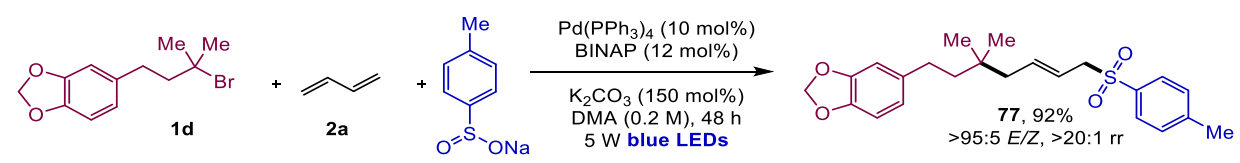

(E)-5-(3,3-Dimethyl-7-tosylhept-5-en-1-yl)benzo[d][1,3]dioxole

(77).

According to the general procedure J, using the corresponding alkyl bromide 1d ( $0.3 \mathrm{mmol}, 1.5$ equiv.), butadiene $2 \mathrm{a}$ ( $0.3 \mathrm{mmol}, 0.15 \mathrm{~mL}, 2 \mathrm{M}$ in THF, 1.5 equiv.), sodium 4-methylbenzenesulfinate ( $0.2 \mathrm{mmol}, 1.0$ equiv.), $\mathrm{Pd}\left(\mathrm{PPh}_{3}\right)_{4}(10 \mathrm{~mol} \%)$, BINAP (12 mol\%), $\mathrm{K}_{2} \mathrm{CO}_{3}$ (150 mol\%), and stirring at room temperature for $48 \mathrm{~h}$ under $5 \mathrm{~W}$ blue LEDs irradiation. The reaction mixture was filtered through a pad of silica gel and concentrated in vacuo, then purified by chromatography (pentane $/$ EtOAc $=4 / 1)$ to give $77(73.2 \mathrm{mg}, 0.183 \mathrm{mmol}, 92 \%,>95: 5 \mathrm{E} / Z,>20: 1$ rr) as a colorless oil. $\mathbf{R}_{\boldsymbol{f}}$ (pentane/EtOAc $\left.=4 / 1\right)$ 0.25. ${ }^{1} \mathbf{H} \mathbf{~ N M R}\left(599 \mathbf{~ M H z}, \mathbf{C D C l}_{3}\right)$ : $\delta$ ppm $7.80-7.68(\mathrm{~m}, 2 \mathrm{H}), 7.31-7.27(\mathrm{~m}, 2 \mathrm{H}), 6.71$ (d, J = $7.8 \mathrm{~Hz}, 1 \mathrm{H}), 6.66-$ $6.52(\mathrm{~m}, 2 \mathrm{H}), 5.90(\mathrm{~s}, 2 \mathrm{H}), 5.65-5.52(\mathrm{~m}, 1 \mathrm{H}), 5.44-5.31(\mathrm{~m}, 1 \mathrm{H}), 3.77(\mathrm{dd}, J=$ 7.3, $1.1 \mathrm{~Hz}, 2 \mathrm{H}), 2.42$ (d, $J=16.0 \mathrm{~Hz}, 5 \mathrm{H}), 1.95$ (dd, $J=7.5,1.3 \mathrm{~Hz}, 2 \mathrm{H}), 1.41-1.30$ (m, 2H), 0.82 (s, 6H). ${ }^{13}$ C NMR (151 MHz, $\mathbf{C D C l}_{3}$ ): $\delta$ ppm 145.5, 144.6, 138.3, 137.0, 135.7, 129.8, 128.5, 120.9, 120.9, 118.5, 108.9, 108.8, 108.2, 100.8, 60.3, 45.1, 44.3, 33.7, 30.4, 26.9, 26.9. HRMS (ESI'): calcd. for $\mathrm{C}_{23} \mathrm{H}_{28} \mathrm{SO}_{4} \mathrm{Na}\left(\mathrm{M}+\mathrm{Na}^{+}\right)$: 423.1601. Found: 423.1593. 


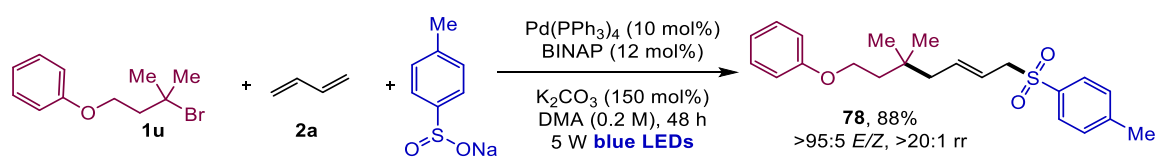

(E)-1-((5,5-Dimethyl-7-phenoxyhept-2-en-1-yl)sulfonyl)-4-methylbenzene

(78). According to the general procedure J, using the corresponding alkyl bromide 1 u ( $0.3 \mathrm{mmol}, 1.5$ equiv.), butadiene $2 \mathrm{a}(0.3 \mathrm{mmol}, 0.15 \mathrm{~mL}, 2 \mathrm{M}$ in THF, 1.5 equiv.), sodium 4-methylbenzenesulfinate ( $0.2 \mathrm{mmol}, 1.0$ equiv.), $\mathrm{Pd}\left(\mathrm{PPh}_{3}\right)_{4}$ (10 mol\%), BINAP (12 mol\%), $\mathrm{K}_{2} \mathrm{CO}_{3}(150 \mathrm{~mol} \%)$, and stirring at room temperature for $48 \mathrm{~h}$ under $5 \mathrm{~W}$ blue LEDs irradiation. The reaction mixture was filtered through a pad of silica gel and concentrated in vacuo, then purified by chromatography $($ pentane $/$ EtOAc $=4 / 1)$ to give $78(65.7 \mathrm{mg}, 0.177 \mathrm{mmol}, 88 \%$, > 95:5 E/Z, $>20: 1 \mathrm{rr}$ ) as a colorless oil. $\mathbf{R}_{\boldsymbol{f}}$ (pentane $/$ EtOAc $=4 / 1$ ) 0.38. ${ }^{\mathbf{1}} \mathbf{H} \mathbf{~ N M R}$ (300 MHz, $\mathbf{C D C l}_{3}$ ): $\delta$ ppm 7.82 - $7.65(\mathrm{~m}, 2 \mathrm{H}), 7.52-7.15(\mathrm{~m}, 4 \mathrm{H}), 7.00-6.69(\mathrm{~m}$, 3H), $5.71-5.56(\mathrm{~m}, 1 \mathrm{H}), 5.46-5.34(\mathrm{~m}, 1 \mathrm{H}), 3.95(\mathrm{t}, J=7.1 \mathrm{~Hz}, 2 \mathrm{H}), 3.77(\mathrm{dd}, J=$ 7.3, $1.0 \mathrm{~Hz}, 2 \mathrm{H}), 2.41$ (s, 3H), $2.06-1.95(\mathrm{~m}, 2 \mathrm{H}), 1.61$ (t, J = 7.1 Hz, 2H), 0.87 (s, 6H). ${ }^{13}$ C NMR (75 MHz, CDCl $_{3}$ ): $\delta$ ppm 159.0, 144.7, 138.1, 135.6, 129.8, 129.5, 128.5, 120.7, 118.8, 114.5, 64.6, 60.3, 45.6, 40.1, 33.0, 27.3, 21.7. HRMS (ESI+): calcd. for $\mathrm{C}_{22} \mathrm{H}_{28} \mathrm{SO}_{3} \mathrm{Na}\left(\mathrm{M}+\mathrm{Na}^{+}\right)$: 395.1651. Found: 395.1652 .
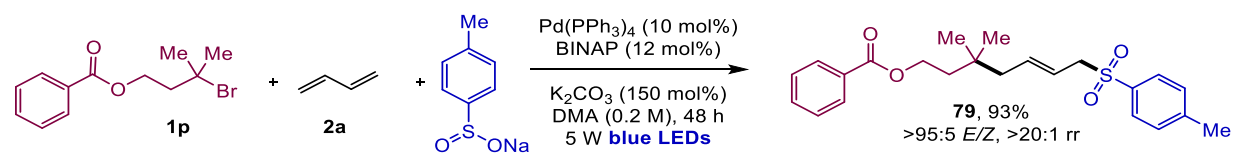

(E)-3,3-Dimethyl-7-tosylhept-5-en-1-yl benzoate (79). According to the general procedure J, using the corresponding alkyl bromide 1p (0.3 mmol, 1.5 equiv.), butadiene 2a (0.3 mmol, $0.15 \mathrm{~mL}, 2 \mathrm{M}$ in THF, 1.5 equiv.), sodium 4methylbenzenesulfinate ( $0.2 \mathrm{mmol}, 1.0$ equiv.), $\mathrm{Pd}\left(\mathrm{PPh}_{3}\right)_{4}(10 \mathrm{~mol} \%)$, BINAP (12 $\mathrm{mol} \%$ ), $\mathrm{K}_{2} \mathrm{CO}_{3}(150 \mathrm{~mol} \%), \mathrm{K}_{2} \mathrm{CO}_{3}(150 \mathrm{~mol} \%)$, and stirring at room temperature for $48 \mathrm{~h}$ under $5 \mathrm{~W}$ blue LEDs irradiation. The reaction mixture was filtered through a pad of silica gel and concentrated in vacuo, then purified by chromatography $($ pentane $/$ EtOAc $=4 / 1)$ to give $79(74.4 \mathrm{mg}, 0.186 \mathrm{mmol}, 93 \%$, > 95:5 E/Z, > 20:1 rr) as a colorless oil. $\mathbf{R}_{\boldsymbol{f}}$ (pentane/EtOAc $=4 / 1$ ) 0.20. ${ }^{\mathbf{1}} \mathbf{H} \mathbf{~ N M R}$ (300 MHz, $\mathbf{C D C l}_{3}$ ): $\delta$ ppm 8.11 - $7.92(\mathrm{~m}, 2 \mathrm{H}), 7.79-7.68(\mathrm{~m}, 2 \mathrm{H}), 7.59-7.51(\mathrm{~m}$, 
1H), 7.46 - $7.37(\mathrm{~m}, 2 \mathrm{H}), 7.33$ - $7.27(\mathrm{~m}, 2 \mathrm{H}), 5.68$ - $5.52(\mathrm{~m}, 1 \mathrm{H}), 5.44-5.26(\mathrm{~m}$, 1H), $4.30(\mathrm{t}, J=7.3 \mathrm{~Hz}, 2 \mathrm{H}), 3.77(\mathrm{dd}, J=7.3,1.0 \mathrm{~Hz}, 2 \mathrm{H}), 2.39(\mathrm{~s}, 3 \mathrm{H}), 2.06-1.93$ $(\mathrm{m}, 2 \mathrm{H}), 1.58(\mathrm{t}, J=7.3 \mathrm{~Hz}, 2 \mathrm{H}), 0.87(\mathrm{~s}, 6 \mathrm{H}) \cdot{ }^{13} \mathrm{C} \mathbf{N M R}\left(75 \mathbf{M H z}, \mathbf{C D C l}_{3}\right): \delta \mathrm{ppm}$ 166.7, 144.7, 137.8, 135.7, 133.0, 130.4, 129.8, 129.6, 128.5, 128.5, 119.0, 62.2, 60.2, 45.6, 39.7, 33.0, 27.1, 21.7. HRMS (ESI $\left.{ }^{+}\right)$: calcd. for $\mathrm{C}_{23} \mathrm{H}_{28} \mathrm{SO}_{4} \mathrm{Na}\left(\mathrm{M}+\mathrm{Na}^{+}\right)$: 423.1601. Found: 423.1603.
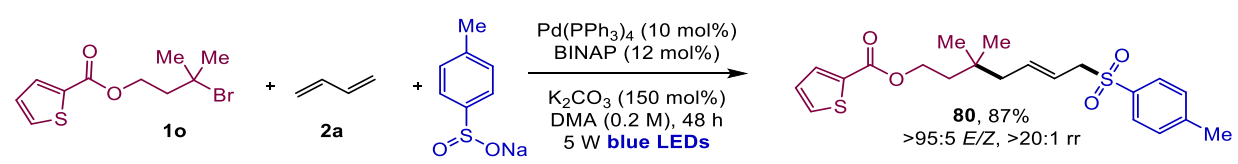

(E)-3,3-Dimethyl-7-tosylhept-5-en-1-yl thiophene-2-carboxylate (80). According to the general procedure J, using the corresponding alkyl bromide 10 ( $0.3 \mathrm{mmol}, 1.5$ equiv.), butadiene $2 \mathrm{a}(0.3 \mathrm{mmol}, 0.15 \mathrm{~mL}, 2 \mathrm{M}$ in THF, 1.5 equiv.), sodium 4-methylbenzenesulfinate (0.2 mmol, 1.0 equiv.), $\mathrm{Pd}\left(\mathrm{PPh}_{3}\right)_{4}(10 \mathrm{~mol} \%)$, BINAP (12 mol\%), $\mathrm{K}_{2} \mathrm{CO}_{3}$ (150 mol\%), and stirring at room temperature for $48 \mathrm{~h}$ under $5 \mathrm{~W}$ blue LEDs irradiation. The reaction mixture was filtered through a pad of silica gel and concentrated in vacuo, then purified by chromatography $($ pentane $/$ EtOAc $=4 / 1)$ to give $80(70.4 \mathrm{mg}, 0.173 \mathrm{mmol}, 87 \%,>95: 5 E / Z,>20: 1$ rr) as a colorless oil. $\mathbf{R}_{\boldsymbol{f}}$ (pentane/EtOAc $\left.=4 / 1\right)$ 0.20. ${ }^{\mathbf{1}} \mathbf{H} \mathbf{~ N M R}\left(\mathbf{3 0 0} \mathbf{~ M H z}, \mathbf{C D C l}_{3}\right)$ : $\delta$ ppm $7.77(\mathrm{dd}, J=3.8,1.3 \mathrm{~Hz}, 1 \mathrm{H}), 7.75-7.68(\mathrm{~m}, 2 \mathrm{H}), 7.54(\mathrm{dd}, J=5.0,1.3 \mathrm{~Hz}$, 1H), $7.35-7.27(\mathrm{~m}, 2 \mathrm{H}), 7.09(\mathrm{dd}, J=5.0,3.7 \mathrm{~Hz}, 1 \mathrm{H}), 5.66-5.53(\mathrm{~m}, 1 \mathrm{H}), 5.44-$ $5.31(\mathrm{~m}, 1 \mathrm{H}), 4.27(\mathrm{t}, J=7.3 \mathrm{~Hz}, 2 \mathrm{H}), 3.77(\mathrm{dd}, J=7.3,1.0 \mathrm{~Hz}, 2 \mathrm{H}), 2.40(\mathrm{~s}, 3 \mathrm{H})$, $1.97(\mathrm{dd}, J=7.4,1.2 \mathrm{~Hz}, 2 \mathrm{H}), 1.54(\mathrm{t}, J=7.3 \mathrm{~Hz}, 2 \mathrm{H}), 0.85(\mathrm{~s}, 6 \mathrm{H}) .{ }^{13} \mathrm{C}$ NMR (75 MHz, $\mathbf{C D C l}_{3}$ ): $\delta$ ppm 162.3, 144.7, 134.0, 137.8, 135.6, 133.4, 132.4, 129.8, 128.5, 127.9, 119.0, 62.3, 60.2, 45.5, 39.5, 33.0, 27.1, 21.7. HRMS (ESI'): calcd. for $\mathrm{C}_{21} \mathrm{H}_{26} \mathrm{SO}_{4} \mathrm{Na}\left(\mathrm{M}+\mathrm{Na}^{+}\right): 429.1170$. Found: 429.1164 .
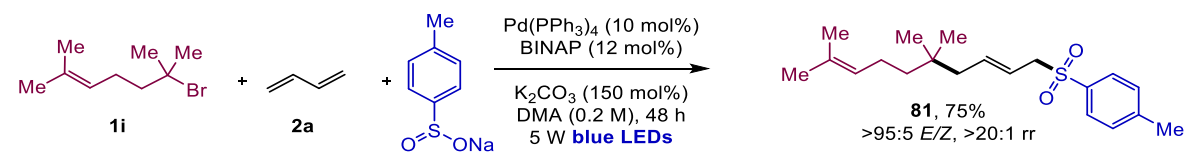

(E)-1-Methyl-4-((5,5,9-trimethyldeca-2,8-dien-1-yl)sulfonyl)benzene (81).

According to the general procedure J, using the corresponding alkyl bromide $\mathbf{1 i}$ S 88 
(0.3 mmol, 1.5 equiv.), butadiene 2 a ( $0.3 \mathrm{mmol}, 0.15 \mathrm{~mL}, 2 M$ in THF, 1.5 equiv.), sodium 4-methylbenzenesulfinate (0.2 mmol, 1.0 equiv.), $\mathrm{Pd}\left(\mathrm{PPh}_{3}\right)_{4}(10 \mathrm{~mol} \%)$, BINAP (12 mol\%), $\mathrm{K}_{2} \mathrm{CO}_{3}$ (150 mol\%), and stirring at room temperature for $48 \mathrm{~h}$ under $5 \mathrm{~W}$ blue LEDs irradiation. The reaction mixture was filtered through a pad of silica gel and concentrated in vacuo, then purified by chromatography $($ pentane $/$ EtOAc $=4 / 1)$ to give $81(50.3 \mathrm{mg}, 0.151 \mathrm{mmol}, 75 \%,>95: 5 E / Z,>20: 1$ rr) as a colorless oil. $\mathbf{R}_{\boldsymbol{f}}$ (pentane/EtOAc $\left.=4 / 1\right)$ 0.81. ${ }^{\mathbf{1}} \mathbf{H} \mathbf{~ N M R}\left(\mathbf{3 0 0} \mathbf{M H z}, \mathbf{C D C l}_{3}\right)$ : $\delta$ ppm $7.83-7.68(\mathrm{~m}, 2 \mathrm{H}), 7.40-7.29(\mathrm{~m}, 2 \mathrm{H}), 5.66-5.48(\mathrm{~m}, 1 \mathrm{H}), 5.46-5.27$ $(\mathrm{m}, 1 \mathrm{H}), 5.04(\mathrm{tt}, J=7.0,1.4 \mathrm{~Hz}, 1 \mathrm{H}), 3.75(\mathrm{dd}, J=7.2,1.0 \mathrm{~Hz}, 2 \mathrm{H}), 2.43(\mathrm{~s}, 3 \mathrm{H})$, $1.90(\mathrm{~d}, J=1.2 \mathrm{~Hz}, 4 \mathrm{H}), 1.67$ (d, $J=1.4 \mathrm{~Hz}, 3 \mathrm{H}), 1.57$ (d, $J=1.3 \mathrm{~Hz}, 3 \mathrm{H}), 1.16-0.98$ (m, 2H), 0.75 (s, 6H). ${ }^{13}$ C NMR (75 MHz, CDCl 3 ): $\delta$ ppm 144.6, 138.7, 135.7, 131.1, 129.8, 128.5, 125.0, 118.2, 60.4, 45.2, 41.9, 33.5, 26.8, 25.8, 22.8, 21.7, 17.7. HRMS (ESI+): calcd. for $\mathrm{C}_{20} \mathrm{H}_{30} \mathrm{SO}_{2} \mathrm{Na}\left(\mathrm{M}+\mathrm{Na}^{+}\right)$: 357.1864. Found: 357.1858.

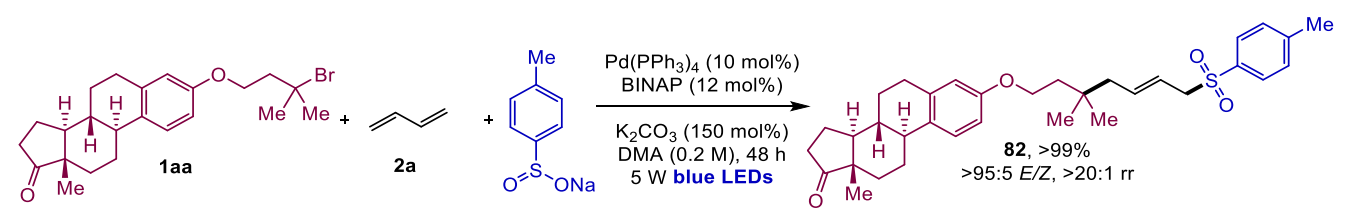

(8R,9S,13S,14S)-3-(((E)-3,3-Dimethyl-7-tosylhept-5-en-1-yl)oxy)-13-

methyl-6,7,8,9,11,12,13,14,15,16-decahydro-17H-

cyclopenta[a]phenanthren-17-one (82). According to the general procedure J, using the corresponding alkyl bromide $\mathbf{1 a a}(0.3 \mathrm{mmol}, 1.5$ equiv.), butadiene $2 \mathbf{a}$ (0.3 mmol, $0.15 \mathrm{~mL}, 2 M$ in THF, 1.5 equiv.), sodium 4-methylbenzenesulfinate (0.2 mmol, 1.0 equiv.), $\mathrm{Pd}\left(\mathrm{PPh}_{3}\right)_{4}(10 \mathrm{~mol} \%)$, BINAP (12 mol\%), $\mathrm{K}_{2} \mathrm{CO}_{3}$ (150 mol\%), and stirring at room temperature for $48 \mathrm{~h}$ under $5 \mathrm{~W}$ blue LEDs irradiation. The reaction mixture was filtered through a pad of silica gel and concentrated in vacuo, then purified by chromatography (pentane $/$ EtOAc $=4 / 1$ ) to give 82 (111.2 mg, $0.200 \mathrm{mmol},>99 \%,>95: 5 \mathrm{E} / Z$, > 20:1 rr) as a colorless oil. $\mathbf{R}_{\boldsymbol{f}}$ (pentane/EtOAc $=4 / 1)$ 0.11. ${ }^{1} \mathbf{H}$ NMR (599 $\left.\mathbf{M H z}_{\mathbf{C}} \mathbf{C D C l}_{3}\right): \delta$ ppm $7.75-7.72(\mathrm{~m}$, $2 \mathrm{H}), 7.31(\mathrm{~d}, J=8.0 \mathrm{~Hz}, 2 \mathrm{H}), 7.21-7.16(\mathrm{~m}, 1 \mathrm{H}), 6.68(\mathrm{dd}, J=8.6,2.8 \mathrm{~Hz}, 1 \mathrm{H}), 6.61$ $(\mathrm{d}, J=2.7 \mathrm{~Hz}, 1 \mathrm{H}), 5.69-5.60(\mathrm{~m}, 1 \mathrm{H}), 5.47-5.31(\mathrm{~m}, 1 \mathrm{H}), 3.92(\mathrm{t}, J=7.0 \mathrm{~Hz}, 2 \mathrm{H})$, 
$3.81-3.74(\mathrm{~m}, 2 \mathrm{H}), 2.93-2.85(\mathrm{~m}, 2 \mathrm{H}), 2.53-2.46(\mathrm{~m}, 1 \mathrm{H}), 2.42(\mathrm{~s}, 4 \mathrm{H}), 2.25(\mathrm{td}$, $J=10.7,4.3 \mathrm{~Hz}, 1 \mathrm{H}), 2.14(\mathrm{dt}, J=18.9,9.0 \mathrm{~Hz}, 1 \mathrm{H}), 2.08-1.92(\mathrm{~m}, 5 \mathrm{H}), 1.65-1.56$ (m, 4H), $1.55-1.38(\mathrm{~m}, 4 \mathrm{H}), 0.91$ (s, 3H), 0.86 (s, 6H). ${ }^{13} \mathrm{C}$ NMR (151 MHz, $\mathbf{C D C l}_{3}$ ): $\delta$ ppm 220.9, 157.0, 144.6, 138.1, 137.8, 135.7, 132.0, 129.8, 129.8, 128.5, 128.4, 126.4, 118.8, 114.5, 112.2, 64.7, 64.6, 60.4, 60.3, 50.5, 48.1, 45.6, 44.1, 40.2, 38.5, 35.9, 33.0, 31.7, 29.7, 27.2, 26.6, 26.0, 21.7, 21.7, 14.0, 13.9. HRMS (ESI ${ }^{+}$): calcd. for $\mathrm{C}_{34} \mathrm{H}_{44} \mathrm{SO}_{4} \mathrm{Na}\left(\mathrm{M}+\mathrm{Na}^{+}\right): 571.2853$. Found: 571.2829 .
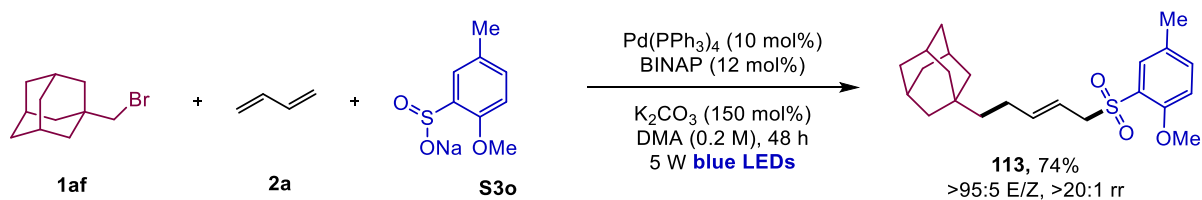

((E)-5-((2-methoxy-5-methylphenyl)sulfonyl)pent-3-en-1-yl)adamantane

(113).

According to the general procedure $\mathrm{J}$, using the bromide 1 af $(0.3 \mathrm{mmol}, 1.5$ equiv. $)$, butadiene 2a (0.3 mmol, $0.150 \mathrm{~mL}, 2 \mathrm{M}$ in THF, 1.5 equiv.), sulfinate $\mathbf{S 3 o}(0.2 \mathrm{mmol}$, 1.0 equiv.), $\mathrm{Pd}\left(\mathrm{PPh}_{3}\right)_{4}(0.020 \mathrm{mmol}, 10 \mathrm{~mol} \%)$, BINAP (0.024 mmol, $\left.12 \mathrm{~mol} \%\right)$ and stirring at room temperature for $48 \mathrm{~h}$ under $5 \mathrm{~W}$ blue LEDs irradiation. The reaction mixture was filtered through a pad of silica gel and concentrated in vacuo, then purified by chromatography (pentane/EtOAc $=5 / 1 \rightarrow 4 / 1)$ to give $113(57.5 \mathrm{mg}$, $0.148 \mathrm{mmol}, 74 \%,>95: 5 \mathrm{E} / \mathrm{Z},>20: 1 \mathrm{rr}$ ) as a pale yellow gum. $\mathbf{R}_{f}$ (pentane/EtOAc $=$ 4/1) 0.35. ${ }^{1} \mathrm{H}$ NMR (400 MHz, $\mathbf{C D C l}_{3}$ ): $\delta$ ppm $7.66(\mathrm{~d}, J=2.3 \mathrm{~Hz}, 1 \mathrm{H}), 7.34(\mathrm{dd}, J=$ 8.5, $2.3 \mathrm{~Hz}, 1 \mathrm{H}), 6.91(\mathrm{~d}, J=8.4 \mathrm{~Hz}, 1 \mathrm{H}), 5.53(\mathrm{dt}, J=15.1,6.8 \mathrm{~Hz}, 1 \mathrm{H}), 5.33$ (ddt, $J$ = 15.3, 7.6, $1.3 \mathrm{~Hz}, 1 \mathrm{H}), 3.97(\mathrm{~d}, J=7.5 \mathrm{~Hz}, 2 \mathrm{H}), 3.94(\mathrm{~s}, 3 \mathrm{H}), 2.31(\mathrm{~s}, 3 \mathrm{H}), 1.94-$ $1.80(\mathrm{~m}, 5 \mathrm{H}), 1.71-1.61(\mathrm{~m}, 4 \mathrm{H}), 1.61-1.51(\mathrm{~m}, 3 \mathrm{H}), 1.35$ (d, J = 2.9 Hz, 6H), 0.88 - $0.79(\mathrm{~m}, 2 \mathrm{H}) .{ }^{\mathbf{1 3}} \mathbf{C}$ NMR (101 MHz, $\left.\mathbf{C D C l}_{3}\right): \delta$ ppm 155.2, 142.4, 135.9, 131.2, $130.4,126.0,115.7,112.2,58.1,56.4,43.7,42.4,37.2,32.2,28.7,26.0,20.4$. HRMS (ESI $\left.{ }^{+}\right)$: calcd. for $\mathrm{C}_{23} \mathrm{H}_{32} \mathrm{O}_{3} \mathrm{SNa}\left(\mathrm{M}+\mathrm{Na}^{+}\right)$: 411.1964. Found: 411.1971 .
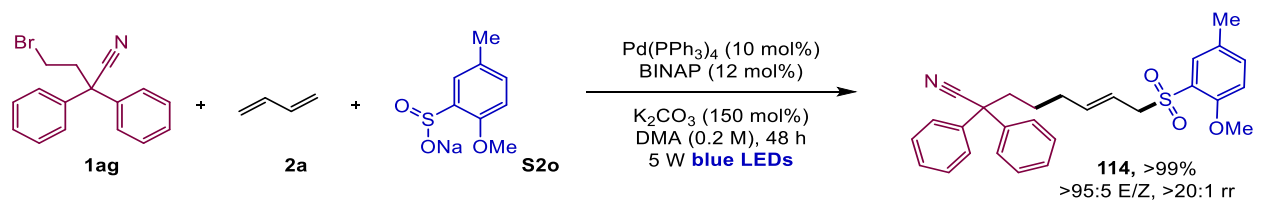

(E)-8-((2-methoxy-5-methylphenyl)sulfonyl)-2,2-diphenyloct-6-enenitrile

(114): According to the general procedure J, using the bromide $\mathbf{1 a g}(0.3 \mathrm{mmol}$, 
1.5 equiv.), butadiene $2 \mathrm{a}(0.3 \mathrm{mmol}, 0.150 \mathrm{~mL}, 2 \mathrm{M}$ in THF, 1.5 equiv.), sulfinate S2o (0.2 mmol, 1.0 equiv.), $\mathrm{Pd}\left(\mathrm{PPh}_{3}\right)_{4}(0.020 \mathrm{mmol}, 10 \mathrm{~mol} \%)$, BINAP (0.024 mmol, $12 \mathrm{~mol} \%$ ) and stirring at room temperature for $48 \mathrm{~h}$ under $5 \mathrm{~W}$ blue LEDs irradiation. The reaction mixture was filtered through a pad of silica gel and concentrated in vacuo, then purified by chromatography (pentane $/$ EtOAc $=3 / 1$ $\rightarrow 5 / 2$ ) to give 114 (91.7 $\mathrm{mg}, 0.200 \mathrm{mmol},>99 \%,>95: 5 E / Z,>20: 1 \mathrm{rr}$ ) as a pale yellow gum. $\mathbf{R}_{\boldsymbol{f}}$ (pentane/EtOAc $\left.=3 / 1\right) 0.20 .{ }^{1} \mathrm{H}$ NMR (400 $\left.\mathbf{M H z}, \mathbf{C D C l}_{3}\right): \delta \mathrm{ppm}$ $7.67(\mathrm{~d}, J=2.3 \mathrm{~Hz}, 1 \mathrm{H}), 7.42-7.34(\mathrm{~m}, 8 \mathrm{H}), 7.34-7.27(\mathrm{~m}, 3 \mathrm{H}), 6.91(\mathrm{~d}, J=8.4 \mathrm{~Hz}$, 1H), $5.59(\mathrm{dt}, J=15.2,6.8 \mathrm{~Hz}, 1 \mathrm{H}), 5.42(\mathrm{ddt}, J=15.3,7.4,1.3 \mathrm{~Hz}, 1 \mathrm{H}), 4.03(\mathrm{~d}, J=$ $7.3 \mathrm{~Hz}, 2 \mathrm{H}), 3.95(\mathrm{~s}, 3 \mathrm{H}), 2.33-2.19(\mathrm{~m}, 5 \mathrm{H}), 2.05(\mathrm{q}, J=7.0 \mathrm{~Hz}, 2 \mathrm{H}), 1.39$ (dtd, $J=$ 14.8, 7.4, 4.2 Hz, 2H). ${ }^{13} \mathbf{C}$ NMR (101 MHz, $\left.\mathbf{C D C l}_{3}\right): \delta$ ppm 155.0, 140.1, 139.8, 136.0, 130.8, 130.4, 128.9, 127.9, 126.8, 126.0, 122.4, 117.4, 112.2, 58.1, 56.4, 51.7, 38.7, 32.2, 24.8, 20.3. HRMS (ESI+): calcd. for $\mathrm{C}_{28} \mathrm{H}_{29} \mathrm{NO}_{3} \mathrm{SNa}\left(\mathrm{M}+\mathrm{Na}^{+}\right)$: 482.1760. Found: 482.1756.

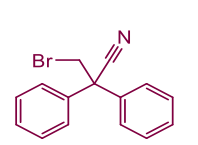

1 ah

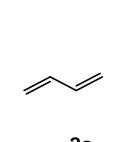

$2 \mathrm{a}$

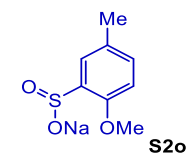

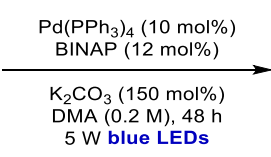

$5 \mathrm{~W}$ blue LEDs

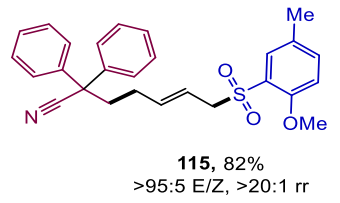

(E)-7-((2-methoxy-5-methylphenyl)sulfonyl)-2,2-diphenylhept-5-

enenitrile (115): According to the general procedure J, using the bromide 1ah ( $0.3 \mathrm{mmol}, 1.5$ equiv.), butadiene $2 \mathrm{a}(0.3 \mathrm{mmol}, 0.150 \mathrm{~mL}, 2 \mathrm{M}$ in THF, 1.5 equiv.), sulfinate S2o (0.2 mmol, 1.0 equiv.), $\mathrm{Pd}\left(\mathrm{PPh}_{3}\right)_{4}(0.020 \mathrm{mmol}, 10 \mathrm{~mol} \%)$, BINAP $(0.024 \mathrm{mmol}, 12 \mathrm{~mol} \%)$ and stirring at room temperature for $48 \mathrm{~h}$ under $5 \mathrm{~W}$ blue LEDs irradiation. The reaction mixture was filtered through a pad of silica gel and concentrated in vacuo, then purified by chromatography (pentane/EtOAc $=3 / 1 \rightarrow 5 / 2)$ to give 115 (73.1 mg, 0.164 mmol, 82\%, >95:5 E/Z, > 20:1 rr) as a pale yellow gum. $\mathbf{R}_{\boldsymbol{f}}$ (pentane/EtOAc $\left.=3 / 1\right)$ 0.20. ${ }^{1} \mathbf{H} \mathbf{~ N M R}\left(400 \mathbf{M H z}, \mathbf{C D C l}_{3}\right): \delta$ ppm $7.68(\mathrm{~d}, J=2.2 \mathrm{~Hz}, 1 \mathrm{H}), 7.37-7.27(\mathrm{~m}, 11 \mathrm{H}), 6.93$ (d, $J=8.4 \mathrm{~Hz}, 1 \mathrm{H}), 5.61(\mathrm{dt}$, $J=15.3,6.5 \mathrm{~Hz}, 1 \mathrm{H}), 5.41$ (ddd, $J=15.1,8.1,6.7 \mathrm{~Hz}, 1 \mathrm{H}), 3.99$ (d, $J=7.4 \mathrm{~Hz}, 2 \mathrm{H}$ ), $3.95(\mathrm{~s}, 3 \mathrm{H}), 2.28(\mathrm{~s}, 3 \mathrm{H}), 2.22-2.15(\mathrm{~m}, 2 \mathrm{H}), 2.13-2.02(\mathrm{~m}, 2 \mathrm{H}) \cdot{ }^{13} \mathbf{C}$ NMR (101 MHz, $\mathbf{C D C l}_{3}$ ): $\delta$ ppm 155.2, 139.7, 138.7, 136.0, 131.0, 130.5, 129.0, 128.1, 126.8, 
125.9, 122.1, 118.0, 112.3, 57.9, 56.5, 51.4, 38.8, 28.8, 20.3. HRMS (ESI+): calcd. for $\mathrm{C}_{27} \mathrm{H}_{27} \mathrm{NO}_{3} \mathrm{SNa}\left(\mathrm{M}+\mathrm{Na}^{+}\right)$: 468.1604. Found: 468.1599 .

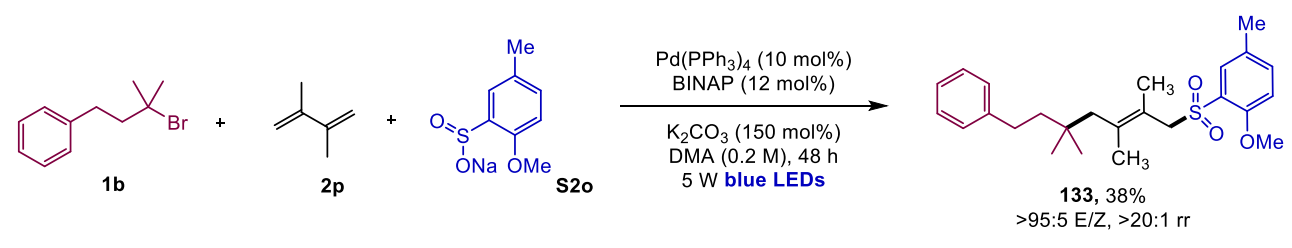

(E)-1-methoxy-4-methyl-2-((2,3,5,5-tetramethyl-7-phenylhept-2-en-1-

yl)sulfonyl)benzene (133): According to the general procedure J, using the bromide $1 \mathbf{b}$ (0.3 mmol, 1.5 equiv.), 2,3-dimethylbutadiene 2p (0.3 mmol, 1.5 equiv.), sulfinate S2o (0.2 mmol, 1.0 equiv.), $\mathrm{Pd}\left(\mathrm{PPh}_{3}\right)_{4}(0.020 \mathrm{mmol}, 10 \mathrm{~mol} \%$ ), BINAP (0.024 mmol, $12 \mathrm{~mol} \%)$ and stirring at room temperature for $48 \mathrm{~h}$ under 5W blue LEDs irradiation. The reaction mixture was filtered through a pad of silica gel and concentrated in vacuo, then purified by chromatography (pentane/EtOAc $=6 / 1 \rightarrow 5 / 1$ ) to give 133 (31.5 mg, $0.076 \mathrm{mmol}, 38 \%,>95: 5$ $E / Z,>20: 1 \mathrm{rr}$ ) as a pale yellow gum. $\mathbf{R}_{\boldsymbol{f}}$ (pentane $/$ EtOAc $=4 / 1$ ) 0.32. The configuration of the double bond was assigned as $E$ using 1D-NOESY. ${ }^{1} \mathbf{H}$ NMR (500 $\mathbf{M H z}, \mathbf{C D C l}_{3}$ ): $\delta$ ppm $7.73(\mathrm{dd}, J=2.3,0.7 \mathrm{~Hz}, 1 \mathrm{H}$ ), $7.33(\mathrm{dd}, J=8.5,0.6 \mathrm{~Hz}$, 1H), $7.31-7.24(\mathrm{~m}, 2 \mathrm{H}), 7.21-7.15(\mathrm{~m}, 3 \mathrm{H}), 6.91$ (d, J = 8.4 Hz, 1H), 4.19 (s, 2H), $3.97(\mathrm{~s}, 3 \mathrm{H}), 2.61-2.54(\mathrm{~m}, 2 \mathrm{H}), 2.30(\mathrm{~s}, 3 \mathrm{H}), 2.12(\mathrm{~s}, 2 \mathrm{H}), 1.83(\mathrm{q}, J=1.4 \mathrm{~Hz}, 3 \mathrm{H})$, $1.69(\mathrm{q}, J=1.5 \mathrm{~Hz}, 3 \mathrm{H}), 1.51-1.46(\mathrm{~m}, 2 \mathrm{H}), 0.87$ (s, 6H). ${ }^{13} \mathrm{C}$ NMR (126 MHz, $\mathbf{C D C l}_{3}$ ): $\delta$ ppm 155.5, 143.3, 138.1, 135.9, 131.4, 130.4, 128.43, 128.39, 127.7, 125.7, 120.1, 112.4, 60.3, 56.5, 46.8, 46.1, 36.4, 30.9, 27.4, 22.3, 21.2, 20.3. HRMS (ESI'): calcd. for $\mathrm{C}_{25} \mathrm{H}_{34} \mathrm{O}_{3} \mathrm{SNa}\left(\mathrm{M}+\mathrm{Na}^{+}\right)$: 437.2121. Found: 437.2116.
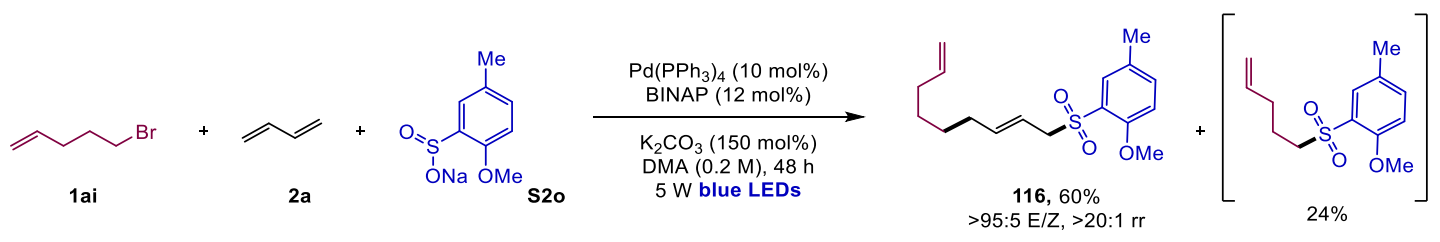

(E)-1-methoxy-4-methyl-2-(nona-2,8-dien-1-ylsulfonyl)benzene

(116):

According to the general procedure J, using the bromide 1ai $(0.45 \mathrm{mmol}, 1.5$ equiv.), butadiene 2a ( $0.45 \mathrm{mmol}, 0.225 \mathrm{~mL}, 2 \mathrm{M}$ in THF, 1.5 equiv.), sulfinate 
S2o (0.3 mmol, 1.0 equiv.), $\mathrm{Pd}\left(\mathrm{PPh}_{3}\right)_{4}(0.030 \mathrm{mmol}, 10 \mathrm{~mol} \%)$, BINAP (0.036 mmol, 12 mol\%) and stirring at room temperature for $48 \mathrm{~h}$ under $5 \mathrm{~W}$ blue LEDs irradiation. The reaction mixture was filtered through a pad of silica gel and concentrated in vacuo, then purified by chromatography (pentane/EtOAc $=6 / 1$ $\rightarrow 5 / 1$ ) to afford two fractions: 1 - pure product (41.2 mg, $0.130 \mathrm{mmol}$ ), 2 - 1:1 product:side product (26.9 mg, $0.048 \mathrm{mmol}$ of product), globally giving 116 (55.9 mg, $0.181 \mathrm{mmol}, 60 \%,>95: 5 \mathrm{E} / Z$, > 20:1 rr) as a pale yellow gum. $\mathbf{R}_{\boldsymbol{f}}$ (pentane $/$ EtOAc $=5 / 1)$ 0.35. ${ }^{1} \mathbf{H}$ NMR (400 $\mathbf{M H z} \mathbf{C D C l}_{3}$, product): $\delta$ ppm $7.67(\mathrm{~d}$, $J=2.3 \mathrm{~Hz}, 1 \mathrm{H}), 7.34(\mathrm{dd}, J=8.5,2.3 \mathrm{~Hz}, 1 \mathrm{H}), 6.91(\mathrm{~d}, J=8.4 \mathrm{~Hz}, 1 \mathrm{H}), 5.72(\mathrm{ddt}, J=$ 17.0, 10.2, $6.7 \mathrm{~Hz}, 1 \mathrm{H}), 5.55(\mathrm{dtt}, J=15.3,6.9,1.2 \mathrm{~Hz}, 1 \mathrm{H}), 5.35(\mathrm{dtt}, J=15.4,7.5$, $1.4 \mathrm{~Hz}, 1 \mathrm{H}), 4.98-4.88(\mathrm{~m}, 2 \mathrm{H}), 3.99$ (dd, $J=7.4,1.1 \mathrm{~Hz}, 2 \mathrm{H}), 3.94(\mathrm{~s}, 3 \mathrm{H}), 2.31$ (s, 3H), $1.98-1.89(\mathrm{~m}, 4 \mathrm{H}), 1.21-1.14(\mathrm{~m}, 4 \mathrm{H}) .{ }^{13} \mathrm{C}$ NMR (101 MHz, CDCl $_{3}$, product): $\delta$ ppm 155.2, 141.1, 138.8, 135.9, 131.1, 130.4, 126.0, 116.5, 114.5,

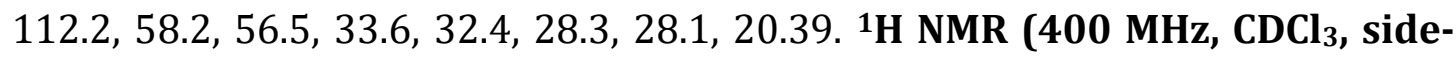
product): $\delta \operatorname{ppm} \delta 7.74(\mathrm{~d}, J=2.3 \mathrm{~Hz}, 1 \mathrm{H}), 7.36(\mathrm{dd}, J=8.5,2.3 \mathrm{~Hz}, 1 \mathrm{H}), 6.92(\mathrm{~d}, J$ $=8.3 \mathrm{~Hz}, 2 \mathrm{H}), 5.03-4.98(\mathrm{~m}, 2 \mathrm{H}), 3.93(\mathrm{~s}, 3 \mathrm{H}), 3.37-3.29(\mathrm{~m}, 2 \mathrm{H}), 2.34(\mathrm{~s}, 3 \mathrm{H})$, 2.13 (qt, $J=7.1,1.4 \mathrm{~Hz}, 2 \mathrm{H}$ ), $1.83-1.74$ (m, 2H). (One olefinic proton overlaps with the product). ${ }^{13} \mathbf{C}$ NMR (101 $\mathbf{~ M H z , ~} \mathbf{C D C l}_{3}$, side-product): $\delta$ ppm 155.3, 136.7, 136.0, 130.7, 130.6, 126.6, 116.3, 112.4, 53.7, 32.2, 31.5, 21.8, 20.42. HRMS (ESI+, product): calcd. for $\mathrm{C}_{17} \mathrm{H}_{24} \mathrm{O}_{3} \mathrm{SNa}\left(\mathrm{M}+\mathrm{Na}^{+}\right)$: 331.1346. Found: 331.1338. HRMS (ESI+, side-product): calcd. for $\mathrm{C}_{13} \mathrm{H}_{18} \mathrm{O}_{3} \mathrm{SNa}\left(\mathrm{M}+\mathrm{Na}^{+}\right)$: 277.0869. Found: 277.0871.
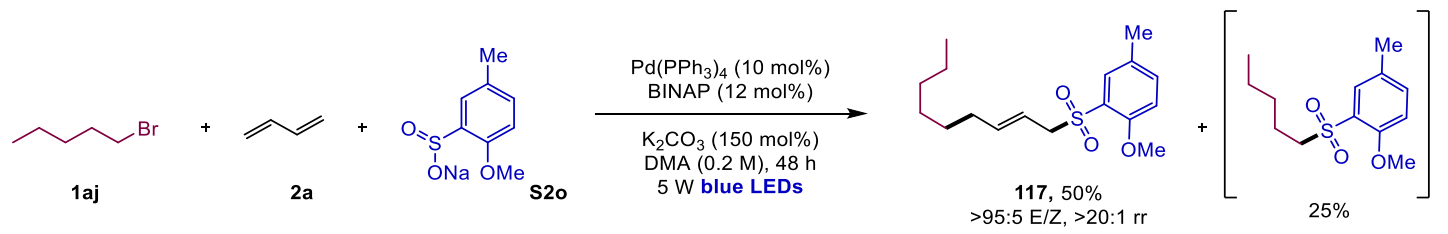

(E)-1-methoxy-4-methyl-2-(non-2-en-1-ylsulfonyl)benzene

(117):

According to the general procedure J, using the bromide 1aj (0.45 mmol, 1.5 equiv.), butadiene $2 \mathrm{a}(0.45 \mathrm{mmol}, 0.225 \mathrm{~mL}, 2 \mathrm{M}$ in THF, 1.5 equiv.), sulfinate S2o (0.3 mmol, 1.0 equiv.), $\mathrm{Pd}\left(\mathrm{PPh}_{3}\right)_{4}$ (0.030 mmol, $\left.10 \mathrm{~mol} \%\right)$, BINAP (0.036 
mmol, $12 \mathrm{~mol} \%$ ) and stirring at room temperature for $48 \mathrm{~h}$ under $5 \mathrm{~W}$ blue LEDs irradiation. The reaction mixture was filtered through a pad of silica gel and concentrated in vacuo, then purified by chromatography (pentane/EtOAc $=6 / 1$ $\rightarrow 5 / 1)$ to afford a inseparable mixture $(66.3 \mathrm{mg}$, ratio product:byproduct $2: 1)$ of product 117 (46.9 mg, $0.151 \mathrm{mmol}, 50 \%,>95: 5 E / Z,>20: 1 \mathrm{rr}$ ) and side-product (19.4 mg, $0.076 \mathrm{mmol}, 25 \%$ ) as a pale yellow gum. $\mathbf{R}_{\boldsymbol{f}}$ (pentane/EtOAc $=5 / 1$ ) 0.35. ${ }^{1} \mathrm{H}$ NMR (599 $\mathbf{M H z}, \mathbf{C D C l}_{3}$, product): $\delta$ ppm $7.66(\mathrm{~d}, J=2.2 \mathrm{~Hz}, 1 \mathrm{H}), 7.34-$ $7.31\left(\mathrm{~m}, 1 \mathrm{H},{ }^{*}\right), 6.90\left(\mathrm{~d}, J=8.5 \mathrm{~Hz}, 1 \mathrm{H},{ }^{*}\right), 5.55(\mathrm{dtt}, J=15.3,6.9,1.0 \mathrm{~Hz}, 1 \mathrm{H}), 5.33$ (dt, $J=15.3,7.4 \mathrm{~Hz}, 1 \mathrm{H}), 3.98(\mathrm{~d}, J=7.5 \mathrm{~Hz}, 2 \mathrm{H}), 3.93$ (s, 3H), 2.30 (s, 3H), 1.91 (q, $J=6.8 \mathrm{~Hz}, 2 \mathrm{H}), 1.24-1.06(\mathrm{~m}, 8 \mathrm{H}), 0.84\left(\mathrm{t}, J=7.2 \mathrm{~Hz}, 3 \mathrm{H},{ }^{*}\right) .{ }^{13} \mathbf{C}$ NMR (151 MHz, $\mathrm{CDCl}_{3}$, product): $\delta$ ppm 155.1, 141.3, 135.8, 131.0, 130.4, 126.0, 116.3, 112.2, 58.2, 56.4 (overlaps with byproduct), 32.5, 31.7, 28.8, 28.5, 22.6, 20.3, 14.1. ${ }^{1} \mathbf{H}$ NMR (599 MHz, $\mathbf{C D C l}_{3}$, byproduct): $\delta$ ppm 7.72 (d, J = $\left.2.2 \mathrm{~Hz}, 1 \mathrm{H}\right), 7.36-7.33$ $\left(\mathrm{m}, 1 \mathrm{H},{ }^{*}\right), 6.91\left(\mathrm{~d}, J=8.6 \mathrm{~Hz}, 1 \mathrm{H},{ }^{*}\right), 3.92(\mathrm{~s}, 3 \mathrm{H}), 3.33-3.28(\mathrm{~m}, 2 \mathrm{H}), 2.32(\mathrm{~s}, 3 \mathrm{H})$, $1.69-1.62(\mathrm{~m}, 2 \mathrm{H}), 1.36-1.30(\mathrm{~m}, 2 \mathrm{H}), 1.30-1.25(\mathrm{~m}, 2 \mathrm{H}), 0.84(\mathrm{t}, J=7.3 \mathrm{~Hz}, 3 \mathrm{H}$, *). ${ }^{13}$ C NMR (151 MHz, $\mathbf{C D C l}_{3}$, byproduct): $\delta$ ppm 155.3, 135.9, 130.6, 130.5, 126.6, 112.4, 56.4 (overlaps with product), 54.4, 30.5, 22.2, 22.1, 20.4, 13.8. *Signals denoted with asterisk show overlap between product and byproduct. HRMS (ESI ${ }^{+}$, product): calcd. for $\mathrm{C}_{17} \mathrm{H}_{26} \mathrm{O}_{3} \mathrm{SNa}\left(\mathrm{M}+\mathrm{Na}^{+}\right)$: 333.1503. Found: 333.1495. HRMS (ESI+, byproduct): calcd. for $\mathrm{C}_{13} \mathrm{H}_{20} \mathrm{O}_{3} \mathrm{SNa}\left(\mathrm{M}+\mathrm{Na}^{+}\right): 279.1039$. Found: 279.1026.

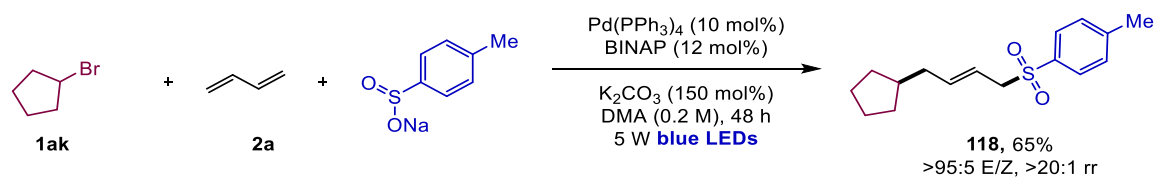

(E)-1-((4-cyclopentylbut-2-en-1-yl)sulfonyl)-4-methylbenzene

(118):

According to the general procedure J, using the bromide 1ak $(0.3 \mathrm{mmol}, 1.5$ equiv.), butadiene 2a ( $0.3 \mathrm{mmol}, 0.150 \mathrm{~mL}, 2 \mathrm{M}$ in THF, 1.5 equiv.), sodium 4methylbenzenesulfinate (0.2 mmol, 1.0 equiv.), $\mathrm{Pd}\left(\mathrm{PPh}_{3}\right)_{4}(0.020 \mathrm{mmol}, 10$ mol\%), BINAP (0.024 mmol, $12 \mathrm{~mol} \%$ ) and stirring at room temperature for $48 \mathrm{~h}$ 
under $5 \mathrm{~W}$ blue LEDs irradiation. The reaction mixture was filtered through a pad of silica gel and concentrated in vacuo, then purified by chromatography (pentane/EtOAc $=18 / 1 \rightarrow 12 / 1)$ to give 118 (36.2 mg, 0.130 mmol, 65\%, >95:5 $E / Z,>20: 1 \mathrm{rr}$ ) as a colorless gum. $\mathbf{R}_{\boldsymbol{f}}$ (pentane/EtOAc $\left.=15 / 1\right)$ 0.35. ${ }^{\mathbf{1}} \mathbf{H}$ NMR (400 MHz, $\mathbf{C D C l}_{3}$ ): $\delta$ ppm $7.72(\mathrm{~d}, J=8.3 \mathrm{~Hz}, 2 \mathrm{H}), 7.32(\mathrm{~d}, J=8.0 \mathrm{~Hz}, 2 \mathrm{H}), 5.49(\mathrm{dt}, J=$ 15.2, $6.9 \mathrm{~Hz}, 1 \mathrm{H}$ ), 5.36 (dtt, $J=15.3,7.1,1.2 \mathrm{~Hz}, 1 \mathrm{H}$ ), 3.72 (d, $J=7.2 \mathrm{~Hz}, 2 \mathrm{H}$ ), 2.43 (s, 3H), 1.98 (t, J = 7.0 Hz, 2H), $1.77-1.65(\mathrm{~m}, 1 \mathrm{H}), 1.63-1.40(\mathrm{~m}, 6 \mathrm{H}), 1.05-$ $0.92(\mathrm{~m}, 2 \mathrm{H}) .{ }^{13} \mathbf{C}$ NMR (101 MHz, $\left.\mathbf{C D C l}_{3}\right): \delta$ ppm 144.6, 141.1, 135.6, 129.7, 128.6, 116.5, 60.4, 39.5, 38.9, 32.3, 25.1, 21.7. HRMS (ESI ${ }^{+}$): calcd. for $\mathrm{C}_{16} \mathrm{H}_{22} \mathrm{O}_{2} \mathrm{SNa}\left(\mathrm{M}+\mathrm{Na}^{+}\right): 301.1233$. Found: 301.1234 .

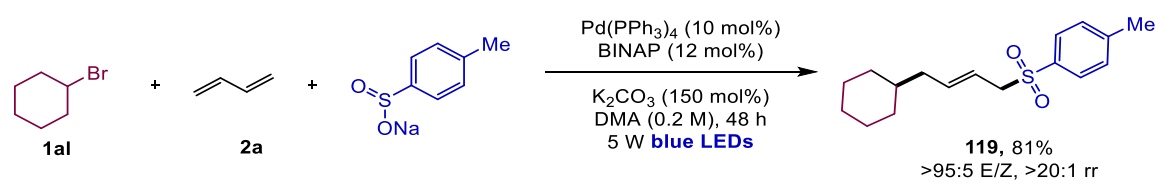

(E)-1-((4-cyclohexylbut-2-en-1-yl)sulfonyl)-4-methylbenzene

(119):

According to the general procedure J, using the bromide 1al (0.3 mmol, 1.5 equiv.), butadiene $2 \mathrm{a}(0.3 \mathrm{mmol}, 0.150 \mathrm{~mL}, 2 \mathrm{M}$ in THF, 1.5 equiv.), sodium 4methylbenzenesulfinate $(0.2 \mathrm{mmol}, 1.0$ equiv. $), \mathrm{Pd}\left(\mathrm{PPh}_{3}\right)_{4}(0.020 \mathrm{mmol}, 10$ mol\%), BINAP (0.024 mmol, $12 \mathrm{~mol} \%$ ) and stirring at room temperature for $48 \mathrm{~h}$ under $5 \mathrm{~W}$ blue LEDs irradiation. The reaction mixture was filtered through a pad of silica gel and concentrated in vacuo, then purified by chromatography $($ pentane/EtOAc $=18 / 1 \rightarrow 12 / 1)$ to give 119 (47.0 mg, 0.161 mmol, 81\%, >95:5 $E / Z,>20: 1 \mathrm{rr}$ ) as a colorless gum. $\mathbf{R}_{\boldsymbol{f}}$ (pentane/EtOAc $\left.=15 / 1\right)$ 0.35. ${ }^{\mathbf{1}} \mathbf{H} \mathbf{N M R}(\mathbf{4 0 0}$ MHz, $\left.\mathbf{C D C l}_{3}\right): \delta$ ppm $7.71(\mathrm{~d}, J=8.3 \mathrm{~Hz}, 2 \mathrm{H}), 7.31(\mathrm{~d}, J=8.0 \mathrm{~Hz}, 2 \mathrm{H}), 5.47(\mathrm{dt}, J=$ 15.3, 7.1 Hz, 1H), 5.34 (dtt, $J=15.3,7.1,0.8 \mathrm{~Hz}, 1 \mathrm{H}$ ), 3.72 (d, $J=7.2 \mathrm{~Hz}, 2 \mathrm{H}$ ), 2.42 (s, 3H), $1.86(\mathrm{t}, J=6.9 \mathrm{~Hz}, 2 \mathrm{H}), 1.69-1.56(\mathrm{~m}, 3 \mathrm{H}), 1.53-1.43(\mathrm{~m}, 2 \mathrm{H}), 1.22-$ $1.01(\mathrm{~m}, 4 \mathrm{H}), 0.75(\mathrm{qd}, J=11.8,3.4 \mathrm{~Hz}, 2 \mathrm{H}) .{ }^{13} \mathbf{C}$ NMR (101 $\left.\mathbf{M H z}, \mathbf{C D C l}_{3}\right): \delta$ ppm 144.6, 140.4, 135.5, 129.7, 128.6, 117.1, 60.3, 40.5, 37.7, 33.0, 26.5, 26.3, 21.7. HRMS (ESI+): calcd. for $\mathrm{C}_{17} \mathrm{H}_{24} \mathrm{O}_{2} \mathrm{SNa}\left(\mathrm{M}+\mathrm{Na}^{+}\right)$: 315.1389. Found: 315.1391. 


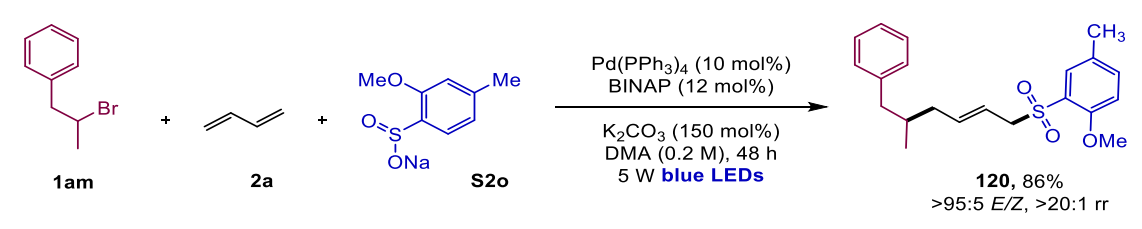

(E)-1-methoxy-4-methyl-2-((5-methyl-6-phenylhex-2-en-1-

yl)sulfonyl)benzene (120): According to the general procedure J, using the bromide 1 am (0.3 mmol, 1.5 equiv.), butadiene 2 a $(0.3 \mathrm{mmol}, 0.150 \mathrm{~mL}, 2 \mathrm{M}$ in THF, 1.5 equiv.), S2o (0.2 mmol, 1.0 equiv.), $\mathrm{Pd}\left(\mathrm{PPh}_{3}\right)_{4}(0.020 \mathrm{mmol}, 10 \mathrm{~mol} \%$ ), BINAP (0.024 mmol, $12 \mathrm{~mol} \%)$ and stirring at room temperature for $48 \mathrm{~h}$ under 5W blue LEDs irradiation. The reaction mixture was filtered through a pad of silica gel and concentrated in vacuo, then purified by chromatography (pentane/EtOAc $=4 / 1)$ to give $120(61.3 \mathrm{mg}, 0.172 \mathrm{mmol}, 86 \%,>95: 5 E / Z$, > 20:1 rr) as a colorless gum. $\mathbf{R}_{\boldsymbol{f}}$ (pentane/EtOAc $\left.=4 / 1\right)$ 0.25. ${ }^{1} \mathbf{H}$ NMR (400 $\mathbf{~ M H z}$, $\mathbf{C D C l}_{3}$ ): $\delta$ ppm $7.69(\mathrm{~d}, J=2.3 \mathrm{~Hz}, 1 \mathrm{H}), 7.30(\mathrm{dd}, J=8.4,2.3 \mathrm{~Hz}, 1 \mathrm{H}), 7.28-7.22(\mathrm{~m}$, 2H), $7.20-7.14(\mathrm{~m}, 1 \mathrm{H}), 7.06-7.00(\mathrm{~m}, 2 \mathrm{H}), 6.92(\mathrm{~d}, J=8.4 \mathrm{~Hz}, 1 \mathrm{H}), 5.58(\mathrm{dt}, J=$ 14.8, 7.2 Hz, 1H), 5.39 (dtt, $J=15.4,7.4,1.4 \mathrm{~Hz}, 1 \mathrm{H}$ ), 4.05 (d, $J=7.4 \mathrm{~Hz}, 2 \mathrm{H}$ ), 3.97 (s, 3H), $2.36(\mathrm{dd}, J=13.4,6.3 \mathrm{~Hz}, 1 \mathrm{H}), 2.24(\mathrm{~s}, 3 \mathrm{H}), 2.18(\mathrm{dd}, J=13.5,8.0 \mathrm{~Hz}, 1 \mathrm{H}$ ), $2.04-1.95(\mathrm{~m}, 1 \mathrm{H}), 1.89-1.79(\mathrm{~m}, 1 \mathrm{H}), 1.69-1.58(\mathrm{~m}, 1 \mathrm{H}), 0.65$ (d, J = 6.7 Hz, 3H). ${ }^{13}$ C NMR (101 MHz, $\mathbf{C D C l}_{3}$ ): $\delta$ ppm 155.1, 141.0, 139.4, 136.0, 131.2, 130.6, 129.2, 128.2, 125.90, 125.86, 118.2, 112.2, 58.2, 56.5, 42.7, 39.4, 34.9, 20.3, 19.0. HRMS (ESI+): calcd. for $\mathrm{C}_{21} \mathrm{H}_{26} \mathrm{O}_{3} \mathrm{SNa}\left(\mathrm{M}+\mathrm{Na}^{+}\right)$: 381.1500 . Found: 381.1493 .

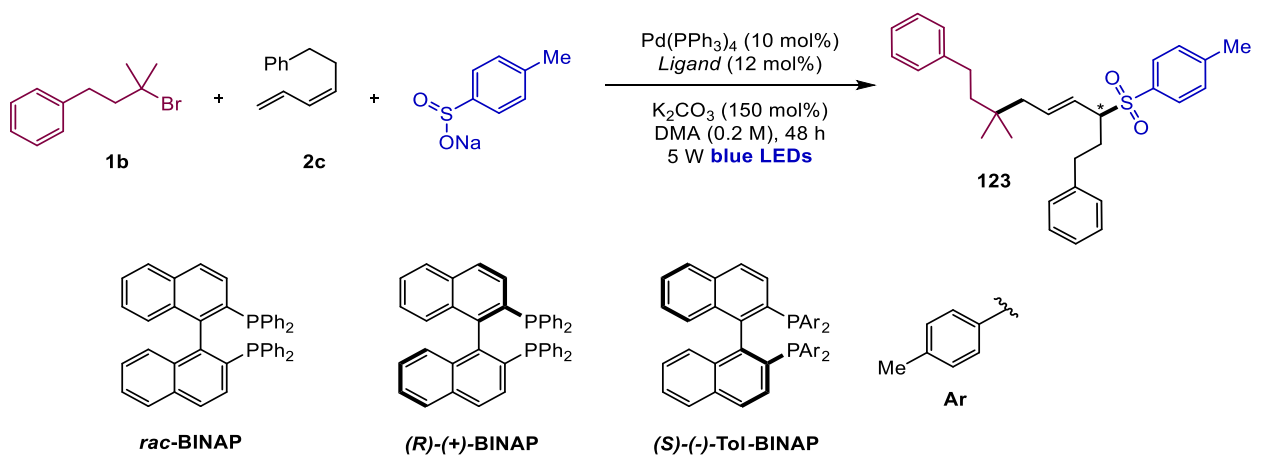

(7,7-dimethyl-3-tosylnon-4-ene-1,9-diyl)dibenzene (123). According to the general procedure xx, using the bromide $\mathbf{1 b}(0.3 \mathrm{mmol}, 1.5$ equiv. $)$, butadiene $\mathbf{2 c}(0.3 \mathrm{mmol}$, 1.5 equiv.), sodium 4-methylbenzenesulfinate ( $0.2 \mathrm{mmol}, 1.0$ equiv.), $\mathrm{Pd}\left(\mathrm{PPh}_{3}\right)_{4}$ 
$(0.020 \mathrm{mmol}, 10 \mathrm{~mol} \%)$, ligand $(0.024 \mathrm{mmol}, 12 \mathrm{~mol} \%)$ and stirring at room temperature for $48 \mathrm{~h}$ under $5 \mathrm{~W}$ blue LEDs irradiation. The reaction mixture was filtered through a pad of silica gel and concentrated in vacuo, then purified by chromatography (pentane/EtOAc $=13 / 1$ ) to give $\mathbf{1 2 3}$ as a colorless gum.

- With rac-BINAP: 123 (63.2 mg, 0.137 mmol, 69\%, >95:5 E/Z, > 20:1 rr);

- With (+)-BINAP: 123 (63.5 mg, 0.138 mmol, 69\%, >95:5 E/Z, > 20:1 rr);

- With (-)-Tol-BINAP: 123 (62.4 mg, $0.135 \mathrm{mmol}, 68 \%,>95: 5$ E/Z, > 20:1 rr). $\mathbf{R}_{f}($ pentane/EtOAc $=15 / 1)$ 0.30. ${ }^{1} \mathbf{H}$ NMR (400 $\left.\mathbf{M H z}, \mathbf{C D C l}_{3}\right): \delta$ ppm $7.64(\mathrm{~d}, J=$ $8.2 \mathrm{~Hz}, 2 \mathrm{H}), 7.29-7.20(\mathrm{~m}, 6 \mathrm{H}), 7.20-7.11(\mathrm{~m}, 4 \mathrm{H}), 7.11-7.05(\mathrm{~m}, 2 \mathrm{H}), 5.53(\mathrm{dt}$, $J=15.1,7.5 \mathrm{~Hz}, 1 \mathrm{H}$ ), 5.26 (dd, $J=15.3,9.3 \mathrm{~Hz}, 1 \mathrm{H}$ ), 3.47 (ddd, $J=11.6,9.2,3.0 \mathrm{~Hz}$, 1H), 2.75 (ddd, $J=13.3,8.9,4.8 \mathrm{~Hz}, 1 \mathrm{H}), 2.56-2.48(\mathrm{~m}, 3 \mathrm{H}), 2.48-2.39(\mathrm{~m}, 1 \mathrm{H})$, 2.37 (s, 3H), $2.06-1.95$ (m, 2H), $1.95-1.85(\mathrm{~m}, 1 \mathrm{H}), 1.46-1.38(\mathrm{~m}, 2 \mathrm{H}), 0.87$ (s, 6H). ${ }^{13}$ C NMR (101 MHz, $\mathbf{C D C l}_{3}$ ): $\delta$ ppm 144.5, 143.2, 140.3, 137.8, 134.8, 129.6, 129.2, 128.6, 128.5, 128.4, 126.4, 125.8, 124.2, 68.6, 45.3, 44.2, 33.8, 32.6, 30.8, 28.9, 27.0, 27.0, 21.7. (One aromatic carbon missing due to overlap). HRMS (ESI ${ }^{+}$): calcd. for $\mathrm{C}_{30} \mathrm{H}_{36} \mathrm{O}_{2} \mathrm{SNa}\left(\mathrm{M}+\mathrm{Na}^{+}\right)$: 483.2328. Found: 483.2327 .

Measurement of enantiomeric ratio.

Enantiomeric excess were obtained using HPLC were performed using an Agilent 1200 LC system equipped with a Daicel Chiralpak ${ }^{\circledR}$ AD-H column $(4.6$ x 250 mm, $5 \mu \mathrm{m}$ particle size), thermostated at $25^{\circ} \mathrm{C}$ using hexane: $\mathrm{PrOH} 95: 5$, flow rate: 1.0 $\mathrm{ml} / \mathrm{min}$, injection volume: $8 \mu \mathrm{L}$. HPLC trace at $230.4 \mathrm{~nm}$ detection was used. Results were analized using Agilent Chemstation Rev. B.02.01-SR2.

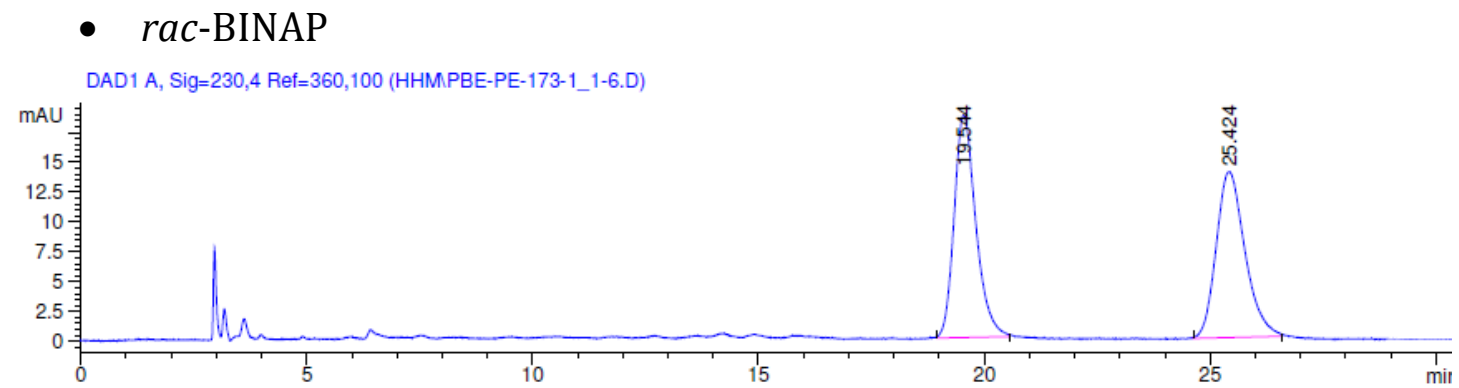


Area Percent Report

\begin{tabular}{|c|c|c|c|}
\hline Sorted By & Signal & & \\
\hline Multiplier & 1.0000 & & \\
\hline Dilution & 1.0000 & & \\
\hline Sample Amount & 1.00000 & {$[\mathrm{ng} / \mathrm{ul}]$} & (not used in calc.) \\
\hline
\end{tabular}

Signal 1: DAD1 A, Sig=230, 4 Ref $=360,100$

\begin{tabular}{|c|c|c|c|c|c|c|}
\hline $\begin{array}{c}\text { Peak } \\
\#\end{array}$ & $\begin{array}{c}\text { RetTime } \\
\text { [min] }\end{array}$ & Type & $\begin{array}{c}\text { Width } \\
\text { [min] }\end{array}$ & $\begin{array}{c}\text { Area } \\
{\left[\mathrm{mAU}^{\star} \mathrm{s}\right]}\end{array}$ & $\begin{array}{l}\text { Height } \\
\text { [mAU] }\end{array}$ & $\begin{array}{c}\text { Area } \\
\quad 8\end{array}$ \\
\hline 1 & $19 \quad 544$ & & 0.4782 & 623.35706 & 18,63641 & 50.3754 \\
\hline 2 & 25.424 & BB & 0.5860 & 614.06641 & 13.88449 & 49.6246 \\
\hline
\end{tabular}

$\begin{array}{lll}\text { Totals : } & 1237.42346 \quad 32.52089\end{array}$

\section{- (+)-BINAP - -7\% ee}

DAD1 A, Sig=230,4 Ref=360,100 (HHMPBE-PE-173-2.D)

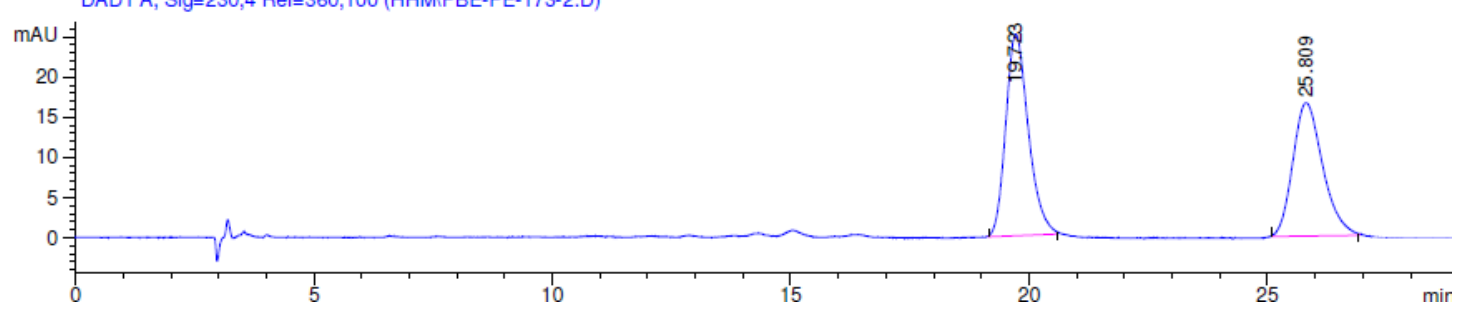

Area Percent Report

$\begin{array}{lccc}\text { Sorted By } & : & \text { Signal } & \\ \text { Multiplier } & : & 1.0000 & \\ \text { Dilution } & : & 1.0000 & \\ \text { Sample Amount } & : & 1.00000 \text { [ng/ul] } & \text { (not used in calc.) } \\ \text { Use Multiplier \& Dilution Factor with ISTDs }\end{array}$

Use Multiplier \& Dilution Factor with ISTDs

Signal 1: DAD1 A, Sig=230, 4 Ref=360, 100

\begin{tabular}{|c|c|c|c|c|c|c|}
\hline $\begin{array}{c}\text { Peak } \\
\#\end{array}$ & $\begin{array}{l}\text { RetTime } \\
\text { [min] }\end{array}$ & Type & $\begin{array}{c}\text { Width } \\
\text { [min] }\end{array}$ & $\begin{array}{c}\text { Area } \\
{\left[\mathrm{mAU}^{\star} \mathrm{s}\right]}\end{array}$ & $\begin{array}{l}\text { Height } \\
\text { [mAU] }\end{array}$ & $\begin{array}{c}\text { Area } \\
\frac{8}{8}\end{array}$ \\
\hline & & & ------ & ---------- & --------- & $--------\mid$ \\
\hline 1 & 19.723 & BB & 0.4463 & 829.38116 & 25.11300 & 53.4044 \\
\hline 2 & 25.809 & $\mathrm{BB}$ & 0.5156 & 723.63776 & 16.60009 & 46.5956 \\
\hline otal & s : & & & 1553.01892 & 41.71309 & \\
\hline
\end{tabular}

- (-)-Tol-BINAP $-12 \%$ ee 


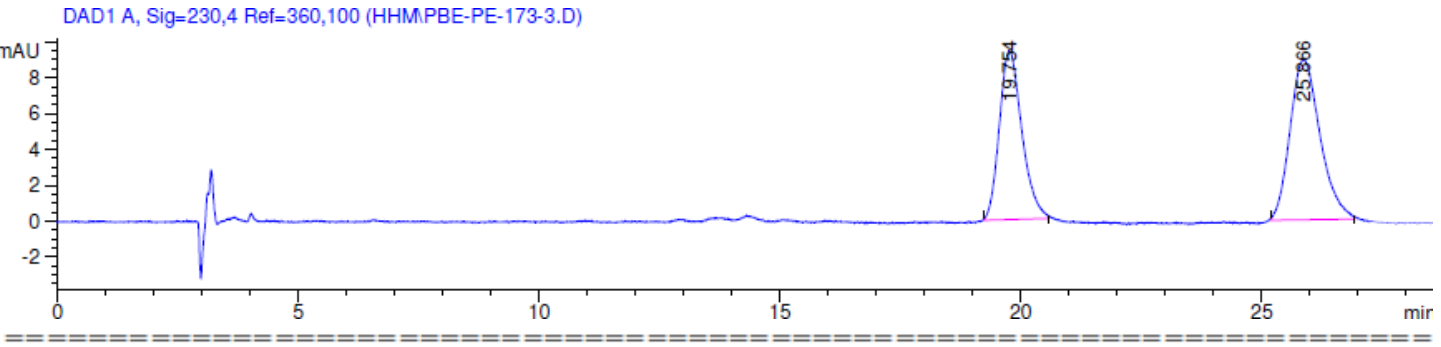

Area Percent Report

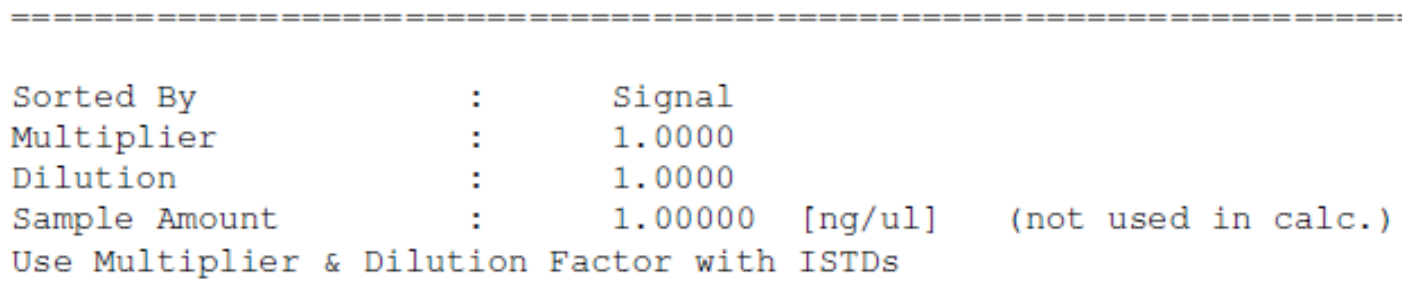

Signal 1: DAD1 A, Sig=230, 4 Ref=360,100

\begin{tabular}{|c|c|c|c|c|c|c|}
\hline $\begin{array}{c}\text { Peak } \\
\#\end{array}$ & $\begin{array}{c}\text { RetTime } \\
\text { [min] }\end{array}$ & Type & $\begin{array}{c}\text { Width } \\
\text { [min] }\end{array}$ & $\begin{array}{c}\text { Area } \\
{\left[\mathrm{mAU}^{\star} \mathrm{s}\right]}\end{array}$ & $\begin{array}{l}\text { Height } \\
\text { [mAU] }\end{array}$ & $\begin{array}{c}\text { Area } \\
8\end{array}$ \\
\hline & & & & ---- & -- & -------- \\
\hline 1 & 19.754 & BB & 0.38 & 305.62912 & 9.42051 & 44.1314 \\
\hline 2 & 25.866 & $\mathrm{BB}$ & 0.5123 & 386.91492 & 8.91572 & 55.8686 \\
\hline
\end{tabular}

Totals :

$692.54404 \quad 18.33623$

\subsection{General Procedure K}

An oven-dried Schlenk tube containing a stir bar was charged under argon with the corresponding alkyl bromide 1 (0.3 mmol, 1.5 equiv.), $\mathrm{Pd}\left(\mathrm{PPh}_{3}\right)_{4}(10 \mathrm{~mol} \%)$, BINAP (12 mol\%), phthalimide 3a (0.2 mmol, 1.0 equiv.), KOAc (150 mol\%) and DMA (0.85 mL). Subsequently, butadiene 2a (0.3 mmol, $0.15 \mathrm{~mL}, 2 \mathrm{M}$ in THF, 1.5 equiv.) was added. The reaction mixture was stirred at room temperate under blue LEDs irradiation (5W, $455 \mathrm{~nm}$ ) for $48 \mathrm{~h}$. Then, the reaction was filtered through a pad of silica gel and washed with EtOAc $(3 \times 5 \mathrm{~mL})$. The obtained solution was concentrated in vacuo and the diastereomeric ratio determined by ${ }^{1} \mathrm{H}$ NMR spectroscopy of the crude reaction mixture. The reaction mixture was purified by column chromatography on silica gel (pentane/EtOAc) to afford the corresponding compounds.

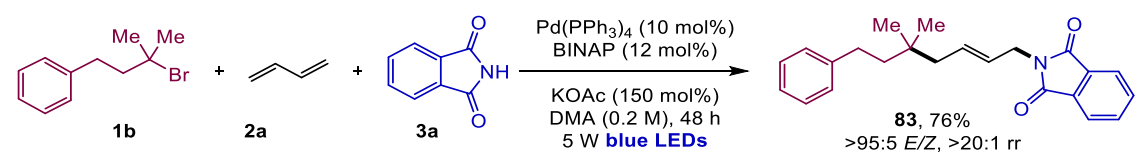


According to the general procedure $\mathrm{K}$, using the corresponding alkyl bromide $\mathbf{1 b}$ ( $0.3 \mathrm{mmol}, 1.5$ equiv.), butadiene $\mathbf{2 a}(0.3 \mathrm{mmol}, 0.15 \mathrm{~mL}, 2 \mathrm{M}$ in THF, 1.5 equiv.), phthalimide 3a (0.2 mmol, 1.0 equiv.), $\mathrm{Pd}\left(\mathrm{PPh}_{3}\right)_{4}(10 \mathrm{~mol} \%)$, BINAP (12 mol\%), KOAc (150 mol\%), and stirring at room temperature for $48 \mathrm{~h}$ under $5 \mathrm{~W}$ blue LEDs irradiation. The reaction mixture was filtered through a pad of silica gel and concentrated in vacuo, then purified by chromatography (pentane $/$ EtOAc $=9 / 1)$ to give $83\left(52.5 \mathrm{mg}, 0.151 \mathrm{mmol}, 76 \%,>95: 5 E / Z,>20: 1 \mathrm{rr}\right.$ ) as a colorless oil. $\mathbf{R}_{f}$ (pentane/EtOAc = 9/1) 0.31. ${ }^{1} \mathbf{H}$ NMR (599 MHz, $\left.\mathbf{C D C l}_{3}\right): \delta \mathrm{ppm} 7.84(\mathrm{dd}, J=5.4$, $3.1 \mathrm{~Hz}, 2 \mathrm{H}), 7.71(\mathrm{dd}, J=5.5,3.0 \mathrm{~Hz}, 2 \mathrm{H}), 7.23-7.16(\mathrm{~m}, 2 \mathrm{H}), 7.16-7.03(\mathrm{~m}, 3 \mathrm{H})$, $5.86-5.77(\mathrm{~m}, 1 \mathrm{H}), 5.60-5.49(\mathrm{~m}, 1 \mathrm{H}), 4.27(\mathrm{dd}, J=6.4,1.2 \mathrm{~Hz}, 2 \mathrm{H}), 2.56-2.46$ (m, 2H), 1.99 (dd, $J=7.5,1.3 \mathrm{~Hz}, 2 \mathrm{H}), 1.49-1.39(\mathrm{~m}, 2 \mathrm{H}), 0.92(\mathrm{~s}, 6 \mathrm{H}) .{ }^{13} \mathbf{C}$ NMR (151 MHz, CDCl $_{3}$ ): $\delta$ ppm 168.0, 143.4, 134.0, 132.3, 132.2, 128.4, 125.7, 125.6,

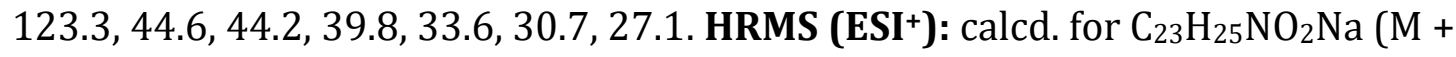
$\left.\mathrm{Na}^{+}\right)$: 370.1778. Found: 370.1779 .

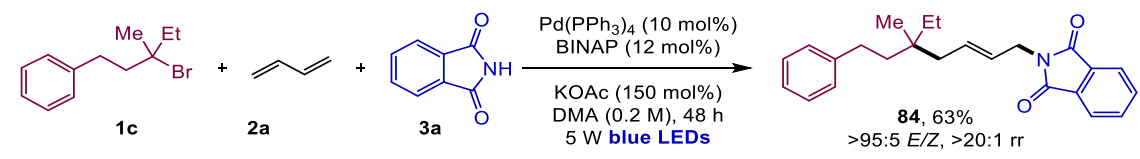

(E)-2-(5-Ethyl-5-methyl-7-phenylhept-2-en-1-yl)isoindoline-1,3-dione (84). According to the general procedure $\mathrm{K}$, using the corresponding alkyl bromide $\mathbf{1 c}$ ( $0.3 \mathrm{mmol}, 1.5$ equiv.), butadiene $\mathbf{2 a}(0.3 \mathrm{mmol}, 0.15 \mathrm{~mL}, 2 \mathrm{M}$ in THF, 1.5 equiv.), phthalimide 3a (0.2 mmol, 1.0 equiv.), $\mathrm{Pd}\left(\mathrm{PPh}_{3}\right)_{4}(10 \mathrm{~mol} \%)$, BINAP (12 mol\%), KOAc (150 mol\%), and stirring at room temperature for $48 \mathrm{~h}$ under $5 \mathrm{~W}$ blue LEDs irradiation. The reaction mixture was filtered through a pad of silica gel and concentrated in vacuo, then purified by chromatography (pentane $/$ EtOAc $=9 / 1)$ to give $84(52.5 \mathrm{mg}, 0.126 \mathrm{mmol}, 63 \%,>95: 5 E / Z,>20: 1 \mathrm{rr})$ as a colorless oil. $\mathbf{R}_{f}$ (pentane/EtOAc = 9/1) 0.39. ${ }^{1} \mathbf{H}$ NMR (599 $\left.\mathbf{M H z}, \mathbf{C D C l}_{3}\right): \delta$ ppm $7.89-7.82(\mathrm{~m}$, 2H), $7.76-7.66(\mathrm{~m}, 2 \mathrm{H}), 7.24-7.16(\mathrm{~m}, 2 \mathrm{H}), 7.15-7.08(\mathrm{~m}, 3 \mathrm{H}), 5.80(\mathrm{dtt}, J=$ 15.1, 7.5, 1.3 Hz, 1H), $5.60-5.54(\mathrm{~m}, 1 \mathrm{H}), 4.27$ (dq, $J=6.4,0.9 \mathrm{~Hz}, 2 \mathrm{H}), 2.55-2.43$ (m, 2H), $1.99(\mathrm{dd}, J=7.5,1.3 \mathrm{~Hz}, 2 \mathrm{H}), 1.48-1.40(\mathrm{~m}, 2 \mathrm{H}), 1.30(\mathrm{qd}, J=7.4,1.9 \mathrm{~Hz}$, 
2H), $0.87(\mathrm{~s}, 3 \mathrm{H}), 0.83(\mathrm{t}, J=7.5 \mathrm{~Hz}, 3 \mathrm{H}) .{ }^{13} \mathbf{C}$ NMR (151 $\left.\mathbf{M H z}, \mathbf{C D C l}_{3}\right): \delta$ ppm $168.1,143.5,134.0,132.3,132.1,128.4,128.4,125.6,125.6,123.4,41.8,41.2$, 39.9, 36.0, 31.5, 30.3, 24.4, 8.1. HRMS (ESI $\left.{ }^{+}\right)$: calcd. for $\mathrm{C}_{24} \mathrm{H}_{27} \mathrm{NO}_{2} \mathrm{Na}\left(\mathrm{M}+\mathrm{Na}^{+}\right)$: 384.1939. Found: 384.1932.

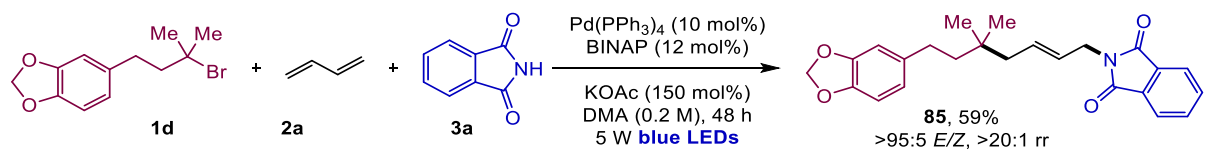

(E)-2-(7-(Benzo[d][1,3]dioxol-5-yl)-5,5-dimethylhept-2-en-1-

yl)isoindoline-1,3-dione (85). According to the general procedure $K$, using the corresponding alkyl bromide 1 d ( $0.3 \mathrm{mmol}$, 1.5 equiv.), butadiene 2 a ( $0.3 \mathrm{mmol}$, $0.15 \mathrm{~mL}, 2 M$ in THF, 1.5 equiv.), phthalimide 3a (0.2 mmol, 1.0 equiv.), $\mathrm{Pd}\left(\mathrm{PPh}_{3}\right)_{4}(10 \mathrm{~mol} \%)$, BINAP (12 mol\%), KOAc (150 mol\%), and stirring at room temperature for $48 \mathrm{~h}$ under $5 \mathrm{~W}$ blue LEDs irradiation. The reaction mixture was filtered through a pad of silica gel and concentrated in vacuo, then purified by chromatography (pentane $/$ EtOAc $=9 / 1)$ to give $85(46.4 \mathrm{mg}, 0.119 \mathrm{mmol}, 59 \%$, > 95:5E/Z, $>20: 1 \mathrm{rr})$ as a colorless oil. $\mathbf{R}_{\boldsymbol{f}}$ (pentane/EtOAc $=9 / 1$ ) 0.39. ${ }^{\mathbf{1}} \mathbf{H} \mathbf{~ N M R}$ (599 $\mathbf{M H z} \mathbf{C D C l}_{3}$ ): $\delta \mathrm{ppm} 7.84(\mathrm{dd}, J=5.4,3.1 \mathrm{~Hz}, 2 \mathrm{H}), 7.70(\mathrm{dd}, J=5.5,3.0 \mathrm{~Hz}$, 2H), $6.68-6.59(\mathrm{~m}, 2 \mathrm{H}), 6.54(\mathrm{dd}, J=7.9,1.8 \mathrm{~Hz}, 1 \mathrm{H}), 5.88(\mathrm{~s}, 2 \mathrm{H}), 5.84-5.73(\mathrm{~m}$, 1H), $5.57-5.43(\mathrm{~m}, 1 \mathrm{H}), 4.26(\mathrm{dd}, J=6.3,1.3 \mathrm{~Hz}, 2 \mathrm{H}), 2.52-2.33(\mathrm{~m}, 2 \mathrm{H}), 2.05-$ $1.88(\mathrm{~m}, 2 \mathrm{H}), 1.43-1.35(\mathrm{~m}, 2 \mathrm{H}), 0.89(\mathrm{~s}, 6 \mathrm{H}) .{ }^{13} \mathbf{C}$ NMR (151 MHz, $\left.\mathbf{C D C l}_{3}\right): \delta$ ppm 168.1, 147.6, 145.4, 137.3, 134.0, 132.3, 132.2, 125.7, 123.4, 120.9, 108.9, 108.2, 100.8, 44.6, 44.4, 39.8, 33.6, 30.5, 27.1. HRMS (ESI+): calcd. for $\mathrm{C}_{24} \mathrm{H}_{25} \mathrm{NO}_{4} \mathrm{Na}\left(\mathrm{M}+\mathrm{Na}^{+}\right): 414.1676$. Found: 414.1677 .

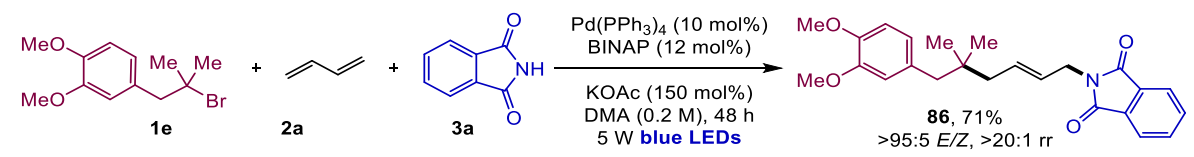

(E)-2-(6-(3,4-Dimethoxyphenyl)-5,5-dimethylhex-2-en-1-yl)isoindoline-

1,3-dione (86). According to the general procedure $\mathrm{K}$, using the corresponding alkyl bromide 1e (0.3 mmol, 1.5 equiv.), butadiene 2a $(0.3 \mathrm{mmol}, 0.15 \mathrm{~mL}, 2 \mathrm{M}$ in THF, 1.5 equiv.), phthalimide $3 a\left(0.2 \mathrm{mmol}, 1.0\right.$ equiv.), $\mathrm{Pd}\left(\mathrm{PPh}_{3}\right)_{4}(10 \mathrm{~mol} \%)$, 
BINAP (12 mol\%), KOAc (150 mol\%), and stirring at room temperature for $48 \mathrm{~h}$ under $5 \mathrm{~W}$ blue LEDs irradiation. The reaction mixture was filtered through a pad of silica gel and concentrated in vacuo, then purified by chromatography (pentane/EtOAc $=4 / 1)$ to give $86(55.5 \mathrm{mg}, 0.141 \mathrm{mmol}, 71 \%,>95: 5 \mathrm{E} / \mathrm{Z},>20: 1$ rr) as a colorless oil. $\mathbf{R}_{\boldsymbol{f}}$ (pentane/EtOAc $=4 / 1$ ) 0.30. ${ }^{\mathbf{1}} \mathbf{H}$ NMR (599 $\mathbf{~ M H z}$, Acetone- $\left.d_{6}\right): \delta$ ppm $7.84(\mathrm{~d}, J=2.8 \mathrm{~Hz}, 4 \mathrm{H}), 6.80(\mathrm{~d}, J=8.1 \mathrm{~Hz}, 1 \mathrm{H}), 6.71(\mathrm{~d}, J=$ $2.0 \mathrm{~Hz}, 1 \mathrm{H}), 6.63(\mathrm{dd}, J=8.1,2.0 \mathrm{~Hz}, 1 \mathrm{H}), 5.83(\mathrm{dtt}, J=15.1,7.5,1.4 \mathrm{~Hz}, 1 \mathrm{H}), 5.54$ $(\mathrm{dtt}, J=15.1,6.1,1.3 \mathrm{~Hz}, 1 \mathrm{H}), 4.25(\mathrm{dq}, J=6.0,1.0 \mathrm{~Hz}, 2 \mathrm{H}), 3.80-3.70(\mathrm{~m}, 6 \mathrm{H})$, $2.42(\mathrm{~s}, 2 \mathrm{H}), 1.94(\mathrm{dq}, J=7.5,1.1 \mathrm{~Hz}, 2 \mathrm{H}), 0.83(\mathrm{~s}, 6 \mathrm{H}) \cdot{ }^{13} \mathrm{C}$ NMR (151 MHz, Acetone-(d6): $\delta$ ppm 168.3, 135.0, 133.1, 132.4, 131.9, 127.2, 123.7, 123.5, 115.6, 115.6, 112.3, 112.3, 56.1, 56.0, 48.2, 45.2, 40.1, 35.2, 27.1. HRMS (ESI+): calcd. for $\mathrm{C}_{24} \mathrm{H}_{27} \mathrm{NO}_{2} \mathrm{Na}\left(\mathrm{M}+\mathrm{Na}^{+}\right): 384.1939$. Found: 384.1942.

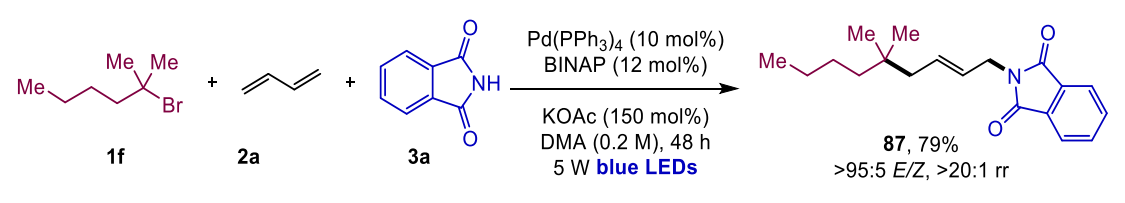

(E)-2-(5,5-Dimethylnon-2-en-1-yl)isoindoline-1,3-dione (87). According to the general procedure $\mathrm{K}$, using the corresponding alkyl bromide $\mathbf{1 f}(0.3 \mathrm{mmol}$, 1.5 equiv.), butadiene $2 \mathrm{a}(0.3 \mathrm{mmol}, 0.15 \mathrm{~mL}, 2 M$ in THF, 1.5 equiv.), phthalimide 3a (0.2 mmol, 1.0 equiv.), $\mathrm{Pd}\left(\mathrm{PPh}_{3}\right)_{4}$ (10 mol\%), BINAP (12 mol\%), KOAc (150 mol\%), and stirring at room temperature for $48 \mathrm{~h}$ under $5 \mathrm{~W}$ blue LEDs irradiation. The reaction mixture was filtered through a pad of silica gel and concentrated in vacuo, then purified by chromatography (pentane/EtOAc $=20 / 1$ ) to give 87 (47.3 mg, 0.158 mmol, 79\%, > 95:5 E/Z, > 20:1 rr) as a colorless oil. $\mathbf{R}_{\boldsymbol{f}}$ (pentane/EtOAc $=20 / 1)$ 0.51. ${ }^{1} \mathbf{H}$ NMR (599 $\left.\mathbf{~ M H z , ~} \mathbf{C D C l}_{3}\right): \delta$ ppm $7.83(\mathrm{dd}, J=$ 5.4, 3.0 Hz, 2H), $7.70(\mathrm{dd}, J=5.4,3.0 \mathrm{~Hz}, 2 \mathrm{H}), 5.84-5.70(\mathrm{~m}, 1 \mathrm{H}), 5.59-5.36(\mathrm{~m}$, $1 \mathrm{H}), 4.24(\mathrm{dd}, J=6.3,1.2 \mathrm{~Hz}, 2 \mathrm{H}), 1.88(\mathrm{dd}, J=7.6,1.2 \mathrm{~Hz}, 2 \mathrm{H}), 1.30-1.07(\mathrm{~m}, 6 \mathrm{H})$, 0.91 - 0.67 (m, 9H). ${ }^{13}$ C NMR (151 MHz, $\mathbf{C D C l}_{3}$ ): $\delta$ ppm 168.1, 134.0, 132.6, 132.4, 125.3, 123.3, 44.7, 41.7, 39.9, 33.3, 27.1, 26.3, 23.6, 14.2. HRMS (ESI ${ }^{+}$): calcd. for $\mathrm{C}_{19} \mathrm{H}_{25} \mathrm{NO}_{2} \mathrm{Na}\left(\mathrm{M}+\mathrm{Na}^{+}\right)$: 322.1778. Found: 322.1778 . 


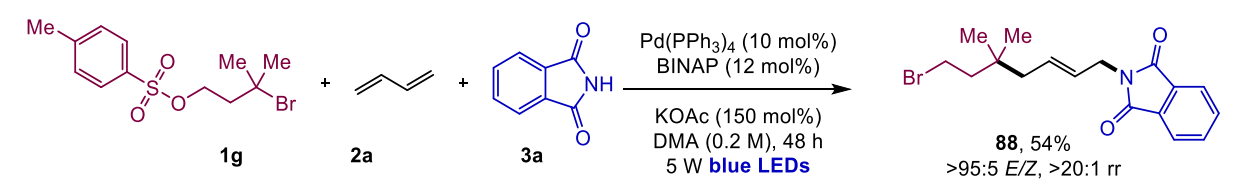

(E)-2-(7-Bromo-5,5-dimethylhept-2-en-1-yl)isoindoline-1,3-dione

(88).

According to the general procedure $\mathrm{K}$, using the corresponding alkyl bromide $\mathbf{1 g}$ ( $0.3 \mathrm{mmol}, 1.5$ equiv.), butadiene $\mathbf{2 a}(0.3 \mathrm{mmol}, 0.15 \mathrm{~mL}, 2 \mathrm{M}$ in THF, 1.5 equiv.), phthalimide 3a (0.2 mmol, 1.0 equiv.), $\mathrm{Pd}\left(\mathrm{PPh}_{3}\right)_{4}(10 \mathrm{~mol} \%)$, BINAP (12 mol\%), KOAc (150 mol\%), and stirring at room temperature for $48 \mathrm{~h}$ under $5 \mathrm{~W}$ blue LEDs irradiation. The reaction mixture was filtered through a pad of silica gel and concentrated in vacuo, then purified by chromatography (pentane $/$ EtOAc $=20 / 1)$ to give 88 (37.7 mg, $0.108 \mathrm{mmol}, 54 \%,>95: 5 E / Z,>20: 1 \mathrm{rr}$ ) as a colorless oil. $\mathbf{R}_{f}$ (pentane/EtOAc = 9/1) 0.67. ${ }^{1} \mathbf{H}$ NMR (599 $\left.\mathbf{M H z}, \mathbf{C D C l}_{3}\right): \delta$ ppm $7.84(\mathrm{dd}, J=5.4$, $3.0 \mathrm{~Hz}, 2 \mathrm{H}), 7.70(\mathrm{dd}, J=5.4,3.0 \mathrm{~Hz}, 2 \mathrm{H}), 5.78-5.69(\mathrm{~m}, 1 \mathrm{H}), 5.54-5.47(\mathrm{~m}, 1 \mathrm{H})$, $4.25(\mathrm{dq}, J=6.3,0.9 \mathrm{~Hz}, 2 \mathrm{H}), 3.41-3.27(\mathrm{~m}, 2 \mathrm{H}), 1.91(\mathrm{dd}, J=7.6,1.2 \mathrm{~Hz}, 2 \mathrm{H})$, $1.84-1.70(\mathrm{~m}, 2 \mathrm{H}), 0.86$ (s, 6H). ${ }^{13} \mathbf{C}$ NMR (151 MHz, $\left.\mathbf{C D C l}_{3}\right): \delta$ ppm 168.1, 134.0, 132.3, 131.3, 126.4, 123.4, 45.5, 44.7, 39.7, 35.0, 29.2, 26.7. HRMS (ESI+): calcd. for $\mathrm{C}_{17} \mathrm{H}_{20} \mathrm{NO}_{2} \mathrm{BrNa}\left(\mathrm{M}+\mathrm{Na}^{+}\right): 372.0575$. Found: 372.0567 .

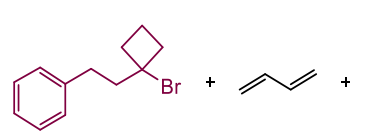

$1 \mathrm{~h}$

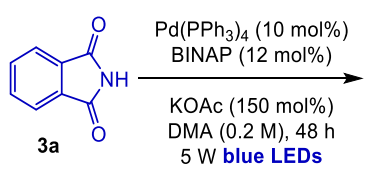

$5 \mathrm{~W}$ blue LEDs

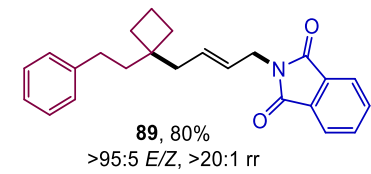

$89,80 \%$
$>95: 5 \mathrm{E} / \mathrm{Z},>20: 1 \mathrm{rr}$

(E)-2-(4-(1-Phenethylcyclobutyl)but-2-en-1-yl)isoindoline-1,3-dione (89). According to the general procedure $\mathrm{K}$, using the bromide $\mathbf{1 h}(0.3 \mathrm{mmol}, 1.5$ equiv.), butadiene $2 \mathrm{a}$ ( $0.3 \mathrm{mmol}, 0.15 \mathrm{~mL}, 2 \mathrm{M}$ in THF, 1.5 equiv.), phthalimide 3a (0.2 mmol, 1.0 equiv.), $\mathrm{Pd}\left(\mathrm{PPh}_{3}\right)_{4}(0.020 \mathrm{mmol}, 10 \mathrm{~mol} \%)$, BINAP (0.024 mmol, $12 \mathrm{~mol} \%)$, KOAc (150 mol\%), and stirring at room temperature for $48 \mathrm{~h}$ under 5W blue LEDs irradiation. The reaction mixture was filtered through a pad of silica gel and concentrated in vacuo, then purified by chromatography (pentane/EtOAc $=15 / 1 \rightarrow 10 / 1)$ to give $89(57.5 \mathrm{mg}, 0.160 \mathrm{mmol}, 80 \%,>95: 5$ 
$E / Z,>20: 1 \mathrm{rr}$ ) as a colorless gum. $\mathbf{R}_{\boldsymbol{f}}$ (pentane/EtOAc $\left.=13 / 1\right)$ 0.30. ${ }^{\mathbf{H}} \mathbf{H} \mathbf{N M R}(\mathbf{4 0 0}$ MHz, $\left.\mathbf{C D C l}_{3}\right): \delta$ ppm $7.88-7.81(\mathrm{~m}, 2 \mathrm{H}), 7.74-7.68(\mathrm{~m}, 2 \mathrm{H}), 7.21-7.14(\mathrm{~m}, 2 \mathrm{H})$, $7.14-7.07(\mathrm{~m}, 3 \mathrm{H}), 5.78(\mathrm{dt}, J=15.1,7.2 \mathrm{~Hz}, 1 \mathrm{H}), 5.61(\mathrm{dt}, J=15.2,6.3 \mathrm{~Hz}, 1 \mathrm{H})$, $4.28(\mathrm{~d}, J=6.3 \mathrm{~Hz}, 2 \mathrm{H}), 2.48-2.40(\mathrm{~m}, 2 \mathrm{H}), 2.23$ (d, $J=7.3 \mathrm{~Hz}, 2 \mathrm{H}), 1.90-1.74(\mathrm{~m}$, 6H), $1.71-1.63(\mathrm{~m}, 2 \mathrm{H}) .{ }^{13} \mathrm{C}$ NMR (101 MHz, $\left.\mathbf{C D C l}_{3}\right): \delta$ ppm 168.0, 143.2, 134.0, 132.3, 132.0, 128.4, 128.3, 125.6, 125.4, 123.3, 41.44, 41.39, 41.1, 39.8, 31.3, 30.4, 15.2. HRMS (ESI+): calcd. for $\mathrm{C}_{24} \mathrm{H}_{33} \mathrm{NO}_{2} \mathrm{Na}\left(\mathrm{M}+\mathrm{Na}^{+}\right)$: 382.1778. Found: 382.1776.

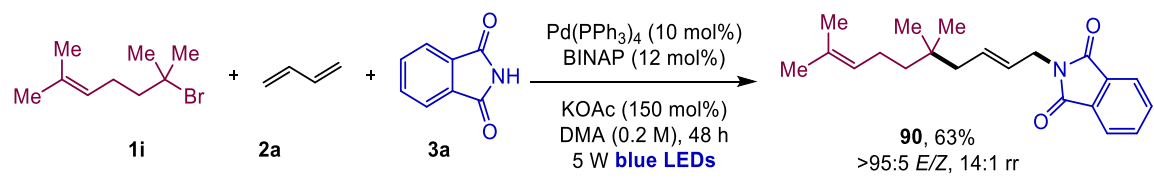

\section{(E)-2-(5,5,9-Trimethyldeca-2,8-dien-1-yl)isoindoline-1,3-dione}

(90).

According to the general procedure $\mathrm{K}$, using the corresponding alkyl bromide 1i ( $0.3 \mathrm{mmol}, 1.5$ equiv.), butadiene $2 \mathrm{a}(0.3 \mathrm{mmol}, 0.15 \mathrm{~mL}, 2 \mathrm{M}$ in THF, 1.5 equiv.), phthalimide 3a (0.2 mmol, 1.0 equiv.), $\mathrm{Pd}\left(\mathrm{PPh}_{3}\right)_{4}$ (10 mol\%), BINAP (12 mol\%), KOAc (150 mol\%), and stirring at room temperature for $48 \mathrm{~h}$ under $5 \mathrm{~W}$ blue LEDs irradiation. The reaction mixture was filtered through a pad of silica gel and concentrated in vacuo, then purified by chromatography (pentane/EtOAc $=20 / 1$ ) to give 90 (41.0 mg, 0.126 mmol, 63\%, > 95:5 E/Z, > 20:1 rr) as a colorless oil. $\mathbf{R}_{\boldsymbol{f}}$ (pentane $/$ EtOAc = 9/1) 0.42. ${ }^{1} \mathbf{H}$ NMR (300 $\left.\mathbf{~ M H z , ~} \mathbf{C D C l}_{3}\right): \delta$ ppm $7.83(\mathrm{dd}, J=5.5$, $3.1 \mathrm{~Hz}, 2 \mathrm{H}), 7.70(\mathrm{dd}, J=5.4,3.1 \mathrm{~Hz}, 2 \mathrm{H}), 5.84-5.69(\mathrm{~m}, 1 \mathrm{H}), 5.58-5.42(\mathrm{~m}, 1 \mathrm{H})$, $5.03(\mathrm{tt}, J=7.2,1.4 \mathrm{~Hz}, 1 \mathrm{H}), 4.24(\mathrm{dd}, J=6.3,1.2 \mathrm{~Hz}, 2 \mathrm{H}), 1.90(\mathrm{dd}, J=7.5,1.2 \mathrm{~Hz}$, 4H), 1.62 (d, $J=1.4 \mathrm{~Hz}, 3 \mathrm{H}), 1.54(\mathrm{~d}, J=1.4 \mathrm{~Hz}, 3 \mathrm{H}), 1.19-1.09(\mathrm{~m}, 2 \mathrm{H}), 0.82$ (s, 6H). ${ }^{13} \mathrm{C}$ NMR (75 MHz, $\mathbf{C D C l}_{3}$ ): $\delta$ ppm 168.1, 134.0, 132.4, 132.3, 131.0, 125.4, 125.2, 123.3, 44.6, 42.0, 39.8, 33.4, 27.0, 25.8, 22.8, 17.6. HRMS (ESI ${ }^{+}$): calcd. for $\mathrm{C}_{21} \mathrm{H}_{27} \mathrm{NO}_{2} \mathrm{BrNa}\left(\mathrm{M}+\mathrm{Na}^{+}\right): 348.1934$. Found: 348.1936 . 


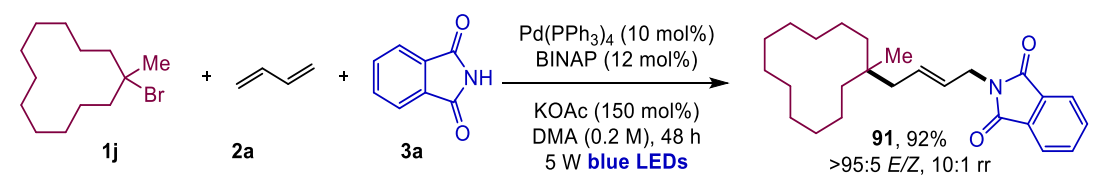

(E)-2-(4-(1-Methylcyclododecyl)but-2-en-1-yl)isoindoline-1,3-dione

(91).

According to the general procedure $K$, using the corresponding alkyl bromide $\mathbf{1 j}$ ( $0.3 \mathrm{mmol}, 1.5$ equiv.), butadiene $\mathbf{2 a}(0.3 \mathrm{mmol}, 0.15 \mathrm{~mL}, 2 \mathrm{M}$ in THF, 1.5 equiv.), phthalimide 3a (0.2 mmol, 1.0 equiv.), $\mathrm{Pd}\left(\mathrm{PPh}_{3}\right)_{4}(10 \mathrm{~mol} \%)$, BINAP (12 mol\%), KOAc (150 mol\%), and stirring at room temperature for $48 \mathrm{~h}$ under $5 \mathrm{~W}$ blue LEDs irradiation. The reaction mixture was filtered through a pad of silica gel and concentrated in vacuo, then purified by chromatography (pentane $/$ EtOAc $=20 / 1$ )

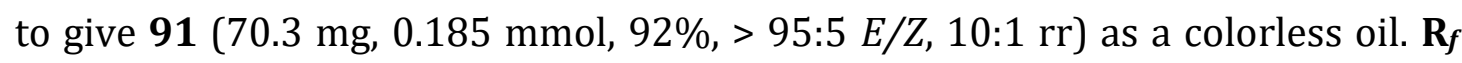
(pentane/EtOAc = 9/1) 0.42. ${ }^{1} \mathbf{H}$ NMR (599 $\mathbf{~ M H z}, \mathbf{C D C l}_{3}$ ): $\delta \mathrm{ppm} 7.84(\mathrm{dd}, J=5.4$, $3.0 \mathrm{~Hz}, 2 \mathrm{H}), 7.70(\mathrm{dd}, J=5.4,3.0 \mathrm{~Hz}, 2 \mathrm{H}), 5.84-5.73(\mathrm{~m}, 1 \mathrm{H}), 5.54-5.44(\mathrm{~m}, 1 \mathrm{H})$, $4.24(\mathrm{dd}, J=6.4,1.2 \mathrm{~Hz}, 2 \mathrm{H}), 1.84(\mathrm{dd}, J=7.7,1.3 \mathrm{~Hz}, 2 \mathrm{H}), 1.35-1.10(\mathrm{~m}, 22 \mathrm{H})$, 0.74 (s, 3H). ${ }^{1} \mathrm{H}$ NMR (599 $\mathbf{M H z}, \mathrm{CDCl}_{3}$, minor isomer, diagnostic peaks): $\delta$ ppm 6.19 (ddd, $J=17.0,10.3,6.5 \mathrm{~Hz}, 1 \mathrm{H}), 5.23(\mathrm{dt}, J=17.2,1.3 \mathrm{~Hz}, 1 \mathrm{H}), 5.16(\mathrm{dt}, J$ $=10.4,1.3 \mathrm{~Hz}, 1 \mathrm{H}), 4.93(\mathrm{pt}, J=7.2,1.5 \mathrm{~Hz}, 1 \mathrm{H}), 1.58(\mathrm{~d}, J=7.1 \mathrm{~Hz}, 3 \mathrm{H}) .{ }^{13} \mathbf{C} \mathbf{~ N M R}$ (151 MHz, $\mathbf{C D C l}_{3}$ ): $\delta$ ppm 168.1, 133.97, 132.6, 132.4, 125.4, 123.3, 43.8, 39.9, 36.0, 34.3, 27.0, 26.3, 25.3, 22.8, 22.3, 19.2. ${ }^{13} \mathbf{C}$ NMR (151 $\mathbf{M H z}, \mathbf{C D C l}_{3}$, minor isomer, diagnostic peaks): $\delta$ ppm 134.00, 116.5, 49.1, 18.4. HRMS (ESI+): calcd. for $\mathrm{C}_{25} \mathrm{H}_{35} \mathrm{NO}_{2} \mathrm{Na}\left(\mathrm{M}+\mathrm{Na}^{+}\right): 404.2560$. Found: 404.2561 .

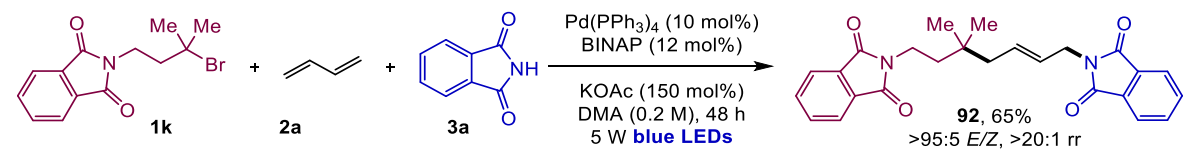

(E)-2,2'-(5,5-Dimethylhept-2-ene-1,7-diyl)bis(isoindoline-1,3-dione) (92).

According to the general procedure $\mathrm{K}$, using the corresponding alkyl bromide $\mathbf{1} \mathbf{k}$ ( $0.3 \mathrm{mmol}, 1.5$ equiv.), butadiene $\mathbf{2 a}(0.3 \mathrm{mmol}, 0.15 \mathrm{~mL}, 2 \mathrm{M}$ in THF, 1.5 equiv.), phthalimide 3a (0.2 mmol, 1.0 equiv.), $\mathrm{Pd}\left(\mathrm{PPh}_{3}\right)_{4}(10 \mathrm{~mol} \%)$, BINAP (12 mol\%), KOAc (150 mol\%), and stirring at room temperature for $48 \mathrm{~h}$ under $5 \mathrm{~W}$ blue LEDs irradiation. The reaction mixture was filtered through a pad of silica gel and 
concentrated in vacuo, then purified by chromatography (pentane $/$ EtOAc $=4 / 1$ ) to give 92 (53.9 mg, $0.130 \mathrm{mmol}, 65 \%$, > 95:5 E/Z, >20:1 rr) as a colorless oil. $\mathbf{R}_{\boldsymbol{f}}$ (pentane/EtOAc $=4 / 1)$ 0.20. ${ }^{1} \mathbf{H}$ NMR (599 $\left.\mathbf{~ M H z}, \mathbf{C D C l}_{3}\right): \delta$ ppm $7.81(\mathrm{dd}, J=5.4$, $3.0 \mathrm{~Hz}, 2 \mathrm{H}), 7.71-7.60(\mathrm{~m}, 6 \mathrm{H}), 5.80(\mathrm{dtt}, J=15.2,7.6,1.3 \mathrm{~Hz}, 1 \mathrm{H}), 5.56(\mathrm{dtt}, J=$ $15.3,6.4,1.3 \mathrm{~Hz}, 1 \mathrm{H}$ ), 4.26 (dd, $J=6.3,1.3 \mathrm{~Hz}, 2 \mathrm{H}$ ), $3.67-3.59$ (m, 2H), 1.98 (dd, $J$ $=7.5,1.3 \mathrm{~Hz}, 2 \mathrm{H}), 1.54-1.47(\mathrm{~m}, 2 \mathrm{H}), 0.94(\mathrm{~s}, 6 \mathrm{H}) .{ }^{13} \mathbf{C}$ NMR (151 MHz, $\left.\mathbf{C D C l}_{3}\right): \delta$ ppm 168.2, 168.1, 133.8, 133.8, 132.5, 132.3, 131.3, 126.3, 123.3, 123.1, 44.5, 39.8, 39.6, 34.3, 33.0, 27.0. HRMS (ESI+): calcd. for $\mathrm{C}_{25} \mathrm{H}_{24} \mathrm{~N}_{2} \mathrm{O}_{4} \mathrm{Na}\left(\mathrm{M}+\mathrm{Na}^{+}\right)$: 439.1628. Found: 439.1633.
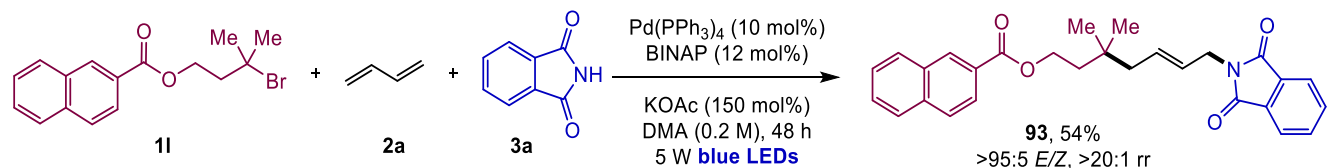

(E)-7-(1,3-Dioxoisoindolin-2-yl)-3,3-dimethylhept-5-en-1-yl 2-naphthoate (93). According to the general procedure $\mathrm{K}$, using the corresponding alkyl bromide 11 ( $0.3 \mathrm{mmol}, 1.5$ equiv.), butadiene $2 \mathbf{a}(0.3 \mathrm{mmol}, 0.15 \mathrm{~mL}, 2 \mathrm{M}$ in THF, 1.5 equiv.), phthalimide 3a ( $0.2 \mathrm{mmol}, 1.0$ equiv.), $\mathrm{Pd}\left(\mathrm{PPh}_{3}\right)_{4}(10 \mathrm{~mol} \%)$, BINAP (12 mol\%), KOAc (150 mol\%), and stirring at room temperature for $48 \mathrm{~h}$ under 5W blue LEDs irradiation. The reaction mixture was filtered through a pad of silica gel and concentrated in vacuo, then purified by chromatography (pentane/EtOAc = 9/1) to give 93 (48.0 mg, $0.109 \mathrm{mmol}, 54 \%,>95: 5 \mathrm{E} / \mathrm{Z},>20: 1$ rr) as a colorless oil. $\mathbf{R}_{f}$ (pentane/EtOAc $\left.=9 / 1\right)$ 0.20. $\left.{ }^{1} \mathbf{H} \mathbf{~ N M R ~ ( 5 9 9 ~} \mathbf{M H z}, \mathbf{C D C l}_{3}\right)$ : $\delta \operatorname{ppm} \delta 8.55(\mathrm{dd}, J=1.6,0.8 \mathrm{~Hz}, 1 \mathrm{H}), 8.00(\mathrm{dd}, J=8.6,1.7 \mathrm{~Hz}, 1 \mathrm{H}), 7.94(\mathrm{ddd}, J=$ 8.1, 1.4, $0.7 \mathrm{~Hz}, 1 \mathrm{H}), 7.88-7.84(\mathrm{~m}, 2 \mathrm{H}), 7.80(\mathrm{dd}, J=5.4,3.0 \mathrm{~Hz}, 2 \mathrm{H}), 7.65(\mathrm{dd}, J=$ 5.5, $3.0 \mathrm{~Hz}, 2 \mathrm{H}$ ), 7.58 (ddd, $J=8.2,6.9,1.3 \mathrm{~Hz}, 1 \mathrm{H}$ ), 7.53 (ddd, $J=8.1,6.8,1.3 \mathrm{~Hz}$, 1H), $5.92-5.74(\mathrm{~m}, 1 \mathrm{H}), 5.62-5.52(\mathrm{~m}, 1 \mathrm{H}), 4.49-4.35(\mathrm{~m}, 2 \mathrm{H}), 4.27(\mathrm{dq}, J=6.2$, $0.9 \mathrm{~Hz}, 2 \mathrm{H}), 2.03(\mathrm{dd}, J=7.6,1.3 \mathrm{~Hz}, 2 \mathrm{H}), 1.74(\mathrm{t}, J=7.4 \mathrm{~Hz}, 2 \mathrm{H}), 0.98(\mathrm{~s}, 6 \mathrm{H}) .{ }^{13} \mathrm{C}$ NMR (151 MHz, $\mathbf{C D C l}_{3}$ ): $\delta$ ppm 168.1, 135.6, 133.9, 132.3, 131.6, 131.1, 129.5, 128.3, 128.2, 127.9, 127.8, 126.7, 126.3, 125.4, 123.3, 62.5, 45.2, 39.9, 39.8, 33.0, 
27.3. HRMS (ESI ${ }^{+}$): calcd. for $\mathrm{C}_{28} \mathrm{H}_{27} \mathrm{~N}_{2} \mathrm{O}_{4} \mathrm{Na}\left(\mathrm{M}+\mathrm{Na}^{+}\right)$: 464.1832. Found: 464.1822.

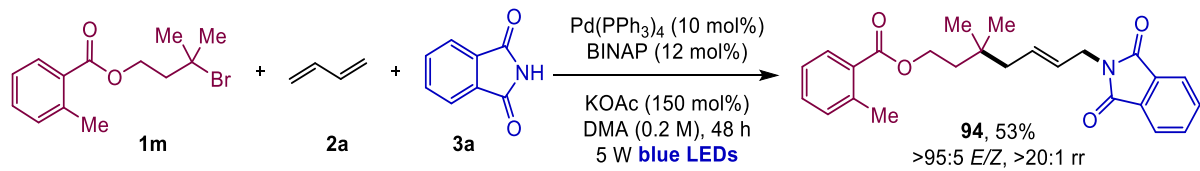

(E)-7-(1,3-Dioxoisoindolin-2-yl)-3,3-dimethylhept-5-en-1-yl

methylbenzoate (94). According to the general procedure $K$, using the corresponding alkyl bromide $\mathbf{1} \mathbf{m}(0.3 \mathrm{mmol}, 1.5$ equiv.), butadiene $\mathbf{2 a}(0.3 \mathrm{mmol}$, $0.15 \mathrm{~mL}, 2 M$ in THF, 1.5 equiv.), phthalimide 3a (0.2 mmol, 1.0 equiv.), $\mathrm{Pd}\left(\mathrm{PPh}_{3}\right)_{4}(10 \mathrm{~mol} \%)$, BINAP (12 mol\%), KOAc (150 mol\%), and stirring at room temperature for $48 \mathrm{~h}$ under $5 \mathrm{~W}$ blue LEDs irradiation. The reaction mixture was filtered through a pad of silica gel and concentrated in vacuo, then purified by chromatography $($ pentane $/$ EtOAc $=9 / 1)$ to give $94(43.1 \mathrm{mg}, 0.106 \mathrm{mmol}, 53 \%$, > 95:5 E/Z, $>20: 1 \mathrm{rr}$ ) as a colorless oil. $\mathbf{R}_{\boldsymbol{f}}$ (pentane/EtOAc $\left.=9 / 1\right)$ 0.30. ${ }^{\mathbf{1}} \mathbf{H} \mathbf{~ N M R}$ (599 $\mathbf{M H z}, \mathrm{CDCl}_{3}$ ): $\delta$ ppm $7.85(\mathrm{dd}, J=8.1,1.5 \mathrm{~Hz}, 1 \mathrm{H}$ ), $7.81(\mathrm{dd}, J=5.4,3.0 \mathrm{~Hz}$, 2H), $7.68(\mathrm{dd}, J=5.5,3.0 \mathrm{~Hz}, 2 \mathrm{H}), 7.37(\mathrm{td}, J=7.5,1.5 \mathrm{~Hz}, 1 \mathrm{H}), 7.24-7.18(\mathrm{~m}, 2 \mathrm{H})$, $5.84-5.75(\mathrm{~m}, 1 \mathrm{H}), 5.58-5.48(\mathrm{~m}, 1 \mathrm{H}), 4.31(\mathrm{dd}, J=8.0,7.1 \mathrm{~Hz}, 2 \mathrm{H}), 4.26(\mathrm{dt}, J=$ 6.3, $1.0 \mathrm{~Hz}, 2 \mathrm{H}), 2.56$ (s, 3H), 1.99 (dd, $J=7.5,1.3 \mathrm{~Hz}, 2 \mathrm{H}), 1.71-1.63(\mathrm{~m}, 2 \mathrm{H})$, $0.94(\mathrm{~s}, 6 \mathrm{H}) .{ }^{13} \mathrm{C}$ NMR (151 MHz, $\mathbf{C D C l}_{3}$ ): $\delta$ ppm 168.1, 167.8, 140.2, 134.0, 132.3, $131.9,131.7,131.6,130.6,129.9,126.2,125.8,123.3,62.0,45.2,39.9,39.8,32.9$, 27.2, 21.8. HRMS (ESI $\left.{ }^{+}\right)$: calcd. for $\mathrm{C}_{25} \mathrm{H}_{27} \mathrm{NO}_{4} \mathrm{Na}\left(\mathrm{M}+\mathrm{Na}^{+}\right): 428.1832$. Found: 428.1833.

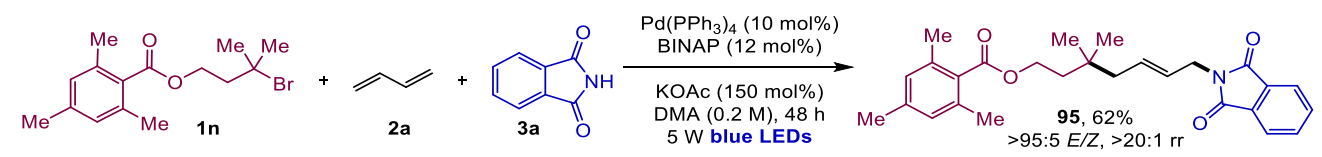

(E)-7-(1,3-Dioxoisoindolin-2-yl)-3,3-dimethylhept-5-en-1-yl 2,4,6trimethylbenzoate (95). According to the general procedure $\mathrm{K}$, using the corresponding alkyl bromide 1 n ( $0.3 \mathrm{mmol}$, 1.5 equiv.), butadiene 2 a $(0.3 \mathrm{mmol}$, $0.15 \mathrm{~mL}, 2 M$ in THF, 1.5 equiv.), phthalimide 3a (0.2 mmol, 1.0 equiv.), 
$\mathrm{Pd}\left(\mathrm{PPh}_{3}\right)_{4}(10 \mathrm{~mol} \%)$, BINAP (12 mol\%), KOAc (150 mol\%), and stirring at room temperature for $48 \mathrm{~h}$ under $5 \mathrm{~W}$ blue LEDs irradiation. The reaction mixture was filtered through a pad of silica gel and concentrated in vacuo, then purified by chromatography $($ pentane $/$ EtOAc $=9 / 1)$ to give $95(53.5 \mathrm{mg}, 0.124 \mathrm{mmol}, 62 \%,>$ 95:5E/Z, >20:1 rr) as a colorless oil. $\mathbf{R}_{\boldsymbol{f}}$ (pentane/EtOAc $=9 / 1$ ) 0.25. ${ }^{\mathbf{1}} \mathbf{H} \mathbf{~ N M R}$

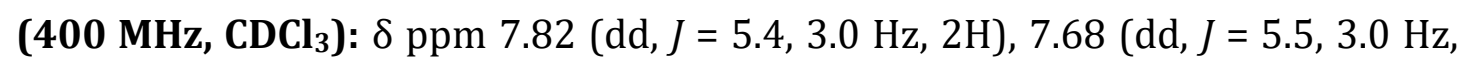
2H), 6.86 - $6.74(\mathrm{~m}, 2 \mathrm{H}), 5.81$ - $5.67(\mathrm{~m}, 1 \mathrm{H}), 5.65$ - $5.44(\mathrm{~m}, 1 \mathrm{H}), 4.39$ - $4.13(\mathrm{~m}$, $4 \mathrm{H}), 2.26(\mathrm{~d}, J=6.9 \mathrm{~Hz}, 9 \mathrm{H}), 1.97(\mathrm{dd}, J=7.5,1.2 \mathrm{~Hz}, 2 \mathrm{H}), 1.67-1.60(\mathrm{~m}, 2 \mathrm{H}), 0.92$ (s, 6H). ${ }^{13} \mathbf{C}$ NMR (101 MHz, $\left.\mathbf{C D C l}_{3}\right): \delta$ ppm 170.3, 168.1, 139.2, 135.1, 134.0, $132.3,131.4,131.2,128.5,126.3,123.3,62.2,45.1,39.8,39.7,32.9,27.2,21.2$, 19.9. HRMS (ESI+): calcd. for $\mathrm{C}_{27} \mathrm{H}_{31} \mathrm{NO}_{4} \mathrm{Na}\left(\mathrm{M}+\mathrm{Na}^{+}\right)$: 456.2145. Found: 456.2151.

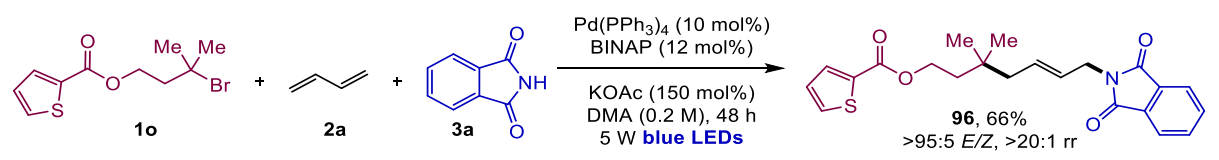

(E)-7-(1,3-Dioxoisoindolin-2-yl)-3,3-dimethylhept-5-en-1-yl thiophene-2-

carboxylate (96). According to the general procedure $K$, using the corresponding alkyl bromide 10 ( $0.3 \mathrm{mmol}, 1.5$ equiv.), butadiene 2 a $(0.3 \mathrm{mmol}$, $0.15 \mathrm{~mL}, 2 M$ in THF, 1.5 equiv.), phthalimide $3 \mathrm{a}$ (0.2 mmol, 1.0 equiv.), $\mathrm{Pd}\left(\mathrm{PPh}_{3}\right)_{4}(10 \mathrm{~mol} \%)$, BINAP (12 mol\%), KOAc (150 mol\%), and stirring at room temperature for $48 \mathrm{~h}$ under $5 \mathrm{~W}$ blue LEDs irradiation. The reaction mixture was filtered through a pad of silica gel and concentrated in vacuo, then purified by chromatography (pentane $/$ EtOAc $=9 / 1)$ to give $96(52.4 \mathrm{mg}, 0.132 \mathrm{mmol}, 66 \%$, > 95:5E/Z, $>20: 1 \mathrm{rr}$ ) as a colorless oil. $\mathbf{R}_{\boldsymbol{f}}$ (pentane/EtOAc $\left.=9 / 1\right)$ 0.27. ${ }^{\mathbf{1}} \mathbf{H} \mathbf{N M R}$ (599 $\mathbf{M H z} \mathbf{C D C l}_{3}$ ): $\delta$ ppm $7.82(\mathrm{dd}, J=5.4,3.0 \mathrm{~Hz}, 2 \mathrm{H}), 7.73(\mathrm{dd}, J=3.7,1.3 \mathrm{~Hz}$, 1H), $7.69(\mathrm{dd}, J=5.4,3.0 \mathrm{~Hz}, 2 \mathrm{H}), 7.51(\mathrm{dd}, J=5.0,1.3 \mathrm{~Hz}, 1 \mathrm{H}), 7.07$ (dd, $J=5.0$, $3.7 \mathrm{~Hz}, 1 \mathrm{H}), 5.83-5.76(\mathrm{~m}, 1 \mathrm{H}), 5.58-5.45(\mathrm{~m}, 1 \mathrm{H}), 4.31(\mathrm{t}, J=7.2 \mathrm{~Hz}, 2 \mathrm{H}), 4.25$ $(\mathrm{dd}, J=6.3,1.2 \mathrm{~Hz}, 2 \mathrm{H}), 1.98(\mathrm{dd}, J=7.4,1.2 \mathrm{~Hz}, 2 \mathrm{H}), 1.65(\mathrm{t}, J=7.2 \mathrm{~Hz}, 2 \mathrm{H}), 0.93$ (s, 6H). ${ }^{13}$ C NMR (151 MHz, CDCl $_{3}$ ): $\delta$ ppm 168.1, 162.3, 134.4, 134.0, 133.3, 
132.3, 132.3, 131.5, 126.2, 123.7, 123.3, 62.5, 45.1, 39.8, 39.7, 32.9, 27.2. HRMS

(ESI'): calcd. for $\mathrm{C}_{22} \mathrm{H}_{23} \mathrm{NO}_{4} \mathrm{Na}\left(\mathrm{M}+\mathrm{Na}^{+}\right): 420.1240$. Found: 420.1245 .
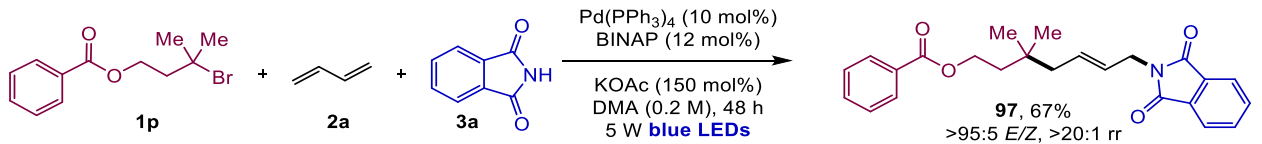

(E)-7-(1,3-Dioxoisoindolin-2-yl)-3,3-dimethylhept-5-en-1-yl benzoate (97). According to the general procedure $\mathrm{K}$, using the corresponding alkyl bromide 1p ( $0.3 \mathrm{mmol}, 1.5$ equiv.), butadiene $\mathbf{2 a}(0.3 \mathrm{mmol}, 0.15 \mathrm{~mL}, 2 \mathrm{M}$ in THF, 1.5 equiv.), phthalimide 3a (0.2 mmol, 1.0 equiv.), $\mathrm{Pd}\left(\mathrm{PPh}_{3}\right)_{4}(10 \mathrm{~mol} \%)$, BINAP (12 mol\%), KOAc (150 mol\%), and stirring at room temperature for $48 \mathrm{~h}$ under $5 \mathrm{~W}$ blue LEDs irradiation. The reaction mixture was filtered through a pad of silica gel and concentrated in vacuo, then purified by chromatography (pentane/EtOAc $=9 / 1)$ to give 97 (52.7 mg, $0.135 \mathrm{mmol}, 67 \%$, > 95:5 E/Z, >20:1 rr) as a colorless oil. $\mathbf{R}_{\boldsymbol{f}}$ (pentane/EtOAc = 9/1) 0.19. ${ }^{1} \mathbf{H}$ NMR (599 $\left.\mathbf{~ M H z , ~} \mathbf{C D C l}_{3}\right): \delta$ ppm $8.05-7.90(\mathrm{~m}$, $2 \mathrm{H}), 7.81(\mathrm{dd}, J=5.4,3.0 \mathrm{~Hz}, 2 \mathrm{H}), 7.68(\mathrm{dd}, J=5.5,3.0 \mathrm{~Hz}, 2 \mathrm{H}), 7.52(\mathrm{t}, J=7.4 \mathrm{~Hz}$, 1H), $7.43-7.36(\mathrm{~m}, 2 \mathrm{H}), 5.87-5.73(\mathrm{~m}, 1 \mathrm{H}), 5.60-5.47(\mathrm{~m}, 1 \mathrm{H}), 4.34(\mathrm{t}, J=7.3$ $\mathrm{Hz}, 2 \mathrm{H}), 4.25$ (dq, $J=6.3,0.9 \mathrm{~Hz}, 2 \mathrm{H}), 1.99$ (dd, $J=7.6,1.3 \mathrm{~Hz}, 2 \mathrm{H}), 1.67(\mathrm{t}, J=7.3$ $\mathrm{Hz}, 2 \mathrm{H}), 0.94(\mathrm{~s}, 6 \mathrm{H}) .{ }^{13} \mathbf{C}$ NMR (151 MHz, $\mathbf{C D C l}_{3}$ ): $\delta \mathrm{ppm}$ 168.0, 166.7, 134.0, 132.9, 132.3, 131.5, 130.5, 129.6, 128.4, 126.2, 123.3, 62.3, 45.2, 39.8, 39.7, 32.9, 27.2. HRMS (ESI+): calcd. for $\mathrm{C}_{24} \mathrm{H}_{25} \mathrm{NO}_{4} \mathrm{Na}\left(\mathrm{M}+\mathrm{Na}^{+}\right)$: 414.1676. Found: 414.1691.

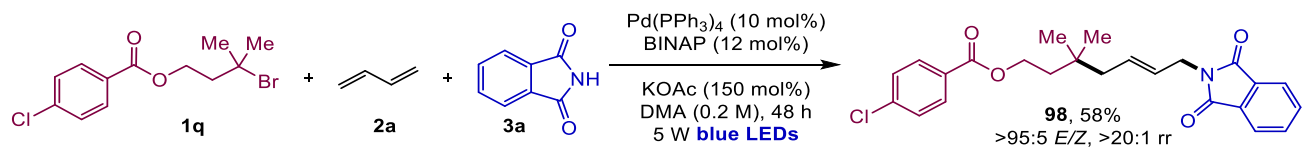

(E)-7-(1,3-Dioxoisoindolin-2-yl)-3,3-dimethylhept-5-en-1-yl

chlorobenzoate (98). According to the general procedure $\mathrm{K}$, using the corresponding alkyl bromide 1q $(0.3 \mathrm{mmol}, 1.5$ equiv.), butadiene $\mathbf{2 a}(0.3 \mathrm{mmol}$, $0.15 \mathrm{~mL}, 2 M$ in THF, 1.5 equiv.), phthalimide 3a ( $0.2 \mathrm{mmol}, 1.0$ equiv.), $\mathrm{Pd}\left(\mathrm{PPh}_{3}\right)_{4}(10 \mathrm{~mol} \%)$, BINAP (12 mol\%), KOAc (150 mol\%), and stirring at room temperature for $48 \mathrm{~h}$ under $5 \mathrm{~W}$ blue LEDs irradiation. The reaction mixture was 
filtered through a pad of silica gel and concentrated in vacuo, then purified by chromatography $($ pentane $/$ EtOAc $=9 / 1)$ to give $98(49.5 \mathrm{mg}, 0.116 \mathrm{mmol}, 58 \%$, > 95:5E/Z, $>20: 1 \mathrm{rr}$ ) as a colorless oil. $\mathbf{R}_{\boldsymbol{f}}$ (pentane/EtOAc $=9 / 1$ ) 0.41. ${ }^{1} \mathbf{H} \mathbf{~ N M R}$ (599 $\mathbf{M H z} \mathbf{C D C l}_{3}$ ): $\delta$ ppm $7.97-7.87(\mathrm{~m}, 2 \mathrm{H}), 7.82(\mathrm{dd}, J=5.4,3.1 \mathrm{~Hz}, 2 \mathrm{H}), 7.69$ (dd, $J=5.5,3.0 \mathrm{~Hz}, 2 \mathrm{H}), 7.44-7.32(\mathrm{~m}, 2 \mathrm{H}), 5.86-5.72(\mathrm{~m}, 1 \mathrm{H}), 5.60-5.46(\mathrm{~m}$, 1H), $4.33(\mathrm{t}, J=7.3 \mathrm{~Hz}, 2 \mathrm{H}), 4.26(\mathrm{dd}, J=6.4,1.2 \mathrm{~Hz}, 2 \mathrm{H}), 1.98$ (dd, $J=7.5,1.2 \mathrm{~Hz}$, 2H), $1.66(\mathrm{t}, J=7.3 \mathrm{~Hz}, 2 \mathrm{H}), 0.94(\mathrm{~s}, 6 \mathrm{H}) .{ }^{13} \mathbf{C}$ NMR (151 $\left.\mathbf{~ M H z}, \mathbf{C D C l}_{3}\right): \delta \mathrm{ppm}$ 168.1, 165.9, 139.3, 134.0, 132.3, 131.5, 131.0, 129.0, 128.8, 126.3, 123.4, 62.6, 45.2, 39.8, 39.7, 32.9, 27.3. HRMS (ESI $\left.{ }^{+}\right)$: calcd. for $\mathrm{C}_{24} \mathrm{H}_{24} \mathrm{NO}_{4} \mathrm{ClNa}\left(\mathrm{M}+\mathrm{Na}^{+}\right)$: 448.1286. Found: 448.1288.

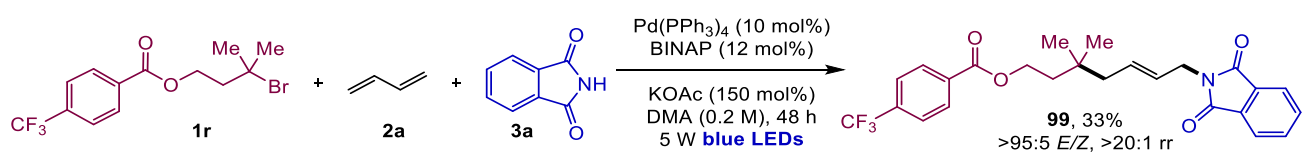

(E)-7-(1,3-Dioxoisoindolin-2-yl)-3,3-dimethylhept-5-en-1-yl 4(trifluoromethyl)benzoate (99). According to the general procedure $\mathrm{K}$, using the corresponding alkyl bromide $1 \mathbf{r}(0.3 \mathrm{mmol}, 1.5$ equiv.), butadiene $2 \mathrm{a}(0.3$ mmol, $0.15 \mathrm{~mL}, 2 M$ in THF, 1.5 equiv.), phthalimide 3a (0.2 mmol, 1.0 equiv.), $\mathrm{Pd}\left(\mathrm{PPh}_{3}\right)_{4}(10 \mathrm{~mol} \%)$, BINAP (12 mol\%), KOAc (150 mol\%), and stirring at room temperature for $48 \mathrm{~h}$ under $5 \mathrm{~W}$ blue LEDs irradiation. The reaction mixture was filtered through a pad of silica gel and concentrated in vacuo, then purified by chromatography $($ pentane $/$ EtOAc $=9 / 1)$ to give $99(30.5 \mathrm{mg}, 0.066 \mathrm{mmol}, 33 \%$, > 95:5E/Z, $>20: 1 \mathrm{rr}$ ) as a colorless oil. $\mathbf{R}_{\boldsymbol{f}}$ (pentane/EtOAc $\left.=9 / 1\right)$ 0.35. ${ }^{\mathbf{1}} \mathbf{H} \mathbf{~ N M R}$ (599 $\mathbf{M H z}, \mathbf{C D C l}_{3}$ ): $\delta$ ppm $8.09(\mathrm{dt}, J=7.9,0.8 \mathrm{~Hz}, 2 \mathrm{H}), 7.82(\mathrm{dd}, J=5.4,3.1 \mathrm{~Hz}$, 2H), $7.73-7.64(\mathrm{~m}, 4 \mathrm{H}), 5.88-5.78(\mathrm{~m}, 1 \mathrm{H}), 5.59-5.47(\mathrm{~m}, 1 \mathrm{H}), 4.38(\mathrm{t}, J=7.3$ $\mathrm{Hz}, 2 \mathrm{H}), 4.31-4.20(\mathrm{~m}, 2 \mathrm{H}), 2.00(\mathrm{dd}, J=7.6,1.2 \mathrm{~Hz}, 2 \mathrm{H}), 1.69$ (t, J = 7.3 Hz, 2H), 0.95 (s, 6H). ${ }^{13} \mathrm{C}$ NMR (151 $\left.\mathbf{M H z}, \mathbf{C D C l}_{3}\right): \delta$ ppm 168.1, 165.5, $137.0 \mathrm{U}=272.5$ $\mathrm{Hz}), 134.4 \mathrm{U}=31.7 \mathrm{~Hz}), 134.0,133.8,132.3,131.5,130.1,126.4,125.5 \mathrm{U}=3.0$ $\mathrm{Hz}), 123.4,62.9,45.2,39.8,39.7,33.0,27.3 .{ }^{19} \mathbf{F}\left\{\mathbf{1}^{\mathbf{H}}\right\}$ NMR (564 $\left.\mathbf{~ M H z}, \mathbf{C D C l}_{3}\right): \delta$ ppm -63.10. HRMS (ESI+): calcd. for $\mathrm{C}_{25} \mathrm{H}_{24} \mathrm{NO}_{4} \mathrm{~F}_{3} \mathrm{Na}\left(\mathrm{M}+\mathrm{Na}^{+}\right)$: 482.1550. Found: 482.1559. 


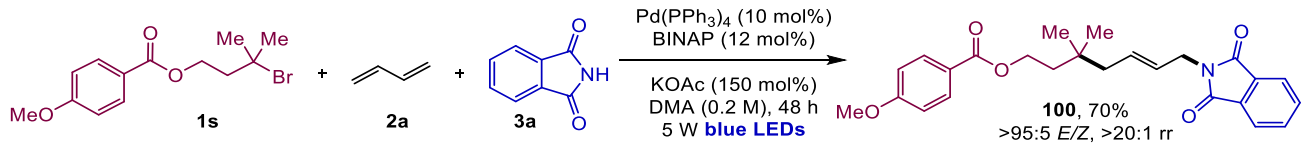

(E)-7-(1,3-Dioxoisoindolin-2-yl)-3,3-dimethylhept-5-en-1-yl

4-

methoxybenzoate (100). According to the general procedure $K$, using the corresponding alkyl bromide $1 \mathrm{~s}(0.3 \mathrm{mmol}, 1.5$ equiv.), butadiene $2 \mathrm{a}(0.3 \mathrm{mmol}$, $0.15 \mathrm{~mL}, 2 M$ in THF, 1.5 equiv.), phthalimide 3a (0.2 mmol, 1.0 equiv.), $\mathrm{Pd}\left(\mathrm{PPh}_{3}\right)_{4}(10 \mathrm{~mol} \%)$, BINAP (12 mol\%), KOAc (150 mol\%), and stirring at room temperature for $48 \mathrm{~h}$ under $5 \mathrm{~W}$ blue LEDs irradiation. The reaction mixture was filtered through a pad of silica gel and concentrated in vacuo, then purified by chromatography (pentane $/$ EtOAc $=9 / 1)$ to give $100(58.9 \mathrm{mg}, 0.140 \mathrm{mmol}$, $70 \%,>95: 5 E / Z,>20: 1 \mathrm{rr}$ ) as a colorless oil. $\mathbf{R}_{\boldsymbol{f}}$ (pentane/EtOAc $=9 / 1$ ) $0.11 .{ }^{1} \mathbf{H}$ NMR (599 MHz, CDCl 3 ): $\delta$ ppm $7.98-7.86(\mathrm{~m}, 2 \mathrm{H}), 7.81(\mathrm{dd}, J=5.5,3.0 \mathrm{~Hz}, 2 \mathrm{H})$, $7.68(\mathrm{dd}, J=5.5,3.0 \mathrm{~Hz}, 2 \mathrm{H}), 6.95-6.80(\mathrm{~m}, 2 \mathrm{H}), 5.79(\mathrm{dt}, J=15.1,7.5 \mathrm{~Hz}, 1 \mathrm{H})$, $5.52(\mathrm{dt}, J=15.1,6.3 \mathrm{~Hz}, 1 \mathrm{H}), 4.30(\mathrm{t}, J=7.3 \mathrm{~Hz}, 2 \mathrm{H}), 4.25(\mathrm{~d}, J=6.3 \mathrm{~Hz}, 2 \mathrm{H}), 3.84$ $(\mathrm{s}, 3 \mathrm{H}), 1.98(\mathrm{~d}, J=7.5 \mathrm{~Hz}, 2 \mathrm{H}), 1.65(\mathrm{t}, J=7.3 \mathrm{~Hz}, 2 \mathrm{H}), 0.93(\mathrm{~s}, 6 \mathrm{H}) .{ }^{13} \mathbf{C}$ NMR (151 MHz, $\mathbf{C D C l}_{3}$ ): $\delta$ ppm 168.1, 166.5, 163.3, 134.3, 134.0, 132.3, 131.6, 131.6, 126.2, 123.6, 123.3, 123.0, 113.6, 62.0, 55.5, 45.2, 39.9, 39.7, 32.9, 27.2. HRMS (ESI+): calcd. for $\mathrm{C}_{25} \mathrm{H}_{27} \mathrm{NO}_{5} \mathrm{Na}\left(\mathrm{M}+\mathrm{Na}^{+}\right): 444.1781$. Found: 444.1784 .

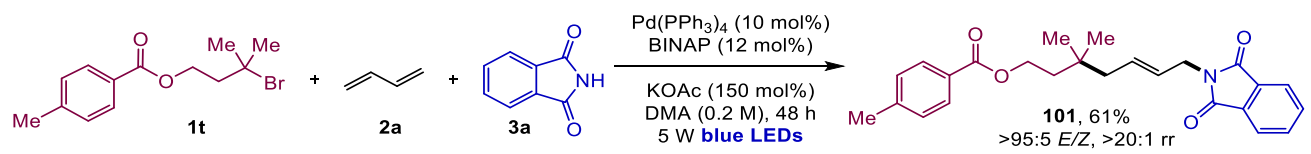

(E)-7-(1,3-Dioxoisoindolin-2-yl)-3,3-dimethylhept-5-en-1-yl 4methylbenzoate (101). According to the general procedure $K$, using the corresponding alkyl bromide $\mathbf{1 t}$ ( $0.3 \mathrm{mmol}, 1.5$ equiv.), butadiene 2 a $(0.3 \mathrm{mmol}$, $0.15 \mathrm{~mL}, 2 M$ in THF, 1.5 equiv.), phthalimide 3a (0.2 mmol, 1.0 equiv.), $\mathrm{Pd}\left(\mathrm{PPh}_{3}\right)_{4}(10 \mathrm{~mol} \%)$, BINAP (12 mol\%), KOAc (150 mol\%), and stirring at room temperature for $48 \mathrm{~h}$ under $5 \mathrm{~W}$ blue LEDs irradiation. The reaction mixture was filtered through a pad of silica gel and concentrated in vacuo, then purified by chromatography (pentane/EtOAc $=9 / 1$ ) to give $101(49.7 \mathrm{mg}, 0.123 \mathrm{mmol}$, 
$61 \%,>95: 5 E / Z,>20: 1 \mathrm{rr}$ ) as a colorless oil. $\mathbf{R}_{\boldsymbol{f}}$ (pentane/EtOAc $\left.=9 / 1\right) 0.21 .{ }^{1} \mathbf{H}$ NMR (400 MHz, $\mathbf{C D C l}_{3}$ ): $\delta$ ppm $7.90-7.86(\mathrm{~m}, 2 \mathrm{H}), 7.82(\mathrm{dd}, J=5.5,3.0 \mathrm{~Hz}, 2 \mathrm{H})$, $7.69(\mathrm{dd}, J=5.5,3.0 \mathrm{~Hz}, 2 \mathrm{H}), 7.23-7.15(\mathrm{~m}, 2 \mathrm{H}), 5.86-5.75(\mathrm{~m}, 1 \mathrm{H}), 5.59-5.50$ (m, 1H), $4.32(\mathrm{t}, J=7.3 \mathrm{~Hz}, 2 \mathrm{H}), 4.26(\mathrm{dd}, J=6.3,1.3 \mathrm{~Hz}, 2 \mathrm{H}), 2.39$ (s, 3H), 1.99 (dd, $J=7.4,1.2 \mathrm{~Hz}, 2 \mathrm{H}), 1.66(\mathrm{t}, J=7.3 \mathrm{~Hz}, 2 \mathrm{H}), 0.94(\mathrm{~s}, 6 \mathrm{H}) .{ }^{13} \mathbf{C}$ NMR (101 MHz, $\mathbf{C D C l}_{3}$ ): $\delta$ ppm 168.1, 166.8, 143.5, 134.0, 132.3, 131.6, 129.7, 129.1, 127.8, 126.2, 123.3, 62.1, 45.2, 39.9, 39.8, 32.9, 27.3, 21.8. HRMS (ESI+): calcd. for $\mathrm{C}_{25} \mathrm{H}_{27} \mathrm{NO}_{4} \mathrm{Na}\left(\mathrm{M}+\mathrm{Na}^{+}\right): 428.1832$. Found: 428.1833 .

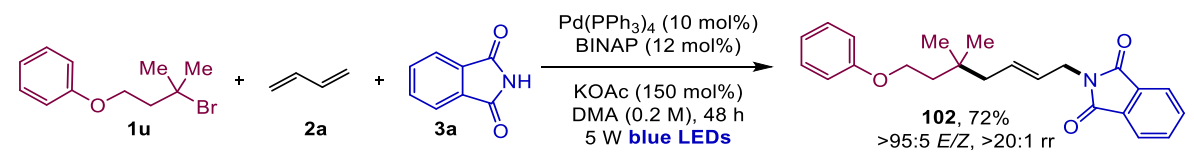

(E)-2-(5,5-Dimethyl-7-phenoxyhept-2-en-1-yl)isoindoline-1,3-dione (102).

According to the general procedure $\mathrm{K}$, using the corresponding alkyl bromide $\mathbf{1} \mathbf{u}$ (0.3 mmol, 1.5 equiv.), butadiene 2 a ( $0.3 \mathrm{mmol}, 0.15 \mathrm{~mL}, 2 M$ in THF, 1.5 equiv.), phthalimide 3a (0.2 mmol, 1.0 equiv.), $\mathrm{Pd}\left(\mathrm{PPh}_{3}\right)_{4}$ (10 mol\%), BINAP (12 mol\%), KOAc (150 mol\%), and stirring at room temperature for $48 \mathrm{~h}$ under $5 \mathrm{~W}$ blue LEDs irradiation. The reaction mixture was filtered through a pad of silica gel and concentrated in vacuo, then purified by chromatography (pentane $/$ EtOAc $=9 / 1$ ) to give 101 (52.5 mg, 0.145 mmol, 72\%, > 95:5 E/Z, >20:1 rr) as a colorless oil. $\mathbf{R}_{\boldsymbol{f}}$ $\left(\right.$ pentane $/$ EtOAc = 9/1) 0.15. ${ }^{1} \mathbf{H}$ NMR (300 $\left.\mathbf{~ M H z , ~} \mathbf{C D C l}_{3}\right): \delta$ ppm $7.84(\mathrm{dd}, J=5.4$, $3.1 \mathrm{~Hz}, 2 \mathrm{H}), 7.70(\mathrm{dd}, J=5.5,3.1 \mathrm{~Hz}, 2 \mathrm{H}), 7.32-7.17(\mathrm{~m}, 2 \mathrm{H}), 6.95-6.77(\mathrm{~m}, 3 \mathrm{H})$, $5.87-5.75(\mathrm{~m}, 1 \mathrm{H}), 5.58-5.47(\mathrm{~m}, 1 \mathrm{H}), 4.26(\mathrm{dd}, J=6.3,1.2 \mathrm{~Hz}, 2 \mathrm{H}), 3.99(\mathrm{t}, J=$ $7.2 \mathrm{~Hz}, 2 \mathrm{H}), 2.00(\mathrm{dd}, J=7.5,1.2 \mathrm{~Hz}, 2 \mathrm{H}), 1.71(\mathrm{t}, J=7.2 \mathrm{~Hz}, 2 \mathrm{H}), 0.94(\mathrm{~s}, 6 \mathrm{H}) .{ }^{13} \mathrm{C}$ NMR (75 MHz, CDCl $_{3}$ ): $\delta$ ppm 168.1, 159.0, 134.0, 132.3, 131.7, 129.5, 126.1, 123.4, 120.6, 114.5, 64.8, 45.3, 40.4, 39.8, 32.9, 27.3. HRMS (ESI'): calcd. for $\mathrm{C}_{23} \mathrm{H}_{25} \mathrm{NO}_{3} \mathrm{Na}\left(\mathrm{M}+\mathrm{Na}^{+}\right): 386.1732$. Found: 386.1738 .

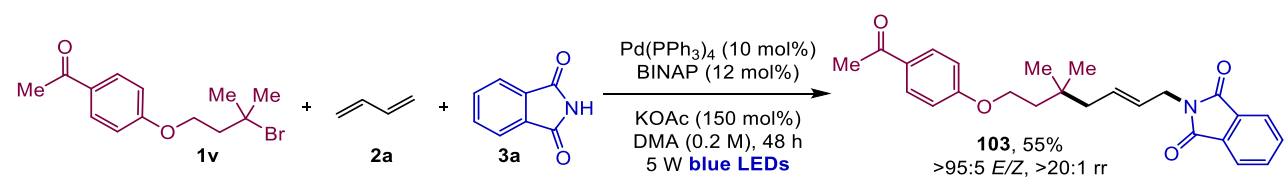




\section{(E)-2-(7-(4-Acetylphenoxy)-5,5-dimethylhept-2-en-1-yl)isoindoline-1,3-}

dione (103). According to the general procedure $K$, using the corresponding alkyl bromide $1 \mathbf{v}(0.3 \mathrm{mmol}, 1.5$ equiv.), butadiene $2 \mathrm{a}(0.3 \mathrm{mmol}, 0.15 \mathrm{~mL}, 2 \mathrm{M}$ in THF, 1.5 equiv.), phthalimide 3a (0.2 mmol, 1.0 equiv.), $\mathrm{Pd}\left(\mathrm{PPh}_{3}\right)_{4}(10 \mathrm{~mol} \%)$, BINAP (12 mol\%), KOAc (150 mol\%), and stirring at room temperature for $48 \mathrm{~h}$ under $5 \mathrm{~W}$ blue LEDs irradiation. The reaction mixture was filtered through a pad of silica gel and concentrated in vacuo, then purified by chromatography (pentane/EtOAc $=9 / 1)$ to give $103(44.7 \mathrm{mg}, 0.110 \mathrm{mmol}, 55 \%,>95: 5$ $E / Z,>20: 1 \mathrm{rr}$ ) as a colorless oil. $\mathbf{R}_{\boldsymbol{f}}$ (pentane/EtOAc $\left.=4 / 1\right)$ 0.17. ${ }^{1} \mathbf{H}$ NMR (599 MHz, $\mathbf{C D C l}_{3}$ ): $\delta$ ppm $7.89(\mathrm{~d}, J=8.9 \mathrm{~Hz}, 2 \mathrm{H}), 7.82(\mathrm{dd}, J=5.4,3.1 \mathrm{~Hz}, 2 \mathrm{H}), 7.70$ (dd, $J=5.5,3.1 \mathrm{~Hz}, 2 \mathrm{H}), 6.87(\mathrm{~d}, J=8.9 \mathrm{~Hz}, 2 \mathrm{H}), 5.87-5.76(\mathrm{~m}, 1 \mathrm{H}), 5.60-5.51(\mathrm{~m}$, 1H), $4.32-4.22(\mathrm{~m}, 2 \mathrm{H}), 4.04(\mathrm{t}, J=7.1 \mathrm{~Hz}, 2 \mathrm{H}), 2.54(\mathrm{~s}, 3 \mathrm{H}), 1.99$ (dd, $J=7.6,1.3$ $\mathrm{Hz}, 2 \mathrm{H}), 1.71(\mathrm{t}, J=7.1 \mathrm{~Hz}, 2 \mathrm{H}), 0.94(\mathrm{~s}, 6 \mathrm{H}) \cdot{ }^{13} \mathbf{C}$ NMR (151 $\left.\mathbf{M H z}, \mathbf{C D C l}_{3}\right): \delta$ ppm 197.0, 168.1, 163.0, 134.0, 132.3, 131.6, 130.7, 130.2, 126.2, 123.4, 114.2, 65.3, 45.3, 40.2, 39.8, 32.9, 27.4, 26.4. HRMS (ESI $\left.{ }^{+}\right)$: calcd. for $\mathrm{C}_{25} \mathrm{H}_{27} \mathrm{NO}_{4} \mathrm{Na}\left(\mathrm{M}+\mathrm{Na}^{+}\right)$: 428.1832. Found: 428.1827.

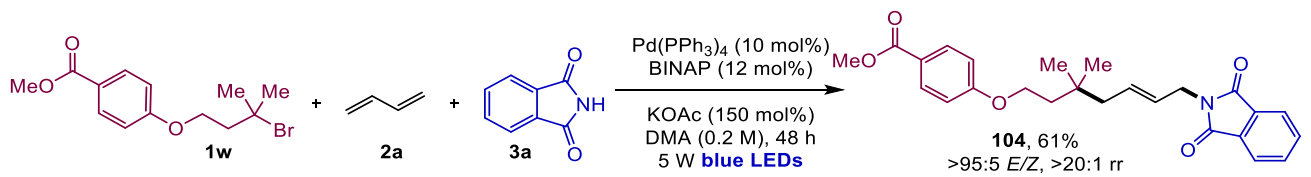

Methyl (E)-4-((7-(1,3-dioxoisoindolin-2-yl)-3,3-dimethylhept-5-en-1yl)oxy)benzoate (104). According to the general procedure $K$, using the corresponding alkyl bromide $\mathbf{1 w}(0.3 \mathrm{mmol}, 1.5$ equiv.), butadiene $\mathbf{2 a}(0.3 \mathrm{mmol}$, $0.15 \mathrm{~mL}, 2 M$ in THF, 1.5 equiv.), phthalimide $3 \mathrm{a}$ (0.2 mmol, 1.0 equiv.), $\mathrm{Pd}\left(\mathrm{PPh}_{3}\right)_{4}(10 \mathrm{~mol} \%)$, BINAP (12 mol\%), KOAc (150 mol\%), and stirring at room temperature for $48 \mathrm{~h}$ under $5 \mathrm{~W}$ blue LEDs irradiation. The reaction mixture was filtered through a pad of silica gel and concentrated in vacuo, then purified by chromatography (pentane $/$ EtOAc $=9 / 1)$ to give $104(51.0 \mathrm{mg}, 0.121 \mathrm{mmol}$, $61 \%,>95: 5 E / Z,>20: 1 \mathrm{rr}$ ) as a colorless oil. $\mathbf{R}_{\boldsymbol{f}}$ (pentane/EtOAc $\left.=4 / 1\right) 0.21 .{ }^{1} \mathbf{H}$ NMR (599 MHz, CDCl 3 ): $\delta$ ppm 7.95 - 7.92 (m, 2H), $7.84-7.80$ (m, 2H), 7.69 (dd, 
$J=5.3,3.0 \mathrm{~Hz}, 2 \mathrm{H}), 6.84(\mathrm{~d}, J=8.8 \mathrm{~Hz}, 2 \mathrm{H}), 5.82-5.77(\mathrm{~m}, 1 \mathrm{H}), 5.54-5.50(\mathrm{~m}$, 1H), $4.28-4.23(\mathrm{~m}, 2 \mathrm{H}), 4.02(\mathrm{t}, J=7.2 \mathrm{~Hz}, 2 \mathrm{H}), 3.87$ (d, $J=0.8 \mathrm{~Hz}, 3 \mathrm{H}), 1.99$ (dd, $J$ = 7.6, $1.3 \mathrm{~Hz}, 2 \mathrm{H}), 1.70(\mathrm{t}, J=7.2 \mathrm{~Hz}, 2 \mathrm{H}), 0.93(\mathrm{~s}, 6 \mathrm{H}) .{ }^{13} \mathbf{C}$ NMR (151 MHz, $\left.\mathbf{C D C l}_{3}\right)$ : $\delta$ ppm 168.1, 167.0, 162.8, 134.0, 132.2, 131.6, 131.6, 126.2, 123.3, 122.4, 114.1, 65.2, 51.9, 45.2, 40.2, 39.7, 32.9, 27.3. HRMS (ESI'): calcd. for $\mathrm{C}_{25} \mathrm{H}_{27} \mathrm{NO}_{5} \mathrm{Na}(\mathrm{M}+$ $\mathrm{Na}^{+}$): 444.1781. Found: 444.1776 .
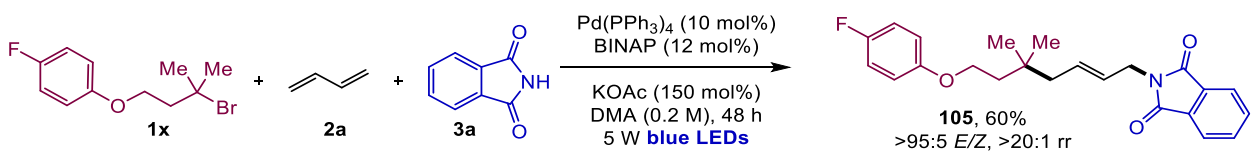

(E)-2-(7-(4-Fluorophenoxy)-5,5-dimethylhept-2-en-1-yl)isoindoline-1,3-

dione (105). According to the general procedure $K$, using the corresponding alkyl bromide $1 x$ (0.3 mmol, 1.5 equiv.), butadiene 2a $(0.3 \mathrm{mmol}, 0.15 \mathrm{~mL}, 2 \mathrm{M}$ in THF, 1.5 equiv.), phthalimide $3 a\left(0.2 \mathrm{mmol}, 1.0\right.$ equiv.), $\mathrm{Pd}\left(\mathrm{PPh}_{3}\right)_{4}\left(10 \mathrm{~mol}^{2}\right)$, BINAP (12 mol\%), KOAc (150 mol\%), and stirring at room temperature for $48 \mathrm{~h}$ under $5 \mathrm{~W}$ blue LEDs irradiation. The reaction mixture was filtered through a pad of silica gel and concentrated in vacuo, then purified by chromatography (pentane/EtOAc $=9 / 1$ ) to give 105 (45.8 mg, 0.120 mmol, 60\%, > 95:5 $E / Z,>20: 1 \mathrm{rr}$ ) as a colorless oil. $\mathbf{R}_{\boldsymbol{f}}$ (pentane/EtOAc $=4 / 1$ ) 0.35. ${ }^{1} \mathbf{H}$ NMR (599 MHz, $\mathbf{C D C l}_{3}$ ): $\delta$ ppm $7.83(\mathrm{dd}, J=5.4,3.1 \mathrm{~Hz}, 2 \mathrm{H}), 7.70(\mathrm{dd}, J=5.5,3.0 \mathrm{~Hz}, 2 \mathrm{H}$ ), $6.92(\mathrm{dd}, J=9.2,8.2 \mathrm{~Hz}, 2 \mathrm{H}), 6.85-6.68(\mathrm{~m}, 2 \mathrm{H}), 5.84-5.75(\mathrm{~m}, 1 \mathrm{H}), 5.55-5.49$ $(\mathrm{m}, 1 \mathrm{H}), 4.25(\mathrm{dt}, J=6.3,1.1 \mathrm{~Hz}, 2 \mathrm{H}), 3.93(\mathrm{t}, J=7.2 \mathrm{~Hz}, 2 \mathrm{H}), 1.98(\mathrm{dt}, J=7.6,1.1$ $\mathrm{Hz}, 2 \mathrm{H}), 1.68(\mathrm{t}, J=7.2 \mathrm{~Hz}, 2 \mathrm{H}), 0.93(\mathrm{~s}, 6 \mathrm{H}) .{ }^{13} \mathbf{C} \mathbf{~ N M R}\left(151 \mathbf{M H z}, \mathbf{C D C l}_{3}\right): \delta \mathrm{ppm}$ 168.1, $157.2 U=237.1 \mathrm{~Hz}), 155.2(U=1.5 \mathrm{~Hz}), 134.0,132.3,131.7,126.1,123.4$, $115.8(\mathrm{~J}=22.7 \mathrm{~Hz}), 115.5 \mathrm{U}=9.1 \mathrm{~Hz}), 65.5,45.3,40.4,39.8,32.9,27.4 .{ }^{19} \mathbf{F}\left\{{ }^{1} \mathbf{H}\right\}$ NMR (564 MHz, $\mathbf{C D C l}_{3}$ ): $\delta$ ppm -124.5. HRMS (ESI+): calcd. for $\mathrm{C}_{23} \mathrm{H}_{24} \mathrm{NO}_{3} \mathrm{Na}(\mathrm{M}$ $+\mathrm{Na}^{+}$): 404.1632. Found: 404.1631 .
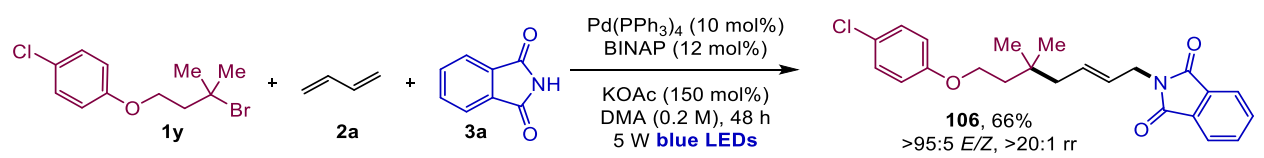


\section{(E)-2-(7-(4-Chlorophenoxy)-5,5-dimethylhept-2-en-1-yl)isoindoline-1,3-}

dione (106). According to the general procedure K, using the corresponding alkyl bromide $1 \mathbf{y}$ ( $0.3 \mathrm{mmol}, 1.5$ equiv.), butadiene $2 \mathbf{a}(0.3 \mathrm{mmol}, 0.15 \mathrm{~mL}, 2 \mathrm{M}$ in THF, 1.5 equiv.), phthalimide за (0.2 mmol, 1.0 equiv.), $\mathrm{Pd}\left(\mathrm{PPh}_{3}\right)_{4}(10 \mathrm{~mol} \%)$, BINAP (12 mol\%), KOAc (150 mol\%), and stirring at room temperature for $48 \mathrm{~h}$ under $5 \mathrm{~W}$ blue LEDs irradiation. The reaction mixture was filtered through a pad of silica gel and concentrated in vacuo, then purified by chromatography (pentane/EtOAc $=9 / 1)$ to give $106(52.7 \mathrm{mg}, 0.133 \mathrm{mmol}, 66 \%$, > 95:5 $E / Z,>20: 1 \mathrm{rr}$ ) as a colorless oil. $\mathbf{R}_{\boldsymbol{f}}($ pentane $/ E t O A c=4 / 1)$ 0.82. ${ }^{1} \mathbf{H}$ NMR (599 MHz, $\mathbf{C D C l}_{3}$ ): $\delta$ ppm $7.83(\mathrm{dd}, J=5.4,3.0 \mathrm{~Hz}, 2 \mathrm{H}), 7.70(\mathrm{dd}, J=5.4,3.0 \mathrm{~Hz}, 2 \mathrm{H})$, $7.18(\mathrm{~d}, J=9.0 \mathrm{~Hz}, 2 \mathrm{H}), 6.76(\mathrm{~d}, J=9.0 \mathrm{~Hz}, 2 \mathrm{H}), 5.83-5.76(\mathrm{~m}, 1 \mathrm{H}), 5.57-5.49(\mathrm{~m}$, 1H), $4.29-4.23(\mathrm{~m}, 2 \mathrm{H}), 3.94(\mathrm{t}, J=7.2 \mathrm{~Hz}, 2 \mathrm{H}), 1.98(\mathrm{dd}, J=7.5,1.3 \mathrm{~Hz}, 2 \mathrm{H}), 1.68$ $(\mathrm{t}, J=7.2 \mathrm{~Hz}, 2 \mathrm{H}), 0.93(\mathrm{~s}, 6 \mathrm{H}) .{ }^{13} \mathbf{C}$ NMR (151 MHz, $\left.\mathbf{C D C l}_{3}\right) \delta$ 168.1, 157.7, 134.0, 132.3, 131.7, 129.3, 126.2, 125.4, 123.4, 115.8, 65.3, 45.3, 40.3, 39.8, 32.9, 27.4. HRMS (ESI+): calcd. for $\mathrm{C}_{23} \mathrm{H}_{24} \mathrm{NO}_{3} \mathrm{ClNa}\left(\mathrm{M}+\mathrm{Na}^{+}\right): 420.1337$. Found: 420.1334 .

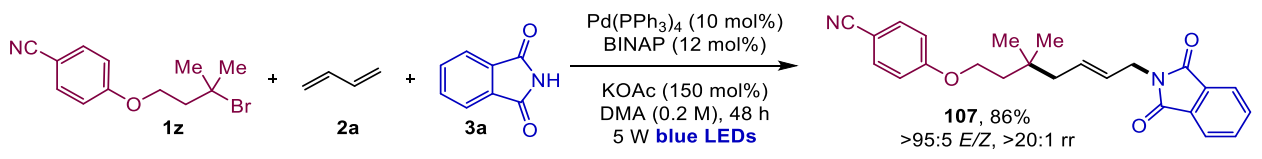

(E)-4-((7-(1,3-Dioxoisoindolin-2-yl)-3,3-dimethylhept-5-en-1-

yl)oxy)benzonitrile (107). According to the general procedure $K$, using the corresponding alkyl bromide $\mathbf{1 z}(0.3 \mathrm{mmol}, 1.5$ equiv.), butadiene $\mathbf{2 a}(0.3 \mathrm{mmol}$, $0.15 \mathrm{~mL}, 2 M$ in THF, 1.5 equiv.), phthalimide 3a (0.2 mmol, 1.0 equiv.), $\mathrm{Pd}\left(\mathrm{PPh}_{3}\right)_{4}(10 \mathrm{~mol} \%)$, BINAP (12 mol\%), KOAc (150 mol\%), and stirring at room temperature for $48 \mathrm{~h}$ under $5 \mathrm{~W}$ blue LEDs irradiation. The reaction mixture was filtered through a pad of silica gel and concentrated in vacuo, then purified by chromatography (pentane/EtOAc $=4 / 1)$ to give $107(66.4 \mathrm{mg}, 0.171 \mathrm{mmol}$, $86 \%,>95: 5 E / Z,>20: 1 \mathrm{rr}$ ) as a colorless oil. $\mathbf{R}_{\boldsymbol{f}}$ (pentane/EtOAc $\left.=4 / 1\right) 0.24 .{ }^{1} \mathbf{H}$ NMR (599 MHz, $\left.\mathbf{C D C l}_{3}\right): \delta$ ppm $7.85-7.80(\mathrm{~m}, 2 \mathrm{H}), 7.71-7.67(\mathrm{~m}, 2 \mathrm{H}), 7.56-$ $7.50(\mathrm{~m}, 2 \mathrm{H}), 6.92-6.84(\mathrm{~m}, 2 \mathrm{H}), 5.83-5.74(\mathrm{~m}, 1 \mathrm{H}), 5.56-5.44(\mathrm{~m}, 1 \mathrm{H}), 4.26-$ $4.21(\mathrm{~m}, 2 \mathrm{H}), 4.01(\mathrm{t}, J=7.1 \mathrm{~Hz}, 2 \mathrm{H}), 1.98(\mathrm{dd}, J=7.5,1.3 \mathrm{~Hz}, 2 \mathrm{H}), 1.70(\mathrm{t}, J=7.1$ 
$\mathrm{Hz}, 2 \mathrm{H}), 0.93$ (s, 6H). ${ }^{13} \mathbf{C}$ NMR (151 MHz, $\mathbf{C D C l}_{3}$ ): $\delta$ ppm 168.0, 162.3, 132.2, 131.4, 126.3, 123.3, 119.4, 115.2, 103.7, 65.4, 45.2, 39.9, 39.7, 32.9, 27.4, 27.4. HRMS (ESI ${ }^{+}$): calcd. for $\mathrm{C}_{24} \mathrm{H}_{24} \mathrm{~N}_{2} \mathrm{O}_{3} \mathrm{Na}\left(\mathrm{M}+\mathrm{Na}^{+}\right)$: 411.1679. Found: 411.1692 .

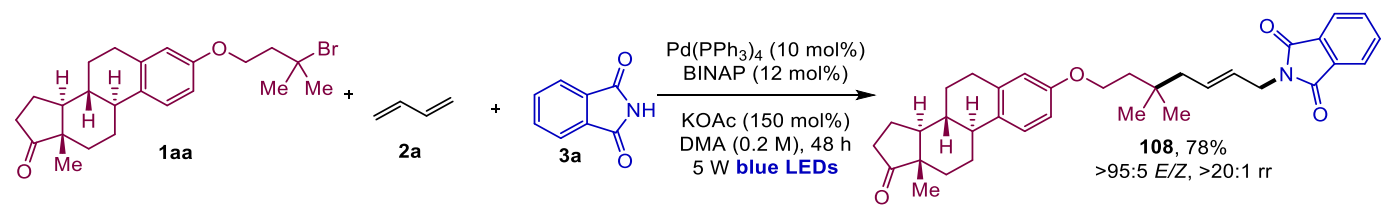

2-((E)-5,5-Dimethyl-7-(((8R,9S,13S,14S)-13-methyl-17-oxo-

\section{$7,8,9,11,12,13,14,15,16,17-d e c a h y d r o-6 H$-cyclopenta[ $a]$ phenanthren-3-}

yl)oxy)hept-2-en-1-yl)isoindoline-1,3-dione (107). According to the general procedure $\mathrm{K}$, using the corresponding alkyl bromide 1aa ( $0.3 \mathrm{mmol}, 1.5$ equiv.), butadiene 2a ( $0.3 \mathrm{mmol}, 0.15 \mathrm{~mL}, 2 \mathrm{M}$ in THF, 1.5 equiv.), phthalimide 3a $(0.2$ mmol, 1.0 equiv.), $\mathrm{Pd}\left(\mathrm{PPh}_{3}\right)_{4}(10 \mathrm{~mol} \%)$, BINAP (12 mol\%), KOAc (150 mol\%), and stirring at room temperature for $48 \mathrm{~h}$ under $5 \mathrm{~W}$ blue LEDs irradiation. The reaction mixture was filtered through a pad of silica gel and concentrated in vacuo, then purified by chromatography (pentane/EtOAc $=4 / 1$ ) to give 107 (84.5 mg, $0.157 \mathrm{mmol}, 78 \%,>95: 5 \mathrm{E} / \mathrm{Z},>20: 1 \mathrm{rr}$ ) as a colorless oil. $\mathbf{R}_{\boldsymbol{f}}$ (pentane/EtOAc = 4/1) 0.08. ${ }^{1} \mathbf{H}$ NMR (599 $\left.\mathbf{M H z}, \mathbf{C D C l}_{3}\right): \delta$ ppm $7.84(\mathrm{dd}, J=5.4$, $3.1 \mathrm{~Hz}, 2 \mathrm{H}), 7.70(\mathrm{dd}, J=5.4,3.0 \mathrm{~Hz}, 2 \mathrm{H}), 7.16(\mathrm{dd}, J=8.8,1.1 \mathrm{~Hz}, 1 \mathrm{H}), 6.67(\mathrm{dd}, J=$ 8.5, $2.9 \mathrm{~Hz}, 1 \mathrm{H}), 6.64-6.54(\mathrm{~m}, 1 \mathrm{H}), 5.87-5.77$ (m, 1H), $5.58-5.50(\mathrm{~m}, 1 \mathrm{H}), 4.26$ $(\mathrm{dd}, J=6.3,1.3 \mathrm{~Hz}, 2 \mathrm{H}), 4.00-3.87(\mathrm{~m}, 2 \mathrm{H}), 2.93-2.84(\mathrm{~m}, 2 \mathrm{H}), 2.55-2.47(\mathrm{~m}$, 1H), $2.43-2.30(\mathrm{~m}, 1 \mathrm{H}), 2.24(\mathrm{dd}, J=10.6,6.0 \mathrm{~Hz}, 1 \mathrm{H}), 2.14(\mathrm{dt}, J=19.0,9.0 \mathrm{~Hz}$, 1H), $2.06-1.91(\mathrm{~m}, 5 \mathrm{H}), 1.68(\mathrm{t}, J=7.1 \mathrm{~Hz}, 2 \mathrm{H}), 1.63-1.39(\mathrm{~m}, 6 \mathrm{H}), 0.93(\mathrm{~s}, 6 \mathrm{H})$, 0.91 (s, 3H). ${ }^{13}$ C NMR (151 MHz, CDCl$\left._{3}\right): \delta$ ppm 221.2, 168.1, 157.1, 137.8, 134.0, 132.3, 131.9, 131.8, 126.4, 126.0, 123.3, 114.6, 112.2, 64.8, 50.5, 45.3, 44.1, 40.5, 39.8, 38.5, 36.0, 32.9, 31.7, 29.7, 27.3, 27.3, 26.7, 26.0, 21.7, 14.0. HRMS (ESI+): calcd. for $\mathrm{C}_{35} \mathrm{H}_{41} \mathrm{NO}_{4} \mathrm{Na}\left(\mathrm{M}+\mathrm{Na}^{+}\right)$: 562.2928. Found: 562.2929 . 


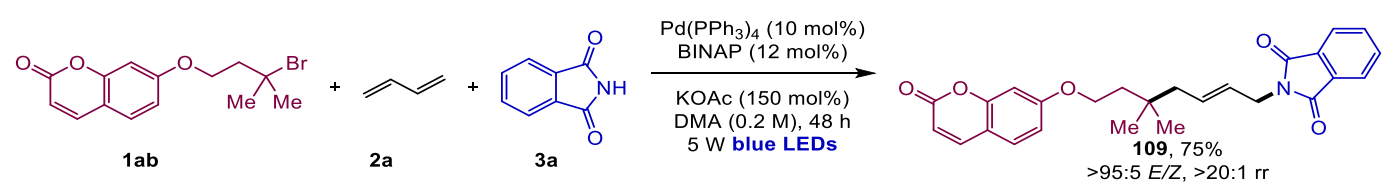

(E)-2-(5,5-Dimethyl-7-((2-oxo-2H-chromen-7-yl)oxy)hept-2-en-1-

yl)isoindoline-1,3-dione (109). According to the general procedure $K$, using the bromide $\mathbf{1} \mathbf{a b}(0.3 \mathrm{mmol}, 1.5$ equiv.), butadiene $\mathbf{2 a}(0.3 \mathrm{mmol}, 0.15 \mathrm{~mL}, 2 \mathrm{M}$ in THF, 1.5 equiv.), phthalimide 3a (0.2 mmol, 1.0 equiv.), $\mathrm{Pd}\left(\mathrm{PPh}_{3}\right)_{4}(0.020 \mathrm{mmol}$, $10 \mathrm{~mol} \%)$, BINAP (0.024 mmol, $12 \mathrm{~mol} \%)$, KOAc (150 mol\%), and stirring at room temperature for $48 \mathrm{~h}$ under $5 \mathrm{~W}$ blue LEDs irradiation. The reaction mixture was filtered through a pad of silica gel and concentrated in vacuo, then purified by chromatography (pentane/EtOAc $=7 / 1 \rightarrow 6 / 1 \rightarrow 4 / 1 \rightarrow 7 / 3 \rightarrow 3 / 2$ ) to give 109 (64.5 mg, $0.149 \mathrm{mmol}, 75 \%,>95: 5 E / Z,>20: 1 \mathrm{rr}$ ) as a colorless gum. $\mathbf{R}_{\boldsymbol{f}}$ (pentane $/$ EtOAc $\left.=3 / 2\right)$ 0.40. ${ }^{1} \mathbf{H}$ NMR $\left(\mathbf{4 0 0} \mathbf{~ M H z}, \mathbf{C D C l}_{3}\right): \delta$ ppm $7.84-7.78$ (m, 2H), $7.72-7.66(\mathrm{~m}, 2 \mathrm{H}), 7.61(\mathrm{~d}, J=9.5 \mathrm{~Hz}, 1 \mathrm{H}), 7.32(\mathrm{~d}, J=8.5 \mathrm{~Hz}, 1 \mathrm{H}), 6.77$ $(\mathrm{dd}, J=8.5,2.4 \mathrm{~Hz}, 1 \mathrm{H}), 6.74(\mathrm{~d}, J=2.4 \mathrm{~Hz}, 1 \mathrm{H}), 6.21(\mathrm{~d}, J=9.5 \mathrm{~Hz}, 1 \mathrm{H}), 5.78(\mathrm{dtt}, J$ $=15.3,7.5,1.1 \mathrm{~Hz}, 1 \mathrm{H}), 5.52(\mathrm{dtt}, J=15.3,6.3,1.3 \mathrm{~Hz}, 1 \mathrm{H}), 4.25(\mathrm{~d}, J=6.3 \mathrm{~Hz}, 2 \mathrm{H})$, $4.03(\mathrm{t}, J=7.1 \mathrm{~Hz}, 2 \mathrm{H}), 1.98(\mathrm{~d}, J=7.5 \mathrm{~Hz}, 2 \mathrm{H}), 1.71(\mathrm{t}, J=7.1 \mathrm{~Hz}, 2 \mathrm{H}), 0.93(\mathrm{~s}, 6 \mathrm{H})$. ${ }^{13}$ C NMR (101 MHz, $\mathrm{CDCl}_{3}$ ): $\delta$ ppm 168.0, 162.3, 161.3, 156.0, 143.5, 134.0, 132.2, 131.5, 128.8, 126.3, 123.3, 113.02, 112.96, 112.5, 101.4, 65.7, 45.3, 40.0, 39.7, 32.9, 27.3. HRMS (ESI $\left.{ }^{+}\right)$: calcd. for $\mathrm{C}_{26} \mathrm{H}_{25} \mathrm{NO}_{5} \mathrm{Na}\left(\mathrm{M}+\mathrm{Na}^{+}\right): 454.1625$. Found: 454.1617.

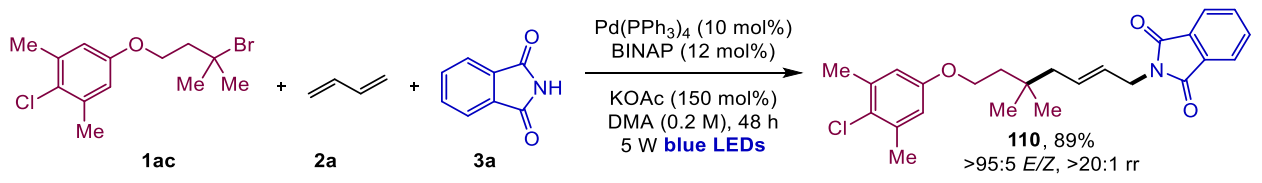

(E)-2-(7-(4-Chloro-3,5-dimethylphenoxy)-5,5-dimethylhept-2-en-1-

yl)isoindoline-1,3-dione (110). According to the general procedure $K$, using the bromide 1 ac $(0.3 \mathrm{mmol}, 1.5$ equiv.), butadiene $2 \mathrm{a}(0.3 \mathrm{mmol}, 0.15 \mathrm{~mL}, 2 \mathrm{M}$ in THF, 1.5 equiv.), phthalimide 3a (0.2 mmol, 1.0 equiv.), $\mathrm{Pd}\left(\mathrm{PPh}_{3}\right)_{4}(0.020 \mathrm{mmol}$, $10 \mathrm{~mol} \%)$, BINAP (0.024 mmol, $12 \mathrm{~mol} \%$ ), KOAc (150 mol\%), and stirring at 
room temperature for $48 \mathrm{~h}$ under $5 \mathrm{~W}$ blue LEDs irradiation. The reaction mixture was filtered through a pad of silica gel and concentrated in vacuo, then purified by chromatography (pentane/EtOAc $=15 / 1 \rightarrow 13 / 1)$ to give $110(75.6$ mg, $0.177 \mathrm{mmol}, 89 \%,>95: 5 E / Z,>20: 1 \mathrm{rr}$ ) as a colorless gum. $\mathbf{R}_{\boldsymbol{f}}$ (pentane/EtOAc = 15/1) 0.30. ${ }^{\mathbf{1}} \mathbf{H}$ NMR (400 $\left.\mathbf{M H z}, \mathbf{C D C l}_{3}\right): \delta$ ppm $7.85-7.79(\mathrm{~m}$, 2H), $7.72-7.66(\mathrm{~m}, 2 \mathrm{H}), 6.58(\mathrm{~s}, 2 \mathrm{H}), 5.80(\mathrm{dtt}, J=15.3,7.5,1.3 \mathrm{~Hz}, 1 \mathrm{H}), 5.53(\mathrm{dtt}$, $J=15.2,6.2,1.2 \mathrm{~Hz}, 1 \mathrm{H}), 4.26(\mathrm{~d}, J=6.3 \mathrm{~Hz}, 2 \mathrm{H}), 3.92(\mathrm{t}, J=7.1 \mathrm{~Hz}, 2 \mathrm{H}), 2.32(\mathrm{~s}$, 6H), $1.98(\mathrm{~d}, J=7.6 \mathrm{~Hz}, 2 \mathrm{H}), 1.67(\mathrm{t}, J=7.1 \mathrm{~Hz}, 2 \mathrm{H}), 0.93(\mathrm{~s}, 6 \mathrm{H}) .{ }^{13} \mathbf{C}$ NMR (101 MHz, $\mathbf{C D C l}_{3}$ ): $\delta$ ppm 168.1, 156.9, 137.1, 134.0, 132.3, 131.7, 126.2, 126.1, 123.3, 114.5, 65.0, 45.2, 40.4, 39.8, 32.9, 27.4, 21.0. HRMS (ESI+): calcd. for $\mathrm{C}_{25} \mathrm{H}_{28} \mathrm{NO}_{3}{ }^{35} \mathrm{ClNa}\left(\mathrm{M}+\mathrm{Na}^{+}\right): 448.1655$. Found: 448.1643.

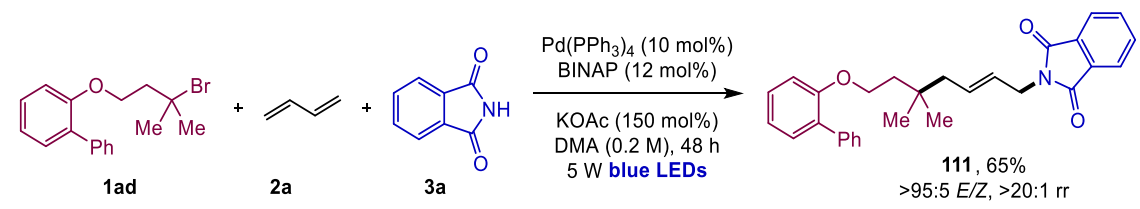

(E)-2-(7-([1,1'-Biphenyl]-2-yloxy)-5,5-dimethylhept-2-en-1-yl)isoindoline-

1,3-dione (111). According to the general procedure $K$, using the bromide 1ad ( $0.3 \mathrm{mmol}, 1.5$ equiv.), butadiene $2 \mathrm{a}(0.3 \mathrm{mmol}, 0.15 \mathrm{~mL}, 2 M$ in THF, 1.5 equiv.), phthalimide 3a (0.2 mmol, 1.0 equiv.), $\mathrm{Pd}\left(\mathrm{PPh}_{3}\right)_{4}(0.020 \mathrm{mmol}, 10 \mathrm{~mol} \%)$, BINAP (0.024 mmol, $12 \mathrm{~mol} \%), \mathrm{KOAc}(150 \mathrm{~mol} \%)$, and stirring at room temperature for $48 \mathrm{~h}$ under $5 \mathrm{~W}$ blue LEDs irradiation. The reaction mixture was filtered through a pad of silica gel and concentrated in vacuo, then purified by chromatography (pentane $/$ EtOAc $=10 / 1)$ to give $111(57.2 \mathrm{mg}, 0.130 \mathrm{mmol}, 65 \%,>95: 5 \mathrm{E} / Z$, > 20:1 rr) as a colorless gum. $\mathbf{R}_{\boldsymbol{f}}$ (pentane/EtOAc $\left.=12 / 1\right)$ 0.20. ${ }^{1} \mathbf{H} \mathbf{~ N M R}(400 \mathbf{~ M H z}$, $\left.\mathbf{C D C l}_{3}\right): \delta$ ppm $7.88-7.81(\mathrm{~m}, 2 \mathrm{H}), 7.74-7.67(\mathrm{~m}, 2 \mathrm{H}), 7.55-7.49(\mathrm{~m}, 2 \mathrm{H}), 7.42$ - $7.36(\mathrm{~m}, 2 \mathrm{H}), 7.34-7.25(\mathrm{~m}, 3 \mathrm{H}), 7.01(\mathrm{td}, J=7.5,1.1 \mathrm{~Hz}, 1 \mathrm{H}), 6.97$ (d, $J=8.2 \mathrm{~Hz}$, 1H), $5.75(\mathrm{dtt}, J=15.0,7.4,1.3 \mathrm{~Hz}, 1 \mathrm{H}), 5.47(\mathrm{dtt}, J=15.1,6.3,1.2 \mathrm{~Hz}, 1 \mathrm{H}), 4.25(\mathrm{~d}$, $J=6.4 \mathrm{~Hz}, 2 \mathrm{H}), 4.00(\mathrm{t}, J=7.2 \mathrm{~Hz}, 2 \mathrm{H}), 1.92(\mathrm{~d}, J=7.3 \mathrm{~Hz}, 2 \mathrm{H}), 1.65(\mathrm{t}, J=7.1 \mathrm{~Hz}$, 2H), 0.86 (s, 6H). ${ }^{13} \mathbf{C}\left\{{ }^{1} \mathbf{H}\right\}$ NMR (101 $\left.\mathbf{M H z}, \mathbf{C D C l}_{3}\right): \delta$ ppm 168.1, 156.1, 138.8, 134.0, 132.3, 131.8, 131.1, 131.0, 129.7, 128.6, 127.9, 126.8, 126.0, 123.4, 120.8, 
112.5, 65.5, 45.2, 40.4, 39.8, 32.8, 27.3. HRMS (ESI+): calcd. for $\left(\mathrm{C}_{29} \mathrm{H}_{29} \mathrm{NO}_{3}\right)_{2} \mathrm{Na}$ $\left(2 \mathrm{M}+\mathrm{Na}^{+}\right):$901.4193. Found: 901.4187.

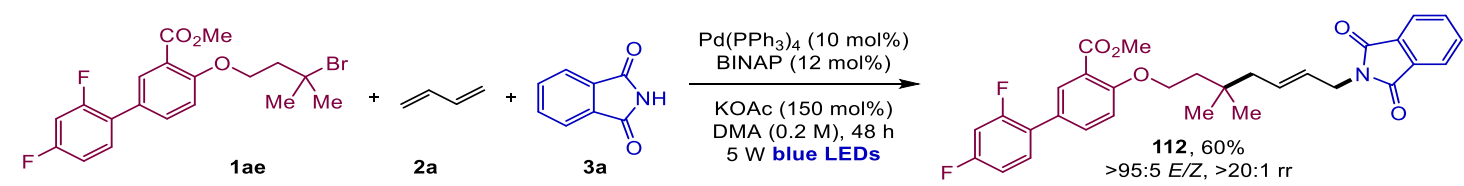

Methyl (E)-4-((7-(1,3-dioxoisoindolin-2-yl)-3,3-dimethylhept-5-en-1yl)oxy)-2',4'-difluoro-[1,1'-biphenyl]-3-carboxylate (112). According to the general procedure $\mathrm{E}$, using the bromide 1ae $(0.3 \mathrm{mmol}, 1.5$ equiv.), butadiene $2 \mathbf{a}$ (0.3 mmol, $0.15 \mathrm{~mL}, 2 M$ in THF, 1.5 equiv.), phthalimide 3a (0.2 mmol, 1.0 equiv.), $\mathrm{Pd}\left(\mathrm{PPh}_{3}\right)_{4}$ (0.020 mmol, $10 \mathrm{~mol} \%$ ), BINAP (0.024 mmol, $12 \mathrm{~mol} \%$ ), KOAc (150 mol\%), and stirring at room temperature for $48 \mathrm{~h}$ under $5 \mathrm{~W}$ blue LEDs irradiation. The reaction mixture was filtered through a pad of silica gel and concentrated in vacuo, then purified by chromatography (pentane/EtOAc $=6 / 1$ $\rightarrow 5 / 1 \rightarrow 4 / 1$ ) to give 112 (64.1 mg, $0.120 \mathrm{mmol}, 60 \%,>95: 5 E / Z,>20: 1 \mathrm{rr}$ ) as a white solid. $\mathbf{R}_{\boldsymbol{f}}$ (pentane/EtOAc $=4 / 1$ ) 0.30. Note: The compound is sparingly soluble in $\mathrm{CDCl}_{3}$, thus causing a broadened ${ }^{1} \mathrm{H}$ NMR spectrum. Alternate solvents must be used. ${ }^{1} \mathbf{H}\left\{{ }^{19} \mathbf{F}\right\}$ NMR (599 MHz, acetone-d $)$ : $\delta$ ppm $7.84-7.79(\mathrm{~m}, 5 \mathrm{H})$, $7.62(\mathrm{dd}, J=8.7,2.4 \mathrm{~Hz}, 1 \mathrm{H}), 7.54(\mathrm{dd}, J=8.4,0.5 \mathrm{~Hz}, 1 \mathrm{H}), 7.21(\mathrm{~d}, J=8.8 \mathrm{~Hz}, 1 \mathrm{H})$, $7.11(\mathrm{dd}, J=2.7,0.5 \mathrm{~Hz}, 1 \mathrm{H}), 7.09$ (dd, $J=8.4,2.6 \mathrm{~Hz}, 1 \mathrm{H}$ ), 5.82 (dtt, $J=15.1,7.5$, $1.4 \mathrm{~Hz}, 1 \mathrm{H}), 5.59(\mathrm{dtt}, J=15.3,6.0,1.3 \mathrm{~Hz}, 1 \mathrm{H}), 4.26-4.23(\mathrm{~m}, 2 \mathrm{H}), 4.18(\mathrm{t}, J=6.9$ $\mathrm{Hz}, 2 \mathrm{H}), 3.82(\mathrm{~s}, 3 \mathrm{H}), 2.08-2.06(\mathrm{~m}, 2 \mathrm{H}), 1.76(\mathrm{t}, J=6.9 \mathrm{~Hz}, 2 \mathrm{H}), 0.97(\mathrm{~s}$, 6H). ${ }^{13}$ C $\left\{{ }^{1} \mathrm{H}\right\}$ NMR (151 MHz, acetone-d 6$): \delta$ ppm 168.3, 167.1, $163.0(\mathrm{dd}, J=$ 247.2, $12.0 \mathrm{~Hz}$ ), 160.5 (dd, $J=248.6,12.1 \mathrm{~Hz}), 158.6,135.00,134.95,134.2$ (d, $J=$ $3.0 \mathrm{~Hz}$ ), 133.1, 132.5 (dd, J = 9.6, $4.8 \mathrm{~Hz}), 132.0$ (d, $J=3.0 \mathrm{~Hz}), 131.6,127.4,127.3$ (d, $J=1.3 \mathrm{~Hz}$ ), 125.1 (dd, $J=13.5,3.8 \mathrm{~Hz}$ ), 123.7 (d, $J=4.6 \mathrm{~Hz}$ ), 122.1, 114.4, $112.6(\mathrm{dd}, J=21.3,3.8 \mathrm{~Hz}$ ), 105.0 (dd, $J=27.1,25.6 \mathrm{~Hz}$ ), 66.8, 52.2, 45.55, 40.9, 40.1, 33.4, 27.6. ${ }^{13} \mathbf{C}\left\{{ }^{1} \mathbf{H},{ }^{19}\right.$ F $\}$ NMR (151 MHz, acetone-d $)$ : $\delta$ ppm 168.3, 167.1, 163.0, 160.5, 158.6, 135.00, 134.95, 134.2, 133.1, 132.5, 132.0, 131.6, 127.4, 127.3, 125.1, 123.8, 123.7, 122.1, 114.4, 112.6, 105.0, 66.8, 52.2, 45.6, 40.9, 40.1, 
33.4, 27.6. ${ }^{19} \mathbf{F}\left\{{ }^{1} \mathbf{H}\right\}$ NMR (564 MHz, acetone-d 6 ): $\delta$ ppm -113.95 (d, ${ }^{3} J_{F-F}=7.2 \mathrm{~Hz}$ ), $-115.89\left(\mathrm{~d},{ }^{3} J_{F-F}=7.2 \mathrm{~Hz}\right)$. HRMS (ESI $\left.{ }^{+}\right)$: calcd. for $\mathrm{C}_{31} \mathrm{H}_{29} \mathrm{NO}_{5} \mathrm{~F}_{2} \mathrm{Na}\left(\mathrm{M}+\mathrm{Na}^{+}\right)$: 556.1906. Found: 556.1912.

\subsection{General Procedure L}

An oven-dried Schlenk tube containing a stir bar was charged under argon with the corresponding alkyl bromide $1 \mathrm{a}\left(0.3 \mathrm{mmol}, 1.5\right.$ equiv.), $\mathrm{Pd}\left(\mathrm{PPh}_{3}\right)_{4}(10 \mathrm{~mol} \%)$, BINAP (12 mol\%), phthalimide 3a (0.2 mmol, 1.0 equiv.), KOAc (150 mol\%) and DMA (0.85 mL). Subsequently, 1,3-diene 2 ( $0.3 \mathrm{mmol}, 1.5$ equiv.) was added. The reaction mixture was stirred at room temperate under blue LEDs irradiation $(5 \mathrm{~W}, 455 \mathrm{~nm})$ for $48 \mathrm{~h}$. Then, the reaction was filtered through a pad of silica gel and washed with EtOAc $(3 \times 5 \mathrm{~mL})$. The obtained solution was concentrated in vacuo and the diastereomeric ratio determined by ${ }^{1} \mathrm{H}$ NMR spectroscopy of the crude reaction mixture. The reaction mixture was purified by column chromatography on silica gel (pentane/EtOAc) to afford the corresponding compounds.

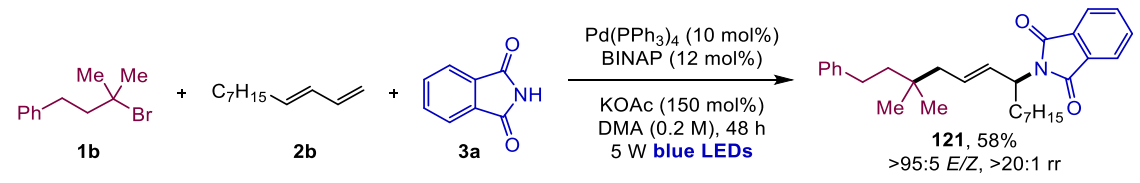

(E)-2-(3,3-Dimethyl-1-phenyltetradec-5-en-7-yl)isoindoline-1,3-dione

(121). According to the general procedure L, using the corresponding alkyl bromide $\mathbf{1 b}$ ( $0.3 \mathrm{mmol}, 1.5$ equiv.), the corresponding diene $\mathbf{2 b}$ ( $0.3 \mathrm{mmol}, 0.15$ $\mathrm{mL}, 2 \mathrm{M}$ in THF, 1.5 equiv.), phthalimide 3a (0.2 mmol, 1.0 equiv.), $\mathrm{Pd}\left(\mathrm{PPh}_{3}\right)_{4}(10$ mol\%), BINAP (12 mol\%), KOAc (150 mol\%), and stirring at room temperature for $48 \mathrm{~h}$ under $5 \mathrm{~W}$ blue LEDs irradiation. The reaction mixture was filtered through a pad of silica gel and concentrated in vacuo, then purified by chromatography (pentane $/$ EtOAc $=9 / 1$ ) to give $121(51.5 \mathrm{mg}, 0.116 \mathrm{mmol}$, $58 \%,>95: 5 E / Z,>20: 1 \mathrm{rr}$ ) as a colorless oil. $\mathbf{R}_{\boldsymbol{f}}$ (pentane/EtOAc $\left.=9 / 1\right) 0.56 .{ }^{1} \mathbf{H}$ NMR (599 MHz, $\mathbf{C D C l}_{3}$ ): $\delta$ ppm $7.82(\mathrm{dd}, J=5.4,3.0 \mathrm{~Hz}, 2 \mathrm{H}), 7.70(\mathrm{dd}, J=5.5,3.0$ 
Hz, 2H), 7.35 - $7.31(\mathrm{~m}, 2 \mathrm{H}), 7.21-7.19(\mathrm{~m}, 1 \mathrm{H}), 7.13$ (d, J = 1.6 Hz, 2H), 5.97 $5.91(\mathrm{~m}, 1 \mathrm{H}), 5.78-5.71(\mathrm{~m}, 1 \mathrm{H}), 4.72(\mathrm{q}, J=8.0 \mathrm{~Hz}, 1 \mathrm{H}), 2.60-2.48(\mathrm{~m}, 2 \mathrm{H})$, $2.04-1.98$ (m, 3H), 1.91 (ddd, $J=13.4,6.4,2.6 \mathrm{~Hz}, 1 \mathrm{H}), 1.49-1.44$ (m, 2H), 1.34 - $1.20(\mathrm{~m}, 12 \mathrm{H}), 0.95-0.90(\mathrm{~m}, 6 \mathrm{H}), 0.86(\mathrm{t}, J=7.1 \mathrm{~Hz}, 3 \mathrm{H}) .{ }^{13} \mathrm{C}$ NMR (151 MHz, $\mathbf{C D C l}_{3}$ ): $\delta$ ppm 168.3, 143.4, 133.9, 133.9, 133.8, 132.2, 131.0, 130.3, 128.8, 128.6, 128.6, 128.4, 125.6, 123.2, 54.1, 44.5, 44.2, 33.6, 32.7, 31.9, 30.8, 29.3, 29.2, 27.2, 27.2, 26.8, 22.7, 14.2. HRMS (ESI $\left.{ }^{+}\right)$: calcd. for $\mathrm{C}_{30} \mathrm{H}_{39} \mathrm{NO}_{2} \mathrm{Na}\left(\mathrm{M}+\mathrm{Na}^{+}\right): 468.2873$. Found: 468.2863.

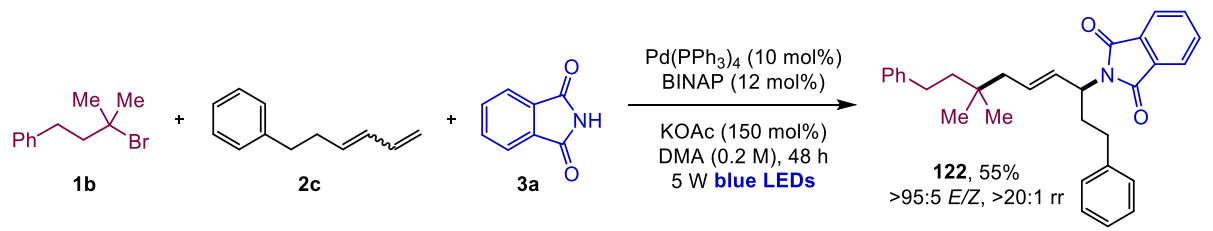

(E)-2-(7,7-Dimethyl-1,9-diphenylnon-4-en-3-yl)isoindoline-1,3-dione (122). According to the general procedure L, using the corresponding alkyl bromide $\mathbf{1 b}$ (0.3 mmol, 1.5 equiv.), the corresponding diene $2 \mathrm{c}(0.3 \mathrm{mmol}, 0.15 \mathrm{~mL}, 2 \mathrm{M}$ in THF, 1.5 equiv.), phthalimide $3 a\left(0.2 \mathrm{mmol}, 1.0\right.$ equiv.), $\mathrm{Pd}\left(\mathrm{PPh}_{3}\right)_{4}(10 \mathrm{~mol} \%)$, BINAP (12 mol\%), KOAc (150 mol\%), and stirring at room temperature for $48 \mathrm{~h}$ under $5 \mathrm{~W}$ blue LEDs irradiation. The reaction mixture was filtered through a pad of silica gel and concentrated in vacuo, then purified by chromatography (pentane/EtOAc $=20 / 1)$ to give 122 (49.8 mg, $0.110 \mathrm{mmol}, 55 \%,>$ 95:5 $E / Z,>20: 1 \mathrm{rr}$ ) as a colorless oil. $\mathbf{R}_{\boldsymbol{f}}$ (pentane/EtOAc $\left.=9 / 1\right)$ 0.63. ${ }^{1} \mathbf{H}$ NMR (500 MHz, $\mathbf{C D C l}_{3}$ ): $\delta$ ppm $7.80(\mathrm{dd}, J=5.4,3.0 \mathrm{~Hz}, 2 \mathrm{H}), 7.69(\mathrm{dd}, J=5.5,3.0 \mathrm{~Hz}, 2 \mathrm{H})$, $7.20(\mathrm{dt}, J=9.1,7.3 \mathrm{~Hz}, 4 \mathrm{H}), 7.15-7.08(\mathrm{~m}, 6 \mathrm{H}), 5.95$ (ddd, $J=15.3,8.3,1.3 \mathrm{~Hz}$, $1 \mathrm{H}), 5.76(\mathrm{dt}, J=15.1,7.4 \mathrm{~Hz}, 1 \mathrm{H}), 4.78(\mathrm{q}, J=8.0 \mathrm{~Hz}, 1 \mathrm{H}), 2.68$ (ddd, $J=13.8,9.9$, $5.7 \mathrm{~Hz}, 1 \mathrm{H}), 2.62-2.49(\mathrm{~m}, 4 \mathrm{H}), 2.48-2.39(\mathrm{~m}, 1 \mathrm{H}), 2.26-2.20(\mathrm{~m}, 1 \mathrm{H}), 2.03-$ $1.98(\mathrm{~m}, 2 \mathrm{H}), 1.50-1.43(\mathrm{~m}, 2 \mathrm{H}), 0.94-0.90(\mathrm{~m}, 6 \mathrm{H}) .{ }^{13} \mathrm{C}$ NMR (126 MHz, $\mathbf{C D C l}_{3}$ ): $\delta$ ppm 168.2, 143.4, 141.1, 133.9, 132.1, 131.4, 130.0, 128.5, 128.5, 128.4, 128.4, 126.0, 125.6, 123.2, 53.9, 44.6, 44.3, 34.2, 33.6, 33.2, 30.8, 27.2, 27.2. HRMS (ESI'): calcd. for $\mathrm{C}_{31} \mathrm{H}_{33} \mathrm{NO}_{2} \mathrm{Na}\left(\mathrm{M}+\mathrm{Na}^{+}\right)$: 474.2404. Found: 474.2399. 


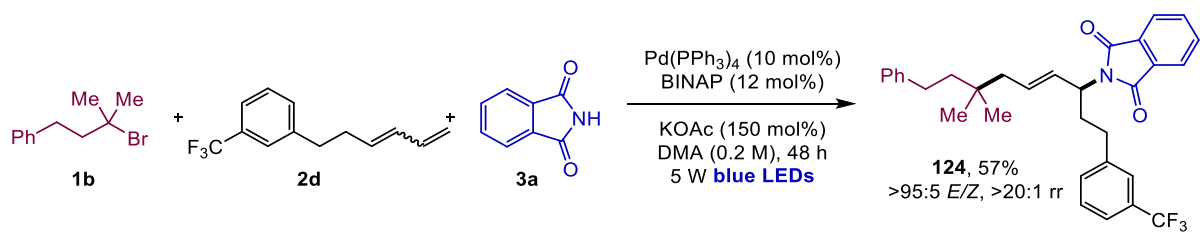

(E)-2-(7,7-Dimethyl-9-phenyl-1-(3-(trifluoromethyl)phenyl)non-4-en-3-

yl)isoindoline-1,3-dione (124). According to the general procedure L, using the bromide $\mathbf{1 b}$ ( $0.3 \mathrm{mmol}, 1.5$ equiv.), diene $\mathbf{2 d}$ ( $0.3 \mathrm{mmol}$, 1.5 equiv.), phthalimide 3a (0.2 mmol, 1.0 equiv.), $\mathrm{Pd}\left(\mathrm{PPh}_{3}\right)_{4}(0.020 \mathrm{mmol}, 10 \mathrm{~mol} \%), \operatorname{BINAP}(0.024$ mmol, $12 \mathrm{~mol} \%)$, KOAc (150 mol\%), and stirring at room temperature for $48 \mathrm{~h}$ under $5 \mathrm{~W}$ blue LEDs irradiation. The reaction mixture was filtered through a pad of silica gel and concentrated in vacuo, then purified by chromatography (pentane/EtOAc $=17 / 1 \rightarrow 15 / 1$ ) to give 124 (58.6 mg, $0.113 \mathrm{mmol}, 57 \%,>95: 5$ $E / Z,>20: 1 \mathrm{rr}$ ) as a colorless gum. $\mathbf{R}_{\boldsymbol{f}}$ (pentane/EtOAc $\left.=15 / 1\right)$ 0.30. ${ }^{\mathbf{1}} \mathbf{H}$ NMR (599 MHz, $\left.\mathbf{C D C l}_{3}\right): \delta$ ppm $7.82-7.77(\mathrm{~m}, 2 \mathrm{H}), 7.72-7.67(\mathrm{~m}, 2 \mathrm{H}), 7.41-7.37(\mathrm{~m}, 1 \mathrm{H})$, 7.38 - 7.33 (m, 1H), 7.33 - $7.30(\mathrm{~m}, 2 \mathrm{H}), 7.22-7.16(\mathrm{~m}, 2 \mathrm{H}), 7.15-7.09(\mathrm{~m}, 3 \mathrm{H})$, $5.96(\mathrm{ddt}, J=15.2,8.3,1.3 \mathrm{~Hz}, 1 \mathrm{H}), 5.77(\mathrm{dtd}, J=15.1,7.5,0.9 \mathrm{~Hz}, 1 \mathrm{H}), 4.84-4.73$ (m, 1H), 2.75 (ddd, $J=14.1,9.7,5.7 \mathrm{~Hz}, 1 \mathrm{H}), 2.63$ (ddd, $J=14.1,9.7,6.5 \mathrm{~Hz}, 1 \mathrm{H}$ ), $2.56-2.51$ (m, 2H), $2.51-2.43(\mathrm{~m}, 1 \mathrm{H}), 2.29-2.19$ (m, 1H), 2.02 (d, J = $7.5 \mathrm{~Hz}$, 2H), $1.51-1.42(\mathrm{~m}, 2 \mathrm{H}), 0.933(\mathrm{~s}, 3 \mathrm{H}), 0.927(\mathrm{~s}, 3 \mathrm{H}) .{ }^{13} \mathbf{C}\left\{{ }^{\mathbf{1}} \mathrm{H},{ }^{19} \mathbf{F}\right\}$ NMR (151 MHz, $\mathbf{C D C l}_{3}$ ): $\delta$ ppm 168.2, 143.3, 142.0, 134.0, 132.0, 131.8, 131.7, 130.7, 129.7, 128.9, 128.40, 128.36, 125.7, 125.1, 124.3, 123.3, 122.9, 53.8, 44.6, 44.2, 33.8, 33.6, 33.1, 30.7, 27.20, 27.19. ${ }^{19} \mathbf{F}\left\{{ }^{1} \mathbf{H}\right\}$ NMR (564 $\mathbf{M H z}, \mathbf{C D C l}_{3}$ ): $\delta$ ppm -62.58. HRMS (ESI'): calcd. for $\mathrm{C}_{32} \mathrm{H}_{32} \mathrm{NO}_{2} \mathrm{~F}_{3} \mathrm{Na}\left(\mathrm{M}+\mathrm{Na}^{+}\right)$: 542.2277. Found: 542.2286.

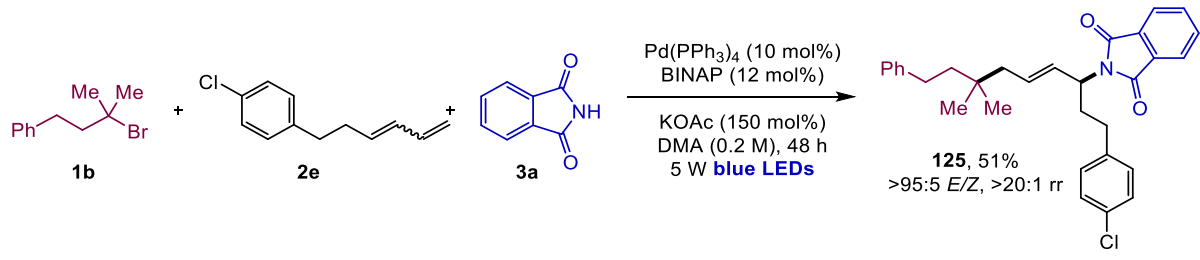

(E)-2-(1-(4-Chlorophenyl)-7,7-dimethyl-9-phenylnon-4-en-3-

yl)isoindoline-1,3-dione (125). According to the general procedure L, using the 
bromide $\mathbf{1 b}$ ( $0.3 \mathrm{mmol}, 1.5$ equiv.), diene $\mathbf{2 e}$ ( $0.3 \mathrm{mmol}, 1.5$ equiv.), phthalimide 3a (0.2 mmol, 1.0 equiv.), $\mathrm{Pd}\left(\mathrm{PPh}_{3}\right)_{4}(0.020 \mathrm{mmol}, 10 \mathrm{~mol} \%)$, BINAP $(0.024$ mmol, $12 \mathrm{~mol} \%)$, KOAc (150 mol\%), and stirring at room temperature for $48 \mathrm{~h}$ under $5 \mathrm{~W}$ blue LEDs irradiation. The reaction mixture was filtered through a pad of silica gel and concentrated in vacuo, then purified by chromatography (pentane/EtOAc $=17 / 1 \rightarrow 15 / 1)$ to give $125(49.4 \mathrm{mg}, 0.102 \mathrm{mmol}, 51 \%$, > 95:5 $E / Z,>20: 1 \mathrm{rr}$ ) as a colorless gum. $\mathbf{R}_{\boldsymbol{f}}$ (pentane/EtOAc $\left.=15 / 1\right)$ 0.30. ${ }^{\mathbf{1}} \mathbf{H} \mathbf{N M R}(\mathbf{5 0 0}$ MHz, $\left.\mathbf{C D C l}_{3}\right): \delta$ ppm $\delta 7.82-7.76(\mathrm{~m}, 2 \mathrm{H}), 7.73-7.67(\mathrm{~m}, 2 \mathrm{H}), 7.22-7.18(\mathrm{~m}$, 2H), $7.17-7.09(\mathrm{~m}, 5 \mathrm{H}), 7.07-7.03(\mathrm{~m}, 2 \mathrm{H}), 5.94(\mathrm{ddt}, J=15.2,8.2,1.2 \mathrm{~Hz}, 1 \mathrm{H})$, $5.75(\mathrm{dtd}, J=15.3,7.5,0.6 \mathrm{~Hz}, 1 \mathrm{H}), 4.88-4.67(\mathrm{~m}, 1 \mathrm{H}), 2.65$ (ddd, $J=14.7,9.4,5.8$ $\mathrm{Hz}, 1 \mathrm{H}$ ), $2.59-2.49$ (m, 3H), $2.49-2.38(\mathrm{~m}, 1 \mathrm{H}), 2.19$ (dddd, $J=13.7,9.4,6.8,5.8$ Hz, 1H), $2.03-1.98(\mathrm{~m}, 2 \mathrm{H}), 1.51-1.43(\mathrm{~m}, 2 \mathrm{H}), 0.93$ (d, J = 2.1 Hz, 6H). ${ }^{13} \mathbf{C}$ NMR (126 MHz, $\mathbf{C D C l}_{3}$ ): $\delta$ ppm 168.2, 143.4, 139.5, 134.0, 132.0, 131.8, 131.5, 129.81, 129.79, 128.5, 128.40, 128.36, 125.7, 123.2, 53.7, 44.6, 44.2, 33.9, 33.6, 32.6, 30.7, 27.22, 27.20. HRMS (ESI+): calcd. for $\mathrm{C}_{31} \mathrm{H}_{32} \mathrm{NO}_{2}{ }^{35} \mathrm{ClNa}\left(\mathrm{M}+\mathrm{Na}^{+}\right)$: 508.2014. Found: 508.2012; calcd. for $\mathrm{C}_{31} \mathrm{H}_{32} \mathrm{NO}_{2}{ }^{37} \mathrm{ClNa}\left(\mathrm{M}+\mathrm{Na}^{+}\right)$: 510.1987. Found: 510.1998.

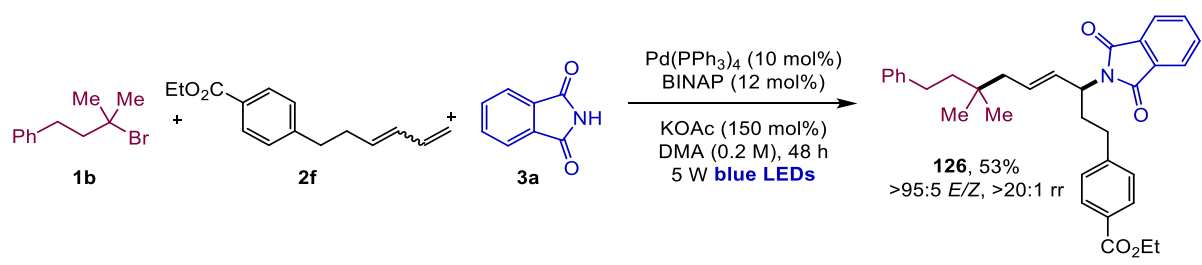

Ethyl (E)-4-(3-(1,3-dioxoisoindolin-2-yl)-7,7-dimethyl-9-phenylnon-4-en-1yl)benzoate (126). According to the general procedure $L$, using the bromide $\mathbf{1 b}$ ( $0.3 \mathrm{mmol}, 1.5$ equiv.), diene $\mathbf{2 f}$ ( $0.3 \mathrm{mmol}, 1.5$ equiv.), phthalimide $3 \mathbf{a}(0.2 \mathrm{mmol}$, 1.0 equiv.), $\mathrm{Pd}\left(\mathrm{PPh}_{3}\right)_{4}(0.020 \mathrm{mmol}, 10 \mathrm{~mol} \%)$, BINAP $(0.024 \mathrm{mmol}, 12 \mathrm{~mol} \%)$, KOAc (150 mol\%), and stirring at room temperature for $48 \mathrm{~h}$ under $5 \mathrm{~W}$ blue LEDs irradiation. The reaction mixture was filtered through a pad of silica gel and concentrated in vacuo, then purified by chromatography (pentane $/$ EtOAc $=$ $10 / 1 \rightarrow 8 / 1$ ) to give 126 (54.8 mg, $0.105 \mathrm{mmol}, 53 \%,>95: 5 E / Z,>20: 1 \mathrm{rr}$ ) as a 
colorless gum. $\mathbf{R}_{\boldsymbol{f}}$ (pentane/EtOAc $\left.=10 / 1\right)$ 0.20. ${ }^{\mathbf{1}} \mathbf{H}$ NMR (500 $\left.\mathbf{M H z}, \mathbf{C D C l}_{3}\right): \delta$ ppm $\delta 7.91-7.84(\mathrm{~m}, 2 \mathrm{H}), 7.82-7.75(\mathrm{~m}, 2 \mathrm{H}), 7.72-7.65(\mathrm{~m}, 2 \mathrm{H}), 7.23-7.16$ (m, 4H), $7.12(\mathrm{tt}, J=7.9,1.4 \mathrm{~Hz}, 3 \mathrm{H}), 5.95(\mathrm{ddt}, J=15.3,8.3,1.2 \mathrm{~Hz}, 1 \mathrm{H}), 5.76(\mathrm{ddt}$, $J=15.3,7.5,0.6 \mathrm{~Hz}, 1 \mathrm{H}), 4.82-4.72(\mathrm{~m}, 1 \mathrm{H}), 4.34(\mathrm{q}, J=7.1 \mathrm{~Hz}, 2 \mathrm{H}), 2.74(\mathrm{ddd}, J=$ 14.7, 9.4, $5.8 \mathrm{~Hz}, 1 \mathrm{H}), 2.62$ (ddd, $J=14.0,9.3,6.6 \mathrm{~Hz}, 1 \mathrm{H}), 2.57-2.50(\mathrm{~m}, 2 \mathrm{H})$, $2.50-2.41(\mathrm{~m}, 1 \mathrm{H}), 2.28-2.18(\mathrm{~m}, 1 \mathrm{H}), 2.01(\mathrm{~d}, J=7.5 \mathrm{~Hz}, 2 \mathrm{H}), 1.50-1.43(\mathrm{~m}$, 2H), $1.38(\mathrm{t}, J=7.1 \mathrm{~Hz}, 3 \mathrm{H}), 0.92(\mathrm{~d}, J=2.2 \mathrm{~Hz}, 6 \mathrm{H}) \cdot{ }^{13} \mathbf{C}$ NMR (126 $\left.\mathbf{M H z}, \mathbf{C D C l}_{3}\right): \delta$ ppm 168.2, 166.6, 146.5, 143.3, 134.0, 132.0, 131.6, 129.79, 129.75, 128.42, 128.40, 128.35, 125.6, 123.3, 60.8, 53.8, 44.6, 44.2, 33.7, 33.6, 33.3, 30.7, 27.21, 27.19, 14.5. HRMS (ESI $\left.{ }^{+}\right)$: calcd. for $\mathrm{C}_{31} \mathrm{H}_{32} \mathrm{NO}_{2}{ }^{35} \mathrm{ClNa}\left(\mathrm{M}+\mathrm{Na}^{+}\right): 508.2014$. Found: 508.2012; calcd. for $\mathrm{C}_{34} \mathrm{H}_{37} \mathrm{NO}_{4} \mathrm{Na}\left(\mathrm{M}+\mathrm{Na}^{+}\right)$: 546.2615. Found: 546.2633.

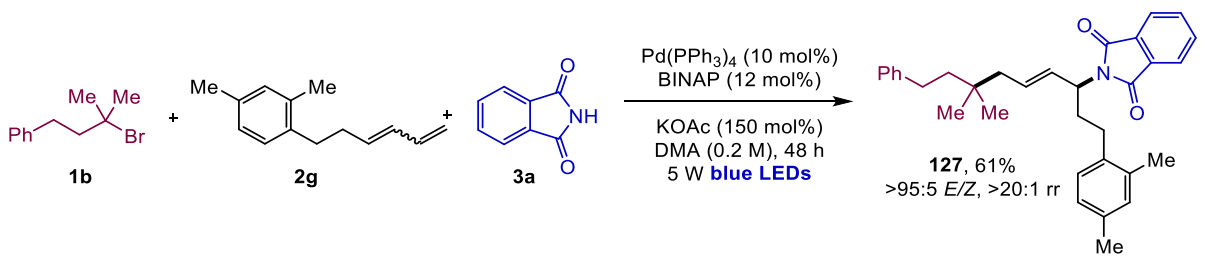

(E)-2-(1-(2,4-Dimethylphenyl)-7,7-dimethyl-9-phenylnon-4-en-3-

yl)isoindoline-1,3-dione (127). According to the general procedure L, using the bromide 1 b ( $0.3 \mathrm{mmol}, 1.5$ equiv.), diene $2 \mathrm{~g}$ ( $0.3 \mathrm{mmol}, 1.5$ equiv.), phthalimide 3a (0.2 mmol, 1.0 equiv.), $\mathrm{Pd}\left(\mathrm{PPh}_{3}\right)_{4}(0.020 \mathrm{mmol}, 10 \mathrm{~mol} \%)$, BINAP (0.024 mmol, $12 \mathrm{~mol} \%)$, KOAc (150 mol\%), and stirring at room temperature for $48 \mathrm{~h}$ under $5 \mathrm{~W}$ blue LEDs irradiation. The reaction mixture was filtered through a pad of silica gel and concentrated in vacuo, then purified by chromatography (pentane/EtOAc $=25 / 1 \rightarrow 20 / 1$ ) to give 127 (58.3 mg, 0.122 mmol, 61\%, > 20:1 $E / Z,>20: 1 \mathrm{rr}$ ) as a colorless gum. $\mathbf{R}_{\boldsymbol{f}}$ (pentane/EtOAc $\left.=20 / 1\right)$ 0.30. ${ }^{\mathbf{1}} \mathbf{H}$ NMR (500 MHz, $\left.\mathbf{C D C l}_{3}\right): \delta$ ppm $\delta 7.85-7.79(\mathrm{~m}, 2 \mathrm{H}), 7.74-7.68(\mathrm{~m}, 2 \mathrm{H}), 7.24-7.16(\mathrm{~m}$, 2H), $7.13(\mathrm{td}, J=7.0,1.3 \mathrm{~Hz}, 3 \mathrm{H}), 6.99(\mathrm{~d}, J=7.6 \mathrm{~Hz}, 1 \mathrm{H}), 6.94-6.85(\mathrm{~m}, 2 \mathrm{H}), 5.95$ (ddt, $J=15.2,8.2,1.2 \mathrm{~Hz}, 1 \mathrm{H}), 5.79(\mathrm{dt}, J=15.1,7.4 \mathrm{~Hz}, 1 \mathrm{H}), 4.83(\mathrm{q}, J=7.9 \mathrm{~Hz}$, 1H), $2.69-2.58(\mathrm{~m}, 1 \mathrm{H}), 2.57-2.46(\mathrm{~m}, 3 \mathrm{H}), 2.44-2.31(\mathrm{~m}, 1 \mathrm{H}), 2.24(\mathrm{~s}, 3 \mathrm{H})$, $2.23(\mathrm{~s}, 3 \mathrm{H}), 2.20-2.08(\mathrm{~m}, 1 \mathrm{H}), 2.07-1.97(\mathrm{~m}, 2 \mathrm{H}), 1.52-1.43(\mathrm{~m}, 2 \mathrm{H}), 0.97-$ 
0.89 (m, 6H). ${ }^{13} \mathrm{C}$ NMR (101 MHz, $\left.\mathbf{C D C l}_{3}\right): \delta$ ppm 168.2, 143.4, 136.4, 135.7, 135.6, 133.9, 132.1, 131.4, 131.1, 130.0, 128.9, 128.39, 128.37, 126.7, 125.6, 123.3, 54.1, 44.6, 44.3, 33.6, 33.1, 30.8, 30.3, 27.2, 21.0, 19.3. Note: Two aliphatic signals overlap in the recorded spectrum. HRMS (ESI+): calcd. for $\mathrm{C}_{33} \mathrm{H}_{37} \mathrm{NO}_{2} \mathrm{Na}(\mathrm{M}$ $+\mathrm{Na}^{+}$): 502.2717. Found: 502.2712.

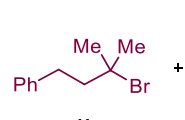

$1 \mathrm{~b}$

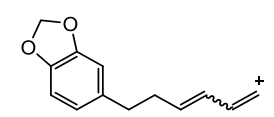

2h

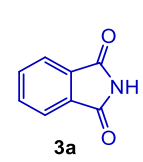

3a $\mathrm{O}$
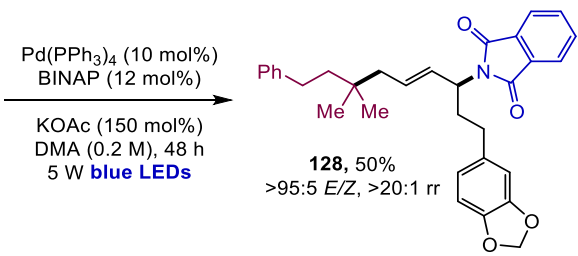

(E)-2-(1-(Benzo[d][1,3]dioxol-5-yl)-7,7-dimethyl-9-phenylnon-4-en-3-

yl)isoindoline-1,3-dione (128). According to the general procedure L, using the bromide $\mathbf{1 b}$ ( $0.3 \mathrm{mmol}, 1.5$ equiv.), diene $\mathbf{2 h}$ ( $0.3 \mathrm{mmol}, 1.5$ equiv.), phthalimide 3a (0.2 mmol, 1.0 equiv.), $\mathrm{Pd}\left(\mathrm{PPh}_{3}\right)_{4}(0.020 \mathrm{mmol}, 10 \mathrm{~mol} \%)$, BINAP (0.024 mmol, $12 \mathrm{~mol} \%$ ), KOAc (150 mol\%), and stirring at room temperature for $48 \mathrm{~h}$ under $5 \mathrm{~W}$ blue LEDs irradiation. The reaction mixture was filtered through a pad of silica gel and concentrated in vacuo, then purified by chromatography (pentane/EtOAc $=10 / 1 \rightarrow 8 / 1)$ to give $128(49.4 \mathrm{mg}, 0.100 \mathrm{mmol}, 50 \%,>95: 5$ $E / Z,>20: 1 \mathrm{rr}$ ) as a colorless gum. $\mathbf{R}_{\boldsymbol{f}}$ (pentane/EtOAc $\left.=7 / 1\right) 0.40 .{ }^{\mathbf{1}} \mathbf{H}$ NMR (400 MHz, $\left.\mathbf{C D C l}_{3}\right): \delta$ ppm $7.80(\mathrm{dd}, J=5.4,3.0 \mathrm{~Hz}, 2 \mathrm{H}), 7.73-7.66(\mathrm{~m}, 2 \mathrm{H}), 7.23-7.16$ (m, 2H), $7.13(\mathrm{td}, J=7.0,1.3 \mathrm{~Hz}, 3 \mathrm{H}), 6.66-6.61(\mathrm{~m}, 2 \mathrm{H}), 6.56(\mathrm{dd}, J=7.9,1.7 \mathrm{~Hz}$, 1H), $5.94(\mathrm{ddt}, J=15.2,8.2,1.2 \mathrm{~Hz}, 1 \mathrm{H}), 5.87-5.83(\mathrm{~m}, 2 \mathrm{H}), 5.75(\mathrm{dt}, J=15.1,7.4$ $\mathrm{Hz}, 1 \mathrm{H}), 4.76(\mathrm{q}, J=8.1 \mathrm{~Hz}, 1 \mathrm{H}), 2.67-2.34(\mathrm{~m}, 5 \mathrm{H}), 2.17$ (ddt, $J=13.6,9.2,5.7 \mathrm{~Hz}$, 1H), $2.01(\mathrm{~d}, J=7.4 \mathrm{~Hz}, 2 \mathrm{H}), 1.51-1.41(\mathrm{~m}, 2 \mathrm{H}), 1.01-0.83(\mathrm{~m}, 6 \mathrm{H}) .{ }^{13} \mathbf{C}$ NMR (101 MHz, CDCl 3 ): $\delta$ ppm 168.2, 147.6, 145.7, 143.4, 134.9, 133.9, 132.1, 131.4, 130.0, 128.4, 128.4, 125.6, 123.2, 121.2, 108.9, 108.2, 100.8, 53.7, 44.6, 44.2, 34.2, 33.6, 32.9, 30.7, 27.25 - $27.14(\mathrm{~m})$. HRMS (ESI+): calcd. for $\mathrm{C}_{32} \mathrm{H}_{33} \mathrm{NO}_{4} \mathrm{Na}(\mathrm{M}+$ $\mathrm{Na}^{+}$): 518.2302. Found: 518.2294. 


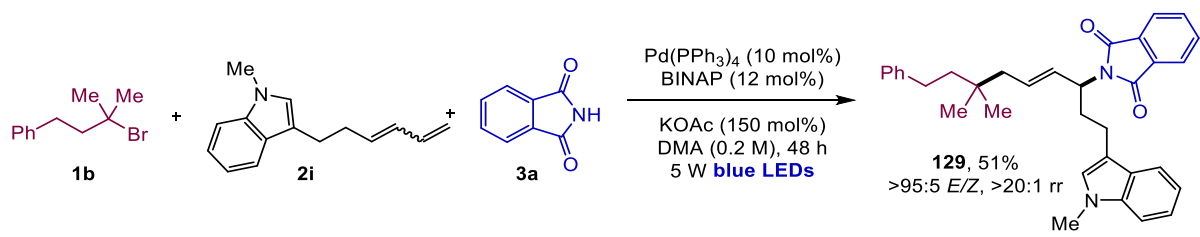

(E)-2-(7,7-Dimethyl-1-(1-methyl-1H-indol-3-yl)-9-phenylnon-4-en-3-

yl)isoindoline-1,3-dione (129). According to the general procedure $L$, using the bromide $\mathbf{1 b}$ ( $0.3 \mathrm{mmol}, 1.5$ equiv.), diene $2 \mathbf{i}$ ( $0.3 \mathrm{mmol}, 1.5$ equiv.), phthalimide 3a (0.2 mmol, 1.0 equiv.), $\mathrm{Pd}\left(\mathrm{PPh}_{3}\right)_{4}(0.020 \mathrm{mmol}, 10 \mathrm{~mol} \%)$, BINAP (0.024 mmol, $12 \mathrm{~mol} \%$ ), KOAc (150 mol\%), and stirring at room temperature for $48 \mathrm{~h}$ under $5 \mathrm{~W}$ blue LEDs irradiation. The reaction mixture was filtered through a pad of silica gel and concentrated in vacuo, then purified by chromatography (pentane/EtOAc $=9 / 1 \rightarrow 8 / 1 \rightarrow 7 / 1)$ to give $129(51.7 \mathrm{mg}, 0.102 \mathrm{mmol}, 51 \%,>$ 95:5E/Z, $>20: 1 \mathrm{rr}$ ) as a light yellow gum. $\mathbf{R}_{\boldsymbol{f}}$ (pentane $/$ EtOAc $=6 / 1$ ) 0.35. ${ }^{1} \mathbf{H}$ NMR (500 MHz, $\mathbf{C D C l}_{3}$ ): $\delta$ ppm $\delta 7.84-7.74(\mathrm{~m}, 2 \mathrm{H}), 7.73-7.65(\mathrm{~m}, 2 \mathrm{H}), 7.56$ $(\mathrm{dt}, J=7.9,1.0 \mathrm{~Hz}, 1 \mathrm{H}), 7.24-7.17(\mathrm{~m}, 4 \mathrm{H}), 7.16-7.11(\mathrm{~m}, 3 \mathrm{H}), 7.08$ (ddd, $J=7.9$, 6.2, $1.9 \mathrm{~Hz}, 1 \mathrm{H}), 6.81(\mathrm{~s}, 1 \mathrm{H}), 5.97(\mathrm{ddt} J=15.4,8.2,1.1 \mathrm{~Hz}, 1 \mathrm{H}), 5.77$ (ddt, $J=15.3$, 7.4, $0.6 \mathrm{~Hz}, 1 \mathrm{H}), 4.99-4.79(\mathrm{~m}, 1 \mathrm{H}), 3.69(\mathrm{~s}, 3 \mathrm{H}), 2.86-2.68(\mathrm{~m}, 2 \mathrm{H}), 2.65-2.49$ (m, 3H), $2.29(\mathrm{ddt}, J=13.2,8.7,6.5 \mathrm{~Hz}, 1 \mathrm{H}), 2.02(\mathrm{~d}, J=7.4 \mathrm{~Hz}, 2 \mathrm{H}), 0.97-0.90(\mathrm{~m}$, 6H). ${ }^{13}$ C NMR (101 MHz, CDCl 3 ): $\delta$ ppm 168.3, 143.4, 137.1, 133.9, 132.1, 131.1, 130.2, 128.4, 127.7, 126.4, 125.6, 123.1, 121.6, 119.1, 118.7, 113.8, 109.1, 53.9, 44.6, 44.2, 33.6, 32.9, 32.6, 30.7, 27.22 - 27.20 (m), 22.5. HRMS (ESI+): calcd. for $\mathrm{C}_{34} \mathrm{H}_{36} \mathrm{~N}_{2} \mathrm{O}_{2} \mathrm{Na}\left(\mathrm{M}+\mathrm{Na}^{+}\right): 527.2669$. Found: 527.2672 .

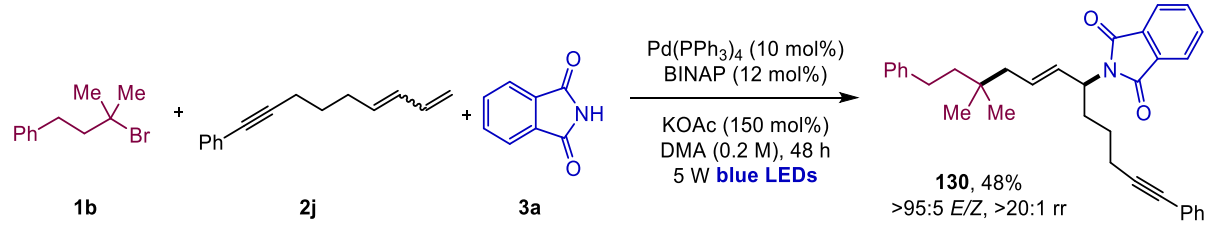

(E)-2-(10,10-Dimethyl-1,12-diphenyldodec-7-en-1-yn-6-yl)isoindoline-1,3-

dione (130). According to the general procedure $\mathrm{L}$, using the bromide $\mathbf{1 b}(0.3$ mmol, 1.5 equiv.), diene $\mathbf{2 j}$ ( $0.3 \mathrm{mmol}, 1.5$ equiv.), phthalimide $3 \mathbf{a}$ ( $0.2 \mathrm{mmol}, 1.0$ equiv.), $\mathrm{Pd}\left(\mathrm{PPh}_{3}\right)_{4}$ (0.020 mmol, $10 \mathrm{~mol} \%$ ), BINAP (0.024 mmol, $12 \mathrm{~mol} \%$ ), KOAc (150 mol\%), and stirring at room temperature for $48 \mathrm{~h}$ under $5 \mathrm{~W}$ blue LEDs 
irradiation. The reaction mixture was filtered through a pad of silica gel and concentrated in vacuo, then purified by chromatography (pentane $/$ EtOAc $=10 / 1$ ) to give 130 (47.0 mg, $0.096 \mathrm{mmol}, 48 \%,>95: 5 \mathrm{E} / Z$, > 20:1 rr) as a colorless gum. $\mathbf{R}_{\boldsymbol{f}}($ pentane $/$ EtOAc $=10 / 1)$ 0.30. ${ }^{\mathbf{1}} \mathbf{H}$ NMR (400 $\left.\mathbf{~ M H z , ~} \mathbf{C D C l}_{3}\right): \delta$ ppm $7.88-7.77$ (m, 2H), $7.75-7.65(\mathrm{~m}, 2 \mathrm{H}), 7.43-7.34(\mathrm{~m}, 2 \mathrm{H}), 7.32-7.17(\mathrm{~m}, 6 \mathrm{H}), 7.17-7.03$ (m, 4H), $5.96(\mathrm{ddt}, J=15.2,8.3,1.1 \mathrm{~Hz}, 1 \mathrm{H}), 5.78(\mathrm{dt}, J=15.0,7.4 \mathrm{~Hz}, 1 \mathrm{H}), 4.79$ (q, $J=8.0 \mathrm{~Hz}, 1 \mathrm{H}), 2.58-2.49(\mathrm{~m}, 2 \mathrm{H}), 2.45(\mathrm{t}, J=7.1 \mathrm{~Hz}, 2 \mathrm{H}), 2.30-2.17(\mathrm{~m}, 1 \mathrm{H})$, $2.17-2.06(\mathrm{~m}, 1 \mathrm{H}), 2.01(\mathrm{dd}, J=7.5,1.2 \mathrm{~Hz}, 2 \mathrm{H}), 1.72-1.51(\mathrm{~m}, 2 \mathrm{H}), 1.52-1.42$ (m, 2H), $0.96-0.89(\mathrm{~m}, 6 \mathrm{H}) .{ }^{13} \mathrm{C}$ NMR (101 MHz, CDCl 3 ): $\delta$ ppm 168.2, 143.4, 134.0, 132.1, 131.7, 131.4, 129.9, 128.38, 128.36, 128.3, 127.7, 125.6, 124.0, 123.3, 89.6, 81.2, 53.6, 44.5, 44.3, 33.6, 31.9, 30.7, 27.2, 25.9, 19.1. Note: The two geminal $\mathrm{CH}_{3}$ signals overlap. HRMS (ESI+): calcd. for $\mathrm{C}_{34} \mathrm{H}_{35} \mathrm{NO}_{2} \mathrm{Na}\left(\mathrm{M}+\mathrm{Na}^{+}\right)$: 512.2565. Found: 512.2557.

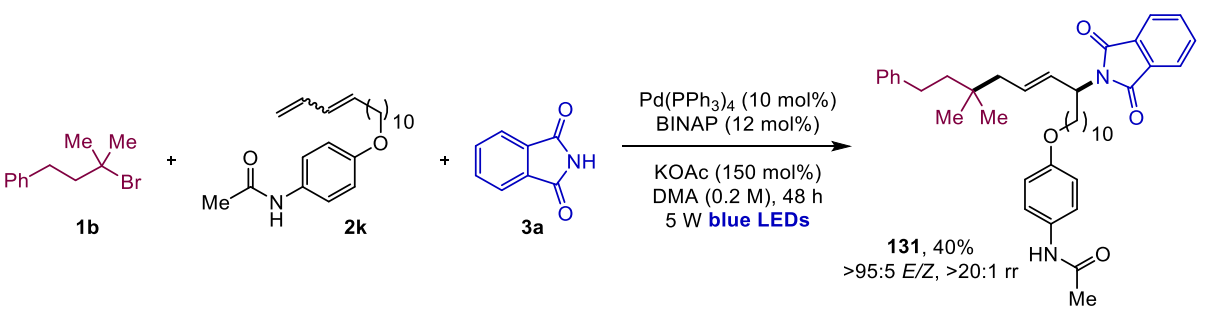

\section{(E)-N-(4-((11-(1,3-Dioxoisoindolin-2-yl)-15,15-dimethyl-17-}

phenylheptadec-12-en-1-yl)oxy)phenyl)acetamide (131). According to the general procedure $\mathrm{L}$, using the bromide $\mathbf{1 b}$ ( $0.3 \mathrm{mmol}, 1.5$ equiv.), diene $\mathbf{2 k}(0.3$ mmol, 1.5 equiv.), phthalimide $3 a$ ( $0.2 \mathrm{mmol}, 1.0$ equiv.), $\mathrm{Pd}\left(\mathrm{PPh}_{3}\right)_{4}(0.020 \mathrm{mmol}$, $10 \mathrm{~mol} \%)$, BINAP (0.024 mmol, $12 \mathrm{~mol} \%$ ), KOAc (150 mol\%), and stirring at room temperature for $48 \mathrm{~h}$ under $5 \mathrm{~W}$ blue LEDs irradiation. The reaction mixture was filtered through a pad of silica gel and concentrated in vacuo, then purified by chromatography (pentane/EtOAc $=3 / 2 \rightarrow 1 / 1)$ to give $132(50.3 \mathrm{mg}$, $0.079 \mathrm{mmol}, 40 \%,>95: 5 \mathrm{E} / \mathrm{Z},>20: 1 \mathrm{rr}$ ) as a colorless gum. $\mathbf{R}_{\boldsymbol{f}}$ (pentane $/$ EtOAc $=$ 1/1) 0.30. 1H NMR (400 MHz, CDCl$\left._{3}\right): \delta$ ppm $7.84-7.78(\mathrm{~m}, 2 \mathrm{H}), 7.73-7.66(\mathrm{~m}$, 2H), $7.40-7.33(\mathrm{~m}, 2 \mathrm{H}), 7.24-7.16(\mathrm{~m}, 2 \mathrm{H}), 7.16-7.06(\mathrm{~m}, 3 \mathrm{H}), 6.87-6.79(\mathrm{~m}$, 2H), $5.92(\mathrm{ddt}, J=15.4,8.3,1.2 \mathrm{~Hz}, 1 \mathrm{H}), 5.72(\mathrm{dt}, J=15.1,7.4 \mathrm{~Hz}, 1 \mathrm{H}), 4.70(\mathrm{q}, J=$ $8.0 \mathrm{~Hz}, 1 \mathrm{H}), 3.91(\mathrm{t}, J=6.5 \mathrm{~Hz}, 2 \mathrm{H}), 2.58-2.45(\mathrm{~m}, 2 \mathrm{H}), 2.14(\mathrm{~s}, 3 \mathrm{H}), 2.05-1.82$ 
$(\mathrm{m}, 4 \mathrm{H}), 1.74(\mathrm{p}, J=6.8 \mathrm{~Hz}, 2 \mathrm{H}), 1.52-1.36(\mathrm{~m}, 4 \mathrm{H}), 1.36-1.14(\mathrm{~m}, 12 \mathrm{H}), 0.91(\mathrm{~s}$, 6H). ${ }^{13}$ C NMR (101 MHz, CDCl 3 ): $\delta$ ppm 168.33, 168.27, 156.16, 143.43, 133.95, 132.14, 131.04, 130.91, 130.24, 128.38, 125.61, 123.25, 121.98, 114.92, 68.40, 54.10, 44.52, 44.24, 33.62, 32.72, 30.75, 29.59, 29.58 - 29.52 (m), 29.44, 29.37, 29.25, 27.21, 26.74, 26.10, 24.49. Note: One aromatic signal missing, most likely due to overlap. HRMS (ESI ${ }^{+}$): calcd. for $\mathrm{C}_{41} \mathrm{H}_{52} \mathrm{~N}_{2} \mathrm{O}_{4} \mathrm{Na}\left(\mathrm{M}+\mathrm{Na}^{+}\right)$: 659.3819. Found: 659.3820 .
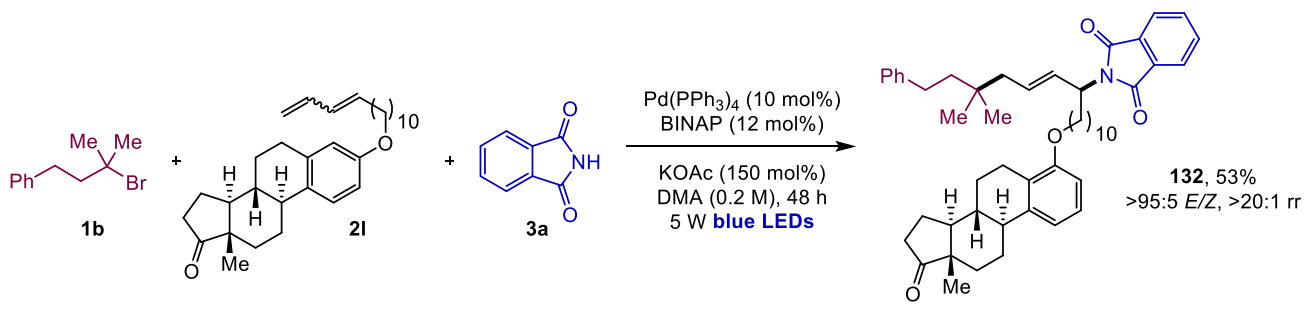

2-((E)-3,3-Dimethyl-17-(((8R,9S,13S,14S)-13-methyl-17-oxo-

\section{$7,8,9,11,12,13,14,15,16,17-d e c a h y d r o-6 H$-cyclopenta[ $a]$ phenanthren-3-}

yl)oxy)-1-phenylheptadec-5-en-7-yl)isoindoline-1,3-dione (132). According to the general procedure $\mathrm{L}$, using the bromide $\mathbf{1 b}$ ( $0.3 \mathrm{mmol}, 1.5$ equiv.), diene $\mathbf{2 l}$ (0.3 mmol, 1.5 equiv.), phthalimide 3a (0.2 mmol, 1.0 equiv.), $\mathrm{Pd}\left(\mathrm{PPh}_{3}\right)_{4}(0.020$ mmol, 10 mol\%), BINAP (0.024 mmol, 12 mol\%), KOAc (150 mol\%), and stirring at room temperature for $48 \mathrm{~h}$ under $5 \mathrm{~W}$ blue LEDs irradiation. The reaction mixture was filtered through a pad of silica gel and concentrated in vacuo, then purified by chromatography (pentane/EtOAc $=7 / 1 \rightarrow 6 / 1 \rightarrow 5 / 1$ ) to give 132 (79.2 mg, $0.105 \mathrm{mmol}, 53 \%,>95: 5 \mathrm{E} / \mathrm{Z},>20: 1 \mathrm{rr}$ ) as a colorless gum. $\mathbf{R}_{\boldsymbol{f}}$ (pentane/EtOAc = 7/1) 0.25. ${ }^{1} \mathbf{H}$ NMR (400 $\left.\mathbf{~ M H z , ~} \mathbf{C D C l}_{3}\right): \delta$ ppm $7.90-7.80(\mathrm{~m}$, 2H), $7.78-7.67(\mathrm{~m}, 2 \mathrm{H}), 7.24-7.07$ (m, 6H), 6.72 (dd, J = 8.6, 2.7 Hz, 1H), 6.65 (d, $J=2.7 \mathrm{~Hz}, 1 \mathrm{H}), 5.94(\mathrm{dd}, J=15.3,8.3 \mathrm{~Hz}, 1 \mathrm{H}), 5.74(\mathrm{dt}, J=15.1,7.4 \mathrm{~Hz}, 1 \mathrm{H}), 4.72$ $(\mathrm{q}, J=8.0 \mathrm{~Hz}, 1 \mathrm{H}), 3.92(\mathrm{t}, J=6.6 \mathrm{~Hz}, 2 \mathrm{H}), 2.98-2.82(\mathrm{~m}, 2 \mathrm{H}), 2.55-1.18(\mathrm{~m}$, 36H), $0.96-0.87(\mathrm{~m}, 9 \mathrm{H}) .{ }^{13}$ C NMR (101 MHz, $\left.\mathbf{C D C l}_{3}\right): \delta$ ppm 221.1, 168.3, 143.4, 137.7, 134.4, 133.9, 132.1, 131.9, 131.0, 130.2, 128.3, 126.4, 125.6, 123.6, 123.2, 114.6, 112.2, 68.0, 54.0, 50.5, 48.1, 44.5, 44.2, 44.1, 38.5, 36.0, 33.6, 32.7, 31.7, $30.7,29.8,29.59,29.57,29.54,29.45,29.42,29.21,27.19,26.70,26.67,26.13$, 
26.02, 21.7, 14.0. HRMS (ESI+): calcd. for $\mathrm{C}_{51} \mathrm{H}_{65} \mathrm{NO}_{4} \mathrm{Na}\left(\mathrm{M}+\mathrm{Na}^{+}\right): 778.4806$. Found: 778.4814. 


\section{Gram-scale Experiments}

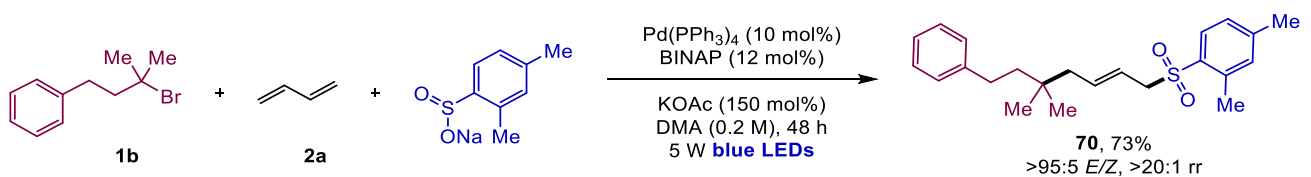

In an oven-dried $50 \mathrm{ml}$ Schlenk tube equipped with a PTFE-coated stirring bar, sulfinate S3m (700 mg, $3.64 \mathrm{mmol}, 1.0$ equiv.), $\mathrm{Pd}\left(\mathrm{PPh}_{3}\right)_{4}(210.0 \mathrm{mg}, 0.182 \mathrm{mmol}$, 5 mol\%), rac-BINAP (135.7 mg, $0.218 \mathrm{mmol}, 6 \mathrm{~mol} \%$ ) and $\mathrm{K}_{2} \mathrm{CO}_{3}(755 \mathrm{mg}, 5.46$ mmol, 1.5 equiv.) were charged under air, then the vessel was evacuated and back-filled with argon four times. Dry DMA (15.5 ml) was charged under argon, followed by bromide $\mathbf{1 b}$ (1.070 g, $4.73 \mathrm{mmol}, 1.3$ equiv.) and butadiene $2 \mathrm{M}$ in THF (2.73 ml, $5.46 \mathrm{mmol}, 1.5$ equiv.), then the vessel was sealed and irradiated at $455 \mathrm{~nm}$ using two Kessil ${ }^{\circledR}$ H150 blue lights (see picture). After 48 hours the solvent was removed in vacuo $\left(<1\right.$ mbar @ $\left.60^{\circ} \mathrm{C}\right)$, then the residue was taken-up with $\mathrm{CH}_{2} \mathrm{Cl}_{2}(60 \mathrm{ml})$, then organic layer washed twice with water $(50 \mathrm{ml}$ each time), dried over $\mathrm{MgSO}_{4}$ and the solvent removed under reduced pressure. The crude was purified by flash column chromatography on silica (pentane/EtOAc $12 / 1 \rightarrow 10 / 1$ ), to give 70 (980 $\mathrm{mg}, 2.64 \mathrm{mmol}, 73 \%$ ) as an orange gum.

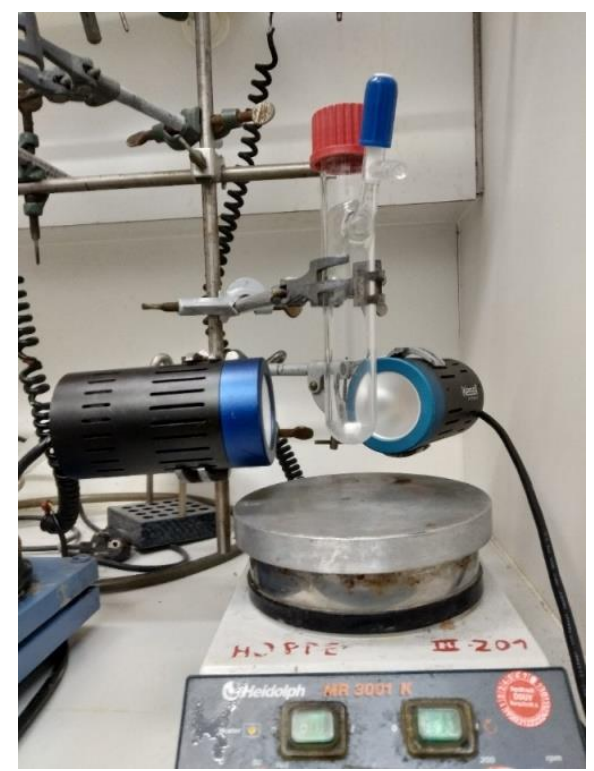

Reaction set-up: Two Kessil ${ }^{\circledR}$ H150 455 nm LEDs lights are placed $4 \mathrm{~cm}$ away from a magnetically stirred Schlenk tube. 


\section{Step by step procedure:}
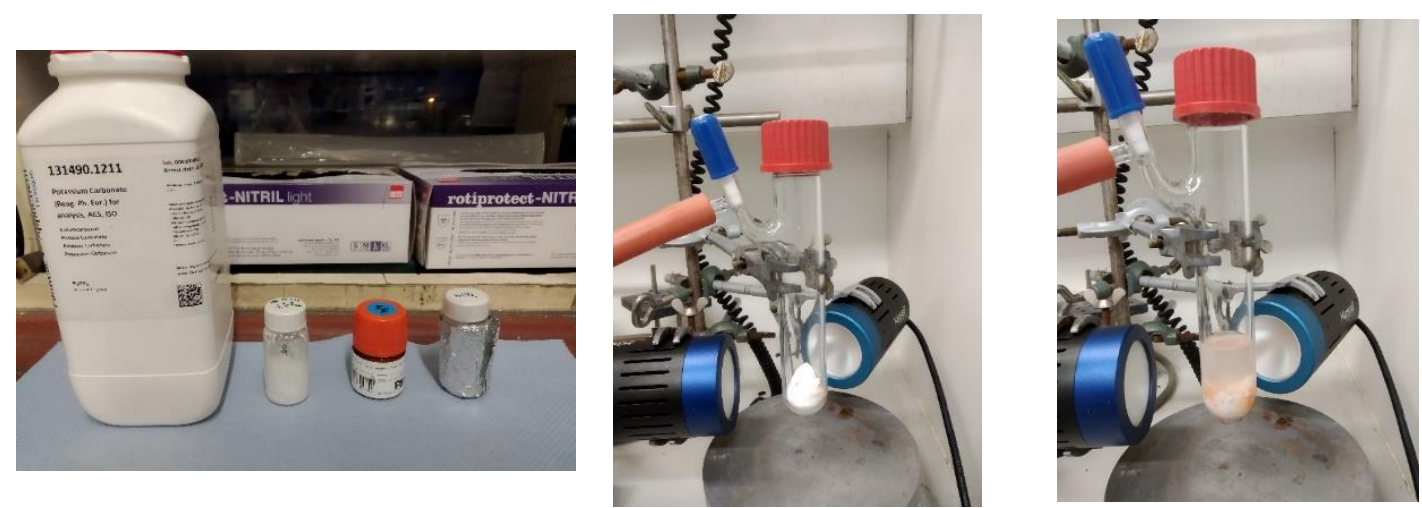

Step 1. Solid substances (from the left: $\mathrm{K}_{2} \mathrm{CO}_{3}$, Sulfinate, rac-BINAP, $\left.\mathrm{Pd}\left(\mathrm{PPh}_{3}\right)_{4}\right)$ are weighted and added to the Schlenk tube under air; Step 2. The Schlenk tube is connected to the vacuum-argon line and a purging-refilling process is repeated four times; Step 3. The solvent is added under argon counterflow.
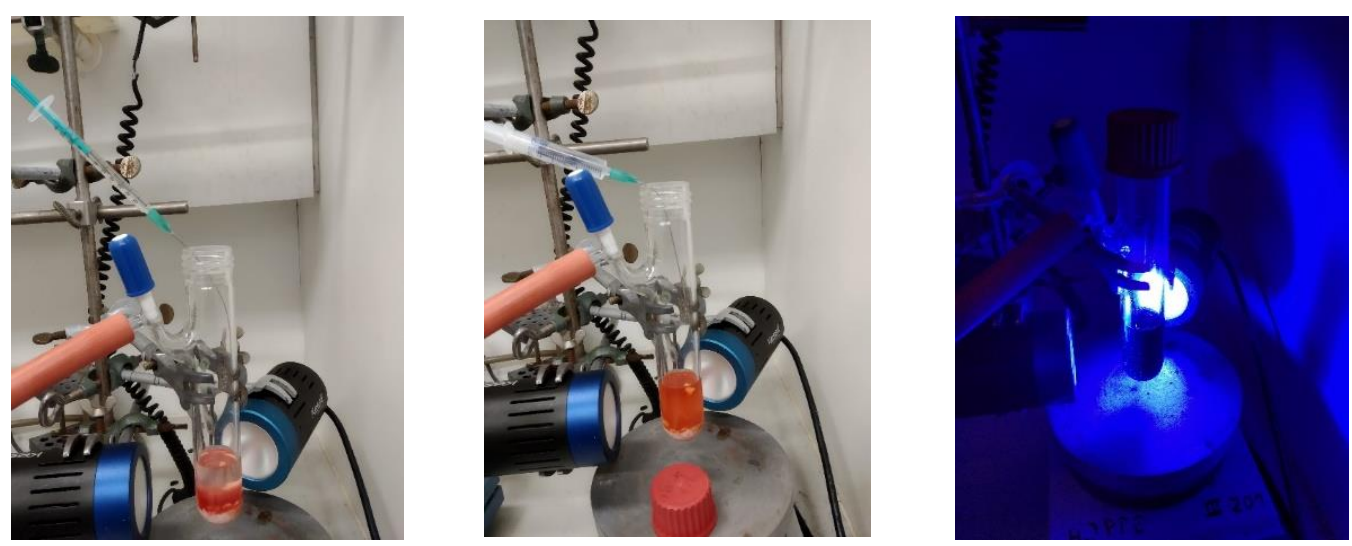

Step 4. The bromide (in this case a liquid) is added under argon counterflow;

Step 5. Butadiene solution is added under argon counterflow; Step 6. The reaction is irradiated at $455 \mathrm{~nm}$. 


\section{Manipulation of Products}

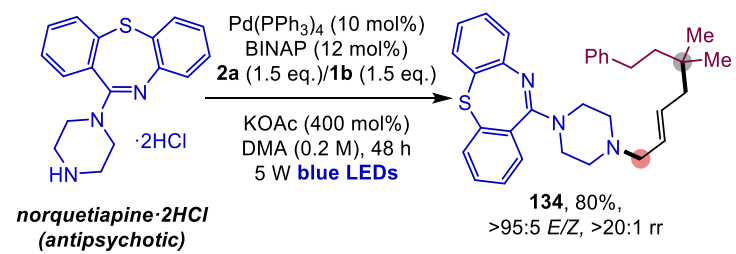

(E)-11-(4-(5,5-Dimethyl-7-phenylhept-2-en-1-yl)piperazin-1-

yl)dibenzo $[b, f][1,4]$ thiazepine (134). According to the general procedure $G$, using the bromide $\mathbf{1 b}(0.3 \mathrm{mmol}, 1.5$ equiv.), butadiene $2 \mathbf{a}(0.3 \mathrm{mmol}, 0.15 \mathrm{~mL}, 2$ $M$ in THF, 1.5 equiv.), norquietapine. $2 \mathrm{HCl}$ ( $0.2 \mathrm{mmol}, 1.0$ equiv.), $\mathrm{Pd}\left(\mathrm{PPh}_{3}\right)_{4}$ (0.020 mmol, $10 \mathrm{~mol} \%$ ), BINAP (0.024 mmol, $12 \mathrm{~mol} \%$ ), KOAc (150 mol\%), and stirring at room temperature for $48 \mathrm{~h}$ under $5 \mathrm{~W}$ blue LEDs irradiation. The reaction mixture was filtered through a pad of silica gel and concentrated in vacuo, then purified by chromatography (pentane/EtOAc $=7 / 3+1 \% \mathrm{Et}_{3} \mathrm{~N} \rightarrow 3 / 2$ $\left.+1 \% \mathrm{Et}_{3} \mathrm{~N}\right)$ to give $134(79.1 \mathrm{mg}, 0.160 \mathrm{mmol}, 80 \%,>95: 5 E / Z,>20: 1 \mathrm{rr}$ ) as a colorless gum. $\mathbf{R}_{\boldsymbol{f}}$ (pentane $/ \mathrm{EtOAc}=7 / 3+1 \% \mathrm{Et}_{3} \mathrm{~N}$ ) 0.35. ${ }^{\mathbf{1}} \mathbf{H} \mathbf{~ N M R}(\mathbf{3 0 0} \mathbf{~ M H z}$, $\left.\mathbf{C D C l}_{3}\right): \delta \mathrm{ppm} 7.58-7.51(\mathrm{~m}, 1 \mathrm{H}), 7.43(\mathrm{dd}, J=7.8,1.5 \mathrm{~Hz}, 1 \mathrm{H}), 7.39-7.25(\mathrm{~m}$, $5 \mathrm{H}), 7.25-7.14(\mathrm{~m}, 4 \mathrm{H}), 7.12(\mathrm{dd}, J=8.0,1.6 \mathrm{~Hz}, 1 \mathrm{H}), 6.91(\mathrm{td}, J=7.4,1.6 \mathrm{~Hz}, 1 \mathrm{H})$, $5.69(\mathrm{dt}, J=14.4,7.0 \mathrm{~Hz}, 1 \mathrm{H}), 5.57(\mathrm{dt}, J=15.3,6.3 \mathrm{~Hz}, 1 \mathrm{H}), 3.90-3.30(\mathrm{br}, 4 \mathrm{H})$, $3.14-2.98(\mathrm{~m}, 2 \mathrm{H}), 2.68-2.43(\mathrm{~m}, 6 \mathrm{H}), 2.06(\mathrm{~d}, J=7.0 \mathrm{~Hz}, 2 \mathrm{H}), 1.58-1.47(\mathrm{~m}$, 2H), 0.97 (s, 6H). ${ }^{13} \mathbf{C}$ NMR (75 MHz, $\mathbf{C D C l}_{3}$ ): $\delta$ ppm 160.8, 149.1, 143.4, 140.0, 134.3, 132.3, 132.2, 131.6, 130.8, 129.2, 129.1, 128.8, 128.4, 128.34, 128.33, 128.1, 125.7, 125.4, 122.8, 61.2, 53.0, 48.3 - 46.9 (br), 44.9, 44.2, 33.6, 30.7, 27.2. HRMS (ESI ${ }^{+}$): calcd. for $\mathrm{C}_{32} \mathrm{H}_{38} \mathrm{O}_{3} \mathrm{~N}_{3} \mathrm{~S}\left(\mathrm{M}+\mathrm{H}^{+}\right)$: 496.2781. Found: 496.2789.

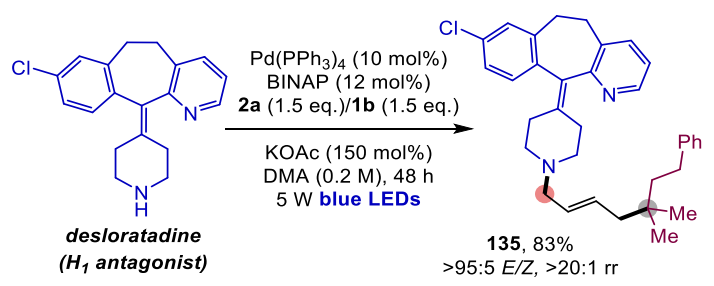

(E)-8-Chloro-11-(1-(5,5-dimethyl-7-phenylhept-2-en-1-yl)piperidin-4ylidene)-6,11-dihydro-5H-benzo[5,6]cyclohepta[1,2-b]pyridine 
According to the general procedure G, using the bromide $\mathbf{1 b}$ (0.4 mmol, 2.0 equiv.), butadiene 2a (0.4 mmol, $0.20 \mathrm{~mL}, 2 \mathrm{M}$ in THF, 2.0 equiv.), desloratadine (0.2 mmol, 1.0 equiv.), $\mathrm{Pd}\left(\mathrm{PPh}_{3}\right)_{4}(0.020 \mathrm{mmol}, 10 \mathrm{~mol} \%)$, BINAP (0.024 mmol, $12 \mathrm{~mol} \%), \mathrm{KOAc}(150 \mathrm{~mol} \%)$, and stirring at room temperature for $48 \mathrm{~h}$ under 5W blue LEDs irradiation. The reaction mixture was filtered through a pad of silica gel and concentrated in vacuo, then purified by chromatography (pentane $\left./ \mathrm{EtOAc}=1 / 1+4 \% \mathrm{Et}_{3} \mathrm{~N} \rightarrow 5 \% \mathrm{Et}_{3} \mathrm{~N}\right)$ to give $135(84.9 \mathrm{mg}, 0.166 \mathrm{mmol}$, $83 \%,>95: 5 E / Z,>20: 1 \mathrm{rr}$ ) as a yellow-orange gum. $\mathbf{R}_{\boldsymbol{f}}$ (pentane/EtOAc $=1 / 1+4 \%$ $\mathrm{Et}_{3} \mathrm{~N}$ ) 0.30. ${ }^{1} \mathrm{H}$ NMR (400 $\left.\mathbf{M H z}, \mathbf{C D C l}_{3}\right): \delta \operatorname{ppm} \delta 8.44(\mathrm{dd}, J=4.8,1.7 \mathrm{~Hz}, 1 \mathrm{H})$, $7.45(\mathrm{dd}, J=7.7,1.7 \mathrm{~Hz}, 1 \mathrm{H}), 7.32-7.22(\mathrm{~m}, 2 \mathrm{H}), 7.22-7.14(\mathrm{~m}, 6 \mathrm{H}), 7.10(\mathrm{dd}, J=$ 7.7, $4.8 \mathrm{~Hz}, 1 \mathrm{H}), 5.70-5.52(\mathrm{~m}, 2 \mathrm{H}), 3.50-3.33(\mathrm{~m}, 2 \mathrm{H}), 3.03-2.95(\mathrm{~m}, 2 \mathrm{H}), 2.91$ $-2.76(\mathrm{~m}, 4 \mathrm{H}), 2.63-2.53(\mathrm{~m}, 3 \mathrm{H}), 2.51-2.33(\mathrm{~m}, 3 \mathrm{H}), 2.16-2.07(\mathrm{~m}, 2 \mathrm{H}), 2.04$ (d, $J=6.3 \mathrm{~Hz}, 2 \mathrm{H}), 1.55-1.46(\mathrm{~m}, 2 \mathrm{H}), 0.95(\mathrm{~s}, 6 \mathrm{H}) .{ }^{13} \mathbf{C}$ NMR (101 MHz, $\left.\mathbf{C D C l}_{3}\right)$ : $\delta \operatorname{ppm} \delta 157.7,146.7,143.3,139.5,139.0,137.9,137.2,133.4,132.64,132.60$, 131.1, 130.9, 129.2, 129.0, 128.3, 128.2, 126.0, 125.5, 122.1, 61.0, 54.8, 44.8, 44.0, 33.5, 31.9, 31.5, 31.0, 30.7, 30.6, 27.1. HRMS (ESI+): calcd. for $\mathrm{C}_{34} \mathrm{H}_{40} \mathrm{~N}_{2}{ }^{35} \mathrm{Cl}(\mathrm{M}+$ $\mathrm{H}^{+}$): 511.2875. Found: 511.2882: calcd. for $\mathrm{C}_{34} \mathrm{H}_{40} \mathrm{~N}_{2}{ }^{37} \mathrm{Cl}\left(\mathrm{M}+\mathrm{H}^{+}\right)$: 513.2848. Found: 513.2866 .
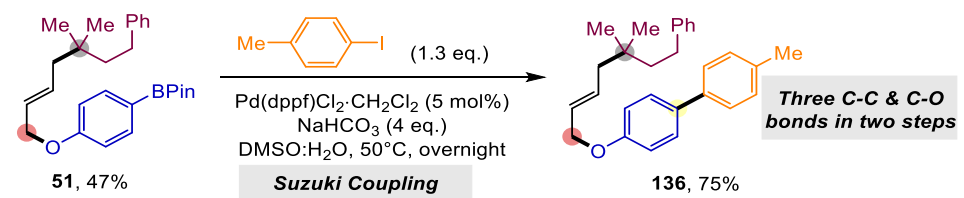

(E)-4-((5,5-Dimethyl-7-phenylhept-2-en-1-yl)oxy)-4'-methyl-1,1'-biphenyl

(136). In a Schlenk tube equipped with a PTFE-coated stirring bar, substrate $\mathbf{5 1}$ (38.0 mg, $90 \mu \mathrm{mol}, 1.0$ equiv.), 4-iodotoluene (25.5 mg, $117 \mu \mathrm{mol}, 1.3$ equiv.), $\mathrm{Pd}(\mathrm{dppf}) \mathrm{Cl}_{2} \cdot \mathrm{CH}_{2} \mathrm{Cl}_{2}(3.7 \mathrm{mg}, 4.5 \mu \mathrm{mol}, 5 \mathrm{~mol} \%)$ and $\mathrm{NaHCO}_{3}(30.2 \mathrm{mg}, 360 \mu \mathrm{mol}$, 4.0 equiv.) were charged, then the vessel was evacuated and back-filled with argon four times. A mixture of DMSO: $\mathrm{H}_{2} \mathrm{O}(20: 1,950 \mu \mathrm{L})$ was added and the system was degassed by freeze-pump-thaw (three times), then heated at $50^{\circ} \mathrm{C}$ overnight. The solvent was removed in vacuo, then the residue was purified by 
flash column chromatography on silica (pentane/EtOAc $=200 / 3 \rightarrow 50 / 1$ ), to give 136 (26.1 mg, $67.9 \mu \mathrm{mol}, 75 \%$ ) as a white waxy solid. $\mathbf{R}_{\boldsymbol{f}}$ (pentane $/$ EtOAc $=$ 100/1) 0.25. ${ }^{1} \mathrm{H}$ NMR (400 MHz, $\mathrm{CDCl}_{3}$ ): $\delta$ ppm $7.53-7.48(\mathrm{~m}, 2 \mathrm{H}), 7.48-7.42$ (m, 2H), $7.32-7.11(\mathrm{~m}, 7 \mathrm{H}), 7.02-6.95(\mathrm{~m}, 2 \mathrm{H}), 5.90(\mathrm{dtt}, J=15.4,7.4,1.2 \mathrm{~Hz}$, $1 \mathrm{H}), 5.76(\mathrm{dtt}, J=15.2,5.8,1.2 \mathrm{~Hz}, 1 \mathrm{H}), 4.56(\mathrm{~d}, J=5.8 \mathrm{~Hz}, 2 \mathrm{H}), 2.62-2.53(\mathrm{~m}$, 2H), $2.40(\mathrm{~s}, 3 \mathrm{H}), 2.10(\mathrm{~d}, J=7.2 \mathrm{~Hz}, 2 \mathrm{H}), 1.56-1.47(\mathrm{~m}, 2 \mathrm{H}), 0.97(\mathrm{~s}, 6 \mathrm{H}) .{ }^{13} \mathrm{C}$ NMR (101 MHz, $\mathrm{CDCl}_{3}$ ): $\delta$ ppm $\delta$ 158.2, 143.5, 138.1, 136.4, 133.8, 132.2, 129.6, 128.5, 128.0, 127.5, 126.7, 125.7, 115.2, 68.9, 44.9, 44.3, 33.7, 30.8, 27.2, 21.2. Note: one aromatic signal is missing, most likely due to overlap. HRMS (ESI+): calcd. for $\mathrm{C}_{28} \mathrm{H}_{32} \mathrm{ONa}\left(\mathrm{M}+\mathrm{Na}^{+}\right)$: 407.2345. Found: 407.2338.

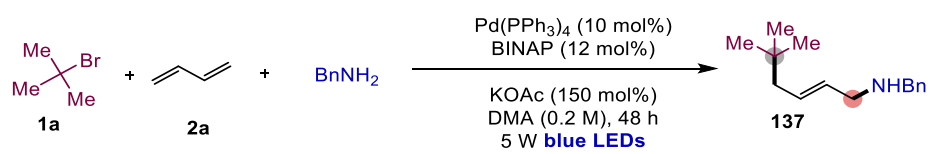

(E)-N-Benzyl-5,5-dimethylhex-2-en-1-amine (137). According to the general procedure G, using the bromide $1 \mathrm{a}$ (7.5 mmol, 1.5 equiv.), butadiene 2a (7.5 mmol, 1.5 equiv.), benzylamine (5.0 mmol, 1.0 equiv.), $\mathrm{Pd}\left(\mathrm{PPh}_{3}\right)_{4}(0.5 \mathrm{mmol}, 10$ mol\%), BINAP (0.6 mmol, $12 \mathrm{~mol} \%$ ), KOAc (150 mol\%), and stirring at room temperature for $48 \mathrm{~h}$ under $5 \mathrm{~W}$ blue LEDs irradiation. The reaction mixture was filtered through a pad of silica gel and concentrated in vacuo, then purified by chromatography (pentane $/$ EtOAc $\left.=1 / 1+2 \% \mathrm{Et}_{3} \mathrm{~N}\right)$ to give $137(0.712 \mathrm{~g}, 3.28$ mmol, 66\%, >95:5 E/Z, > 20:1 rr) as a yellow-orange gum. $\mathbf{R}_{\boldsymbol{f}}$ (pentane $/$ EtOAc $=$

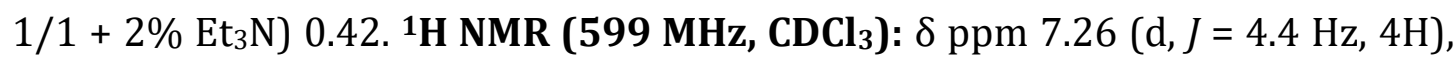
7.22 - $7.16(\mathrm{~m}, 1 \mathrm{H}), 5.62$ - $5.53(\mathrm{~m}, 1 \mathrm{H}), 5.50$ - $5.44(\mathrm{~m}, 1 \mathrm{H}), 3.73(\mathrm{~s}, 2 \mathrm{H}), 3.22$ $3.14(\mathrm{~m}, 2 \mathrm{H}), 1.86$ (dd, $J=7.4,1.2 \mathrm{~Hz}, 2 \mathrm{H}), 0.82$ (s, 9H). ${ }^{13}$ C NMR (151 MHz, $\mathbf{C D C l}_{3}$ ): $\delta$ ppm 140.5, 130.6, 130.2, 128.5, 128.4, 127.0, 53.3, 51.3, 47.1, 31.0, 29.4. HRMS (ESI ${ }^{+}$): calcd. for $\mathrm{C}_{15} \mathrm{H}_{24} \mathrm{~N}\left(\mathrm{M}+\mathrm{H}^{+}\right)$: 218.1903. Found: 218.1925. 


\section{Mechanistic Studies}

\subsection{Radical Inhibitor}

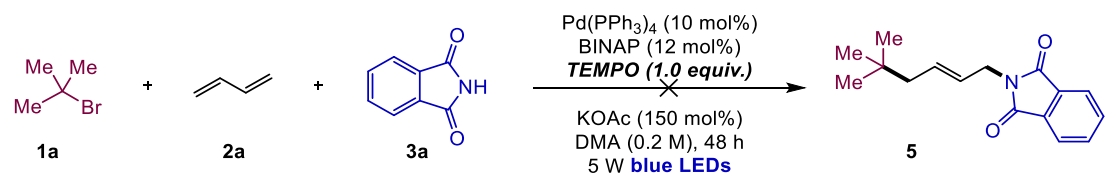

An oven-dried Schlenk tube containing a stir bar was charged under argon with the tertbutyl bromide 1a ( $0.3 \mathrm{mmol}, 1.5$ equiv.), radical inhibitor (TEMPO, $0.2 \mathrm{mmol}, 1.0$ equiv.), $\mathrm{Pd}\left(\mathrm{PPh}_{3}\right)_{4}(10 \mathrm{~mol} \%)$, BINAP (12 mol\%), phthalimide 3a (0.2 mmol, 1.0 equiv.), KOAc (150 mol\%) and DMA (0.85 mL). Subsequently, butadiene $2 \mathbf{a}(0.3 \mathrm{mmol}, 0.15 \mathrm{~mL}, 2 \mathrm{M}$ in THF, 1.5 equiv.) was added. The reaction mixture was stirred at room temperate under blue LEDs irradiation (5W, $455 \mathrm{~nm}$ ) for $48 \mathrm{~h}$. The desired product 5 could not be detected by GC-MS and ${ }^{1} \mathrm{H}$ NMR.

\subsection{EPR Study}

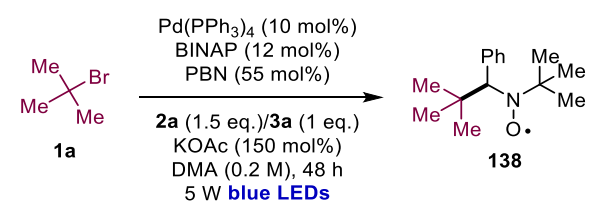

An oven-dried Schlenk tube containing a stir bar was charged under argon with the tertbutyl bromide $1 \mathrm{a}\left(0.3 \mathrm{mmol}, 1.5\right.$ equiv.), $\mathrm{Pd}\left(\mathrm{PPh}_{3}\right)_{4}$ (10 mol\%), BINAP (12 mol\%), phthalimide 3a (0.2 mmol, 1.0 equiv.), KOAc (150 mol\%) and DMA (0.85 mL). Subsequently, butadiene 2a ( $0.3 \mathrm{mmol}, 0.15 \mathrm{~mL}, 2 \mathrm{M}$ in THF, 1.5 equiv.) was added. The reaction mixture was stirred at room temperate under blue LEDs irradiation (5W, 455 $\mathrm{nm}$ ) for $2 \mathrm{~h}$. Then $0.1 \mathrm{~mL}$ reaction mixture was added to EPR tube which containing $2 \mathrm{mg}$ PBN (N-tert-Butyl- $\alpha$-phenylnitrone) under argon condition. Subsequently, the reaction mixture was analyzed by ESR spectrometry.

The red-labeled nuclei induce the characteristic line shape of the EPR spectra. The EPR spectra are dominated by the hyperfine interaction with the ${ }^{14} \mathrm{~N}$ nucleus which results in a triplet with a hyperfine coupling constant $A_{N}$ with 14.58 G. Furthermore, the EPR spectra show hyperfine interactions with any other nuclei with $I \neq 0$, in this case with the proton (doublet, $A_{H}=2.55 \mathrm{G}$ ). 
In comparison with the reported data, ${ }^{56,57}$ the hyperfine coupling constants fits well with their results for tert-butyl radical spin trapped by PBN.
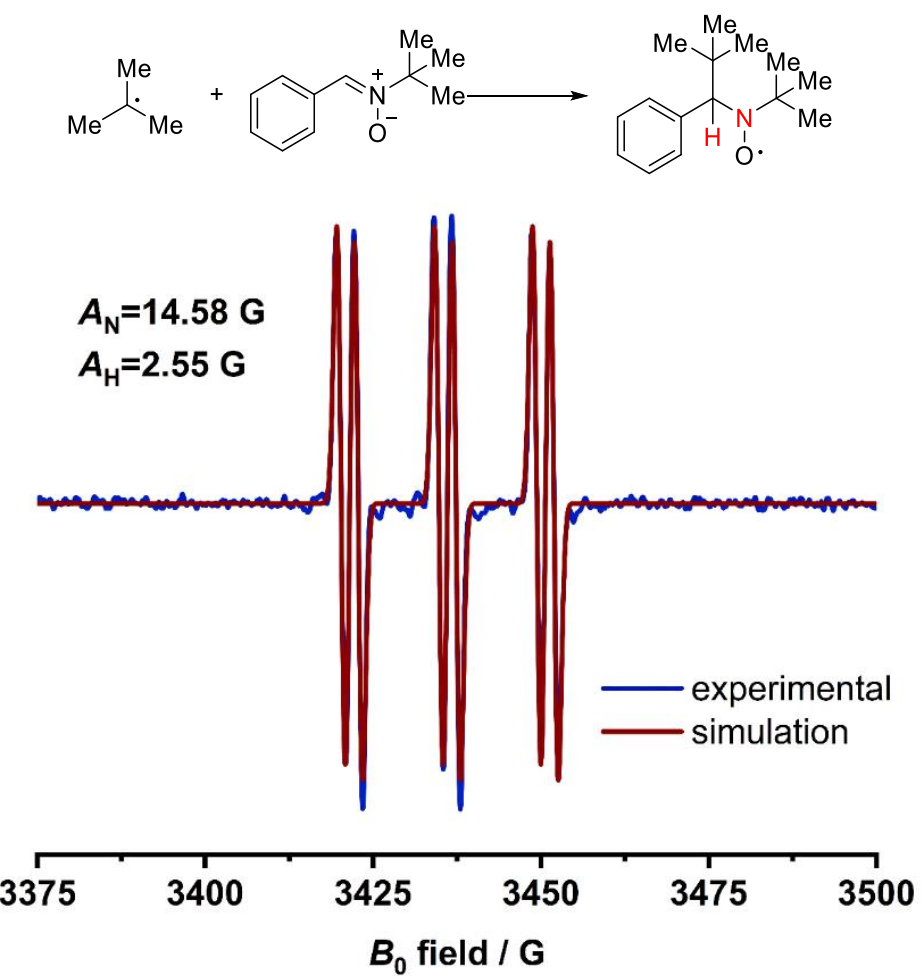

Fig. S3 EPR spectra of the tert-butyl radical spin trapped by PBN. A typical hyperfine splitting of the ${ }^{14} \mathrm{~N}$ nucleus (triplet, $\mathrm{A}_{\mathrm{N}}=14.58 \mathrm{G}$ ) and the ${ }^{1} \mathrm{H}$ nucleus (doublet, $A_{H}=2.55$ G) can be observed and calculated using SpinFit (Bruker). $(g$ factor $=2.00623$; Line width $=1.291 \mathrm{G}$ ).

\subsection{X-Ray Photoelectron Spectroscopy (XPS)}

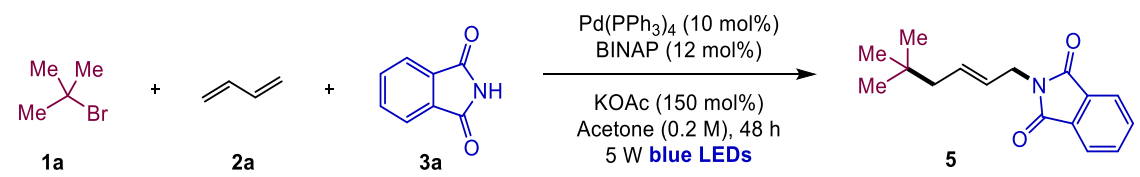

An oven-dried Schlenk tube containing a stir bar was charged under argon with the tertbutyl bromide 1a (0.3 mmol, 1.5 equiv.), $\mathrm{Pd}\left(\mathrm{PPh}_{3}\right)_{4}$ (10 mol\%), BINAP (12 mol\%), phthalimide 3a (0.2 mmol, 1.0 equiv.), KOAc (150 mol\%) and acetone (0.85 mL). Subsequently, butadiene 2a ( $0.3 \mathrm{mmol}, 0.15 \mathrm{~mL}, 2 \mathrm{M}$ in THF, 1.5 equiv.) was added. The reaction mixture was stirred at room temperate under blue LEDs irradiation (5W, 455 $\mathrm{nm}$ ) for $12 \mathrm{~h}$. Then, the reaction was concentrated under argon, then the resulting powder was analyzed by the following procedure. 


\section{Experimental}

XPS spectra of the samples were measured with an Axis Ultra DLD spectrometer (Kratos). The reaction mixture was pre-dried in the Schlenk tubes and transported to a glovebox connected to the spectrometer. Inside the glovebox, the samples were put onto a sample holder for powder samples and moved into the device, where they were dried for $12 \mathrm{~h}$ under ultra-high vacuum conditions $\left(10^{-8}\right.$ mbar $)$. Afterwards, the samples were immediately moved into the analysis chamber. Spectra were acquired using a monochromatic $\mathrm{Al} \mathrm{K} \alpha$ source at an emission current of $10 \mathrm{~mA}$ using an accelerating voltage of $12 \mathrm{kV}$. For survey spectra, a pass energy of $160 \mathrm{eV}$ was chosen and 2 sweeps were made. For high resolution core spectra, a pass energy of $20 \mathrm{eV}$ was chosen and 5 sweeps were made. A charge neutralizer was used to prevent charging of the sample. To minimize the reductive influence of prolonged x-ray exposition on Pd species, the Pd $3 d$ spectra were collected first.

The spectra were exported from the Vision2 software (Kratos) to CasaXPS (Casa). Shirley-type backgrounds were used for all spectra (average width $=5$ or 1 for core or survey). Peak fitting was performed using $\mathrm{LA}(2,2,100)$ line shapes, which consist of a symmetric Lorentzian, convoluted with a Gaussian. Energy calibration was performed using the $\mathrm{C} 1 \mathrm{~s}$ C-C peak at $284.8 \mathrm{eV}$.

\section{$\underline{\text { Results }}$}

The Pd 3d spectra indicate the presence of three distinct Pd oxidation states, namely $\operatorname{Pd}(0), \operatorname{Pd}(\mathrm{I})$ and Pd(II). (According to Alexander V. Naumkin, Anna Kraut-Vass, Stephen W. Gaarenstroom, Cedric J. Powell, NIST Standard Reference Database 20, Version 4.1 (http:/srdata.nist.gov/xps/), 2012.) This is also the case for their counterparts, which arise from spin-orbit-splitting and are shifted by $\sim 5.3 \mathrm{eV}$ towards higher binding energy. The Pd(II) and Pd(I) peak areas make up 51.4 at.\% and 44.4 at\% of the total area, respectively. 


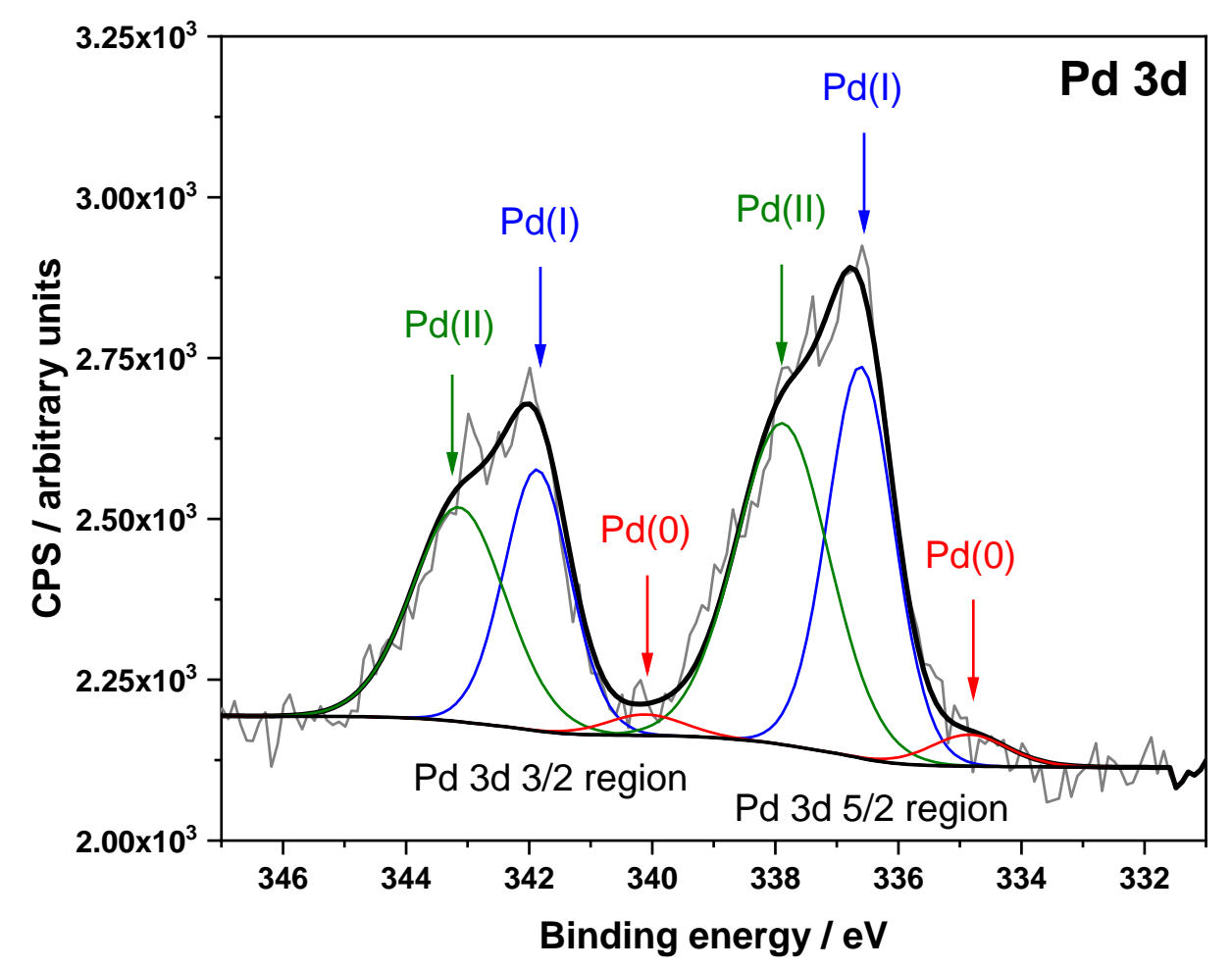

\begin{tabular}{cccc}
\hline Species & At.\% & Pos. / eV & FWHM / eV \\
\hline Pd(0) & 4.2 & 334.9 & 1.3 \\
Pd(I) & 44.4 & 336.6 & 1.5 \\
Pd(II) & 51.4 & 337.9 & 1.8 \\
\hline
\end{tabular}

Fig. S4 Three distinct oxidation states of $\mathrm{Pd}, \mathrm{Pd}(0)$ (red), $\mathrm{Pd}(\mathrm{I})$ (blue) and $\operatorname{Pd}(I I)$ (green), can be identified in the Pd 3d core spectrum.

While it would have also been possible carry out the peak fitting with only two different oxidation states, i.e. $\operatorname{Pd}(0)$ and $\operatorname{Pd}(\mathrm{II})$, this would have required very broad peaks with unusually high full width at half maximum. It can, however, not fully be excluded, that the peak associated to $\mathrm{Pd}(\mathrm{I})$ stems from $\mathrm{x}$-ray-induced reduction of $\mathrm{Pd}(\mathrm{II})$ species. When repeatedly measuring on the same position, the higher oxidized Pd species are readily reduced and the proportion of $\operatorname{Pd}(0)$ species increases notably. 


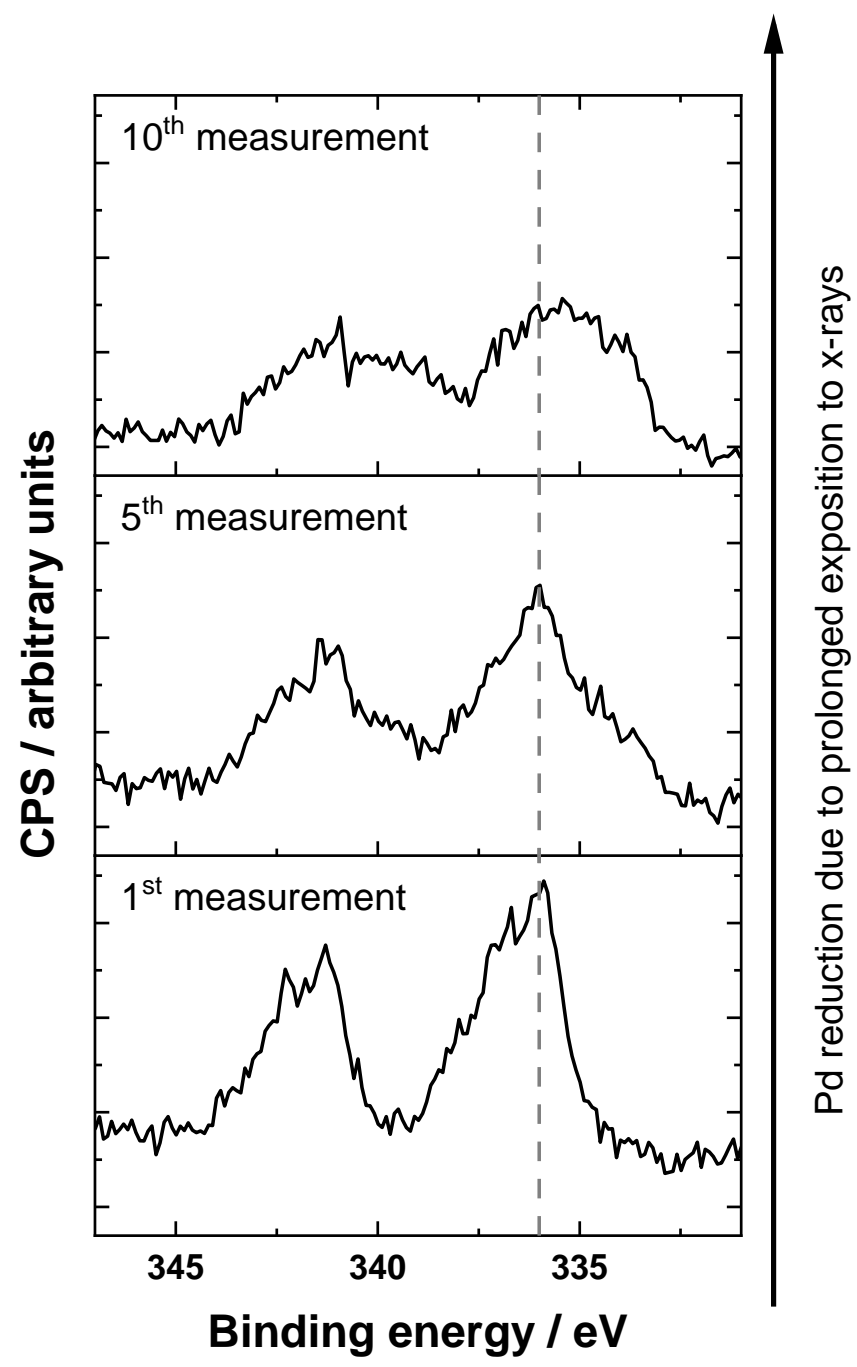

Fig. S5 Measuring Pd 3d core spectra repeatedly on the same position leads to a reduction of $\mathrm{Pd}(\mathrm{II})$ to $\mathrm{Pd}(\mathrm{I})$ and $\mathrm{Pd}(0)$. 


\subsection{UV-visible Absorption Analysis}

UV/vis absorption spectra were recorded on a Jasco V-650 spectrophotometer, equipped with a temperature control unit at $25{ }^{\circ} \mathrm{C}$. The samples were measured in Hellma fluorescence QS quartz cuvettes (chamber volume $=1.4 \mathrm{~mL}, \mathrm{H} \times \mathrm{W} \times \mathrm{D}$ $=46 \mathrm{~mm} \times 12.5 \mathrm{~mm}, 12.5 \mathrm{~mm}$ ) fitted with a PTFE stopper. The UV-visible absorption of the substrates $\operatorname{tBuBr}(\mathbf{1 a},-)$, 1,3-butadiene $(\mathbf{2 a},-)$, phthalimide $(3 \mathrm{a},-), \mathrm{Pd}\left(\mathrm{PPh}_{3}\right)_{4}$ and rac-BINAP $(-)$, and reaction mixture $(-)$ were determined at the standard reaction concentration (0.2 M) in DMA as a solvent.

According to the UV/vis spectra, the only absorbing species at $455 \mathrm{~nm}$ consists in the pre-catalytic system $\mathrm{Pd}\left(\mathrm{PPh}_{3}\right)_{4}$ and rac-BINAP, while the other components of the reaction mixture have negligible absorption in the visible range.

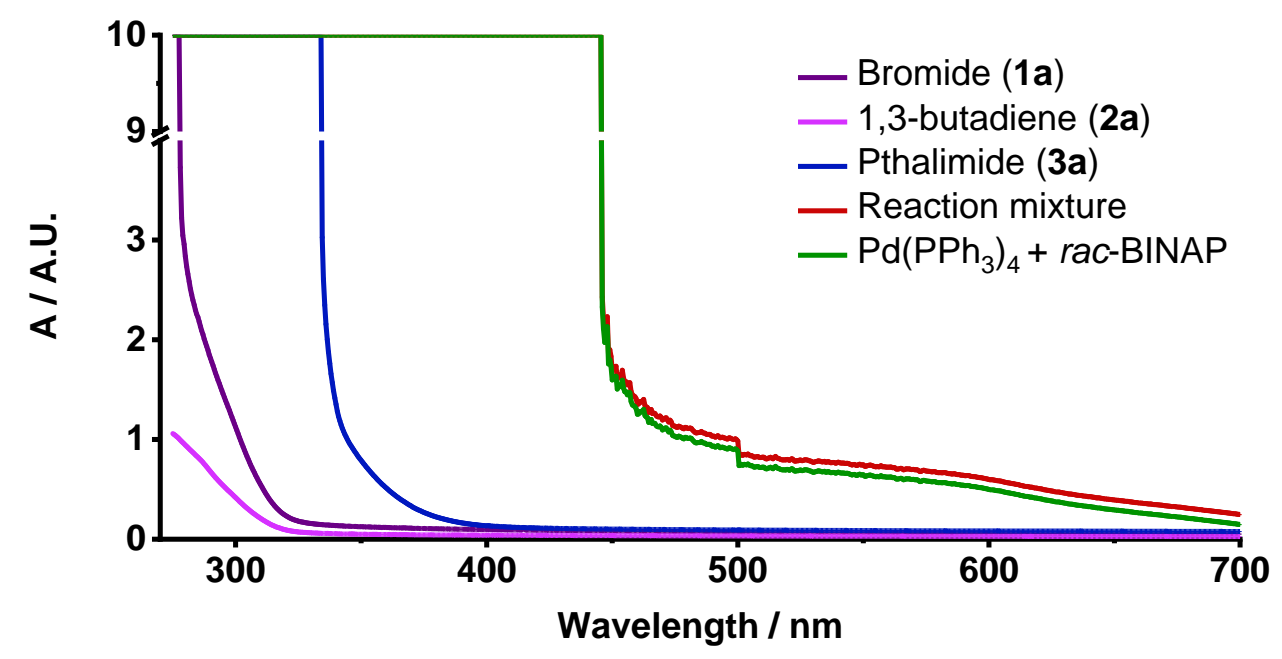

Fig. S6 UV-visible absorption spectra.

\subsection{Stern-Volmer Luminescence Quenching Analysis}

All samples used in the luminescence quenching-based screening studies were prepared under oxygen-free conditions. The palladium catalyst and potential quenchers were weighed into vials and placed inside a glovebox under a positive pressure of argon. DMA was degassed by argon sparging for one hour and placed inside along with micropipettes and their tips, cuvettes, empty vials, waste containers and parafilm. Stern-Volmer luminescence quenching studies were carried out using a $10^{-4} \mathrm{M} \mathrm{Pd}\left(\mathrm{PPh}_{3}\right)_{4}+1.2 \cdot 10^{-4} \mathrm{M}$ rac-BINAP solution and 
variable concentrations of $t \mathrm{BuBr}(\mathbf{1 a})$ butadiene (2a) and phthalimide (3a) in dry degassed DMA at room temperature under an argon atmosphere. The samples were prepared in $1.4 \mathrm{~mL}$ quartz cuvettes, equipped with PTFE stoppers and sealed with parafilm inside an argon filled glovebox. The solutions were irradiated at $420 \mathrm{~nm}$ and the luminescence was measured at $457 \mathrm{~nm}$. The ratio of $\mathrm{I}_{0} / \mathrm{I}$ was plotted as a function of the quencher concentration $\left(\mathrm{I}_{0}=\right.$ emission intensity of the photocatalyst in isolation at the specified wavelength; $\mathrm{I}=$ observed emission intensity of the photocatalyst with added quencher). The results clearly verify that only the $\operatorname{BuBr}(\mathbf{1 a})$ quenches the photocatalyst, while butadiene (2a), phthalimide (3a) does not react with the excited palladium catalyst.

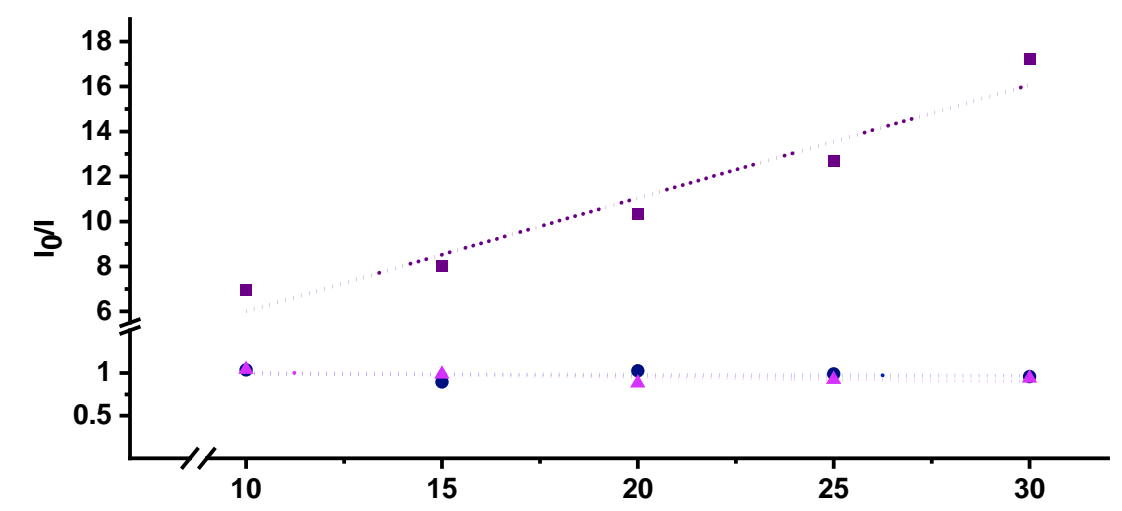

\begin{tabular}{|c|c|c|c|c|c|}
\hline Equation & $y=a+b^{*} x$ & Equation & $y=a+b^{*} x$ & Equation & $y=a+b^{*} x$ \\
\hline Plot & phthalimide & Plot & bromide & Pot & 1,3-butadiene \\
\hline Weight & No Weighting & Weight & No Weighting & & \\
\hline Intercept & $1,00015 \pm 0,0937$ & Intercept & $1 \pm 0,0194$ & Weight & No Weighting \\
\hline Slope & $0,00111 \pm 0,0044$ & Slope & $-0,02319 \pm 9,14686 \mathrm{E}-$ & Intercept & $\begin{array}{l}1,06082 \pm 5,47782 E-17 \\
-0,00529+263126 E-18\end{array}$ \\
\hline Residual Sum of Square & 0,01464 & Residual Sum of Square & $6,27488 \mathrm{E}-4$ & Residual Sum of Squares & $\begin{array}{c}-0,00529 \pm 2,63126 \mathrm{E}-18 \\
2,30783 \mathrm{E}-28\end{array}$ \\
\hline Pearson's r & 0,14372 & Pearson's r & $-0,99768$ & Pearson's r & -1 \\
\hline R-Square (COD) & 0,02066 & R-Square (COD) & 0,99536 & R-Square (COD) & 1 \\
\hline Adj. R-Square & $-0,30579$ & Adj. R-Square & 0,99381 & Adj. R-Square & 1 \\
\hline
\end{tabular}

Fig. S7 Stern-Volmer Luminescence Quenching Analysis. Legend: $\mathbf{a}=$ $t \operatorname{BuBr}(\mathbf{1 a}), \mathbf{\Delta}=1,3$-butadiene (2a),$\bullet=$ phthalimide (3a).

\subsection{Kinetic Analysis}

Preliminary information. The quantification of bromide $\mathbf{1 b}$, phthalimide $\mathbf{3 a}$ and product 83 was performed by GC-FID using an Agilent Technologies 6890A equipment equipped with a HP-5 quartz column (0.32 mm x $30 \mathrm{~m}$, film: $0.25 \mu \mathrm{m}$ ) using flame ionization detection was employed. The following method 
(50_30_40_280_3) was used: Initial temperature $50^{\circ} \mathrm{C}$, hold $3 \mathrm{~min}$, increment $20^{\circ} \mathrm{C} \cdot \mathrm{min}^{-1}$ for $11.5 \mathrm{~min}$, final temperature $280^{\circ} \mathrm{C}$, hold $3 \mathrm{~min}$.

Calibration. The calibration curves were obtained using known amounts of the corresponding compounds and $n$-dodecane as standard. The used concentrations were chosen to roughly match the experimental concentration window to be obtained by sampling of the reaction.

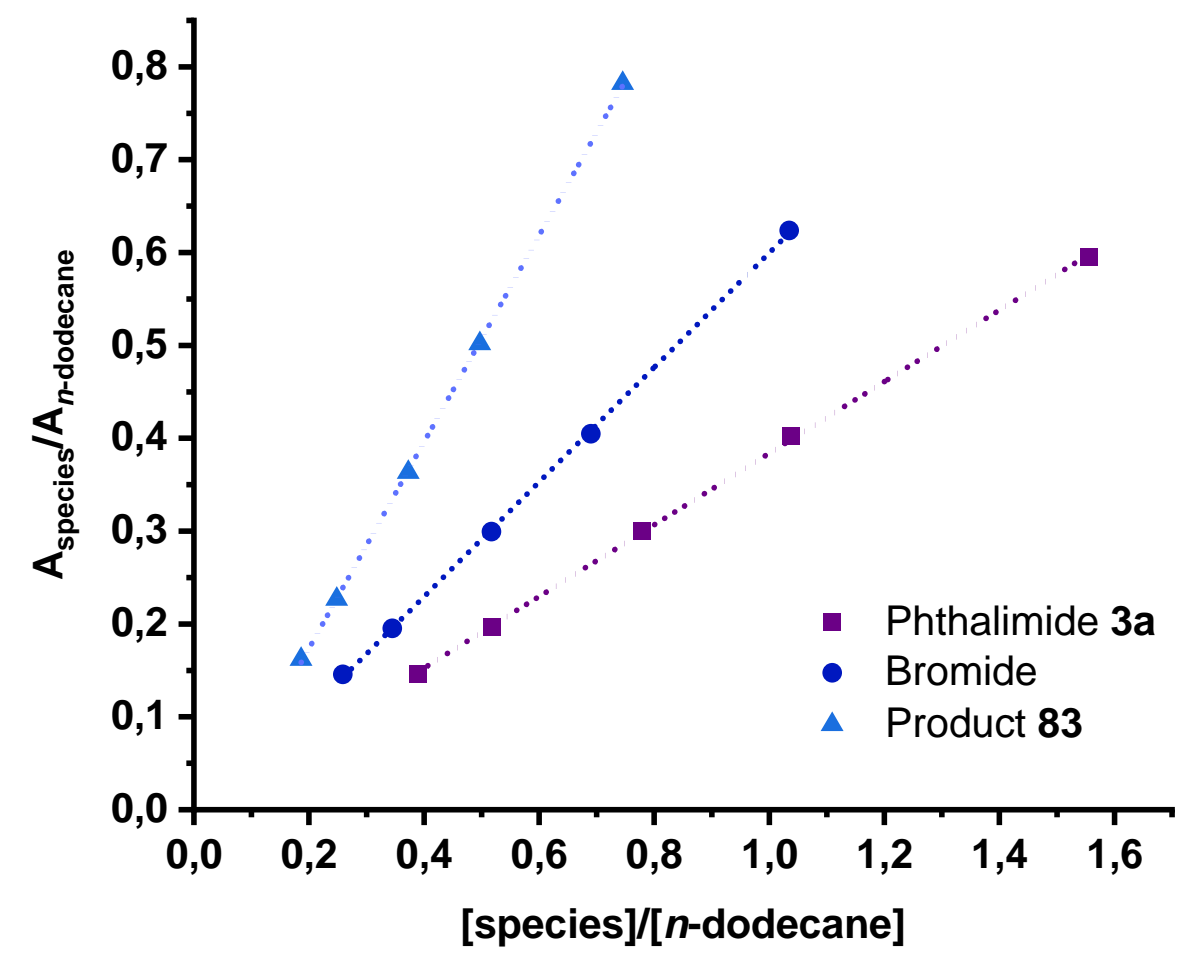

\begin{tabular}{|c|c|c|c|c|c|}
\hline Equation & $y=a+b^{*} x$ & Equation & $y=a+b^{*} x$ & Equation & $y=a+b^{\star} x$ \\
\hline Plot & phth & Plot & bromide & Plot & product \\
\hline Weight & No Weighting & Weight & No Weighting & Weight & No Weighting \\
\hline Intercept & $-0,00165 \pm 0,0034$ & Intercept & $-0,01725 \pm 0,0036$ & Intercept & $-0,0486 \pm 0,0030$ \\
\hline Slope & $0,38552 \pm 0,00363$ & Slope & $0,61707 \pm 0,00575$ & Slope & $1,11172 \pm 0,0067$ \\
\hline Residual Sum of Square & $3,3966 \mathrm{E}-5$ & Residual Sum of Square & $3,77083 \mathrm{E}-5$ & Residual Sum of Square & $2,68671 \mathrm{E}-5$ \\
\hline Pearson's $r$ & 0,99987 & Pearson's r & 0,99987 & Pearson's $r$ & 0,99994 \\
\hline R-Square (COD) & 0,99973 & R-Square (COD) & 0,99974 & R-Square (COD) & 0,99989 \\
\hline Adj. R-Square & 0,99965 & Adj. R-Square & 0,99965 & Adj. R-Square & 0,99985 \\
\hline
\end{tabular}

Fig. $\mathbf{S 8}$ Calibration curves for $\mathbf{a}=$ phthalimide $(\mathbf{3 a}), \Delta=\mathbf{8 3}, \bullet=$ bromide. The shape of the calibration curves confirmed that the typical sample concentrations are within the range of linearity of the analytical technique. The calibration coefficients reported above were used for the quantification of the analytes. For the determination of the uncertainty of the measure, a confidence 
level of $3 \sigma$ (approx. 99.5\%) was employed and the following formula was used to estimate $\sigma$ :

$$
\sigma=\frac{\sqrt{\frac{\sum\left(y_{i}-m x_{i}-b\right)^{2}}{n-2}}}{|m|} \cdot \sqrt{\frac{1}{n}+1+\frac{\left(y_{u n k}-y_{a v}\right)^{2}}{m^{2}}}
$$

where: $\sqrt{\frac{\sum\left(y_{i}-m x_{i}-b\right)^{2}}{n-2}}$ is the standard deviation of the residuals of the calibration curve, $m$ the slope of the calibration curve, $n$ the number of samples to build the calibration curve, $y_{u n k}$ the measurement of the unknown, $y_{a v}$ the average value of the standard.

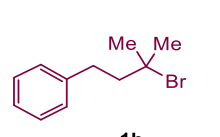

$1 \mathrm{~b}$

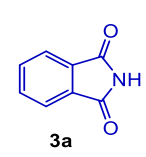

2a

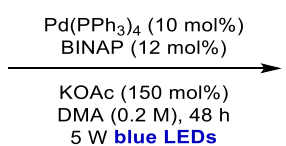

$5 \mathrm{~W}$ blue LEDs

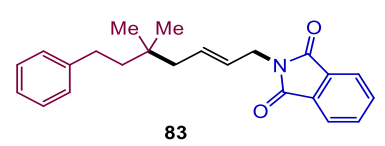

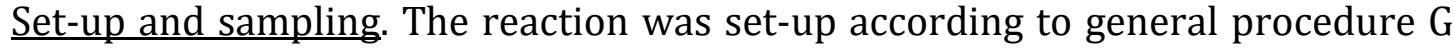
on $0.4 \mathrm{mmol}$ scale (total volume: $2.0 \mathrm{ml}$ ), with the addition of $n$-dodecane as standard $(0.20 \mathrm{M})$. Before collecting the sample at $t=0$, the reaction was stirred for 5 minutes without light irradiation, to allow the dissolution of the solids. The reaction was irradiated using the usual set-up, while maintaining the connection to the argon manifold of the Schlenk line. Sampling was performed under argon counterflow using a micropipette (approx. $30 \mu \mathrm{L}$ for each aliquot). The sampling was performed as fast as possible in order to avoid substantial 1,3-butadiene (2a) loss during the procedure. The sample was transferred to a short plug of silica and eluted with EtOAc, then analysed by GC-FID to quantify the analytes.

\begin{tabular}{cccc} 
Time / hours & {$[\mathbf{2 b}] / M$} & {$[\mathbf{8 3}] / M$} & {$[\mathbf{3 a}] / M$} \\
\hline 0 & $0.312 \pm 0.005$ & $0 \pm 0.002$ & $0.213 \pm 0.006$ \\
0.458 & $0.290 \pm 0.005$ & $0.034 \pm 0.002$ & $0.188 \pm 0.006$ \\
0.978 & $0.254 \pm 0.004$ & $0.061 \pm 0.002$ & $0.171 \pm 0.006$ \\
1.992 & $0.207 \pm 0.004$ & $0.076 \pm 0.002$ & $0.144 \pm 0.006$ \\
2.967 & $0.158 \pm 0.004$ & $0.092 \pm 0.002$ & $0.135 \pm 0.006$ \\
3.917 & $0.142 \pm 0.004$ & $0.102 \pm 0.002$ & $0.125 \pm 0.006$ \\
4.883 & $0.116 \pm 0.004$ & $0.108 \pm 0.002$ & $0.118 \pm 0.006$ \\
6.700 & $0.076 \pm 0.004$ & $0.110 \pm 0.002$ & $0.108 \pm 0.006$ \\
8.300 & $0.064 \pm 0.004$ & $0.116 \pm 0.002$ & $0.106 \pm 0.006$
\end{tabular}




$\begin{array}{llll}9.500 & 0.051 \pm 0.004 & 0.119 \pm 0.002 & 0.104 \pm 0.006 \\ 10.983 & 0.043 \pm 0.004 & 0.122 \pm 0.002 & 0.104 \pm 0.006 \\ 12.392 & 0.034 \pm 0.004 & 0.125 \pm 0.002 & 0.101 \pm 0.006 \\ 14.758 & 0.027 \pm 0.004 & 0.128 \pm 0.002 & 0.102 \pm 0.006 \\ 24.375 & 0.012 \pm 0.004 & 0.129 \pm 0.002 & 0.103 \pm 0.006 \\ 26.058 & 0.010 \pm 0.004 & 0.129 \pm 0.002 & 0.103 \pm 0.006\end{array}$

Confidence was set at $3 \sigma$ (approx. 99.5\% probability level)

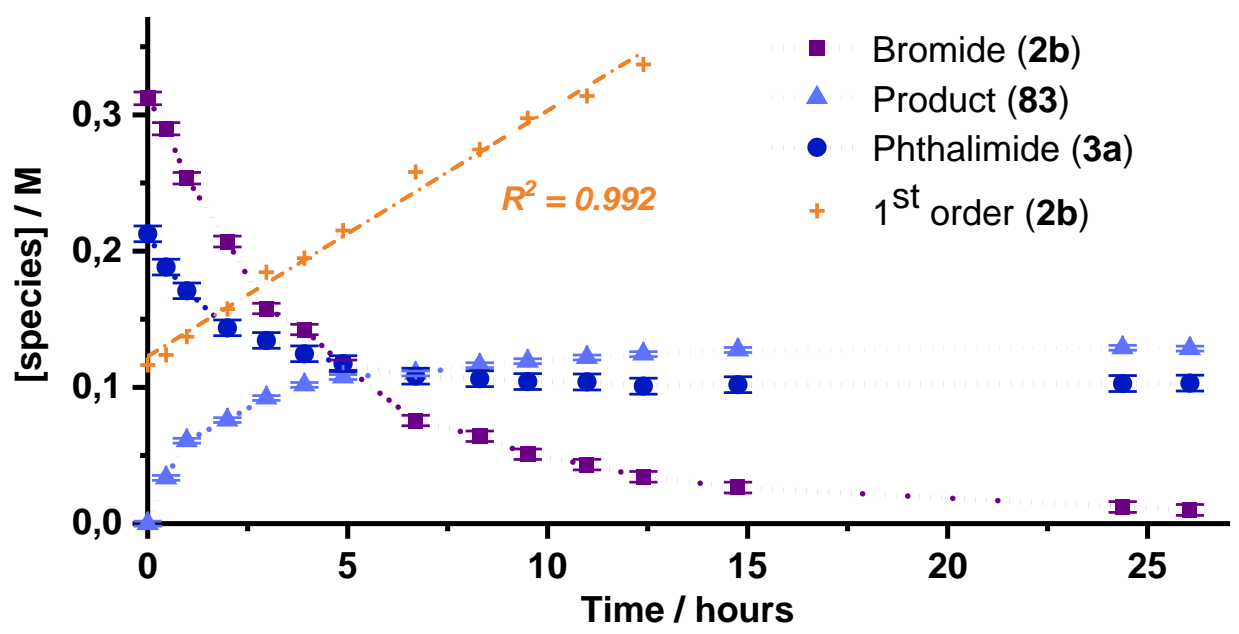

Fig. S9 Kinetic analysis of the reaction upon irradiation. In orange, the values $-0.1 \cdot \ln [\mathbf{2} \mathbf{b}]$ (the coefficients being used to improve graphical visualization) were plotted to show the $1^{\text {st }}$ order kinetic of $\mathbf{2 b}$ consumption.

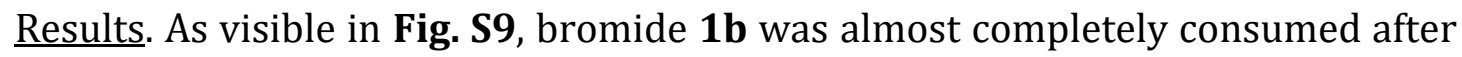
approximately 24 hours from the beginning of the irradiation. The incomplete reaction of phthalimide $\mathbf{3 a}$ is most likely caused by the loss of 1,3-butadiene $\mathbf{2 a}$, which was due to the unavoidability of reaction sampling under argon stream, as well as polymerization processes. The kinetic profile of $\mathbf{1 b}$ revealed a pseudo $1^{\text {st }}$ order consumption of the bromide $\mathbf{1 b}$, while $\mathbf{3} \mathbf{a}$ and the formation of $\mathbf{8 3}$ revealed a more complex behaviour over time. Formation of $\mathbf{8 3}$ had a specular profile compared to consumption of $\mathbf{3 a}$, suggesting that the Tsuji-Trost step of the overall process determines the overall kinetic of the product formation. 


\subsection{On-Off Experiment}

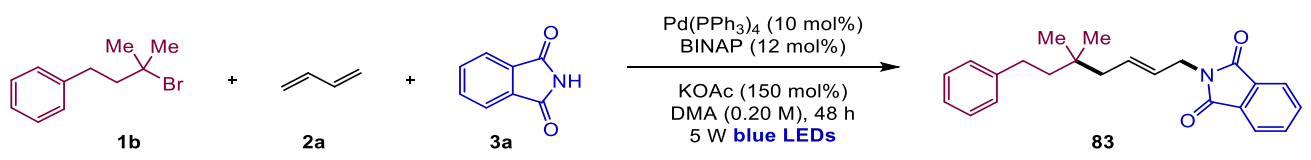

Set-up and sampling. The reaction was set-up according to general procedure $G$ on $0.4 \mathrm{mmol}$ scale (total volume: $2.0 \mathrm{~mL}$ ), with the addition of $n$-dodecane as standard $(0.20 \mathrm{M})$. Before collecting the sample at $t=0$, the reaction was stirred for 5 minutes without light irradiation, to allow the dissolution of the solids. The reaction was irradiated using the usual set-up, while maintaining the connection to the argon manifold of the Schlenk line. Sampling was performed under argon counterflow using a micropipette (approx. $30 \mu \mathrm{L}$ for each aliquot). The sampling was performed as fast as possible in order to avoid substantial 1,3-butadiene loss during the procedure. The sample was transferred to a short plug of silica and eluted with EtOAc, then analysed by GC-FID to quantify the analytes.

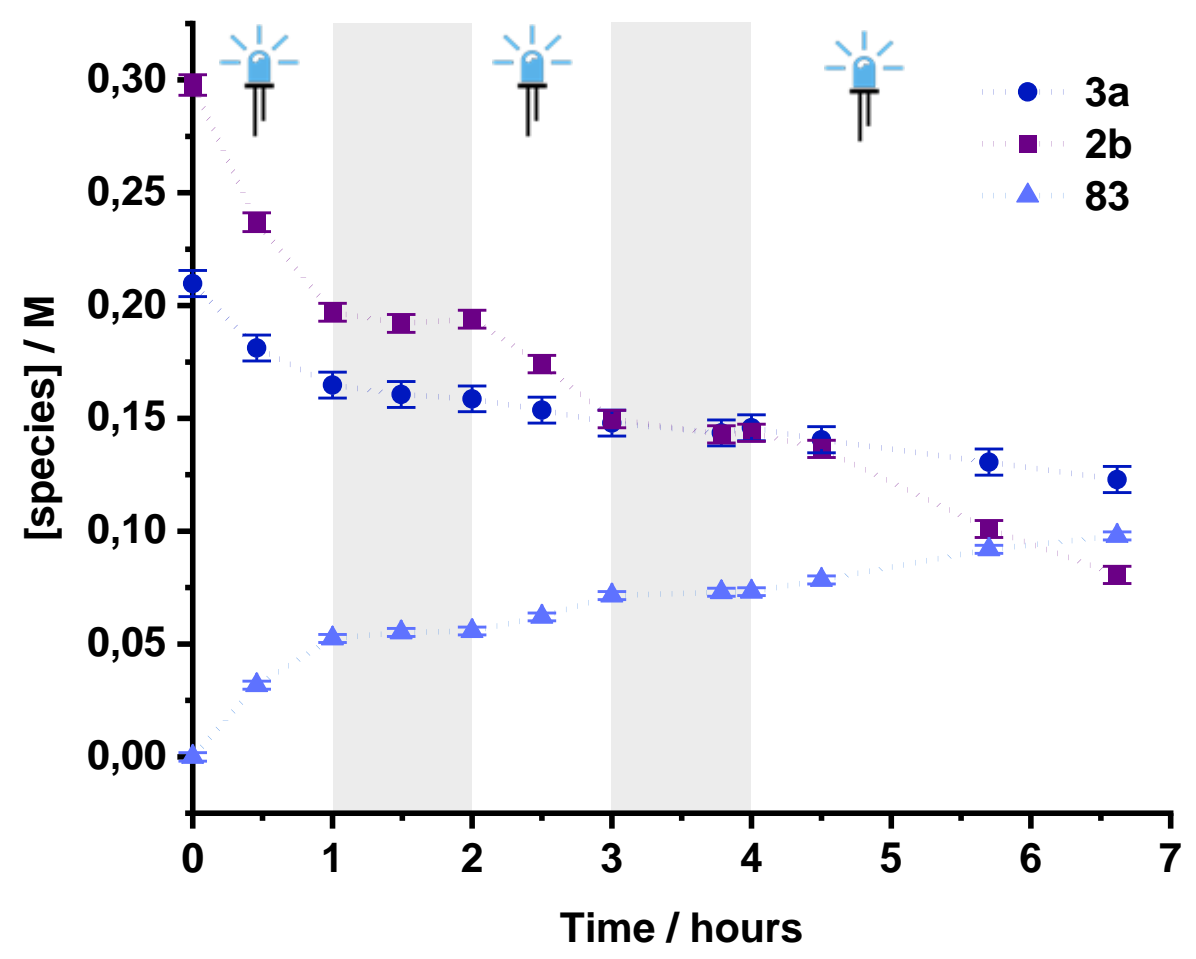

Fig. S10 Behavour of the catalytic reaction upon ON-OFF cycles. 


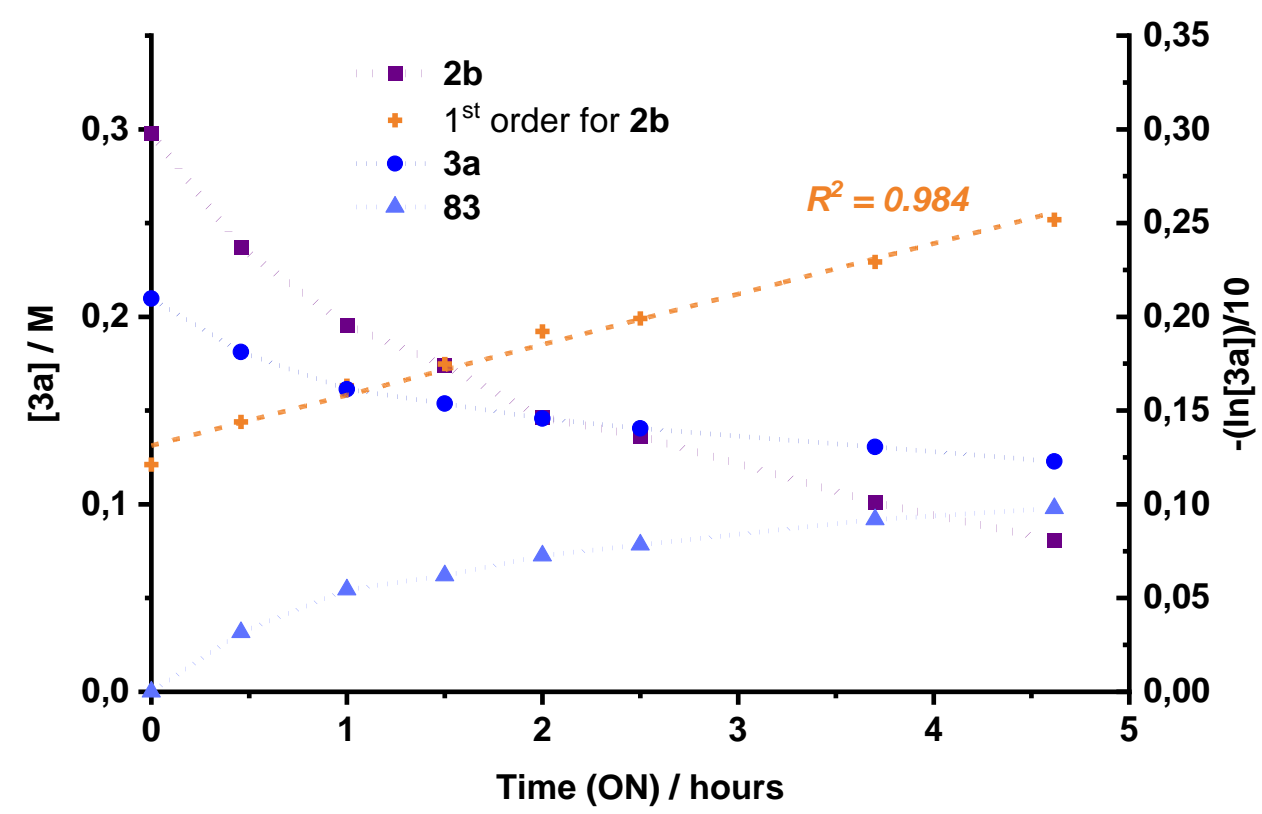

Fig. S11 Extrapolation of the kinetic data for the analytes during the ON phases. As visible from the plot of $\ln [\mathbf{2} \mathbf{b}]$ (corrected with numeric coefficients to improve visualization), the $1^{\text {st }}$ order rate was maintained.

\begin{tabular}{ccccc} 
Light & Time / hours & {$[\mathbf{2 b}] / M$} & {$[\mathbf{8 3}] / M$} & {$[\mathbf{3 a}] / M$} \\
\hline \multirow{2}{*}{ ON } & 0 & $0.298 \pm 0.005$ & $0 \pm 0.002$ & $0.210 \pm 0.006$ \\
& 0.458 & $0.237 \pm 0.004$ & $0.032 \pm 0.002$ & $0.181 \pm 0.006$ \\
\multirow{2}{*}{ OFF } & 1.000 & $0.197 \pm 0.004$ & $0.052 \pm 0.002$ & $0.165 \pm 0.006$ \\
& 1.492 & $0.192 \pm 0.004$ & $0.055 \pm 0.002$ & $0.161 \pm 0.006$ \\
ON & 2.000 & $0.194 \pm 0.004$ & $0.056 \pm 0.002$ & $0.159 \pm 0.006$ \\
& 2.500 & $0.174 \pm 0.004$ & $0.062 \pm 0.002$ & $0.154 \pm 0.006$ \\
OFF & 3.000 & $0.150 \pm 0.004$ & $0.071 \pm 0.002$ & $0.148 \pm 0.006$ \\
& 3.783 & $0.143 \pm 0.004$ & $0.073 \pm 0.002$ & $0.144 \pm 0.006$ \\
& 4.000 & $0.144 \pm 0.004$ & $0.073 \pm 0.002$ & $0.146 \pm 0.006$ \\
& 4.500 & $0.136 \pm 0.004$ & $0.078 \pm 0.002$ & $0.141 \pm 0.006$ \\
& 5.700 & $0.101 \pm 0.004$ & $0.092 \pm 0.002$ & $0.131 \pm 0.006$ \\
& 6.617 & $0.081 \pm 0.004$ & $0.098 \pm 0.002$ & $0.123 \pm 0.006$
\end{tabular}

Confidence was set at $3 \sigma$ (approx. 99.5\% probability level)

$\underline{\text { Results. }}$ As visible in Fig. S10, only upon light irradiation the starting materials are consumed, and the product formed. The catalytic activity can be restablished after absence of irradiation. Interestingly, no decrease in the catalytic 
turnover on the bromide $\mathbf{1 b}$ could be observed after an on-off cycle, as the same $1^{\text {st }}$ order kinetic is followed during multiple illumination cycles (see Fig. S11).

\subsection{Radical Probing Experiment}
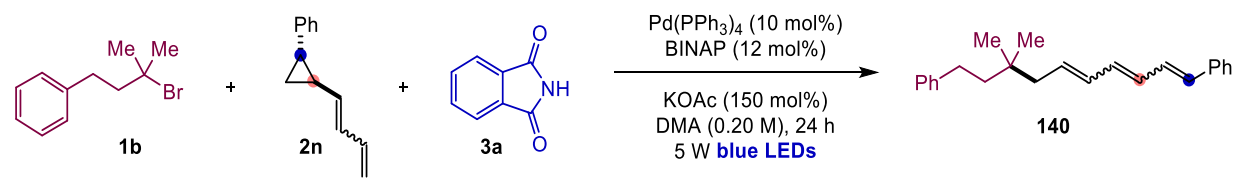

An oven-dried Schlenk tube containing a stir bar was charged under argon with

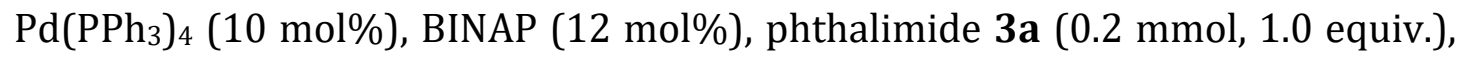
KOAc (150 mol\%) and DMA (0.85 mL). Subsequently, the bromide $\mathbf{1 b}(0.3 \mathrm{mmol}$, 1.5 equiv.) and diene $2 \mathrm{n}$ ( $0.3 \mathrm{mmol}, 1.5$ equiv.) was added. The reaction mixture was stirred at room temperate under blue LEDs irradiation (5W, $455 \mathrm{~nm}$ ) for 24 h. The solvent was removed in vacuo, then the residue was purified by multiple preparative TLC on silica (first TLC: pentane/EtOAc $=15 / 1$, fractions with Rf $>$ 0.7 submitted for second TLC: pentane/EtOAc $=200 / 1$ ), affording the product $\mathbf{1 4 0}$ as a colorless viscous oil ( $8.5 \mathrm{mg}, 26.9 \mu \mathrm{mol}, 9 \%$ to $\mathbf{1 b})$. While most of the diene 2n was recovered intact, no product arising from the nucleophilic attack could be detected in any of the fractions. $\mathbf{R}_{\boldsymbol{f}}$ (pentane/EtOAc $=200 / 1$ ) $0.40 .{ }^{1} \mathbf{H}$ NMR (599 MHz, $\mathbf{C D C l}_{3}$ ): (major isomer only) $\delta$ ppm $7.39(\mathrm{~d}, J=7.5 \mathrm{~Hz}, 2 \mathrm{H}$ ), 7.33 - $7.26(\mathrm{~m}, 5 \mathrm{H}), 7.18(\mathrm{~d}, J=7.5 \mathrm{~Hz}, 3 \mathrm{H}), 6.81(\mathrm{dd}, J=15.6,10.0 \mathrm{~Hz}, 1 \mathrm{H}), 6.54(\mathrm{t}, J=$ $16.6 \mathrm{~Hz}, 1 \mathrm{H}), 6.43-6.24(\mathrm{~m}, 2 \mathrm{H}), 6.16(\mathrm{dd}, J=15.0,10.0 \mathrm{~Hz}, 1 \mathrm{H}), 5.81(\mathrm{dt}, J=15.2$, $7.8 \mathrm{~Hz}, 1 \mathrm{H}), 2.63-2.52(\mathrm{~m}, 2 \mathrm{H}), 2.10(\mathrm{~d}, J=7.8 \mathrm{~Hz}, 2 \mathrm{H}), 1.53-1.50(\mathrm{~m}, 2 \mathrm{H}), 0.96$ (s, 6H). ${ }^{13}$ C NMR (151 MHz, CDCl $_{3}$ ): $\delta$ ppm 143.5, 137.7, 133.8, 133.0, 132.6, 131.8, 131.2, 129.4, 128.7, 128.5, 127.4, 126.5, 126.4, 125.7, 45.7, 44.4, 34.3, 30.9, 27.2.

\section{Comment on the result:}

As shown in Fig. S12, the hybrid alkyl Pd(I) radical species may form Pd(II)-alkyl intermediate via radical recombination (path a), then the same $\operatorname{Pd}(\mathrm{II})$ intermediate could be formed through a carbopalladation step between 1,3- 
dienes and $\mathrm{Pd}(\mathrm{II})$-alkyl intermediate. After $\beta$-carbon elimination and $\beta$-hydride elimination, the product 141 could be obtained. However, only product 140 could be obtained, which clearly shows that the reaction must occur through path $b$. Therefore, we can assume that after radical addition to 1,3-diene, ringopening and ß-hydride elimination cascade yields the isolated product $\mathbf{1 4 0 .}$

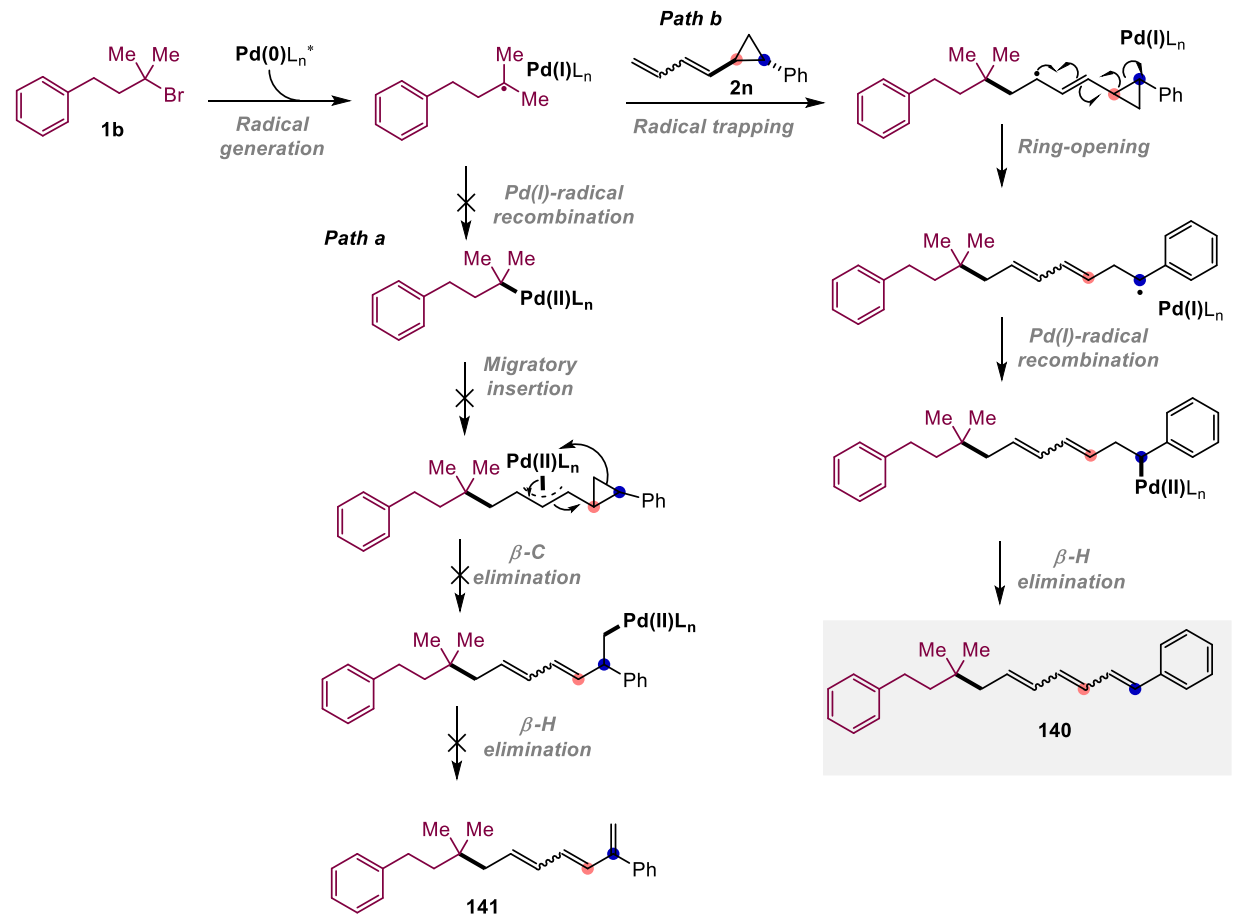

Fig. S12 Possible catalytic pathways in the formal insertion step of the 1,3diene.

\subsection{ESI-MS Analysis}

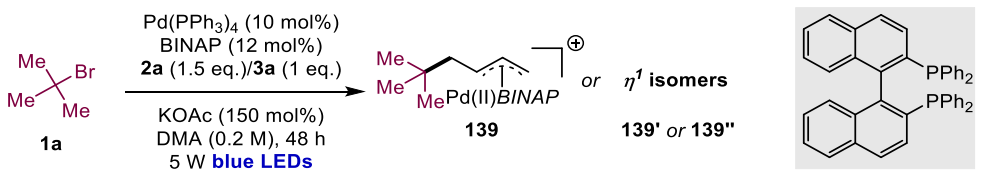

An oven-dried Schlenk tube containing a stir bar was charged under argon with the tert-butyl bromide $1 \mathrm{a}\left(0.3 \mathrm{mmol}, 1.5\right.$ equiv.), $\mathrm{Pd}\left(\mathrm{PPh}_{3}\right)_{4}(10 \mathrm{~mol} \%)$, BINAP (12 mol\%), phthalimide 3a (0.2 mmol, 1.0 equiv.), KOAc (150 mol\%) and DMA (0.85 mL). Subsequently, butadiene $2 \mathbf{a}(0.3 \mathrm{mmol}, 0.15 \mathrm{~mL}, 2 M$ in THF, 1.5 equiv.) was added. The reaction mixture was stirred at room temperate under blue LEDs 
irradiation (5W, $455 \mathrm{~nm}$ ) for $2 \mathrm{~h}$. Then the palladium intermediate (139 or 139' or 139") was detected and confirmed by high-resolution mass spectrometry (HRMS). HRMS (ESI+): calcd. for $\mathrm{C}_{52} \mathrm{H}_{47} \mathrm{P}_{2} \mathrm{Pd}\left(\mathrm{M}^{+}\right)$: 839.22006. Found: 839.22014.

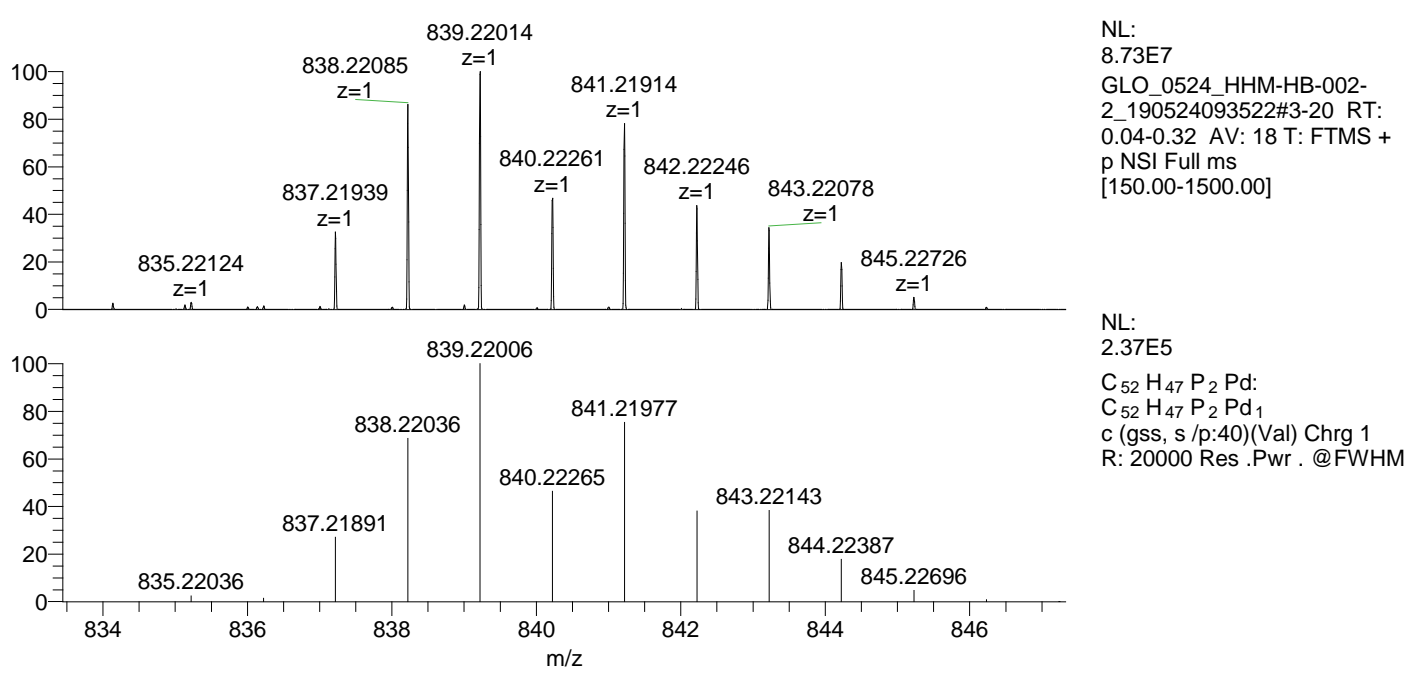

Fig. S13 High-resolution mass spectra of palladium intermediate.

\subsection{Reaction of Pd(II)-allyl complexes with nucleophiles}
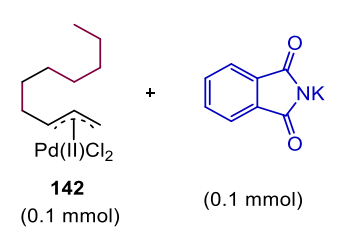

$\underset{\text { DMA (0.2 M), RT, } 24 \mathrm{~h}}{\stackrel{\text { Ligand (1.0 equiv.) }}{\longrightarrow}}$
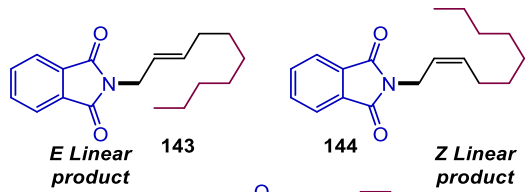

(0.1 mmol)

(0.1 mmol)

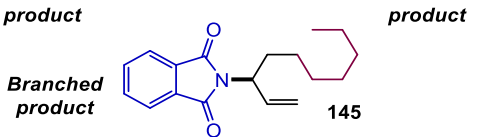

In an oven-dried Schlenk tube equipped with a PTFE-coated stirring bar, the Pd(II)-allyl complex 142 (28.1 mg, 0.1 mmol, 1.0 equiv.), potassium phthalimide (18.3 $\mathrm{mg}, 0.1 \mathrm{mmol}, 1.0$ equiv.) and the appropriate ligand $(0.1 \mathrm{mmol})$ were charged under argon, then DMA $(0.5 \mathrm{ml})$ was added and the reaction was stirred at room temperature for 24 hours. The solvent was removed in vacuo, then the product was purified by flash column chromatography on silica (pentane/EtOAc $=9 / 1)$ and the product ratio analyzed by ${ }^{1} \mathrm{H}$ NMR. ${ }^{1} \mathrm{H}$ NMR $\left(400 \mathrm{MHz}, \mathbf{C D C l}_{3}, \boldsymbol{E}\right.$ linear product, diagnostic peaks): $\delta$ ppm $5.74(\mathrm{dtt}, J=15.2,6.7,1.0 \mathrm{~Hz}, 1 \mathrm{H})$, $5.50(\mathrm{dtt}, J=15.4,6.2,1.5 \mathrm{~Hz}, 1 \mathrm{H}), 4.23(\mathrm{dd}, J=6.2,1.2 \mathrm{~Hz}, 2 \mathrm{H}), 1.99(\mathrm{q}, J=7.8,7.1$ $\mathrm{Hz}, 2 \mathrm{H}$ ). ${ }^{1} \mathrm{H}$ NMR (400 MHz, $Z$ linear product, diagnostic peaks): $\delta$ ppm 5.64 - 
$5.54(\mathrm{~m}, 1 \mathrm{H}), 4.31(\mathrm{dd}, J=6.9,1.4 \mathrm{~Hz}, 2 \mathrm{H}), 2.25(\mathrm{q}, J=7.5 \mathrm{~Hz}, 2 \mathrm{H}) .{ }^{\mathbf{1}} \mathbf{H}$ NMR (400 MHz, branched product, diagnostic peaks): $\delta$ ppm 6.21 (ddd, $J=17.5,10.2,7.6$ $\mathrm{Hz}, 1 \mathrm{H}), 5.22$ (dd, $J=17.2,1.3 \mathrm{~Hz}, 1 \mathrm{H}), 5.15(\mathrm{~d}, J=10.2 \mathrm{~Hz}, 1 \mathrm{H}), 4.71(\mathrm{q}, J=7.8 \mathrm{~Hz}$, $1 \mathrm{H})$.

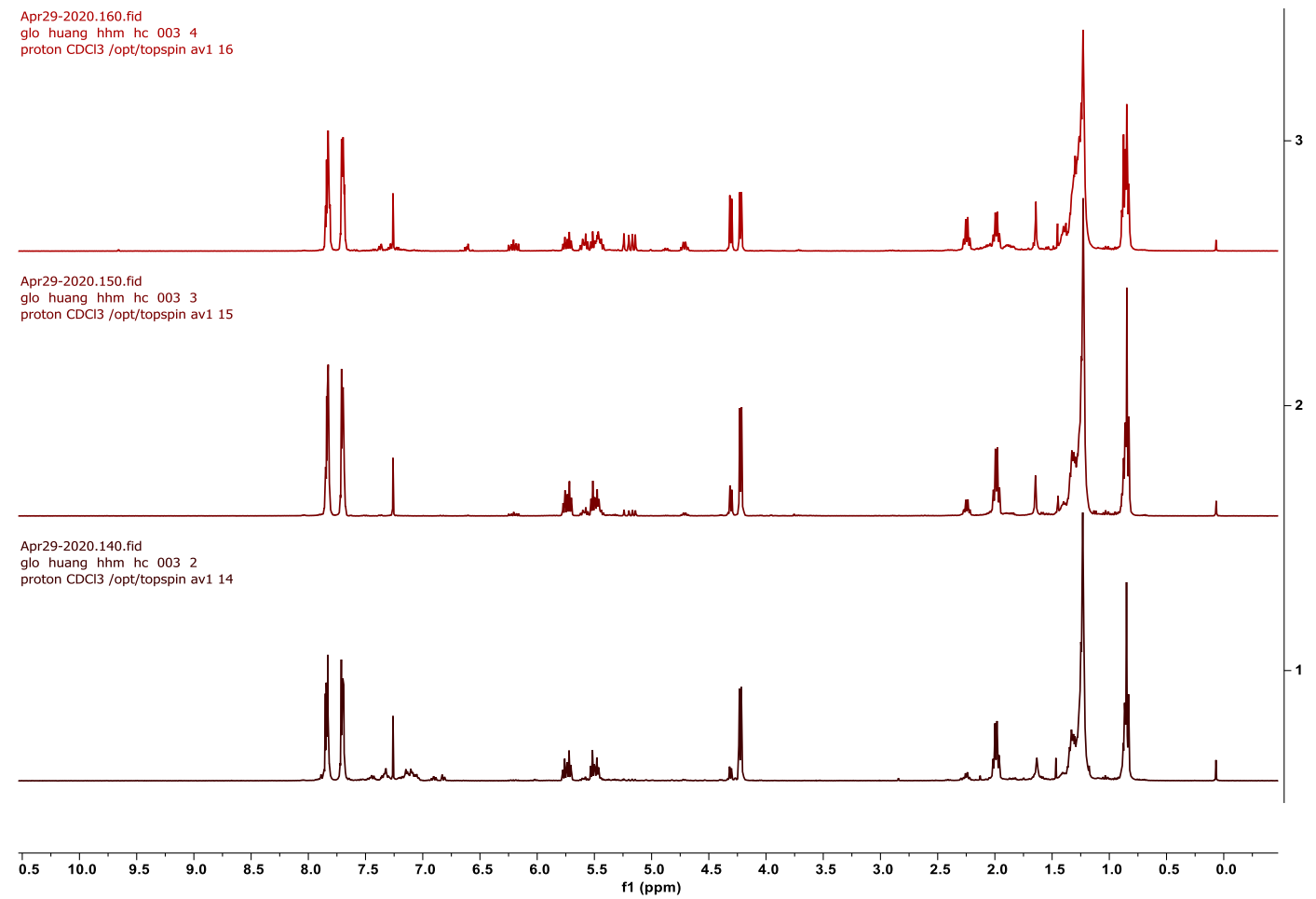

Fig. S14 ${ }^{1} \mathrm{H}$ NMR spectra of the product 143, 144, 145 upon reaction using BINAP (1), DPEPhos (2) and XantPhos (3).

\begin{tabular}{ccccc} 
Ligand & Yield \% & $\boldsymbol{E}$ linear \% & $\boldsymbol{Z}$ Linear \% & Branched \% \\
\hline BINAP & 77 & 83 & 13 & 4 \\
DPEPhos & 74 & 75 & 18 & 7 \\
XantPhos & 67 & 43 & 37 & 20
\end{tabular}

Comment:

The results are in accordance with early reports from Van Leeuwen, ${ }^{58}$ which observed a reduced regioselectivity for the terminal position, as well as diminished $E / Z$ stereoselectivity, when bidentate phosphines with increasing 
bite angle were used in stoichiometric reaction between $\mathrm{Pd}(\mathrm{II})$-allyl species and nucleophiles. As summarized by Fig. S15, this accounts for the presence of both syn and anti $\eta^{3}$-allyl species. While the anti isomer is higher in energy than the syn, the energetic difference becomes increasingly smaller in presence of ligands with large bite angle. The syn complex can deliver the $E$ linear product with high selectivity (path b), because the other possible attack is highly unfavorable due to steric bulk. On the other hand, the anti complex does not show such high preference for the terminal position (path d), thus offering possibility of forming both the branched product (path c) and $Z$ linear one.

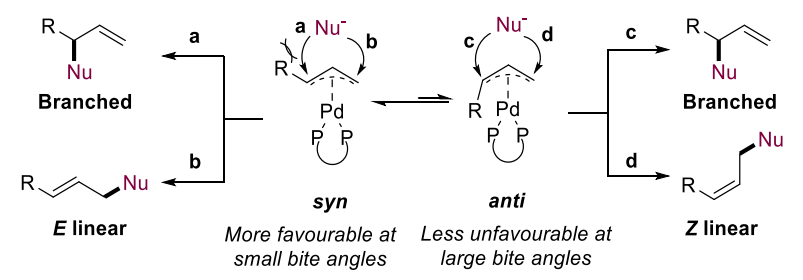

Fig. S15 Regio and stereoselectivity of the nucleophilic attack.

We can therefore conclude that the choice of BINAP as ligand has a strong impact on the reaction outcome, in particular concerning the regio and stereoselectivity of the product formation.

\subsection{Alternative Proposed Mechanism}

Based the mechanism we proposed in main text, butadiene 2a may pre-associate to the Pd species that would accelerate the rate of alkyl radical addition to $\mathbf{2 a}$ and recombination of allylic radical with $\mathrm{Pd}(\mathrm{I})$ radical species ${ }^{59,60}$, so an alternative mechanism has been proposed. Please see it below. 


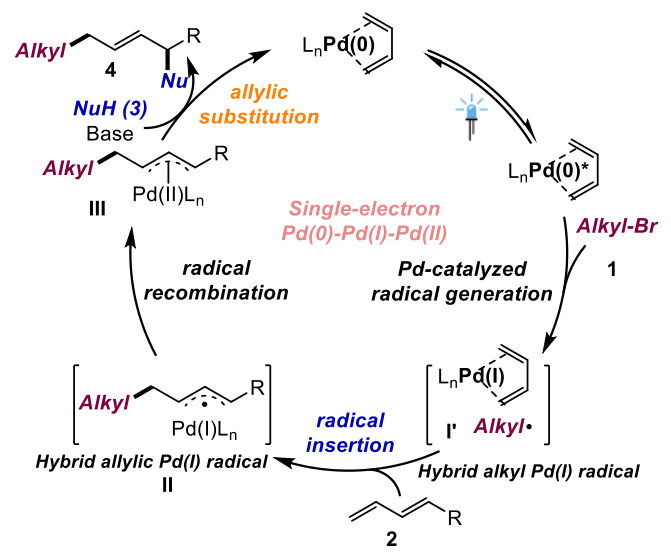

Fig. S16 Alternative possible mechanism

\section{Computational Details}

All computations were carried out using ORCA 4.1.1 software package. ${ }^{61}$ The geometries were optimized with the M06 meta-GGA-hybrid functional ${ }^{62}$ on a recontracted Ahlrichs double- $\zeta$-basis (ZORA-def2-SVP) ${ }^{63}$ using ZORA ${ }^{64}$ for scalar relativistic approximations, applying the CPCM continuum solvation model for DMF.65,66 Optimized geometries were characterized to be a minima on the respective potential energy surface by harmonic frequency analysis on the same level of theory. For further energy evaluation single point calculations were performed on the optimized geometries using the M06 meta-GGA-hybrid functional on a recontracted triple- $\zeta$-basis set (ZORA-def2-TZVP) with the CPCM for DMF. No symmetry or internal coordinate constraints were applied. Optimized structures are given in the .xyz format and visualized using Jmol.67 Redox potentials were determined adapting a protocol reported by Nicewicz and coworkers $^{68}$ using the M06 meta-GGA-hybrid functional on a triple- $\zeta$-basis set (def2-TZVPP) with the CPCM for DMF. 


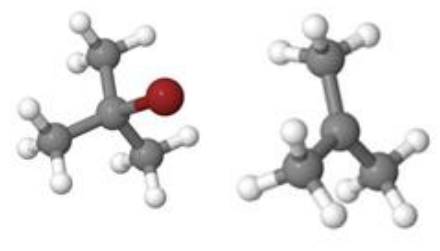

A

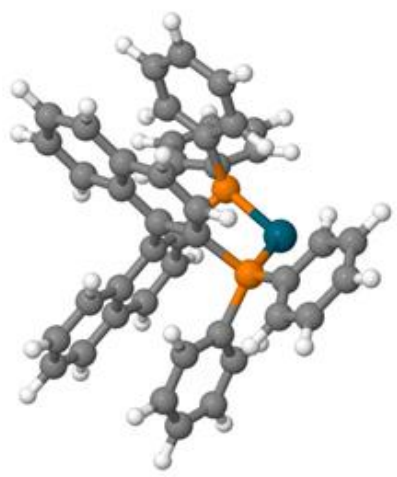

$E_{0}$

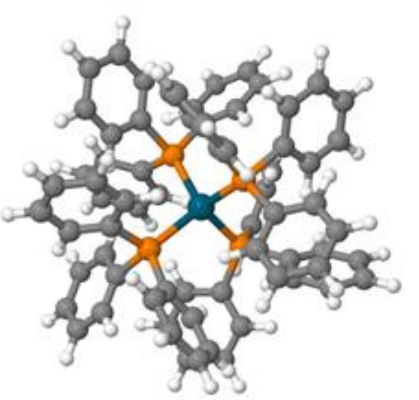

$F_{0}$
B

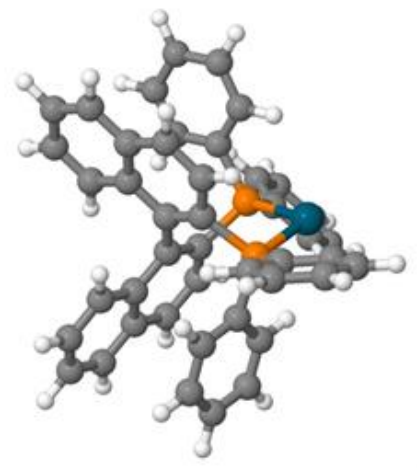

$\mathrm{E}_{1}$

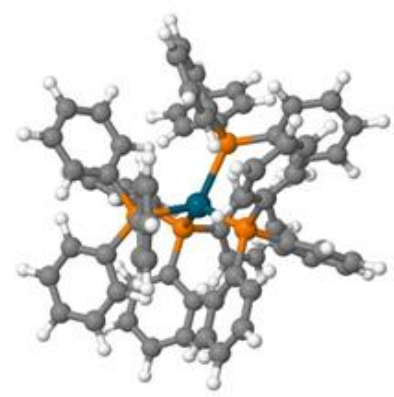

$\mathbf{F}_{1}$

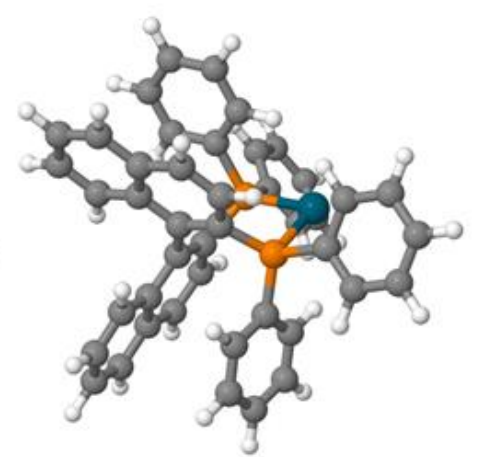

$E_{2}$

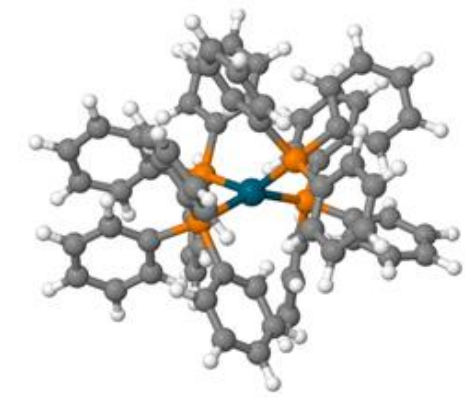

$F_{2}$ 


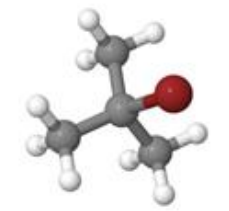

A

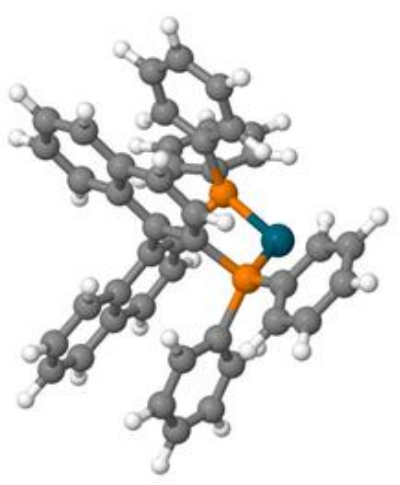

$E_{0}$

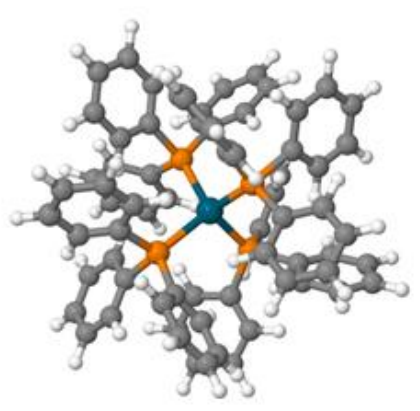

$\mathbf{F}_{0}$

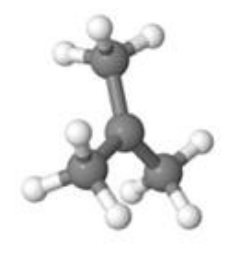

B

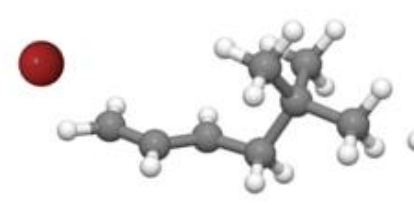

C

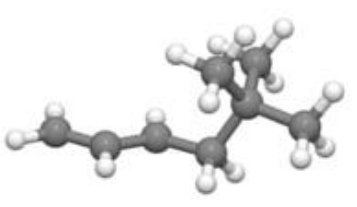

D

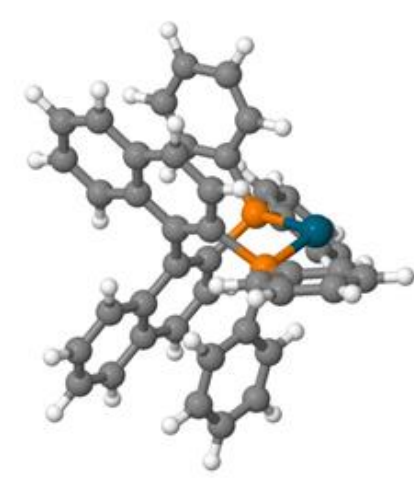

$\mathrm{E}_{1}$

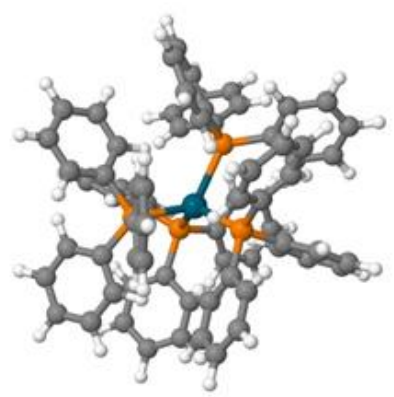

$\mathbf{F}_{1}$

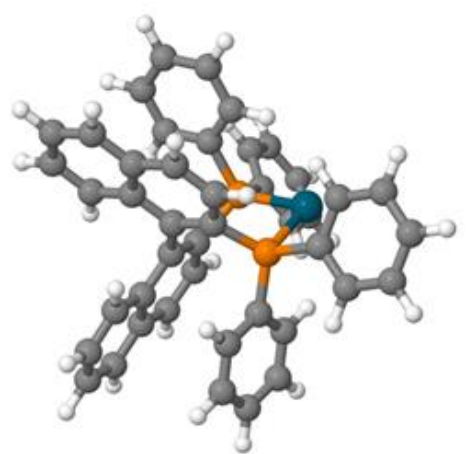

$E_{2}$

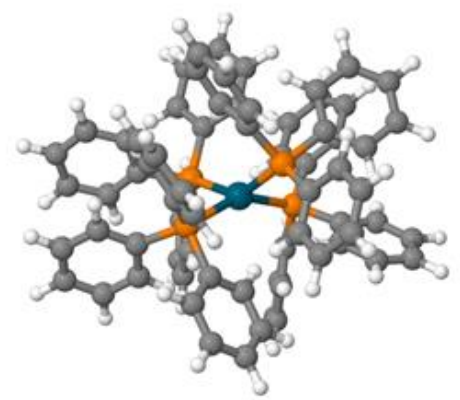

$\mathbf{F}_{2}$

Fig. S17 Optimized geometries.

Free energies at $298 \mathrm{~K}\left(\mathrm{G}_{298}\right)$ were obtained as the sum of the electronic energies (SCF energies) and the corresponding free energy corrections (ZPVE, thermal corrections, enthalpy correction, entropic corrections), as obtained from the frequency calculation.

Table S1. Determination of the Free Enthalpy (in Hartree) at 298 K (M06 /TZVPP) / CPCM (DMF) for diverse species.

\begin{tabular}{|l|l|l|l|l|l|}
\hline & $\begin{array}{l}\text { Electronic } \\
\text { Energy }\end{array}$ & $\begin{array}{l}\text { Thermal } \\
\text { Corrections }\end{array}$ & ZPVE & $\begin{array}{l}\text { Enthalpy } \\
\text { Corrections }\end{array}$ & $\begin{array}{l}\text { Entropy } \\
\text { Corrections }\end{array}$ \\
\hline
\end{tabular}




\begin{tabular}{|l|l|l|l|l|l|}
\hline$A$ & -313.723211 & 0.01029256 & 0.20802532 & 0.00094421 & -0.04532433 \\
\hline$A^{\text {red }}(B)$ & -313.903906 & 0.01024589 & 0.2059016 & 0.00094421 & -0.04572756 \\
\hline $\mathbf{C}$ & -2782.22687 & 0.00667594 & 0.12098994 & 0.00094421 & -0.03823676 \\
\hline $\mathbf{C}^{\text {red }}(\mathbf{D})$ & -2782.33307 & 0.00875988 & 0.11604744 & 0.00094421 & -0.04471573 \\
\hline $\mathbf{E}_{\mathbf{0}}$ & -7485.63544 & 0.03840319 & 0.62022605 & 0.00094421 & -0.10242641 \\
\hline $\mathbf{E}_{\mathbf{1}}$ & -7485.48034 & 0.03939681 & 0.62114627 & 0.00094421 & -0.10566064 \\
\hline $\mathbf{E}_{\mathbf{2}}$ & -7485.29897 & 0.0384439 & 0.62185644 & 0.00094421 & -0.10273389 \\
\hline $\mathbf{F}_{\mathbf{0}}$ & -9255.13435 & 0.06751600 & 1.09411792 & 0.00094421 & -0.15766620 \\
\hline $\mathbf{F}_{\mathbf{1}}$ & -9254.97898 & 0.06851186 & 1.09772733 & 0.00094421 & -0.16021401 \\
\hline $\mathbf{F}_{\mathbf{2}}$ & -9254.79951 & 0.06910879 & 1.10089521 & 0.00094421 & -0.16031032 \\
\hline
\end{tabular}

Redox potentials were then calculated from the free Energies of the respective oxidized and reduced species; the values were referenced to saturated calomel electrode (SCE) by subtraction of its absolute potential in acetonitrile $\left(\mathrm{E}_{\text {ref }}=4.422\right.$ V). ${ }^{69}$

$$
E^{c a l c}=\frac{G_{298}(r e d)-G_{298}(o x)}{F}-E_{r e f}
$$

Table S2. Redox potential determination for diverse species.

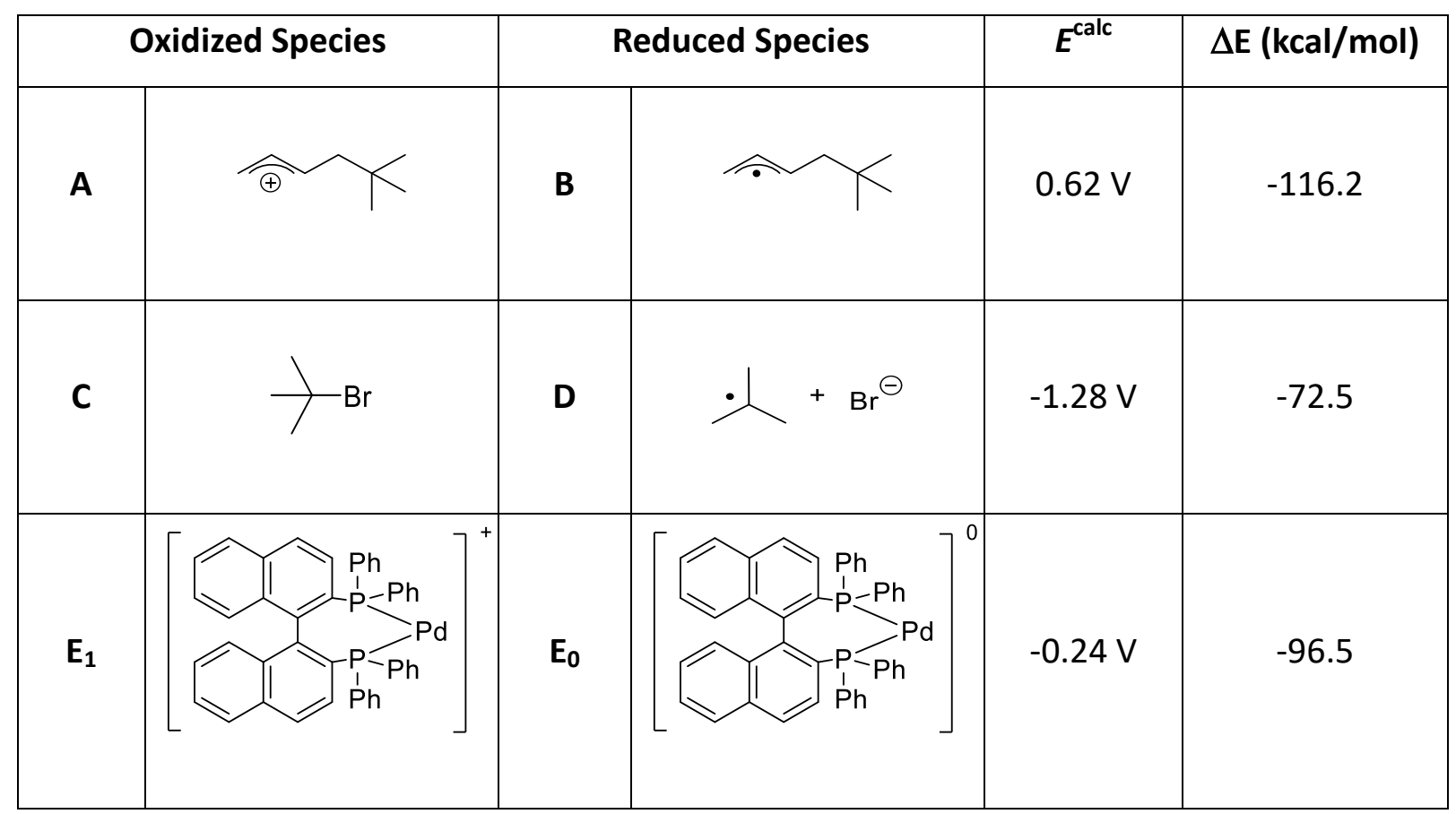




\begin{tabular}{|c|c|c|c|c|c|}
\hline$E_{2}$ & ${ }_{P}^{P h}$ & $E_{1}$ & $\mathrm{~N}$ & $0.56 \mathrm{~V}$ & -115.5 \\
\hline$F_{1}$ & {$\left[\begin{array}{c}\mathrm{PPh}_{3} \\
\mid \mathrm{Ph}_{3} \mathrm{P}-\mathrm{Pd}-\mathrm{PPh}_{3} \\
\mid \mathrm{PPh}_{3}\end{array}\right]^{+}$} & $F_{0}$ & {$\left[\begin{array}{c}\mathrm{PPh}_{3} \\
\mid \mathrm{Ph}_{3} \mathrm{P}-\mathrm{Pd}-\mathrm{PPh}_{3} \\
\mid \\
\mathrm{PPh}_{3}\end{array}\right]^{0}$} & $-0.14 \mathrm{~V}$ & -98.7 \\
\hline$F_{2}$ & {$\left[\begin{array}{l}\mathrm{PPh}_{3} \\
\mathrm{Ph}_{3} \mathrm{P}-\mathrm{Pd}-\mathrm{PPh}_{3} \\
\mid \\
\mathrm{PPh}_{3}\end{array}\right]^{2+}$} & $F_{1}$ & {$\left[\begin{array}{c}\mathrm{PPh}_{3} \\
\mathrm{Ph}_{3} \mathrm{P}-\mathrm{Pd}-\mathrm{PPh}_{3} \\
\mid \mathrm{PPh}_{3}\end{array}\right]^{+}$} & $0.56 \mathrm{~V}$ & -114.9 \\
\hline
\end{tabular}

To disprove a radical chain process, redox potentials of $\mathbf{A} / \mathbf{B}$ as well as $\mathbf{C} / \mathbf{D}, \mathbf{E}_{\mathbf{0}} / \mathbf{E}_{\mathbf{1}}$, $\mathbf{E}_{1} / \mathbf{E}_{2}, \mathbf{F}_{\mathbf{0}} / \mathbf{F}_{1}$ and $\mathbf{F}_{\mathbf{1}} / \mathbf{F}_{2}$ were calculated and compared. An oxidation of species $\mathbf{B}$ by $\mathbf{E}_{1}$ or $\mathbf{F}_{\mathbf{1}}$ forming $\mathbf{A}$ and $\mathbf{E}_{\mathbf{0}}$ or $\mathbf{F}_{\mathbf{0}}$ is thermodynamically not feasible due to an energy difference of $19.7 \mathrm{kcal} / \mathrm{mol}$ or $17.5 \mathrm{kcal} / \mathrm{mol}$. Furthermore, a reduction of $\mathbf{C}$ by $\mathbf{B}$ is thermodynamically not possible due to a high energy difference of $43.7 \mathrm{kcal} / \mathrm{mol}$.
A
C $\quad-0.450469-1.392974 \quad-0.766962$
C $\quad-0.593275 \quad-0.654154 \quad 0.372466$
H $\quad-1.261716 \quad-2.004523 \quad-1.161195$
H $\quad 0.492425 \quad-1.388849 \quad-1.317149$
C $\quad 0.488581 \quad 0.114251 \quad 0.811243$
C $\quad 0.469334 \quad 0.977107 \quad 1.970536$
$\begin{array}{llll}\mathrm{H} & -0.401721 & 0.773494 & 2.608722\end{array}$
C $\quad 0.463329 \quad 2.508037 \quad 1.576382$
$\begin{array}{llll}\mathrm{H} & 1.401370 & 0.817018 & 2.539794\end{array}$
$\begin{array}{llll}\mathrm{H} & -1.527485 & -0.651791 & 0.937585\end{array}$
$\begin{array}{llll}\mathrm{H} & 1.403777 & 0.078034 & 0.207288\end{array}$
$\begin{array}{llll}\text { C } & -0.799099 & 2.817661 & 0.780126\end{array}$
C $\quad 1.707243 \quad 2.862240 \quad 0.771359$
$\begin{array}{llll}\text { C } & 0.454888 & 3.284342 & 2.887583\end{array}$ 


$\begin{array}{lrrr}\mathrm{H} & -0.424833 & 3.035110 & 3.497583 \\ \mathrm{H} & 0.424506 & 4.363071 & 2.675227 \\ \mathrm{H} & 1.357148 & 3.079759 & 3.481096 \\ \mathrm{H} & 1.724125 & 2.373784 & -0.213103 \\ \mathrm{H} & 2.626336 & 2.588816 & 1.309729 \\ \mathrm{H} & 1.732502 & 3.947390 & 0.596321 \\ \mathrm{H} & -0.804329 & 2.314364 & -0.198453 \\ \mathrm{H} & -0.866380 & 3.899148 & 0.594430 \\ \mathrm{H} & -1.703725 & 2.514135 & 1.327811\end{array}$

B

C $\quad-0.509593 \quad-1.489264 \quad-0.800991$

$\begin{array}{llll}\text { C } & -0.530717 & -0.653350 & 0.299135\end{array}$

$\mathrm{H} \quad-1.389957 \quad-2.051381-1.111306$

$\mathrm{H} \quad 0.401467 \quad-1.607939-1.392814$

$\begin{array}{llll}\text { C } & 0.553085 & 0.093785 & 0.754191\end{array}$

$\begin{array}{llll}\text { C } & 0.507122 & 1.004774 & 1.929739\end{array}$

$\mathrm{H} \quad-0.378505 \quad 0.767562 \quad 2.545125$

C $\quad 0.466308 \quad 2.516954 \quad 1.598896$

$\begin{array}{llll}\mathrm{H} & 1.392301 & 0.832047 & 2.569125\end{array}$

$\mathrm{H} \quad-1.468085 \quad-0.564045 \quad 0.863507$

$\mathrm{H} \quad 1.496799 \quad 0.010237 \quad 0.200503$

$\begin{array}{llll}\text { C } & -0.799148 & 2.842745 & 0.810293\end{array}$

$\begin{array}{llll}\text { C } & 1.696053 & 2.916650 & 0.787503\end{array}$

$\begin{array}{llll}\text { C } & 0.455823 & 3.290554 & 2.914813\end{array}$

$\mathrm{H} \quad-0.417346 \quad 3.020231 \quad 3.528109$

$\mathrm{H} \quad 0.415570 \quad 4.375027 \quad 2.732003$

$\mathrm{H} \quad 1.360638 \quad 3.082654 \quad 3.506156$

$\mathrm{H} \quad 1.720489 \quad 2.410816 \quad-0.188925$

$\mathrm{H} \quad 2.624850 \quad 2.666054 \quad 1.323228$

$\mathrm{H} \quad 1.699554 \quad 4.000998 \quad 0.599076$

$\begin{array}{llll}\mathrm{H} & -0.817281 & 2.320159 & -0.157829\end{array}$

$\mathrm{H} \quad-0.865602 \quad 3.922904 \quad 0.610145$ 


\begin{tabular}{|c|c|c|c|}
\hline & -1.701296 & 2.547299 & 1.368736 \\
\hline \multicolumn{4}{|l|}{ C } \\
\hline $\mathrm{C}$ & -0.234458 & 3.190347 & -0.011779 \\
\hline C & 1.277209 & 3.201435 & -0.077261 \\
\hline C & -0.769592 & 4.317479 & 0.844175 \\
\hline C & -0.769522 & 1.845251 & 0.428525 \\
\hline $\mathrm{Br}$ & -0.907641 & 3.505638 & -1.888882 \\
\hline $\mathrm{H}$ & -0.416617 & 1.646036 & 1.452939 \\
\hline $\mathrm{H}$ & -0.409702 & 1.033948 & -0.217777 \\
\hline $\mathrm{H}$ & -1.867267 & 1.828626 & 0.439055 \\
\hline $\mathrm{H}$ & 1.672867 & 3.030061 & 0.936528 \\
\hline $\mathrm{H}$ & 1.661203 & 4.166788 & -0.432297 \\
\hline $\mathrm{H}$ & 1.661324 & 2.406460 & -0.729875 \\
\hline $\mathrm{H}$ & -0.417213 & 4.170204 & 1.877526 \\
\hline $\mathrm{H}$ & -1.867326 & 4.330183 & 0.859083 \\
\hline $\mathrm{H}$ & -0.409215 & 5.295573 & 0.499341 \\
\hline \\
\hline C & -0.080477 & 3.116106 & 0.424287 \\
\hline C & 1.348511 & 3.184552 & 0.019183 \\
\hline C & -0.748844 & 4.328338 & 0.967081 \\
\hline C & -0.748195 & 1.792800 & 0.542787 \\
\hline $\mathrm{Br}$ & -1.185130 & 3.658269 & -2.737294 \\
\hline $\mathrm{H}$ & -0.568041 & 1.296285 & 1.517321 \\
\hline $\mathrm{H}$ & -0.395235 & 1.097770 & -0.235373 \\
\hline $\mathrm{H}$ & -1.840150 & 1.886225 & 0.433415 \\
\hline $\mathrm{H}$ & 2.051719 & 3.049312 & 0.865162 \\
\hline $\mathrm{H}$ & 1.586274 & 4.158599 & -0.436793 \\
\hline $\mathrm{H}$ & 1.589838 & 2.405235 & -0.720646 \\
\hline $\mathrm{H}$ & -0.572354 & 4.478606 & 2.051009 \\
\hline $\mathrm{H}$ & -1.840362 & 4.276667 & 0.829144 \\
\hline $\mathrm{H}$ & -0.393505 & 5.239265 & 0.460017 \\
\hline
\end{tabular}




\begin{tabular}{|c|c|c|c|}
\hline & 1.113243 & -0.163995 & -0.642974 \\
\hline & -1.550499 & 4.900559 & -4.445597 \\
\hline & -2.871850 & 4.421868 & -4.586014 \\
\hline & -3.139495 & 3.091053 & -4.358888 \\
\hline & -2.105112 & 2.191486 & -3.997205 \\
\hline & -0.767978 & 2.667176 & -3.846959 \\
\hline & -0.531809 & 4.050556 & -4.076910 \\
\hline & -2.367633 & 0.816399 & -3.779598 \\
\hline & -1.346474 & -0.049692 & -3.487363 \\
\hline & 0.004399 & 0.392673 & -3.377049 \\
\hline & 0.283263 & 1.753634 & -3.490691 \\
\hline & 1.643788 & 2.334875 & -3.272599 \\
\hline & 2.121685 & 2.654884 & -1.998506 \\
\hline & 3.384867 & 3.293034 & -1.874707 \\
\hline & 4.151991 & 3.591646 & -2.974550 \\
\hline & 3.702245 & 3.273496 & -4.276553 \\
\hline & 2.428040 & 2.648914 & -4.428600 \\
\hline & 4.481360 & 3.570167 & -5.424953 \\
\hline & 4.020517 & 3.272517 & -6.685963 \\
\hline & 2.756376 & 2.659087 & -6.844694 \\
\hline & 1.984700 & 2.353773 & -5.747325 \\
\hline & 1.226147 & 2.113479 & -0.450829 \\
\hline & 1.211549 & -0.899946 & -2.806390 \\
\hline & 0.698022 & -2.384920 & -3.779993 \\
\hline & 0.908826 & -3.629513 & -3.175057 \\
\hline & 0.610510 & -4.812880 & -3.851995 \\
\hline & 0.083006 & -4.761965 & -5.141490 \\
\hline & -0.141387 & -3.525941 & -5.751643 \\
\hline & 0.166456 & -2.345644 & -5.077518 \\
\hline & 2.227530 & 2.906331 & 0.877390 \\
\hline & 2.023495 & 4.188402 & 1.399961 \\
\hline
\end{tabular}




\begin{tabular}{|c|c|c|c|}
\hline & 2.873283 & 4.690053 & 2.3 \\
\hline & 3.938951 & 3.921971 & 2.856736 \\
\hline & 4.143883 & 2.637485 & 2.349564 \\
\hline & 3.283833 & 2.130915 & 1.377735 \\
\hline & -0.341687 & 3.062715 & -0.505846 \\
\hline & -1.518226 & 2.372354 & -0.193623 \\
\hline & -2.751290 & 3.021748 & -0.230806 \\
\hline & -2.815929 & 4.369419 & -0.584316 \\
\hline & -1.648562 & 5.063139 & -0.906959 \\
\hline & -0.417651 & 4.411445 & -0.876286 \\
\hline $\mathrm{c}$ & 2.847466 & -0.537303 & -3.585541 \\
\hline & 3.902457 & -0.176971 & -2.738063 \\
\hline & 5.170719 & 0.088015 & -3.251885 \\
\hline & 5.400839 & -0.016734 & -4.623451 \\
\hline & 4.359331 & -0.386766 & -5.475483 \\
\hline & 3.090702 & -0.646062 & -4.960214 \\
\hline I & -1.335839 & 5.956119 & -4.619721 \\
\hline 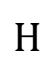 & -3.672944 & 5.105270 & -4.870651 \\
\hline 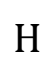 & -4.154620 & 2.700 & \\
\hline & & & \\
\hline & -3.394683 & & \\
\hline$\Pi$ & -1.577 & -1.10 & -3.331883 \\
\hline$\Pi$ & 3.762422 & 3.556744 & -0.885978 \\
\hline $\mathrm{H}$ & 5.119541 & 4.084323 & -2.854481 \\
\hline $\mathrm{H}$ & 5.454545 & 4.045789 & -5.283574 \\
\hline $\mathrm{H}$ & 4.624203 & 3.509033 & -7.563171 \\
\hline $\mathrm{H}$ & 2.392513 & 2.421507 & -7.845412 \\
\hline $\mathrm{H}$ & 1.016956 & 1.868189 & -5.886682 \\
\hline $\mathrm{H}$ & 1.307763 & -3.669143 & -2.157460 \\
\hline $\mathrm{H}$ & 0.782629 & -5.774623 & -3.366850 \\
\hline 11 & -0.159531 & -5.684340 & -5.670792 \\
\hline $\mathrm{H}$ & -0.558157 & -3.480679 & -6.758833 \\
\hline $\mathrm{H}$ & -0.019527 & -1.385932 & -5.566397 \\
\hline & & & \\
\hline
\end{tabular}




$\begin{array}{lrrr}\mathrm{H} & 1.198254 & 4.806264 & 1.041602 \\ \mathrm{H} & 2.702916 & 5.689993 & 2.789289 \\ \mathrm{H} & 4.604277 & 4.320107 & 3.624027 \\ \mathrm{H} & 4.968103 & 2.026029 & 2.719319 \\ \mathrm{H} & 3.423205 & 1.115987 & 0.990863 \\ \mathrm{H} & -1.454399 & 1.311210 & 0.062607 \\ \mathrm{H} & -3.663107 & 2.473479 & 0.010420 \\ \mathrm{H} & -3.779539 & 4.879852 & -0.618334 \\ \mathrm{H} & -1.699313 & 6.112133 & -1.203046 \\ \mathrm{H} & 0.488248 & 4.952377 & -1.164191 \\ \mathrm{H} & 3.704964 & -0.088059 & -1.664685 \\ \mathrm{H} & 5.979874 & 0.377064 & -2.579151 \\ \mathrm{H} & 6.392285 & 0.188832 & -5.030167 \\ \mathrm{H} & 4.532543 & -0.464113 & -6.550253 \\ \mathrm{H} & 2.286760 & -0.929231 & -5.643018\end{array}$

$\mathbf{E}_{1}$

Pd $\quad 1.252791 \quad-0.306455 \quad-0.595059$

$\begin{array}{llll}\text { C } & -1.550671 & 5.066915 & -4.597646\end{array}$

$\begin{array}{llll}\text { C } & -2.868873 & 4.575809 & -4.730837\end{array}$

$\begin{array}{llll}\text { C } & -3.126868 & 3.242152 & -4.512967\end{array}$

$\begin{array}{llll}\text { C } & -2.082965 & 2.348056 & -4.165289\end{array}$

$\begin{array}{llll}\text { C } & -0.750028 & 2.838202 & -4.024936\end{array}$

$\begin{array}{llll}\text { C } & -0.521517 & 4.223438 & -4.245402\end{array}$

$\begin{array}{llll}\text { C } & -2.337096 & 0.970584 & -3.955728\end{array}$

$\begin{array}{llll}\text { C } & -1.315570 & 0.107885 & -3.652086\end{array}$

$\begin{array}{llll}\text { C } & 0.021496 & 0.576264 & -3.526165\end{array}$

$\begin{array}{llll}\text { C } & 0.302768 & 1.933630 & -3.662983\end{array}$

$\begin{array}{llll}\text { C } & 1.666664 & 2.494059 & -3.427405\end{array}$

$\begin{array}{llll}\text { C } & 2.162200 & 2.685745 & -2.137178\end{array}$

$\begin{array}{llll}\text { C } & 3.444907 & 3.263531 & -1.946251\end{array}$

$\begin{array}{llll}\text { C } & 4.218260 & 3.629650 & -3.019880\end{array}$

$\begin{array}{llll}\text { C } & 3.758191 & 3.435837 & -4.343828\end{array}$ 


\begin{tabular}{lrrr}
$\mathrm{C}$ & 2.462753 & 2.873432 & -4.553398 \\
$\mathrm{C}$ & 4.560004 & 3.781217 & -5.462035 \\
$\mathrm{C}$ & 4.101824 & 3.584426 & -6.743693 \\
$\mathrm{C}$ & 2.817916 & 3.032138 & -6.956710 \\
$\mathrm{C}$ & 2.020533 & 2.684921 & -5.890670 \\
$\mathrm{P}$ & 1.261090 & 2.045038 & -0.666521 \\
$\mathrm{P}$ & 1.255823 & -0.623401 & -2.902302 \\
$\mathrm{C}$ & 0.727624 & -2.271745 & -3.480105 \\
$\mathrm{C}$ & 0.808651 & -3.324781 & -2.561895 \\
$\mathrm{C}$ & 0.492278 & -4.625919 & -2.952534 \\
$\mathrm{C}$ & 0.087589 & -4.877187 & -4.262131 \\
$\mathrm{C}$ & -0.003038 & -3.829186 & -5.181237 \\
$\mathrm{C}$ & 0.316189 & -2.530005 & -4.795630 \\
$\mathrm{C}$ & 2.202527 & 2.652724 & 0.774230 \\
$\mathrm{C}$ & 1.947560 & 3.878619 & 1.399219 \\
$\mathrm{C}$ & 2.744101 & 4.292813 & 2.466165 \\
$\mathrm{C}$ & 3.795167 & 3.490828 & 2.912256 \\
$\mathrm{C}$ & 4.047791 & 2.263356 & 2.298609 \\
$\mathrm{C}$ & 3.249201 & 1.842754 & 1.237812 \\
$\mathrm{C}$ & -0.360837 & 2.863655 & -0.639070 \\
$\mathrm{C}$ & -1.476481 & 2.119010 & -0.239443 \\
$\mathrm{C}$ & -2.734557 & 2.717696 & -0.194876 \\
$\mathrm{C}$ & -2.880010 & 4.057670 & -0.556056 \\
$\mathrm{C}$ & -1.769591 & 4.801777 & -0.959031 \\
$\mathrm{C}$ & -0.510672 & 4.208072 & -1.002920 \\
$\mathrm{C}$ & 2.864714 & -0.331390 & -3.714572 \\
$\mathrm{C}$ & 3.994103 & -0.137599 & -2.911628 \\
$\mathrm{C}$ & 5.244739 & 0.055114 & -3.496205 \\
$\mathrm{C}$ & 5.371728 & 0.046207 & -4.884400 \\
$\mathrm{C}$ & 4.249376 & -0.157073 & -5.689364 \\
$\mathrm{C}$ & 2.997709 & -0.343862 & -5.109172 \\
& -1.347583 & 6.124732 & -4.768571 \\
& & $\mathrm{~S} 162$ & -5.005629 \\
\hline
\end{tabular}




\begin{tabular}{lrrr}
$\mathrm{H}$ & -4.139243 & 2.845359 & -4.611744 \\
$\mathrm{H}$ & 0.485826 & 4.626300 & -4.134325 \\
$\mathrm{H}$ & -3.360185 & 0.601733 & -4.049333 \\
$\mathrm{H}$ & -1.536258 & -0.950049 & -3.501307 \\
$\mathrm{H}$ & 3.825481 & 3.421965 & -0.936564 \\
$\mathrm{H}$ & 5.202796 & 4.074376 & -2.862705 \\
$\mathrm{H}$ & 5.549057 & 4.207012 & -5.281262 \\
$\mathrm{H}$ & 4.724952 & 3.853422 & -7.597260 \\
$\mathrm{H}$ & 2.459467 & 2.875256 & -7.974701 \\
$\mathrm{H}$ & 1.037552 & 2.247493 & -6.072579 \\
$\mathrm{H}$ & 1.124274 & -3.126450 & -1.532943 \\
$\mathrm{H}$ & 0.559552 & -5.440617 & -2.231281 \\
$\mathrm{H}$ & -0.162879 & -5.892863 & -4.569997 \\
$\mathrm{H}$ & -0.325012 & -4.024990 & -6.204426 \\
$\mathrm{H}$ & 0.231524 & -1.718948 & -5.521998 \\
$\mathrm{H}$ & 1.129049 & 4.515392 & 1.059243 \\
$\mathrm{H}$ & 2.542853 & 5.249131 & 2.949697 \\
$\mathrm{H}$ & 4.416365 & 3.821160 & 3.745311 \\
$\mathrm{H}$ & 4.863465 & 1.630290 & 2.648406 \\
$\mathrm{H}$ & 3.443878 & 0.877385 & 0.758681 \\
$\mathrm{H}$ & -1.358142 & 1.065033 & 0.026640 \\
$\mathrm{H}$ & -3.602660 & 2.134504 & 0.113402 \\
$\mathrm{H}$ & -3.865366 & 4.524281 & -0.529754 \\
$\mathrm{H}$ & -1.885737 & 5.845776 & -1.252369 \\
$\mathrm{H}$ & 0.354316 & 4.788552 & -1.334537 \\
$\mathrm{H}$ & 3.886386 & -0.125644 & -1.823071 \\
$\mathrm{H}$ & 6.119713 & 0.213260 & -2.864825 \\
$\mathrm{H}$ & 6.349553 & 0.197693 & -5.343356 \\
$\mathrm{H}$ & 4.346550 & -0.158647 & -6.775895 \\
& 2.125472 & -0.483539 & -5.751173 \\
& & & \\
\hline & & & \\
\hline
\end{tabular}




\begin{tabular}{lrrr}
$\mathrm{C}$ & -1.506374 & 5.284280 & -4.744750 \\
$\mathrm{C}$ & -2.801675 & 4.752716 & -4.944739 \\
$\mathrm{C}$ & -3.023179 & 3.405048 & -4.785669 \\
$\mathrm{C}$ & -1.963727 & 2.535499 & -4.420216 \\
$\mathrm{C}$ & -0.650796 & 3.066363 & -4.224783 \\
$\mathrm{C}$ & -0.458085 & 4.464399 & -4.397704 \\
$\mathrm{C}$ & -2.187150 & 1.150727 & -4.239852 \\
$\mathrm{C}$ & -1.166286 & 0.317780 & -3.855795 \\
$\mathrm{C}$ & 0.135459 & 0.835918 & -3.639235 \\
$\mathrm{C}$ & 0.408846 & 2.191629 & -3.830063 \\
$\mathrm{C}$ & 1.769913 & 2.773513 & -3.617530 \\
$\mathrm{C}$ & 2.292367 & 2.971382 & -2.336772 \\
$\mathrm{C}$ & 3.568627 & 3.557017 & -2.134912 \\
$\mathrm{C}$ & 4.321622 & 3.933548 & -3.217944 \\
$\mathrm{C}$ & 3.845828 & 3.737162 & -4.537104 \\
$\mathrm{C}$ & 2.551945 & 3.162777 & -4.744917 \\
$\mathrm{C}$ & 4.627464 & 4.113853 & -5.657602 \\
$\mathrm{C}$ & 4.153733 & 3.932235 & -6.935668 \\
$\mathrm{C}$ & 2.876519 & 3.362365 & -7.145647 \\
$\mathrm{C}$ & 2.095336 & 2.985518 & -6.079015 \\
$\mathrm{P}$ & 1.444128 & 2.249374 & -0.929396 \\
$\mathrm{P}$ & 1.399812 & -0.276387 & -2.953868 \\
$\mathrm{C}$ & 0.792254 & -1.977287 & -2.938435 \\
$\mathrm{C}$ & -0.201878 & -2.320509 & -2.006463 \\
$\mathrm{C}$ & -0.676733 & -3.626163 & -1.951423 \\
$\mathrm{C}$ & -0.152604 & -4.593004 & -2.811750 \\
$\mathrm{C}$ & 0.846882 & -4.257343 & -3.724668 \\
$\mathrm{C}$ & 1.328573 & -2.951837 & -3.789999 \\
$\mathrm{C}$ & 2.279938 & 2.436038 & 0.652167 \\
$\mathrm{C}$ & 1.597810 & 2.962897 & 1.761330 \\
& 2.209644 & 2.943530 & 3.009361 \\
& 3.479925 & 2.382744 & 3.163752 \\
\hline & 4.155220 & 1.843503 & 2.068921 \\
\hline & $\mathrm{S} 164$ & \\
\hline
\end{tabular}




\begin{tabular}{lrrr}
$\mathrm{C}$ & 3.562910 & 1.864966 & 0.809804 \\
$\mathrm{C}$ & -0.282623 & 2.705655 & -0.816284 \\
$\mathrm{C}$ & -1.219344 & 1.768430 & -0.360799 \\
$\mathrm{C}$ & -2.553380 & 2.142952 & -0.237229 \\
$\mathrm{C}$ & -2.943356 & 3.444108 & -0.559829 \\
$\mathrm{C}$ & -2.004967 & 4.379343 & -0.998944 \\
$\mathrm{C}$ & -0.668521 & 4.015366 & -1.133469 \\
$\mathrm{C}$ & 2.892275 & -0.158266 & -3.959044 \\
$\mathrm{C}$ & 4.151234 & -0.078069 & -3.352670 \\
$\mathrm{C}$ & 5.293586 & -0.003186 & -4.145622 \\
$\mathrm{C}$ & 5.177252 & -0.020479 & -5.536231 \\
$\mathrm{C}$ & 3.922130 & -0.115581 & -6.140080 \\
$\mathrm{C}$ & 2.774208 & -0.177401 & -5.355553 \\
$\mathrm{H}$ & -1.337413 & 6.354431 & -4.866093 \\
$\mathrm{H}$ & -3.621963 & 5.415111 & -5.221732 \\
$\mathrm{H}$ & -4.017431 & 2.979870 & -4.932647 \\
$\mathrm{H}$ & 0.530560 & 4.897861 & -4.241291 \\
$\mathrm{H}$ & -3.188179 & 0.750466 & -4.407559 \\
$\mathrm{H}$ & -1.362146 & -0.746760 & -3.727063 \\
$\mathrm{H}$ & 3.944993 & 3.722761 & -1.125449 \\
$\mathrm{H}$ & 5.299827 & 4.393932 & -3.072367 \\
$\mathrm{H}$ & 5.609297 & 4.556935 & -5.481777 \\
$\mathrm{H}$ & 4.760420 & 4.228955 & -7.791355 \\
$\mathrm{H}$ & 2.509941 & 3.219088 & -8.162167 \\
$\mathrm{H}$ & 1.115699 & 2.540597 & -6.257689 \\
$\mathrm{H}$ & -0.611826 & -1.566908 & -1.328438 \\
$\mathrm{H}$ & -1.452713 & -3.889856 & -1.233464 \\
$\mathrm{H}$ & -0.522317 & -5.617416 & -2.765040 \\
& 1.257338 & -5.015346 & -4.391455 \\
$\mathrm{H}$ & 2.113475 & -2.699301 & -4.503999 \\
\hline & 0.601684 & 3.392926 & 1.647217 \\
\hline
\end{tabular}




$\begin{array}{lrrr}\mathrm{H} & 5.145034 & 1.405744 & 2.191424 \\ \mathrm{H} & 4.112973 & 1.463687 & -0.046847 \\ \mathrm{H} & -0.906513 & 0.752298 & -0.104422 \\ \mathrm{H} & -3.288921 & 1.418497 & 0.111063 \\ \mathrm{H} & -3.989910 & 3.733529 & -0.462949 \\ \mathrm{H} & -2.314128 & 5.395929 & -1.241930 \\ \mathrm{H} & 0.066049 & 4.746612 & -1.477513 \\ \mathrm{H} & 4.242165 & -0.067634 & -2.262928 \\ \mathrm{H} & 6.274287 & 0.069980 & -3.676220 \\ \mathrm{H} & 6.072419 & 0.040288 & -6.155530 \\ \mathrm{H} & 3.834324 & -0.134034 & -7.226636 \\ \mathrm{H} & 1.791307 & -0.240218 & -5.830179\end{array}$

$\mathbf{F}_{\mathbf{0}}$

$\begin{array}{llll}\text { Pd } & 0.001598 \quad 0.001566 & 0.010580\end{array}$

$\begin{array}{llll}\mathrm{P} & -1.315369 & 1.933334 & 0.787795\end{array}$

$\begin{array}{llll}\mathrm{P} & -1.005530 & -2.099047 & 0.802137\end{array}$

$\begin{array}{llll}\mathrm{P} & 2.331691 & 0.177739 & 0.776129\end{array}$

$\begin{array}{llll}\mathrm{P} & -0.001907 & -0.013622 & -2.448598\end{array}$

$\begin{array}{llll}\text { C } & 1.616262 & -0.293943 & -3.298124\end{array}$

C $\quad 2.159188 \quad 0.565224 \quad-4.261316$

C $\quad 2.362551 \quad-1.411683 \quad-2.897973$

C $\quad 3.432442 \quad 0.329739 \quad-4.784772$

$\begin{array}{llll}\mathrm{H} & 1.595205 & 1.436690 & -4.599669\end{array}$

C $\quad 3.621048 \quad-1.662203 \quad-3.438464$

H $\quad 1.955695 \quad-2.092989 \quad-2.144529$

$\begin{array}{llll}\text { C } & 4.168281 & -0.780745 & -4.372838\end{array}$

H $3.847432 \quad 1.018084 \quad-5.522856$

$\mathrm{H} \quad 4.184380 \quad-2.537457 \quad-3.107284$

$\mathrm{H} \quad 5.163575 \quad-0.961950 \quad-4.781015$

C $\quad-0.567205 \quad 1.531160 \quad-3.291912$

$\begin{array}{llll}\text { C } & -1.558700 & 1.575959 & -4.279342\end{array}$

$\begin{array}{llll}\text { C } & 0.009641 & 2.734697 & -2.862624\end{array}$ 


\begin{tabular}{lrrr}
$\mathrm{C}$ & -1.986818 & 2.799740 & -4.798404 \\
$\mathrm{H}$ & -2.015107 & 0.652370 & -4.640831 \\
$\mathrm{C}$ & -0.399474 & 3.954012 & -3.396281 \\
$\mathrm{H}$ & 0.784223 & 2.716970 & -2.089981 \\
$\mathrm{C}$ & -1.412973 & 3.991238 & -4.355918 \\
$\mathrm{H}$ & -2.771758 & 2.818873 & -5.556231 \\
$\mathrm{H}$ & 0.060256 & 4.878901 & -3.040382 \\
$\mathrm{H}$ & -1.751409 & 4.946358 & -4.760016 \\
$\mathrm{C}$ & -1.058806 & -1.274776 & -3.292126 \\
$\mathrm{C}$ & -0.591423 & -2.190068 & -4.242932 \\
$\mathrm{C}$ & -2.402697 & -1.343767 & -2.897569 \\
$\mathrm{C}$ & -1.439723 & -3.171944 & -4.759599 \\
$\mathrm{H}$ & 0.447309 & -2.152582 & -4.576809 \\
$\mathrm{C}$ & -3.256660 & -2.304359 & -3.432751 \\
$\mathrm{H}$ & -2.785065 & -0.638105 & -2.153839 \\
$\mathrm{C}$ & -2.772253 & -3.234785 & -4.354306 \\
$\mathrm{H}$ & -1.054662 & -3.888063 & -5.487442 \\
$\mathrm{H}$ & -4.298798 & -2.339271 & -3.107485 \\
$\mathrm{H}$ & -3.433302 & -4.003305 & -4.757459 \\
$\mathrm{C}$ & -3.103377 & 1.939727 & 0.304389 \\
$\mathrm{C}$ & -3.381186 & 1.882579 & -1.069602 \\
$\mathrm{C}$ & -4.174596 & 1.970126 & 1.203154 \\
$\mathrm{C}$ & -4.692189 & 1.848970 & -1.535693 \\
$\mathrm{H}$ & -2.554262 & 1.872964 & -1.784926 \\
$\mathrm{C}$ & -5.492140 & 1.925514 & 0.737897 \\
$\mathrm{H}$ & -3.989455 & 2.027791 & 2.277814 \\
$\mathrm{C}$ & -5.756267 & 1.863767 & -0.629466 \\
$\mathrm{H}$ & -4.881847 & 1.804845 & -2.610511 \\
$\mathrm{H}$ & -6.316037 & 1.945615 & 1.453254 \\
$\mathrm{H}$ & -6.785764 & 1.829266 & -0.988691 \\
& -0.872647 & 3.660071 & 0.279779 \\
& 0.484452 & 3.992736 & 0.216001 \\
\hline & -1.821368 & 4.658007 & 0.025432 \\
\hline
\end{tabular}




\begin{tabular}{lrrr}
$\mathrm{C}$ & 0.890284 & 5.291910 & -0.088067 \\
$\mathrm{H}$ & 1.237353 & 3.222395 & 0.399056 \\
$\mathrm{C}$ & -1.419448 & 5.955772 & -0.290266 \\
$\mathrm{H}$ & -2.887354 & 4.427279 & 0.080633 \\
$\mathrm{C}$ & -0.062931 & 6.277526 & -0.346938 \\
$\mathrm{H}$ & 1.955813 & 5.527010 & -0.128464 \\
$\mathrm{H}$ & -2.171699 & 6.721598 & -0.486545 \\
$\mathrm{H}$ & 0.250163 & 7.293898 & -0.590427 \\
$\mathrm{C}$ & -1.453262 & 2.180987 & 2.620157 \\
$\mathrm{C}$ & -1.775780 & 1.062203 & 3.400818 \\
$\mathrm{C}$ & -1.209359 & 3.399113 & 3.267381 \\
$\mathrm{C}$ & -1.871402 & 1.157003 & 4.786975 \\
$\mathrm{H}$ & -1.942451 & 0.095727 & 2.917583 \\
$\mathrm{C}$ & -1.283867 & 3.491308 & 4.659615 \\
$\mathrm{H}$ & -0.953599 & 4.288719 & 2.688404 \\
$\mathrm{C}$ & -1.618763 & 2.373874 & 5.423247 \\
$\mathrm{H}$ & -2.124951 & 0.269249 & 5.370114 \\
$\mathrm{H}$ & -1.084019 & 4.446856 & 5.147378 \\
$\mathrm{H}$ & -1.679074 & 2.449269 & 6.510000 \\
$\mathrm{C}$ & -0.109035 & -3.649157 & 0.331114 \\
$\mathrm{C}$ & 0.080314 & -3.871904 & -1.041039 \\
$\mathrm{C}$ & 0.410101 & -4.578924 & 1.237741 \\
$\mathrm{C}$ & 0.776493 & -4.987006 & -1.497718 \\
$\mathrm{H}$ & -0.334081 & -3.162762 & -1.762873 \\
$\mathrm{C}$ & 1.119391 & -5.694049 & 0.781893 \\
$\mathrm{H}$ & 0.266803 & -4.439617 & 2.311243 \\
$\mathrm{C}$ & 1.306531 & -5.902012 & -0.583659 \\
$\mathrm{H}$ & 0.909577 & -5.137442 & -2.571480 \\
$\mathrm{H}$ & 1.521792 & -6.407134 & 1.503368 \\
$\mathrm{H}$ & 1.860367 & -6.773674 & -0.935151 \\
& -2.714719 & -2.575379 & 0.267610 \\
& -3.678184 & -1.564153 & 0.195927 \\
\hline & -103291 & -3.893737 & 0.000718 \\
\hline
\end{tabular}




\begin{tabular}{lrrr}
$\mathrm{C}$ & -5.002873 & -1.858302 & -0.126004 \\
$\mathrm{H}$ & -3.387074 & -0.528790 & 0.388108 \\
$\mathrm{C}$ & -4.424049 & -4.190054 & -0.335435 \\
$\mathrm{H}$ & -2.372738 & -4.703171 & 0.062363 \\
$\mathrm{C}$ & -5.378430 & -3.174193 & -0.398243 \\
$\mathrm{H}$ & -5.738188 & -1.052037 & -0.170492 \\
$\mathrm{H}$ & -4.710616 & -5.222676 & -0.541439 \\
$\mathrm{H}$ & -6.412354 & -3.407986 & -0.656472 \\
$\mathrm{C}$ & -1.174060 & -2.345539 & 2.632476 \\
$\mathrm{C}$ & -2.358241 & -2.750106 & 3.261950 \\
$\mathrm{C}$ & -0.054816 & -2.066499 & 3.429719 \\
$\mathrm{C}$ & -2.419269 & -2.869486 & 4.652748 \\
$\mathrm{H}$ & -3.248228 & -2.972058 & 2.669675 \\
$\mathrm{C}$ & -0.107455 & -2.206553 & 4.814601 \\
$\mathrm{H}$ & 0.871822 & -1.722883 & 2.961229 \\
$\mathrm{C}$ & -1.294772 & -2.603405 & 5.432898 \\
$\mathrm{H}$ & -3.352391 & -3.179394 & 5.126214 \\
$\mathrm{H}$ & 0.779485 & -1.984841 & 5.411654 \\
$\mathrm{H}$ & -1.343665 & -2.700218 & 6.518525 \\
$\mathrm{C}$ & 3.224732 & 1.727795 & 0.299390 \\
$\mathrm{C}$ & 3.317488 & 2.000382 & -1.073825 \\
$\mathrm{C}$ & 3.776536 & 2.642287 & 1.202274 \\
$\mathrm{C}$ & 3.936895 & 3.158102 & -1.535389 \\
$\mathrm{H}$ & 2.905688 & 1.286710 & -1.792561 \\
$\mathrm{C}$ & 4.389782 & 3.811103 & 0.741511 \\
$\mathrm{H}$ & 3.730246 & 2.450730 & 2.276348 \\
$\mathrm{C}$ & 4.471655 & 4.074198 & -0.625028 \\
$\mathrm{H}$ & 3.996301 & 3.346768 & -2.609590 \\
$\mathrm{H}$ & 4.811108 & 4.516556 & 1.459663 \\
$\mathrm{H}$ & 4.951012 & 4.987493 & -0.980290 \\
& 2.620593 & 0.160324 & 2.607009 \\
& 3.569024 & -0.650008 & 3.243957 \\
\hline & 1.802372 & 0.979671 & 3.397305 \\
\hline & $\mathrm{S} 169$ & \\
\hline
\end{tabular}




$\begin{array}{lrrr}\mathrm{C} & 3.690385 & -0.641812 & 4.635891 \\ \mathrm{H} & 4.220749 & -1.300213 & 2.656813 \\ \mathrm{C} & 1.936923 & 1.005667 & 4.783246 \\ \mathrm{H} & 1.036942 & 1.598637 & 2.920986 \\ \mathrm{C} & 2.879324 & 0.187632 & 5.409427 \\ \mathrm{H} & 4.429883 & -1.285048 & 5.115752 \\ \mathrm{H} & 1.287087 & 1.654060 & 5.374662 \\ \mathrm{H} & 2.977879 & 0.194514 & 6.495978 \\ \mathrm{C} & 3.603169 & -1.063804 & 0.251349 \\ \mathrm{C} & 3.217474 & -2.407486 & 0.206094 \\ \mathrm{C} & 4.934689 & -0.737533 & -0.033328 \\ \mathrm{C} & 4.137219 & -3.406723 & -0.110514 \\ \mathrm{H} & 2.179604 & -2.676889 & 0.417045 \\ \mathrm{C} & 5.854510 & -1.732772 & -0.363246 \\ \mathrm{H} & 5.265071 & 0.302419 & 0.007997 \\ \mathrm{C} & 5.459612 & -3.070374 & -0.402090 \\ \mathrm{H} & 3.812457 & -4.449080 & -0.134857 \\ \mathrm{H} & 6.888260 & -1.461386 & -0.583696 \\ \mathrm{H} & 6.181073 & -3.848443 & -0.655817\end{array}$

$\begin{array}{lrrr}\mathbf{F}_{1} & & & \\ \mathrm{Pd} & 1.376712 & -1.144068 & 1.343747 \\ \mathrm{P} & -0.322676 & 0.660764 & 1.003492 \\ \mathrm{P} & -0.098339 & -3.086596 & 2.004669 \\ \mathrm{P} & 3.147425 & -0.077449 & 2.793537 \\ \mathrm{P} & 2.895245 & -2.224524 & -0.316636 \\ \mathrm{C} & 3.542123 & -0.921485 & -1.447904 \\ \mathrm{C} & 2.888295 & 0.312876 & -1.483720 \\ \mathrm{C} & 4.598715 & -1.158308 & -2.336665 \\ \mathrm{C} & 3.281560 & 1.307713 & -2.378266 \\ \mathrm{H} & 2.075780 & 0.508374 & -0.779144 \\ \mathrm{C} & 5.002508 & -0.162147 & -3.223484 \\ \mathrm{H} & 5.113421 & -2.121958 & -2.337617 \\ & & \mathrm{~S} 170 & \end{array}$




\begin{tabular}{lrrr}
$\mathrm{C}$ & 4.345985 & 1.071071 & -3.246839 \\
$\mathrm{H}$ & 2.761065 & 2.267419 & -2.381844 \\
$\mathrm{H}$ & 5.831487 & -0.351453 & -3.906496 \\
$\mathrm{H}$ & 4.665597 & 1.845503 & -3.944995 \\
$\mathrm{C}$ & 4.374269 & -3.149417 & 0.228618 \\
$\mathrm{C}$ & 4.242506 & -4.496594 & 0.594403 \\
$\mathrm{C}$ & 5.593921 & -2.499026 & 0.458326 \\
$\mathrm{C}$ & 5.313996 & -5.183759 & 1.161153 \\
$\mathrm{H}$ & 3.294617 & -5.016643 & 0.441390 \\
$\mathrm{C}$ & 6.663642 & -3.188198 & 1.028425 \\
$\mathrm{H}$ & 5.711588 & -1.444343 & 0.201370 \\
$\mathrm{C}$ & 6.528271 & -4.531107 & 1.379302 \\
$\mathrm{H}$ & 5.195145 & -6.233546 & 1.435182 \\
$\mathrm{H}$ & 7.605974 & -2.666060 & 1.202215 \\
$\mathrm{H}$ & 7.366621 & -5.068387 & 1.823907 \\
$\mathrm{C}$ & 2.084435 & -3.312898 & -1.563555 \\
$\mathrm{C}$ & 0.775078 & -2.970322 & -1.913048 \\
$\mathrm{C}$ & 2.716431 & -4.359434 & -2.245550 \\
$\mathrm{C}$ & 0.093620 & -3.660361 & -2.913495 \\
$\mathrm{H}$ & 0.282371 & -2.147369 & -1.389082 \\
$\mathrm{C}$ & 2.030076 & -5.063183 & -3.235308 \\
$\mathrm{H}$ & 3.746528 & -4.635207 & -2.013059 \\
$\mathrm{C}$ & 0.719206 & -4.719917 & -3.570112 \\
$\mathrm{H}$ & -0.928566 & -3.371348 & -3.171321 \\
$\mathrm{H}$ & 2.528293 & -5.882944 & -3.754278 \\
$\mathrm{H}$ & 0.190360 & -5.272816 & -4.347061 \\
$\mathrm{C}$ & -1.568046 & 0.172980 & -0.255925 \\
$\mathrm{C}$ & -1.118783 & -0.107612 & -1.555395 \\
$\mathrm{C}$ & -2.936723 & 0.096075 & 0.021085 \\
$\mathrm{C}$ & -2.021696 & -0.444292 & -2.560394 \\
$\mathrm{H}$ & -0.055465 & -0.020803 & -1.798842 \\
& -3.838575 & -0.253404 & -0.984751 \\
& -3.310149 & 0.313237 & 1.023591 \\
& $\mathrm{~S} 171$ & \\
\hline
\end{tabular}




\begin{tabular}{lrrr}
$\mathrm{C}$ & -3.386522 & -0.520460 & -2.275009 \\
$\mathrm{H}$ & -1.658645 & -0.637363 & -3.571348 \\
$\mathrm{H}$ & -4.903109 & -0.313269 & -0.754099 \\
$\mathrm{H}$ & -4.095293 & -0.785628 & -3.060369 \\
$\mathrm{C}$ & 0.328023 & 2.240111 & 0.303970 \\
$\mathrm{C}$ & 1.432515 & 2.829962 & 0.932593 \\
$\mathrm{C}$ & -0.233929 & 2.883185 & -0.806420 \\
$\mathrm{C}$ & 1.978334 & 4.020990 & 0.460951 \\
$\mathrm{H}$ & 1.869141 & 2.359919 & 1.812515 \\
$\mathrm{C}$ & 0.316511 & 4.073691 & -1.285047 \\
$\mathrm{H}$ & -1.107655 & 2.463137 & -1.306794 \\
$\mathrm{C}$ & 1.425060 & 4.643099 & -0.659106 \\
$\mathrm{H}$ & 2.841472 & 4.455296 & 0.969773 \\
$\mathrm{H}$ & -0.131333 & 4.560156 & -2.152347 \\
$\mathrm{H}$ & 1.851845 & 5.571800 & -1.039106 \\
$\mathrm{C}$ & -1.362905 & 1.224281 & 2.404779 \\
$\mathrm{C}$ & -1.816712 & 0.254525 & 3.306139 \\
$\mathrm{C}$ & -1.729944 & 2.560545 & 2.595798 \\
$\mathrm{C}$ & -2.616831 & 0.606811 & 4.389928 \\
$\mathrm{H}$ & -1.540136 & -0.790922 & 3.158631 \\
$\mathrm{C}$ & -2.530202 & 2.915472 & 3.682662 \\
$\mathrm{H}$ & -1.387850 & 3.331488 & 1.902038 \\
$\mathrm{C}$ & -2.971189 & 1.943755 & 4.581657 \\
$\mathrm{H}$ & -2.958066 & -0.162807 & 5.085014 \\
$\mathrm{H}$ & -2.809214 & 3.959756 & 3.827988 \\
$\mathrm{H}$ & -3.592621 & 2.228029 & 5.431465 \\
$\mathrm{C}$ & 0.629368 & -4.762195 & 1.715371 \\
$\mathrm{C}$ & 0.350229 & -5.541050 & 0.585603 \\
$\mathrm{C}$ & 1.571638 & -5.235592 & 2.642701 \\
$\mathrm{C}$ & 0.989615 & -6.767058 & 0.394620 \\
$\mathrm{H}$ & -0.378621 & -5.212162 & -0.155608 \\
& 2.199243 & -6.464794 & 2.456376 \\
\hline & 1.817492 & -4.643224 & 3.527924 \\
& $\mathrm{~S} 172$ & \\
\hline
\end{tabular}




\begin{tabular}{|c|c|c|c|}
\hline & 1.912626 & -7.235395 & 1.329027 \\
\hline & 0.755222 & -7.359893 & -0.490569 \\
\hline & 2.920469 & -6.817859 & 3.195619 \\
\hline & 2.405314 & -8.196926 & 1.180149 \\
\hline & -1.851019 & -3.221642 & 1.444902 \\
\hline & -2.114261 & -3.367091 & 0.075550 \\
\hline & -2.938887 & -3.122010 & 2.324356 \\
\hline & -3.418839 & -3.477637 & -0.397180 \\
\hline & -1.291960 & -3.392384 & -0.638266 \\
\hline & -4.247528 & -3.206801 & 1.847428 \\
\hline & -2.778123 & -2.984459 & 3.394943 \\
\hline & -4.492392 & -3.401705 & 0.489520 \\
\hline & -3.593701 & -3.607688 & -1.466600 \\
\hline & -5.078650 & -3.128954 & 2.549413 \\
\hline & -5.516087 & -3.479526 & 0.121271 \\
\hline & -0.239752 & -3.121694 & 3.840642 \\
\hline & -0.857975 & -4.185570 & 4.516590 \\
\hline & 0.329292 & -2.091203 & 4.587576 \\
\hline & -0.918534 & -4.190819 & 5.907066 \\
\hline & -1.291188 & -5.016778 & 3.955298 \\
\hline & 0.271733 & -2.089754 & 5.981108 \\
\hline & 0.838616 & -1.285573 & 4.059539 \\
\hline & -0.357430 & -3.141592 & 6.641968 \\
\hline & -1.403643 & -5.020157 & 6.423009 \\
\hline & 0.728018 & -1.271221 & 6.541299 \\
\hline & -0.407321 & -3.152706 & 7.731320 \\
\hline & 4.786285 & 0.387832 & 2.109753 \\
\hline & 4.814140 & 1.228053 & 0.988758 \\
\hline & 5.992735 & -0.022399 & 2.687625 \\
\hline & 6.027073 & 1.640690 & 0.442818 \\
\hline & 3.879246 & 1.569072 & 0.538111 \\
\hline & 7.207563 & 0.393764 & 2.141801 \\
\hline & 5.990777 & -0.674609 & 3.563646 \\
\hline & & S1 & \\
\hline
\end{tabular}




\begin{tabular}{|c|c|c|c|}
\hline $\mathrm{C}$ & 7.227643 & 1.218436 & 1.017183 \\
\hline $\mathrm{H}$ & 6.032866 & 2.291028 & -0.43316 \\
\hline & 8.143307 & 0.067551 & 2.59786 \\
\hline & 8.179349 & 1.537324 & 0.59070 \\
\hline & 2.717087 & 1.426330 & 3.76984 \\
\hline & 1.430898 & 1.526199 & 4.31710 \\
\hline & 3.611967 & 2.489320 & 3.94560 \\
\hline & 1.038539 & 2.666673 & 5.01283 \\
\hline & 0.706145 & 0.722964 & 4.17626 \\
\hline & 3.215645 & 3.635749 & 4.63567 \\
\hline & 4.621941 & 2.434774 & 3.53514 \\
\hline & 1.929208 & 3.729894 & 5.16532 \\
\hline $\mathrm{H}$ & 0.027573 & 2.727698 & 5.41866 \\
\hline$H$ & 3.919572 & 4.459713 & 4.75817 \\
\hline$H$ & 1.620908 & 4.630661 & 5.69692 \\
\hline$r$ & 3.581822 & -1.285087 & 4.11090 \\
\hline$r$ & 3.719261 & -0.909744 & 5.45265 \\
\hline${ }^{2}$ & 3.810213 & -2.619024 & 3.74717 \\
\hline C & 4.066328 & -1.857342 & 6.41609 \\
\hline $\mathrm{H}$ & 3.554413 & 0.126393 & 5.75468 \\
\hline $\mathrm{C}$ & 4.174328 & -3.558911 & 4.70892 \\
\hline $\mathrm{H}$ & 3.713994 & -2.931043 & 2.70361 \\
\hline $\mathrm{C}$ & 4.294043 & -3.182246 & 6.04730 \\
\hline $\mathrm{H}$ & 4.163524 & -1.554499 & 7.45934 \\
\hline $\mathrm{H}$ & 4.364255 & -4.591944 & 4.41035 \\
\hline $\mathrm{H}$ & 4.568985 & -3.921127 & 6.80074 \\
\hline \multicolumn{4}{|l|}{$\mathbf{F}_{2}$} \\
\hline $\mathrm{Pd}$ & 1.509588 & -1.105462 & 1.24889 \\
\hline$P$ & -0.199409 & 0.543856 & 0.48904 \\
\hline $\mathrm{P}$ & 0.095218 & -2.577848 & 2.58082 \\
\hline $\mathrm{P}$ & 3.378817 & -0.765466 & 2.88660 \\
\hline $\mathrm{C}$ & -1.078939 & 0.242136 & -1.09591 \\
\hline
\end{tabular}




\begin{tabular}{lrrr}
$\mathrm{C}$ & -0.531358 & -0.710560 & -1.964324 \\
$\mathrm{C}$ & -2.283196 & 0.868689 & -1.444984 \\
$\mathrm{C}$ & -1.178696 & -1.039343 & -3.154780 \\
$\mathrm{H}$ & 0.408545 & -1.197892 & -1.692451 \\
$\mathrm{C}$ & -2.931772 & 0.539090 & -2.634571 \\
$\mathrm{H}$ & -2.732029 & 1.607691 & -0.777602 \\
$\mathrm{C}$ & -2.382807 & -0.418550 & -3.488576 \\
$\mathrm{H}$ & -0.745928 & -1.787772 & -3.819911 \\
$\mathrm{H}$ & -3.871629 & 1.029376 & -2.892147 \\
$\mathrm{H}$ & -2.896076 & -0.681703 & -4.414492 \\
$\mathrm{C}$ & 0.514951 & 2.236170 & 0.356798 \\
$\mathrm{C}$ & 1.562096 & 2.551478 & 1.227511 \\
$\mathrm{C}$ & 0.058727 & 3.212099 & -0.533065 \\
$\mathrm{C}$ & 2.135824 & 3.821253 & 1.232881 \\
$\mathrm{H}$ & 1.930199 & 1.789048 & 1.916037 \\
$\mathrm{C}$ & 0.639182 & 4.481218 & -0.540352 \\
$\mathrm{H}$ & -0.745865 & 2.988352 & -1.236176 \\
$\mathrm{C}$ & 1.674777 & 4.791694 & 0.342861 \\
$\mathrm{H}$ & 2.948537 & 4.042326 & 1.928636 \\
$\mathrm{H}$ & 0.277848 & 5.234004 & -1.242638 \\
$\mathrm{H}$ & 2.122151 & 5.786437 & 0.333482 \\
$\mathrm{C}$ & -1.598140 & 0.863915 & 1.665324 \\
$\mathrm{C}$ & -2.784061 & 0.114955 & 1.608747 \\
$\mathrm{C}$ & -1.454144 & 1.796468 & 2.703788 \\
$\mathrm{C}$ & -3.796223 & 0.304975 & 2.549758 \\
$\mathrm{H}$ & -2.929910 & -0.624036 & 0.818925 \\
$\mathrm{C}$ & -2.462798 & 1.979641 & 3.649443 \\
$\mathrm{H}$ & -0.546687 & 2.400983 & 2.776837 \\
$\mathrm{H}$ & -3.639857 & 1.235647 & 3.576821 \\
& -4.712549 & -0.283596 & 2.477319 \\
$\mathrm{H}$ & 0.801158 & -4.278119 & 2.599613 \\
& $\mathrm{~S} 175$ & \\
\hline
\end{tabular}




$\begin{array}{lrrr}\mathrm{C} & 0.315516 & -5.276760 & 3.454611 \\ \mathrm{C} & 1.790302 & -4.593691 & 1.664545 \\ \mathrm{C} & 0.831411 & -6.568725 & 3.384660 \\ \mathrm{H} & -0.467949 & -5.045332 & 4.180398 \\ \mathrm{C} & 2.299941 & -5.891038 & 1.589366 \\ \mathrm{H} & 2.190464 & -3.813410 & 1.008724 \\ \mathrm{C} & 1.824409 & -6.877747 & 2.450777 \\ \mathrm{H} & 0.452873 & -7.340677 & 4.056251 \\ \mathrm{H} & 3.077741 & -6.123168 & 0.859562 \\ \mathrm{H} & 2.223362 & -7.891710 & 2.396280 \\ \mathrm{C} & -1.563184 & -2.954632 & 1.868143 \\ \mathrm{C} & -1.649689 & -2.938686 & 0.470959 \\ \mathrm{C} & -2.694596 & -3.303127 & 2.615400 \\ \mathrm{C} & -2.849699 & -3.236439 & -0.172568 \\ \mathrm{H} & -0.768058 & -2.669046 & -0.117166 \\ \mathrm{C} & -3.897449 & -3.595951 & 1.972839 \\ \mathrm{H} & -2.650924 & -3.339516 & 3.705792 \\ \mathrm{C} & -3.980591 & -3.556132 & 0.580178 \\ \mathrm{H} & -2.903711 & -3.206073 & -1.262581 \\ \mathrm{H} & -4.775487 & -3.856445 & 2.565612 \\ \mathrm{H} & -4.925317 & -3.778916 & 0.082635 \\ \mathrm{C} & -0.203334 & -2.197472 & 4.348622 \\ \mathrm{C} & 0.704358 & -2.631221 & 5.324931 \\ \mathrm{C} & -1.230664 & -1.318816 & 4.723095 \\ \mathrm{C} & 0.571137 & -2.217748 & 6.649892 \\ \mathrm{H} & 1.531549 & -3.290934 & 5.052673 \\ \mathrm{C} & -1.363953 & -0.910632 & 6.049170 \\ \mathrm{H} & -1.933855 & -0.942937 & 3.975551 \\ \mathrm{C} & -0.466074 & -1.361395 & 7.017790 \\ \mathrm{H} & 1.287251 & -2.567924 & 7.395651 \\ & -2.174641 & -0.233886 & 6.325547 \\ & 4.959792 & -0.222513 & 2.117418 \\ & \mathrm{~S} 176 & -1.041611 & 8.055137 \\ \end{array}$




\begin{tabular}{llll}
$\mathrm{C}$ & 4.907444 & 0.829417 & 1.190357 \\
$\mathrm{C}$ & 6.183476 & -0.860094 & 2.346649 \\
$\mathrm{C}$ & 6.053806 & 1.236117 & 0.511940 \\
$\mathrm{H}$ & 3.956921 & 1.327861 & 0.981833 \\
$\mathrm{C}$ & 7.330365 & -0.457882 & 1.659582 \\
$\mathrm{H}$ & 6.246846 & -1.684224 & 3.060032 \\
$\mathrm{C}$ & 7.268842 & 0.587844 & 0.739797 \\
$\mathrm{H}$ & 5.993007 & 2.050809 & -0.211739 \\
$\mathrm{H}$ & 8.276073 & -0.969218 & 1.845507 \\
$\mathrm{H}$ & 8.163570 & 0.894202 & 0.196067 \\
$\mathrm{C}$ & 2.974364 & 0.648779 & 3.995580 \\
$\mathrm{C}$ & 1.634223 & 0.848807 & 4.350237 \\
$\mathrm{C}$ & 3.945745 & 1.530965 & 4.486567 \\
$\mathrm{C}$ & 1.267290 & 1.908792 & 5.177306 \\
$\mathrm{H}$ & 0.864553 & 0.181188 & 3.954410 \\
$\mathrm{C}$ & 3.579074 & 2.594394 & 5.311480 \\
$\mathrm{H}$ & 4.997212 & 1.387487 & 4.225575 \\
$\mathrm{C}$ & 2.240059 & 2.786325 & 5.656834 \\
$\mathrm{H}$ & 0.215304 & 2.052871 & 5.432923 \\
$\mathrm{H}$ & 4.343628 & 3.276535 & 5.685751 \\
$\mathrm{H}$ & 1.956160 & 3.621774 & 6.297986 \\
$\mathrm{C}$ & 3.903883 & -2.075769 & 4.063369 \\
$\mathrm{C}$ & 3.990786 & -1.875114 & 5.445800 \\
$\mathrm{C}$ & 4.168015 & -3.349696 & 3.538328 \\
$\mathrm{C}$ & 4.327771 & -2.933656 & 6.290740 \\
$\mathrm{H}$ & 3.776952 & -0.893043 & 5.873169 \\
$\mathrm{C}$ & 4.504236 & -4.404603 & 4.384128 \\
$\mathrm{H}$ & 4.111394 & -3.503570 & 2.457693 \\
$\mathrm{H}$ & 4.578483 & -4.200242 & 5.763556 \\
& 4.387229 & -2.767572 & 7.367557 \\
$\mathrm{H}$ & 4.703442 & -5.392815 & 3.965210 \\
& 4.834240 & -5.028250 & 6.426172 \\
& & $\mathrm{~S} 177$ & \\
\hline
\end{tabular}




$\begin{array}{lrrr}\mathrm{O} & 2.246979 & 0.855995 & -1.666288 \\ \mathrm{C} & 4.942757 & 0.425590 & -3.180960 \\ \mathrm{H} & 6.712726 & 0.475980 & -4.419704 \\ \mathrm{C} & 6.192883 & -0.040337 & -3.611515 \\ \mathrm{C} & 3.036059 & -0.042319 & -1.427286 \\ \mathrm{C} & 4.329825 & -0.267327 & -2.150626 \\ \mathrm{C} & 6.787340 & -1.162162 & -3.023298 \\ \mathrm{~N} & 2.901613 & -1.006892 & -0.459663 \\ \mathrm{C} & 4.920606 & -1.380657 & -1.567298 \\ \mathrm{H} & 7.760159 & -1.499767 & -3.383321 \\ \mathrm{C} & 6.151718 & -1.856114 & -1.984695 \\ \mathrm{C} & 3.985689 & -1.844110 & -0.495050 \\ \mathrm{H} & 6.608177 & -2.730591 & -1.517435 \\ \mathrm{O} & 4.171821 & -2.816198 & 0.222634\end{array}$

\section{Condition-based Sensitivity Screen}

The assessment of the reaction-condition-based sensitivity was performed following an adapted literature procedure. ${ }^{70}$

The following reaction parameters have been screened:

\begin{tabular}{lcc} 
& Description & Additives $/$ procedures \\
\cline { 2 - 3 } 2 & Control reaction & --- \\
3 & High $c$ & Volume $=\mathrm{V}_{0}-10 \%$ \\
4 & Low $c$ & Volume $=\mathrm{V}_{0}+10 \%$ \\
5 & Low $\mathrm{O}_{2}$ & Freeze-pump-thaw $(3 \mathrm{x})$ \\
6 & High $\mathrm{O}_{2}$ & 5 ml of air injected in the vessel \\
7 & High $T$ & Encapsulated and wrapped with $\mathrm{Al}$ \\
8 & Low intensity & Distance: $8.8 \mathrm{~cm}\left(\mathrm{I} \simeq \mathrm{I}_{0} / 16\right)$ \\
9 & High intensity & Distance $0.5 \mathrm{~cm}\left(\mathrm{I} \simeq 16 \mathrm{I}_{0}\right)$
\end{tabular}


Procedure for the Reaction-Condition-Based Sensitivity:
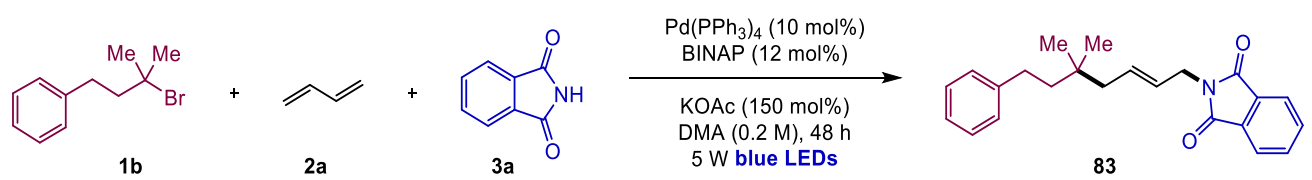

Step 1. Preparation of the stock solution (1b, 3a, rac-BINAP).

In an oven-dried Schlenk tube equipped with a PTFE-coated stirring bar, bromide 1a (33.9 $\mathrm{mg}$ for each reaction, 1.5 equiv.), rac-BINAP (7.47 $\mathrm{mg}$ for each reaction, $12 \mathrm{~mol} \%$ ) and phthalimide 3a (14.7 $\mathrm{mg}$ for each reaction, 1.0 equiv.) were charged under air, then the vessel was purged four times and re-filled with argon. Subsequently, dry DMA (300 $\mu \mathrm{L}$ for each reaction) was added.

Step 2. Reaction set-up (except large scale, low temperature reaction/control). In an oven-dried Schlenk tube equipped with a PTFE-coated stirring bar, $\mathrm{Pd}\left(\mathrm{PPh}_{3}\right)_{4}(11.6 \mathrm{mg}, 10 \mathrm{~mol} \%)$ and KOAc (14.7 mg, 1.5 equiv.) were charged under air, then the vessel was purged and re-filled four times with argon. Under argon counterflow, $300 \mu \mathrm{L}$ of stock solution were added, followed by the appropriate amount of dry DMA (0.2 M) and $75 \mu \mathrm{L}$ of $2 M$ solution of 1,3butadiene in THF. If required, either additives were added or procedures were performed at this point ${ }^{1}$, then the vessel was sealed, and the reaction irradiated for 48 hours using the shown set-up (see Fig. S18). Upon ending of the irradiation, the reaction was filtered through a short pad of silica, rinsing thoroughly with $\mathrm{CH}_{2} \mathrm{Cl}_{2}$. The filtrate was concentrated in vacuo, then

\footnotetext{
${ }^{1}$ Exception is the "freeze-pump-thaw", which was performed before the addition of the butadiene solution, due to the substantial loss of the volatile reagent that the procedure might cause.
} 
dibromoethane $(0.1 \mathrm{mmol})$ was added as standard and the mixture was analysed by ${ }^{1} \mathrm{H}$ NMR $\left(\mathrm{d}_{1}=30 \mathrm{~s}\right)$.

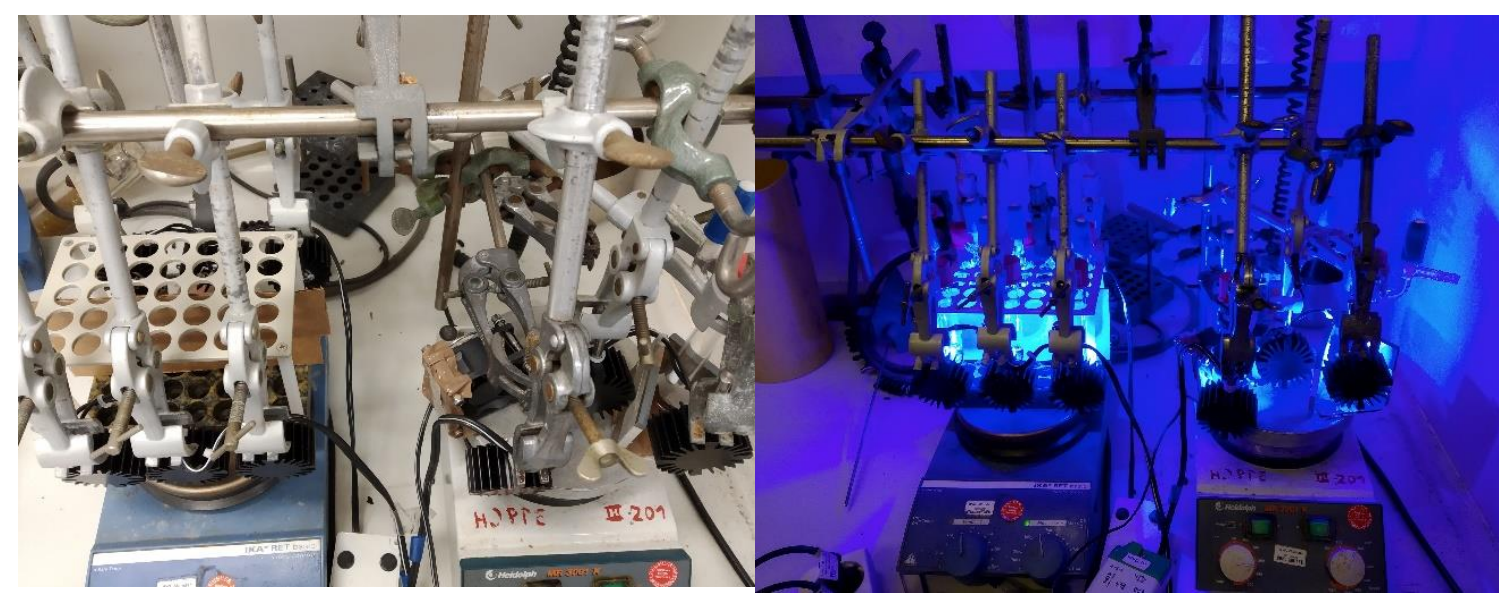

Fig. S18 Sensitivity screening set-up. From the left: six Schlenk tubes holder, LED placed at $2.2 \mathrm{~cm}$ from the vessel; set-up for high intensity, LED placed at 0.5 $\mathrm{cm}$ from the vessel, cooling fan used to avoid any excessive increase of the temperature; set-up for low intensity, LED placed at $8.8 \mathrm{~cm}$ from the vessel; setup for high temperature, LED placed at $2.2 \mathrm{~cm}$ from the vessel, vessel and LED wrapped with a plastic box and aluminum foil. Stirring was set at $\sim 1000 \mathrm{~s}^{-1}$.

Step 2'. Reaction set-up for large scale reaction.

In an oven-dried $50 \mathrm{ml}$ Schlenk tube equipped with a PTFE-coated stirring bar, phthalimide (147.1 mg, 1.0 mmol, 1.0 equiv.), $\mathrm{Pd}\left(\mathrm{PPh}_{3}\right)_{4}(115.5 \mathrm{mg}, 0.1 \mathrm{mmol}, 10$ mol\%), rac-BINAP (74.7 mg, $0.12 \mathrm{mmol}, 12 \mathrm{~mol} \%$ ) and KOAc (147.2 mg, 1.5 mmol, 1.5 equiv.) were charged under air, then the vessel was evacuated and back-filled with argon four times. Dry DMA (4.25 ml) was charged under argon, followed by bromide $\mathbf{1 b}$ (339.2 $\mathrm{mg}, 1.5 \mathrm{mmol}, 1.5$ equiv.) and butadiene $2 \mathrm{M}$ in THF $(0.75 \mathrm{ml})$, then the vessel was sealed and irradiated at $455 \mathrm{~nm}$ using an array of 6 circularly assembled LEDs. After 48 hours the solvent was removed in vacuo $\left(<1\right.$ mbar @ $\left.60^{\circ} \mathrm{C}\right)$, then the residue was taken-up with $\mathrm{CH}_{2} \mathrm{Cl}_{2}(60 \mathrm{ml})$, then organic layer washed twice with water (50 ml each time), dried over $\mathrm{MgSO}_{4}$ and the solvent removed under reduced pressure. The crude was purified by 
flash column chromatography on silica (pentane/EtOAc 14/1 $\rightarrow 12 / 1$ ), to give 83 (247.4 mg, $0.712 \mathrm{mmol}, 71 \%$, E/Z > 95:5, rr > 20:1) as a colorless gum.

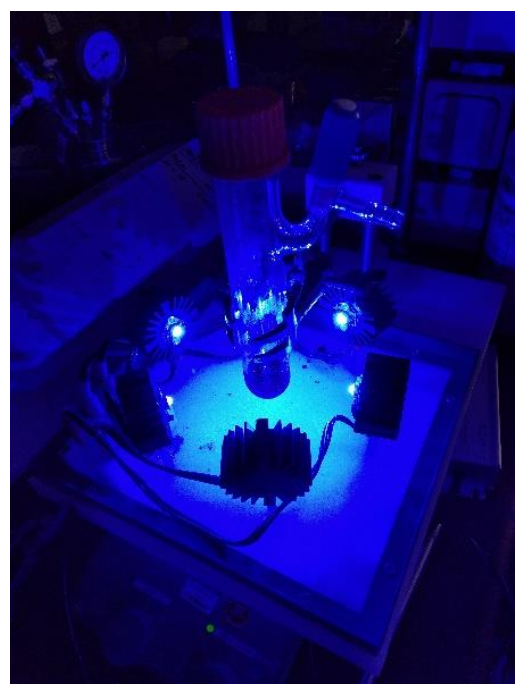

Fig. S19 Set-up for the scale-up reaction of the sensitivity screening. The 6-LEDs array used for the photochemical reaction was used, with the sole exception of the employment of a 50 $\mathrm{ml}$ Schlenk tube. Magnetic stirring was set at $1000 \mathrm{~s}^{-1}$.

Step 2". Reaction set-up for the low temperature reaction/control reaction In an oven-dried jacketed Schlenk tube equipped with a PTFE-coated stirring bar, phthalimide (14.7 mg, $0.1 \mathrm{mmol}, 1.0$ equiv.), $\mathrm{Pd}\left(\mathrm{PPh}_{3}\right)_{4}(11.6 \mathrm{mg}, 10 \mu \mathrm{mol}, 10$ mol\%) and KOAc (14.7 mg, $0.15 \mathrm{mmol}, 1.5$ equiv.) were charged under air, then the vessel was purged and re-filled four times with argon. Under argon counterflow, dry DMA (425 $\mu \mathrm{L})$, bromide $\mathbf{1 b}$ (33.9 mg, $0.15 \mathrm{mmol}, 1.5$ equiv.) and 1,3-butadiene $2 M$ in THF (75 $\mu \mathrm{L}, 0.15 \mathrm{mmol}, 1.5$ equiv.) were consecutively added, then the vessel was sealed and irradiated at $455 \mathrm{~nm}$ as pictorially described below. The reaction was cooled using flowing water at room temperature $\left(20^{\circ} \mathrm{C}-\right.$ measured temperature of the cooling medium), while the reference jacket contained water which did not flow (see picture below). Upon ending of the irradiation, the reaction was filtered through a short pad of silica, rinsing thoroughly with $\mathrm{CH}_{2} \mathrm{Cl}_{2}$. The filtrate was concentrated in vacuo, then dibromoethane $(0.1 \mathrm{mmol})$ was added as standard and the mixture was analysed by ${ }^{1} \mathrm{H}$ NMR $\left(\mathrm{d}_{1}=30 \mathrm{~s}\right)$. 


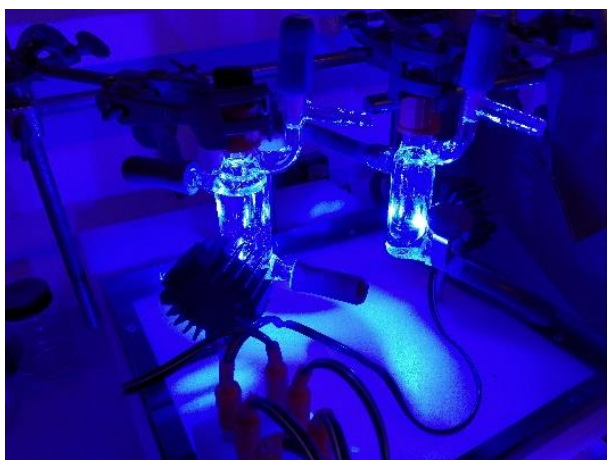

Fig. S20 Set-up for the cooled reaction and control reaction. The right jacketed vessel was connected to the water supply $\left(20^{\circ} \mathrm{C}-\right.$ measured temperature of the cooling medium), while the left jacketed vessel was filled with water. The light sources were placed at $2.2 \mathrm{~cm}$ away from the vessels. Magnetic stirring was set at $\sim 1000 \mathrm{~s}^{-1}$.

\section{Results:}

The yields (except in the case of the large-scale reaction) were obtained by ${ }^{1} \mathrm{H}$ NMR analysis (see below) of the crude reaction mixture, using $\mathrm{CH}_{2} \mathrm{Br}_{2}$ as standard. The following signals and integrations were used: for $\mathrm{CH}_{2} \mathrm{Br}_{2}(\mathrm{~s}, 2 \mathrm{H})$ from 4.915 to $4.760 \mathrm{ppm}$; for product $-\mathrm{CH}_{2} \mathrm{~N}-(\mathrm{d}, 2 \mathrm{H})$ from 4.300 to $4.070 \mathrm{ppm}$.

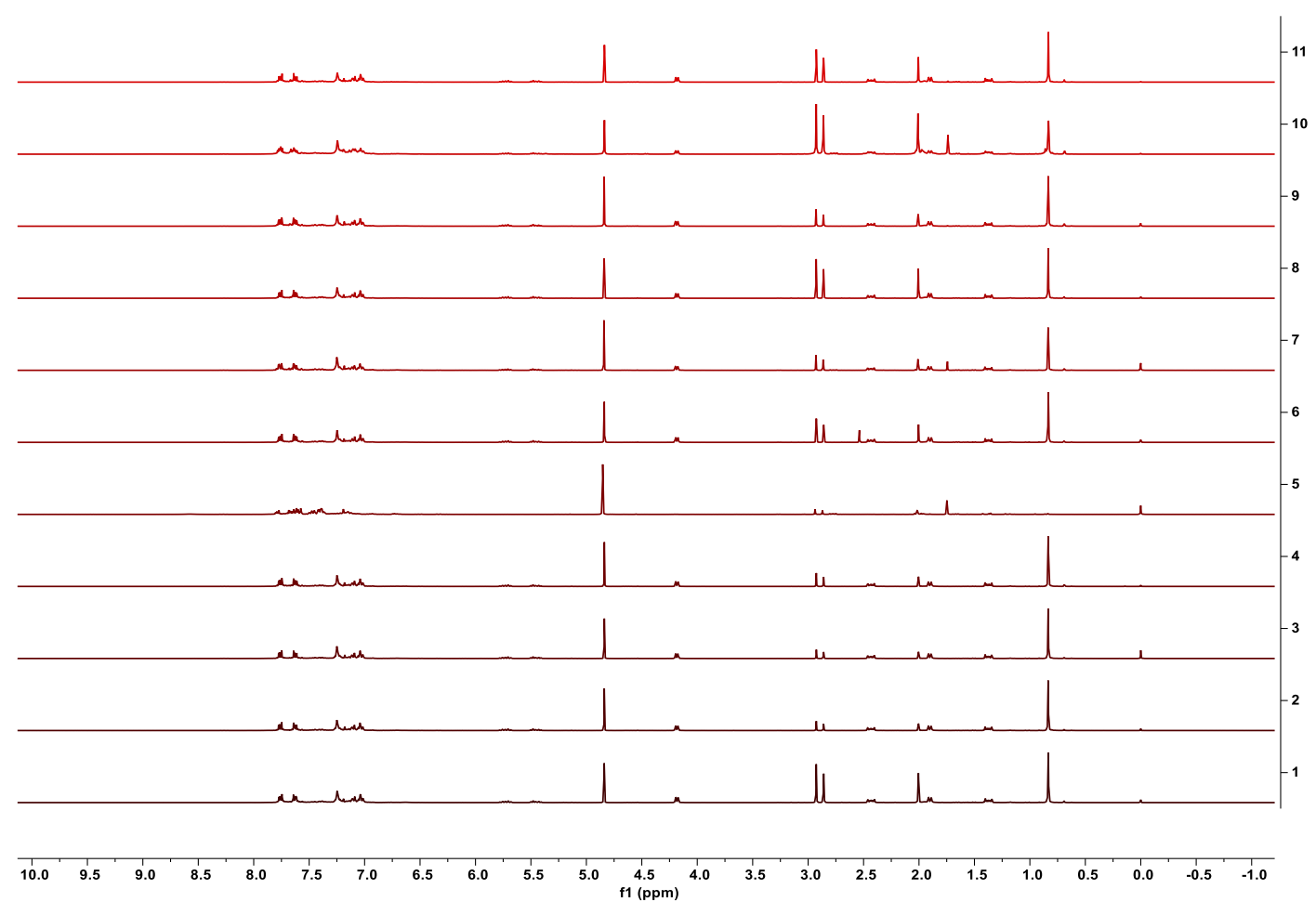

Fig. S21 ${ }^{1} \mathrm{H}$ NMR analysis of the reactions.

Table S3. Results of the condition-based sensitivity screening 


\begin{tabular}{lccc}
\cline { 2 - 3 } 2 & Control reaction & 74 & --- \\
2 & High C $(+10 \%)$ & 74 & 0.0 \\
3 & Low C $(-10 \%)$ & 73 & -1.5 \\
4 & Low $0_{2}$ & 71 & -3.6 \\
5 & High $\mathrm{O}_{2}$ & 4 & -95.1 \\
6 & High T $\left(>35^{\circ} \mathrm{C}\right)$ & 74 & -0.7 \\
7 & Low intensity & 52 & -30.4 \\
8 & High intensity & 73 & -1.5 \\
9 & Water & 65 & -12.9 \\
10 & Control low T & 78 & --- \\
11 & Low T $\left(20^{\circ} \mathrm{C}\right)$ & 49 & -37.4 \\
12 & Large scale & 71 & $-6.3^{2}$
\end{tabular}

As shown in Table S3, either small variations in the reaction volume (entry 2, 3), thorough degassing of the reaction (entry 4), scaling-up (entry 12) and higher reaction temperature (entry 6) had a negligible to small effect on the formation of product. To some extent, the presence of water had a negative impact, which was markedly observed at low temperature (entry 11) particularly in the presence of oxygen (entry 5), which completely inhibited the reaction.

\footnotetext{
${ }^{2}$ The isolated yield of the $1.0 \mathrm{mmol}$ scale reaction was compared to the one performed at $0.2 \mathrm{mmol}$ scale.
} 
High $c$

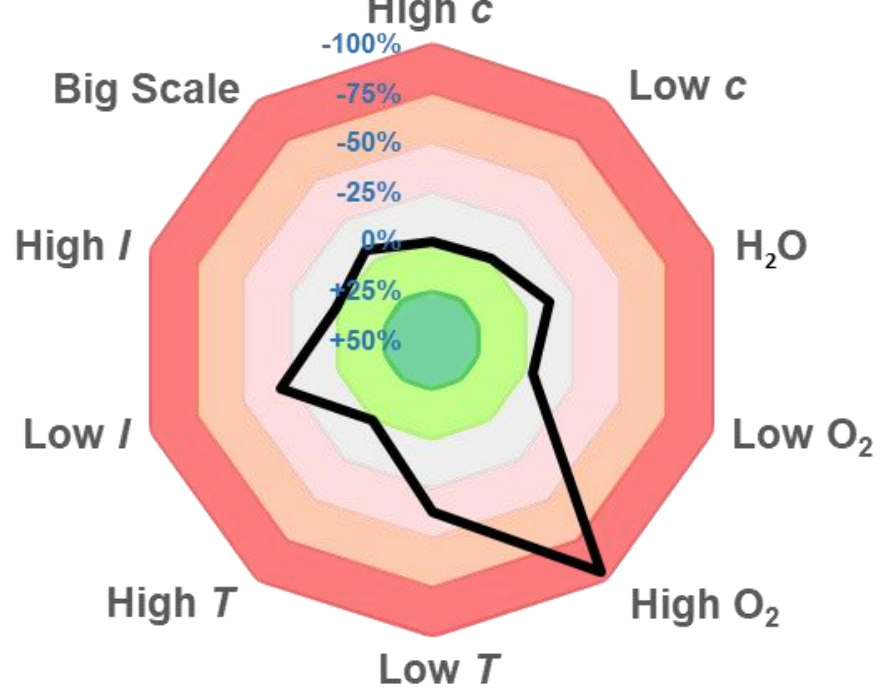


9. ${ }^{1} \mathrm{H},{ }^{13} \mathrm{C}$ and ${ }^{19} \mathrm{~F}$ NMR Spectra of Compounds

${ }^{1} \mathrm{H}$ NMR (400 MHz, $\mathrm{CDCl}_{3}$ )
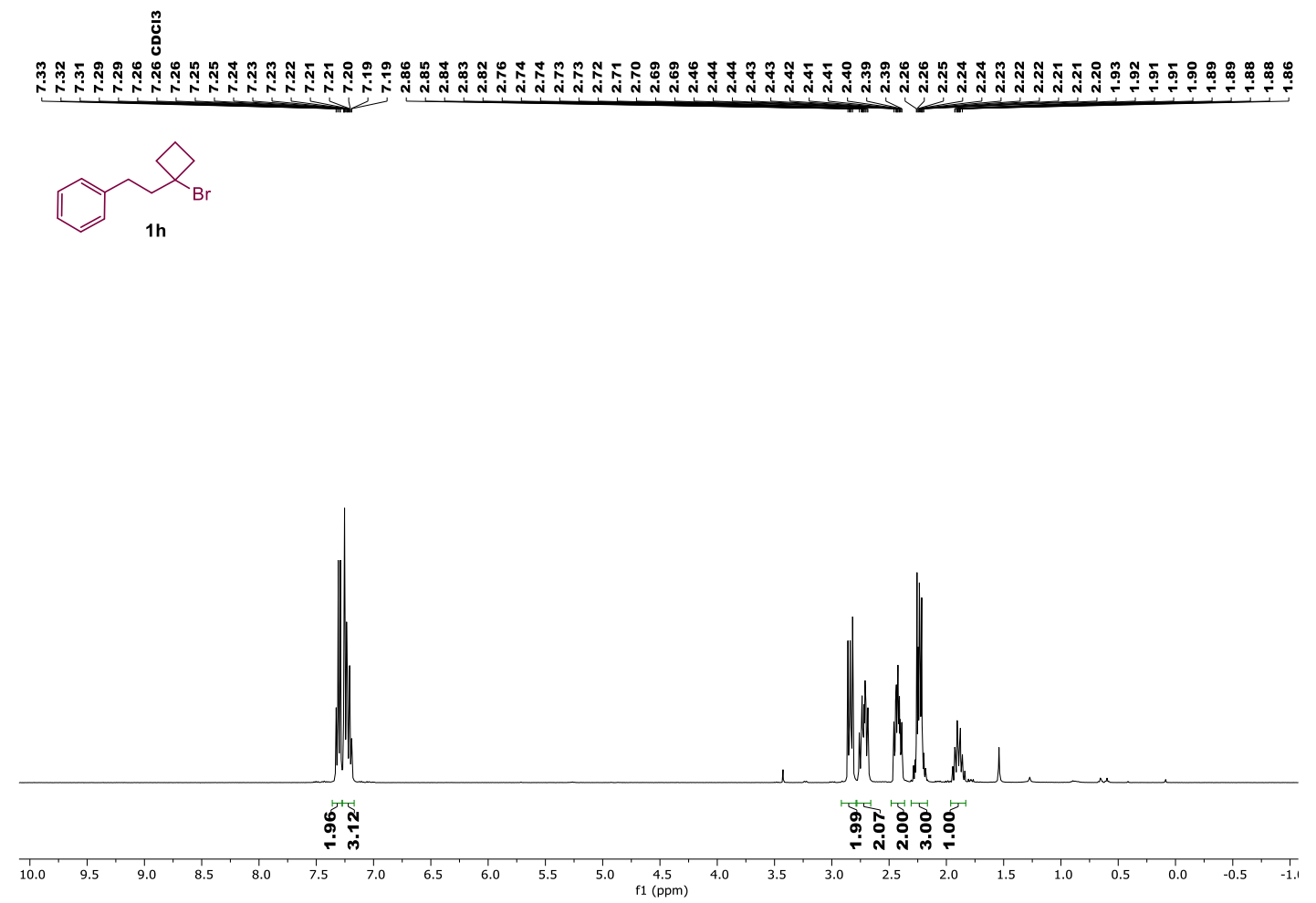

${ }^{13} \mathrm{C}$ NMR (101 MHz, $\mathrm{CDCl}_{3}$ )
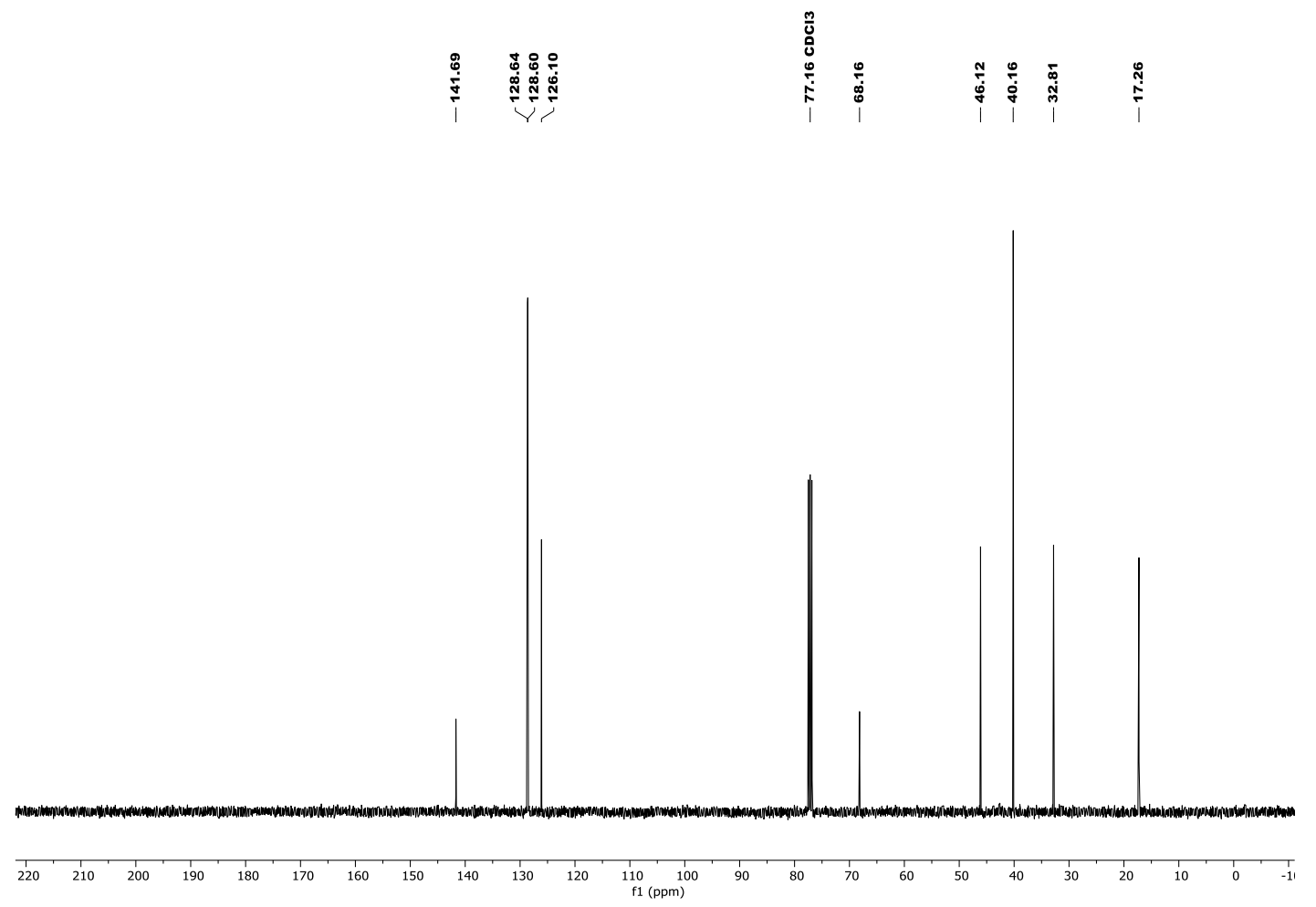
${ }^{1} \mathrm{H}$ NMR (400 MHz, $\mathrm{CDCl}_{3}$ )
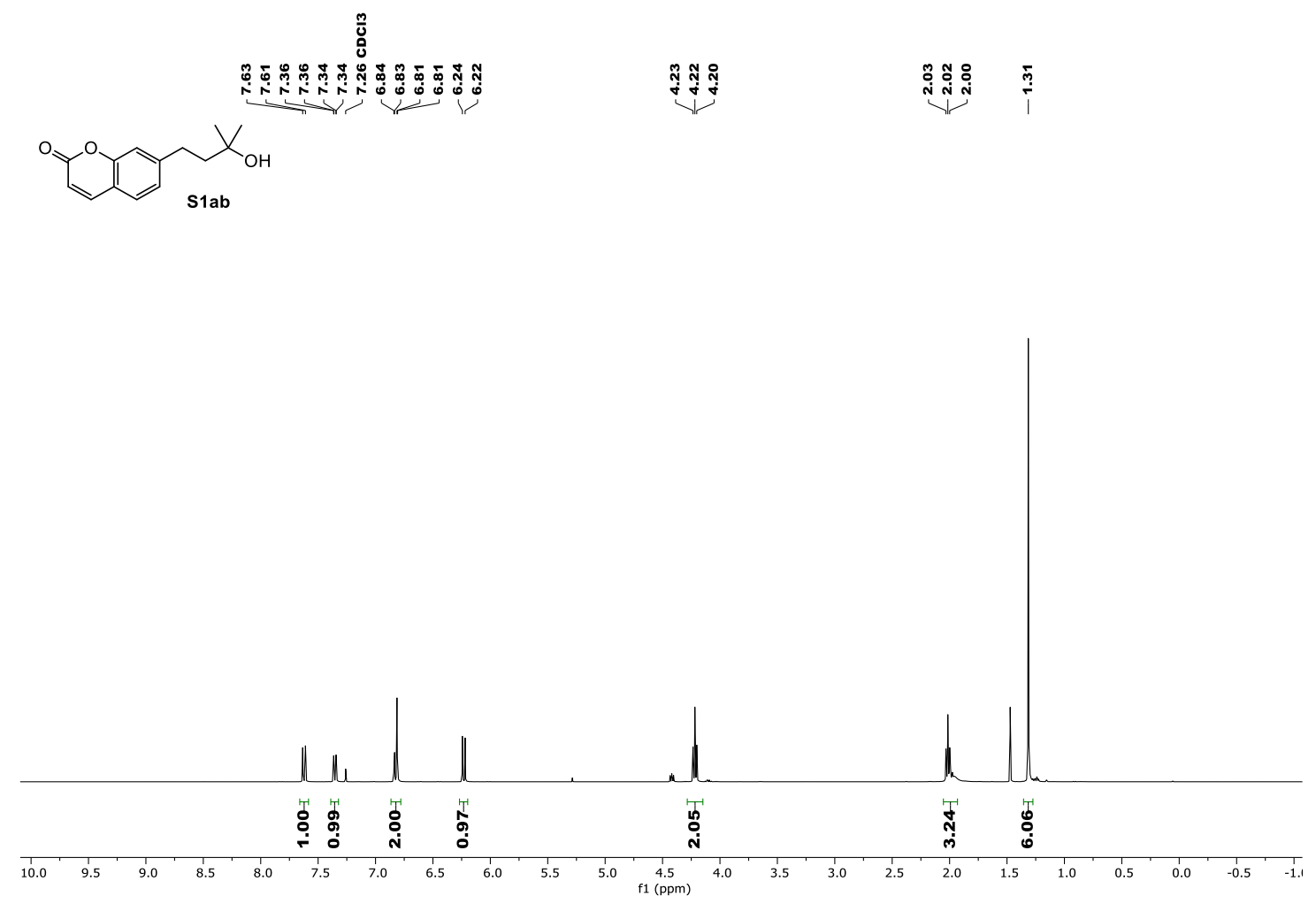

${ }^{13} \mathrm{C}$ NMR (101 MHz, $\mathrm{CDCl}_{3}$ )
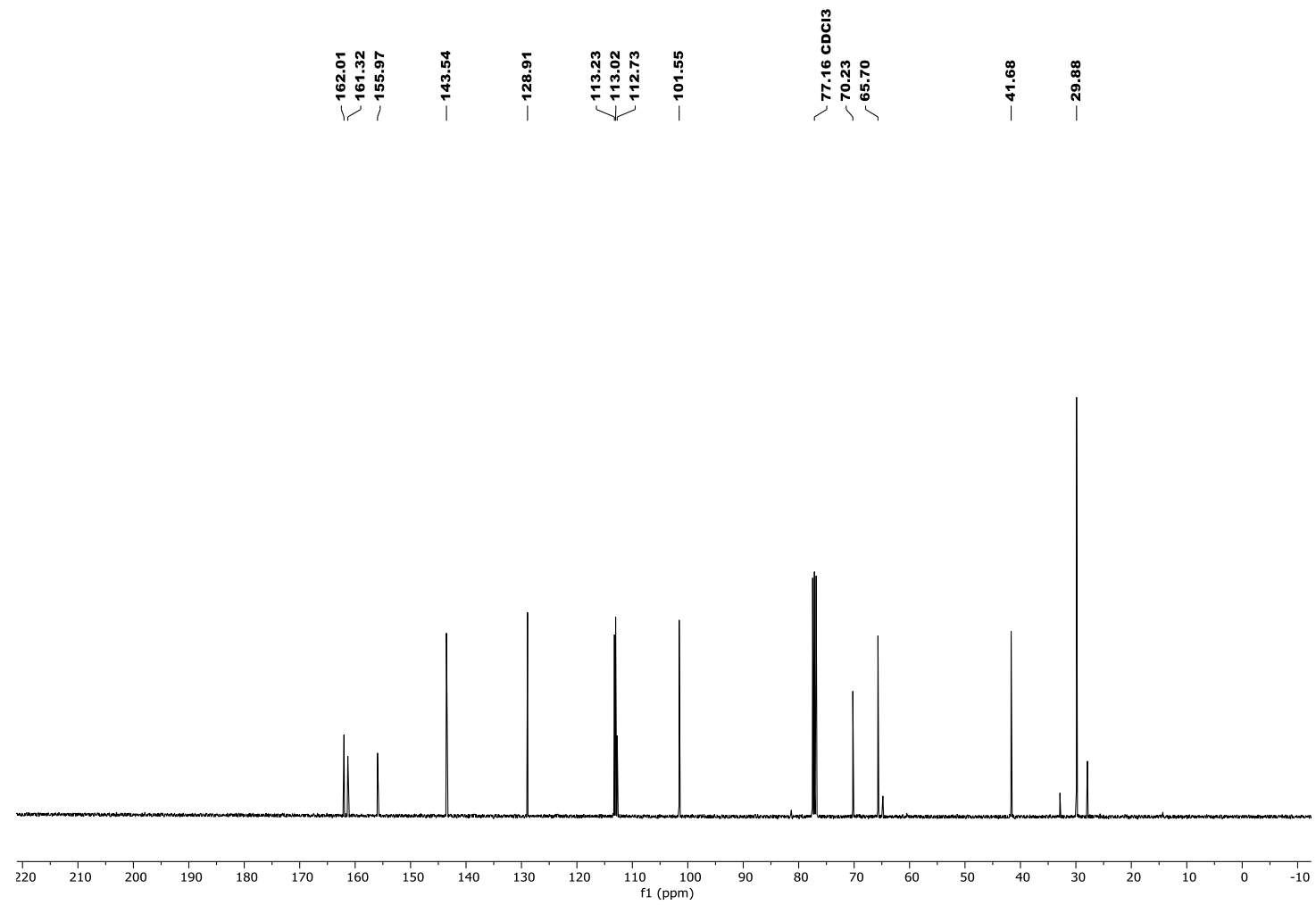
${ }^{1} \mathrm{H}$ NMR (400 MHz, $\mathrm{CDCl}_{3}$ )

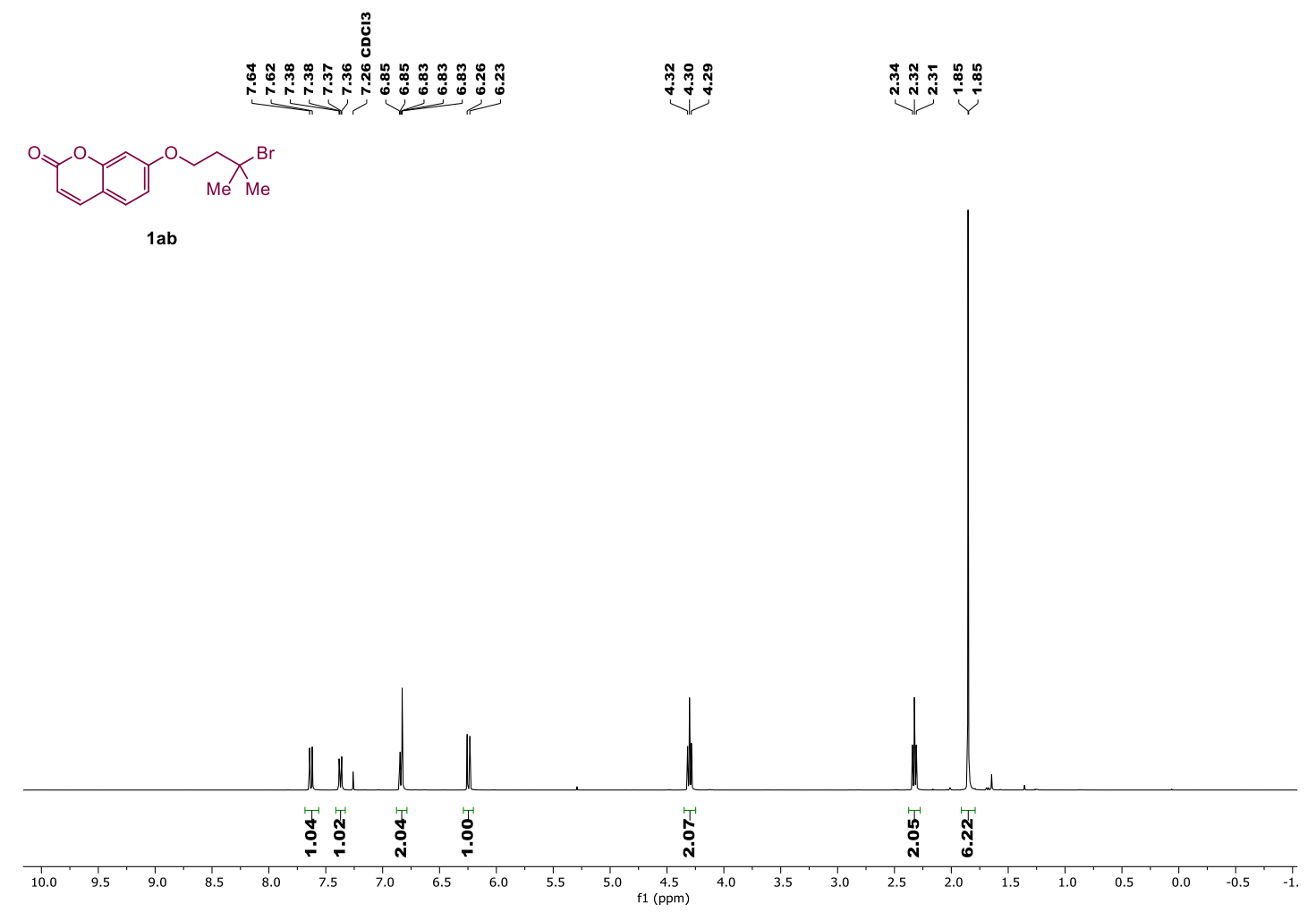

${ }^{13} \mathrm{C}$ NMR (101 MHz, $\mathrm{CDCl}_{3}$ )

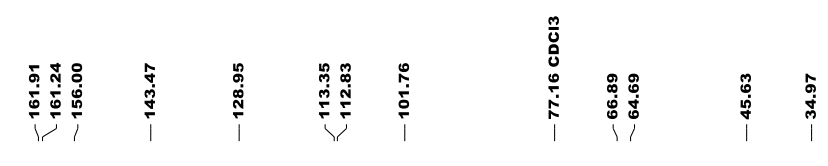

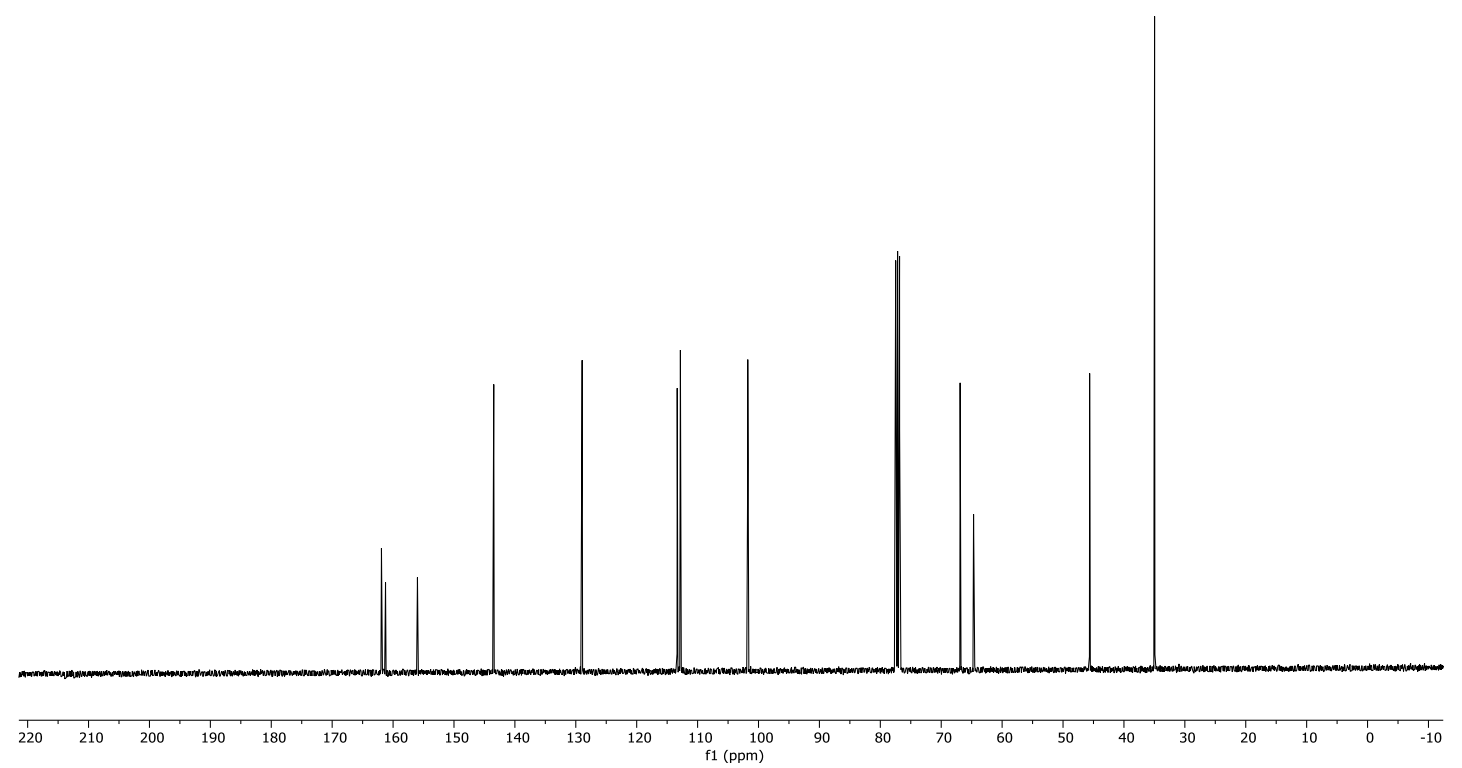


${ }^{1} \mathrm{H}$ NMR (300 MHz, $\mathrm{CDCl}_{3}$ )

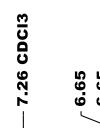

$\overbrace{\mathbf{1 a c}}^{\mathrm{Br}}$

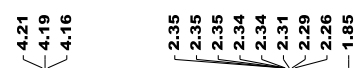

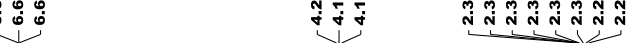

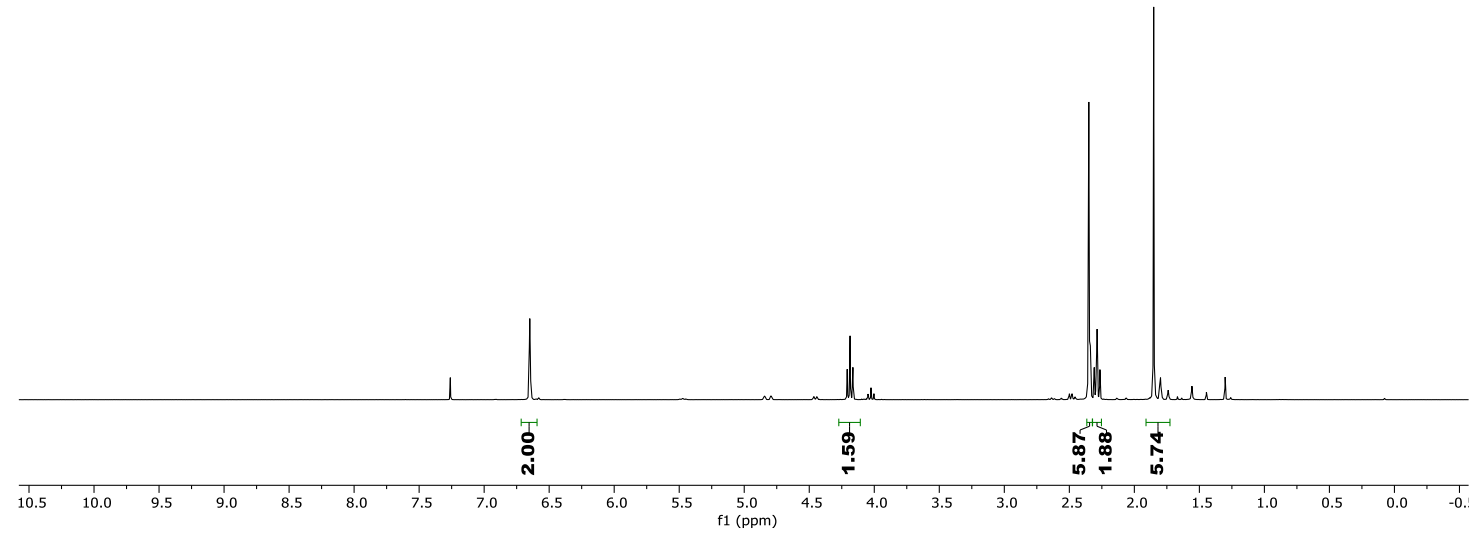

${ }^{13} \mathrm{C}$ NMR $\left(75 \mathrm{MHz}, \mathrm{CDCl}_{3}\right)$
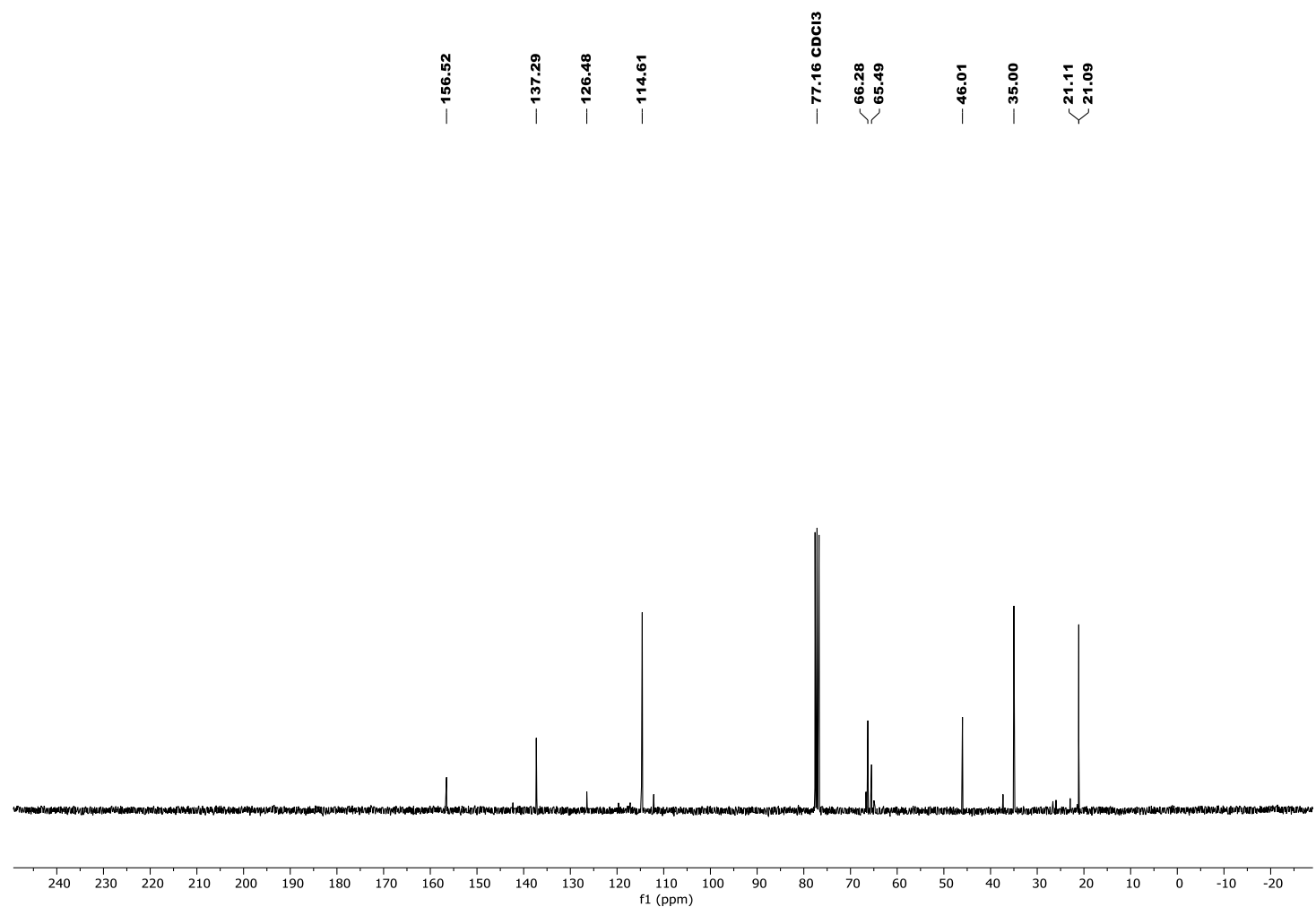
${ }^{1} \mathrm{H} \mathrm{NMR}\left(300 \mathrm{MHz}, \mathrm{CDCl}_{3}\right)$
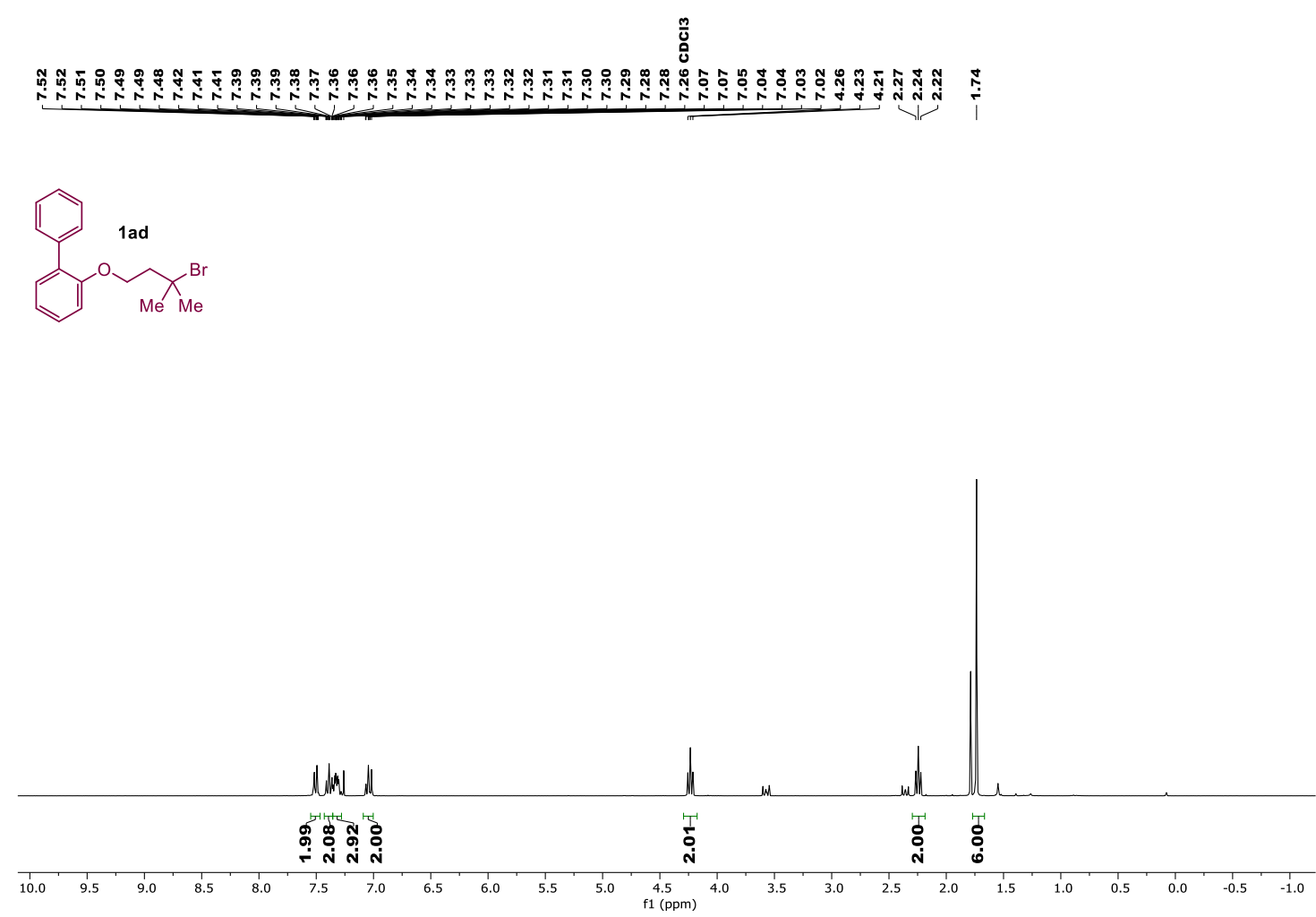

${ }^{13} \mathrm{C}$ NMR (101 MHz, $\left.\mathrm{CDCl}_{3}\right)$
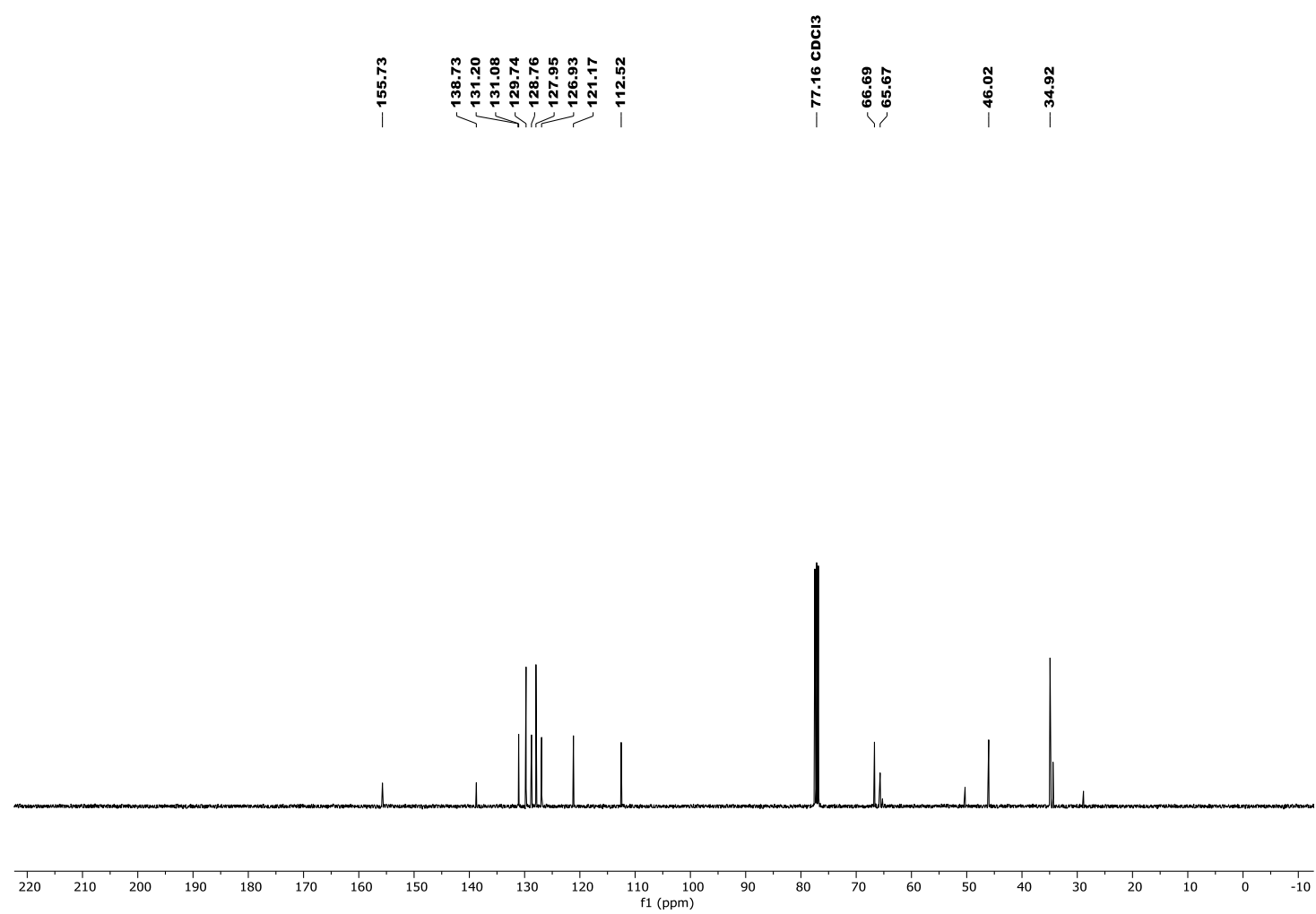
${ }^{1} \mathrm{H}$ NMR (400 MHz, $\mathrm{CDCl}_{3}$ )
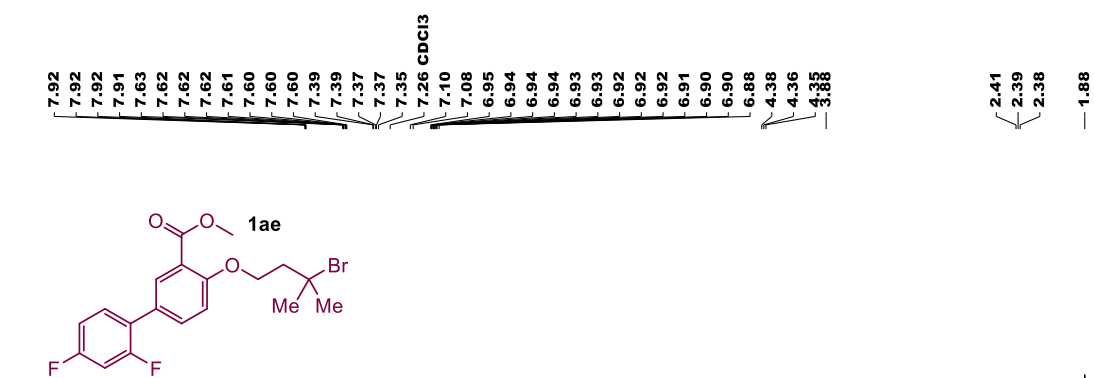

${ }^{13} \mathrm{C}$ NMR (101 MHz, $\mathrm{CDCl}_{3}$ )
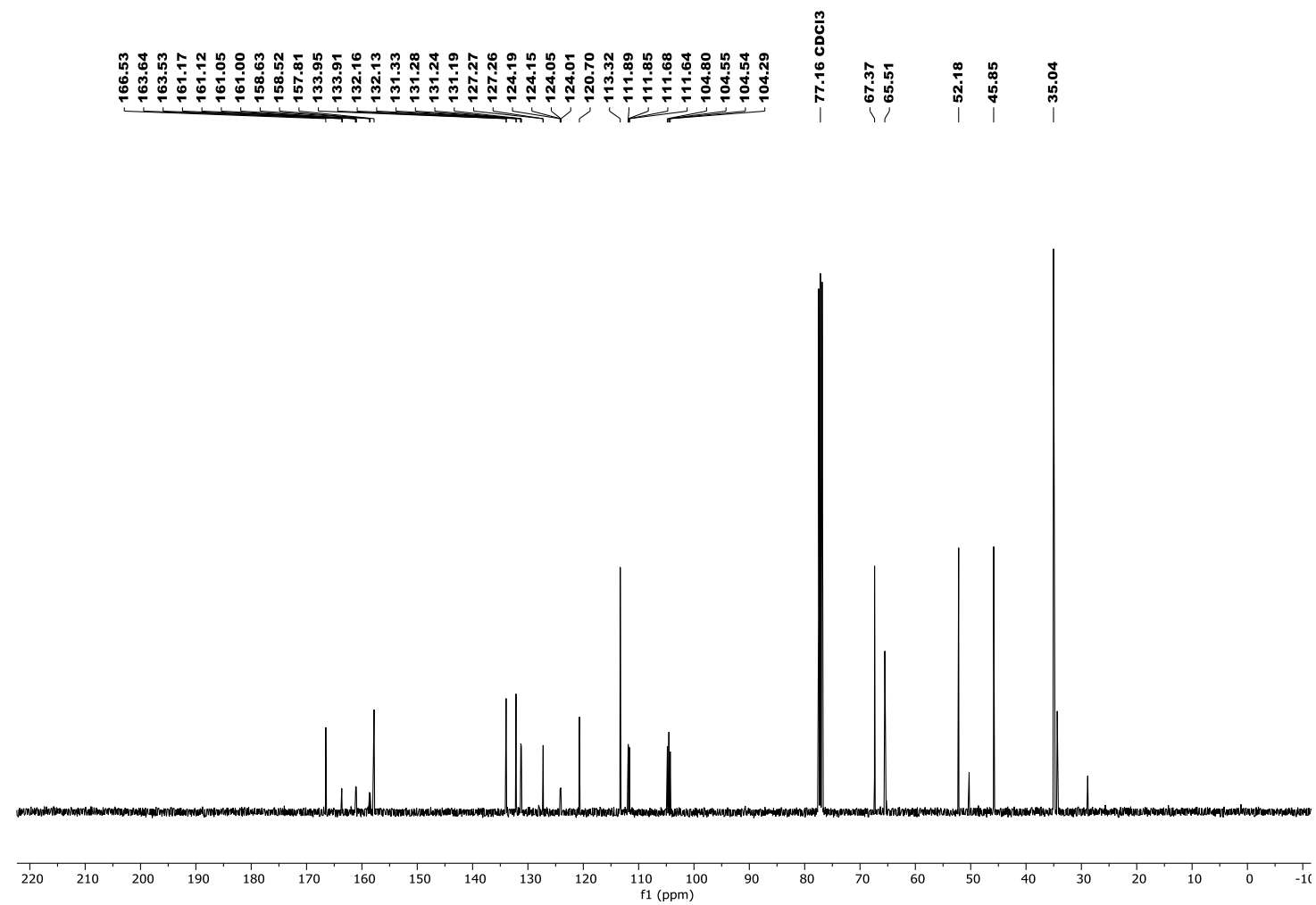
${ }^{19} \mathrm{~F}\left\{{ }^{1} \mathrm{H}\right\} \mathrm{NMR}\left(376 \mathrm{MHz}, \mathrm{CDCl}_{3}\right)$
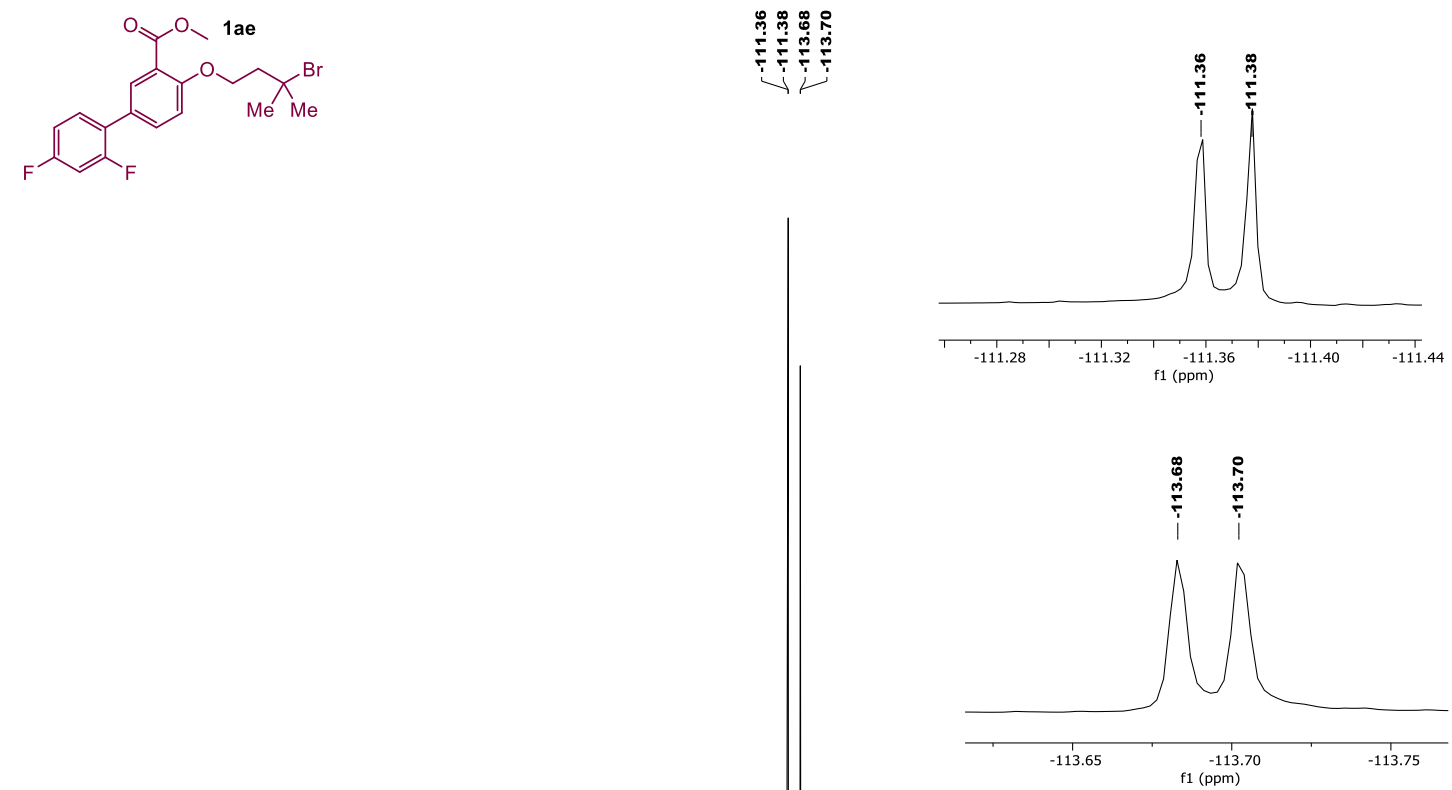

\begin{tabular}{rllllllllllllllllllllllllllllllll}
\hline & 1 \\
30 & 20 & 10 & 0 & -10 & -20 & -30 & -40 & -50 & -60 & -70 & -80 & -90 & -100 & -110 & -120 & -130 & -140 & -150 & -160 & -170 & -180 & -190 & -200 & -210 & -220 & -230
\end{tabular} 
${ }^{1} \mathrm{H}$ NMR $\left(300 \mathrm{MHz}, \mathrm{CDCl}_{3}\right)$
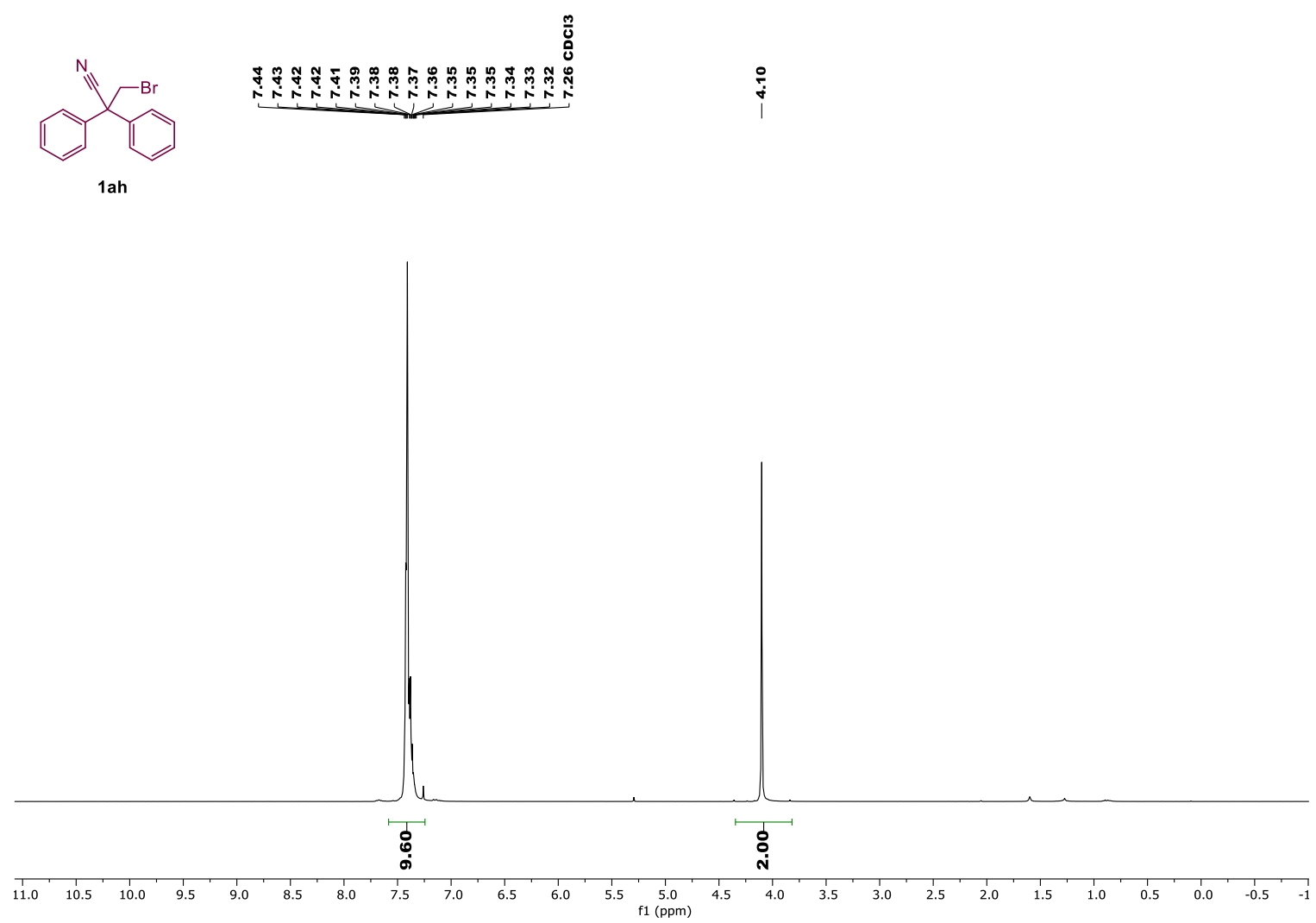

${ }^{13} \mathrm{C}$ NMR $\left(75 \mathrm{MHz}, \mathrm{CDCl}_{3}\right.$ )
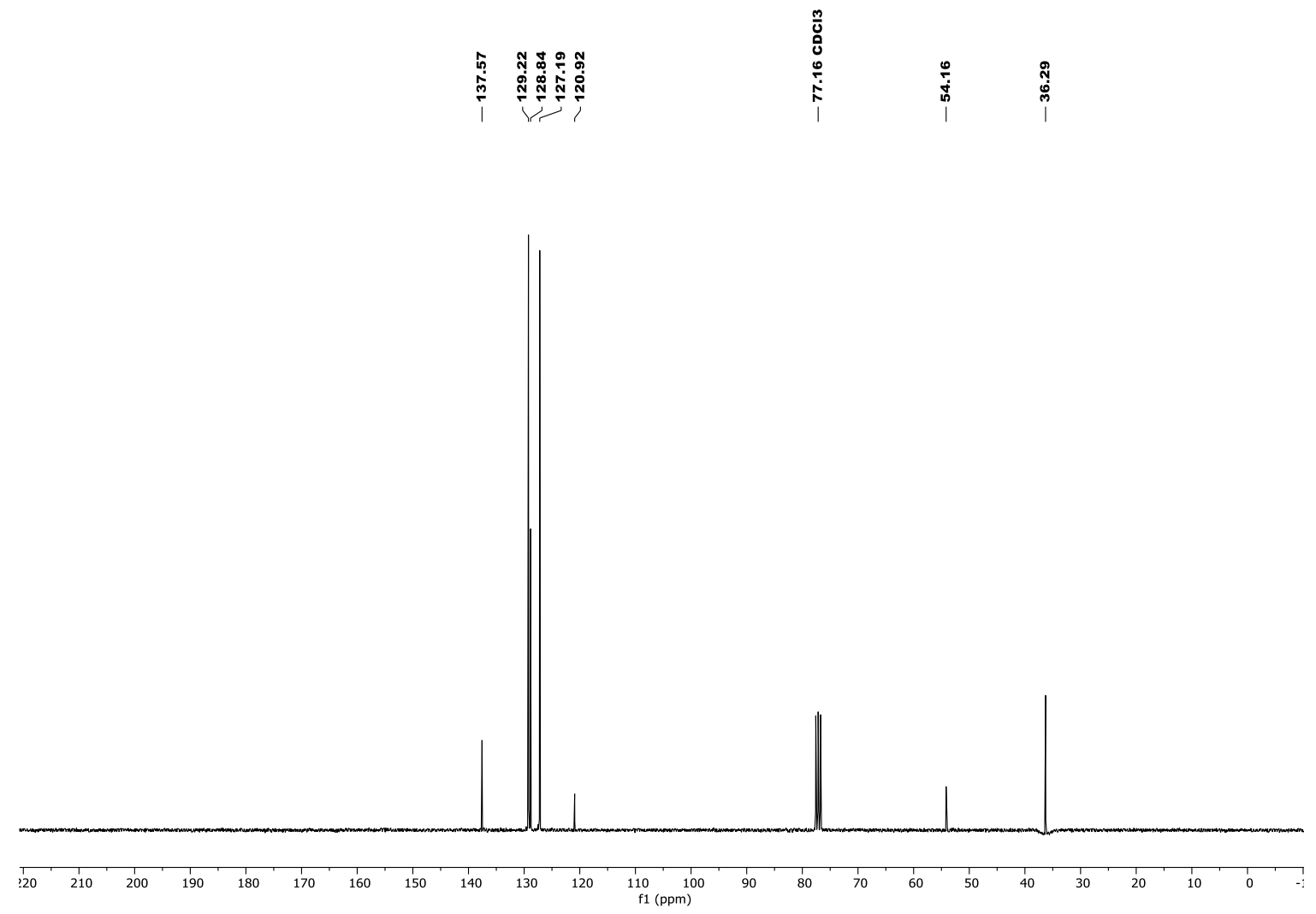
${ }^{1} \mathrm{H}$ NMR (400 MHz, $\mathrm{CDCl}_{3}$ )

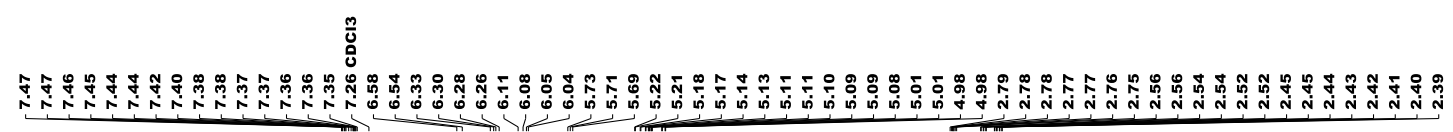

$\underbrace{F_{3} C}_{2 d}$

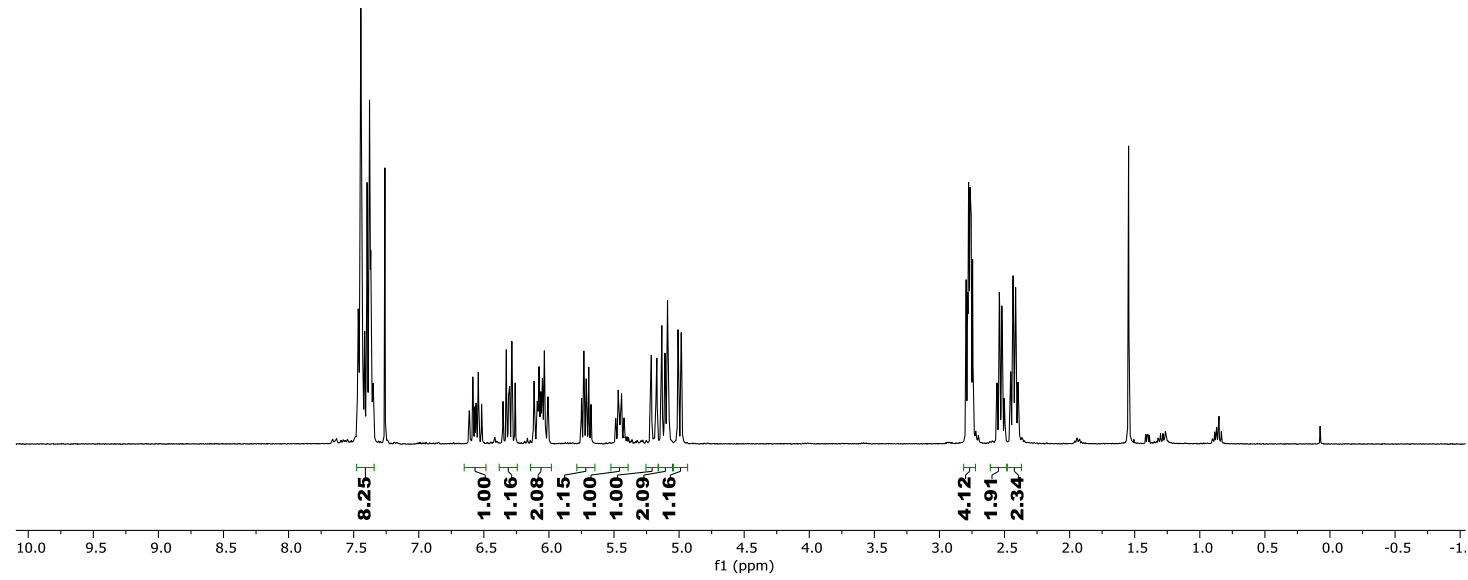

${ }^{13} \mathrm{C}$ NMR (101 MHz, $\mathrm{CDCl}_{3}$ )

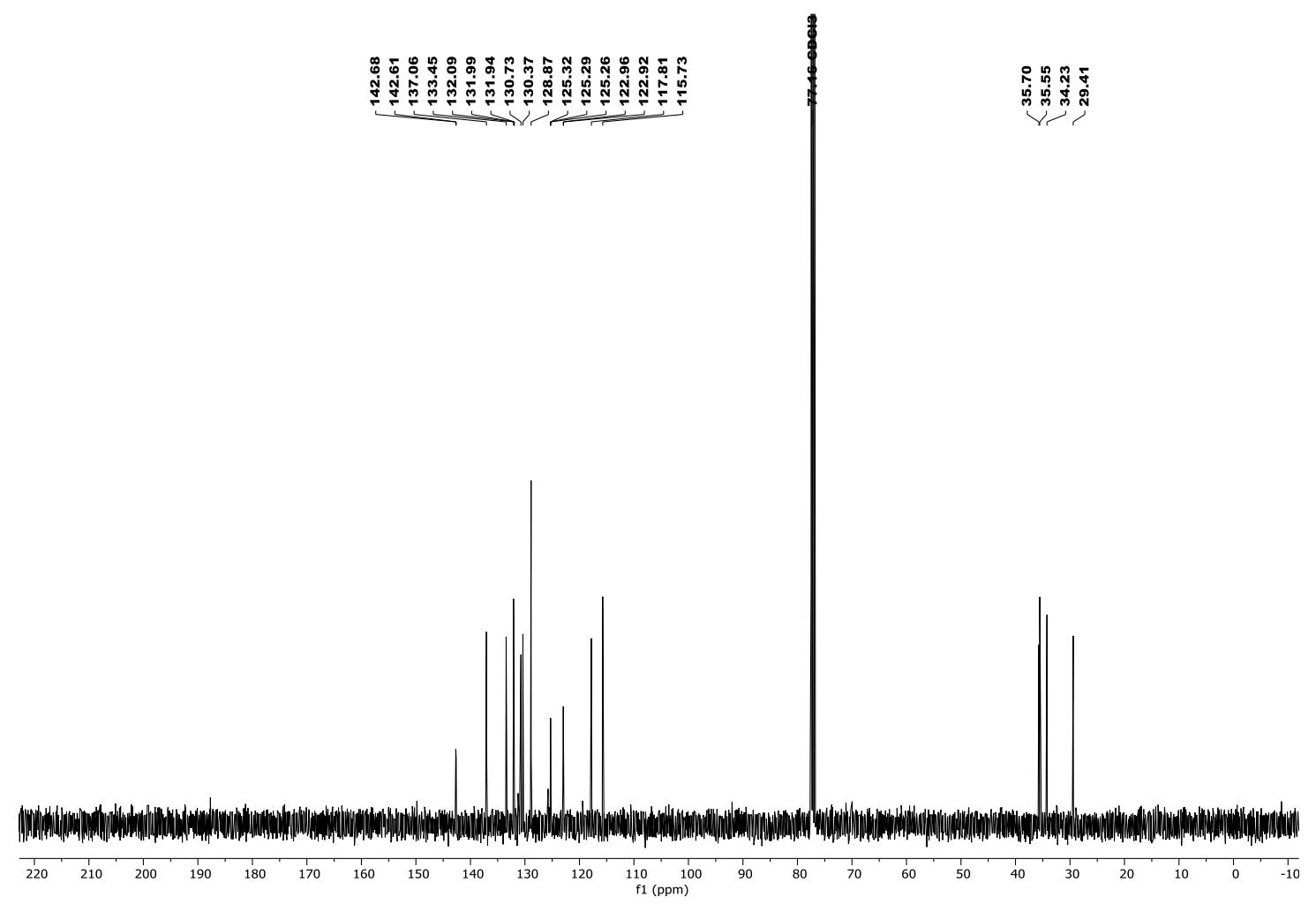


${ }^{19} \mathrm{~F}\left\{{ }^{1} \mathrm{H}\right\} \mathrm{NMR}\left(376 \mathrm{MHz}, \mathrm{CDCl}_{3}\right)$

:

$\overbrace{2 d}^{\mathrm{F}_{3} \mathrm{C}}$

$\stackrel{\leftrightarrow}{\leftrightarrow}$

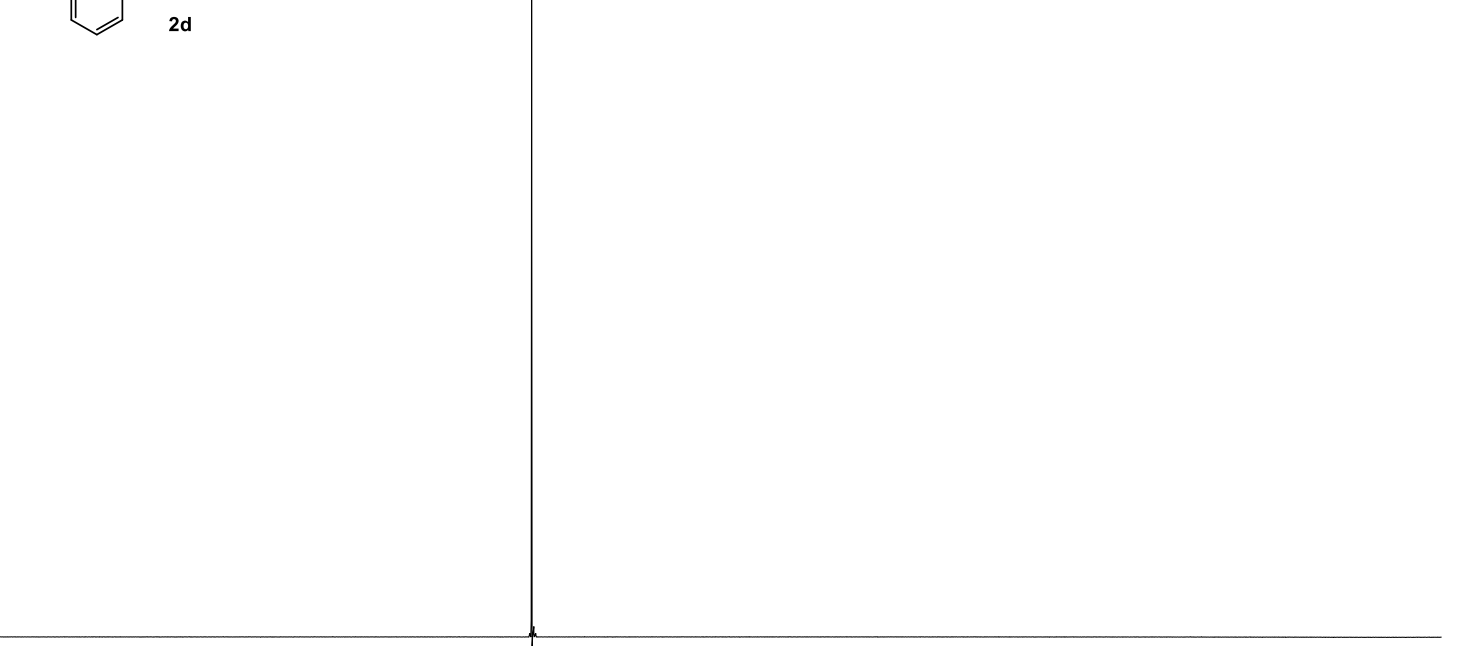

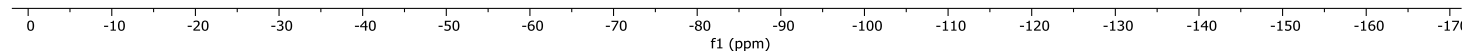


${ }^{1} \mathrm{H}$ NMR (300 MHz, $\left.\mathrm{CDCl}_{3}\right)$

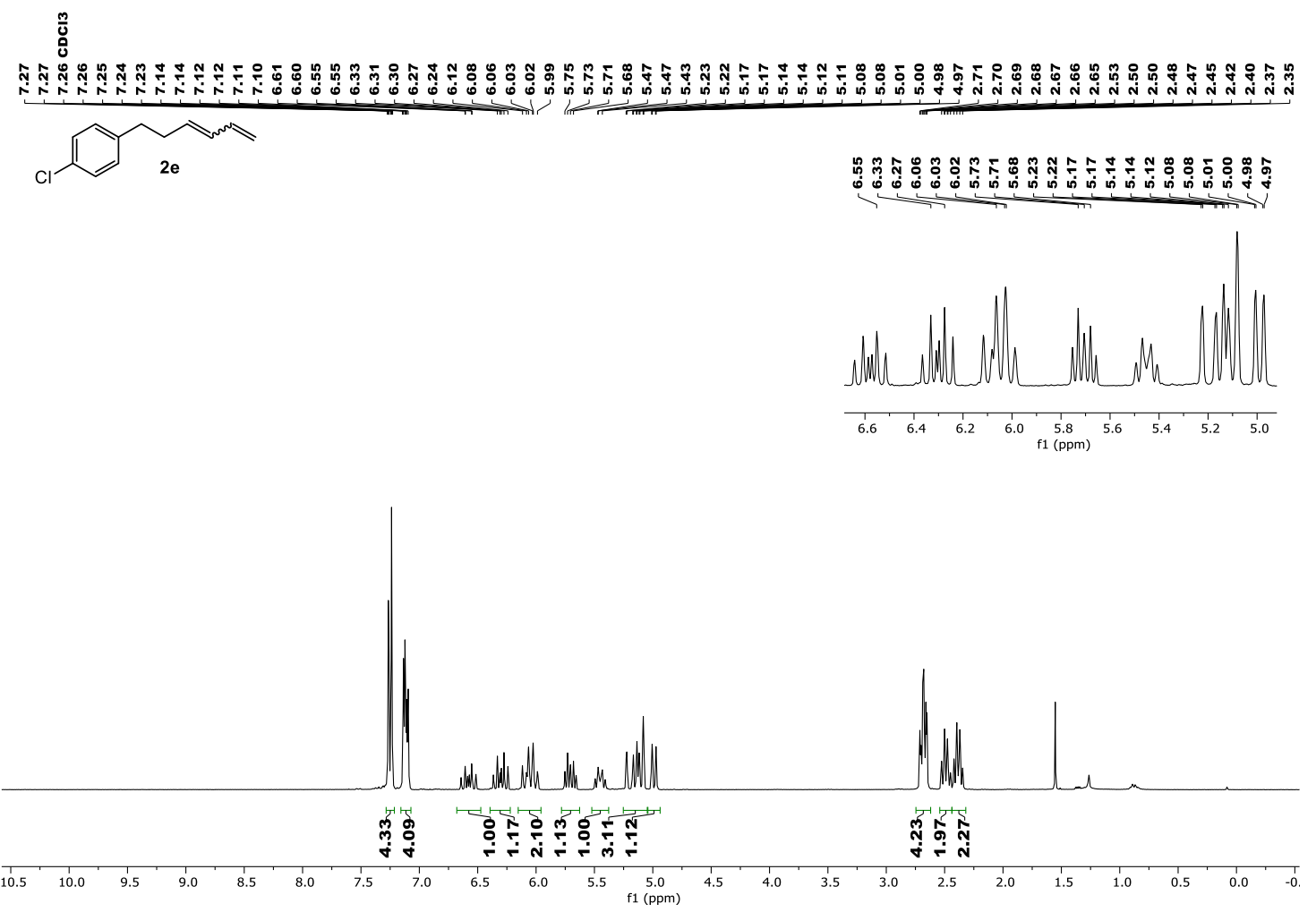

${ }^{13} \mathrm{C}$ NMR $\left(75 \mathrm{MHz}, \mathrm{CDCl}_{3}\right)$
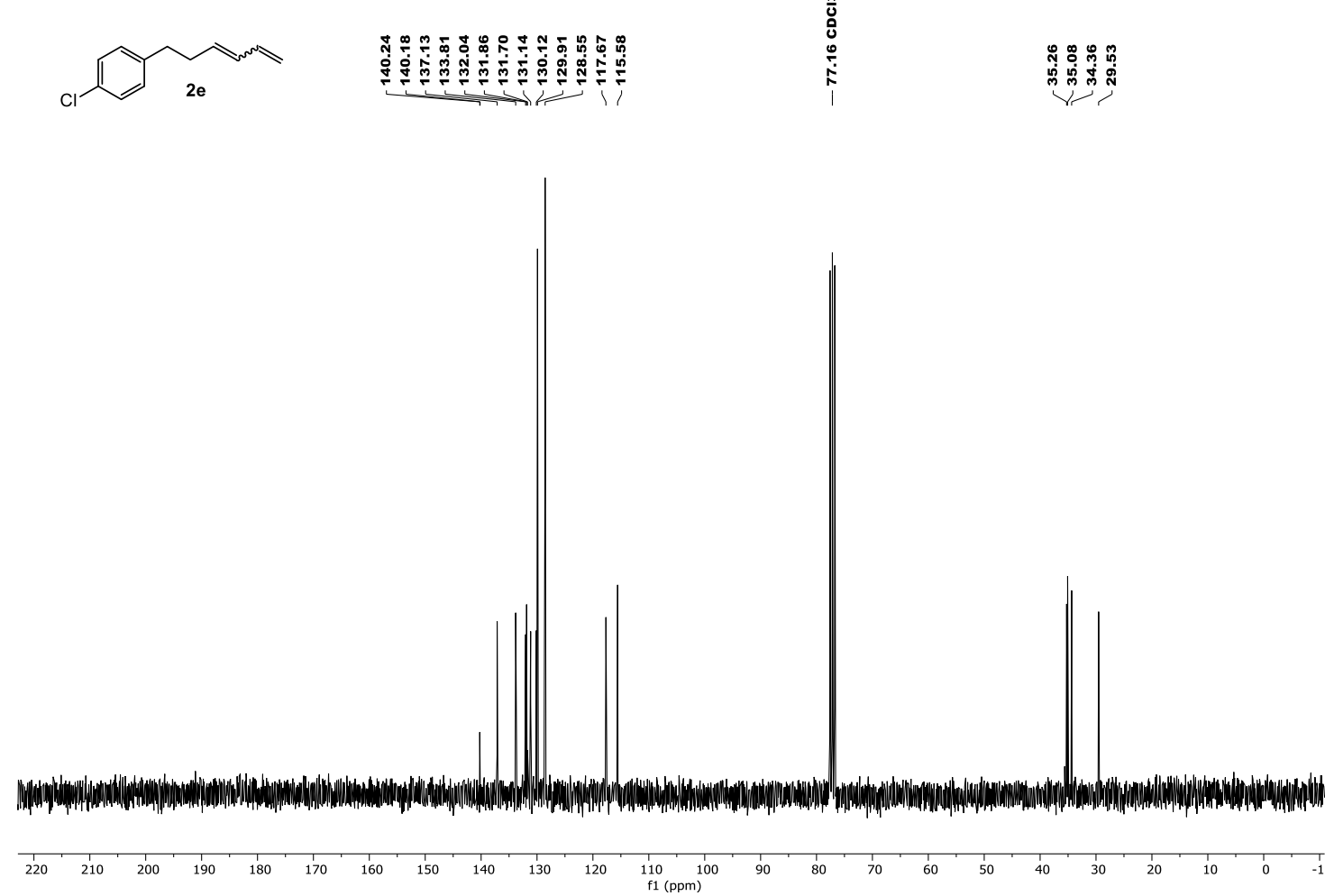
${ }^{1} \mathrm{H}$ NMR $\left(300 \mathrm{MHz}, \mathrm{CDCl}_{3}\right)$

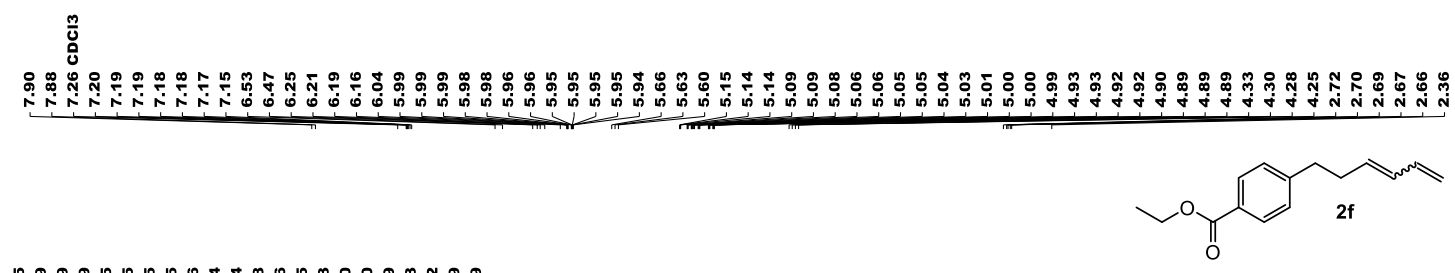

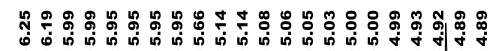
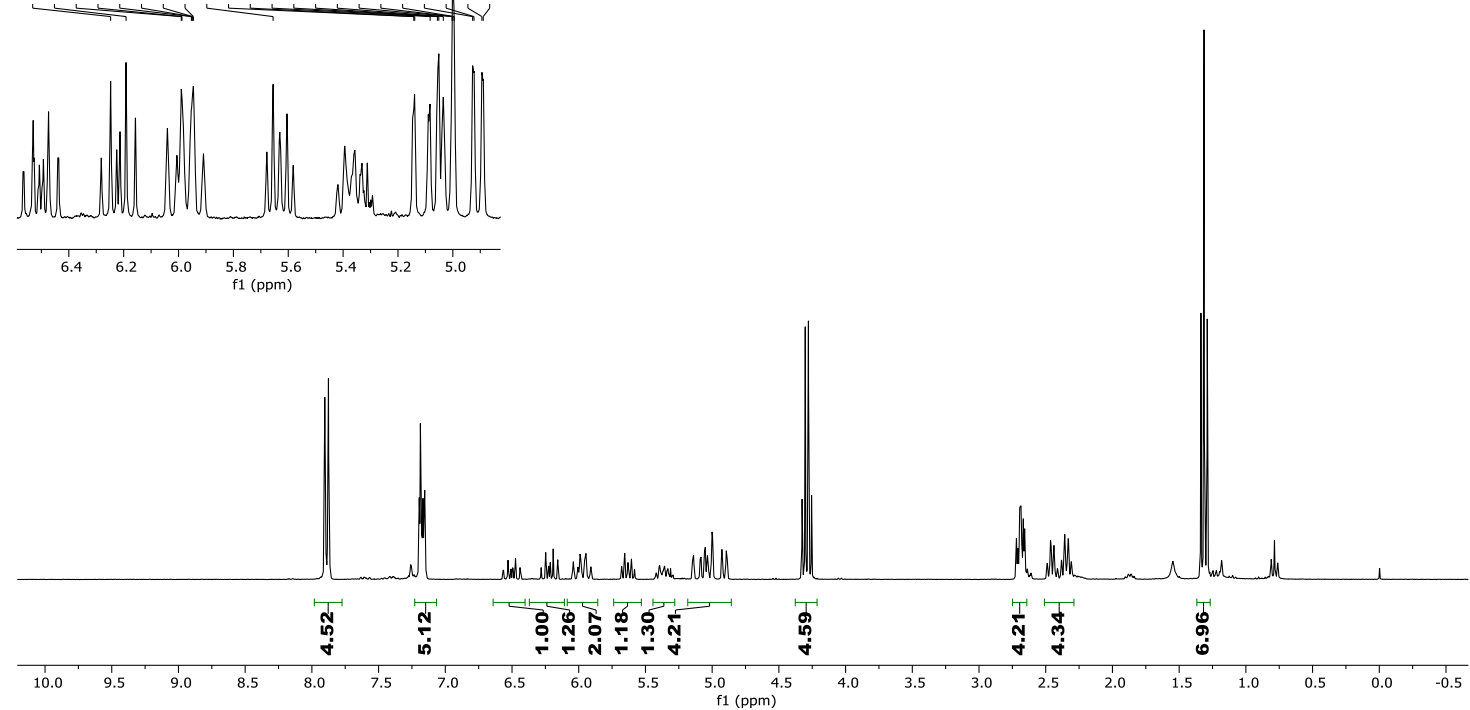

${ }^{13} \mathrm{C}$ NMR $\left(75 \mathrm{MHz}, \mathrm{CDCl}_{3}\right)$
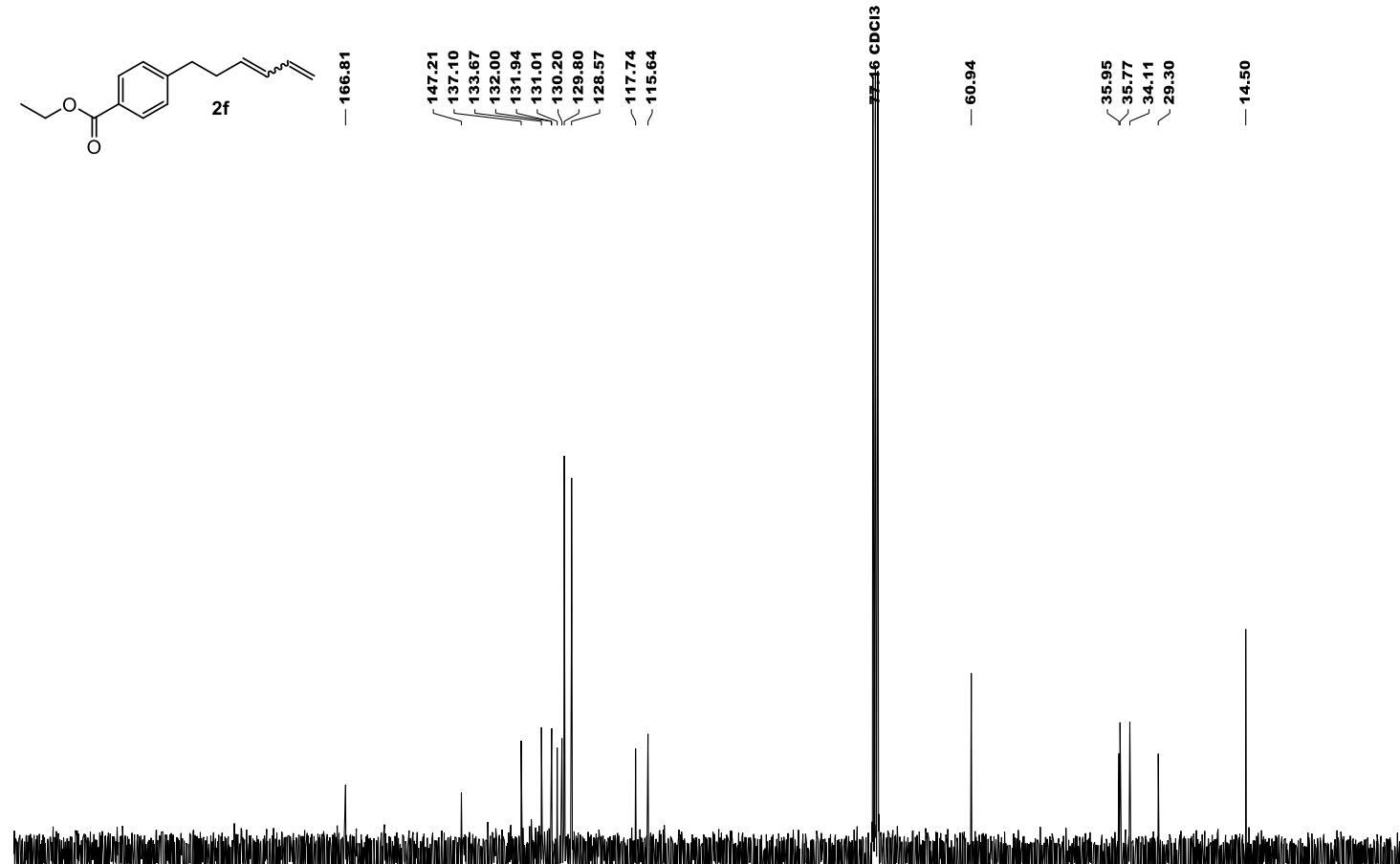

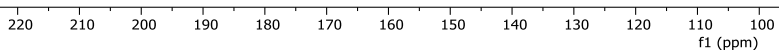


${ }^{1} \mathrm{H} \mathrm{NMR}\left(400 \mathrm{MHz}, \mathrm{CDCl}_{3}\right)$

営

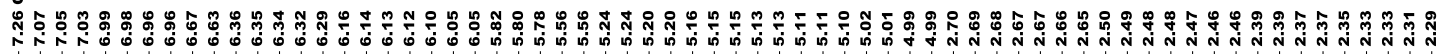
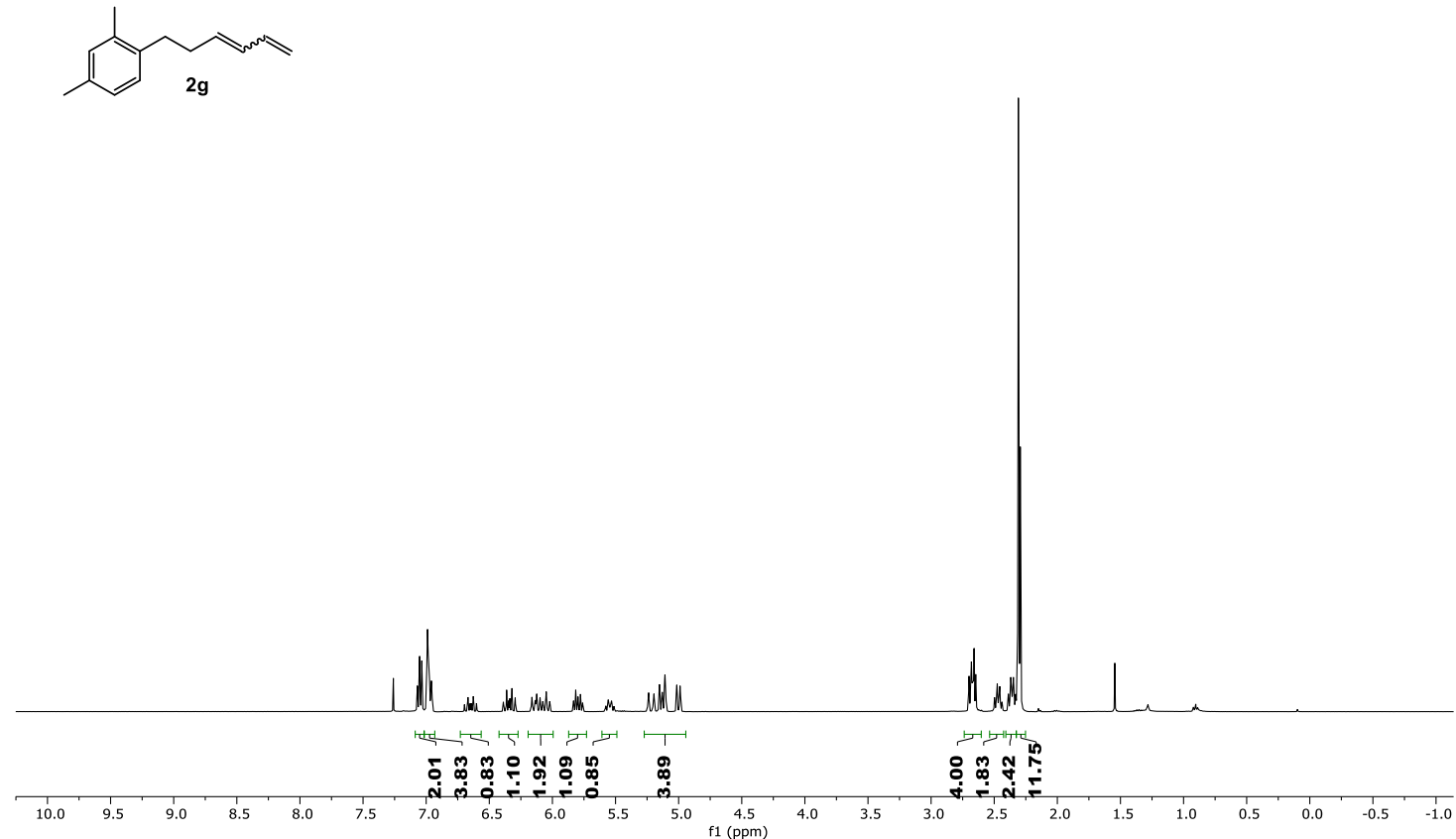

${ }^{13} \mathrm{C}$ NMR (101 MHz, $\mathrm{CDCl}_{3}$ )

7.

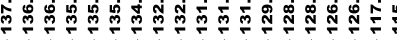

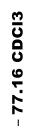

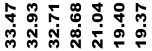

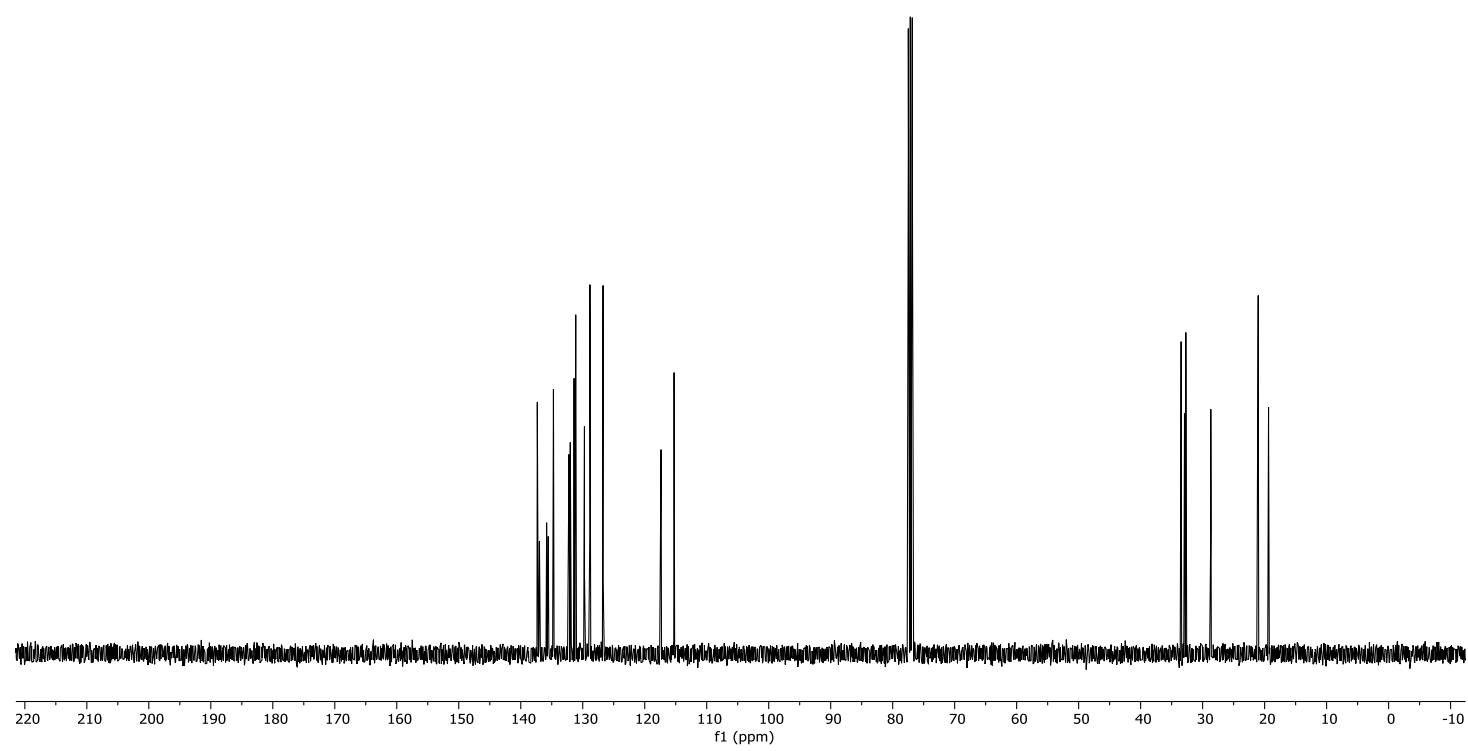


${ }^{1} \mathrm{H}$ NMR (400 MHz, $\left.\mathrm{CDCl}_{3}\right)$

$\frac{2}{\grave{a}}$

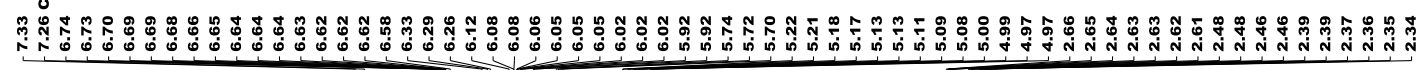

CNI

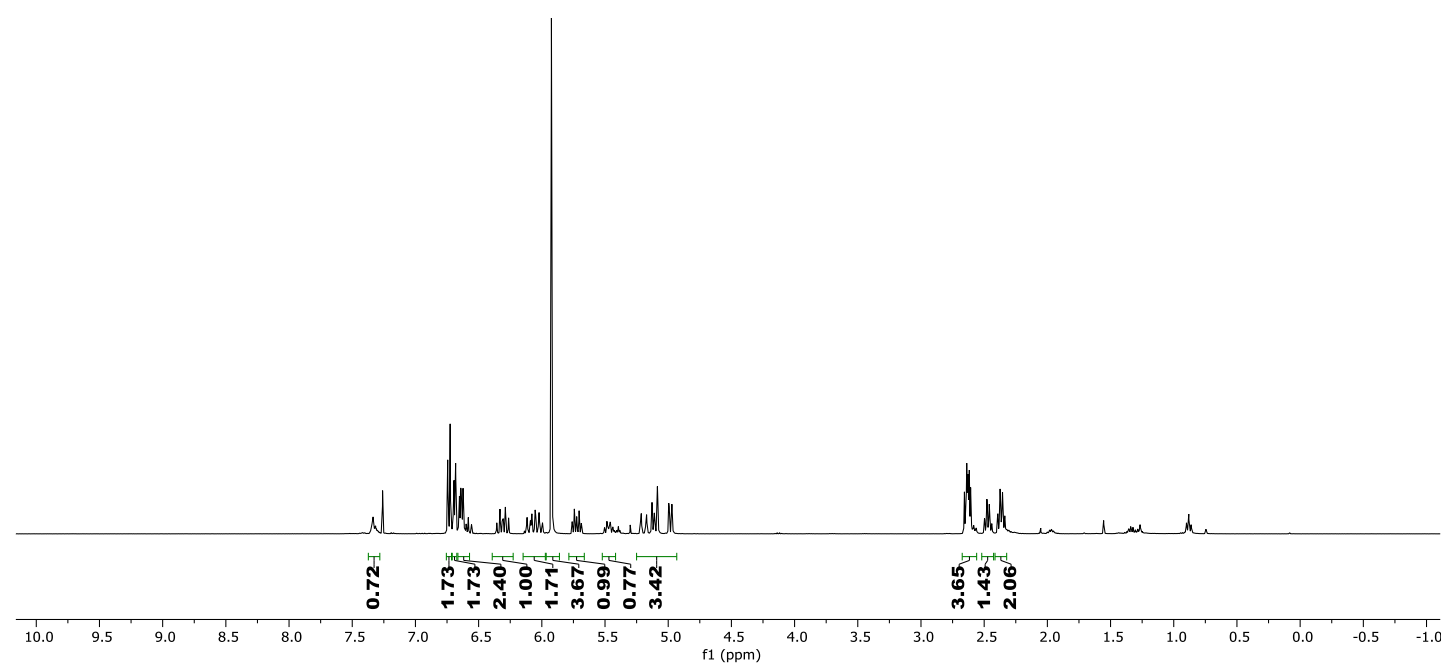

${ }^{13} \mathrm{C} \mathrm{NMR}\left(101 \mathrm{MHz}, \mathrm{CDCl}_{3}\right)$

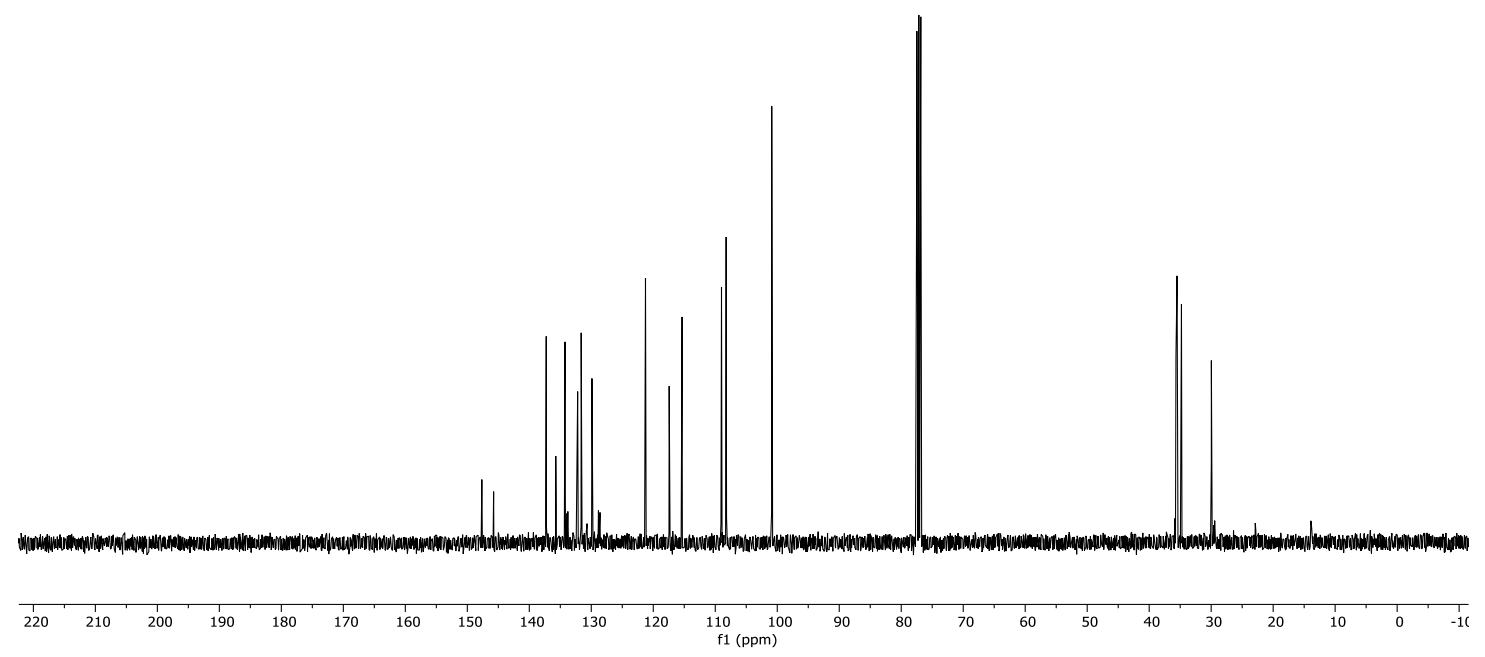


${ }^{1} \mathrm{H}$ NMR (400 MHz, $\mathrm{CDCl}_{3}$ )
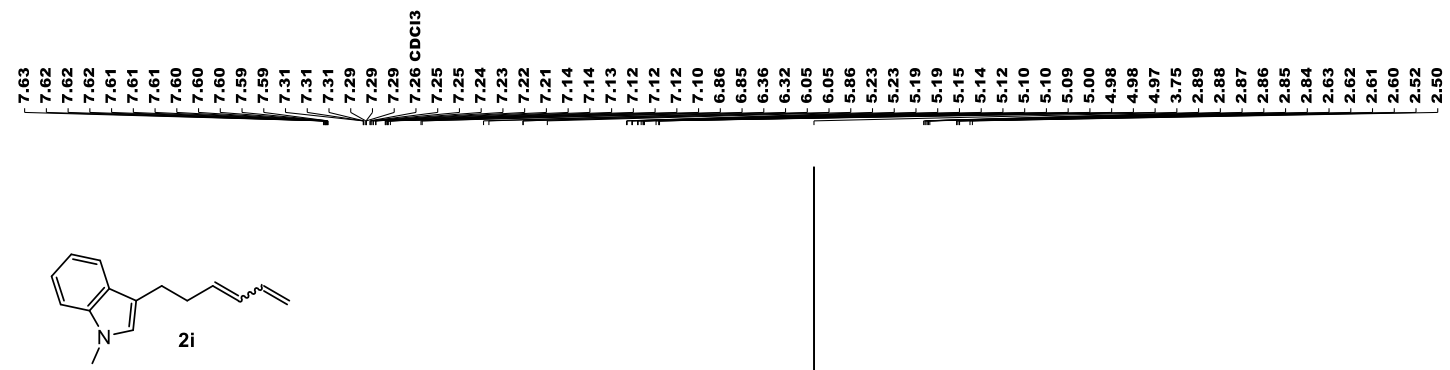

${ }^{13} \mathrm{C}$ NMR (101 MHz, $\mathrm{CDCl}_{3}$ )

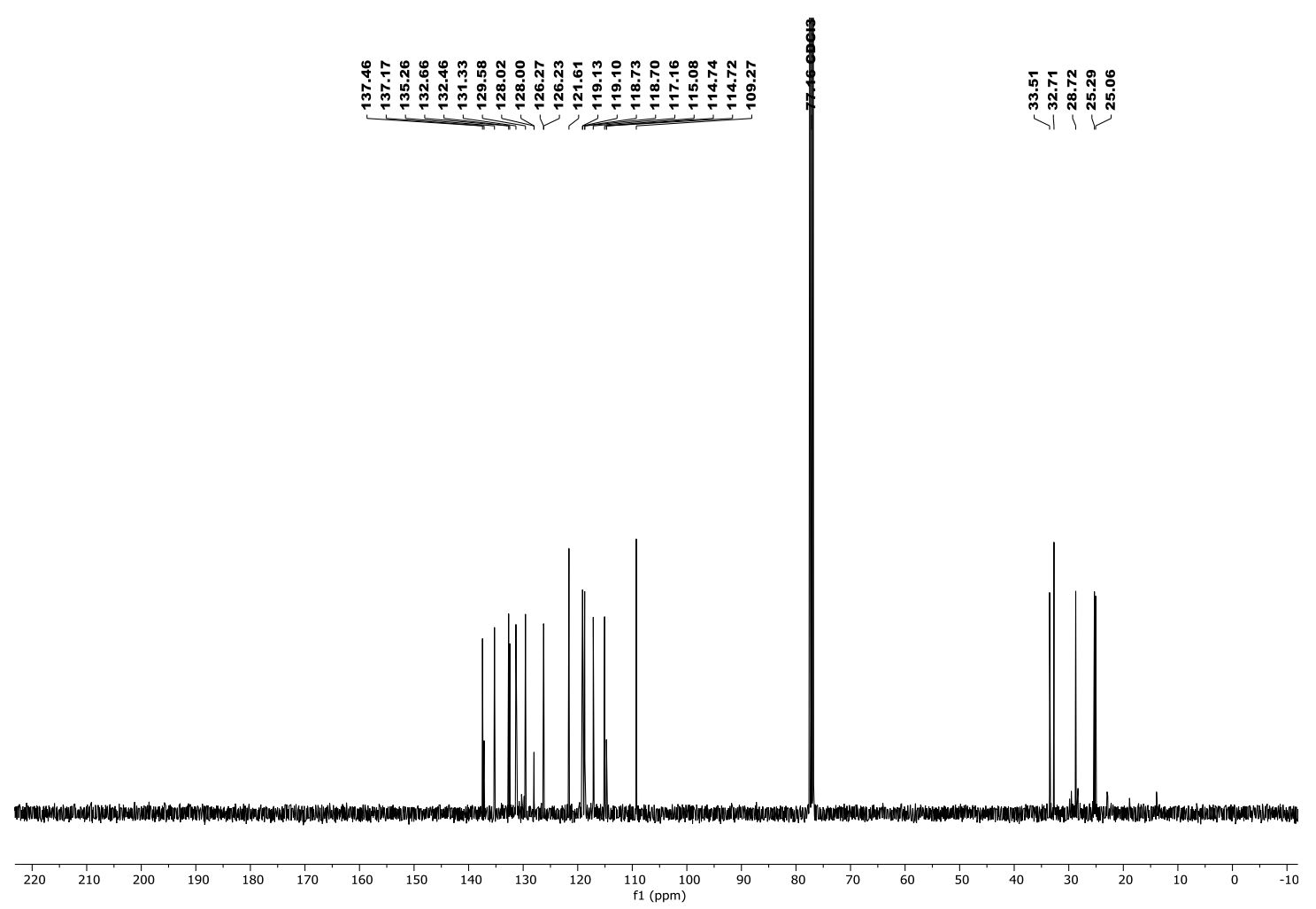


${ }^{1} \mathrm{H}$ NMR (400 MHz, $\left.\mathrm{CDCl}_{3}\right)$

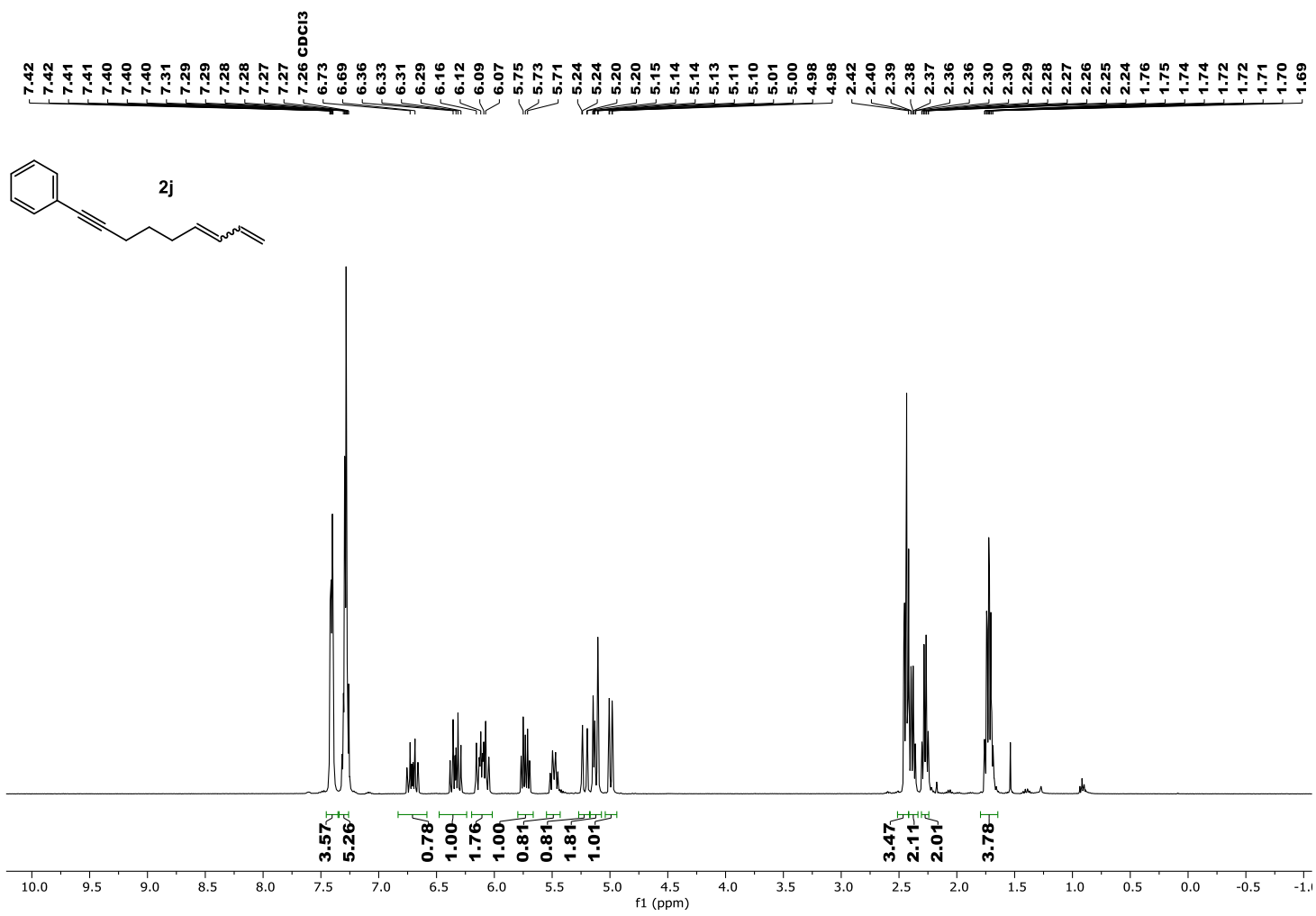

${ }^{13} \mathrm{C}$ NMR (101 MHz, $\mathrm{CDCl}_{3}$ )

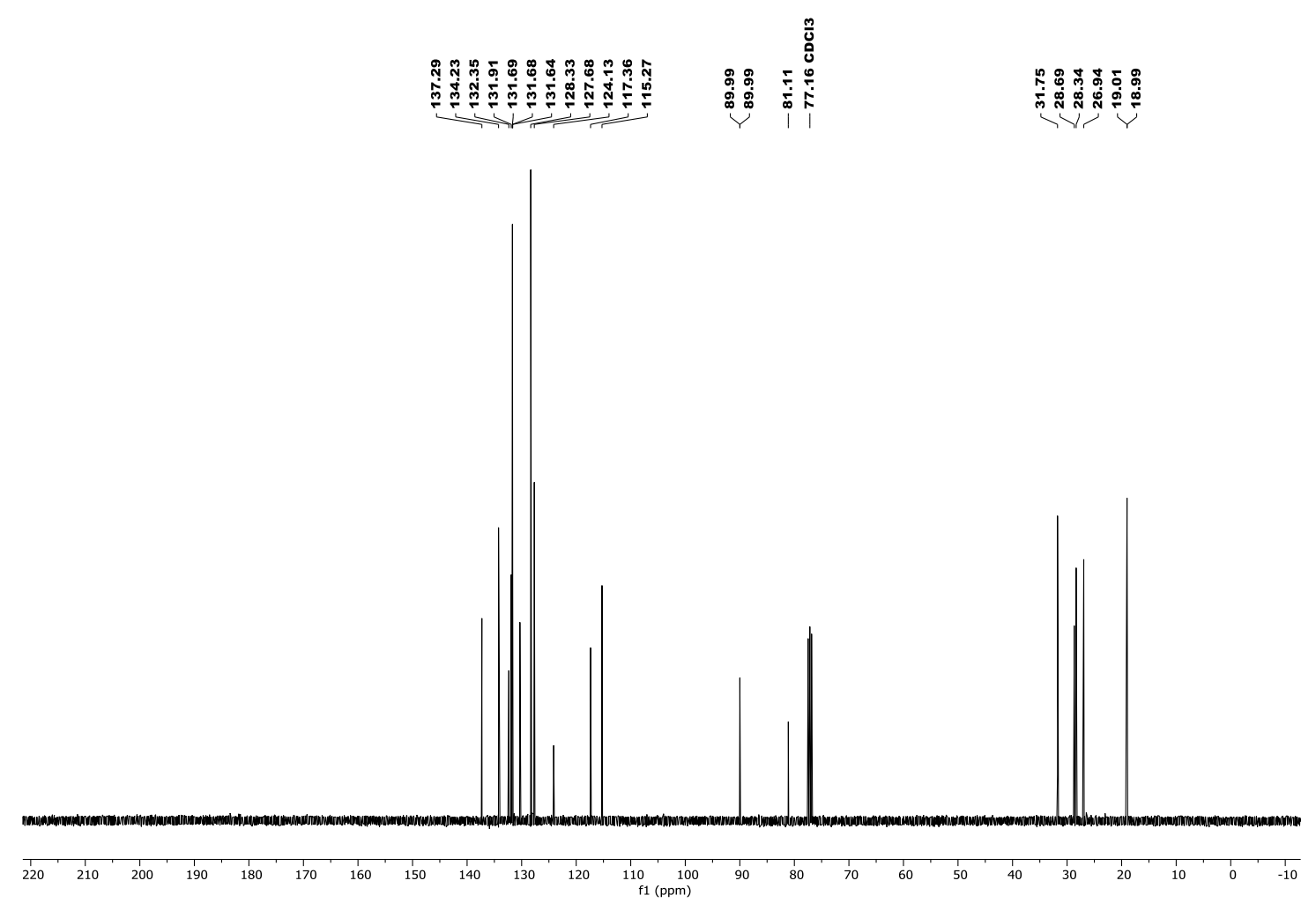


${ }^{1} \mathrm{H} \mathrm{NMR}\left(400 \mathrm{MHz}, \mathrm{CDCl}_{3}\right)$

品

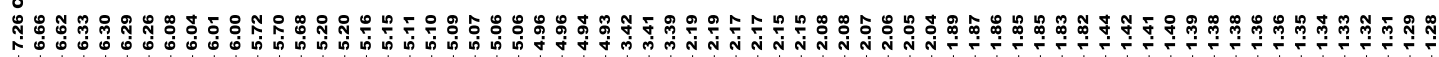

$\mathrm{Br}_{8}$

S2k

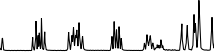

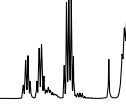

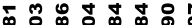

0 :

$\begin{array}{ccc}5.0 & 4.5 & 4.0 \\ & f 1(\mathrm{ppm}) & \end{array}$

${ }^{13} \mathrm{C}$ NMR $\left(101 \mathrm{MHz}, \mathrm{CDCl}_{3}\right)$
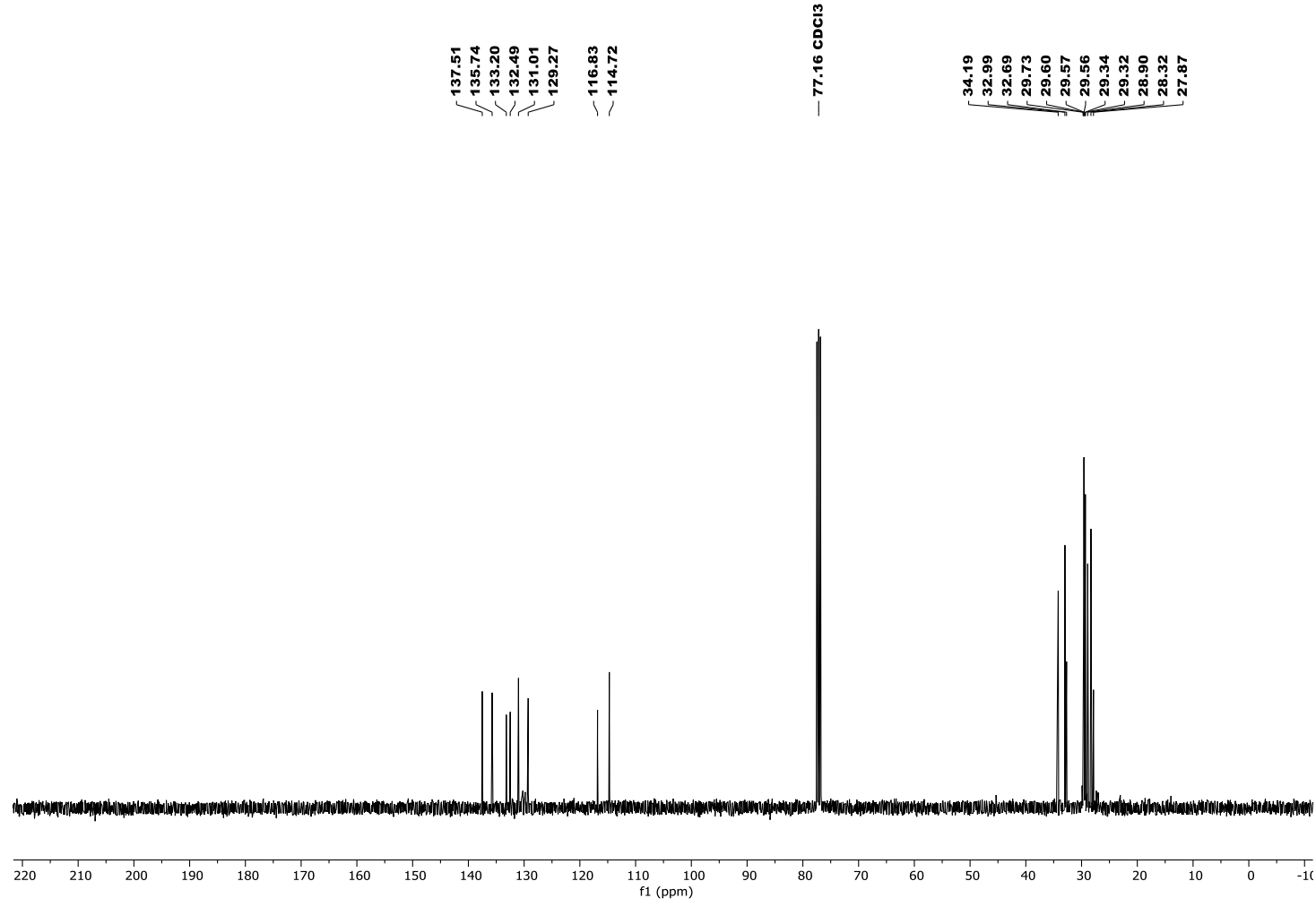
${ }^{1} \mathrm{H} \mathrm{NMR}\left(400 \mathrm{MHz}, \mathrm{CDCl}_{3}\right)$

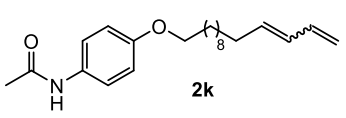

${ }^{13} \mathrm{C}$ NMR $\left(101 \mathrm{MHz}, \mathrm{CDCl}_{3}\right)$
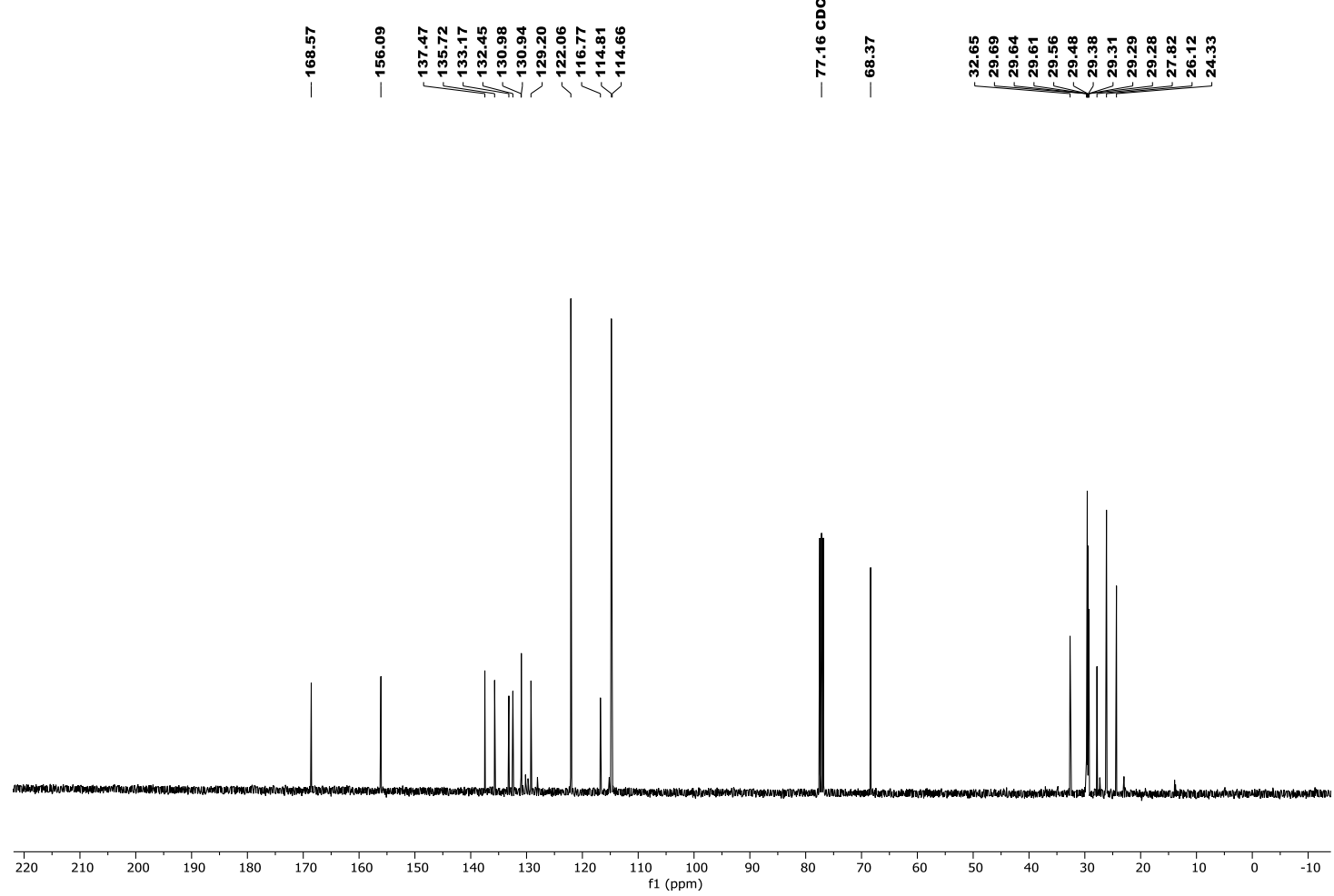
${ }^{1} \mathrm{H} \mathrm{NMR}\left(400 \mathrm{MHz}, \mathrm{CDCl}_{3}\right)$

范

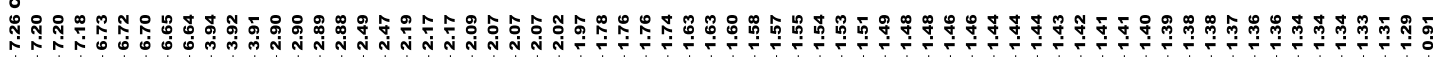

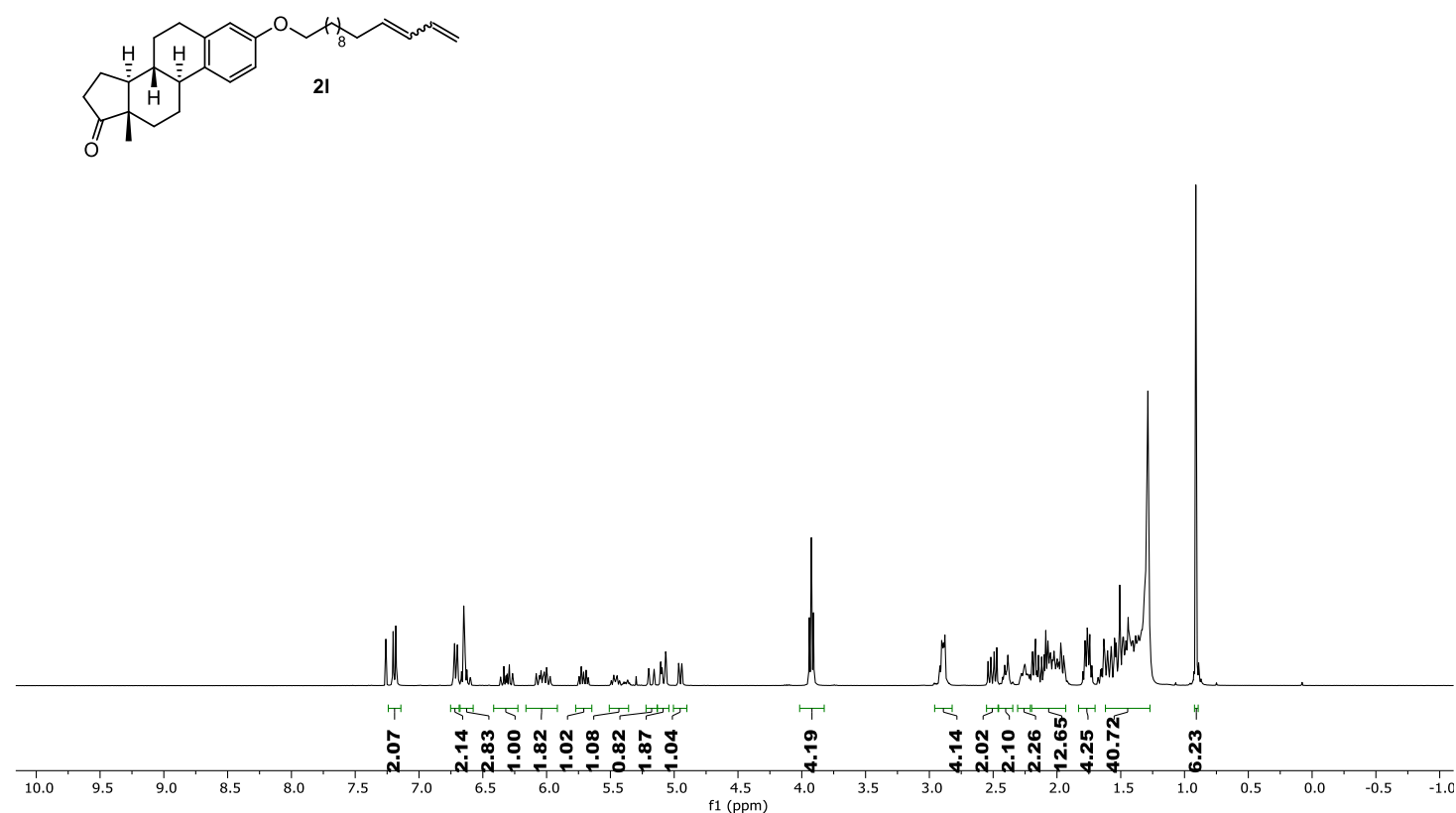

${ }^{13} \mathrm{C}$ NMR (101 MHz, $\left.\mathrm{CDCl}_{3}\right)$

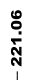

作

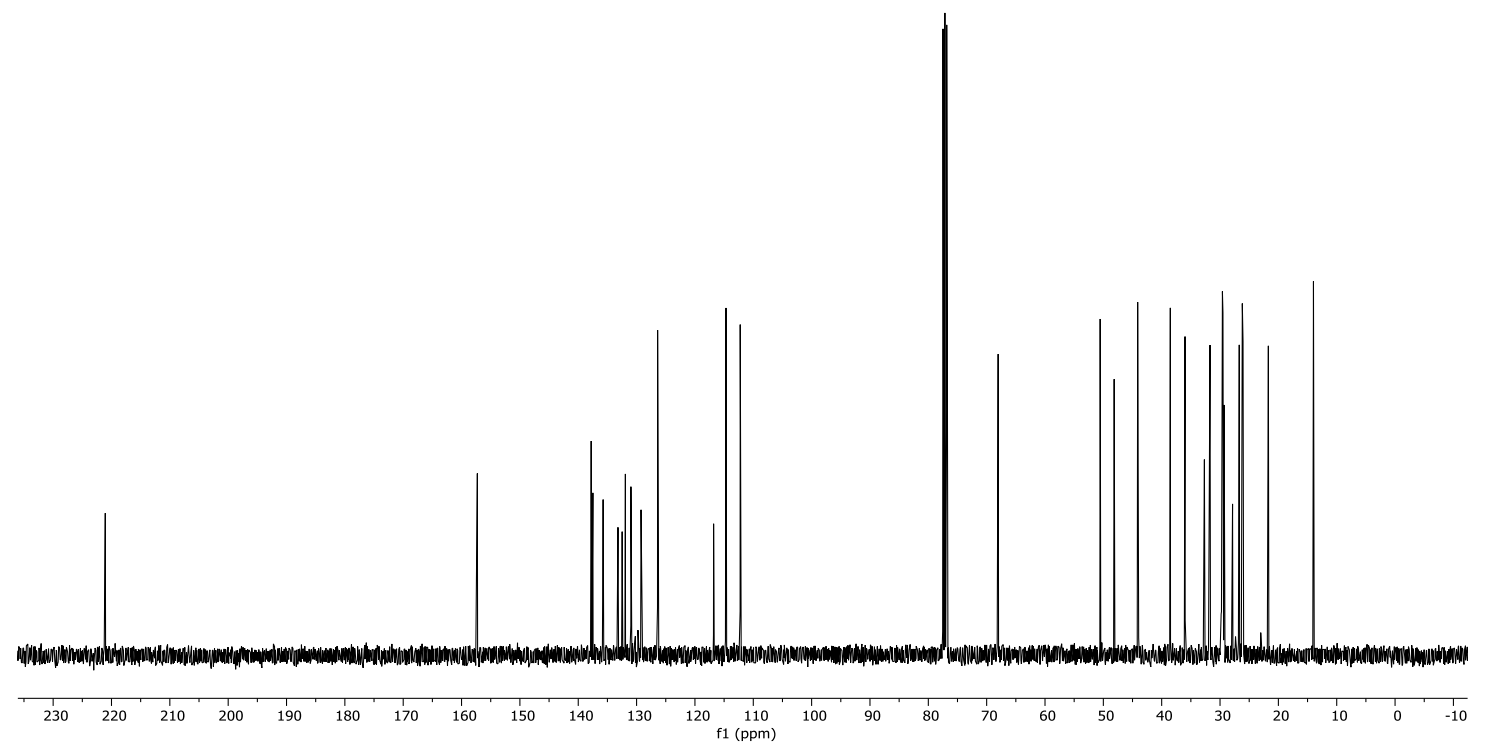


${ }^{1} \mathrm{H}$ NMR (500 MHz, $\left.\mathrm{D}_{2} \mathrm{O}\right)$

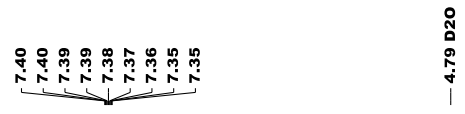
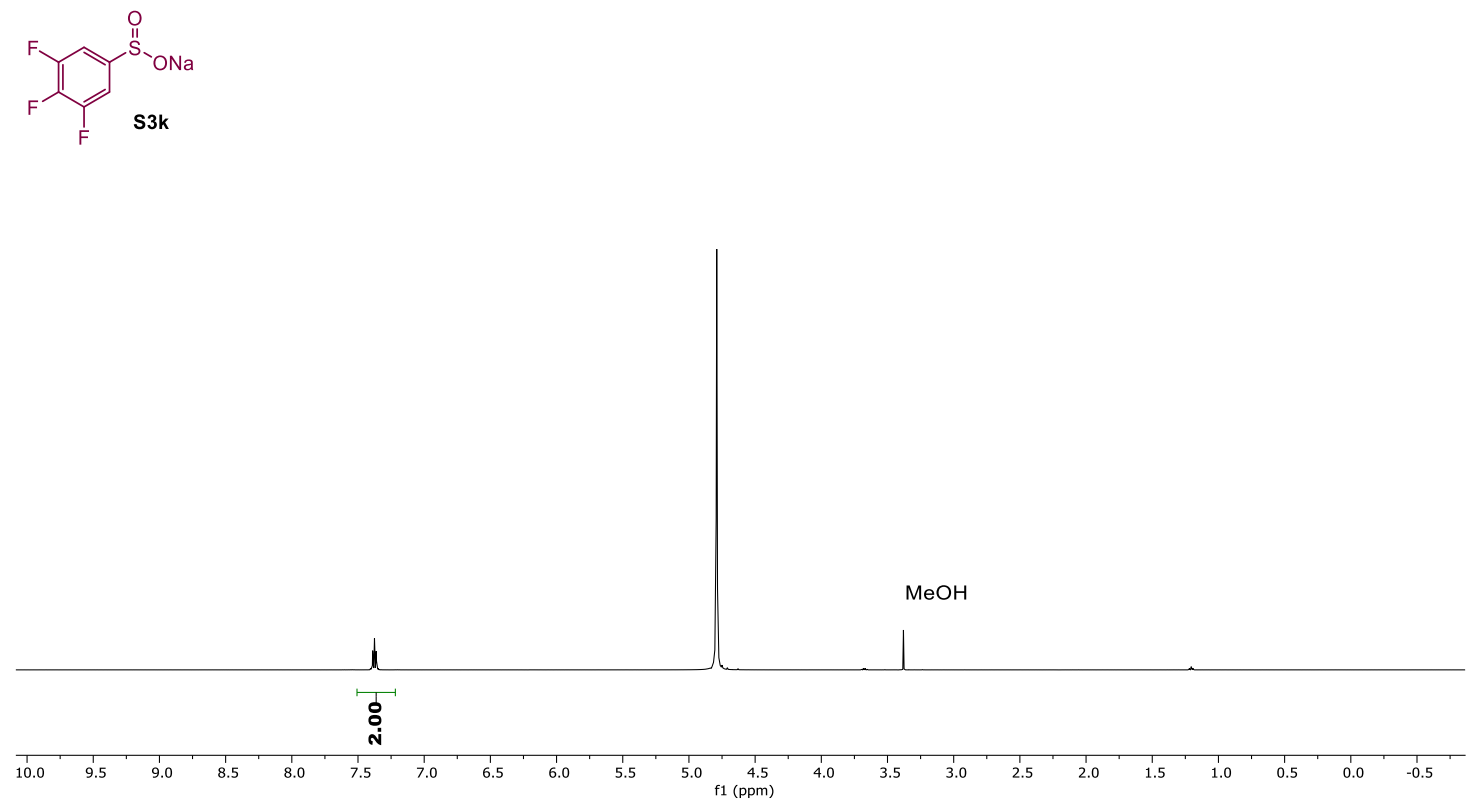

${ }^{13} \mathrm{C}$ NMR $\left(126 \mathrm{MHz}, \mathrm{D}_{2} \mathrm{O}\right)$

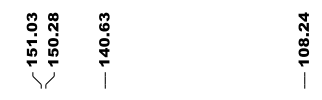

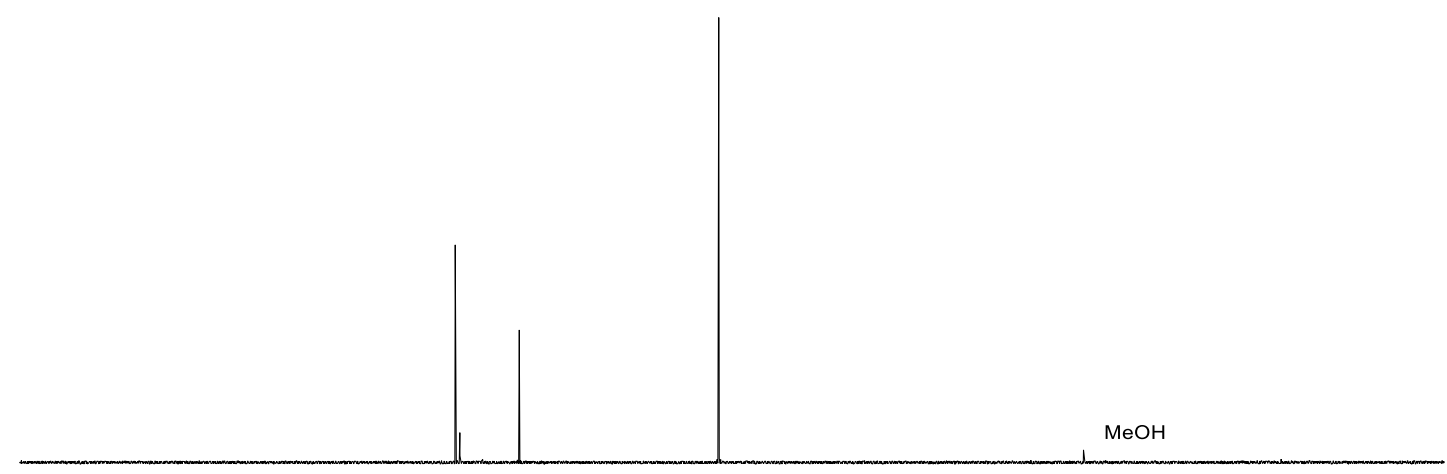

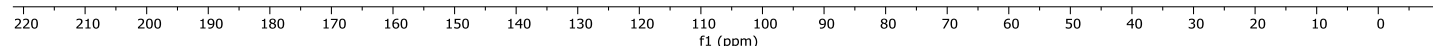


${ }^{19} \mathrm{~F}\left\{{ }^{1} \mathrm{H}\right\}$ NMR (400 MHz, $\left.\mathrm{D}_{2} \mathrm{O}\right)$

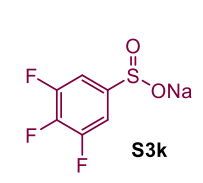

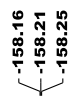
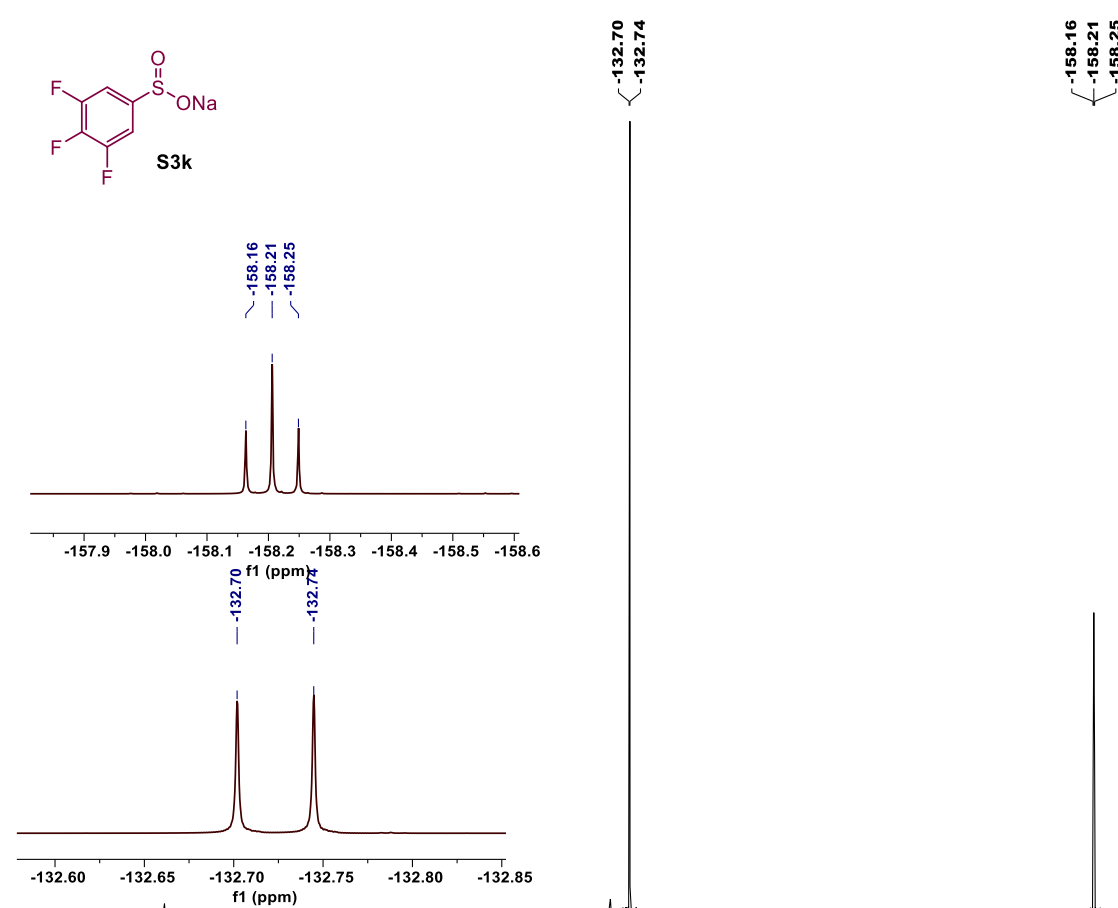

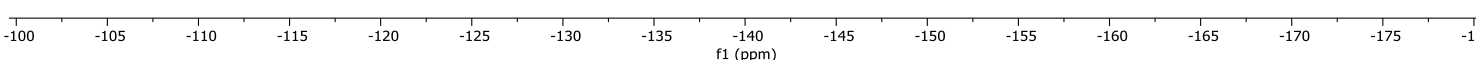


${ }^{1} \mathrm{H}$ NMR (400 MHz, $\mathrm{D}_{2} \mathrm{O}$ )

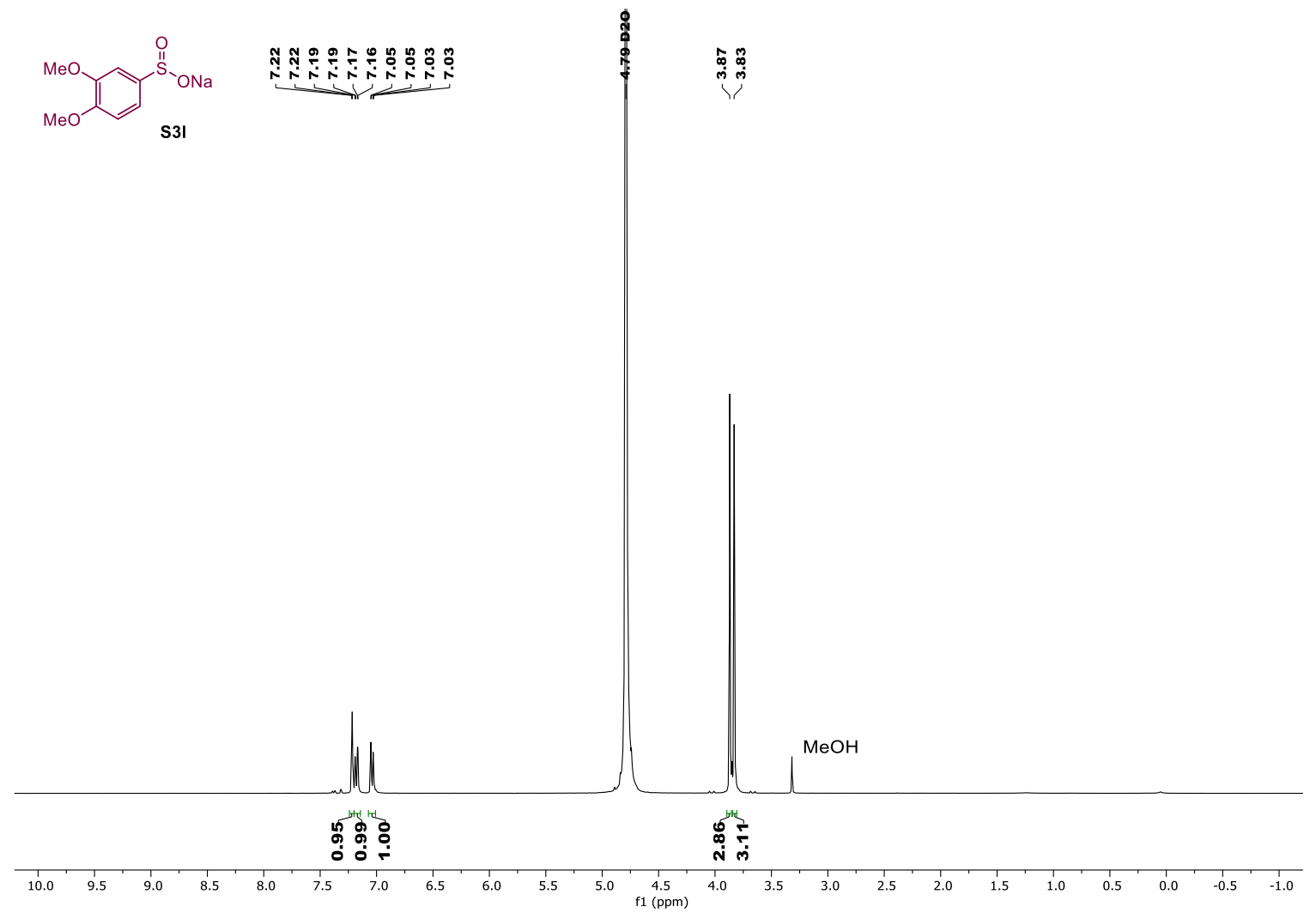

${ }^{13} \mathrm{C}$ NMR (100 MHz, $\left.\mathrm{D}_{2} \mathrm{O}\right)$
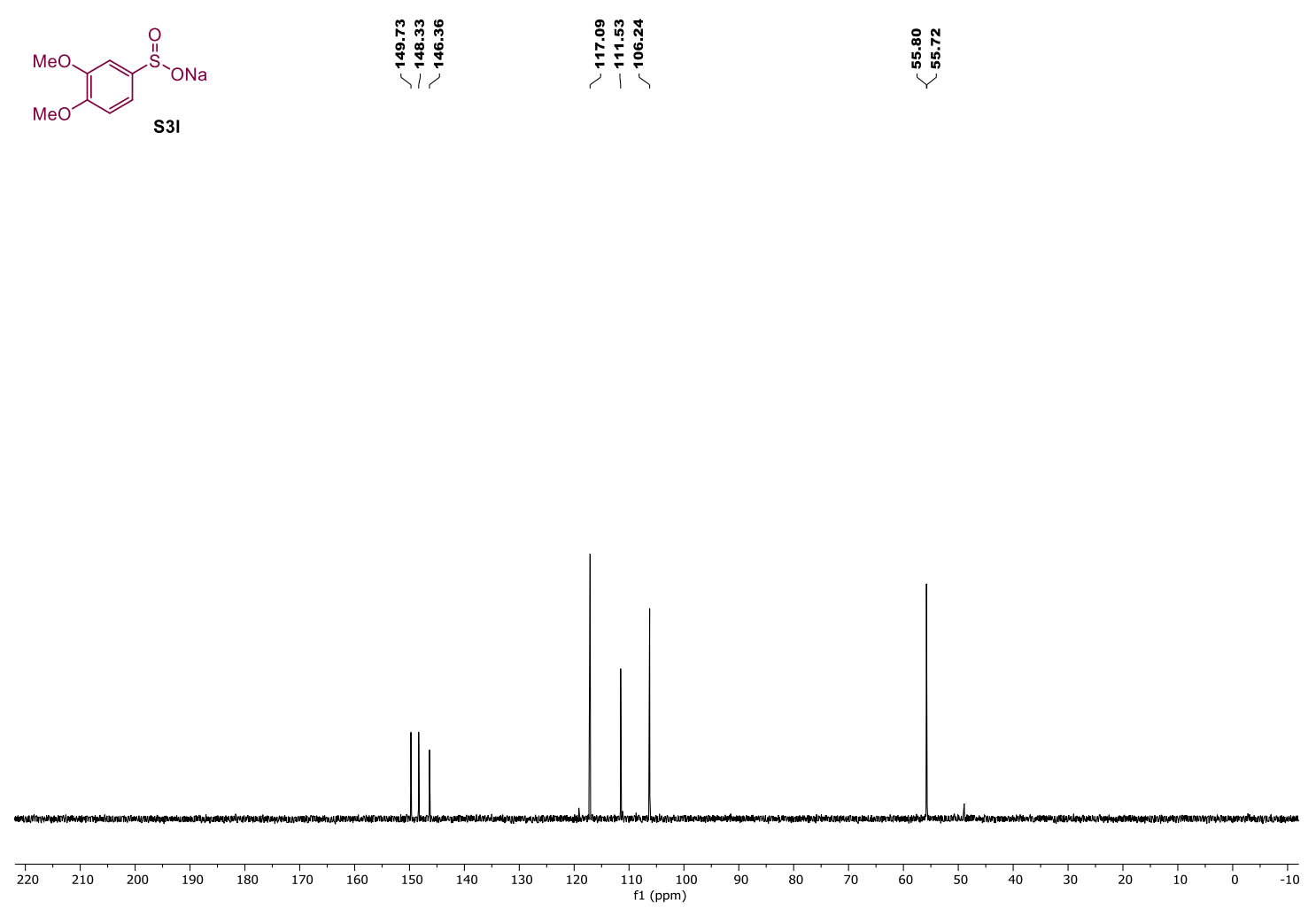
${ }^{1} \mathrm{H}$ NMR (400 MHz, $\mathrm{D}_{2} \mathrm{O}$ )

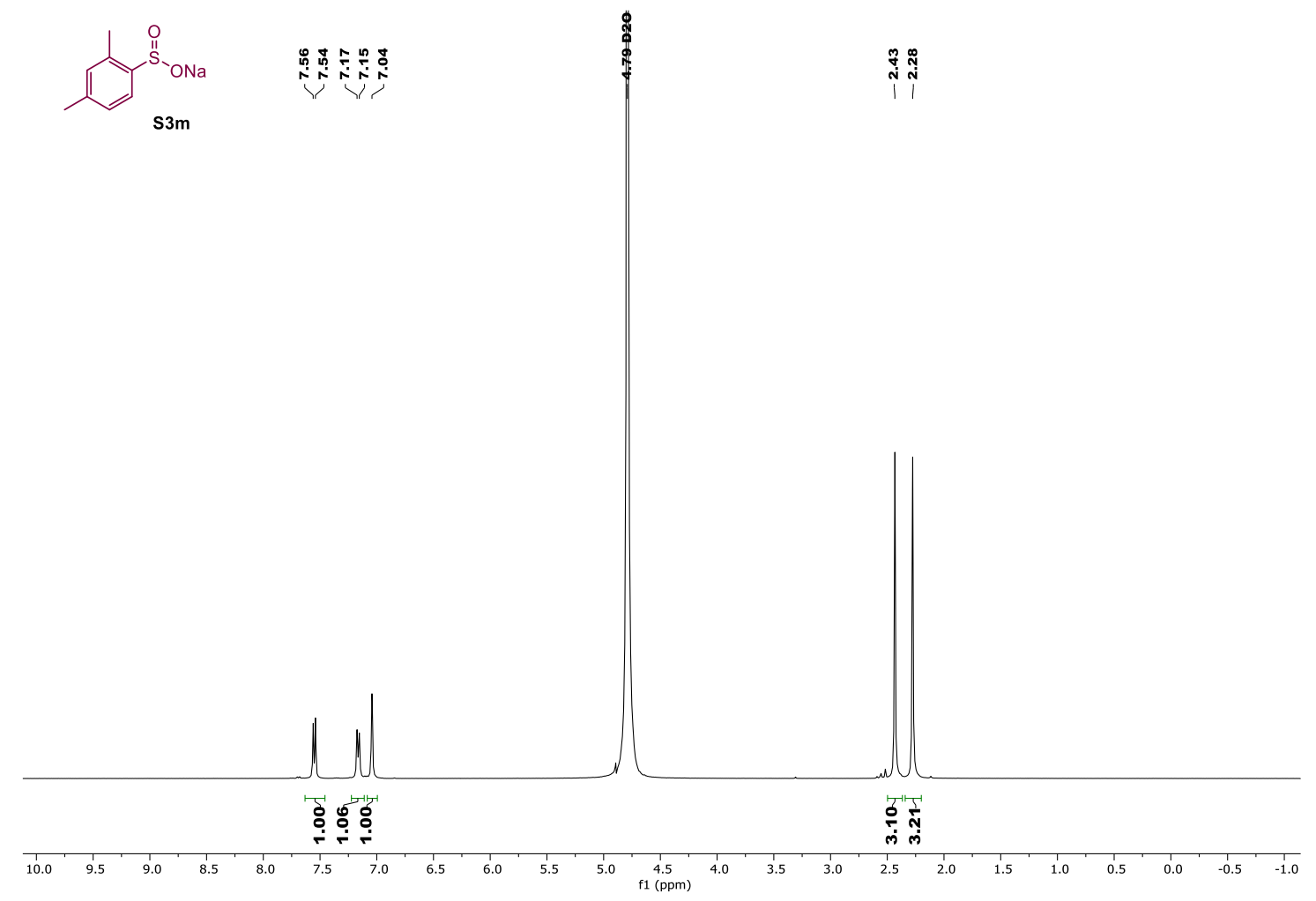

${ }^{13} \mathrm{C}$ NMR (100 MHz, $\mathrm{D}_{2} \mathrm{O}$ )

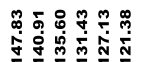

广这

ฝึ่ง

$\underbrace{11}$ ina

s3m 
${ }^{1} \mathrm{H}$ NMR (500 MHz, DMSO- $\left.d_{6}\right)$
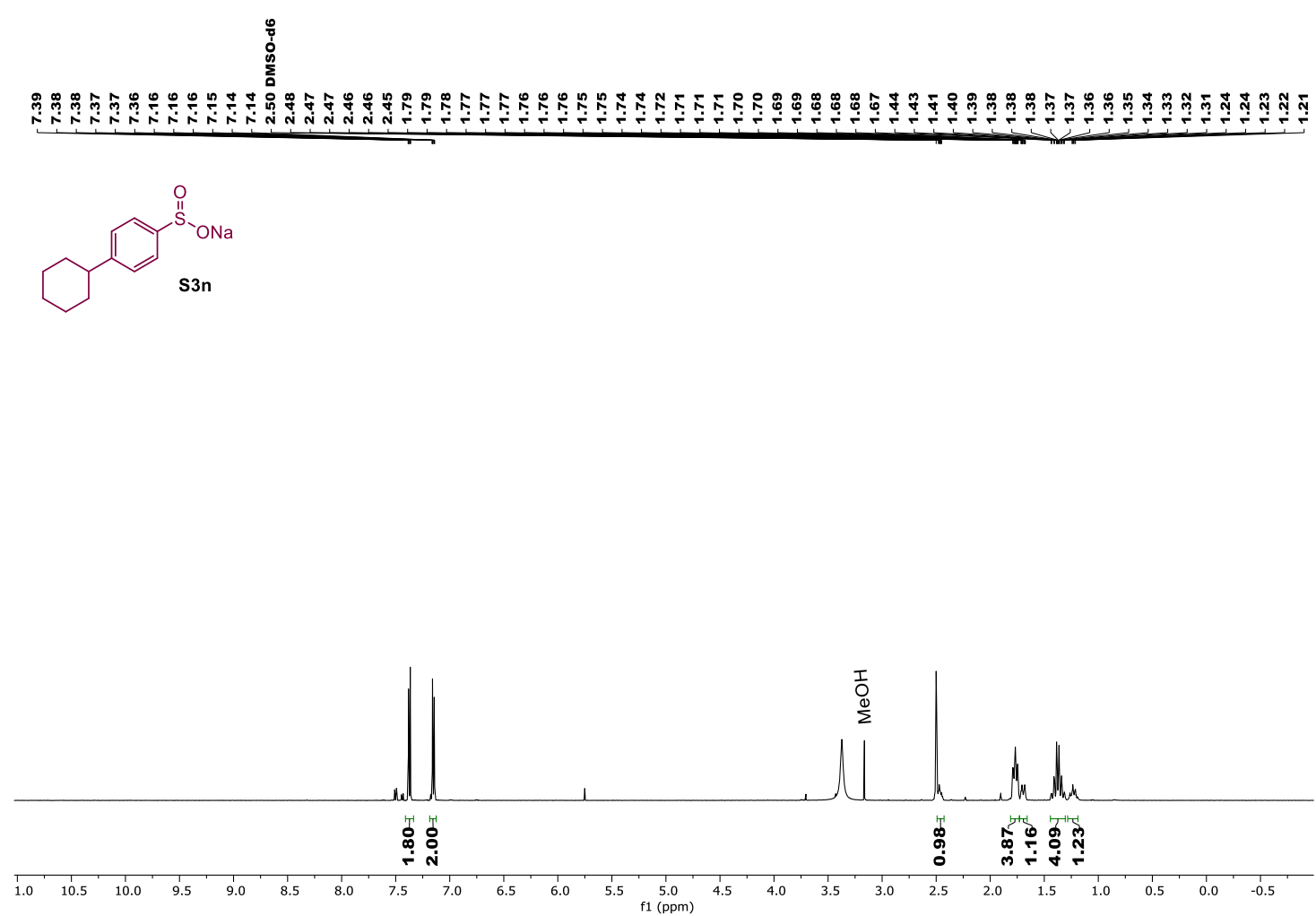

${ }^{13} \mathrm{C}$ NMR $\left(126 \mathrm{MHz}\right.$, DMSO- $\left.d_{6}\right)$

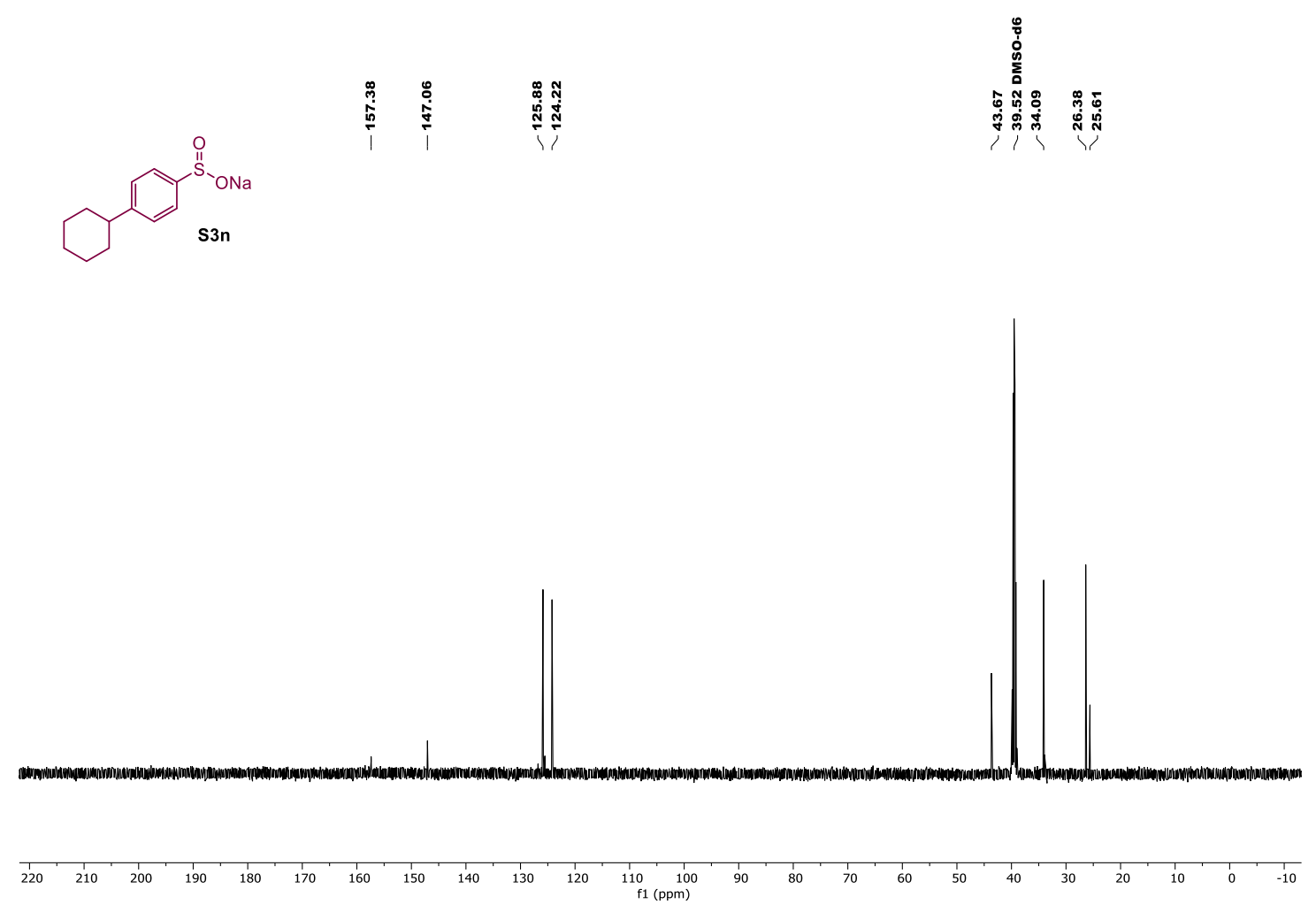


${ }^{1} \mathrm{H}$ NMR (500 MHz, $\mathrm{D}_{2} \mathrm{O}$ )

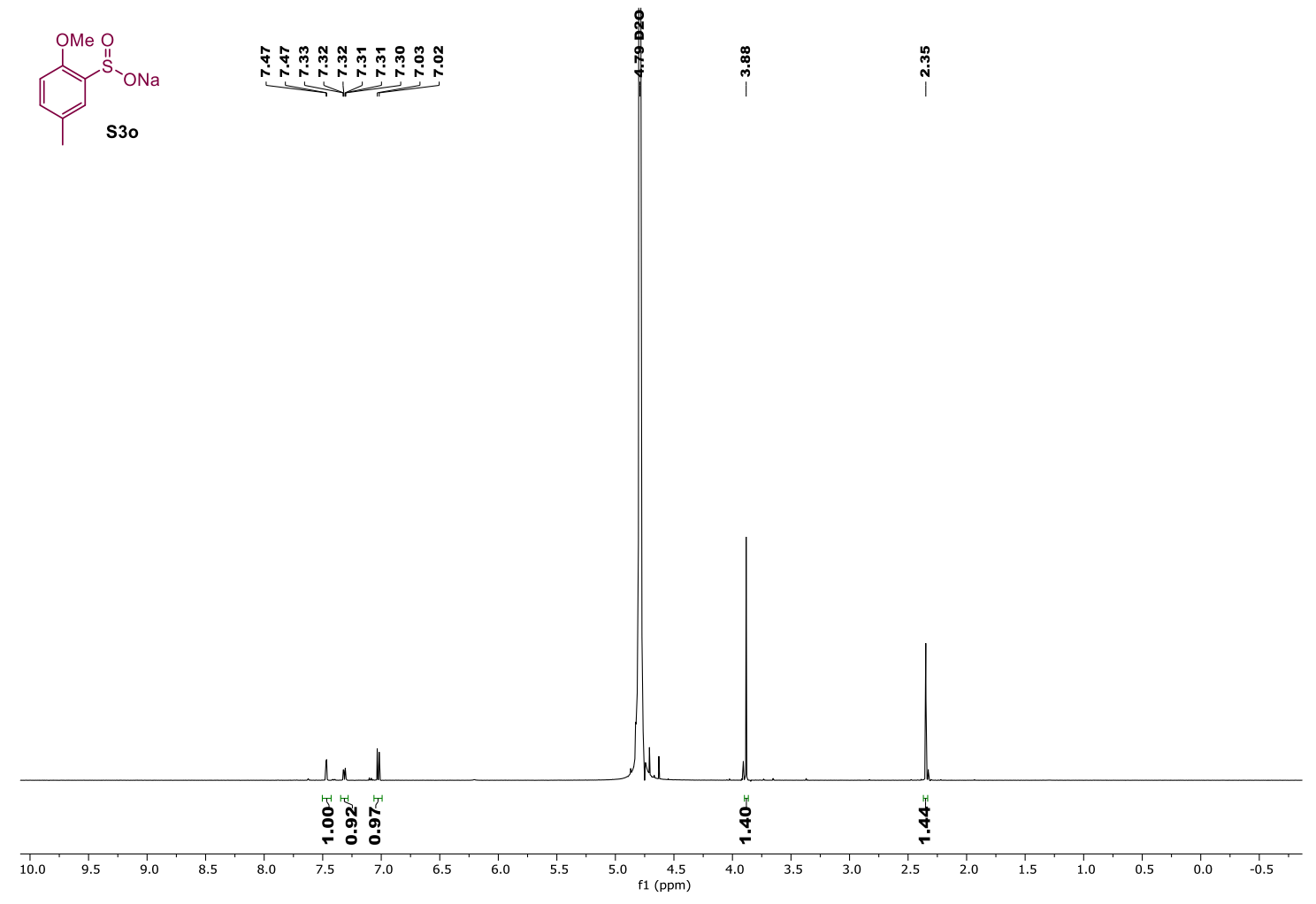

${ }^{13} \mathrm{C}$ NMR (126 MHz, $\mathrm{D}_{2} \mathrm{O}$ )
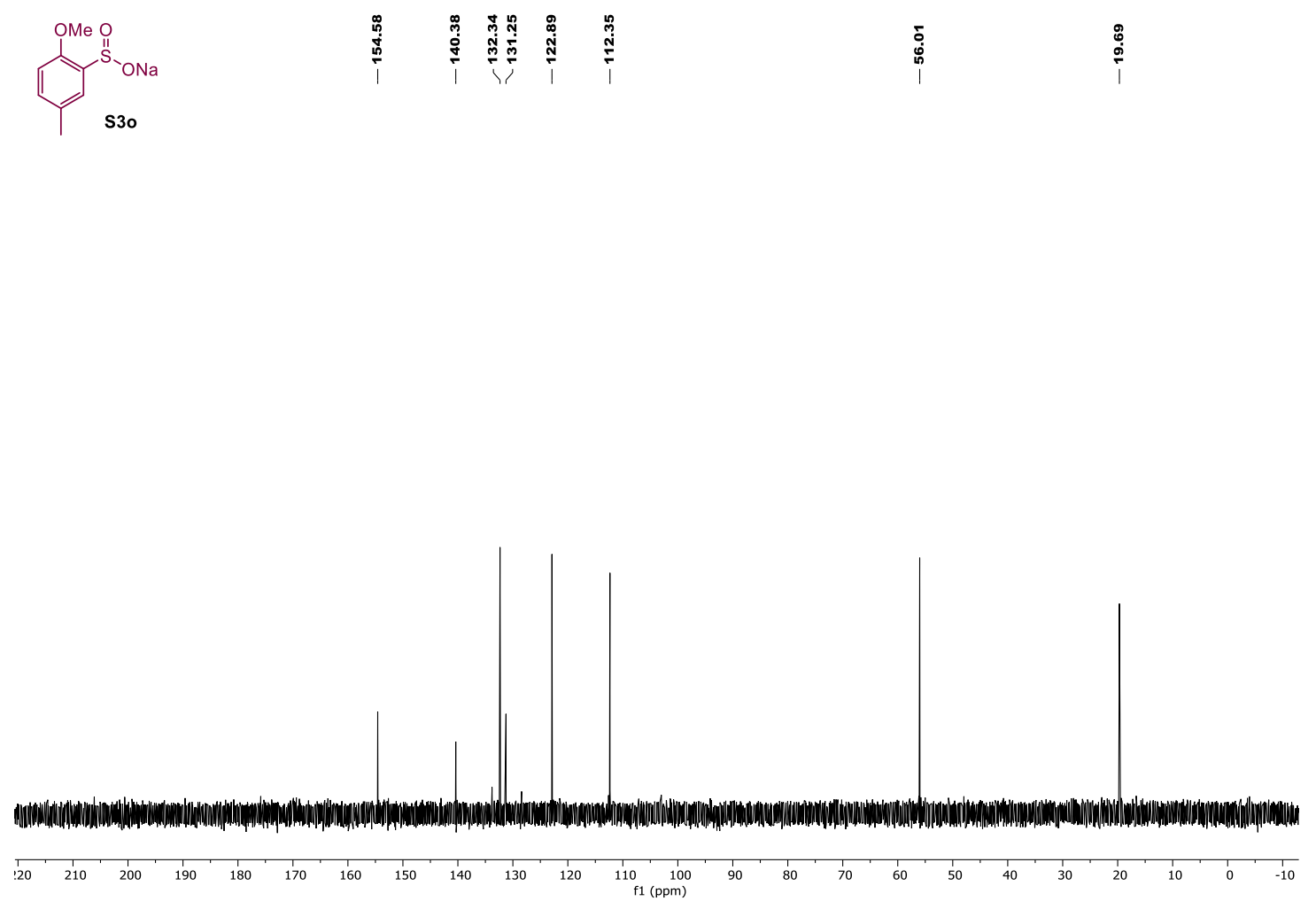
${ }^{1} \mathrm{H}$ NMR (400 MHz, $\mathrm{CDCl}_{3}$ )

䔽

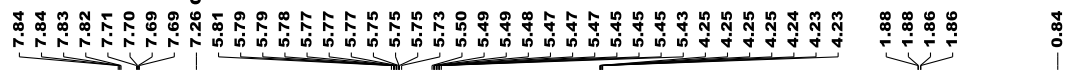
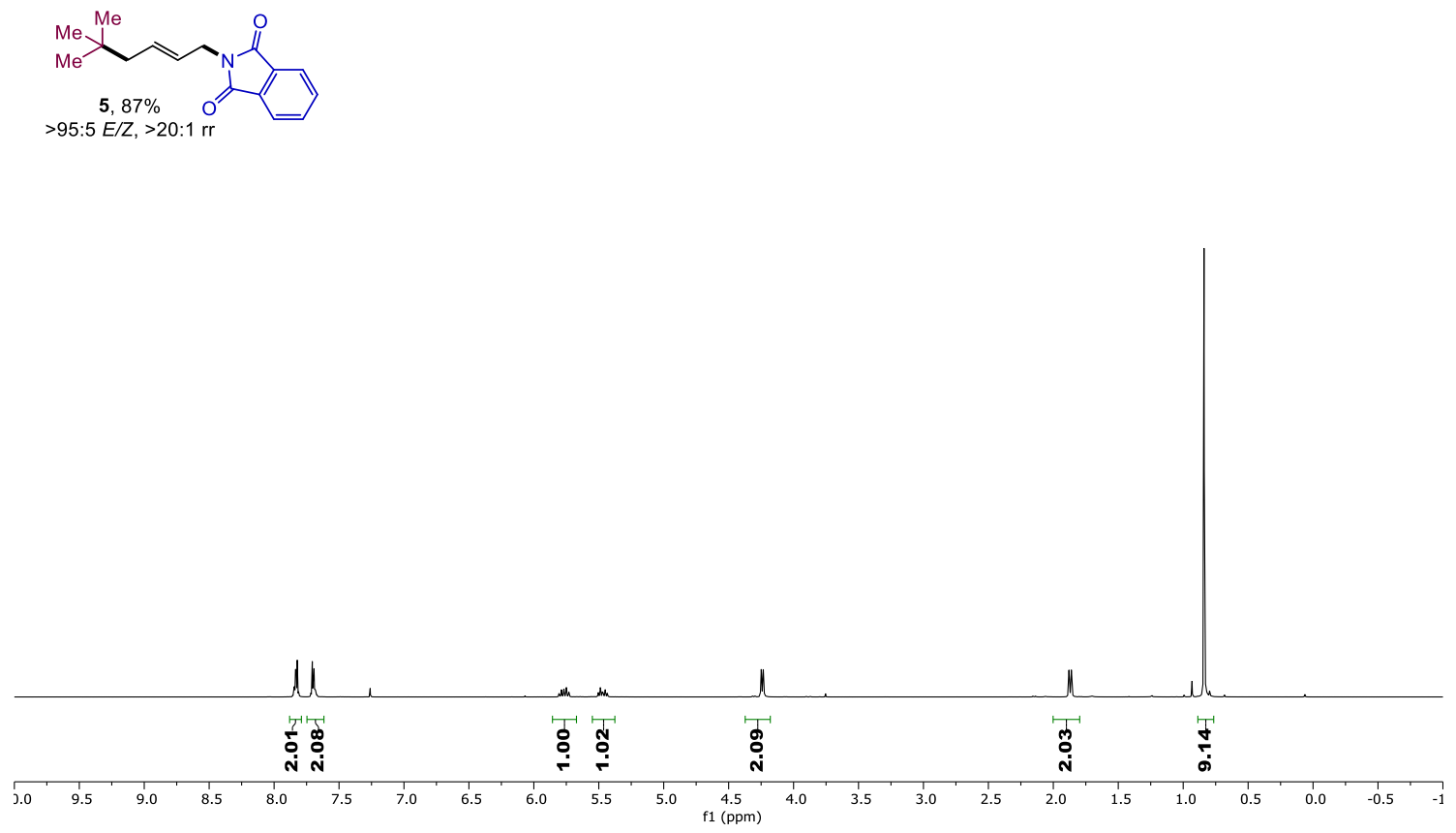

${ }^{13} \mathrm{C}$ NMR (101 MHz, $\left.\mathrm{CDCl}_{3}\right)$

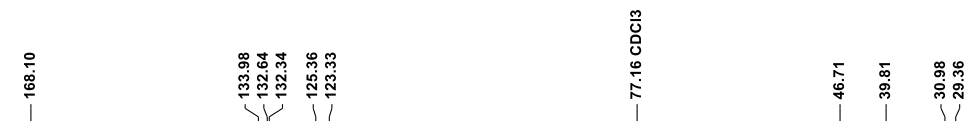

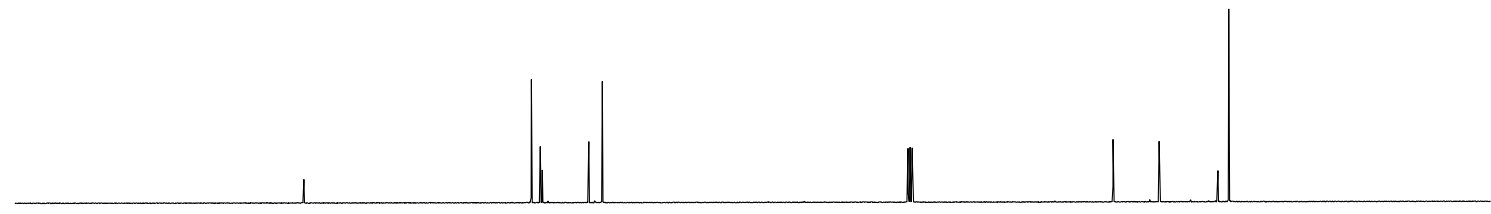

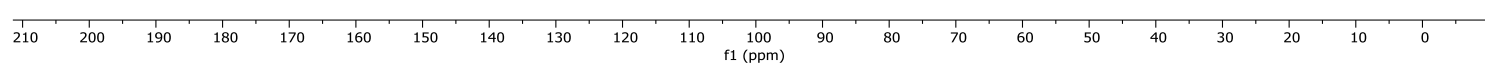


${ }^{1} \mathrm{H}$ NMR (599 MHz, $\mathrm{CDCl}_{3}$ )
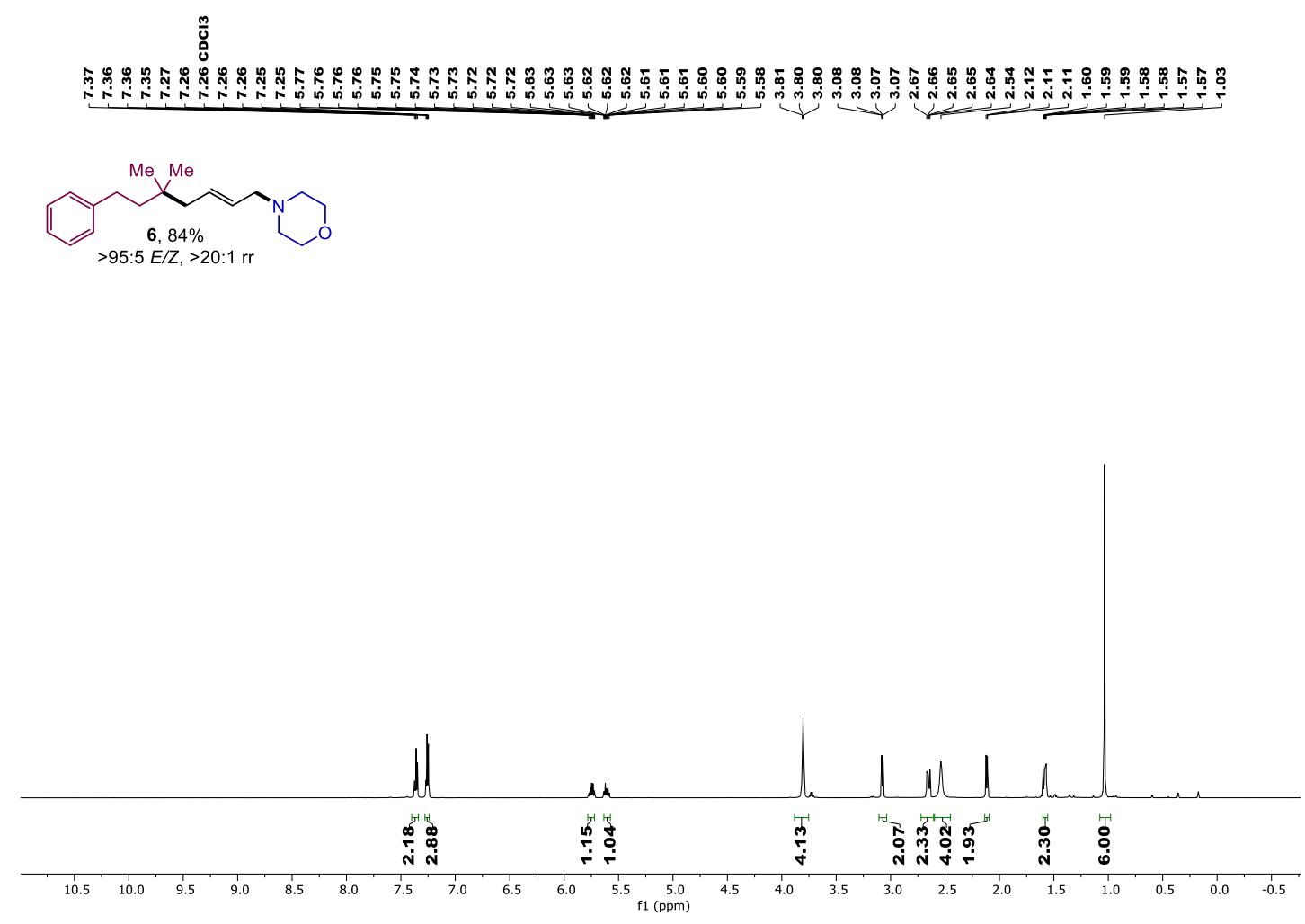

${ }^{13} \mathrm{C}$ NMR (151 MHz, $\mathrm{CDCl}_{3}$ )

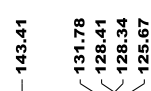

高

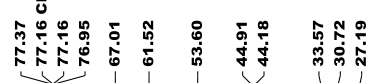

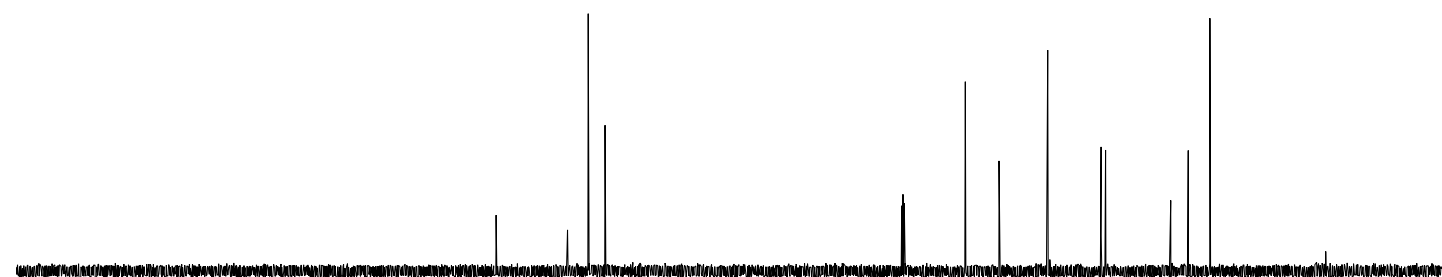

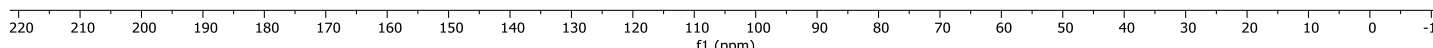


${ }^{1} \mathrm{H}$ NMR $\left(599 \mathrm{MHz}, \mathrm{CDCl}_{3}\right)$

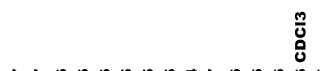

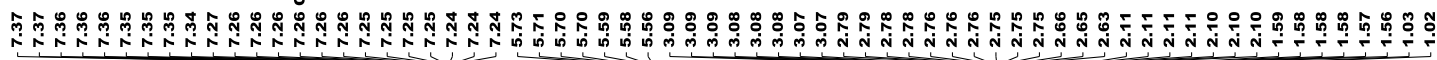

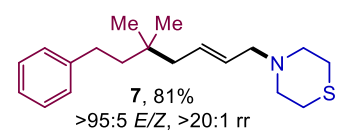

$>95: 5 \mathrm{E} / \mathrm{Z},>20: 1 \mathrm{rr}$

${ }^{13} \mathrm{C} \mathrm{NMR}\left(151 \mathrm{MHz}, \mathrm{CDCl}_{3}\right)$
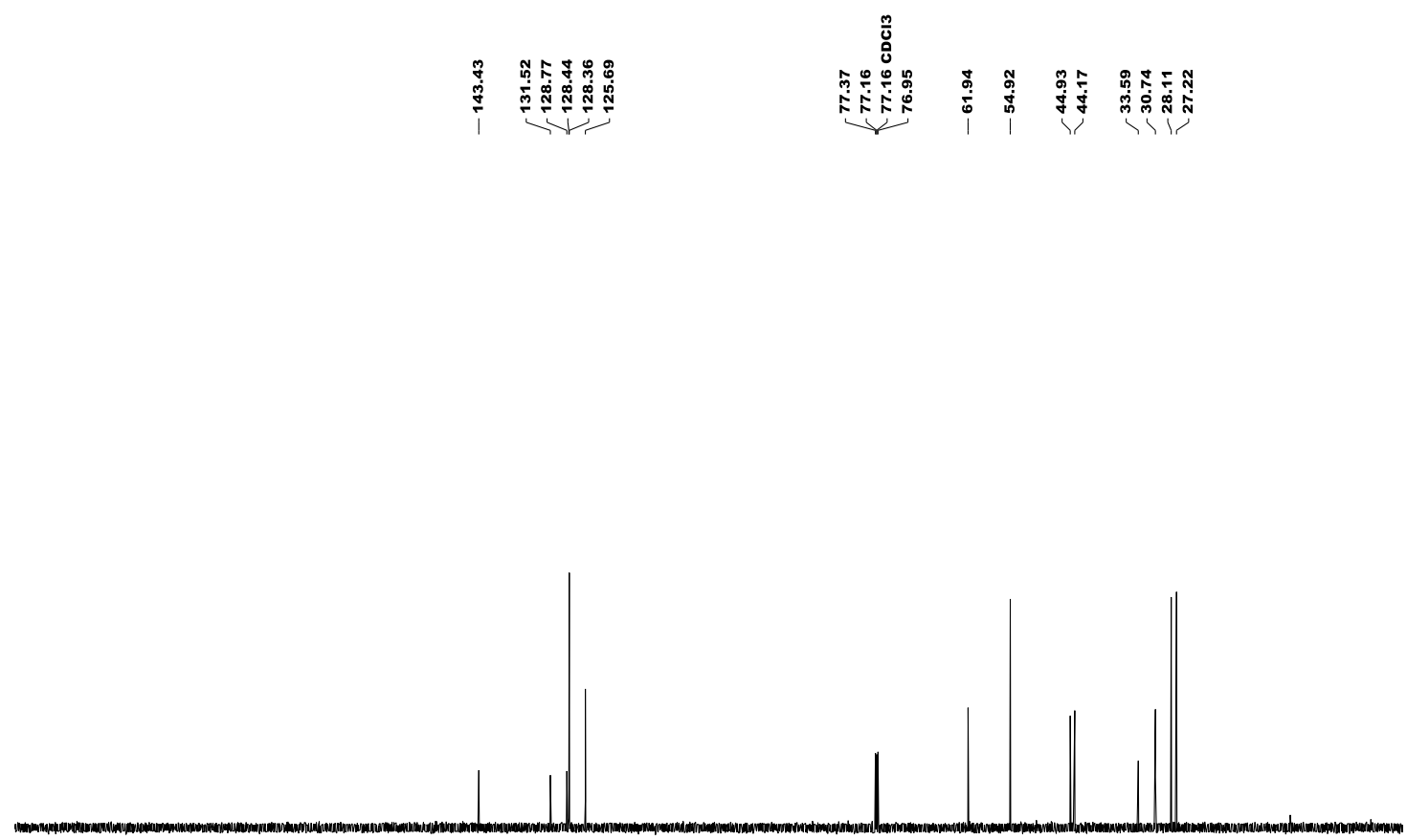

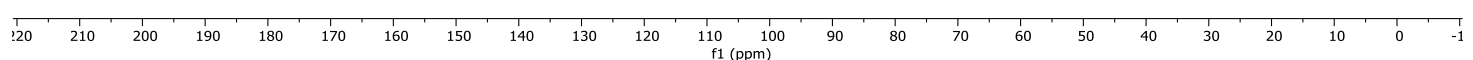


${ }^{1} \mathrm{H}$ NMR (599 MHz, $\mathrm{CDCl}_{3}$ )
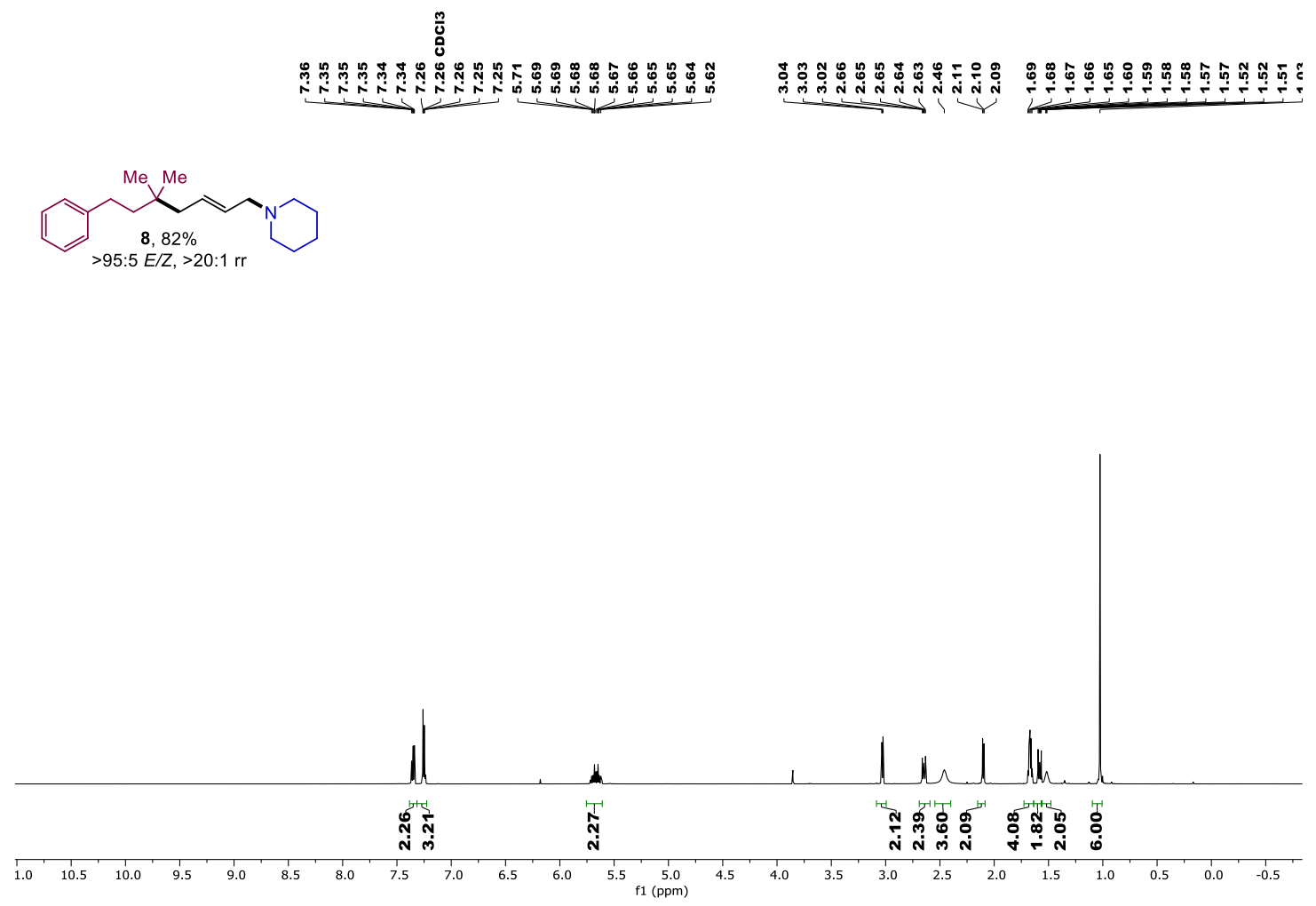

${ }^{13} \mathrm{C} \mathrm{NMR}\left(151 \mathrm{MHz}, \mathrm{CDCl}_{3}\right)$
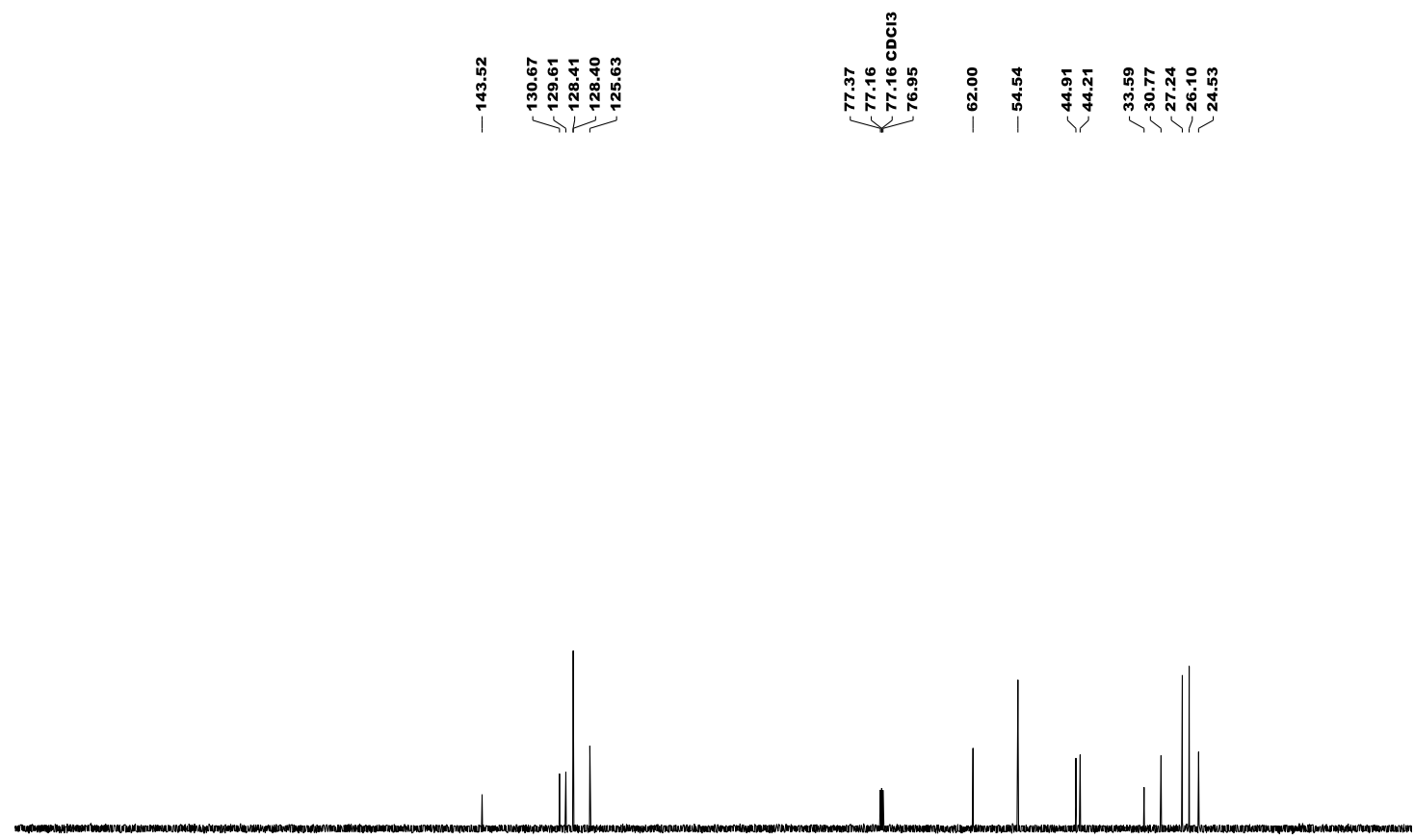

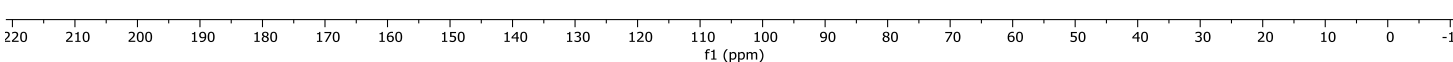


${ }^{1} \mathrm{H}$ NMR $\left(599 \mathrm{MHz}, \mathrm{CDCl}_{3}\right)$
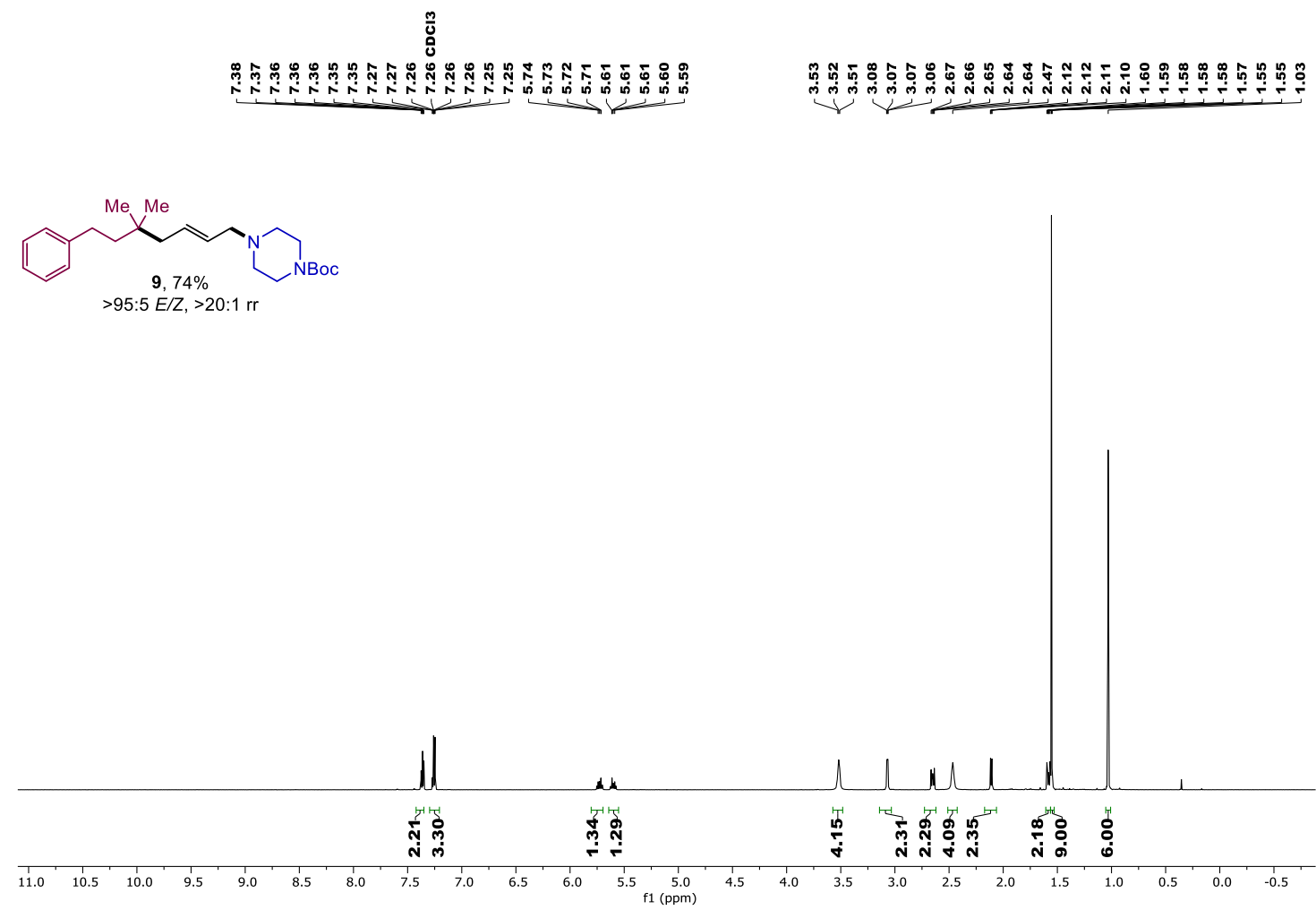

${ }^{13} \mathrm{C}$ NMR (151 MHz, $\mathrm{CDCl}_{3}$ )
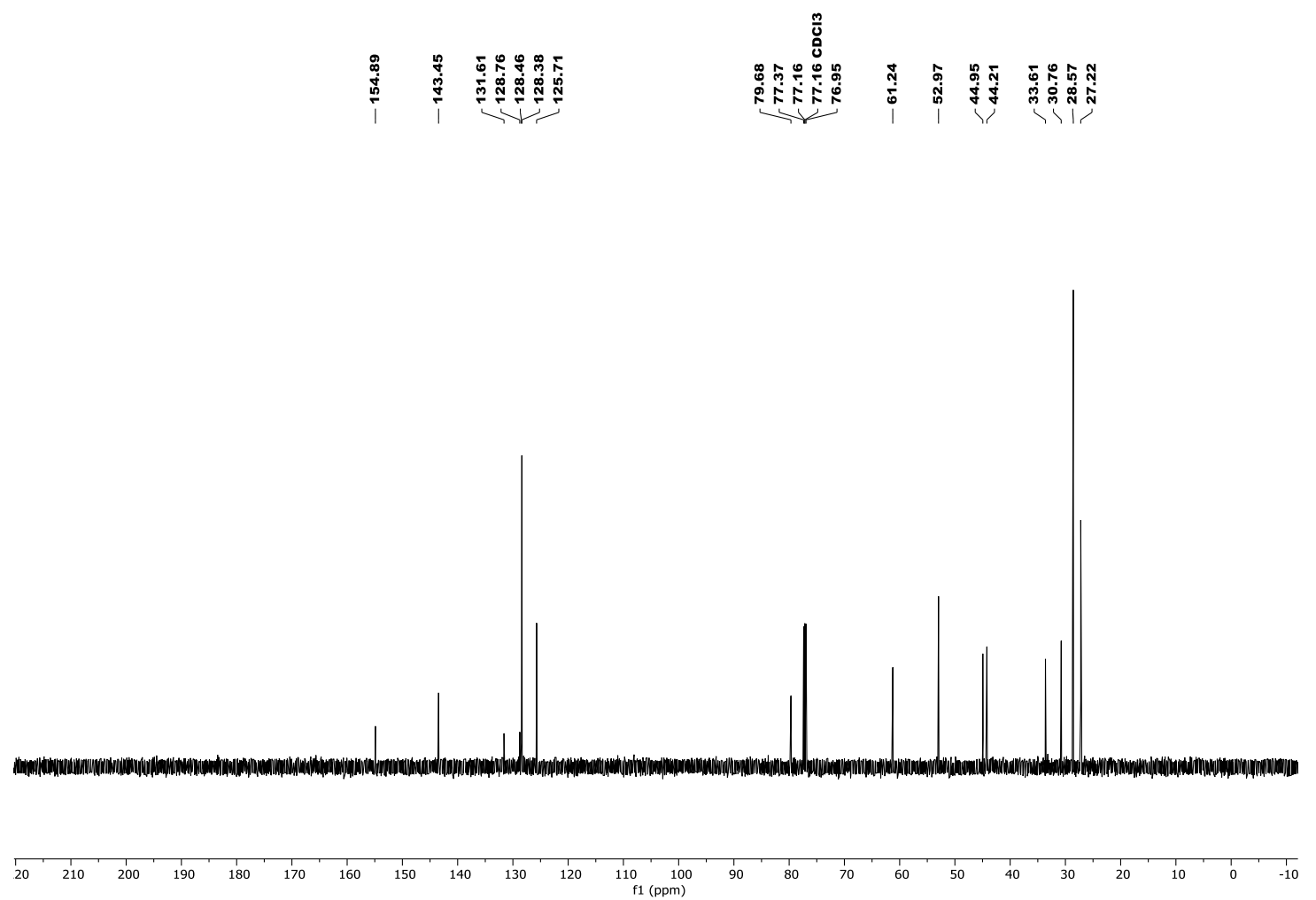
${ }^{1} \mathrm{H}$ NMR $\left(400 \mathrm{MHz}, \mathrm{CDCl}_{3}\right)$

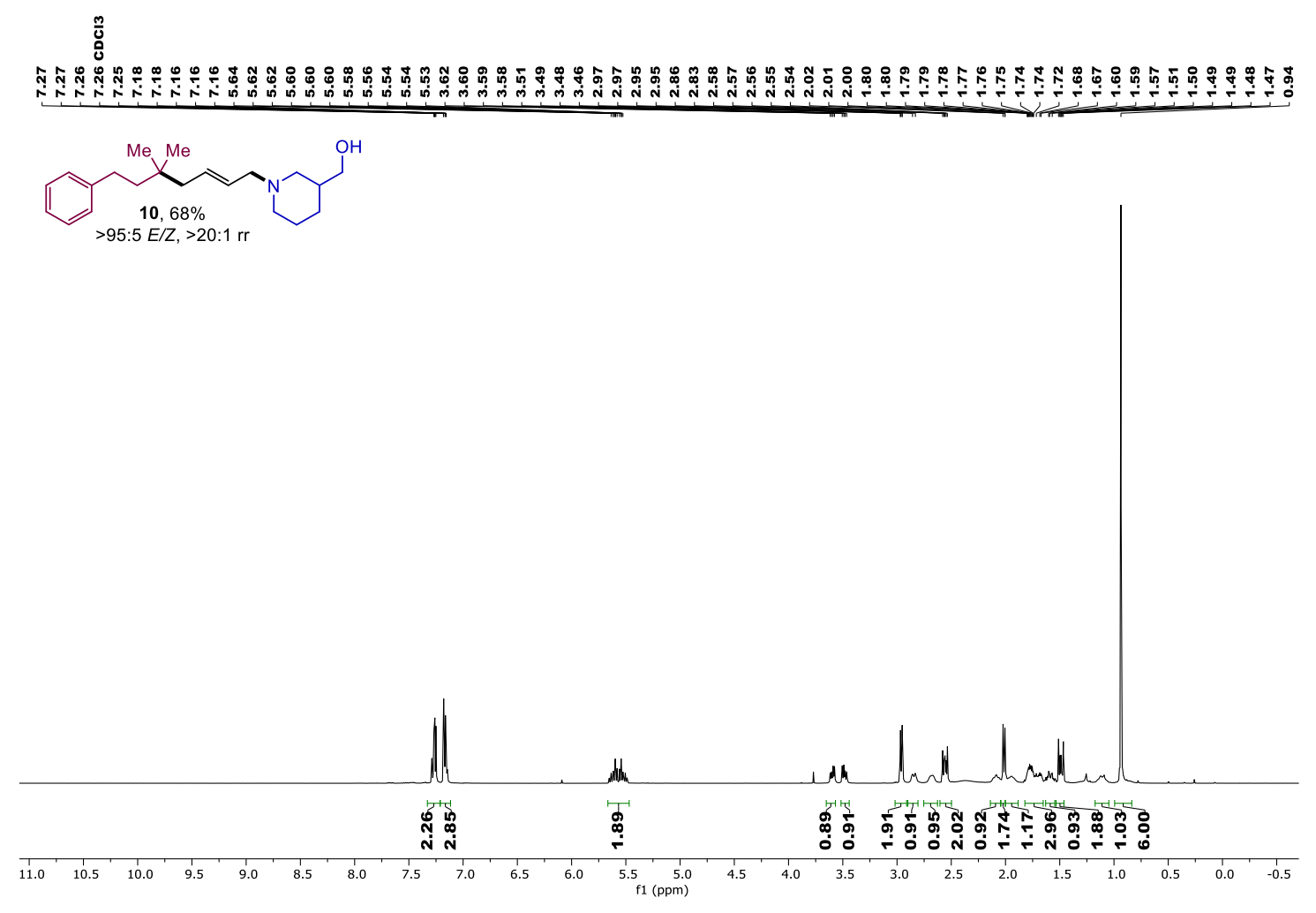

${ }^{13} \mathrm{C}$ NMR $\left(101 \mathrm{MHz}, \mathrm{CDCl}_{3}\right)$
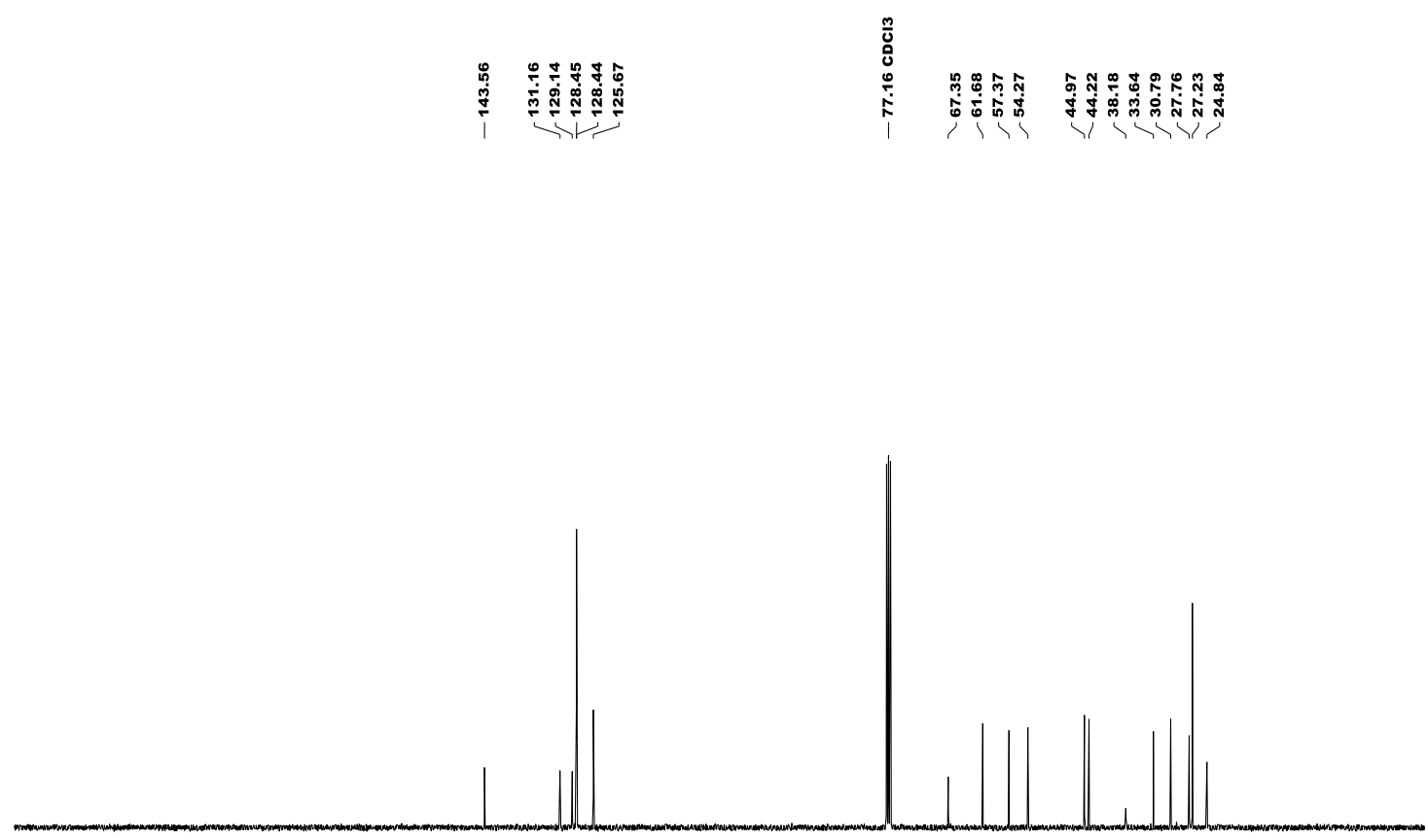

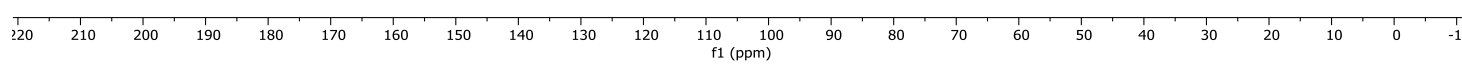


${ }^{1} \mathrm{H}$ NMR (599 MHz, $\mathrm{CDCl}_{3}$ )
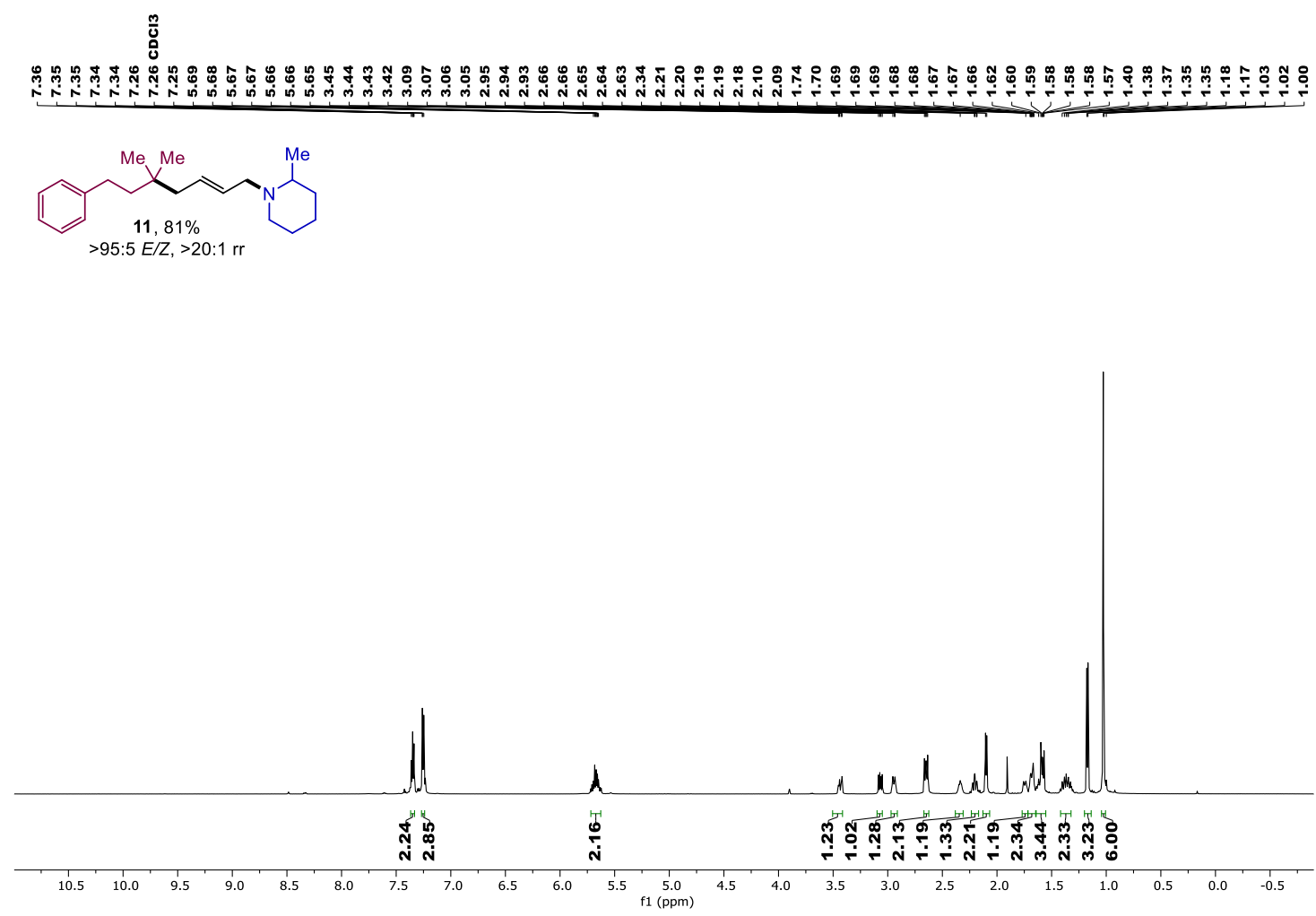

${ }^{13} \mathrm{C}$ NMR $\left(151 \mathrm{MHz}, \mathrm{CDCl}_{3}\right)$
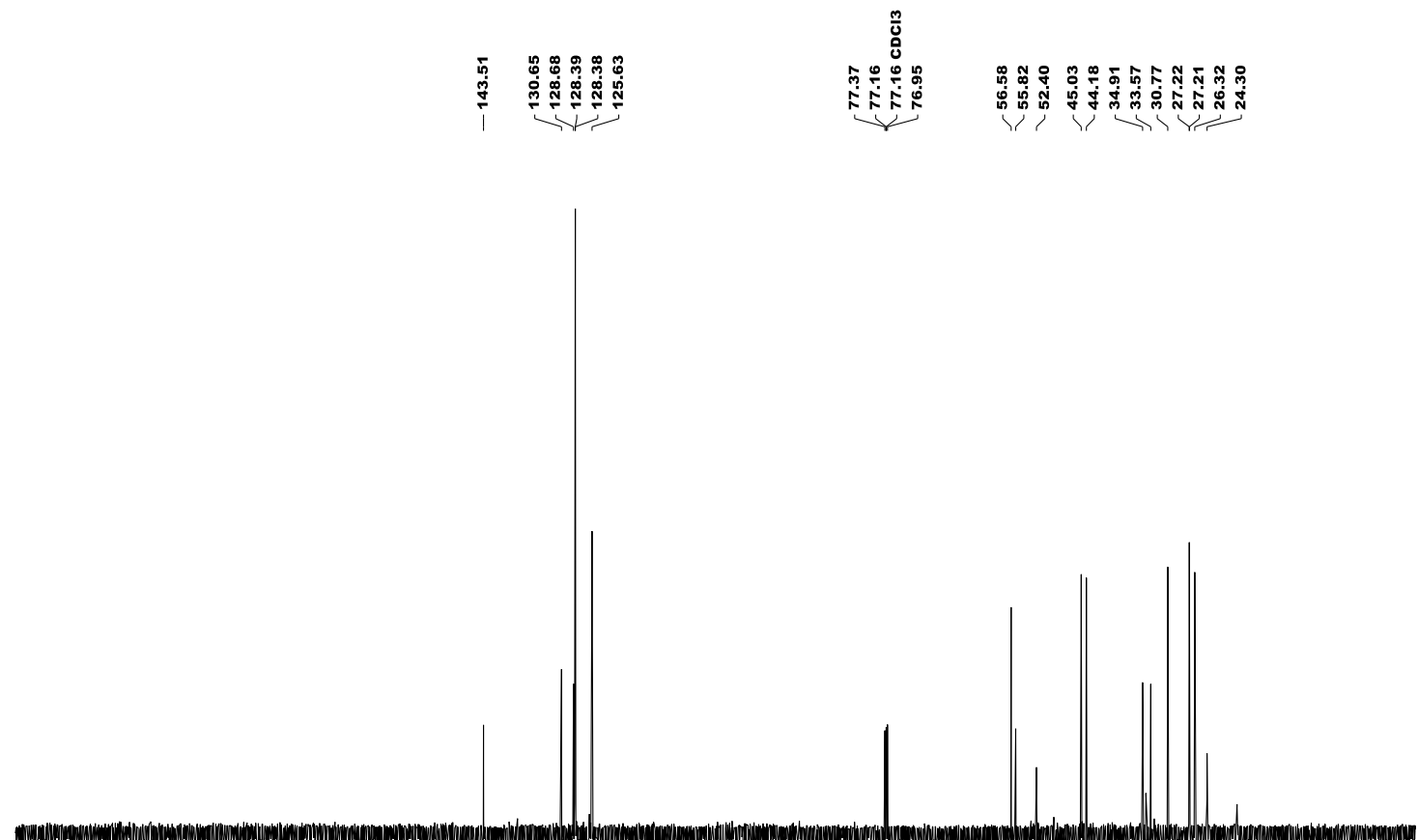

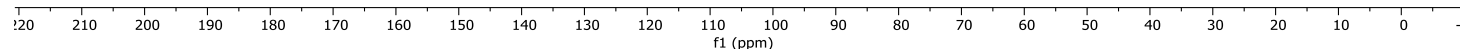


${ }^{1} \mathrm{H}$ NMR (599 MHz, $\mathrm{CDCl}_{3}$ )
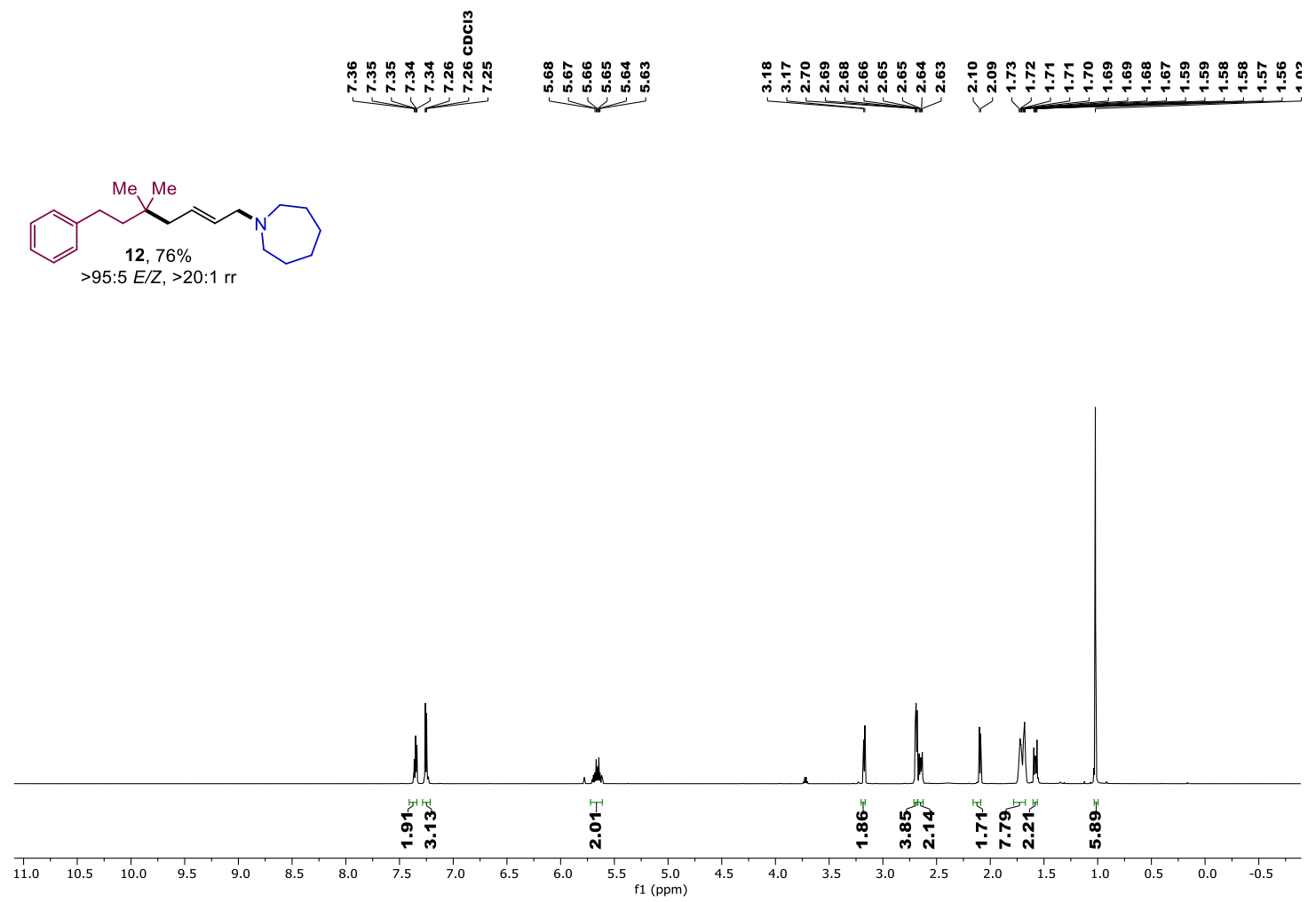

${ }^{13} \mathrm{C}$ NMR $\left(151 \mathrm{MHz}, \mathrm{CDCl}_{3}\right)$
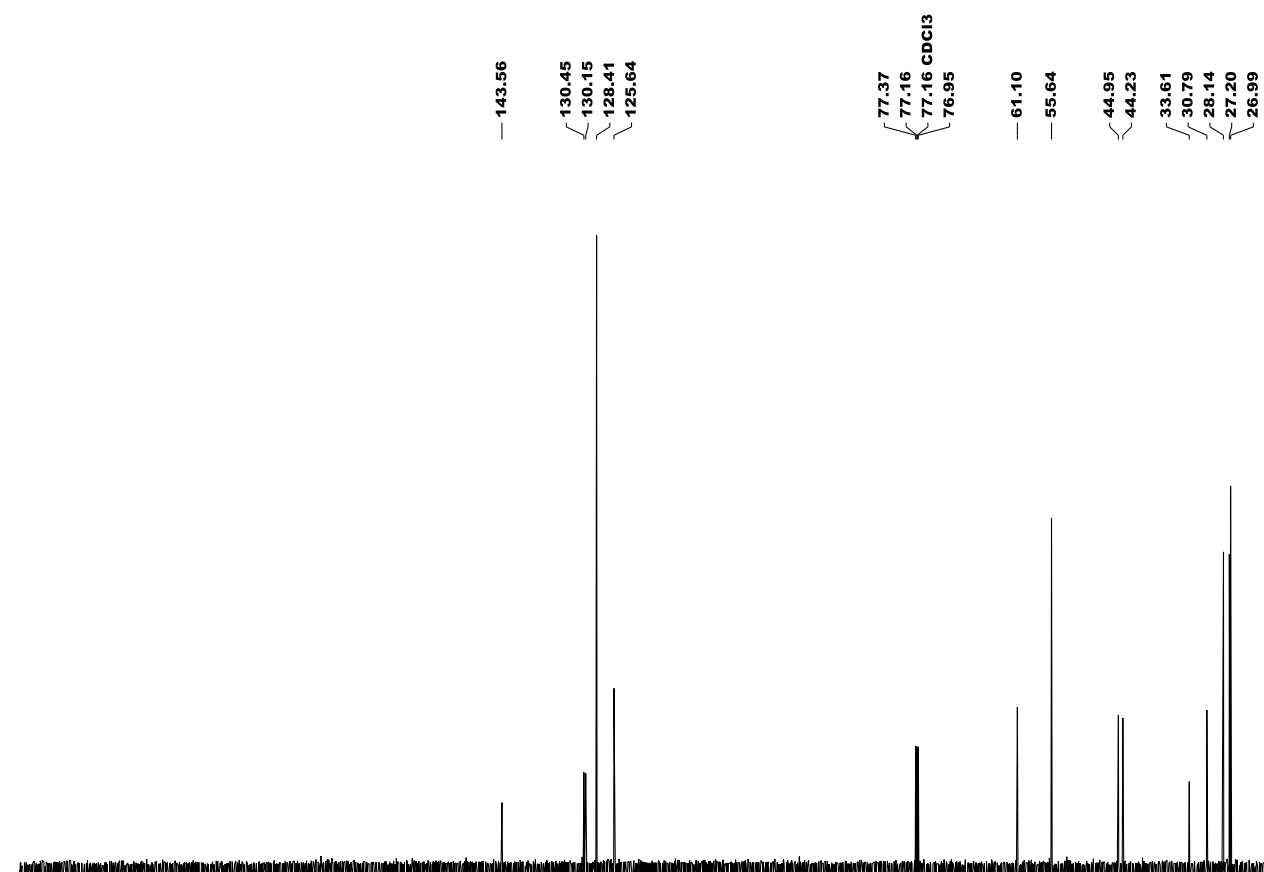

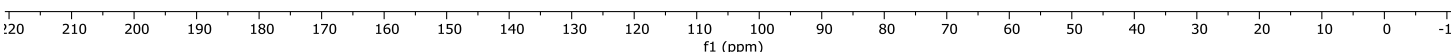


${ }^{1} \mathrm{H} \mathrm{NMR}\left(599 \mathrm{MHz}, \mathrm{CDCl}_{3}\right.$ )
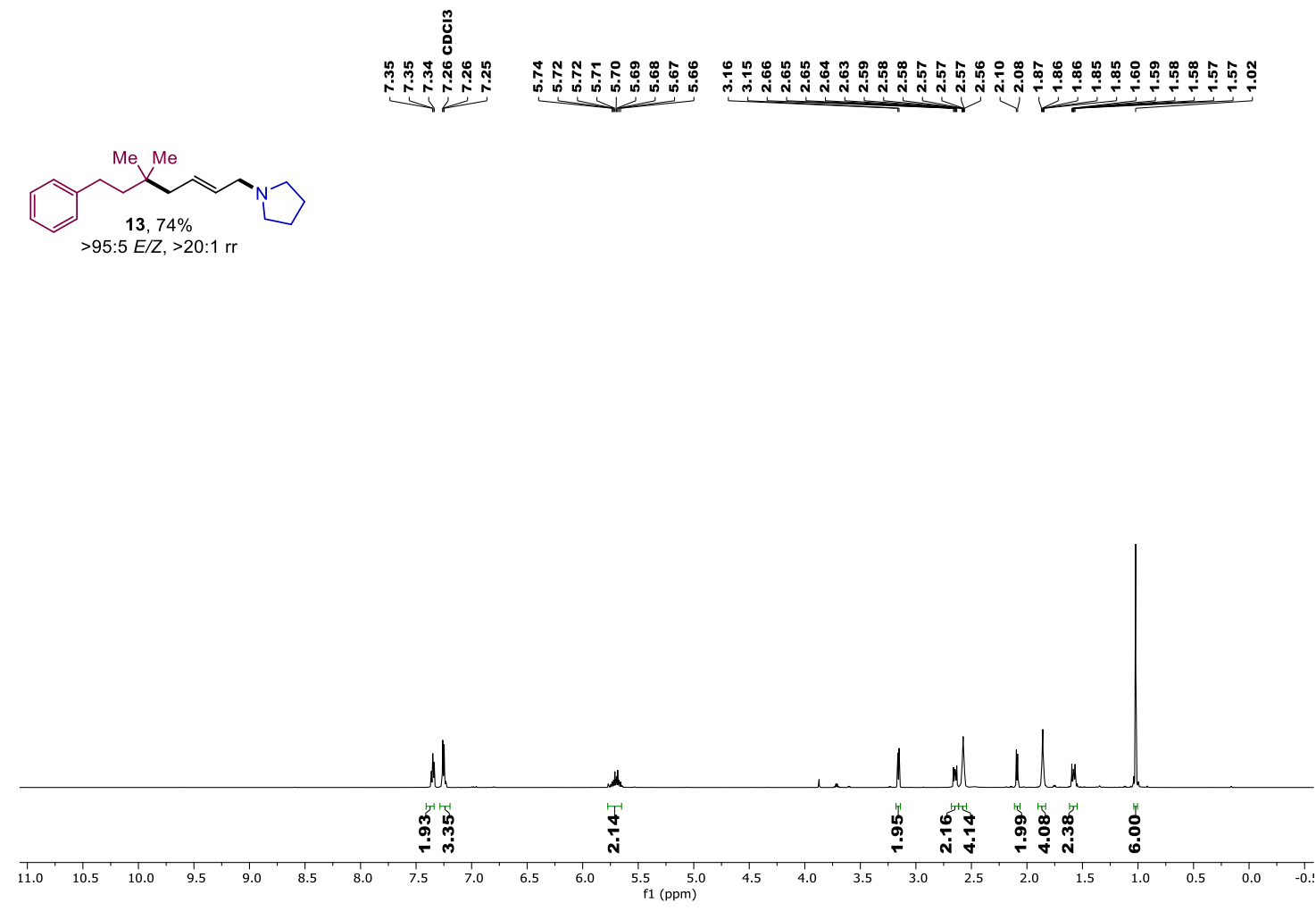

${ }^{13} \mathrm{C}$ NMR $\left(151 \mathrm{MHz}, \mathrm{CDCl}_{3}\right)$
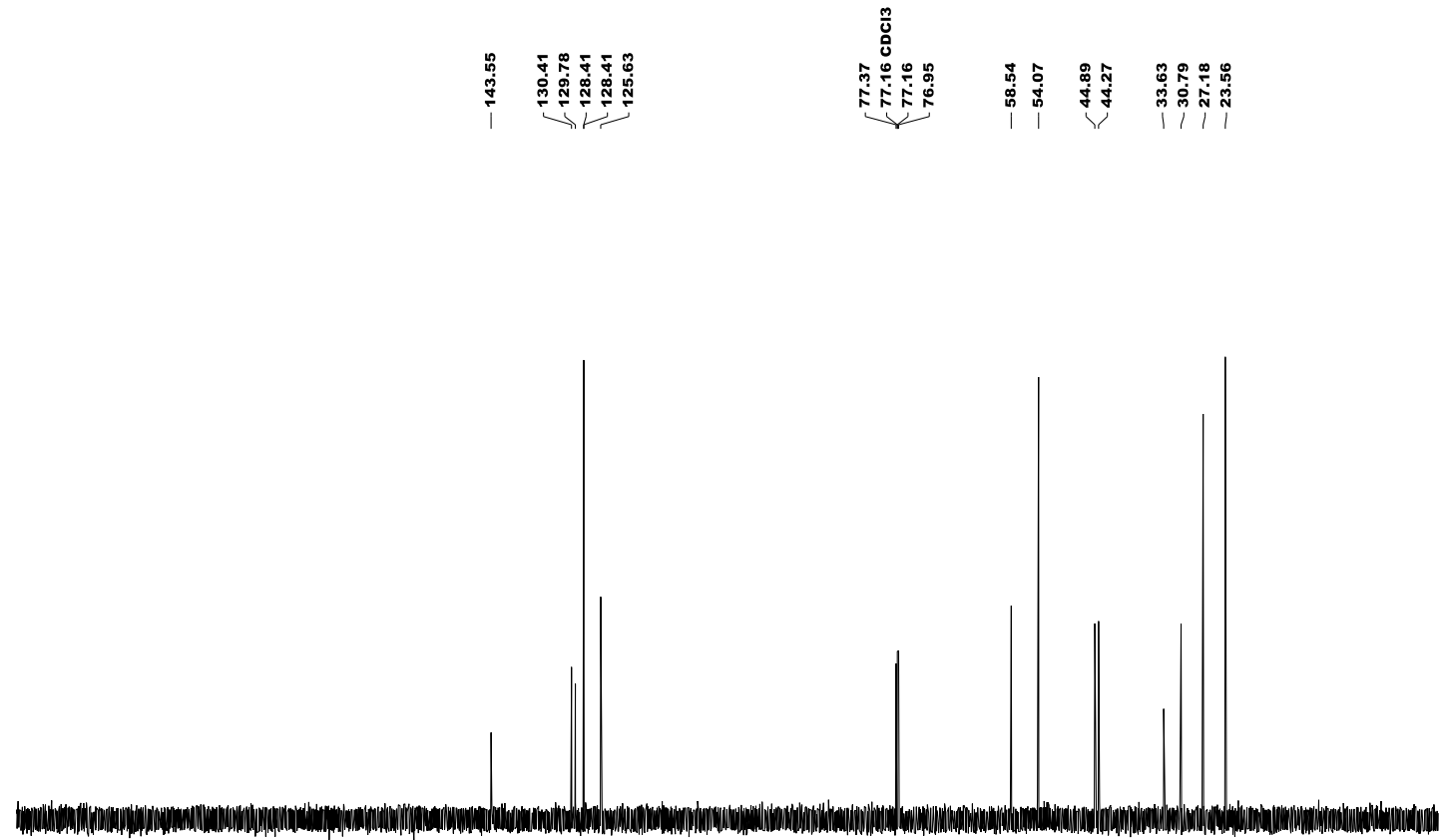

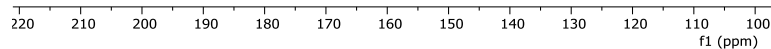


${ }^{1} \mathrm{H}$ NMR (599 MHz, $\mathrm{CDCl}_{3}$ )
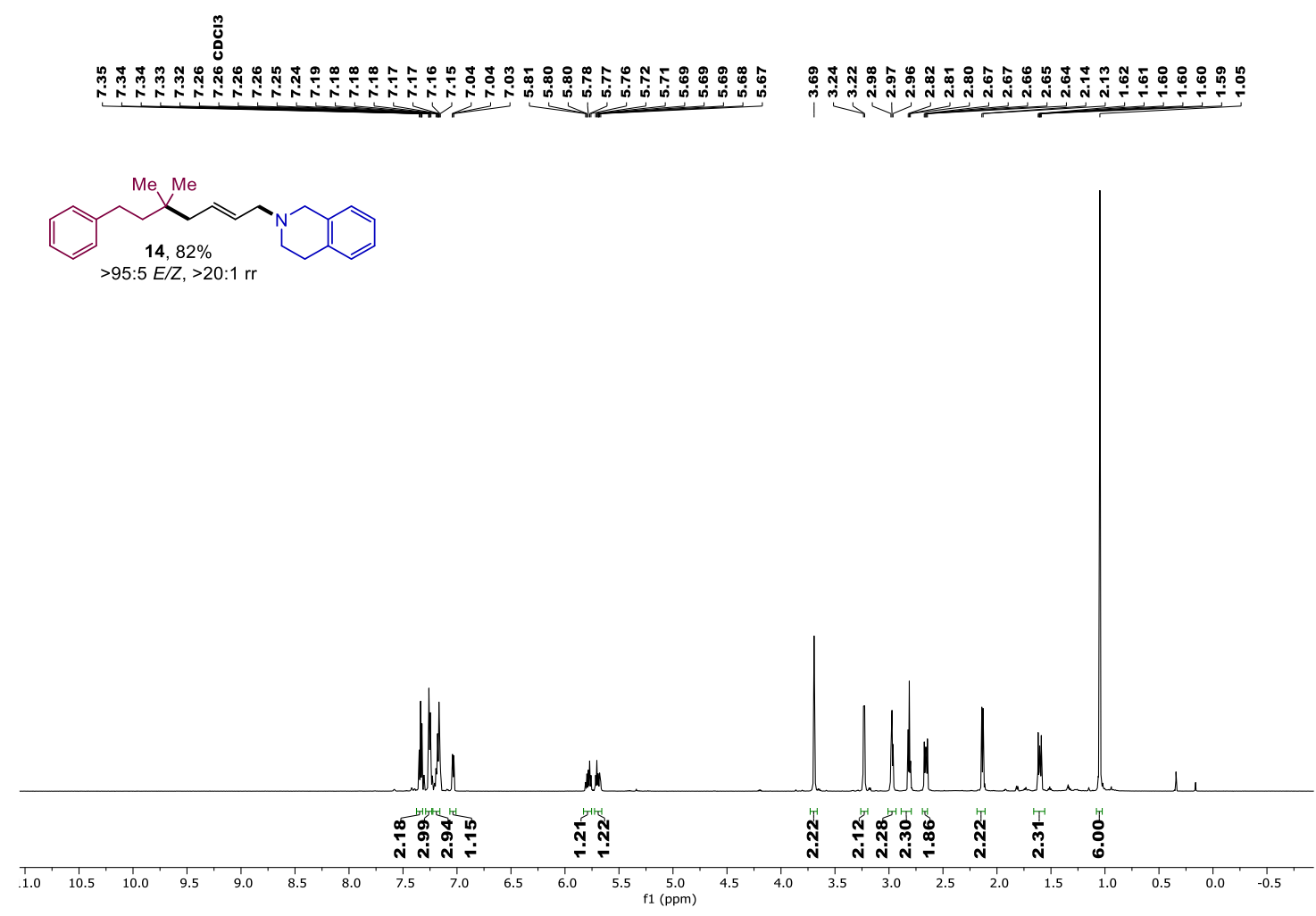

${ }^{13} \mathrm{C}$ NMR $\left(151 \mathrm{MHz}, \mathrm{CDCl}_{3}\right)$
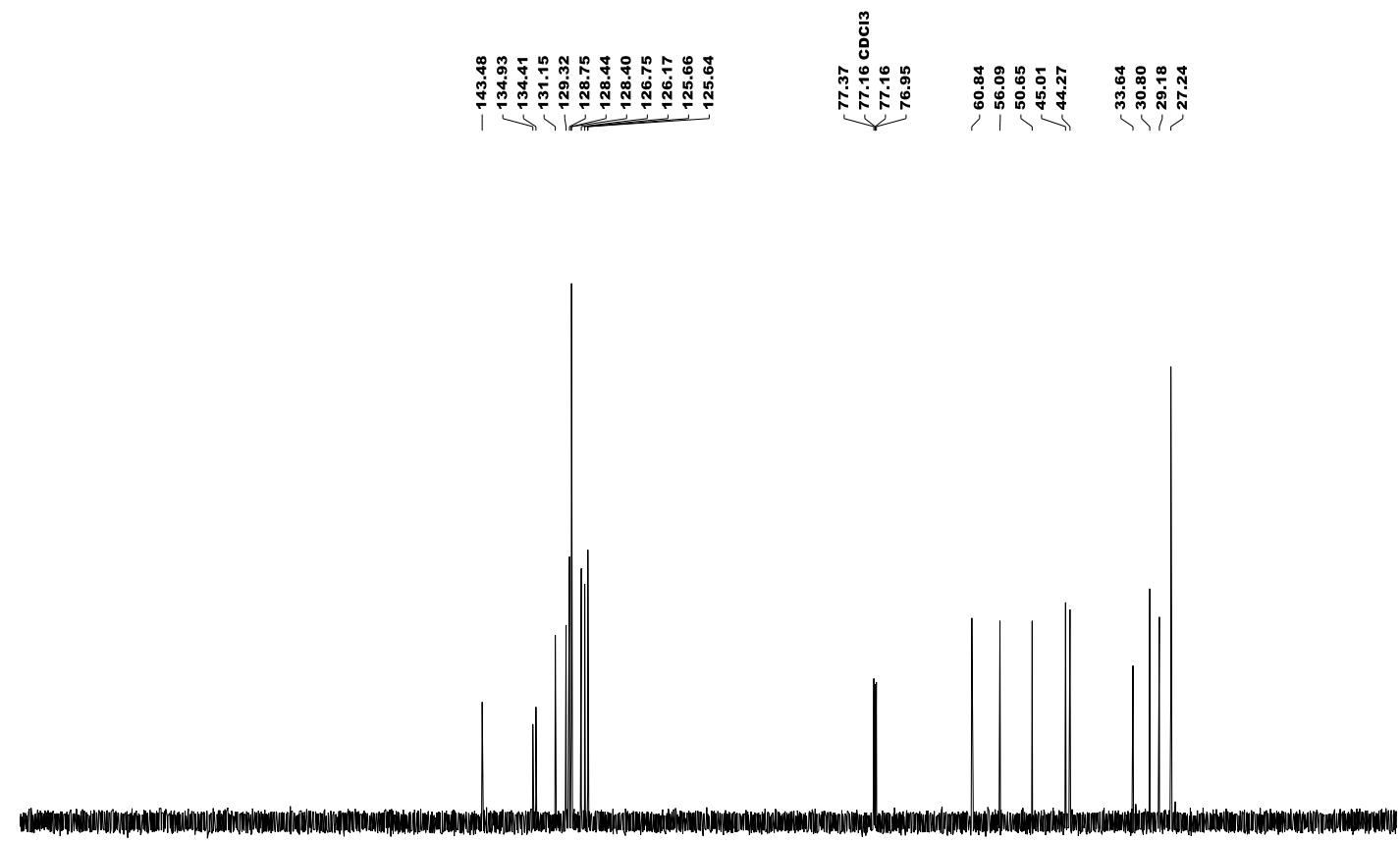

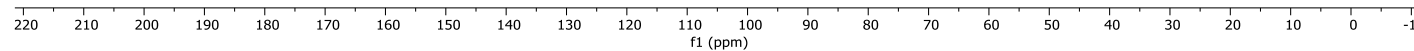


${ }^{1} \mathrm{H}$ NMR $\left(599 \mathrm{MHz}, \mathrm{CDCl}_{3}\right.$ )

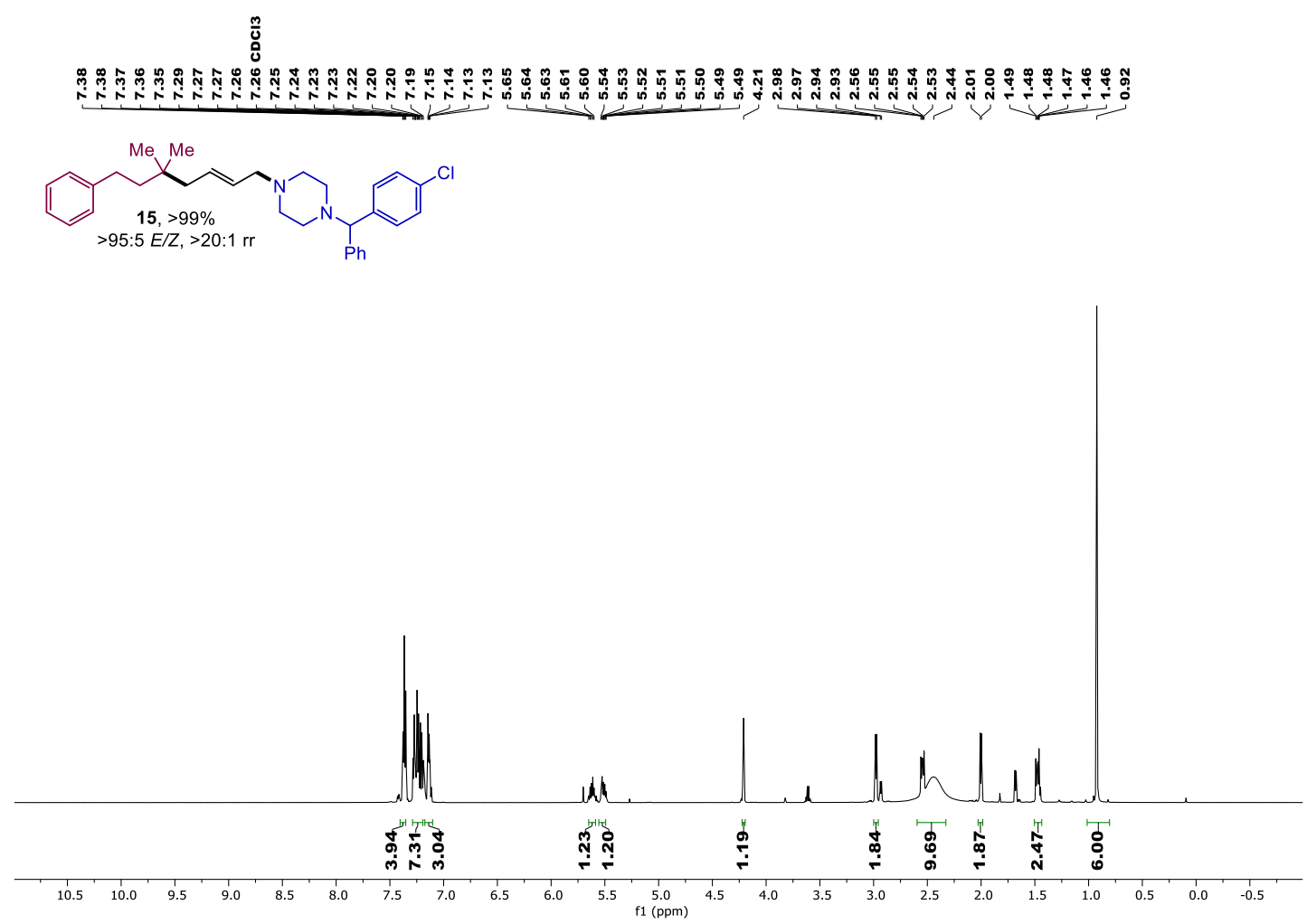

${ }^{13} \mathrm{C}$ NMR $\left(151 \mathrm{MHz}, \mathrm{CDCl}_{3}\right)$
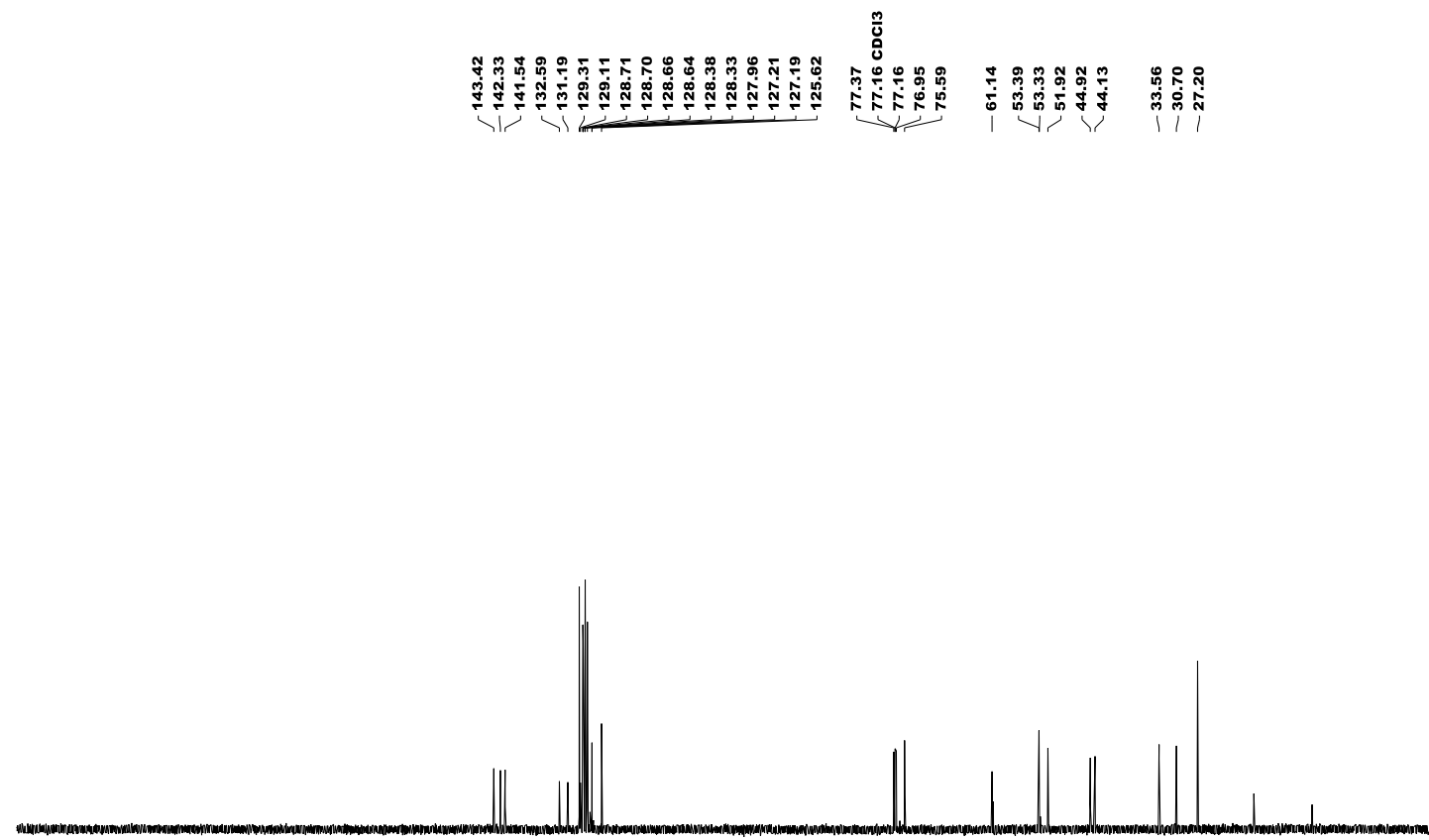

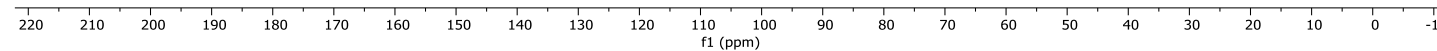


${ }^{1} \mathrm{H}$ NMR (599 MHz, $\mathrm{CDCl}_{3}$ )
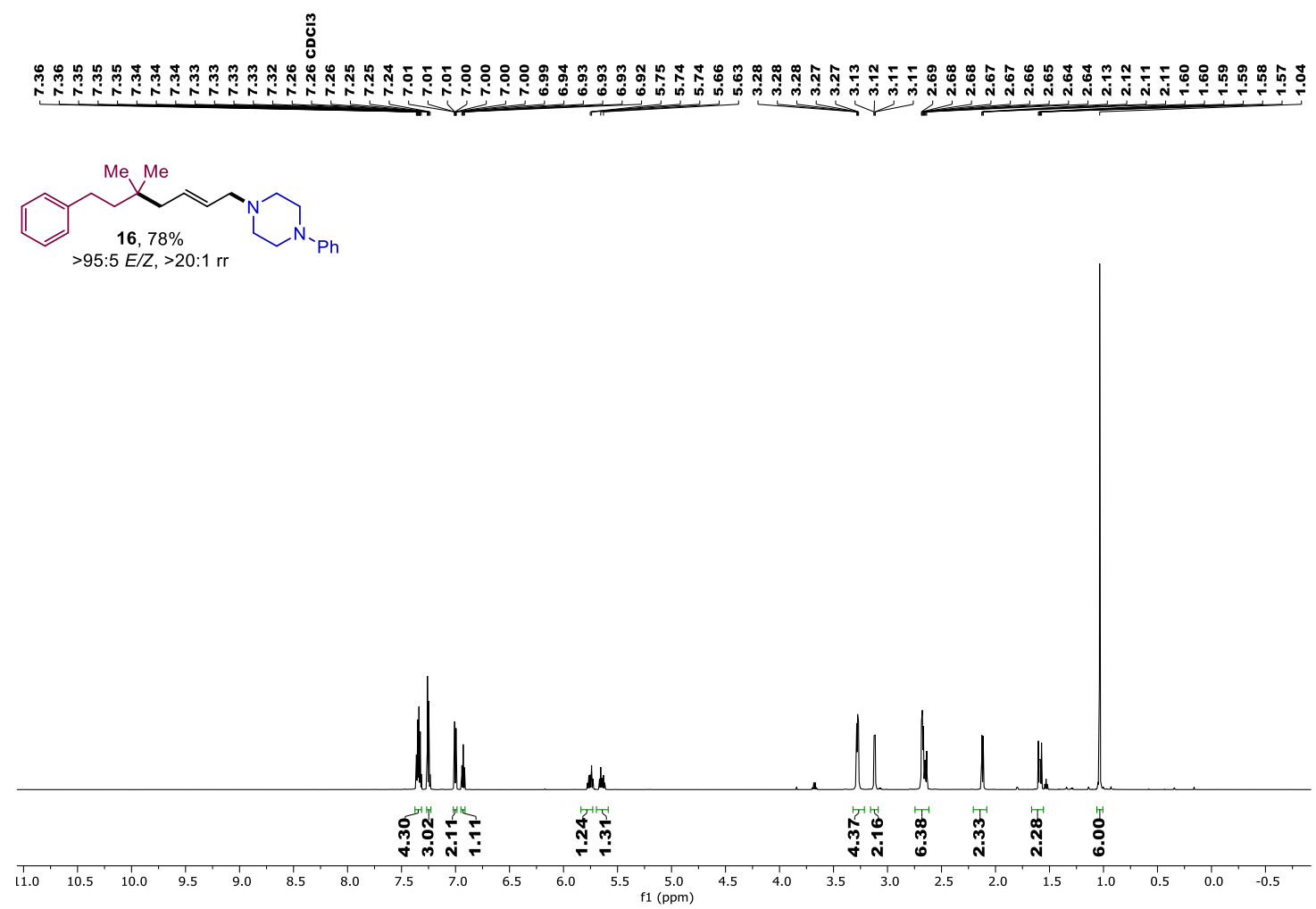

${ }^{13} \mathrm{C}$ NMR $\left(151 \mathrm{MHz}, \mathrm{CDCl}_{3}\right)$
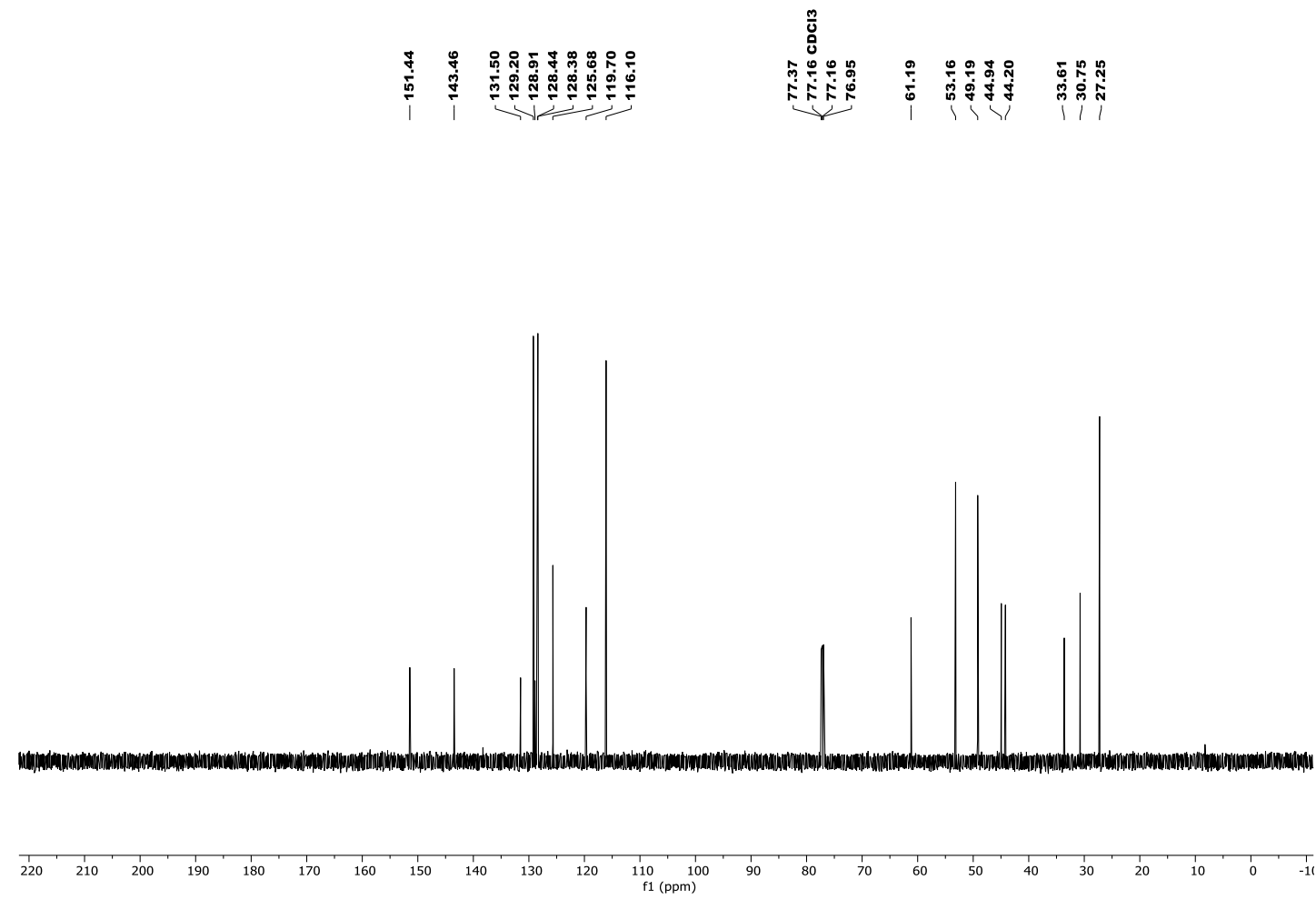
${ }^{1} \mathrm{H}$ NMR $\left(599 \mathrm{MHz}, \mathrm{CDCl}_{3}\right)$

\section{$\frac{m}{\Delta}$}

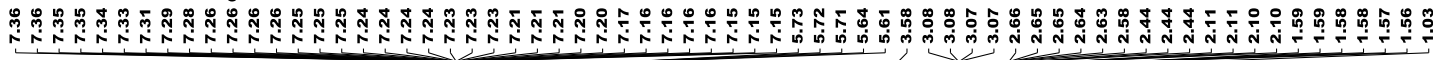<smiles>Cc1cccc(CN2CCN(C/C=C/CC(C)(C)CCc3ccccc3)CC2)c1</smiles>

${ }^{13} \mathrm{C}$ NMR $\left(151 \mathrm{MHz}, \mathrm{CDCl}_{3}\right)$
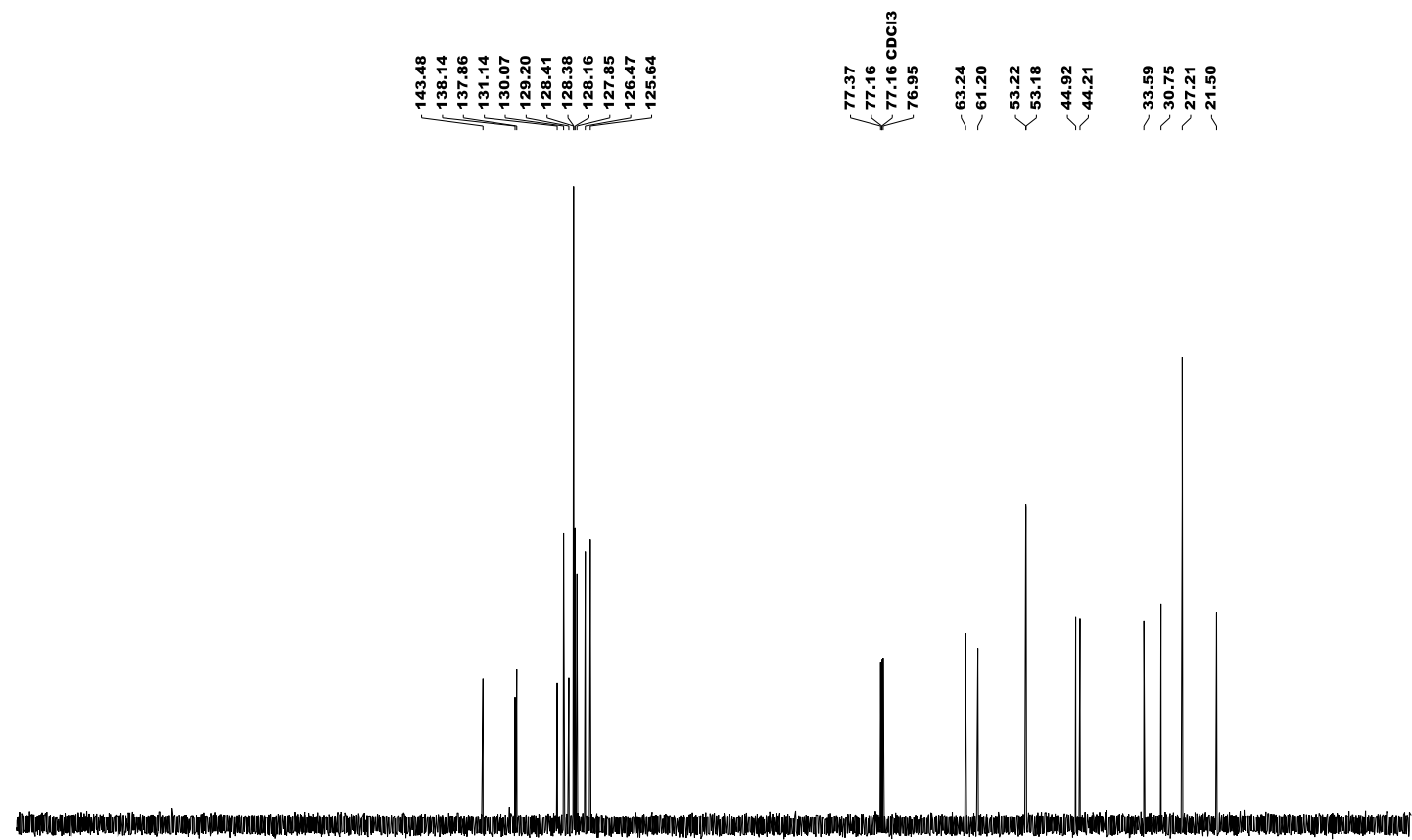

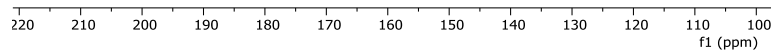


${ }^{1} \mathrm{H}$ NMR $\left(599 \mathrm{MHz}, \mathrm{CDCl}_{3}\right)$

$\frac{0}{9}$

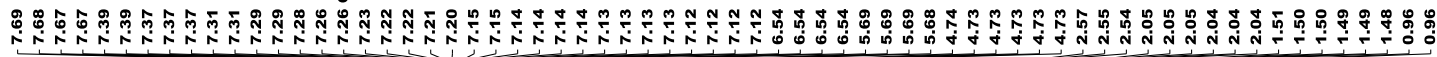
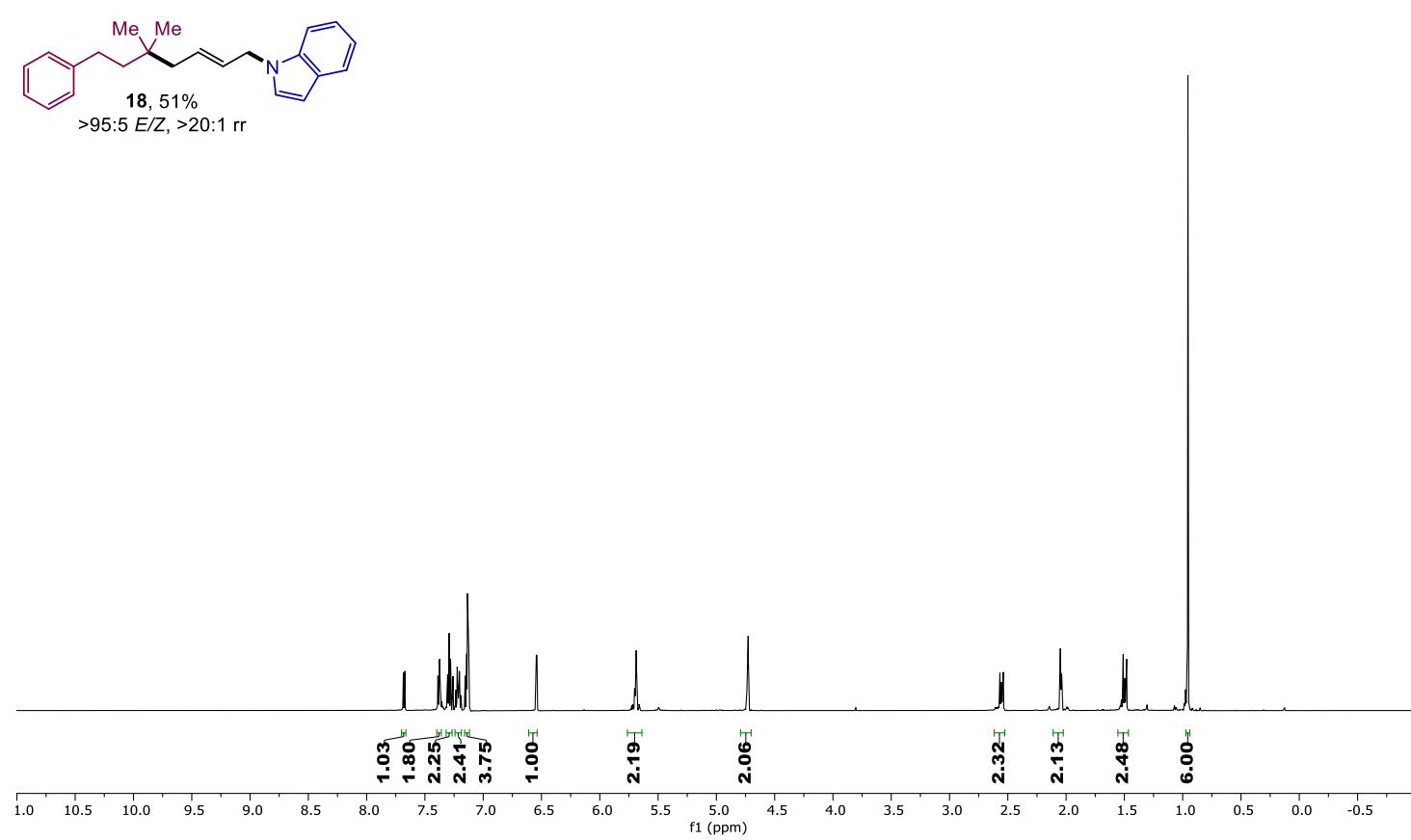

${ }^{13} \mathrm{C}$ NMR $\left(151 \mathrm{MHz}, \mathrm{CDCl}_{3}\right)$
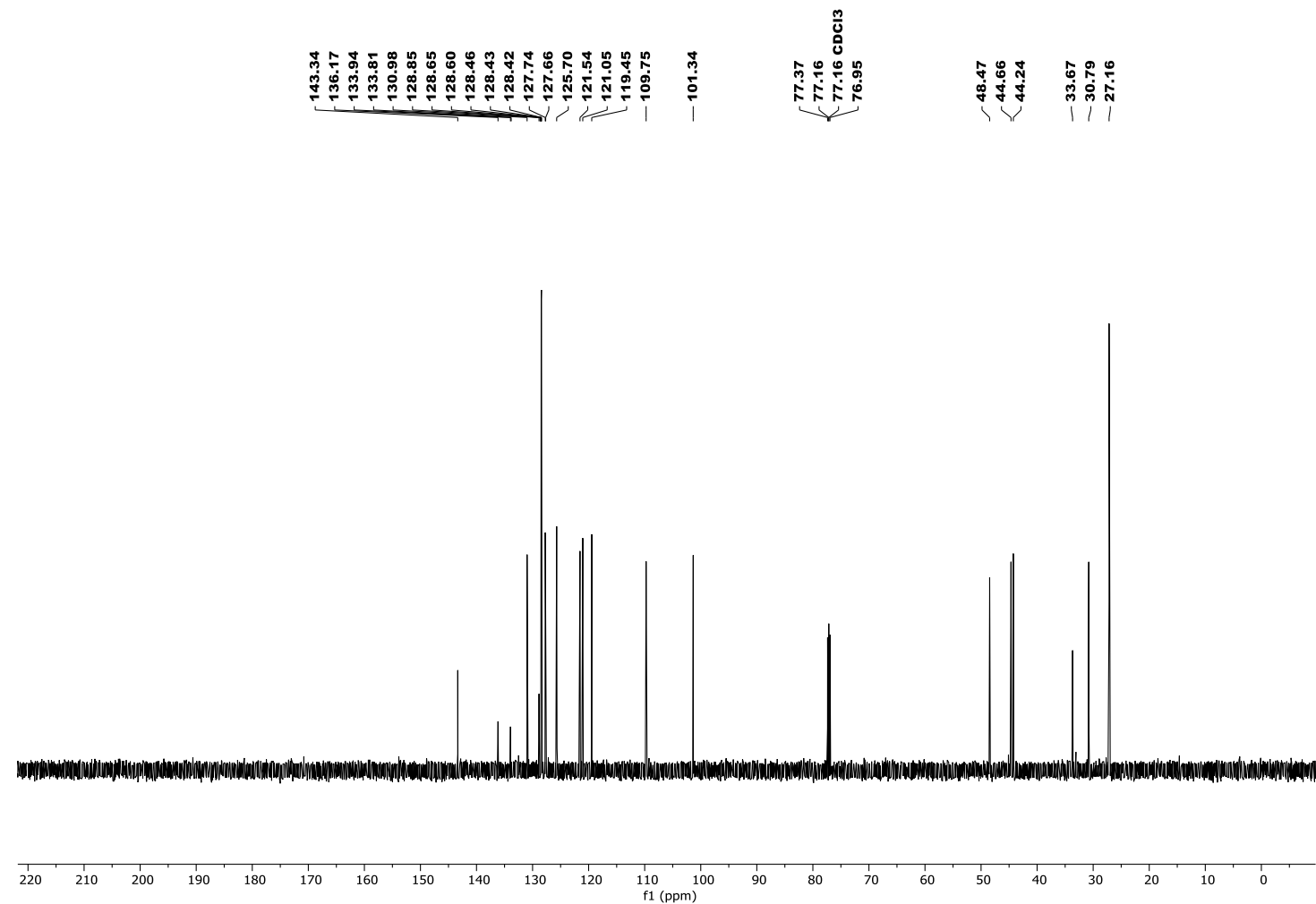
${ }^{1} \mathrm{H}$ NMR (599 MHz, $\mathrm{CDCl}_{3}$ )
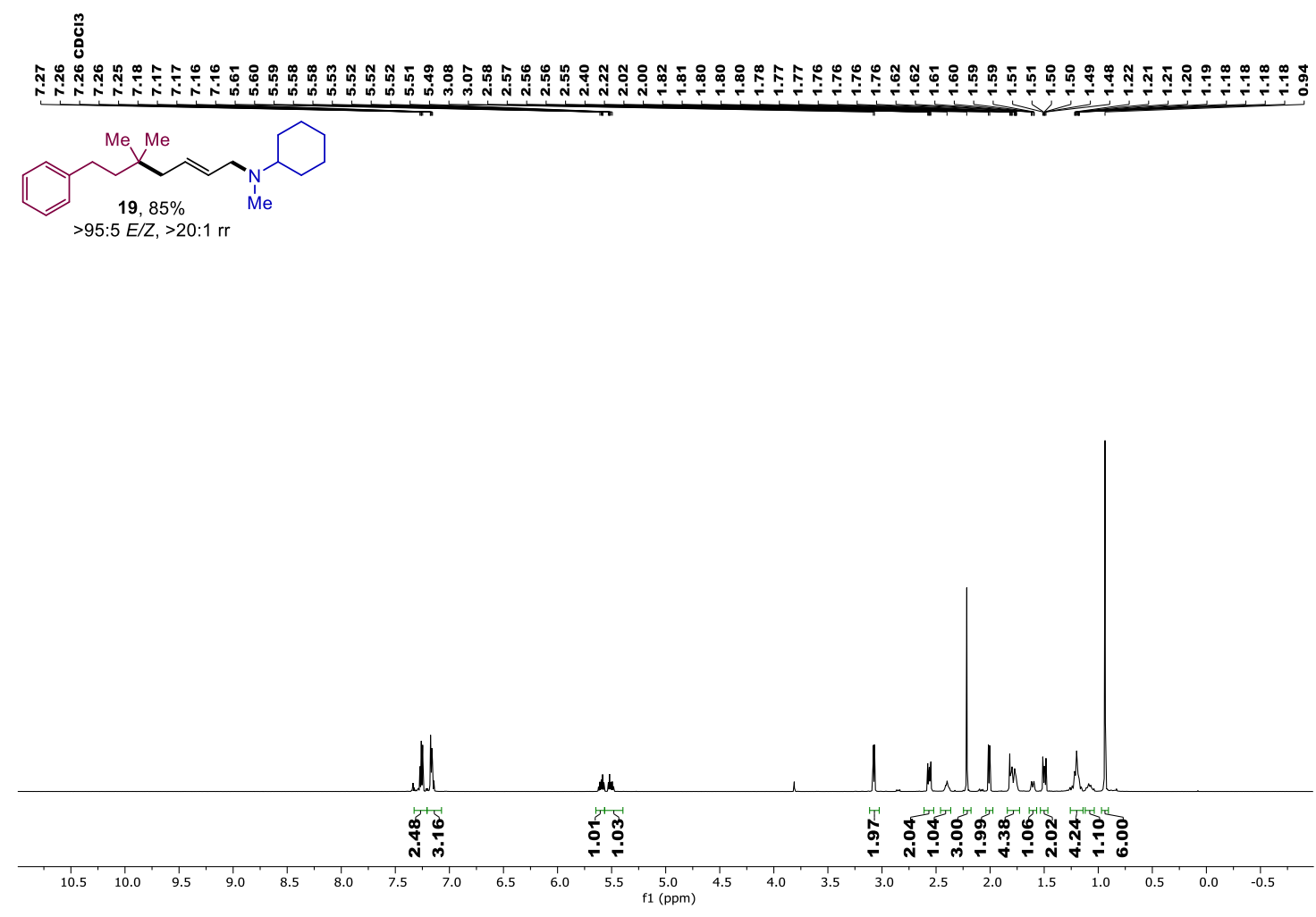

${ }^{13} \mathrm{C}$ NMR (151 MHz, $\mathrm{CDCl}_{3}$ )
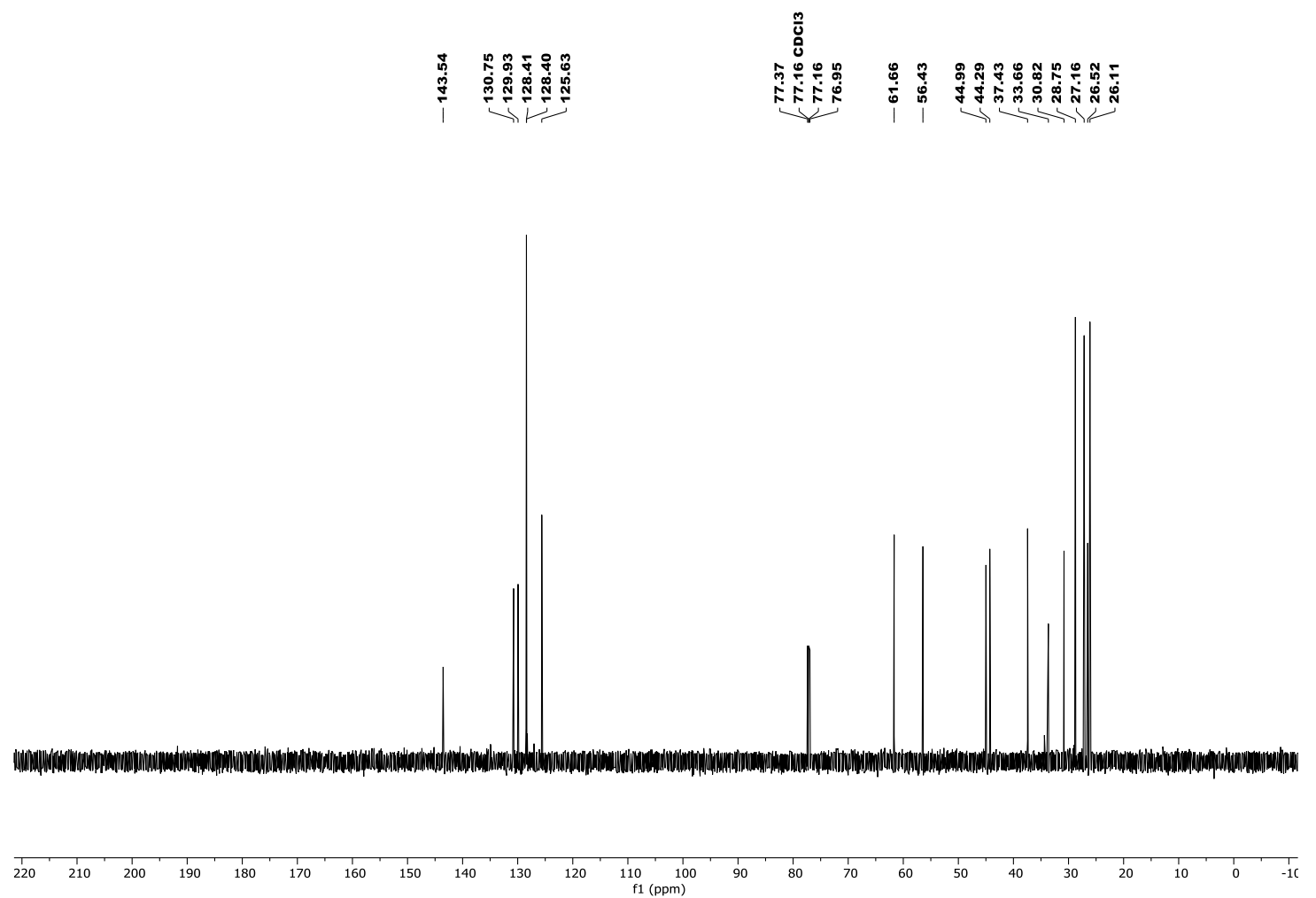
${ }^{1} \mathrm{H}$ NMR $\left(599 \mathrm{MHz}, \mathrm{CDCl}_{3}\right)$
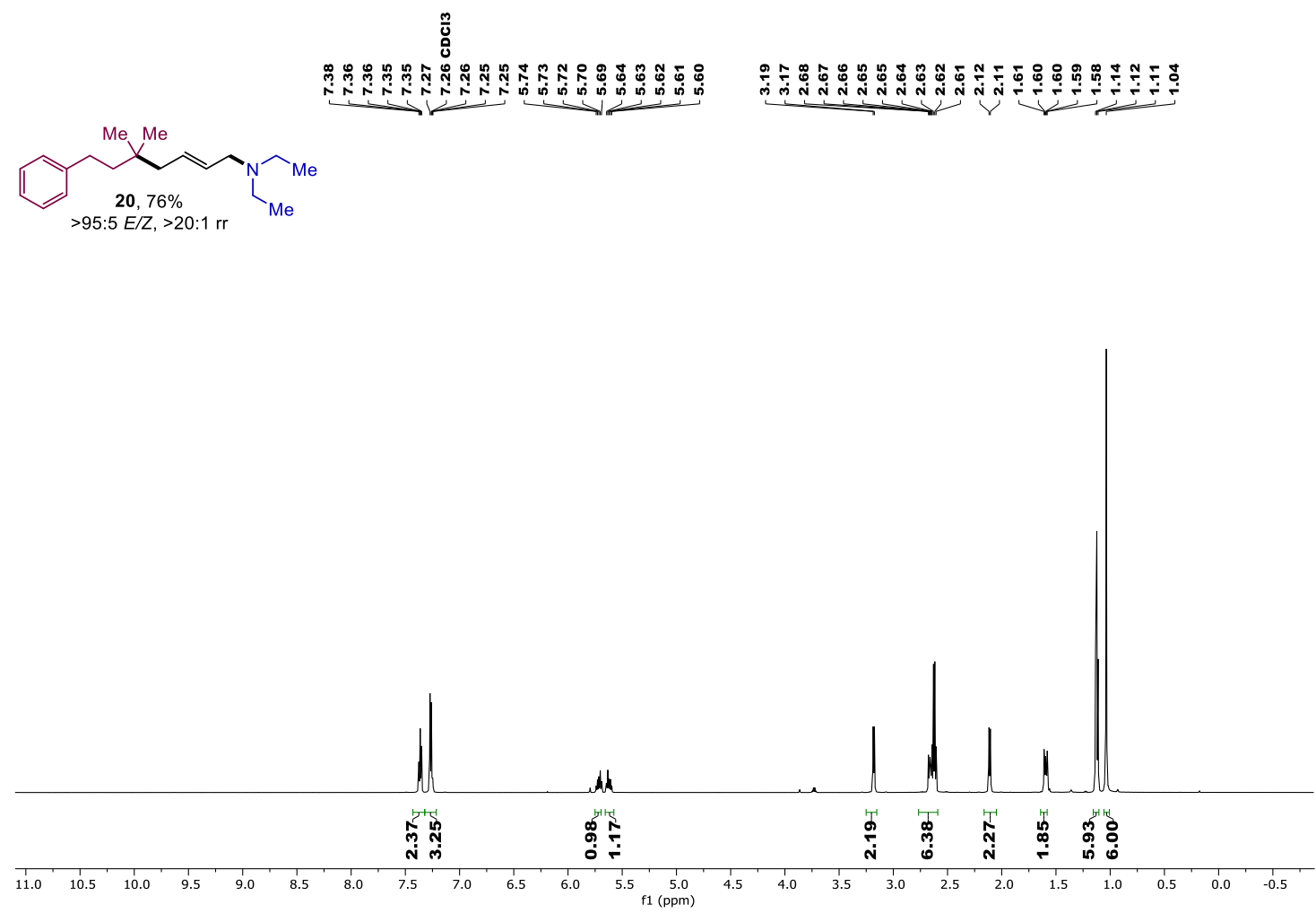

${ }^{13} \mathrm{C}$ NMR $\left(151 \mathrm{MHz}, \mathrm{CDCl}_{3}\right)$
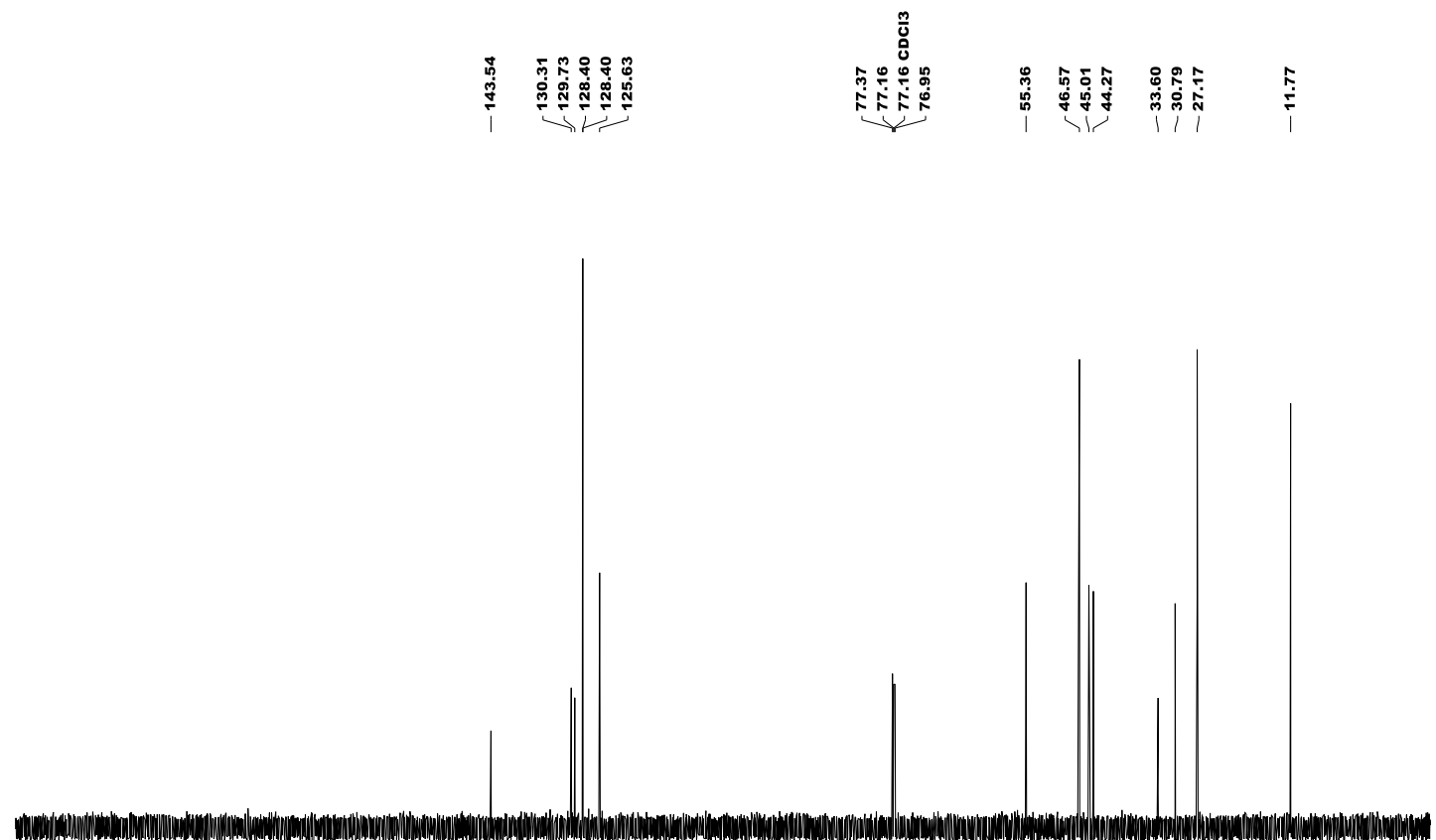

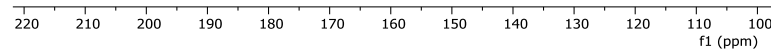


${ }^{1} \mathrm{H}$ NMR (599 MHz, $\mathrm{CDCl}_{3}$ )

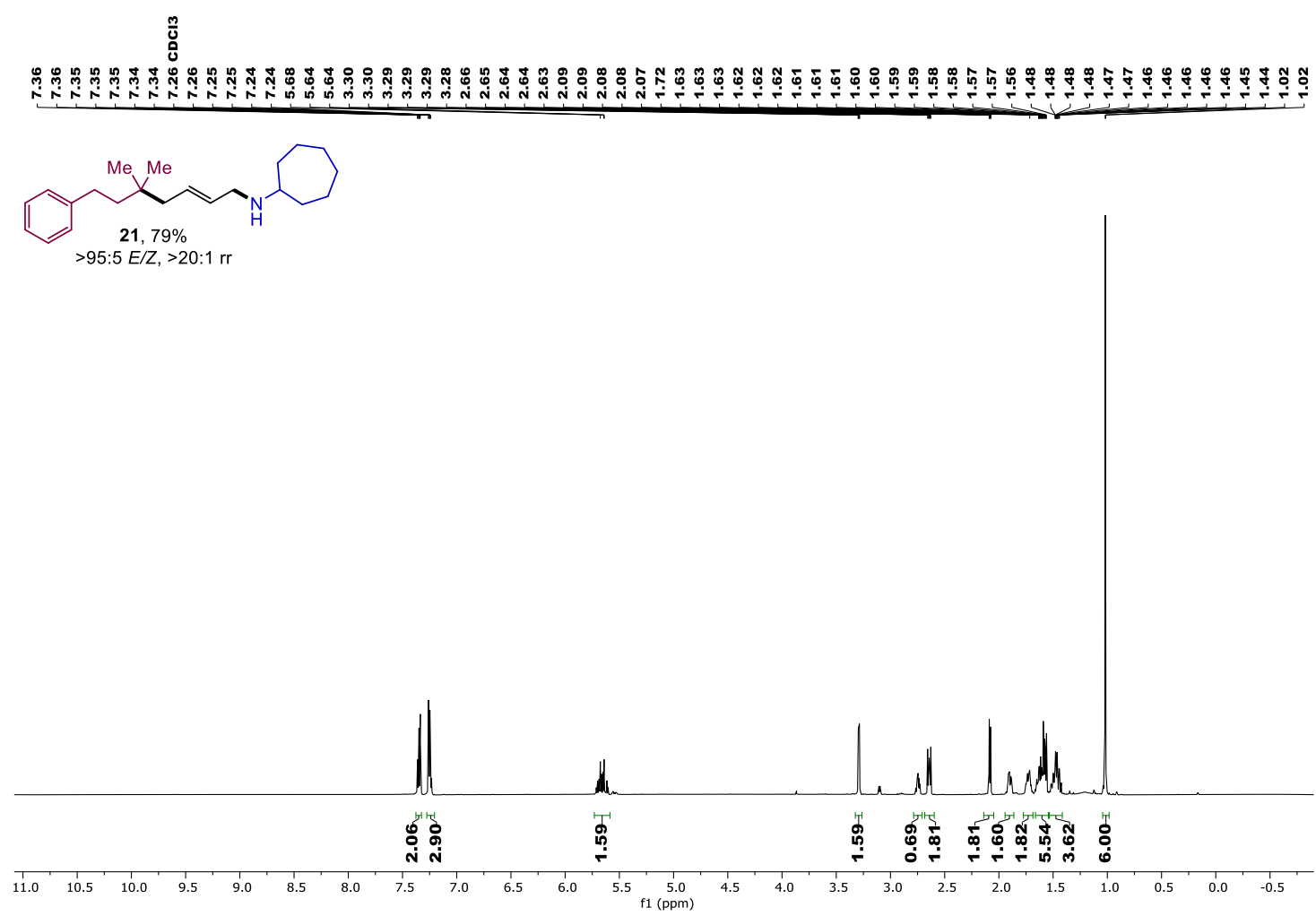

${ }^{13} \mathrm{C}$ NMR (151 MHz, $\mathrm{CDCl}_{3}$ )
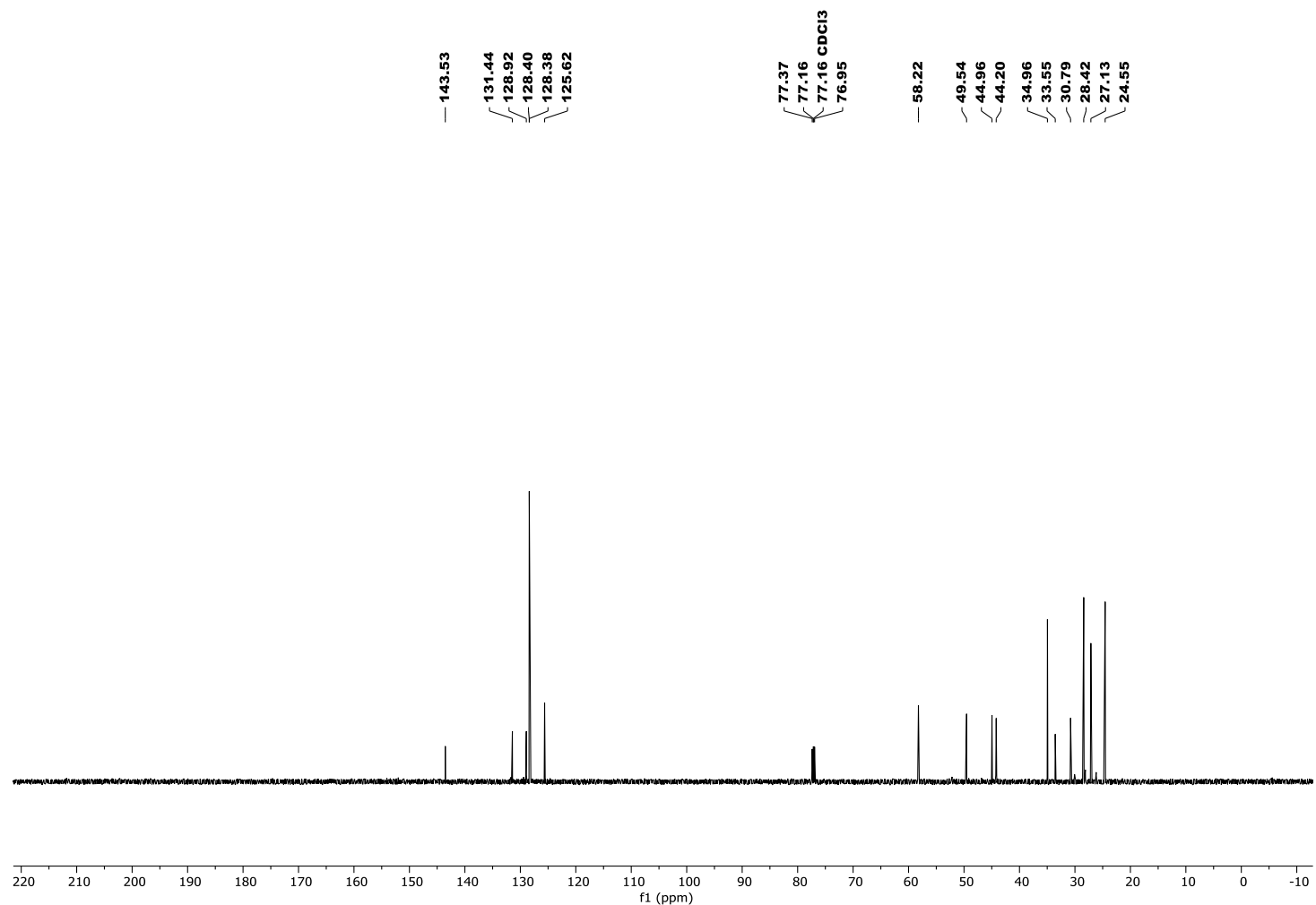
${ }^{1} \mathrm{H} \mathrm{NMR}\left(599 \mathrm{MHz}, \mathrm{CDCl}_{3}\right)$
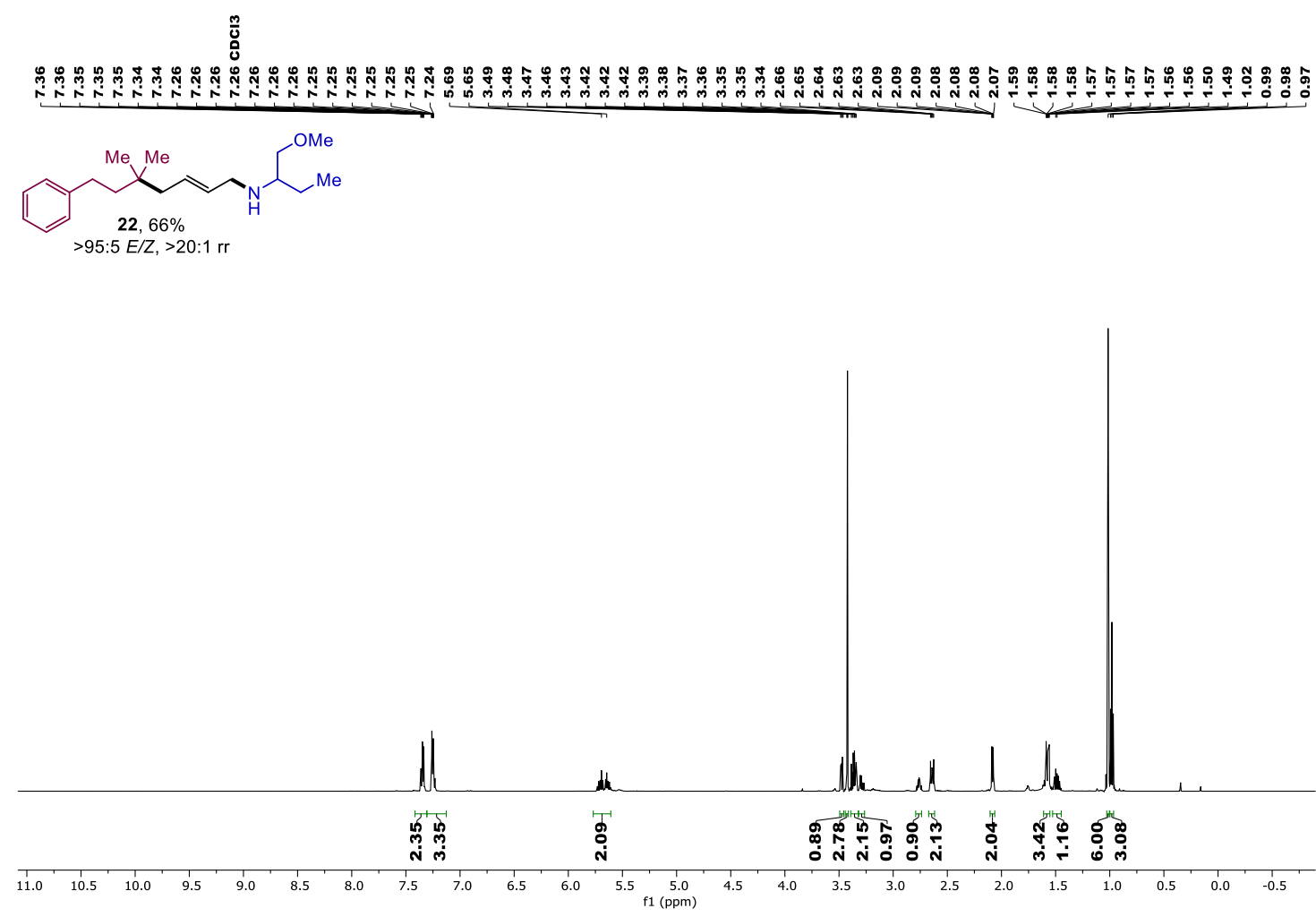

${ }^{13} \mathrm{C}$ NMR (151 MHz, $\mathrm{CDCl}_{3}$ )
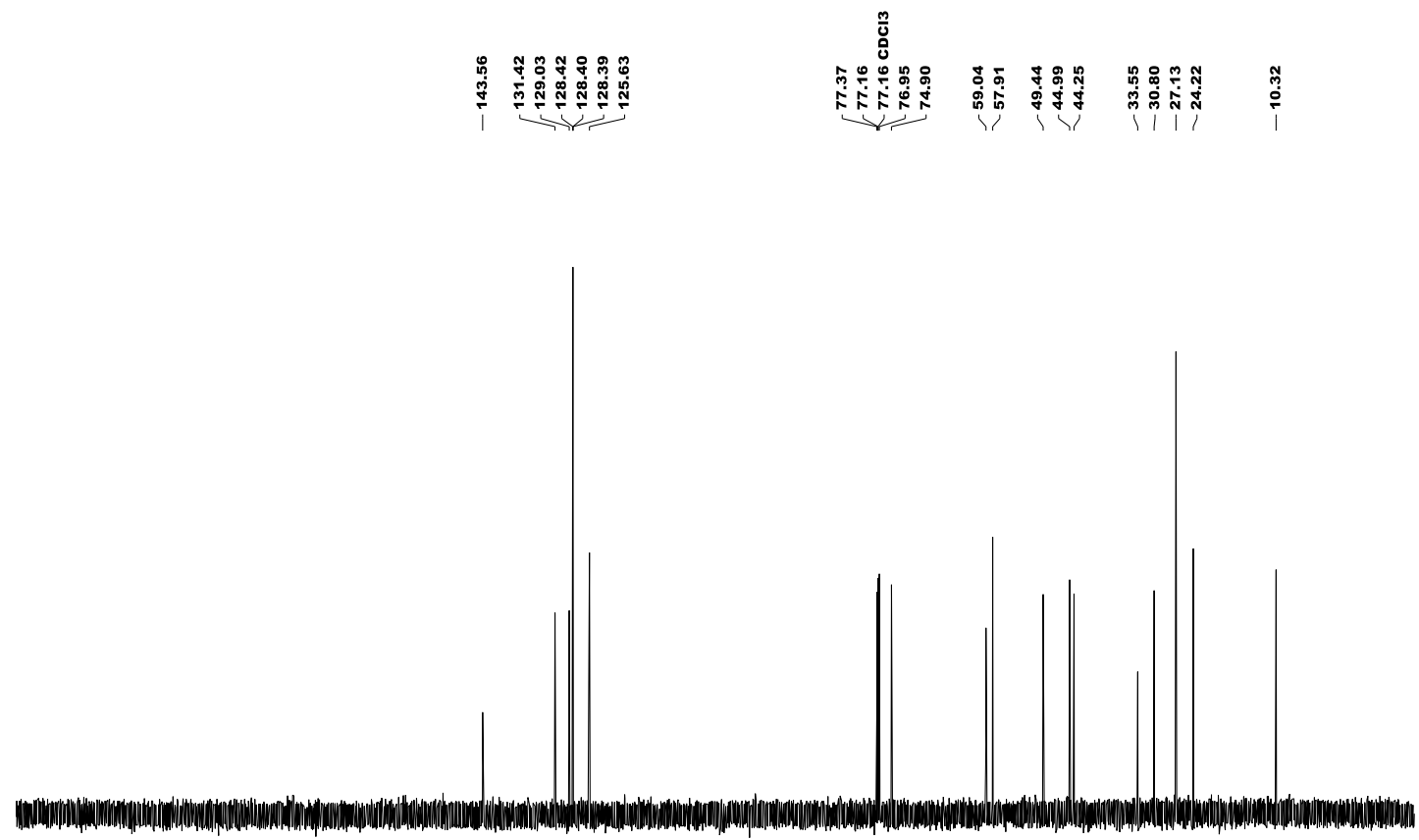

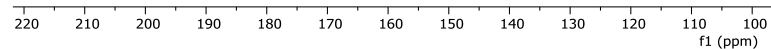


${ }^{1} \mathrm{H}$ NMR (599 MHz, $\mathrm{CDCl}_{3}$ )
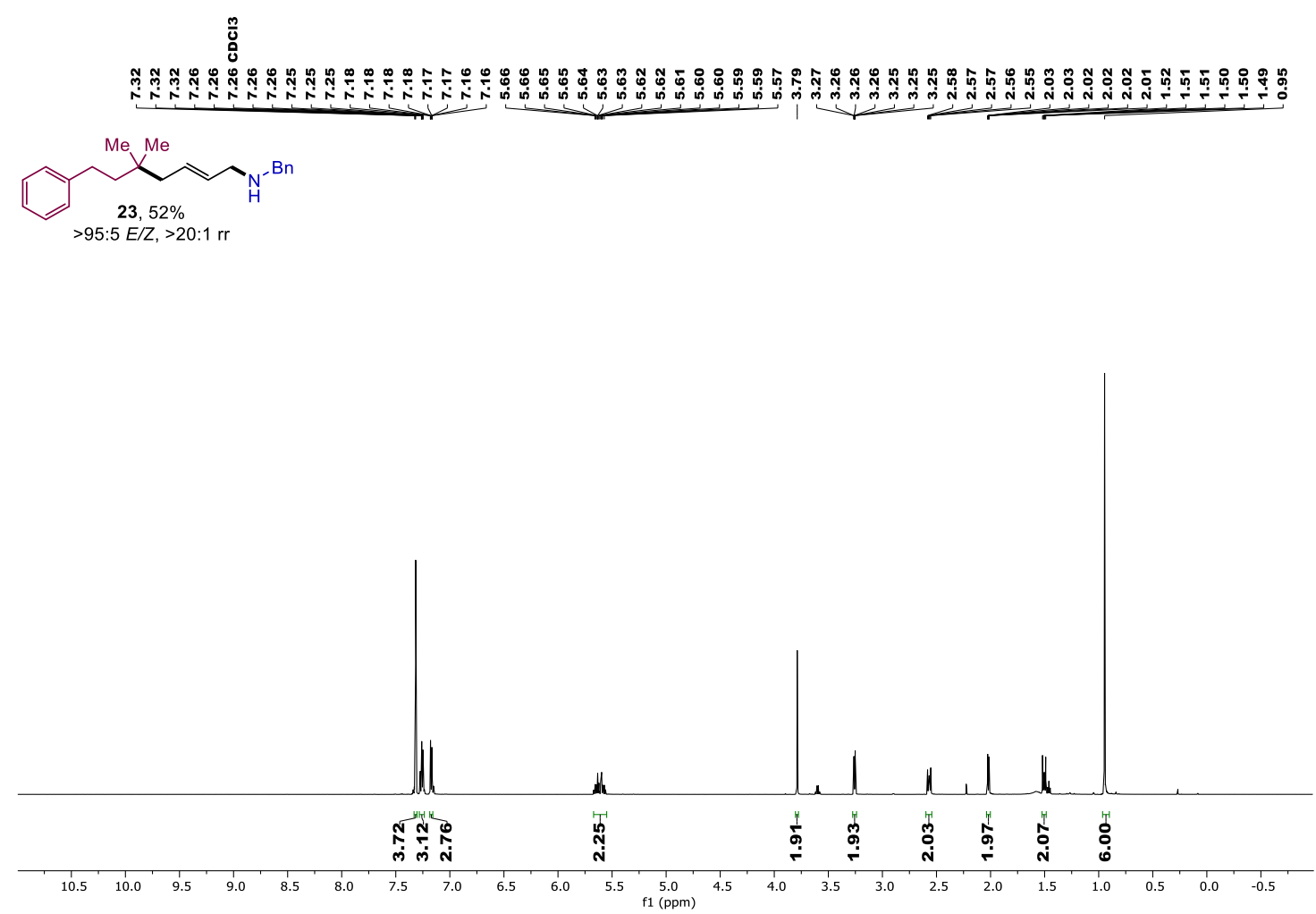

${ }^{13} \mathrm{C}$ NMR (151 MHz, $\left.\mathrm{CDCl}_{3}\right)$
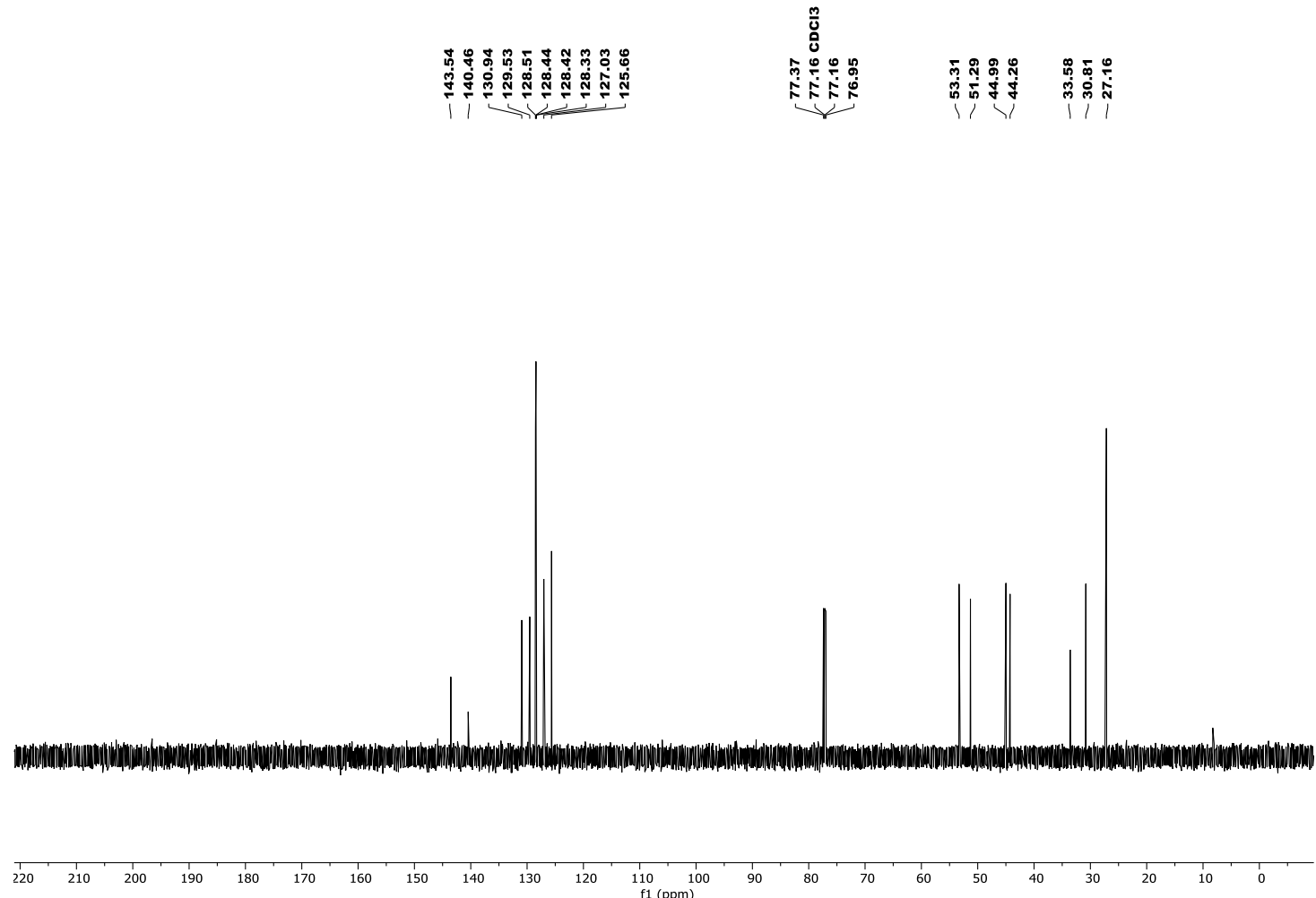
${ }^{1} \mathrm{H}$ NMR (599 MHz, $\mathrm{CDCl}_{3}$ )
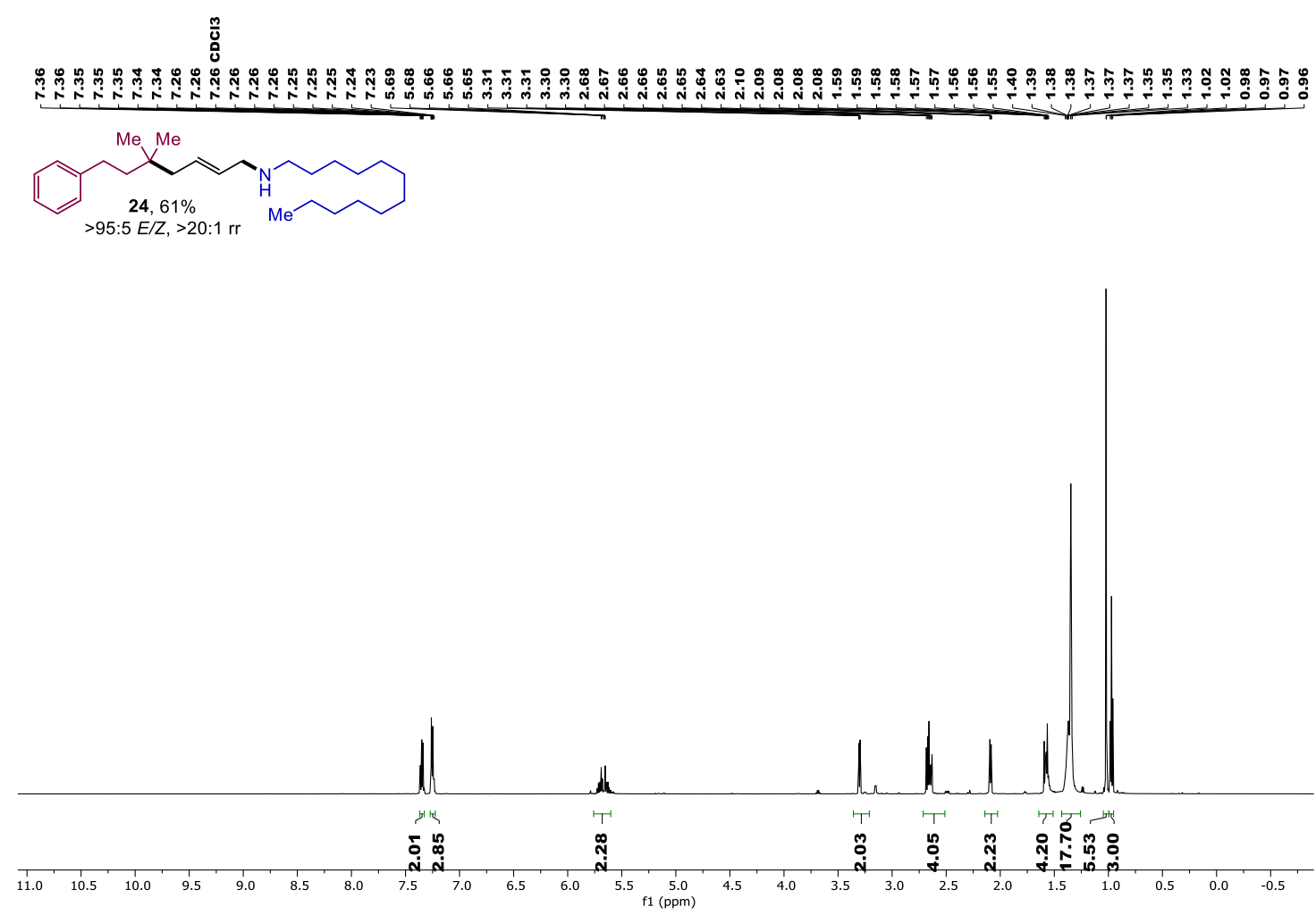

${ }^{13} \mathrm{C}$ NMR $\left(151 \mathrm{MHz}, \mathrm{CDCl}_{3}\right)$
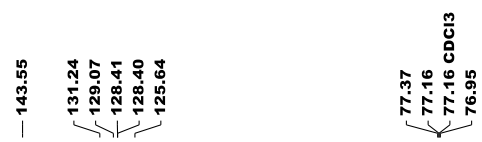

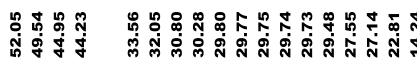

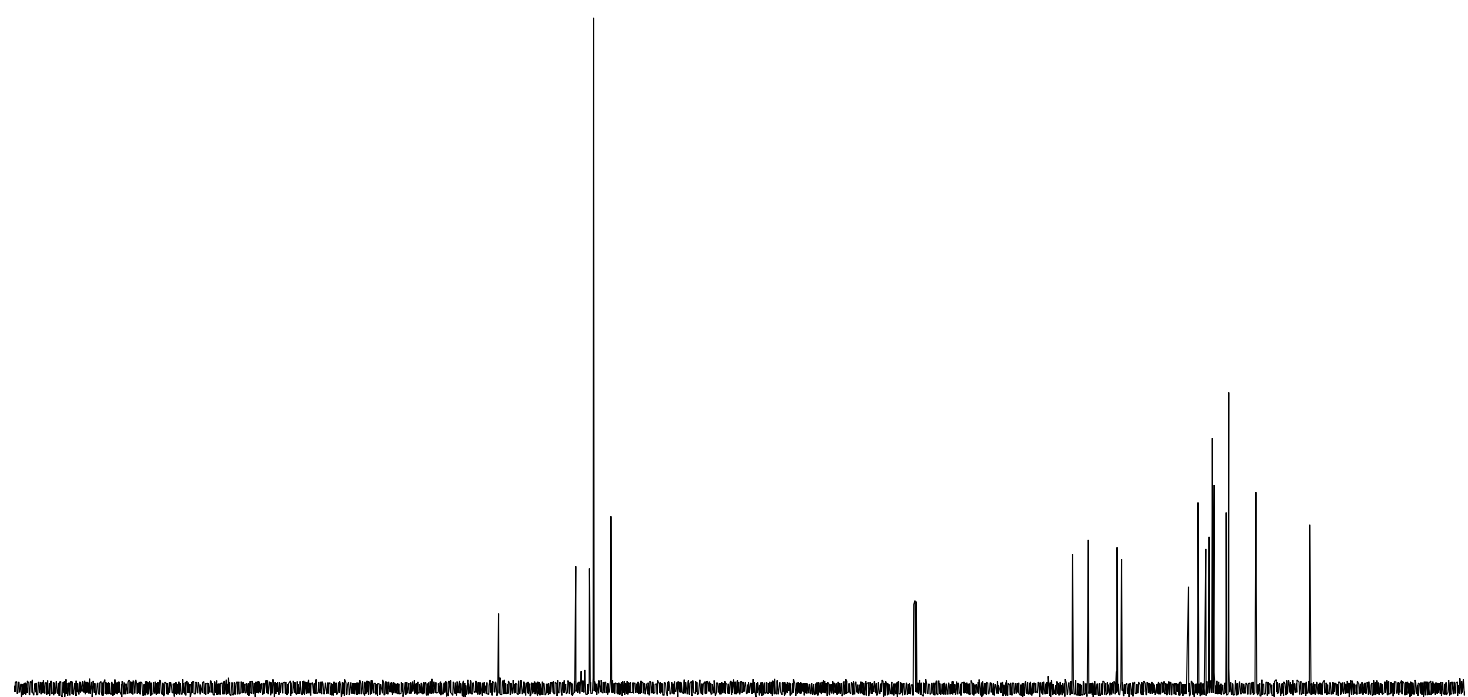

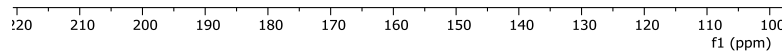


${ }^{1} \mathrm{H} \mathrm{NMR}\left(599 \mathrm{MHz}, \mathrm{CDCl}_{3}\right)$

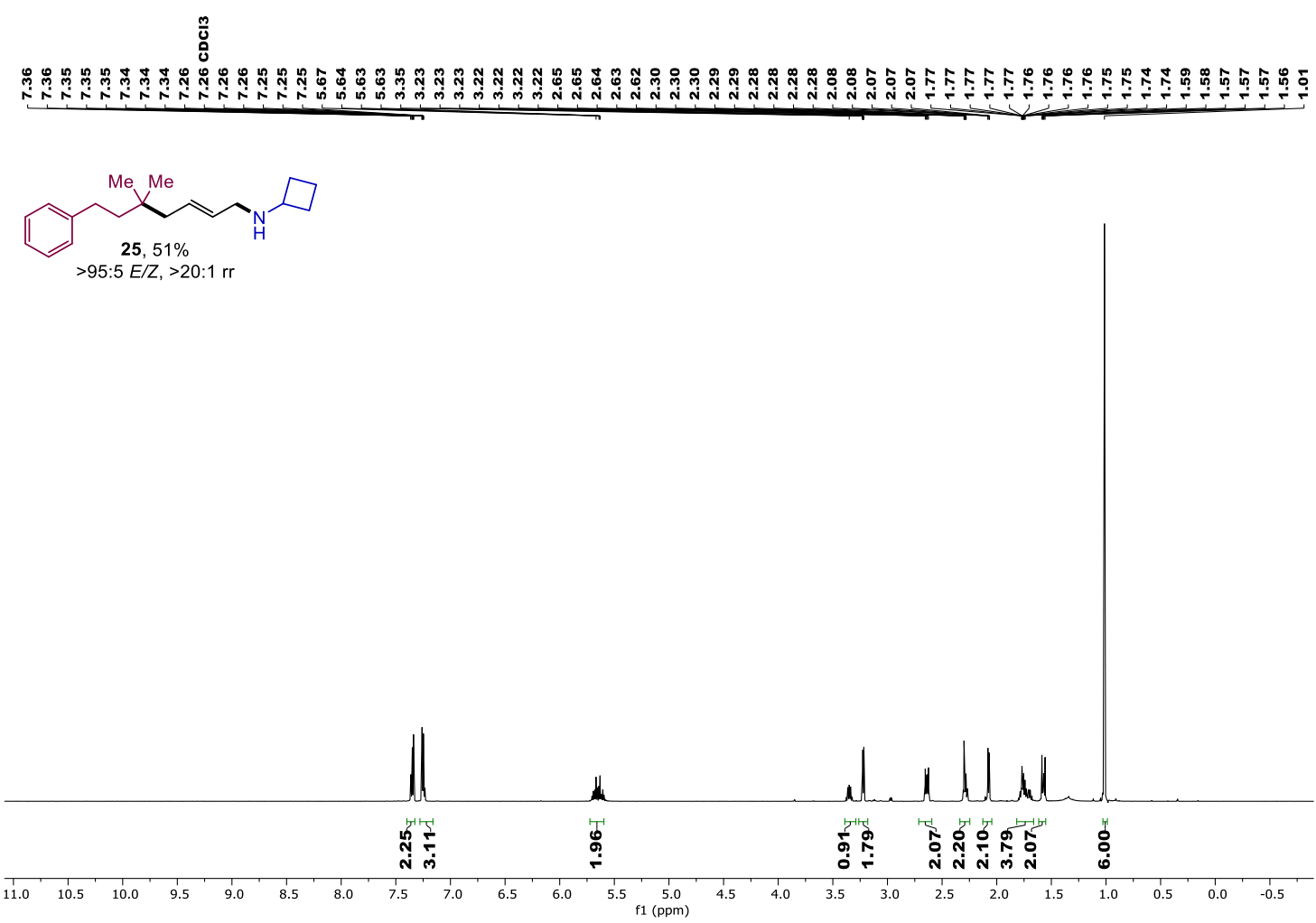

${ }^{13} \mathrm{C}$ NMR (151 MHz, $\mathrm{CDCl}_{3}$ )

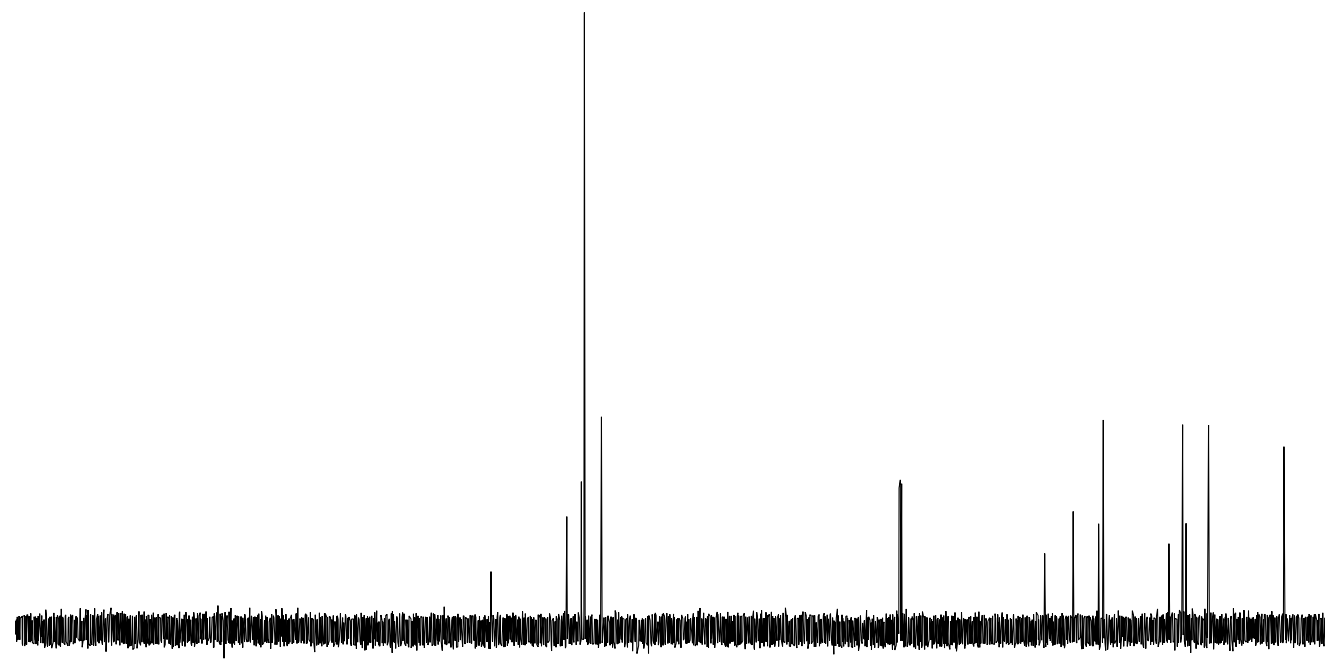

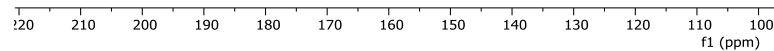


${ }^{1} \mathrm{H}$ NMR $\left(599 \mathrm{MHz}, \mathrm{CDCl}_{3}\right)$
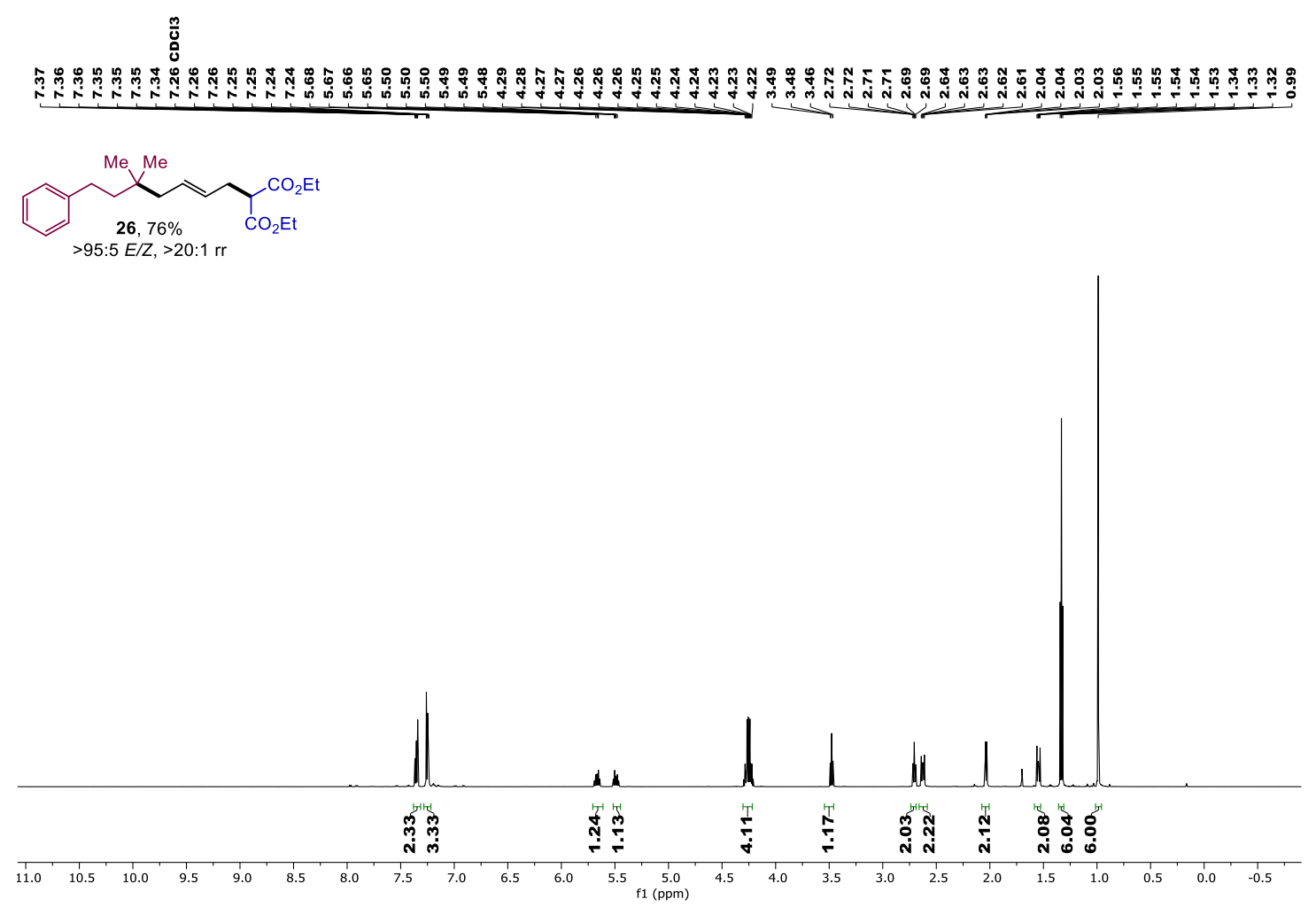

${ }^{13} \mathrm{C}$ NMR $\left(151 \mathrm{MHz}, \mathrm{CDCl}_{3}\right)$
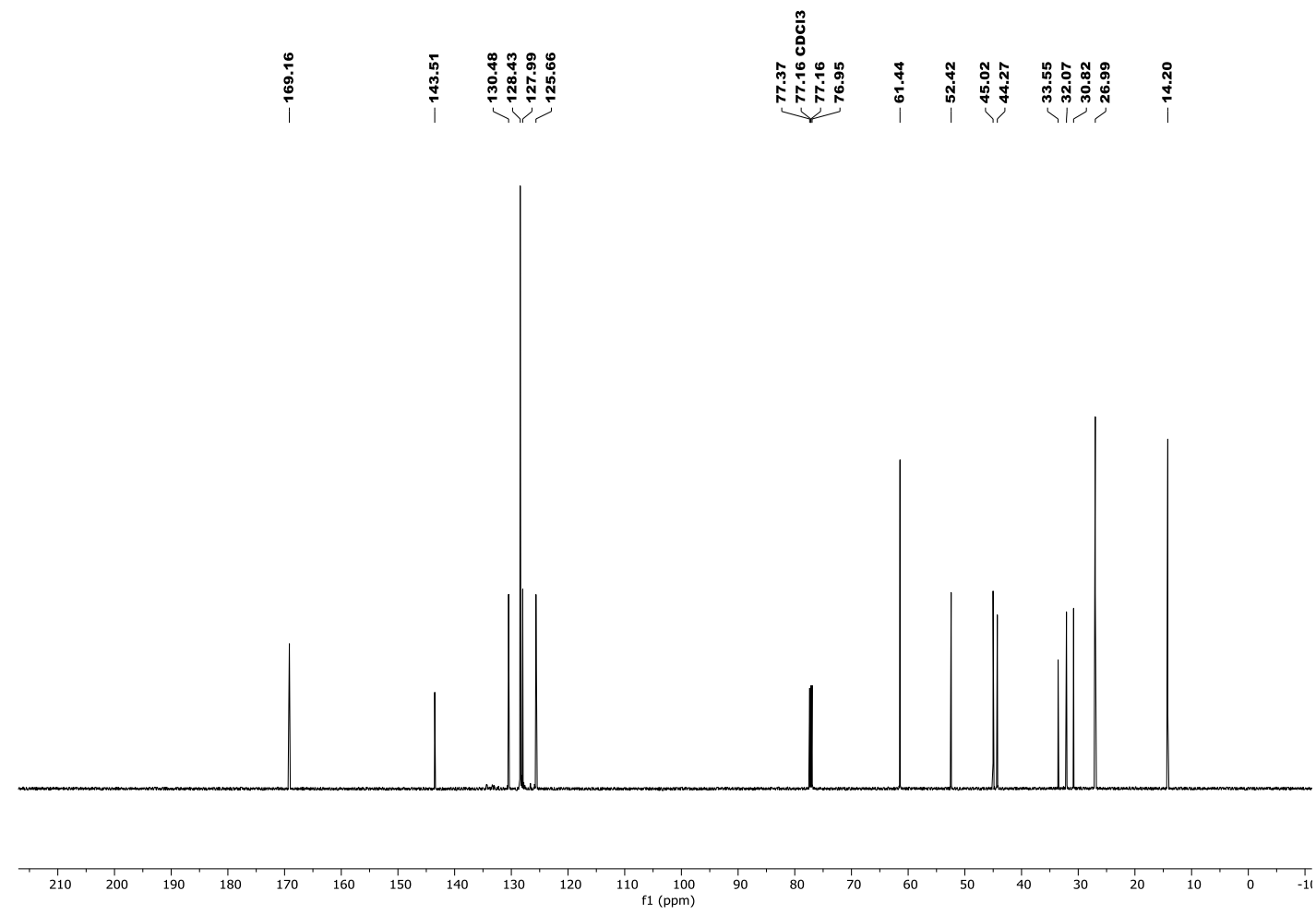
${ }^{1} \mathrm{H}$ NMR $\left(400 \mathrm{MHz}, \mathrm{CDCl}_{3}\right)$

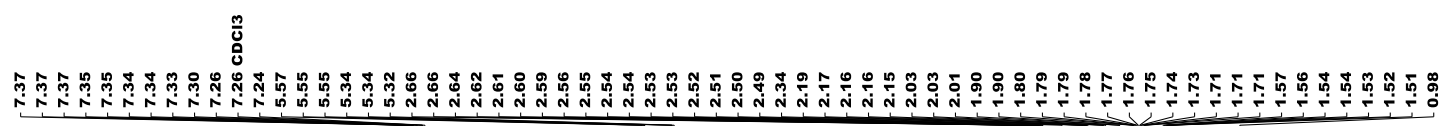
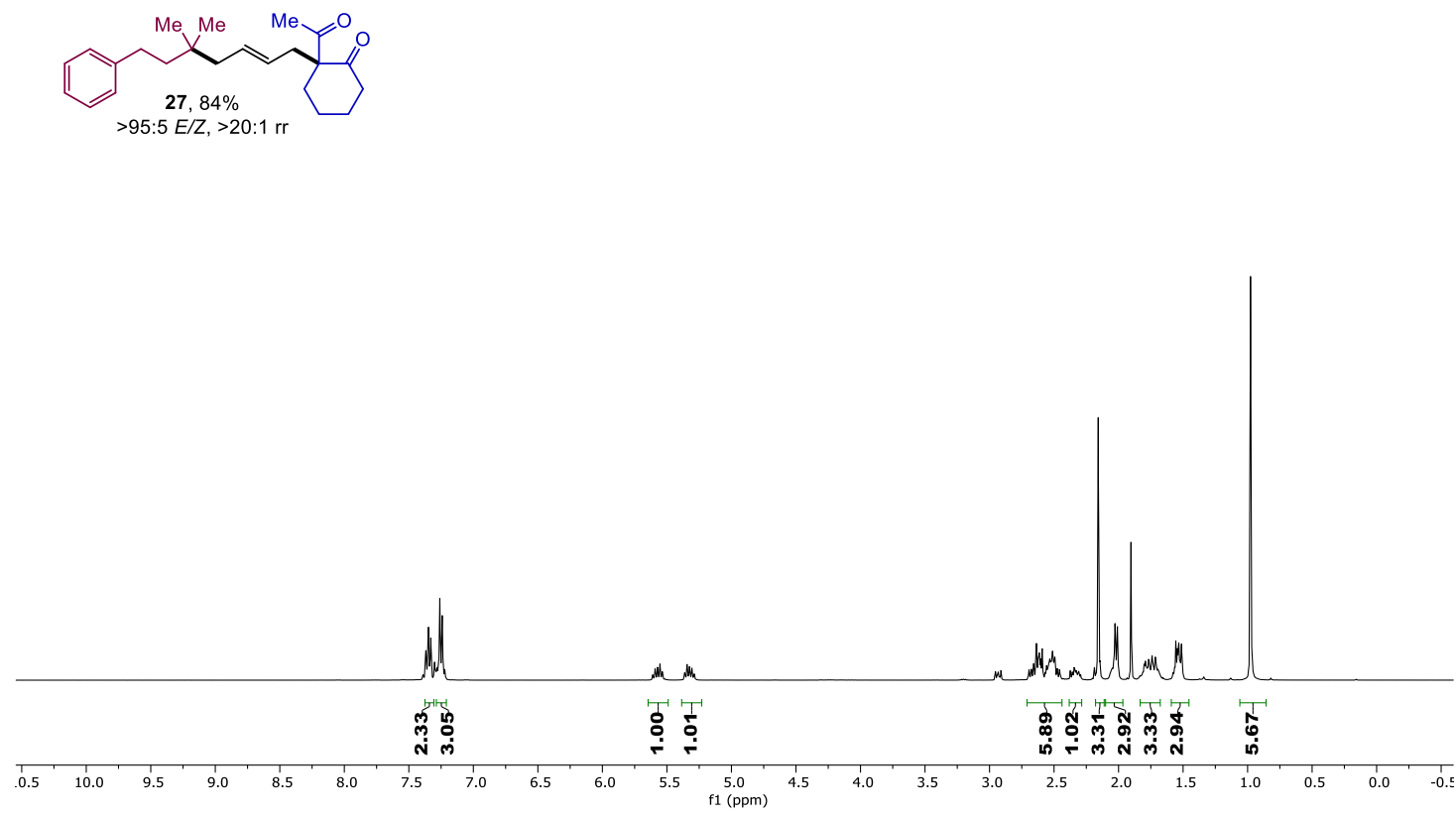

${ }^{13} \mathrm{C}$ NMR (101 MHz, $\left.\mathrm{CDCl}_{3}\right)$

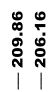

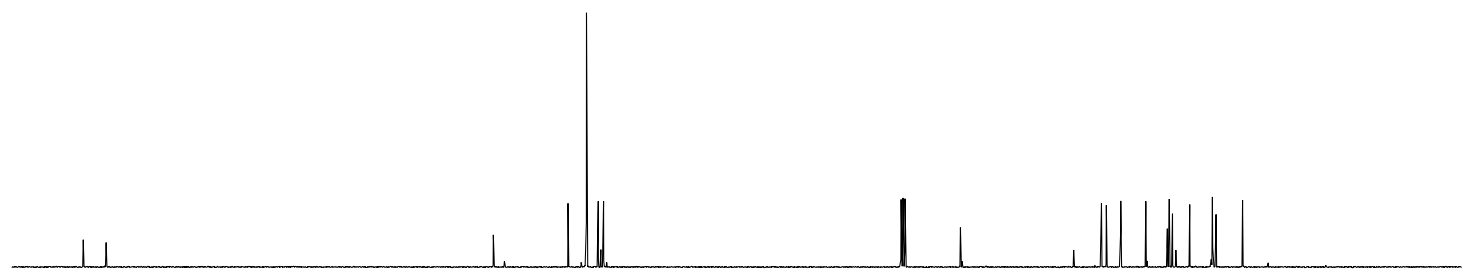

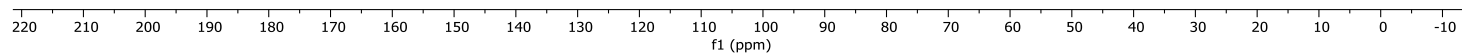


${ }^{1} \mathrm{H}$ NMR $\left(599 \mathrm{MHz}, \mathrm{CDCl}_{3}\right)$

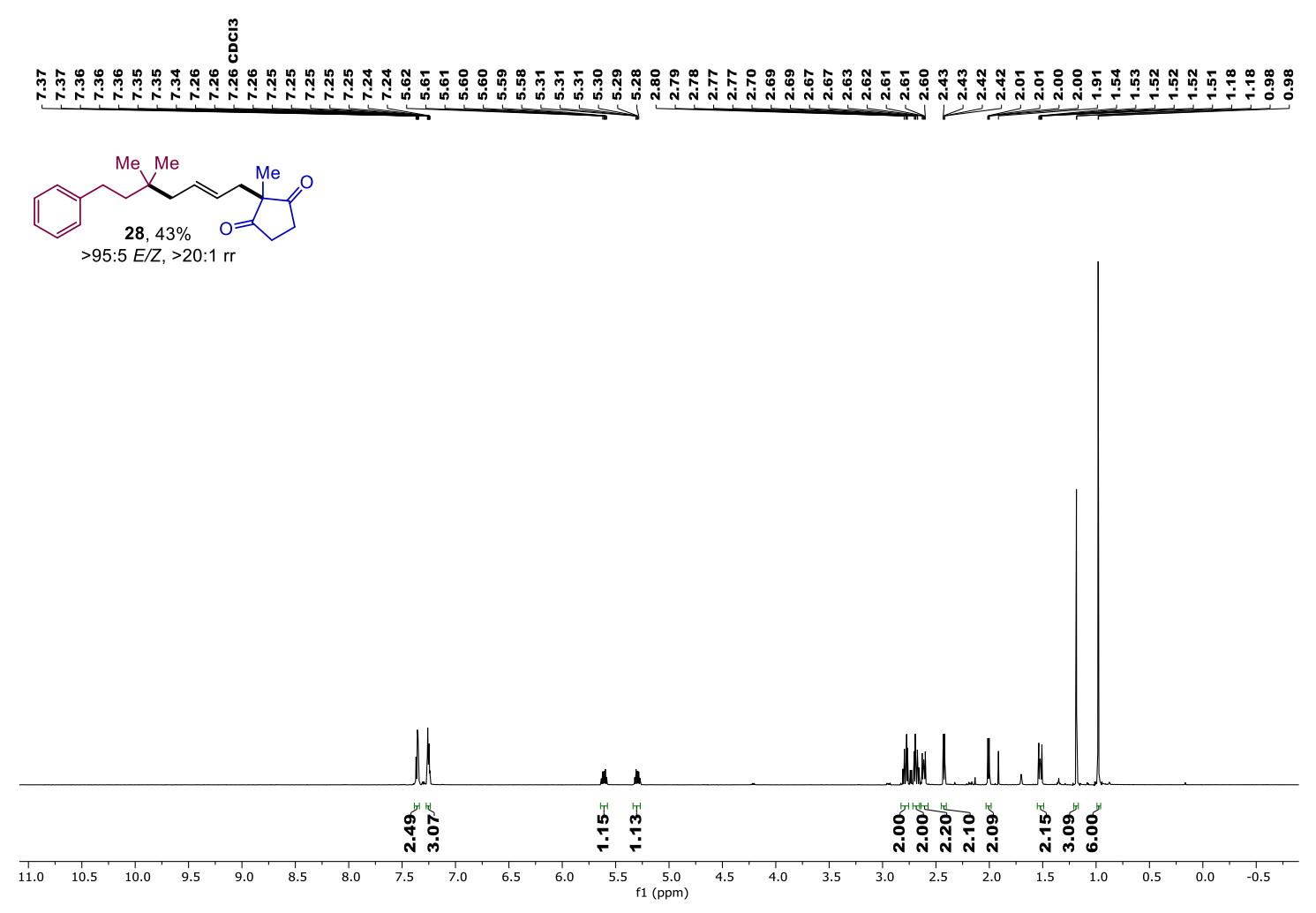

${ }^{13} \mathrm{C}$ NMR $\left(151 \mathrm{MHz}, \mathrm{CDCl}_{3}\right)$

ํํำ

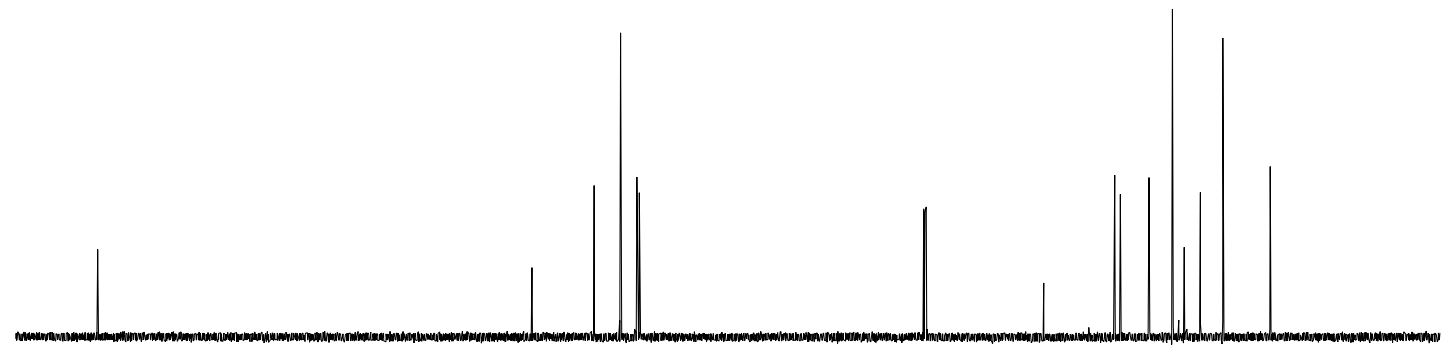

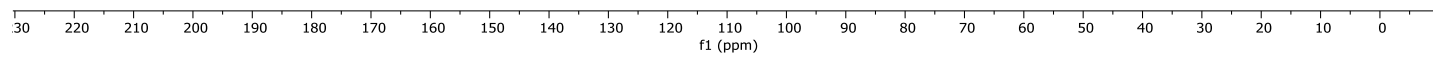


${ }^{1} \mathrm{H}$ NMR $\left(400 \mathrm{MHz}, \mathrm{CDCl}_{3}\right)$
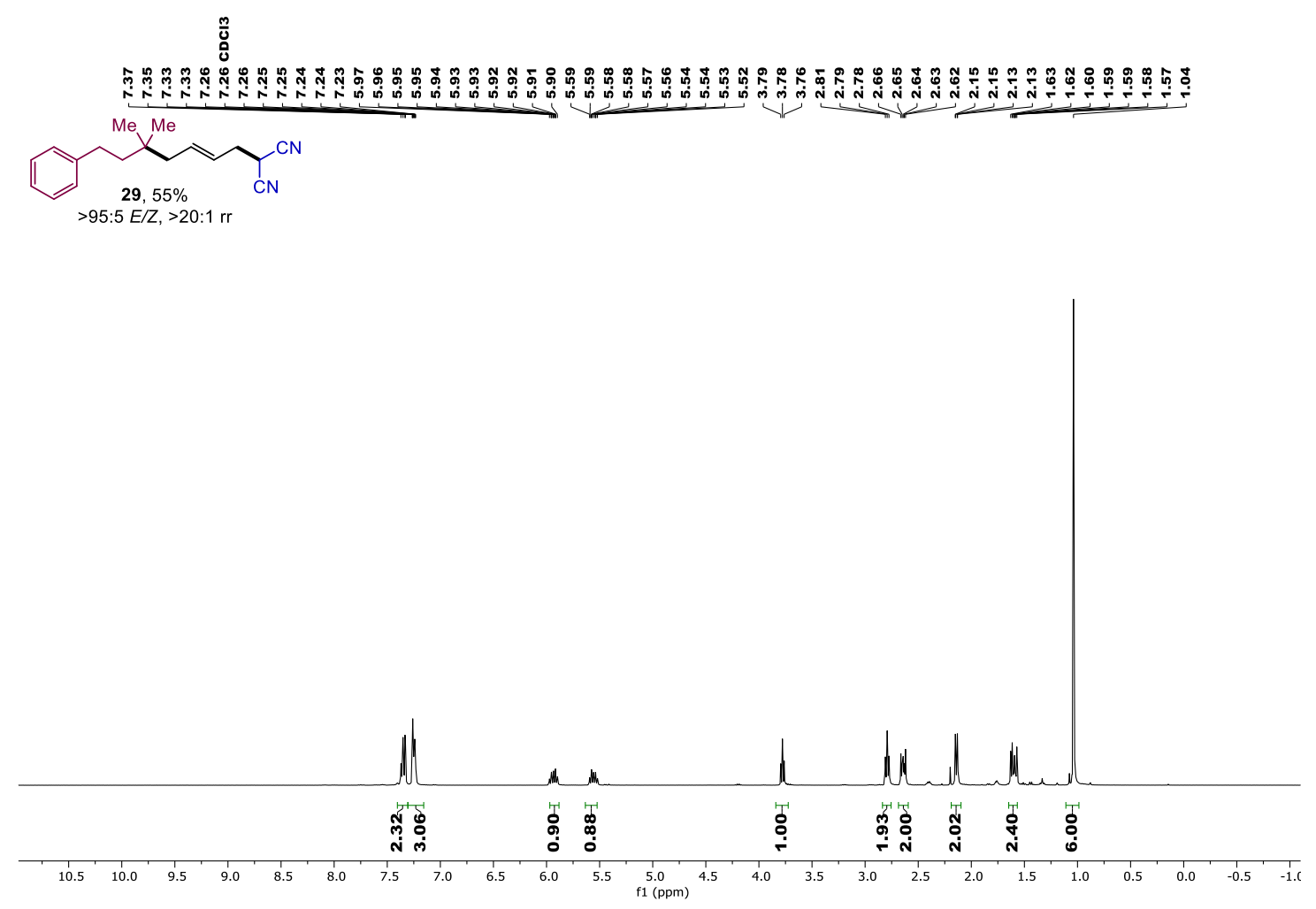

${ }^{13} \mathrm{C}$ NMR $\left(101 \mathrm{MHz}, \mathrm{CDCl}_{3}\right)$
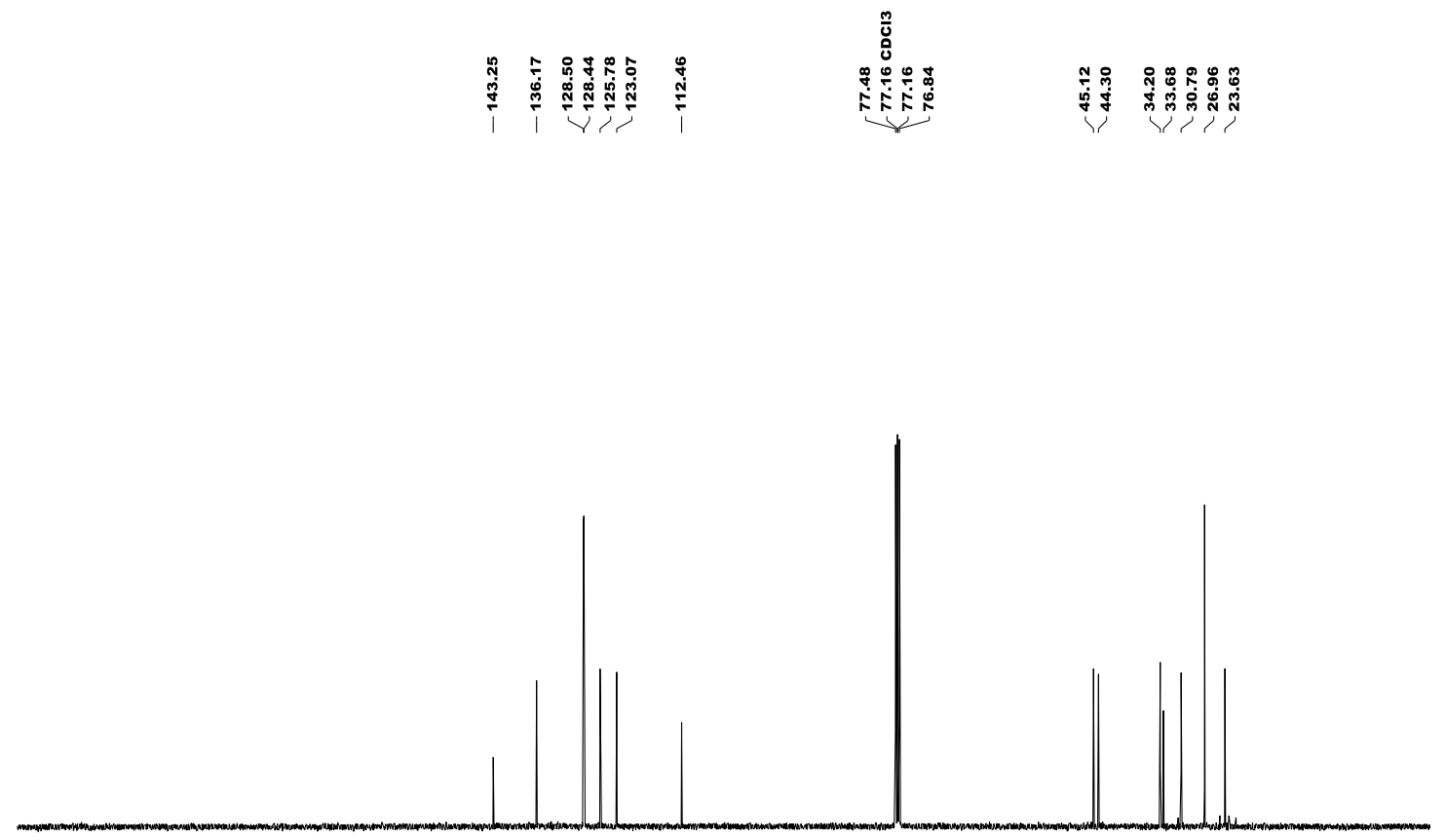

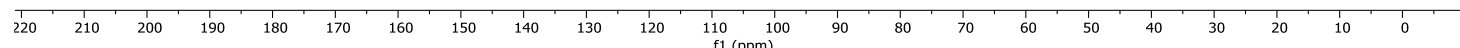


${ }^{1} \mathrm{H}$ NMR (599 MHz, $\mathrm{CDCl}_{3}$ )
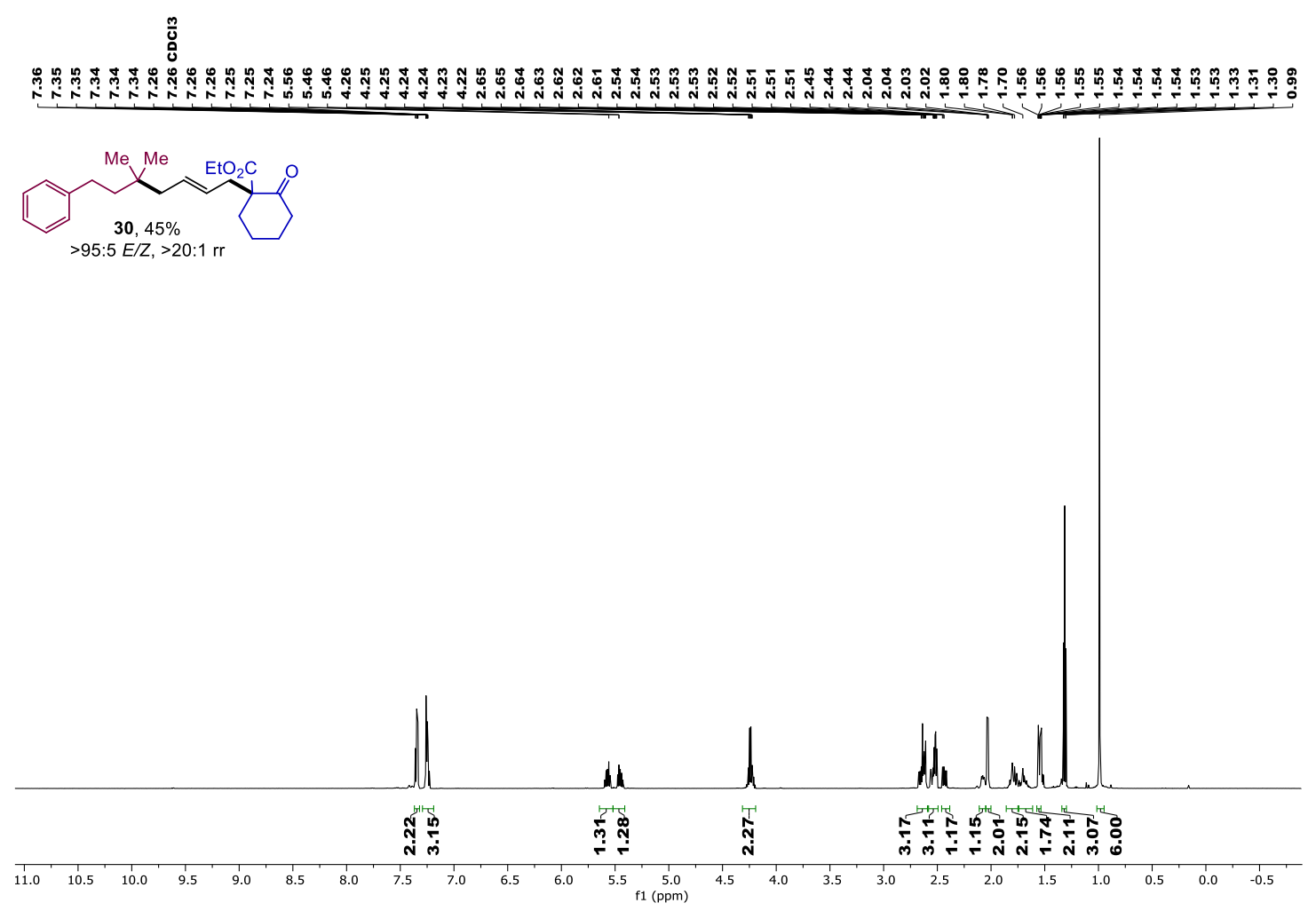

${ }^{13} \mathrm{C} \mathrm{NMR}\left(151 \mathrm{MHz}, \mathrm{CDCl}_{3}\right)$

望

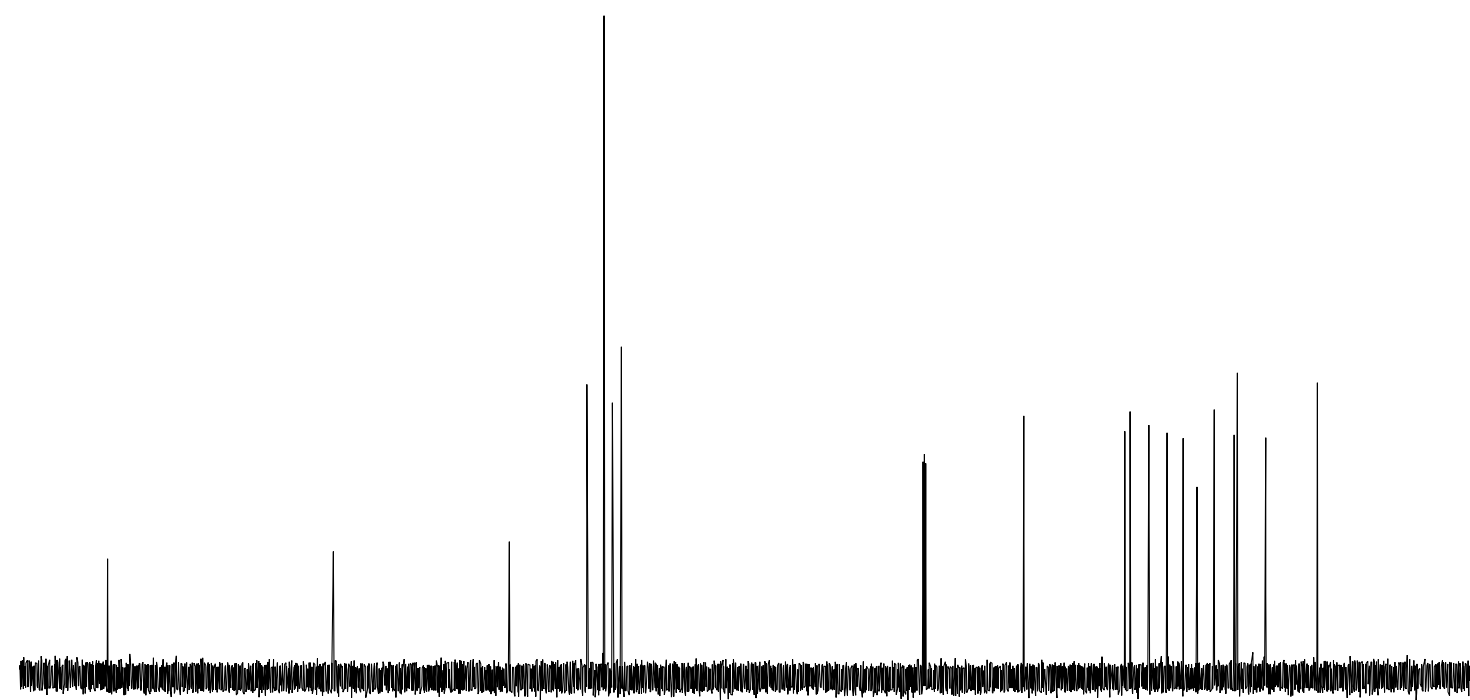

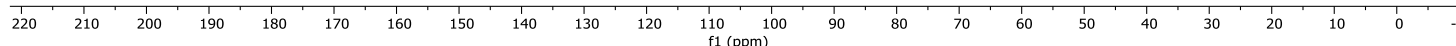


${ }^{1} \mathrm{H}$ NMR $\left(400 \mathrm{MHz}, \mathrm{CDCl}_{3}\right)$
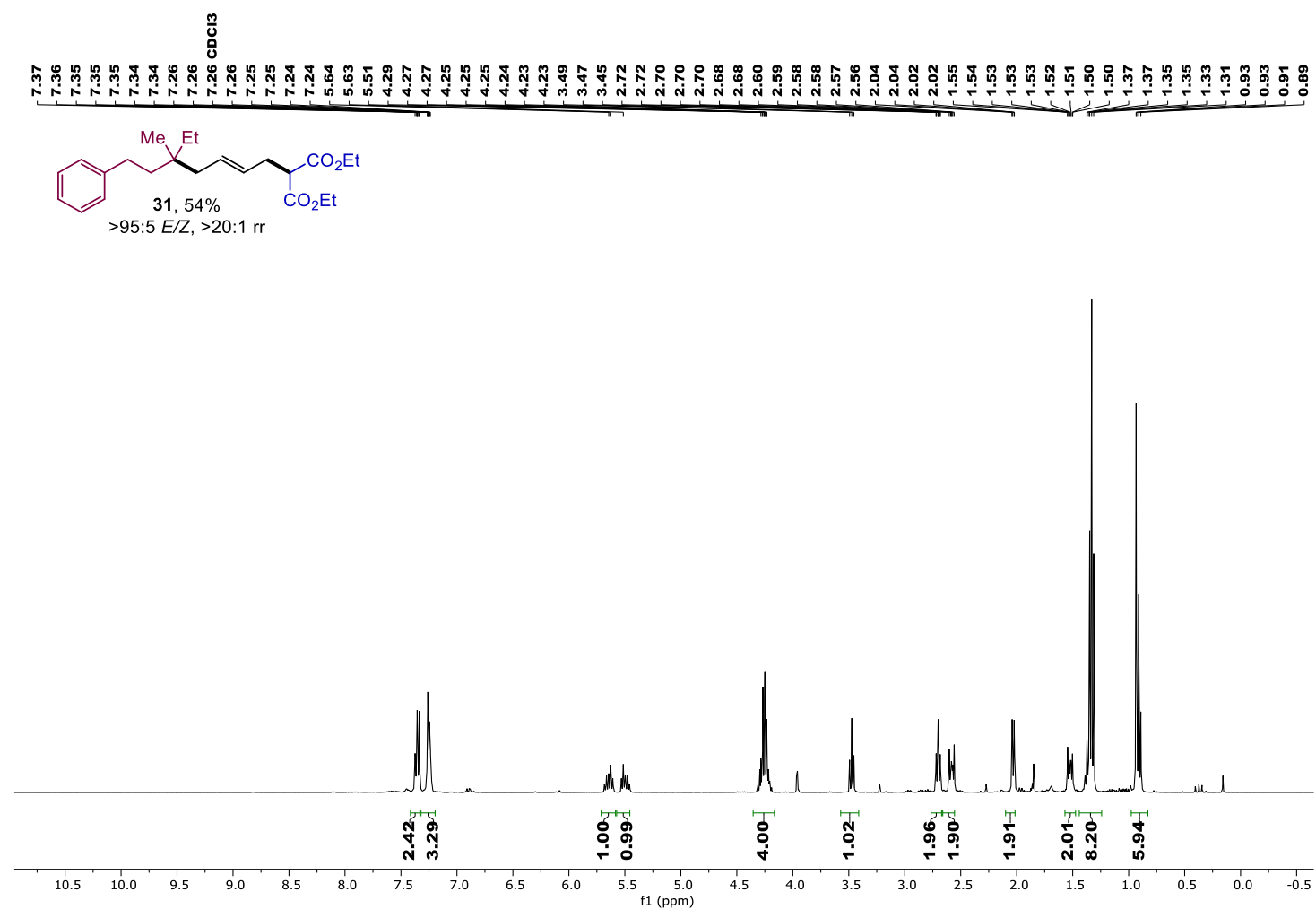

${ }^{13} \mathrm{C}$ NMR $\left(101 \mathrm{MHz}, \mathrm{CDCl}_{3}\right)$
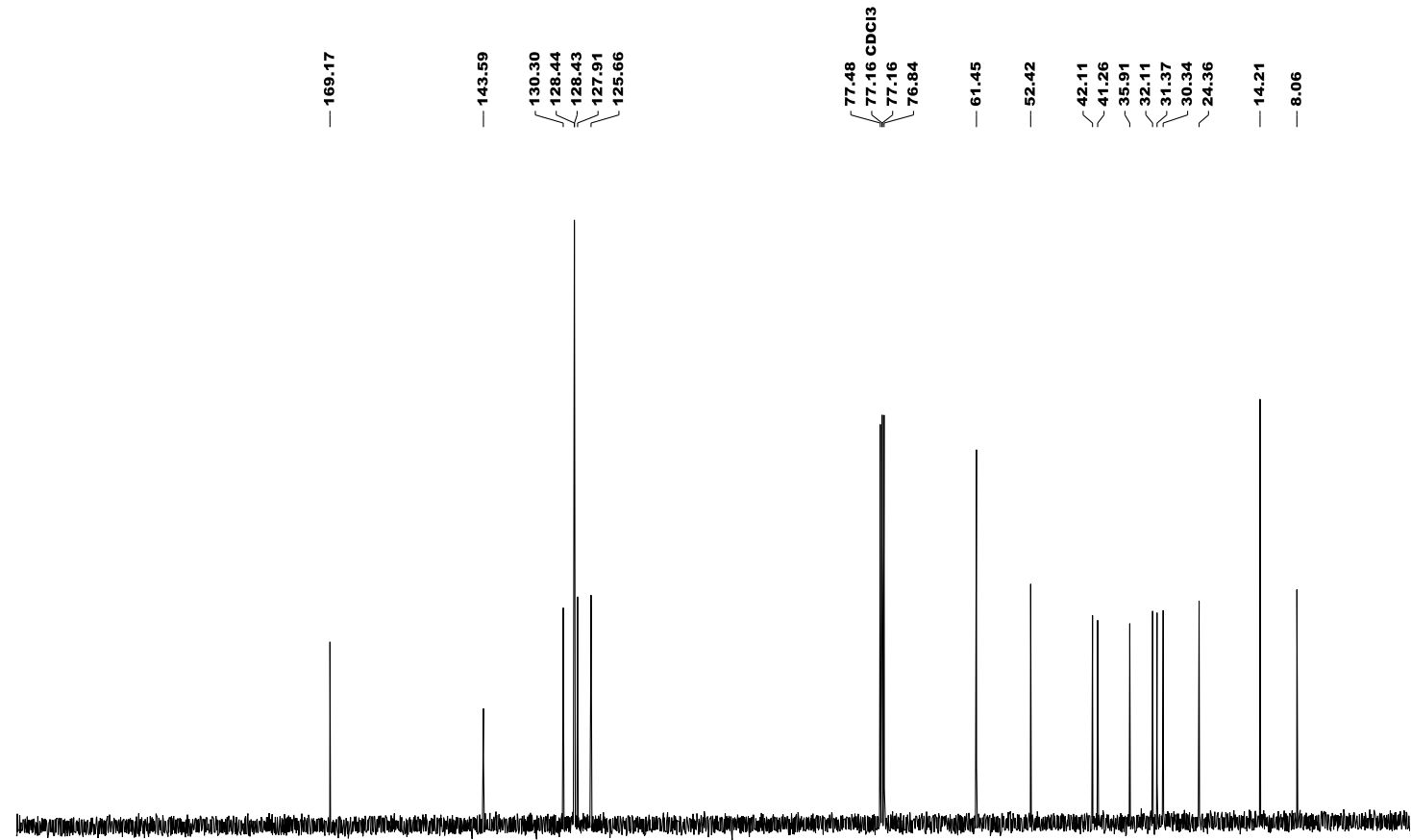

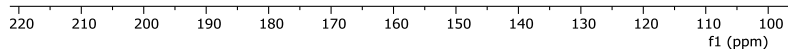


${ }^{1} \mathrm{H}$ NMR $\left(599 \mathrm{MHz}, \mathrm{CDCl}_{3}\right)$
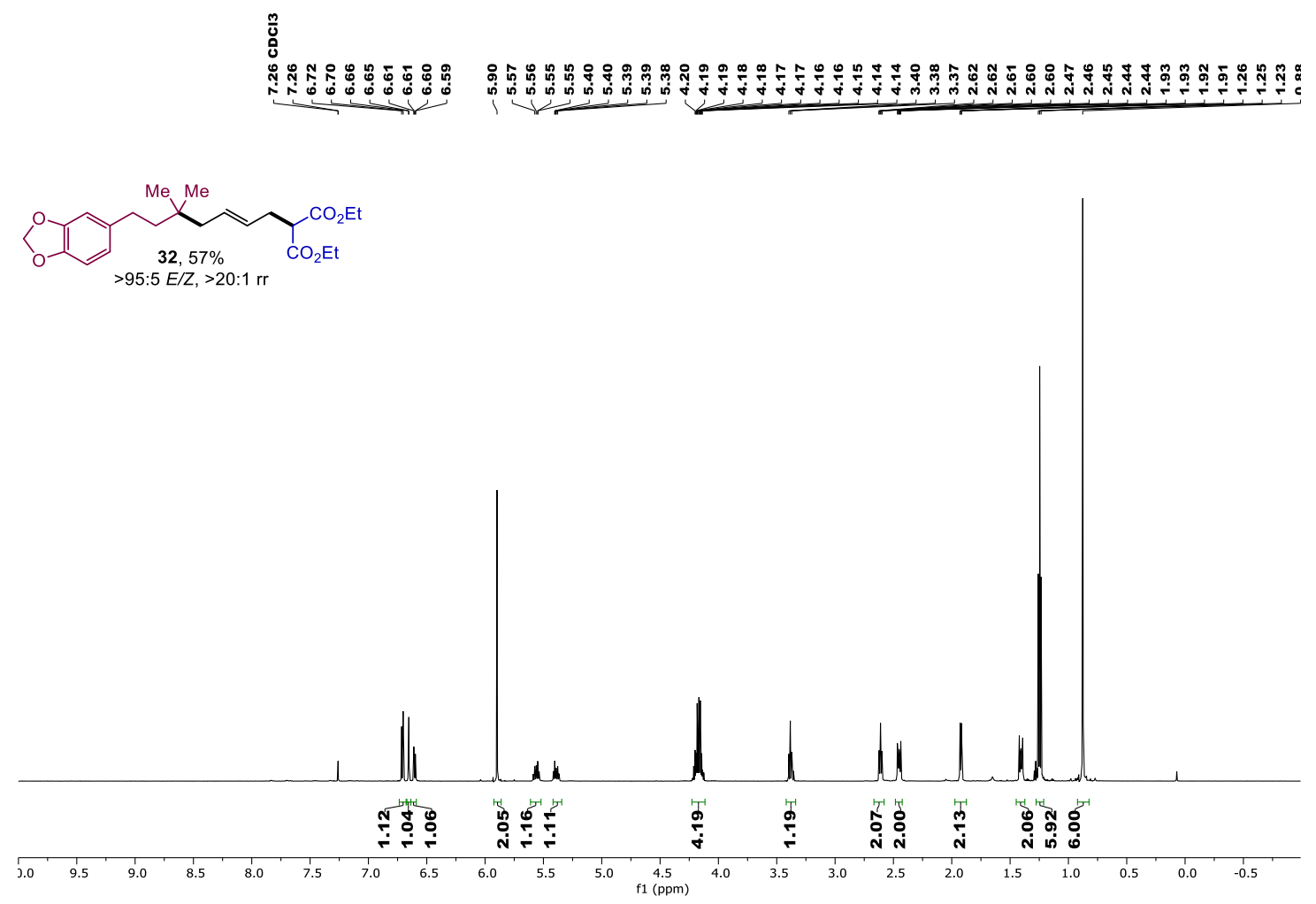

${ }^{13} \mathrm{C}$ NMR (151 MHz, $\mathrm{CDCl}_{3}$ )
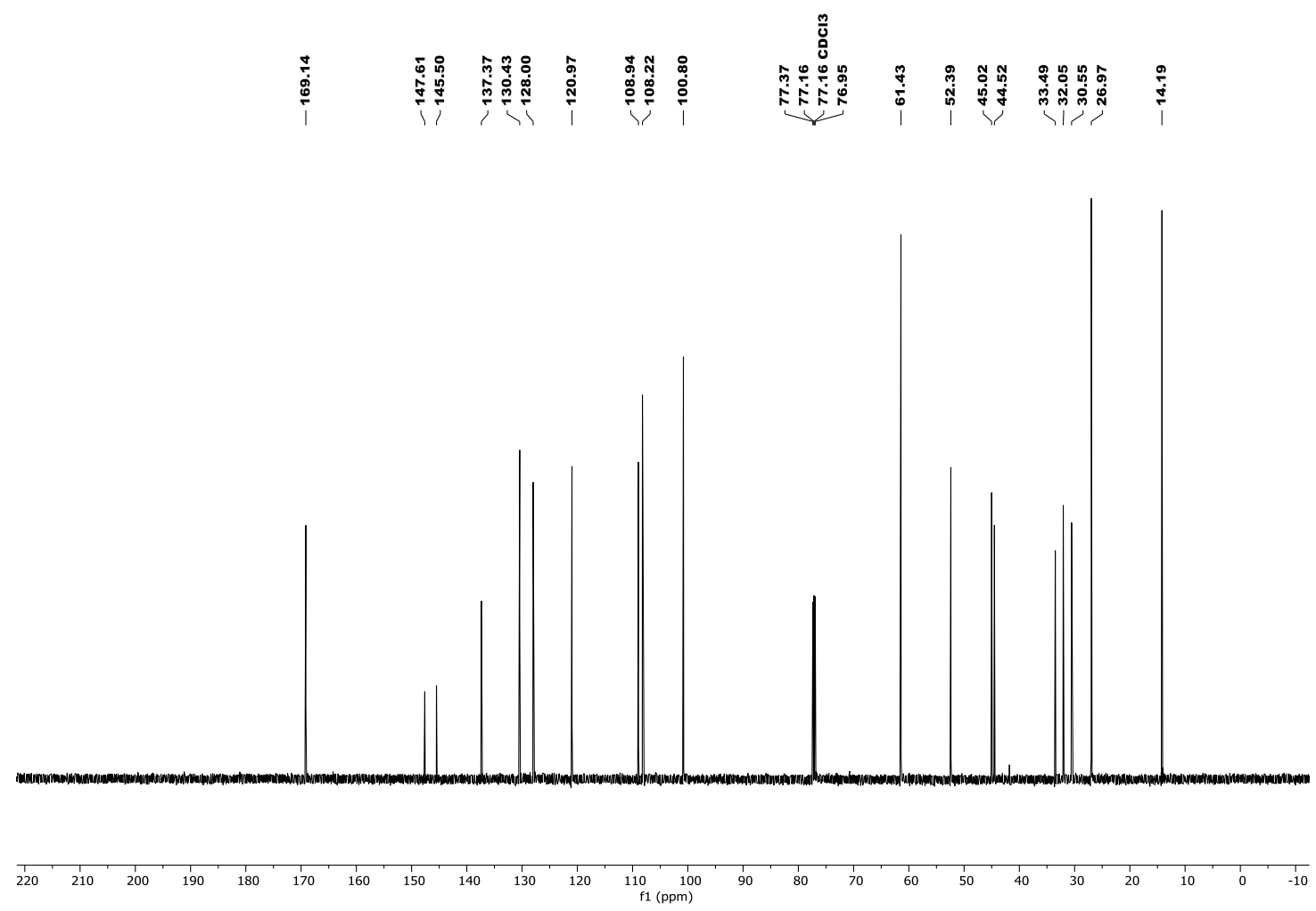
${ }^{1} \mathrm{H}$ NMR $\left(599 \mathrm{MHz}, \mathrm{CDCl}_{3}\right)$
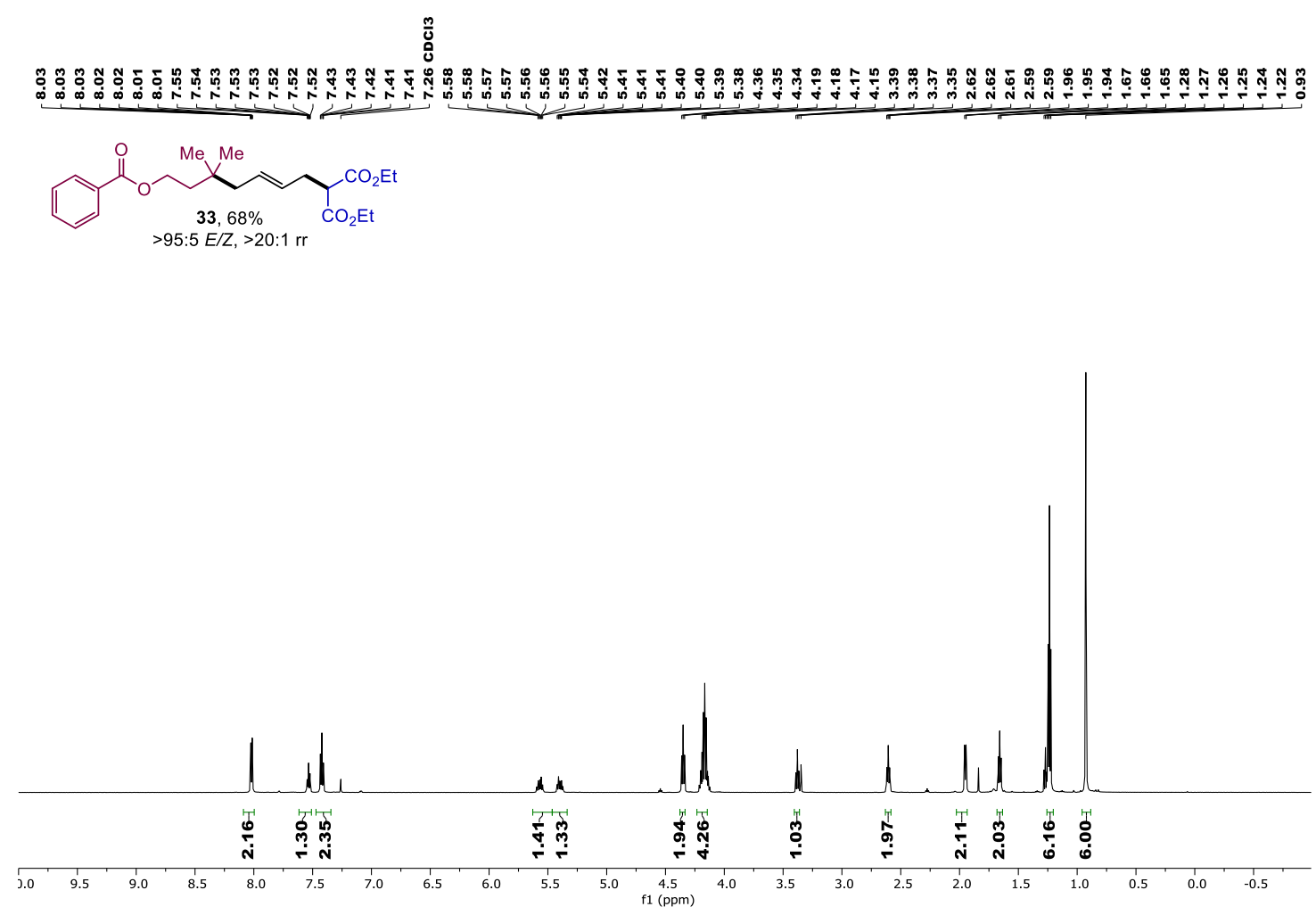

${ }^{13} \mathrm{C}$ NMR (151 MHz, $\mathrm{CDCl}_{3}$ )

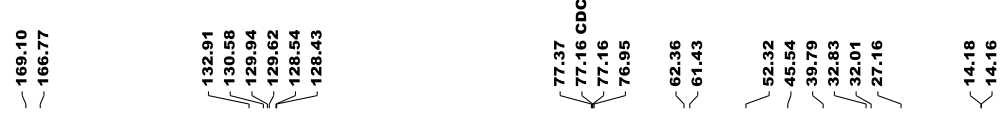

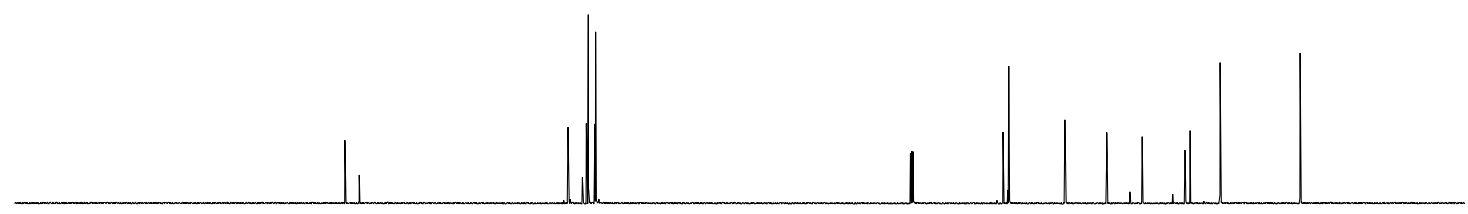

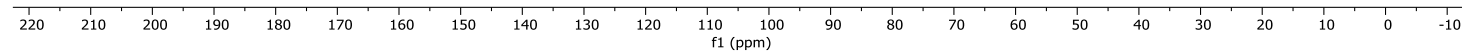


${ }^{1} \mathrm{H}$ NMR (599 MHz, $\mathrm{CDCl}_{3}$ )

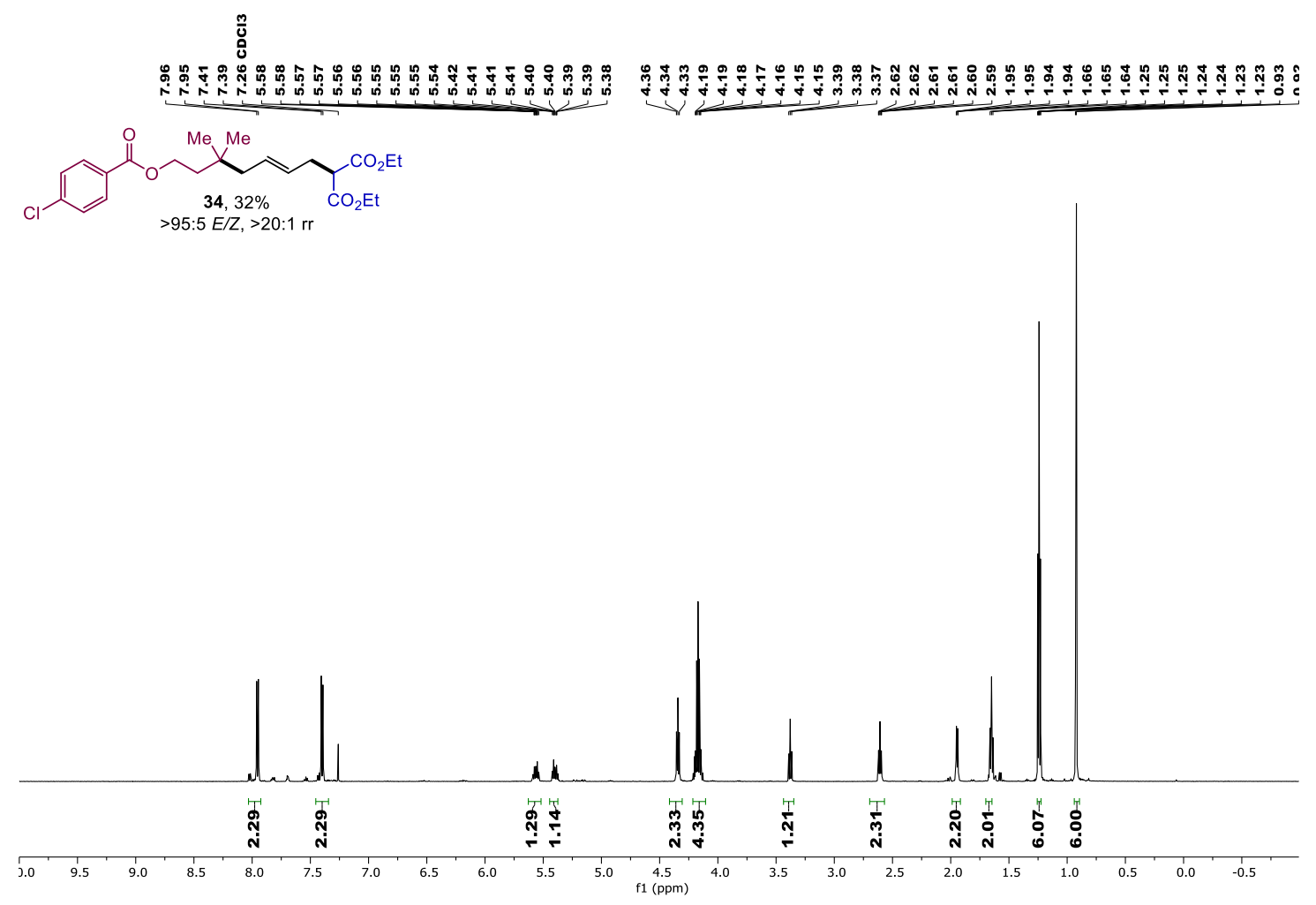

${ }^{13} \mathrm{C}$ NMR (151 MHz, $\mathrm{CDCl}_{3}$ )

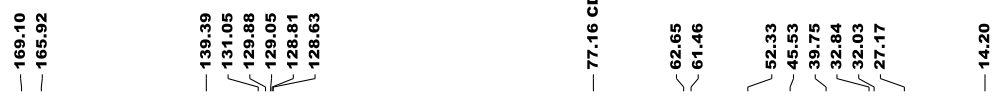

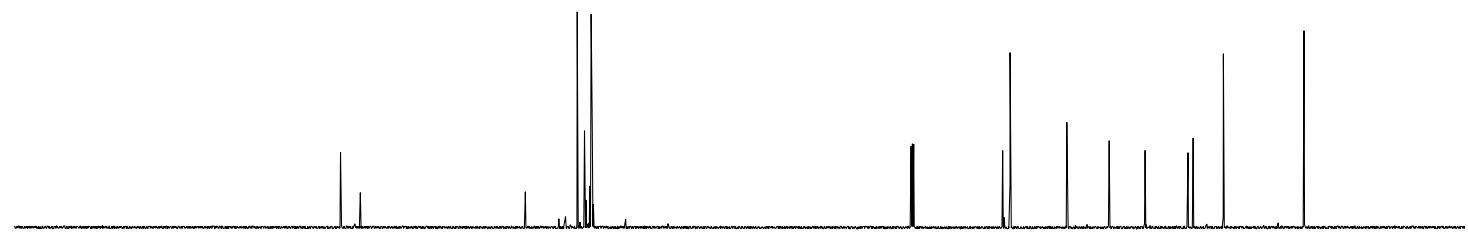

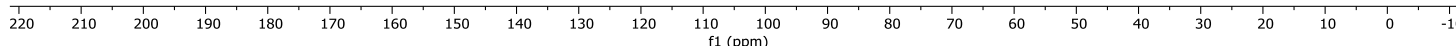


${ }^{1} \mathrm{H}$ NMR $\left(300 \mathrm{MHz}, \mathrm{CDCl}_{3}\right)$

$\frac{m}{0}$

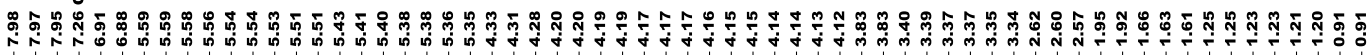
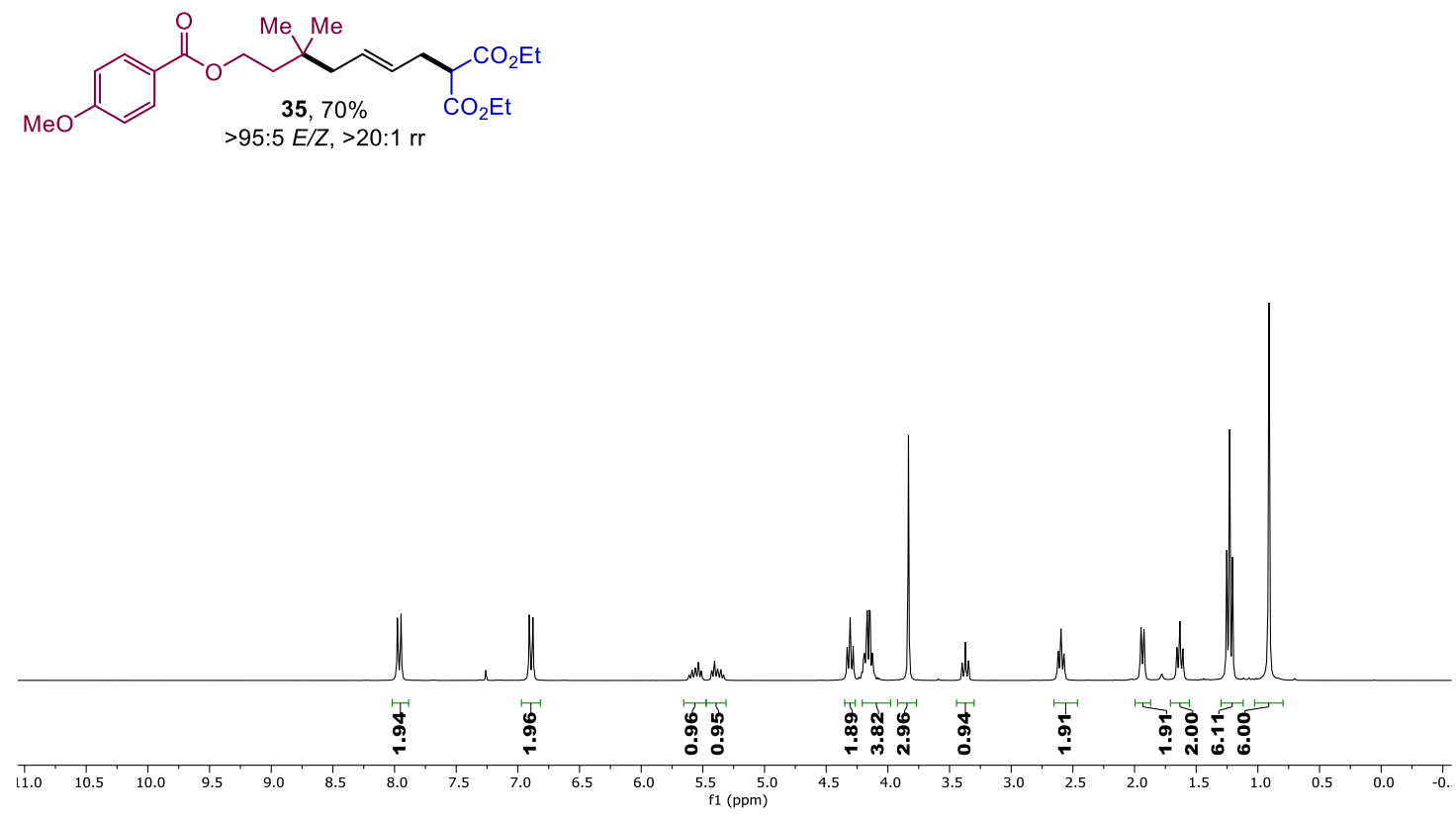

${ }^{13} \mathrm{C}$ NMR $\left(75 \mathrm{MHz}, \mathrm{CDCl}_{3}\right.$ )

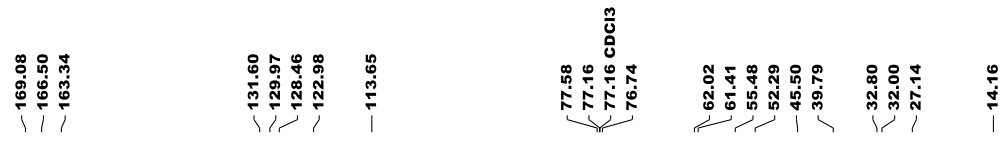

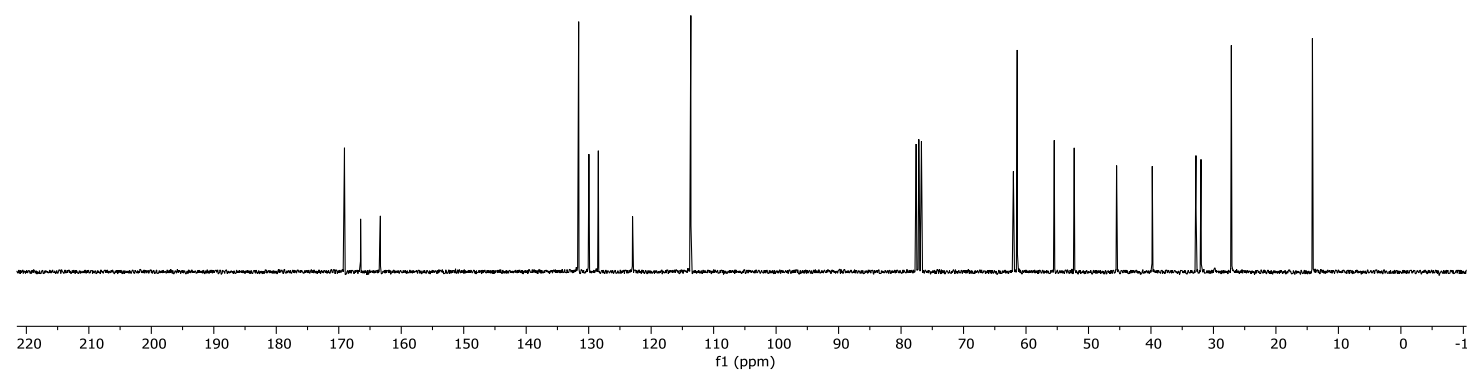


${ }^{1} \mathrm{H}$ NMR $\left(300 \mathrm{MHz}, \mathrm{CDCl}_{3}\right)$

\section{\%

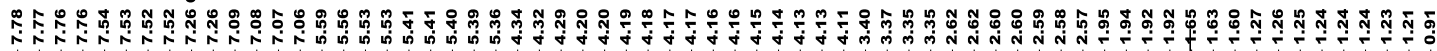
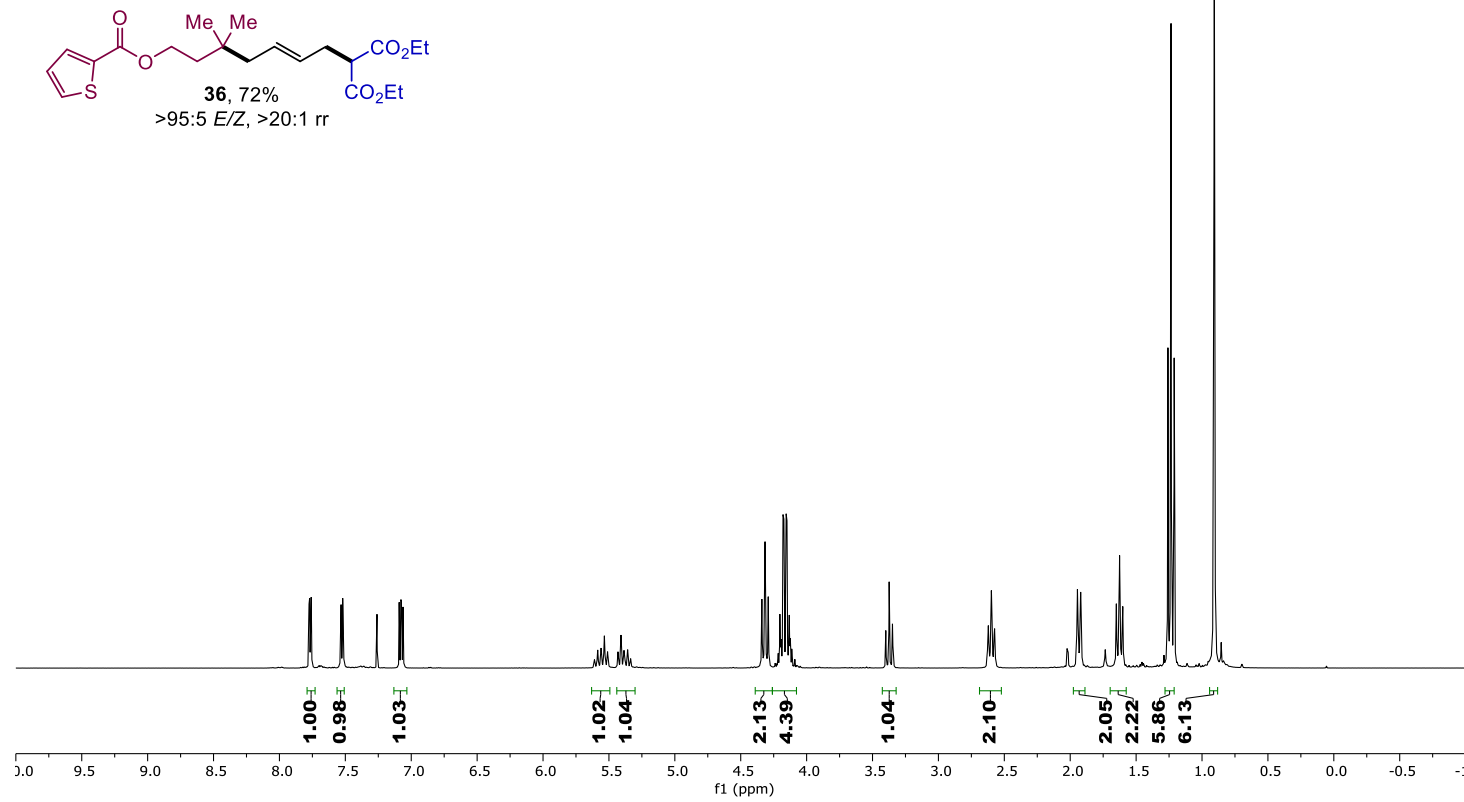

${ }^{13} \mathrm{C} \mathrm{NMR}\left(75 \mathrm{MHz}, \mathrm{CDCl}_{3}\right)$

I
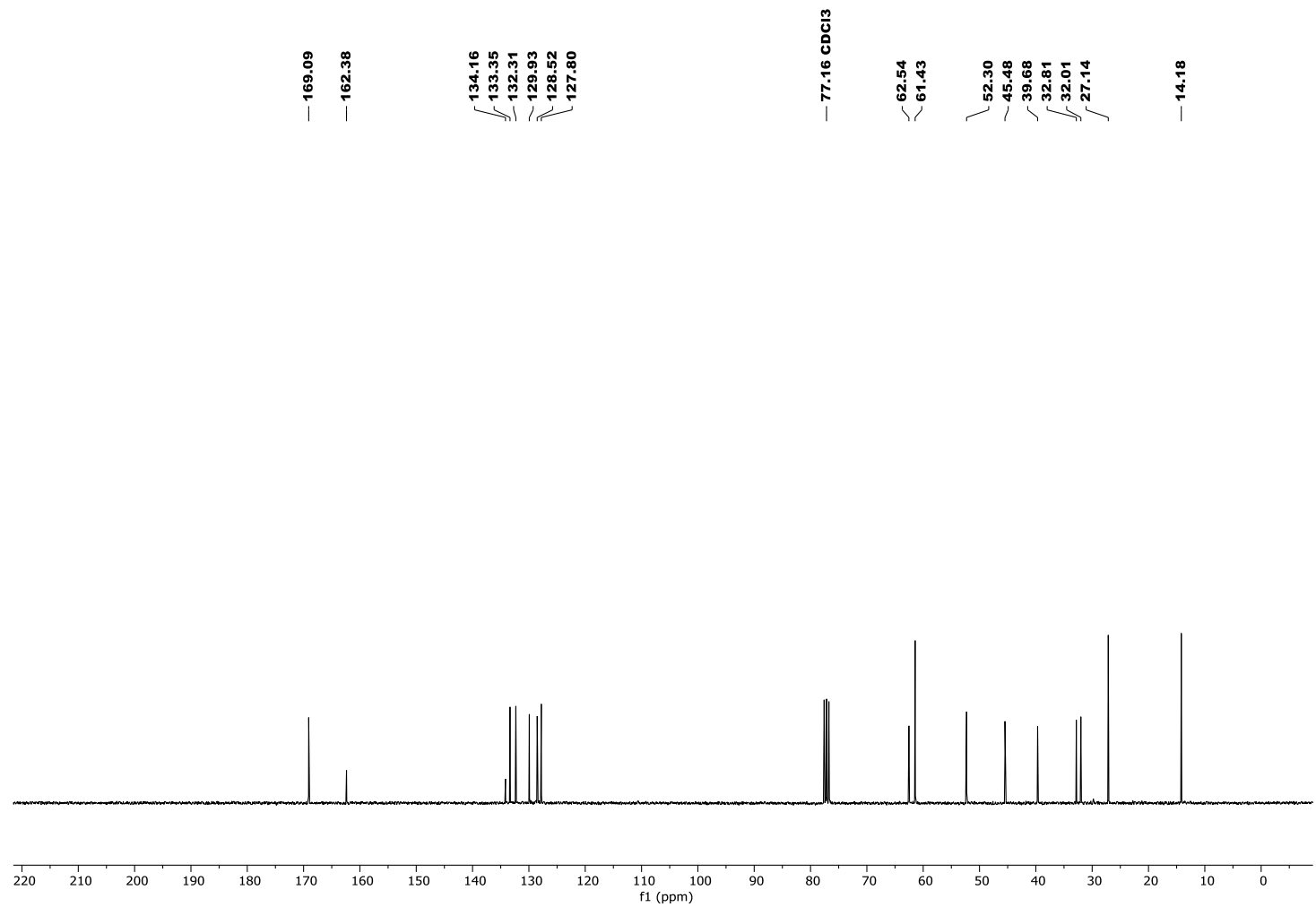
${ }^{1} \mathrm{H}$ NMR $\left(599 \mathrm{MHz}, \mathrm{CDCl}_{3}\right)$
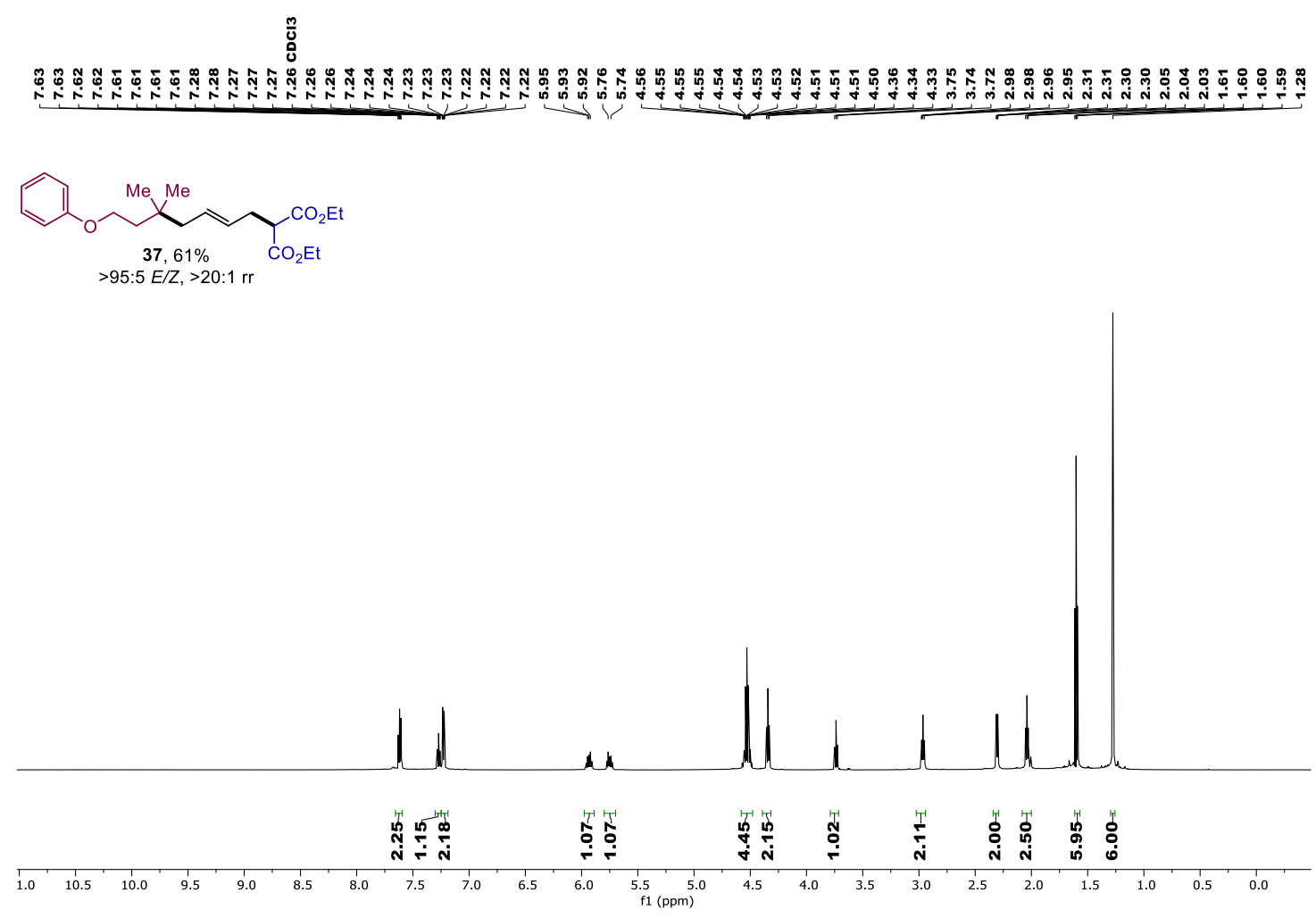

${ }^{13} \mathrm{C}$ NMR $\left(151 \mathrm{MHz}, \mathrm{CDCl}_{3}\right)$
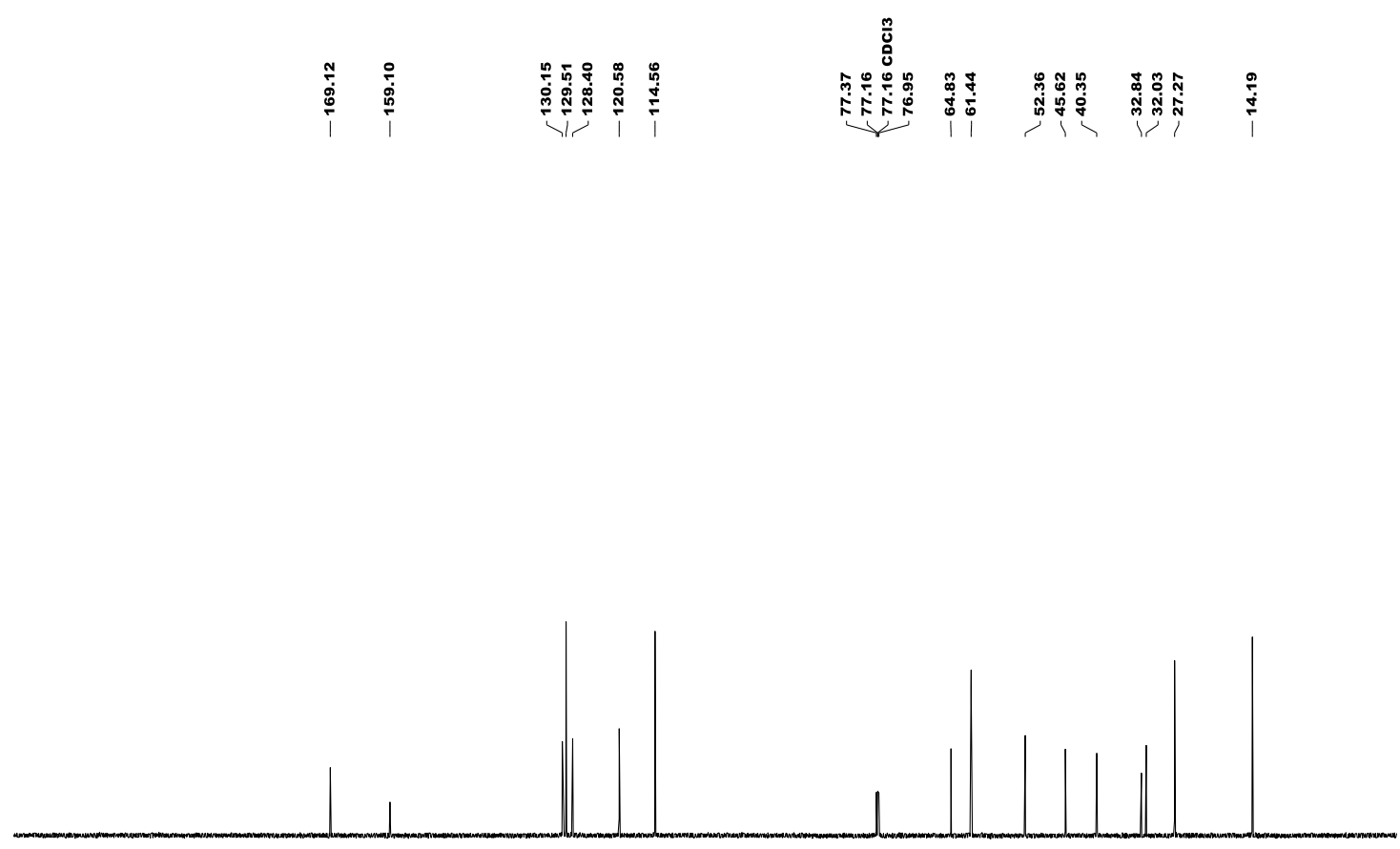

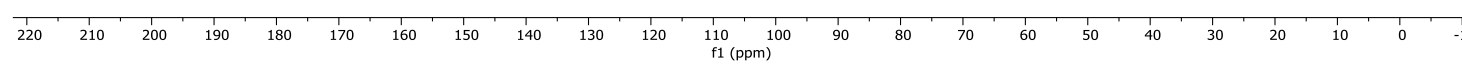


${ }^{1} \mathrm{H}$ NMR (599 MHz, $\mathrm{CDCl}_{3}$ )
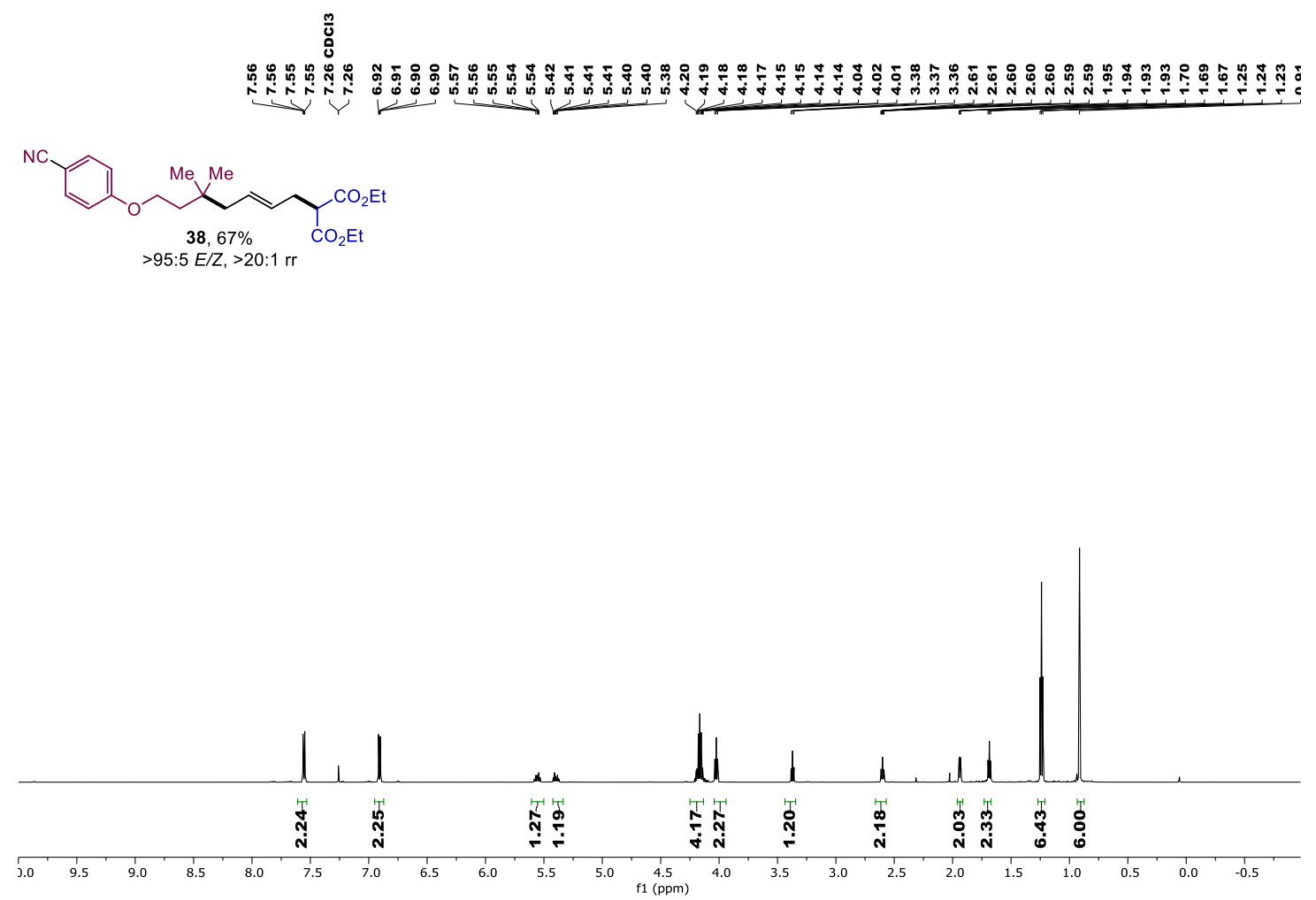

${ }^{13} \mathrm{C}$ NMR $\left(151 \mathrm{MHz}, \mathrm{CDCl}_{3}\right)$
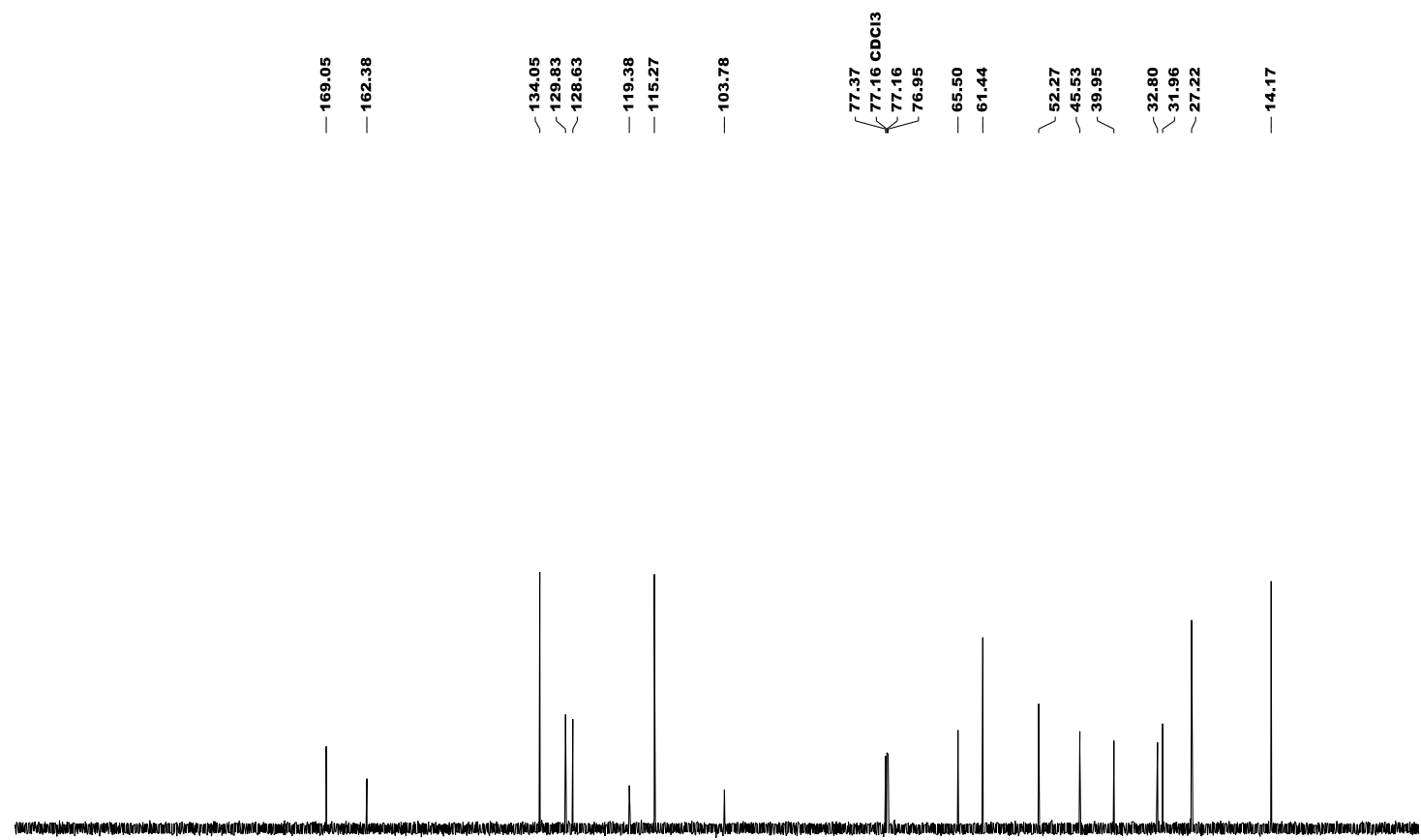

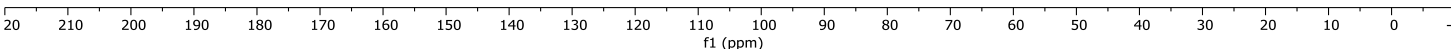


${ }^{1} \mathrm{H}$ NMR $\left(599 \mathrm{MHz}, \mathrm{CDCl}_{3}\right)$

摘

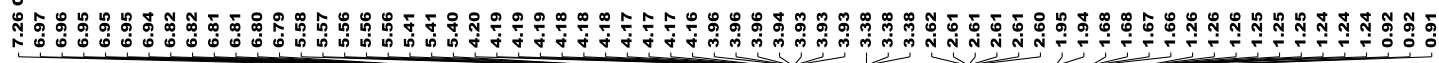

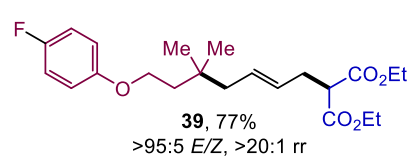

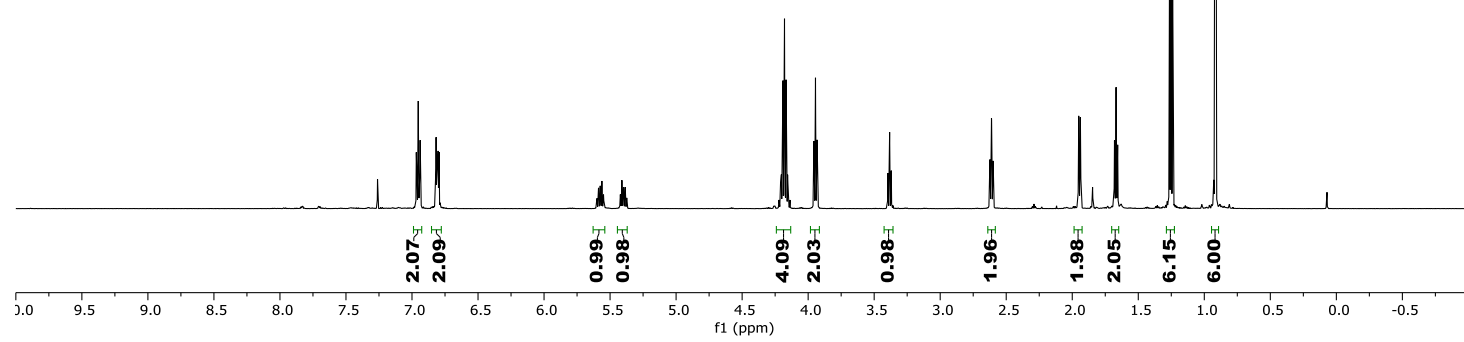

${ }^{13} \mathrm{C}$ NMR $\left(151 \mathrm{MHz}, \mathrm{CDCl}_{3}\right)$

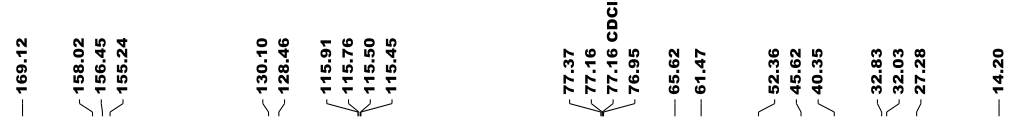

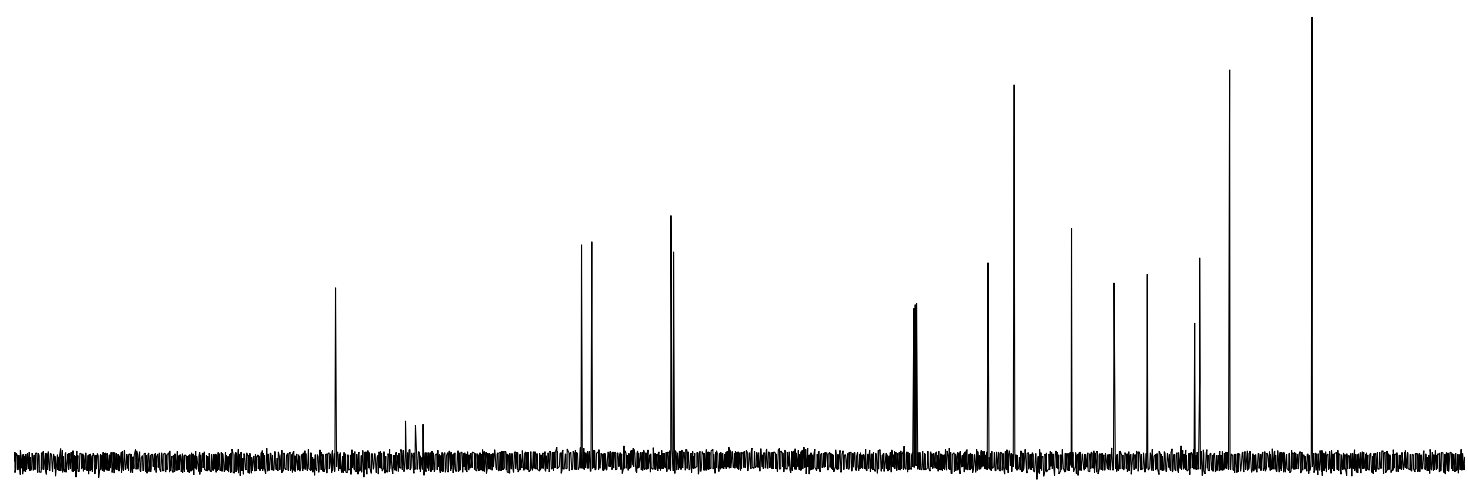

\begin{tabular}{llllllllllll}
\hline 20 & 210 & 200 & 190 & 180 & 170 & 160 & 150 & 140 & 130 & 120 & $\underset{110}{\mid} 100$ \\
$\mathrm{f} 1(\mathrm{ppm})$ & 100
\end{tabular} 
${ }^{19} \mathrm{~F}\left\{{ }^{1} \mathrm{H}\right\} \mathrm{NMR}\left(564 \mathrm{MHz}, \mathrm{CDCl}_{3}\right)$

$\stackrel{\substack{\mathfrak{d} \\ \grave{i}}}{i}$

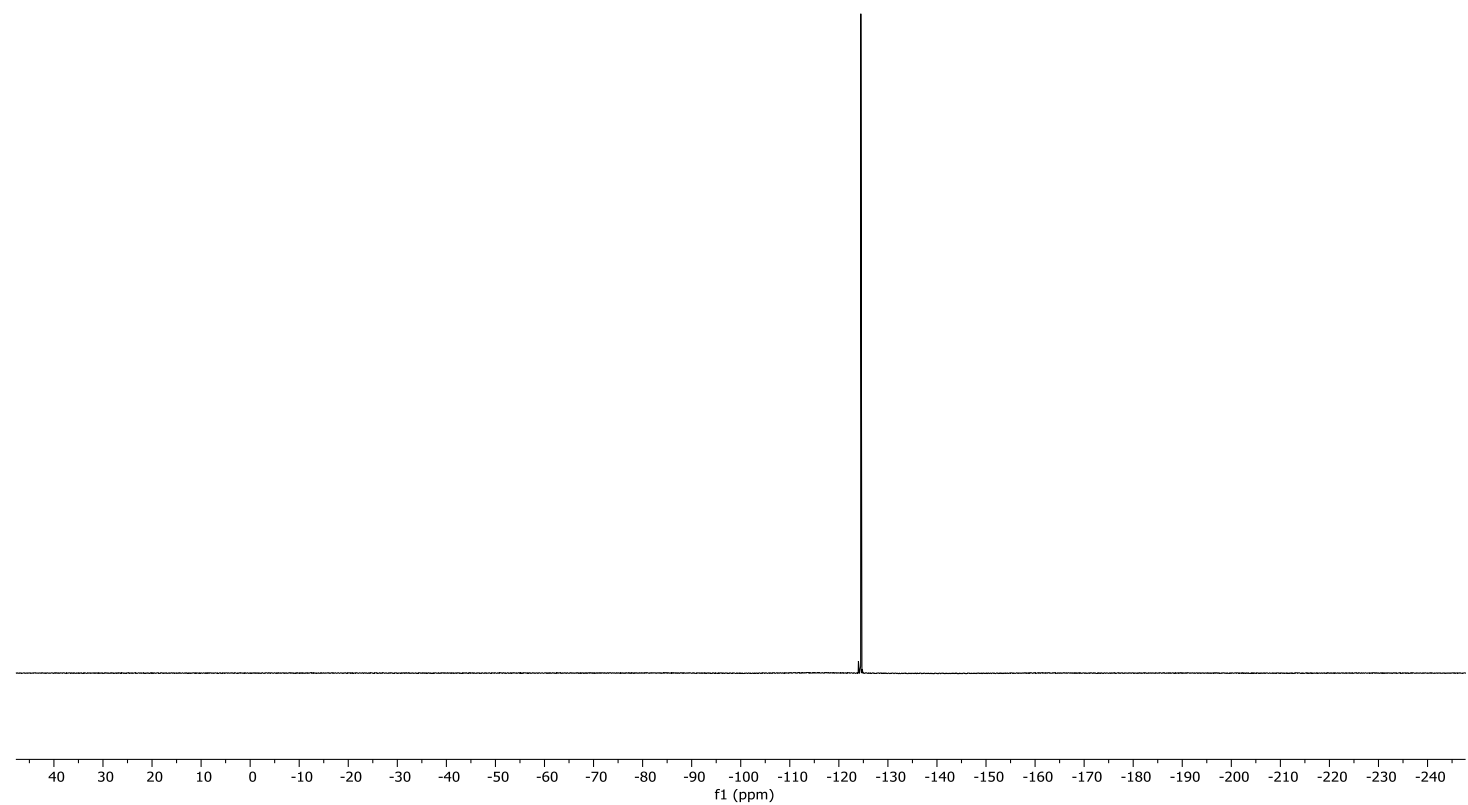


${ }^{1} \mathrm{H}$ NMR (599 MHz, $\mathrm{CDCl}_{3}$ )
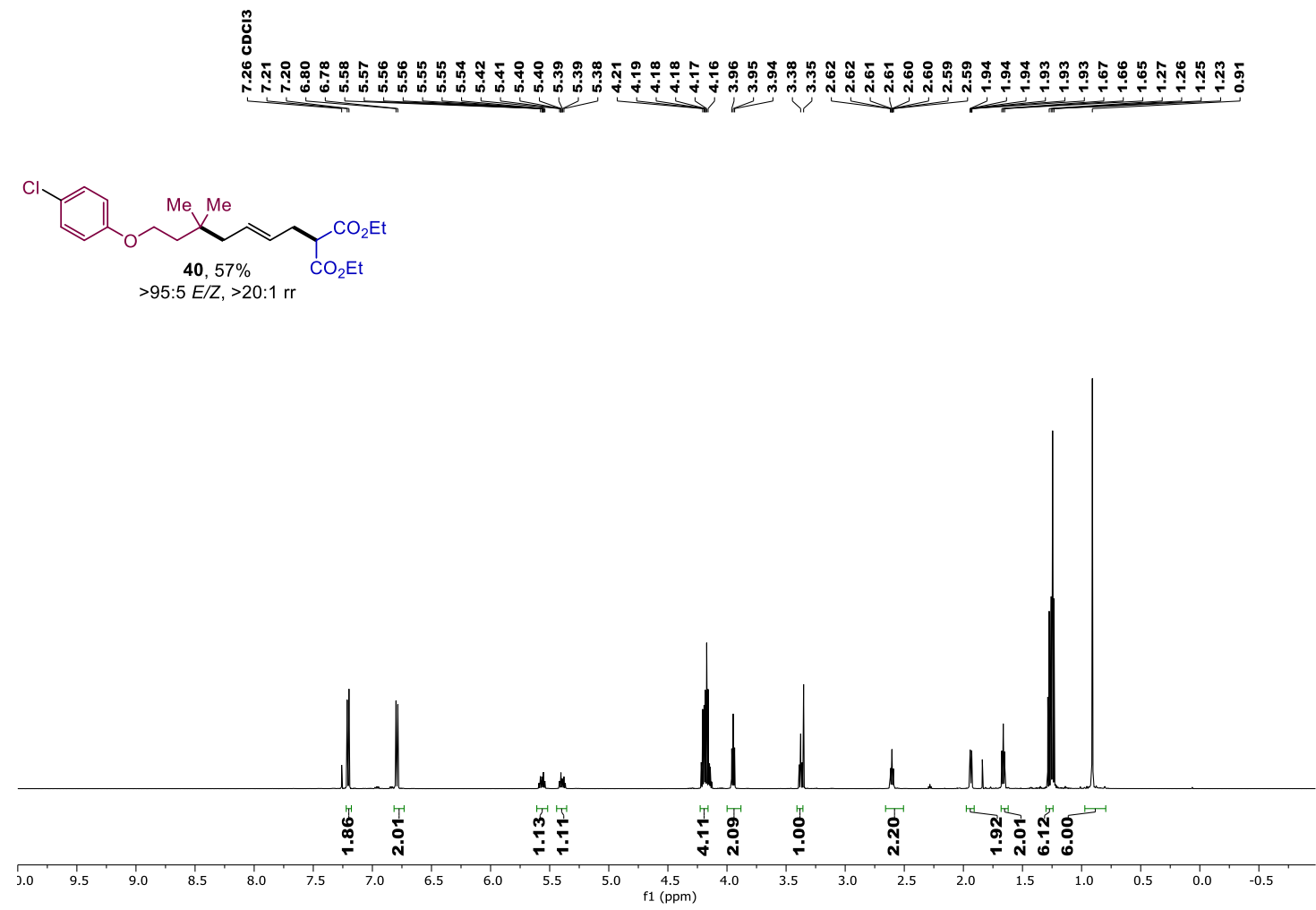

${ }^{13} \mathrm{C}$ NMR $\left(151 \mathrm{MHz}, \mathrm{CDCl}_{3}\right)$
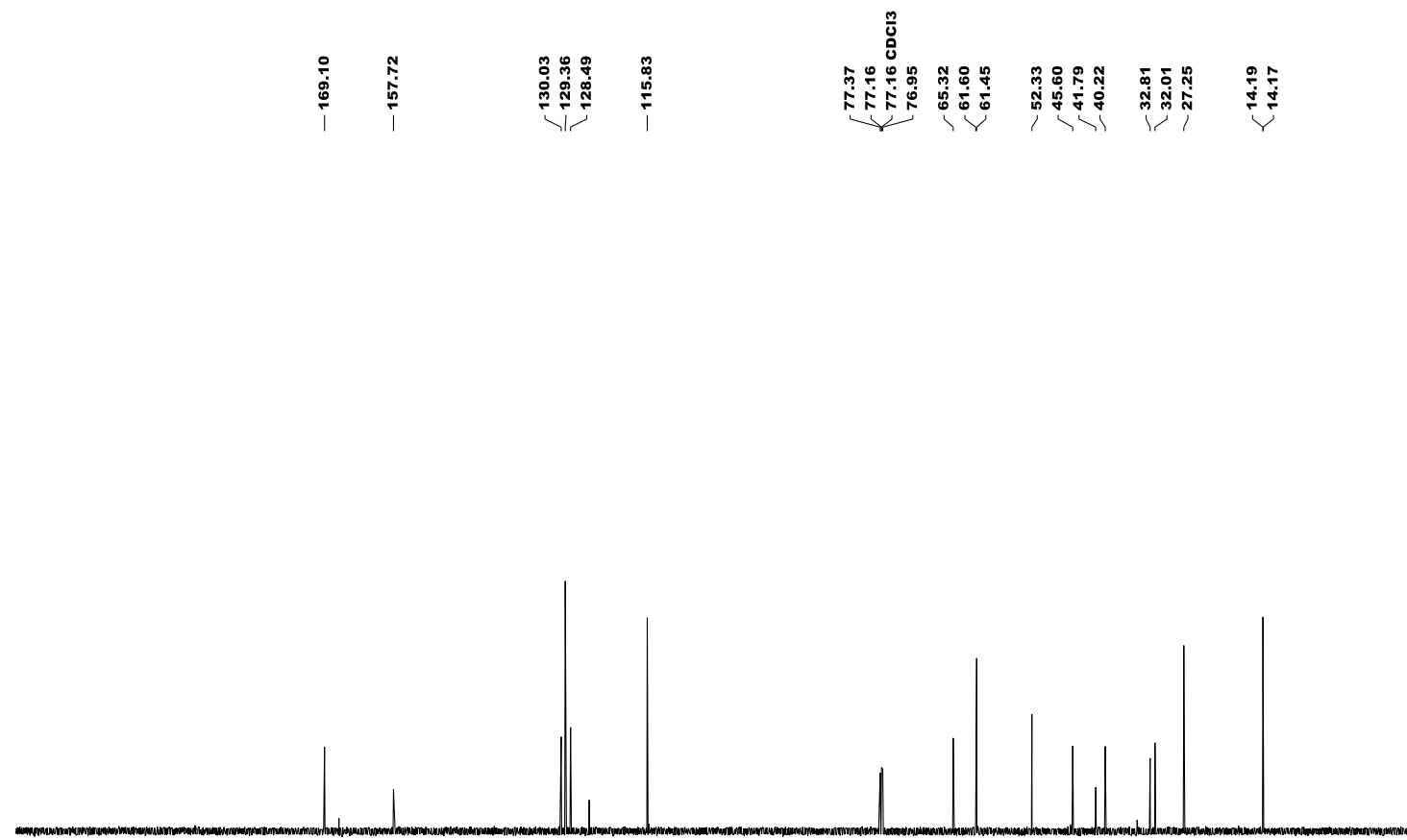

\begin{tabular}{llllllllllll}
\hline 20 & 210 & 200 & 190 & 180 & 170 & 160 & 150 & 140 & 130 & 120 & 110 \\
$\mathrm{f} 1(\mathrm{ppm})$ & 100
\end{tabular} 
${ }^{1} \mathrm{H}$ NMR $\left(400 \mathrm{MHz}, \mathrm{CDCl}_{3}\right)$

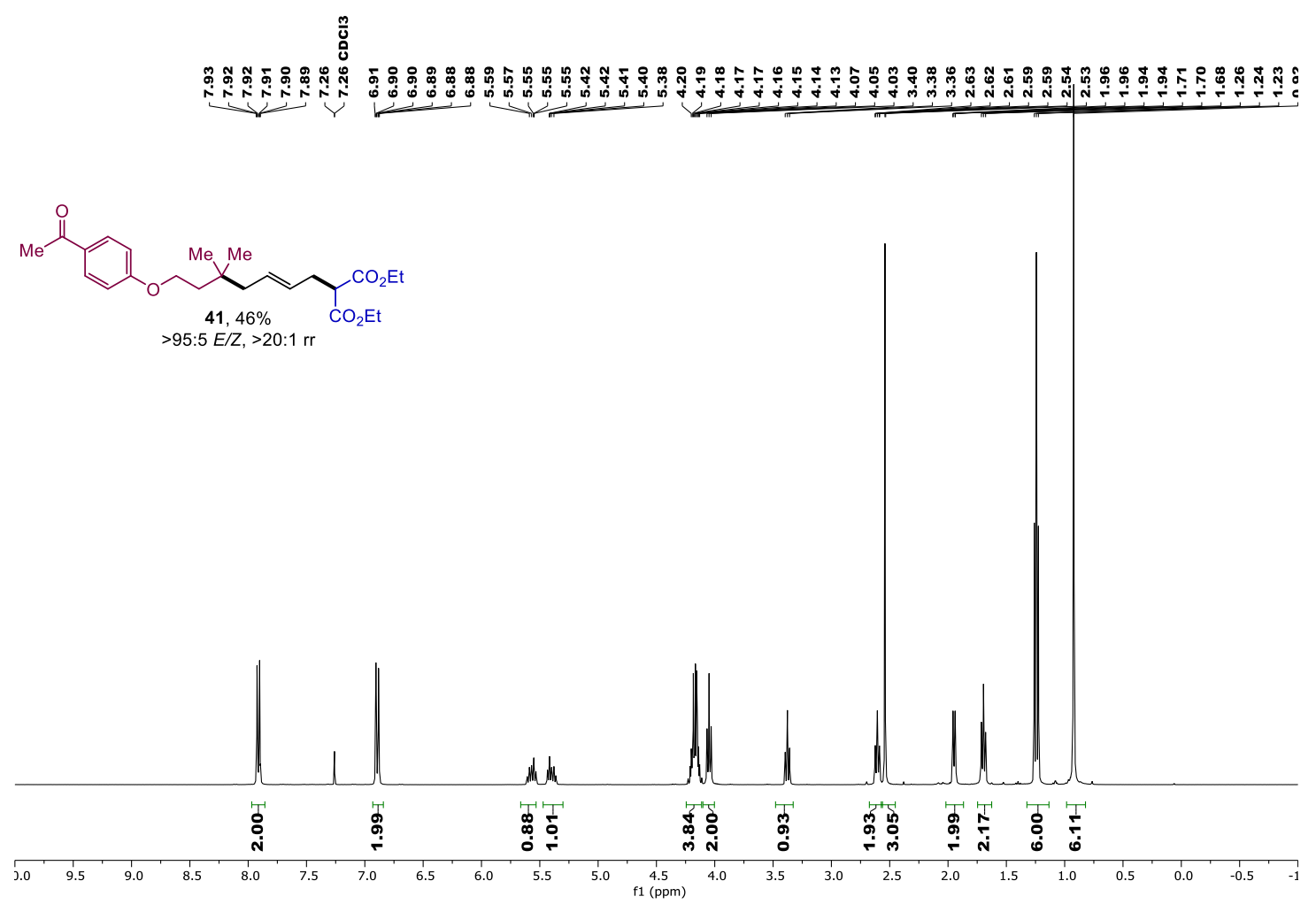

${ }^{13} \mathrm{C}$ NMR (101 MHz, $\left.\mathrm{CDCl}_{3}\right)$

\begin{tabular}{|c|c|c|}
\hline 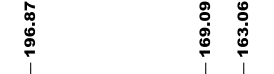 & 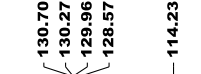 & 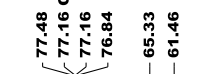 \\
\hline
\end{tabular}

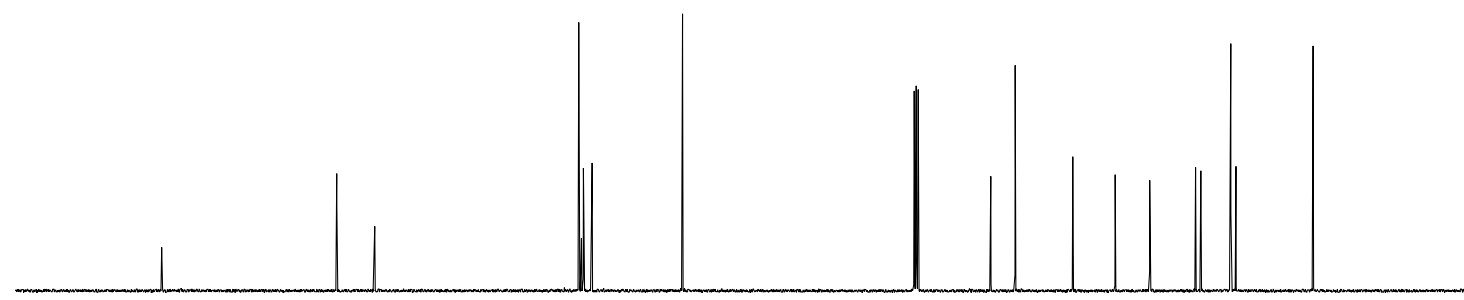

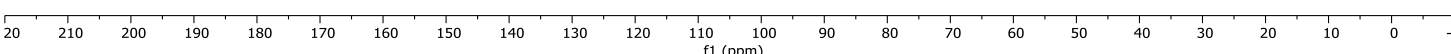


${ }^{1} \mathrm{H}$ NMR (400 MHz, $\mathrm{CDCl}_{3}$ )

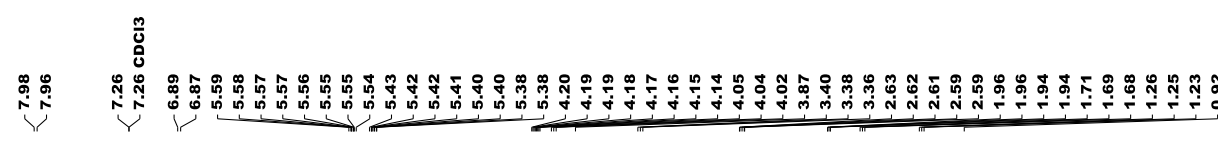
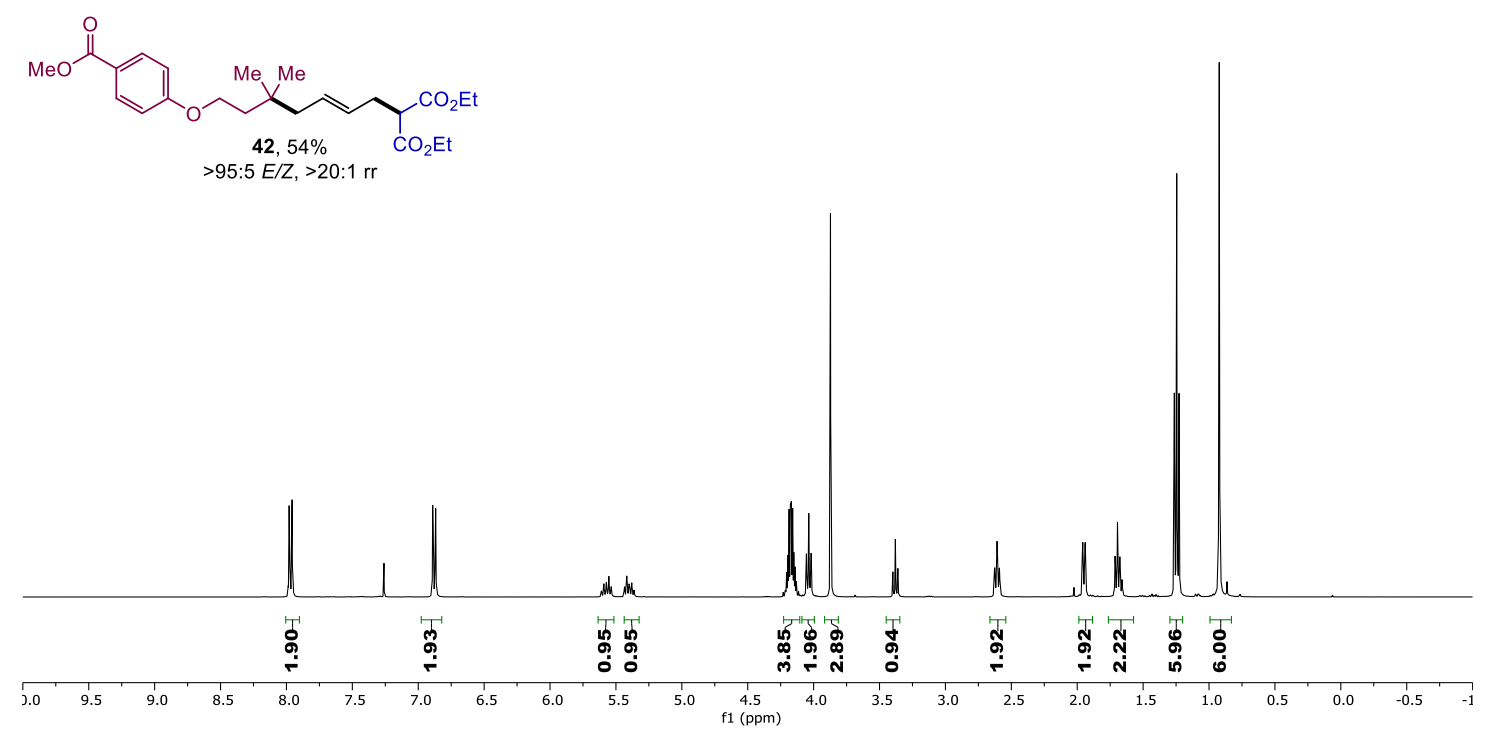

${ }^{13} \mathrm{C}$ NMR (101 MHz, $\mathrm{CDCl}_{3}$ )
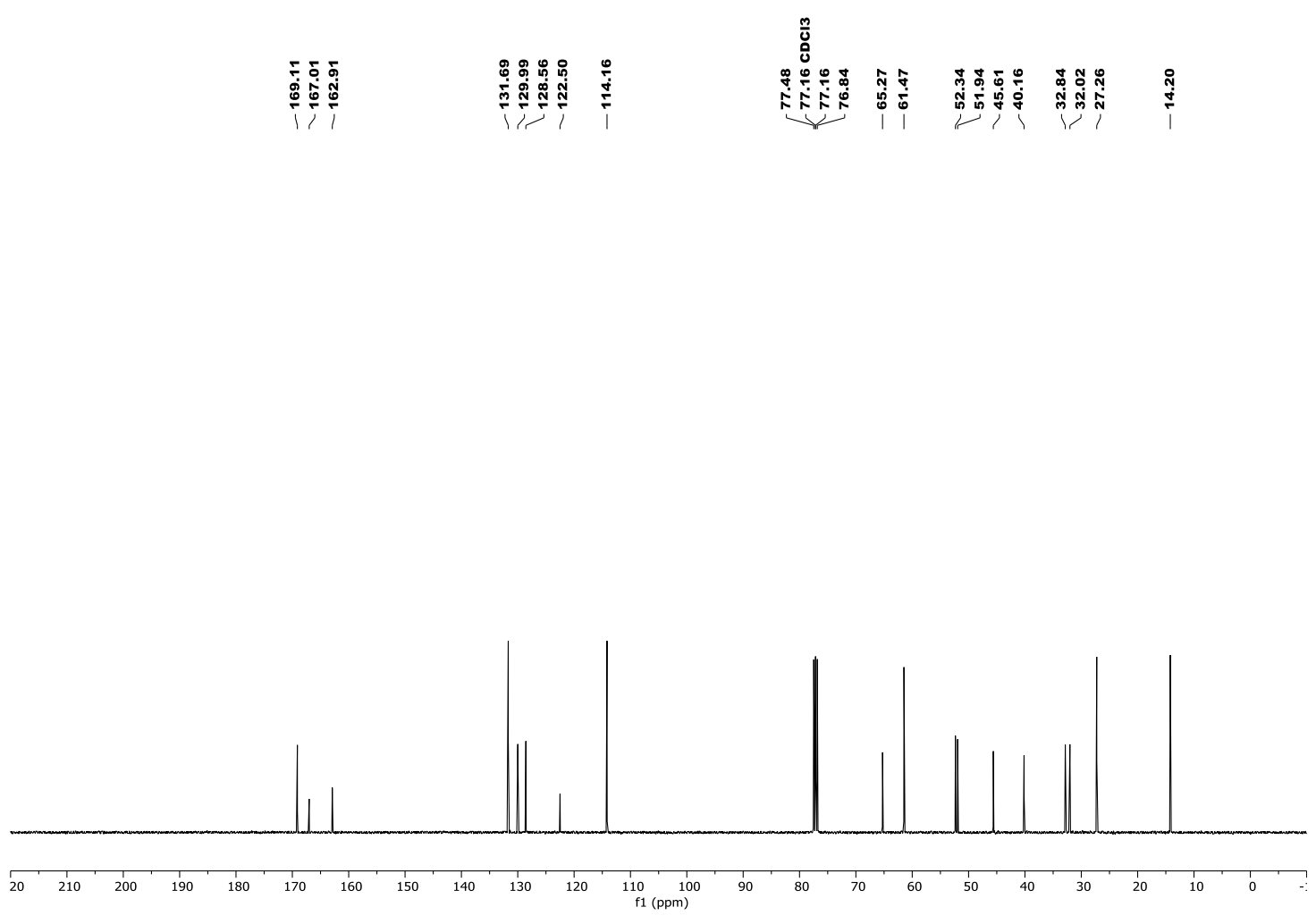

S 248 
${ }^{1} \mathrm{H} \mathrm{NMR}\left(400 \mathrm{MHz}, \mathrm{CDCl}_{3}\right)$

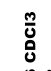

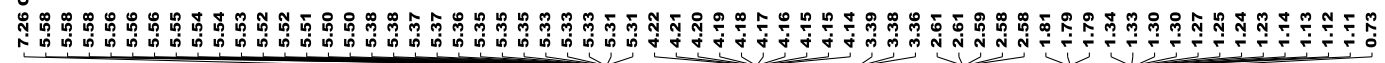
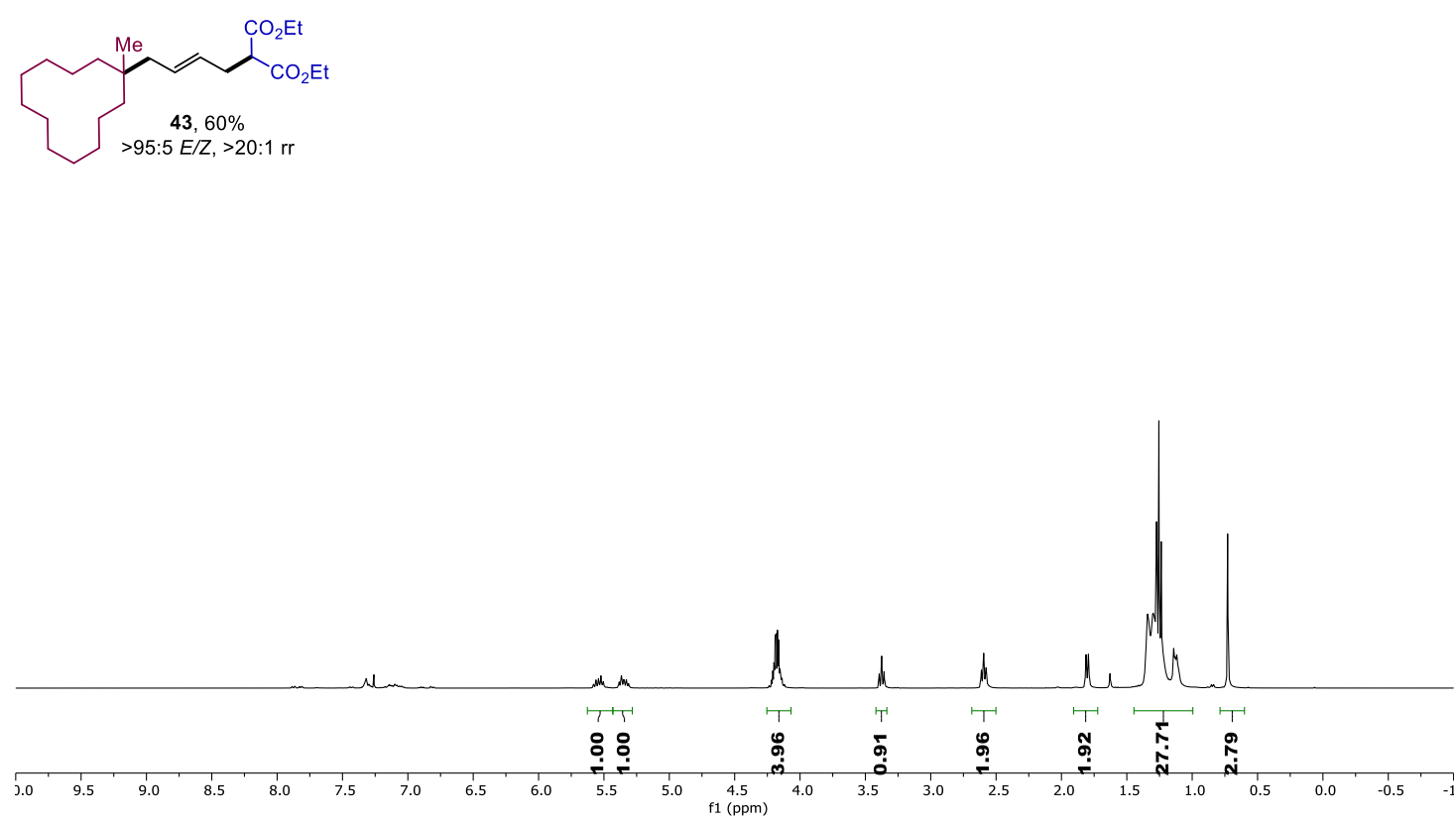

${ }^{13} \mathrm{C}$ NMR $\left(101 \mathrm{MHz}, \mathrm{CDCl}_{3}\right)$
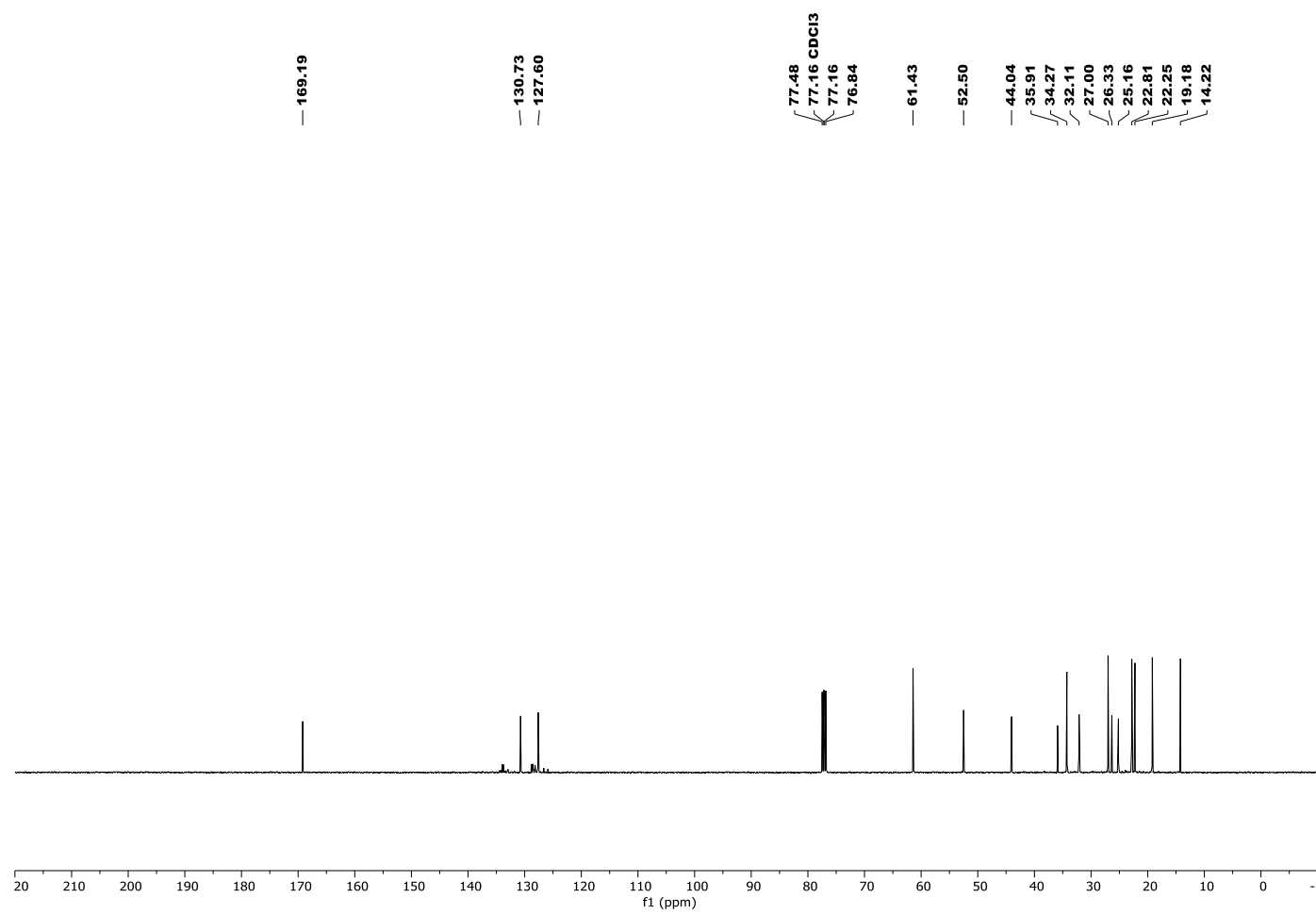
${ }^{1} \mathrm{H}$ NMR $\left(400 \mathrm{MHz}, \mathrm{CDCl}_{3}\right)$

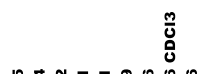

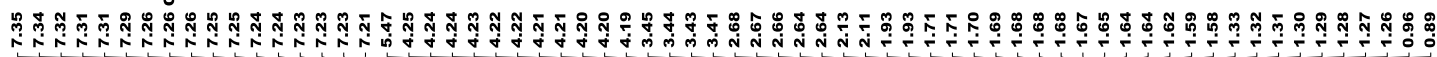
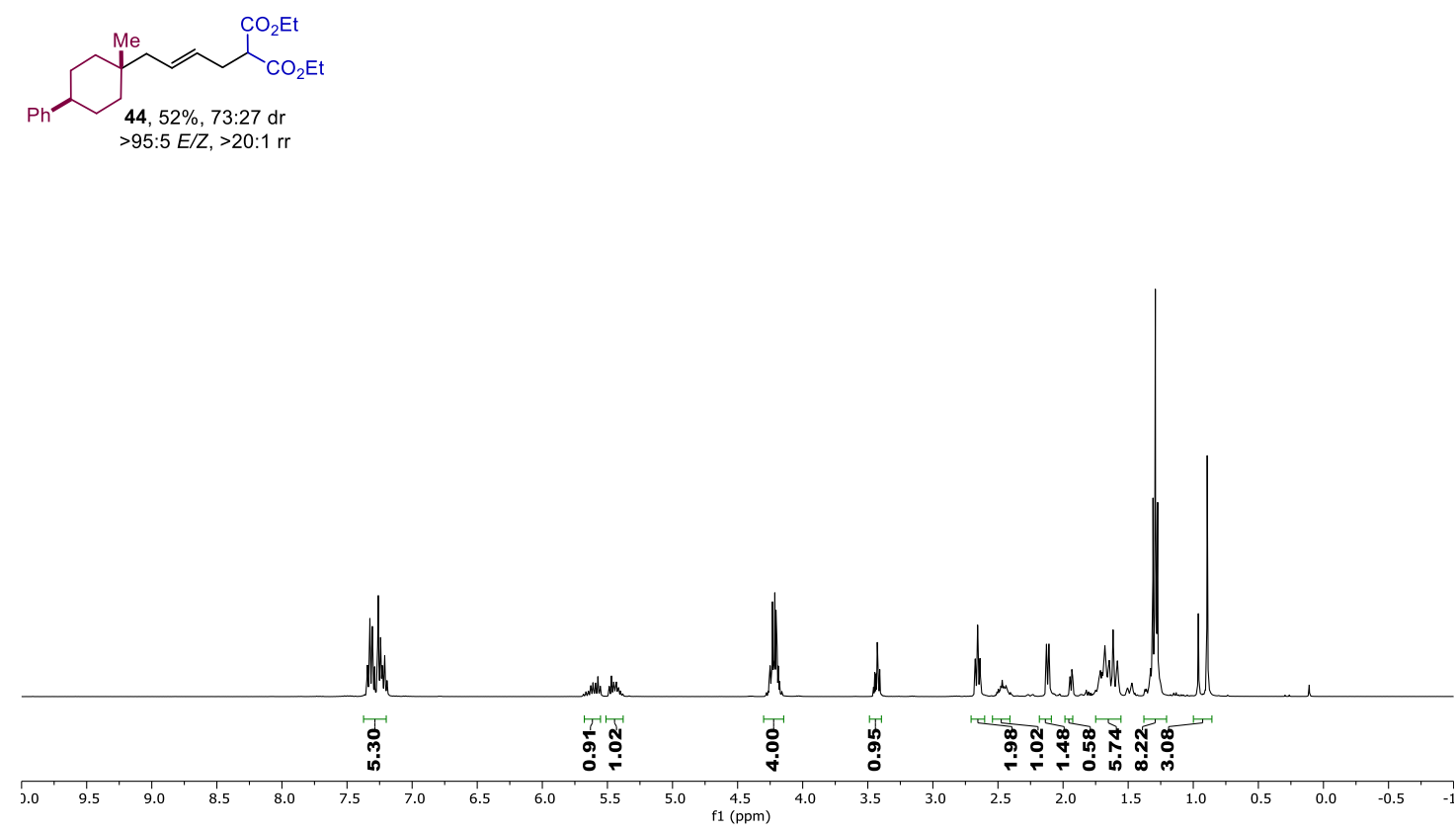

${ }^{13} \mathrm{C}$ NMR $\left(101 \mathrm{MHz}, \mathrm{CDCl}_{3}\right)$
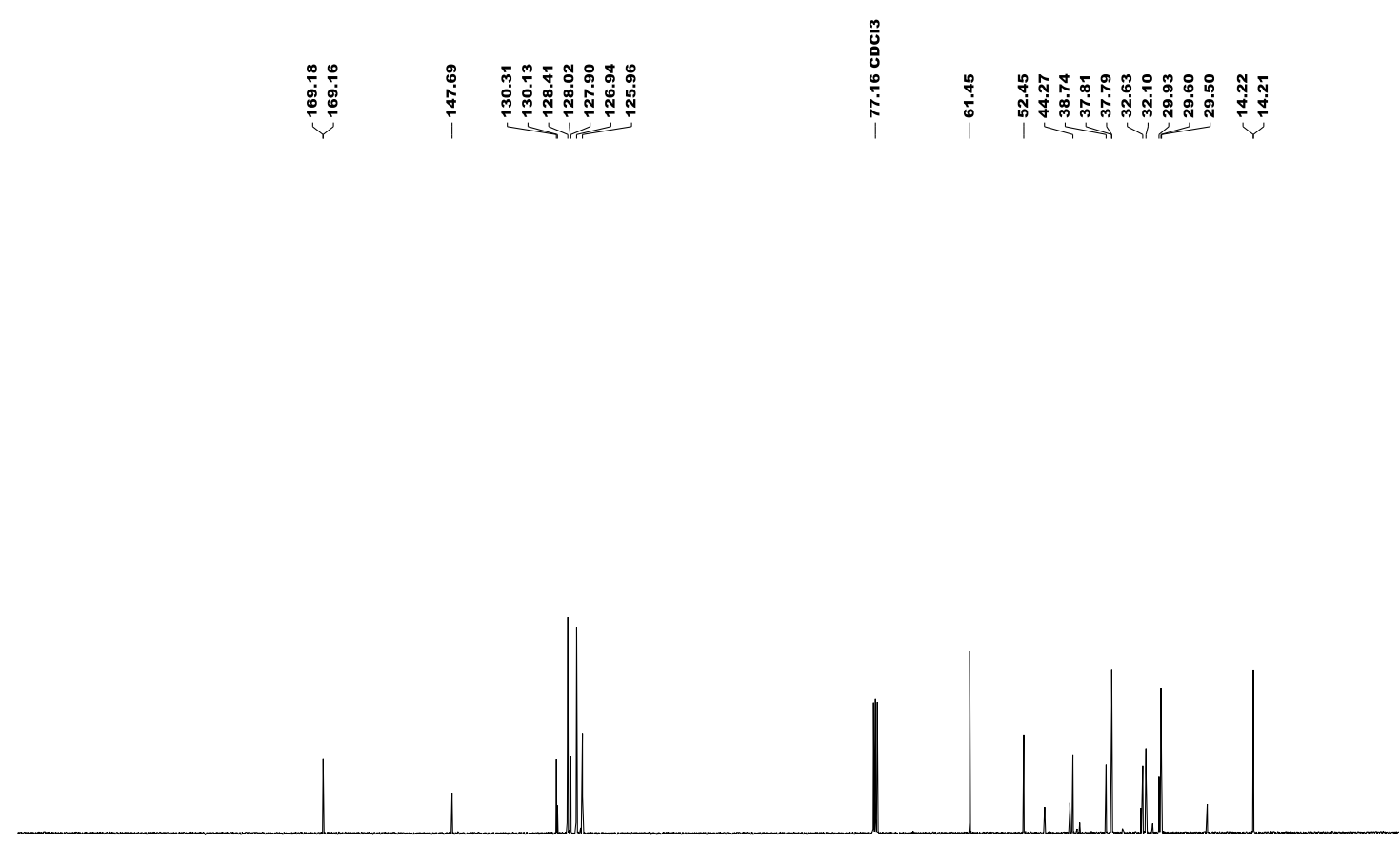

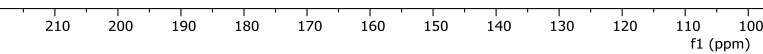


${ }^{1} \mathrm{H}$ NMR $\left(599 \mathrm{MHz}, \mathrm{CDCl}_{3}\right)$

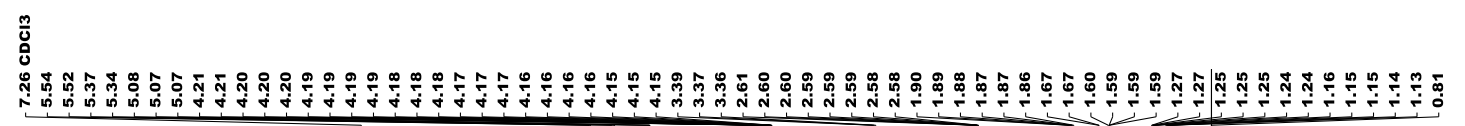

$\overbrace{\substack{45,91 \% \\>95: 5 E / Z,>20: 1 \mathrm{rr}}}^{\mathrm{CO}_{2} \mathrm{Et}}$

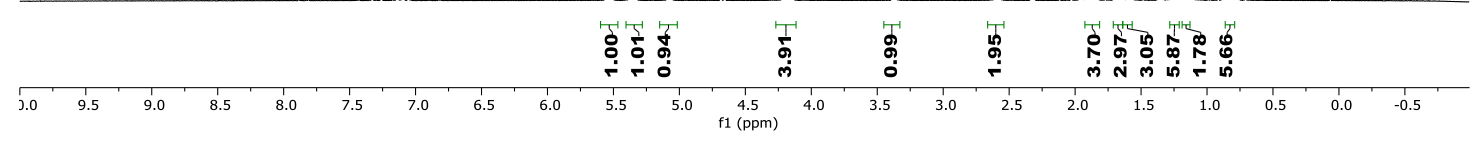

${ }^{13} \mathrm{C}$ NMR $\left(101 \mathrm{MHz}, \mathrm{CDCl}_{3}\right)$

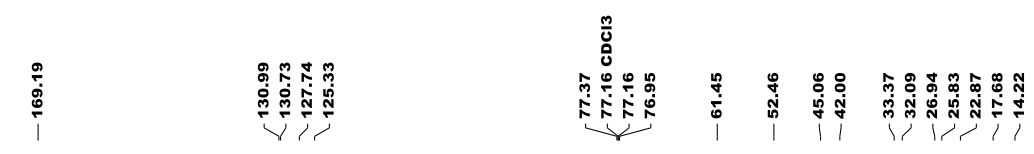

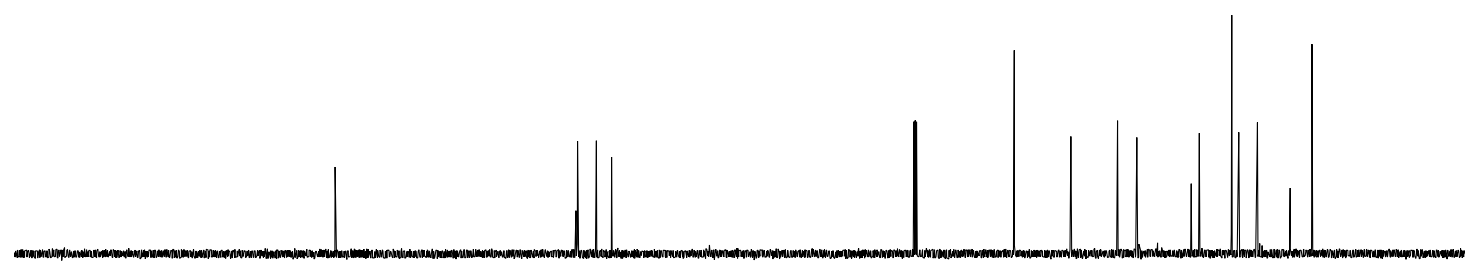

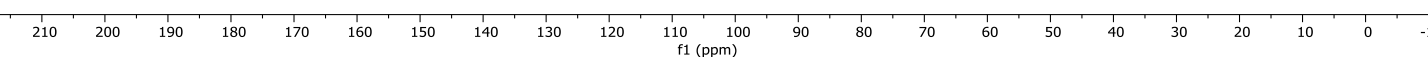


${ }^{1} \mathrm{H}$ NMR $\left(599 \mathrm{MHz}, \mathrm{CDCl}_{3}\right)$

高

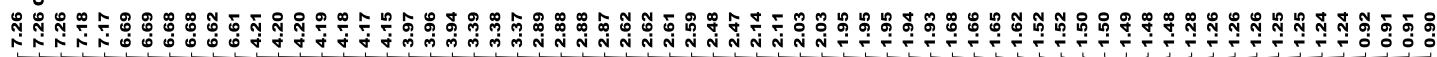
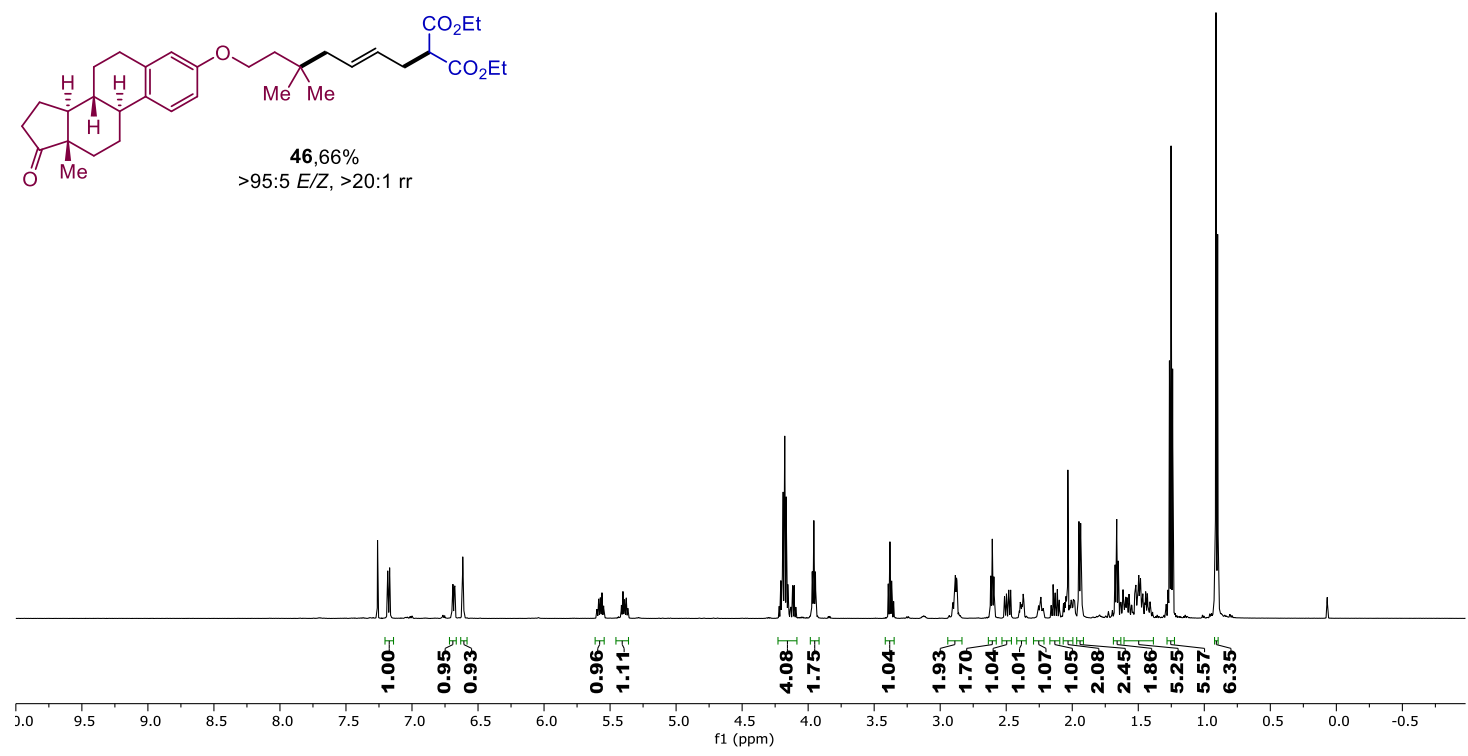

${ }^{13} \mathrm{C}$ NMR (151 MHz, $\mathrm{CDCl}_{3}$ )
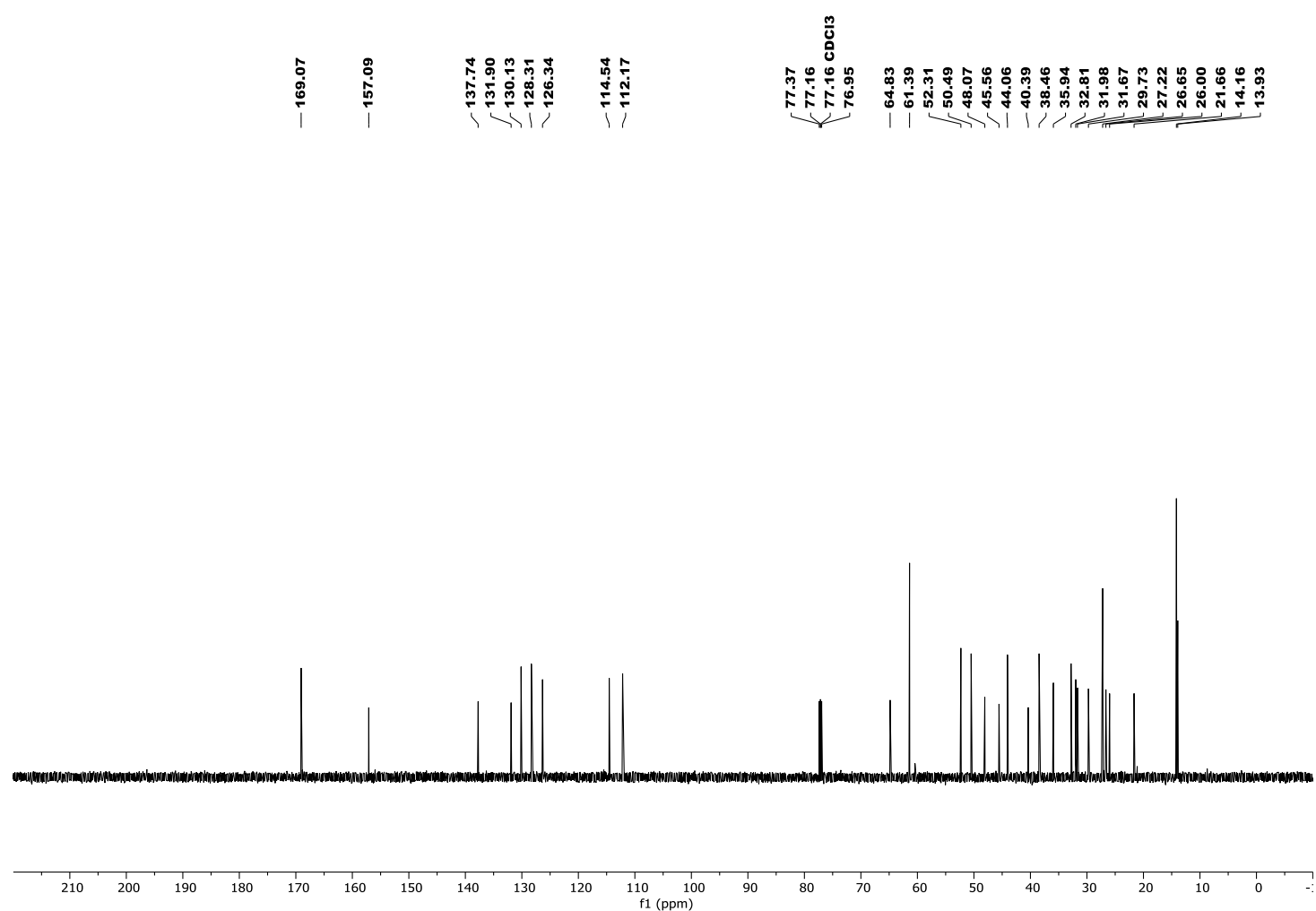
${ }^{1} \mathrm{H} \mathrm{NMR}\left(599 \mathrm{MHz}, \mathrm{CDCl}_{3}\right)$

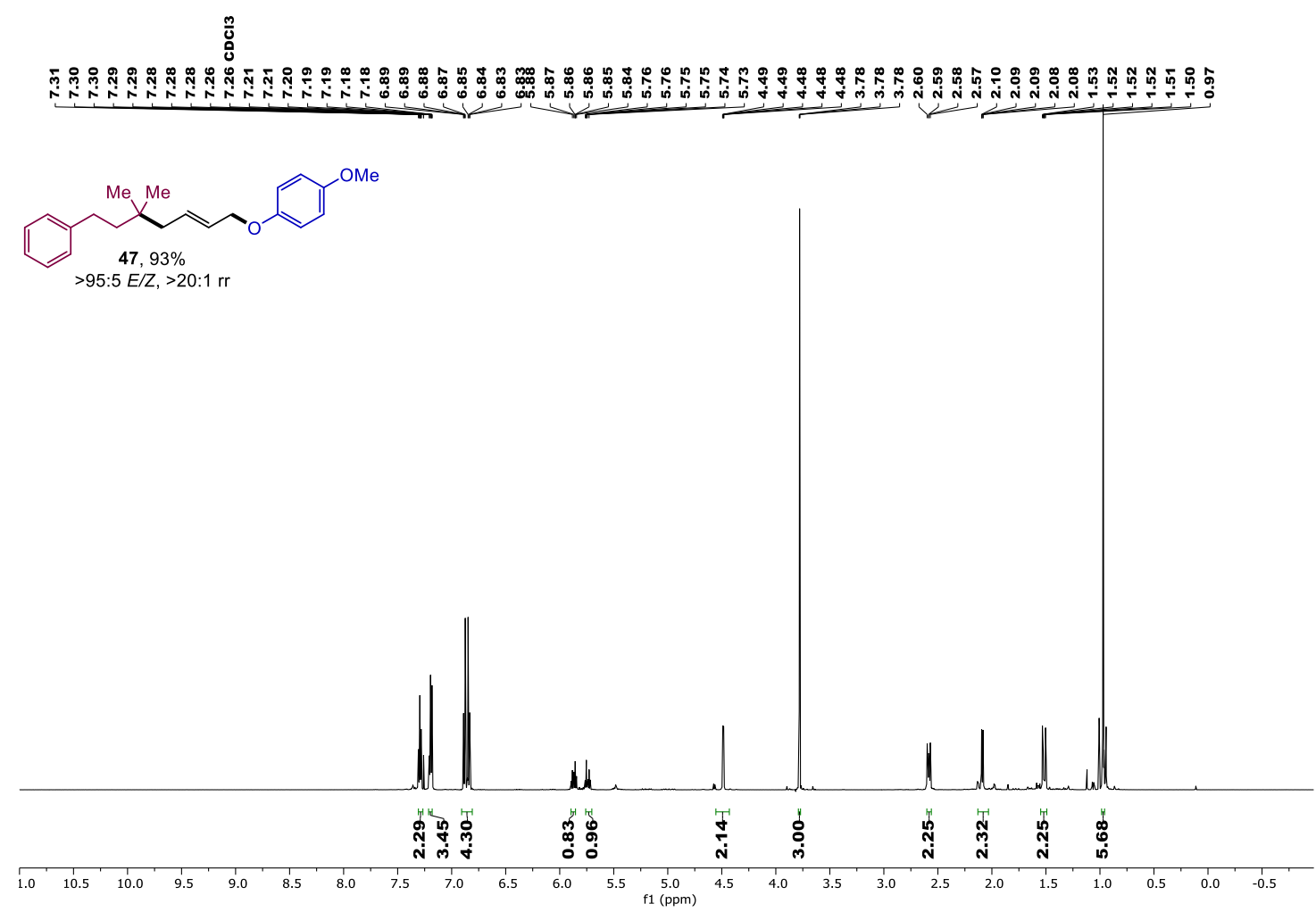

${ }^{13} \mathrm{C}$ NMR $\left(151 \mathrm{MHz}, \mathrm{CDCl}_{3}\right)$
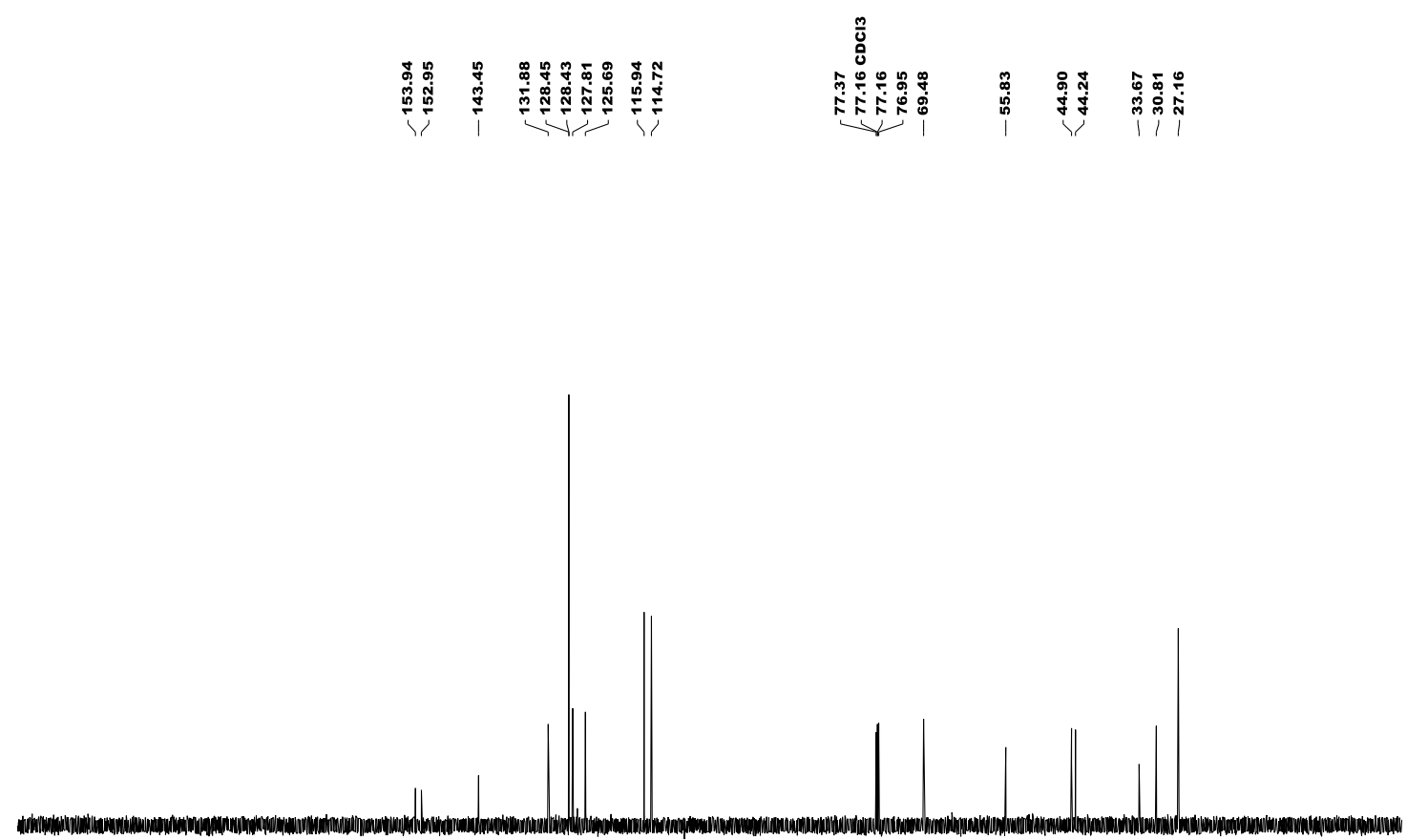

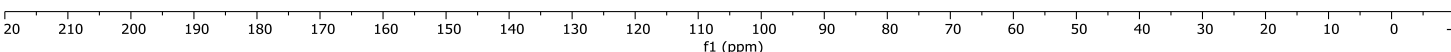


${ }^{1} \mathrm{H}$ NMR (400 MHz, $\mathrm{CDCl}_{3}$ )

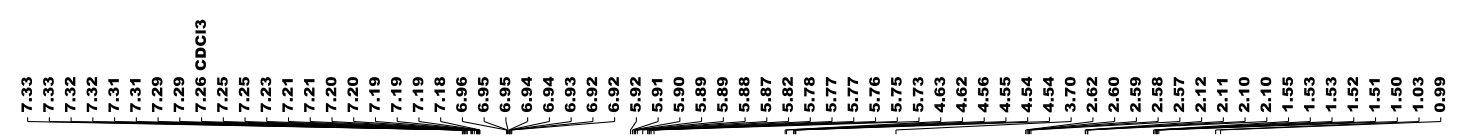

$\underbrace{\mathrm{Cu}}_{\substack{48,34 \% \\ 91: 9 E / Z,>20: 1 \mathrm{rr}}}$

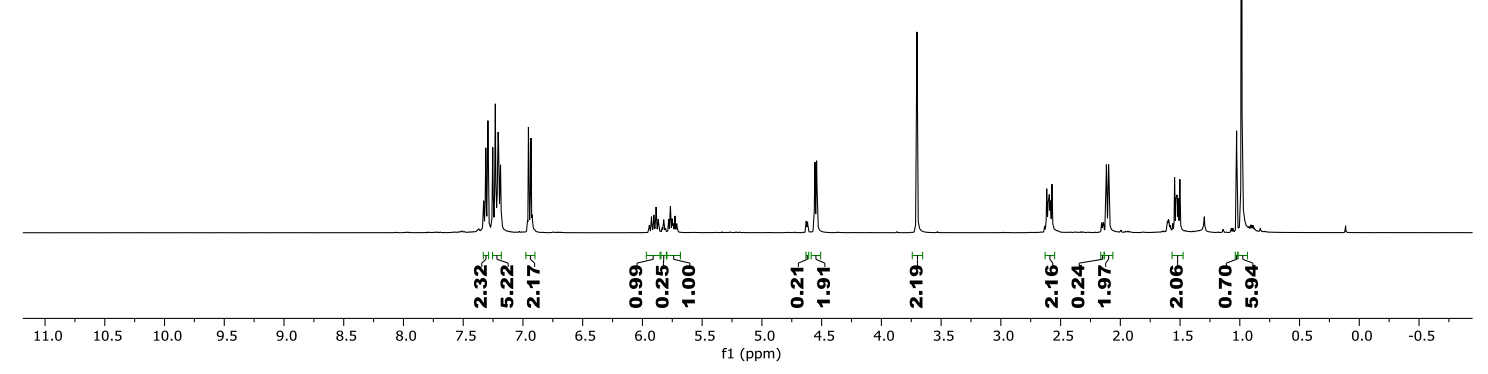

${ }^{13} \mathrm{C}$ NMR $\left(101 \mathrm{MHz}, \mathrm{CDCl}_{3}\right)$
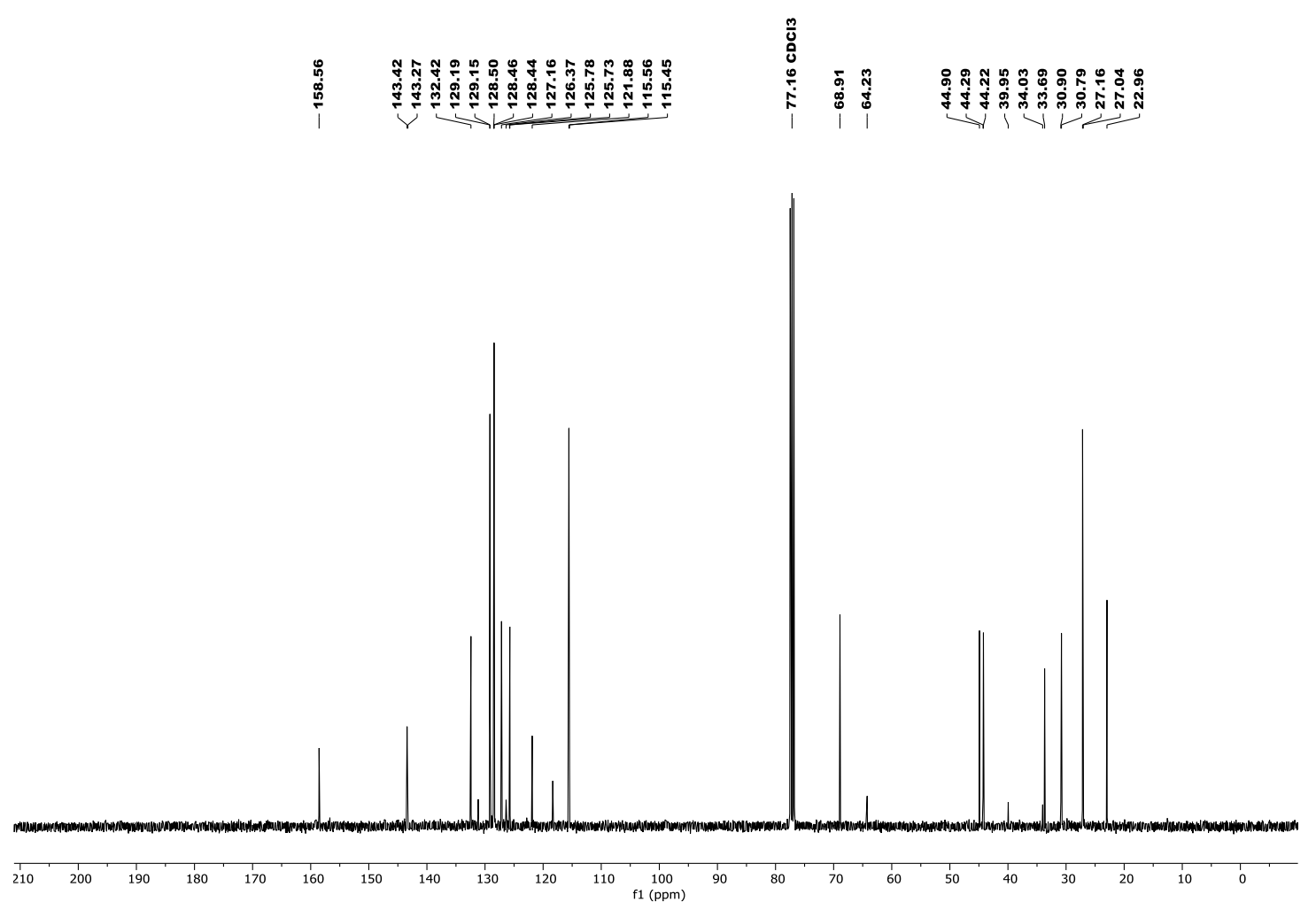
${ }^{1} \mathrm{H}$ NMR $\left(300 \mathrm{MHz}, \mathrm{CDCl}_{3}\right)$
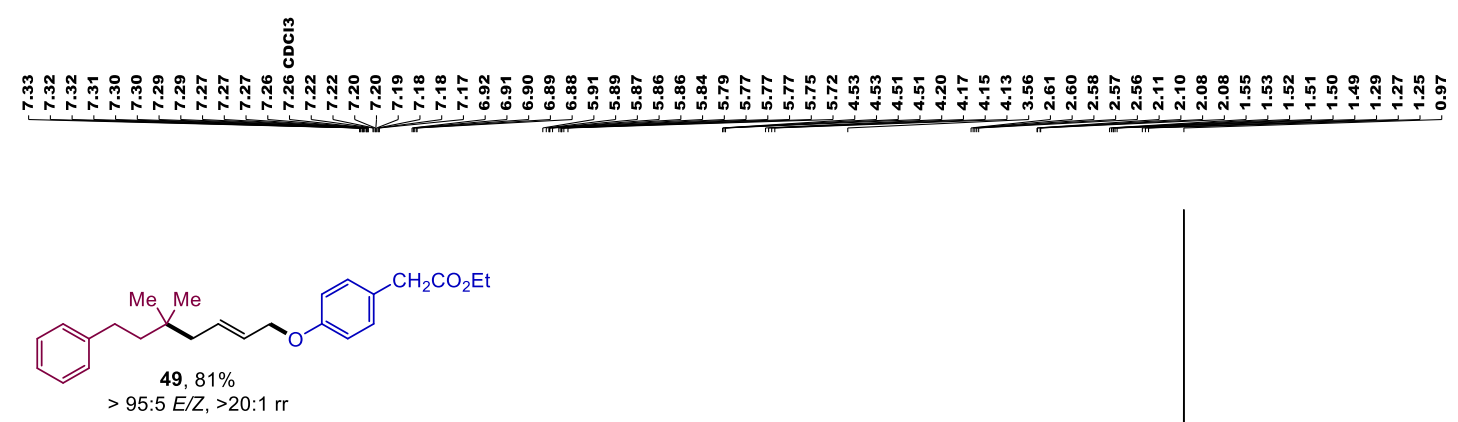

${ }^{13} \mathrm{C}$ NMR $\left(75 \mathrm{MHz}, \mathrm{CDCl}_{3}\right.$ )
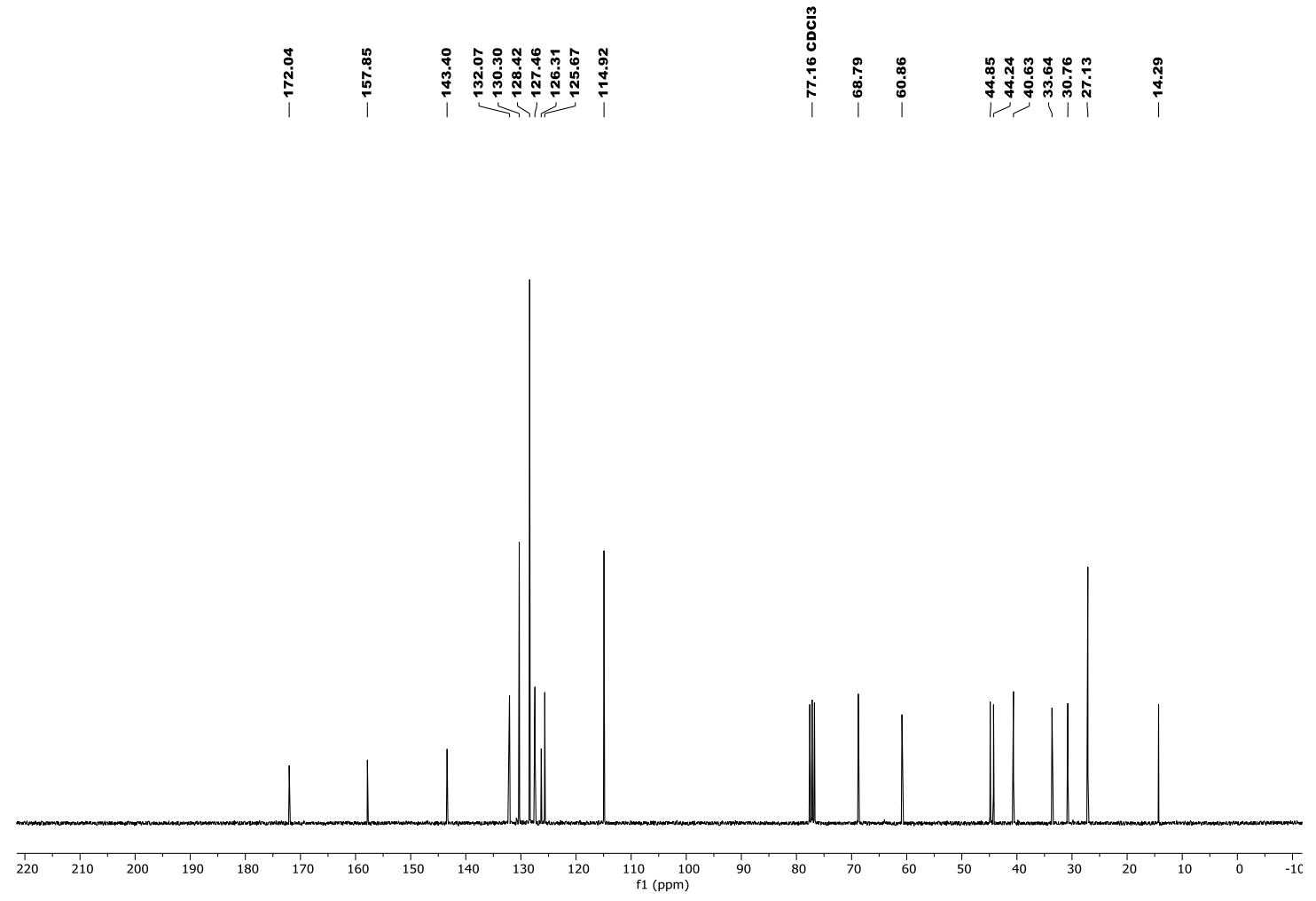
${ }^{1} \mathrm{H} \mathrm{NMR}\left(400 \mathrm{MHz}, \mathrm{CDCl}_{3}\right)$
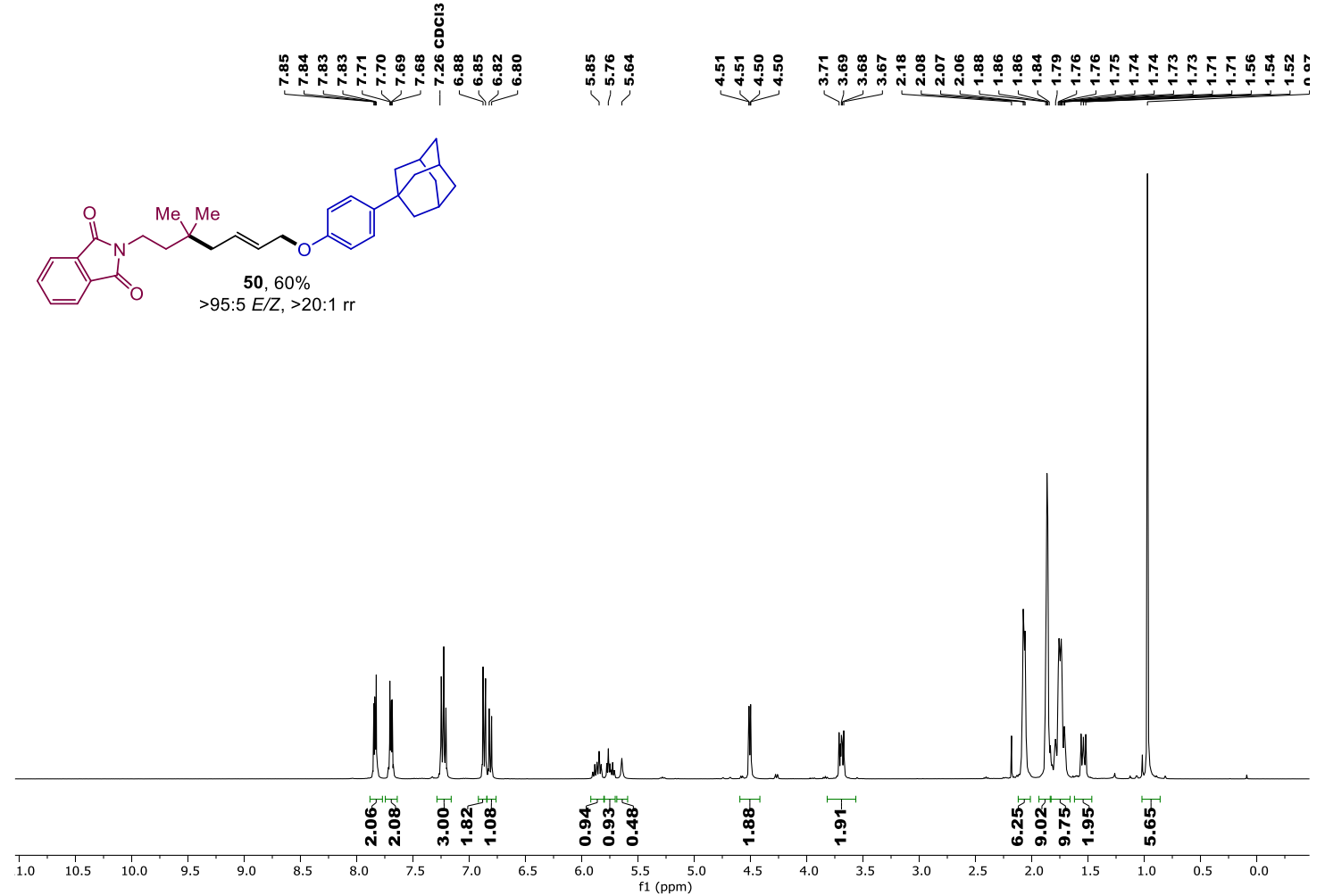

${ }^{13} \mathrm{C}$ NMR $\left(101 \mathrm{MHz}, \mathrm{CDCl}_{3}\right)$

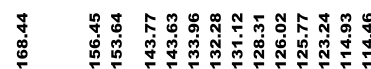

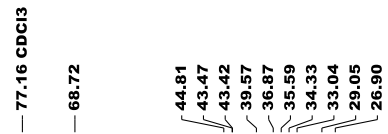

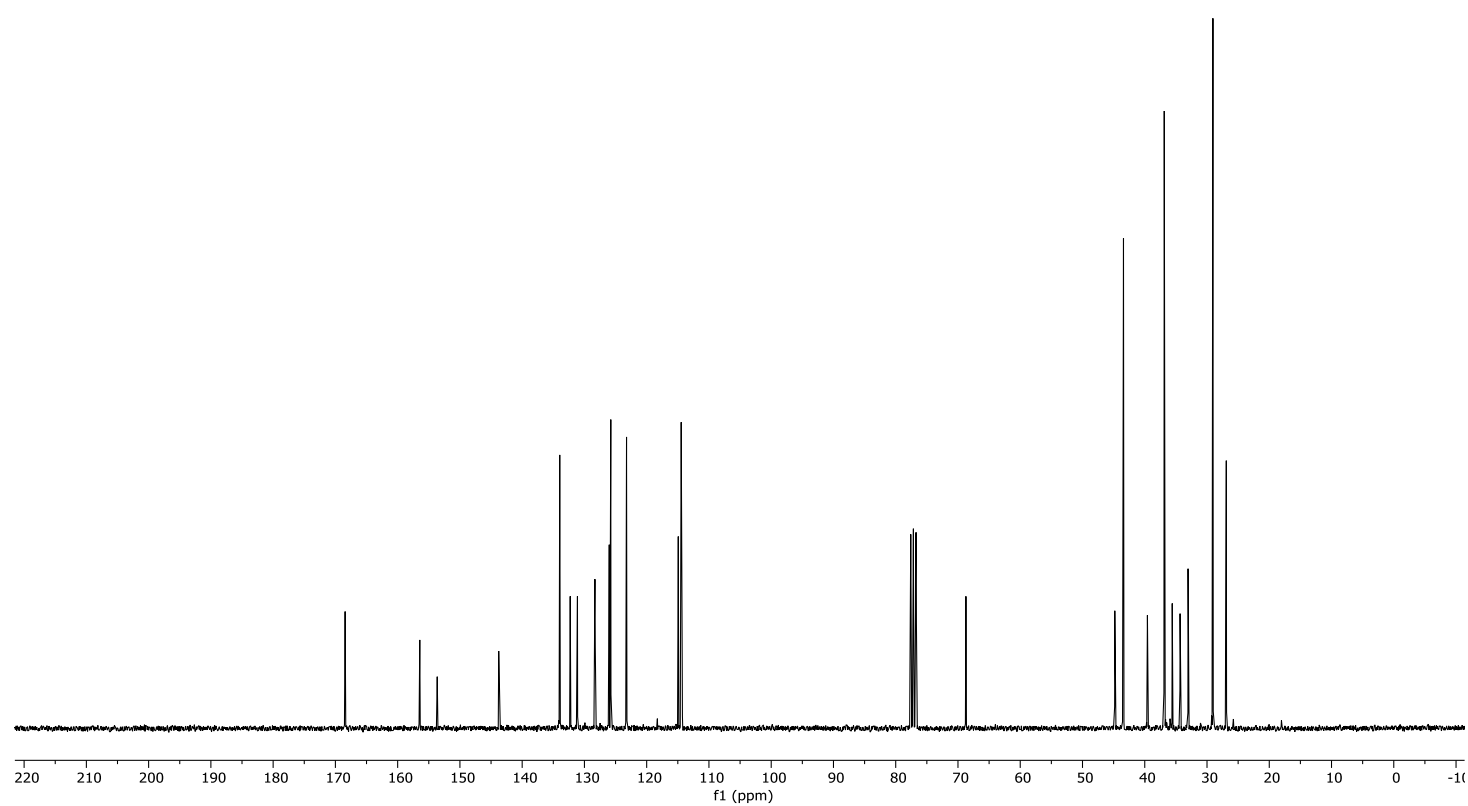


${ }^{1} \mathrm{H} \mathrm{NMR}\left(400 \mathrm{MHz}, \mathrm{CDCl}_{3}\right)$
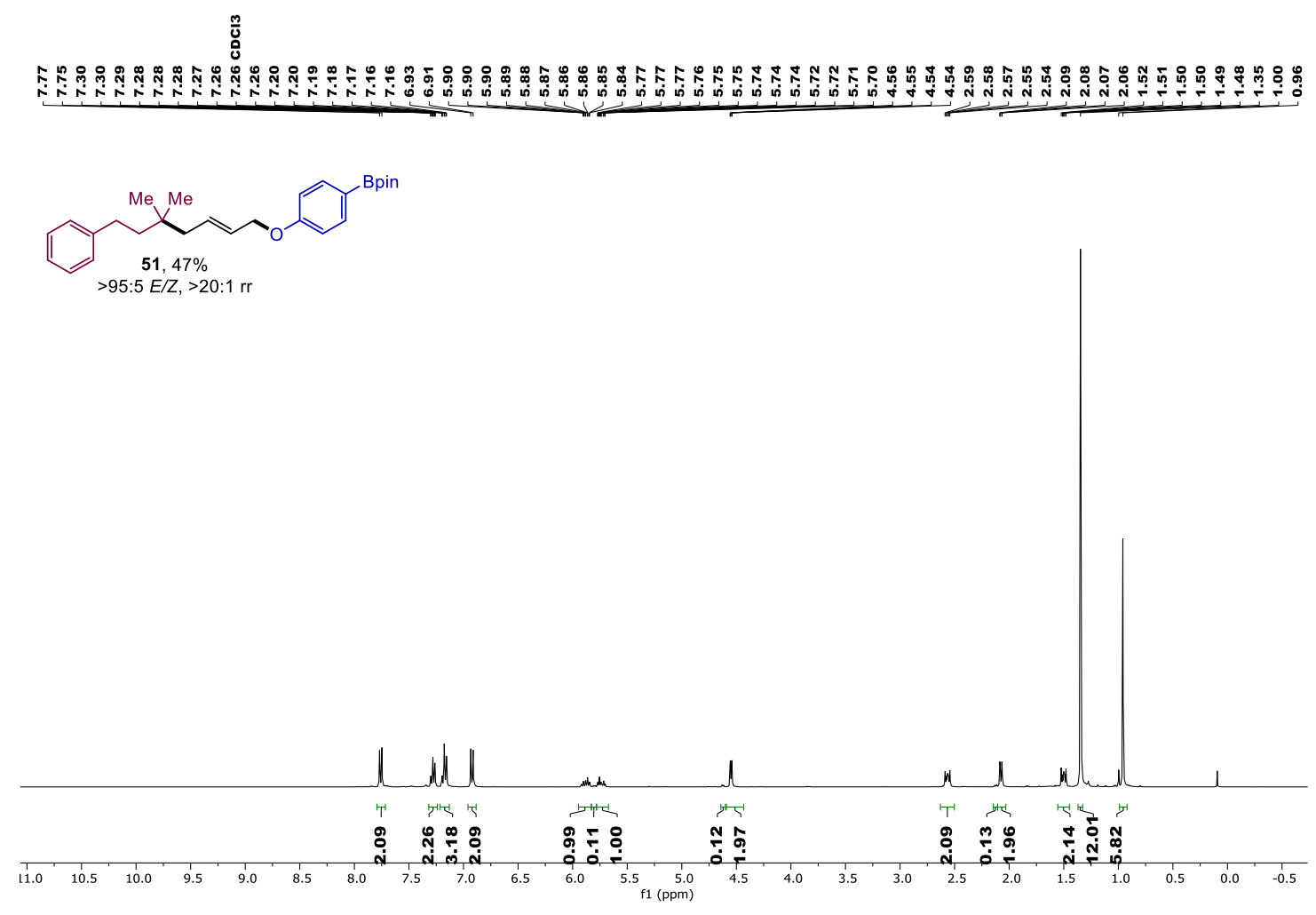

${ }^{13} \mathrm{C}$ NMR $\left(101 \mathrm{MHz}, \mathrm{CDCl}_{3}\right)$
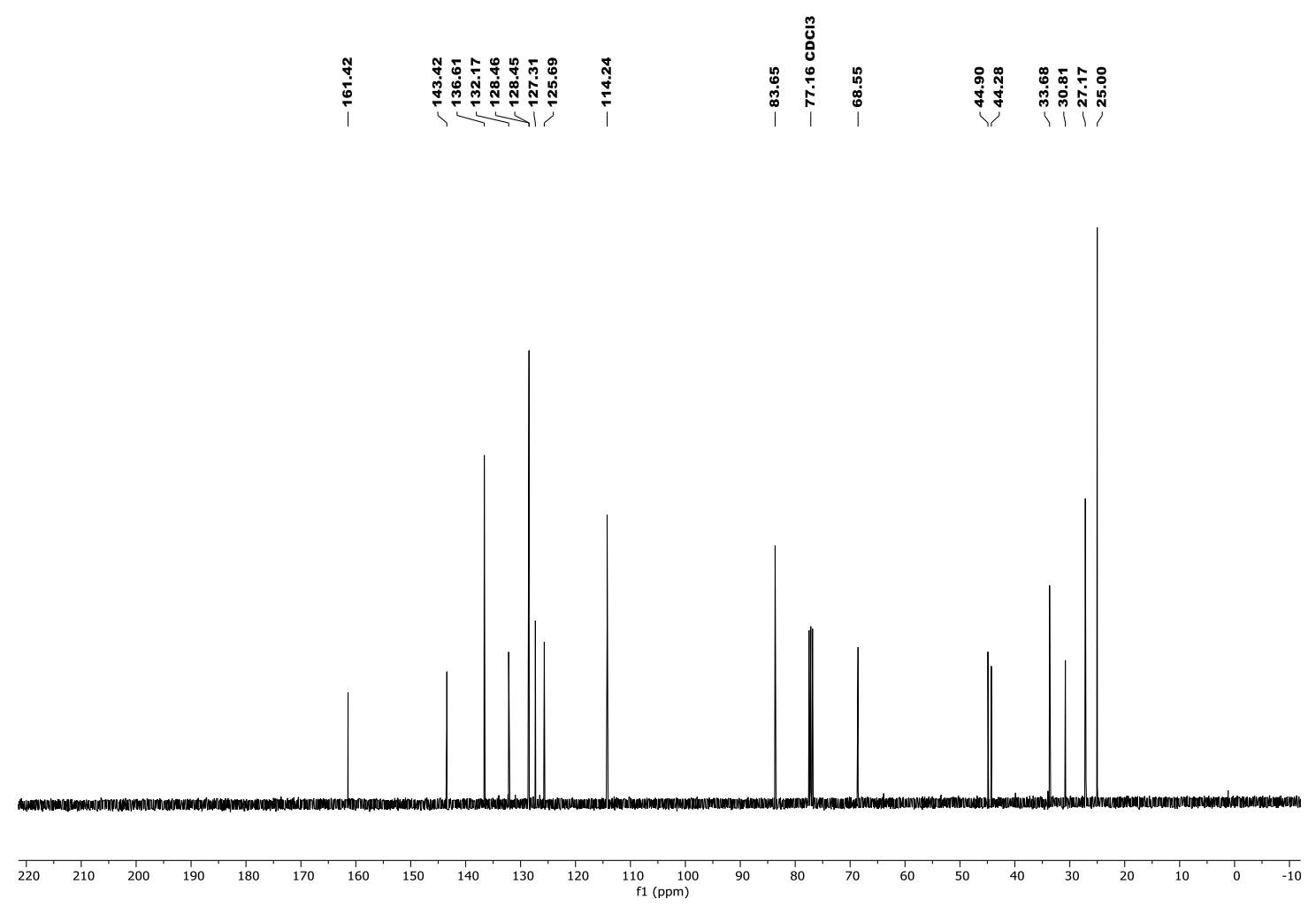
${ }^{1} \mathrm{H}$ NMR $\left(300 \mathrm{MHz}, \mathrm{CDCl}_{3}\right)$

\section{䓪

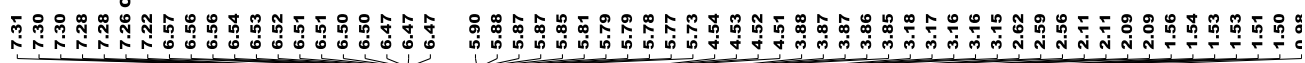
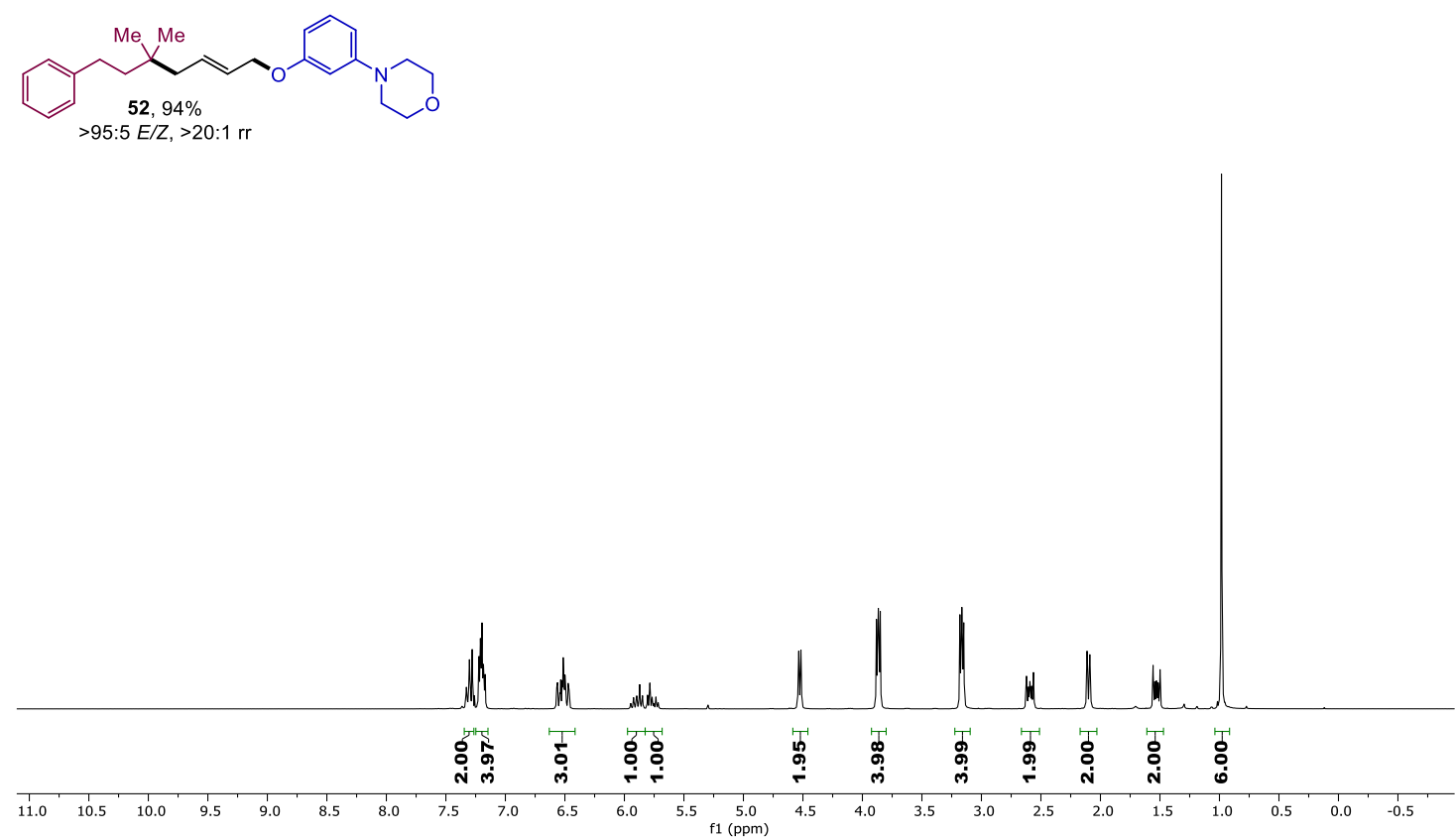

${ }^{13} \mathrm{C}$ NMR $\left(75 \mathrm{MHz}, \mathrm{CDCl}_{3}\right)$

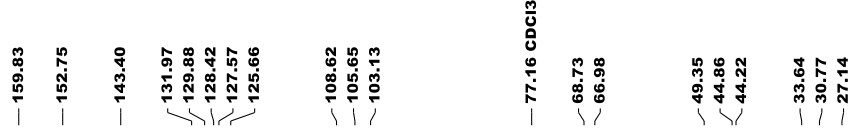

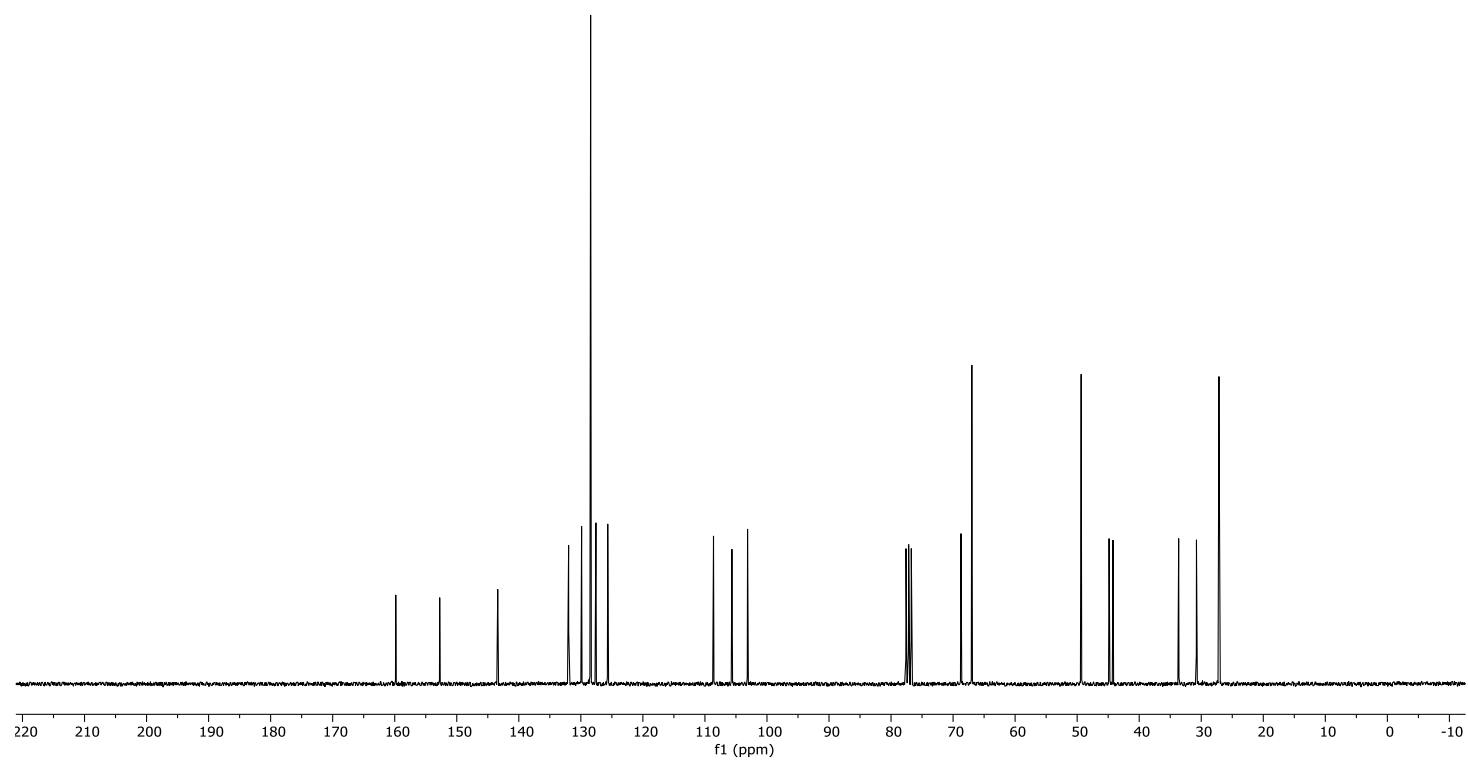


${ }^{1} \mathrm{H}$ NMR $\left(400 \mathrm{MHz}, \mathrm{CDCl}_{3}\right)$

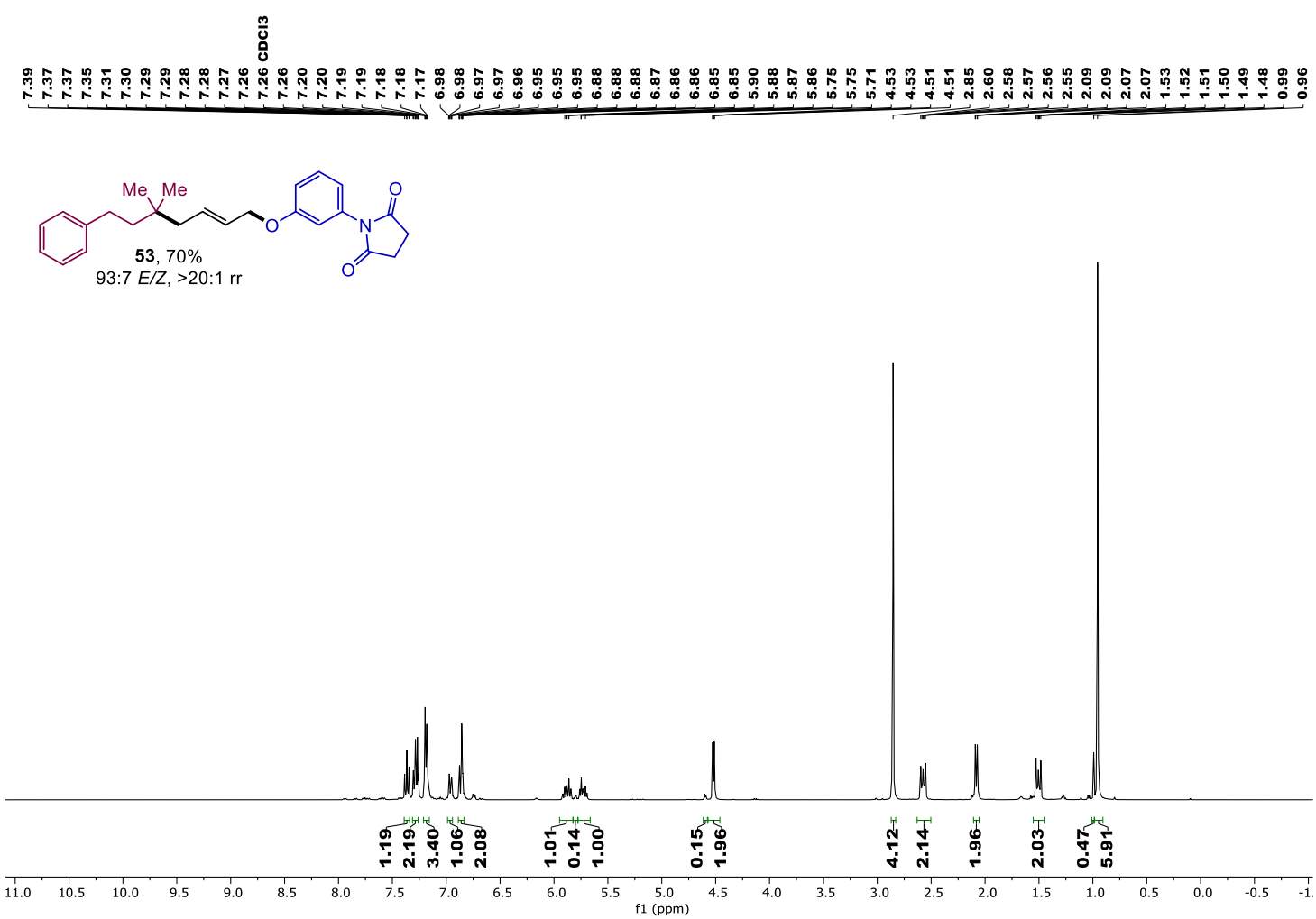

${ }^{13} \mathrm{C}$ NMR $\left(101 \mathrm{MHz}, \mathrm{CDCl}_{3}\right)$

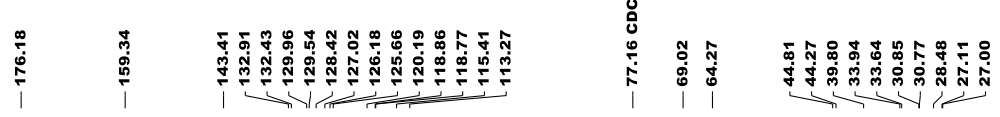

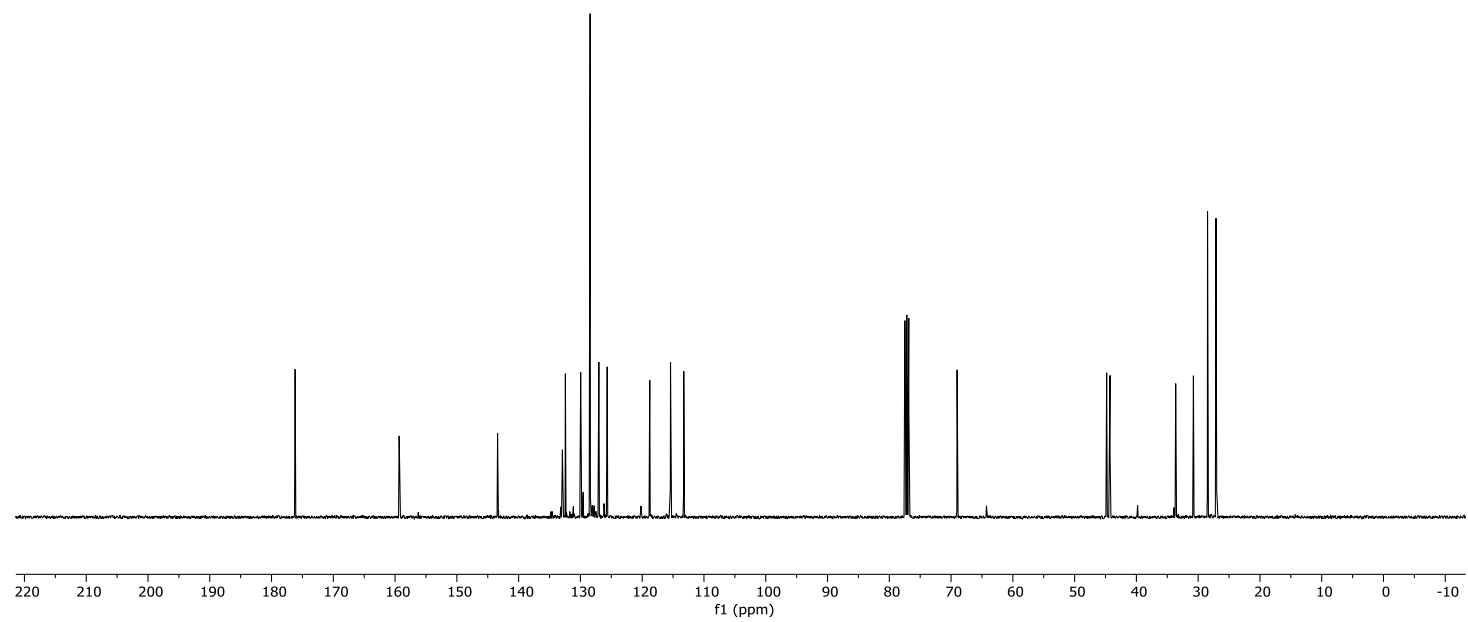


${ }^{1} \mathrm{H}$ NMR (400 MHz, $\left.\mathrm{CDCl}_{3}\right)$

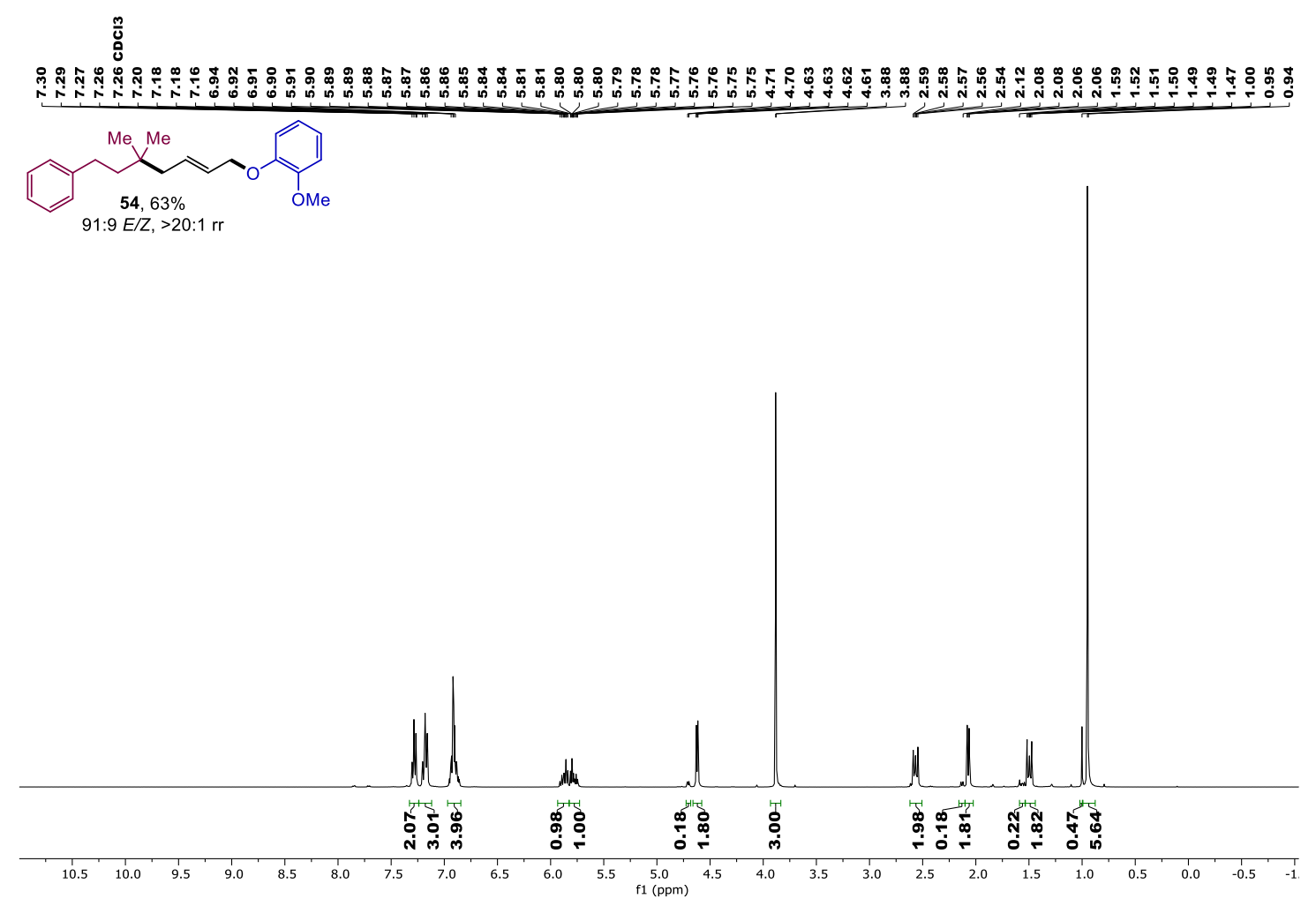

${ }^{13} \mathrm{C}$ NMR (101 MHz, $\mathrm{CDCl}_{3}$ )

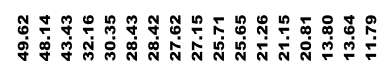

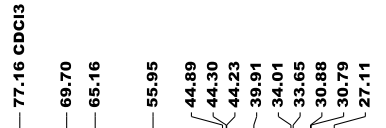

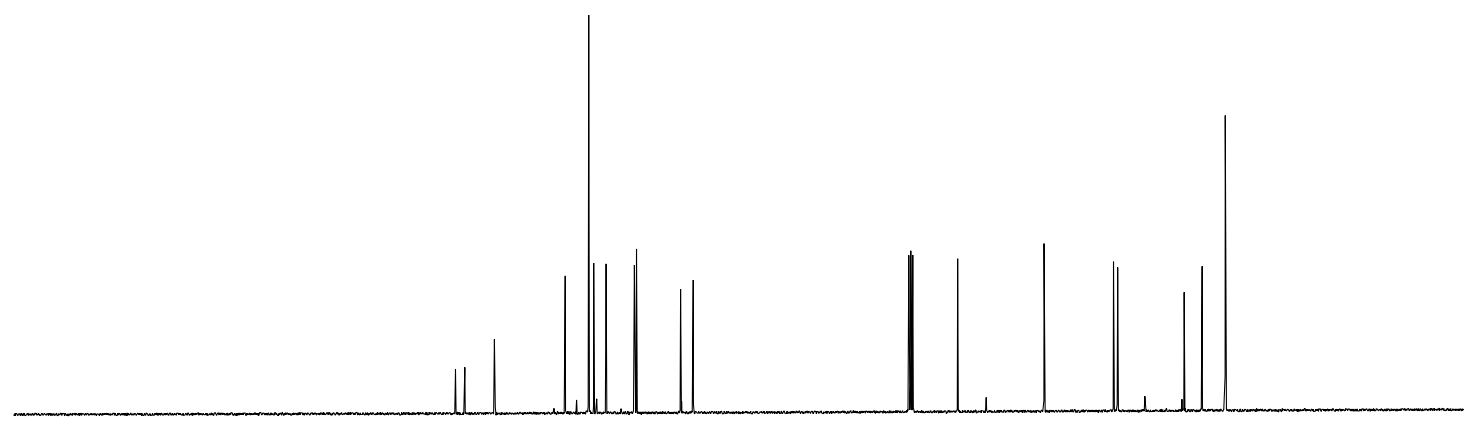


${ }^{1} \mathrm{H}$ NMR (599 MHz, $\mathrm{CDCl}_{3}$ )
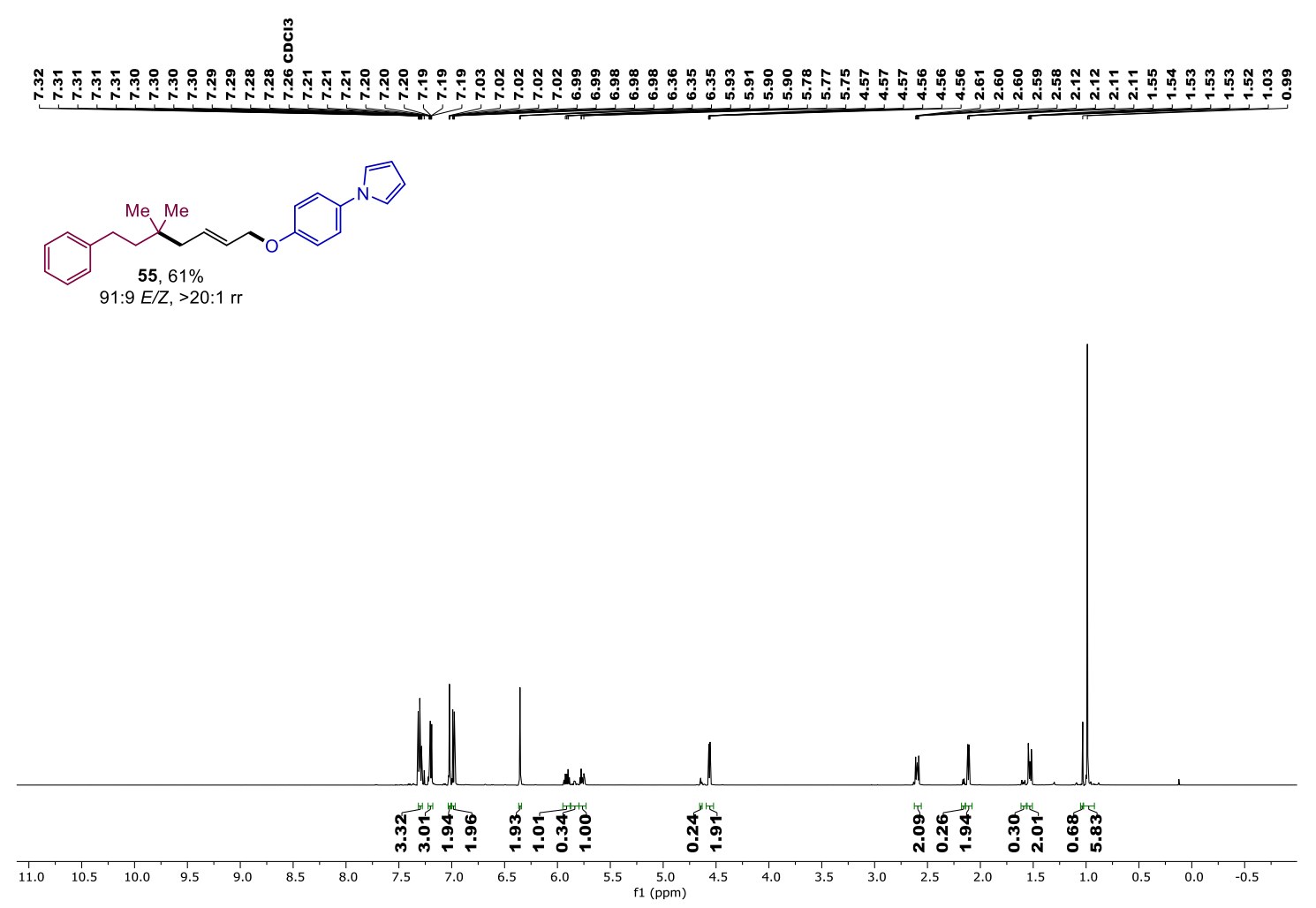

${ }^{13} \mathrm{C}$ NMR $\left(151 \mathrm{MHz}, \mathrm{CDCl}_{3}\right.$ )

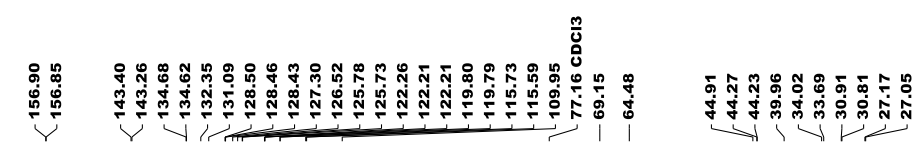

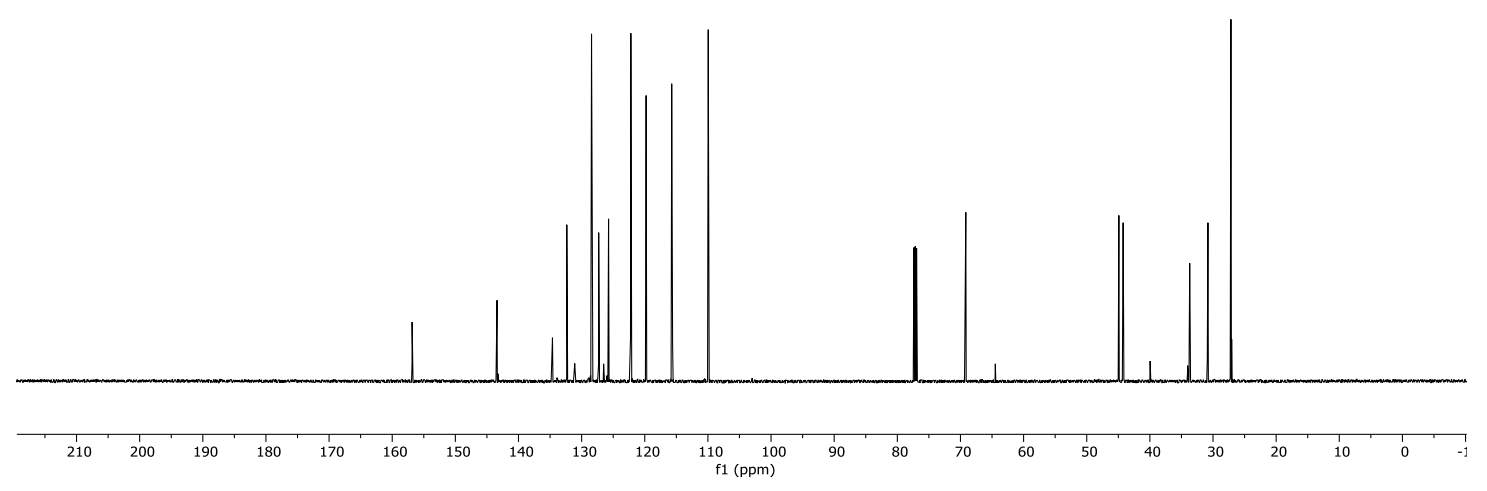


${ }^{1} \mathrm{H}$ NMR (599 MHz, $\left.\mathrm{CDCl}_{3}\right)$
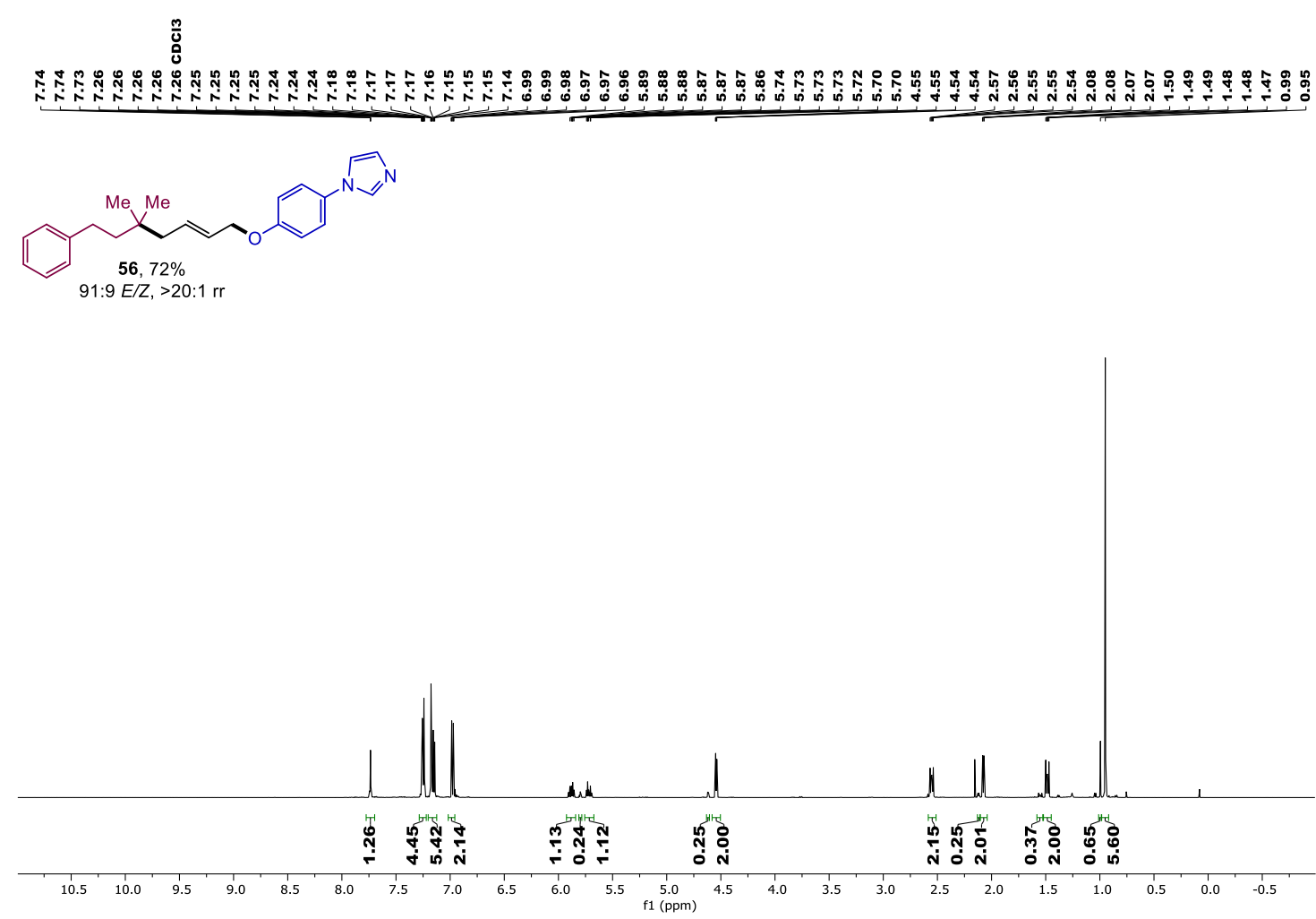

${ }^{13} \mathrm{C} \mathrm{NMR}\left(151 \mathrm{MHz}, \mathrm{CDCl}_{3}\right)$
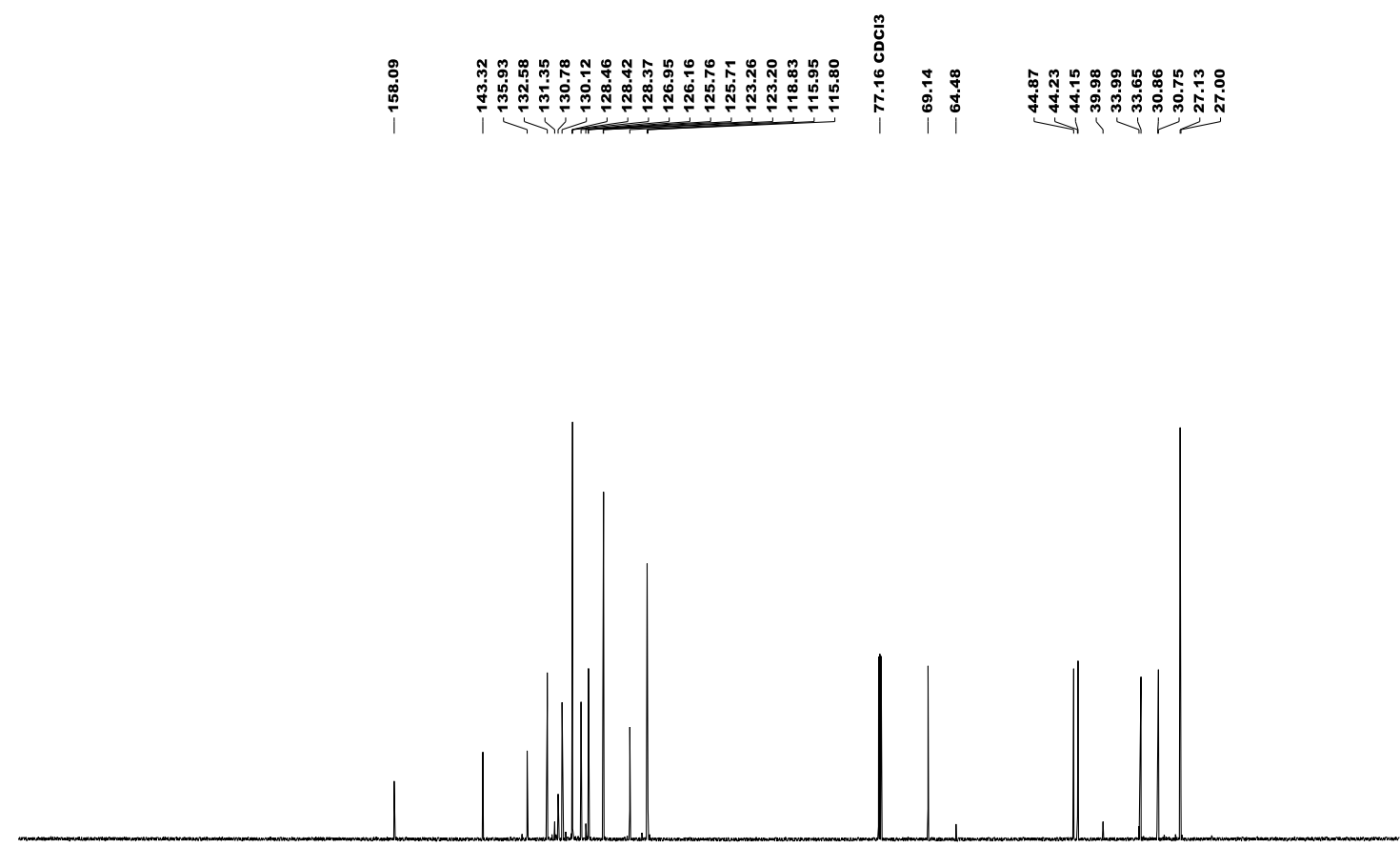

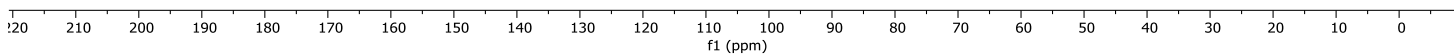


${ }^{1} \mathrm{H}^{-1} \mathrm{H} \operatorname{COSY}\left(599 \mathrm{MHz}, \mathrm{CDCl}_{3}\right)$

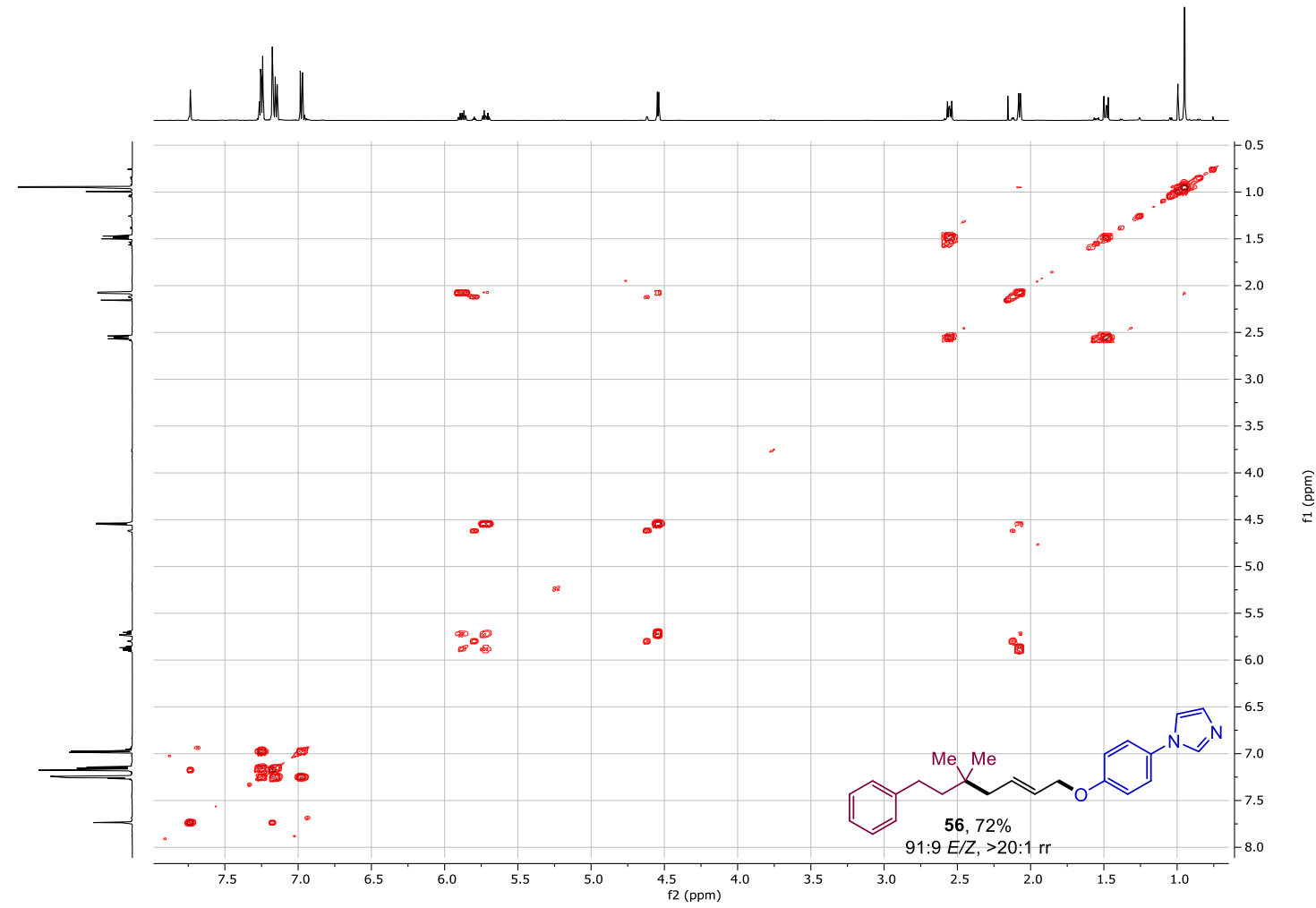

${ }^{1} \mathrm{H}-{ }^{13} \mathrm{C}$ HSQC (599 MHz, $\mathrm{CDCl}_{3}$ )

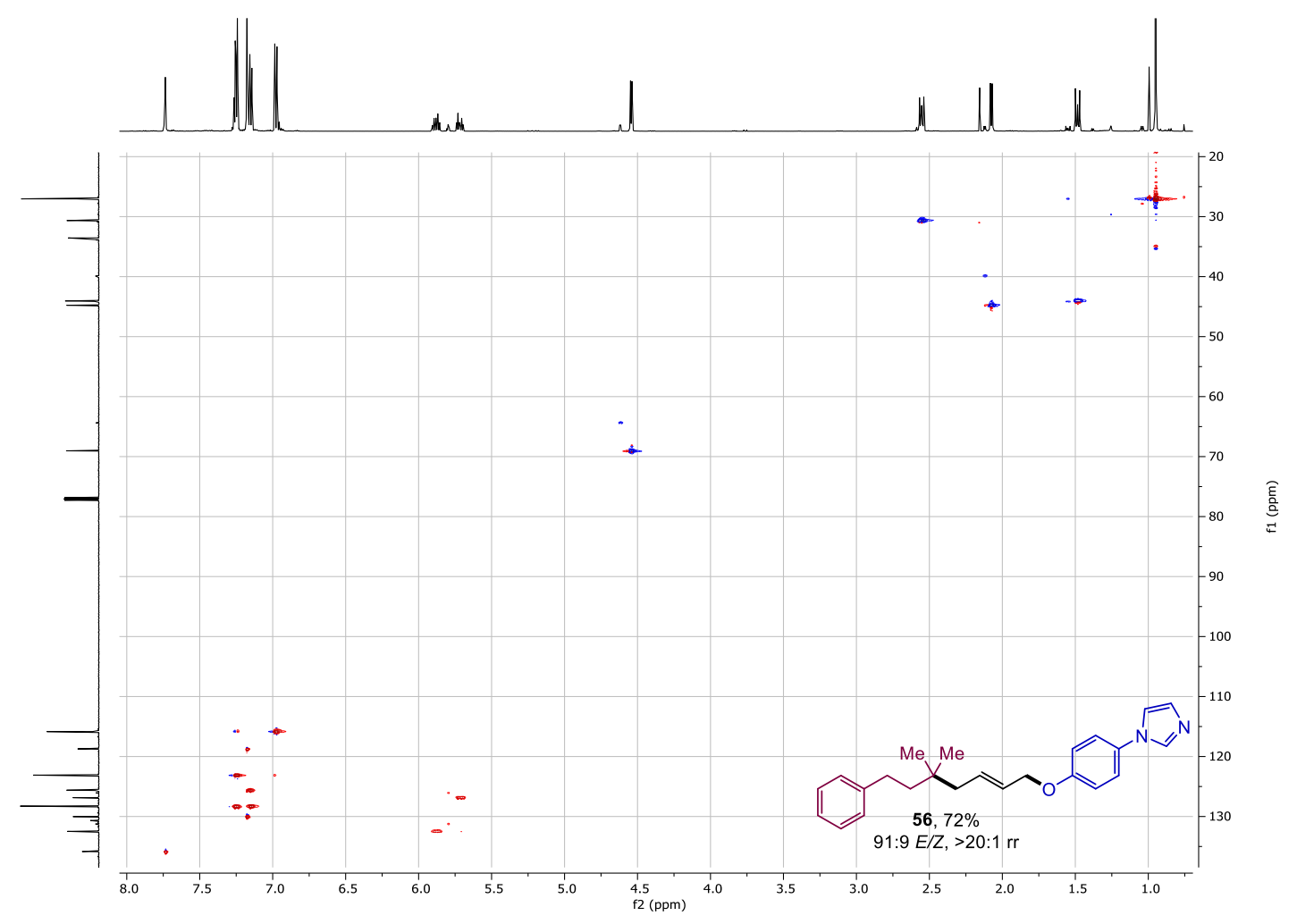


${ }^{1} \mathrm{H}-{ }^{13} \mathrm{C} \mathrm{HMBC}\left(599 \mathrm{MHz}, \mathrm{CDCl}_{3}\right)$

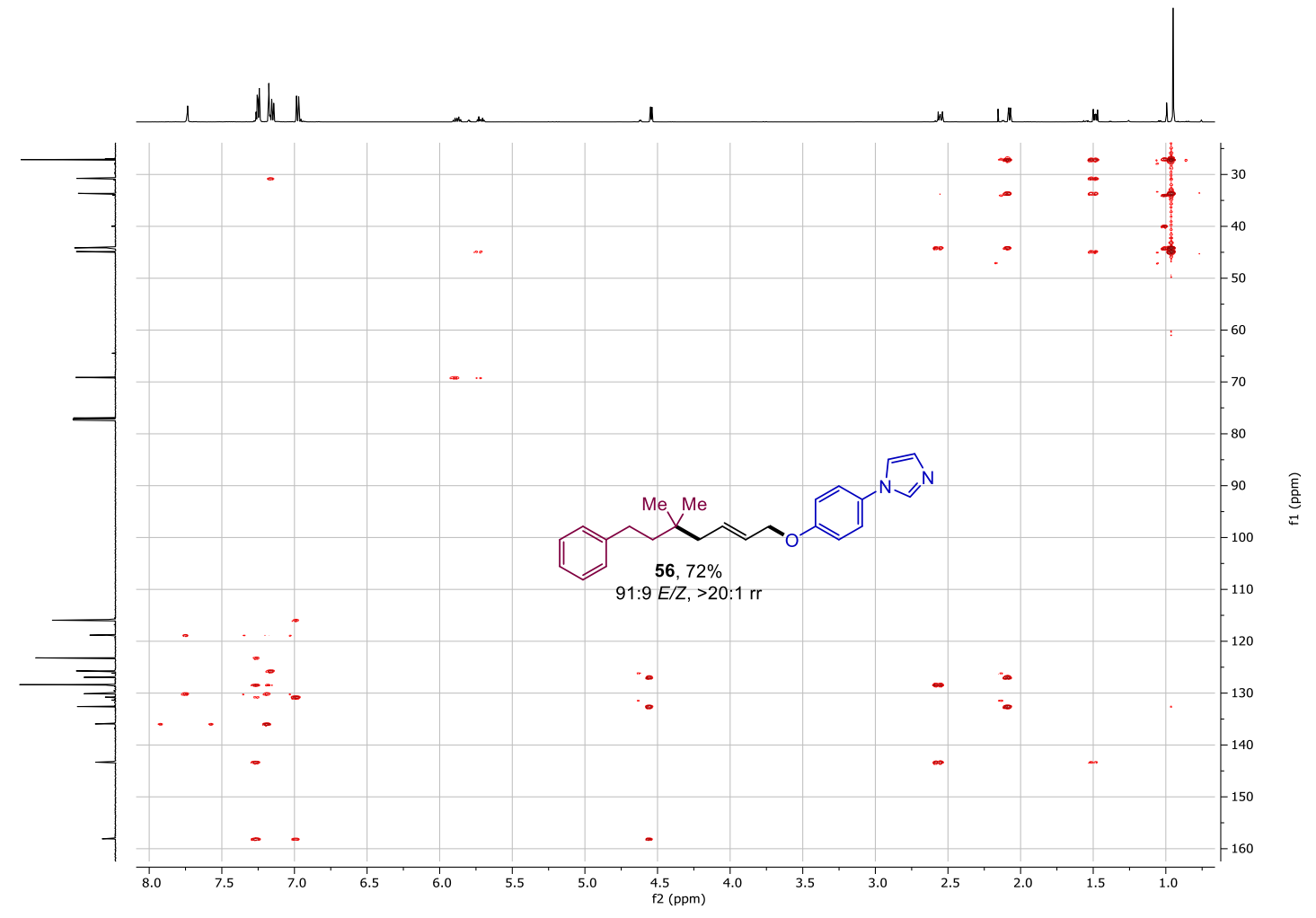


${ }^{1} \mathrm{H}$ NMR $\left(300 \mathrm{MHz}, \mathrm{CDCl}_{3}\right)$

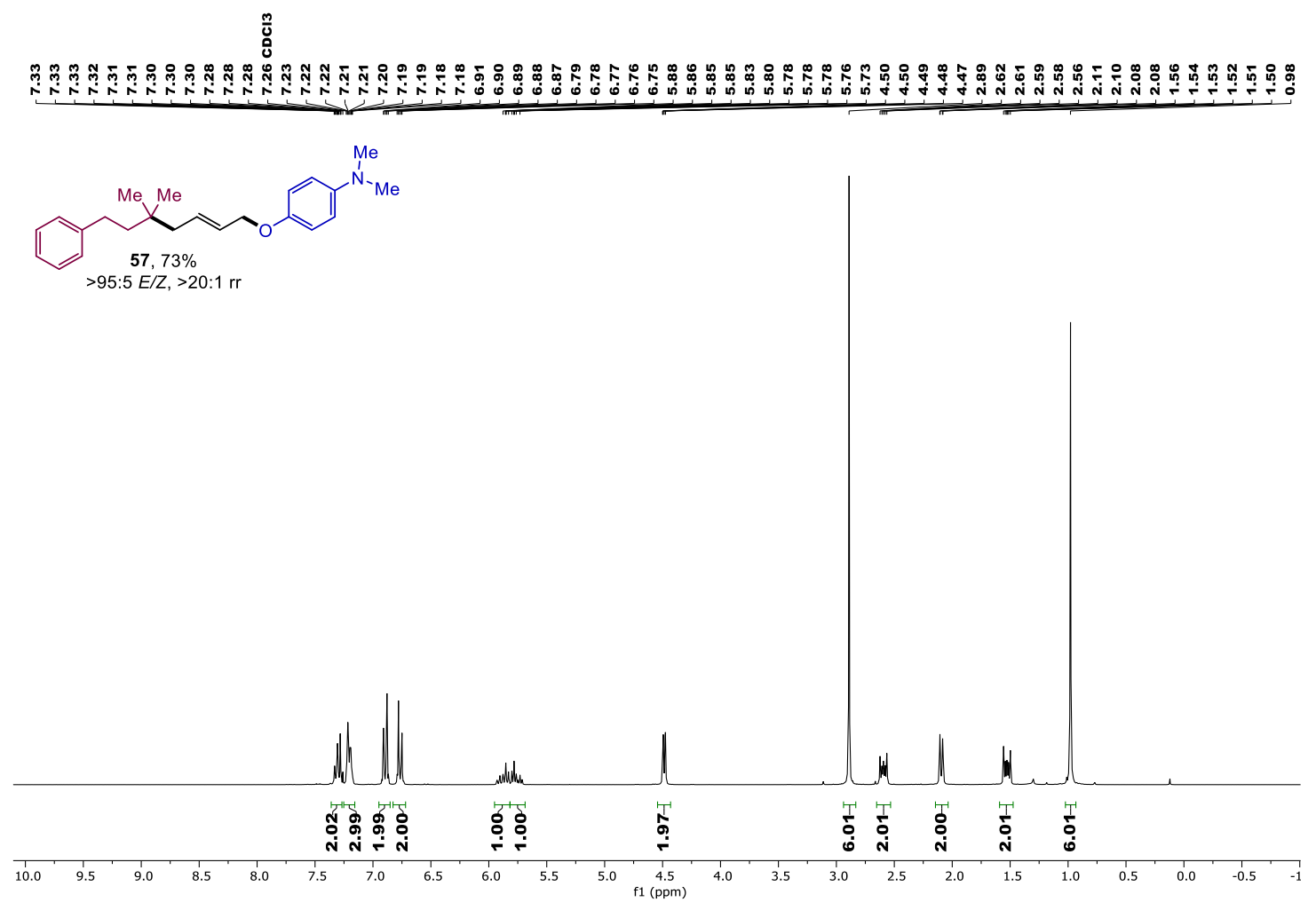

${ }^{13} \mathrm{C}$ NMR $\left(75 \mathrm{MHz}, \mathrm{CDCl}_{3}\right)$
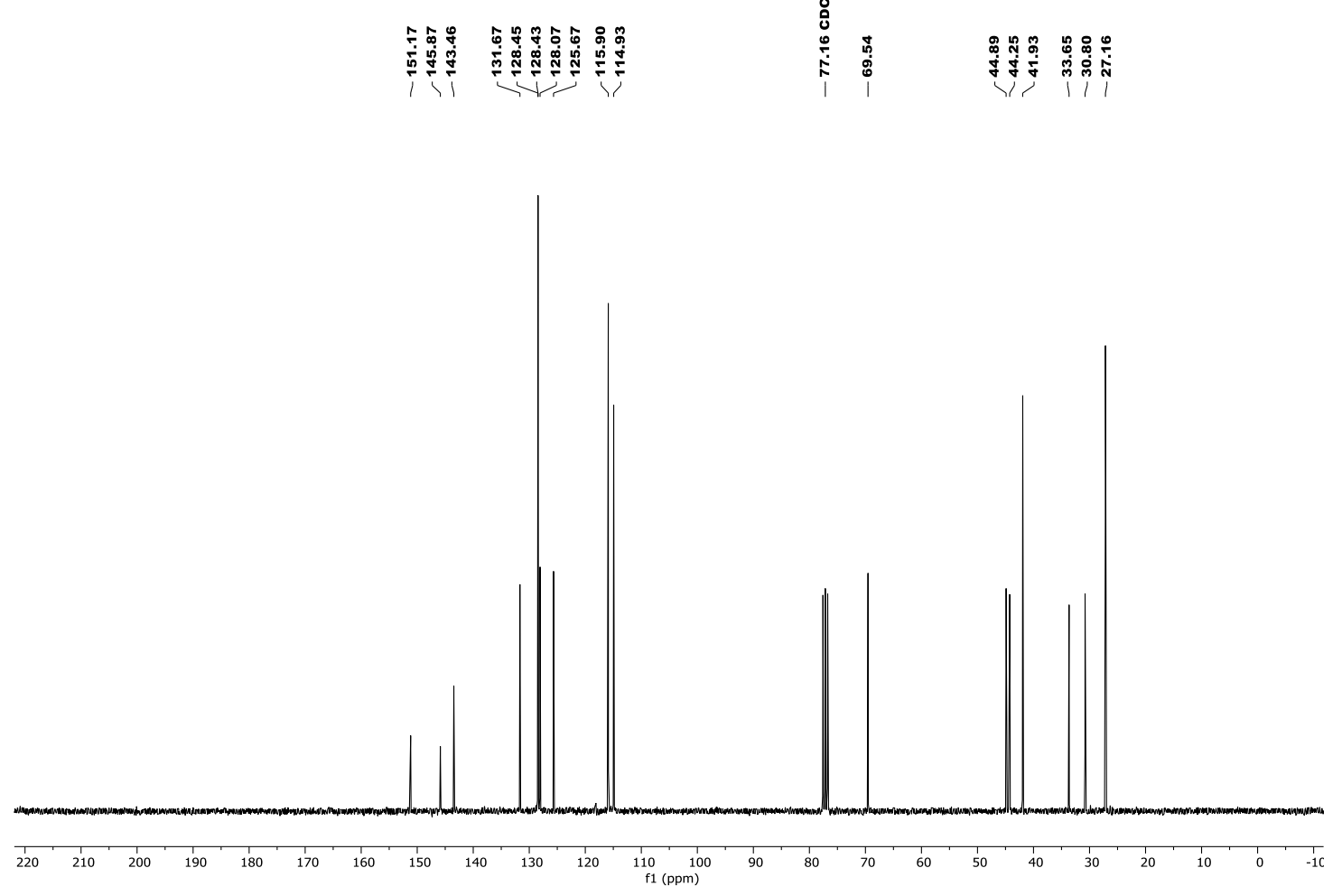
${ }^{1} \mathrm{H}$ NMR $\left(300 \mathrm{MHz}, \mathrm{CDCl}_{3}\right.$ )
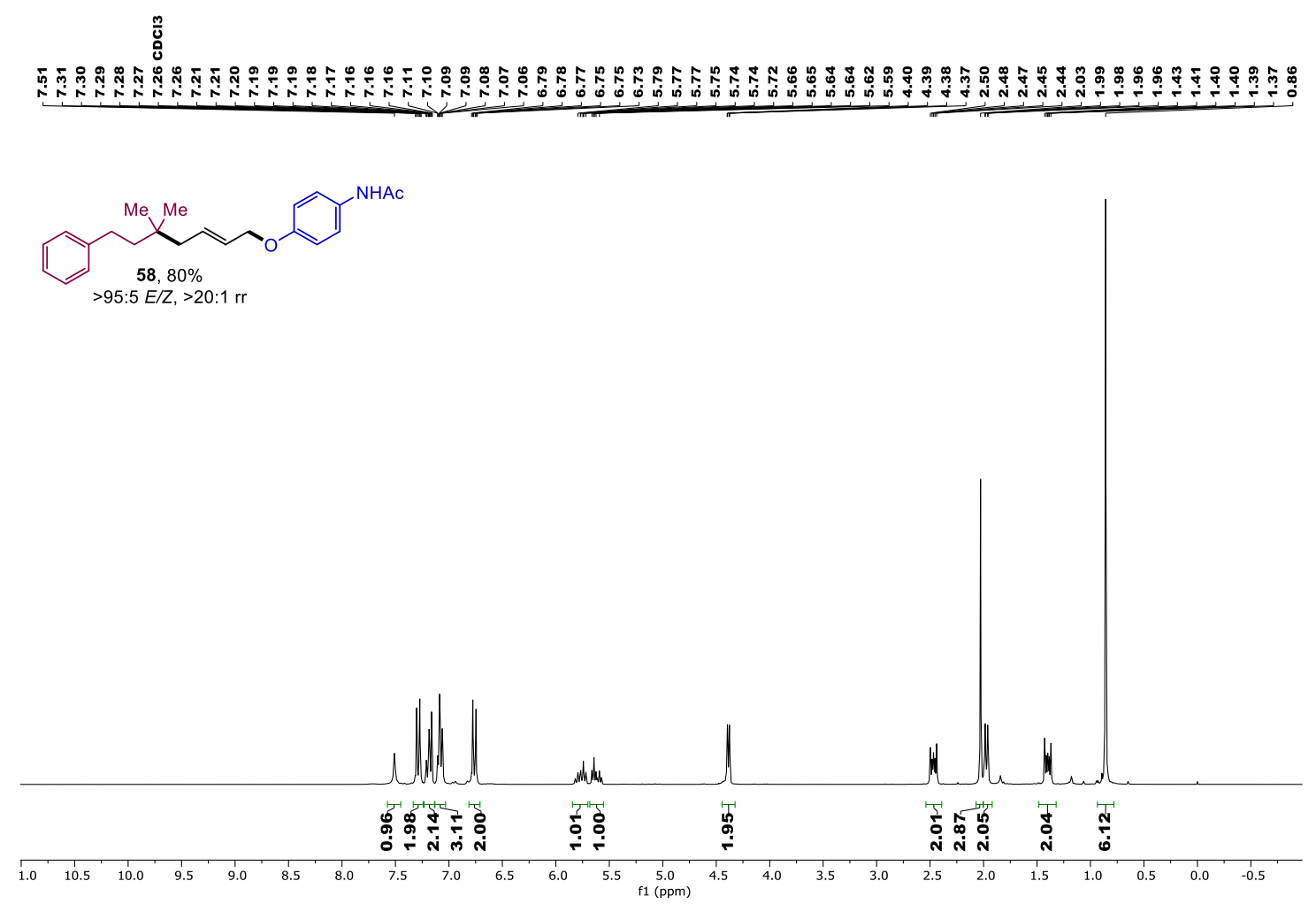

${ }^{13} \mathrm{C}$ NMR $\left(75 \mathrm{MHz}, \mathrm{CDCl}_{3}\right.$ )
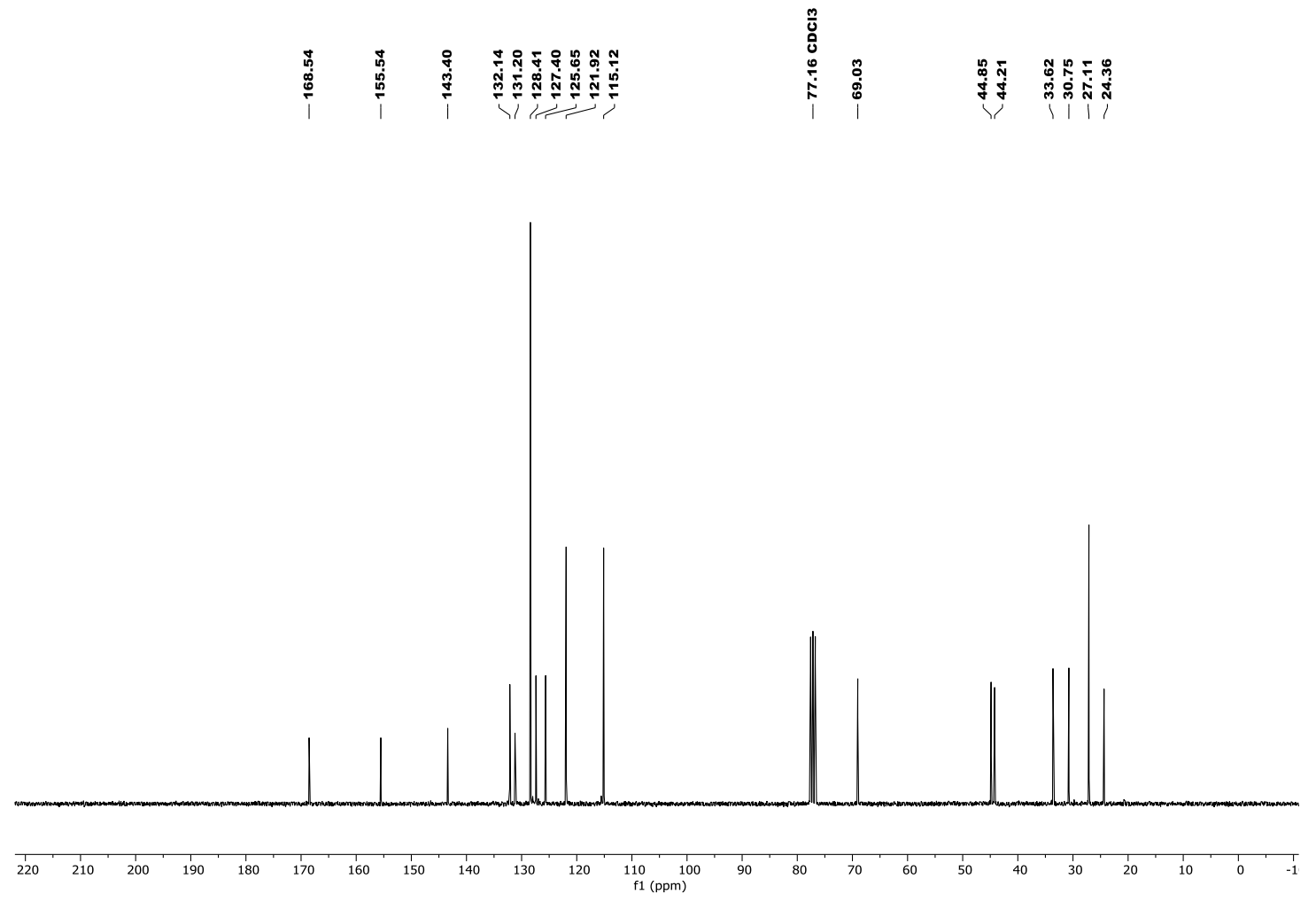
${ }^{1} \mathrm{H} \mathrm{NMR}\left(400 \mathrm{MHz}, \mathrm{CDCl}_{3}\right)$

\section{g.8.

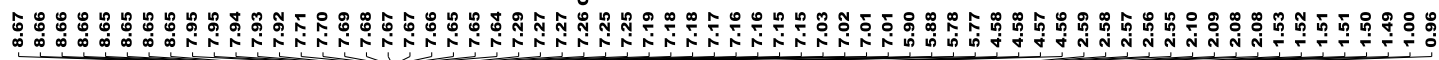
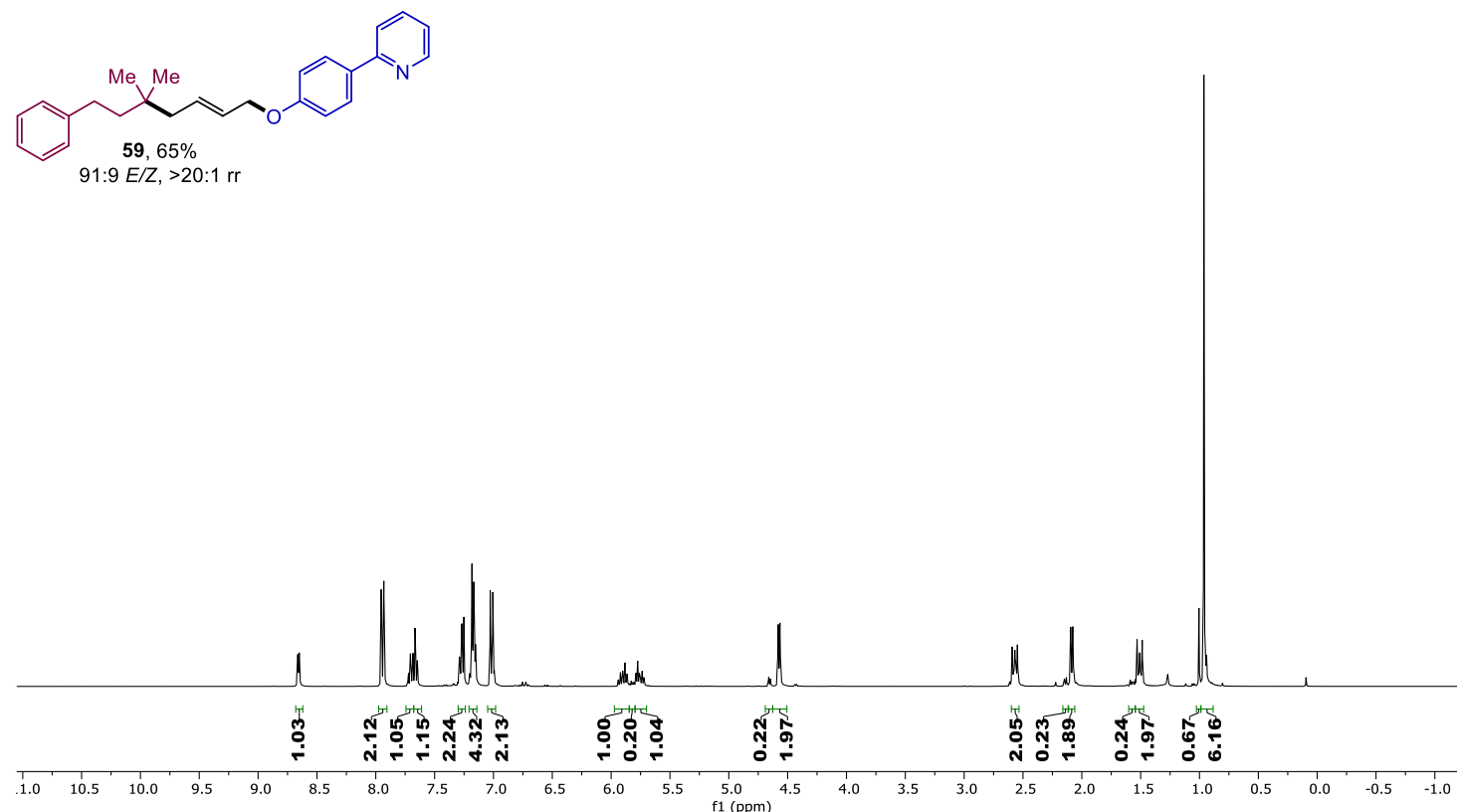

${ }^{13} \mathrm{C}$ NMR $\left(101 \mathrm{MHz}, \mathrm{CDCl}_{3}\right)$
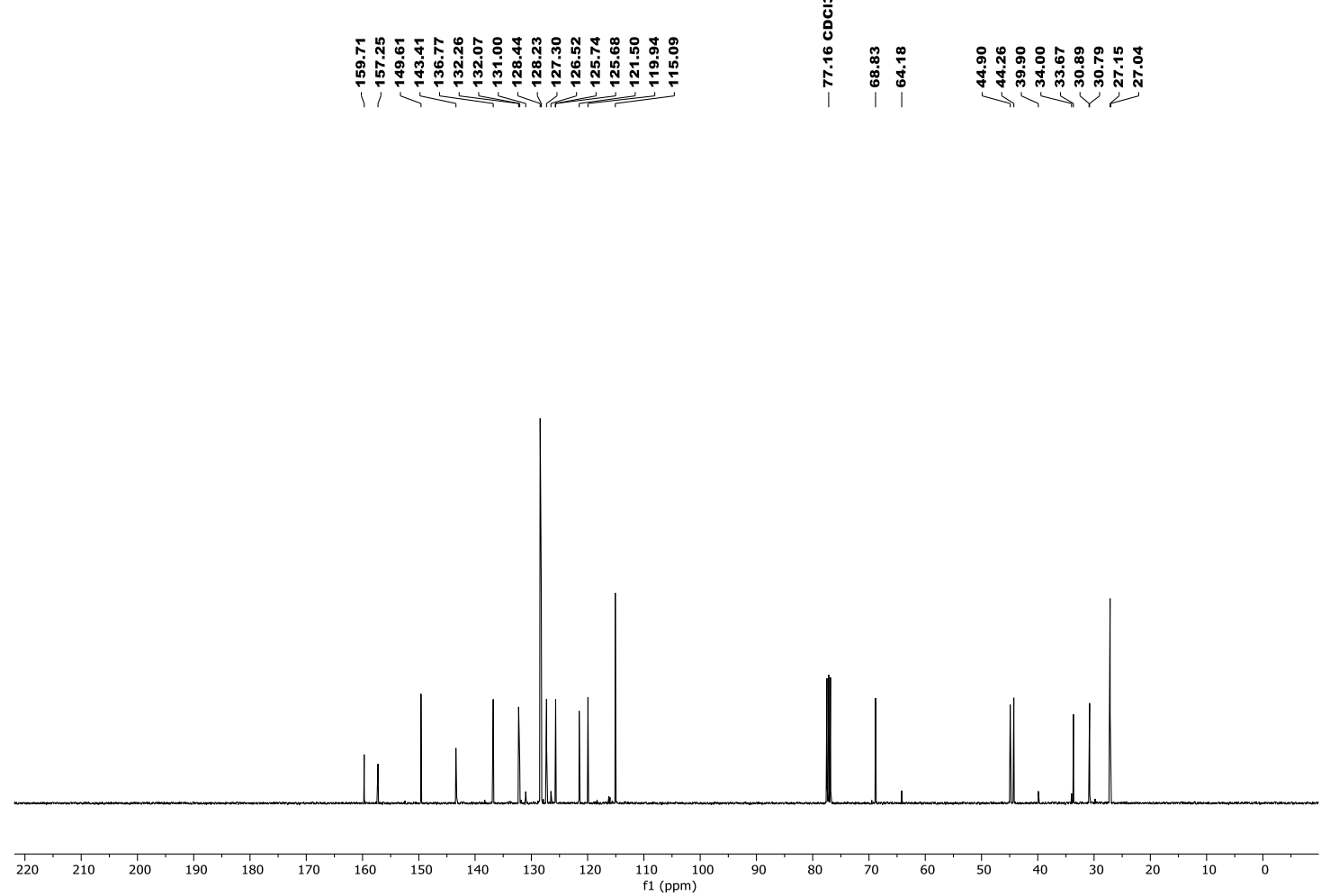
${ }^{1} \mathrm{H} \mathrm{NMR}\left(300 \mathrm{MHz}, \mathrm{CDCl}_{3}\right)$

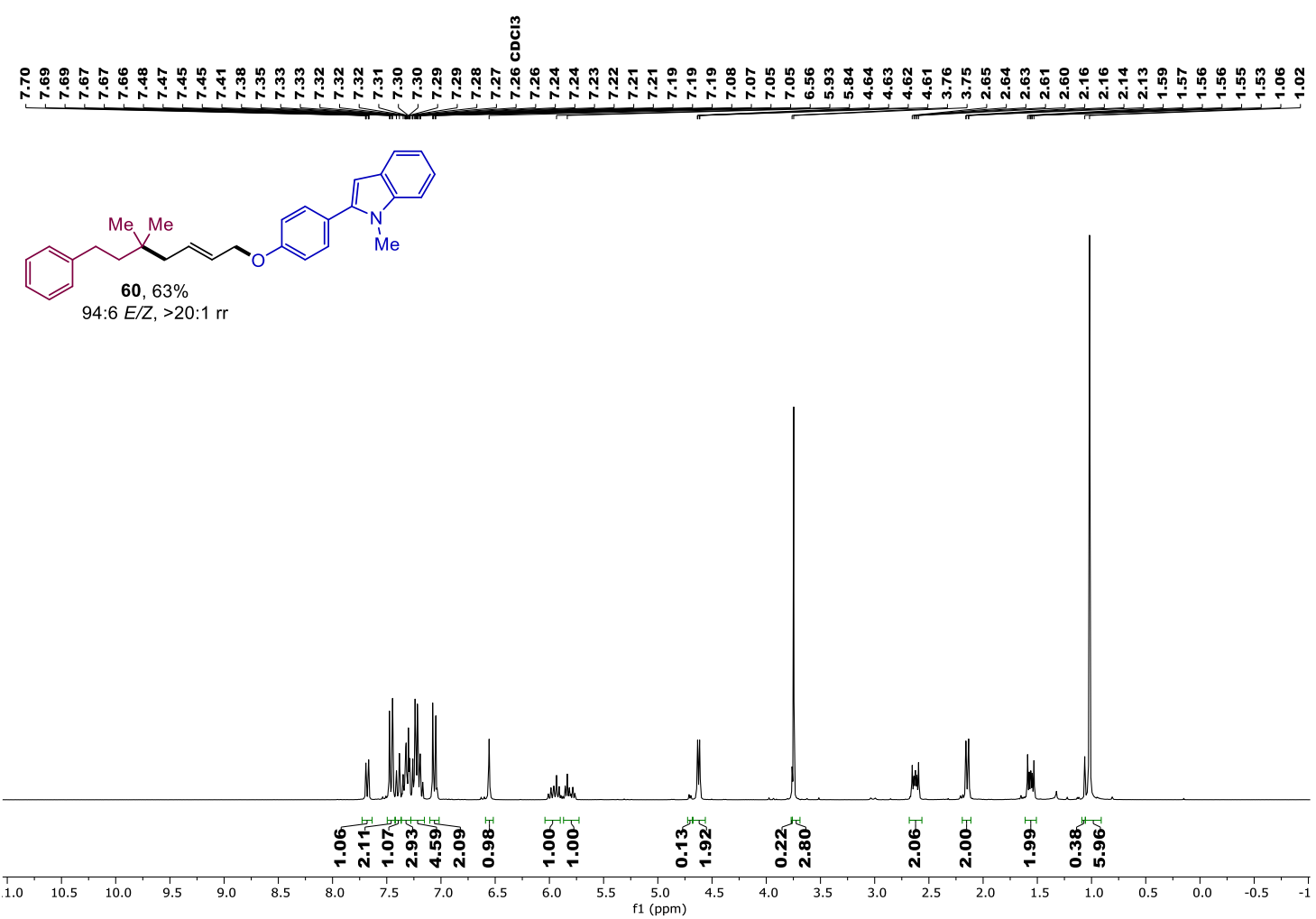

${ }^{13} \mathrm{C}$ NMR $\left(75 \mathrm{MHz}, \mathrm{CDCl}_{3}\right)$
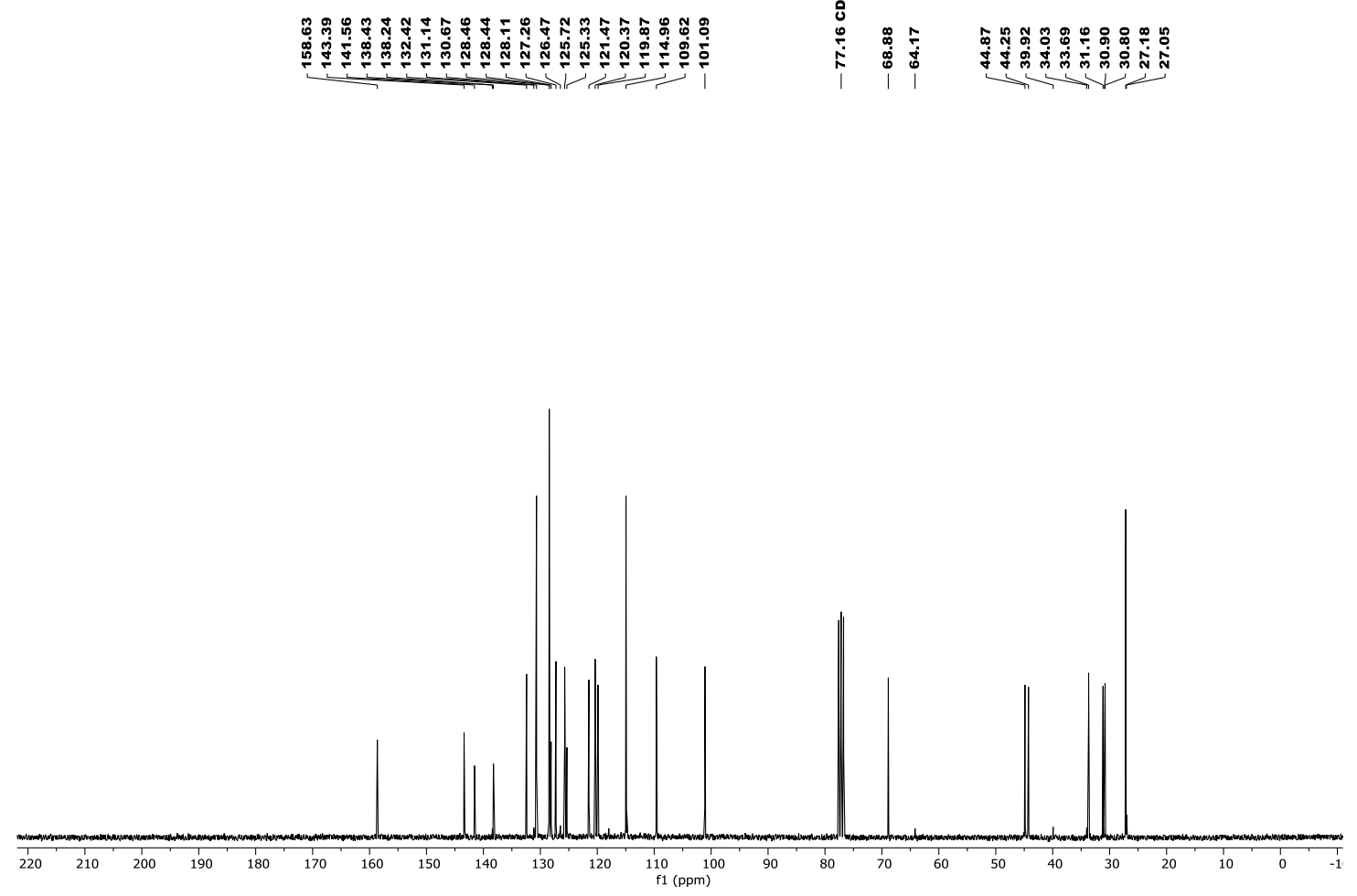
${ }^{1} \mathrm{H}$ NMR $\left(300 \mathrm{MHz}, \mathrm{CDCl}_{3}\right)$

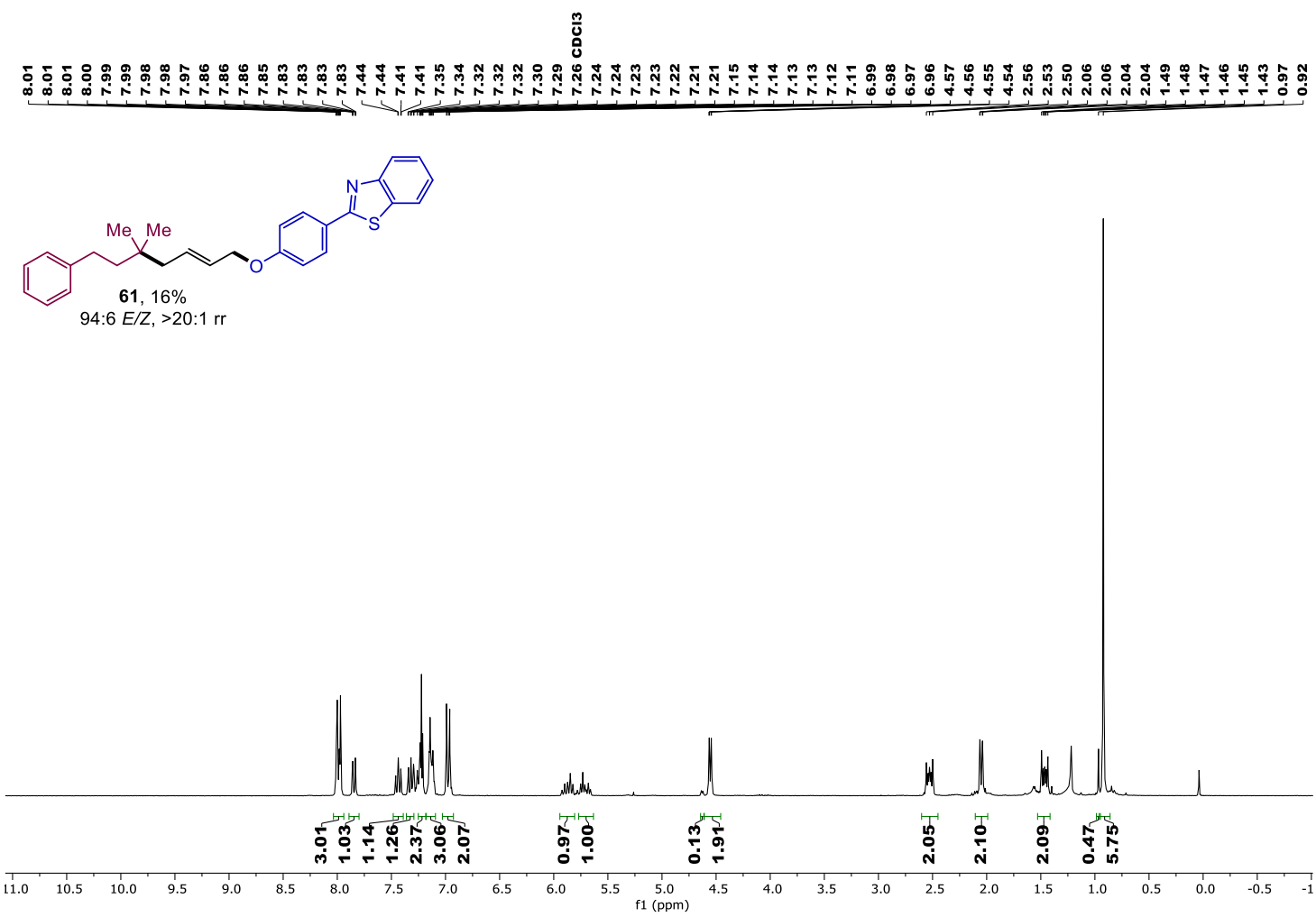

${ }^{13} \mathrm{C}$ NMR $\left(75 \mathrm{MHz}, \mathrm{CDCl}_{3}\right)$

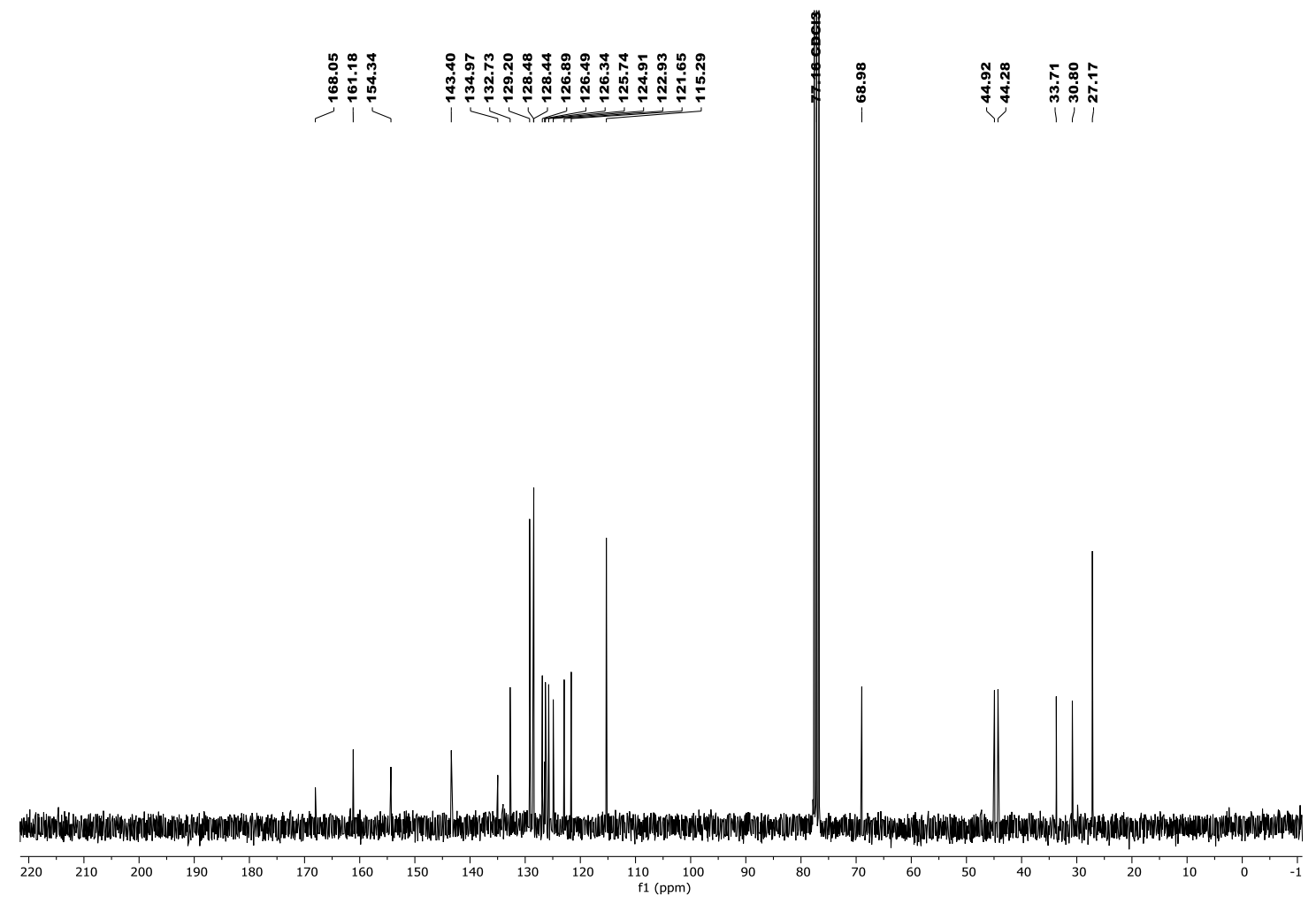


${ }^{1} \mathrm{H}$ NMR $\left(300 \mathrm{MHz}, \mathrm{CDCl}_{3}\right)$
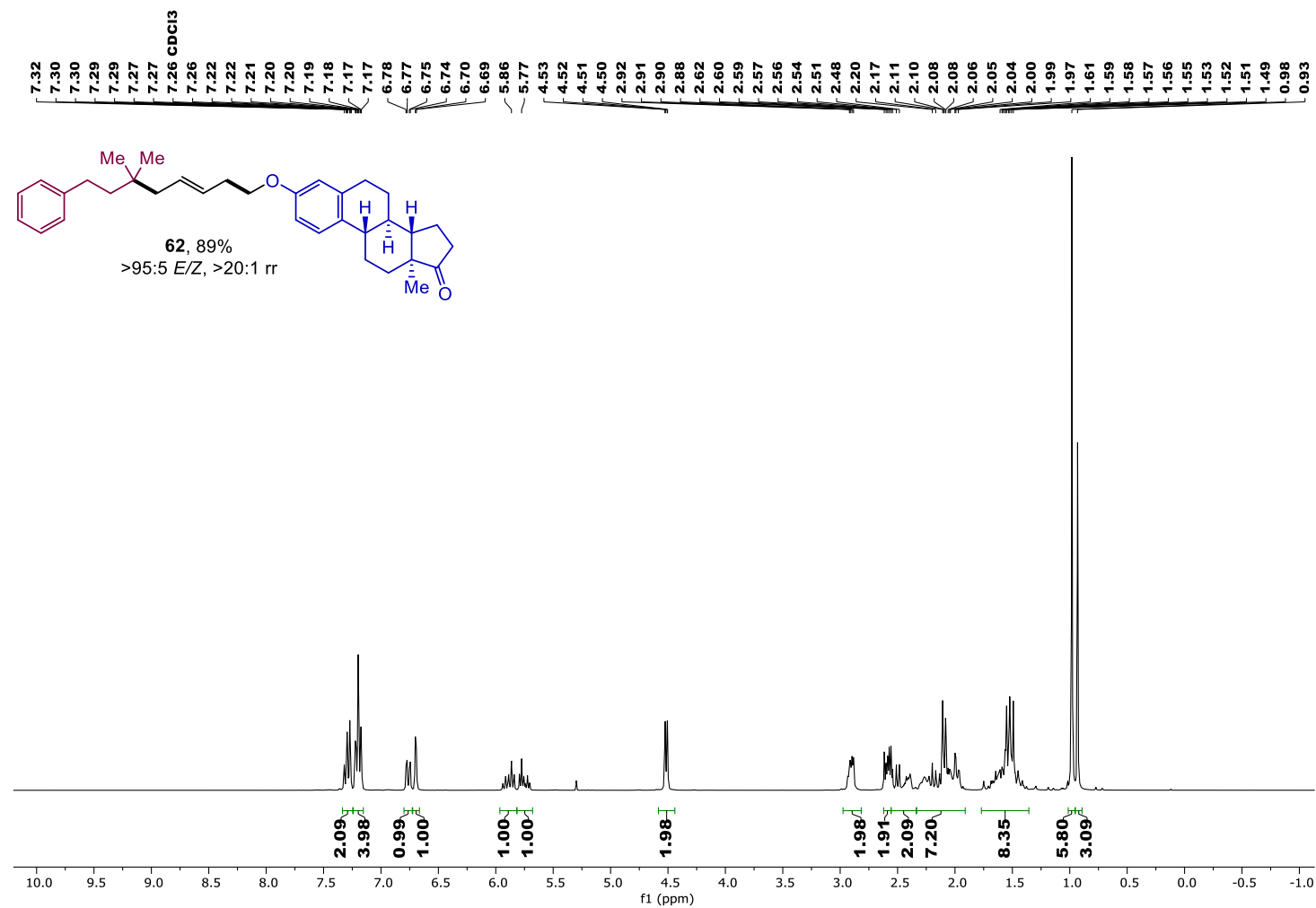

${ }^{13} \mathrm{C}$ NMR $\left(75 \mathrm{MHz}, \mathrm{CDCl}_{3}\right)$

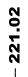

$\frac{m}{0}$

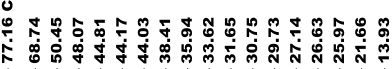

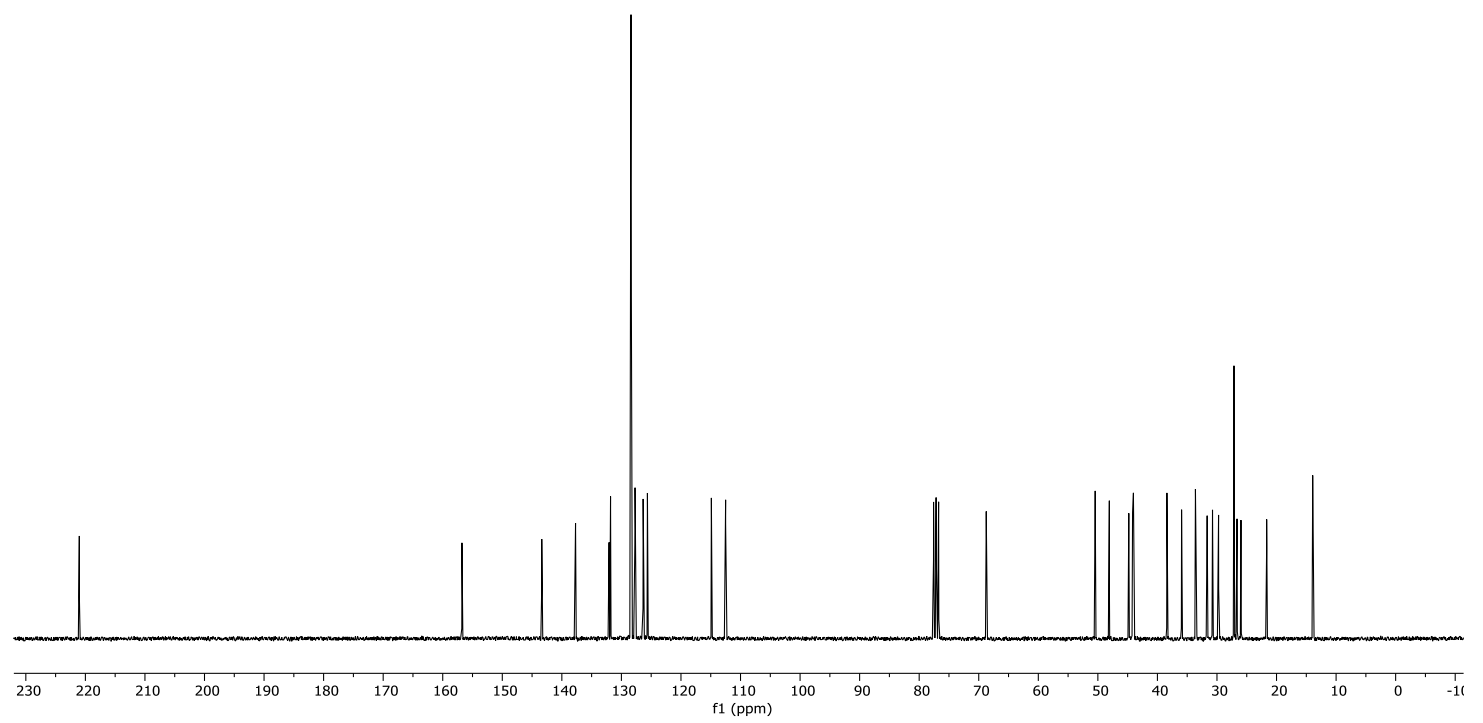


${ }^{1} \mathrm{H} \mathrm{NMR}\left(400 \mathrm{MHz}, \mathrm{CDCl}_{3}\right)$
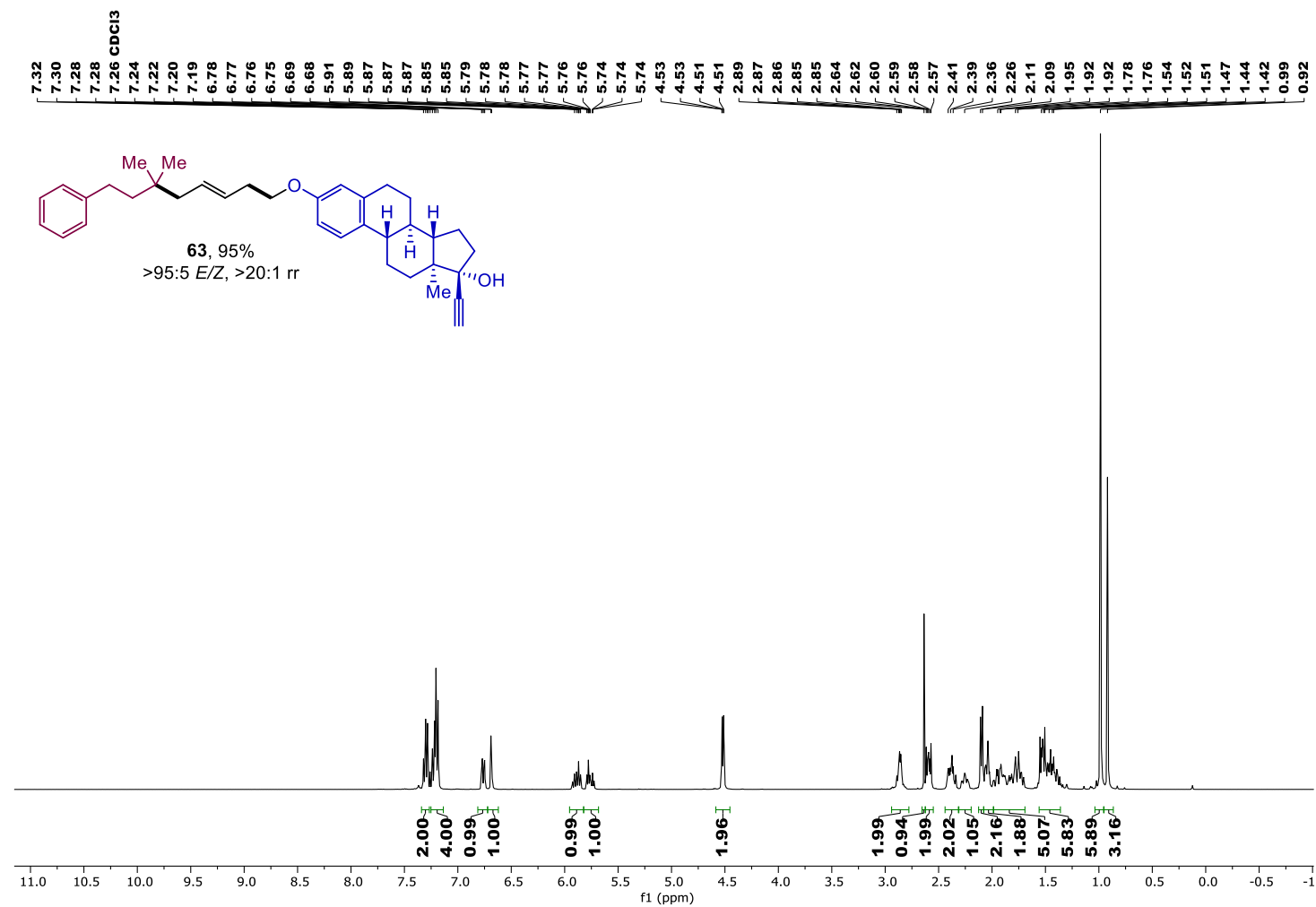

${ }^{13} \mathrm{C}$ NMR (101 MHz, $\mathrm{CDCl}_{3}$ )
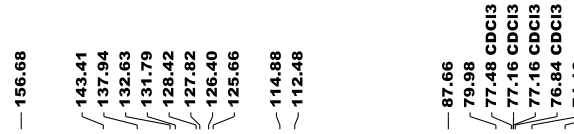

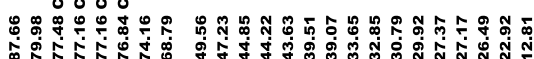

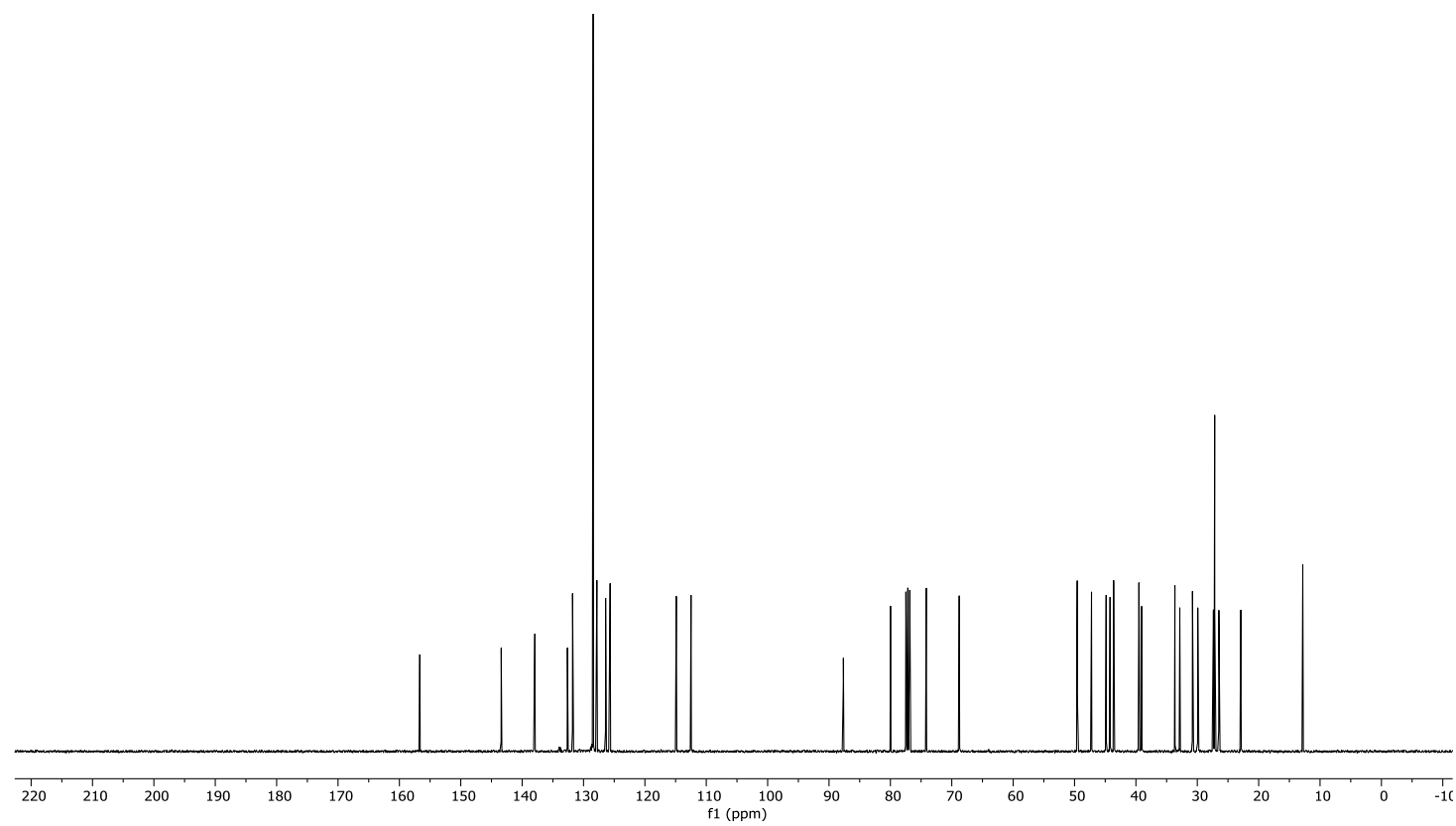


${ }^{1} \mathrm{H}$ NMR $\left(599 \mathrm{MHz}, \mathrm{CDCl}_{3}\right)$

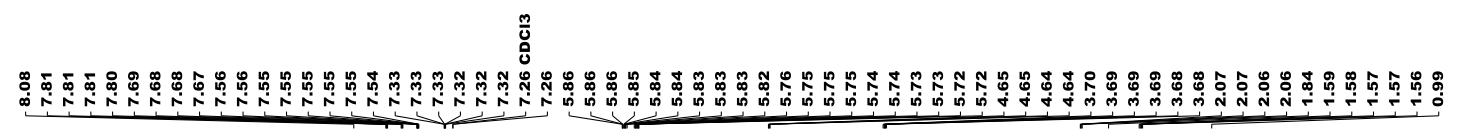

(c)

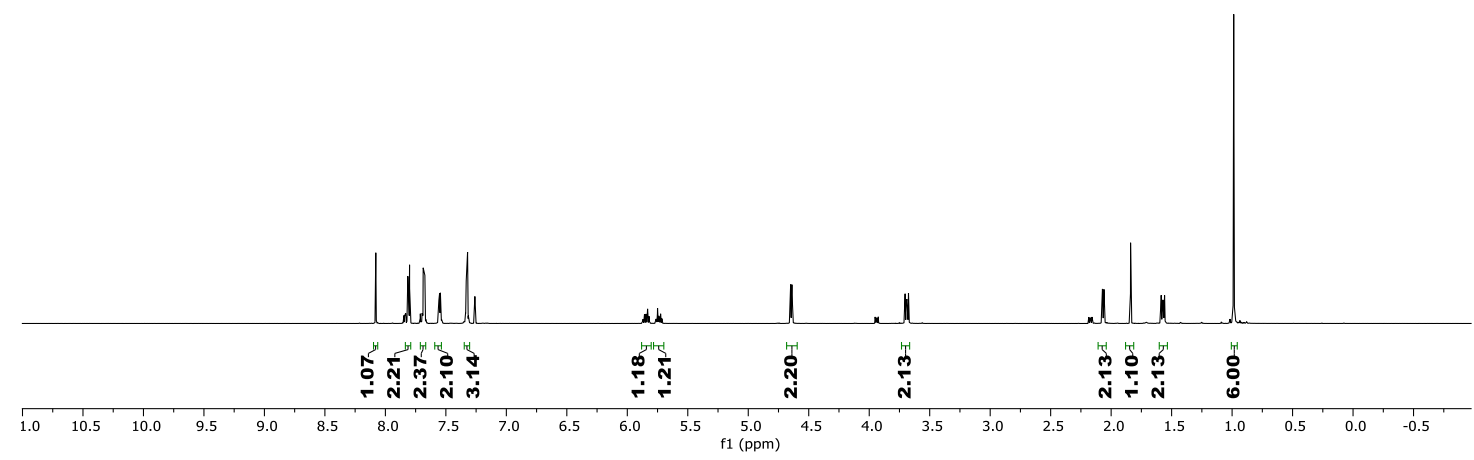

${ }^{13} \mathrm{C}$ NMR (151 MHz, $\mathrm{CDCl}_{3}$ )
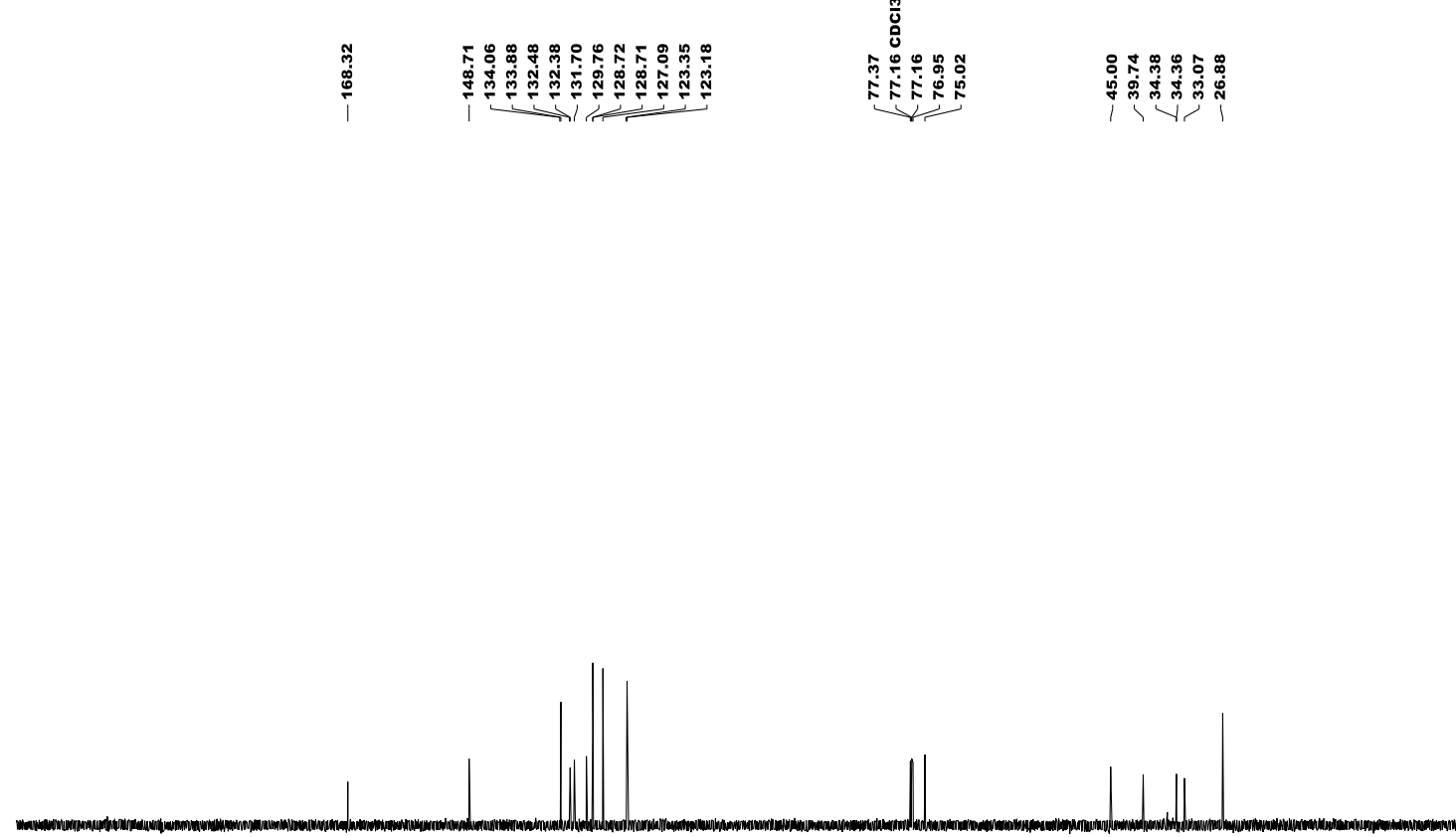

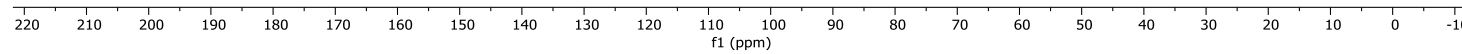


${ }^{1} \mathrm{H} \mathrm{NMR}\left(599 \mathrm{MHz}, \mathrm{CDCl}_{3}\right)$
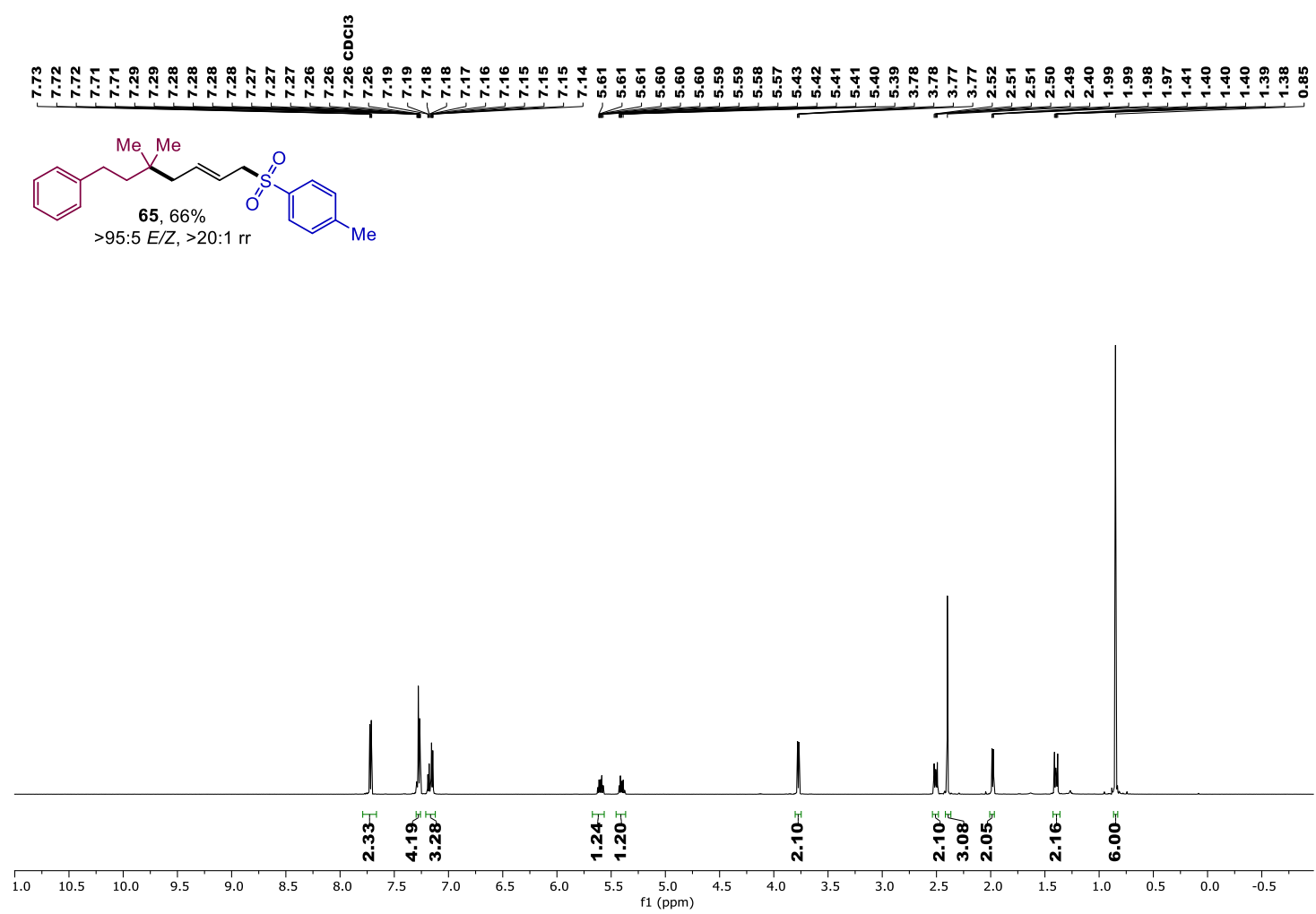

${ }^{13} \mathrm{C}$ NMR $\left(151 \mathrm{MHz}, \mathrm{CDCl}_{3}\right)$
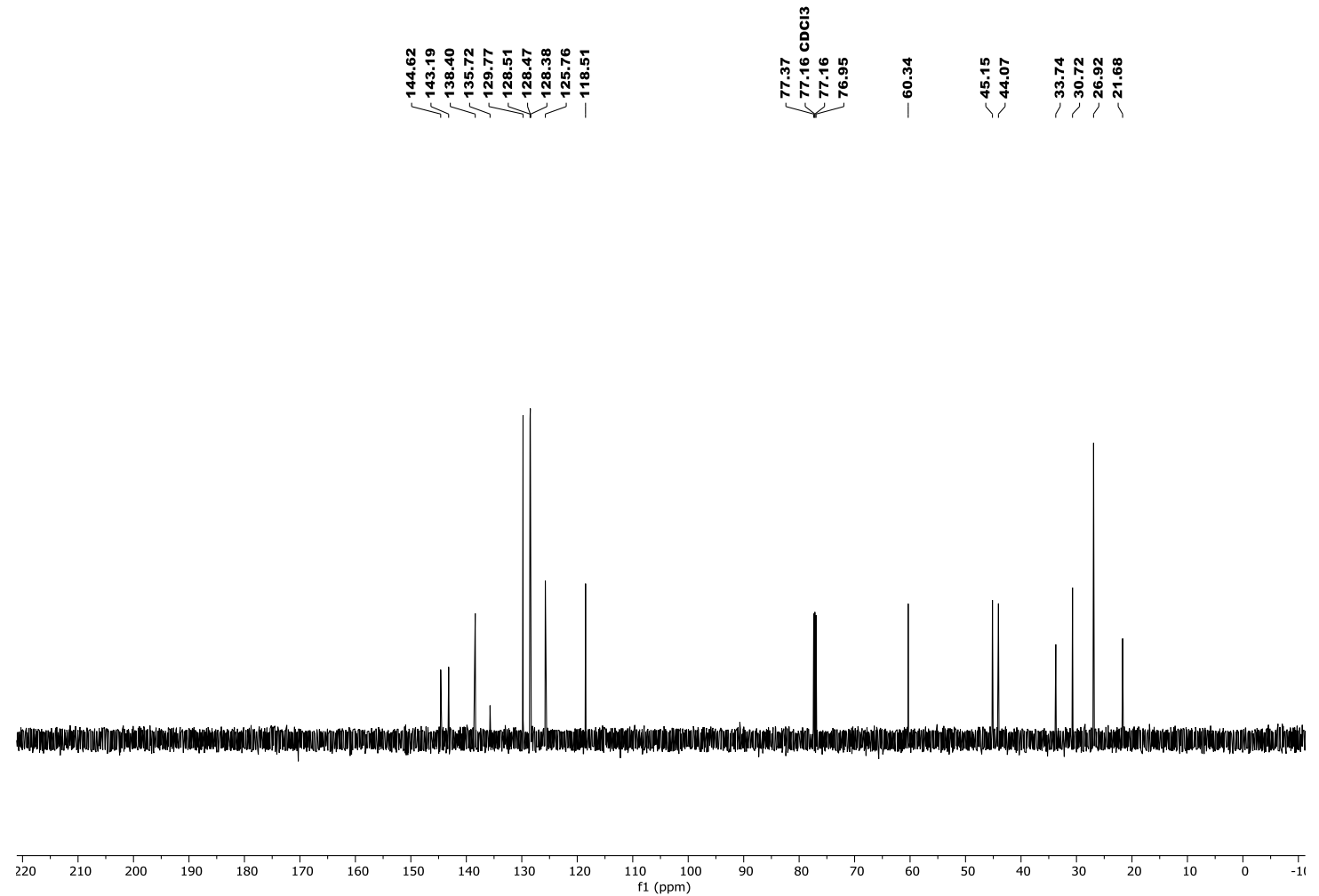
${ }^{1} \mathrm{H}$ NMR $\left(500 \mathrm{MHz}, \mathrm{CDCl}_{3}\right)$
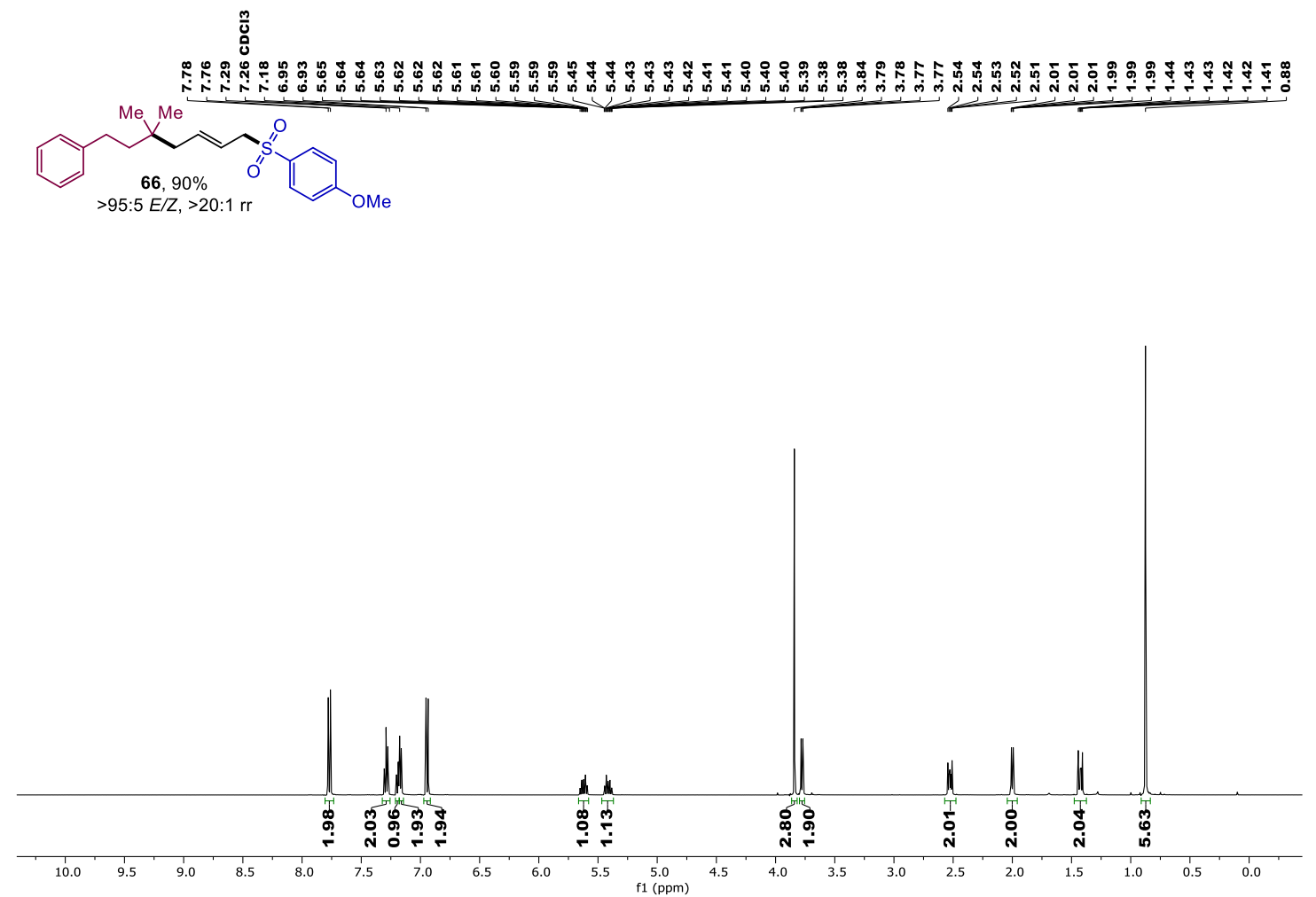

${ }^{13} \mathrm{C}$ NMR $\left(126 \mathrm{MHz}, \mathrm{CDCl}_{3}\right)$
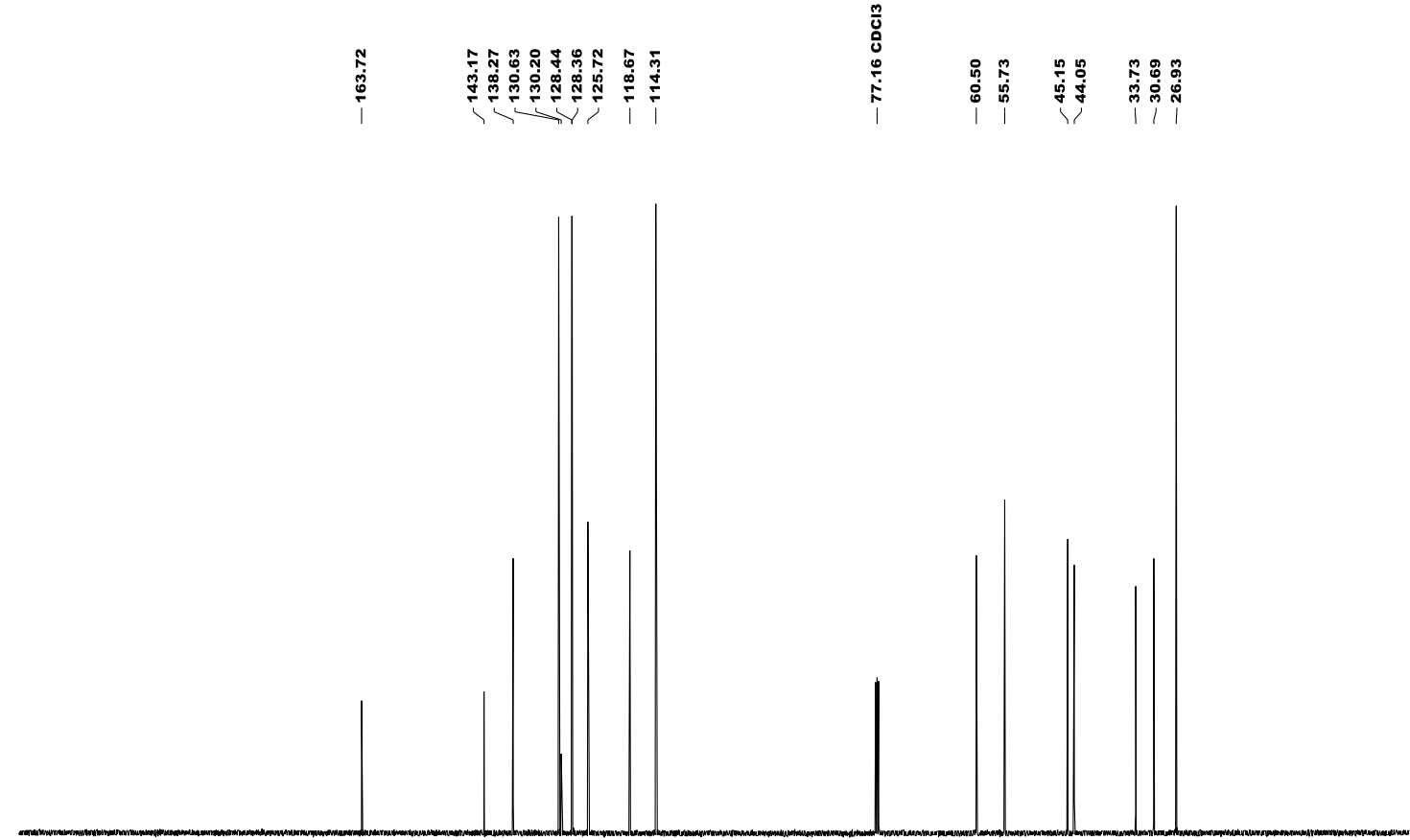

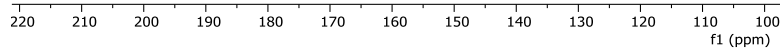


${ }^{1} \mathrm{H}$ NMR (600 MHz, $\mathrm{CDCl}_{3}$ )

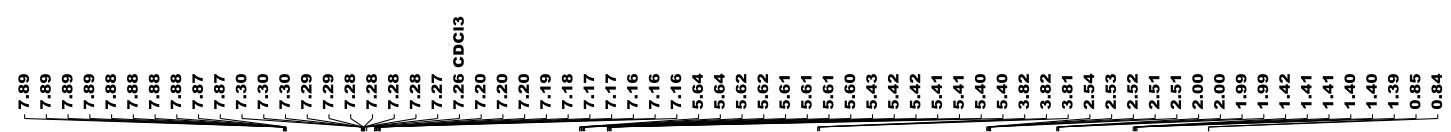
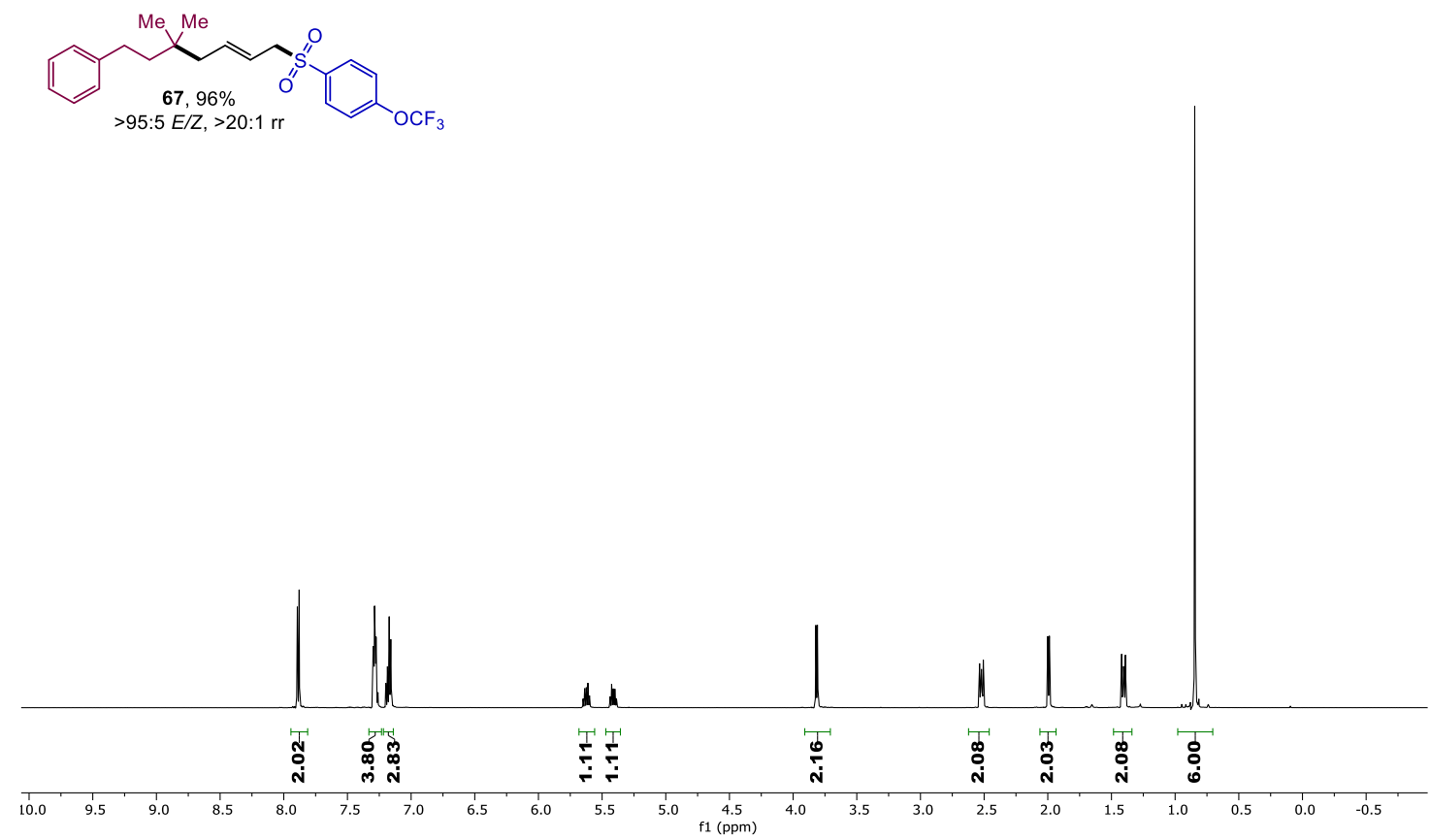

${ }^{13} \mathrm{C}$ NMR (151 MHz, $\left.\mathrm{CDCl}_{3}\right)$

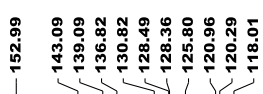

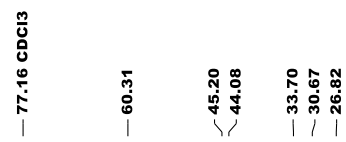

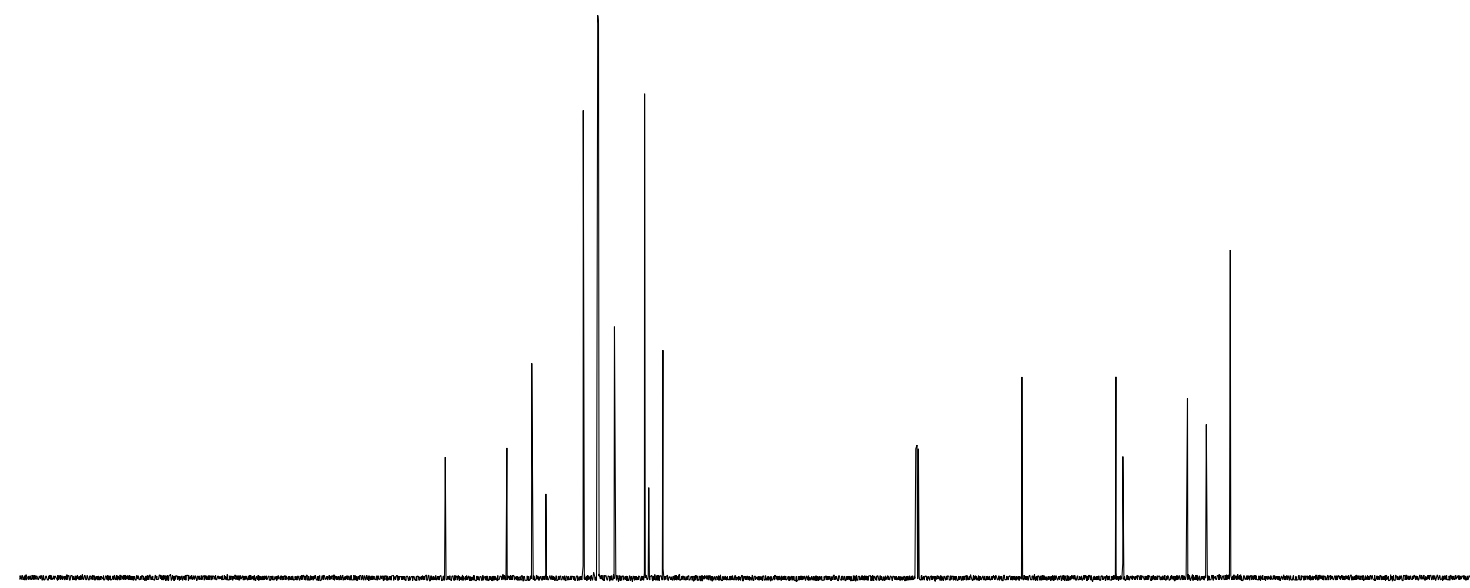

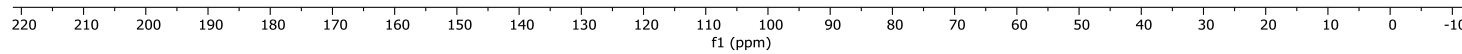


${ }^{19} \mathrm{~F}\left\{{ }^{1} \mathrm{H}\right\} \mathrm{NMR}\left(564 \mathrm{MHz}, \mathrm{CDCl}_{3}\right)$
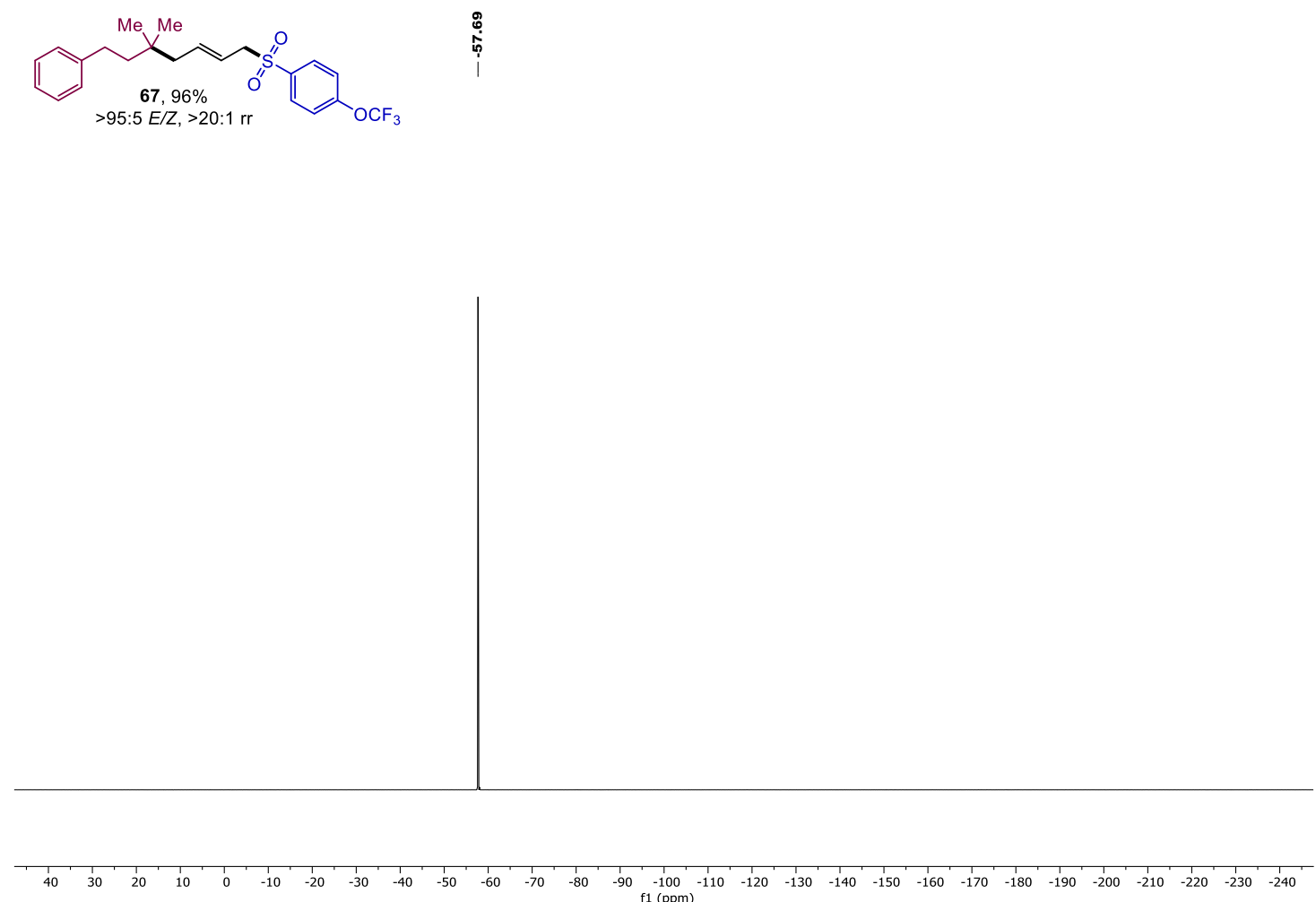
${ }^{1} \mathrm{H}$ NMR $\left(300 \mathrm{MHz}, \mathrm{CDCl}_{3}\right)$

응

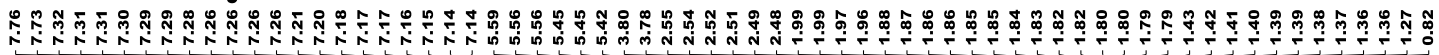
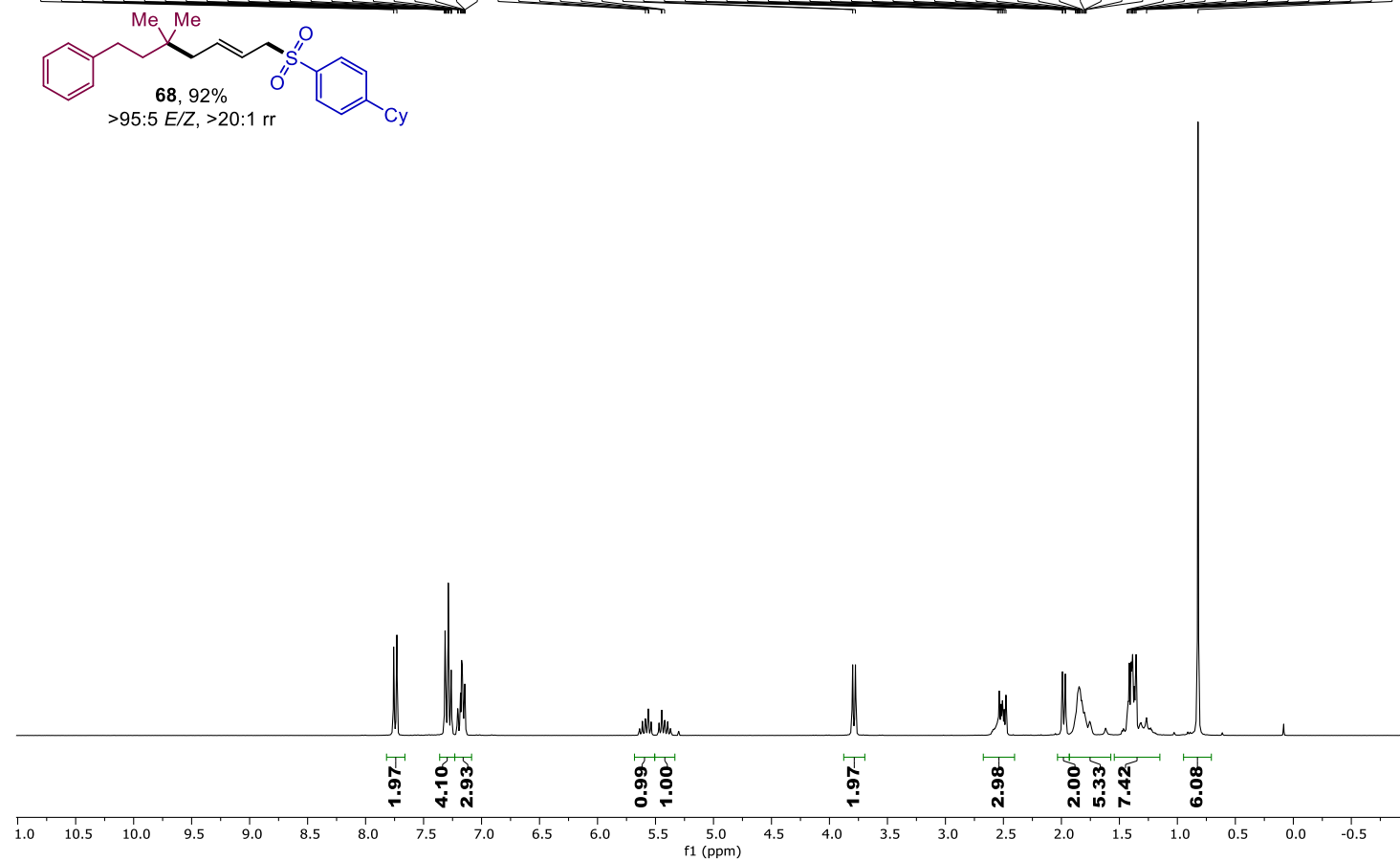

${ }^{13} \mathrm{C}$ NMR $\left(75 \mathrm{MHz}, \mathrm{CDCl}_{3}\right)$
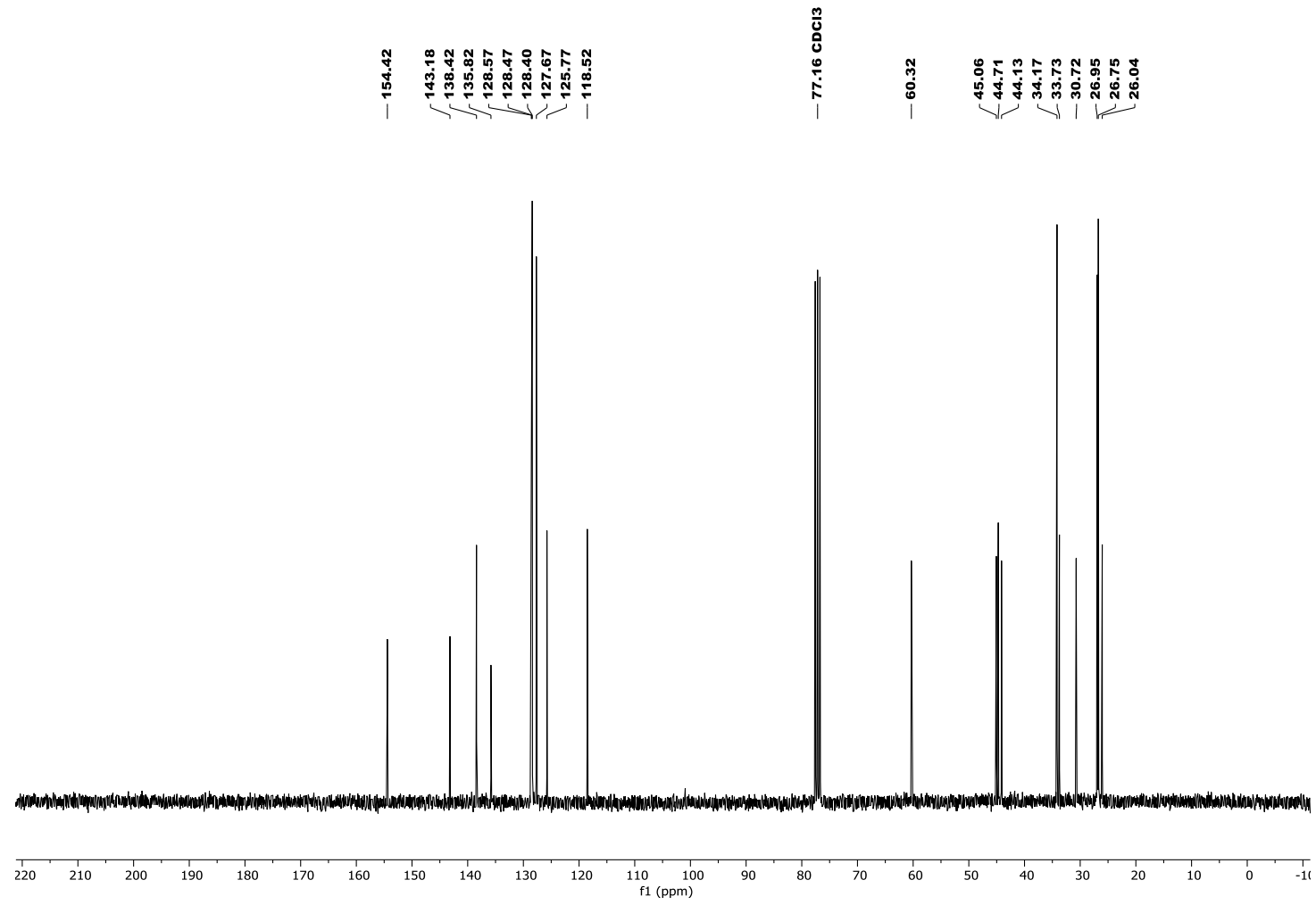
${ }^{1} \mathrm{H}$ NMR $\left(500 \mathrm{MHz}, \mathrm{CDCl}_{3}\right)$
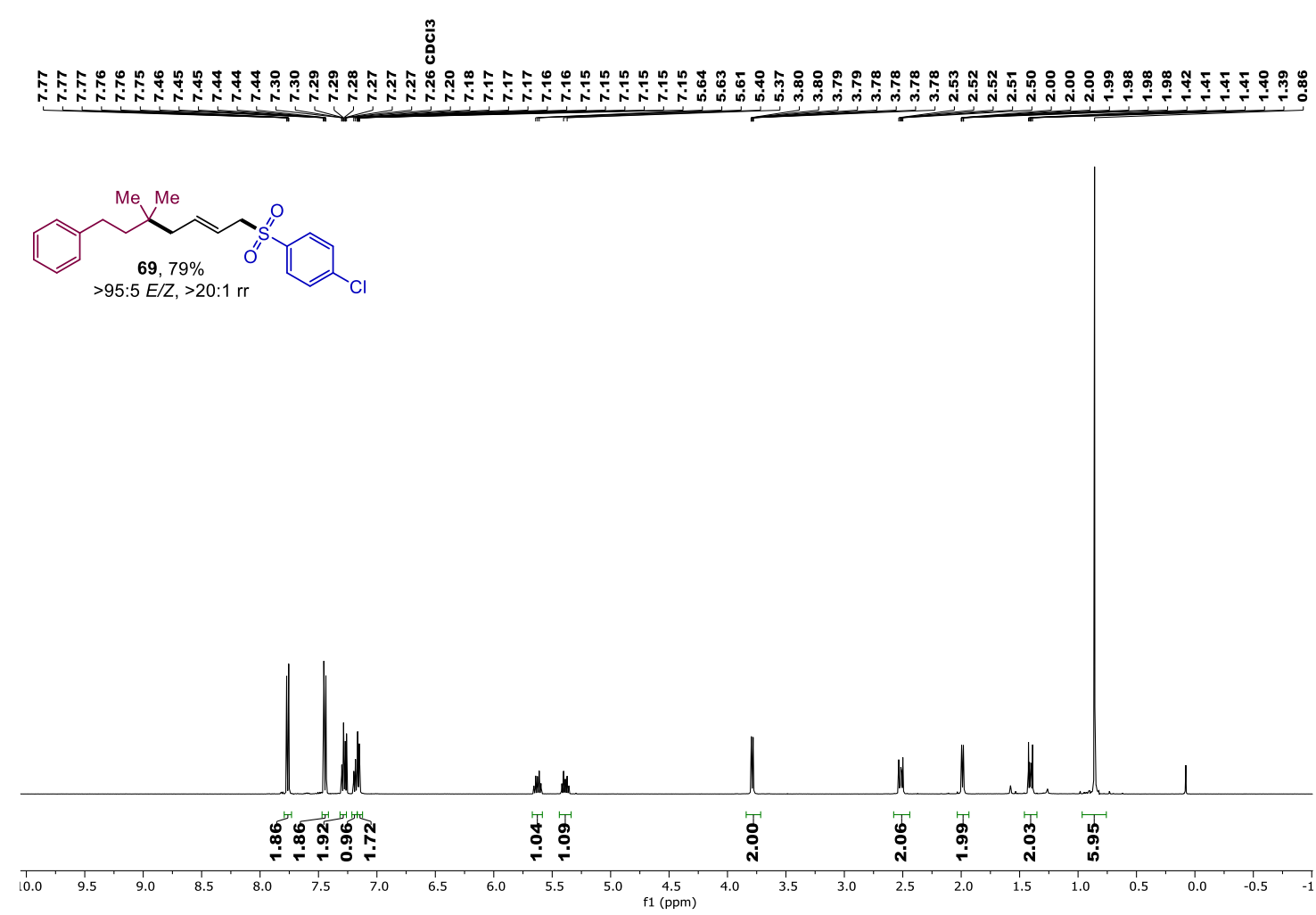

${ }^{13} \mathrm{C}$ NMR $\left(126 \mathrm{MHz}, \mathrm{CDCl}_{3}\right)$
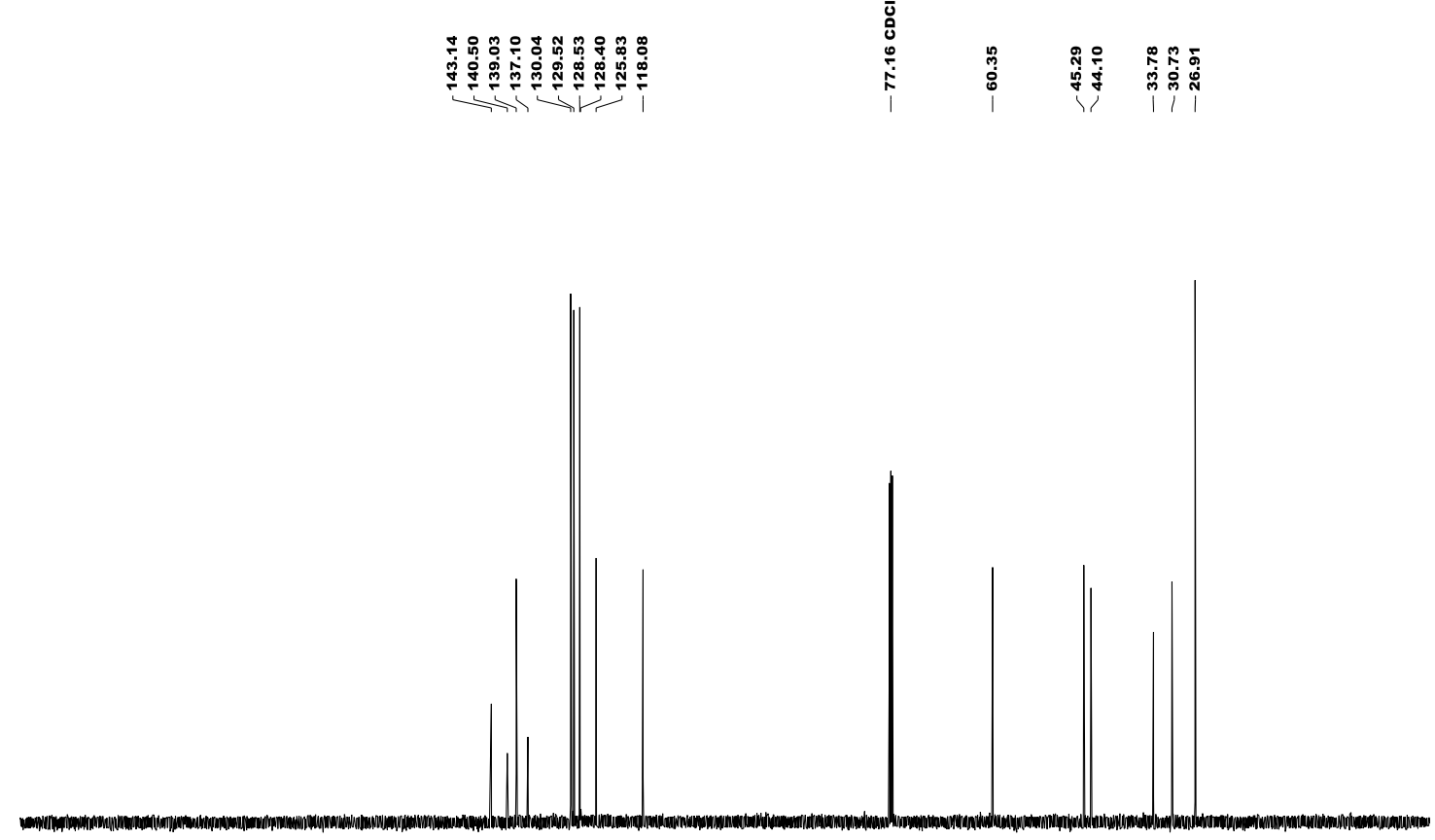

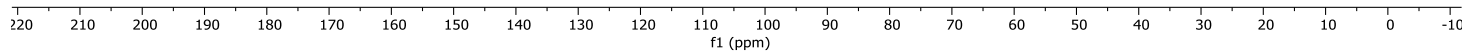


${ }^{1} \mathrm{H} \mathrm{NMR}\left(500 \mathrm{MHz}, \mathrm{CDCl}_{3}\right)$
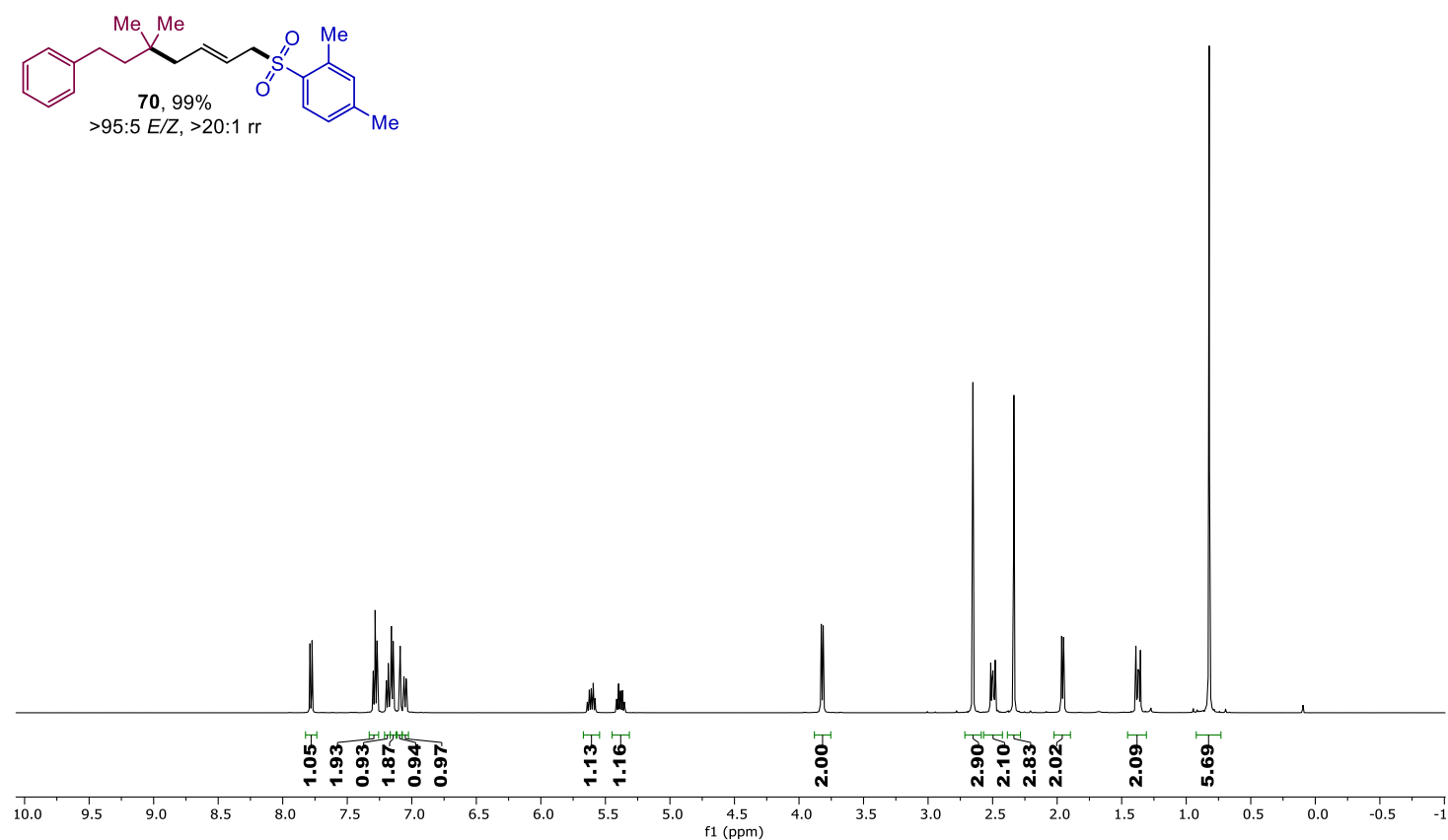

${ }^{13} \mathrm{C}$ NMR $\left(126 \mathrm{MHz}, \mathrm{CDCl}_{3}\right)$

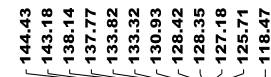
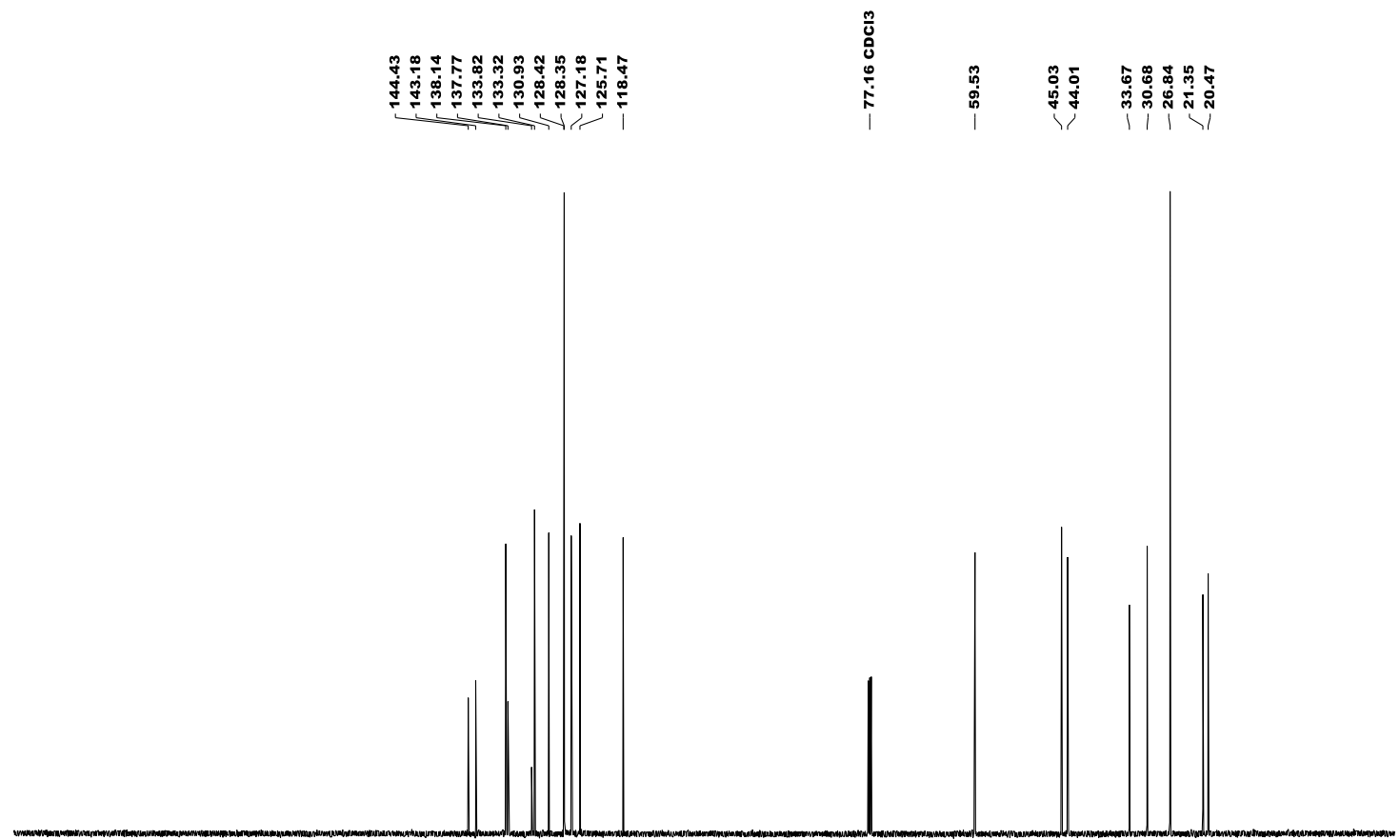

$\begin{array}{lllllllllllll}20 & 210 & 200 & 190 & 180 & 170 & 160 & 150 & 140 & 130 & 120 & \underset{110}{1} 100 \\ \mathrm{f} 1(\mathrm{ppm}) & 100\end{array}$ 
${ }^{1} \mathrm{H}$ NMR (400 MHz, $\mathrm{CDCl}_{3}$ )
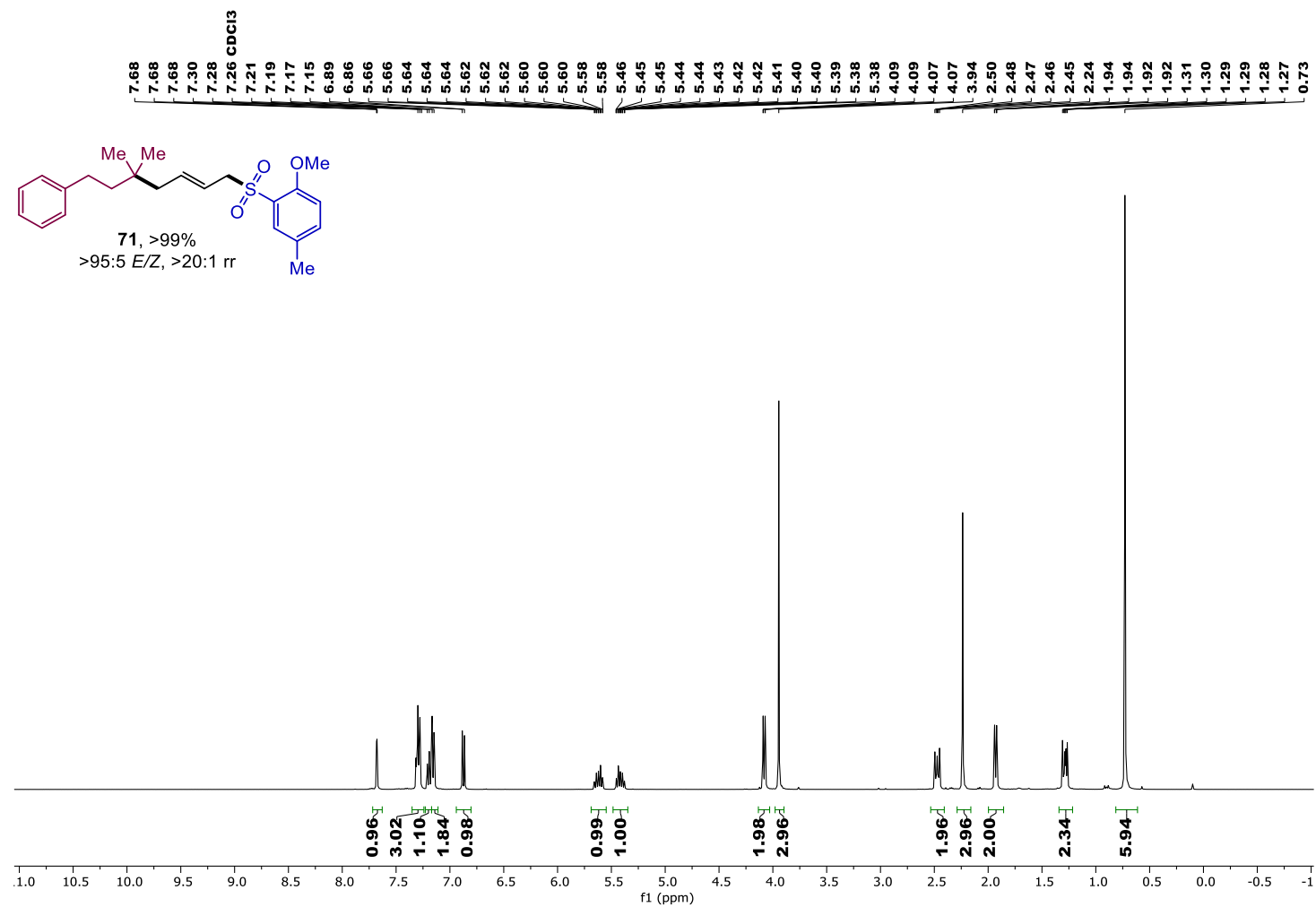

${ }^{13} \mathrm{C}$ NMR $\left(101 \mathrm{MHz}, \mathrm{CDCl}_{3}\right)$

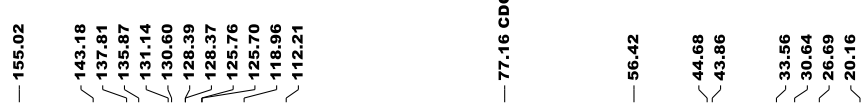

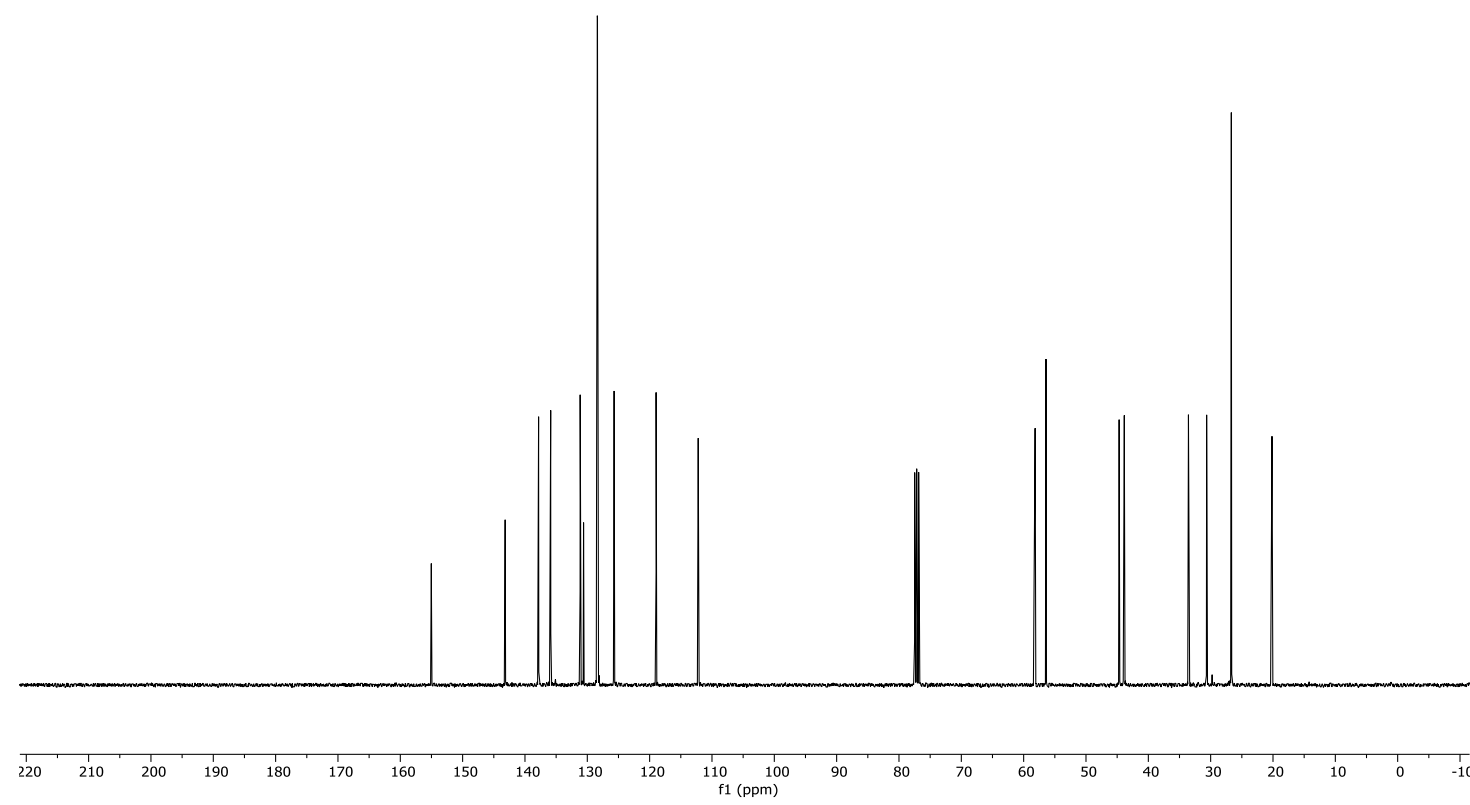


${ }^{1} \mathrm{H}$ NMR $\left(500 \mathrm{MHz}, \mathrm{CDCl}_{3}\right)$

竞

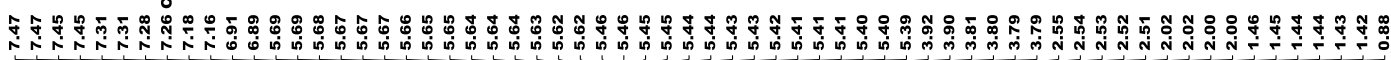
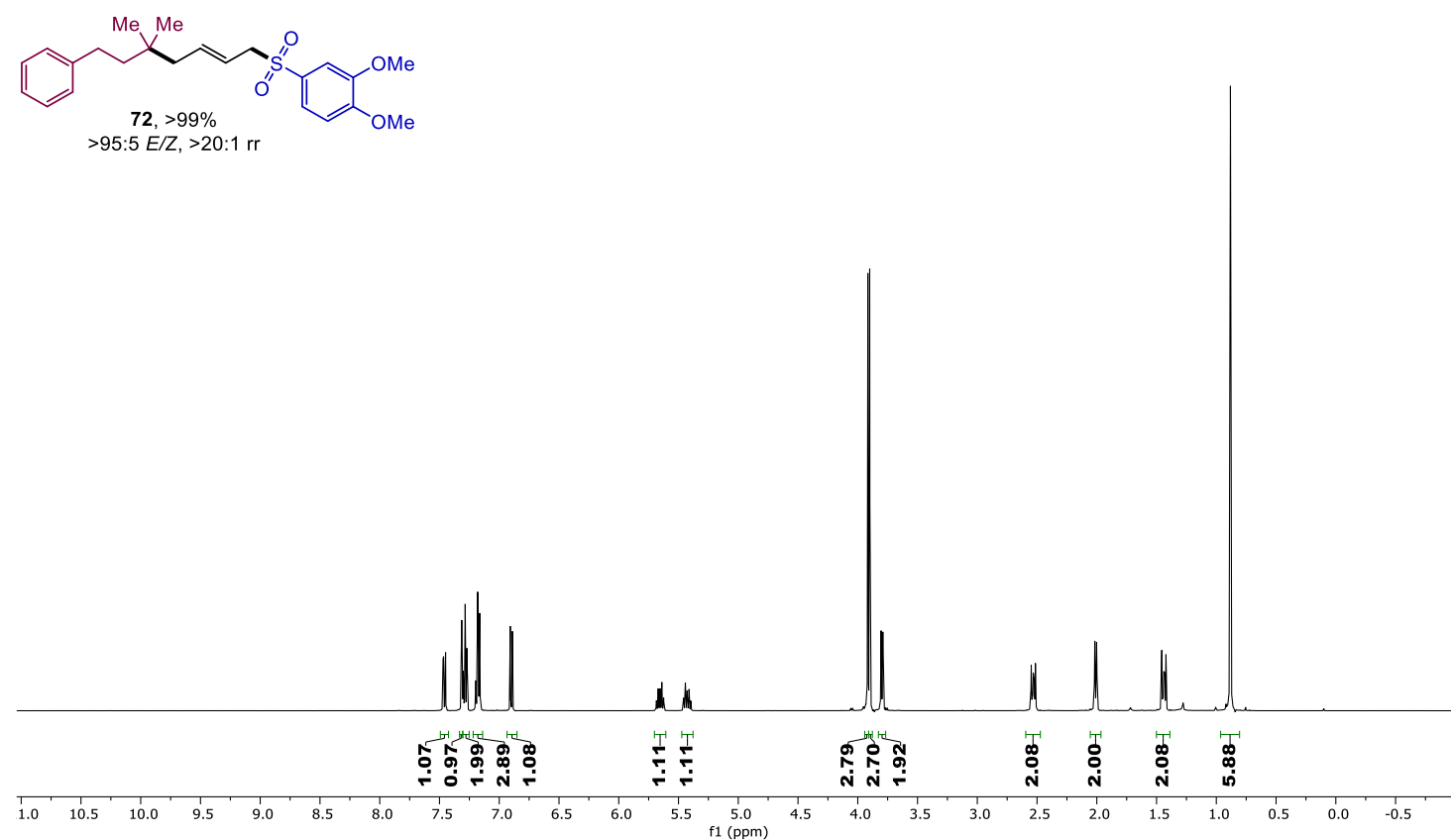

${ }^{13} \mathrm{C}$ NMR $\left(126 \mathrm{MHz}, \mathrm{CDCl}_{3}\right)$
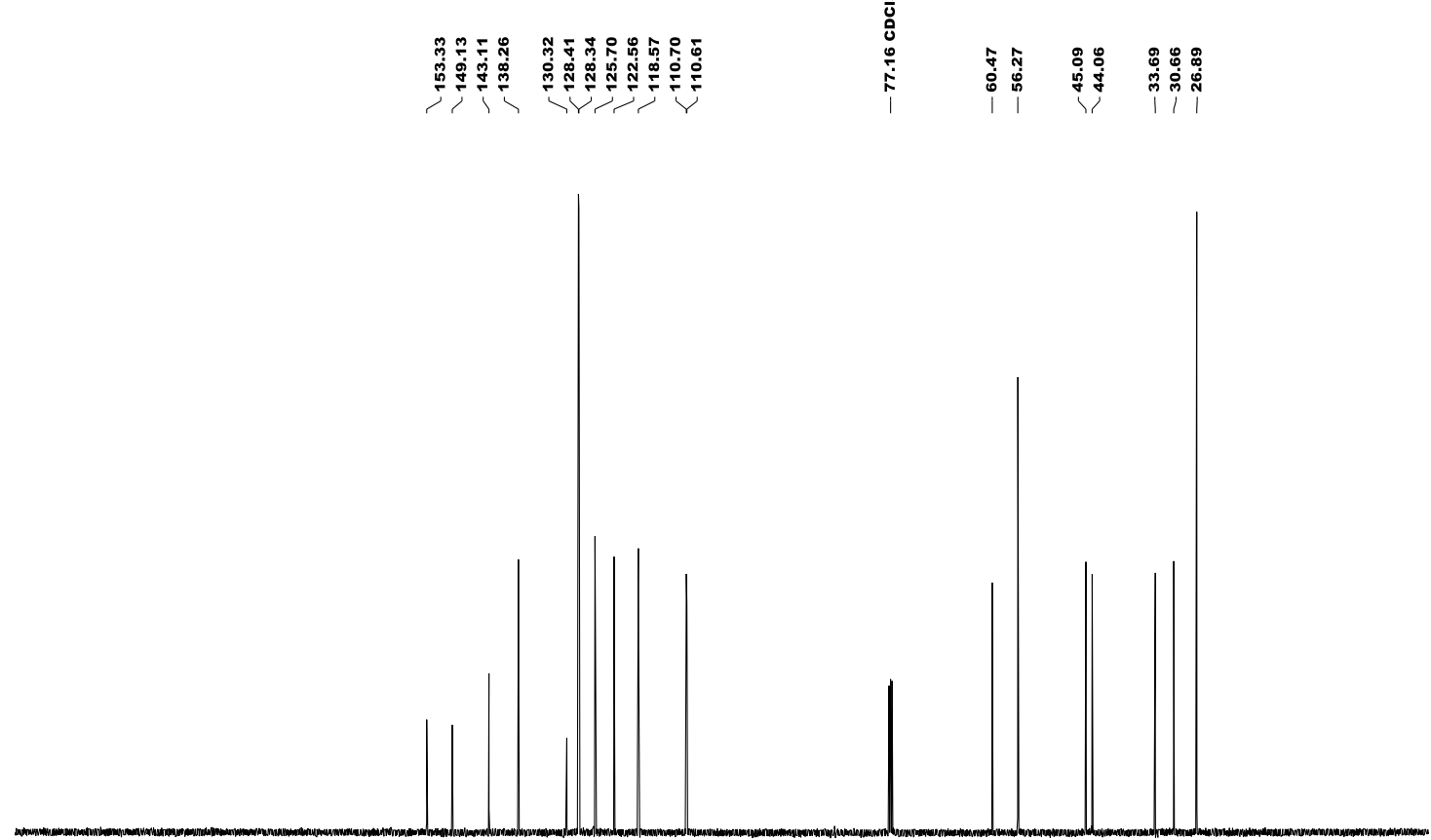

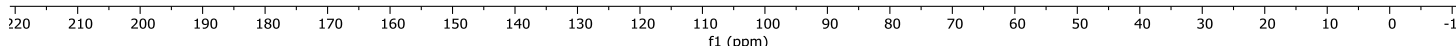


${ }^{1} \mathrm{H}$ NMR (599 MHz, $\mathrm{CDCl}_{3}$ )
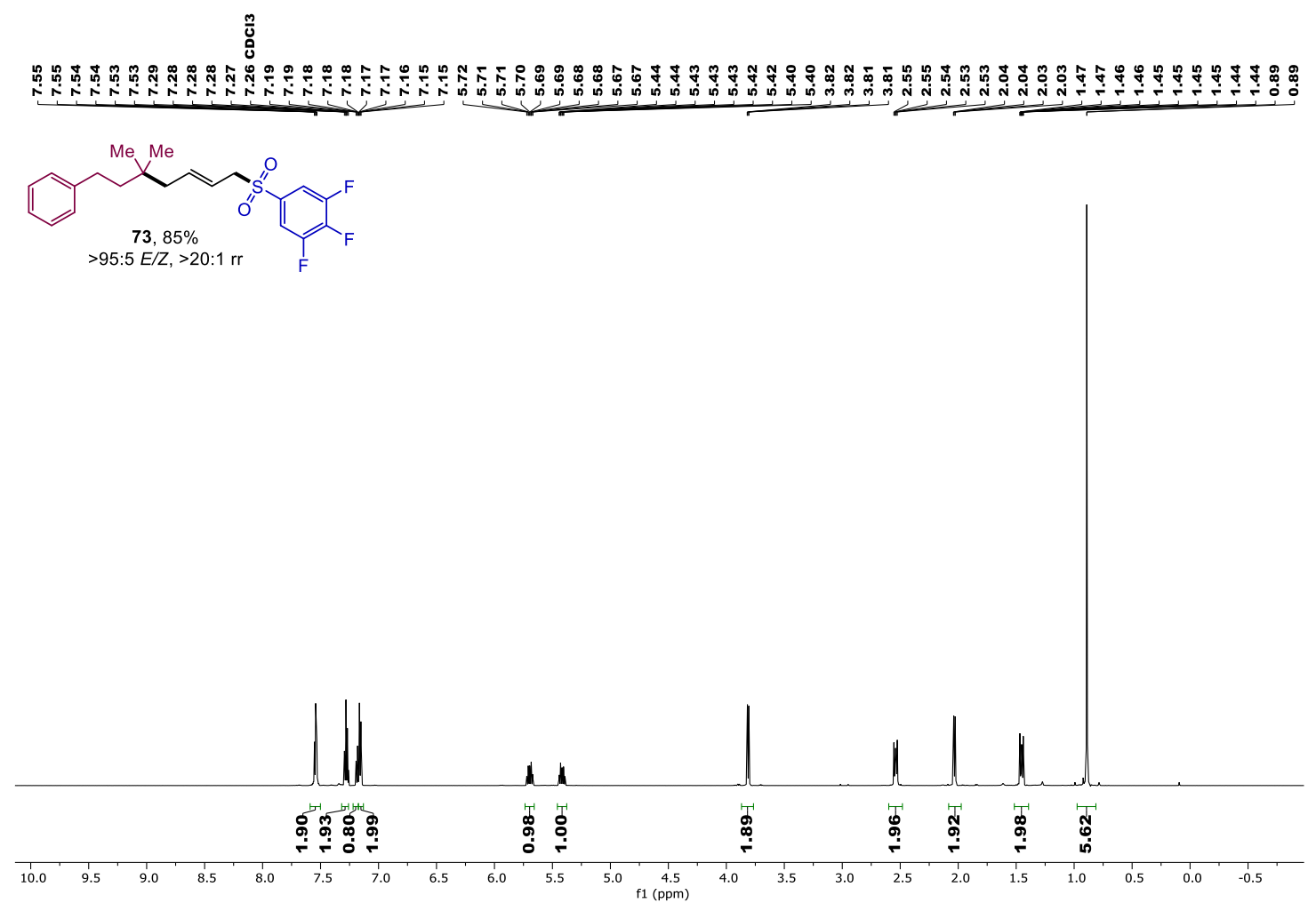

${ }^{13} \mathrm{C}\left\{{ }^{1} \mathrm{H},{ }^{19} \mathrm{~F}\right\}$ NMR $\left(151 \mathrm{MHz}, \mathrm{CDCl}_{3}\right)$

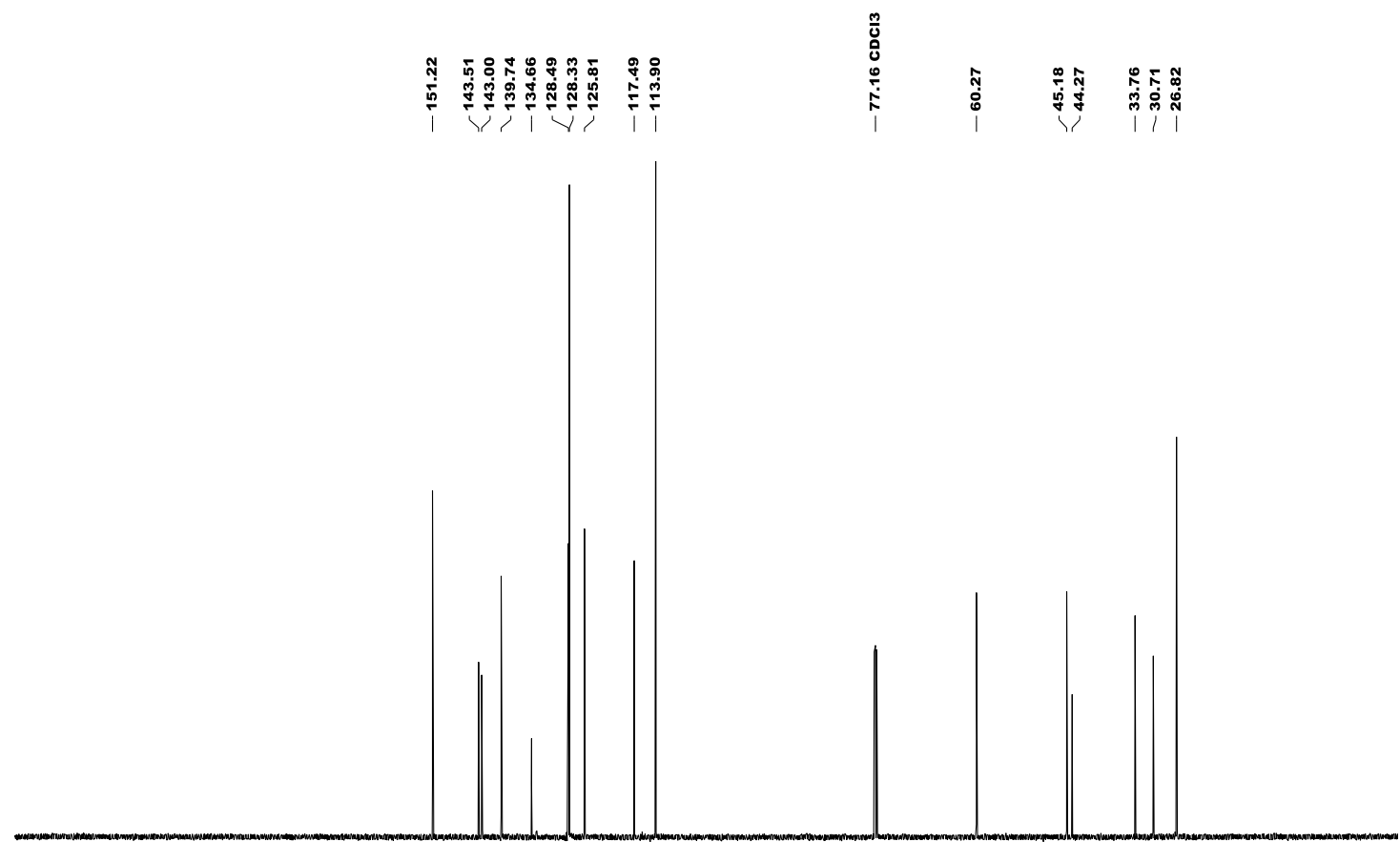

$\begin{array}{llllllllllll}220 & 210 & 200 & 190 & 180 & 170 & 160 & 150 & 140 & 130 & 120 & \underset{110}{1} 100 \\ f 1(\mathrm{ppm})\end{array}$ 
${ }^{19} \mathrm{~F}\left\{{ }^{1} \mathrm{H}\right\} \mathrm{NMR}\left(564 \mathrm{MHz}, \mathrm{CDCl}_{3}\right)$

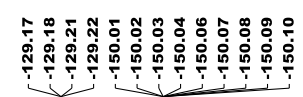
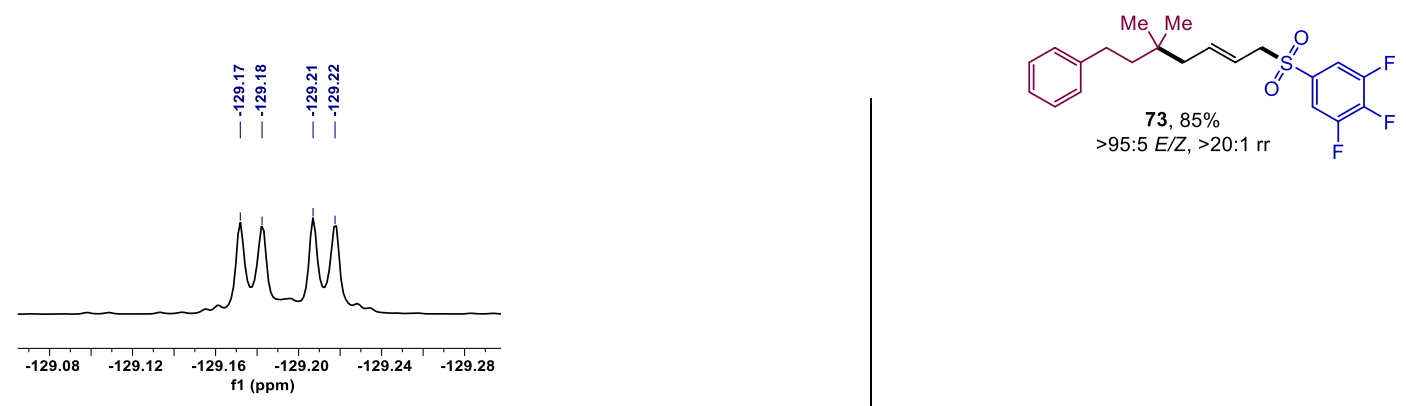

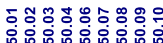

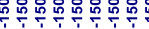

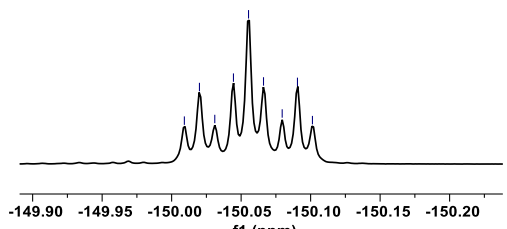

$\begin{array}{lllllll}-149.90 & -149.95 & -150.00 & -150.05 & -150.10 & -150.15 & -150.20\end{array}$ $11(\mathrm{ppm})$

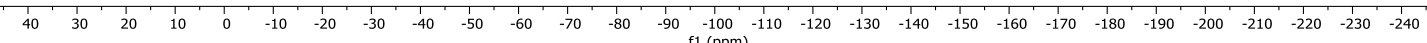


${ }^{1} \mathrm{H}$ NMR (400 MHz, $\left.\mathrm{CDCl}_{3}\right)$

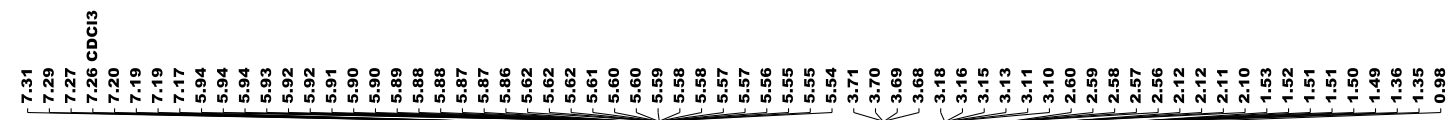

$\underbrace{\mathrm{O}}_{\substack{74,>99 \% \\>95: 5 E / Z,>20: 1 \mathrm{rr}}}$

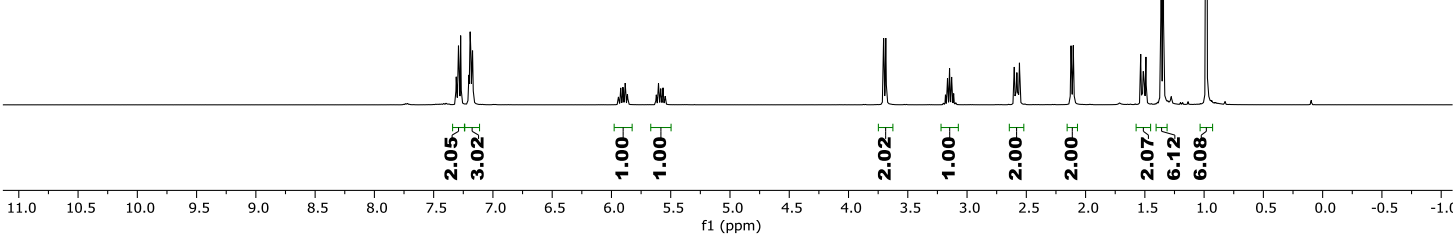

${ }^{13} \mathrm{C}$ NMR (101 MHz, $\mathrm{CDCl}_{3}$ )

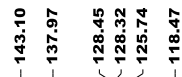

每

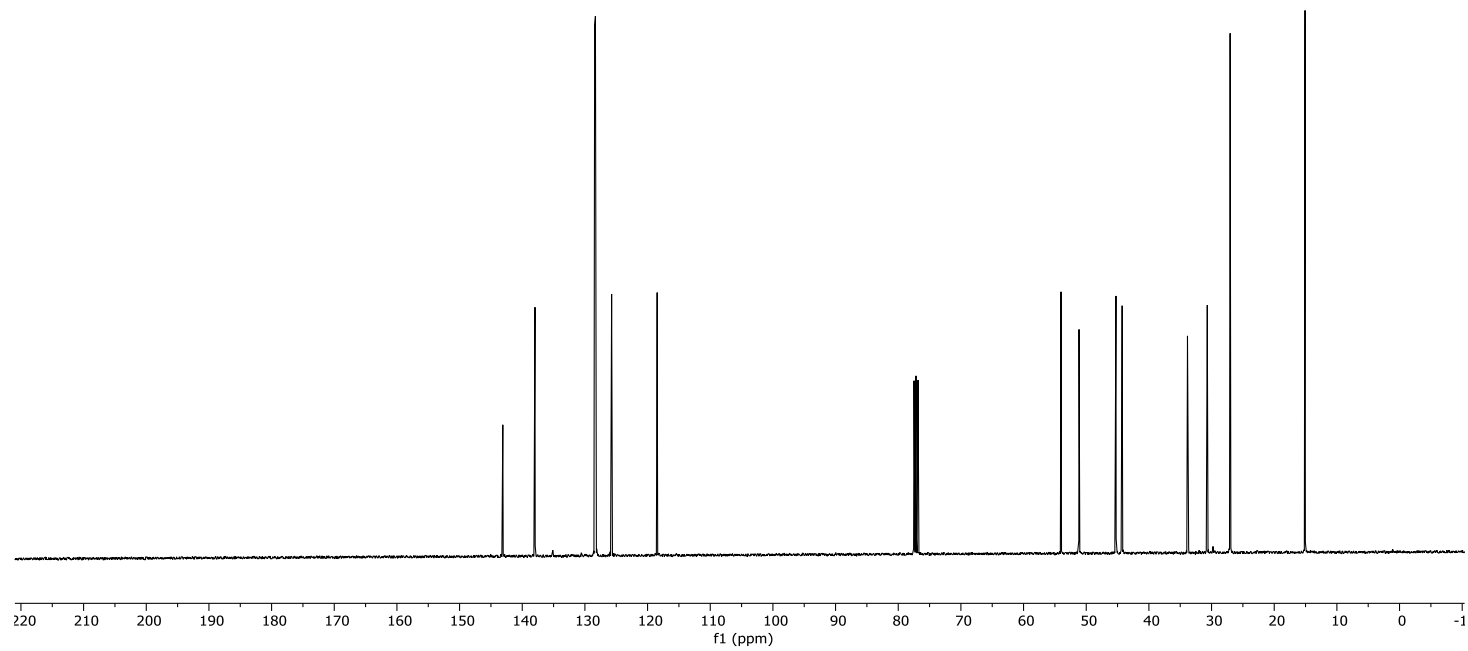


${ }^{1} \mathrm{H}$ NMR (400 MHz, $\mathrm{CDCl}_{3}$ )

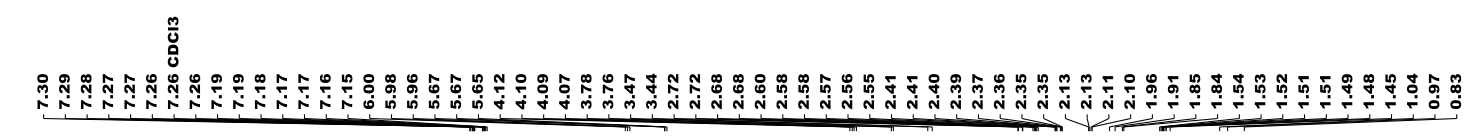

(15,55\%,

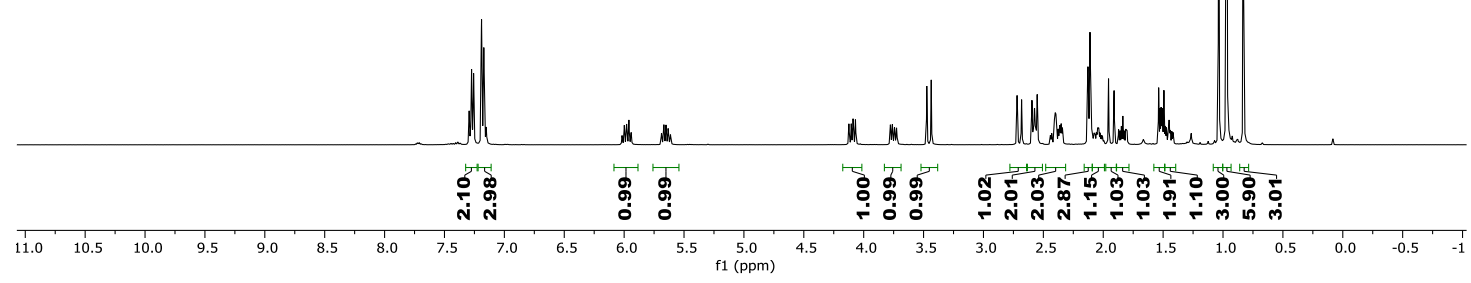

${ }^{13} \mathrm{C}$ NMR (101 MHz, $\mathrm{CDCl}_{3}$ )
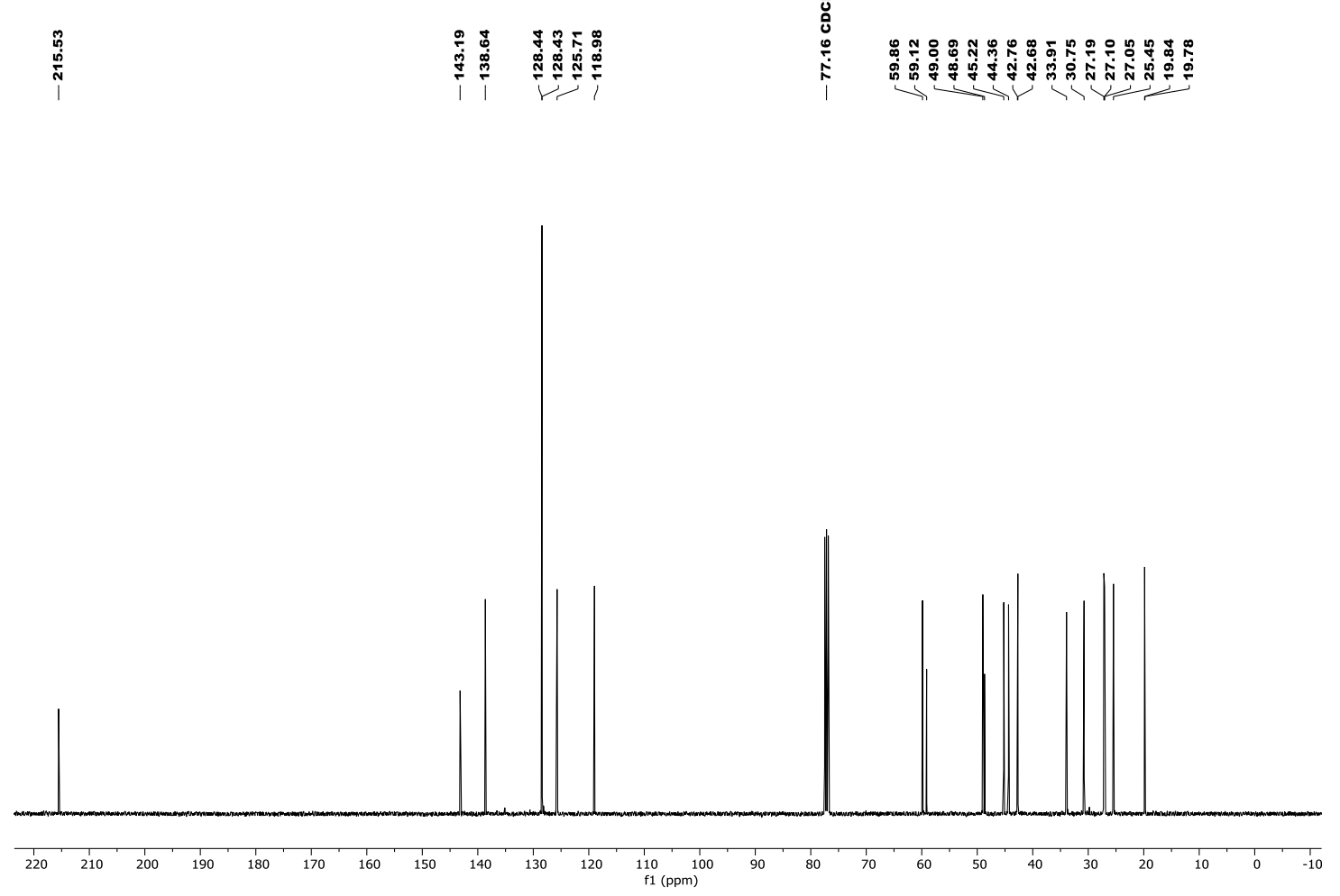
${ }^{1} \mathrm{H}$ NMR $\left(599 \mathrm{MHz}, \mathrm{CDCl}_{3}\right)$

尊

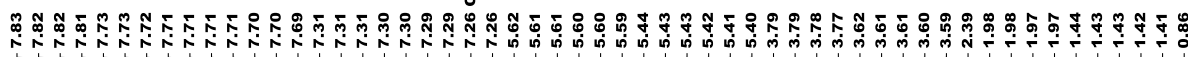
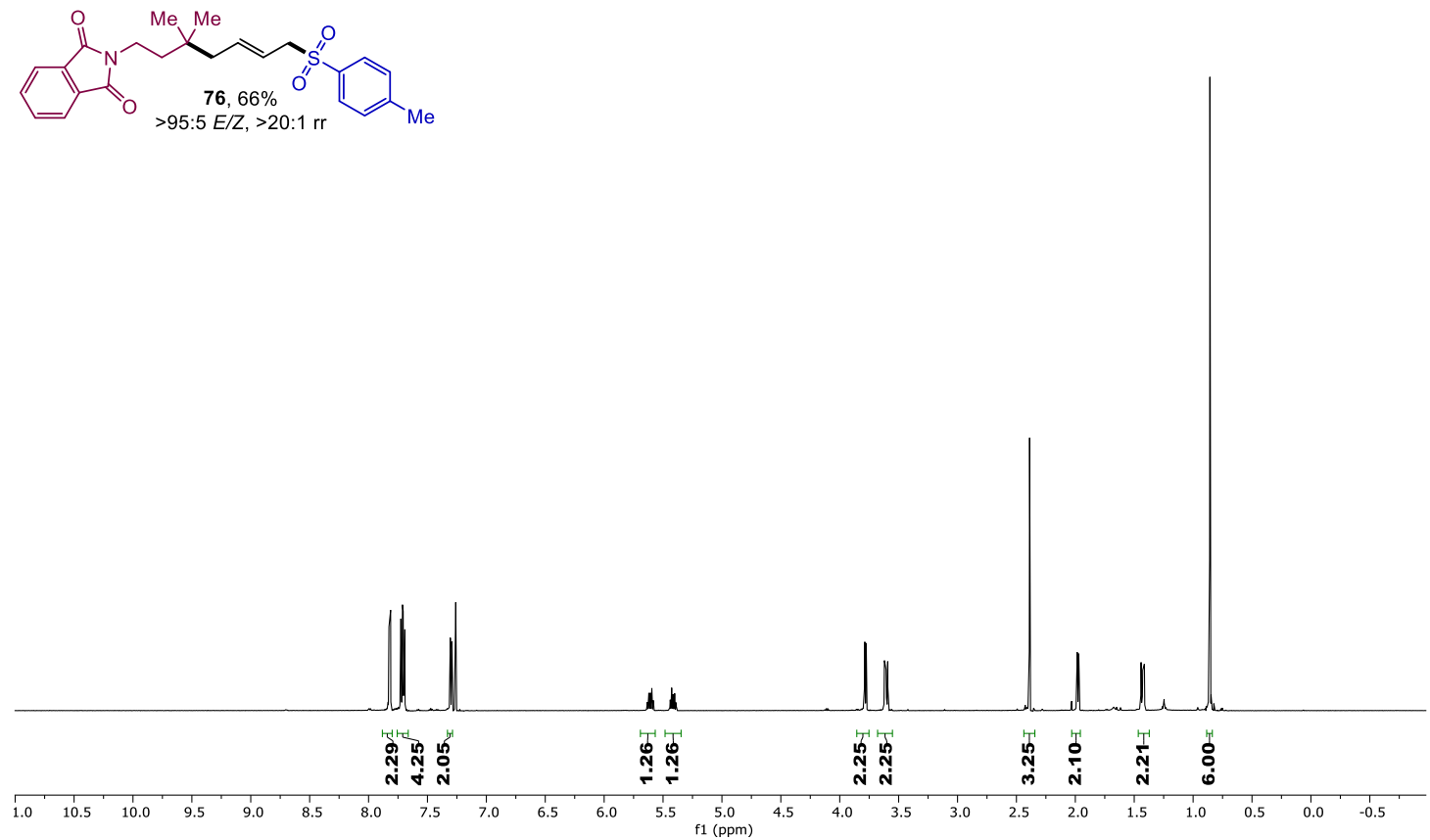

${ }^{13} \mathrm{C}$ NMR $\left(151 \mathrm{MHz}, \mathrm{CDCl}_{3}\right)$

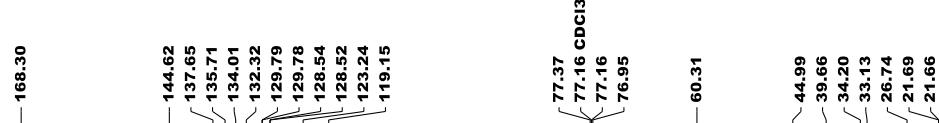

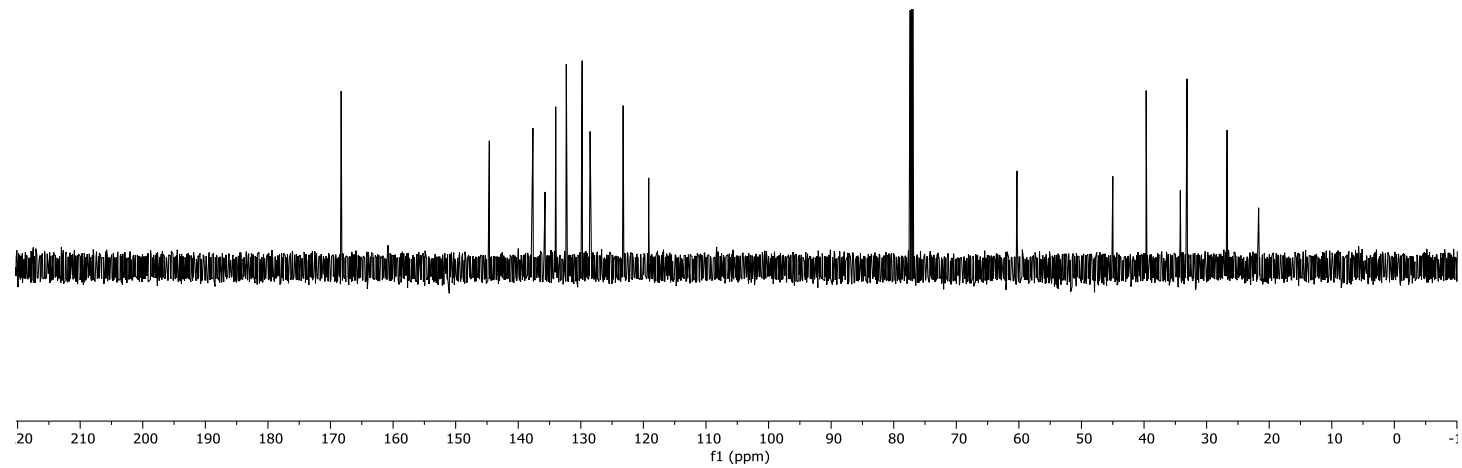


${ }^{1} \mathrm{H} \mathrm{NMR}\left(599 \mathrm{MHz}, \mathrm{CDCl}_{3}\right)$
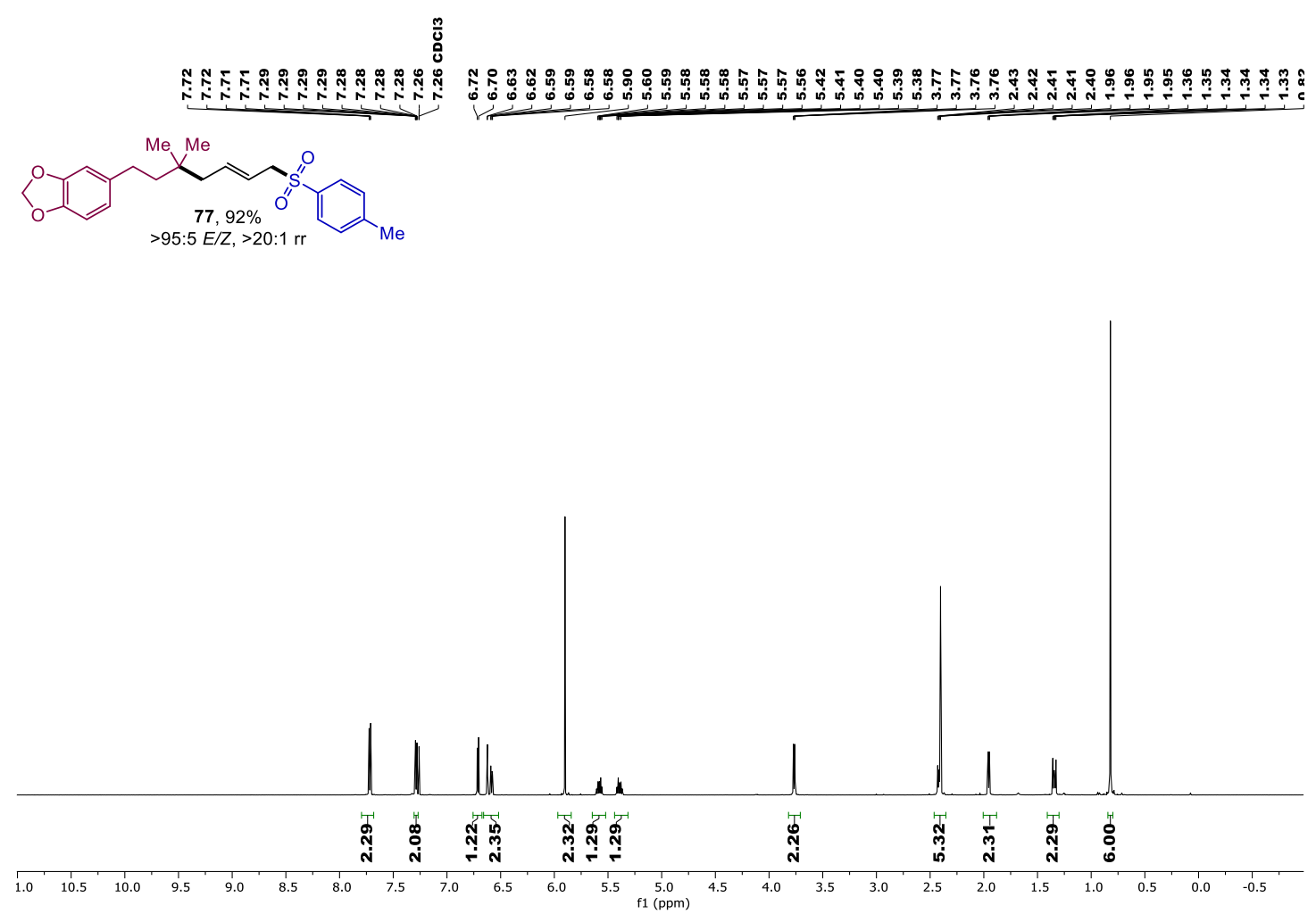

${ }^{13} \mathrm{C}$ NMR (151 MHz, $\left.\mathrm{CDCl}_{3}\right)$
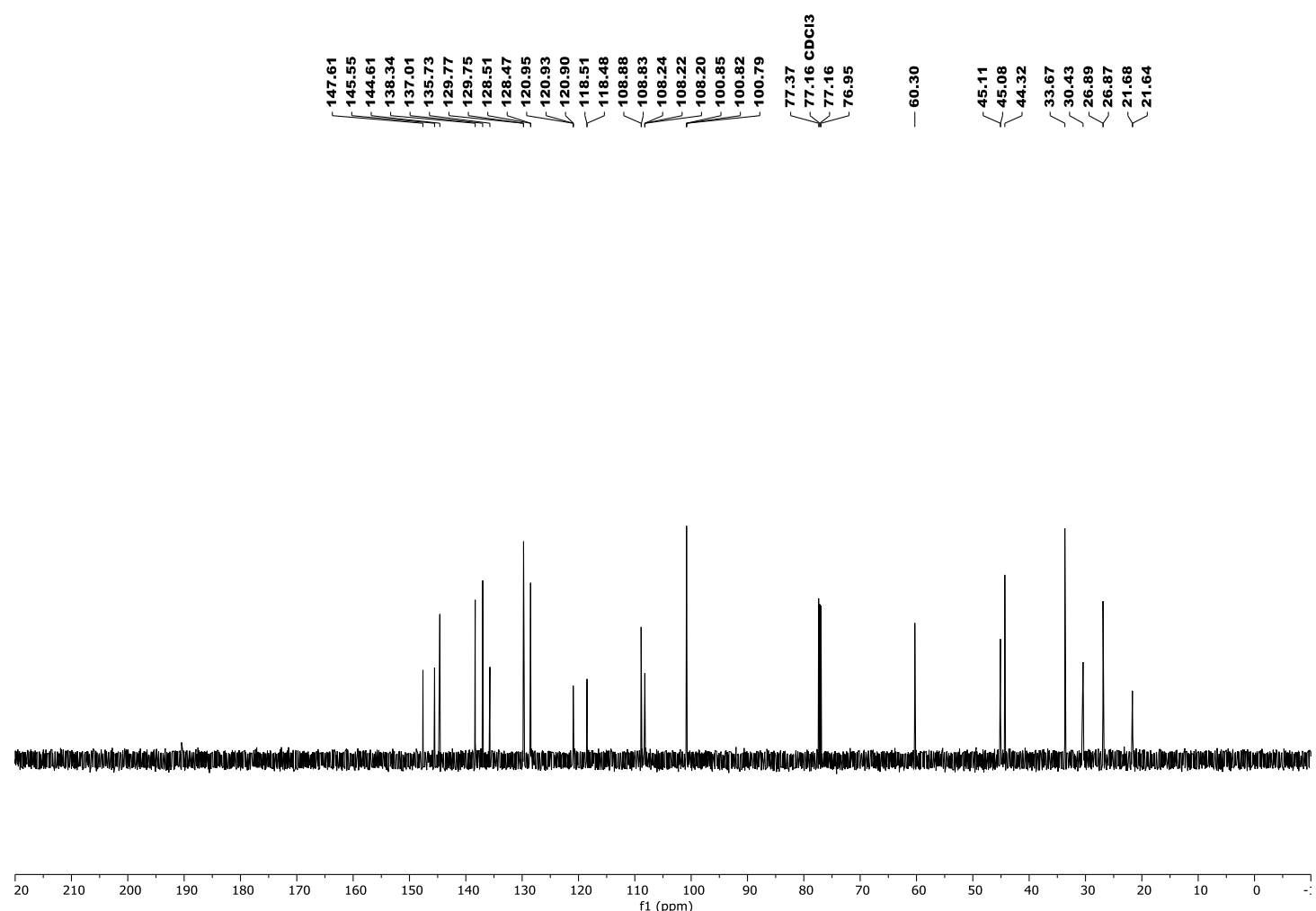
${ }^{1} \mathrm{H}$ NMR $\left(300 \mathrm{MHz}, \mathrm{CDCl}_{3}\right.$ )

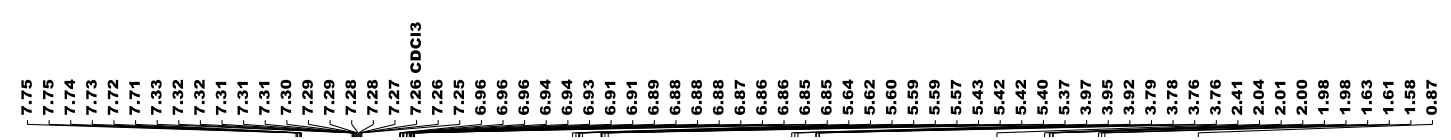

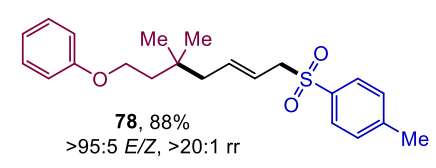

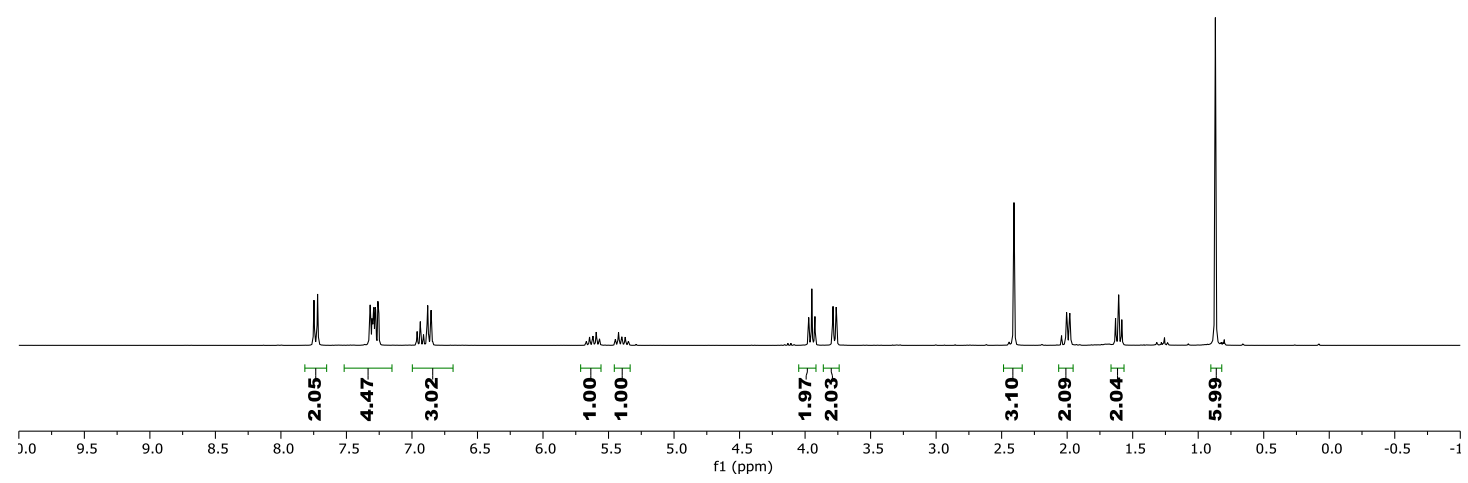

${ }^{13} \mathrm{C}$ NMR $\left(75 \mathrm{MHz}, \mathrm{CDCl}_{3}\right.$ )
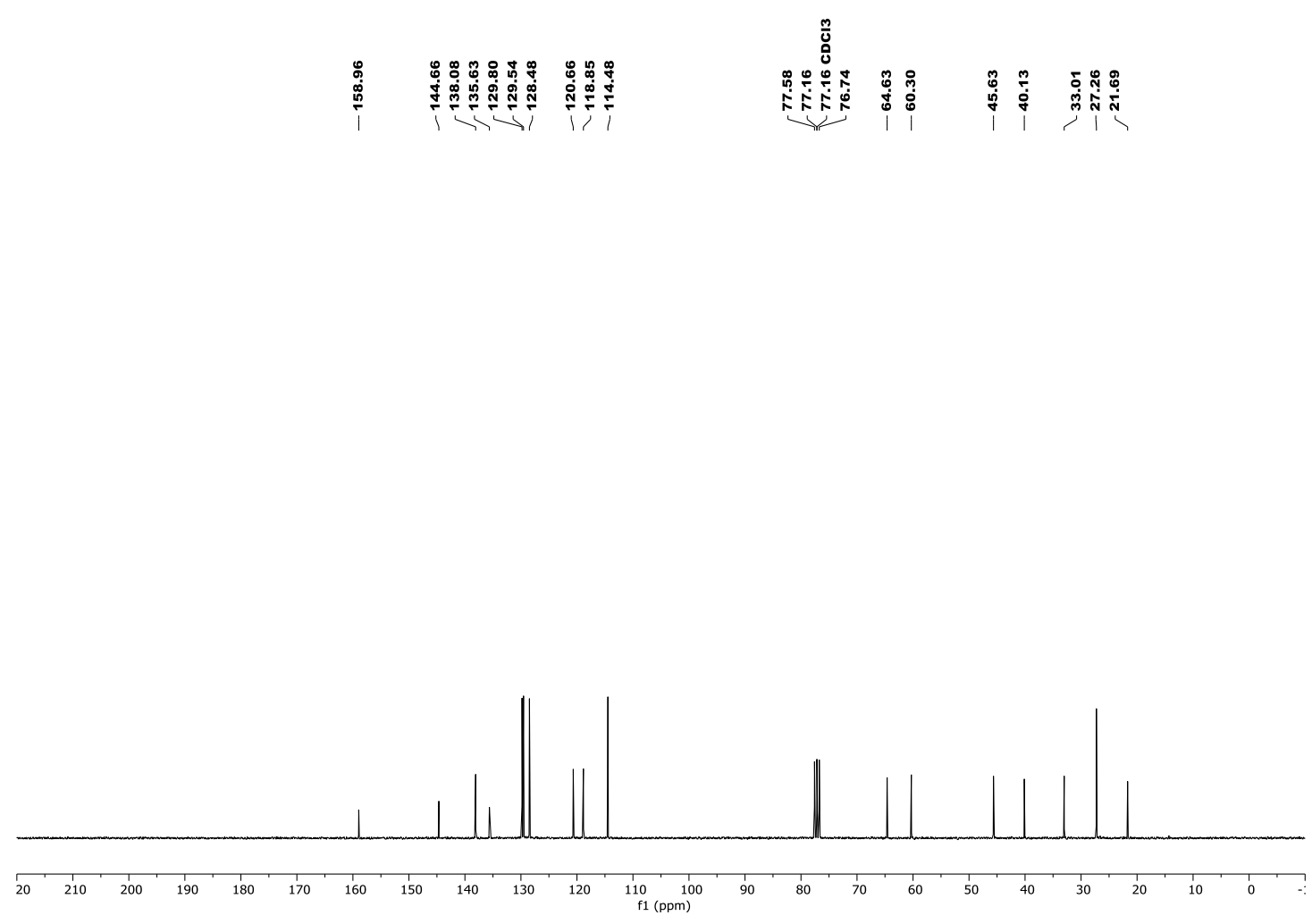
${ }^{1} \mathrm{H}$ NMR $\left(300 \mathrm{MHz}, \mathrm{CDCl}_{3}\right)$

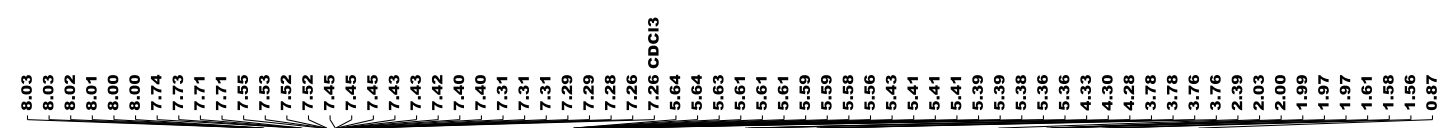
(195:5\%,

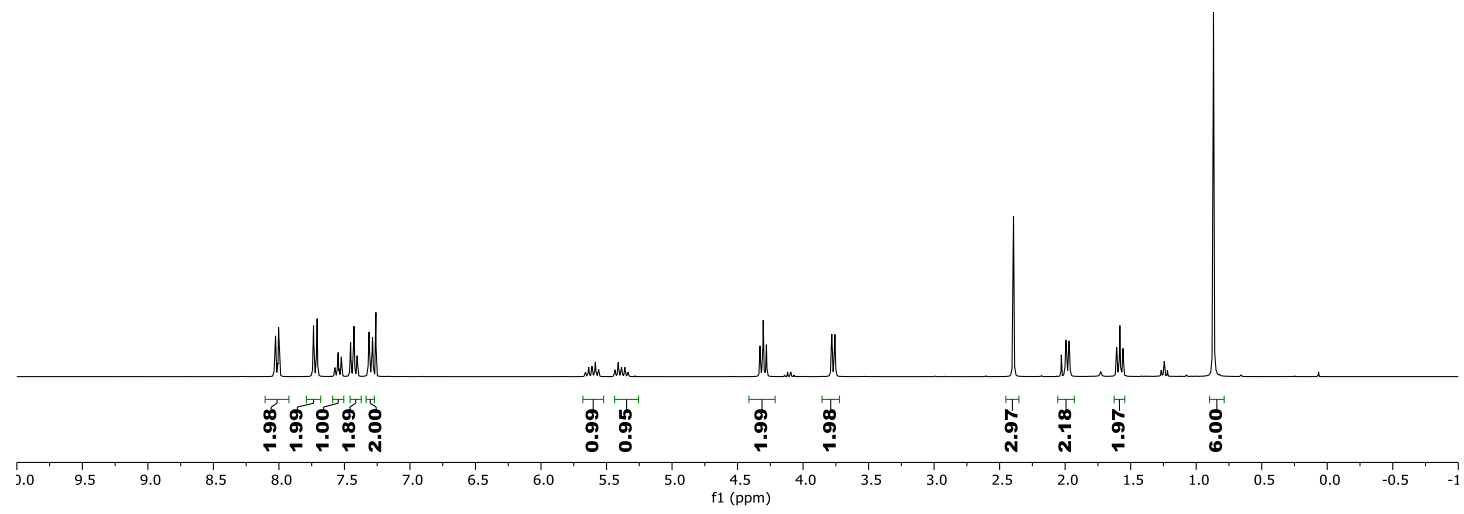

${ }^{13} \mathrm{C}$ NMR $\left(75 \mathrm{MHz}, \mathrm{CDCl}_{3}\right.$ )
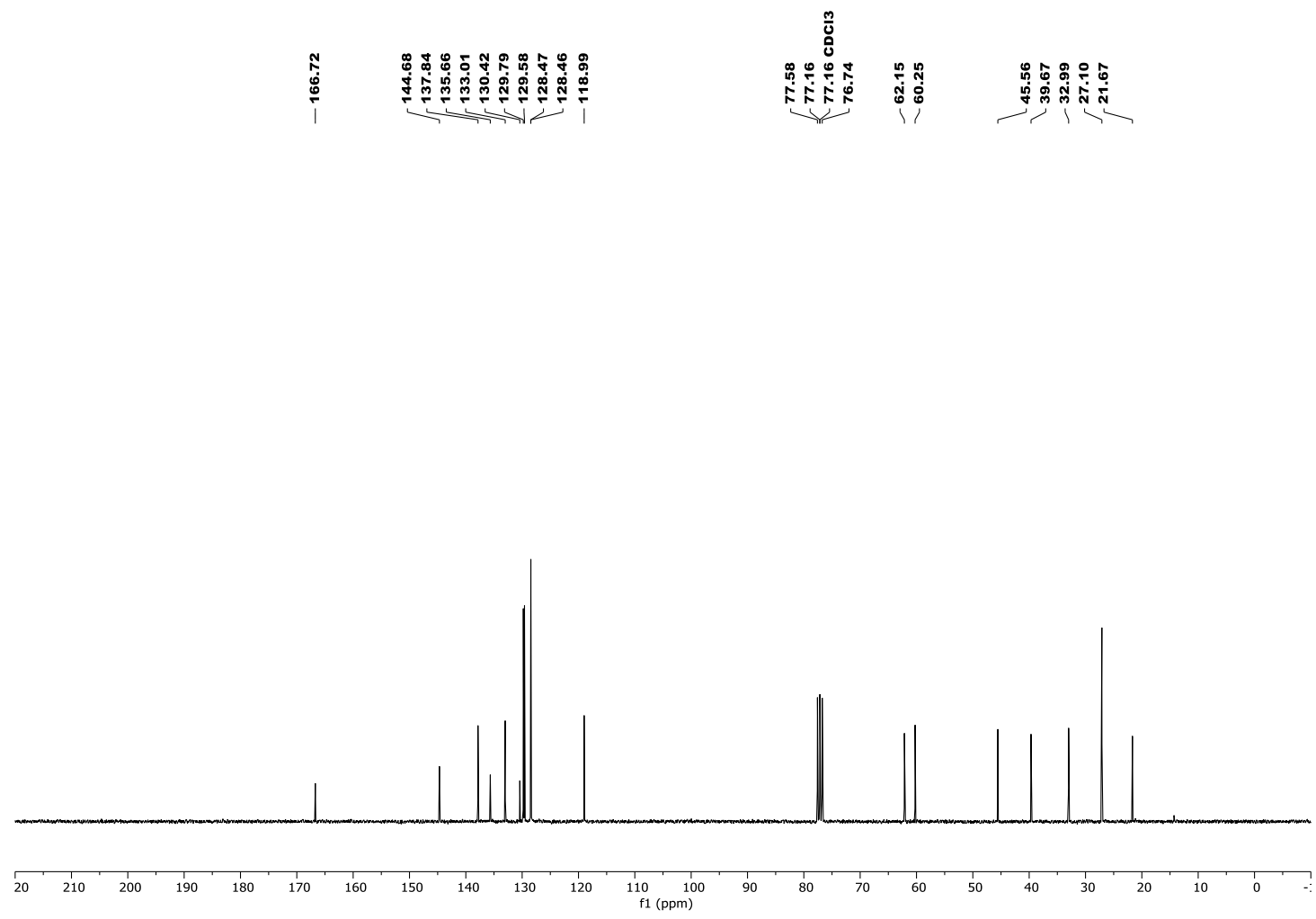
${ }^{1} \mathrm{H}$ NMR $\left(300 \mathrm{MHz}, \mathrm{CDCl}_{3}\right)$
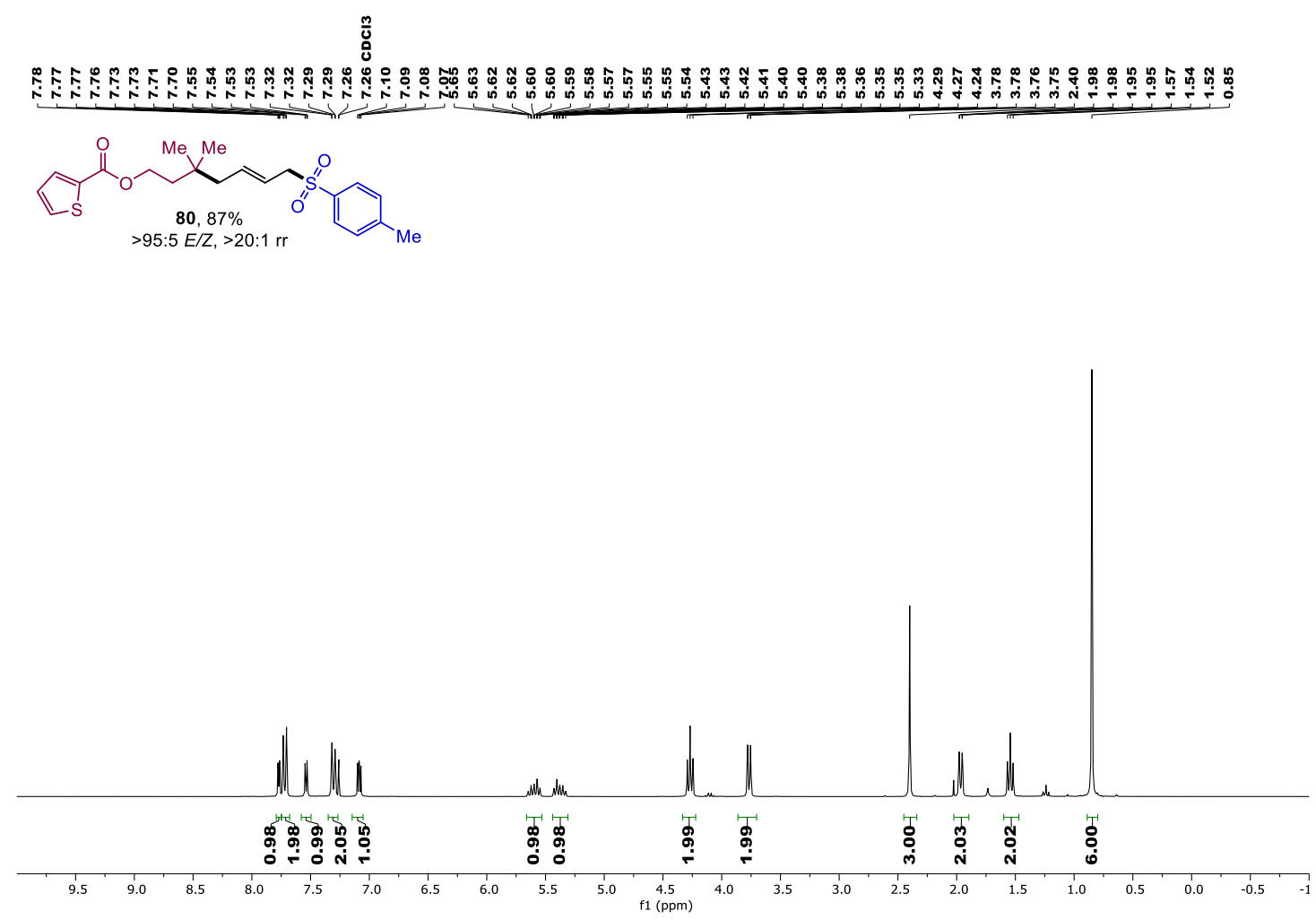

${ }^{13} \mathrm{C}$ NMR $\left(75 \mathrm{MHz}, \mathrm{CDCl}_{3}\right.$ )
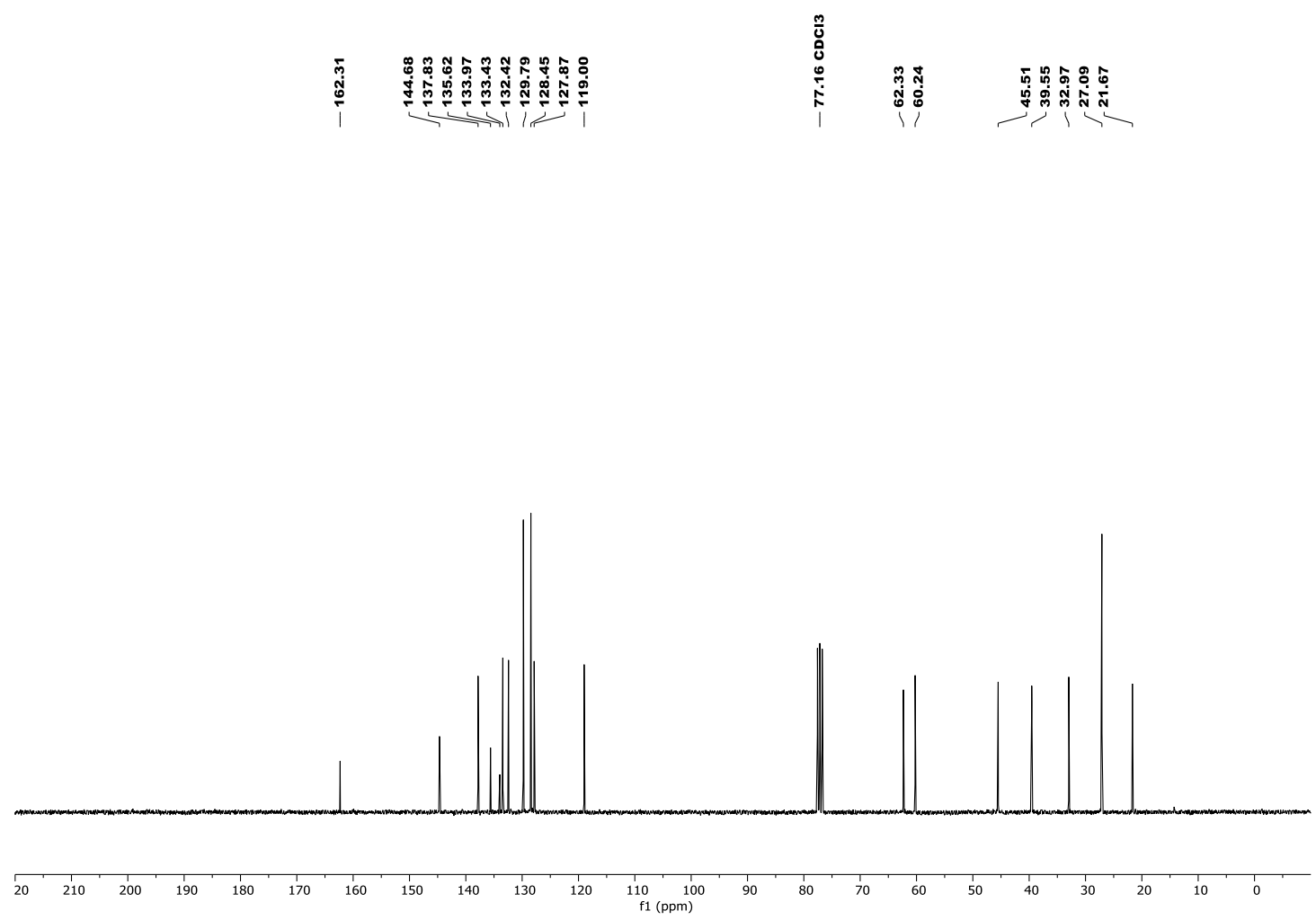
${ }^{1} \mathrm{H} \mathrm{NMR}\left(300 \mathrm{MHz}, \mathrm{CDCl}_{3}\right)$
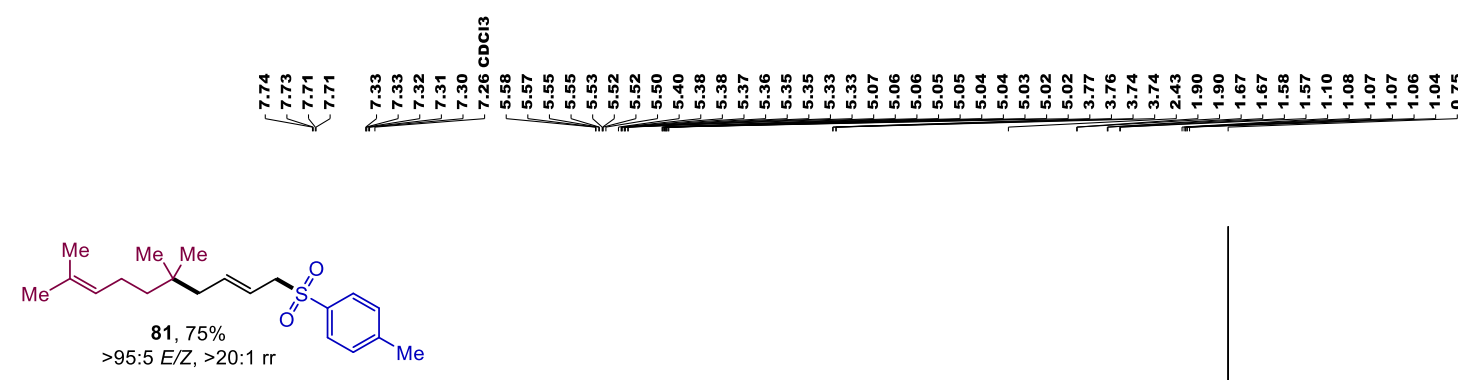

${ }^{13} \mathrm{C}$ NMR (75 MHz, $\mathrm{CDCl}_{3}$ )

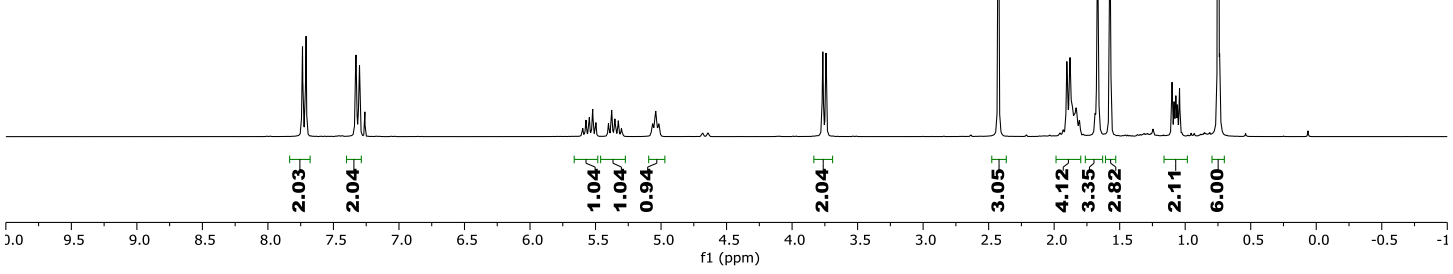

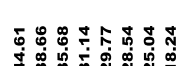

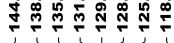

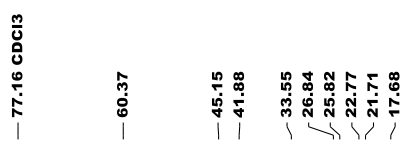

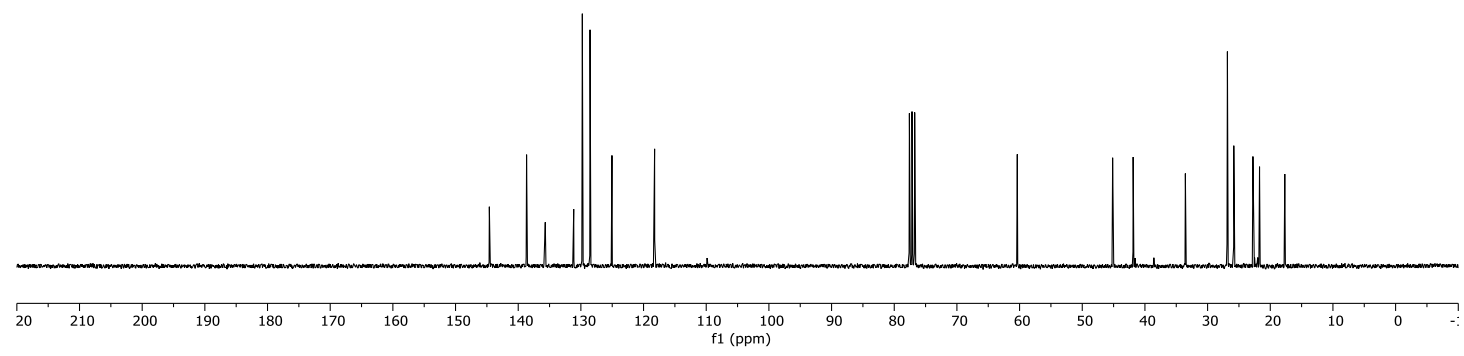

S 291 
${ }^{1} \mathrm{H}$ NMR (599 MHz, $\mathrm{CDCl}_{3}$ )

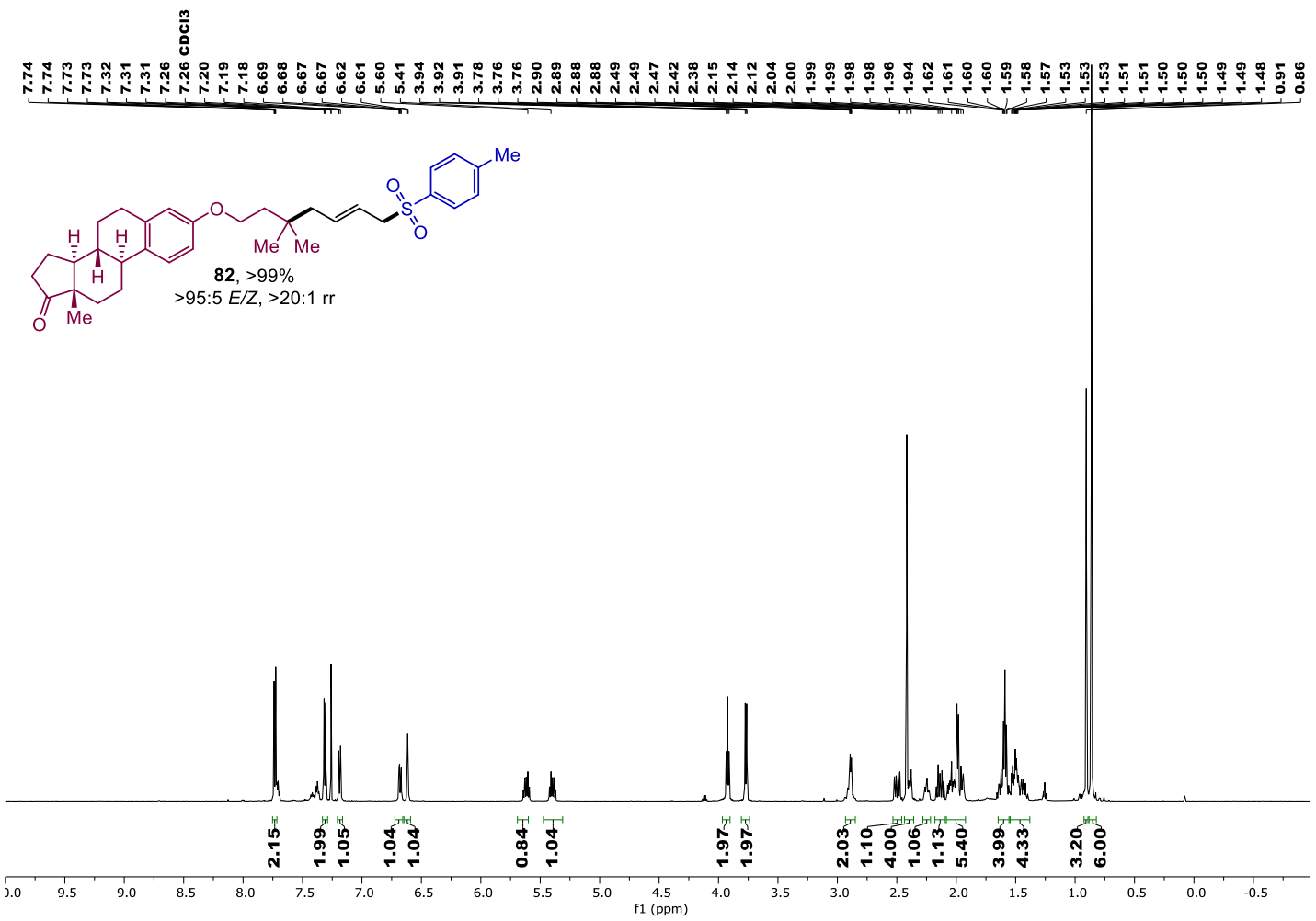

${ }^{13} \mathrm{C}$ NMR $\left(151 \mathrm{MHz}, \mathrm{CDCl}_{3}\right)$

ถุ.

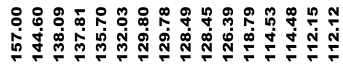
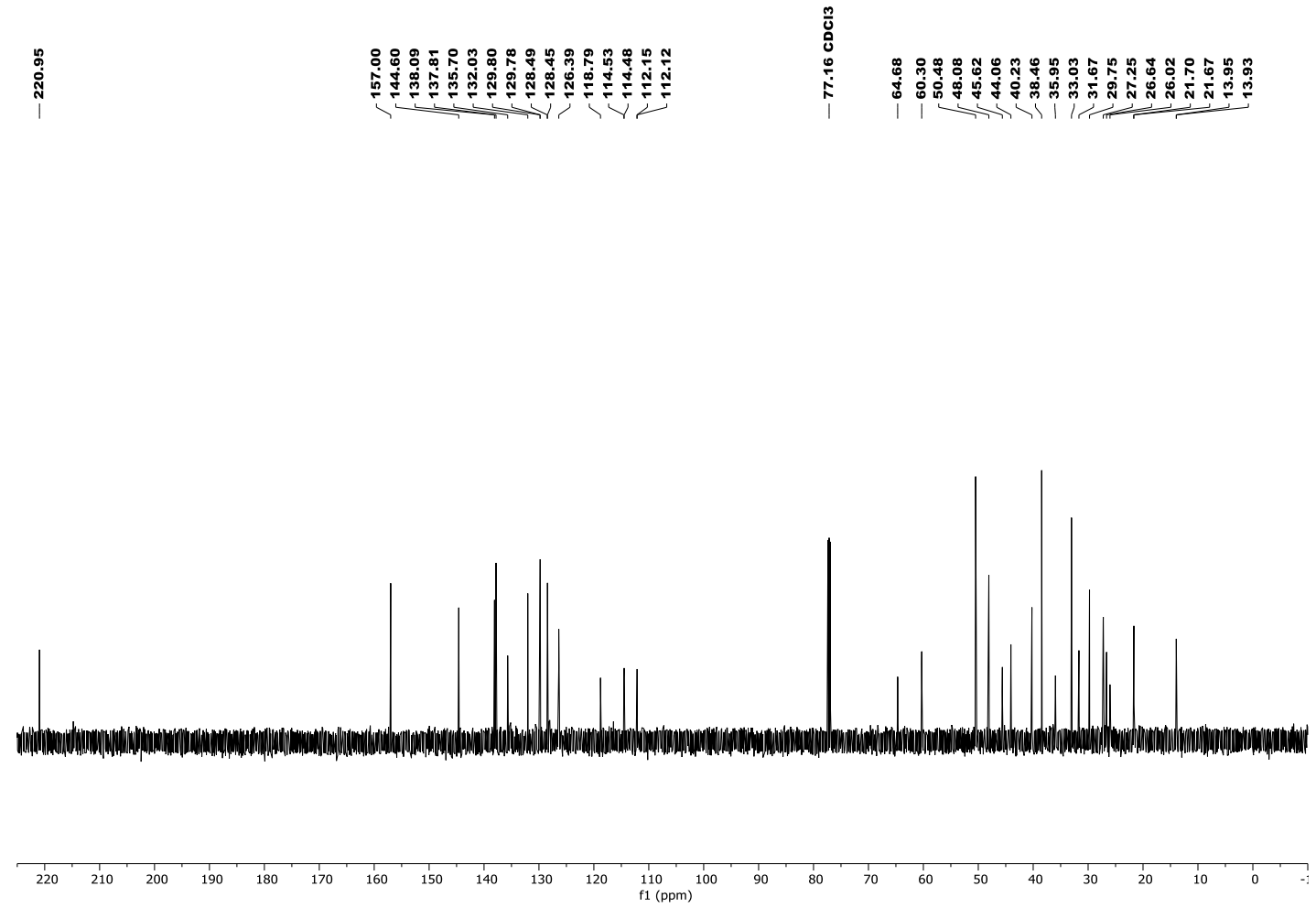
${ }^{1} \mathrm{H}$ NMR $\left(400 \mathrm{MHz}, \mathrm{CDCl}_{3}\right)$

ัั.

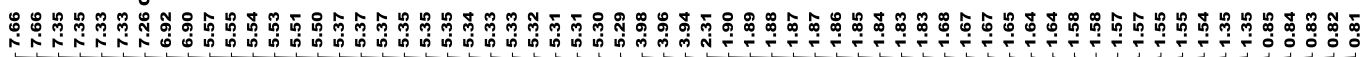

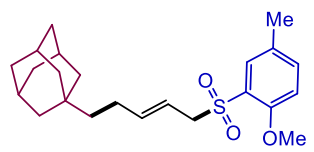

$113,74 \%$

$>95: 5 \mathrm{E} / \mathrm{Z},>20: 1 \mathrm{rr}$

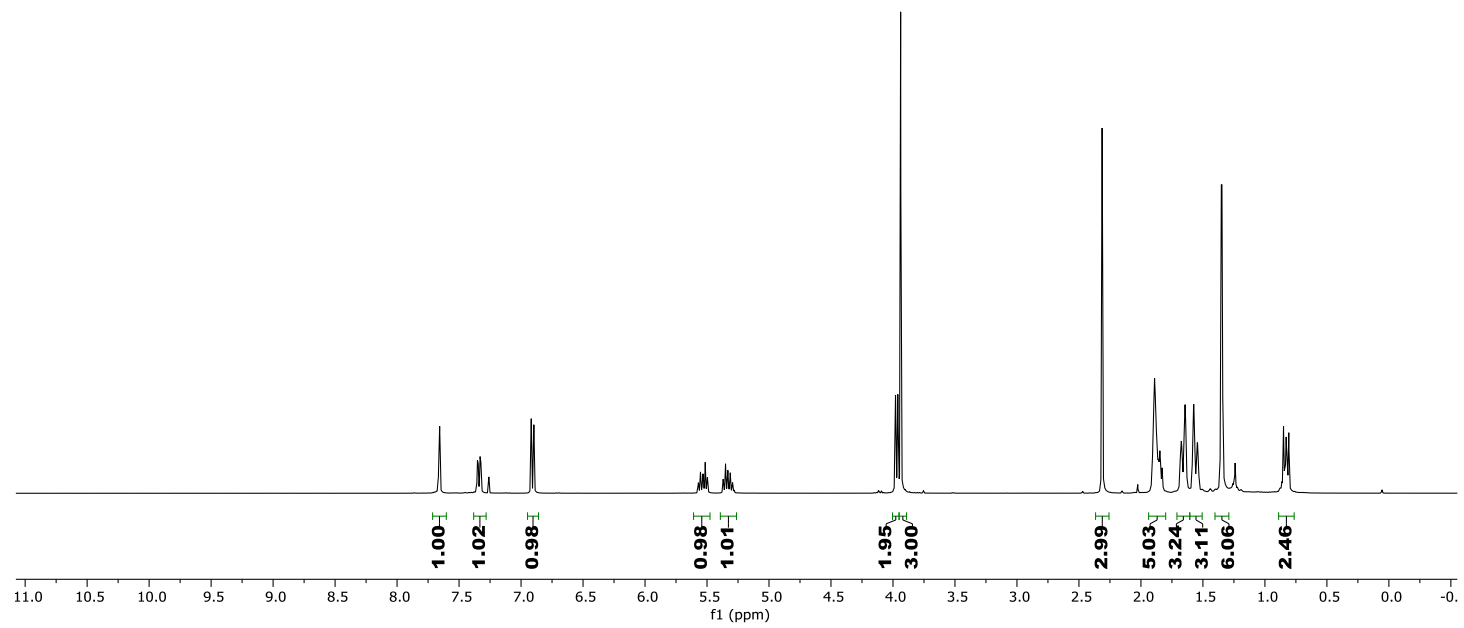

${ }^{13} \mathrm{C}$ NMR $\left(101 \mathrm{MHz}, \mathrm{CDCl}_{3}\right)$
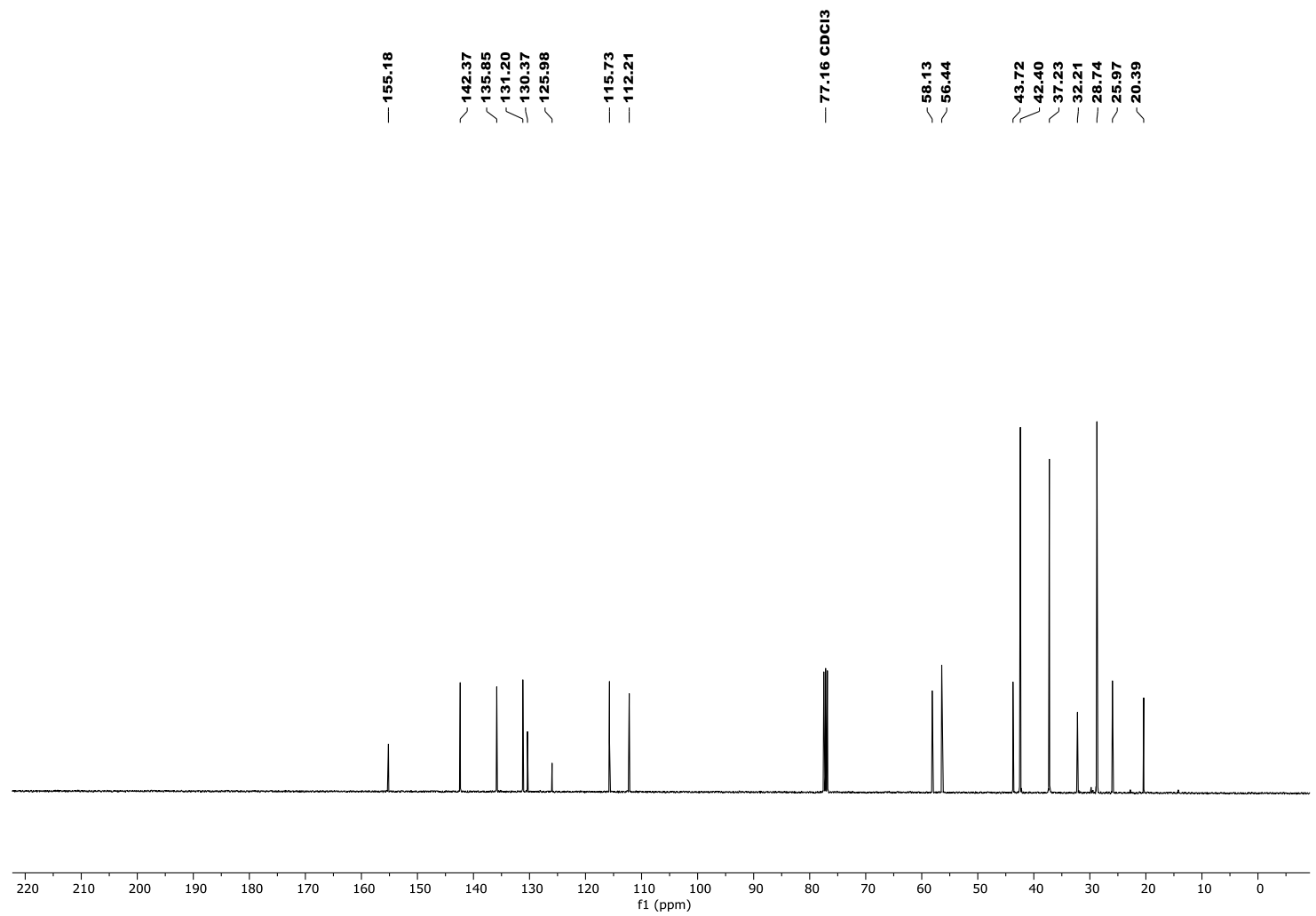
${ }^{1} \mathrm{H}$ NMR $\left(400 \mathrm{MHz}, \mathrm{CDCl}_{3}\right)$

\%ั0

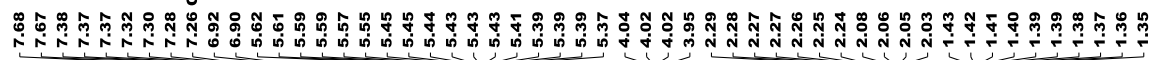
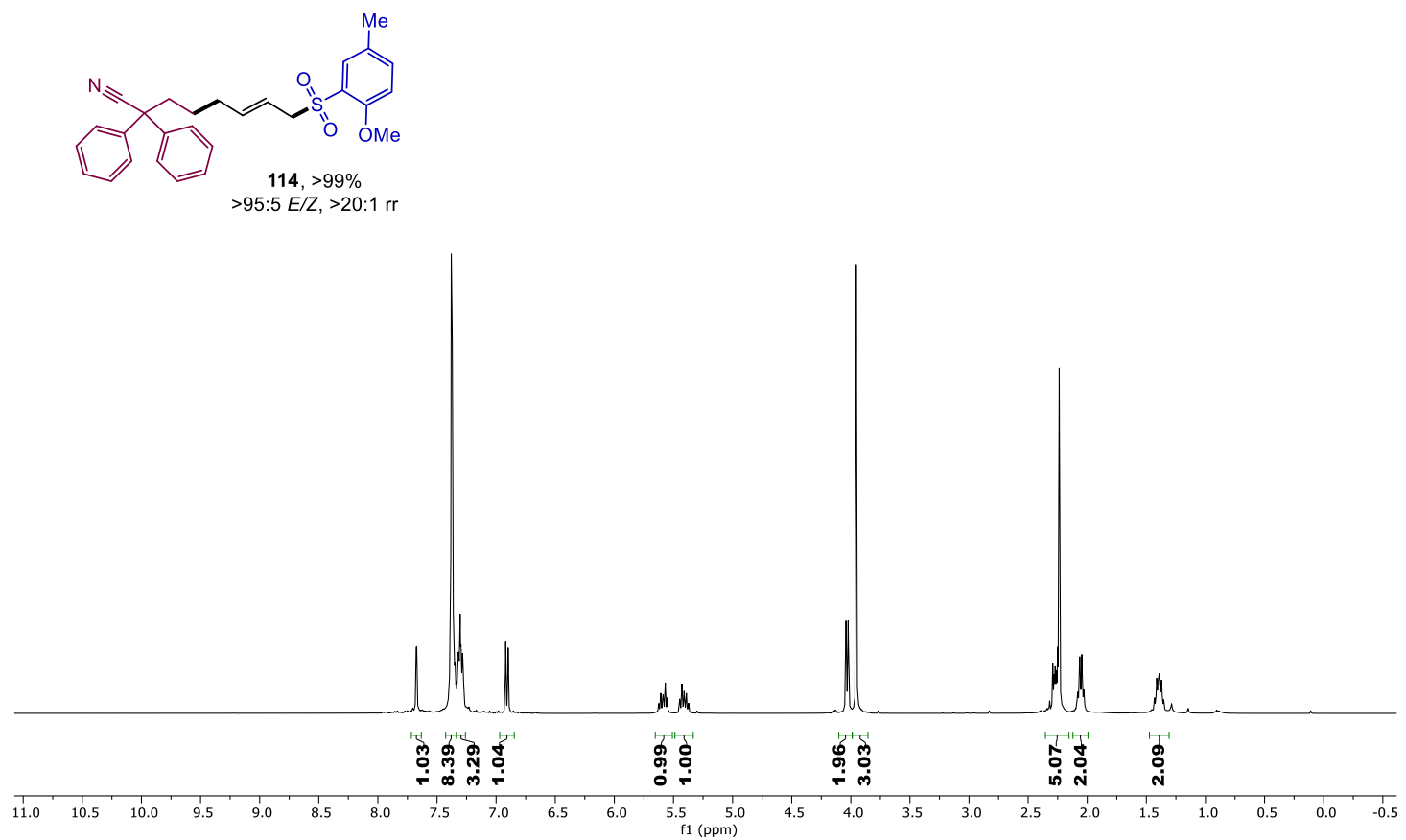

${ }^{13} \mathrm{C}$ NMR $\left(101 \mathrm{MHz}, \mathrm{CDCl}_{3}\right)$
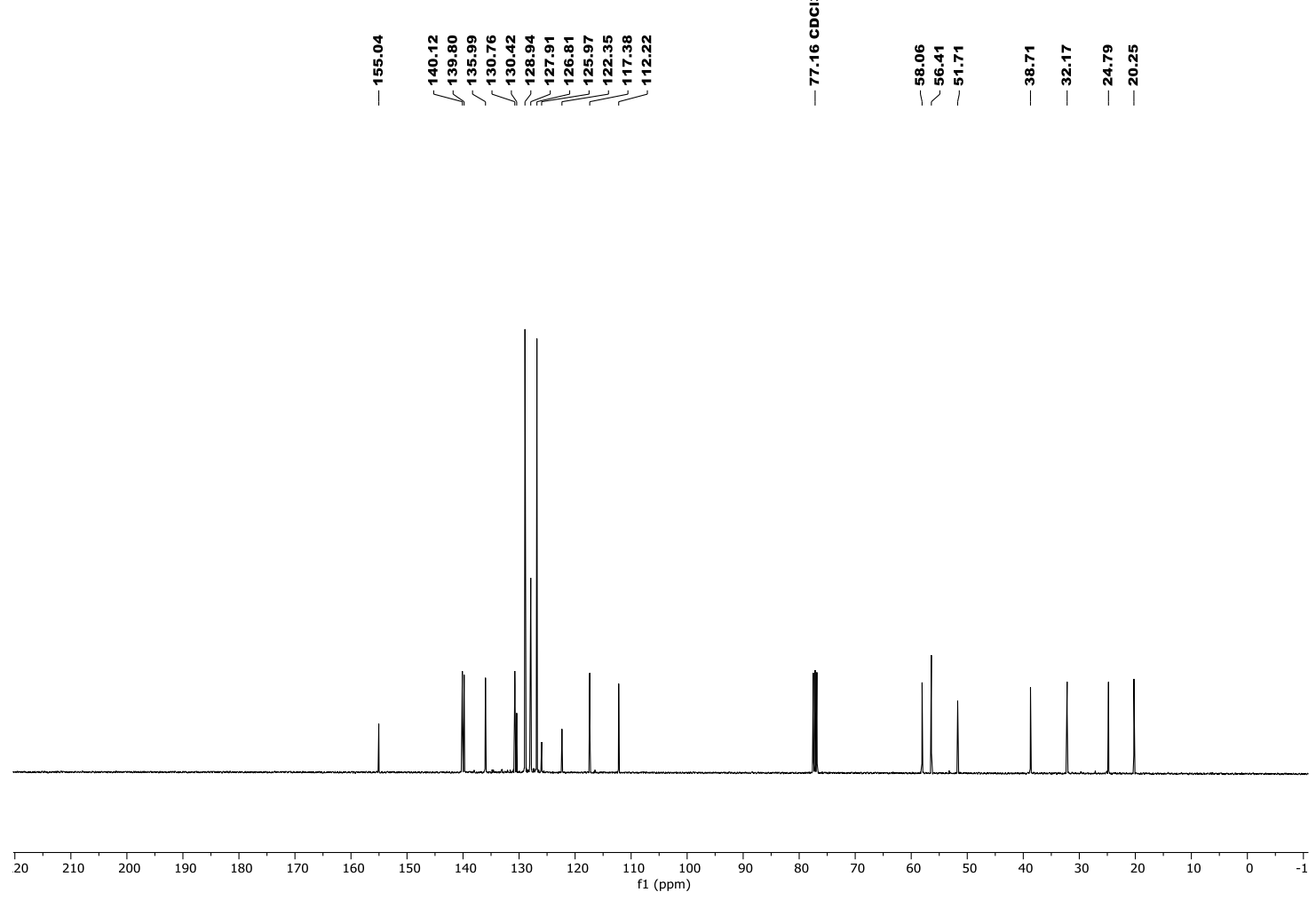
${ }^{1} \mathrm{H}$ NMR $\left(400 \mathrm{MHz}, \mathrm{CDCl}_{3}\right)$

\section{$\frac{0}{0}$

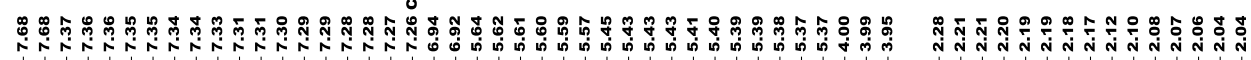
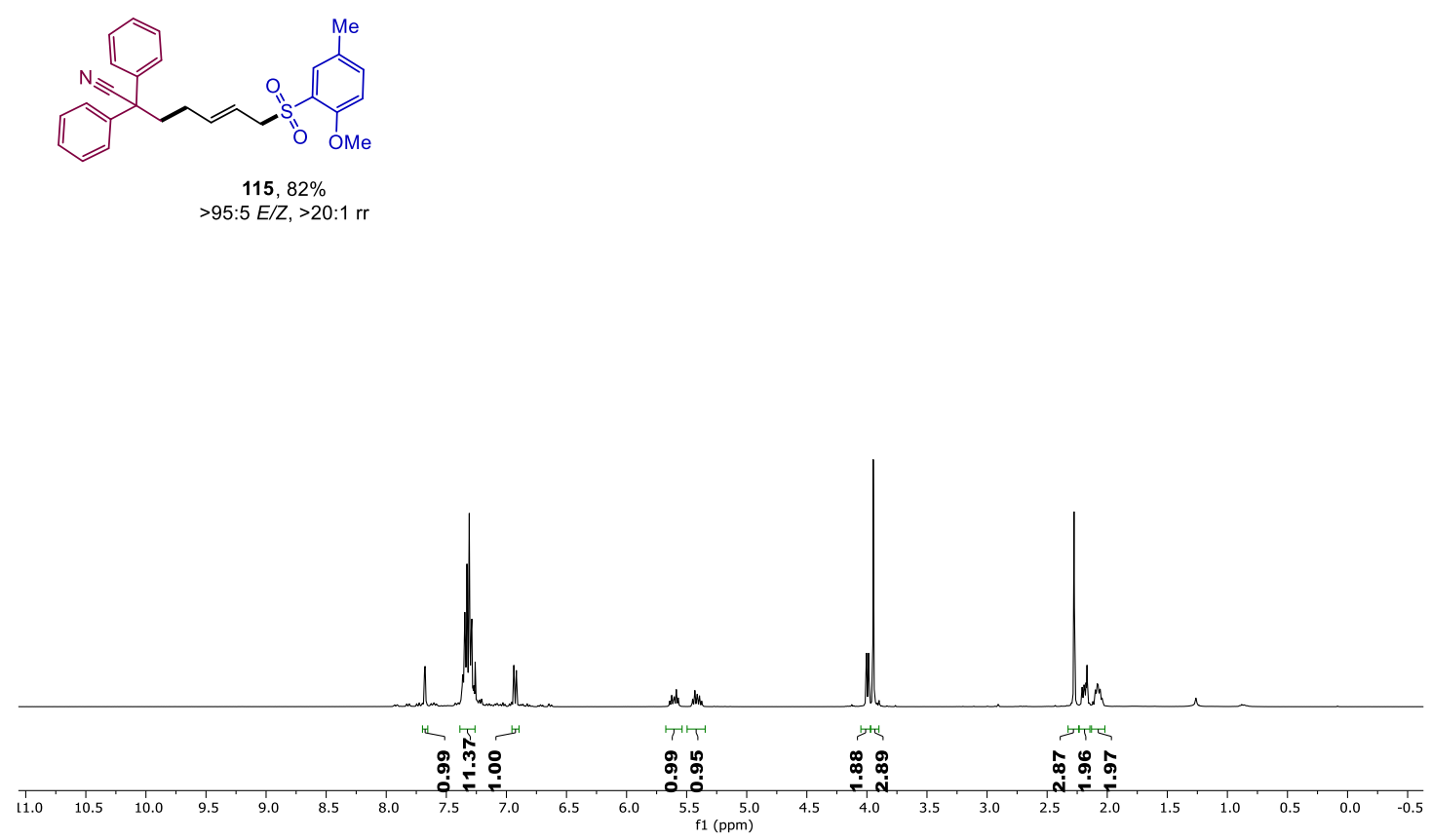

${ }^{13} \mathrm{C}$ NMR $\left(101 \mathrm{MHz}, \mathrm{CDCl}_{3}\right)$
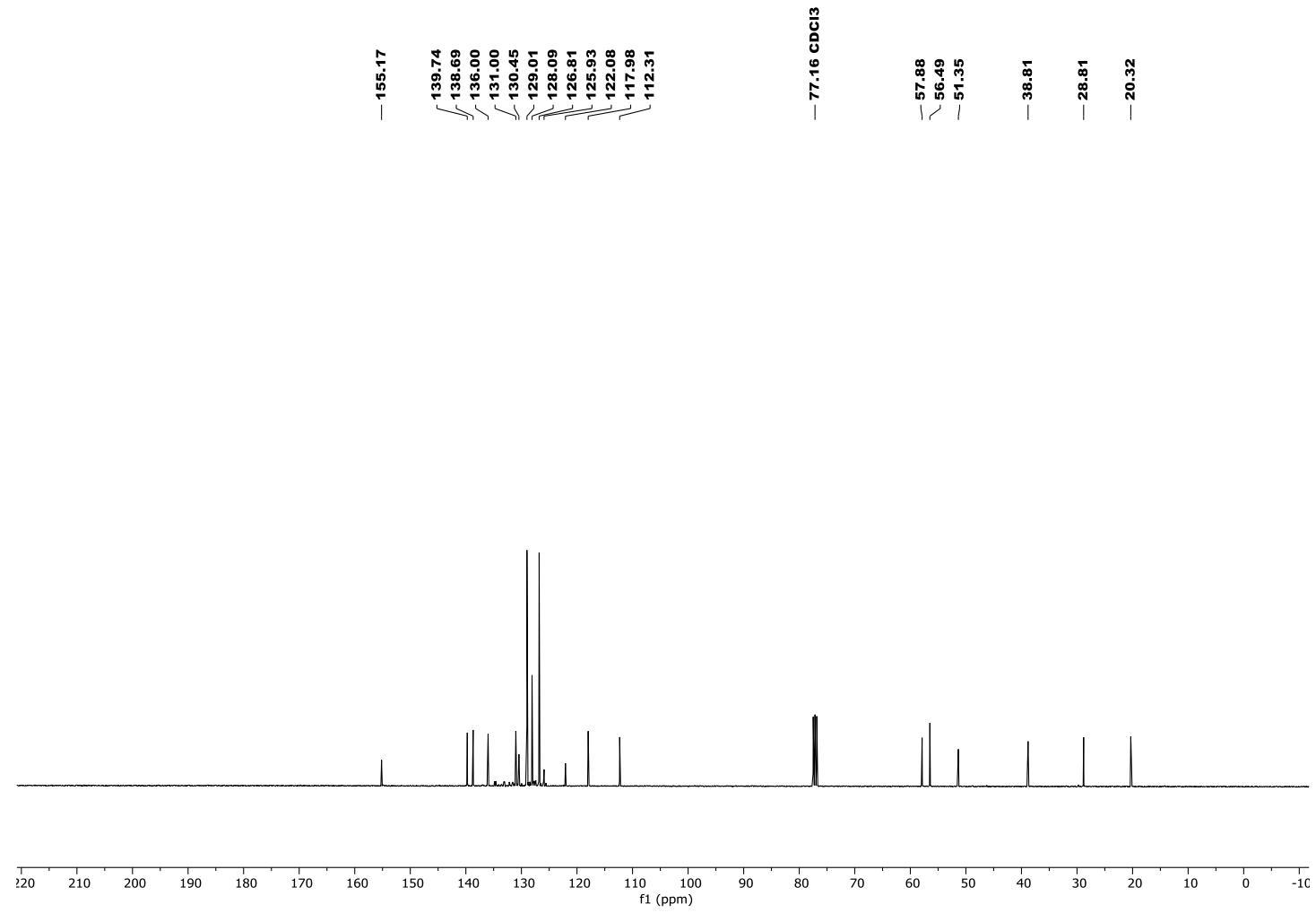
${ }^{1} \mathrm{H}$ NMR $\left(400 \mathrm{MHz}, \mathrm{CDCl}_{3}\right)$

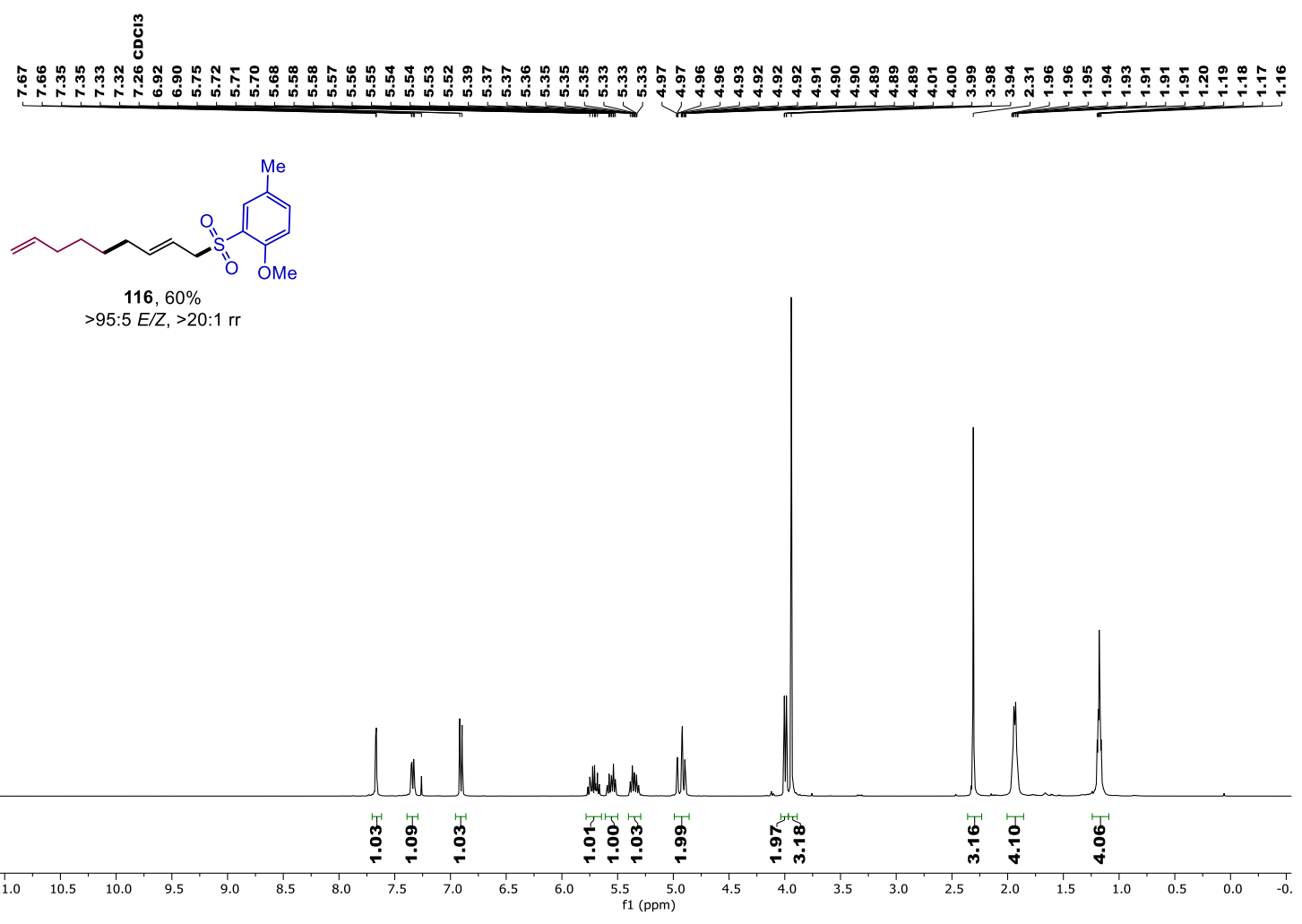

${ }^{13} \mathrm{C}$ NMR (101 MHz, $\mathrm{CDCl}_{3}$ )
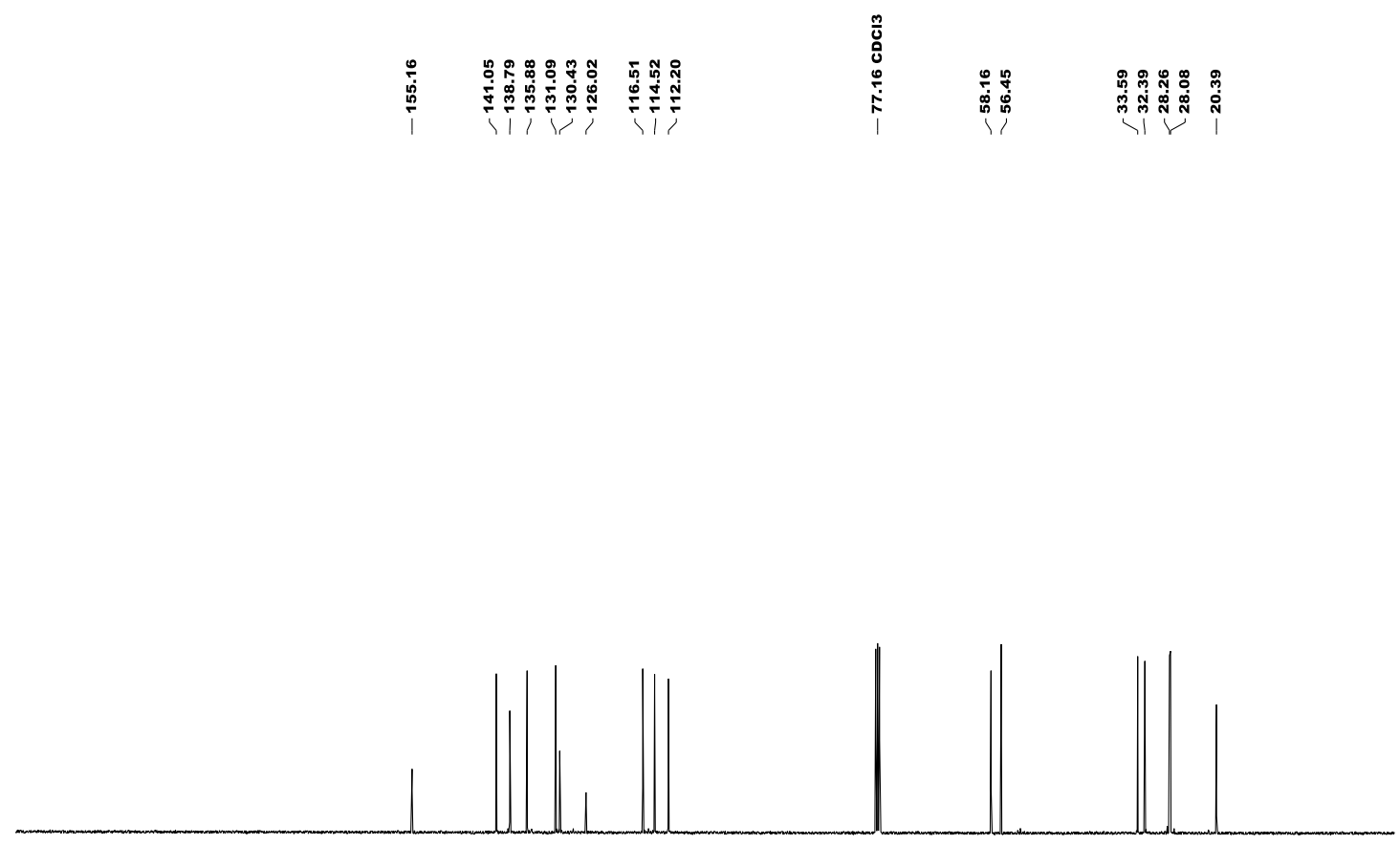

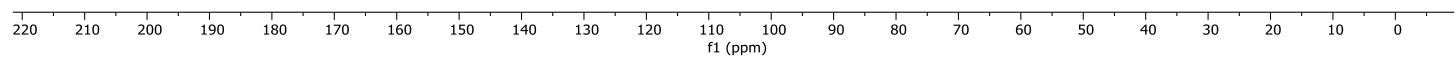


${ }^{1} \mathrm{H}$ NMR $\left(400 \mathrm{MHz}, \mathrm{CDCl}_{3}\right)$ - Contains $50 \%$ of product (see above)

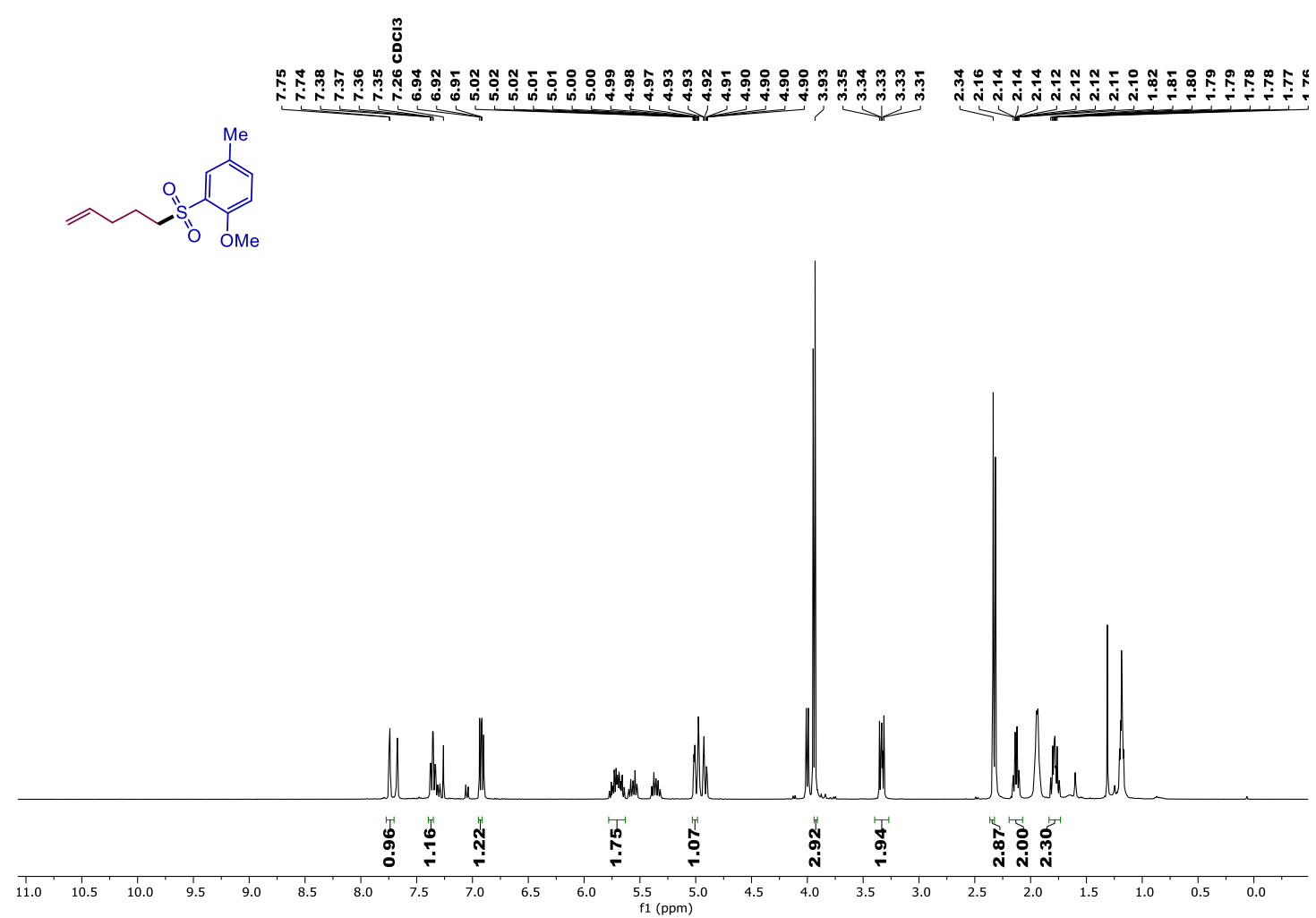

${ }^{13} \mathrm{C}$ NMR (101 MHz, $\mathrm{CDCl}_{3}$ ) - Contains $50 \%$ of product (see above)
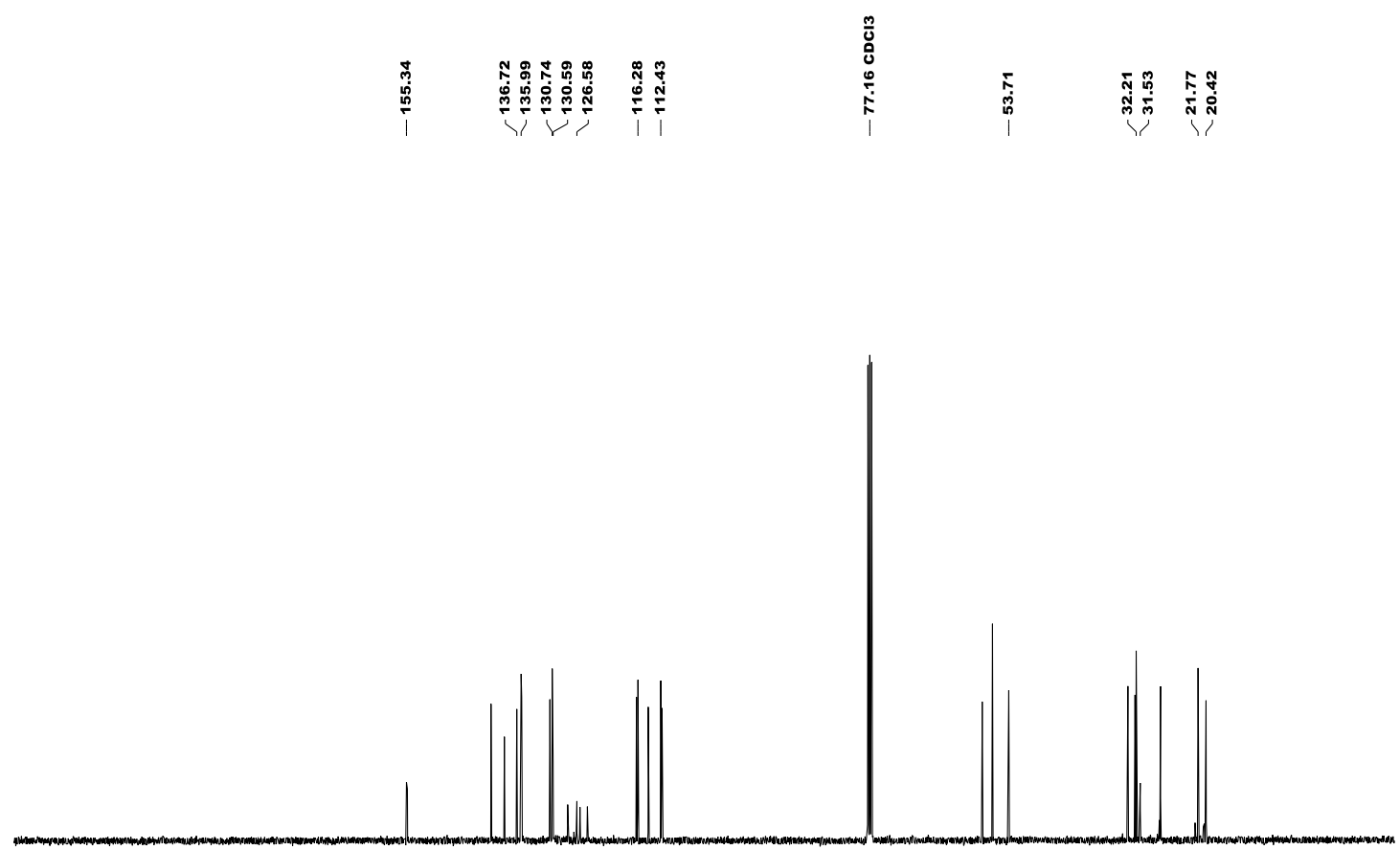

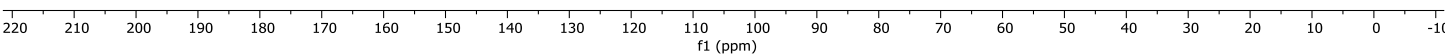


${ }^{1} \mathrm{H}$ NMR $\left(599 \mathrm{MHz}, \mathrm{CDCl}_{3}\right.$ )

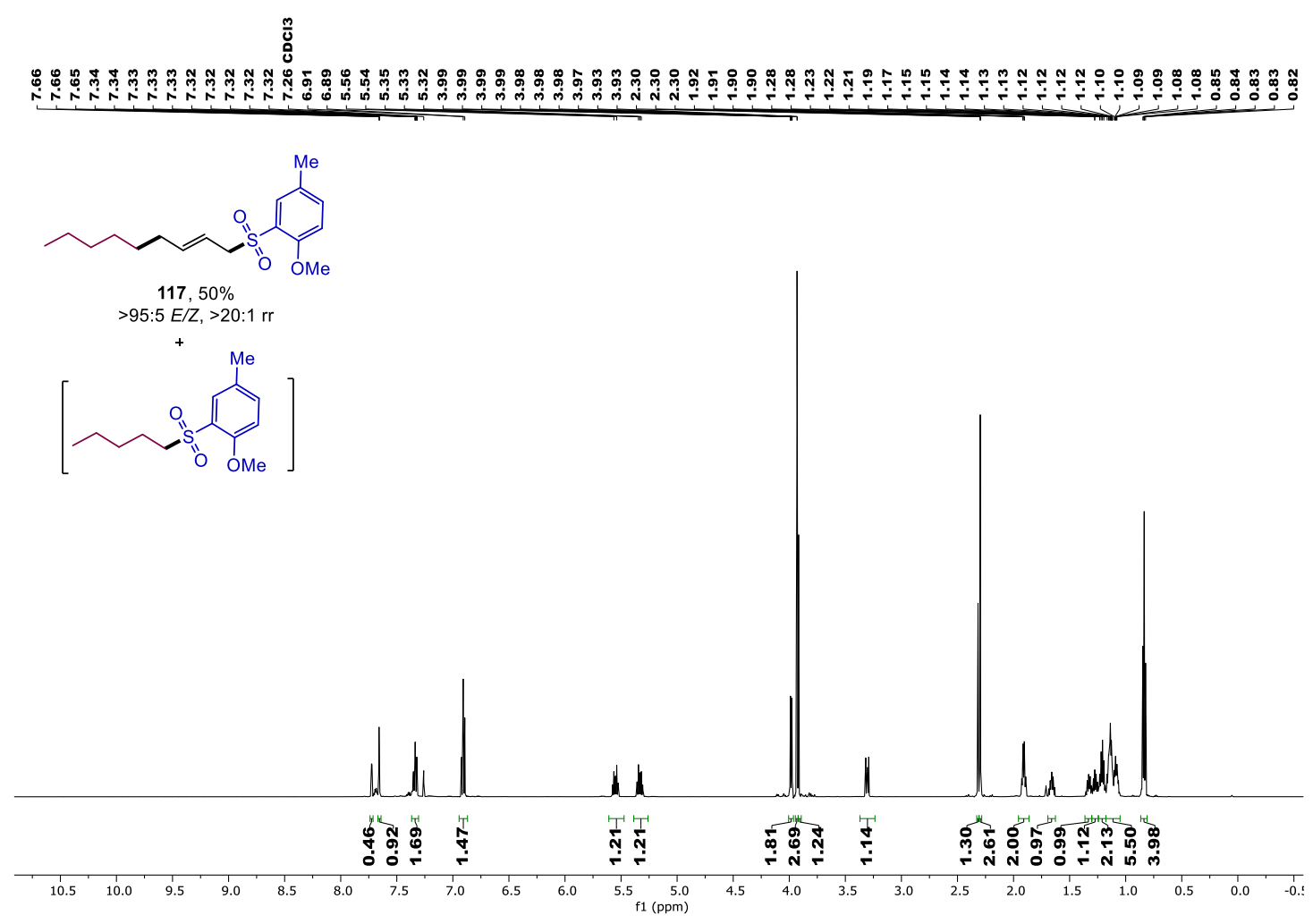

${ }^{13} \mathrm{C}$ NMR (150 MHz, $\mathrm{CDCl}_{3}$ )
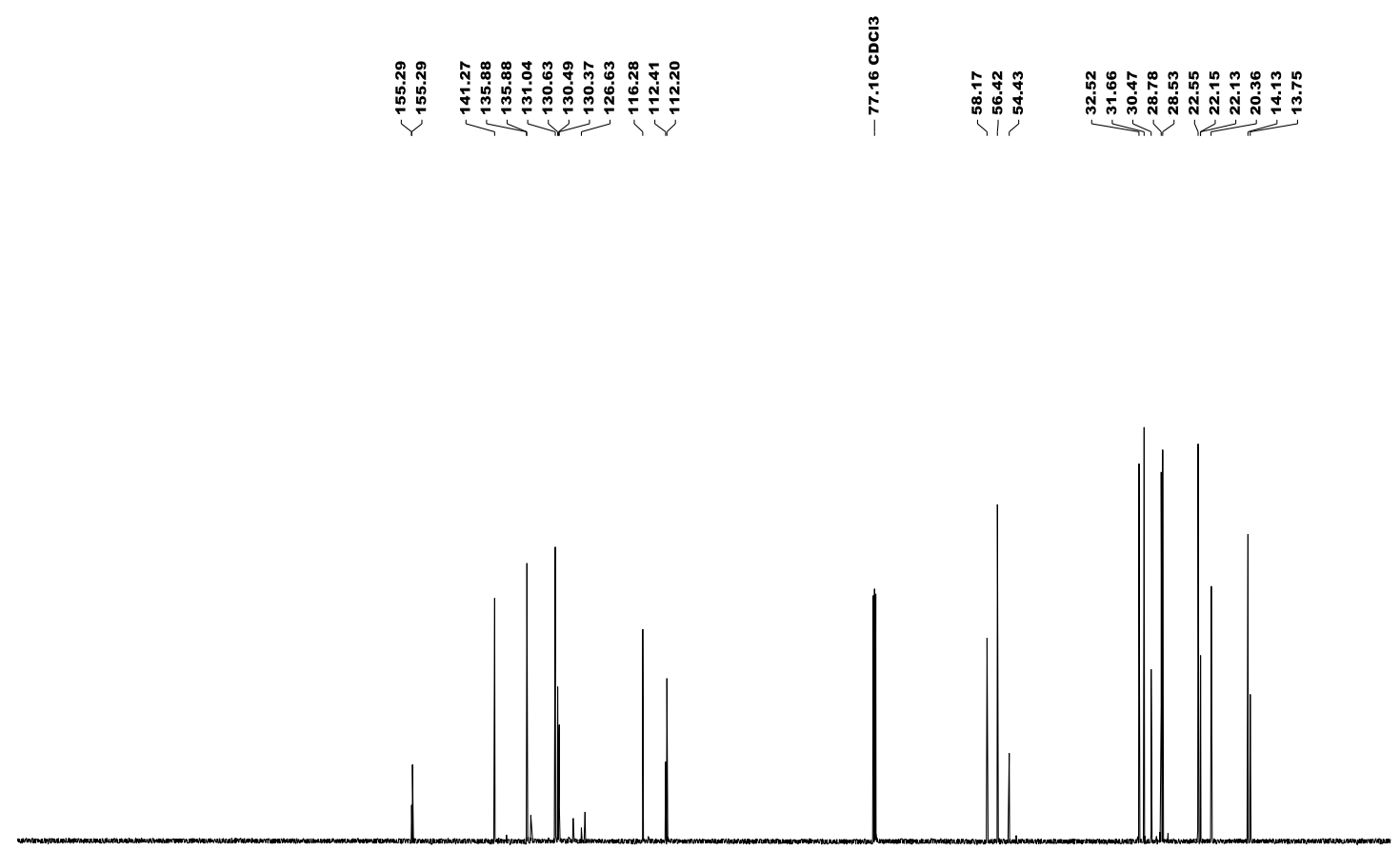

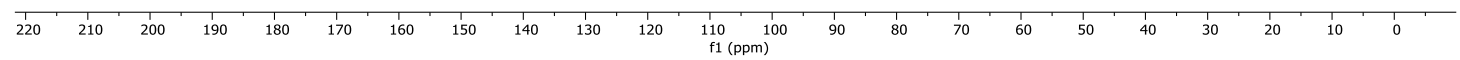


${ }^{1} \mathrm{H}-{ }^{1} \mathrm{H}-\mathrm{COSY}\left(599 \mathrm{MHz}, \mathrm{CDCl}_{3}\right)$

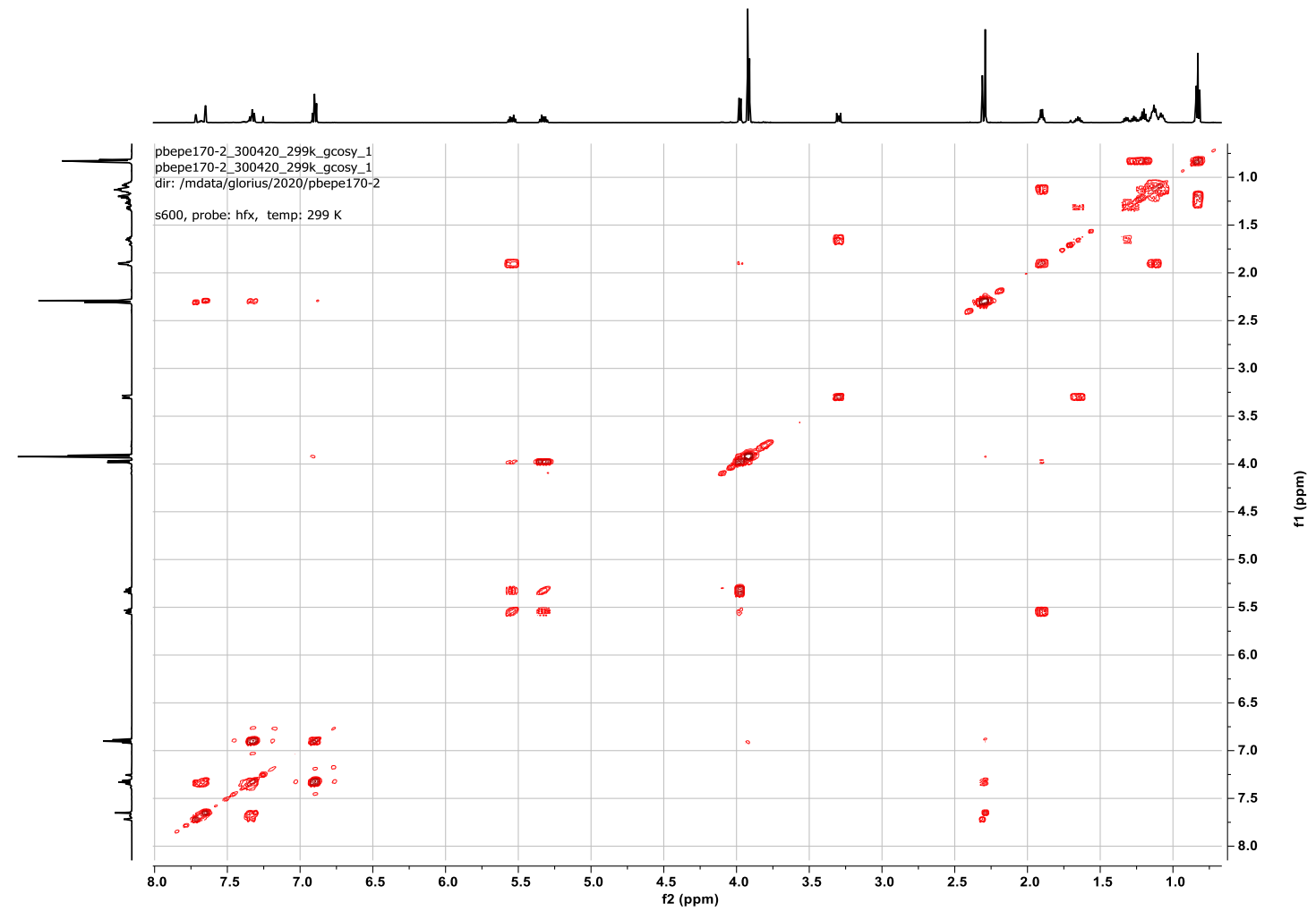

${ }^{1} \mathrm{H}-{ }^{13} \mathrm{C}-\mathrm{HSQC}\left(599 \mathrm{MHz}, \mathrm{CDCl}_{3}\right)$

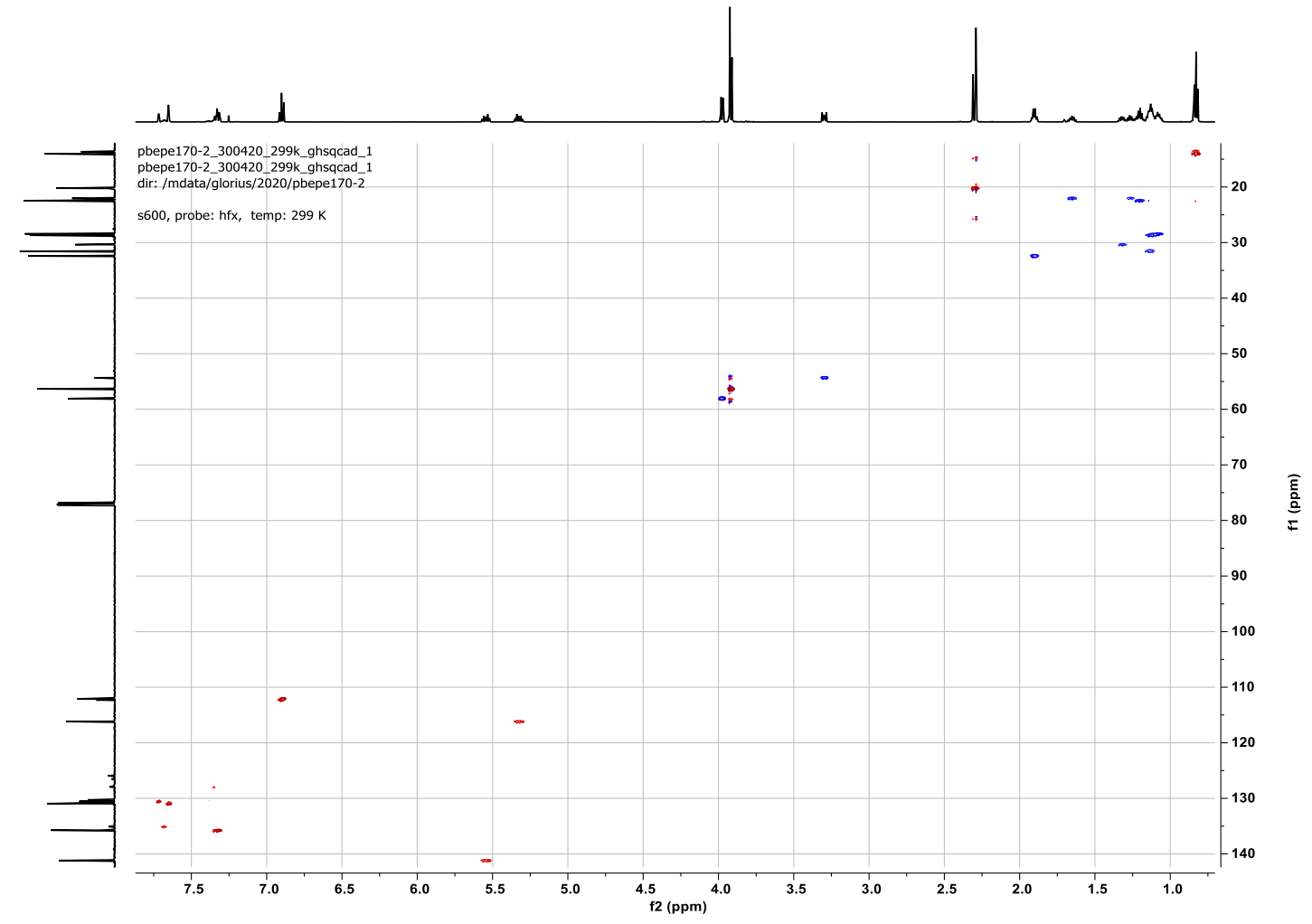


${ }^{1} \mathrm{H}-{ }^{13} \mathrm{C}-\mathrm{HMBC}\left(599 \mathrm{MHz}, \mathrm{CDCl}_{3}\right)$

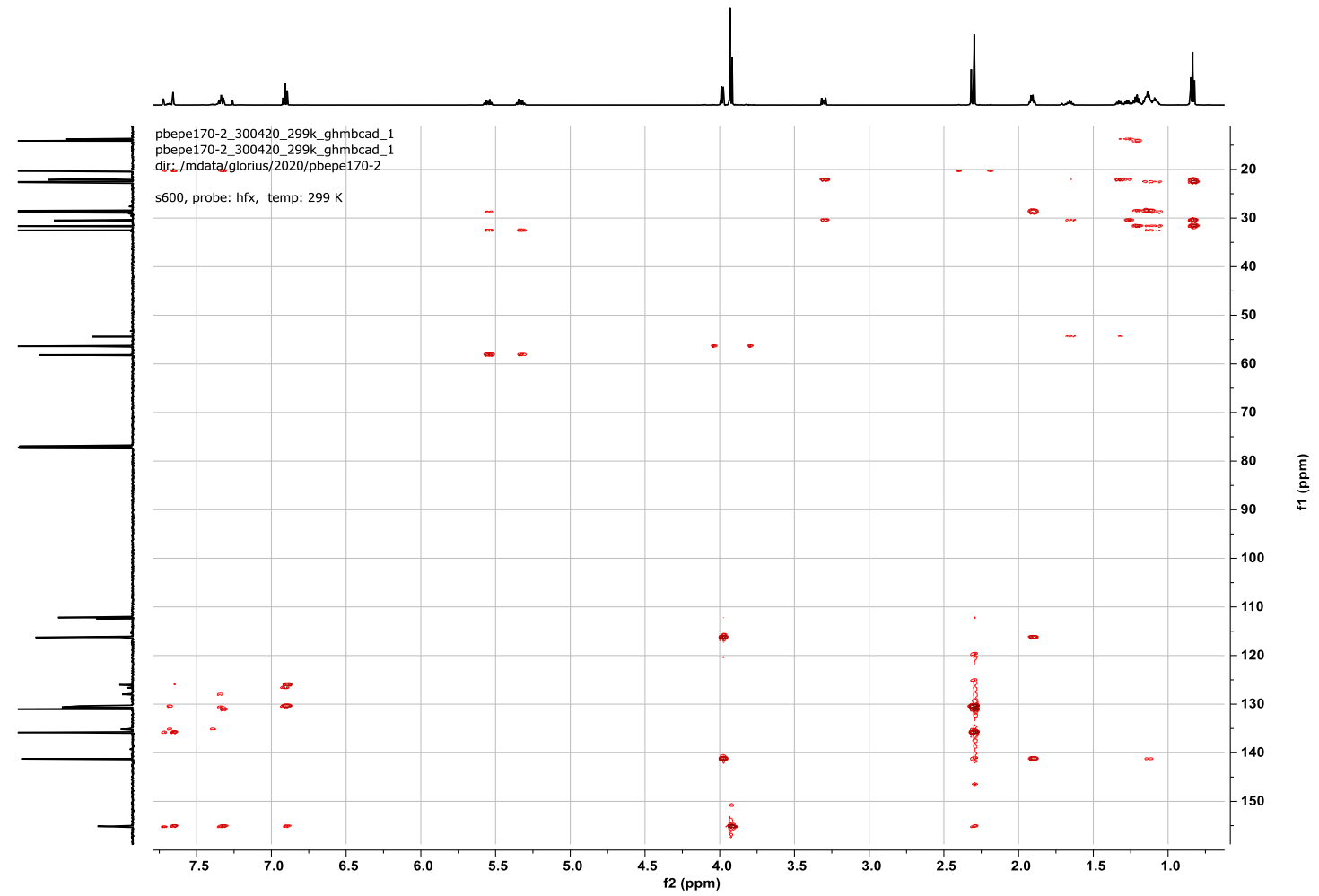


${ }^{1} \mathrm{H}$ NMR $\left(400 \mathrm{MHz}, \mathrm{CDCl}_{3}\right)$
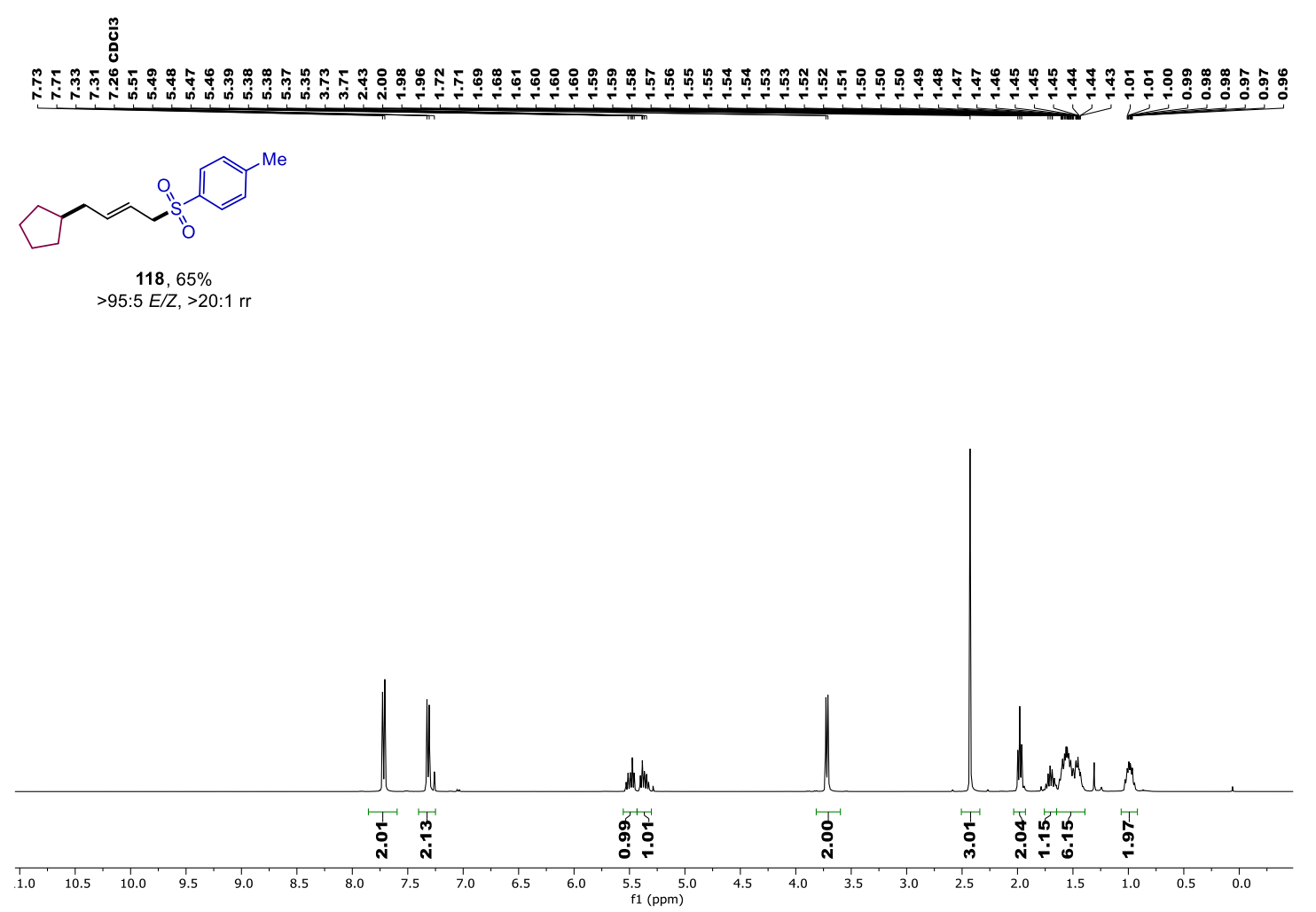

${ }^{13} \mathrm{C}$ NMR $\left(101 \mathrm{MHz}, \mathrm{CDCl}_{3}\right)$

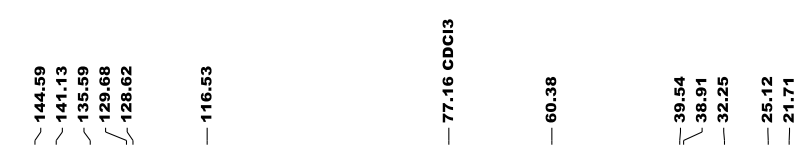

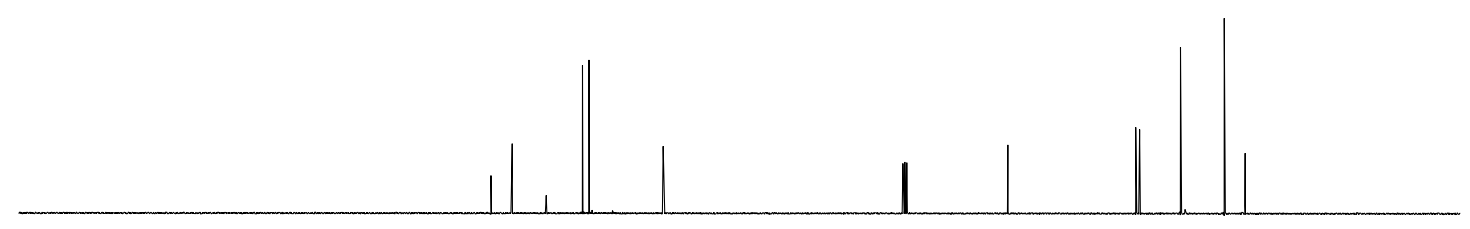

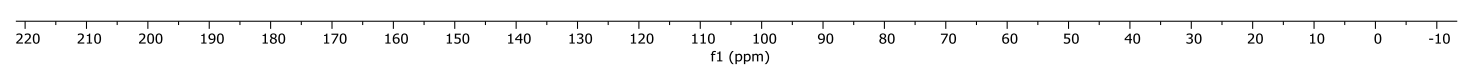


${ }^{1} \mathrm{H}$ NMR (400 MHz, $\mathrm{CDCl}_{3}$ )

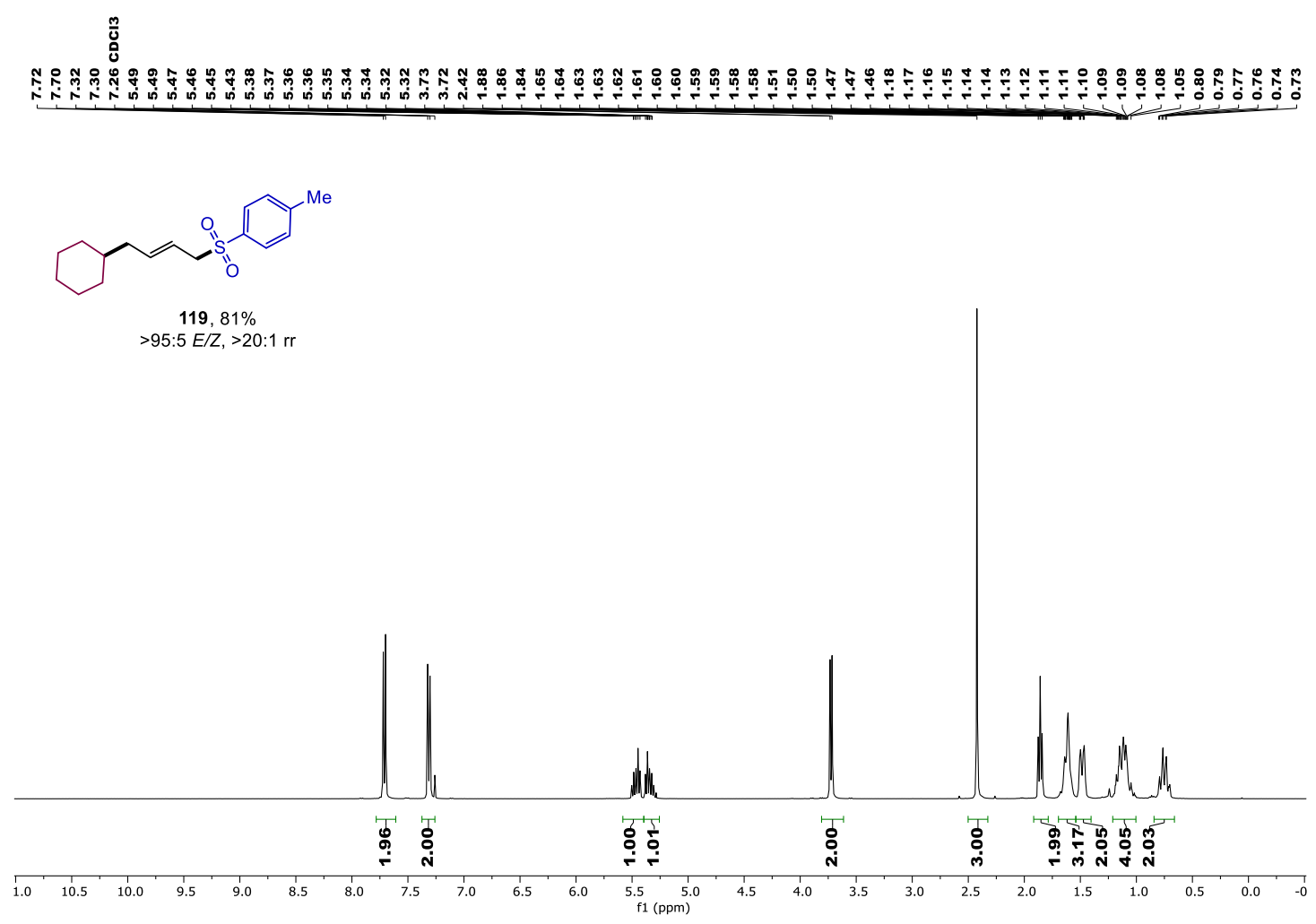

${ }^{13} \mathrm{C}$ NMR $\left(101 \mathrm{MHz}, \mathrm{CDCl}_{3}\right)$
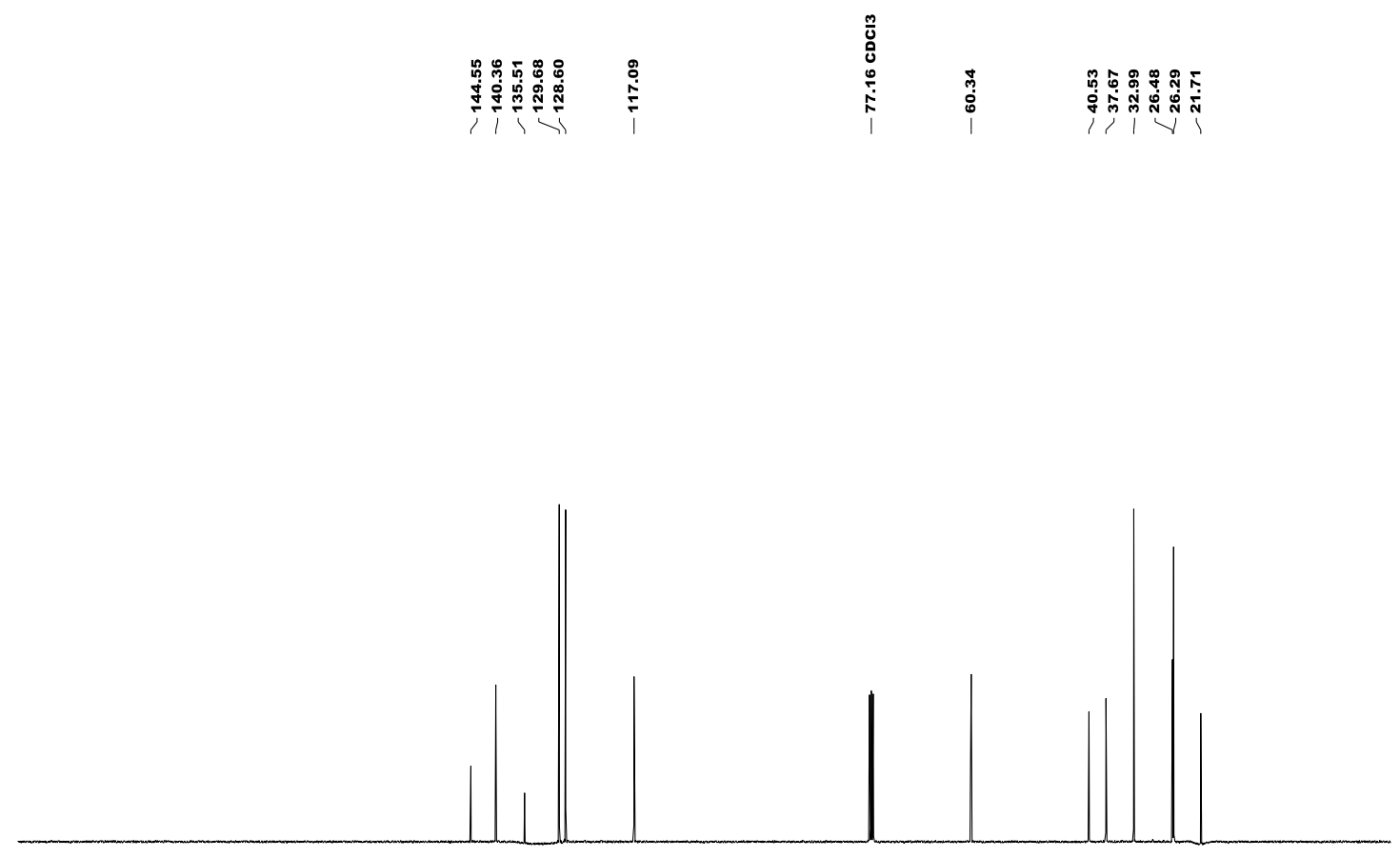

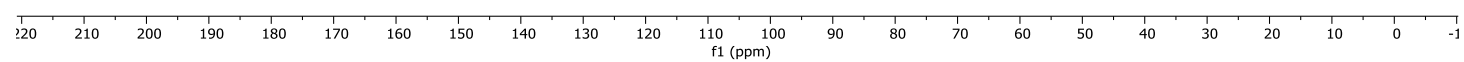


${ }^{1} \mathrm{H}$ NMR $\left(400 \mathrm{MHz}, \mathrm{CDCl}_{3}\right)$
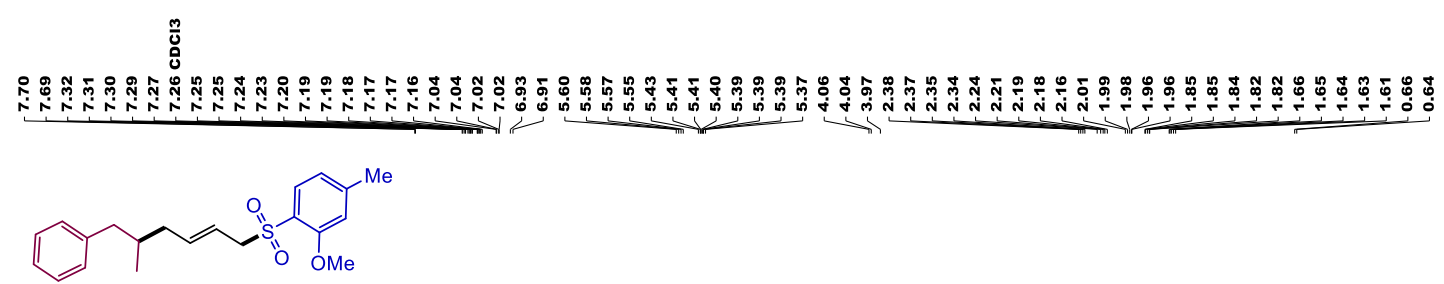

$120,86 \%$

$>95: 5 E / Z,>20: 1 \mathrm{rr}$

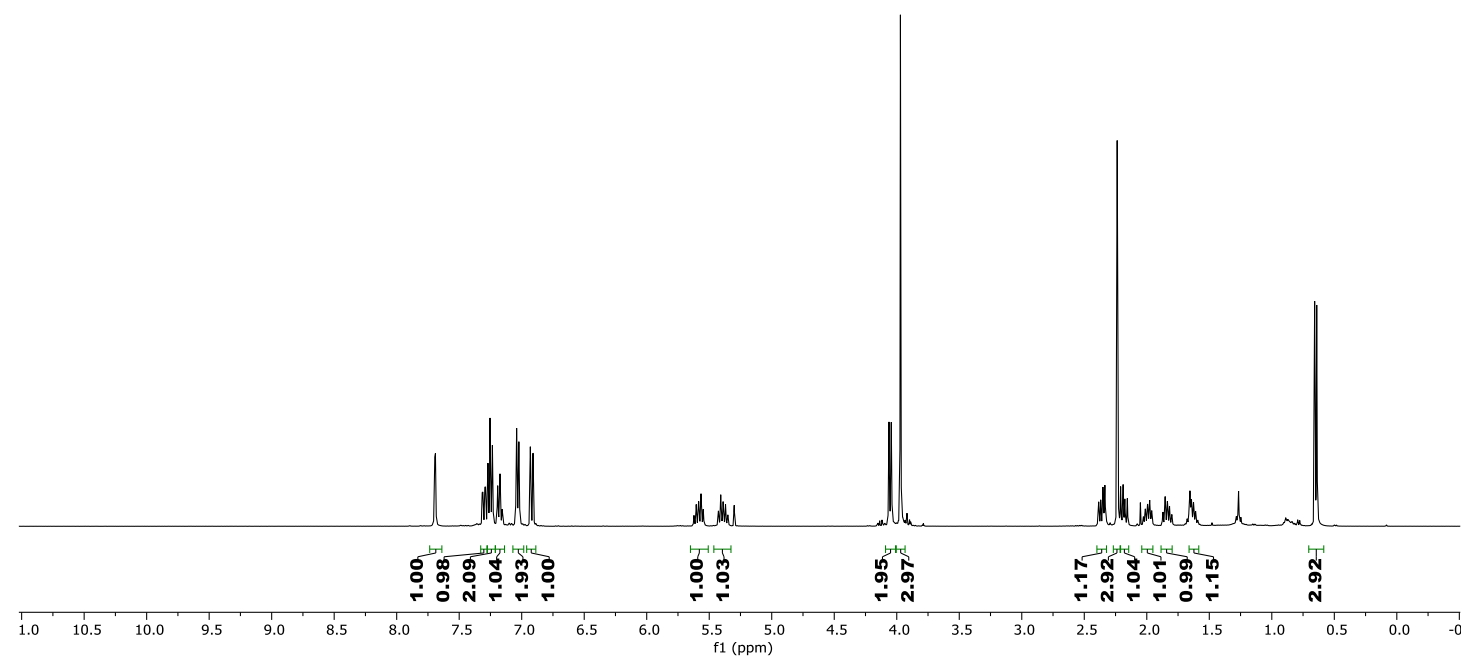

${ }^{13} \mathrm{C}$ NMR (101 MHz, $\left.\mathrm{CDCl}_{3}\right)$

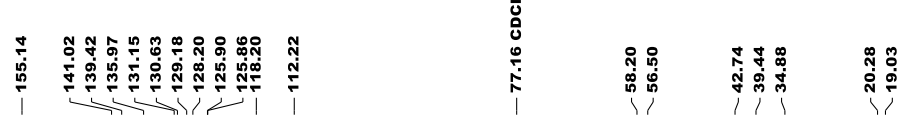

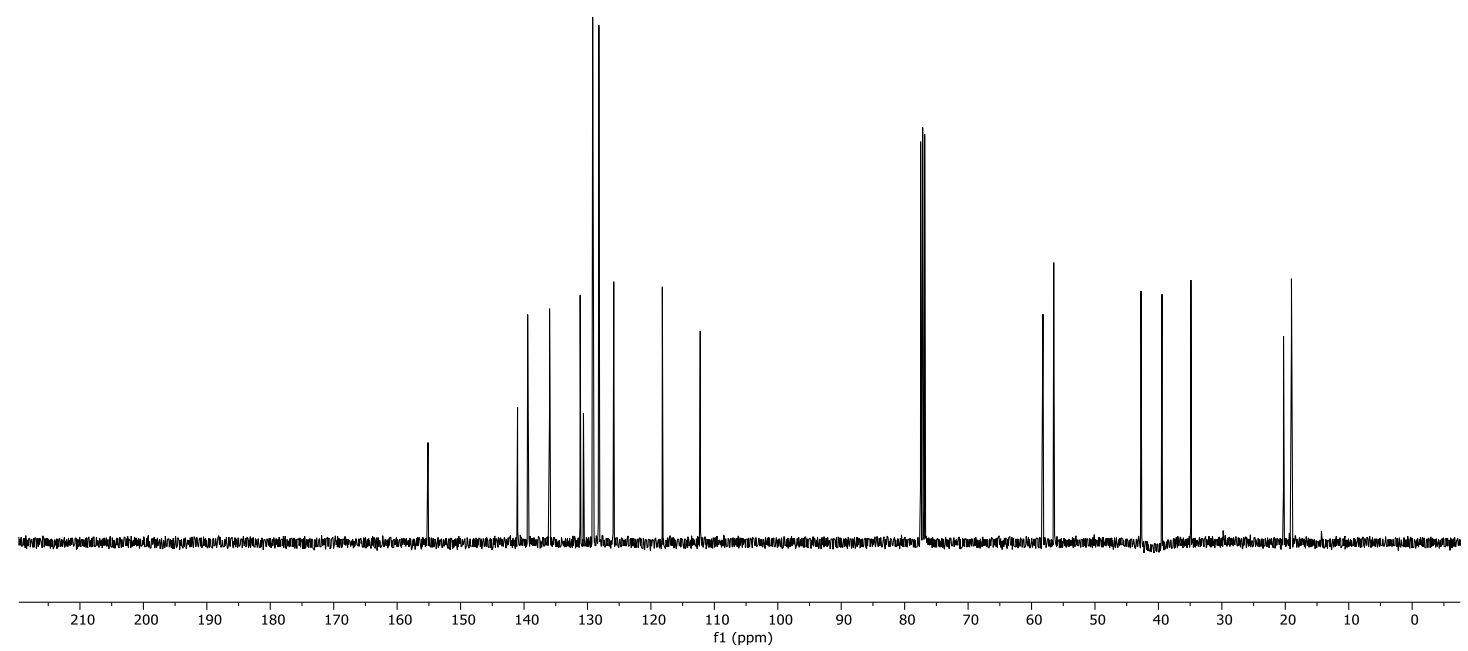


${ }^{1} \mathrm{H}$ NMR $\left(500 \mathrm{MHz}, \mathrm{CDCl}_{3}\right)$

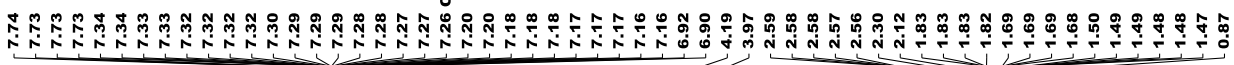

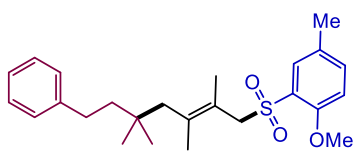

$133,38 \%$

$>95: 5 \mathrm{E} / \mathrm{Z},>20: 1 \mathrm{rr}$

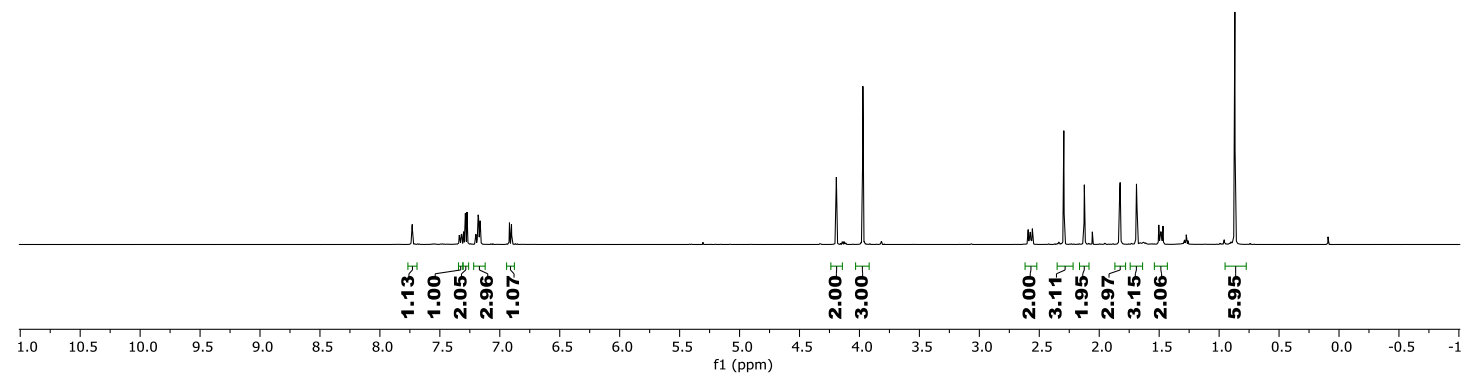

${ }^{13} \mathrm{C} \mathrm{NMR}\left(126 \mathrm{MHz}, \mathrm{CDCl}_{3}\right)$
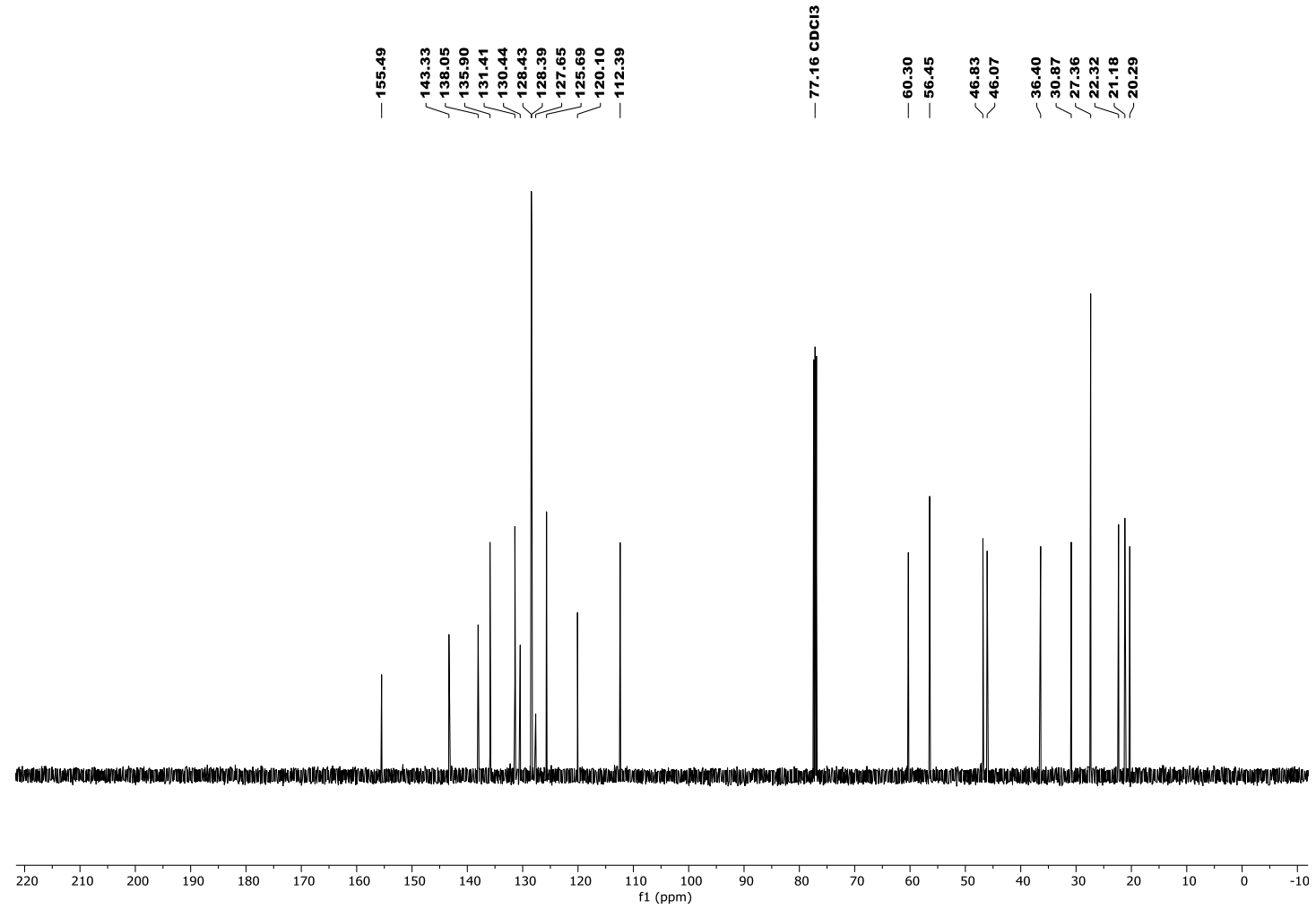
1D NOESY (alkene $\mathrm{CH}_{3}$ substituents) (500 MHz, $\mathrm{CDCl}_{3}$ )

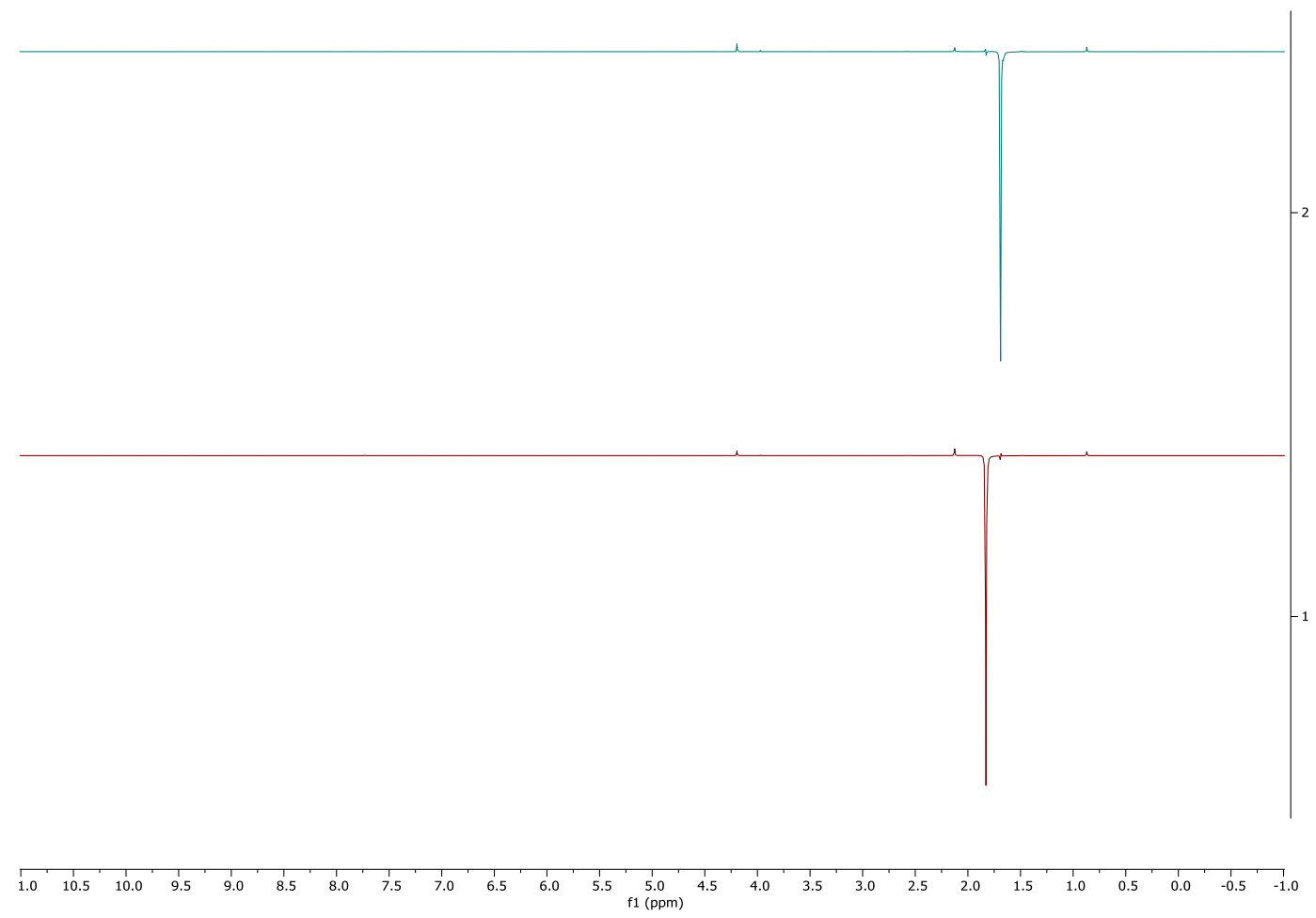


${ }^{1} \mathrm{H}$ NMR (400 MHz, $\mathrm{CDCl}_{3}$ )
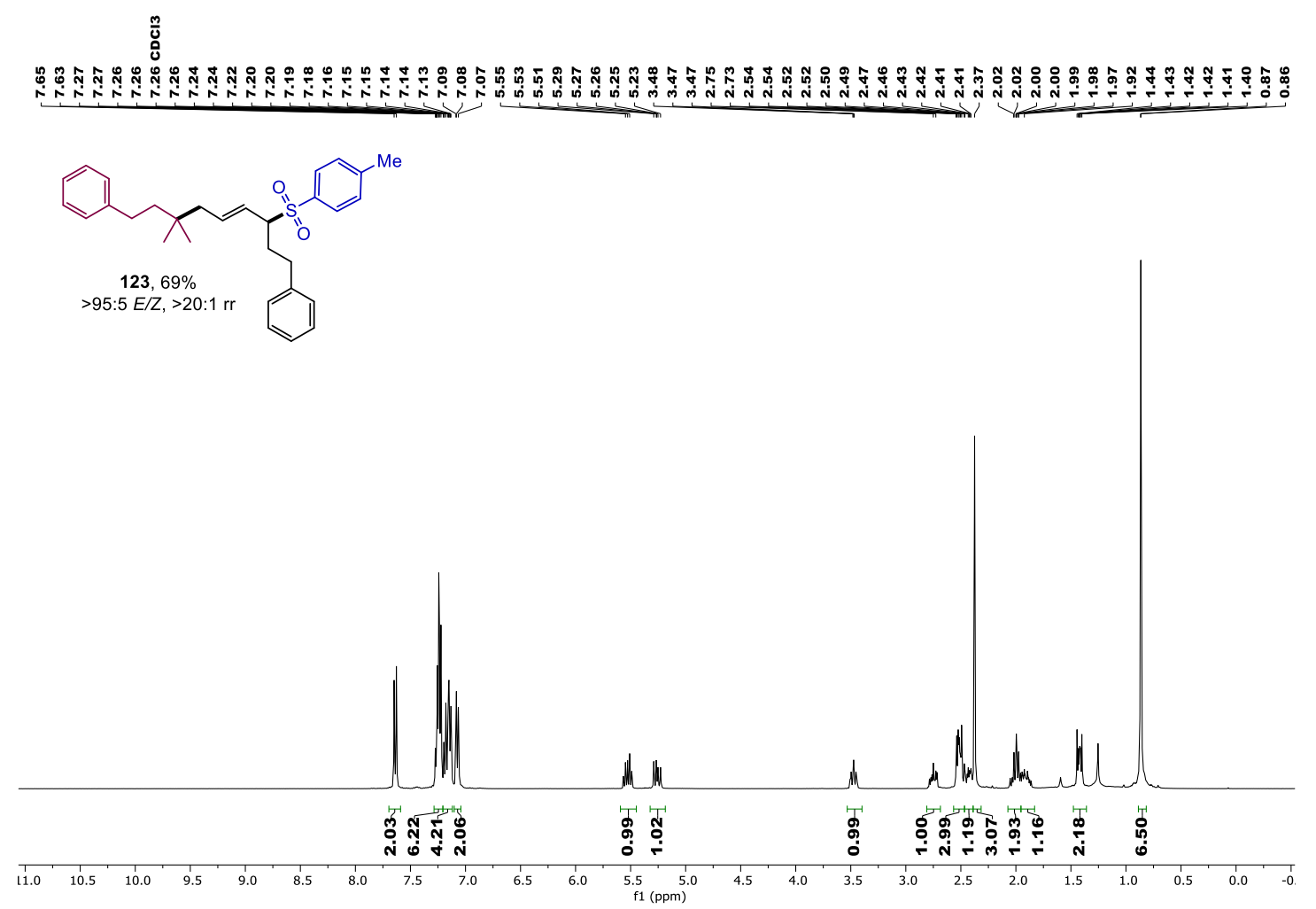

${ }^{13} \mathrm{C}$ NMR $\left(101 \mathrm{MHz}, \mathrm{CDCl}_{3}\right)$
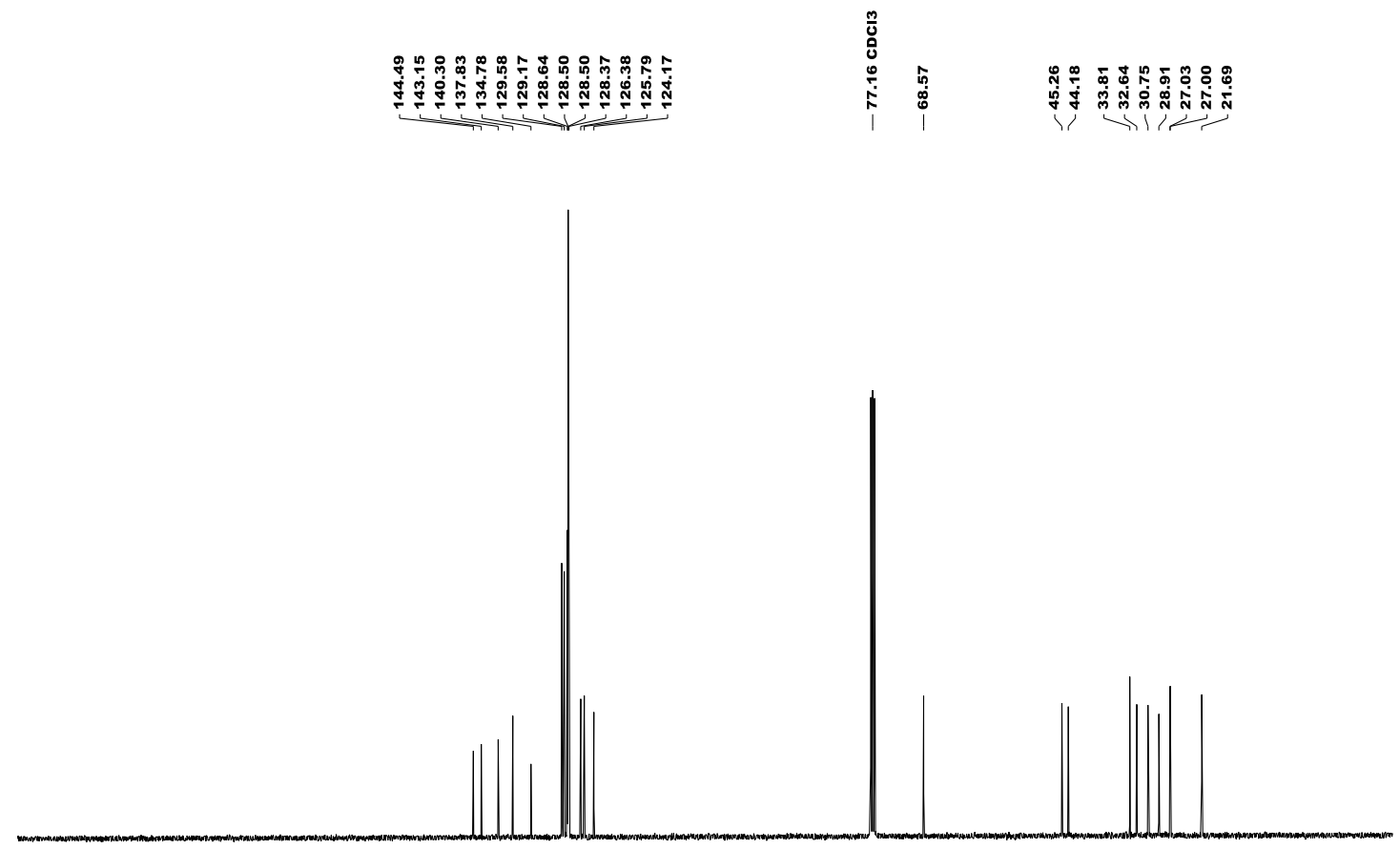

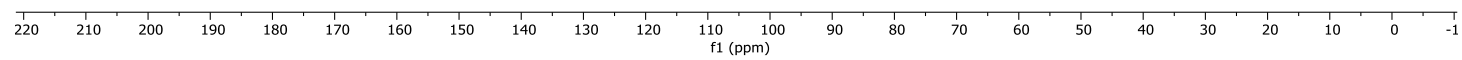


${ }^{1} \mathrm{H}$ NMR $\left(599 \mathrm{MHz}, \mathrm{CDCl}_{3}\right)$

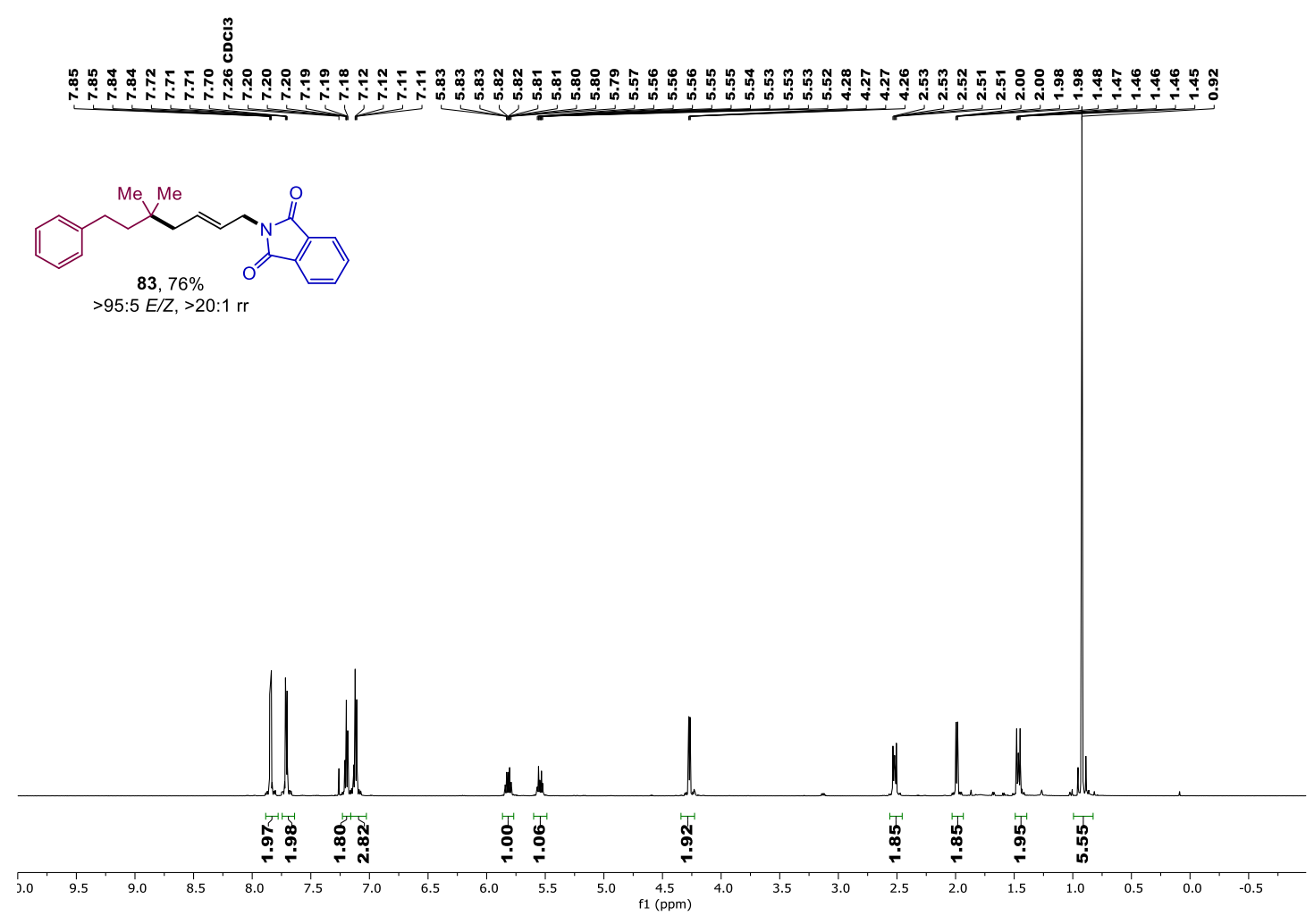

${ }^{13} \mathrm{C}$ NMR $\left(151 \mathrm{MHz}, \mathrm{CDCl}_{3}\right)$
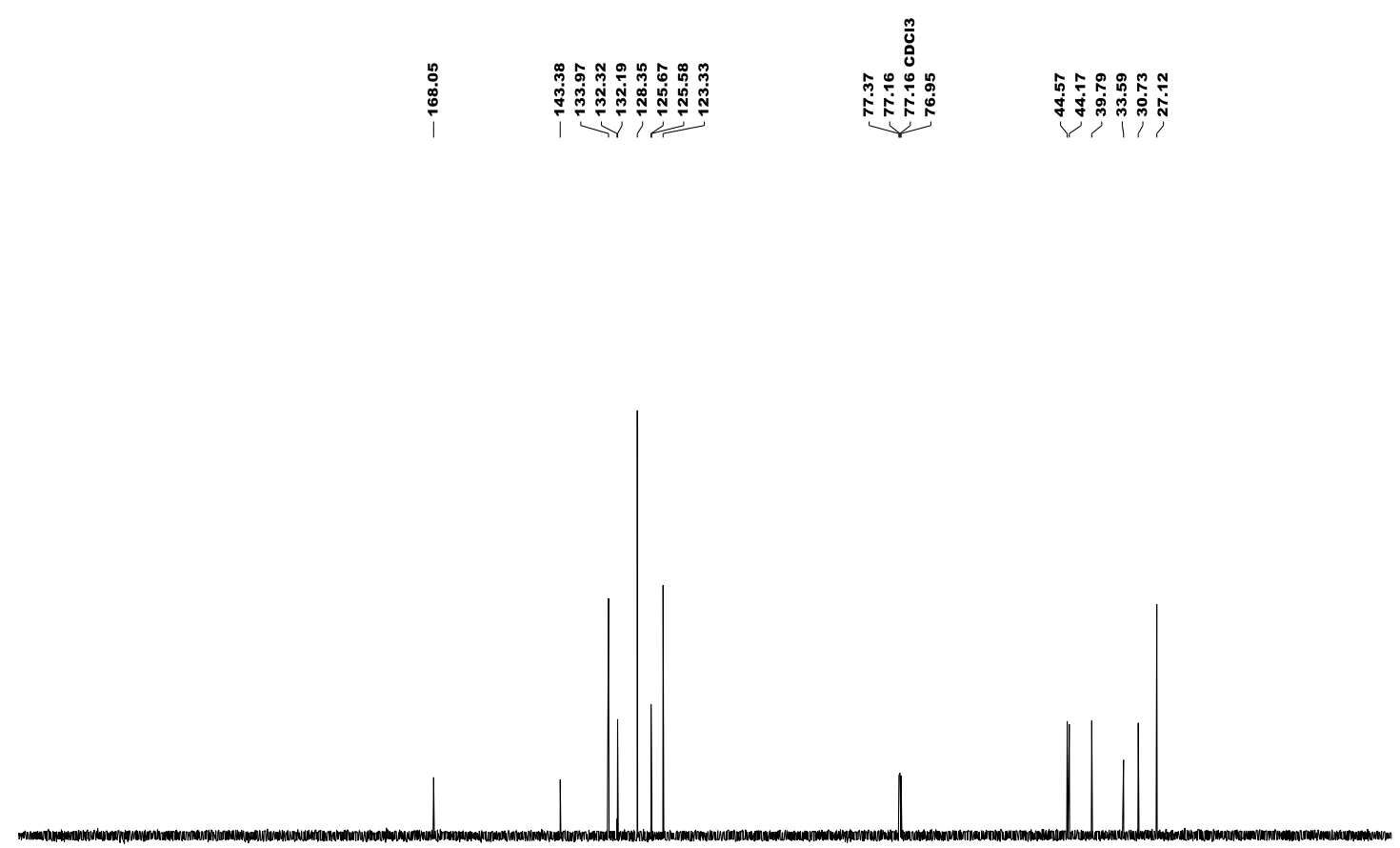

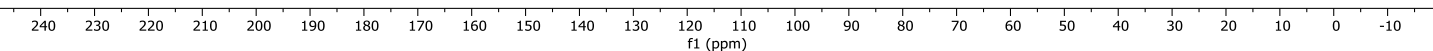


${ }^{1} \mathrm{H}$ NMR $\left(599 \mathrm{MHz}, \mathrm{CDCl}_{3}\right)$

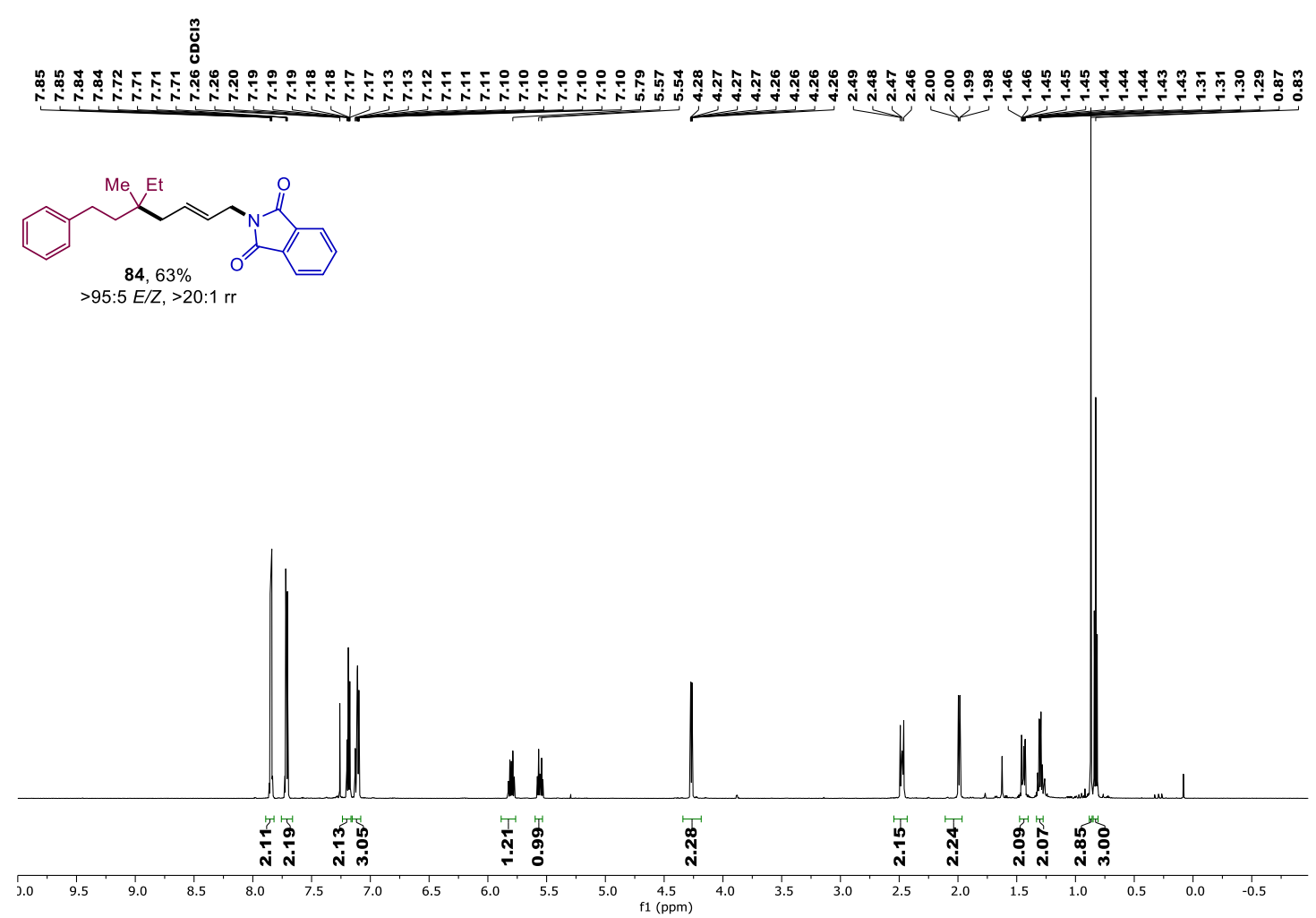

${ }^{13} \mathrm{C}$ NMR (151 MHz, $\left.\mathrm{CDCl}_{3}\right)$
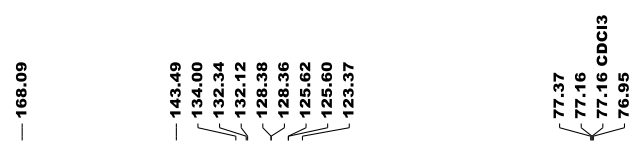

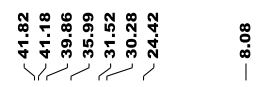

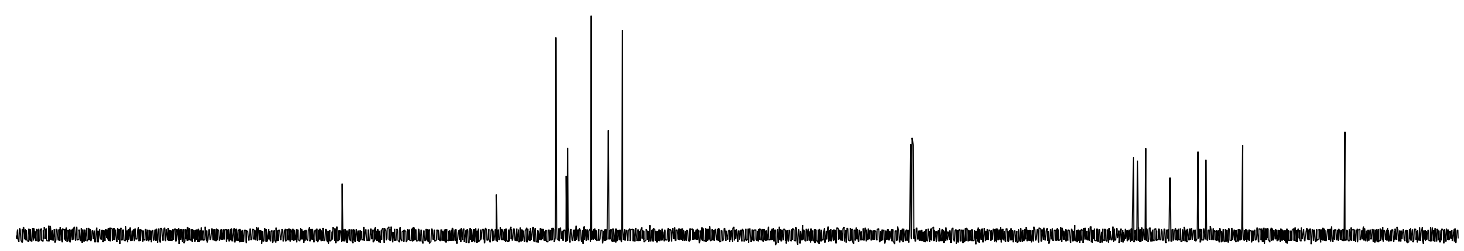

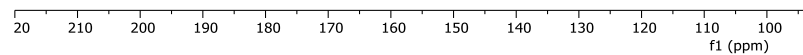


${ }^{1} \mathrm{H} \mathrm{NMR}\left(599 \mathrm{MHz}, \mathrm{CDCl}_{3}\right)$

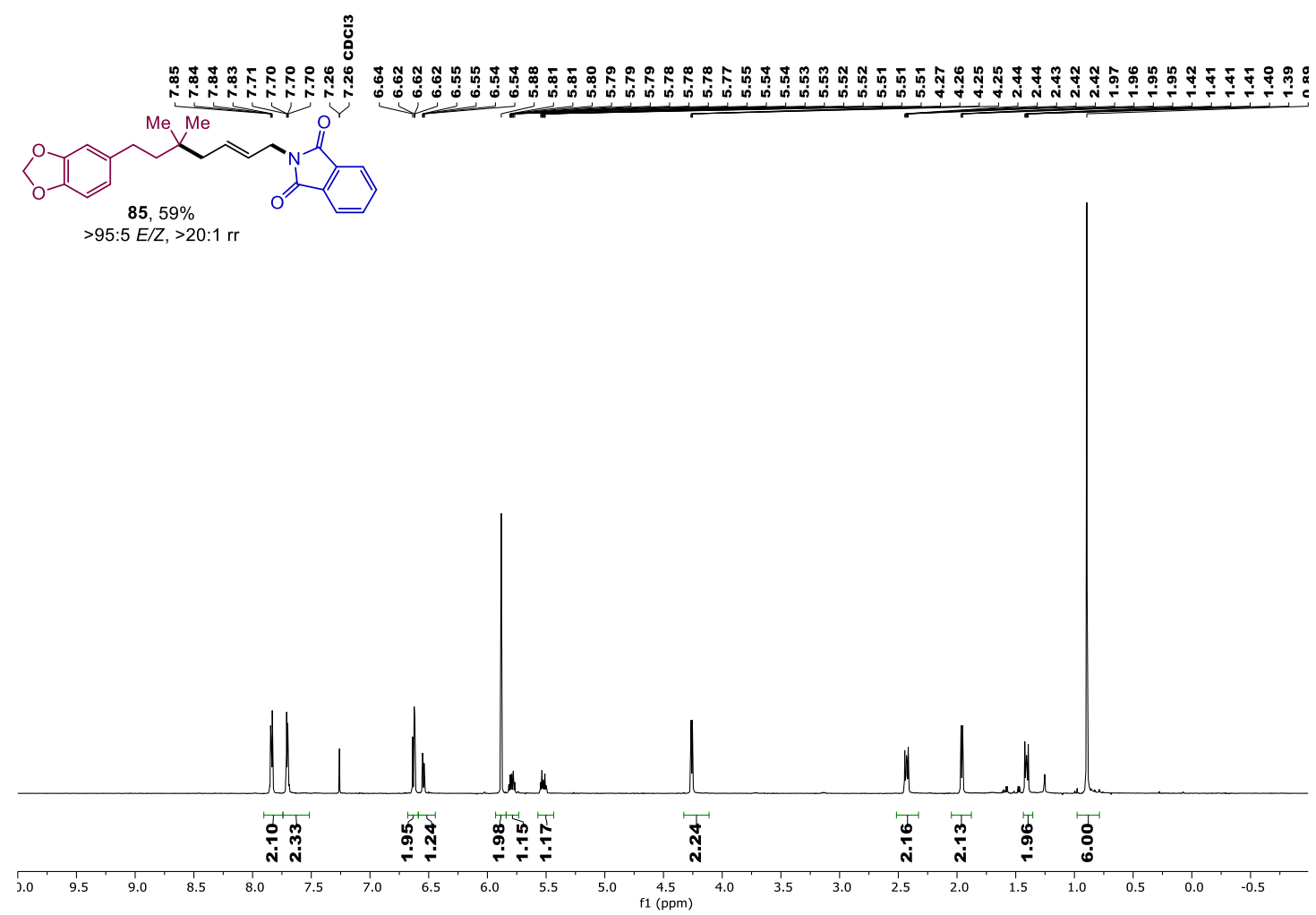

${ }^{13} \mathrm{C}$ NMR (151 MHz, $\mathrm{CDCl}_{3}$ )
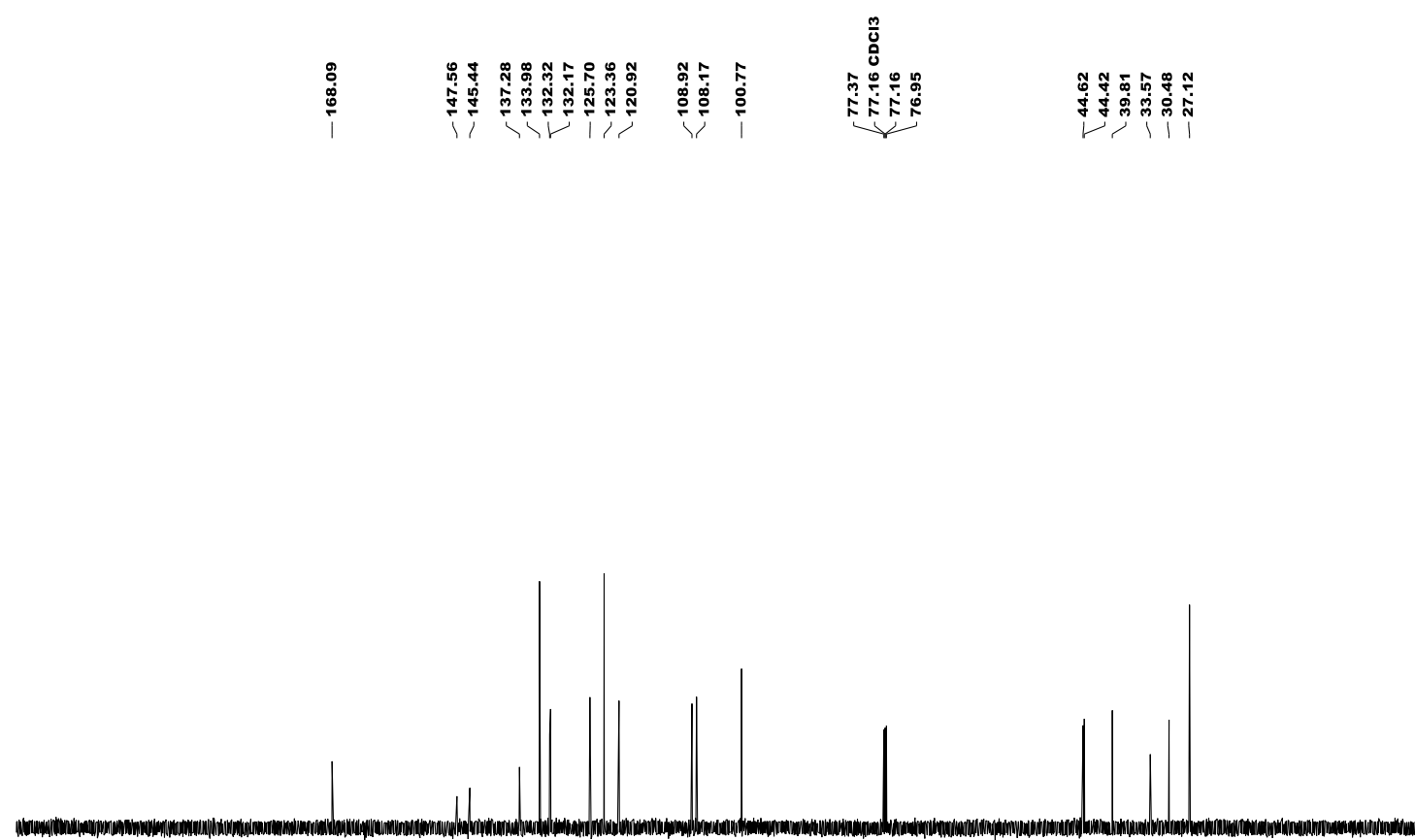

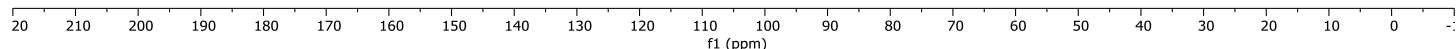


${ }^{1} \mathrm{H}$ NMR (599 MHz, Acetone- $d_{6}$ )

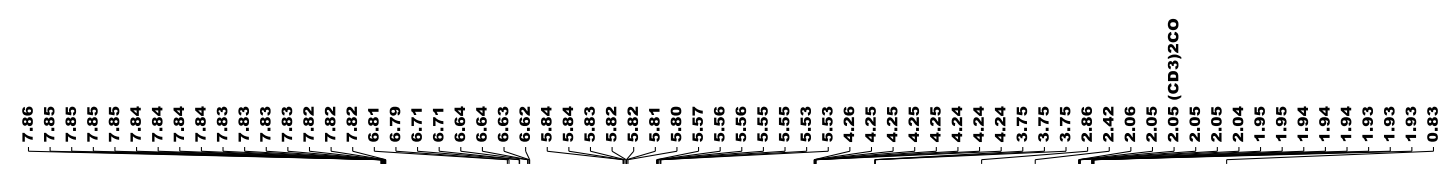

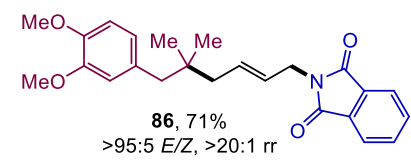

95:5 E/Z, >20:1 rr

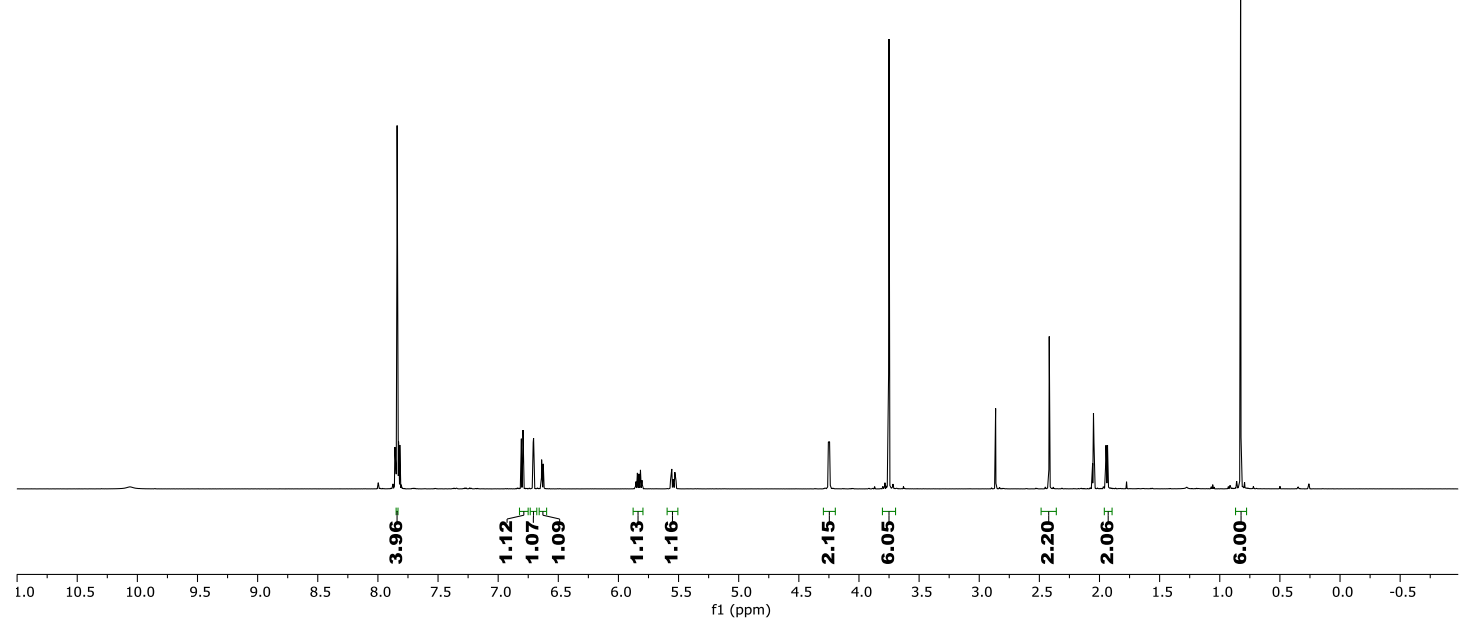

${ }^{13} \mathrm{C}$ NMR (151 MHz, Acetone- $d_{6}$ )
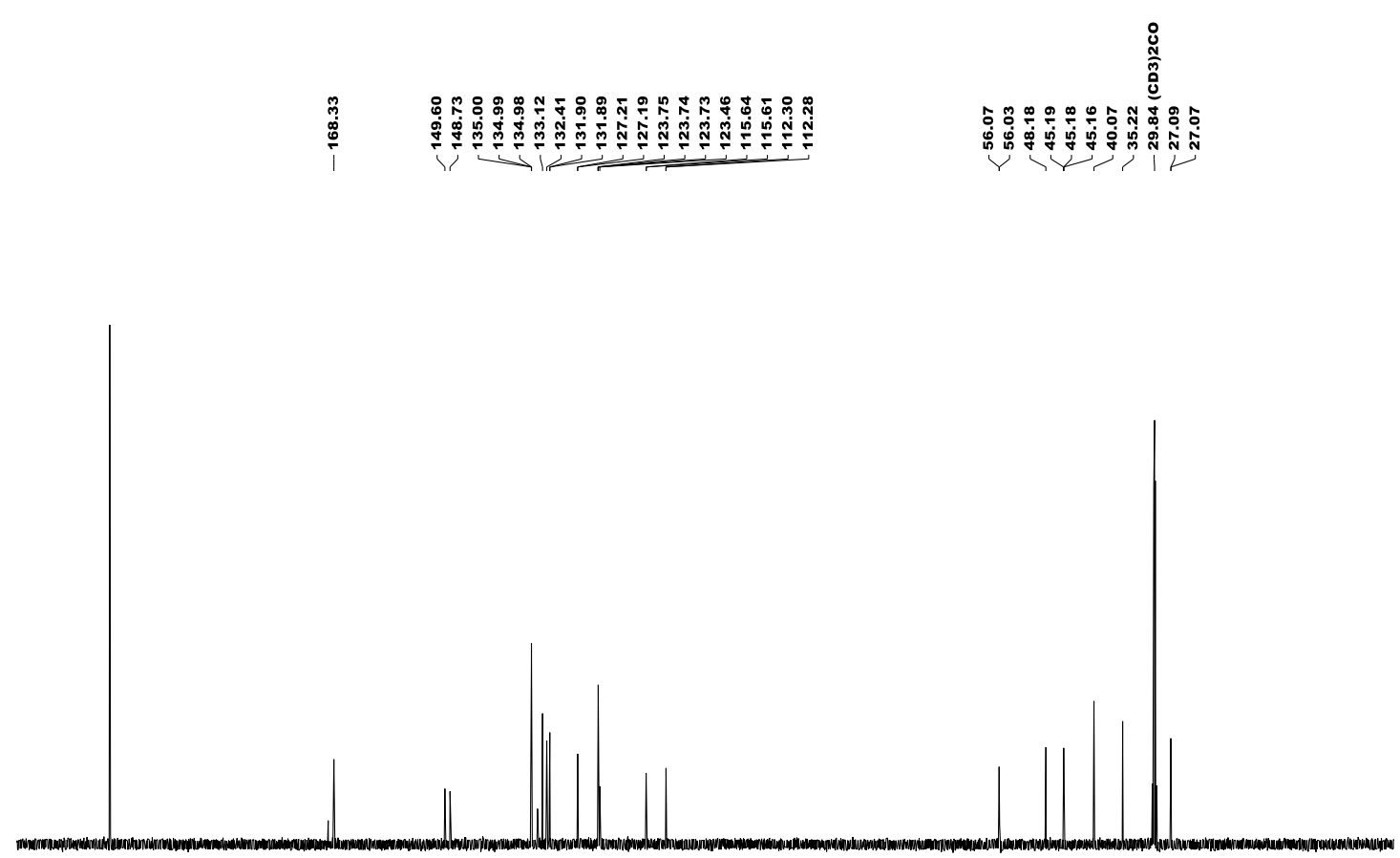

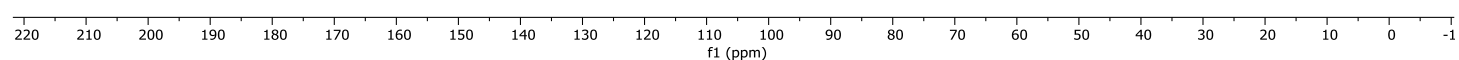


${ }^{1} \mathrm{H} \mathrm{NMR}\left(599 \mathrm{MHz}, \mathrm{CDCl}_{3}\right)$
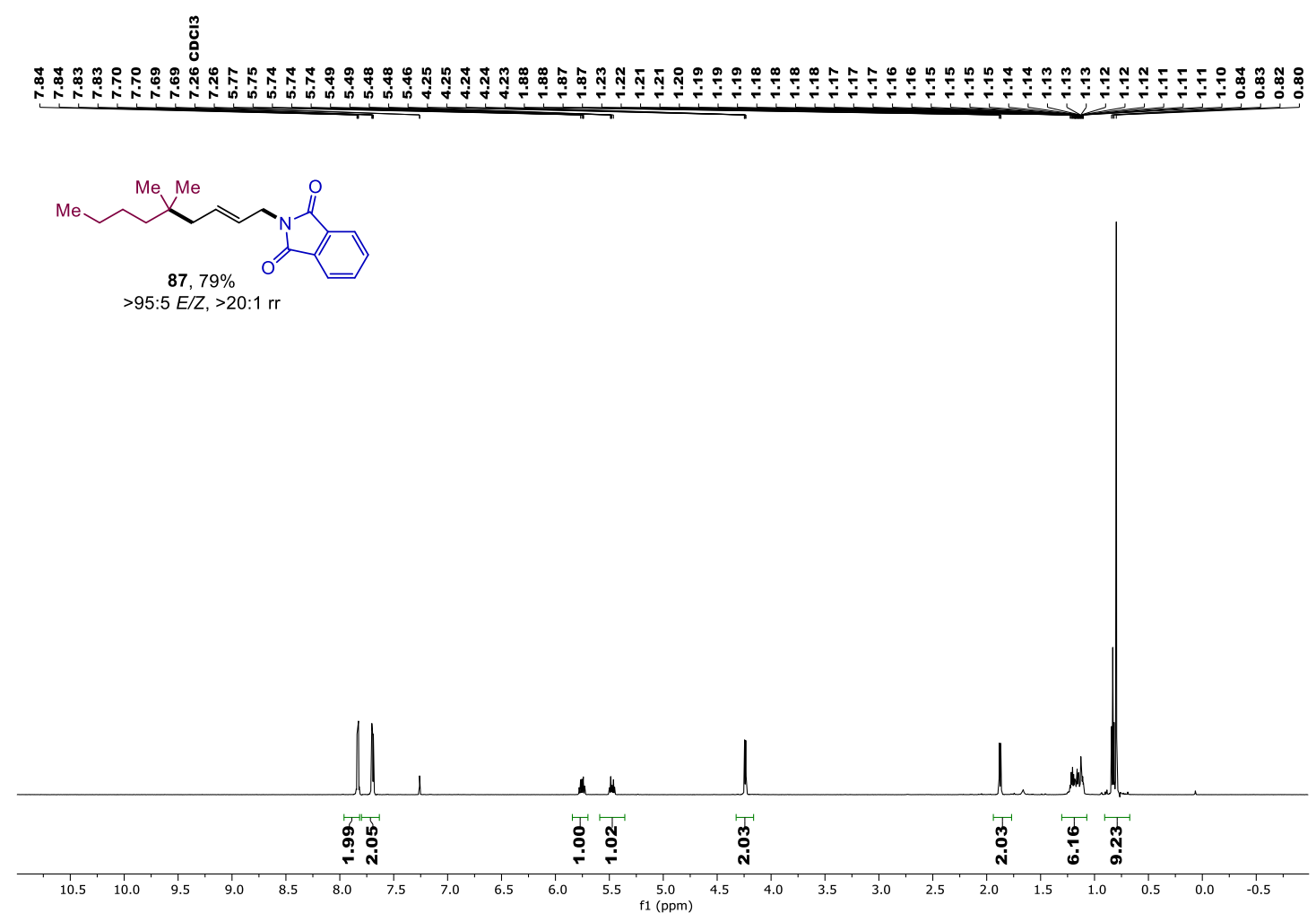

${ }^{13} \mathrm{C}$ NMR (151 MHz, $\mathrm{CDCl}_{3}$ )
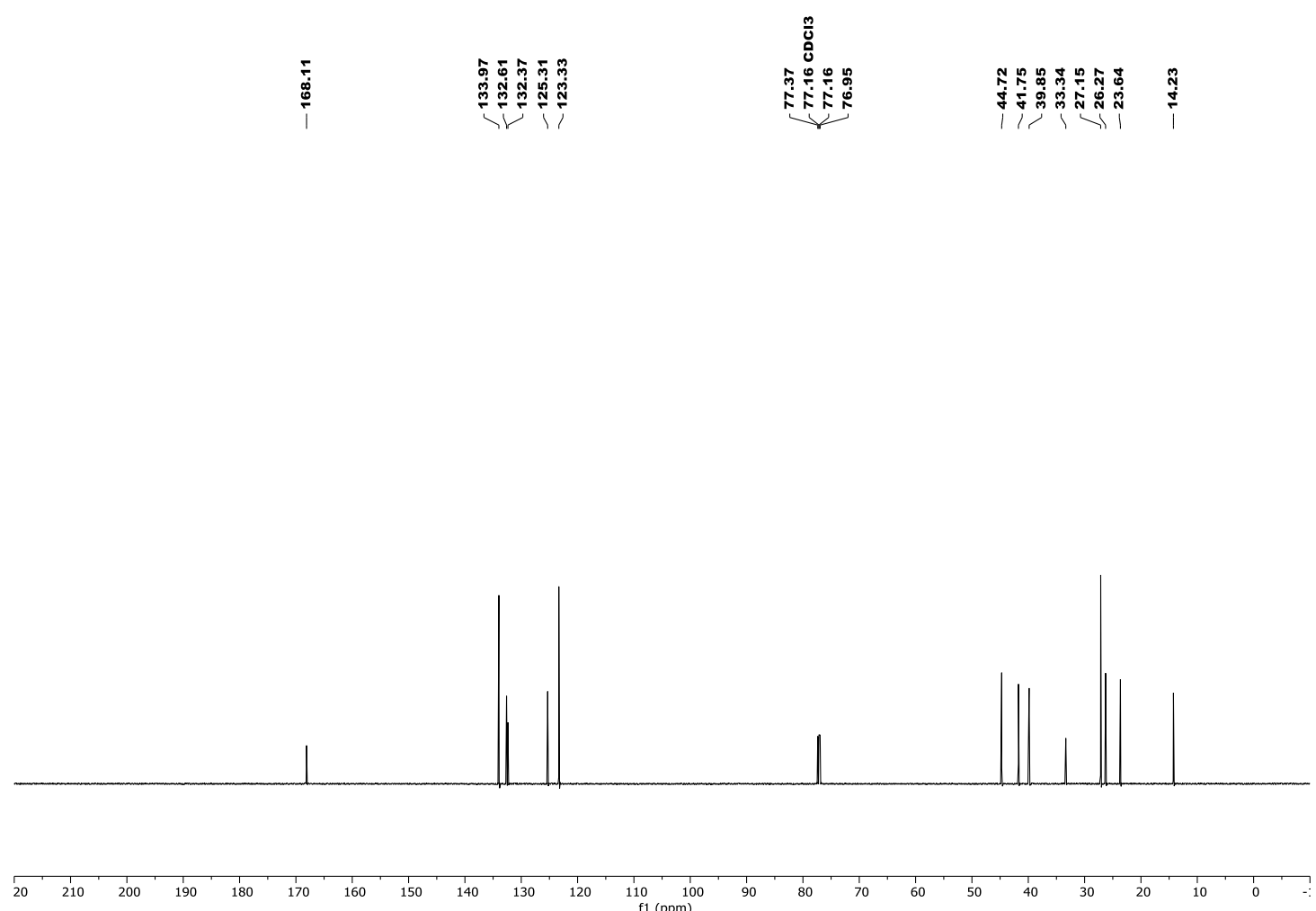
${ }^{1} \mathrm{H}$ NMR $\left(599 \mathrm{MHz}, \mathrm{CDCl}_{3}\right.$ )

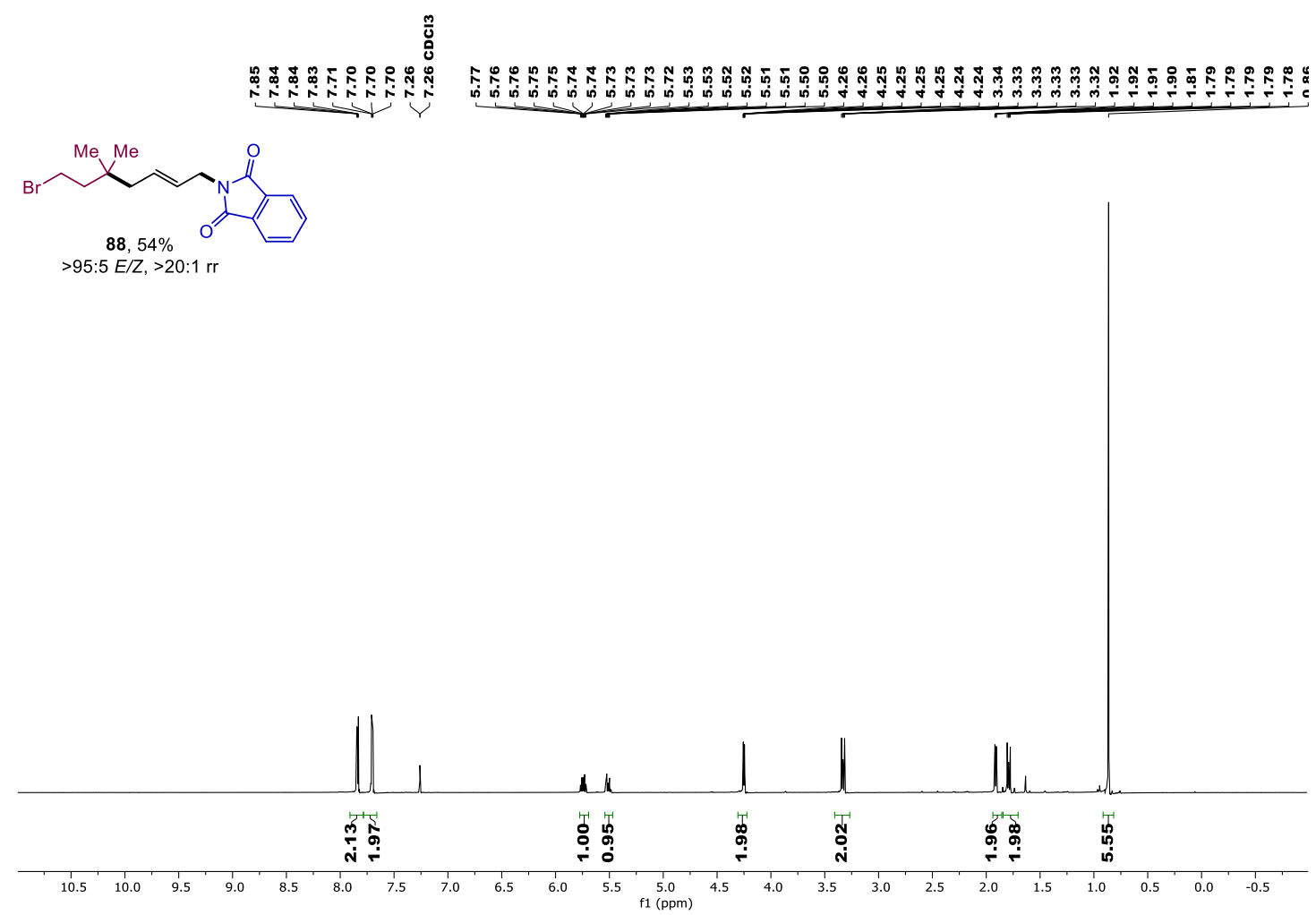

${ }^{13} \mathrm{C}$ NMR (151 MHz, $\mathrm{CDCl}_{3}$ )
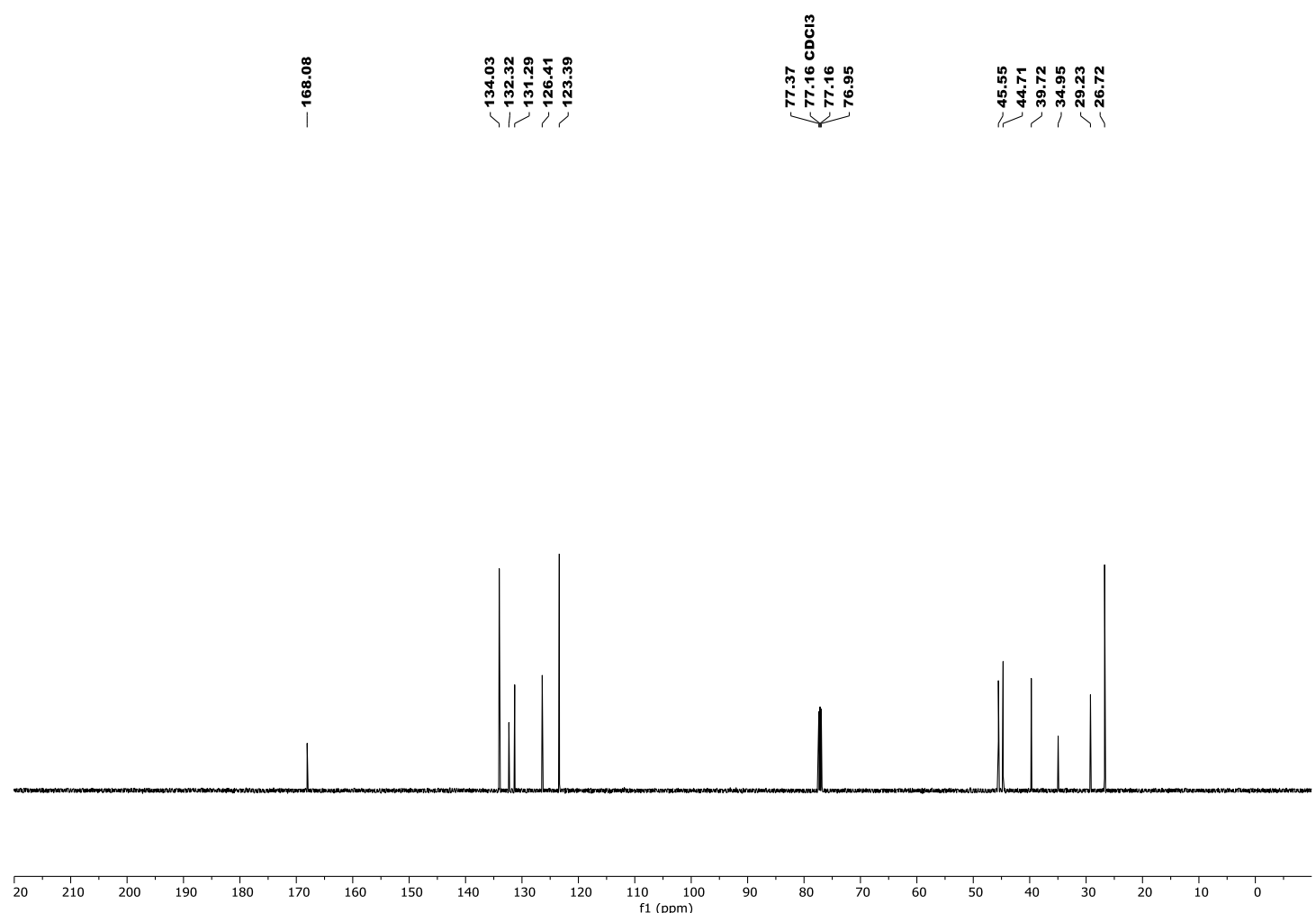
${ }^{1} \mathrm{H} \mathrm{NMR}\left(400 \mathrm{MHz}, \mathrm{CDCl}_{3}\right)$
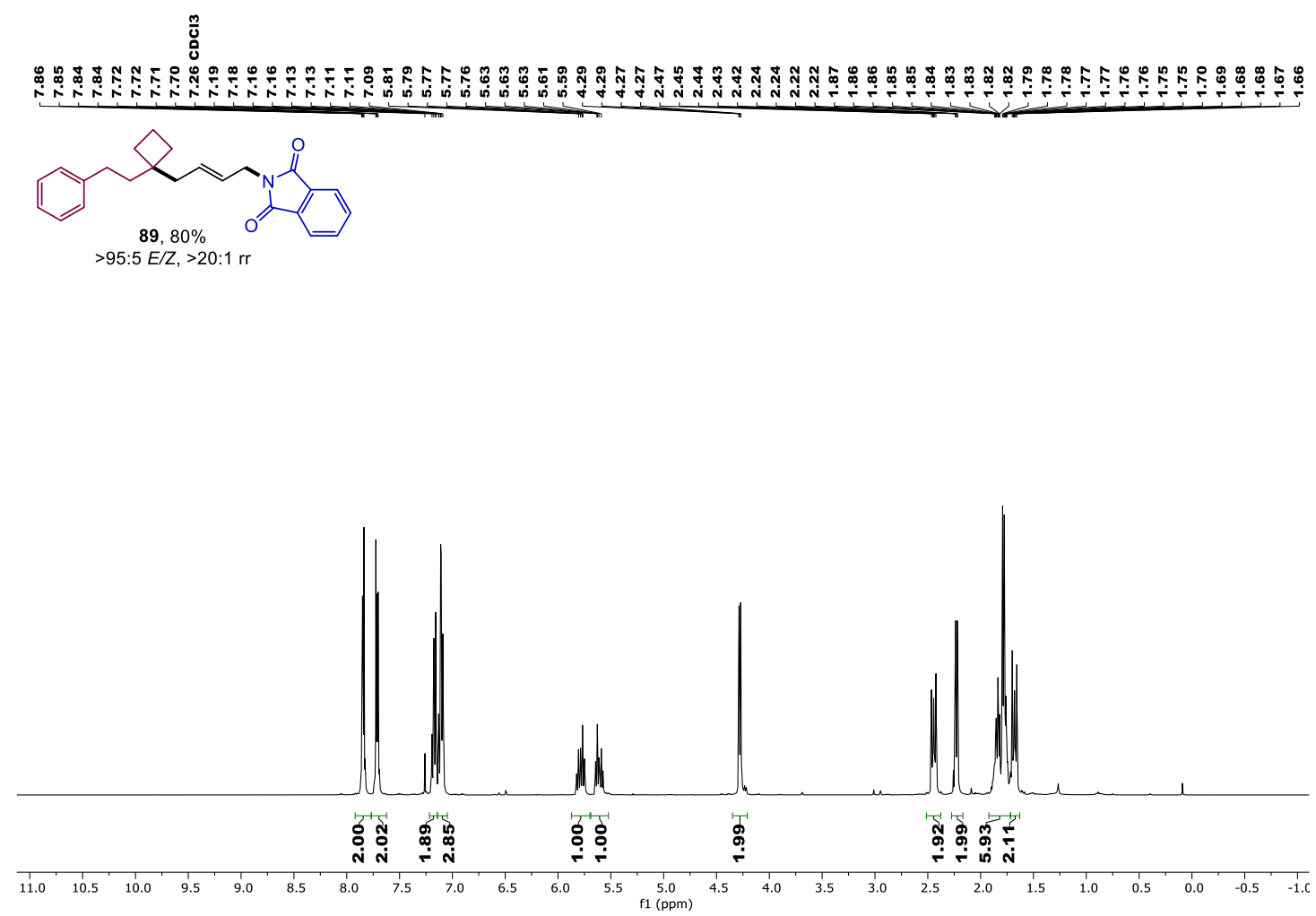

${ }^{13} \mathrm{C}$ NMR $\left(101 \mathrm{MHz}, \mathrm{CDCl}_{3}\right)$

\begin{tabular}{|c|}
\hline 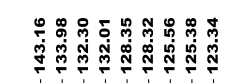 \\
\hline
\end{tabular}

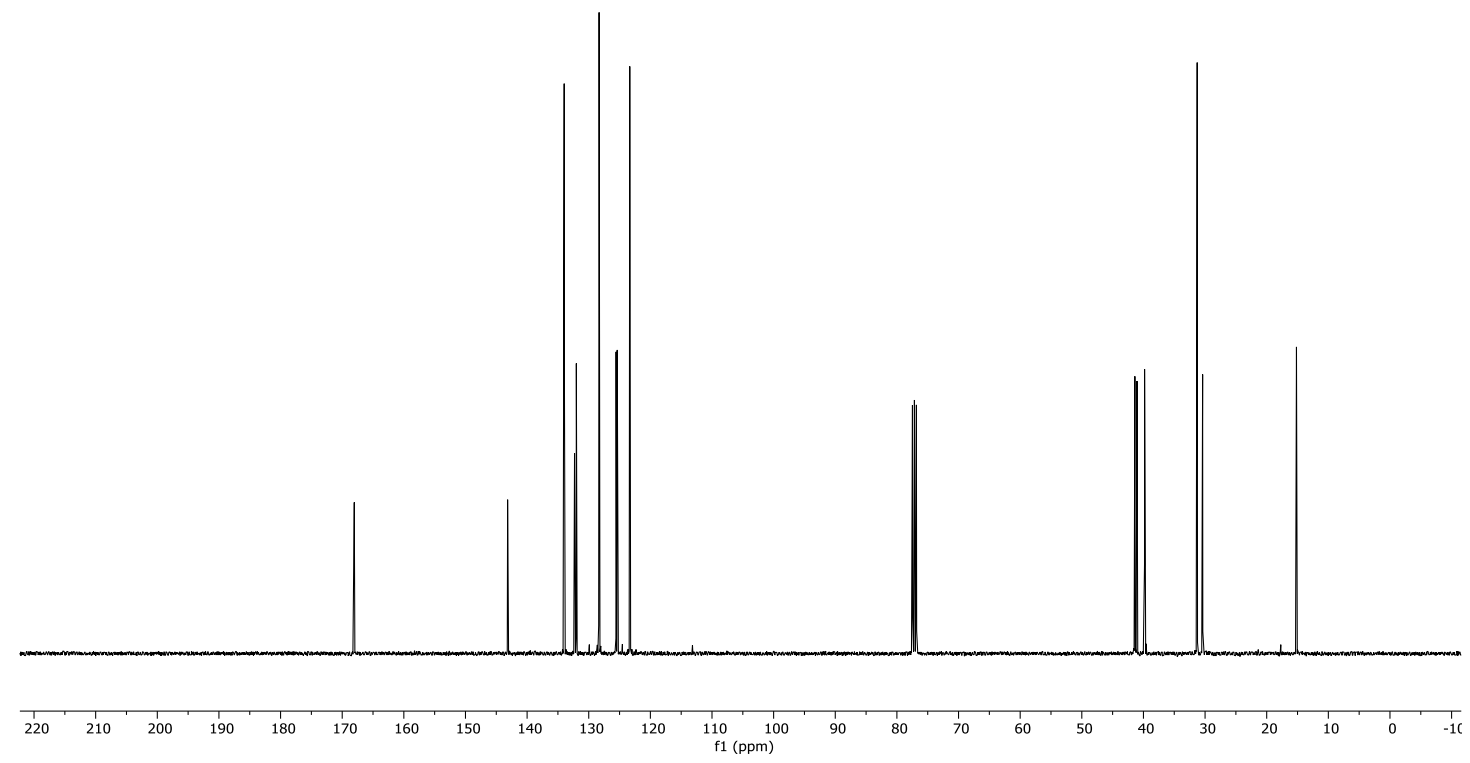


${ }^{1} \mathrm{H}$ NMR $\left(300 \mathrm{MHz}, \mathrm{CDCl}_{3}\right)$
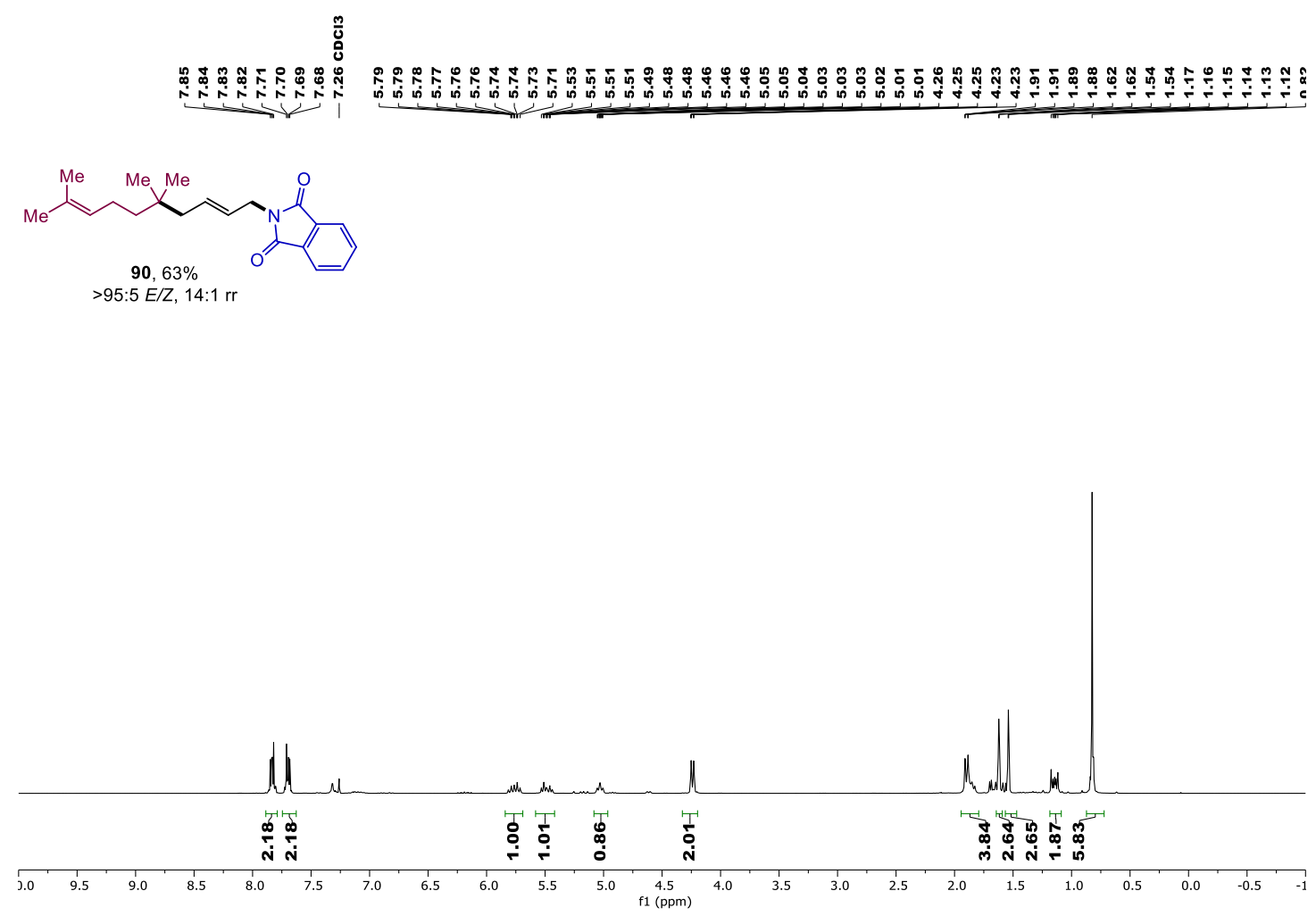

${ }^{13} \mathrm{C}$ NMR $\left(75 \mathrm{MHz}, \mathrm{CDCl}_{3}\right)$
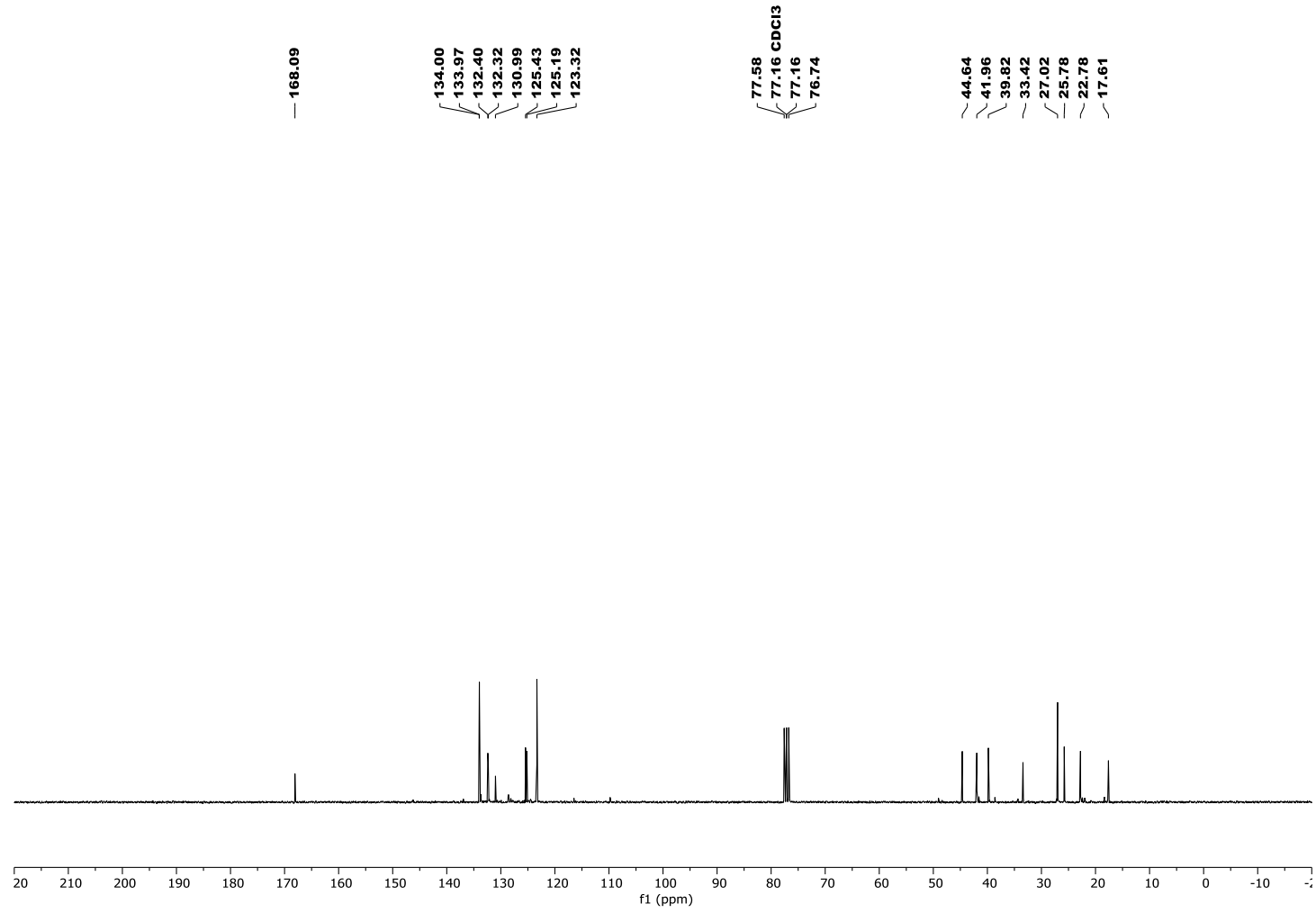
${ }^{1} \mathrm{H}$ NMR $\left(599 \mathrm{MHz}, \mathrm{CDCl}_{3}\right)$
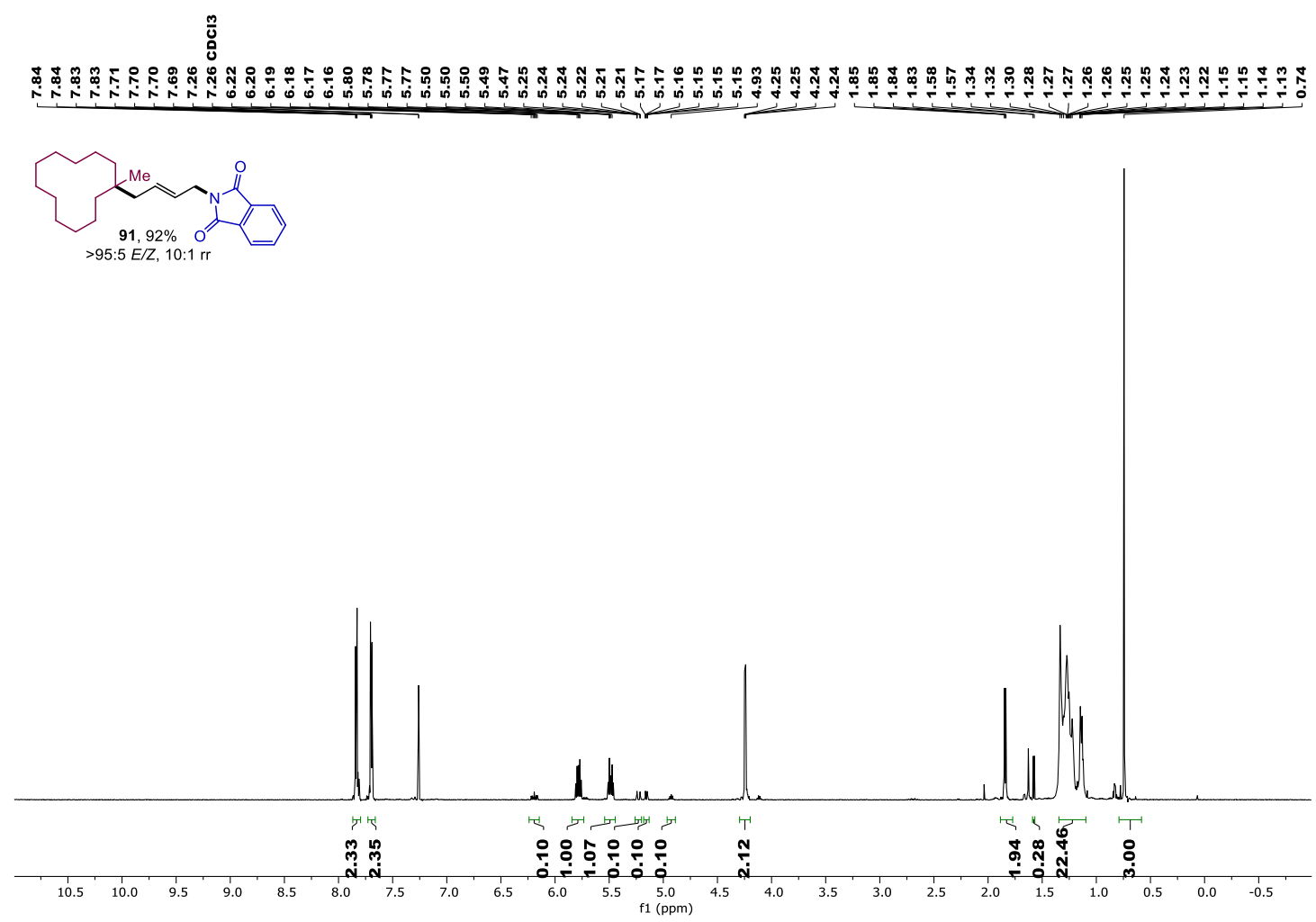

${ }^{13} \mathrm{C} \mathrm{NMR}\left(151 \mathrm{MHz}, \mathrm{CDCl}_{3}\right)$
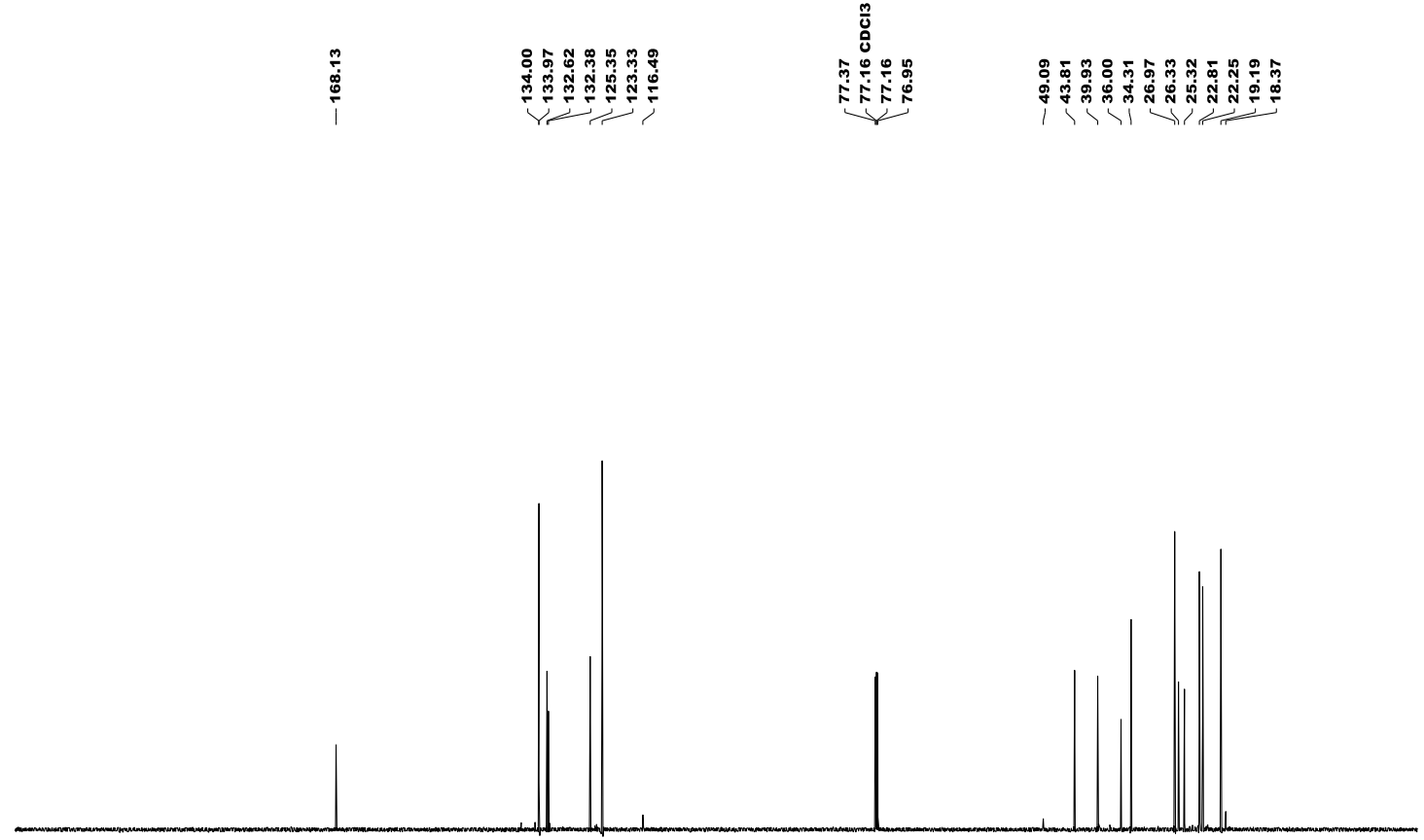

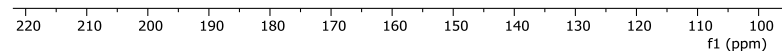


${ }^{1} \mathrm{H} \mathrm{NMR}\left(599 \mathrm{MHz}, \mathrm{CDCl}_{3}\right.$ )
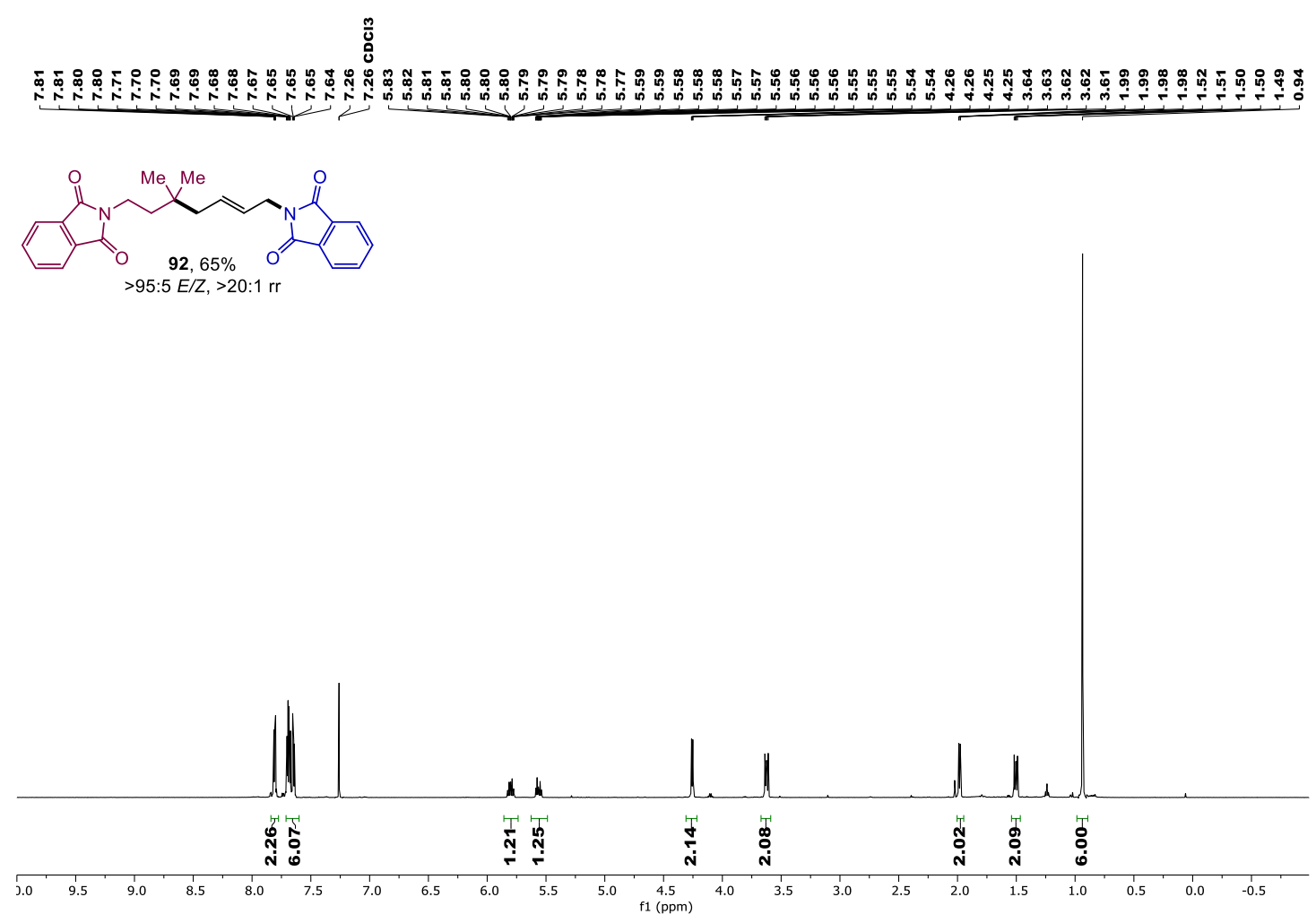

${ }^{13} \mathrm{C} \mathrm{NMR}\left(151 \mathrm{MHz}, \mathrm{CDCl}_{3}\right)$

충ำ
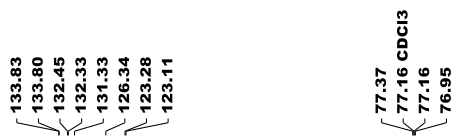

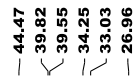

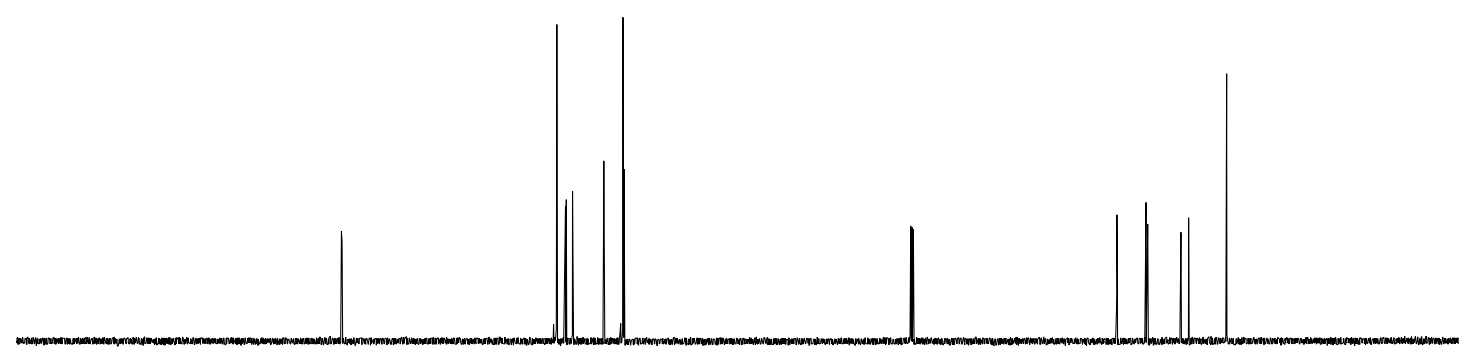

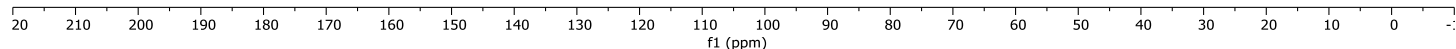


${ }^{1} \mathrm{H} \mathrm{NMR}\left(599 \mathrm{MHz}, \mathrm{CDCl}_{3}\right)$

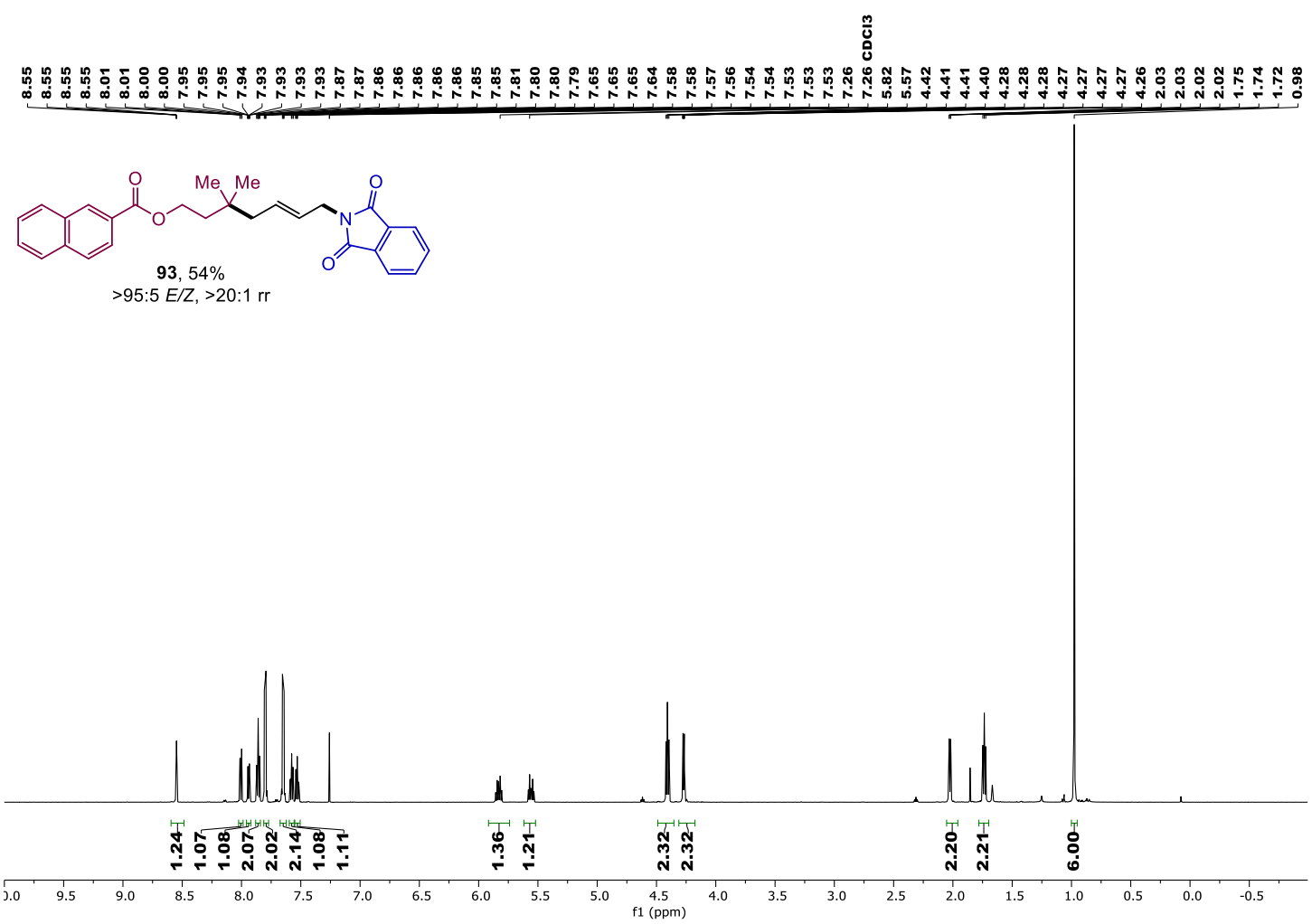

${ }^{13} \mathrm{C}$ NMR $\left(151 \mathrm{MHz}, \mathrm{CDCl}_{3}\right)$
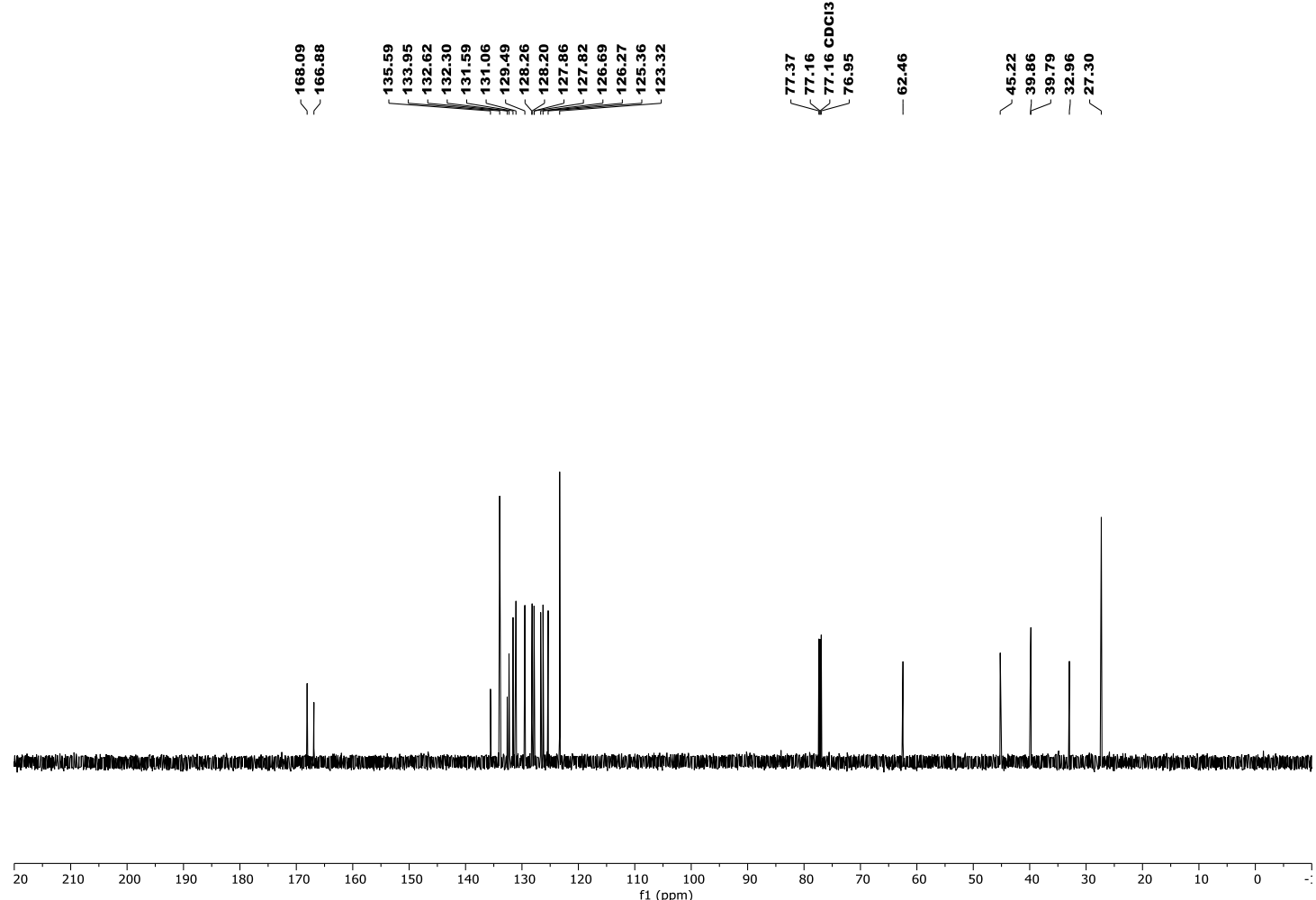
${ }^{1} \mathrm{H}$ NMR $\left(599 \mathrm{MHz}, \mathrm{CDCl}_{3}\right)$
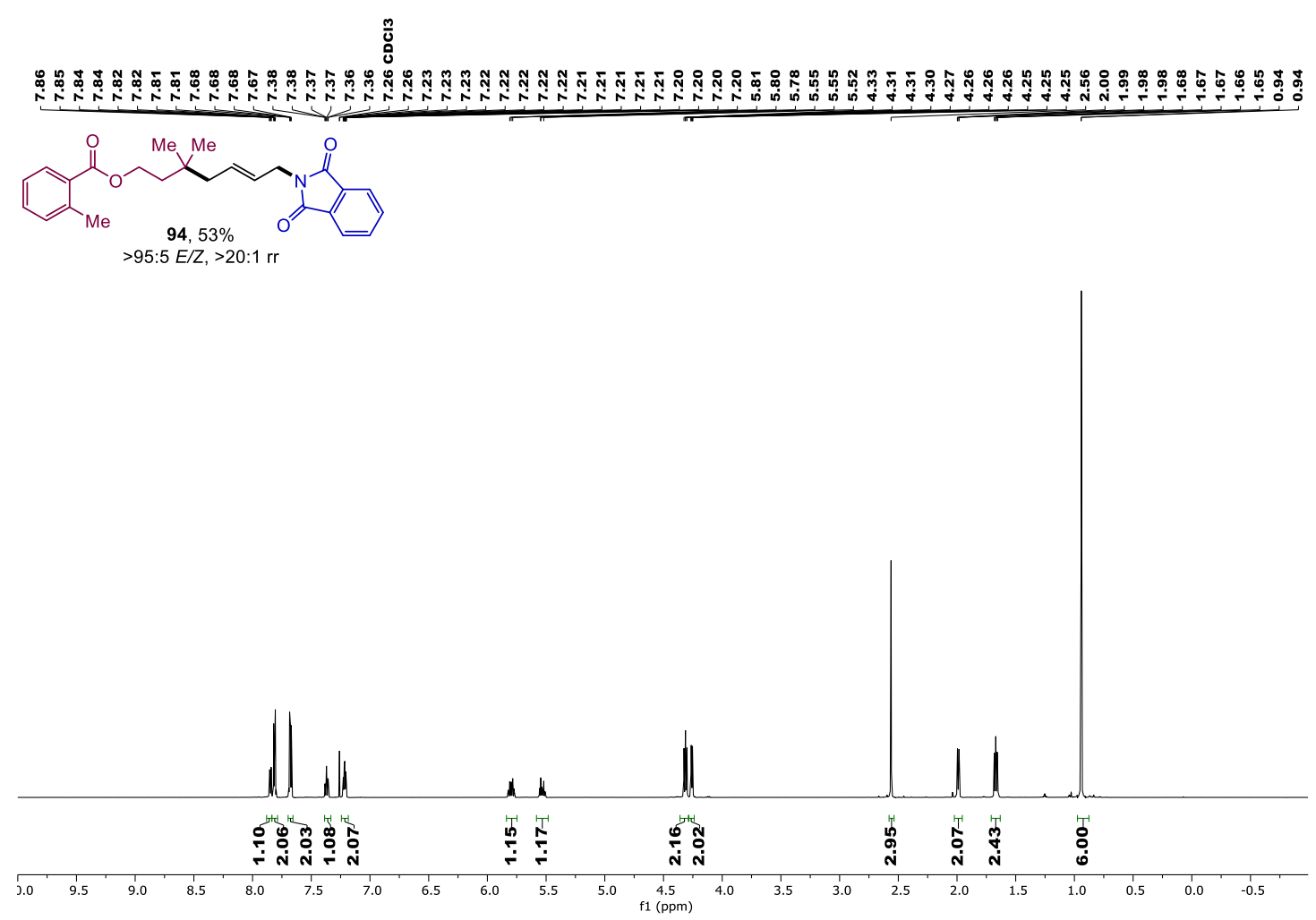

${ }^{13} \mathrm{C}$ NMR (151 MHz, $\mathrm{CDCl}_{3}$ )
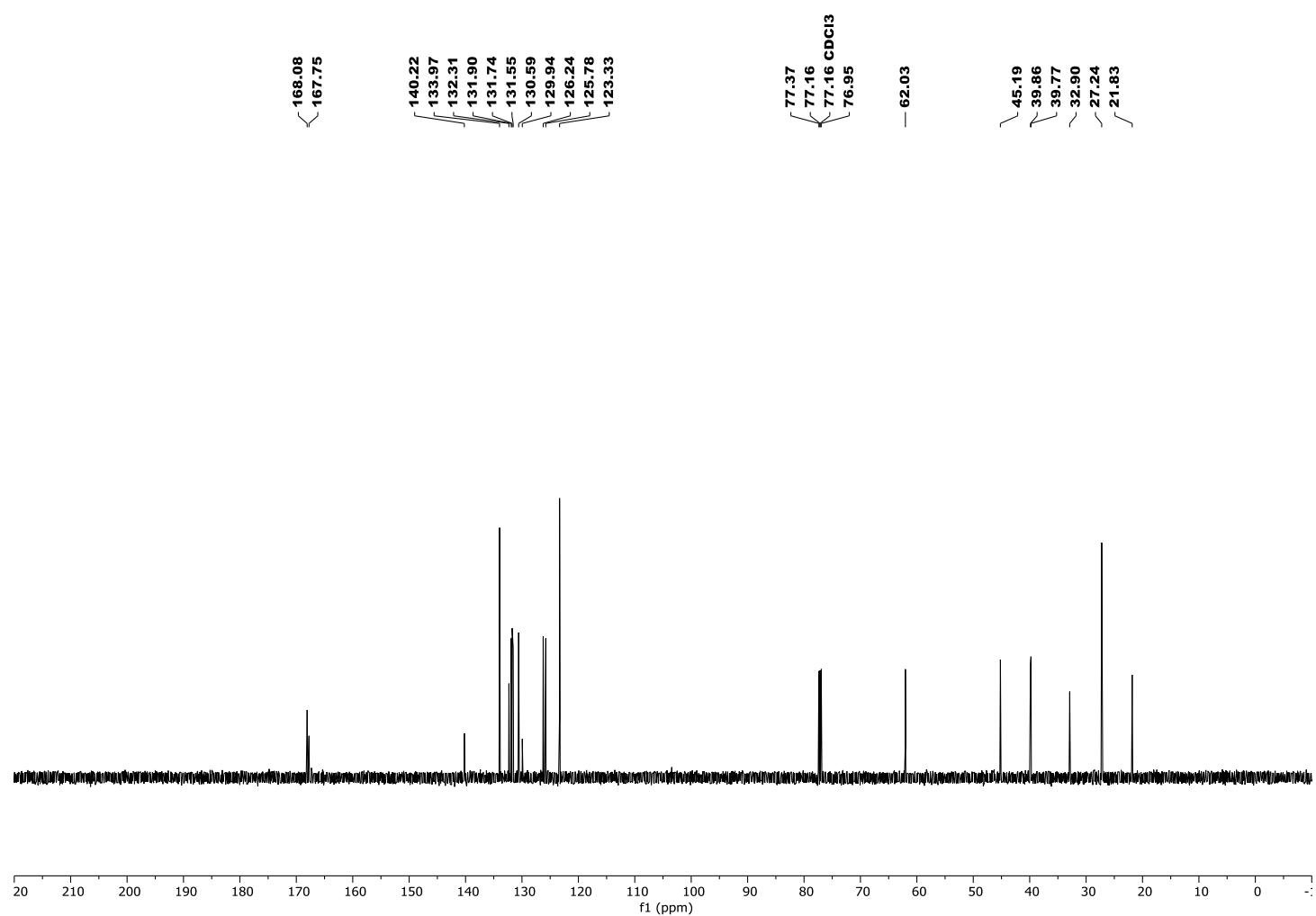
${ }^{1} \mathrm{H} \mathrm{NMR}\left(400 \mathrm{MHz}, \mathrm{CDCl}_{3}\right)$
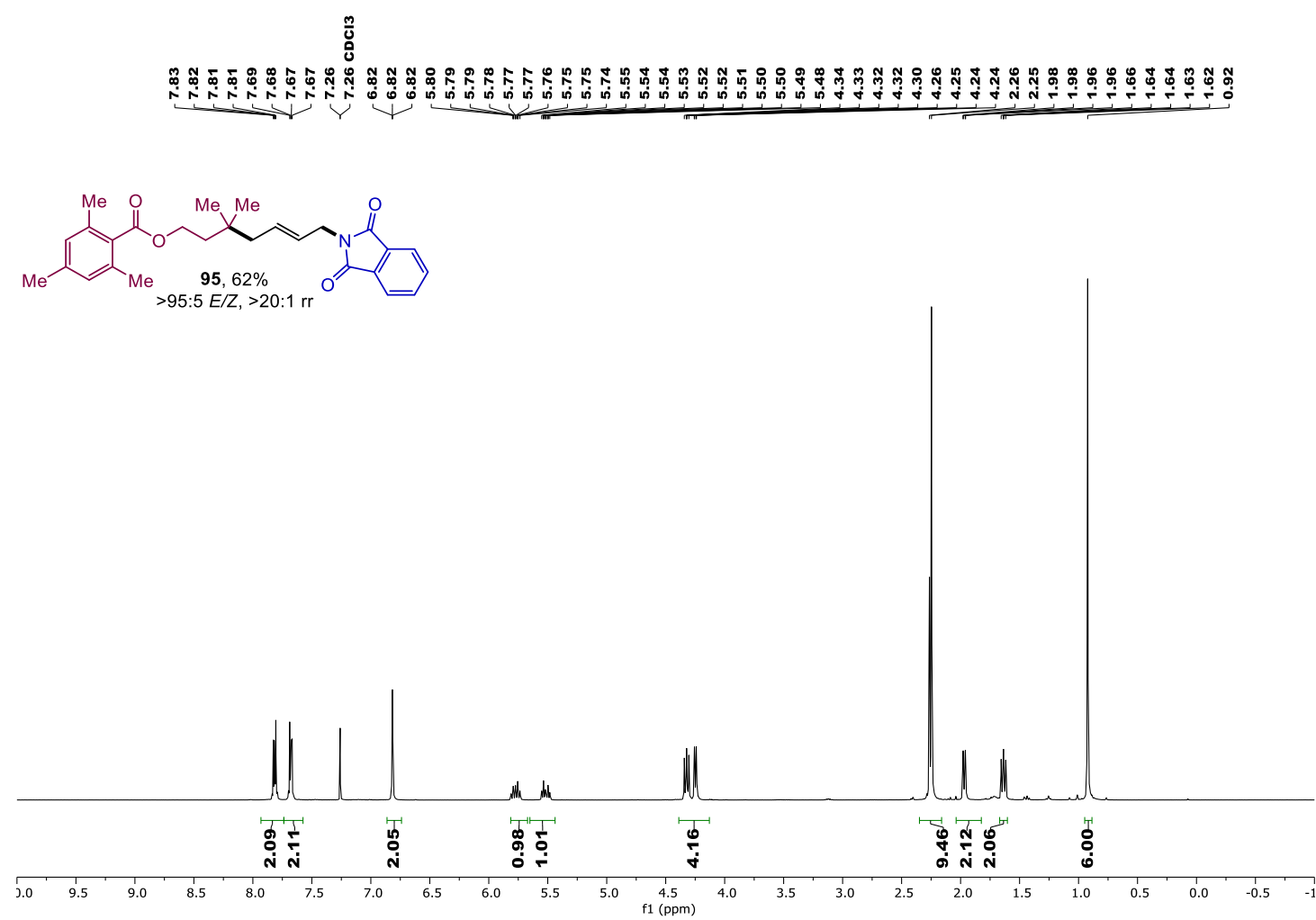

${ }^{13} \mathrm{C}$ NMR (101 MHz, $\mathrm{CDCl}_{3}$ )
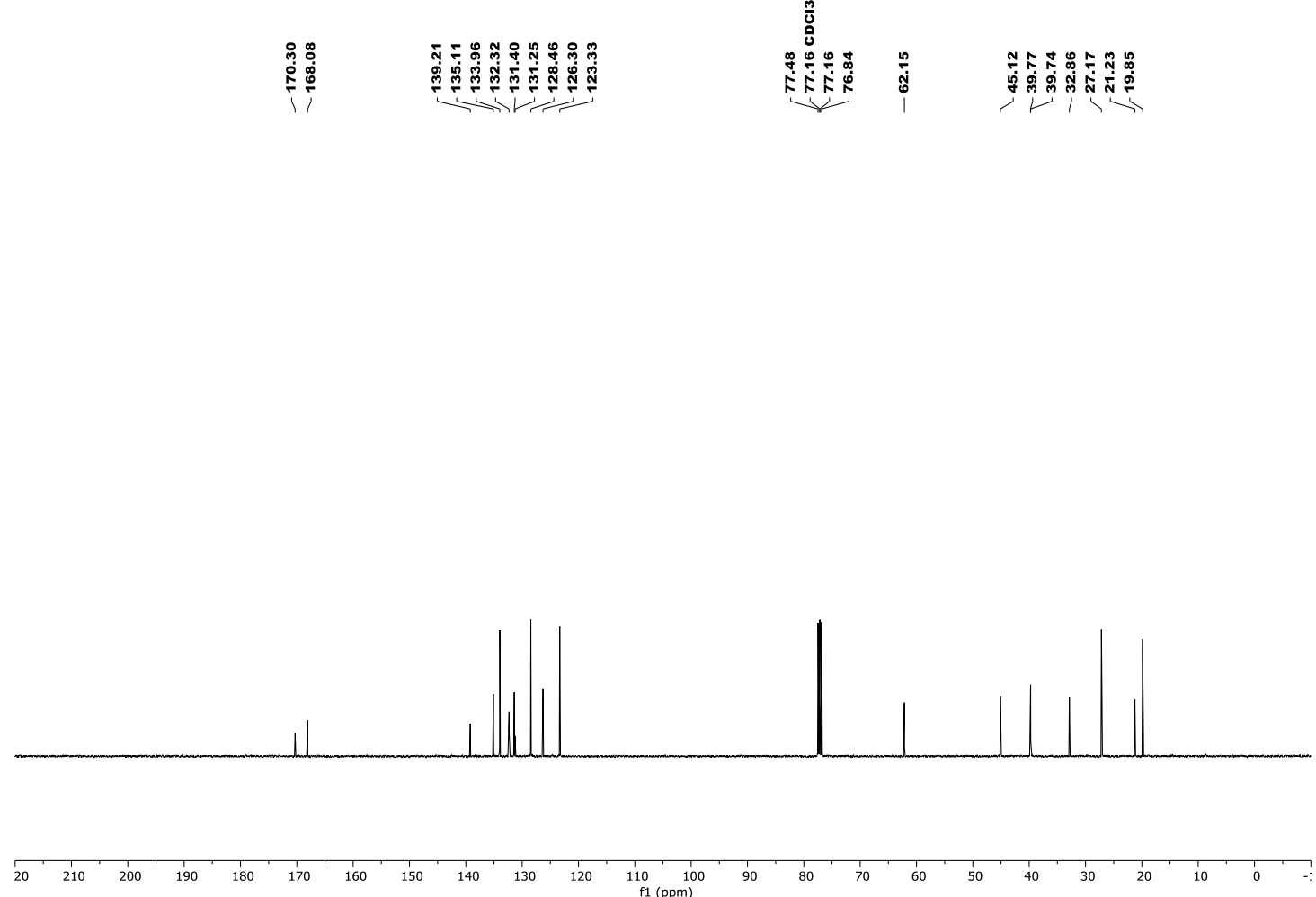
${ }^{1} \mathrm{H}$ NMR $\left(599 \mathrm{MHz}, \mathrm{CDCl}_{3}\right)$

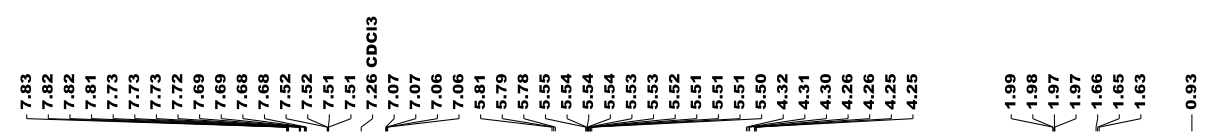
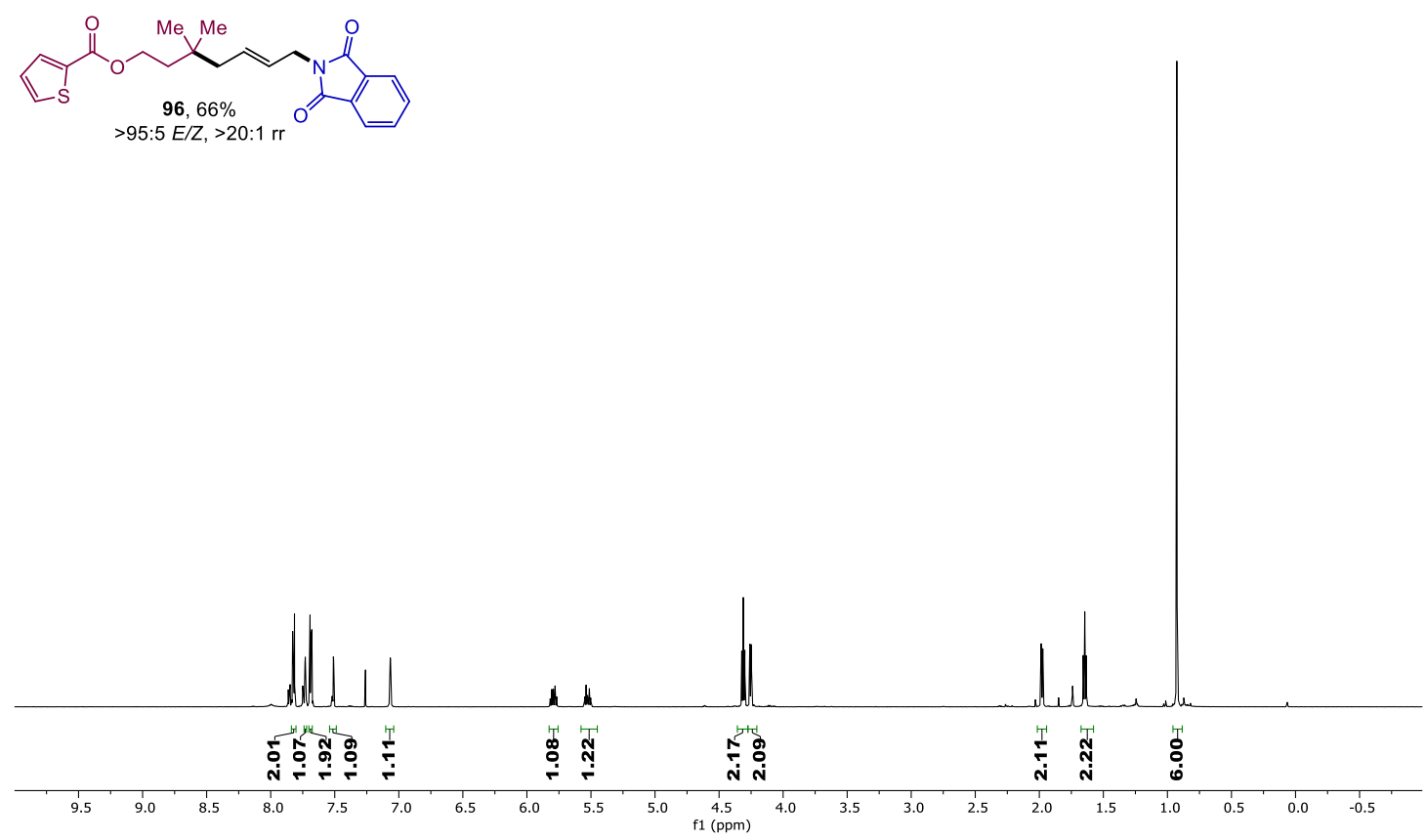

${ }^{13} \mathrm{C}$ NMR (151 MHz, $\left.\mathrm{CDCl}_{3}\right)$
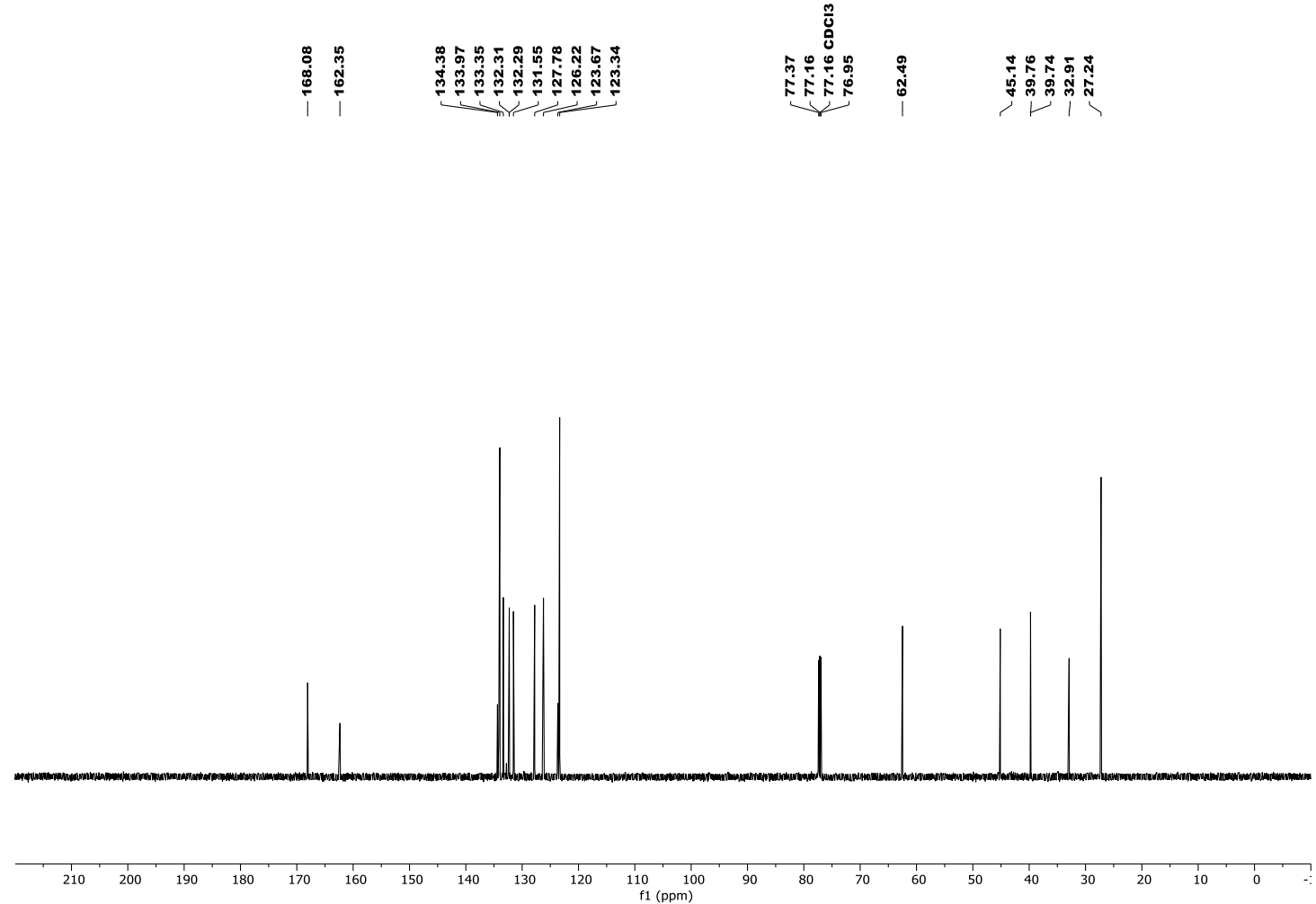
${ }^{1} \mathrm{H}$ NMR $\left(599 \mathrm{MHz}, \mathrm{CDCl}_{3}\right)$

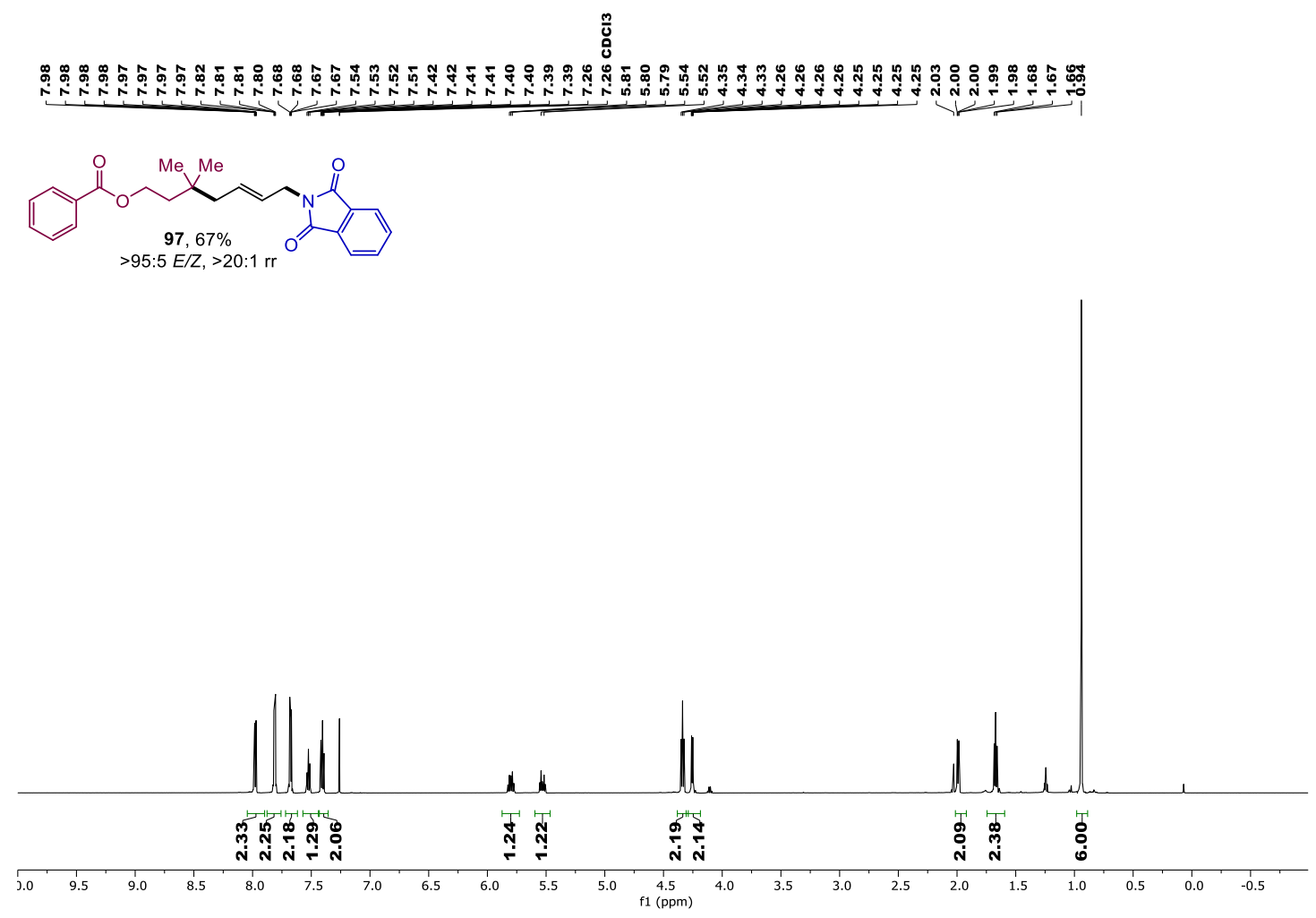

${ }^{13} \mathrm{C}$ NMR $\left(151 \mathrm{MHz}, \mathrm{CDCl}_{3}\right)$
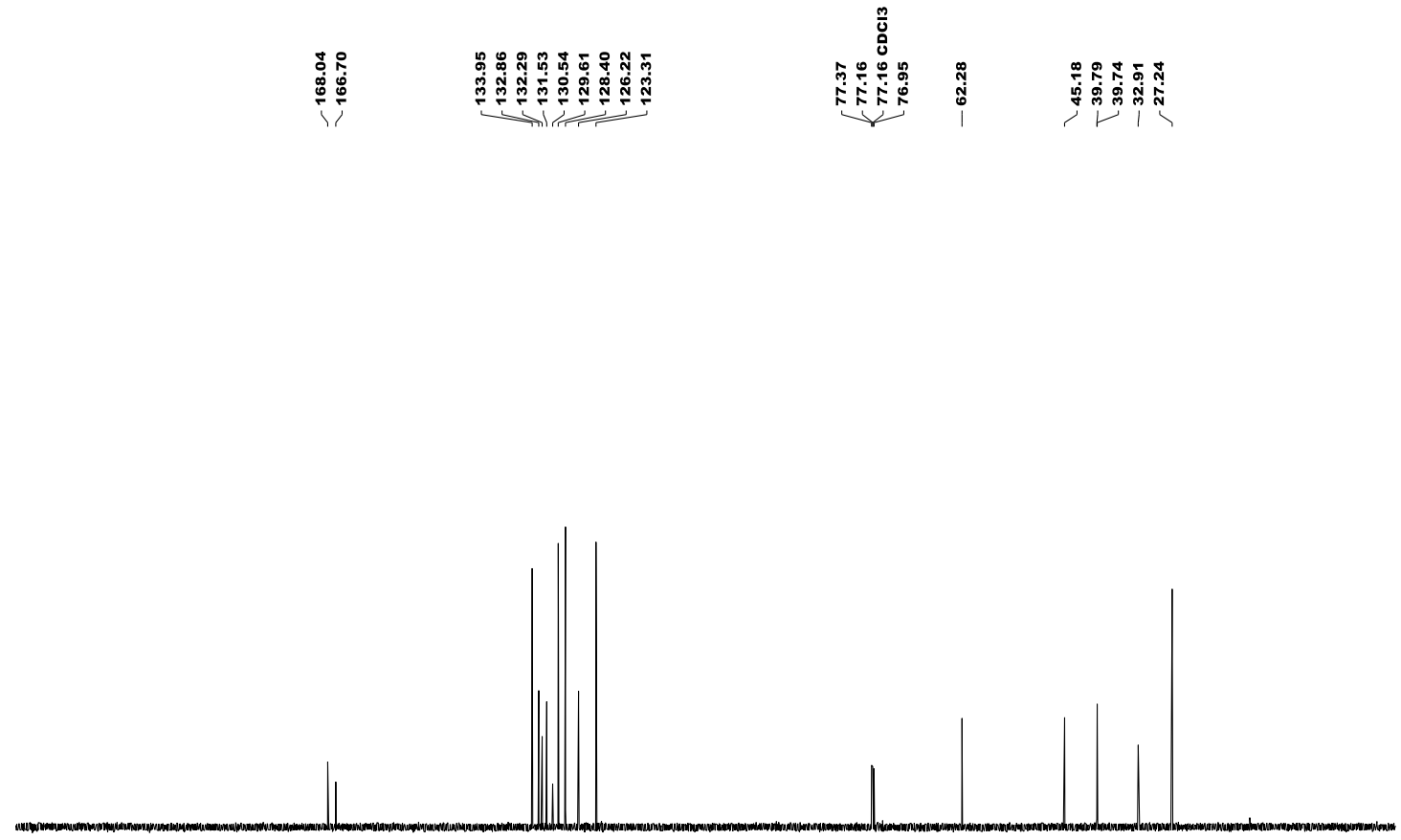

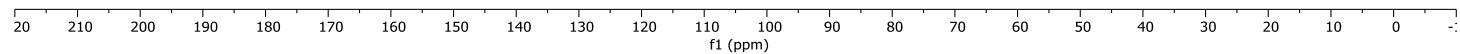


${ }^{1} \mathrm{H} \mathrm{NMR}\left(599 \mathrm{MHz}, \mathrm{CDCl}_{3}\right)$
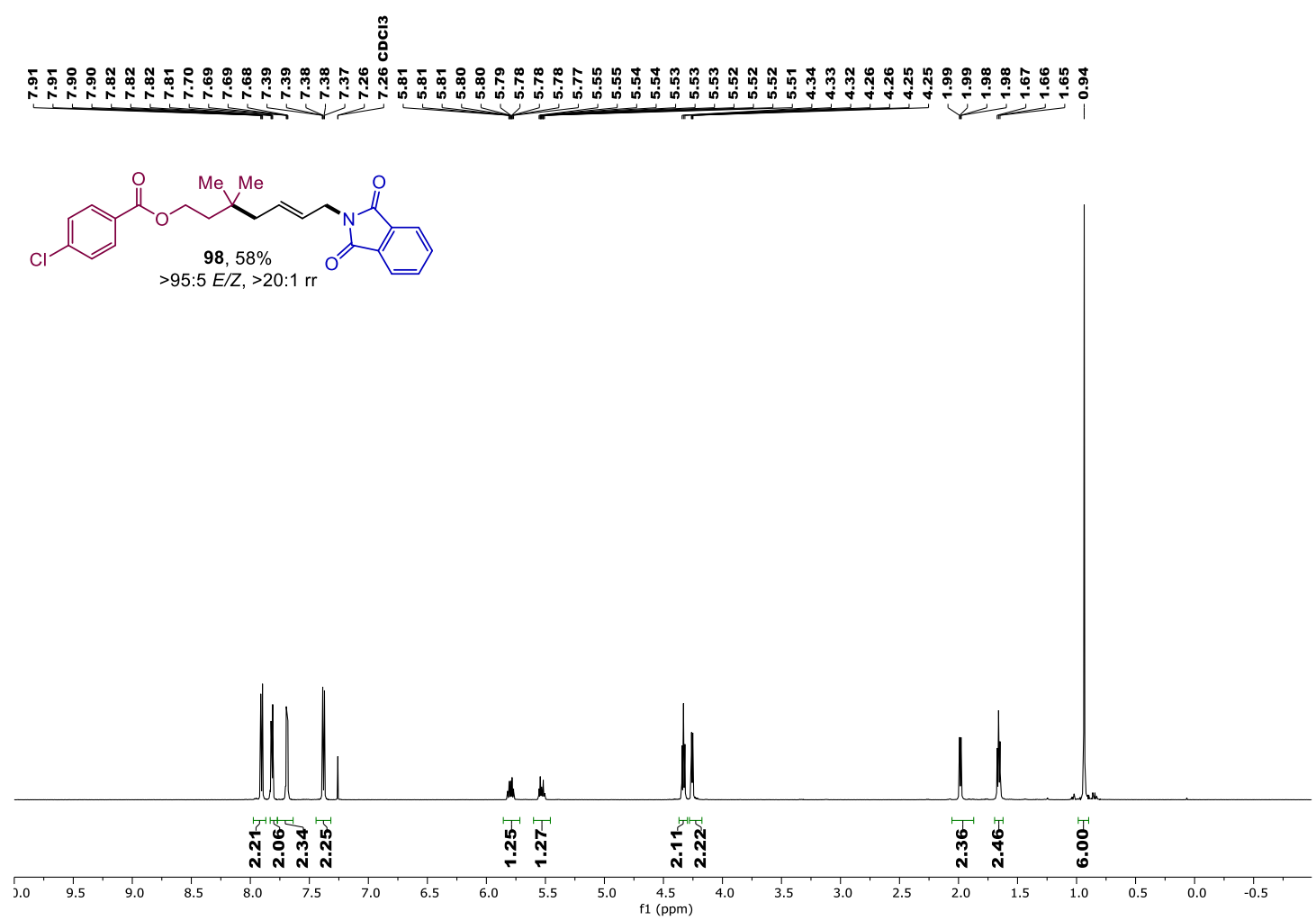

${ }^{13} \mathrm{C}$ NMR (151 MHz, $\mathrm{CDCl}_{3}$ )

\begin{tabular}{|c|c|}
\hline 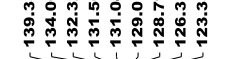 & 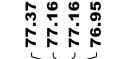 \\
\hline
\end{tabular}

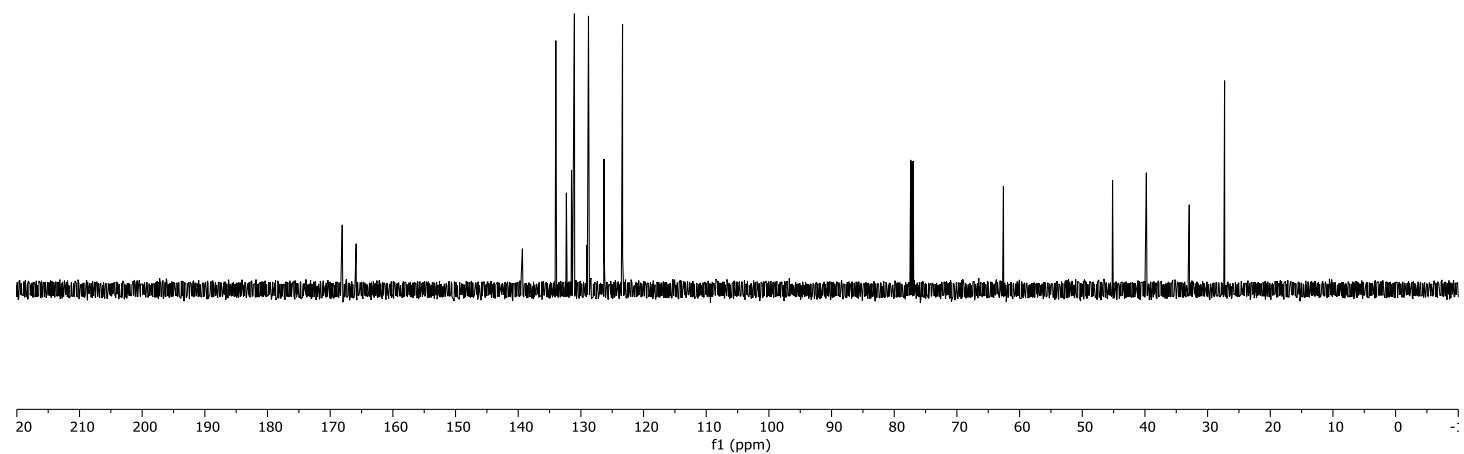


${ }^{1} \mathrm{H}$ NMR (599 MHz, $\mathrm{CDCl}_{3}$ )
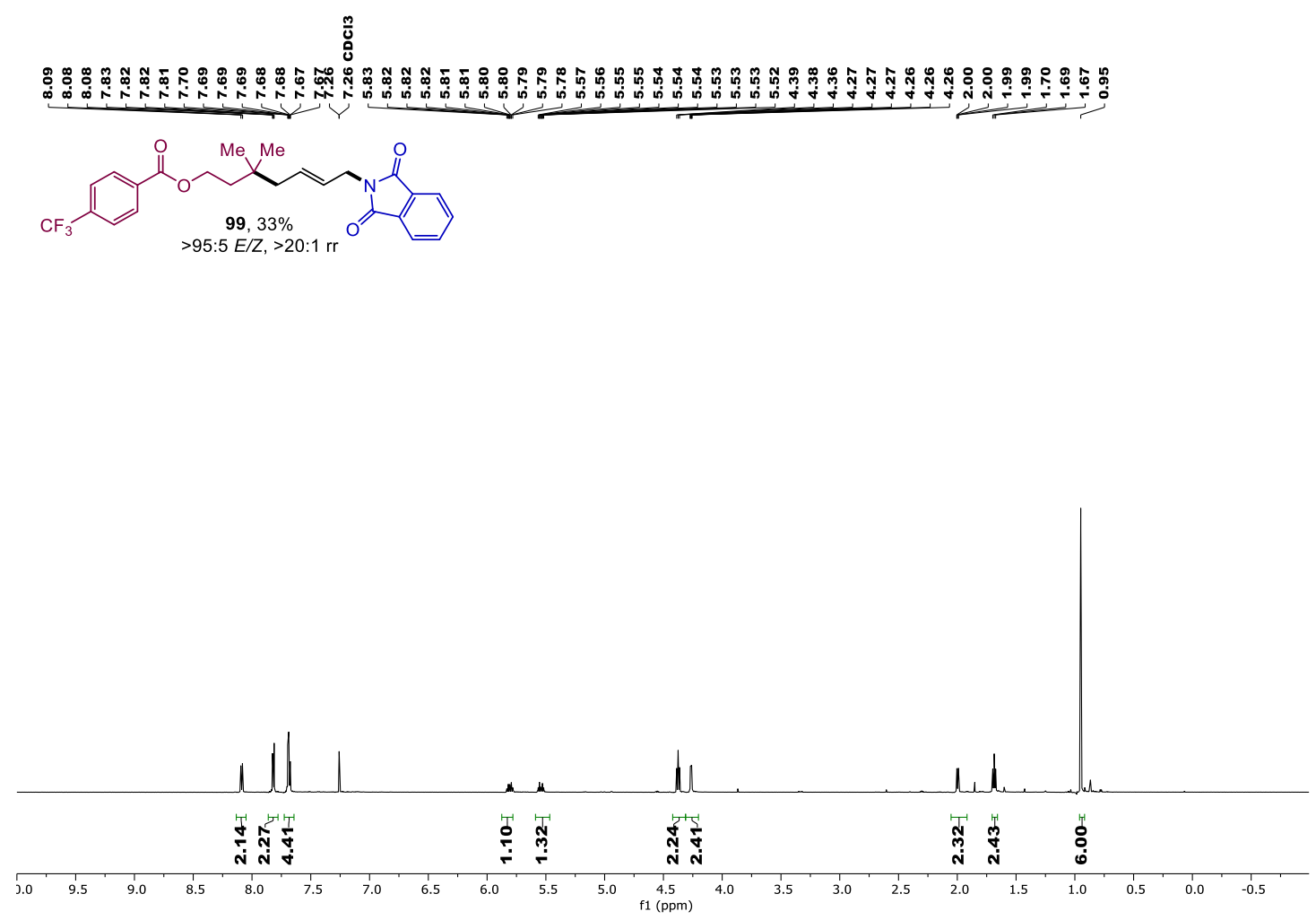

${ }^{13} \mathrm{C}\left\{{ }^{1} \mathrm{H},{ }^{19} \mathrm{~F}\right\} \mathrm{NMR}\left(151 \mathrm{MHz}, \mathrm{CDCl}_{3}\right)$
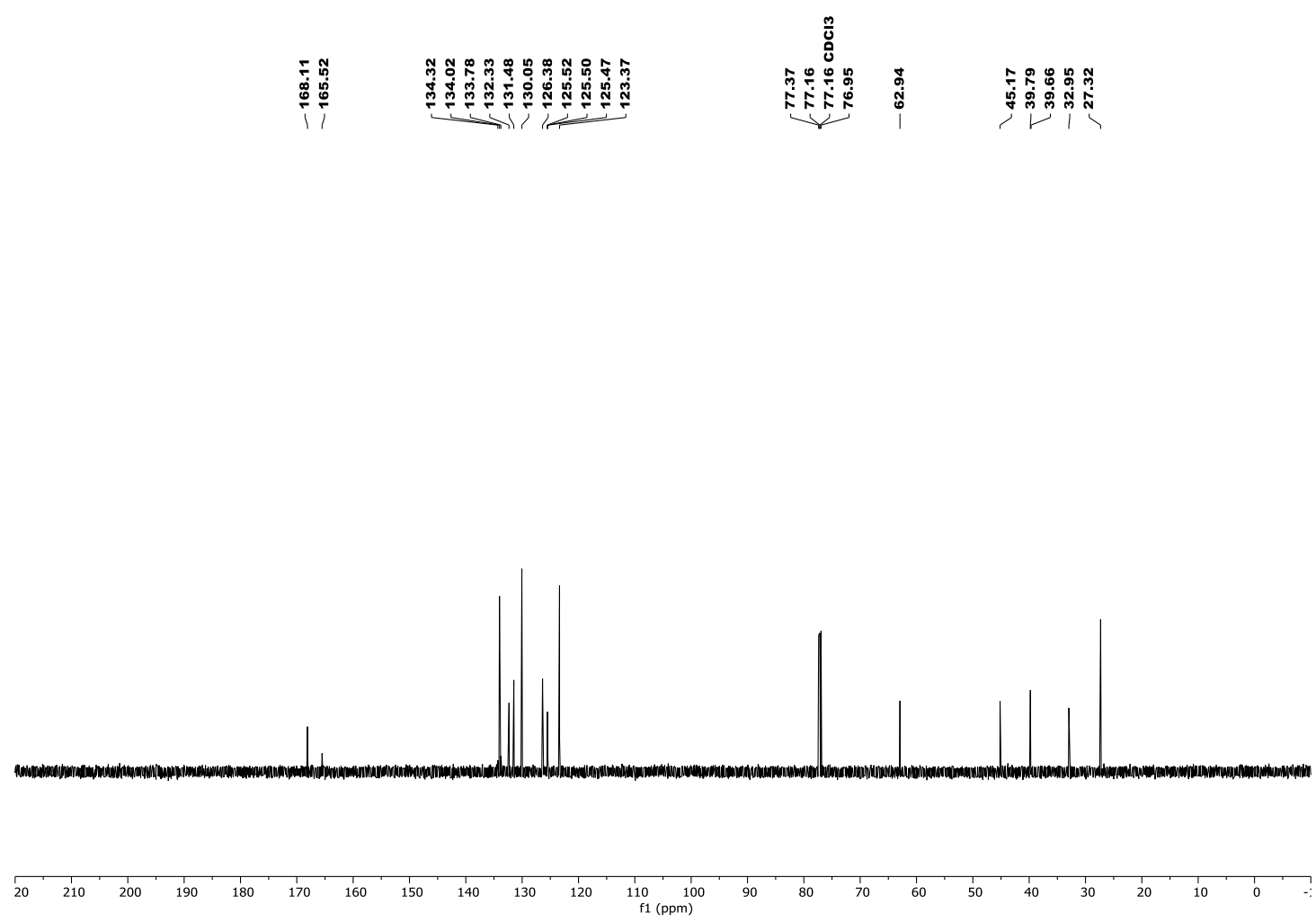
${ }^{19} \mathrm{~F}\left\{{ }^{1} \mathrm{H}\right\} \mathrm{NMR}\left(564 \mathrm{MHz}, \mathrm{CDCl}_{3}\right)$

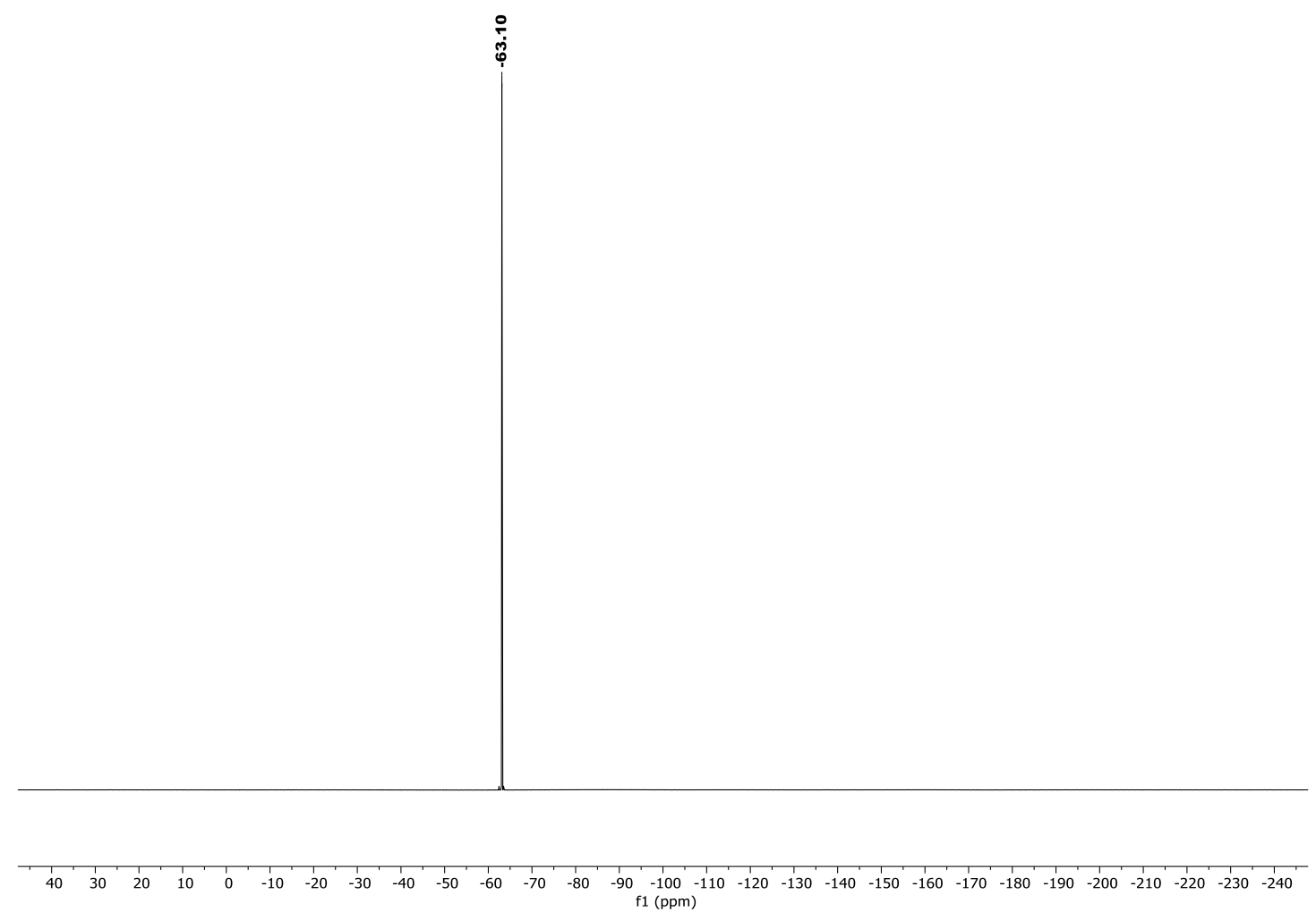


${ }^{1} \mathrm{H} \mathrm{NMR}\left(599 \mathrm{MHz}, \mathrm{CDCl}_{3}\right)$
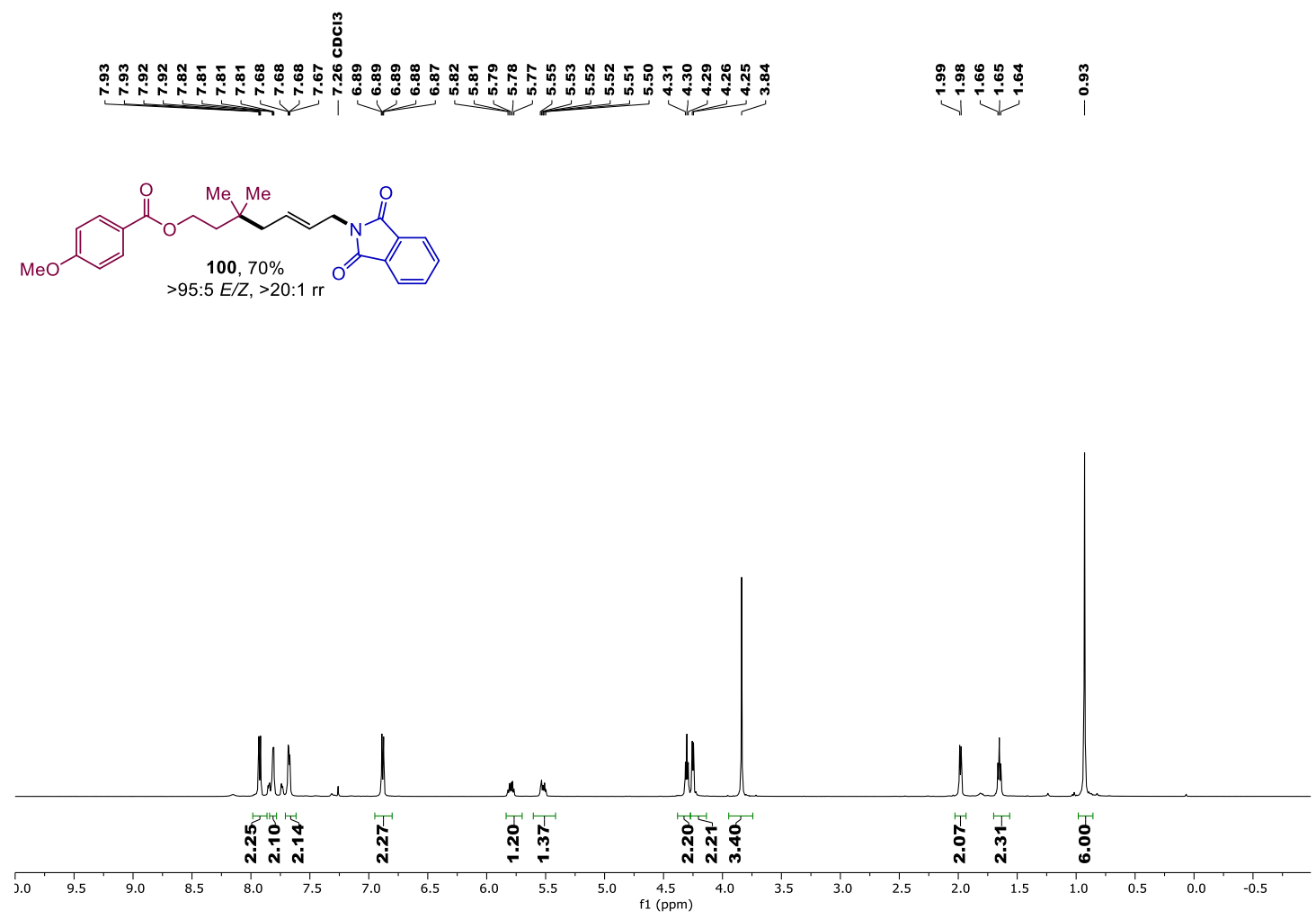

${ }^{13} \mathrm{C}$ NMR (151 MHz, $\mathrm{CDCl}_{3}$ )
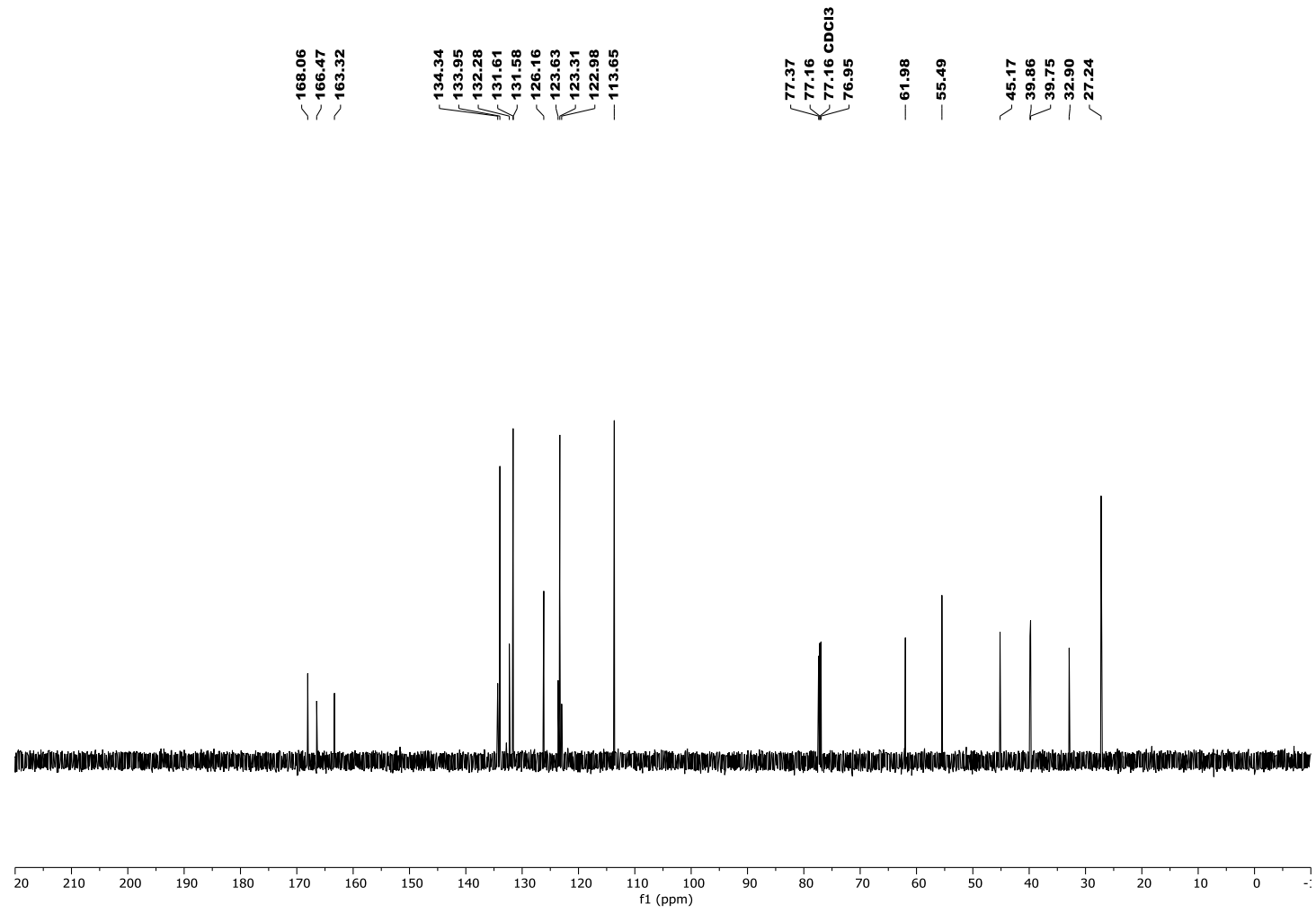
${ }^{1} \mathrm{H} \mathrm{NMR}\left(400 \mathrm{MHz}, \mathrm{CDCl}_{3}\right)$

\section{范

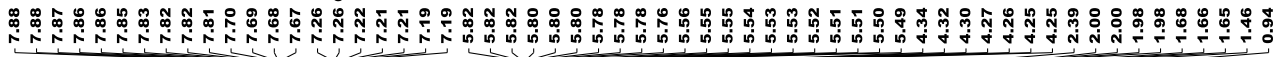
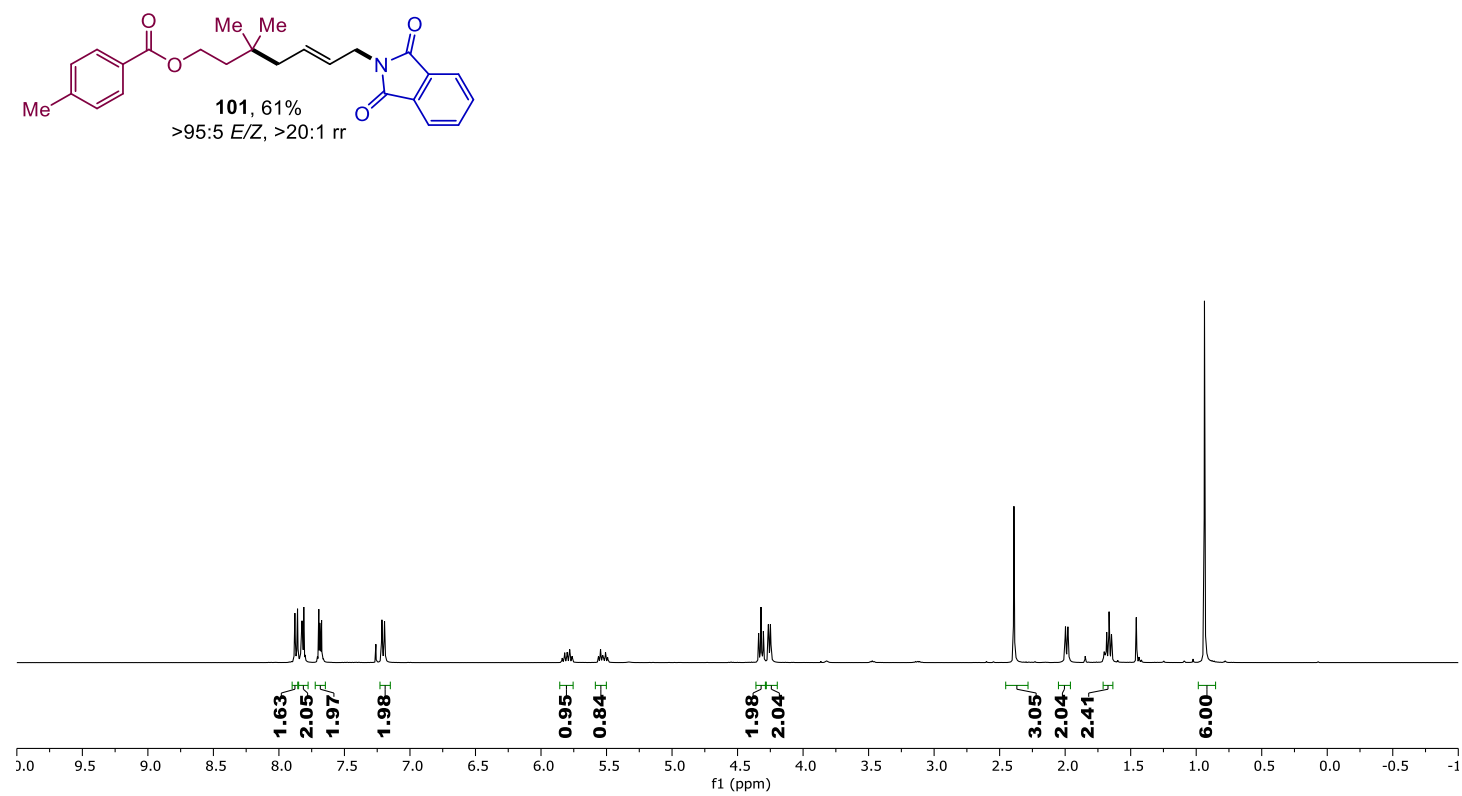

${ }^{13} \mathrm{C}$ NMR $\left(101 \mathrm{MHz}, \mathrm{CDCl}_{3}\right)$

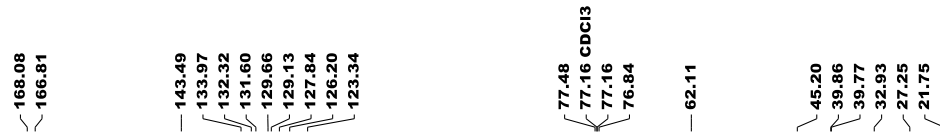
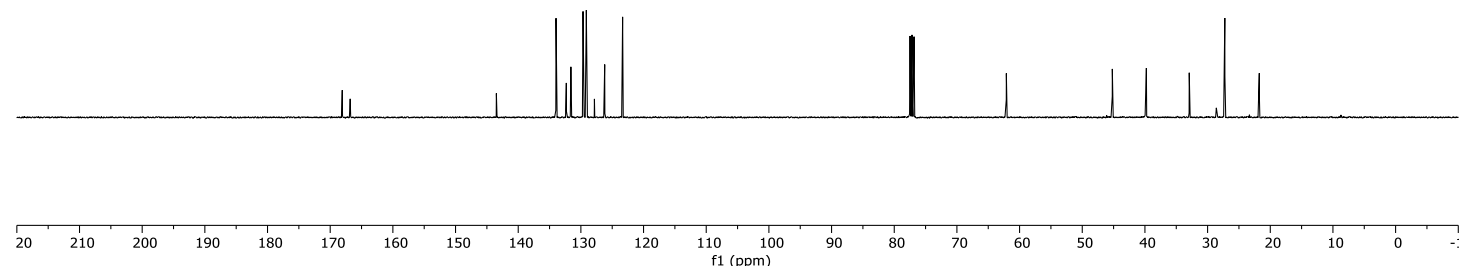
${ }^{1} \mathrm{H}$ NMR $\left(300 \mathrm{MHz}, \mathrm{CDCl}_{3}\right)$
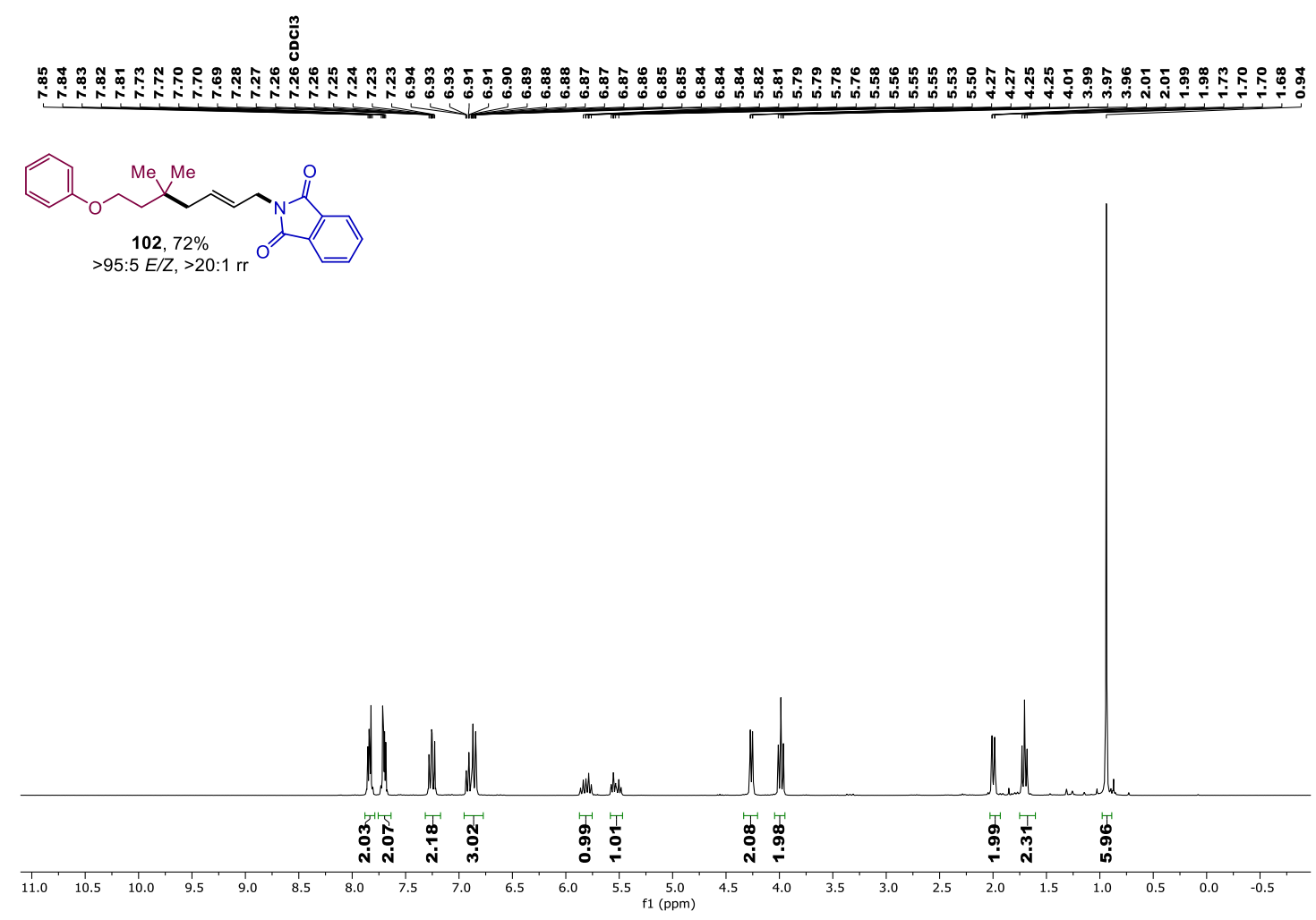

${ }^{13} \mathrm{C}$ NMR $\left(75 \mathrm{MHz}, \mathrm{CDCl}_{3}\right.$ )
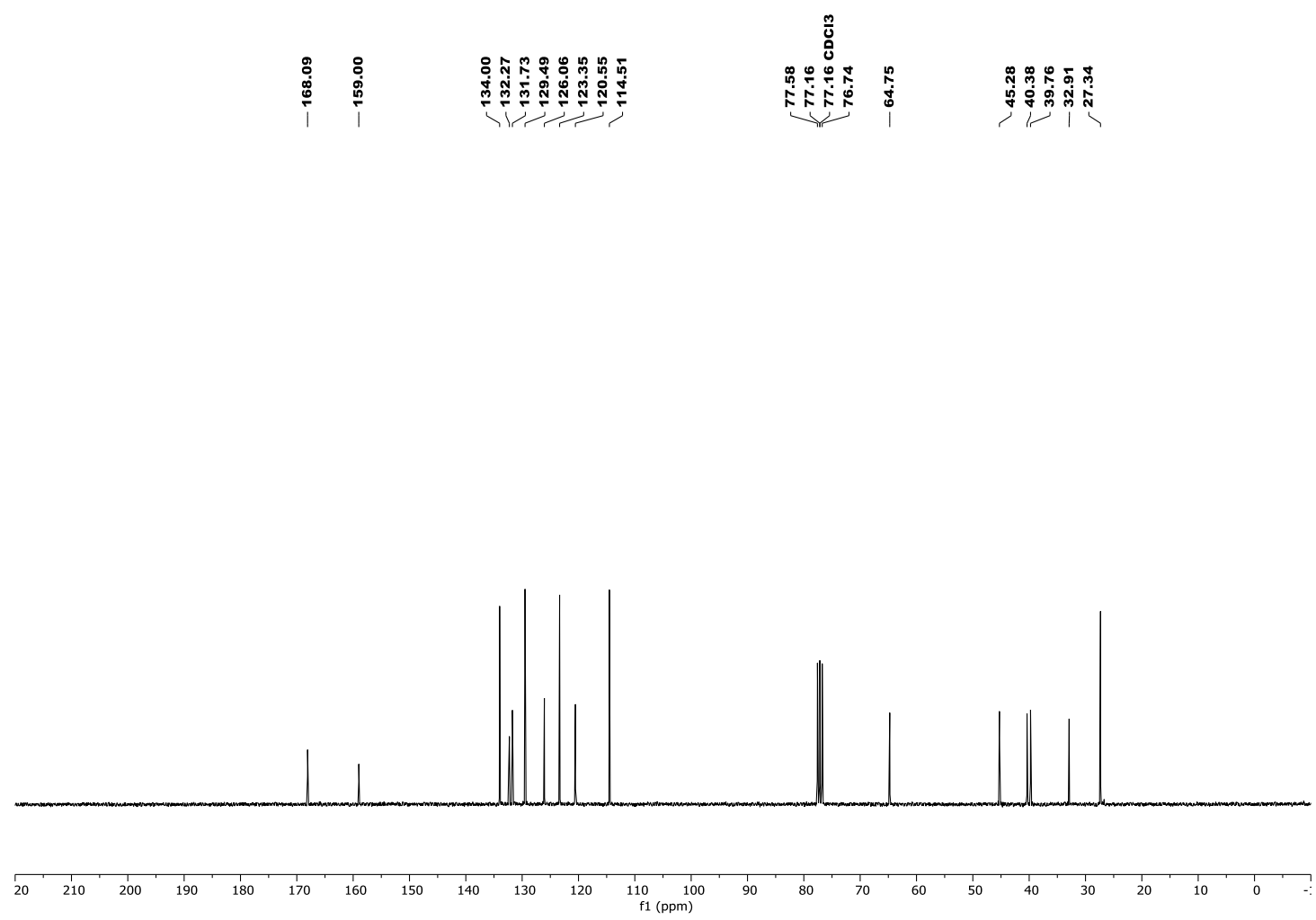
${ }^{1} \mathrm{H}$ NMR $\left(599 \mathrm{MHz}, \mathrm{CDCl}_{3}\right)$
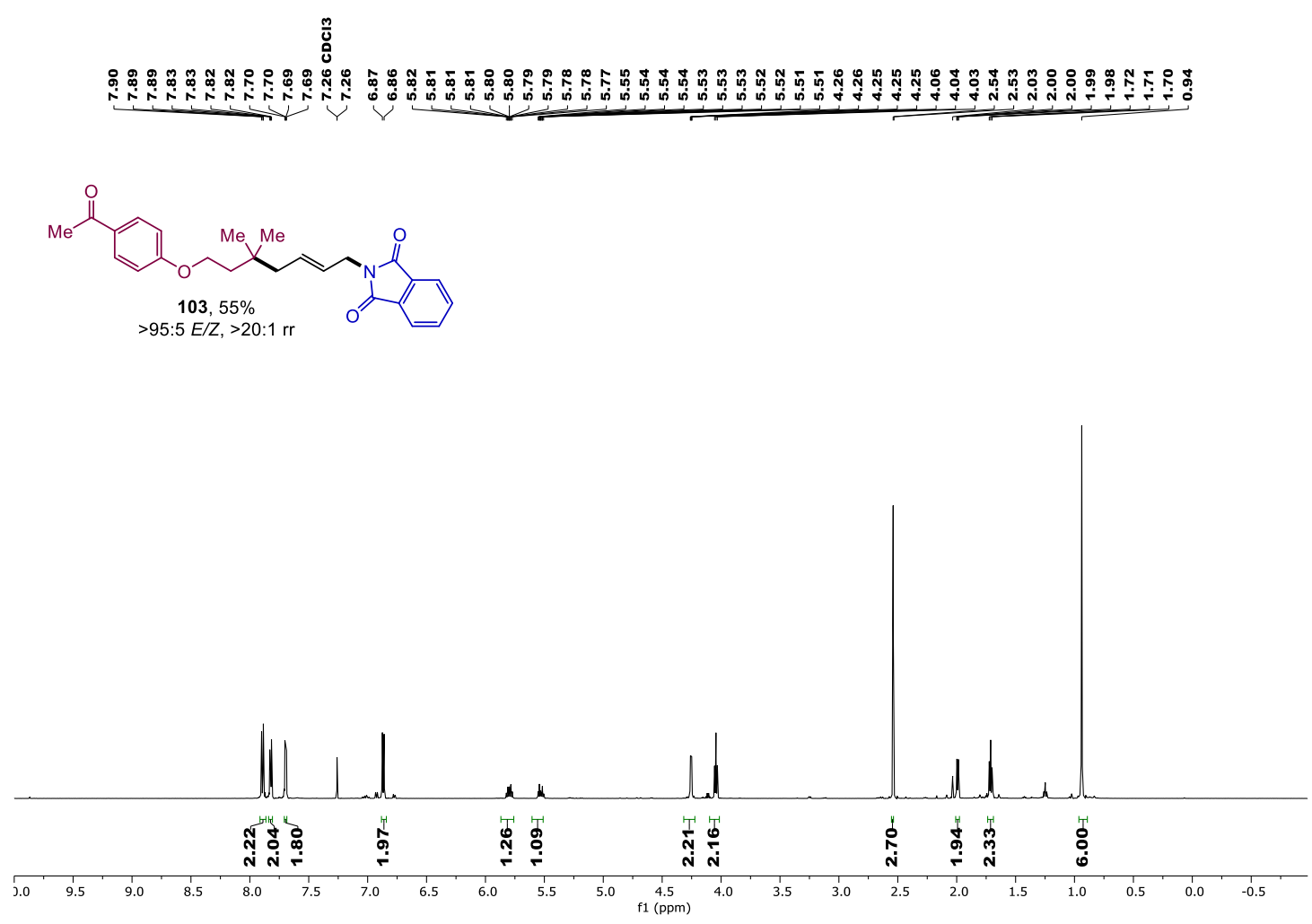

${ }^{13} \mathrm{C}$ NMR (151 MHz, $\mathrm{CDCl}_{3}$ )

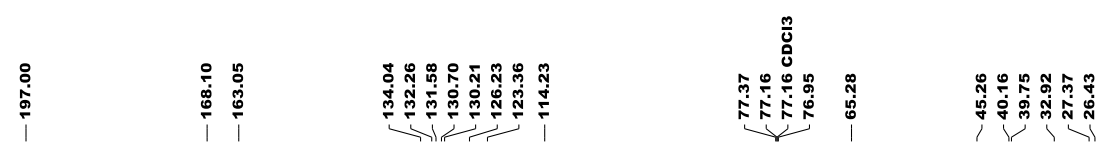

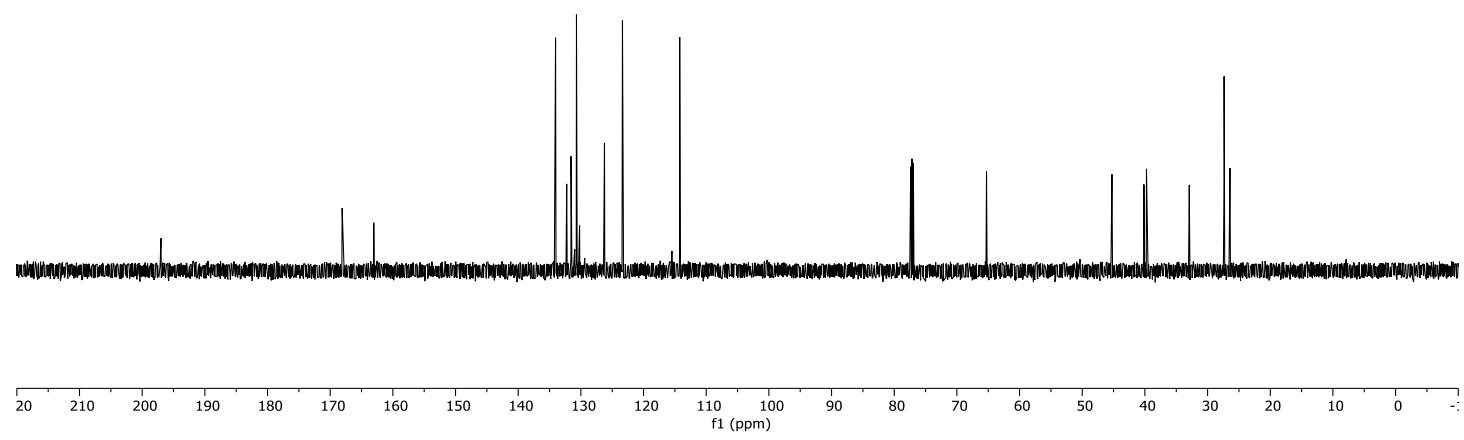


${ }^{1} \mathrm{H} \mathrm{NMR}\left(599 \mathrm{MHz}, \mathrm{CDCl}_{3}\right)$

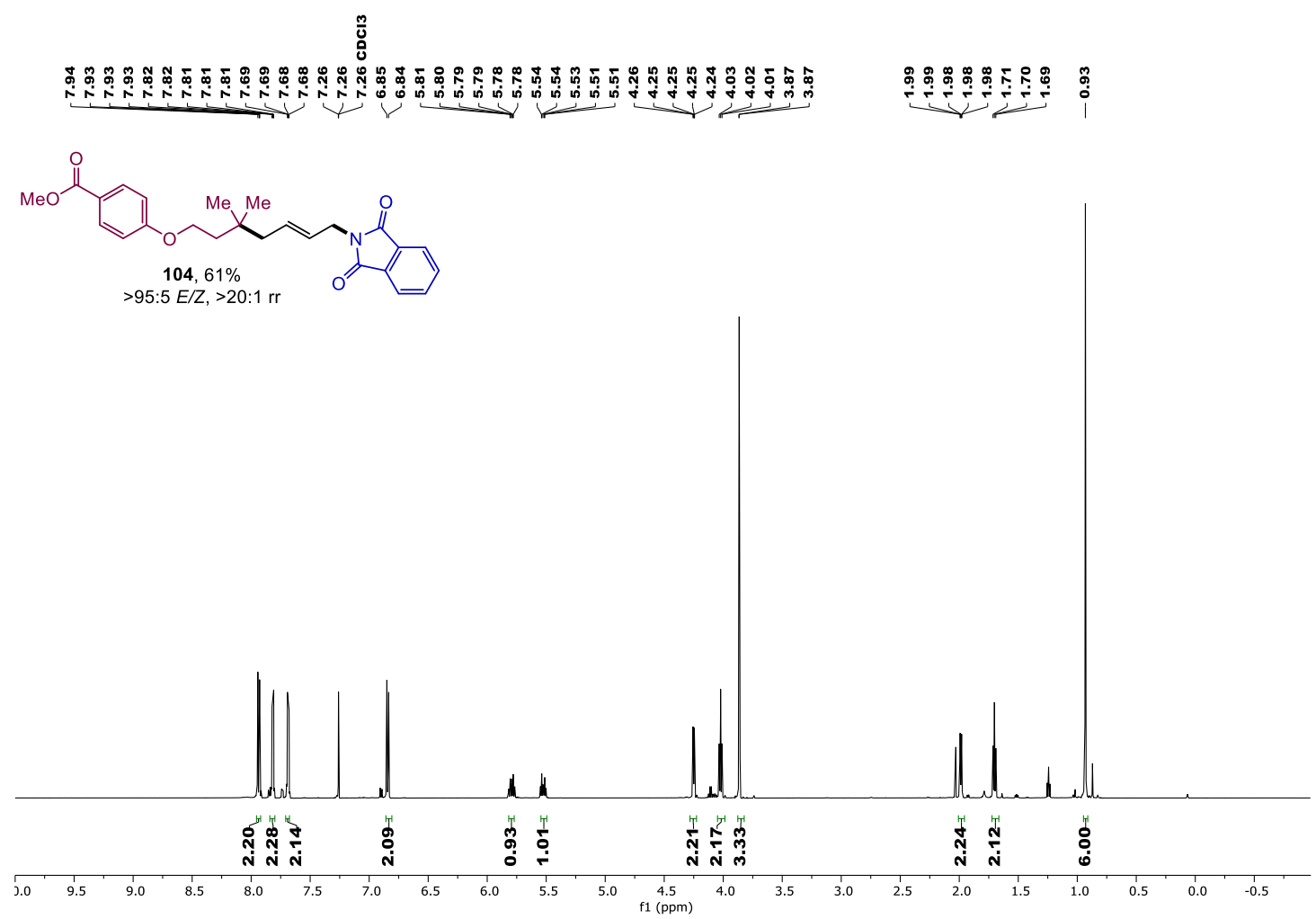

${ }^{13} \mathrm{C}$ NMR (151 MHz, $\left.\mathrm{CDCl}_{3}\right)$

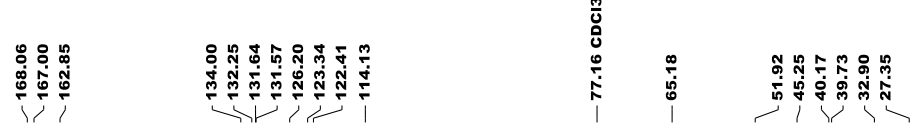
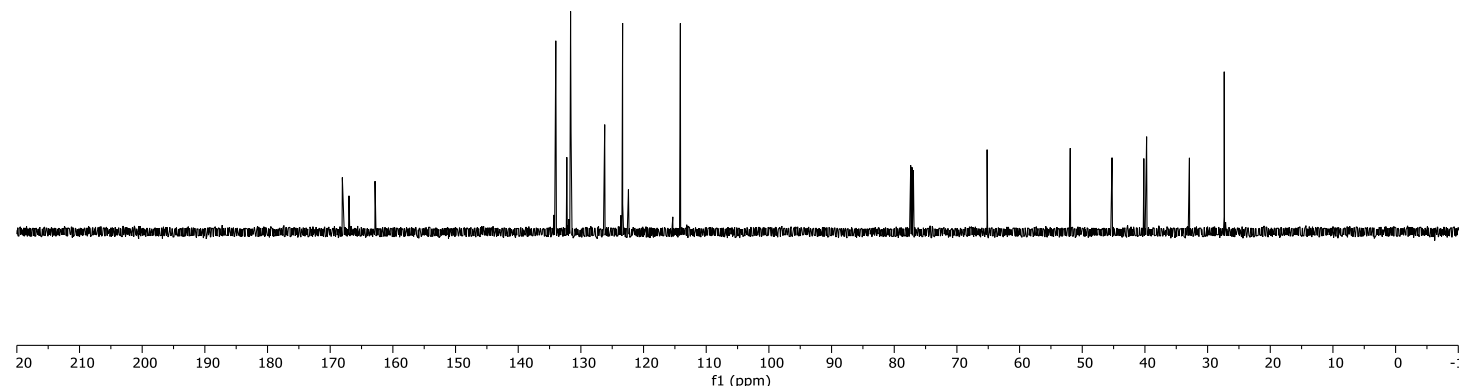
${ }^{1} \mathrm{H}$ NMR $\left(599 \mathrm{MHz}, \mathrm{CDCl}_{3}\right)$
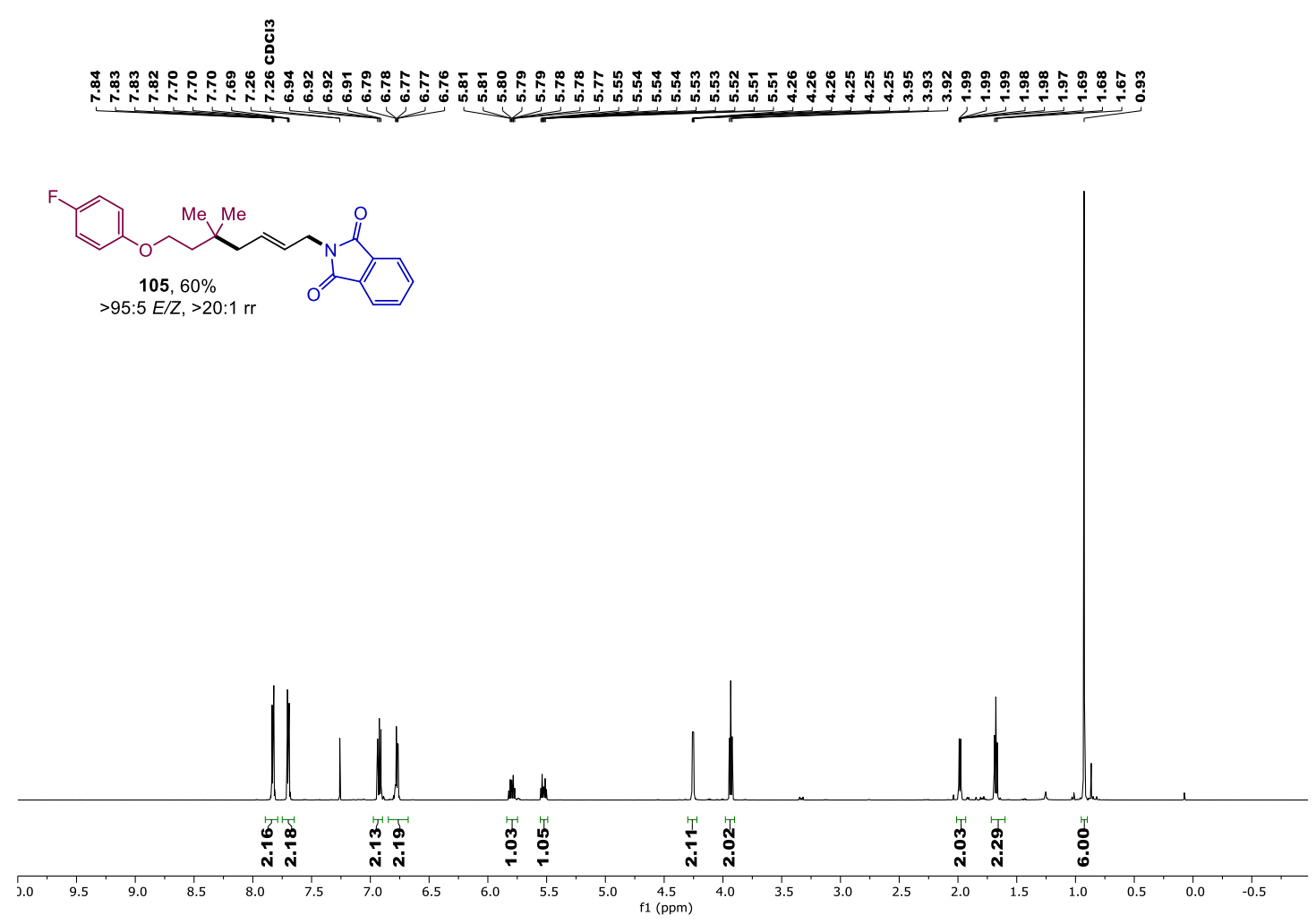

${ }^{13} \mathrm{C}$ NMR $\left(151 \mathrm{MHz}, \mathrm{CDCl}_{3}\right)$
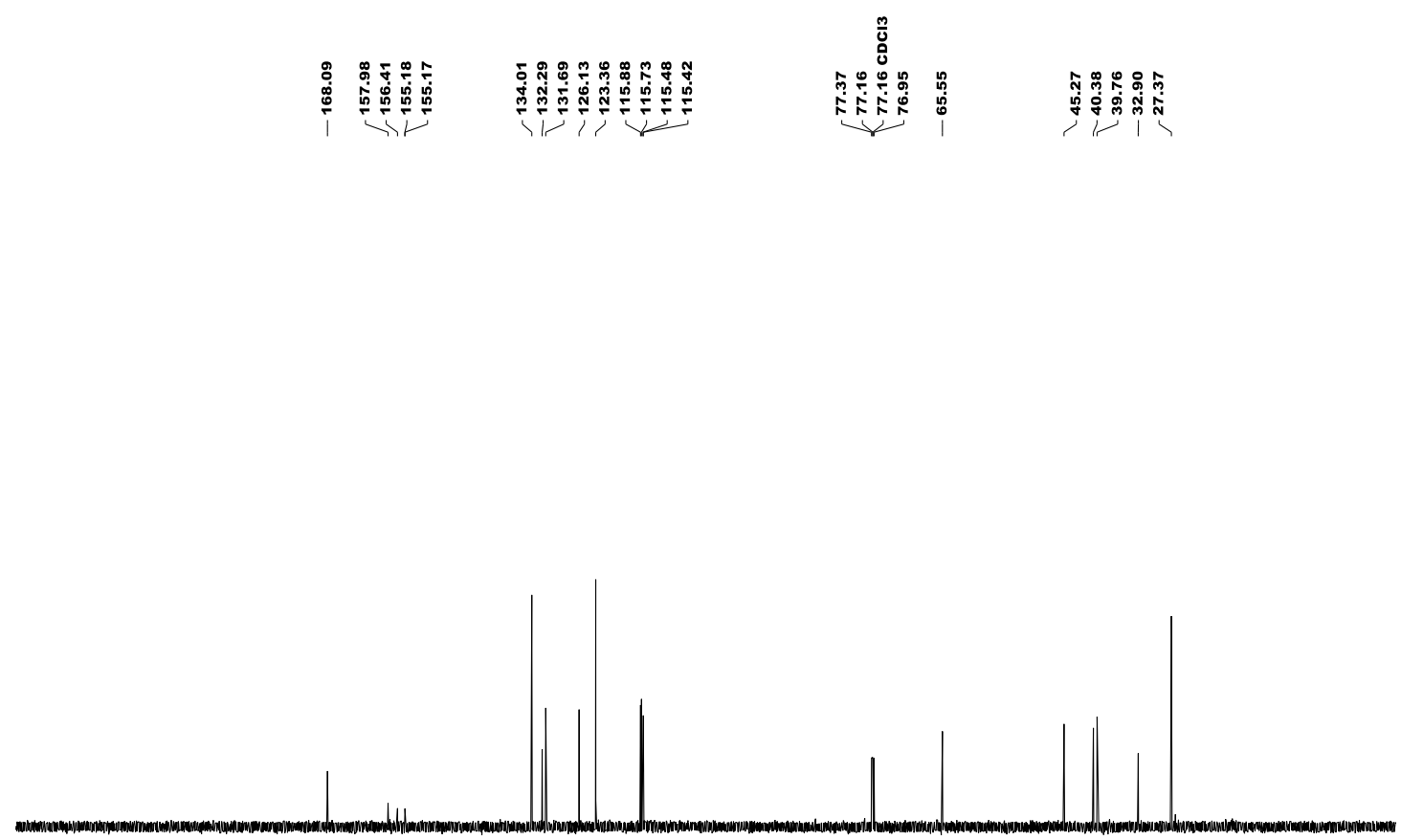

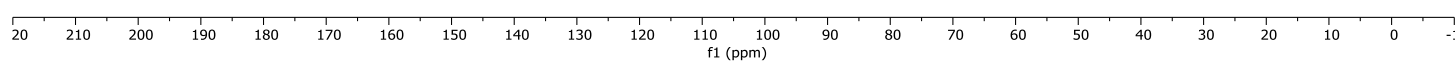


${ }^{19} \mathrm{~F}\left\{{ }^{1} \mathrm{H}\right\} \mathrm{NMR}\left(564 \mathrm{MHz}, \mathrm{CDCl}_{3}\right)$

$\stackrel{i}{\stackrel{i}{d}}$

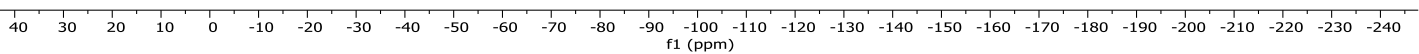


${ }^{1} \mathrm{H} \mathrm{NMR}\left(599 \mathrm{MHz}, \mathrm{CDCl}_{3}\right.$ )

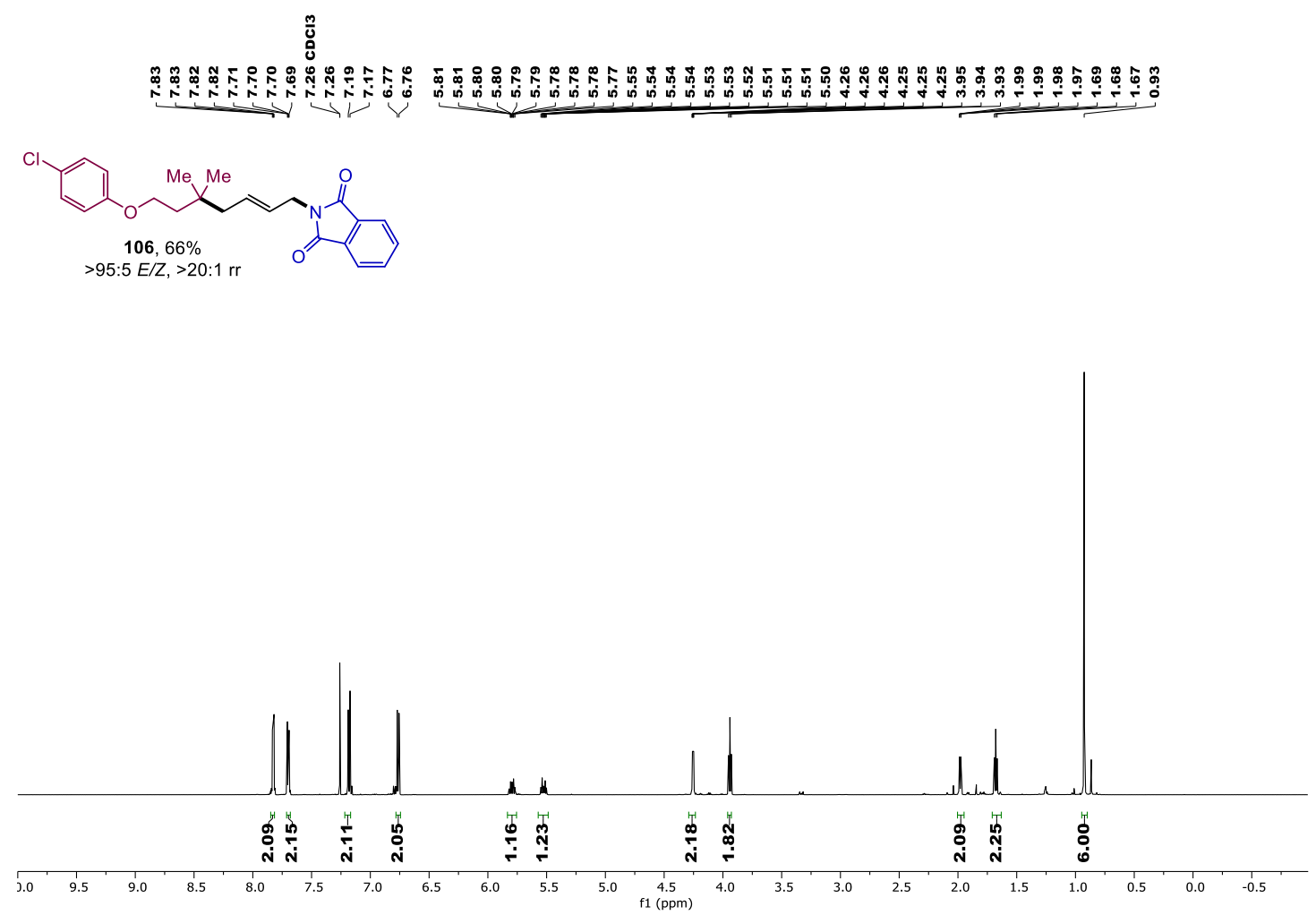

${ }^{13} \mathrm{C}$ NMR $\left(151 \mathrm{MHz}, \mathrm{CDCl}_{3}\right)$
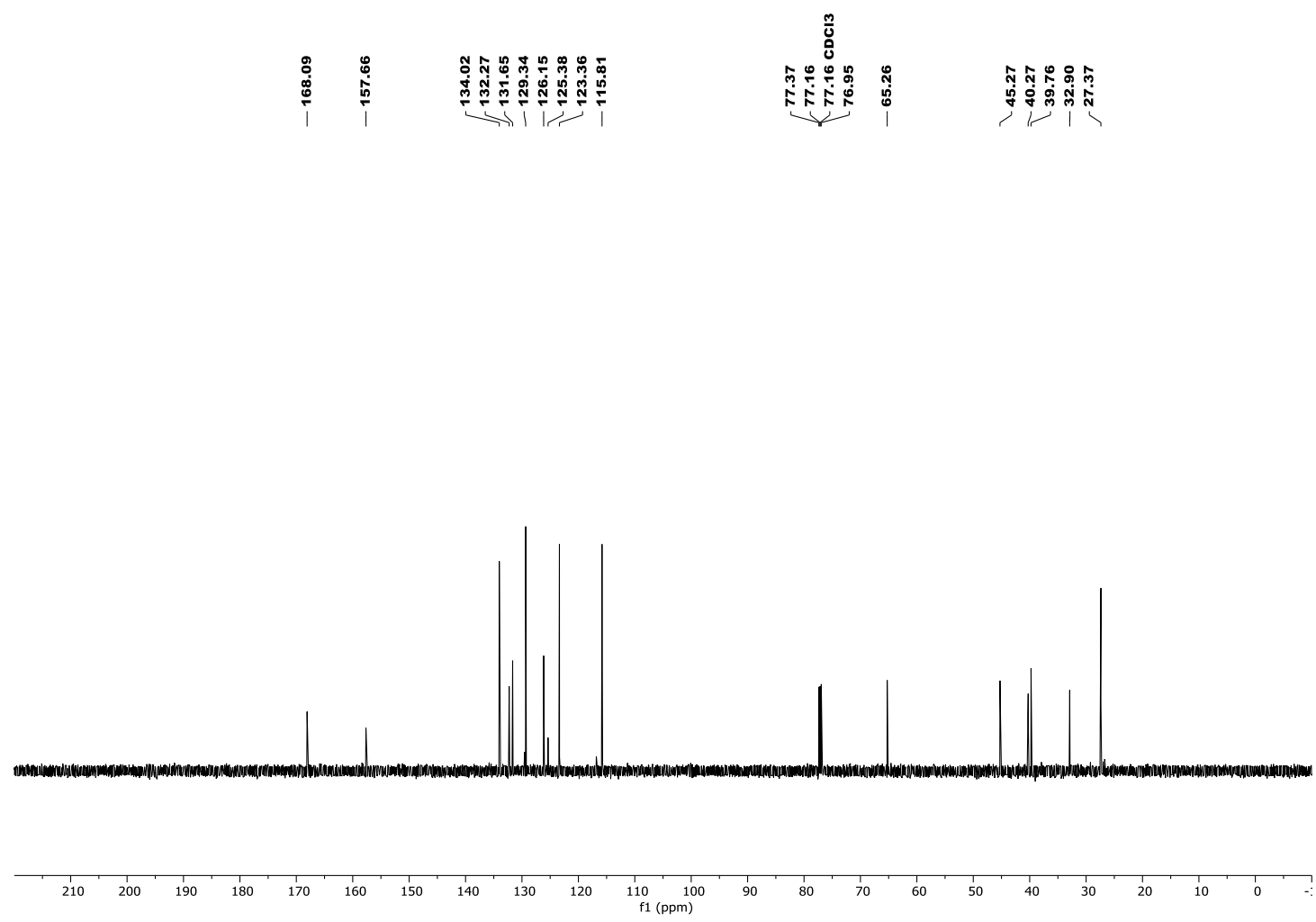
${ }^{1} \mathrm{H}$ NMR (599 MHz, $\mathrm{CDCl}_{3}$ )

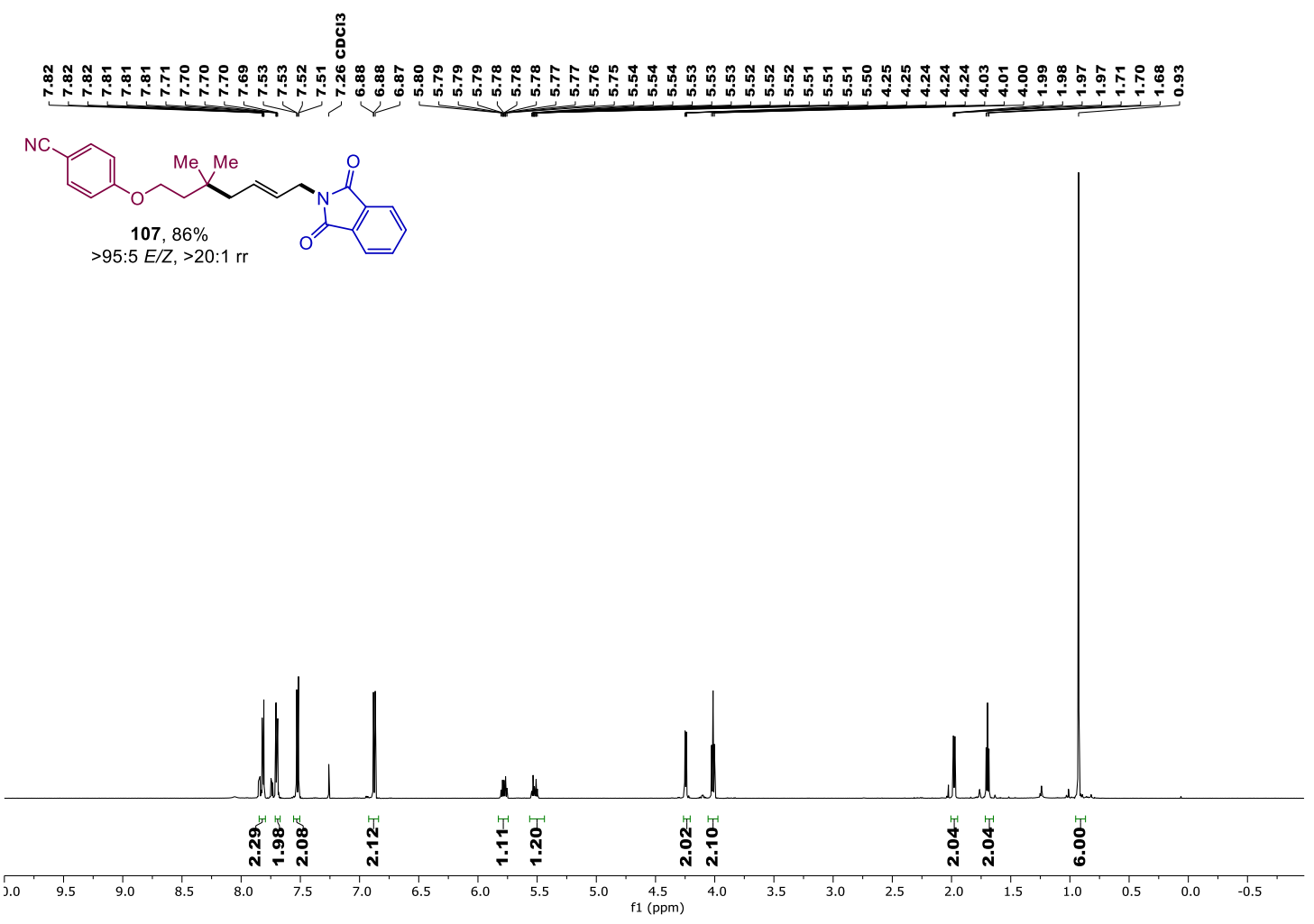

${ }^{13} \mathrm{C}$ NMR (151 MHz, $\mathrm{CDCl}_{3}$ )
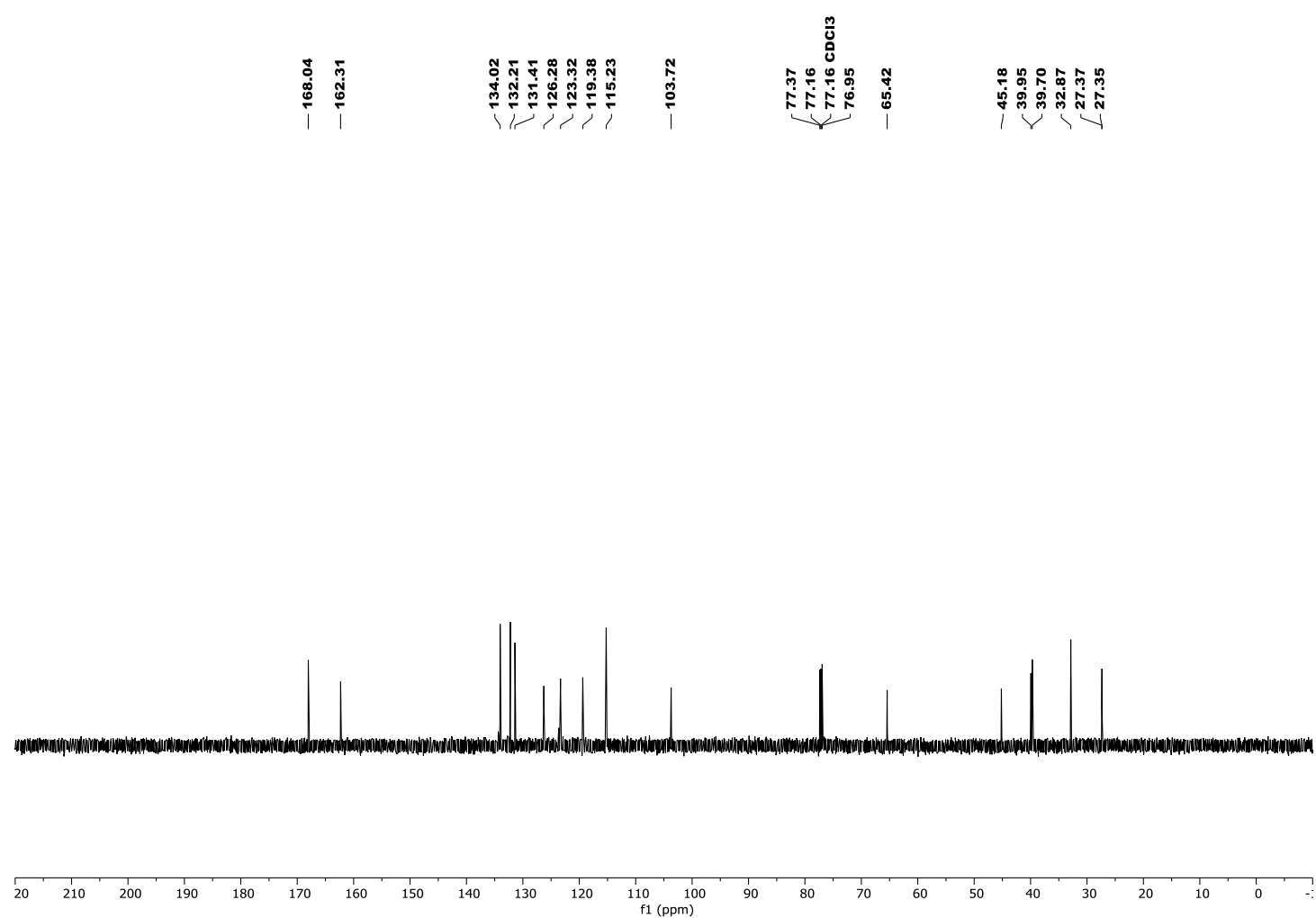
${ }^{1} \mathrm{H}$ NMR $\left(599 \mathrm{MHz}, \mathrm{CDCl}_{3}\right)$
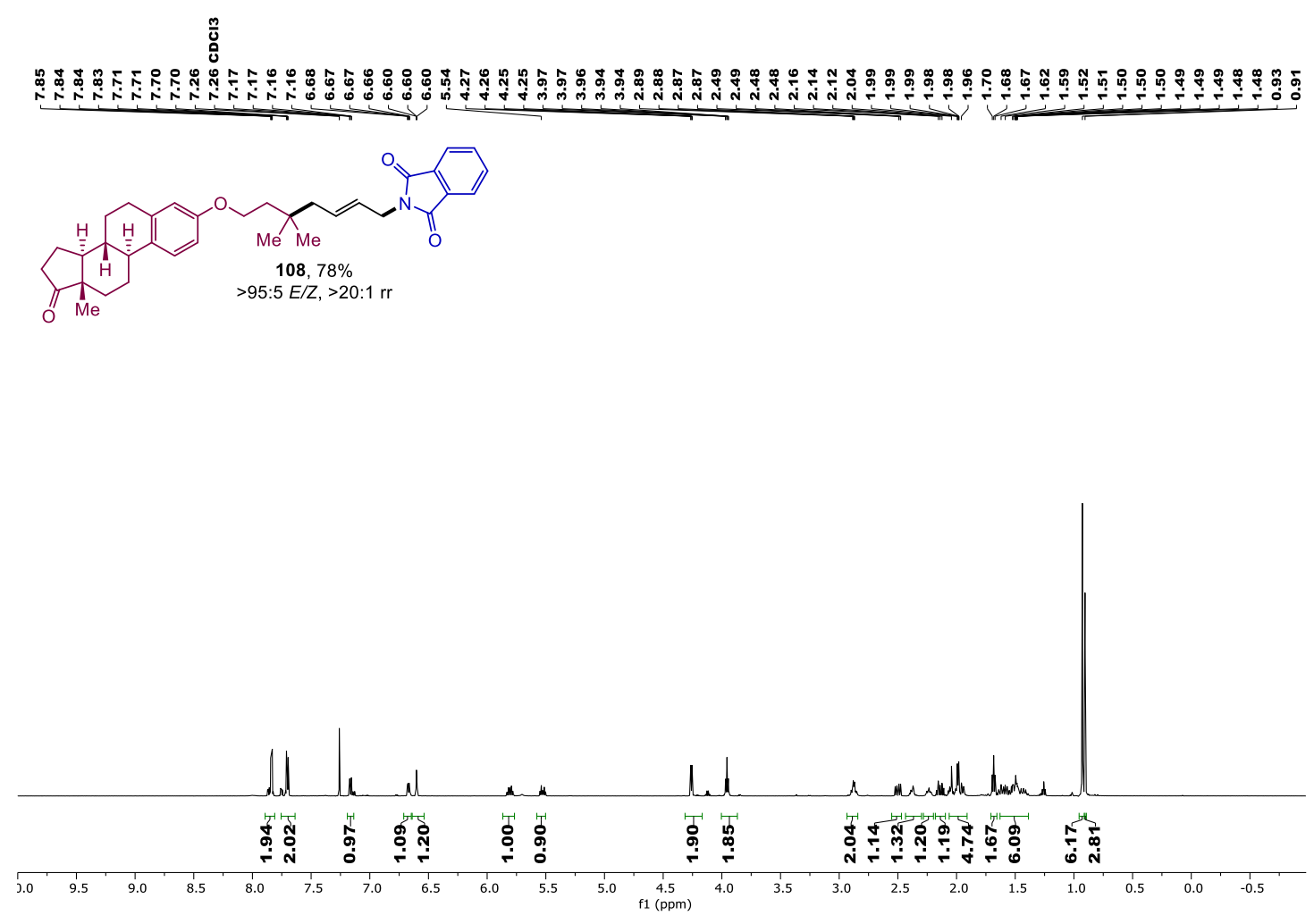

${ }^{13} \mathrm{C}$ NMR (151 MHz, $\mathrm{CDCl}_{3}$ )
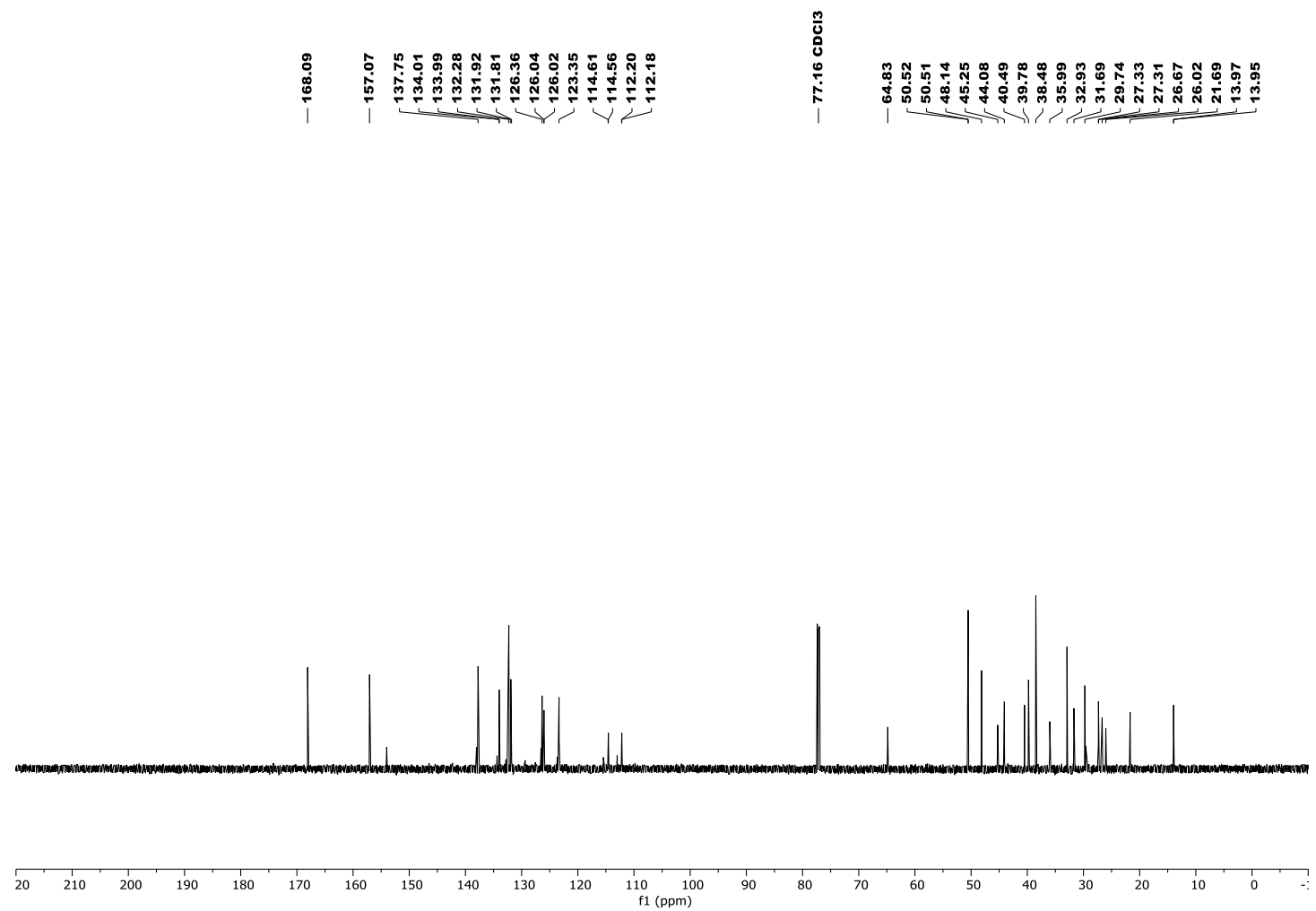
${ }^{1} \mathrm{H}$ NMR (400 MHz, $\mathrm{CDCl}_{3}$ )
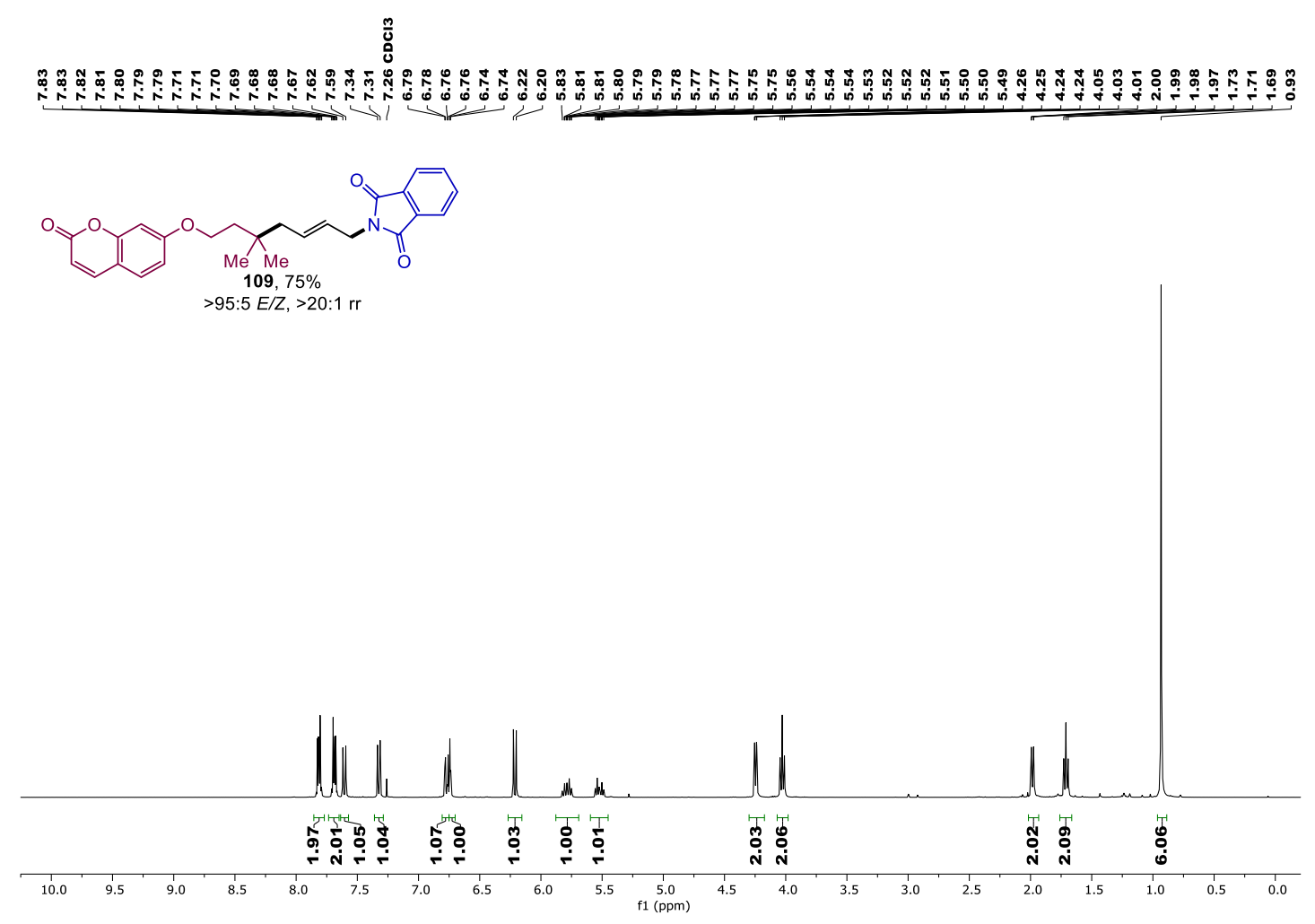

${ }^{13} \mathrm{C}$ NMR (101 MHz, $\mathrm{CDCl}_{3}$ )

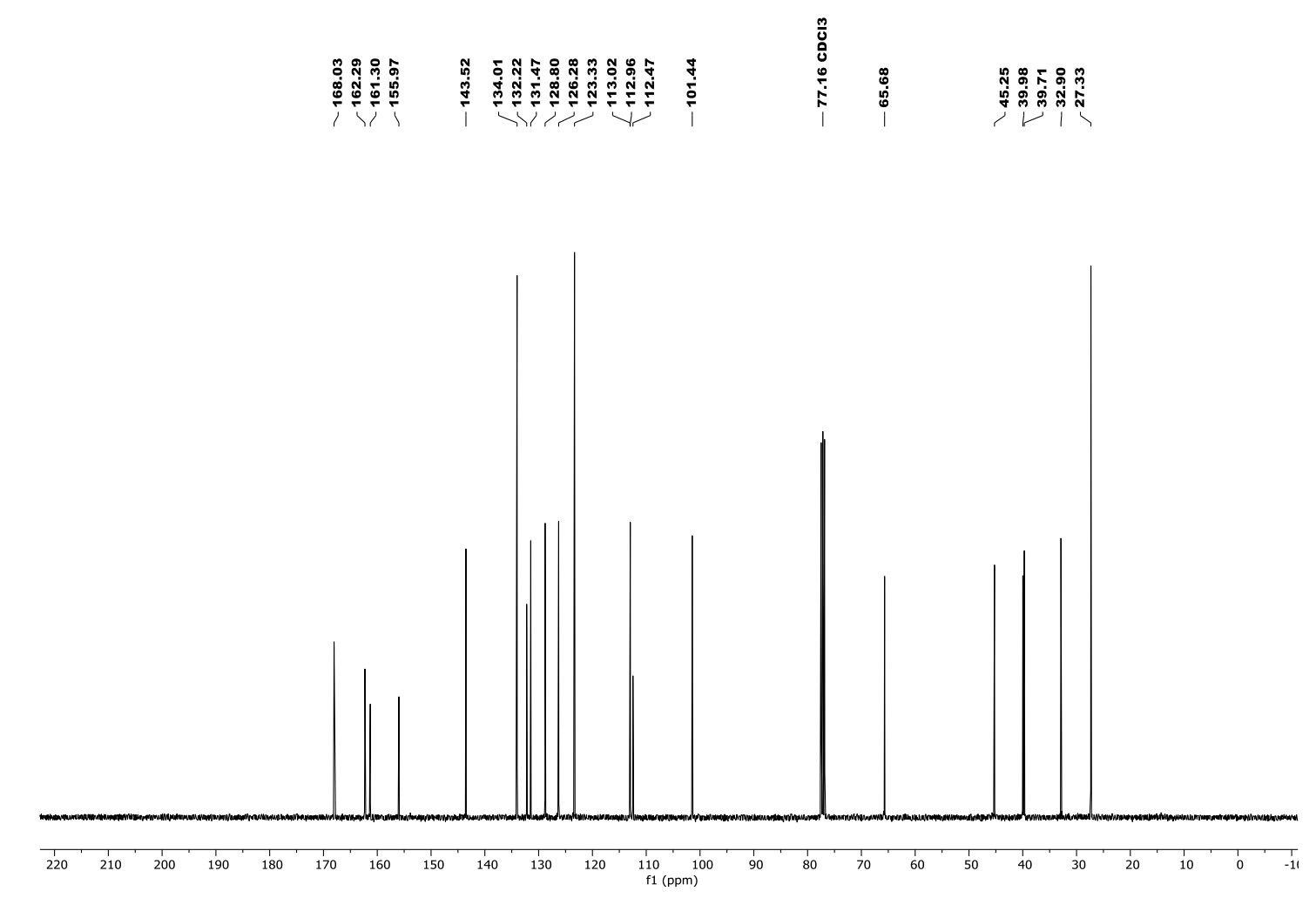


${ }^{1} \mathrm{H}$ NMR (400 MHz, $\mathrm{CDCl}_{3}$ )

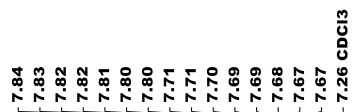

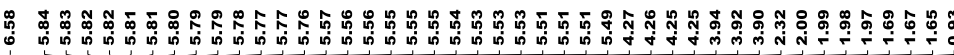<smiles>CC(C)(CC=CCN1C(=O)c2ccccc2C1=O)CCOc1cc([N+](=O)[O-])c(Cl)c([N+](=O)[O-])c1</smiles>

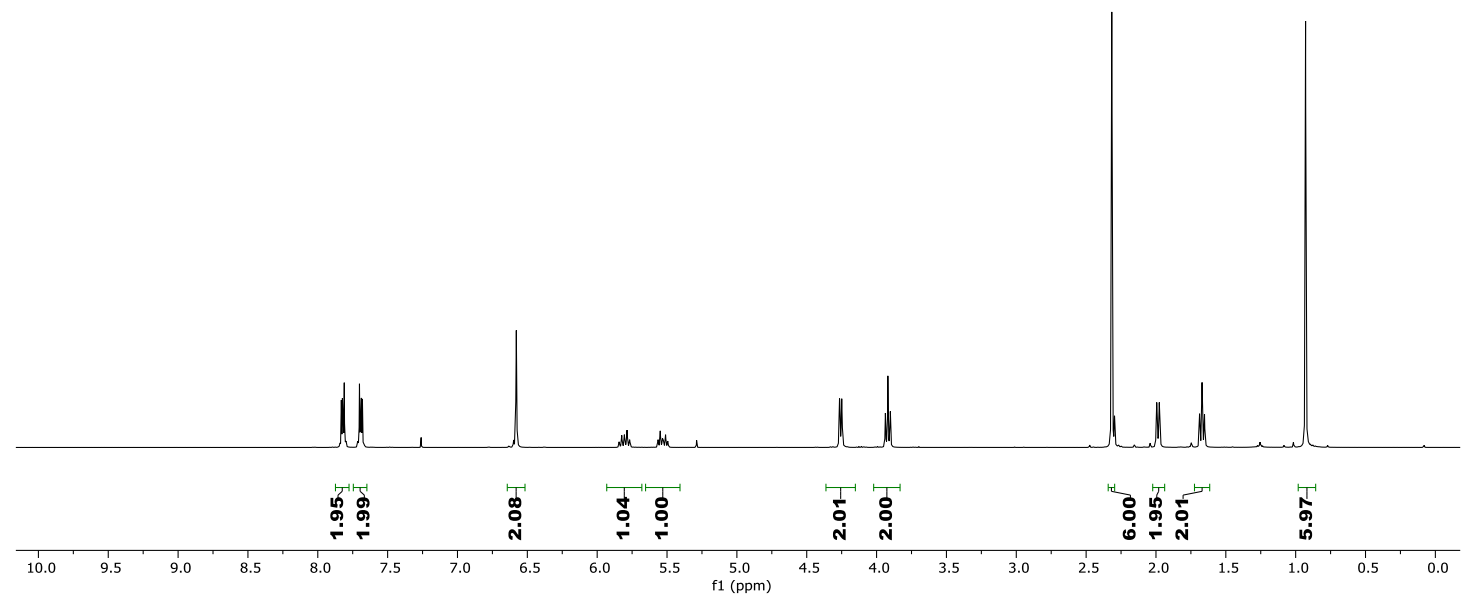

${ }^{13} \mathrm{C}$ NMR $\left(101 \mathrm{MHz}, \mathrm{CDCl}_{3}\right)$

萬
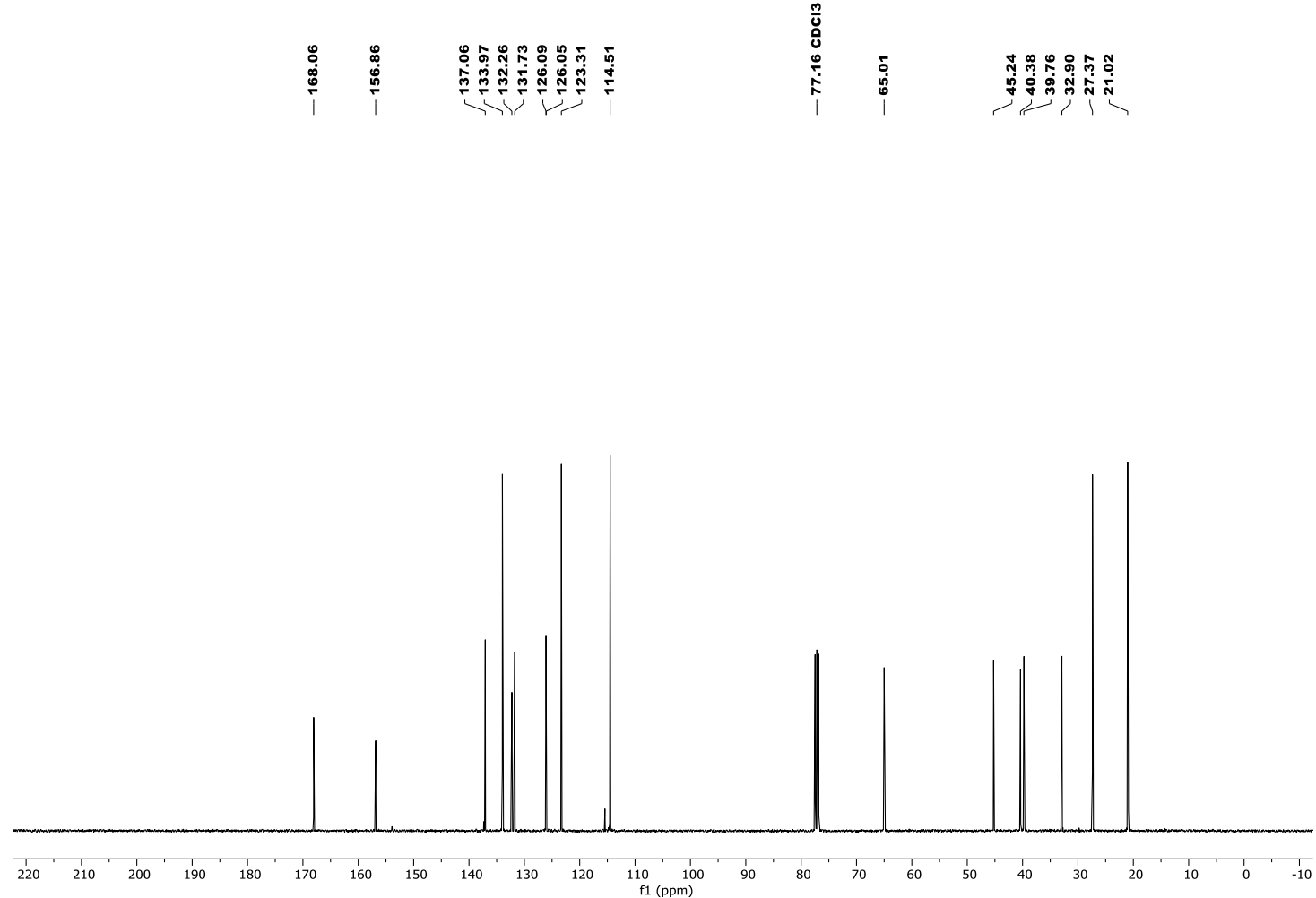
${ }^{1} \mathrm{H}$ NMR (400 MHz, $\mathrm{CDCl}_{3}$ )
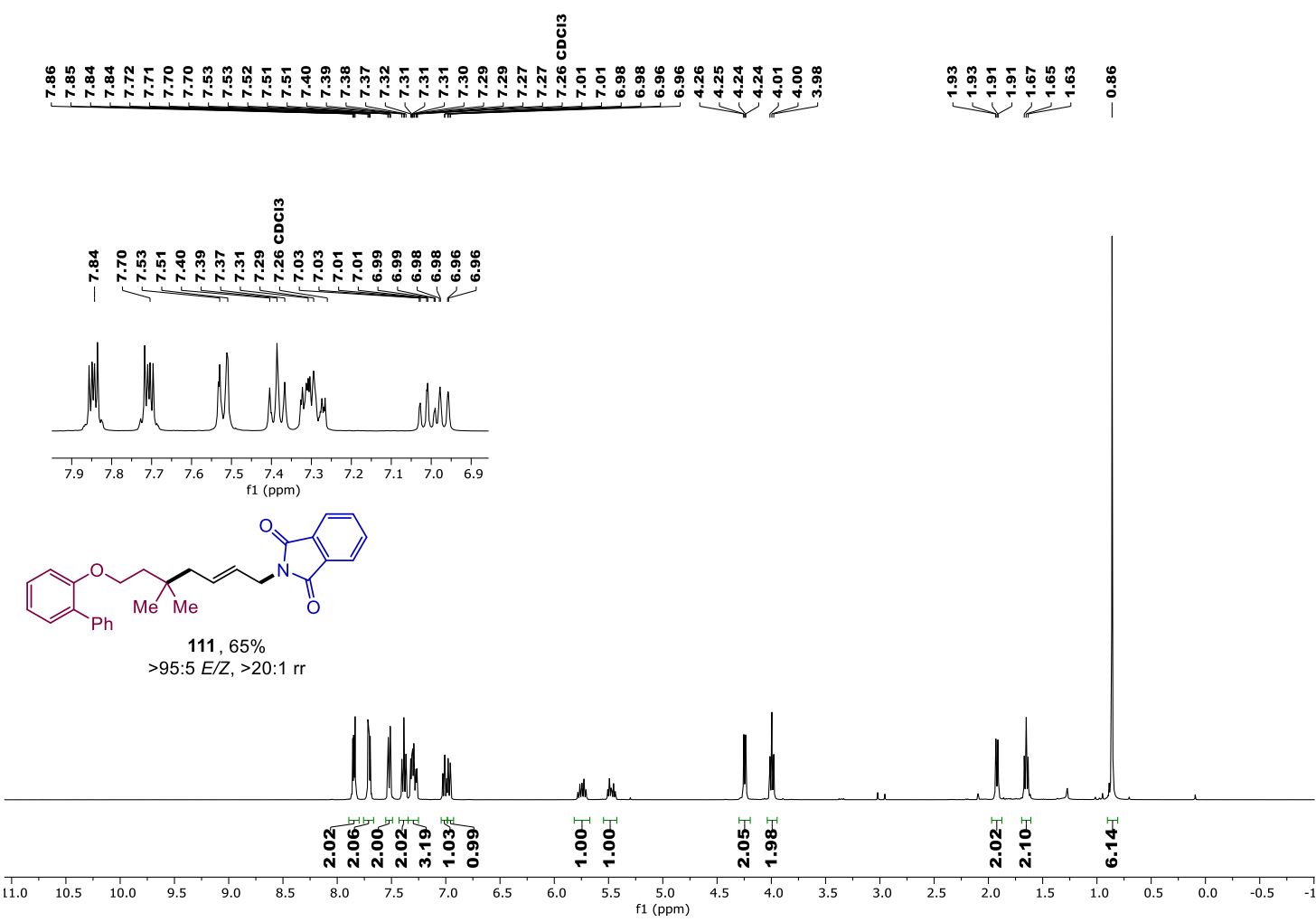

${ }^{13} \mathrm{C}$ NMR (101 MHz, $\left.\mathrm{CDCl}_{3}\right)$

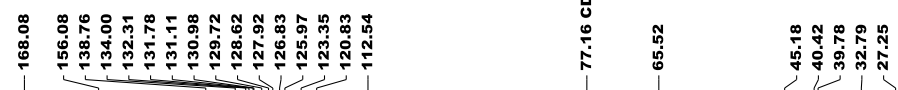

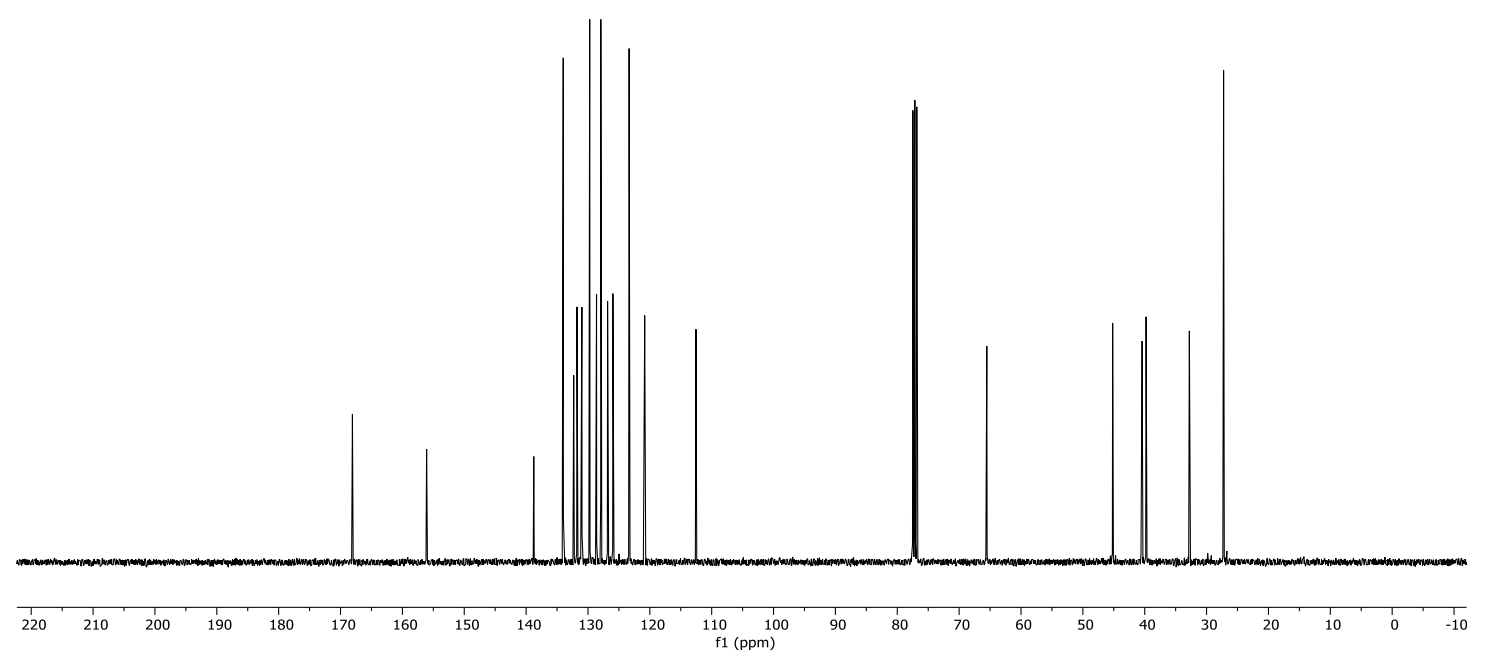


${ }^{1} \mathrm{H}$ NMR (599 MHz, acetone- $\left.d_{6}\right)$

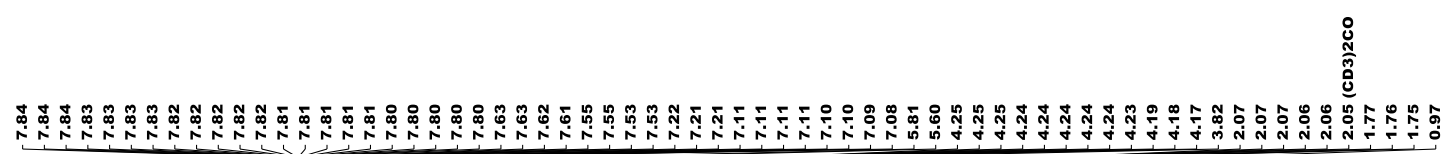
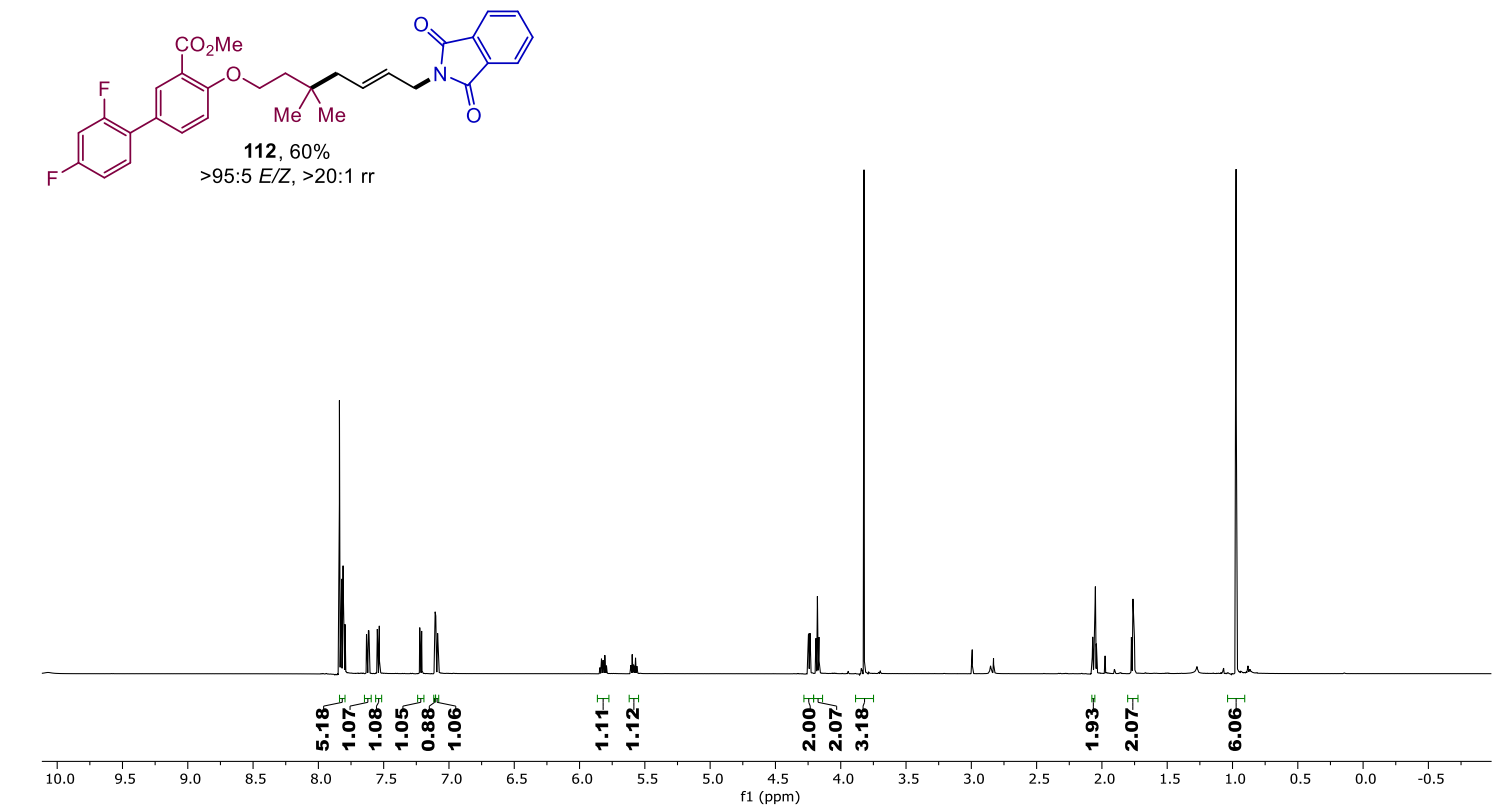

${ }^{13} \mathrm{C}$ NMR $\left(151 \mathrm{MHz}\right.$, acetone- $\left.d_{6}\right)$

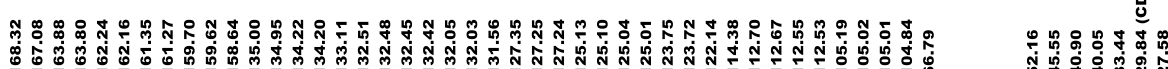

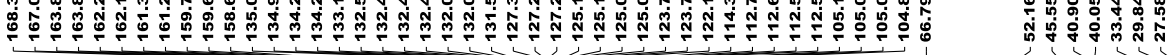

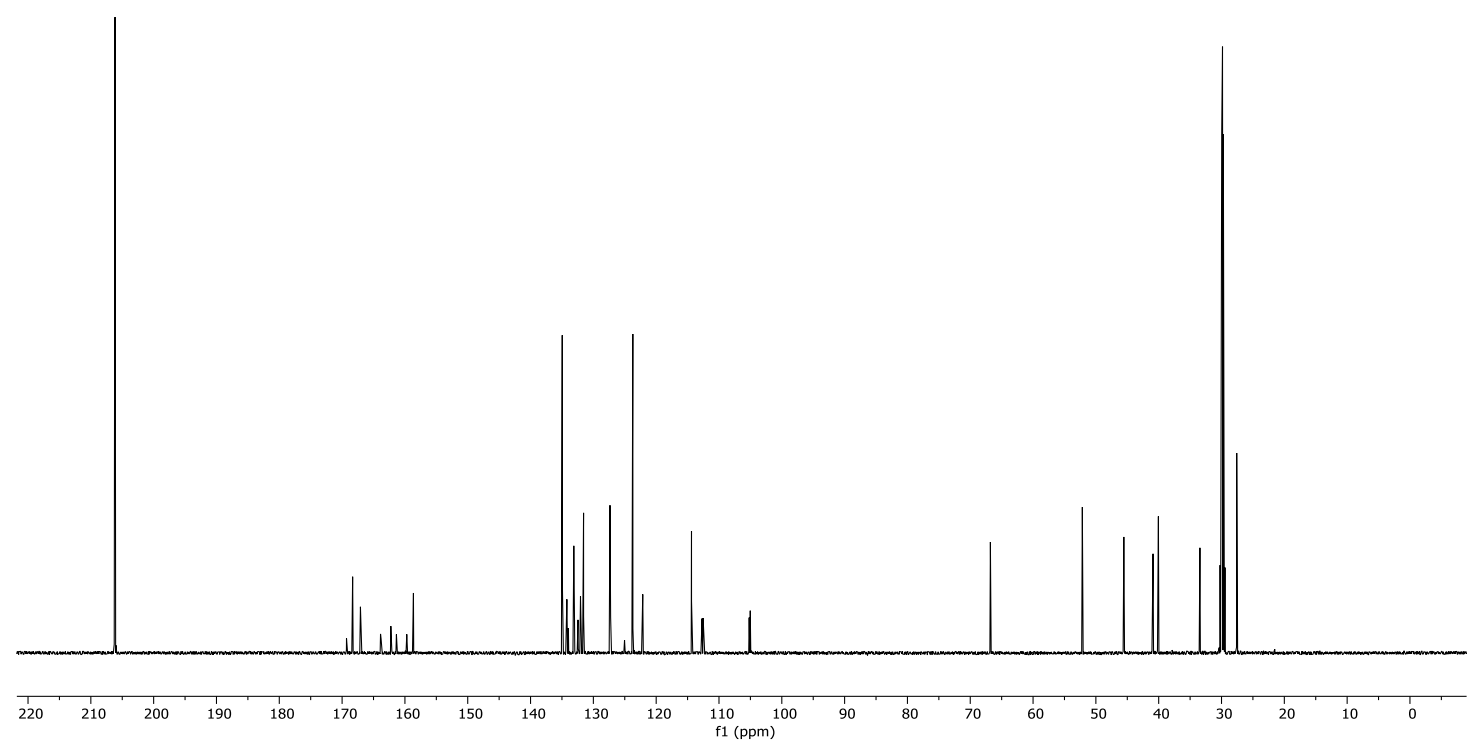


${ }^{19} \mathrm{~F}\left\{{ }^{1} \mathrm{H}\right\} \mathrm{NMR}\left(564 \mathrm{MHz}\right.$, acetone- $\left.d_{6}\right)$

ن
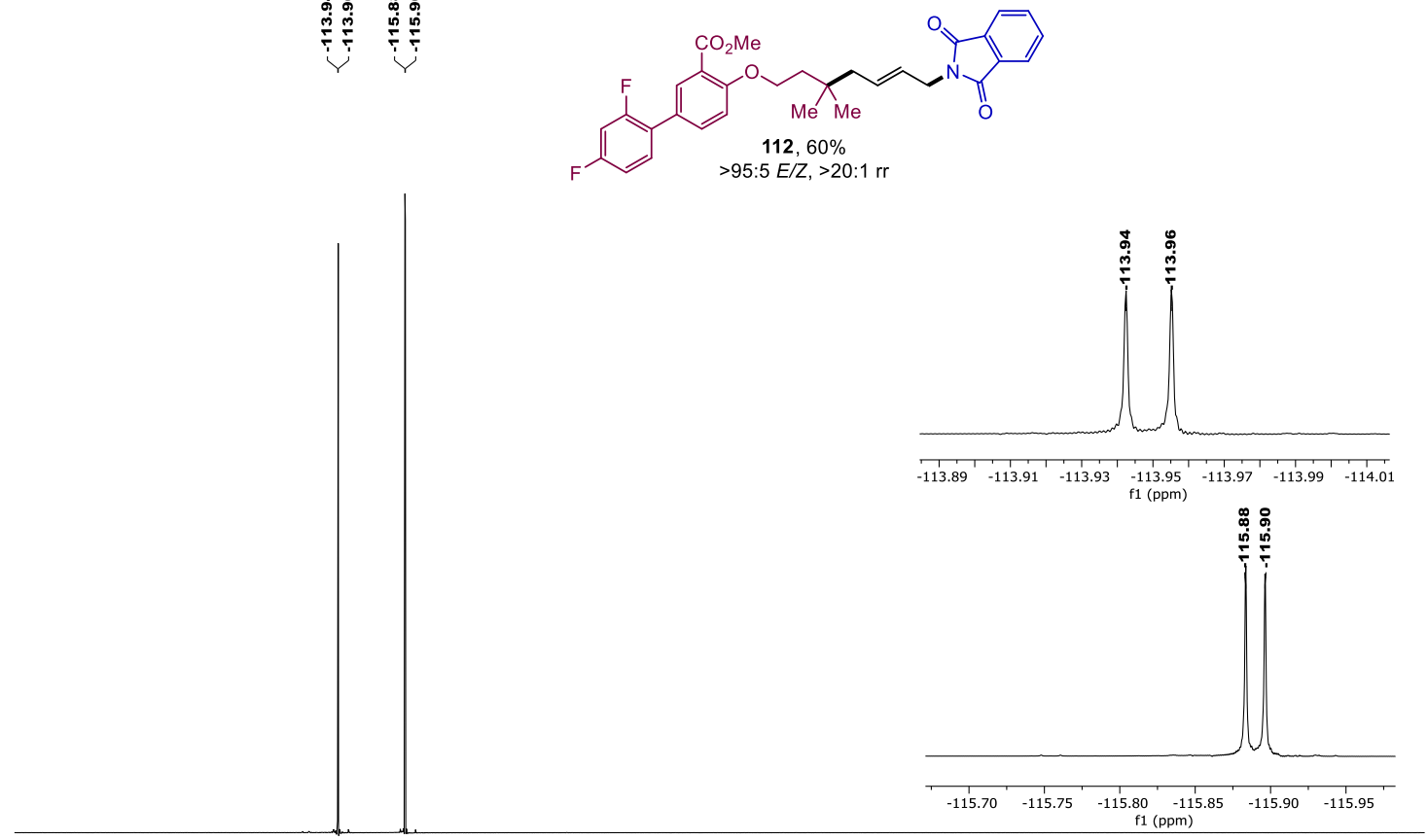

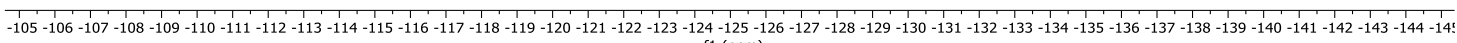
f1 (ppm) 
${ }^{1} \mathrm{H}$ NMR (599 MHz, $\mathrm{CDCl}_{3}$ )
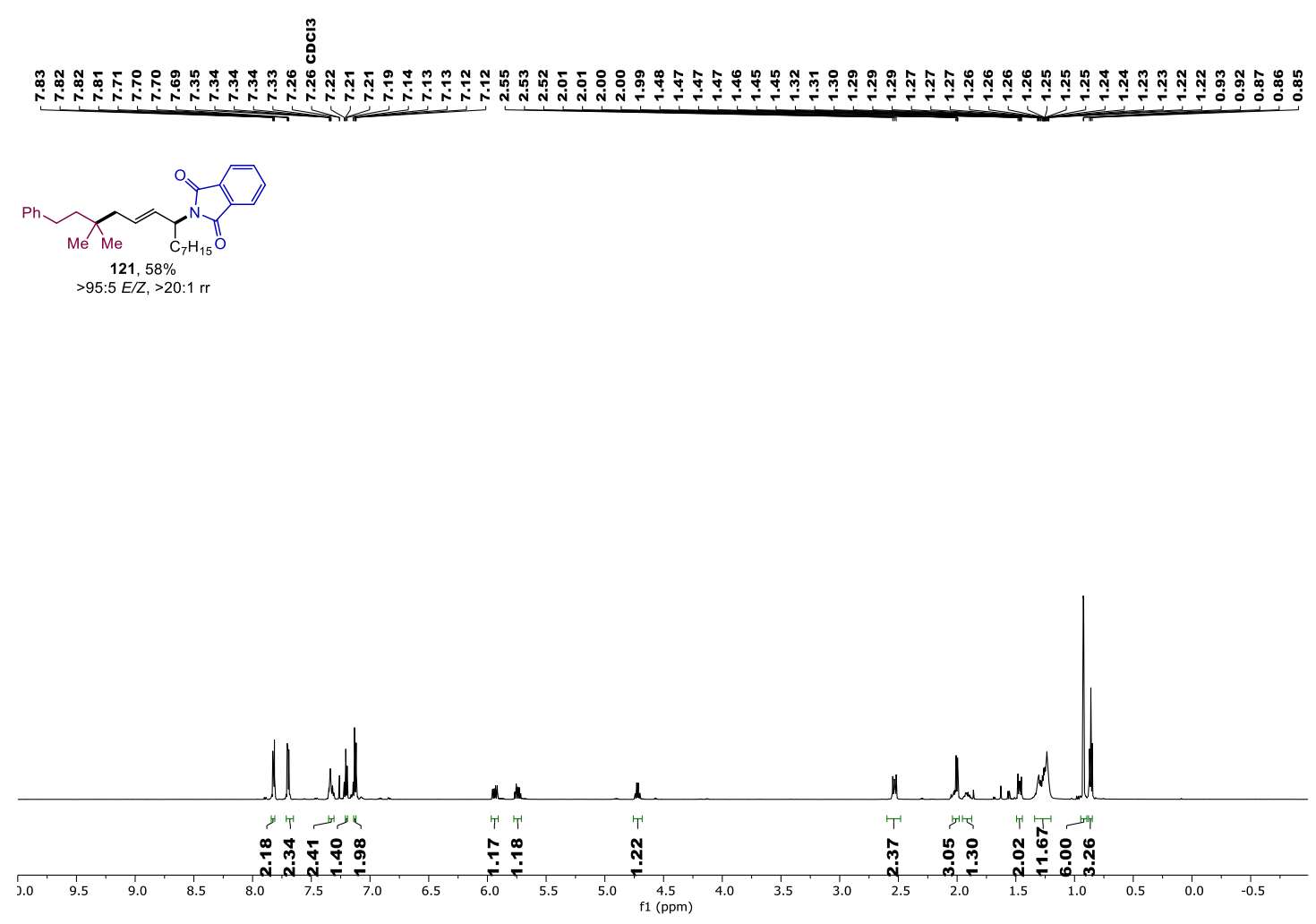

${ }^{13} \mathrm{C}$ NMR $\left(151 \mathrm{MHz}, \mathrm{CDCl}_{3}\right)$
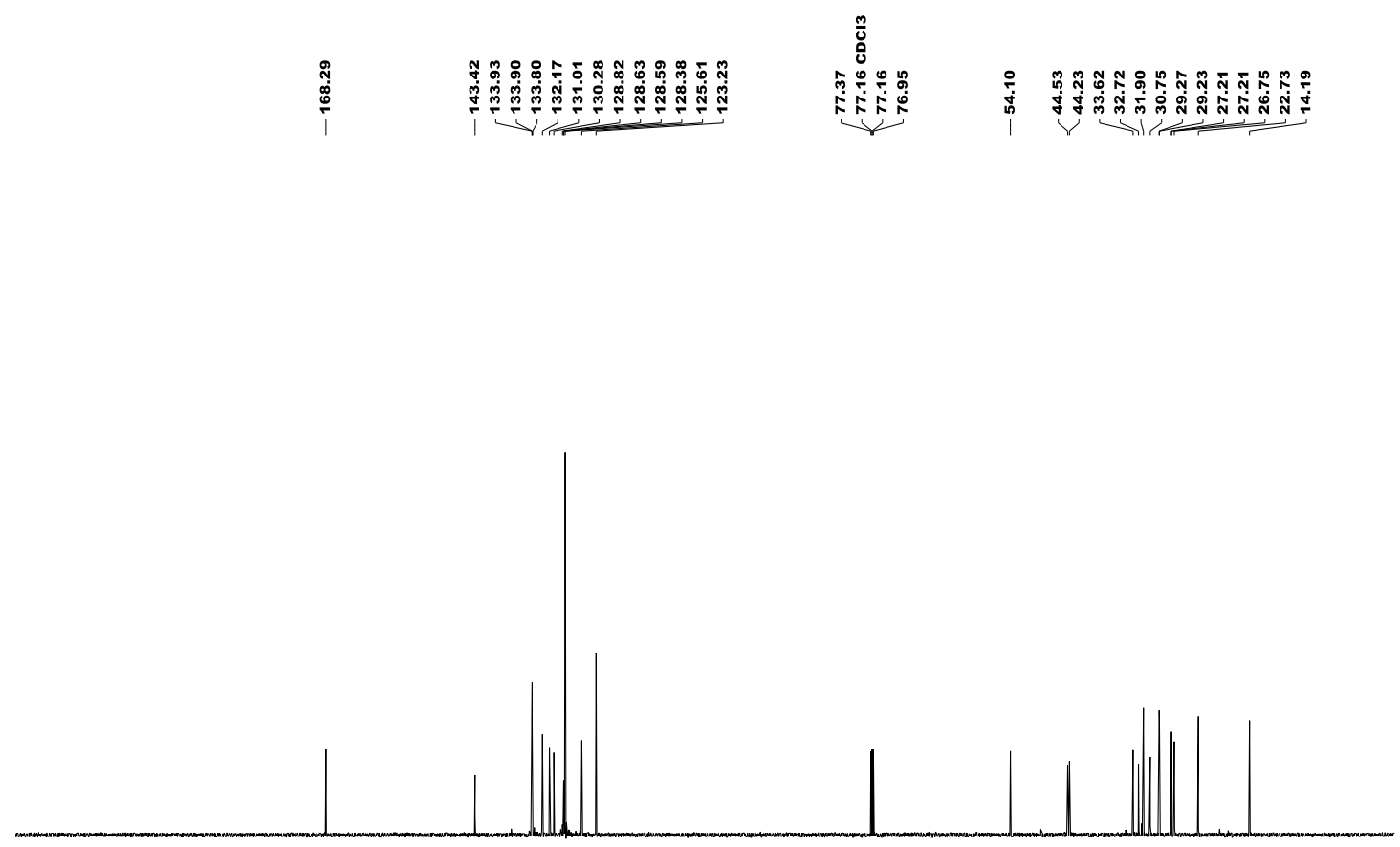

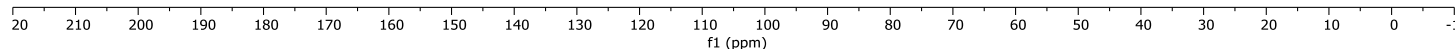


${ }^{1} \mathrm{H} \mathrm{NMR}\left(500 \mathrm{MHz}, \mathrm{CDCl}_{3}\right)$

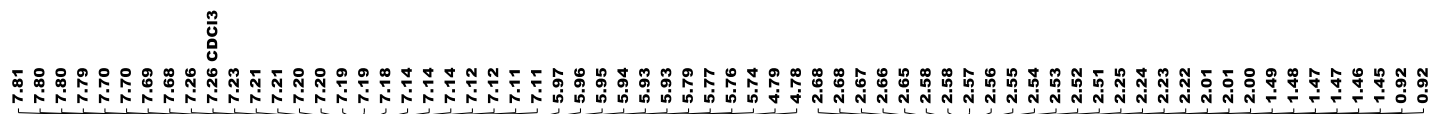
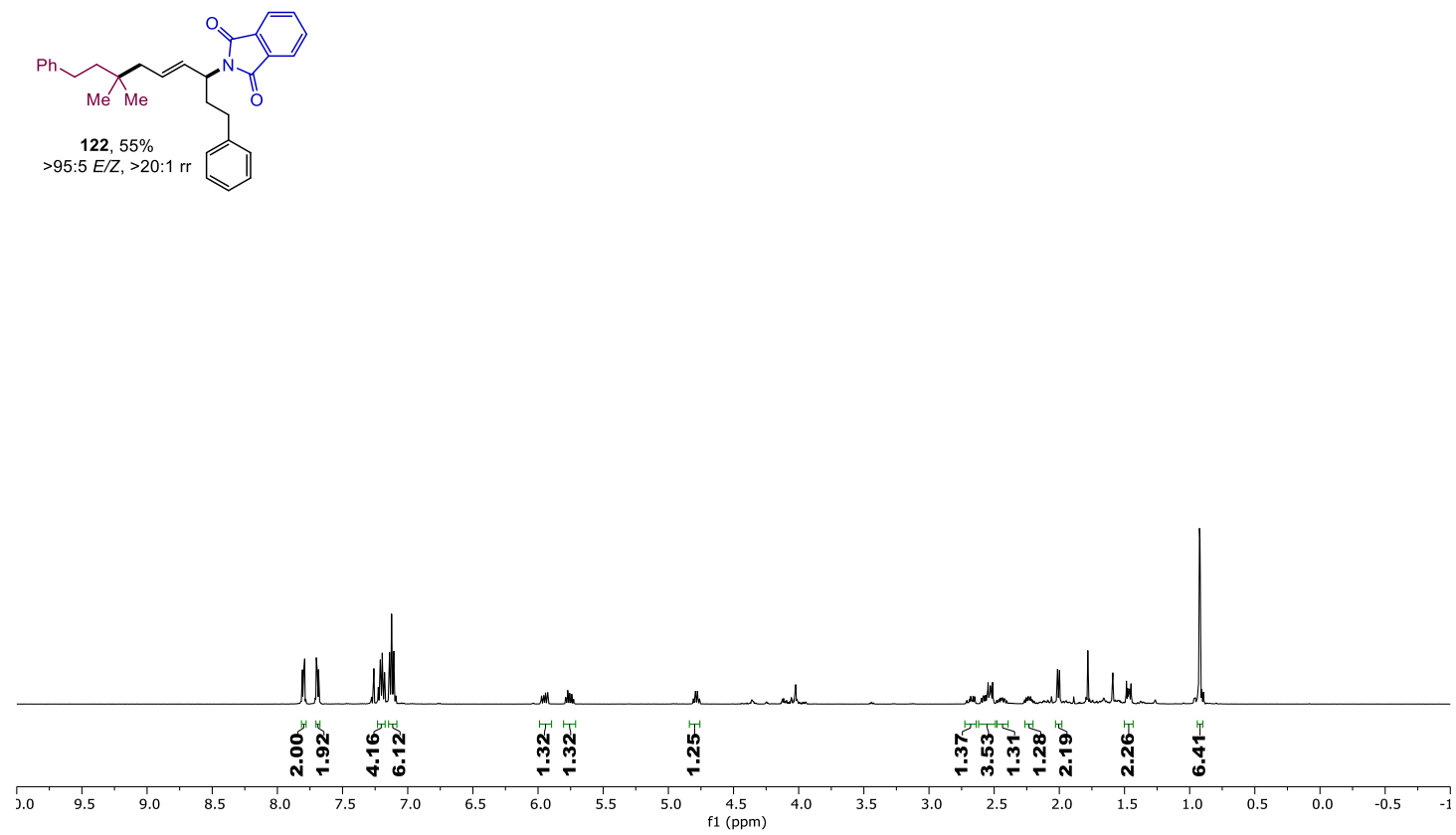

${ }^{13} \mathrm{C}$ NMR $\left(126 \mathrm{MHz}, \mathrm{CDCl}_{3}\right)$
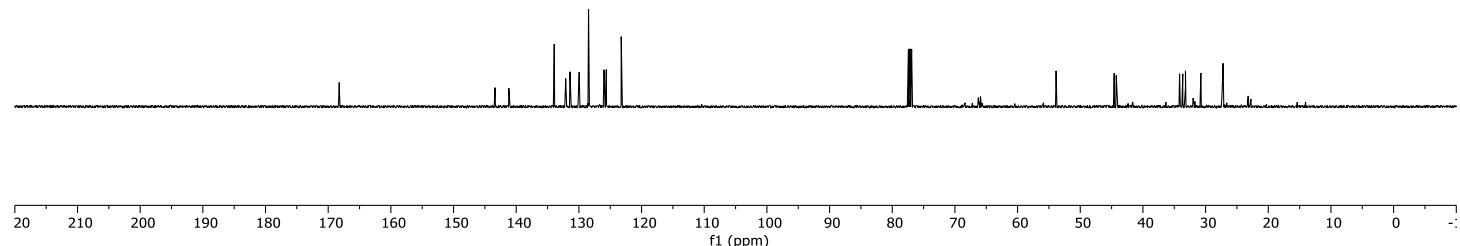
${ }^{1} \mathrm{H}$ NMR $\left(599 \mathrm{MHz}, \mathrm{CDCl}_{3}\right)$

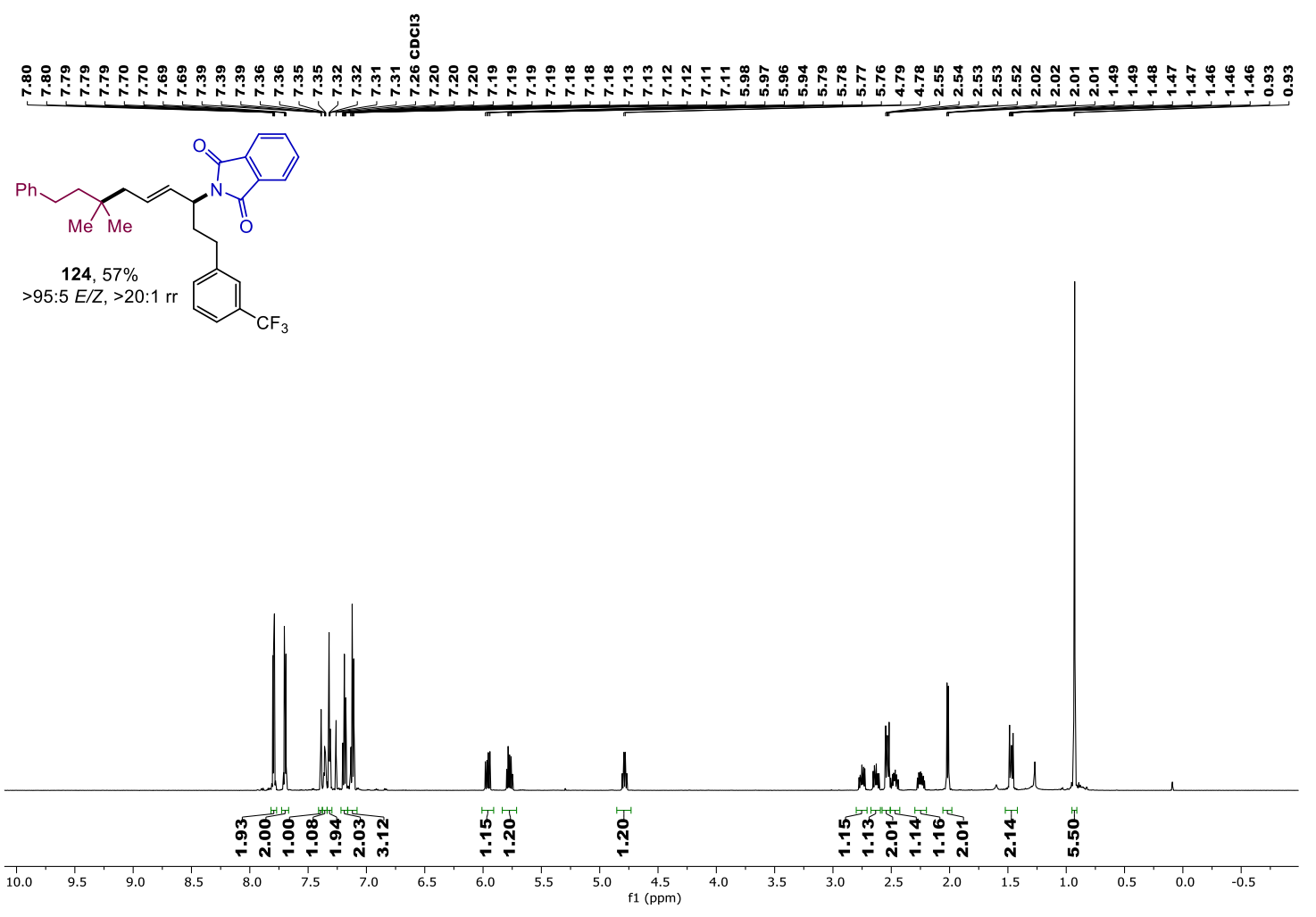

${ }^{13} \mathrm{C}\left\{{ }^{1} \mathrm{H},{ }^{19} \mathrm{~F}\right\}$ NMR $\left(151 \mathrm{MHz}, \mathrm{CDCl}_{3}\right)$
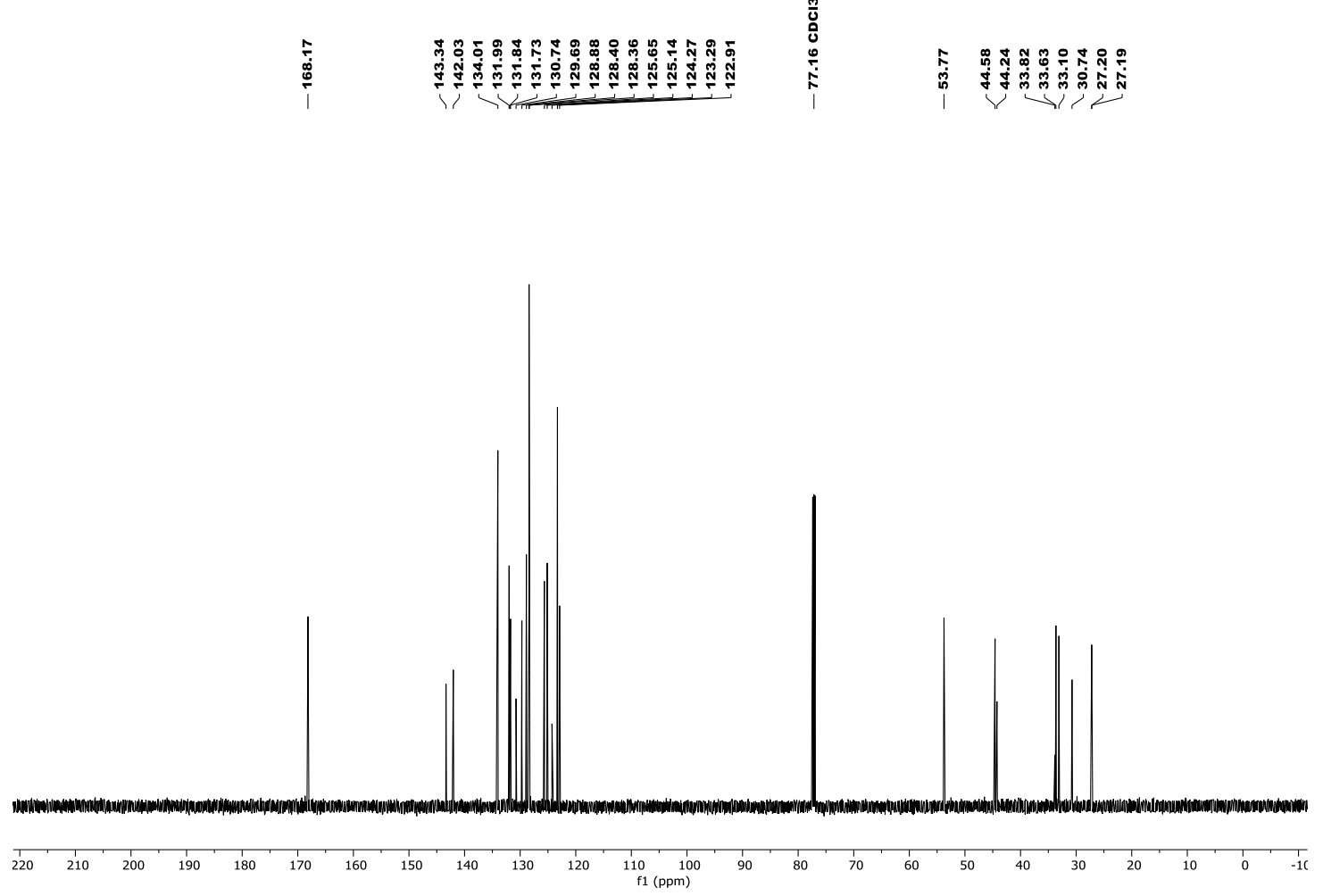
${ }^{19} \mathrm{~F}\left\{{ }^{1} \mathrm{H}\right\}$ NMR (564 MHz, $\left.\mathrm{CDCl}_{3}\right)$

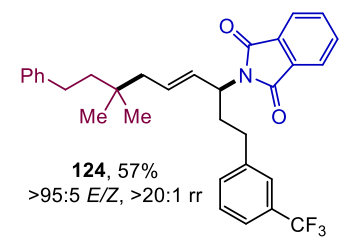

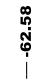

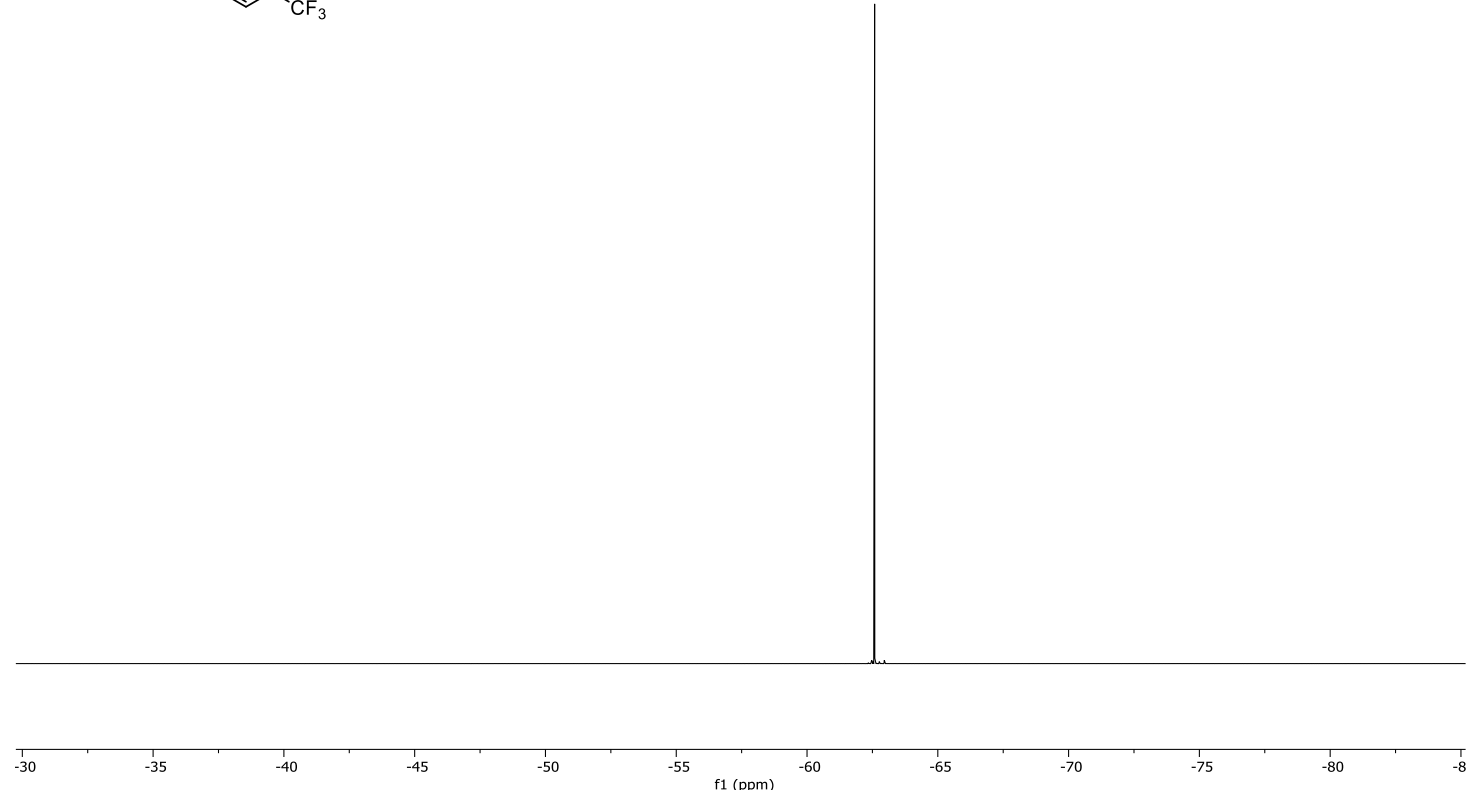


${ }^{1} \mathrm{H}$ NMR $\left(500 \mathrm{MHz}, \mathrm{CDCl}_{3}\right)$
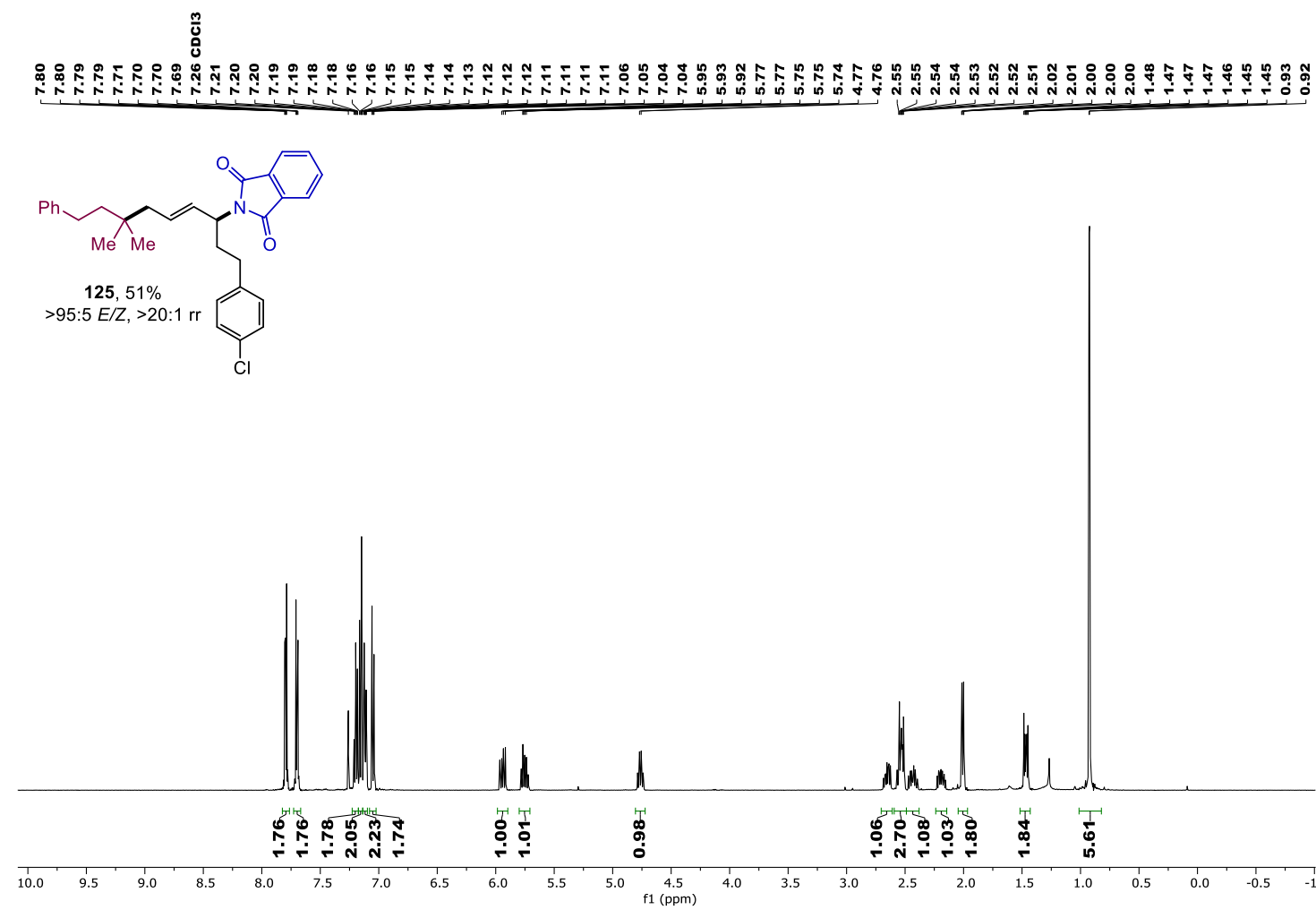

${ }^{13} \mathrm{C}$ NMR $\left(126 \mathrm{MHz}, \mathrm{CDCl}_{3}\right)$
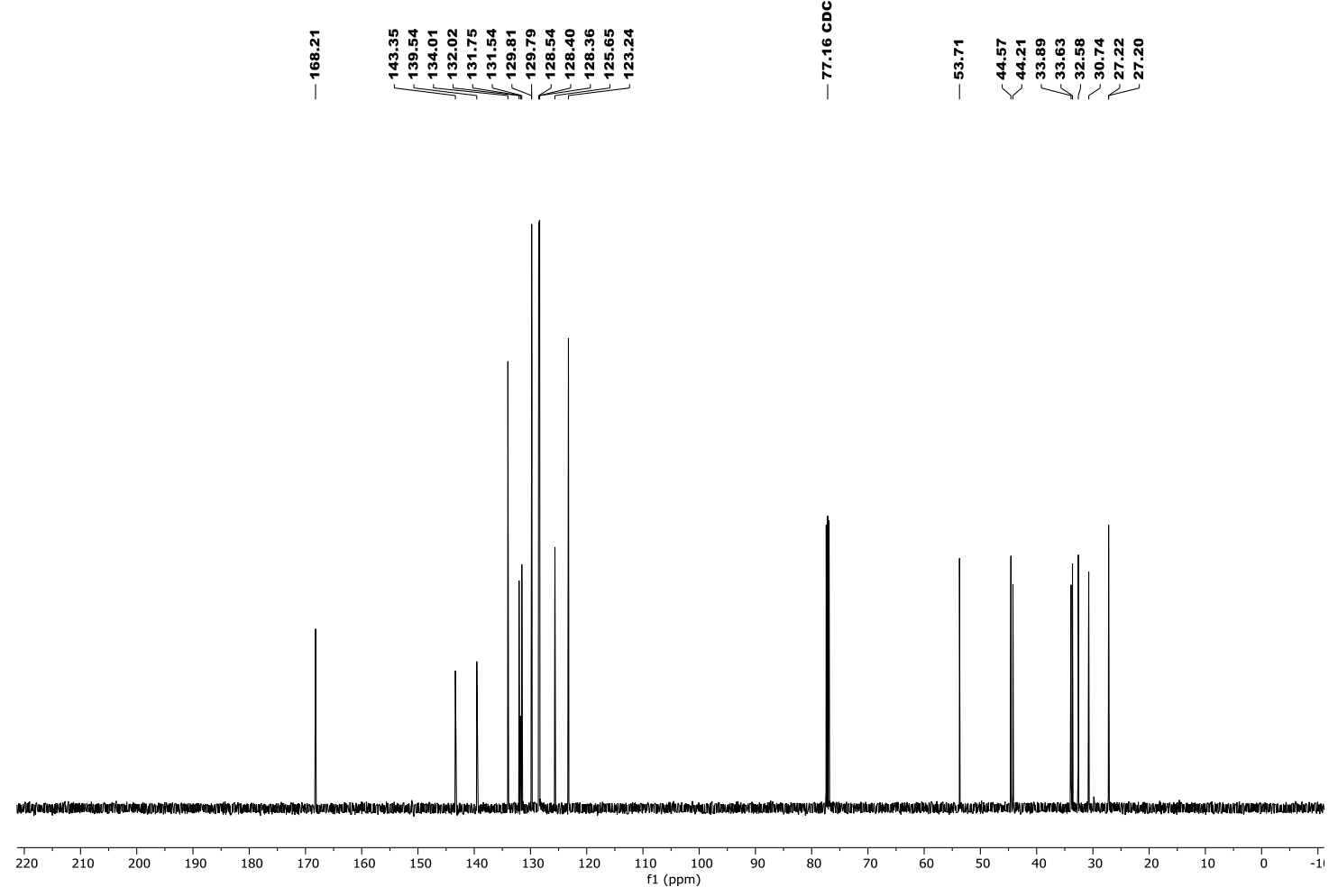
${ }^{1} \mathrm{H}$ NMR $\left(500 \mathrm{MHz}, \mathrm{CDCl}_{3}\right)$

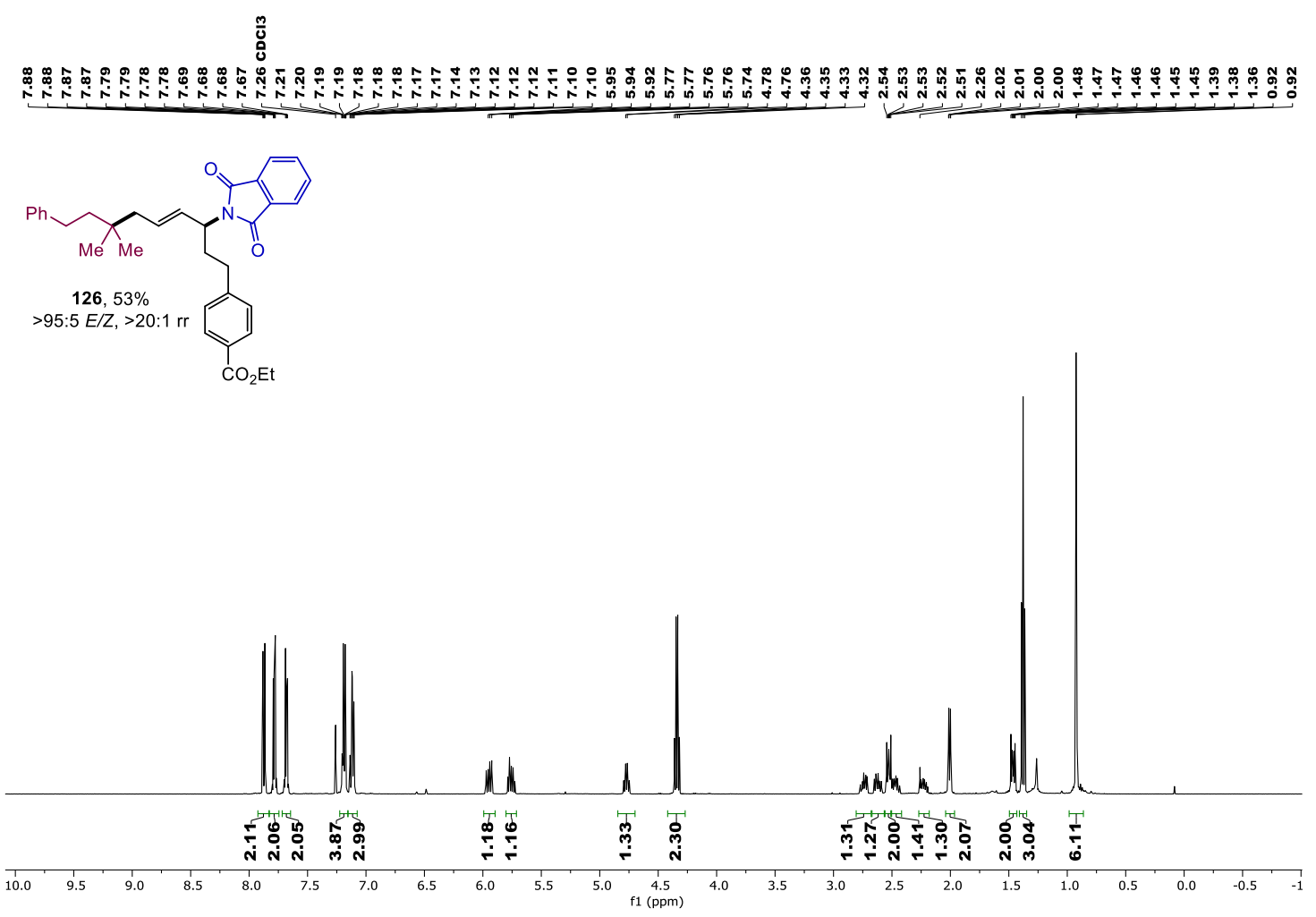

${ }^{13} \mathrm{C}$ NMR $\left(126 \mathrm{MHz}, \mathrm{CDCl}_{3}\right)$

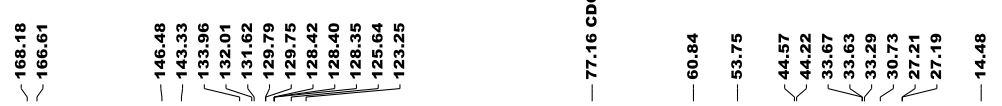

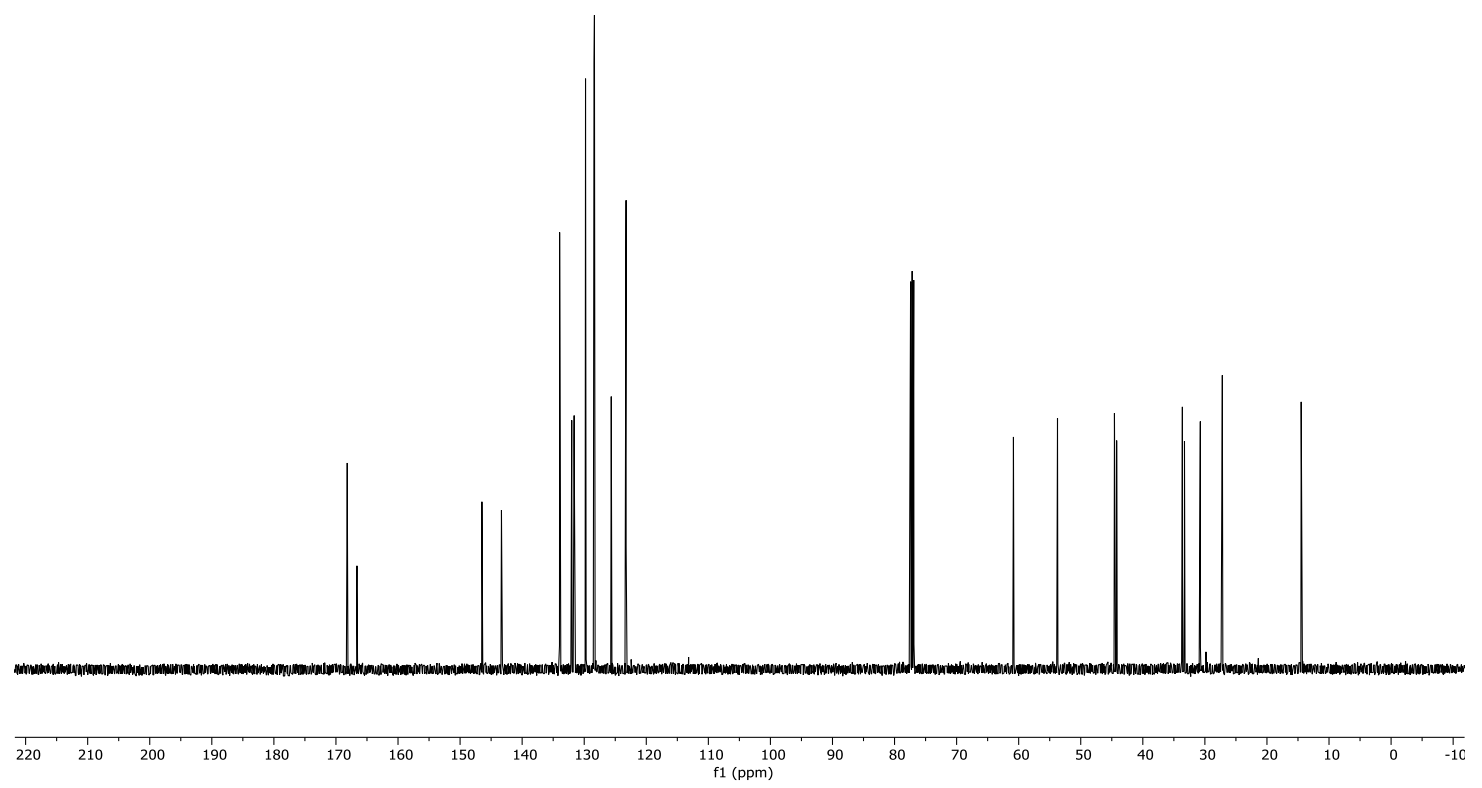


${ }^{1} \mathrm{H}$ NMR $\left(500 \mathrm{MHz}, \mathrm{CDCl}_{3}\right)$
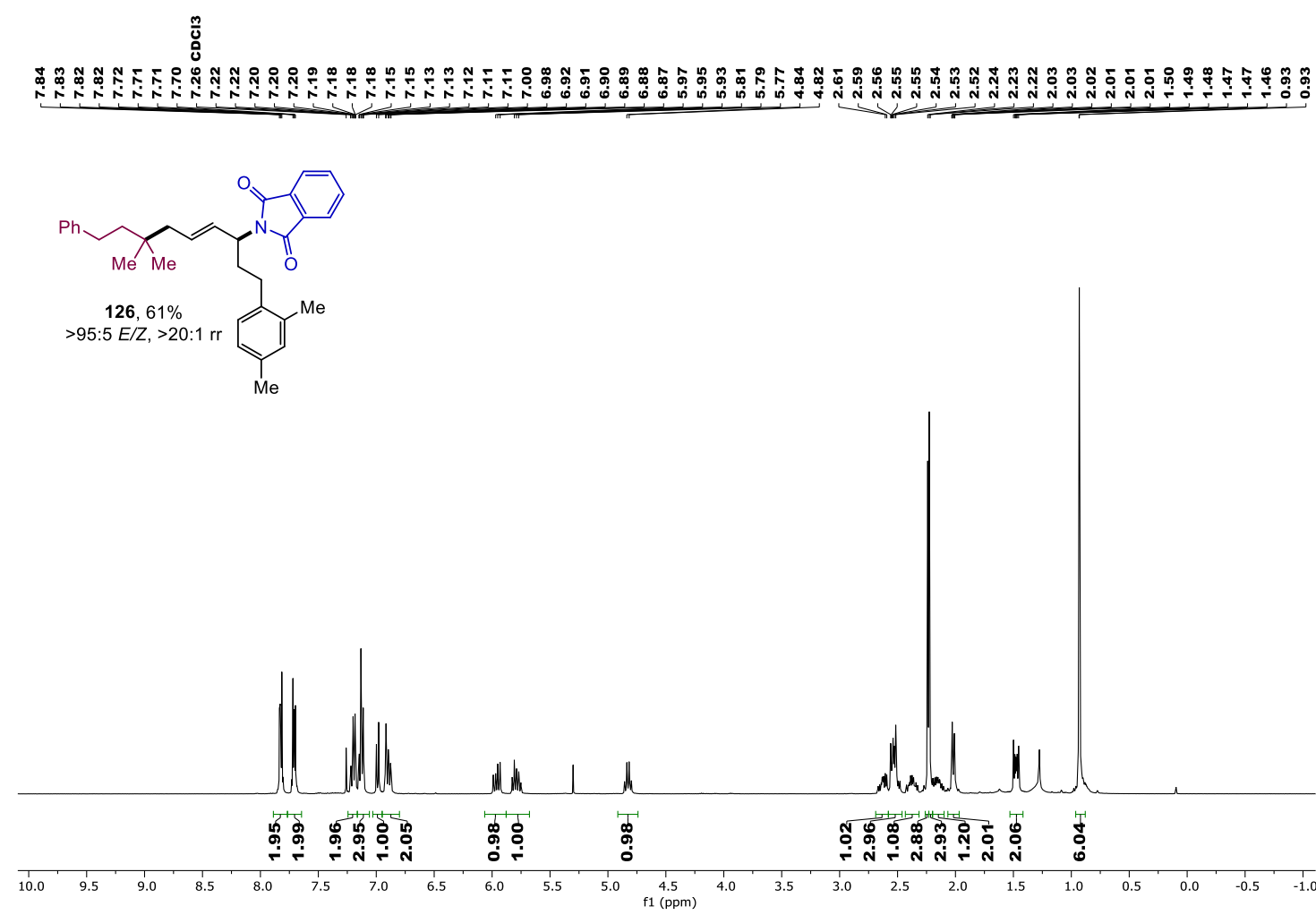

${ }^{13} \mathrm{C} \mathrm{NMR}\left(126 \mathrm{MHz}, \mathrm{CDCl}_{3}\right)$

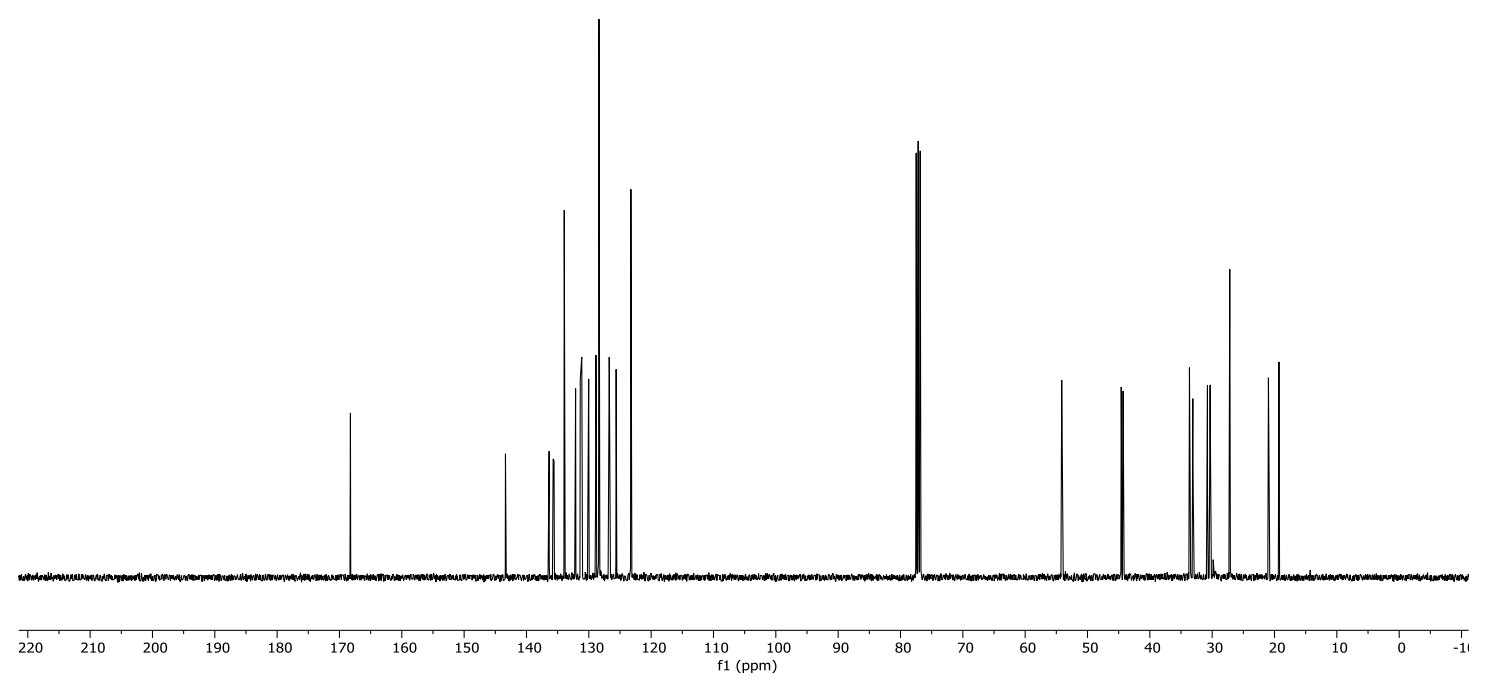


${ }^{1} \mathrm{H} \mathrm{NMR}\left(400 \mathrm{MHz}, \mathrm{CDCl}_{3}\right)$
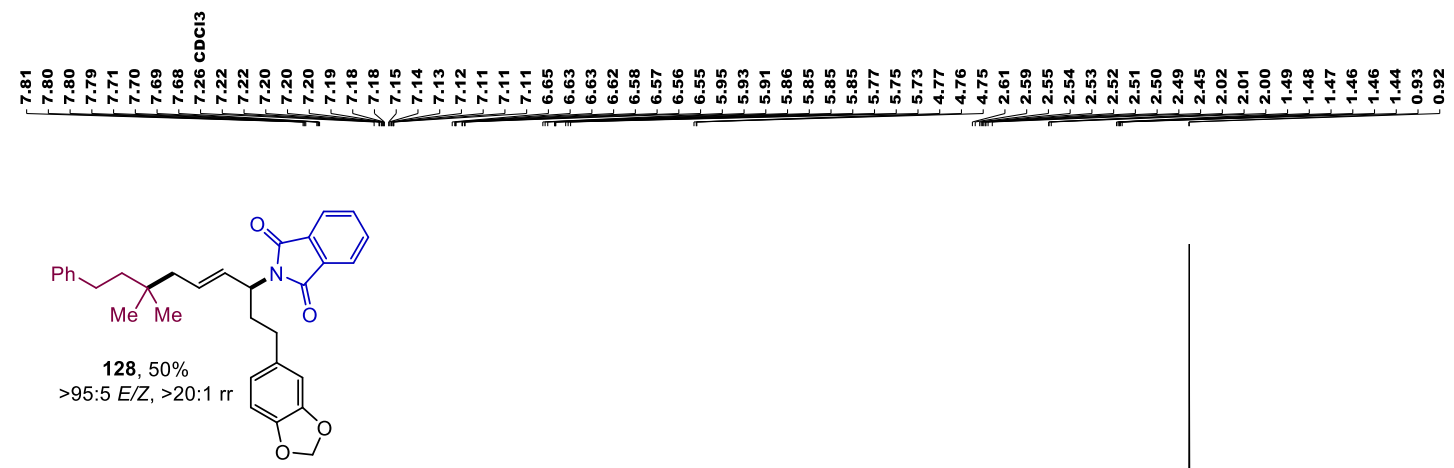

${ }^{13} \mathrm{C}$ NMR $\left(101 \mathrm{MHz}, \mathrm{CDCl}_{3}\right)$
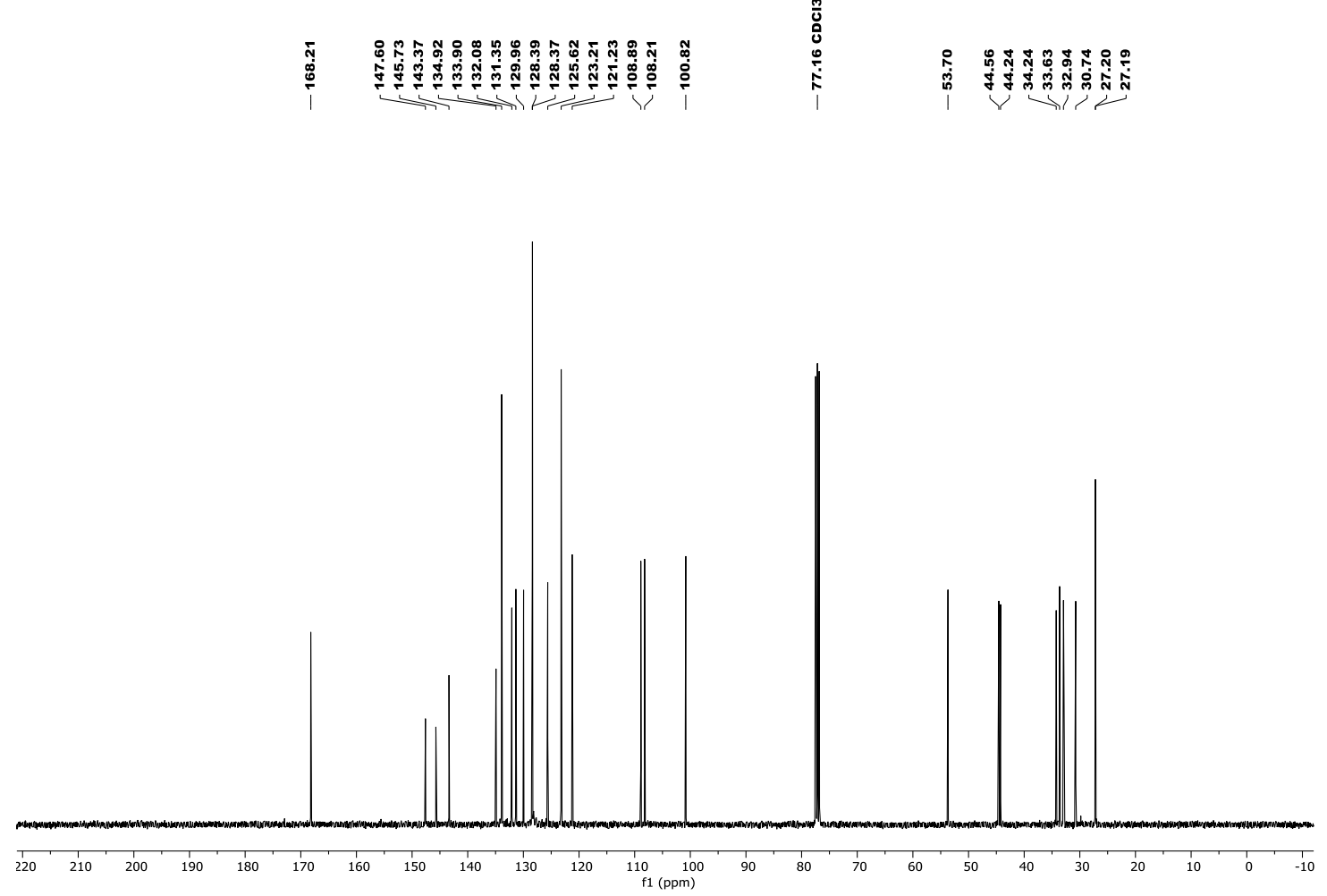
${ }^{1} \mathrm{H} \mathrm{NMR}\left(400 \mathrm{MHz}, \mathrm{CDCl}_{3}\right)$

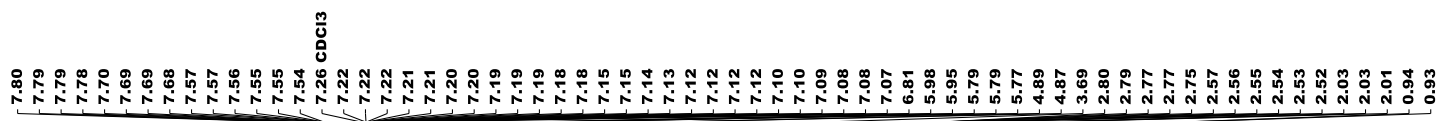
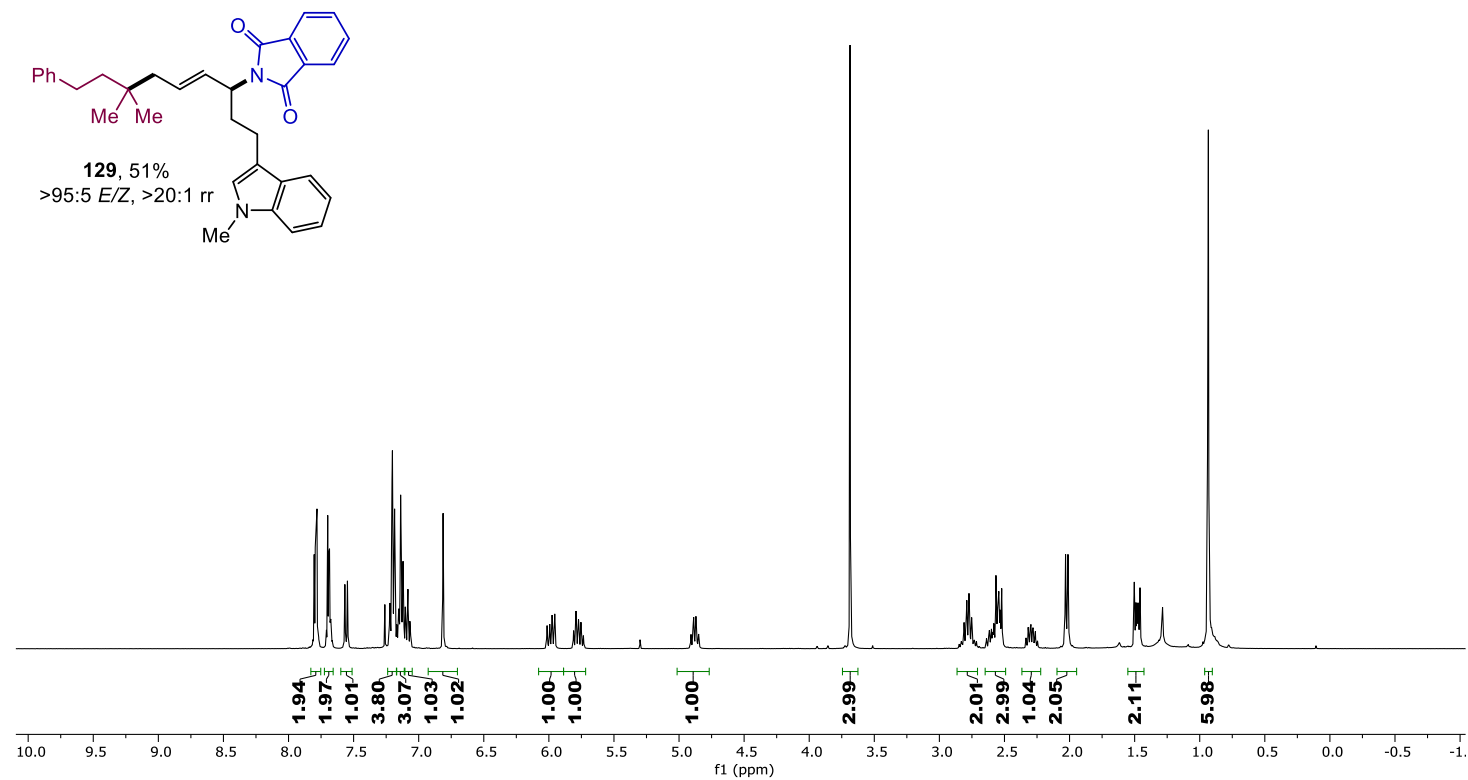

${ }^{13} \mathrm{C}$ NMR (101 MHz, $\mathrm{CDCl}_{3}$ )
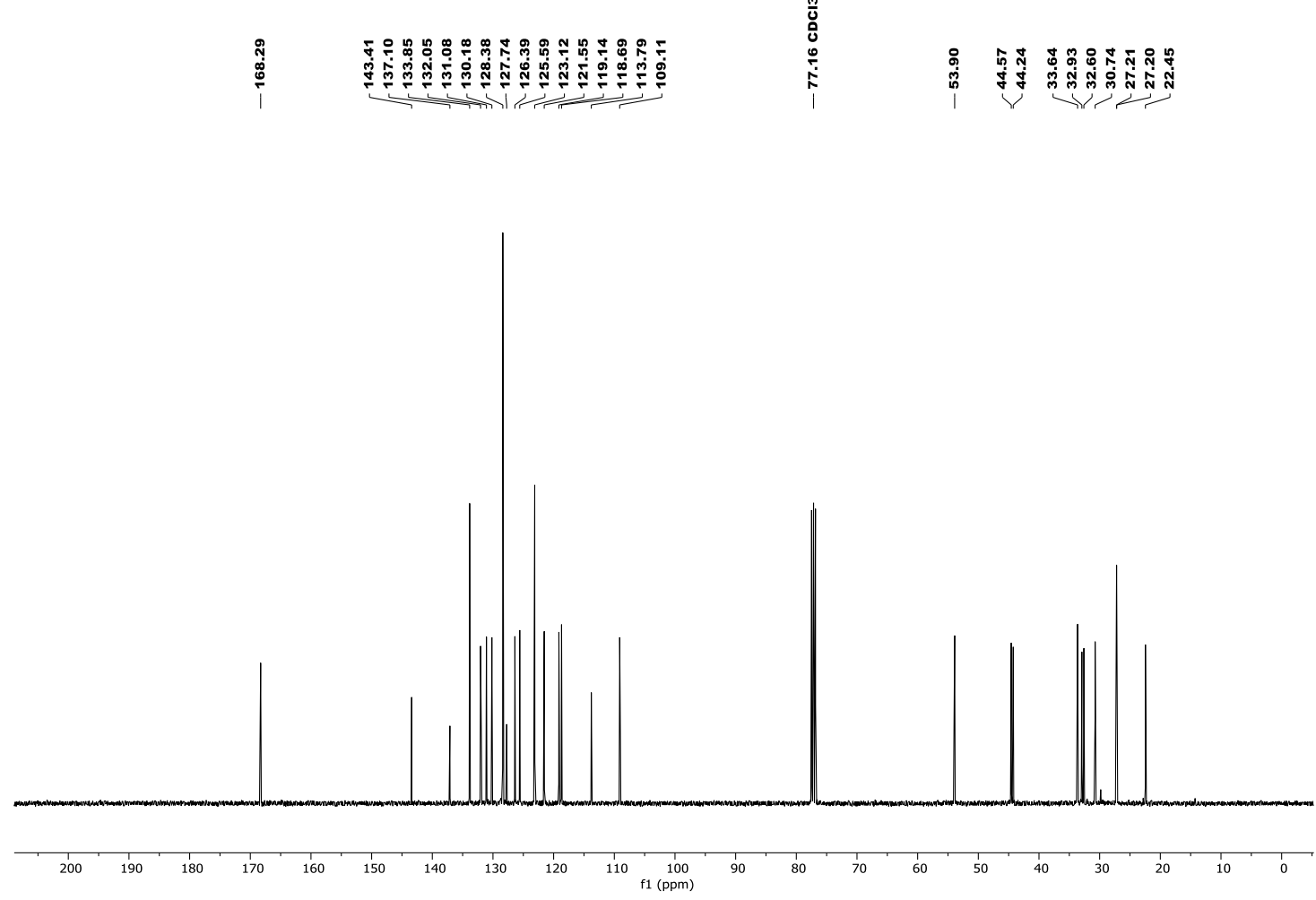
${ }^{1} \mathrm{H} \mathrm{NMR}\left(400 \mathrm{MHz}, \mathrm{CDCl}_{3}\right)$

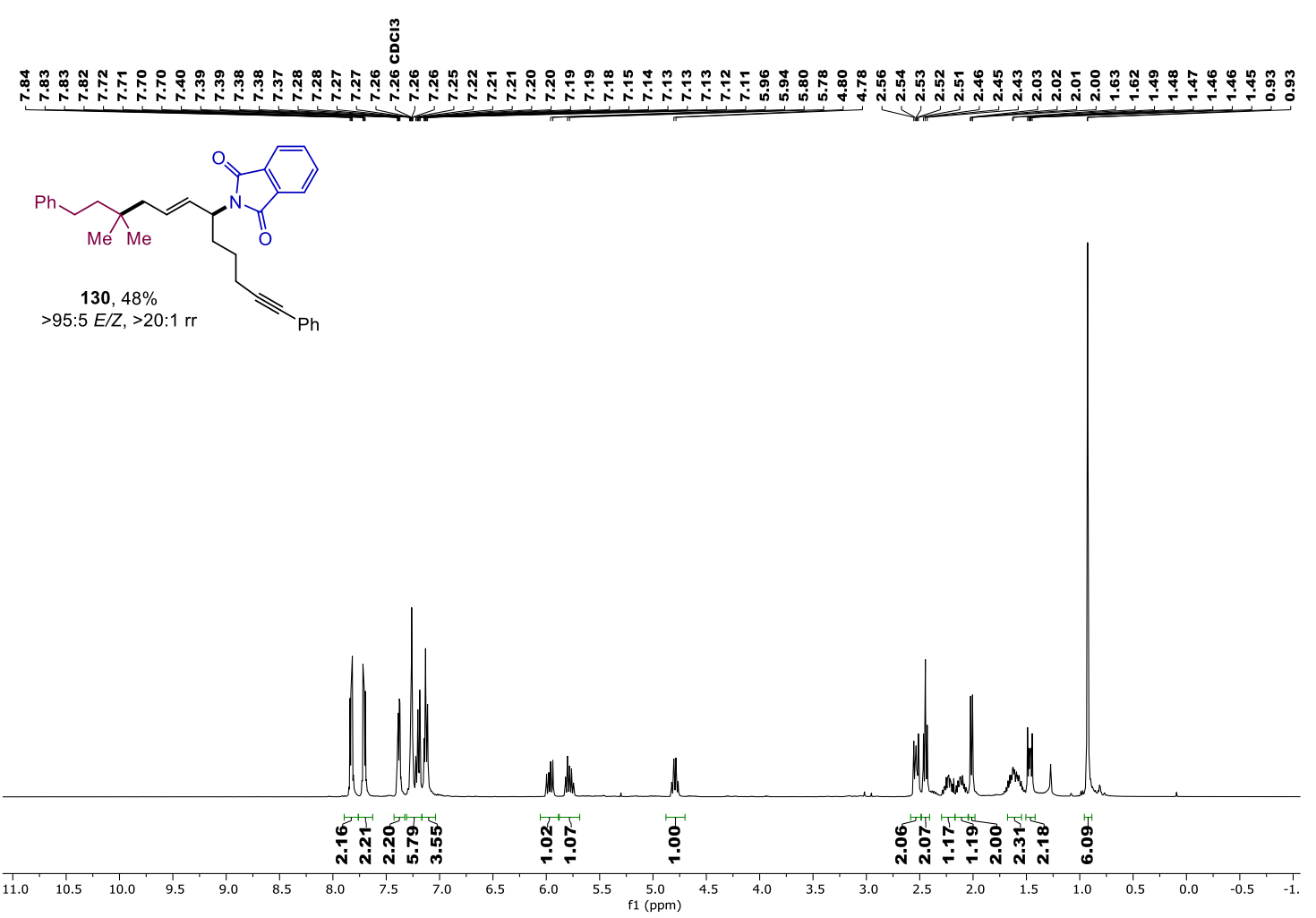

${ }^{13} \mathrm{C}$ NMR (101 MHz, $\mathrm{CDCl}_{3}$ )

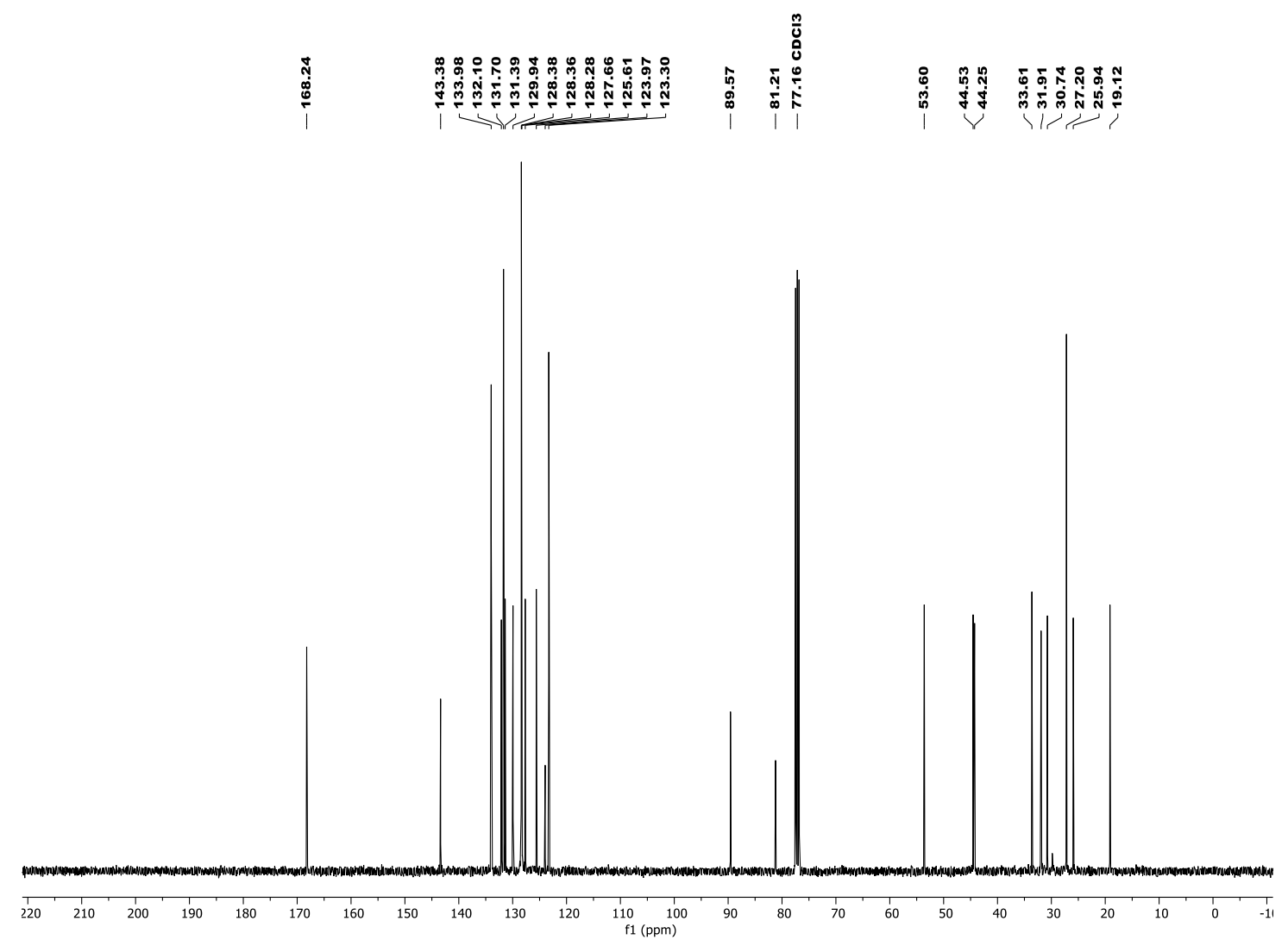


${ }^{1} \mathrm{H} \mathrm{NMR}\left(400 \mathrm{MHz}, \mathrm{CDCl}_{3}\right)$
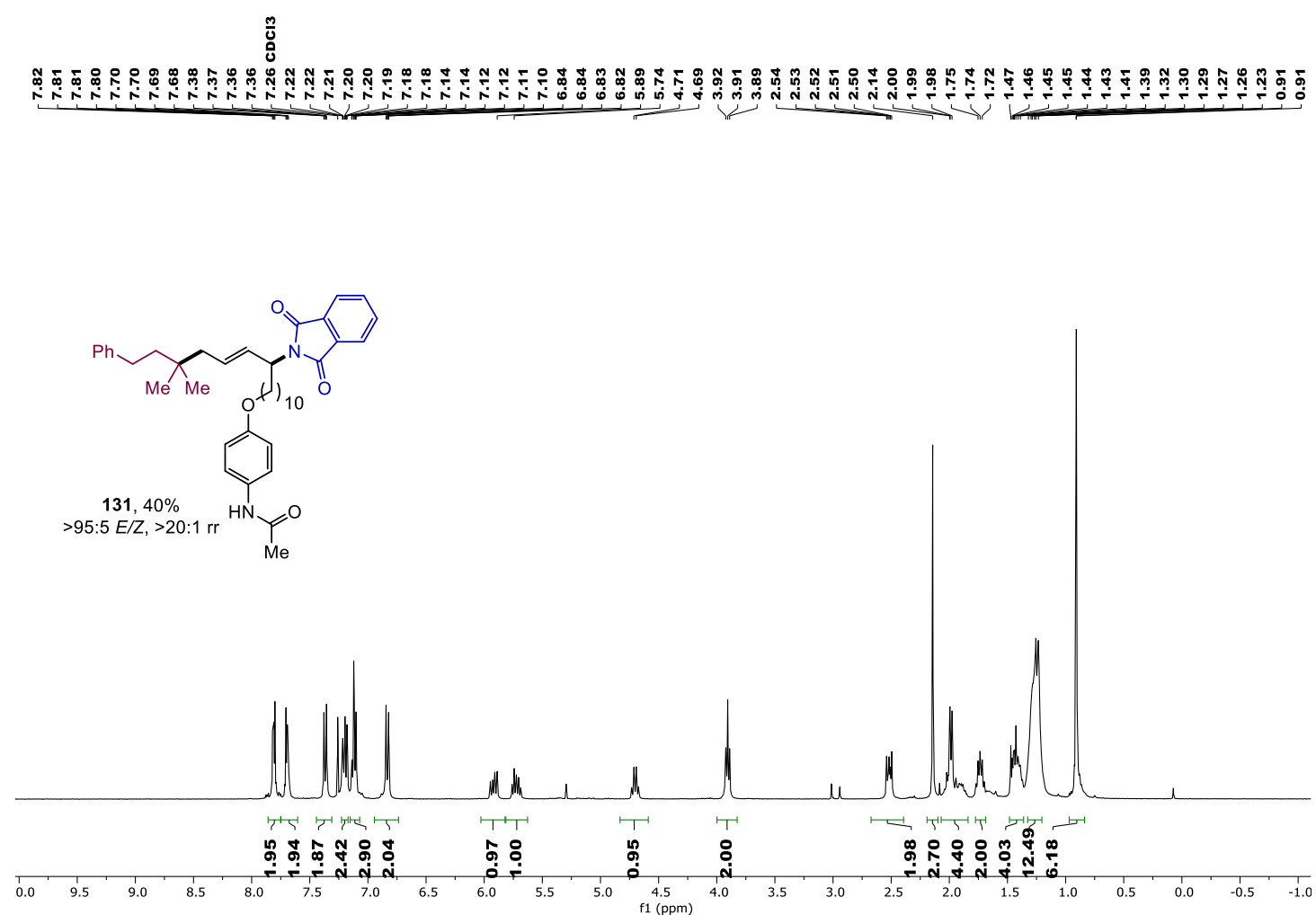

${ }^{13} \mathrm{C}$ NMR (101 MHz, $\left.\mathrm{CDCl}_{3}\right)$
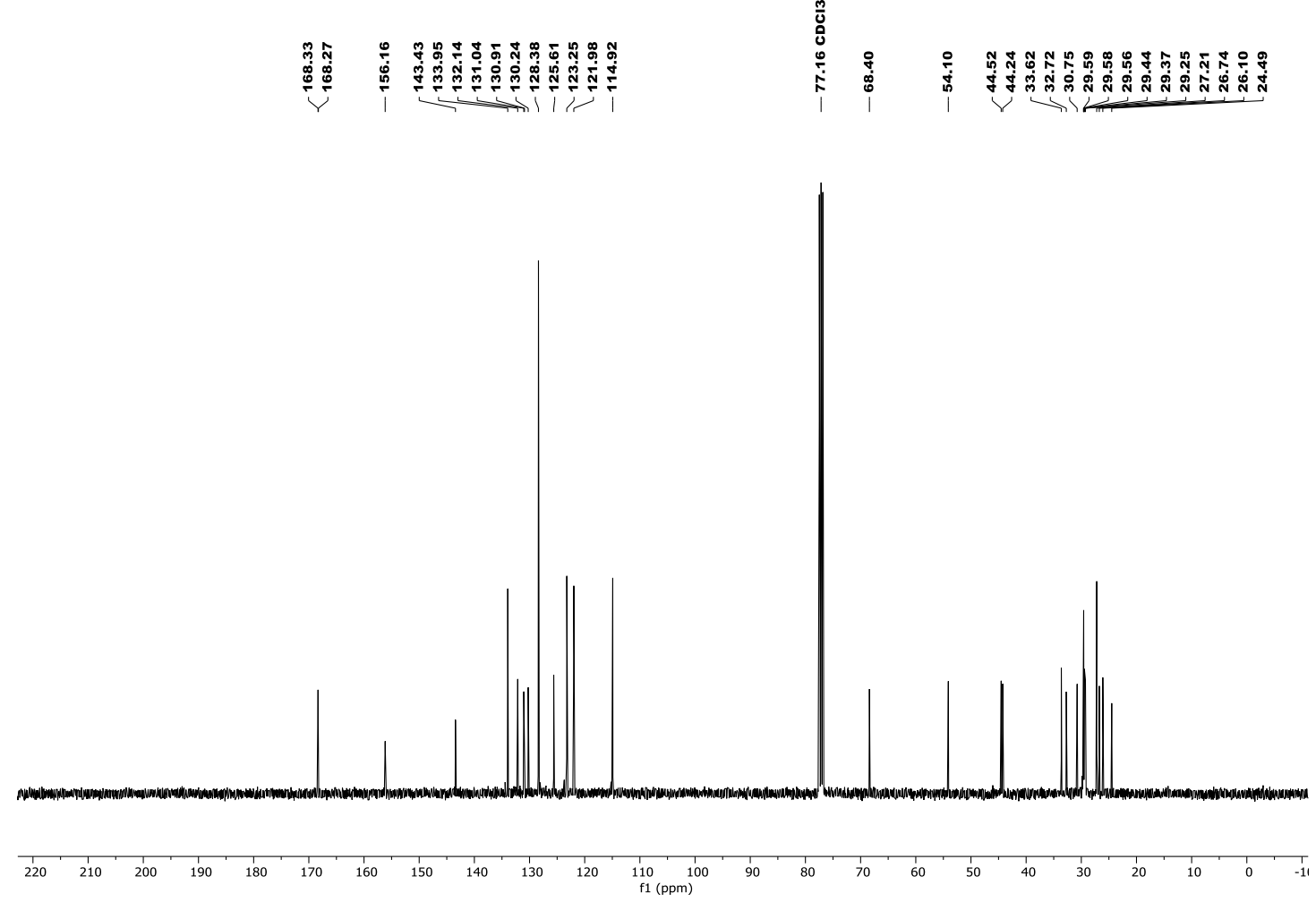
${ }^{1} \mathrm{H} \mathrm{NMR}\left(400 \mathrm{MHz}, \mathrm{CDCl}_{3}\right)$

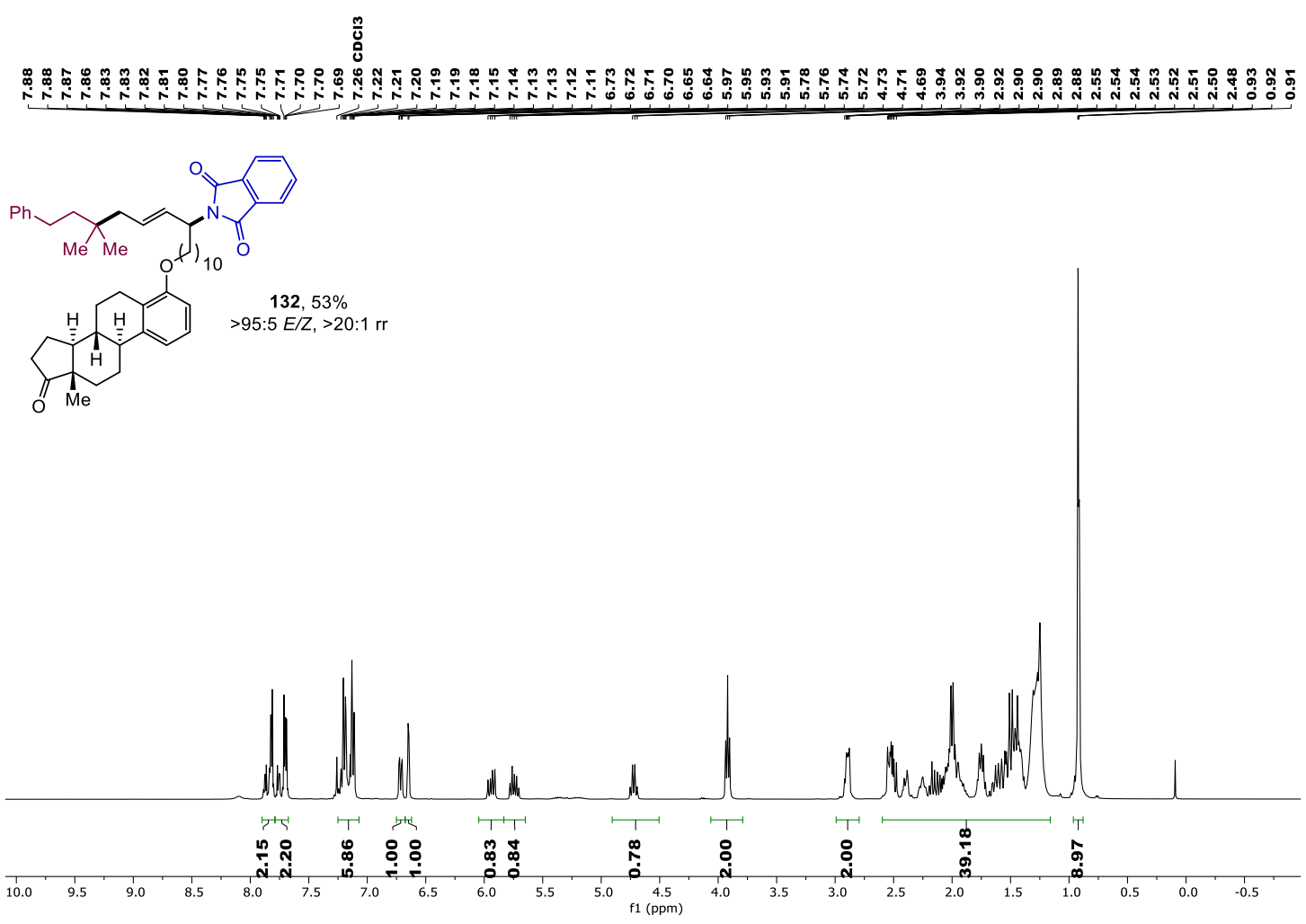

${ }^{13} \mathrm{C}$ NMR (101 MHz, $\left.\mathrm{CDCl}_{3}\right)$
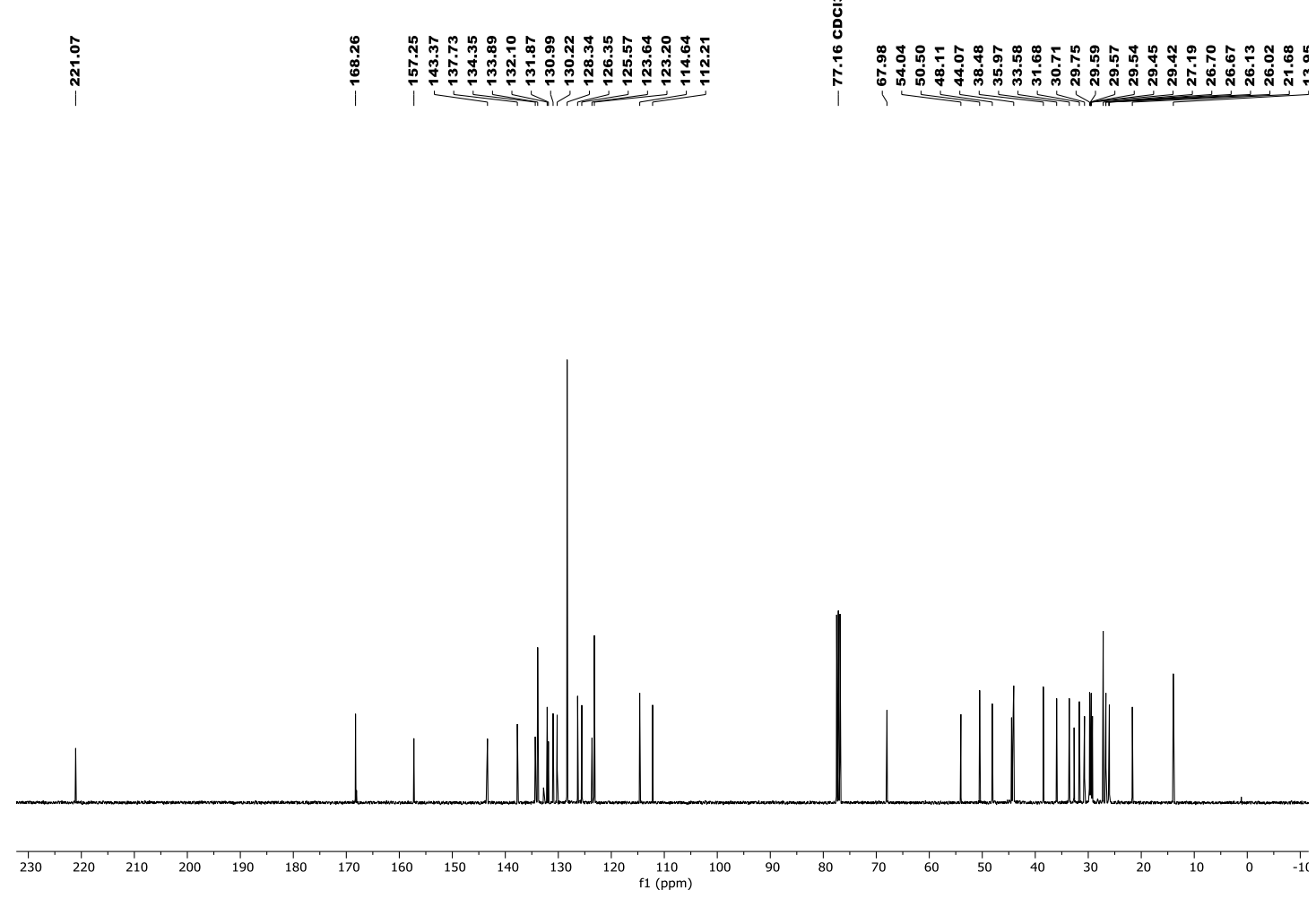
${ }^{1} \mathrm{H}$ NMR $\left(300 \mathrm{MHz}, \mathrm{CDCl}_{3}\right)$
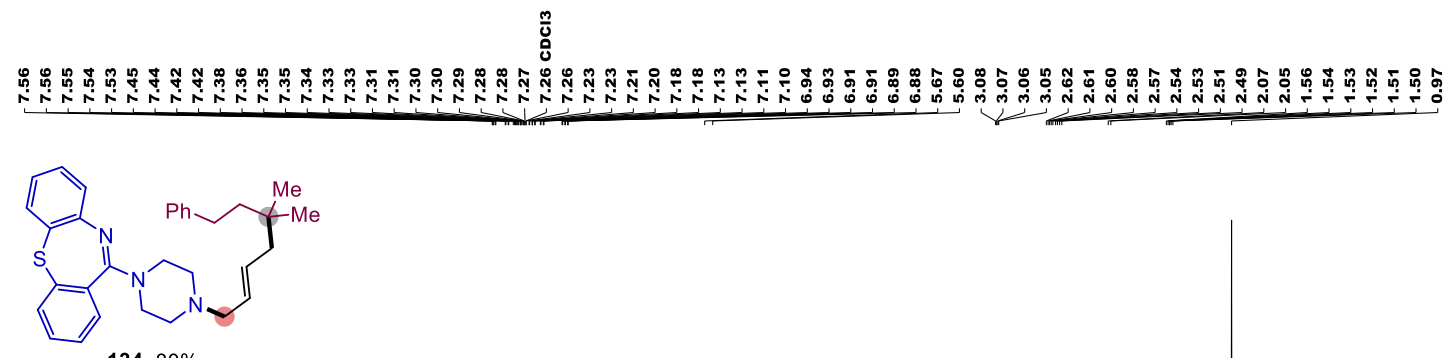

$134,80 \%$,

$>95: 5 \mathrm{E} / \mathrm{Z},>20: 1 \mathrm{rr}$

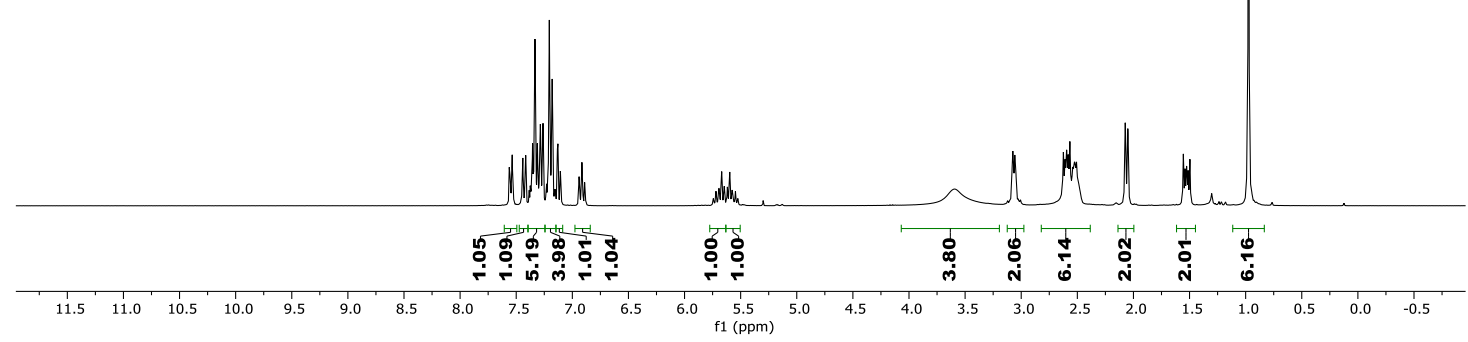

${ }^{13} \mathrm{C}$ NMR $\left(75 \mathrm{MHz}, \mathrm{CDCl}_{3}\right)$
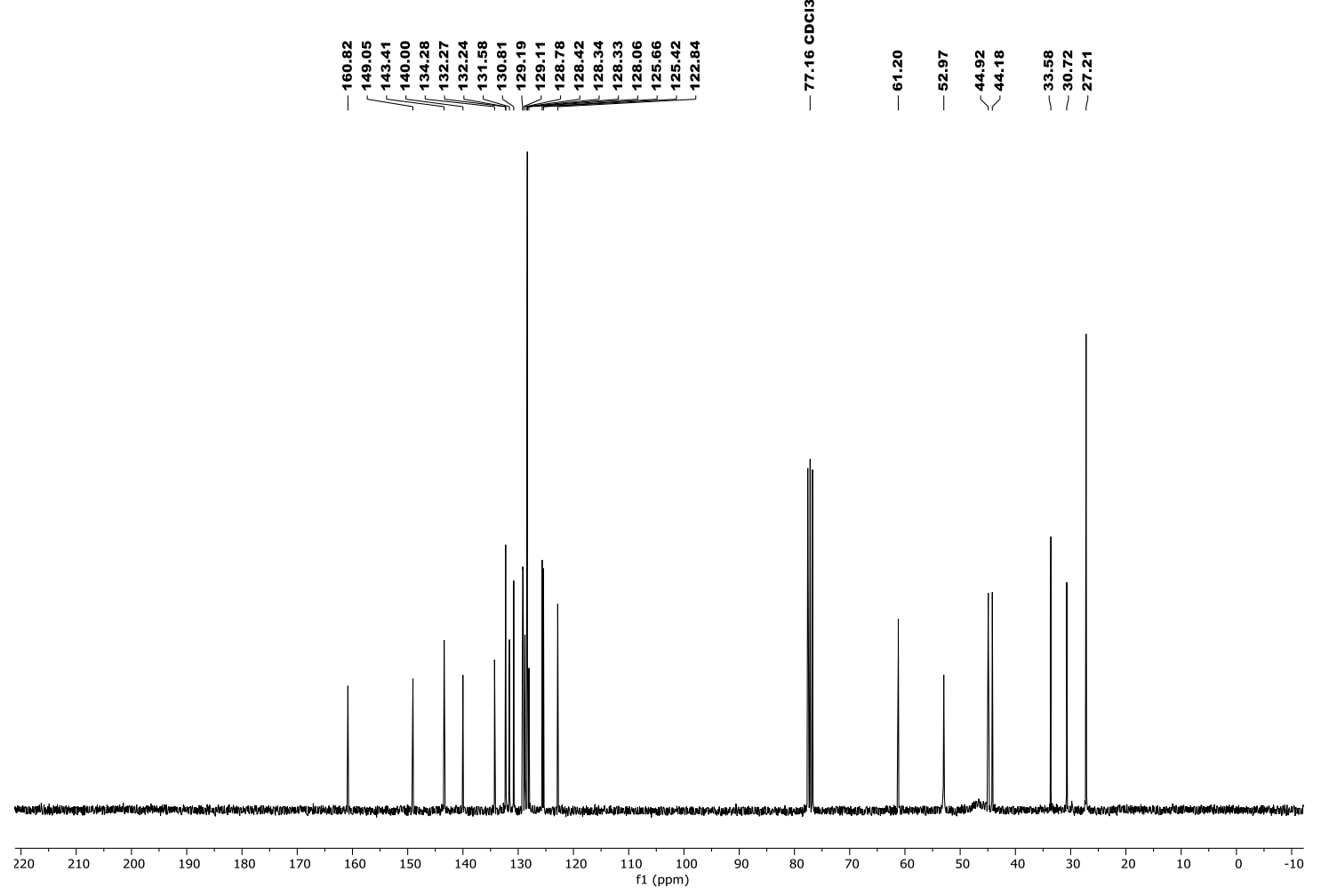
${ }^{1} \mathrm{H} \mathrm{NMR}\left(400 \mathrm{MHz}, \mathrm{CDCl}_{3}\right)$
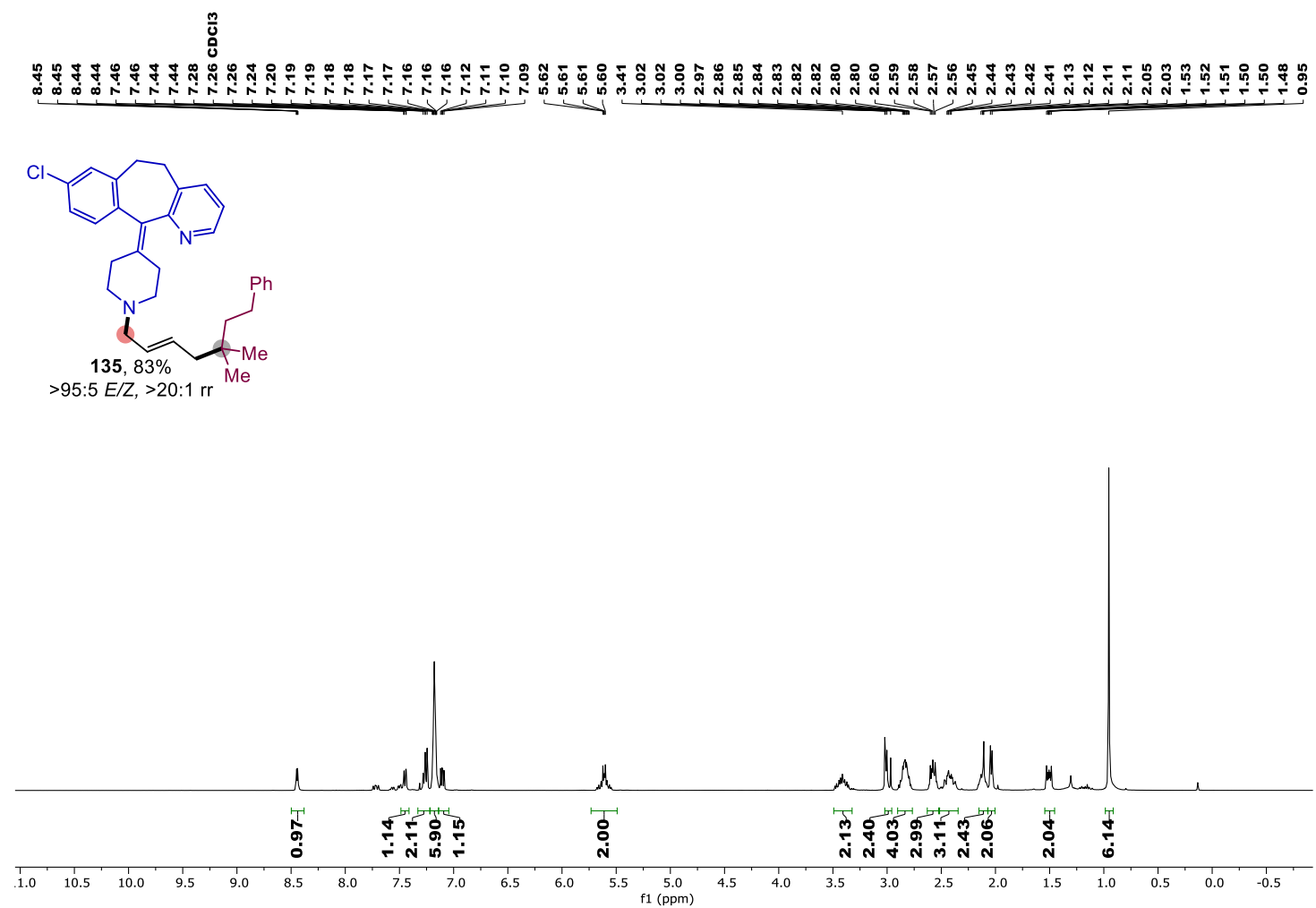

${ }^{13} \mathrm{C}$ NMR $\left(101 \mathrm{MHz}, \mathrm{CDCl}_{3}\right)$
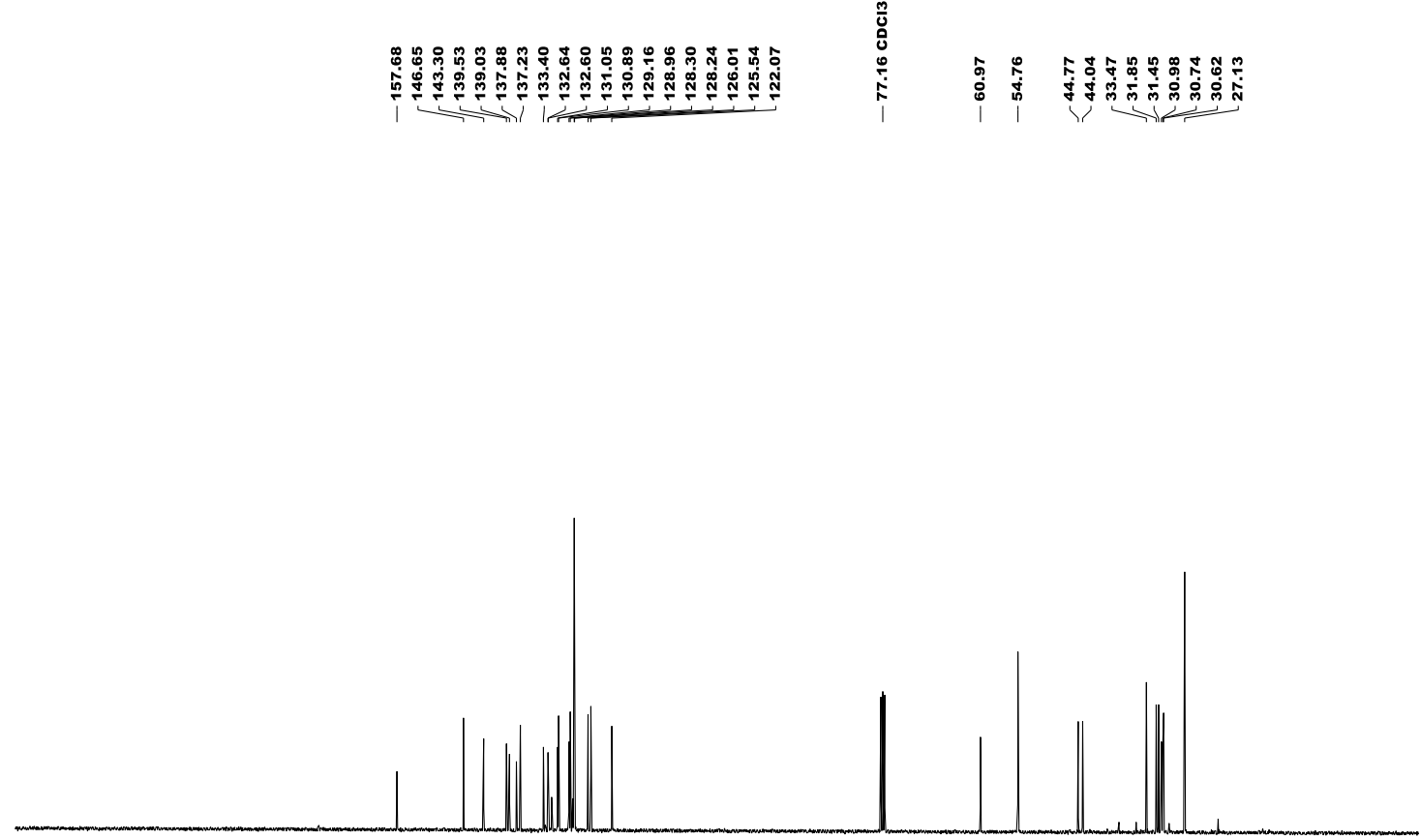

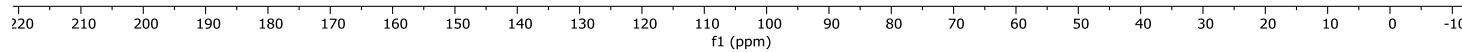


${ }^{1} \mathrm{H}$ NMR (400 MHz, $\mathrm{CDCl}_{3}$ )

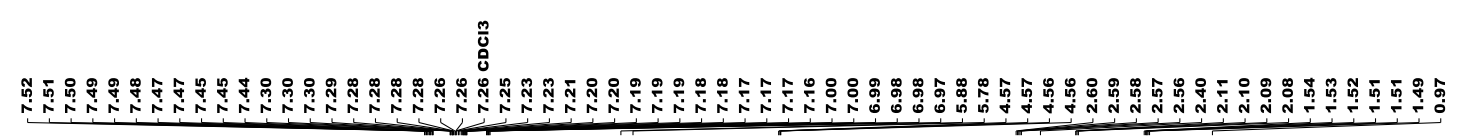

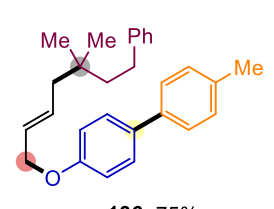

$136,75 \%$

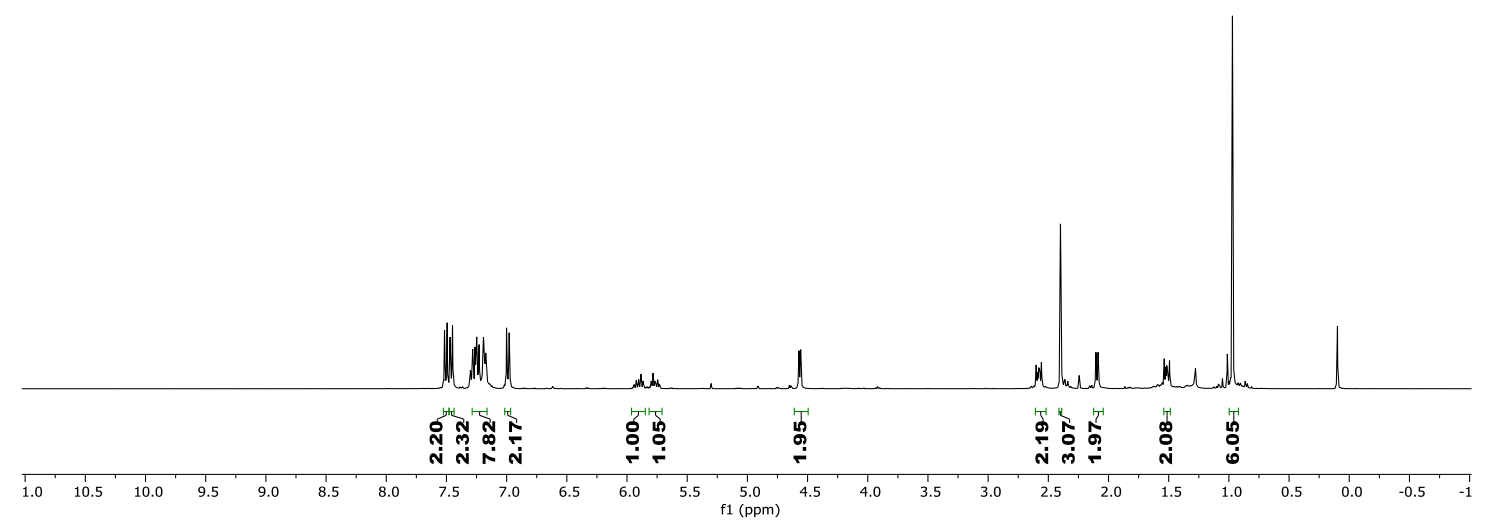

${ }^{13} \mathrm{C}$ NMR (101 MHz, $\mathrm{CDCl}_{3}$ )
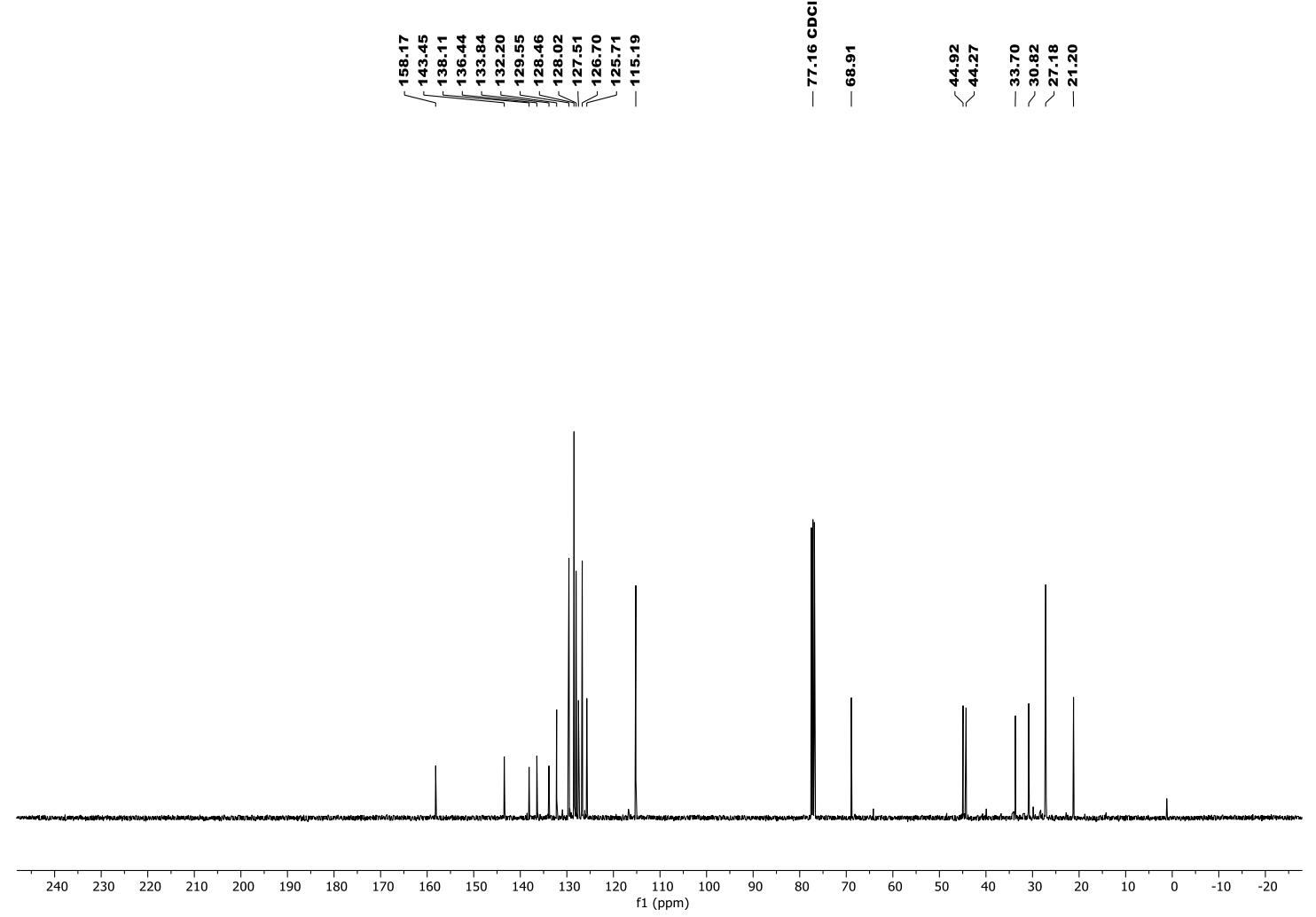
${ }^{1} \mathrm{H}$ NMR $\left(599 \mathrm{MHz}, \mathrm{CDCl}_{3}\right.$ )
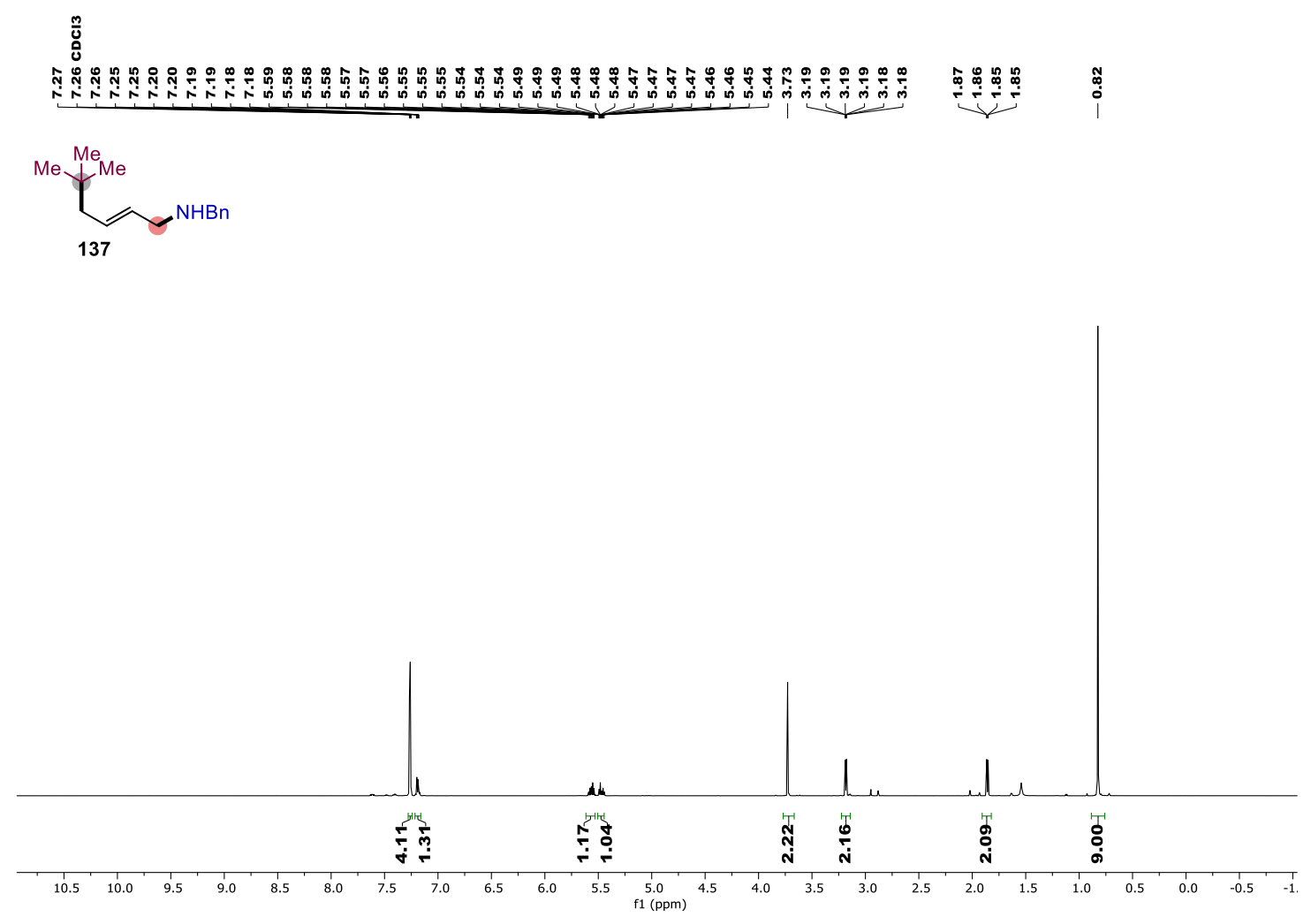

${ }^{13} \mathrm{C}$ NMR $\left(151 \mathrm{MHz}, \mathrm{CDCl}_{3}\right)$
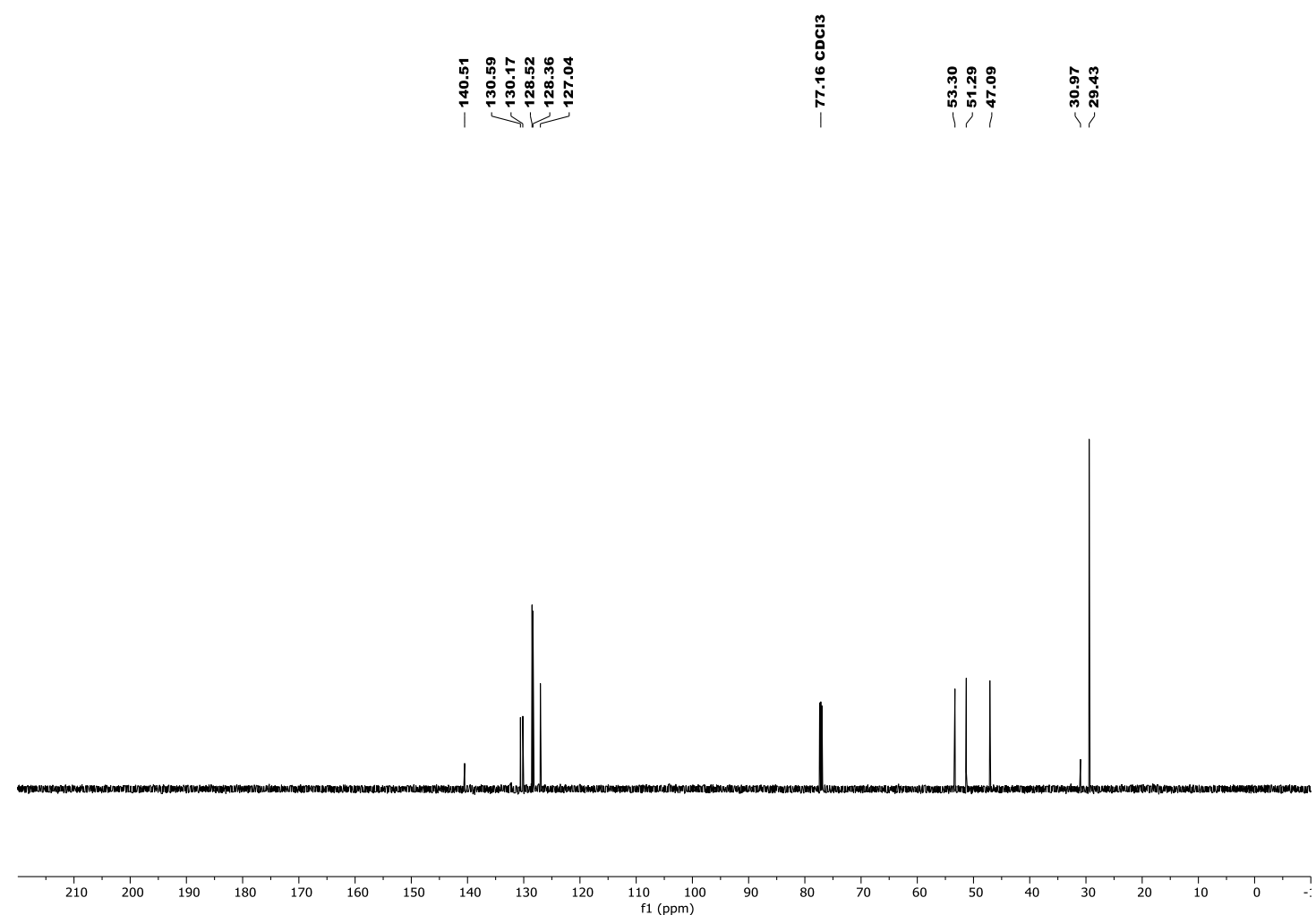
${ }^{1} \mathrm{H}$ NMR (599 MHz, $\mathrm{CDCl}_{3}$ )
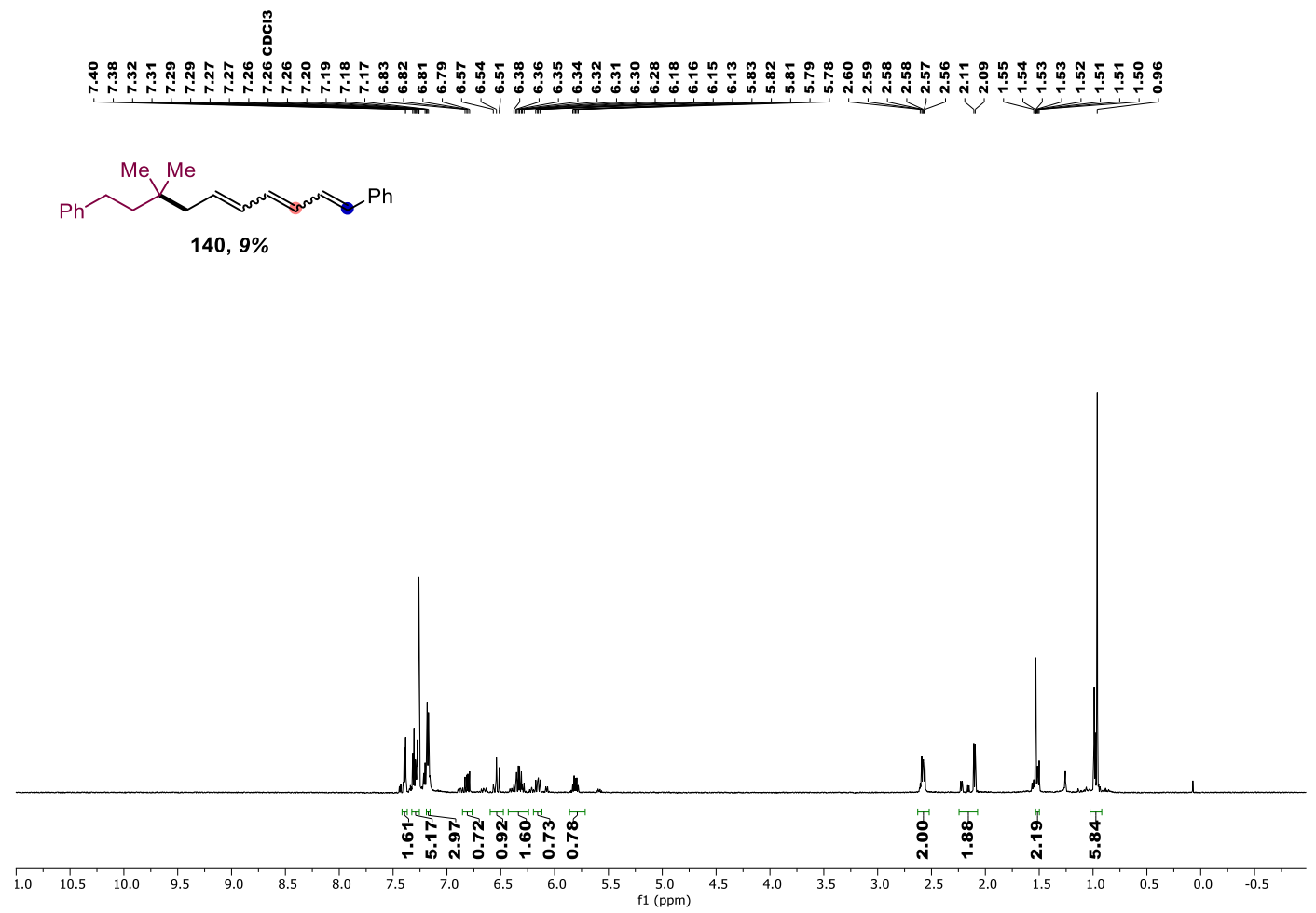

${ }^{13} \mathrm{C} \mathrm{NMR}\left(151 \mathrm{MHz}, \mathrm{CDCl}_{3}\right)$
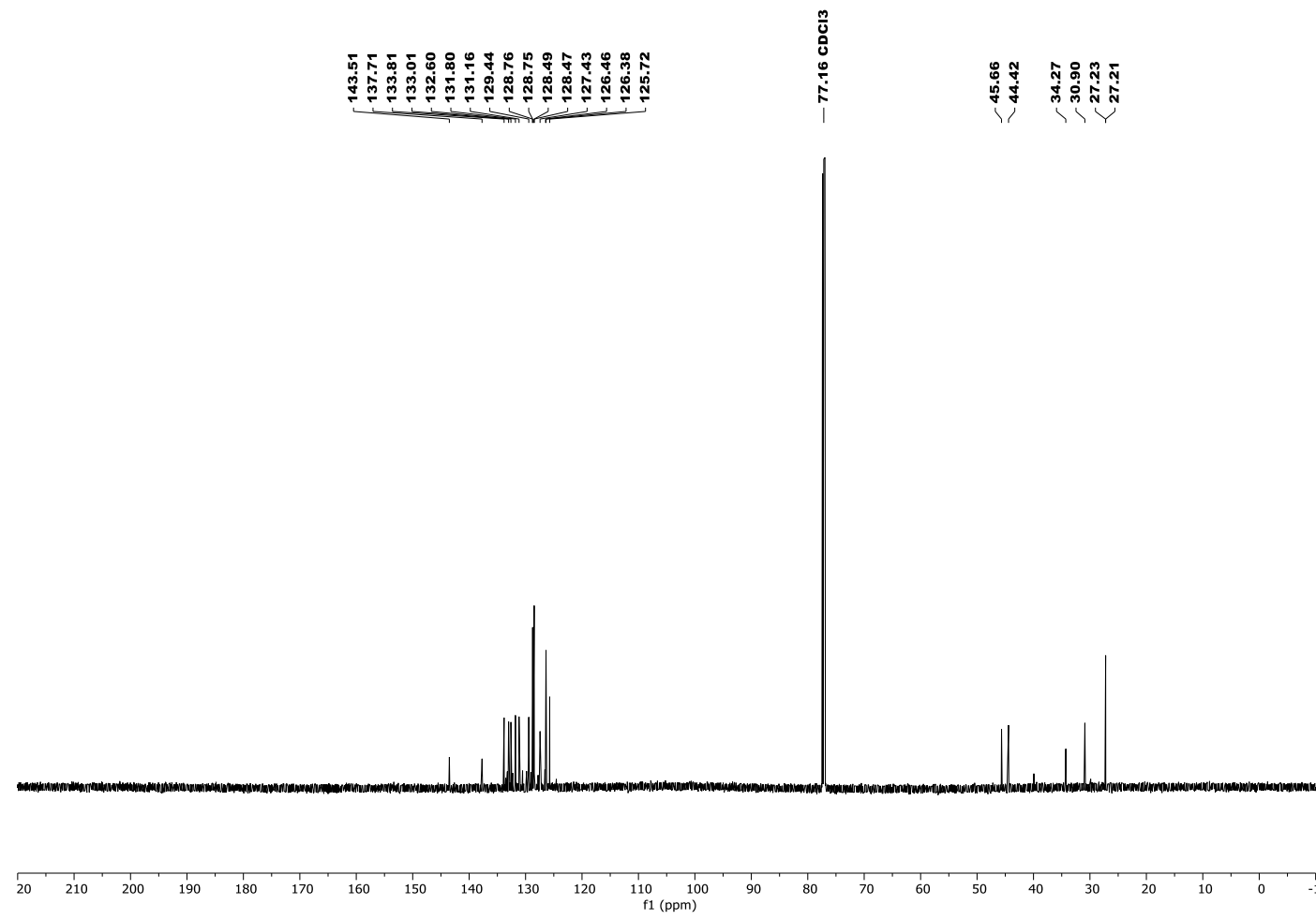


\section{References}

(1) Dudnik, A. S.; Fu, G. C. Nickel-Catalyzed Coupling Reactions of Alkyl Electrophiles, Including Unactivated Tertiary Halides, to Generate Carbon-Boron Bonds. J. Am. Chem. Soc. 2012, 134, 10693-10697.

(2) Chen, H.; Jia, X.; Yu, Y.; Qian, Q.; Gong, H. Nickel-Catalyzed Reductive Allylation of Tertiary Alkyl Halides with Allylic Carbonates. Angew. Chem. Int. Ed. 2017, 56, 1310313106.

(3) Wang, X.; Wang, S.; Xue, W.; Gong, H. Nickel-Catalyzed Reductive Coupling of Aryl Bromides with Tertiary Alkyl Halides. J. Am. Chem. Soc. 2015, 137, 11562-11565.

(4) Yu, W.; Chen, L.; Tao, J.; Wang, T.; Fu, J. Dual Nickel- and Photoredox-Catalyzed Reductive Cross-Coupling of Aryl Vinyl Halides and Unactivated Tertiary Alkyl Bromides. Chem. Commun. 2019, 55, 5918-5921.

(5) Chen, J.; Lin, J.-H.; Xiao, J.-C. Halogenation through Deoxygenation of Alcohols and Aldehydes. Org. Lett. 2018, 20, 3061-3064.

(6) Gandeepan, P.; Koeller, J.; Korvorapun, K.; Mohr, J.; Ackermann, L. Visible-LightEnabled Ruthenium-Catalyzed Meta-C-H Alkylation at Room Temperature. Angew. Chem. Int. Ed. 2019, 9820-9825.

(7) Wang, G. Z.; Shang, R.; Cheng, W. M.; Fu, Y. Irradiation-Induced Heck Reaction of Unactivated Alkyl Halides at Room Temperature. J. Am. Chem. Soc. 2017, 139, 1830718312.

(8) Tenbrink, Ruth; Maillard, Michel; Warpehoski, M. Preparation of Substituted Hydroxyethylamines as $\beta$-Secretase Inhibitors. WO 2003050073, 2003.

(9) Hurkes, N.; Ehmann, H. M. A.; List, M.; Spirk, S.; Bussiek, M.; Belaj, F.; Pietschnig, R. Silanol-Based Surfactants: Synthetic Access and Properties of an Innovative Class of Environmentally Benign Detergents. Chem. Eur. J. 2014, 20, 9330-9335.

(10) Lu, X.; Wang, Y.; Zhang, B.; Pi, J. J.; Wang, X. X.; Gong, T. J.; Xiao, B.; Fu, Y. NickelCatalyzed Defluorinative Reductive Cross-Coupling of Gem-Difluoroalkenes with Unactivated Secondary and Tertiary Alkyl Halides. J. Am. Chem. Soc. 2017, 139, 1263212637.

(11) Matsubara, H.; Tsukida, M.; Ishihara, D.; Kuniyoshi, K.; Ryu, I. A Photoirradiative Phase-Vanishing Method: Efficient Generation of $\mathrm{HBr}$ from Alkanes and Molecular Bromine and Its Use for Subsequent Radical Addition to Terminal Alkenes. Synlett 2010, 2010, 2014-2018.

(12) Coulomb, J.; Certal, V.; Larraufie, M.-H.; Ollivier, C.; Corbet, J.-P.; Mignani, G.; Fensterbank, L.; Lacôte, E.; Malacria, M. Intramolecular Homolytic Substitution of Sulfinates and Sulfinamides. Chem. Eur. J. 2009, 15, 10225-10232.

(13) Lu, X.; Wang, X.-X.; Gong, T.-J.; Pi, J.-J.; He, S.-J.; Fu, Y. Nickel-Catalyzed Allylic Defluorinative Alkylation of Trifluoromethyl Alkenes with Reductive Decarboxylation of Redox-Active Esters. Chem. Sci. 2019, 10, 809-814.

(14) Chen, Y.; Su, L.; Gong, H. Copper-Catalyzed and Indium-Mediated Methoxycarbonylation of Unactivated Alkyl Iodides with Balloon CO. Org. Lett. 2019, 21, 4689-4693.

(15) Gu, Y.; Wu, F.; Yang, J. Oxidative [3+3] Annulation of Atropaldehyde Acetals with 1,3-Bisnucleophiles: An Efficient Method of Constructing Six-Membered Aromatic Rings, Including Salicylates and Carbazoles. Adv. Synth. Catal. 2018, 360, 2727-2741. 
(16) Bochmann, M.; Wilkinson, G.; Young, G. B. Preparation and Properties of 1Adamantylmethyl and Adamantyl Complexes of Transition Metals. J. Chem. Soc. Dalt. Trans. 1980, 10, 1879-1887.

(17) Hou, X.; Ge, Z.; Wang, T.; Guo, W.; Wu, J.; Cui, J.; Lai, C.; Li, R. Synthesis and Structure-Activity Relationships of A Novel Class of Dithiocarbamic Acid Esters as Anticancer Agent. Arch. Pharm. 2011, 344, 320-332.

(18) Batory, L. A.; McInnis, C. E.; Njardarson, J. T. Copper-Catalyzed Rearrangement of Vinyl Oxiranes. J. Am. Chem. Soc. 2006, 128, 16054-16055.

(19) Yao, Z.; Li, J.; Yu, Z. Rh-Catalyzed [7 + 1] Cycloaddition of Buta-1,3Dienylcyclopropanes and CO for the Synthesis of Cyclooctadienones. Org. Lett. 2011, 13, 134-137.

(20) Lee, J.-H.; Shin, S. C.; Seo, S. H.; Seo, Y. H.; Jeong, N.; Kim, C.-W.; Kim, E. E.; Keum, G. Synthesis and in Vitro Antiproliferative Activity of C5-Benzyl Substituted 2-AminoPyrrolo[2,3- d ]Pyrimidines as Potent Hsp90 Inhibitors. Bioorg. Med. Chem. Lett. 2017, 27, 237-241.

(21) Takakura, H.; Kojima, R.; Kamiya, M.; Kobayashi, E.; Komatsu, T.; Ueno, T.; Terai, T.; Hanaoka, K.; Nagano, T.; Urano, Y. New Class of Bioluminogenic Probe Based on Bioluminescent Enzyme-Induced Electron Transfer: BioLeT. J. Am. Chem. Soc. 2015, 137, 4010-4013.

(22) Mitchell-Ryan, S.; Wang, Y.; Raghavan, S.; Ravindra, M. P.; Hales, E.; Orr, S.; Cherian, C.; Hou, Z.; Matherly, L. H.; Gangjee, A. Discovery of 5-Substituted Pyrrolo[2,3d ]Pyrimidine Antifolates as Dual-Acting Inhibitors of Glycinamide Ribonucleotide Formyltransferase and 5-Aminoimidazole-4-Carboxamide Ribonucleotide Formyltransferase in de Novo Purine Nucleotide Biosynthesis: Impl. J. Med. Chem. 2013, $56,10016-10032$.

(23) Skouroumounis, G.; Winter, B. Synthesis of 1,3,4,5-Tetrahydro-2-Benzoxepin Derivatives as Conformationally Restricted Analogues of Cyclamenaldehyde-Type Compounds and as Intermediates for Highly Odour-Active Homologues. Helv. Chim. Acta 1996, 79, 1095-1109.

(24) Mahto, P.; Rana, N. K.; Shukla, K.; Das, B. G.; Joshi, H.; Singh, V. K. Asymmetric Multifunctional Modular Organocatalysis: One-Pot Direct Strategy to Enantiopure $\alpha, \beta-$ Disubstituted $\gamma$-Butyrolactones. Org. Lett. 2019, 21, 5962-5966.

(25) Ferreira, E. M.; Stoltz, B. M. Catalytic C-H Bond Functionalization with Palladium(II): Aerobic Oxidative Annulations of Indoles. J. Am. Chem. Soc. 2003, 125, 9578-9579.

(26) Schiffner, J. A.; Wöste, T. H.; Oestreich, M. Enantioselective Fujiwara-Moritani Indole and Pyrrole Annulations Catalyzed by Chiral Palladium(II)-NicOx Complexes. Eur. J. Org. Chem. 2010, 2010, 174-182.

(27) Gauthier, R.; Mamone, M.; Paquin, J. F. Gold-Catalyzed Hydrofluorination of Internal Alkynes Using Aqueous HF. Org. Lett. 2019, 21-24.

(28) Farndon, J. J.; Young, T. A.; Bower, J. F. Stereospecific Alkene Aziridination Using a Bifunctional Amino-Reagent: An Aza-Prilezhaev Reaction. J. Am. Chem. Soc. 2018, 140, 17846-17850.

(29) Körner, C.; Starkov, P.; Sheppard, T. D. An Alternative Approach to Aldol Reactions: Gold-Catalyzed Formation of Boron Enolates from Alkynes. J. Am. Chem. Soc. 2010, 132, 5968-5969.

(30) Tortajada, A.; Ninokata, R.; Martin, R. Ni-Catalyzed Site-Selective Dicarboxylation of 1,3-Dienes with $\mathrm{CO}_{2}$. J. Am. Chem. Soc. 2018, 140, 2050-2053. 
(31) Heckenbichler, K.; Schweiger, A.; Brandner, L. A.; Binter, A.; Toplak, M.; Macheroux, P.; Gruber, K.; Breinbauer, R. Asymmetric Reductive Carbocyclization Using Engineered Ene Reductases. Angew. Chem. Int. Ed. 2018, 57, 7240-7244.

(32) Ryland, B. L.; McCann, S. D.; Brunold, T. C.; Stahl, S. S. Mechanism of Alcohol Oxidation Mediated by Copper(II) and Nitroxyl Radicals. J. Am. Chem. Soc. 2014, 136, 12166-12173.

(33) Um, C.; Chemler, S. R. Synthesis of 2-Aryl- and 2-Vinylpyrrolidines via CopperCatalyzed Coupling of Styrenes and Dienes with Potassium $\beta$-Aminoethyl Trifluoroborates. Org. Lett. 2016, 18, 2515-2518.

(34) Koy, M.; Bellotti, P.; Katzenburg, F.; Daniliuc, C.; Glorius, F. Synthesis of All-Carbon Quaternary Centers by Palladium-Catalyzed Olefin Dicarbofunctionalization. Angew. Chem. Int. Ed. 2019, 18, 463-477.

(35) Arredondo, Y.; Moreno-Mañas, M.; Pleixats, R. Non-Catalyzed C-Alkylation of Phenols With Cyclic Secondary Alkyl Bromides. Synth. Commun. 1996, 26, 3885-3895.

(36) Lebrasseur, N.; Larrosa, I. Room Temperature and Phosphine Free Palladium Catalyzed Direct C-2 Arylation of Indoles. J. Am. Chem. Soc. 2008, 130, 2926-2927.

(37) Das, D.; Bhutia, Z. T.; Chatterjee, A.; Banerjee, M. Mechanochemical Pd(II)-Catalyzed Direct and C-2-Selective Arylation of Indoles. J. Org. Chem. 2019, 84, 10764-10774.

(38) Zhu, L.; Guo, P.; Li, G.; Lan, J.; Xie, R.; You, J. Simple Copper Salt-Catalyzed NArylation of Nitrogen-Containing Heterocycles with Aryl and Heteroaryl Halides. J. Org. Chem. 2007, 72, 8535-8538.

(39) Lanier, M.; Cole, D. C.; Istratiy, Y.; Klein, M. G.; Schwartz, P. A.; Tjhen, R.; Jennings, A.; Hixon, M. S. Repurposing Suzuki Coupling Reagents as a Directed Fragment Library Targeting Serine Hydrolases and Related Enzymes. J. Med. Chem. 2017, 60, 5209-5215.

(40) Xia, S.; Gan, L.; Wang, K.; Li, Z.; Ma, D. Copper-Catalyzed Hydroxylation of (Hetero)Aryl Halides under Mild Conditions. J. Am. Chem. Soc. 2016, 138, 13493-13496.

(41) Ward, J. S.; Lynam, J. M.; Moir, J. W. B.; Sanin, D. E.; Mountford, A. P.; Fairlamb, I. J. S. A Therapeutically Viable Photo-Activated Manganese-Based CO-Releasing Molecule (Photo-CO-RM). Dalt. Trans. 2012, 41, 10514.

(42) Sharma, R.; Patel, N.; Vishwakarma, R. A.; Bharatam, P. V.; Bharate, S. B. Metal-Free Oxidative Cyclization of Acetophenones with Diamines: A Facile Access to Phenylpyridines. Chem. Commun. 2016, 52, 1009-1012.

(43) Qiao, J. X.; Wang, T. C.; Hu, C.; Li, J.; Wexler, R. R.; Lam, P. Y. S. Transformation of Anionically Activated Trifluoromethyl Groups to Heterocycles under Mild Aqueous Conditions. Org. Lett. 2011, 13, 1804-1807.

(44) Huang, L.; Su, T.; Shan, W.; Luo, Z.; Sun, Y.; He, F.; Li, X. Inhibition of Cholinesterase Activity and Amyloid Aggregation by Berberine-Phenyl-Benzoheterocyclic and TacrinePhenyl-Benzoheterocyclic Hybrids. Bioorg. Med. Chem. 2012, 20, 3038-3048.

(45) Yang, C.; Li, X.; Wei, J.; Zhu, F.; Gang, F.; Wei, S.; Zhao, Y.; Zhang, J.; Wu, W. Synthesis and Insecticidal Activity in Vitro and Vivo of Novel Benzenesulfonyl Derivatives Based on Potent Target Subunit H of V-ATPase. Bioorg. Med. Chem. Lett. 2018, 28, 3164-3167.

(46) Mulina, O. M.; Pirgach, D. A.; Nikishin, G. I.; Terent'ev, A. O. Switching of Sulfonylation Selectivity by Nature of Solvent and Temperature: The Reaction of $\beta$ Dicarbonyl Compounds with Sodium Sulfinates under the Action of Iron-Based Oxidants. Eur. J. Org. Chem. 2019, 2019, 4179-4188.

(47) Guzzo, P.; Henderson, A.; Isherwod, M.; Lee, C.; Ghosh, A.; Zhao, H. Benzofuro[3,2-c] Pyridines and Related Analogs as Serotonin Sub-type 6(5-HT6) Modulators for The Treatment of Obesity. US201213352864 20120118, 2012. 
(48) Cremlyn, R. J. W.; Hornby, R. Sulphonohydrazides and Related Compounds. Part XI. Some Substituted Aryl Ether Sulphonhydrazides. J. Chem. Soc. C 1969, 10, 1341.

(49) Madabhushi, S.; Jillella, R.; Sriramoju, V.; Singh, R. Oxyhalogenation of Thiols and Disulfides into Sulfonyl Chlorides/Bromides Using Oxone-KX (X $=\mathrm{Cl}$ or $\mathrm{Br}$ ) in Water. Green Chem. 2014, 16, 3125-3131.

(50) Roemmele, R. C.; Rapoport, H. Removal of N-Arylsulfonyl Groups from Hydroxy .Alpha.-Amino Acids. J. Org. Chem. 1988, 53, 2367-2371.

(51) Jereb, M.; Hribernik, L. Conversion of Thiols into Sulfonyl Halogenides under Aerobic and Metal-Free Conditions. Green Chem. 2017, 19, 2286-2295.

(52) Adhikari, N.; Halder, A. K.; Mallick, S.; Saha, A.; Saha, K. D.; Jha, T. Robust Design of Some Selective Matrix Metalloproteinase-2 Inhibitors over Matrix Metalloproteinase-9 through in Silico/Fragment-Based Lead Identification and de Novo Lead Modification: Syntheses and Biological Assays. Bioorganic Med. Chem. 2016, 24, 4291-4309.

(53) Cesura, A.; Röver, S. Use of N-(4-Aryl-Thiazol-2-Yl)-Sulfonamides. US19970874050 $19970612,1999$.

(54) Waser, J.; Gaspar, B.; Nambu, H.; Carreira, E. M. Hydrazines and Azides via the Metal-Catalyzed Hydrohydrazination and Hydroazidation of Olefins. J. Am. Chem. Soc. 2006, 128, 11693-11712.

(55) Meyer, A. U.; Straková, K.; Slanina, T.; König, B. Eosin Y (EY) Photoredox-Catalyzed Sulfonylation of Alkenes: Scope and Mechanism. Chem. Eur. J. 2016, 22, 8694-8699.

(56) Hwang, J. S.; Tsonis, C. P. ESR Spin Radical Trapping in Dehydrohalogenation of Alkyl Halides Catalyzed by Molybdenum Hexacarbonyl. J. Mol. Catal. 1988, 44, 259-267.

(57) Novakov, C. P.; Feierman, D.; Cederbaum, A. I.; Stoyanovsky, D. A. An ESR and HPLCEC Assay for the Detection of Alkyl Radicals. Chem. Res. Toxicol. 2001, 14, 1239-1246.

(58) van Haaren, R. J.; Oevering, H.; Coussens, B. B.; van Strijdonck, G. P. F.; Reek, J. N. H.; Kamer, P. C. J.; van Leeuwen, P. W. N. M. On the Influence of the Bite Angle of Bidentate Phosphane Ligands on TheRegioselectivity in Allylic Alkylation. Eur. J. Inorg. Chem. 1999, 1999, 1237-1241.

(59) Benn, R.; Jolly, P. W.; Mynott, R.; Raspel, B.; Schenker, G.; Schick, K. P.; Schroth, G. Intermediates in the Palladium-Catalyzed Reactions of 1,3-Dienes. 2. Preparation and Structure of (.Eta.1,.Eta.3-Octadienediyl)Palladium Complexes. Organometallics 1985, 4, 1945-1953.

(60) Jackstell, R.; Grotevendt, A.; Andreu, M. G.; Beller, M. A Practical PalladiumCatalyzed Telomerization for the Synthesis of Functionalized Alcohols. Org. Process Res. Dev. 2009, 13, 349-353.

(61) Neese, F. The ORCA Program System. Wiley Interdiscip. Rev. Comput. Mol. Sci. 2012, $2,73-78$.

(62) Zhao, Y.; Truhlar, D. G. The M06 Suite of Density Functionals for Main Group Thermochemistry, Thermochemical Kinetics, Noncovalent Interactions, Excited States, and Transition Elements: Two New Functionals and Systematic Testing of Four M06Class Functionals and 12 Other Function. Theor. Chem. Acc. 2008, 120, 215-241.

(63) Schäfer, A.; Horn, H.; Ahlrichs, R. Fully Optimized Contracted Gaussian Basis Sets for Atoms Li to Kr. J. Chem. Phys. 1992, 97, 2571-2577.

(64) Lenthe, E. van; Baerends, E. J.; Snijders, J. G. Relativistic Regular Two-component Hamiltonians. J. Chem. Phys. 1993, 99, 4597-4610.

(65) Barone, V.; Cossi, M. Quantum Calculation of Molecular Energies and Energy Gradients in Solution by a Conductor Solvent Model. J. Phys. Chem. A 1998, 102, 19952001. 
(66) Cossi, M.; Rega, N.; Scalmani, G.; Barone, V. Energies, Structures, and Electronic Properties of Molecules in Solution with the C-PCM Solvation Model. J. Comput. Chem. 2003, 24, 669-681.

(67) Jmol: An Open-Source Java Viewer for Chemical Structures in 3D. Http://Www.Jmol.Org/.

(68) Roth, H.; Romero, N.; Nicewicz, D. Experimental and Calculated Electrochemical Potentials of Common Organic Molecules for Applications to Single-Electron Redox Chemistry. Synlett 2015, 27, 714-723.

(69) Isse, A. A.; Gennaro, A. Absolute Potential of the Standard Hydrogen Electrode and the Problem of Interconversion of Potentials in Different Solvents. J. Phys. Chem. B 2010, 114, 7894-7899.

(70) Pitzer, L.; Schäfers, F.; Glorius, F. Rapid Assessment of the Reaction-ConditionBased Sensitivity of Chemical Transformations. Angew. Chem. Int. Ed. 2019, 58, 85728576. 